\title{
Injury Experience in Stone Mining, 1991
}

U.S. Department of Labor

Robert B. Reich, Secretary

Mine Safety and Health Administration

Edward C. Hugler, Acting Assistant Secretary

Information Report

IR 1210

1993
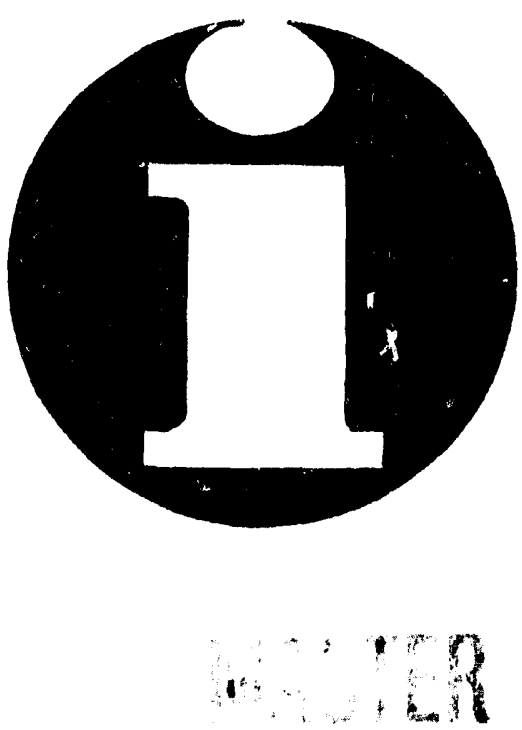
This publication has been reviewed and approved for distribution to the mining public by the office of the Assistant secretary for Mine Safety and Health. 
Introduction-

Scope of statistics-

Definition of terms-

Table reference guide (operalor data)

\section{ILLUSTRATIONS}

Figure 1. Scheduled charges in lost workdays for loss or loss of use involving hand or foot- 10

Figure 2. Quarterly mine employment and coal production report form--

Figure 3. Mine accilent, injury, and illness report form--.-- 12

TABLES FOR STONE OPERATIONS IN THE UNITED STATES, 1991 (OPERATOR DATA)

1. General statistics on injury experience and worktime--- - -

2. Injury experience by degree and worktime for work location---

3. Number of injuries, lost workdays, and average severity by degree for work location- 15

4. Injury experience by degree and worktime for work location and employment size group- 16

5. Number of injuries, lost workdays, and average severity by degree for work location and employment size group---.--

6. Injury experience by degree and worktime for type of mineral, work location, and state-

7. Number of injuries, lost workdays, and average severity by degree for type of mineral, work location, and state----

8. Number of injuries, incidence rates, and severity measures by degree for work location and part of body injured-..--

9. Number of injuries, lost workdays, and average severity by degree for work location and part of body injured------

10. Number of injuries, incidence rates, and severity measures by degree for work location and nature of injury--

11. Number of injuries, lost workdays, and average severity by degree for work location and nature of injury-.......

12. Number of injuries, lost workdays, and average severity by degree for part of body injured and nature of injury----

13. Number of injuries by accident classification and degree for work location and state-..-

14. Number of lost workdays by accident classification and degree for work location and state--

15. Number of injuries by accident classification and degree for work location and type of mineral-

16. Number of lost workdays by accident classification and degree of injury for work Jocation and type of mineral-----

17. Number of injuries, lost workdays, and average severity by degree for work location and occupation at time of injury 
TABLES FOR STONE OPERATIONS IN THE UNITED STATES, 1991

(OPERATOR DATA)--Continued

Page

18. Number of injuries, lost workdays, and average severity by degree for work location and accident classification---- 386

19. Number of injuries, lost workdays, and average severity by degree for accident classification and part of body injured- of injuries, lost workdays, and average severity

20. Number of injuries, lost. workdays, and average severity
by degree for accident classification and nature of injury-... 402

21. Occurrences reported to MSHA as occupational ilinesses--.---- 408

22. Related noncoal mining summary reference tabulation, number of injuries, incidence rates, average number of workers, and employee-hours by work location and mineral industry---

23. Related coal mining summary reference tabulation, number of injuries, incidence rates, average number of workers, employee-hours, and production by type of coal mined and work location--

TABLES FOR STONE OPERATIONS IN THE UNITED STATES, 1991 (CONTRACTOR DATA)

Table reference guide (contractor data)

1. General statistics on injury experience and severity--.----

3. Number of injuries, lost workdays, and average severity by degree for work location-

7. Number of injuries, lost workdays, and average severity by degree for type of mineral, work location, and state----

9. Number of injuries, lost workdays, and average severity by degree for work location and sart of body injured--.---

11. Number of injuries, lost workdays, and average severity by degree for work location and nature of injury-.-.-.--

12. Number of injuries, lost workdays, and average severity by degree for part of body injured and nature of injury----

13. Number of injuries by accident classification and degree for work location and state-...

14. Number of lost workdays by accident classification and degree of injury for work location and state-.....

15. Number of injuries by accident classification and degree for work location and type of mineral-..-

16. Number of lost workdays by accident classification and degree of injury for work location and type of mineral-----
umber of injuries, lost workdays, and average severity

17. Number of injuries, lost workdays, and average severity
by degree for work location and occupation at time of
injury- Number of injuries, lost workdays, and average severity
by degree for work location and accident classification
18. Number of injuries, lost workdays, and average severity
by degree for accident classification and part of body

17. Number of injuries, lost workdays, and average severity
by degree for work location and occupation at time of
injury- Number of injuries, lost workdays, and average severity
by degree for work location and accident classification
18. Number of injuries, lost workdays, and average severity
by degree for accident classification and part of body

17. Number of injuries, lost workdays, and average severity
by degree for work location and occupation at time of
injury- Number of injuries, lost workdays, and average severity
by degree for work location and accident classification----
18. Number of injuries, lost workdays, and average severity
by degree for accident classification and part of body

17. Number of injuries, lost workdays, and average severity
by degree for work location and occupation at time of
injury- Number of injuries, lost workdays, and average severity
by degree for work location and accident classification
18. Number of injuries, lost workdays, and average severity
by degree for accident classification and part of body

17. Number of injuries, lost workdays, and average severity
by degree for work location and occupation at time of
injury- Number of injuries, lost workdays, and average severity
by degree for work location and accident classification
18. Number of injuries, lost workdays, and average severity
by degree for accident classification and part of body injured 
TABLES FOR STONE OPERATIONS IN THE UNITED STATES, 1991 CONTRACTOR DATA)--COntinued

Page

20. Number of injuries, lost workdays, and average severity by degree for accident classification and nature of injury--

21. Occurrences reported to MSHA as occupational illnesses------- 514

22. Related noncoal mining summary reference tabulation, number of injuries, incidence rates, average number of workers, and employee-hours by work location and mineral industry--- 515

23. Related coal mining summary reference tabulation, number of injuries, incidence rates, average number of workers, employee-hours, and production by type of coal mined and work location-- 
INJURY EXPERIENCE IN STONE MINING, 1991

by

Staff, Division of Mining Information systems Denver Safety and Health Technology Center

Technical support, Denver, Colorado

\begin{abstract}
This Mine Safety and Health Administration (MSHA) informational report reviews in detail the occupational injury and illness experience of stone mining in the United States for 1991. Data reported by operators of mining establishments concerning work injuries are summarized by work location, accident classification, part of body injured, nature of injury, occupation, and principal type of mineral. Related information on employment, worktime, and operating activity also is presented. Data reported by independent contractors performing certain work at mining locations are depicted separately in this report. For ease of comparison with other metal and nonmetallic mineral mining industries and with coal mining, summary reference tabulations are included at the end of both the operator and the contractor sections of this report.
\end{abstract}

\title{
INTRODUCTION
}

Data used in compiling this report were reported by operators of stone mines and preparation plants on a mandatory basis as required under the Fedexal Mine safety and Health Act of 1977 , Public Law 91-173 as amended by Law 95-164. Since January 1, 1978, operators of mines or preparation plants or both which are subject to the Act have been required under $30 \mathrm{CFR}$, Part 50, to submit reports of injuries, occupational illnesses, and related data.

Only data reported by the operators and contractors themselves are utilized in this report. No estimates are made of employment, employee-hours, or injuries for nonrespondent operations except as necessary to meet statistical processing requirements by providing minimum employment data for reported unmatched injury data.

Beginning with calendar year 1978, statistical measures for injury experience in mining were changed from the measures used from 1930 through 1977. The statistical measures for injury experience adopted by MSHA in 1978 compare closely with the measures used in the office of Occupational safety and Health Statistics, Bureau of Labor Statistics, U. S. Department of Labor. Therefore, beginning with 1978 data, the mining industry can be compared on a standard basis with other United States industries reporting under the Occupational safety and Health Act of 1970. 
Occurrences identified as occupational illnesses by mine operators were reported to MSHA and were processed in accordance with 30 CFR PART 50.20-6. The reported illnesses are summarized in seven categori ss for each work location, type of mineral, and state. Incidence rates and severity measures are not computed for occupa-tional illnesses.

Because of the modifications in reporting and processing procedures that became effective January 1, 1978, injury rates as currently computed are not precisely comparable to those of previous years, excepi for fatality rates in which the "incidence rate" (the term used after 1977) is one-fifth of the "frequency rate" (the term used prior to 1978) for otherwise similar groupings of fatal injuries and worktimes. Because of this disparity, MSHA annual publications concerning injuries, illnesses, and operating activity will not contain data for years prior to 1978 .

\section{SCOPE OF STATISTICS}

The statistical data in this report cover the work experiense of all personnel engaged in mine exploration, development, production, maintenance, repair, and construction work, including supervisory and technical personnel, working partners, and onsite officeworkers. The classification of industries is in close general agreement with the standard Industrial Classification. If

It should be noted that amendments to legislation that funded MSHA and other government programs from December 16, 1981, through July 18, 1982, prohibited MSHA from administering or enforcing the Federal Mine Safety and Health Act of 1977 (Mine Act) or MSHA standards and regulations with respect to any person engaged in the surface mining or milling of stone, clay, feldspar, colloidal phosphate, or sand and gravel. They also provided that MSHA could not administer or enforce the Mine Act or MSHA standards and regulations on any state or political subdivision thereof. Further, MSHA could not administer or enforce the Mine Act or MSHA standards or regulations with respect to any independent construction contractor who was engaged by an operator for the construction, repair or alteration of structures, facilities, utilities or private ways or roads located on (or appurtenant to) the surface areas of any coal or other mine, and whose employees worked in a specifically demarcated area, separate from actual mining or extraction activities. No data on exempted activities are included for that period of time in Table 1 of this publication.

MSHA issued guidelines which additionally reduced the reporting requirements of independent contractors. Reporting of employment, hours worked, and injury/illness data by independent contractors is limited to the following types of work performed: mine development, including shaft and slope sinking; construction or reconstruction of mine facilities, including building or rebuilding preparation plants

1. Executive Office of the President, office of Management and Budget. Standard Industrial Classification Manual. 1972 revision. 
and mining equipment, and building additions to existing facilities: demclition of mine facilities; construction of dams; excavation or earthmoving activities involving mobile equipment; equipment irstallation, such as crushers and mills; equipment service or repair done underground or in the pit for a period exceeding five consecutive days at a particular mine; material handling within mine property, including haulage of coal, ore, refuse, etc., unless for the sole purpose of direct removal from or delivery to mine property; drilling and blasting. Also, data collected for employment and hours worked in those activities reflect the contractor's total metallic/nonmetallic work locations and total coal work locations and not the individual mine sites. For more detailed information concerning these changes, see the guidelines accompanying MSHA Policy Memoranda Nos. 82-6A MM and 81-35A C.

\section{DEFINITION OF TERMS}

Cccupational Injury.--An occupational injury is an injury to a mine worker which occurs at a mine and for which medical treatment is administered, or which results in death or loss of consciousness, restriction of work or motion, inability to perform all job duties on any day after an injury, lost workdays, temporary assignment to other duties, transfer to another job, or termination. The injury usually results from a recognizable single incident. For example, an explosion of a battery which splattered a worker with sulfuric acid would be a single incident and the worker would be considered to have suffered an injury. Another example would be an injury where a worker was overcome by hydrogen sulfide gas released from an exploding vessel.

occupational illness.--An occupational illness is an illness or disease of a mine worker which may have resulted from work at a mine or for which an award of compensation is made. To be classified as an occupational illness, the disability must result from repeated exposure to the condition or substance which caused the disability. A classic illness example is a pneumoconiosis disability which may have been induced by repeated exposure to the causative dusty condition. Dermatitis resulting from repeated exposure to lime dust or other material in the work environment also should be reported as an illness. In cases where the time of onset of illness is in doubt, the day of diagnosis of illness will be considered as the first day of illness.

Number of Operations and Associated Work Locations.--Number of operations is the number of individual respondents with a unique Federal identification number that have reported worktime data. The count and kind of operation are based on the lowest numbered (primary) subunit on employment report form 7000-2 (see figure 2, p. 11) that contairs worktime data. An associated work location is a subunit reported with a primary subunit, e.g., an "office" is almost always an associated work location. Independent contractors working on mine property performing the types of work described under "Scope of statistics" are assigned an identification number, report to MSHA, but are not included in the count of operations. contractor data are depicted separately in this report. 
Worktime.--Worktime includes the number of workers and the number of employee-hours worked. "Average number of workers" is a summary of the average number of persons working at individual establishments during calendar quarters of active operations. "Employee-hours" is a summary of employee-hours reported.

Employment Size Group.--Reporting operations are classified into size groups according to the average number of workers in specified work locations.

Degree of Injury.--Degree of injury indicates the seriousness of injuries. FATAL injuries are those occurrences resulting in death, NFDL (NonFatal with Days Lost) injuries are nonfatal occurrences that result in days away from work, statutory days charged, or days of restricted work activity. NDL (No Days Lost) injuries are occurrences having no lost workdays; that is, nonfatal injury occurrences resulting only in temporary loss of consciousness or medical treatment other than first aid.

A permanent total disability is caused by any work injury or illness other than death that permanently and totally incapacitates a worker from following any gainful occupation, or that results in the loss, or the complete loss of use, of both or any combination of: hands, arms, legs, feet, or eyes.

A permanent partial disability is caused by any work injury or illness other than death or permanent total disability that results in the loss, or the complete loss of use, of any member or part of a member of the body, or any permanent impairment of functions of the body or part thereof, regardless of any preexisting disability of the injured member or impaired body function.

Incidence Rate.--Incidence rate is defined as the number of injuries per 200,000 employee-hours, rounded to two decimal places. The highest rate given is 99.99 . If an incidence rate is greater than 99.99, then 99.99 is printed. If no data were reported, then a hyphen is printed. If a computation is less than 0.01 after rounding, then an asterisk inside parentheses (*) is printed. The following is the standard incidence rate formula:

$$
I R=\frac{\text { Number of injuries } \times 200,000}{\text { Number of employee-hours }}
$$

Lost Workdays (LWD). --Lost workdays consist of days away from work (DAW) and days of restricted work activity (DRA), or statutory days charged as prescribed from a table of standard charges (see p. 7), e.g., 6,000 days for a fatality or permanent total disability.

Severity Measure.--severity measure is the number of lost workdays per 200,000 employee-hours, rounded to whole numbers. The highest rate given is 99,999 . If the computed rate is greater than 99,999, then 99,999 is printed. If no data were reported, then a 
hyphen is printed. If the computation results in a zero after rounding, then an asterisk inside parentheses (*) is printed. The following is the standard severity measure formula:

$$
\mathrm{SM}=\frac{\text { Number of lost workdays } \times 200,000}{\text { Number of employee-hours }}
$$

Average Severity.--Average severity is the average number of lost workdays per injury and is computed only for injuries classified as FATAL or NFDL. Lost workdays are selected on the same basis as that for computing the severity measure. The following is the standard average severity formula:

$$
\text { AS }=\frac{\text { Number of lost workdays }}{\text { Number of injuries contributing the lost workdays }}
$$

Type of Mineral. - The type of mineral is determined from the standard Industrial Classification (S.I.C.) code assigned to an operation based on information provided by the mine operator. Data for all work locations in the operation are credited to the assigned S.I.C.

Classification of Accident.--Accident classification identifies circumstances which contributed most directly to the resulting accident. The 21 classifications in the following list are designed to categorize all accidents, whether injury-producing or not.

Electrical.--Accidents in which the electric current is most directly responsible for the resulting accident.

Entrapment.--Accidents involving entrapment of persons.

Exploding Vessels Under Pressure.--Accidents involved with bursting of air hoses, air tanks, hydraulic lines, hydraulic hoses, stand pipes, etc., due to internal pressure.

Explosives and Breaking Agents.--Accidents involving the detonation of manufactured explosives; includes Airdox and cardox.

Falling, Rolling, or sliding Rock or Material of Any kind.--Accidents caused directly by falling material other than material from the roof or face. Or, if material was set in motion by machinery, by haulage, by handtools, or while being handled or disturbed, etc., the force that set the material in motion determines the classification. For example, where a rock was pushed over a highwall by a bulldozer and the rock hit another rock which hit and injured a worker--the accident is classified as machinery. Machinery (a bulldozer) most directly caused the resulting accident.

Fall of Face, Rib, Pillar, Side, or Highwall (from in place).--Accidents in this classification include falls of material while barring down or placing props, also pressure bumps and bursts. Not included are accidents in which the motion of machinery or haulage equipment caused the fall either directly or by knocking out support. 
Fall of Roof, Back, or Brow (from in place).--Underground only - Accidents which include falls while barring down or placing props, also pressure bumps and bursts. Not included are accidents in which the motion of machinery or haulage equipment caused the fall either directly or by knocking out support.

Fire.-Accidents related to uncontrolled burning of material or mineral in the mine environment. Not included are fires initiated by electricity or by ignition or explosion of gas or dust.

Handling Material.--Accidents related to handling packaged or loose material while lifting, pulling, pushing, or shoveling.

Handtools.--Accidents related to nonpowered tools.

Nonpowered Haulage.--Accidents related to the motion of nonpowered haulage equipment. Included are accidents involving wheelbarrows, manually pushed mine cars, timber trucks, etc.

Powered Haulage.--Accidents related to the motion of powered haulage equipment. Included are accidents involving conveyors, front-end loaders, forklifts, shuttle cars, loadhaul-dump units, locomotives, railroad cars, haulage trucks, pickups, automobiles, and personnel carriers.

Hoisting.--Accidents involving cages, skips, ore buckets, and elevators. The accident results from the action, motion, or failure of the hoisting equipment or mechanism. Inclided are equipment such as cranes and derricks only when used in shaft sinking; also suspended work platforms in shafts. Not included is equipment such as chain hoists, come-alongs, and winches.

Ignition or Explosion of Gas or Dust.--Accidents resulting as a consequence of the ignition or explosion of gas or dust.

Impoundment.--Accidents caused by an unstable condition or failure of an impoundment, refuse pile, or culm bank requiring emergency preventative action or evacuation of an area.

Inundation.--Accidents caused by inundation of a surface or underground mine by a liquid (or semi-solid) or a gas.

Machinery.--Accidents related to the motion of machinery. Included are all electric and air-powered tools and mining machinery such as drills, tuggers, winches, slushers, draglines, power shovels, loaders, and compressors. 
Slip or Fall of Person (from an elevation or on the same level).--Accidents include slips or falls while getting on or off machinery and haulage equipment which is not moving, and slips or falls while servicing or repairing equipment or machinery.

Stepping or Kneeling on object. Accidents are classified in this category only where the object stepped or kneeled on contributed most directly to the accident.

Striking or Bumping.--This classification is restricted to those accidents in which an individual, while moving about, strikes or bumps an object but is not handling material, using handtools, or operating equipment.

other.--Accidents not elsewhere classified.

Abbreviations.--Space limitations in column headings and stub entries of standard-format tabulations require occasional adaptation of terminology. In this report PLNTS should be interpreted as "plants."

Statutory Days Charged.--Some injuries or illnesses are of such a degree of severity that a standard time charge of lost workdays has been adopted by MSHA as follows:

Disability

Iost Workdays Charged

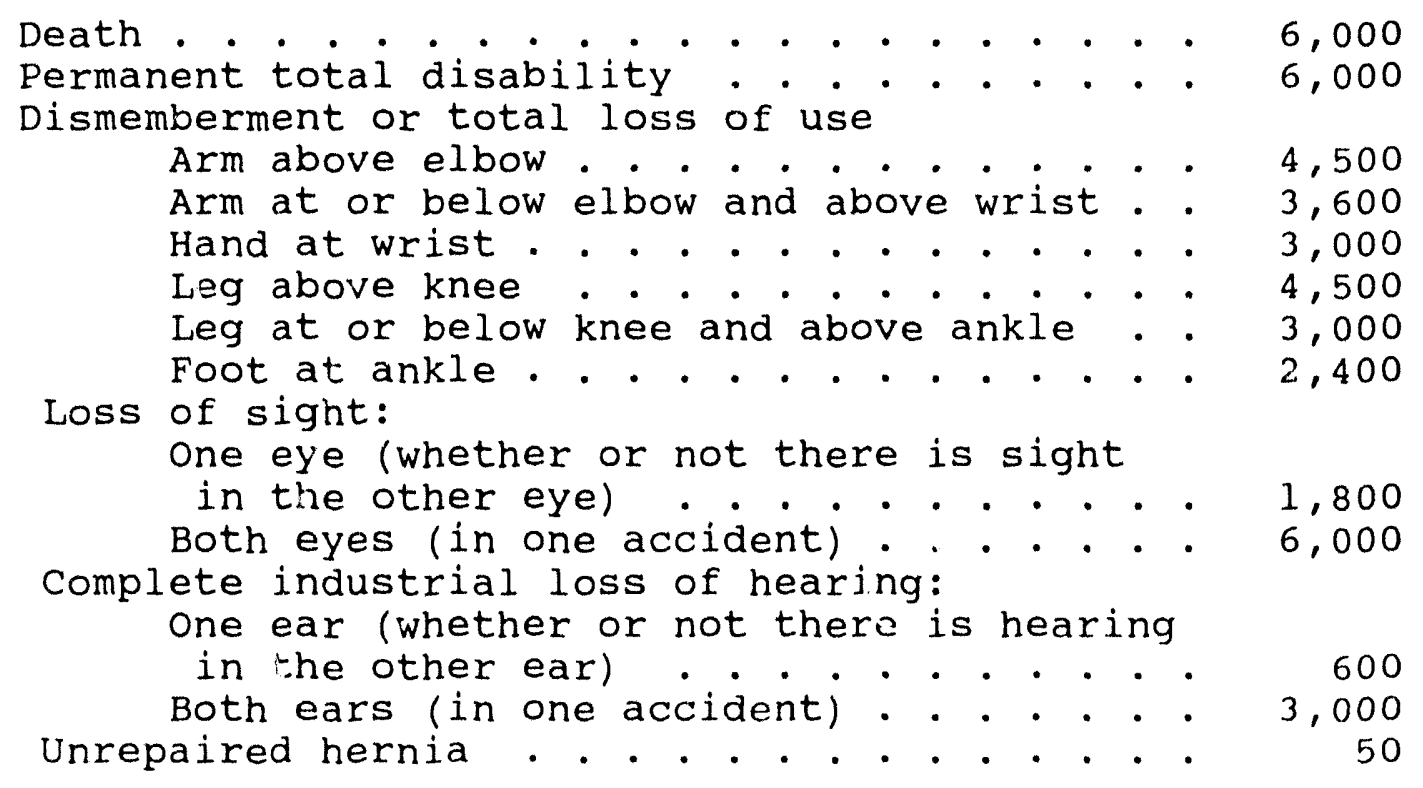

1) Not more than 6,000 lost workdays will be charged in a single accident that results in multiple injuries to a mine worker. (See definition of permanent total disability on page 4.) 


\section{TABLE REFERENCE GUIDE (OPERATOR DATA)}

The following reference guide facilitates locating particular data elements contained in the annual statistical tables for operators. A comparable guide precedes the contractor section of this report. The number appearing in each cell indicates the table number in which that particular element may be found. All elements listed under the stub headings appear as vertical headings in the tables. Elements listed under column headings appear as horizontal headings. The only exception to this is table 1, in which the headings have been reversed.

To use this guide, read down or across the elements listed in both the stub and column headings to determine which combinations are of interest. For example, incidence rates by work location appear in six different tables, numbers $2,4,6,8,10,22$, and 23 . However, only one, table 10, provides a further division by nature of injury as well. The occupation of the injured is found in table 17 and, as seen in the column headings, provides the number of injuries, lost workdays, and average severity. 
REFERENCE GUIDE TO STONE STATISTICAL TABLES (OPERATOR DATA)

\begin{tabular}{|c|c|c|c|c|c|c|c|c|c|c|c|c|c|}
\hline 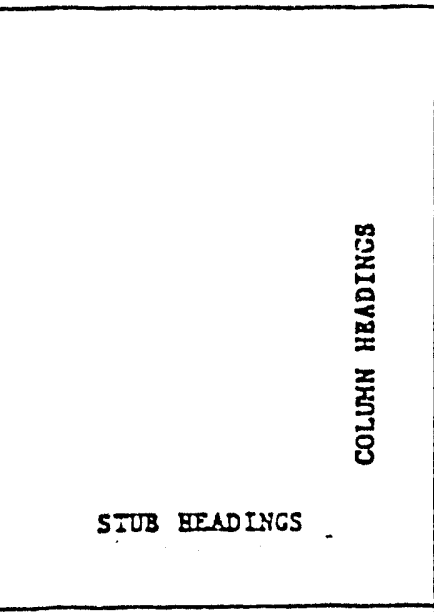 & 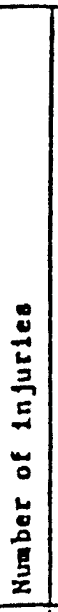 & 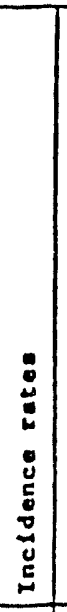 & 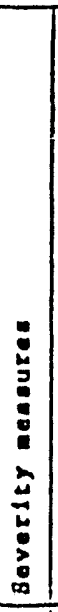 & 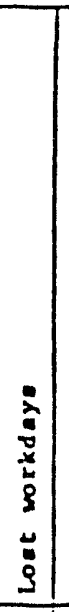 & $\begin{array}{l}\vdots \\
\vdots \\
\vdots \\
\vdots \\
\vdots \\
\vdots \\
\vdots \\
\vdots\end{array}$ & 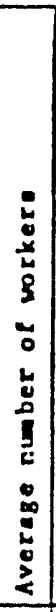 & $\begin{array}{c}0 \\
\vdots \\
3 \\
\vdots \\
5 \\
\vdots \\
\vdots \\
\vdots \\
\vdots \\
0 \\
\vdots \\
\vdots \\
0\end{array}$ & 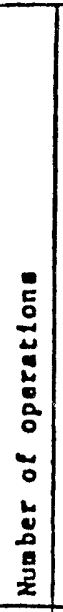 & 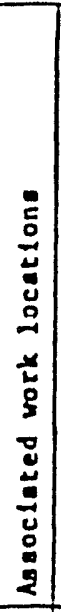 & 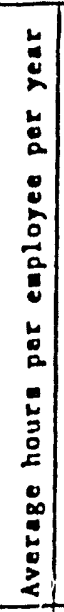 & 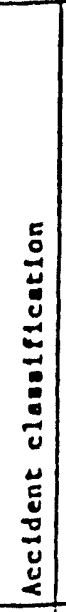 & 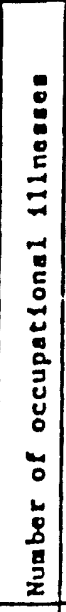 & 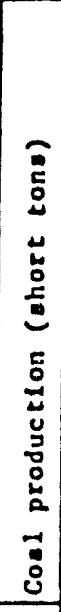 \\
\hline $\begin{array}{l}\text { Prior gear data } \\
\text { weh and without offlce }\end{array}$ & 1 & 1 & 1 & & 1 & 1 & 1 & 1 & 1 & 1 & & & \\
\hline \multirow{18}{*}{ Work locat1on } & 2 & 21 & 2 & & & 2 & 2 & 21 & 2 & & & & \\
\hline & 3 & 1 & & 3 & 31 & & & 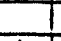 & & & & & \\
\hline & 4 & 4 & 41 & & & 4 & 6 & 4 & 4 & & & & \\
\hline & 5 & & & 51 & 51 & & & & & & & & - \\
\hline & 6 & 61 & 61 & & & 6 & 6 & 6 & 6 & & & & \\
\hline & 7 & & & 71 & 71 & & & & & & & & \\
\hline & 8 & 81 & 81 & 1 & & & & & & & - & & \\
\hline & 9 & & & 91 & 91 & & & & & L & - & & \\
\hline & 10 & 10 & 101 & & & & & & & L & - & & \\
\hline & 11 & & & 111 & 111 & & & & & 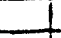 & 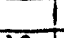 & & \\
\hline & 13 & & & 1 & & & & & & & 13 & & \\
\hline & & & & $14 !$ & & & & & & & 14 & & \\
\hline & 13 & & & $\frac{1}{161}$ & $\frac{1}{t}$ & & & - & & & $\frac{15}{16}$ & & \\
\hline & 27 & & & 171 & 171 & & & & & & 16 & & \\
\hline & 18 & & & 181 & 181 & & & & & & & & \\
\hline & & & & 1 & 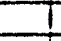 & & & & & & & 21 & \\
\hline & 22 & 22 & & 1 & & 22 & 22 & & & & & & 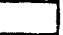 \\
\hline & 23 & \begin{tabular}{l|l}
233 \\
\end{tabular} & & 1 & & 23 & 23 & & & & & & 23 \\
\hline \multirow{2}{*}{ Exploy=ent stze group } & 4 & 61 & 41 & 1 & & 4 & 4 & 4 & 4 & & & & \\
\hline & 51 & & & 31 & 51 & & & & & & & & \\
\hline \multirow{5}{*}{ Iype of Elderal } & 61 & 61 & 61 & 1 & & 6 & 6 & 6 & 6 & & & & \\
\hline & 7 & & & $\pi$ & 21 & & & & & & & & \\
\hline & 151 & 1 & & 1 & 1 & & & & & & 15 & & \\
\hline & & & & $16 !$ & $I$ & & & & & & 16 & & \\
\hline & & & & & 1 & & & & & & & 211 & \\
\hline \multirow{5}{*}{ Srate } & 6 & 6 & 61 & 1 & & 6 & 67 & 6 & 6 & & & & \\
\hline & 7 & & & 31 & 71 & & & & & & & & \\
\hline & 13 & & & 1 & & & & & & & 13 & & \\
\hline & $\rightarrow$ & & & 141 & & & & & & & 14 & & \\
\hline & & & & 1 & & & & & & & & 211 & \\
\hline \multirow{4}{*}{ Part of body lajured } & 8 & 8 & 8 & 1 & & & & & & & & & \\
\hline & 91 & & & 91 & 91 & & & & & & & & \\
\hline & 121 & & & 121 & 121 & & & & & & & & \\
\hline & 191 & & & 191 & 191 & & & & & & & & \\
\hline \multirow{4}{*}{ Hature of Ingury } & 10 & 101 & 101 & & & & & & & & & & \\
\hline & 11 & & & 111 & 111 & & & & & & & & \\
\hline & 121 & & & 131 & 121 & & & & & & & & \\
\hline & 201 & & & 201 & 201 & & & & & & & & \\
\hline $\begin{array}{l}\text { Oceupac100 at tise of } \\
\text { 10fusy }\end{array}$ & 17 & & & 17 & 17 & & & & & & & & \\
\hline \multirow{3}{*}{ Accident clasal\$1cat100 } & 181 & & & 181 & 181 & & & & & & & & \\
\hline & 19 & & & 191 & 191 & & & & & & & & \\
\hline & 201 & & & 201 & 201 & & & & & & & & \\
\hline Hoceal ladusczy & 22 & 221 & & 1 & & 22 & 221 & & & & & & \\
\hline Iype of cos & 23 & 231 & & 1 & & $23]$ & 23 & & & & & & 23 \\
\hline
\end{tabular}

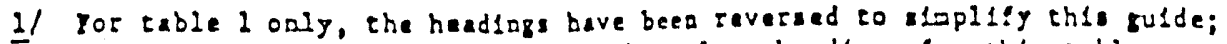
that 18, the data lecots ladicated la the coluso headiags for thls table actually appear as vertical headlags. 

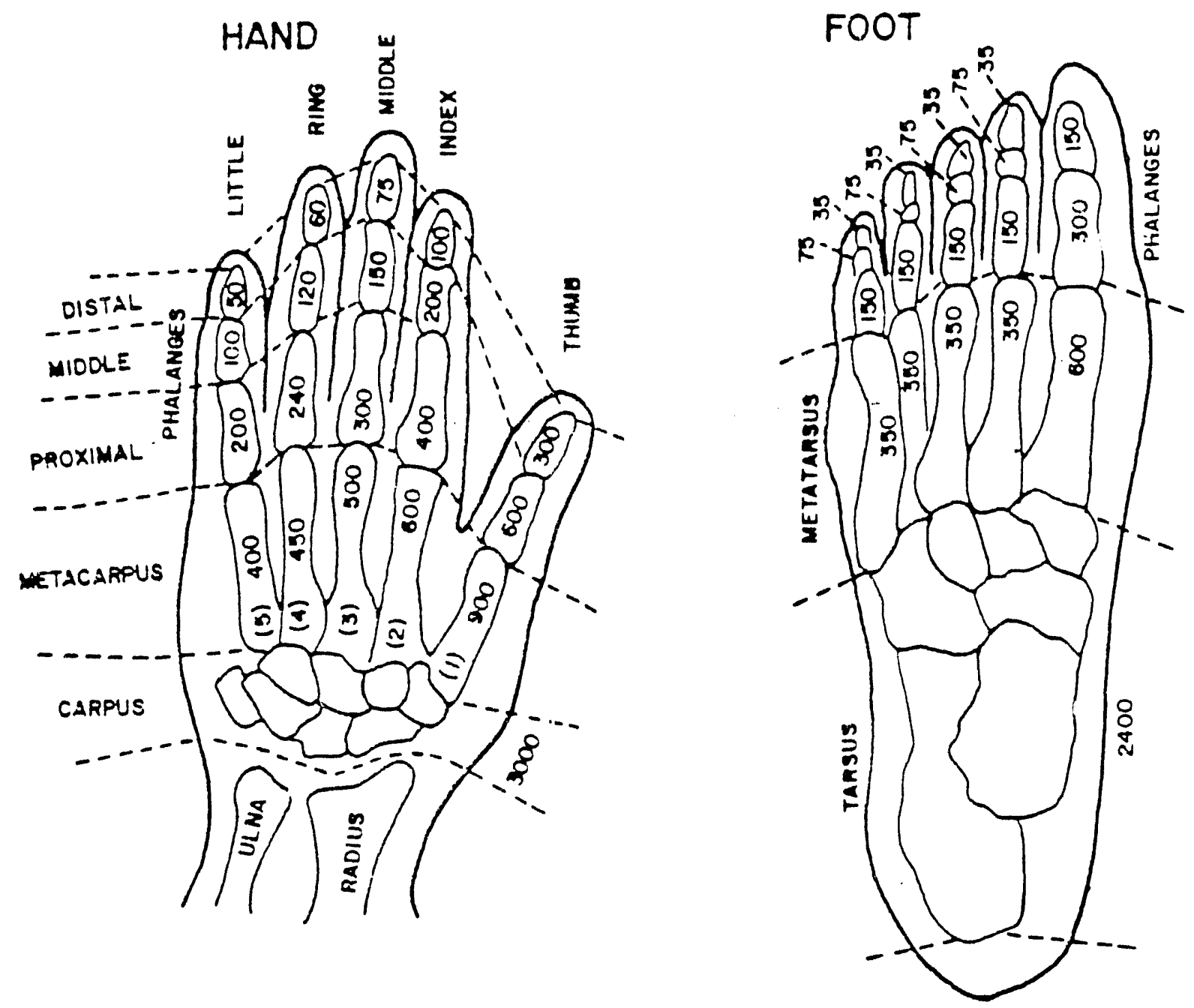

Note:

Numbers shown on diagrams are statutary charges of lost workdays for loss of all or part of bone.

Proportional charges may be computed based on medical determination of percentage loss of use.

FIGURE I. Scheduled charges in lost workdays for loss or loss of use involving hand or foot. 
O.M8. Number 12100000: Aporove Eaplien buly 31.1980

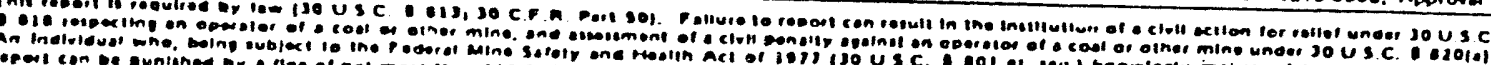

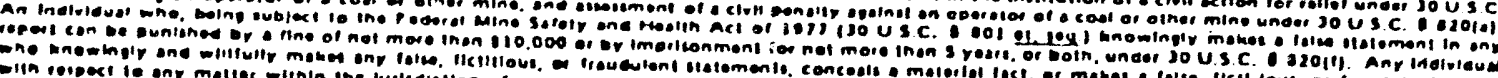

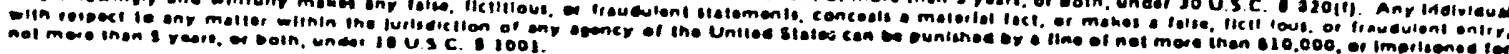

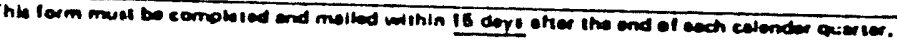

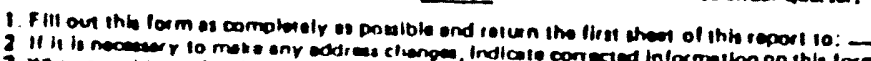

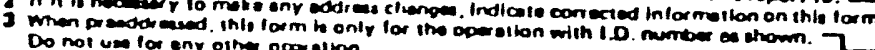

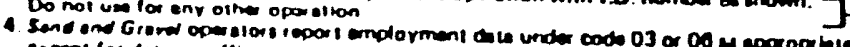

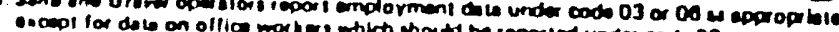

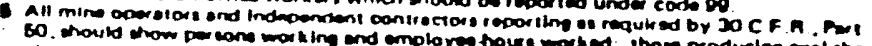

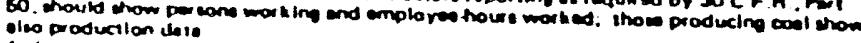

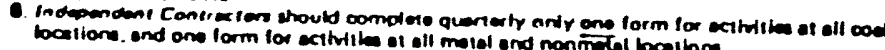

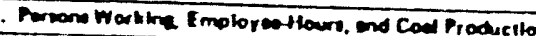

\begin{tabular}{|c|c|c|c|c|c|}
\hline \multirow{2}{*}{\multicolumn{2}{|c|}{ comideroviounty }} & \multirow{3}{*}{01} & \multirow[b]{2}{*}{ (1) A norop number of por } & \multirow[b]{2}{*}{ (3) Tome aroporom Aours } & \multirow[b]{2}{*}{ 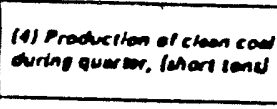 } \\
\hline & & & & & \\
\hline Whe & Undar foume & & & & \\
\hline \multirow{7}{*}{ 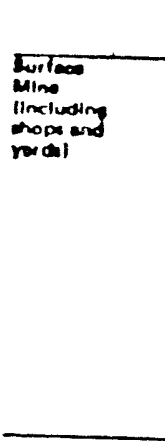 } & $\begin{array}{l}\text { Sumleos Shoon. } \\
\text { rendt. Etc. }\end{array}$ & 02 & & & \\
\hline & $\begin{array}{l}\text { Sulp. Orem Pil. } \\
\text { ox Oniviry }\end{array}$ & 03 & & & \\
\hline & 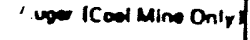 & of & & & \\
\hline & 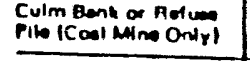 & $\infty$ & & & \\
\hline & Drower & $\infty$ & & & \\
\hline & 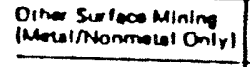 & 12 & & & \\
\hline & Indapendent Shope o & 17 & & & \\
\hline \multicolumn{2}{|c|}{ 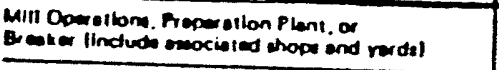 } & 30 & & & \\
\hline \multicolumn{2}{|c|}{ 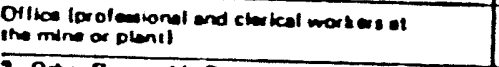 } & $\infty$ & & & \\
\hline \multicolumn{6}{|c|}{ 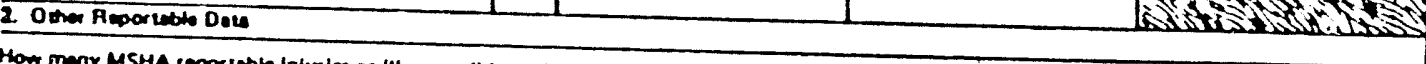 } \\
\hline \multicolumn{6}{|c|}{ 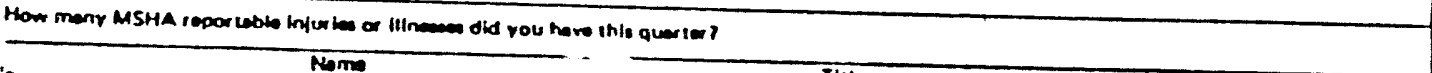 } \\
\hline \multicolumn{3}{|c|}{ 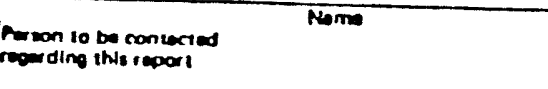 } & \multicolumn{2}{|c|}{ Tils } & Phom lincl. ANe Codel \\
\hline
\end{tabular}

MSHA, Health and Salety Analysis Conter P.O. Box 25367

enver, Coloredo 80225

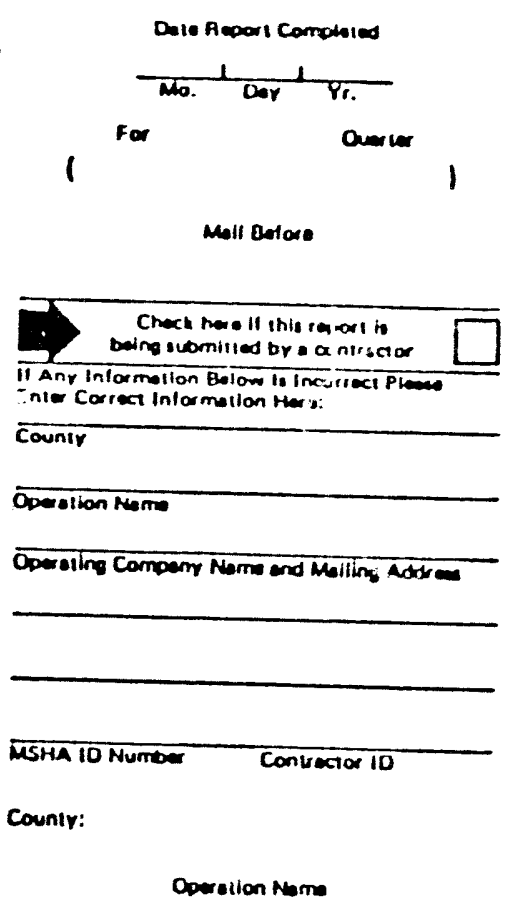

Operaline Compony Nume end todren

Relurn To MSHA 
Mine Accident, Injury and IIIness Report

US Departivent of Labor

Mine Safety and Health Administration

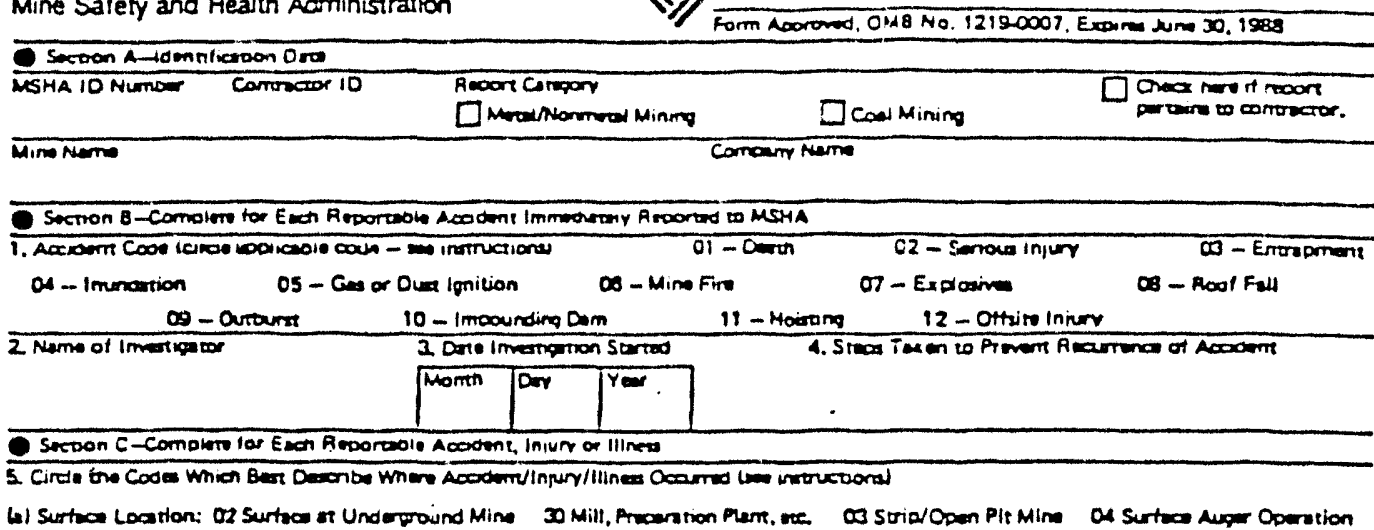

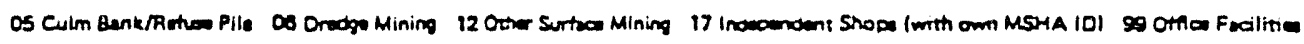

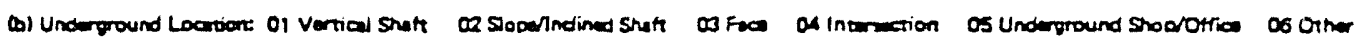

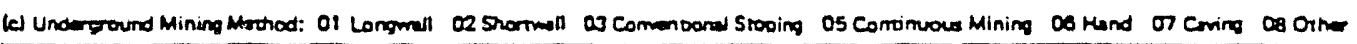

\begin{tabular}{|c|c|c|c|c|}
\hline a Dare & $A \infty$ & & 7. Time of Acosomn & $8 \pi$ w Shitt Sterted \\
\hline Momn & Der & $Y=$ & & \\
\hline
\end{tabular}

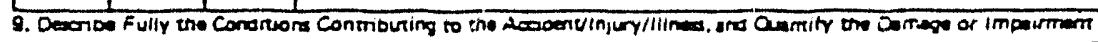

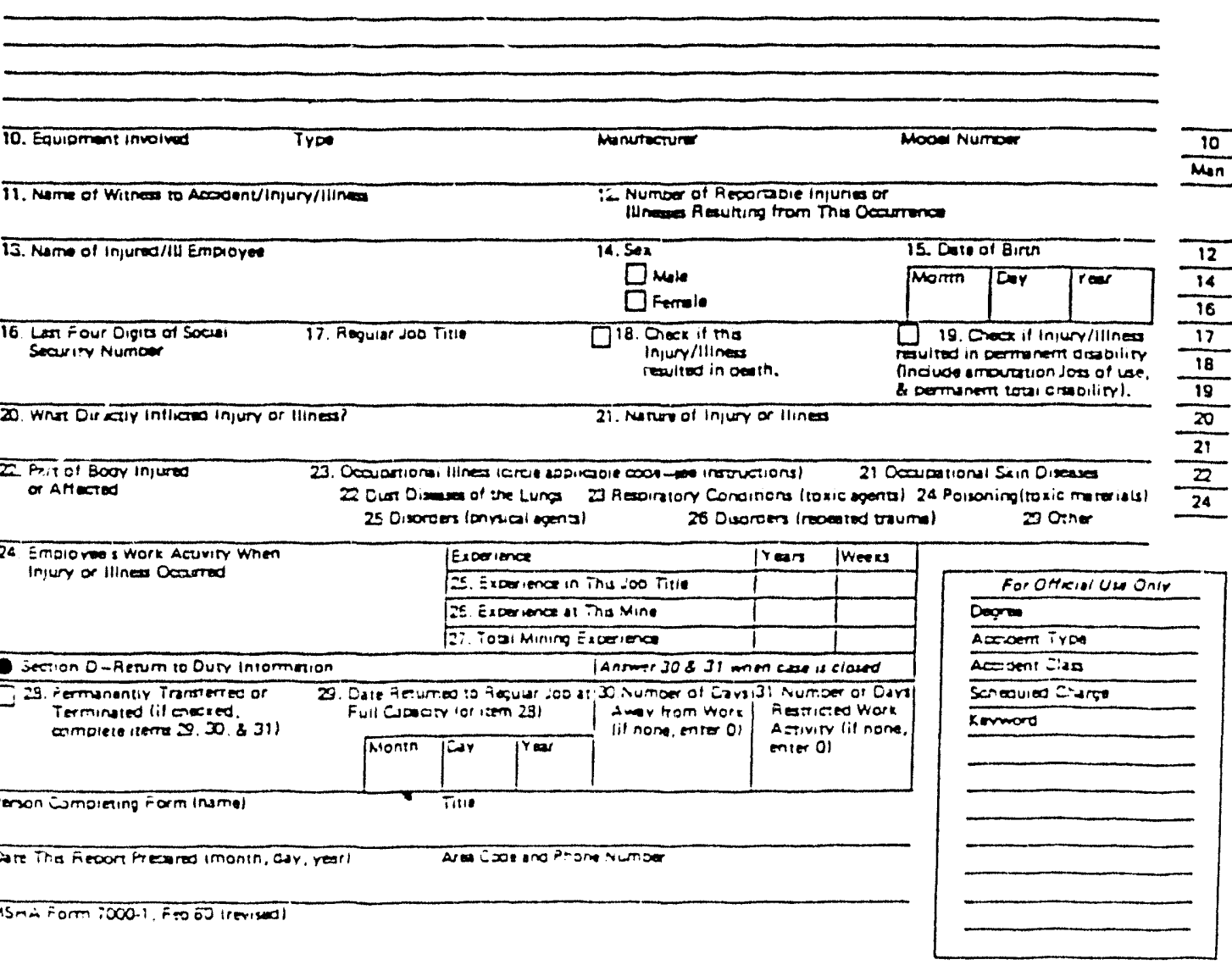

MAIL THIS PAGE TO THE HEALTHAHD SAFET AHALYSIS ESNTER, MINE SAFETY

ANO HEALTH AOMINISTAATICN, PO. BOX $\simeq 367$. OEYVER, COLORADO BE:S

FIGURE 3. Mine accident, injury, and illness report form. 


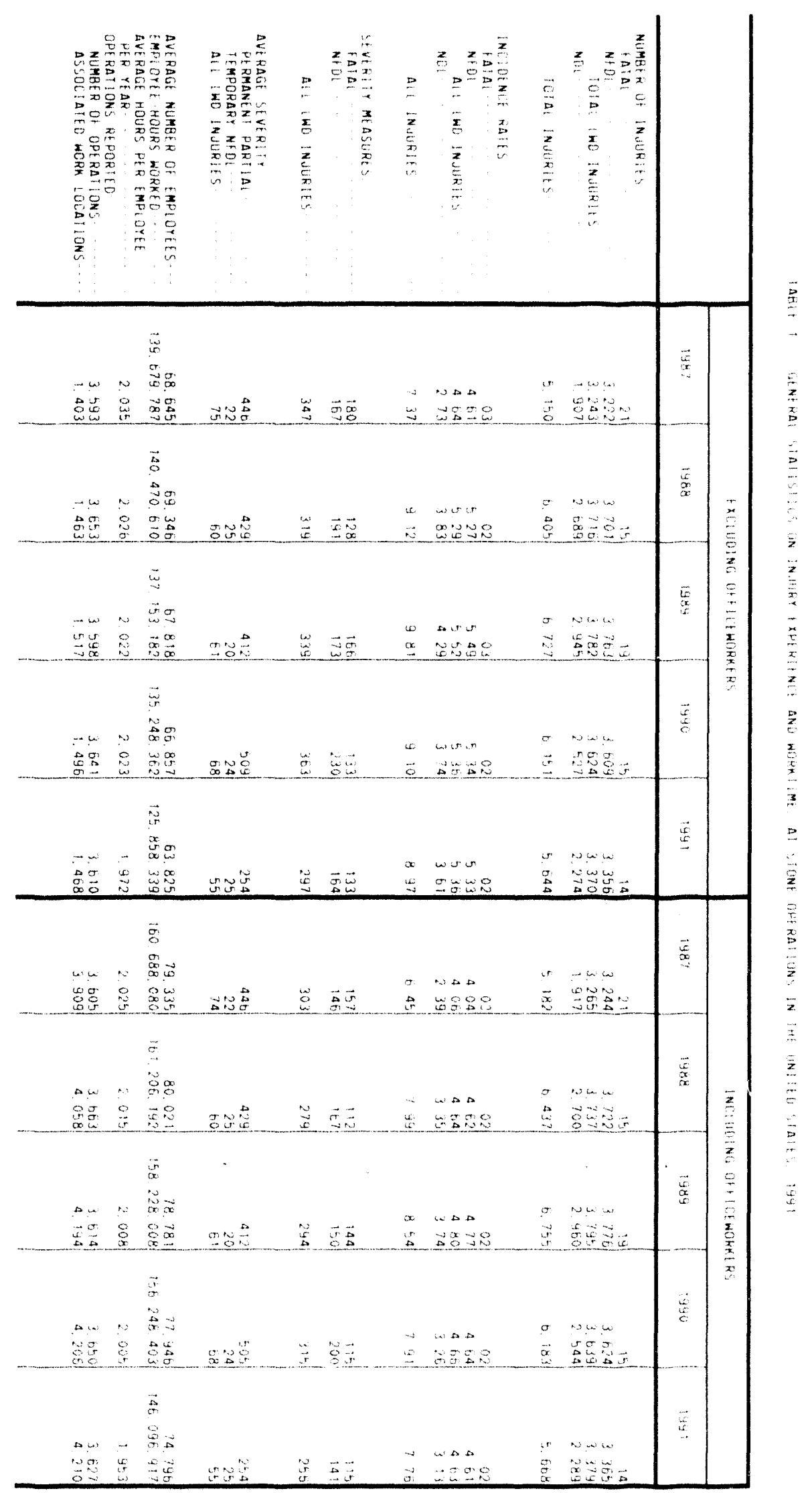




\begin{tabular}{|c|c|c|c|c|c|c|c|c|c|c|c|}
\hline & \multicolumn{2}{|c|}{ 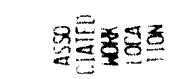 } & $\infty$ & $\approx$ & & & $\mathbb{s}$ & $\stackrel{ }{5}$ & 祃 & $\stackrel{s}{g}$ & 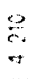 \\
\hline & \multicolumn{2}{|c|}{ 繁管 } & 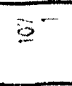 & $\underline{3}$ & 票 & id & 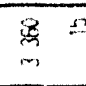 & d & $\stackrel{5}{0}$ & $=$ & 6 \\
\hline \multicolumn{3}{|c|}{ 管簘 } & 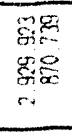 & $\begin{array}{l}8 \\
z \\
z\end{array}$ & 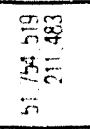 & $\begin{array}{l}8 \\
8 \\
5 \\
5\end{array}$ & 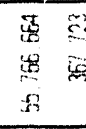 & 8 & 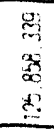 & 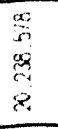 & 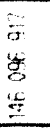 \\
\hline \multicolumn{3}{|c|}{ 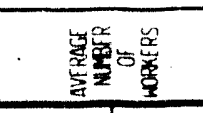 } & $\begin{array}{l}\text { 等等 } \\
-\end{array}$ & 类 & $\begin{array}{l}\bar{S}= \\
E\end{array}$ & 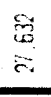 & 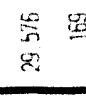 & $\begin{array}{l}8 \\
3 \\
3 \\
\end{array}$ & 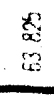 & $\begin{array}{l}3 \\
2\end{array}$ & 等 \\
\hline \multirow{3}{*}{\multicolumn{2}{|c|}{ 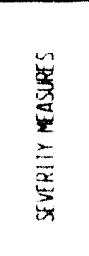 }} & 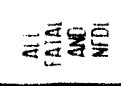 & ऊह & 票 & 9 & $\frac{\mathscr{m}}{m}$ & 7 & \&్ & is & $m$ & s \\
\hline & & $\bar{E}$ & $=8$. & 9 & $\leq \pm$ & 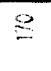 & 97 & $\underline{B}$ & $\underline{z}$ & $m$ & 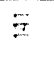 \\
\hline & & 플 & $\frac{9}{7}$ & $\underline{\underline{m}}$ & 专 & 8 & $\therefore$ & 8 & 9 & & 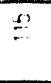 \\
\hline & \multirow{5}{*}{ 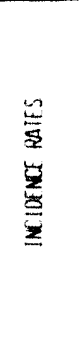 } & 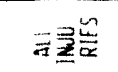 & $\begin{array}{l}8= \\
\infty=5\end{array}$ & gi & $\stackrel{R=}{\infty}$ & $\sum_{\infty}$ & 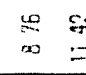 & $\frac{a}{\infty}$ & को & it & 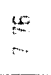 \\
\hline & & $\overline{\underline{z}}$ & $\begin{array}{l}40 \\
40 \\
\end{array}$ & 8 & m- & m & $\begin{array}{ll}5 \\
m\end{array}$ & $\stackrel{g}{g}$ & $\begin{array}{c}i_{0} \\
\infty \\
i\end{array}$ & $?$ & $=$ \\
\hline & & 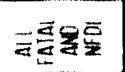 & $\begin{array}{l}78 \\
+\infty\end{array}$ & si & $\begin{array}{l}58 \\
50\end{array}$ & 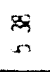 & $\begin{array}{ll}g & 8 \\
\pi & 8\end{array}$ & 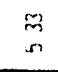 & $\stackrel{4}{s}$ & s & 8 \\
\hline & & $\overline{\bar{z}}$ & $\begin{array}{l}\operatorname{lig}_{-\infty} 8 \\
-\infty\end{array}$ & in & 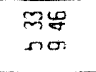 & fin & $\begin{array}{ll}\tilde{m} & g \\
\Omega & 8\end{array}$ & si & s & : & 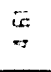 \\
\hline & & $\overline{\underline{z}}$ & 5 & 5 & 3 & $\overline{5}$ & 3 & $\overline{5}$ & 8 & & 5 \\
\hline \multirow{8}{*}{ 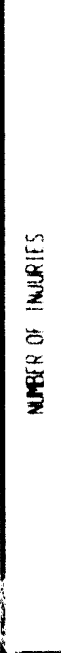 } & \multicolumn{2}{|c|}{ 突点 } & \pm & 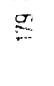 & $\therefore$ & $\underset{c}{z}$ & $\frac{7}{7}$ & $\stackrel{\underline{z}}{m}$ & 浔 & c & 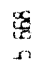 \\
\hline & \multicolumn{2}{|c|}{ 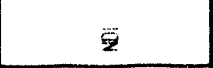 } & $x \alpha_{2}$ & $\underline{E}$ & $g^{\circ}$ & $\mathbb{x}$ & $\overline{8}=$ & 8 & $s$ & $=$ & g. \\
\hline & \multicolumn{2}{|c|}{ 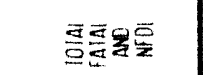 } & 88 & 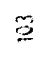 & 92 & 密 & $\xi=$ & 列 & : & $\sigma$ & 箵 \\
\hline & \multicolumn{2}{|c|}{ 홍를 } & BDA? & $\underline{g}$ & 20 & 总 & $g=$ & $\underset{\substack{n \\
\alpha}}{n}$ & 8 & s. & 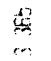 \\
\hline & \multirow{3}{*}{$\tilde{\pi}$} & 垔言 & $\forall \bar{x}$ & 8 & 9 & 䓌 & 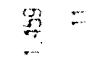 & 等 & $\stackrel{v}{v}$ & 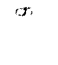 & ? \\
\hline & & \multirow{2}{*}{ 竞 } & $\cdots$ & c. & $\bar{n}$ & $=$ & 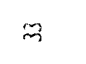 & 7 & $\mathrm{~s}$ & & $E$ \\
\hline & & & & & & & & & & & \\
\hline & \multicolumn{2}{|c|}{$\underline{z}$} & - & - & $\sigma$ & क & 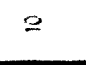 & $\sigma$ & $=$ & & $I$ \\
\hline & & & 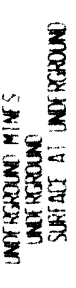 & 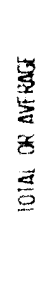 & 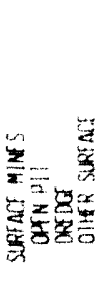 & 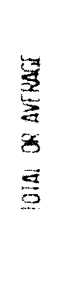 & 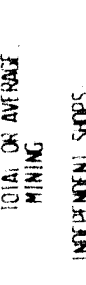 & 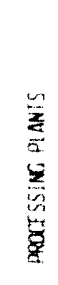 & 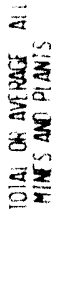 & $\begin{array}{l} \\
y \\
\stackrel{y}{5}\end{array}$ & 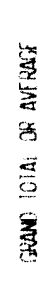 \\
\hline
\end{tabular}




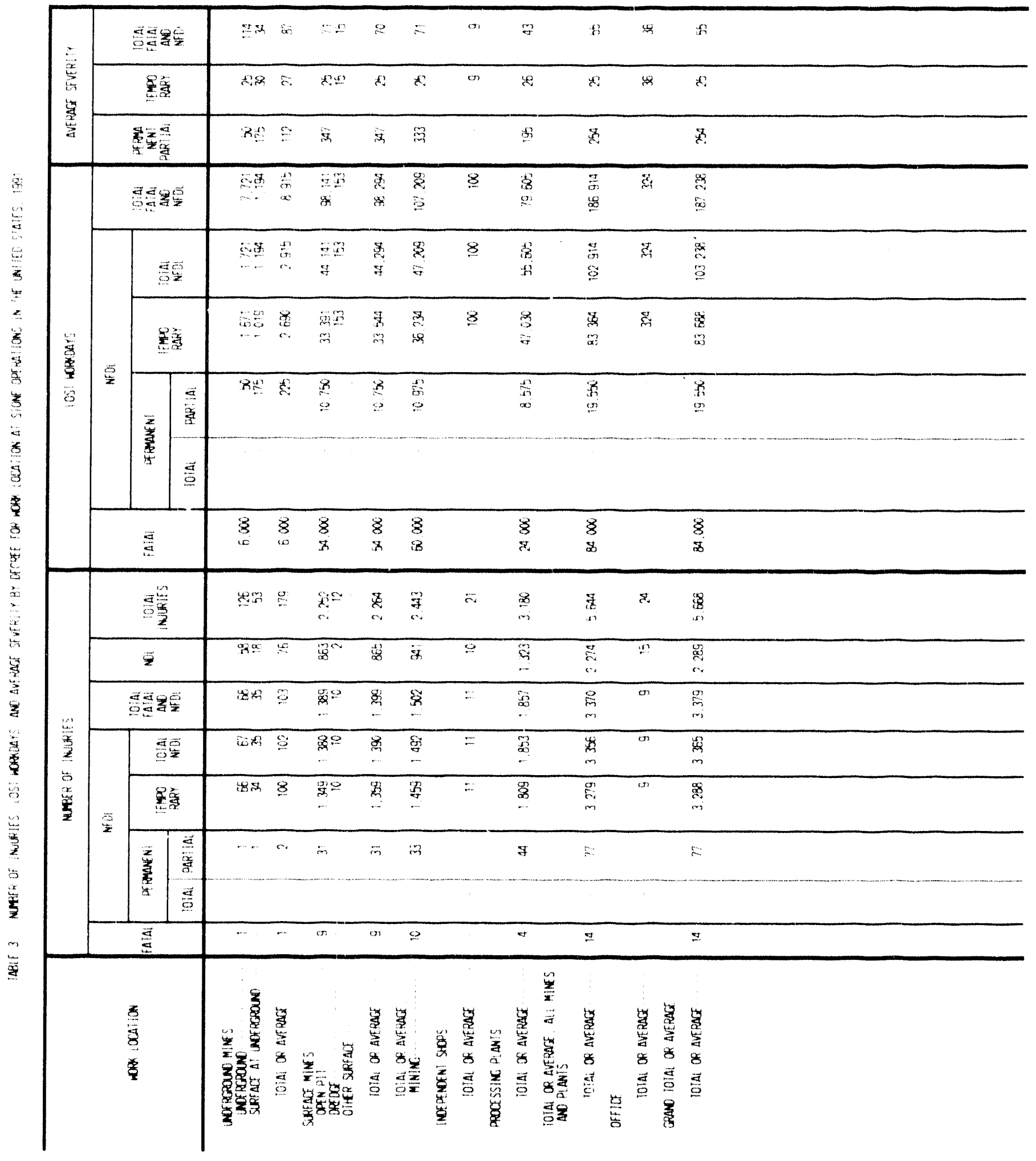




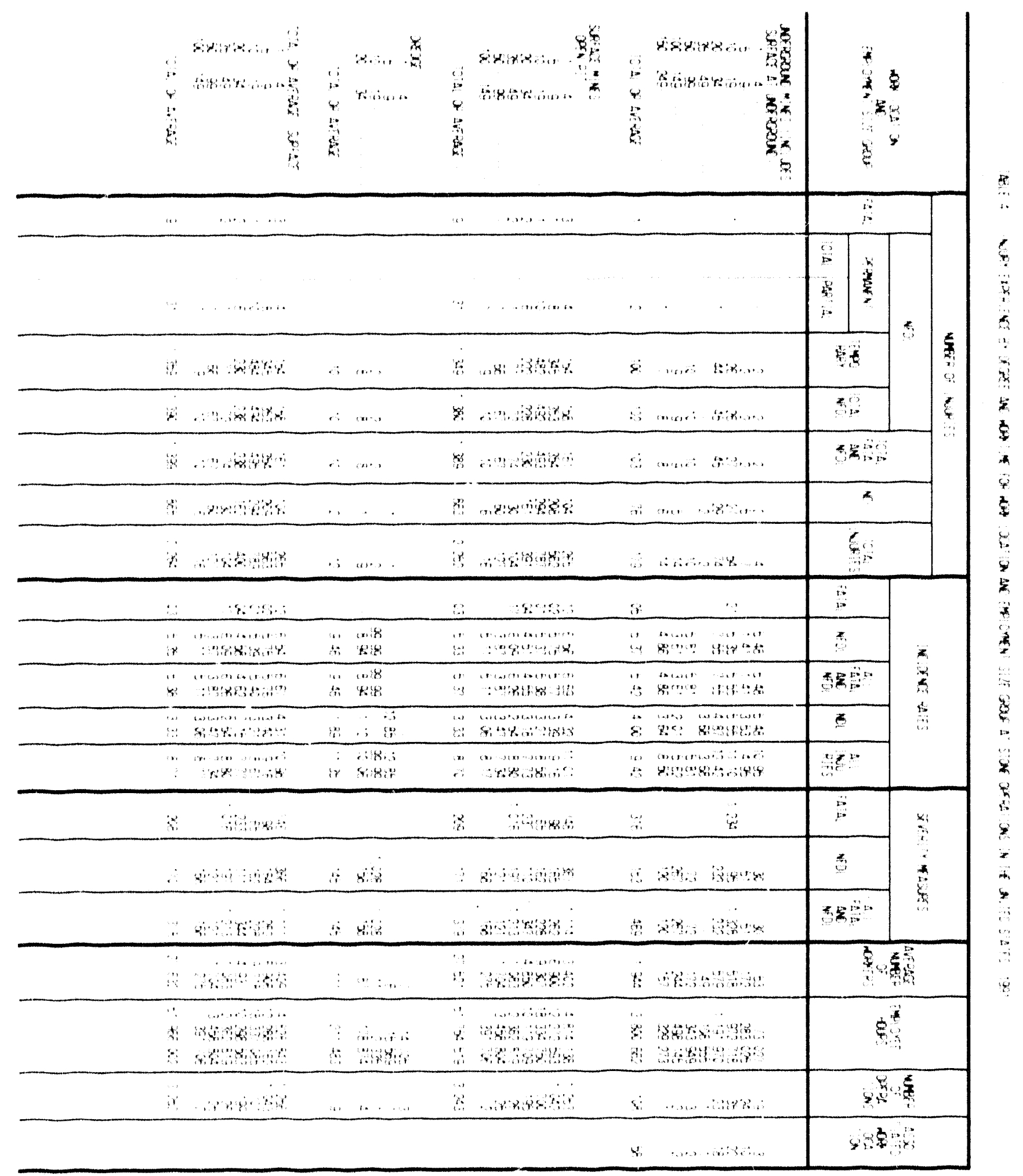




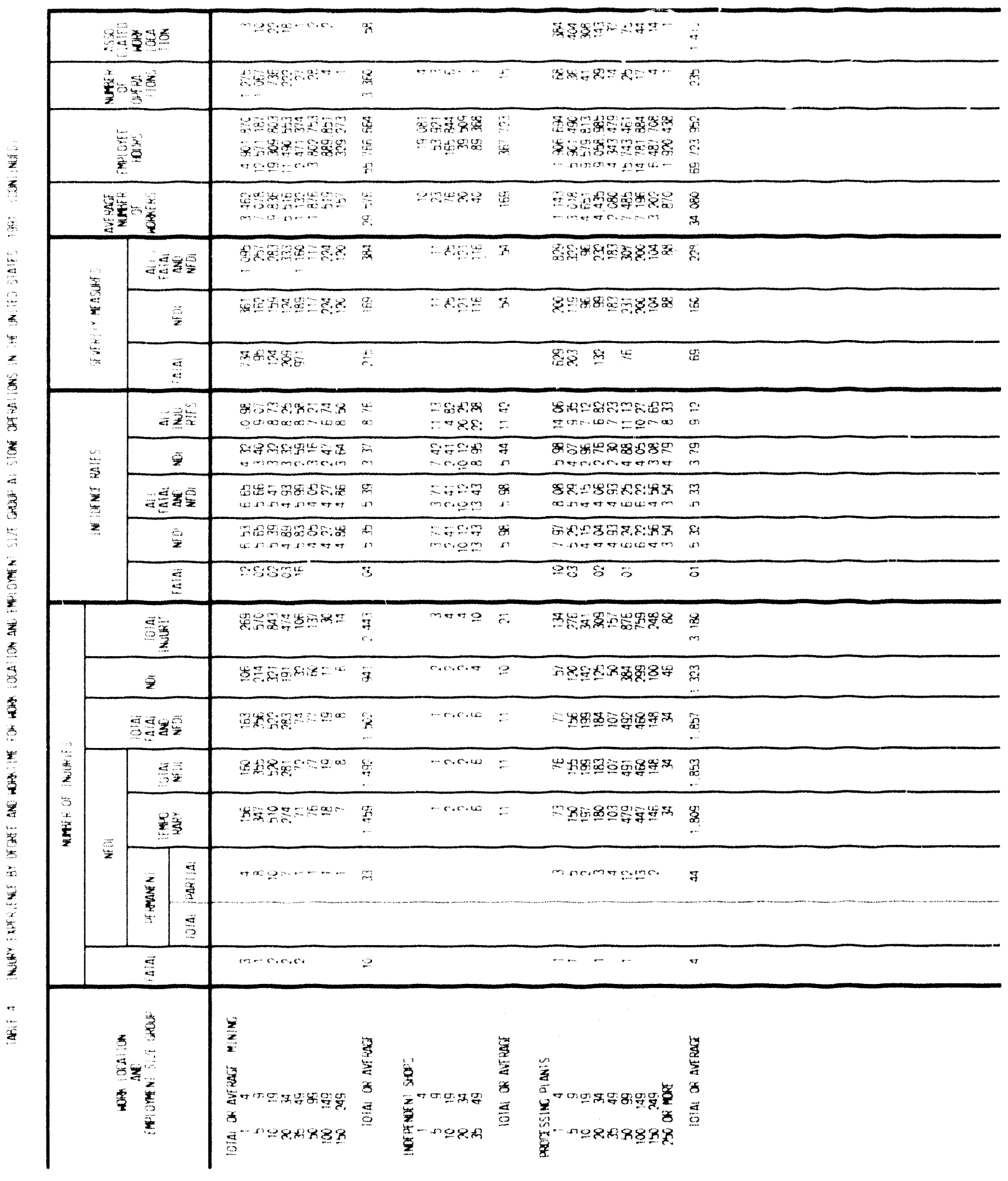




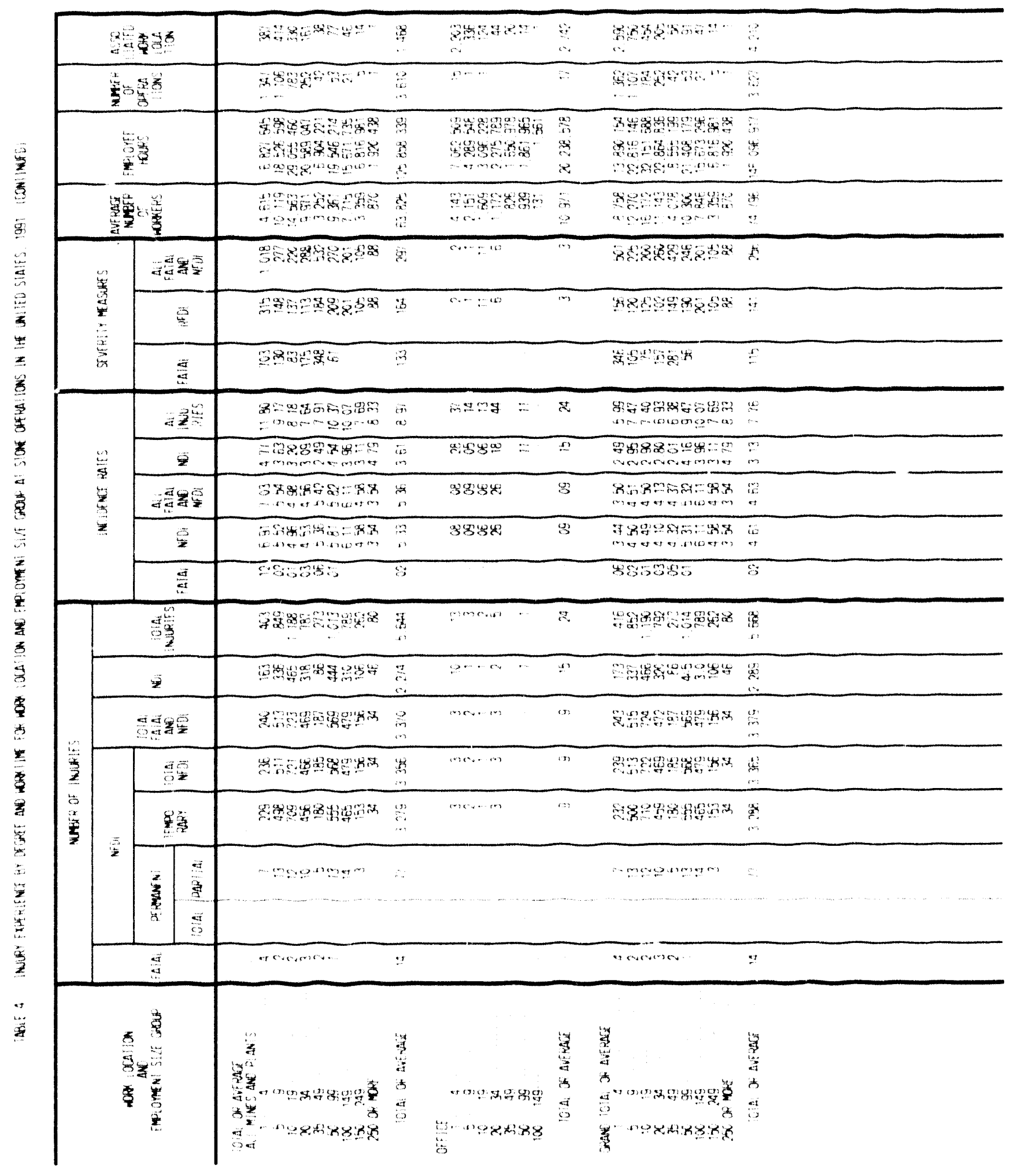




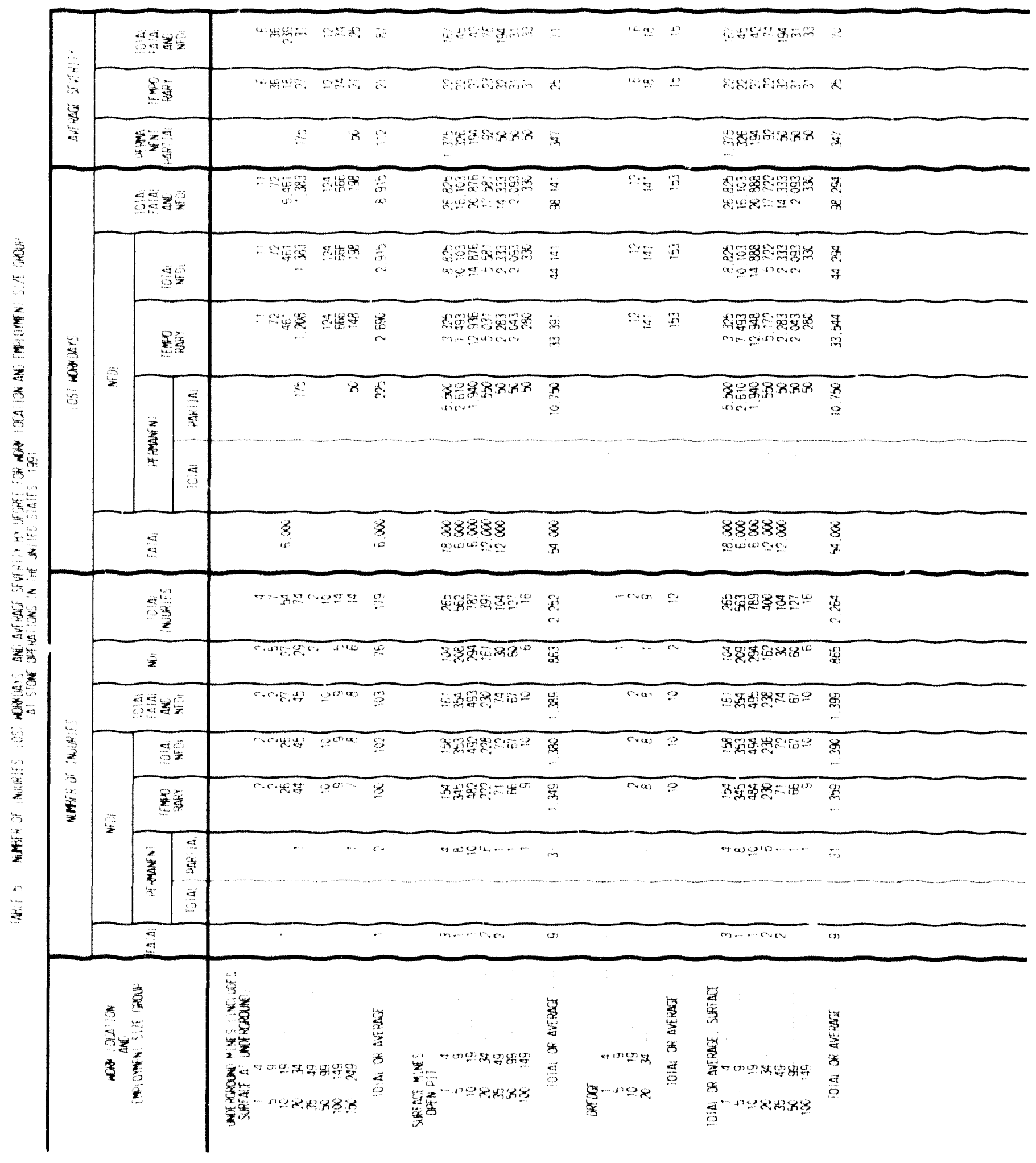




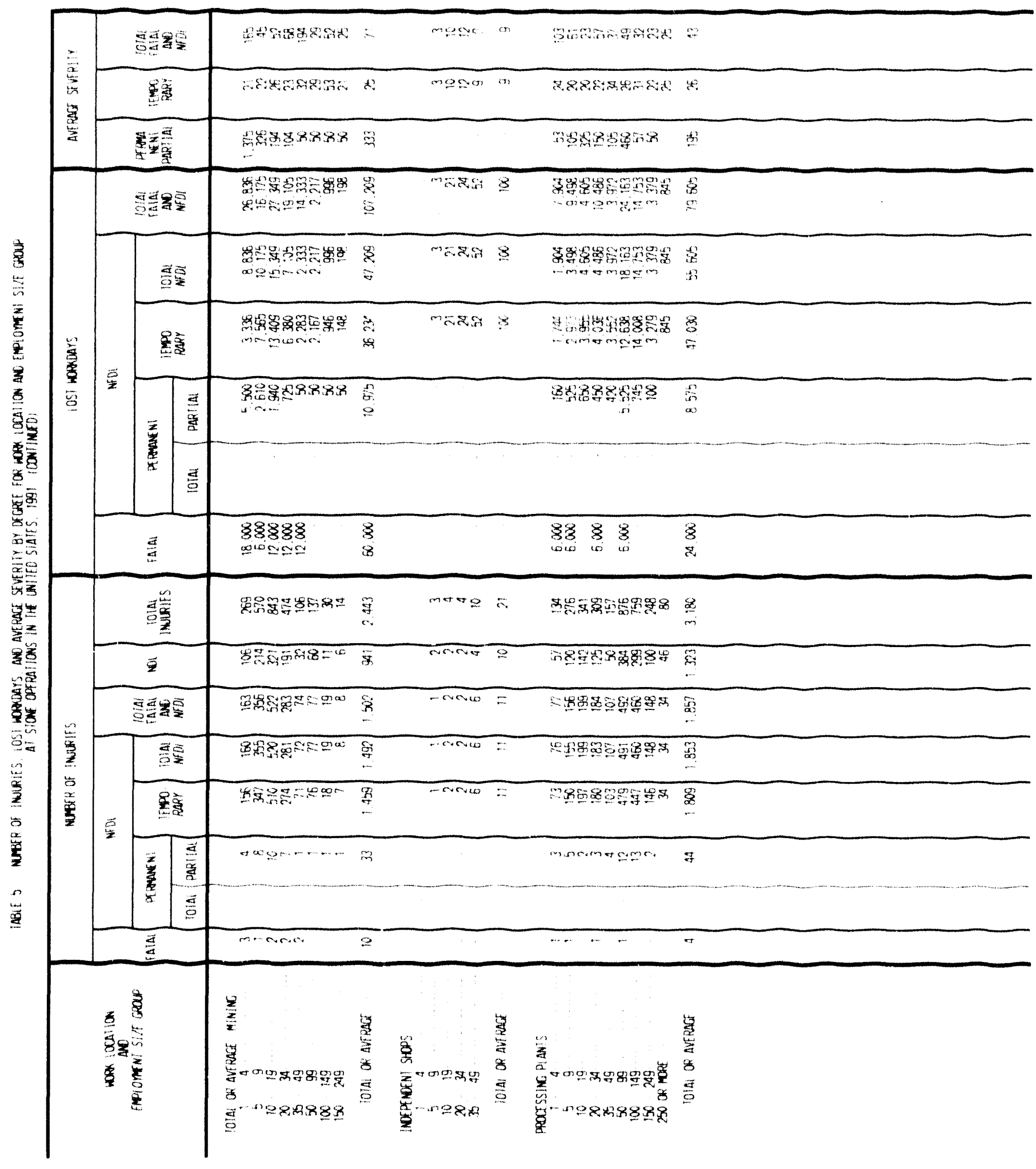




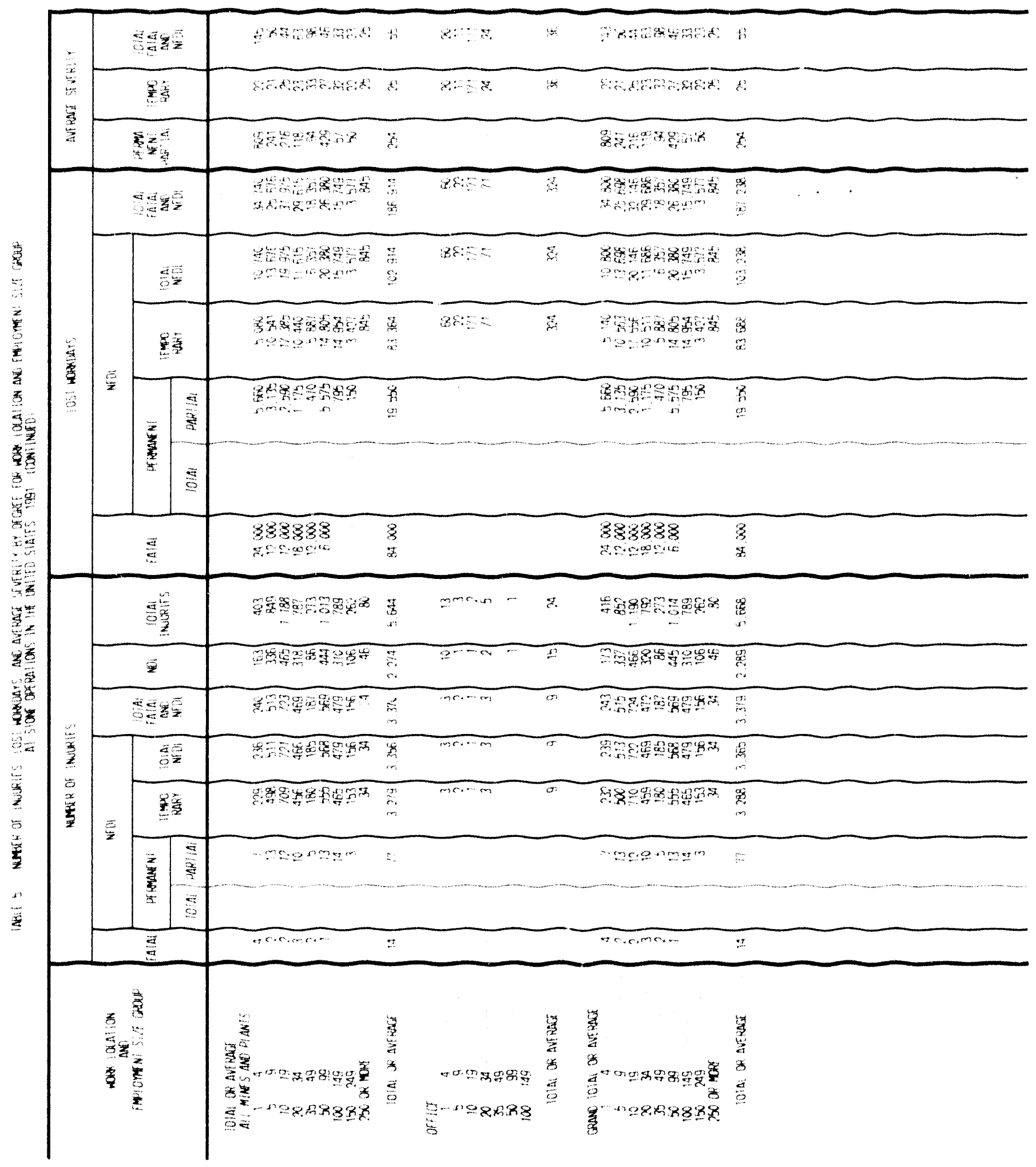




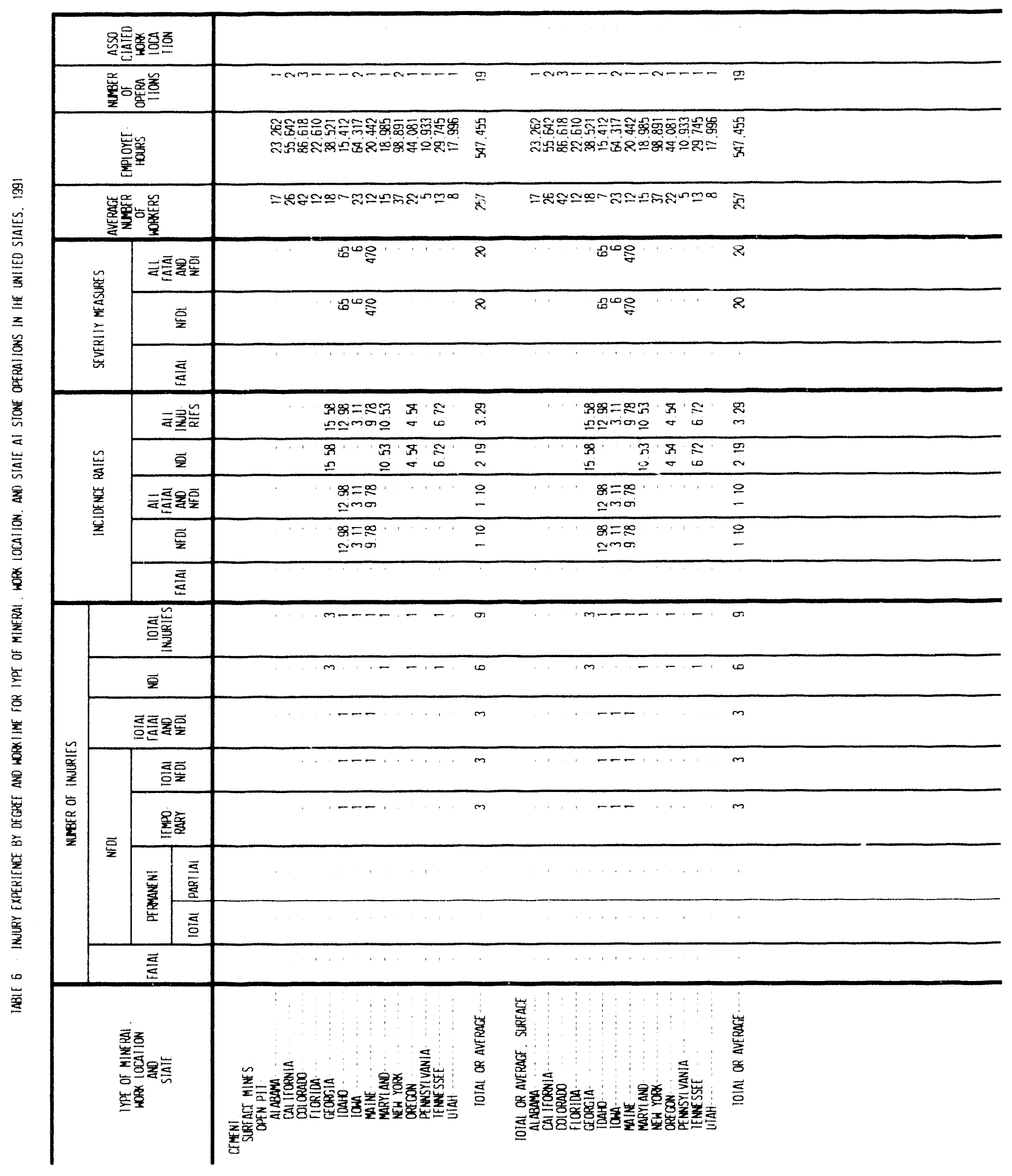




\begin{tabular}{|c|c|c|c|c|c|c|c|}
\hline \multicolumn{4}{|c|}{ 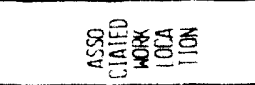 } & & & $-a m-n-a-n-m-\infty$ & on \\
\hline \multicolumn{4}{|c|}{ 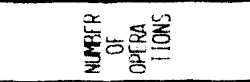 } & $-a m--n+m-n-m$ & g & 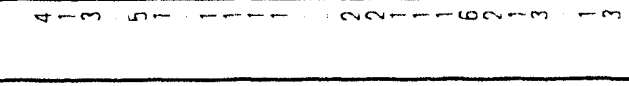 & $\bar{F}$ \\
\hline \multicolumn{4}{|c|}{ 崖金 } & 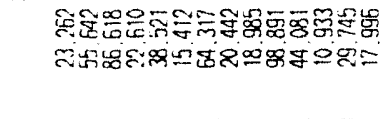 & 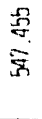 & 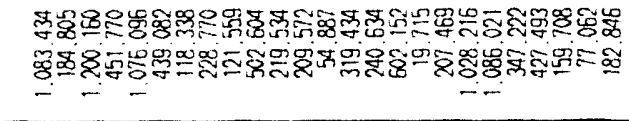 & 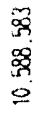 \\
\hline \multicolumn{4}{|c|}{ 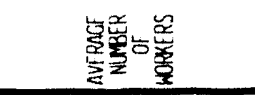 } & 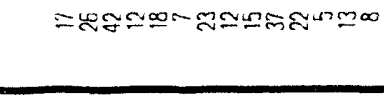 & $\bar{\Omega}$ & 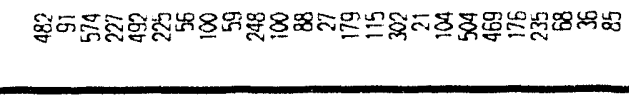 & 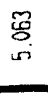 \\
\hline \multirow{3}{*}{\multicolumn{2}{|c|}{ 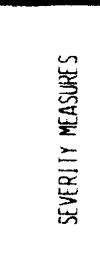 }} & \multicolumn{2}{|c|}{ 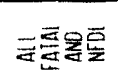 } & $w^{\infty} \%$ & $\&$ & 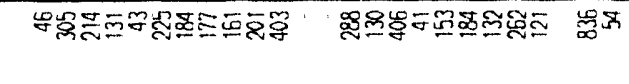 & 兽 \\
\hline & & \multicolumn{2}{|c|}{ 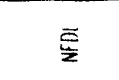 } & $\mathbb{E}^{\infty} \stackrel{8}{\forall}$ & $\&$ & 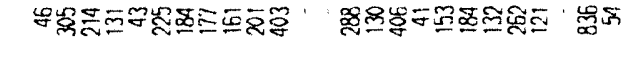 & gg \\
\hline & & \multicolumn{2}{|c|}{$\stackrel{\Xi}{\Xi}$} & ' & $\cdot$ & , 1 : & ' \\
\hline & \multirow{5}{*}{ 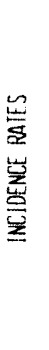 } & \multicolumn{2}{|c|}{ 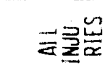 } & 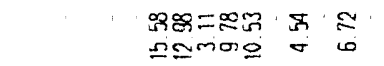 & $\stackrel{g}{m}$ & 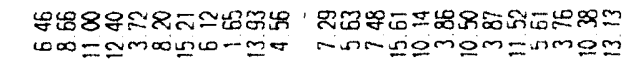 & $\stackrel{\infty}{\infty}$ \\
\hline & & \multicolumn{2}{|c|}{$\overline{\$}$} & 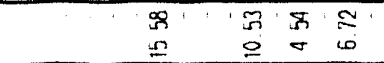 & $\frac{\sigma}{2}$ & 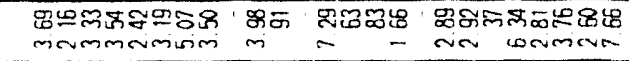 & $\Xi$ \\
\hline & & \multicolumn{2}{|c|}{ 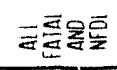 } & $\begin{array}{l}\mathscr{g}=\infty \\
:=20\end{array}$ & $\underline{\underline{-}}$ & 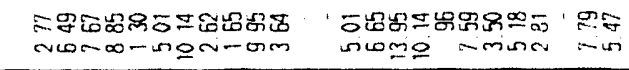 & $\stackrel{9}{\infty}$ \\
\hline & & \multicolumn{2}{|c|}{$\frac{\bar{c}}{\Sigma}$} & $\begin{array}{l}B=\infty \\
\sin \infty\end{array}$ & $\stackrel{ }{-}$ & 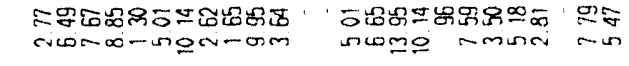 & m \\
\hline & & \multicolumn{2}{|c|}{$\underline{\underline{E}}$} & & & & \\
\hline \multirow{8}{*}{ 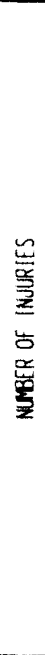 } & \multicolumn{3}{|c|}{ 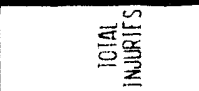 } & $m--\infty-\cdots$ & の & 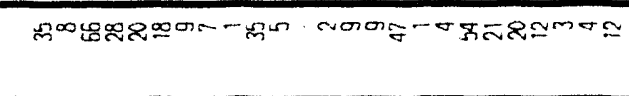 & ঞ্ণ \\
\hline & \multicolumn{3}{|c|}{$\bar{z}$} & $m+-\infty$ & $\infty$ & greomang & $\stackrel{\Omega}{g}$ \\
\hline & \multicolumn{3}{|c|}{ 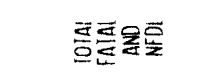 } & $-\cdots$ & 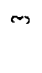 & 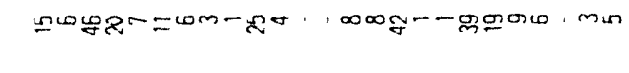 & 胥 \\
\hline & \multirow{4}{*}{$\dot{5}$} & \multicolumn{2}{|c|}{ 흥물 } & $\cdot \quad, \quad+-$ & $m$ & 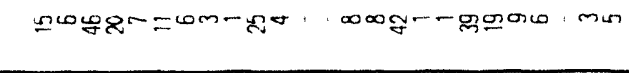 & 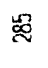 \\
\hline & & \multicolumn{2}{|c|}{ 邆哀 } & 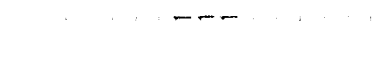 & $m$ & 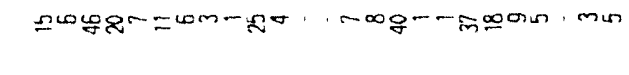 & $\stackrel{\infty}{\approx}$ \\
\hline & & \multirow{2}{*}{ 妾 } & $\overrightarrow{\underline{\Xi}}$ & $\therefore$ & . & $-\infty \cdot a-$ & - \\
\hline & & & 롱 & $\therefore$ & & & ' \\
\hline & \multicolumn{3}{|c|}{$\stackrel{\underline{a}}{a}$} & : : & : & : & , \\
\hline & & $\frac{5}{5}$ & & 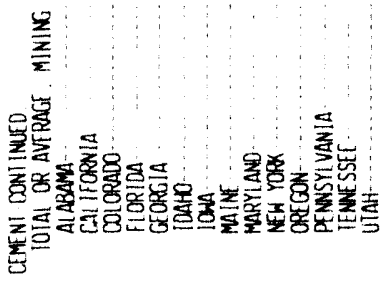 & 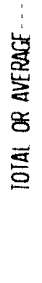 & 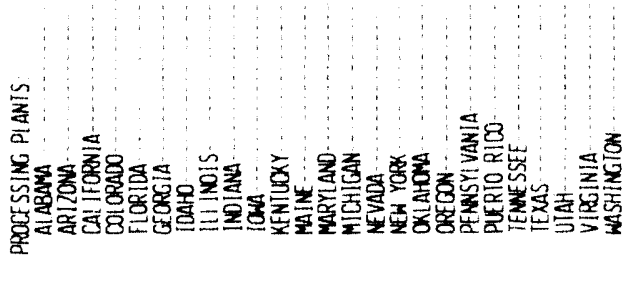 & 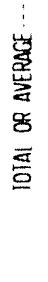 \\
\hline
\end{tabular}




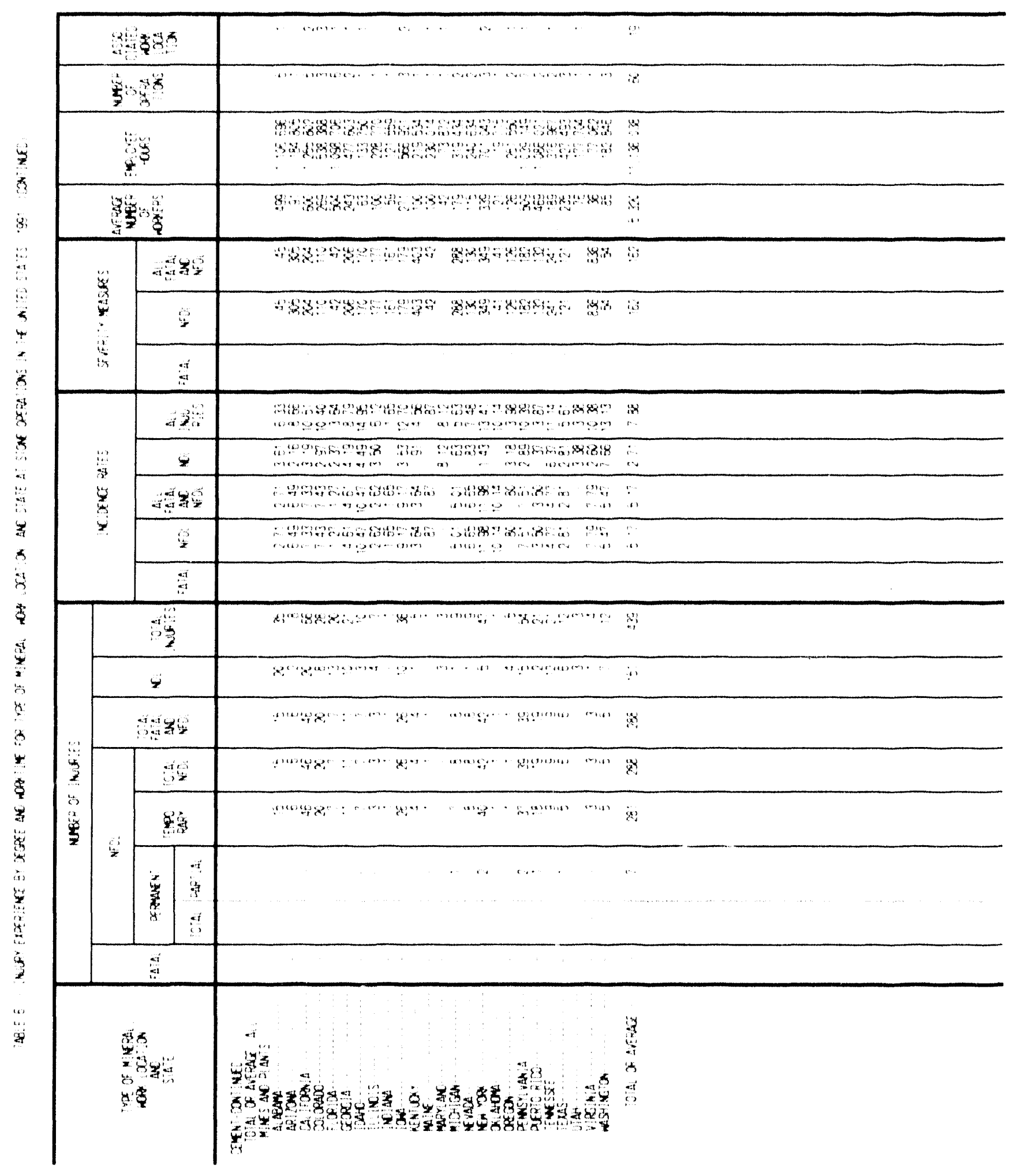




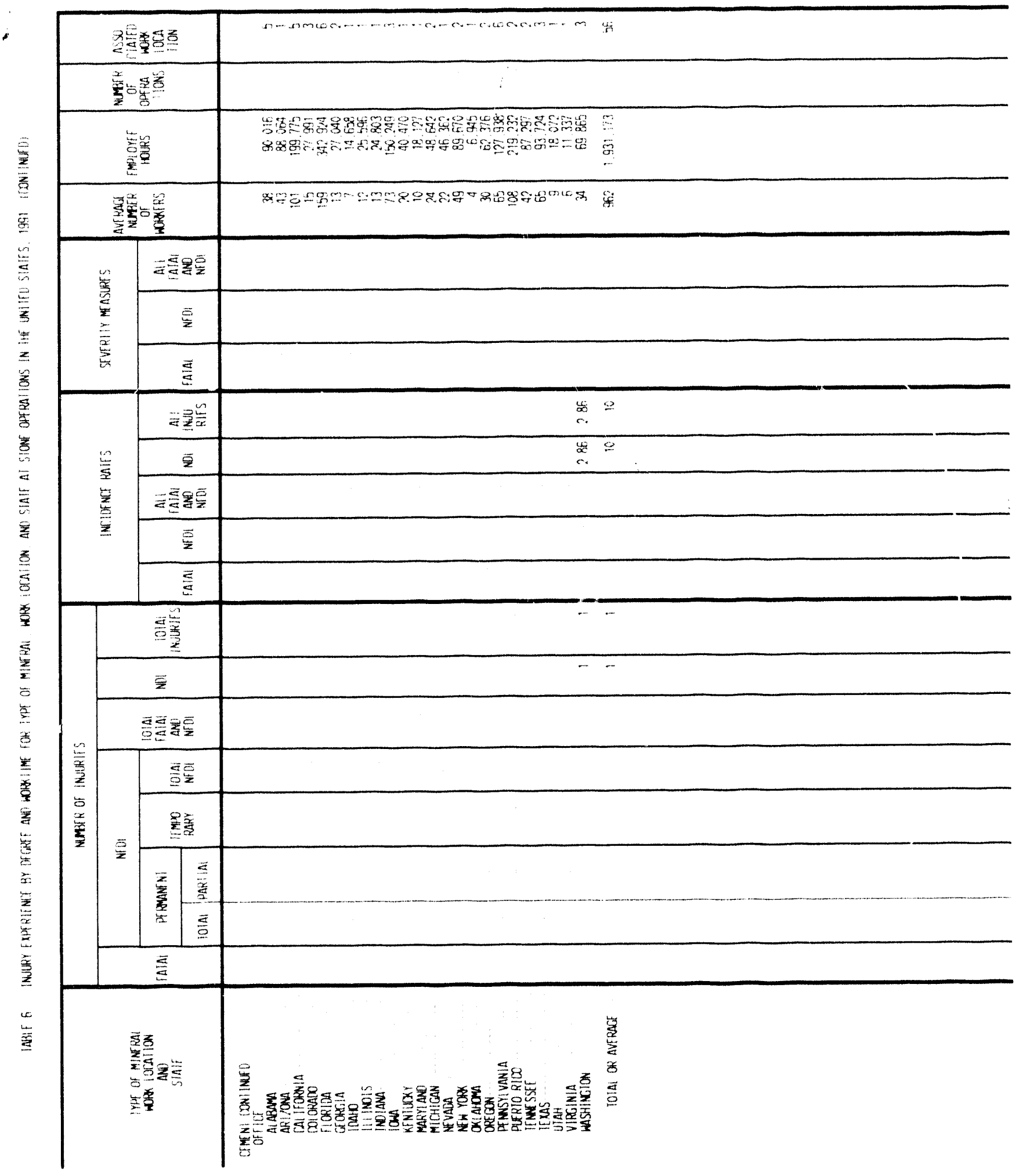



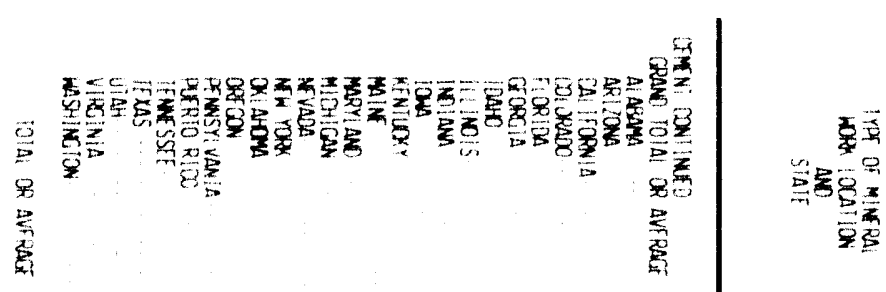

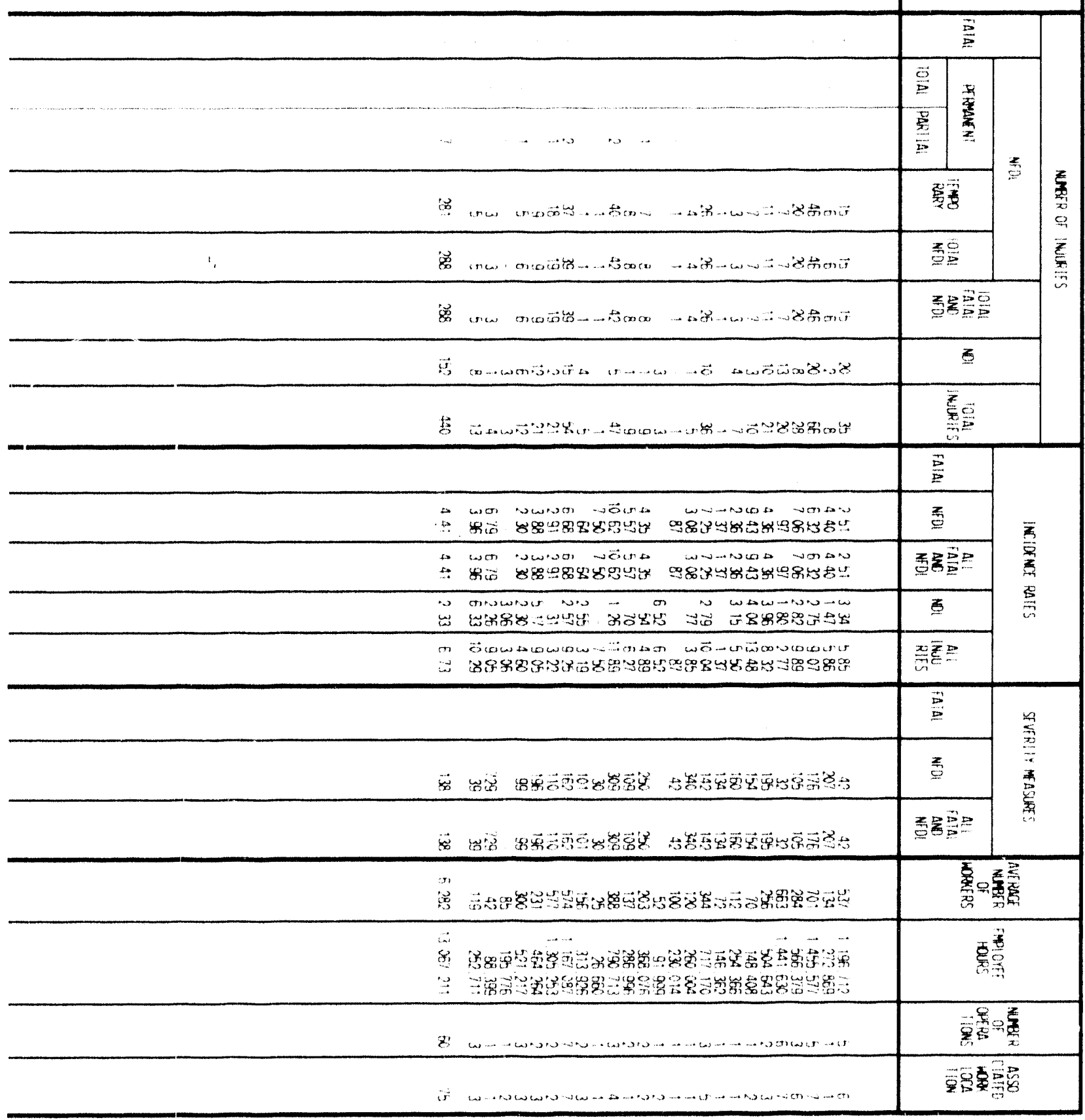




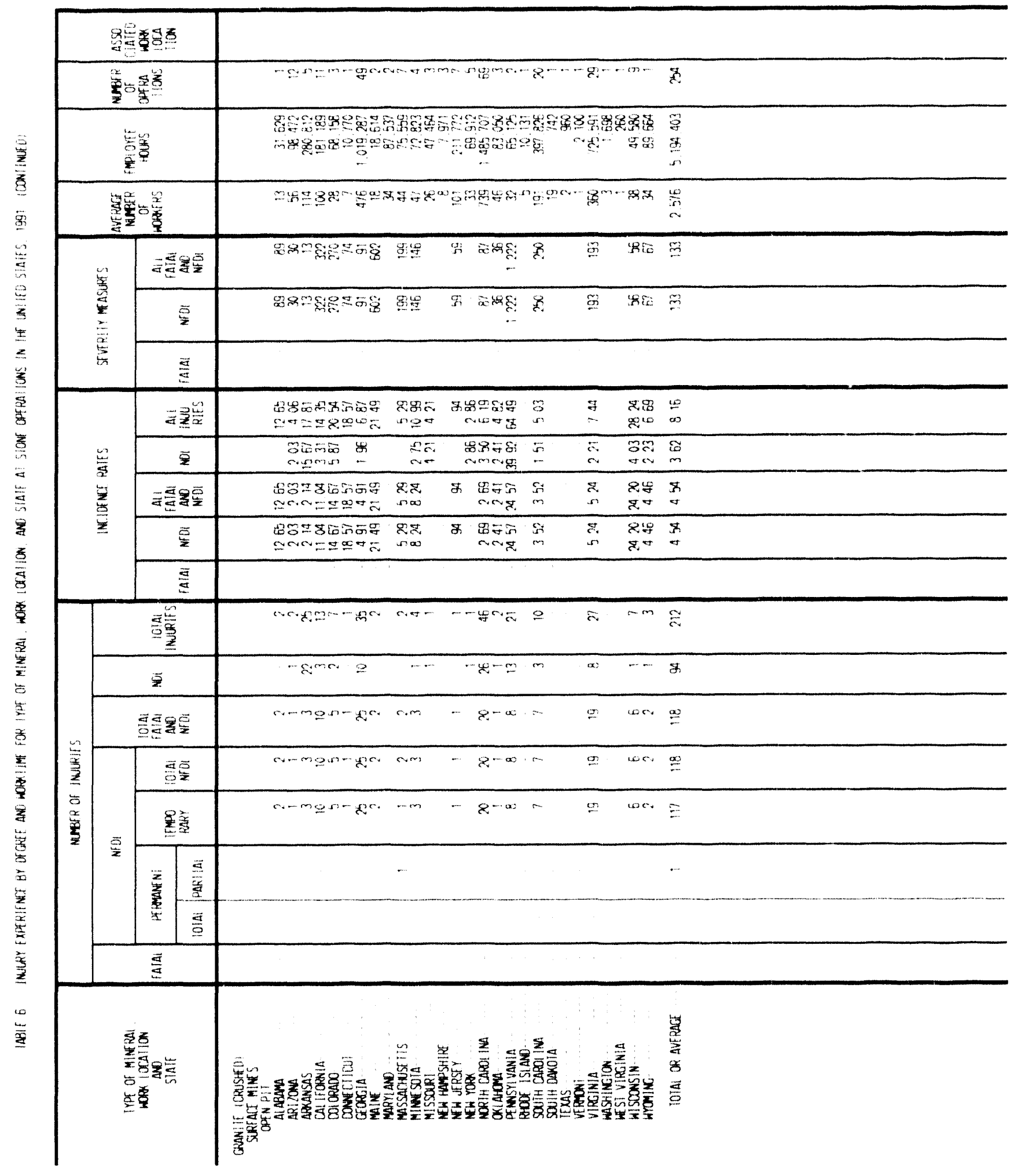




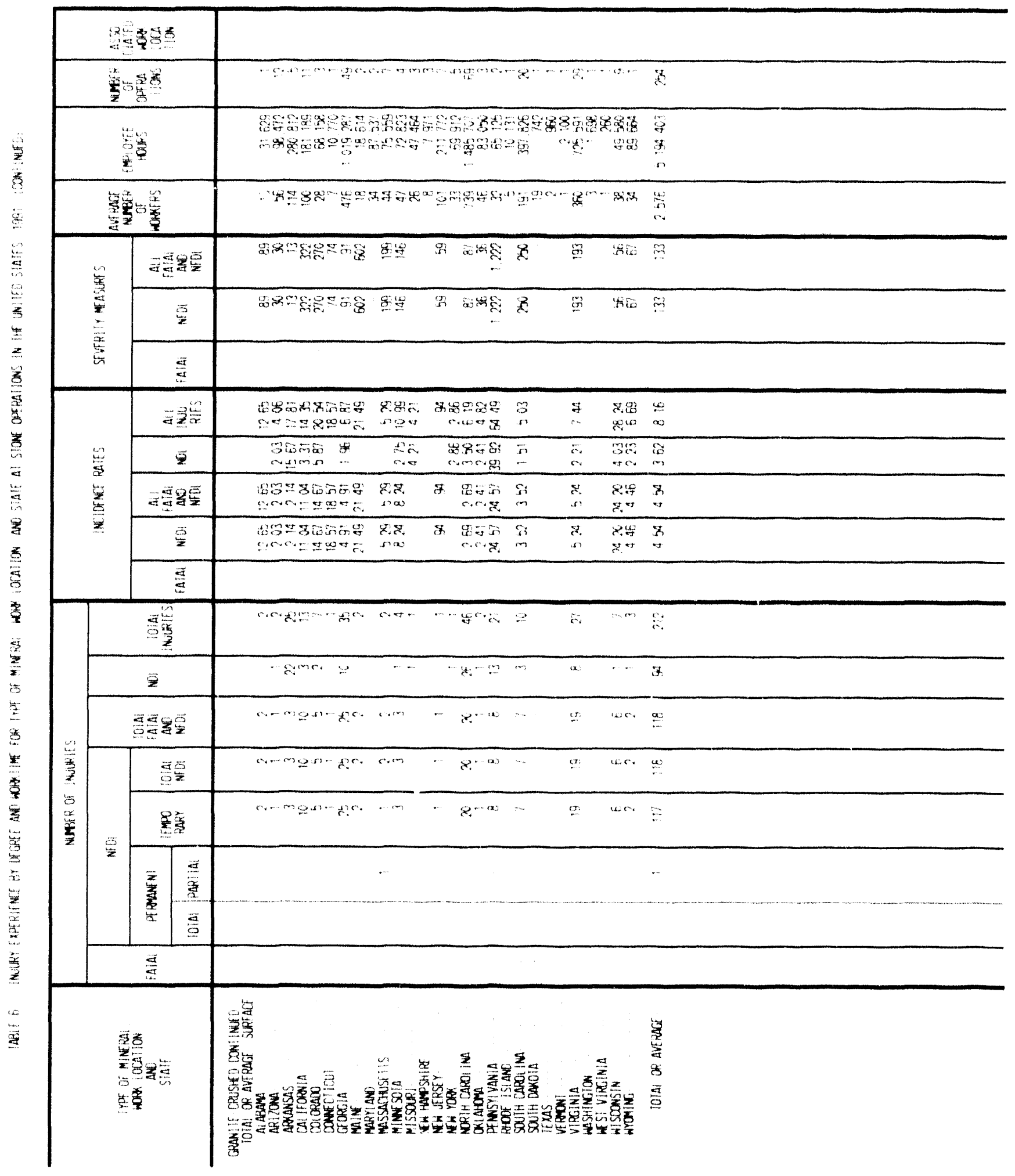




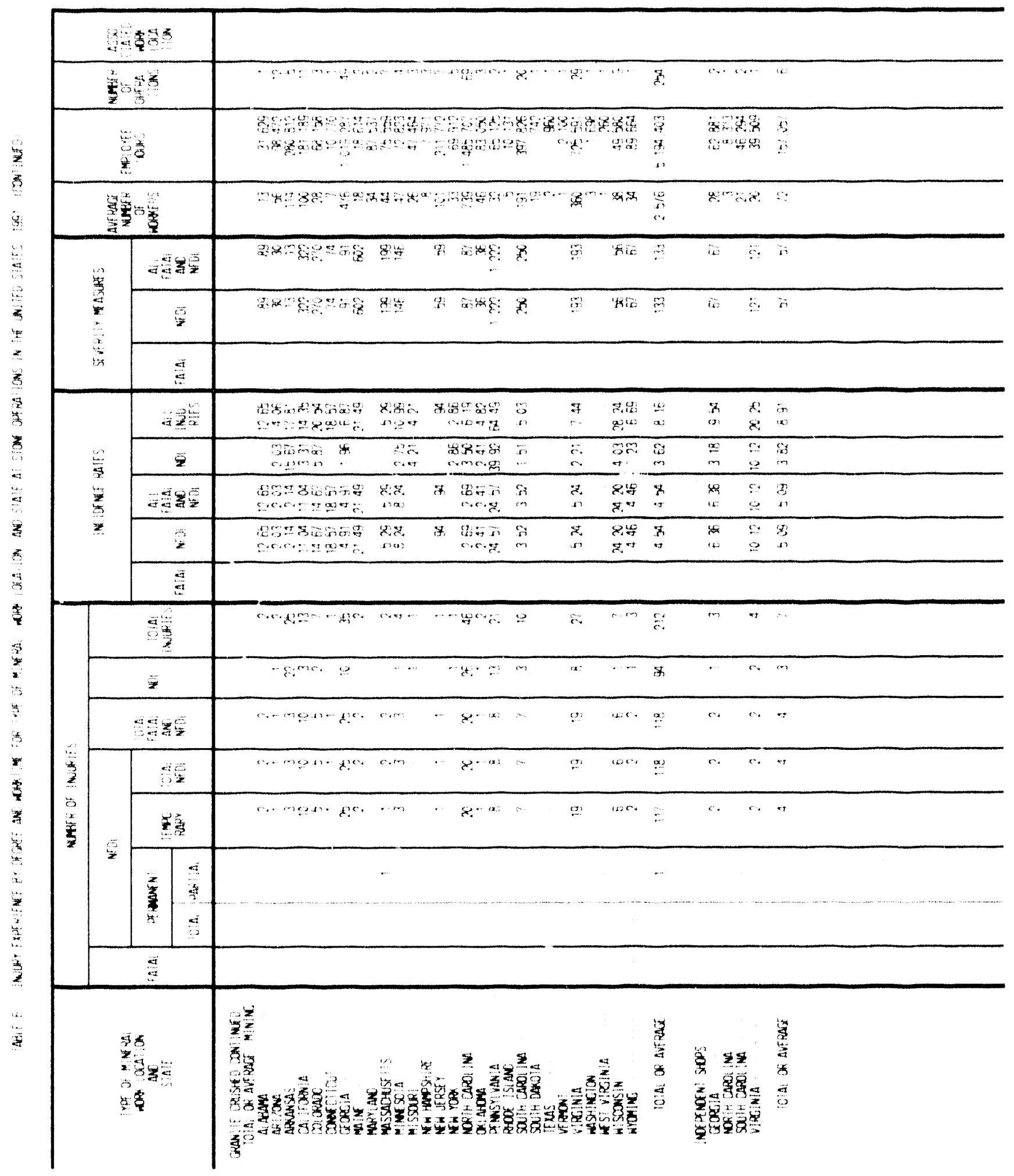




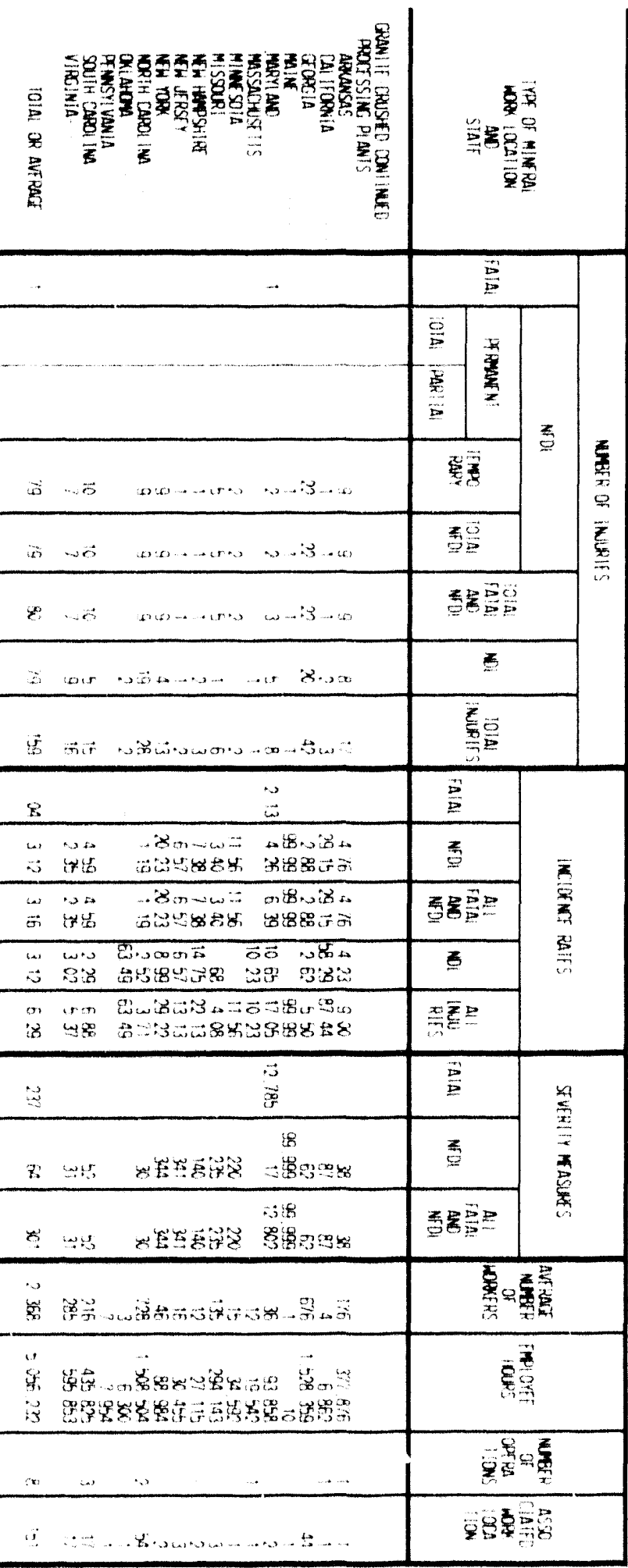




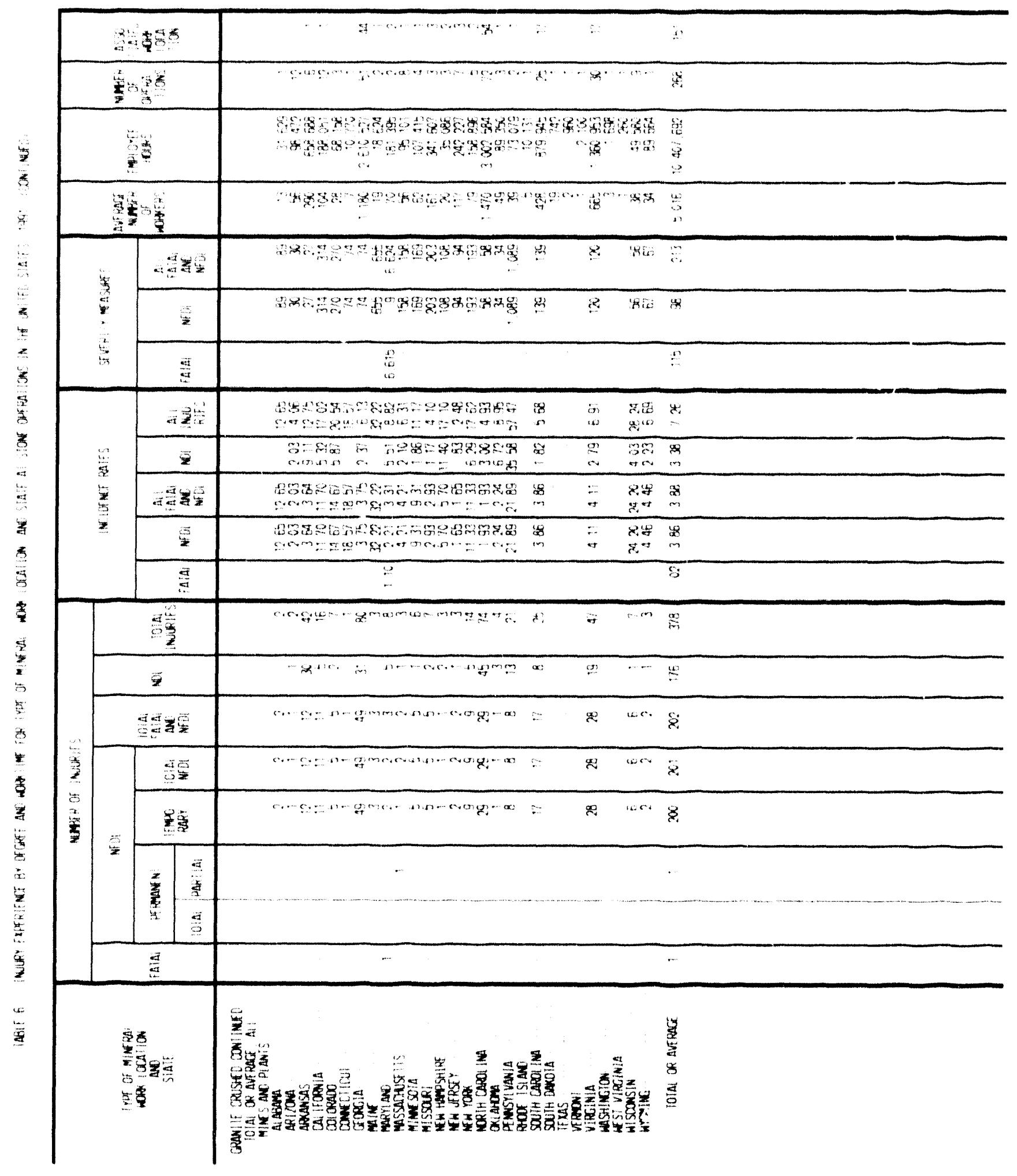




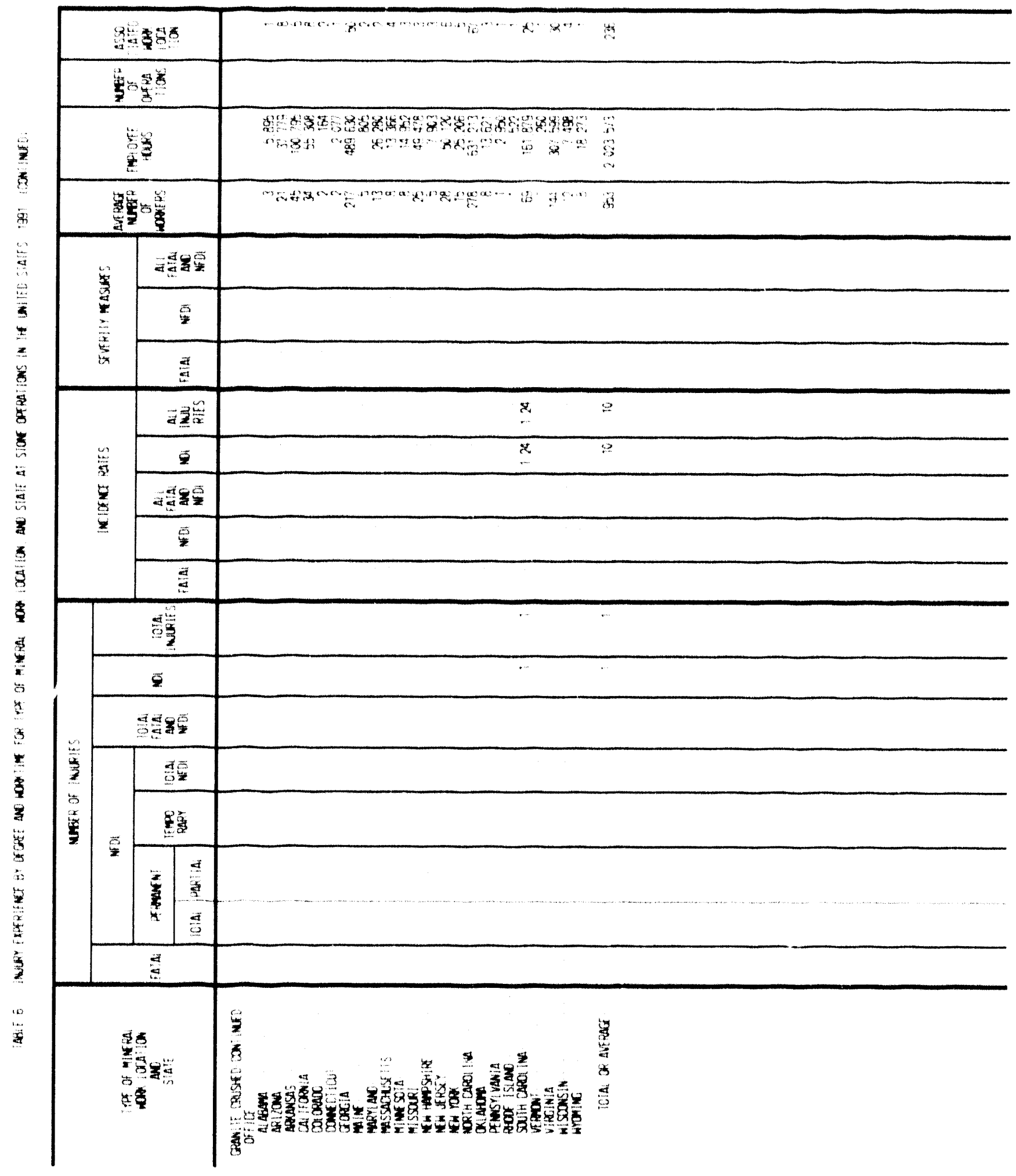




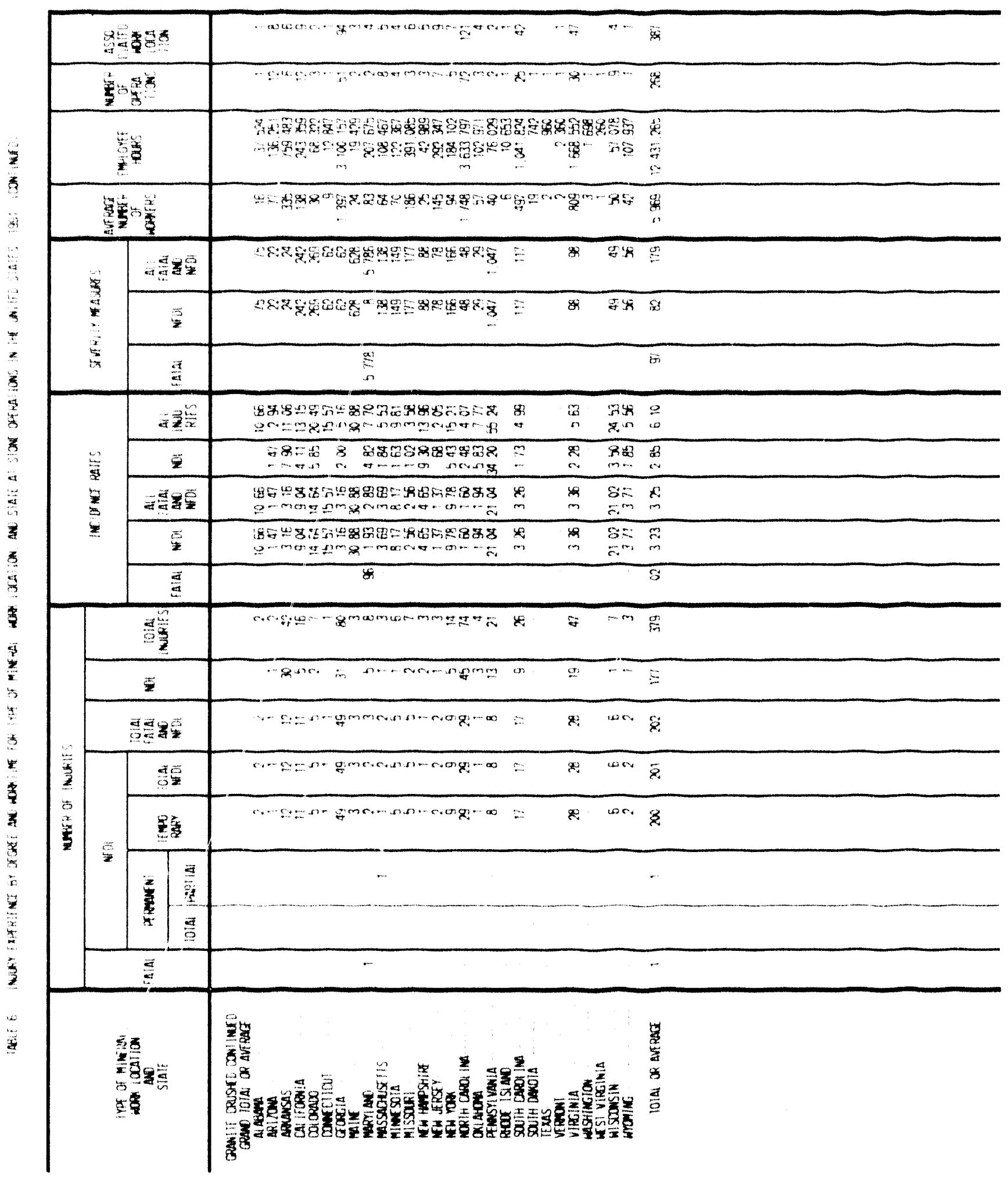




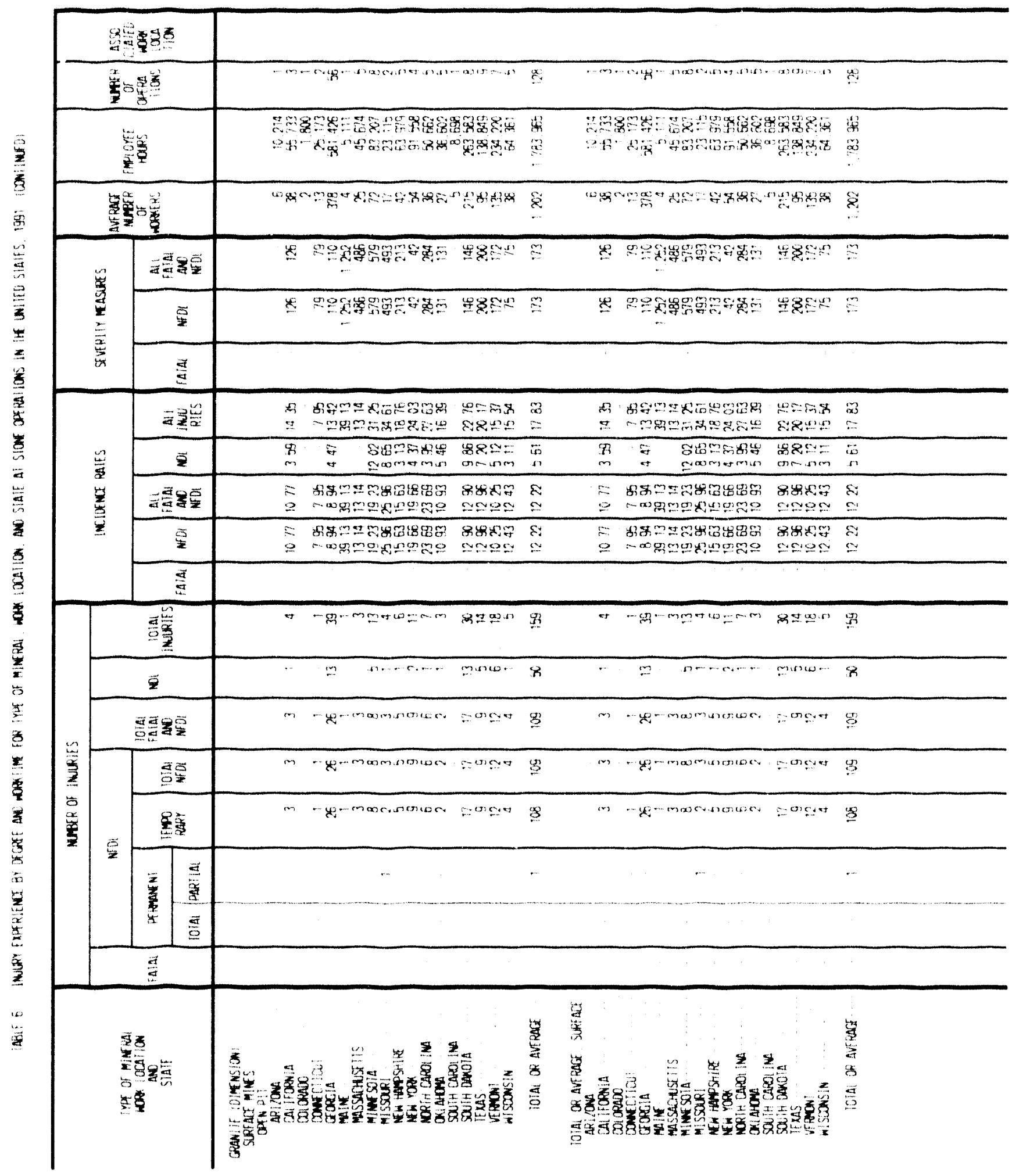




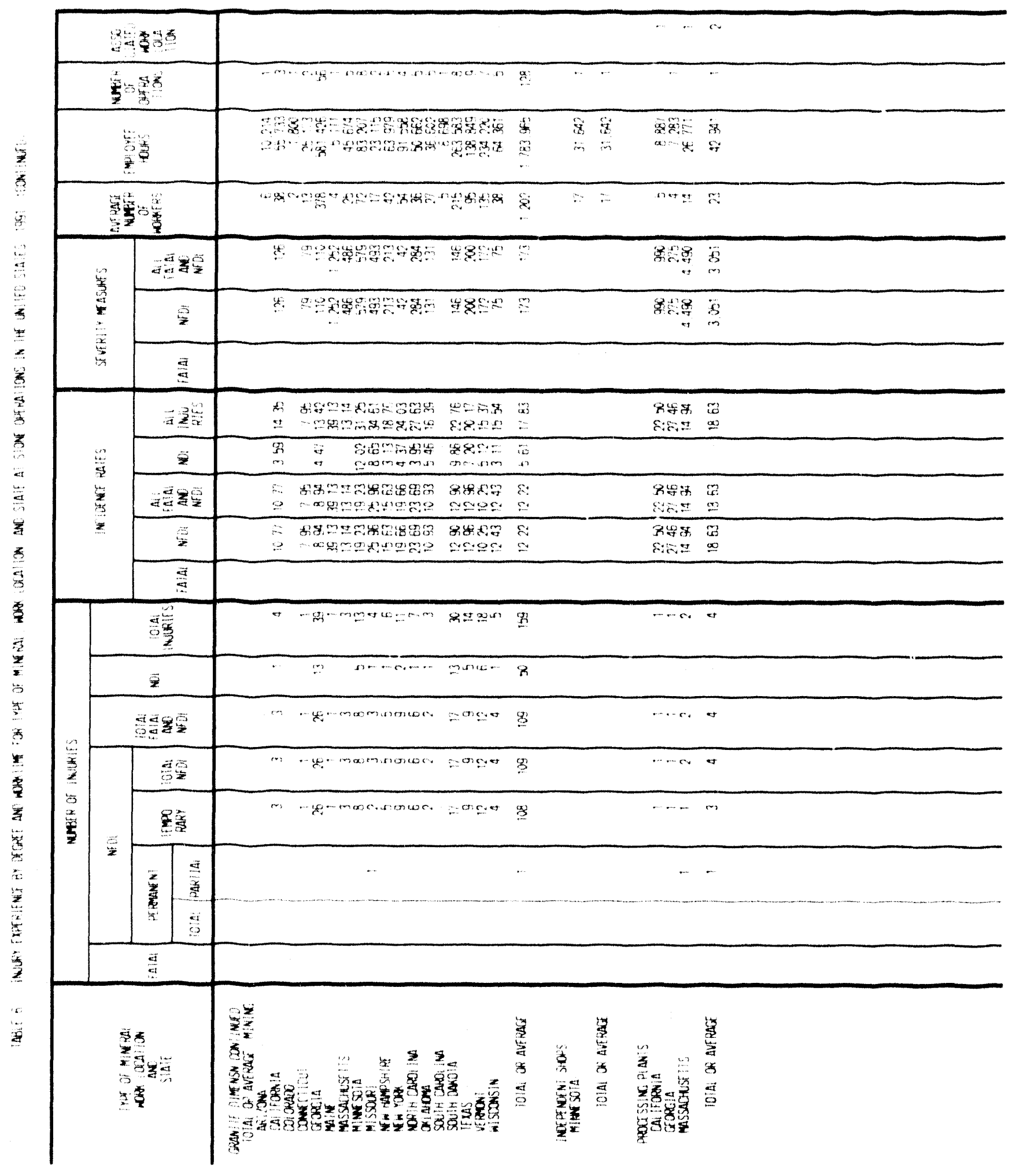




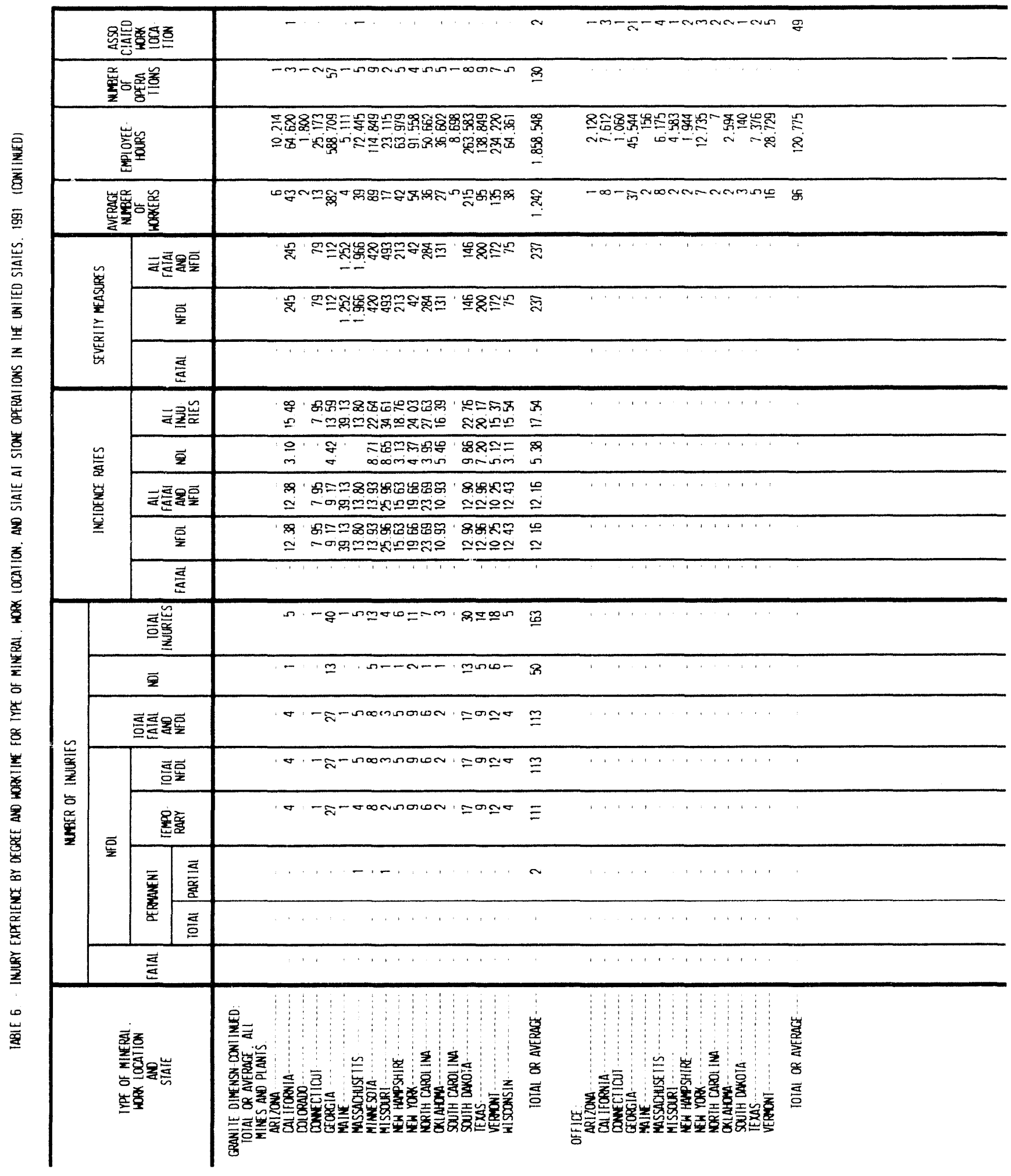




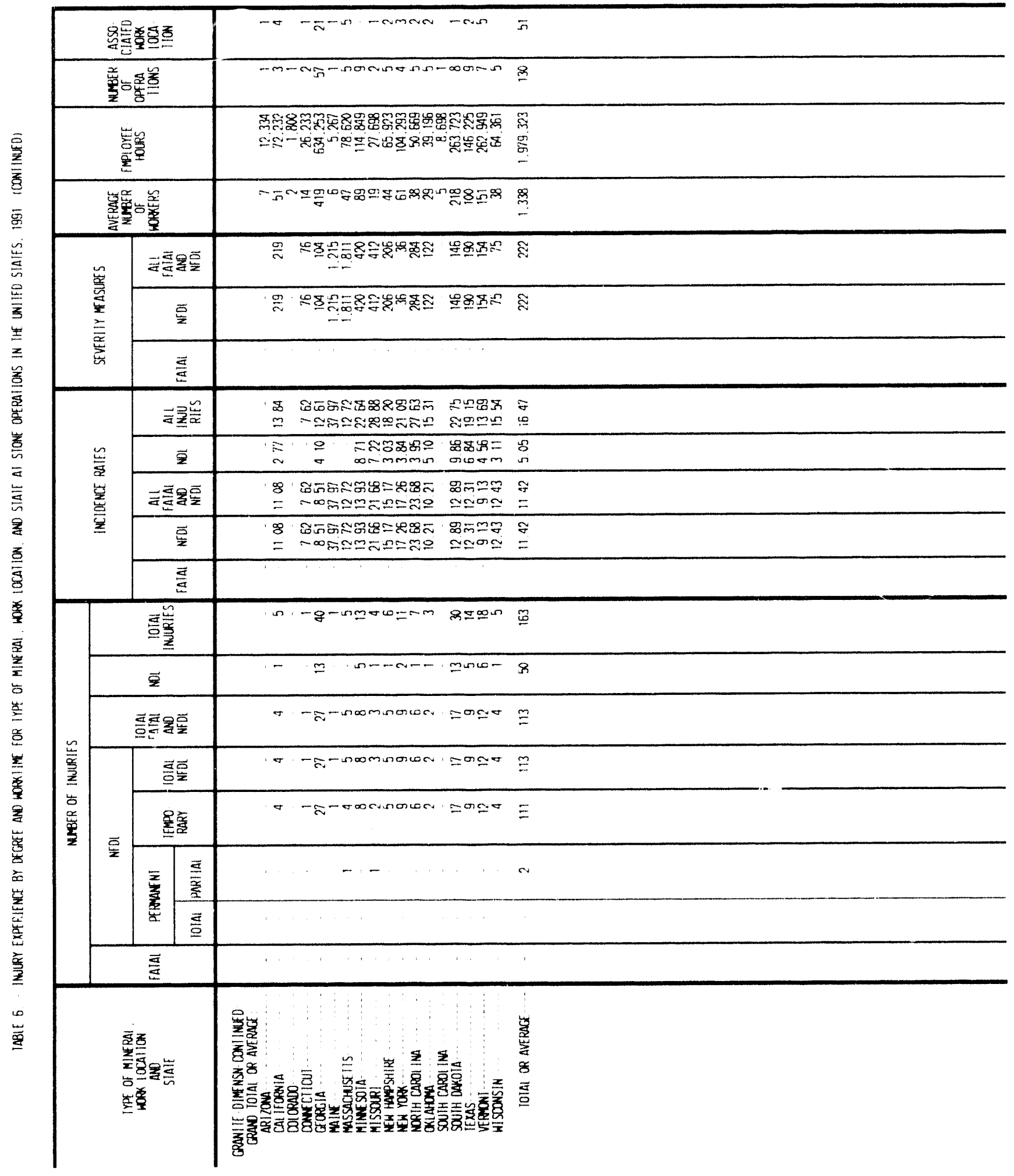




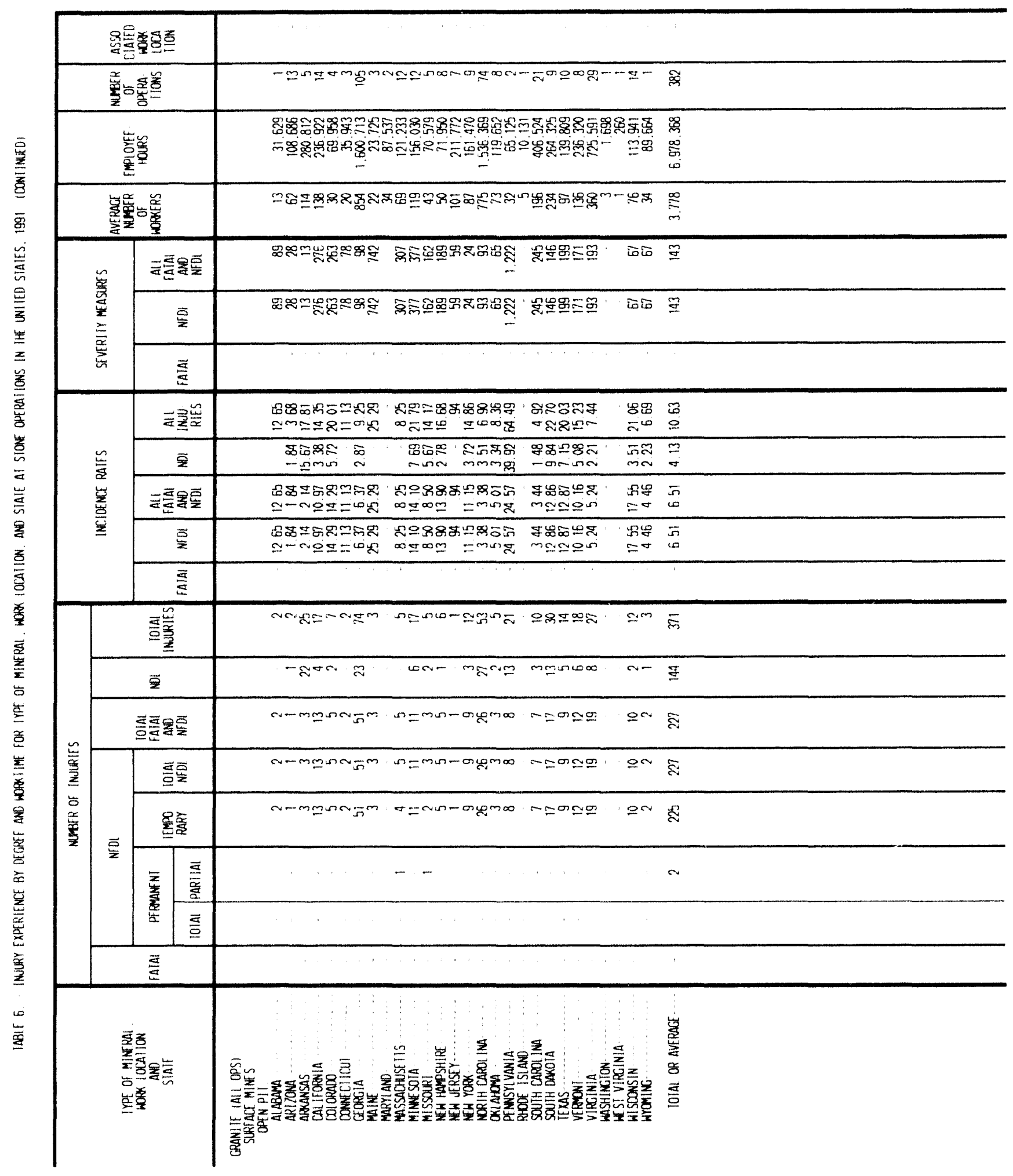




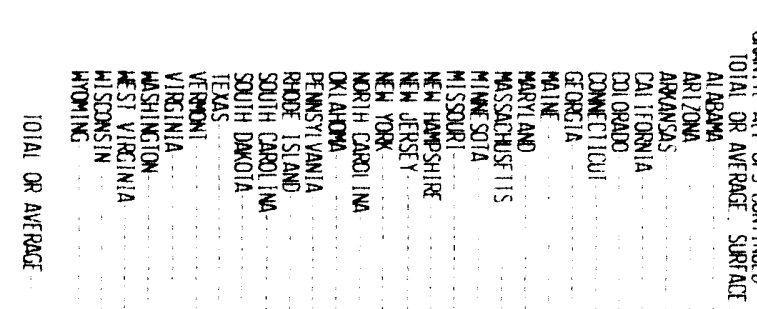

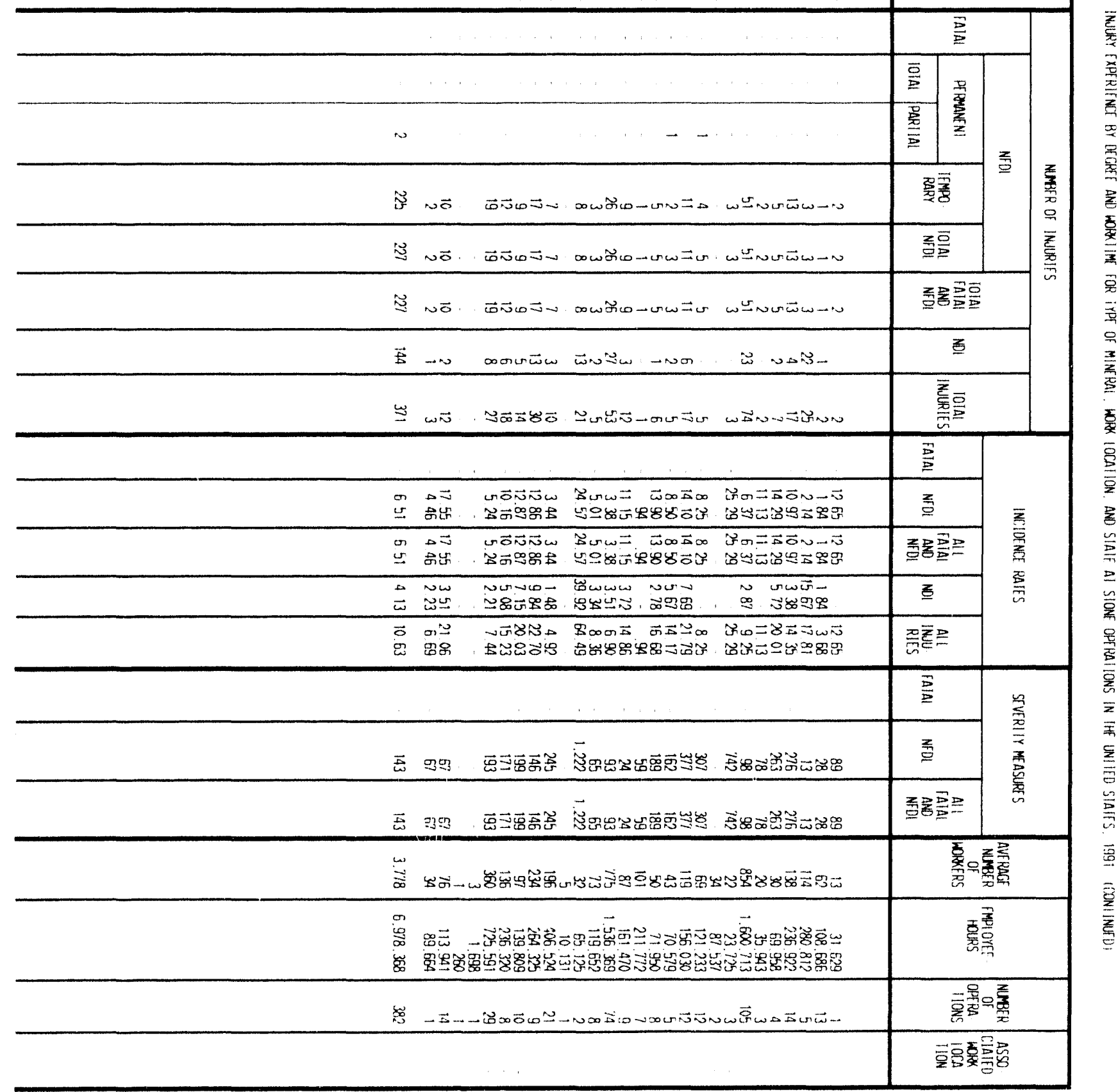




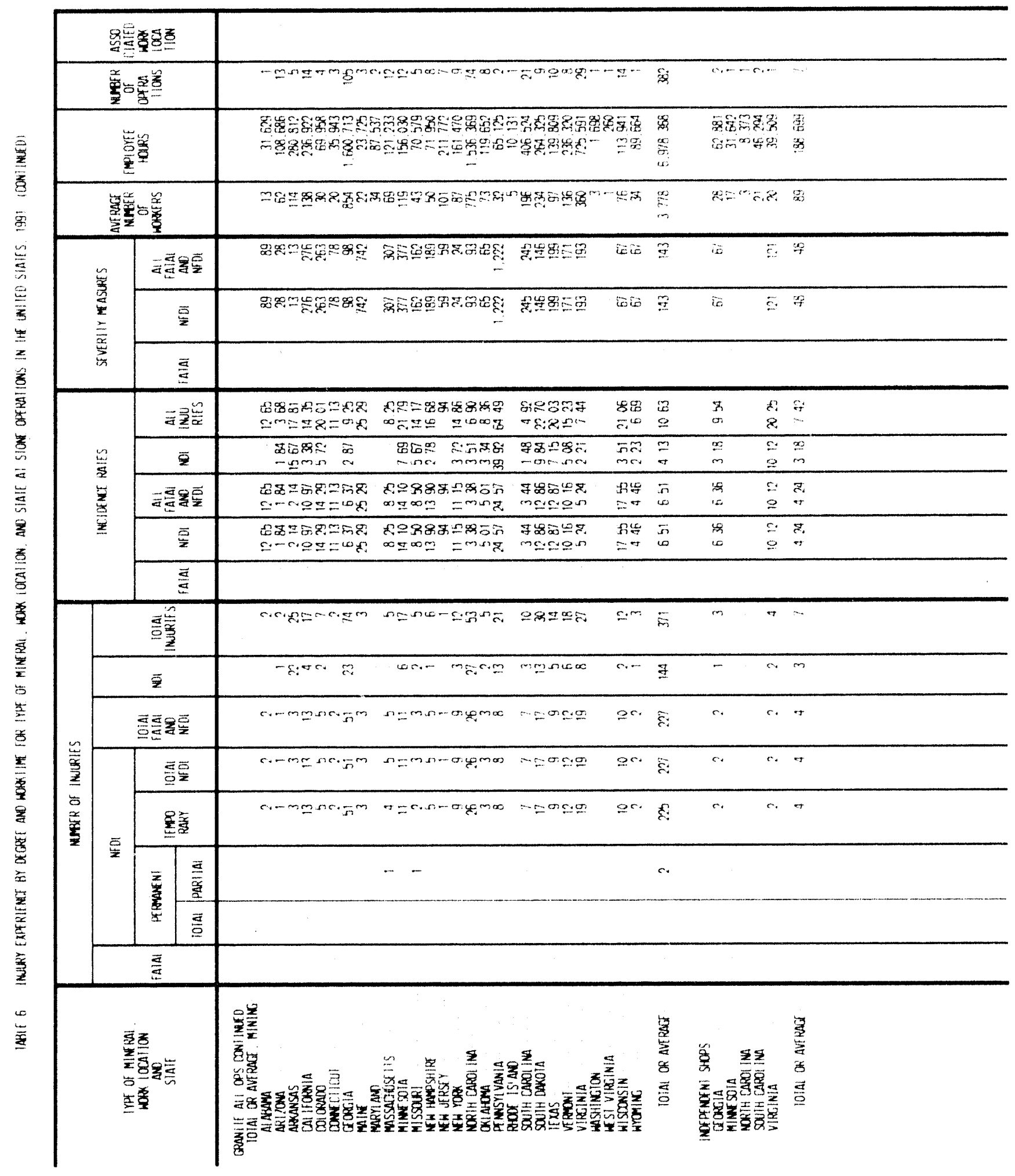




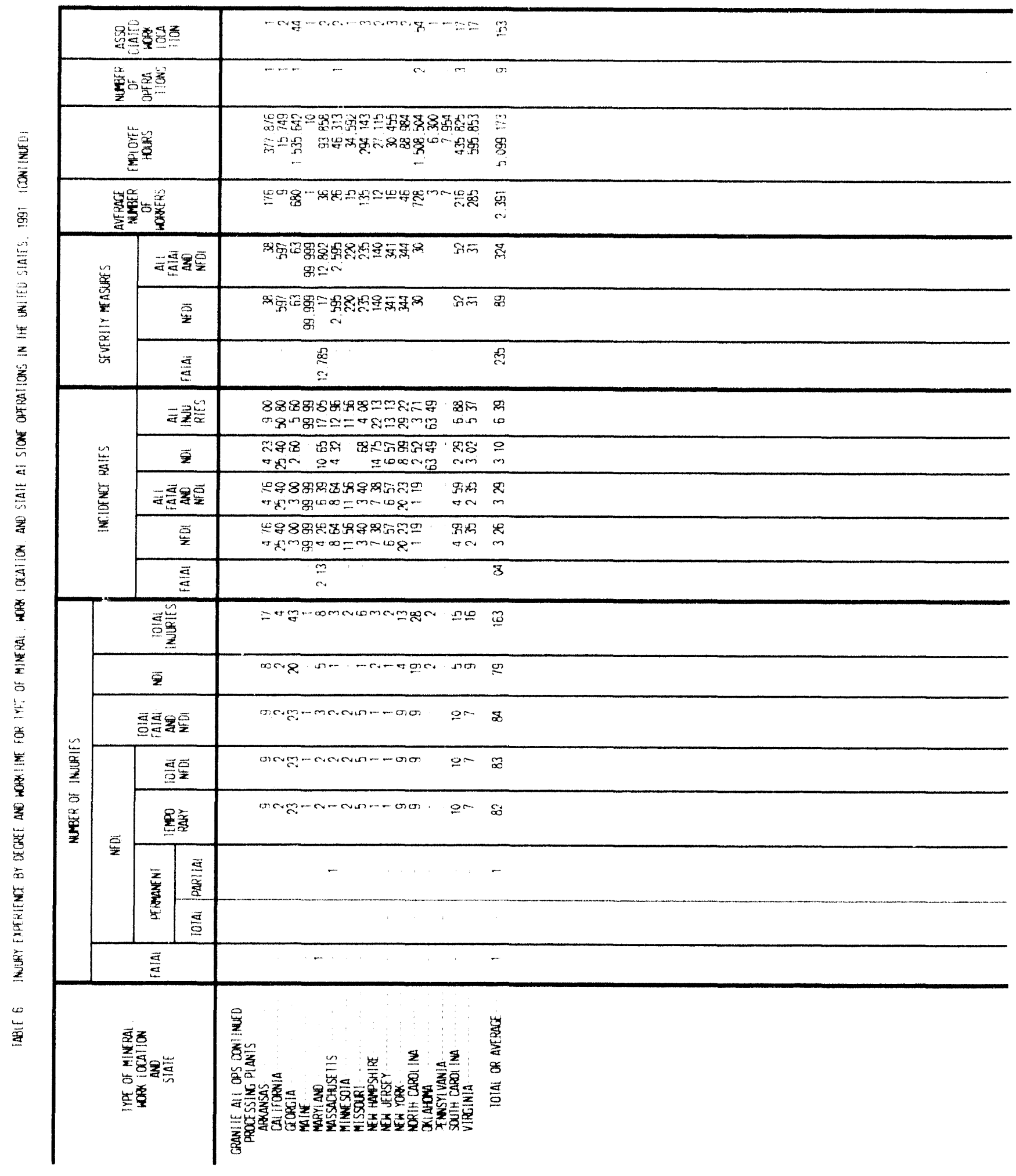




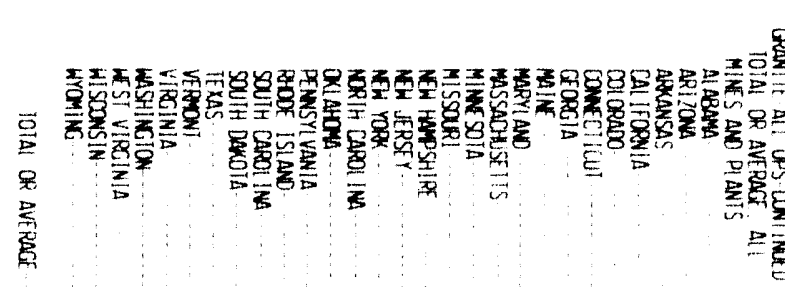

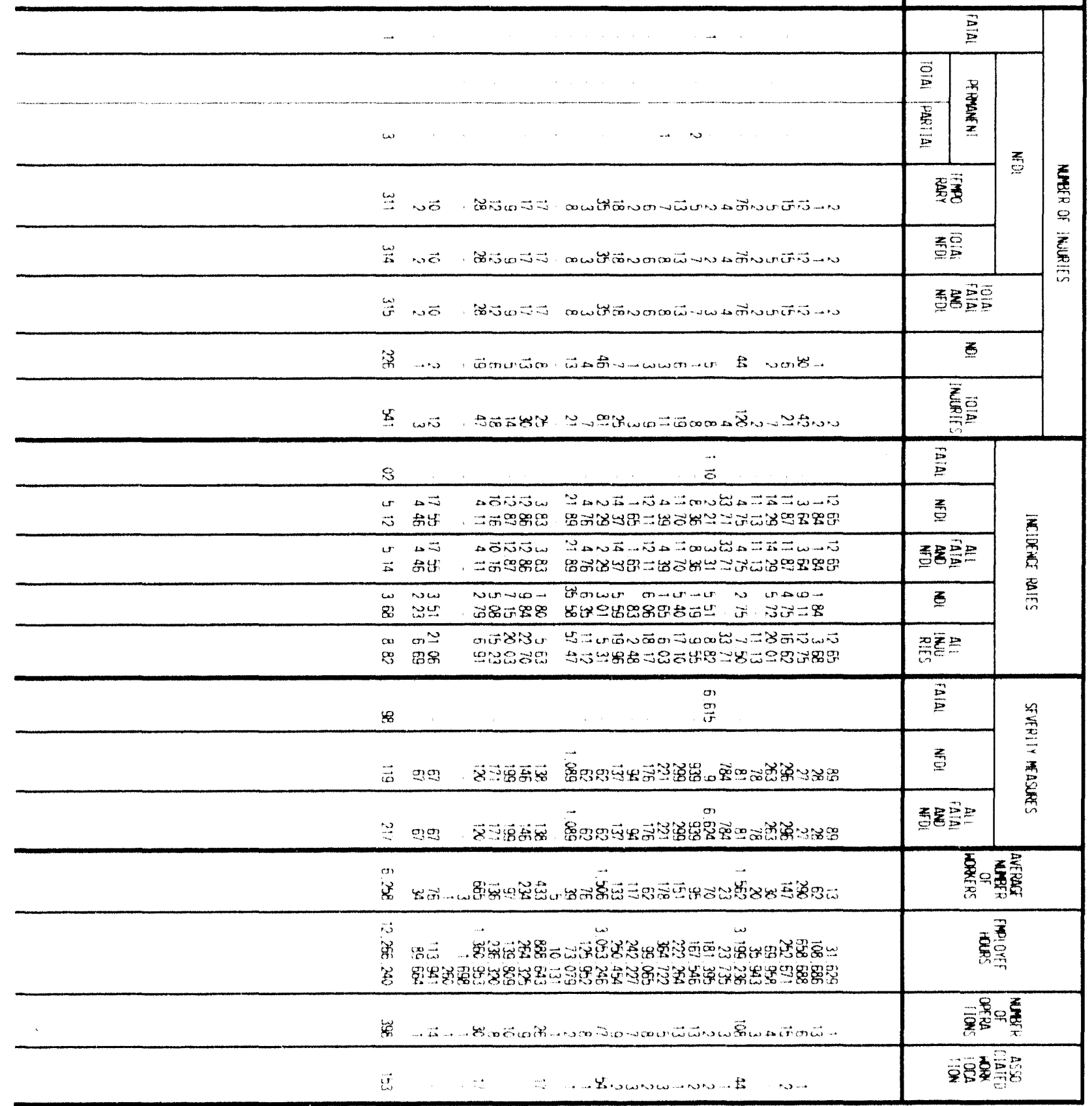




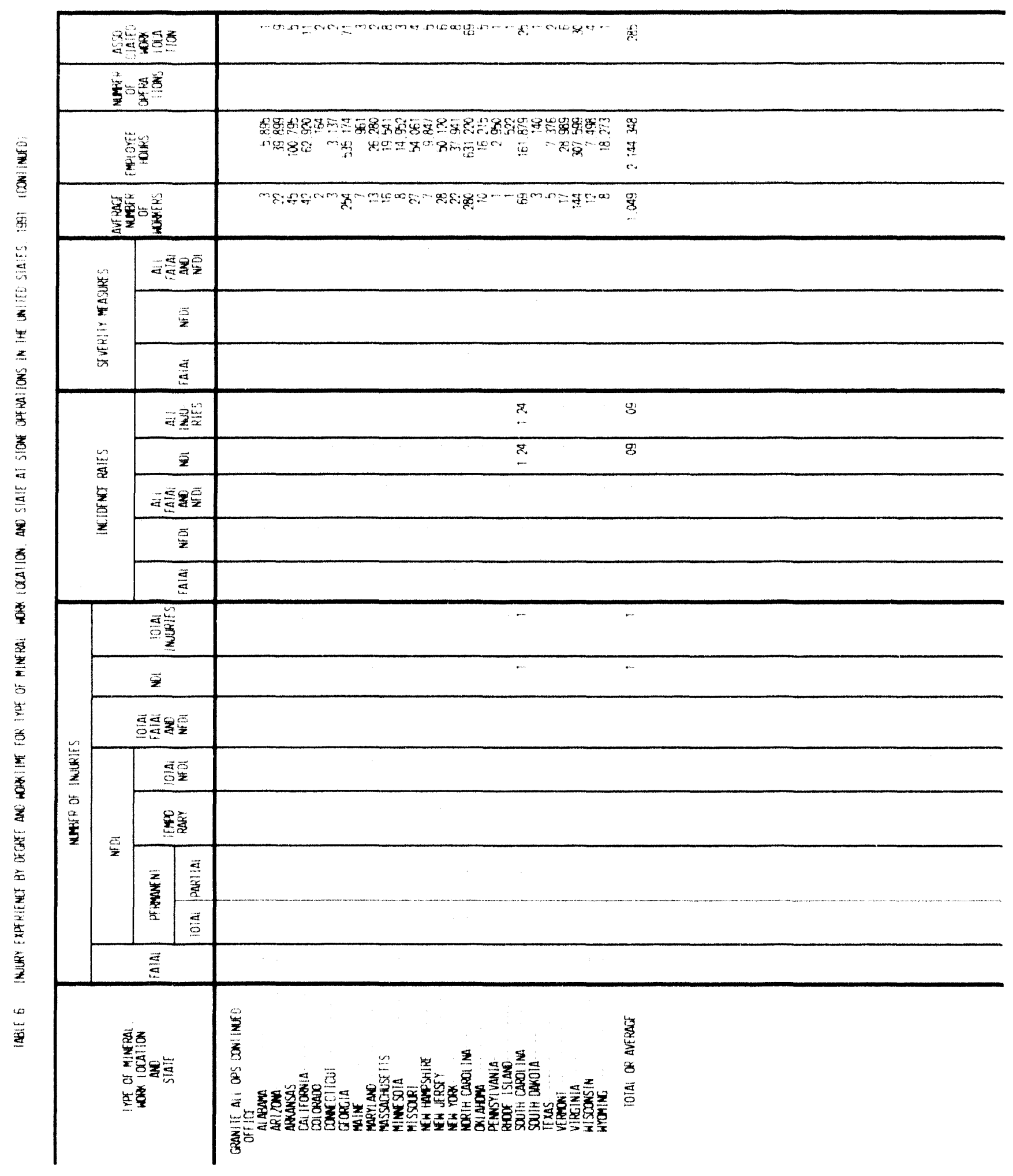




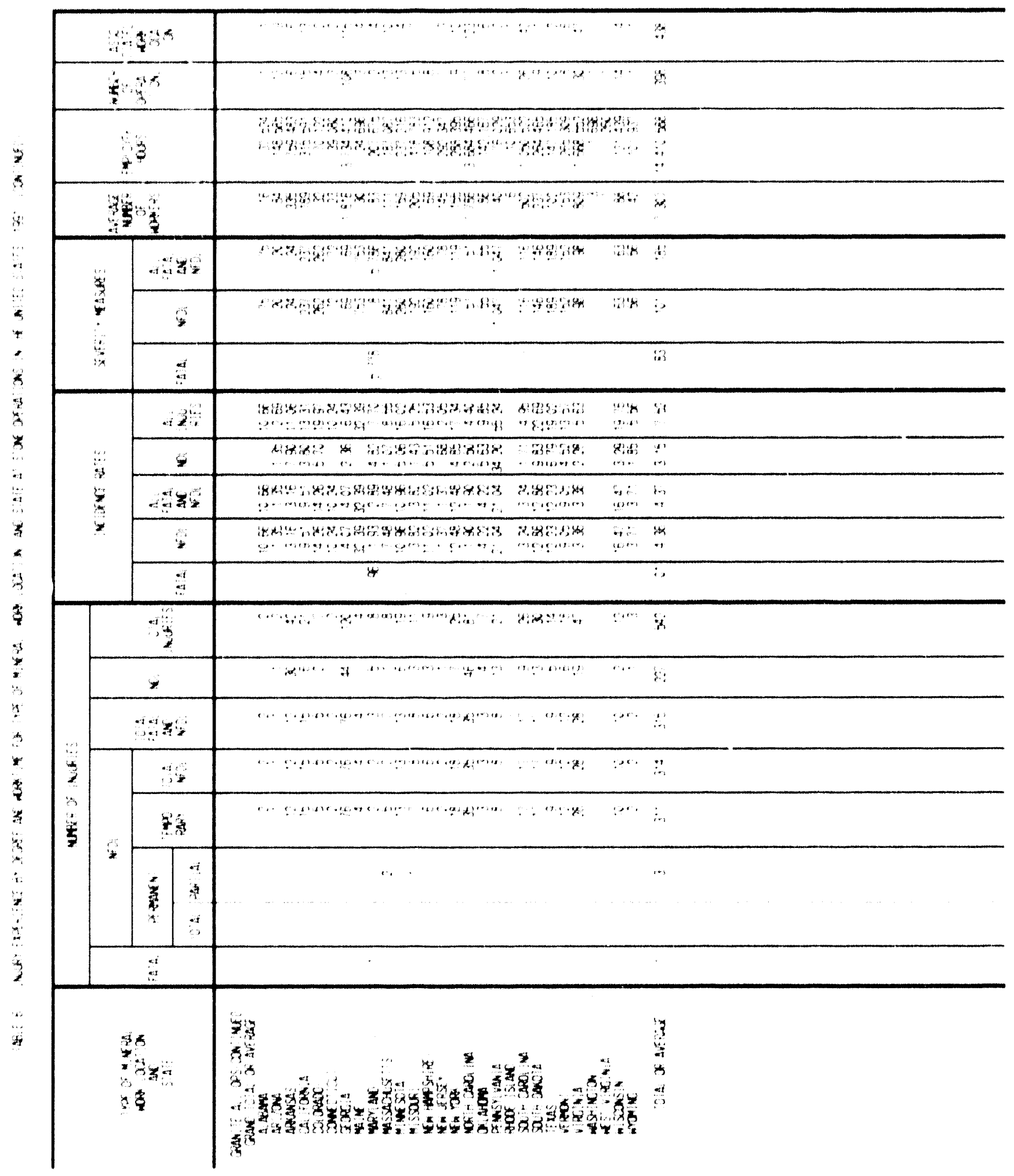




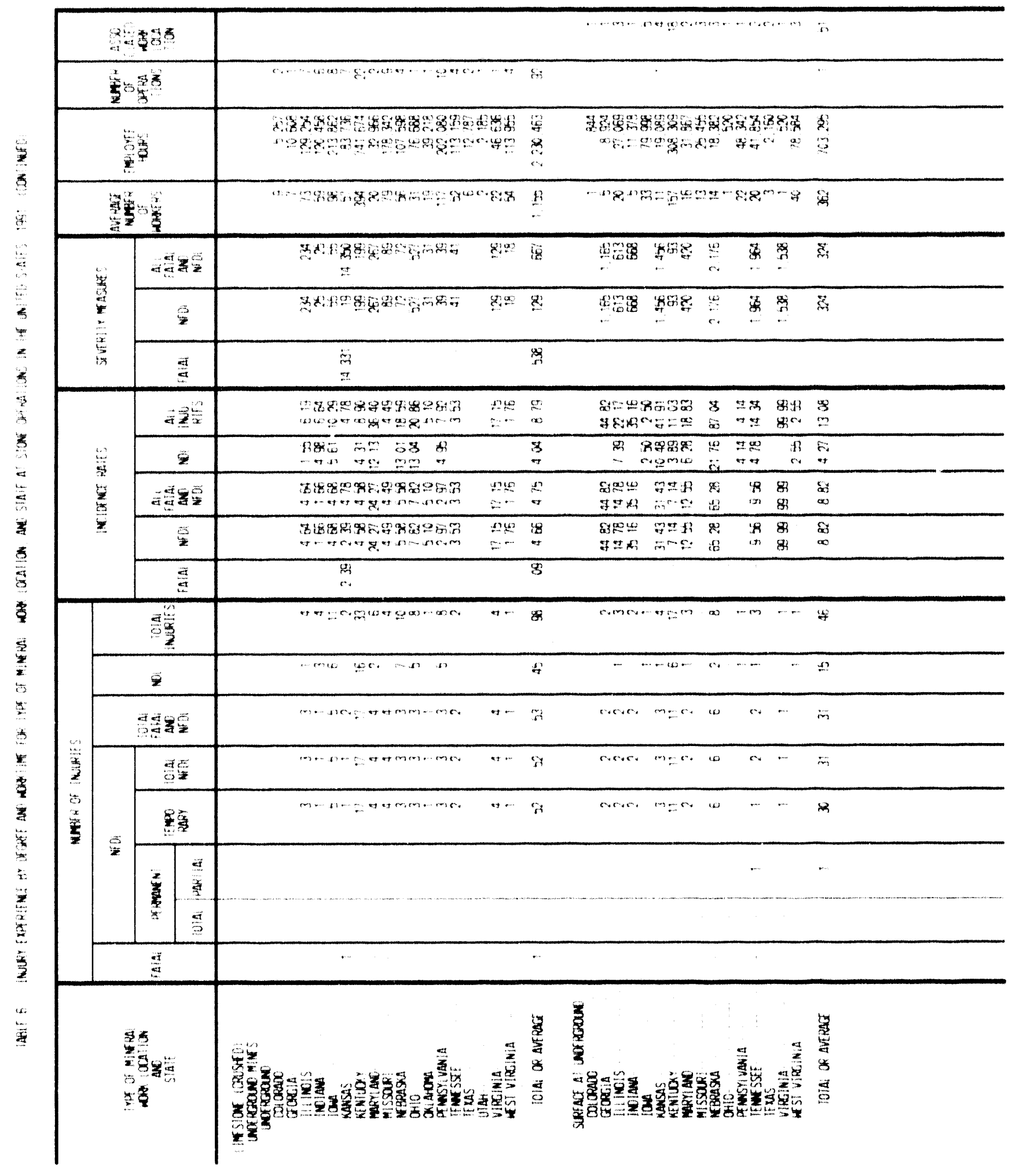




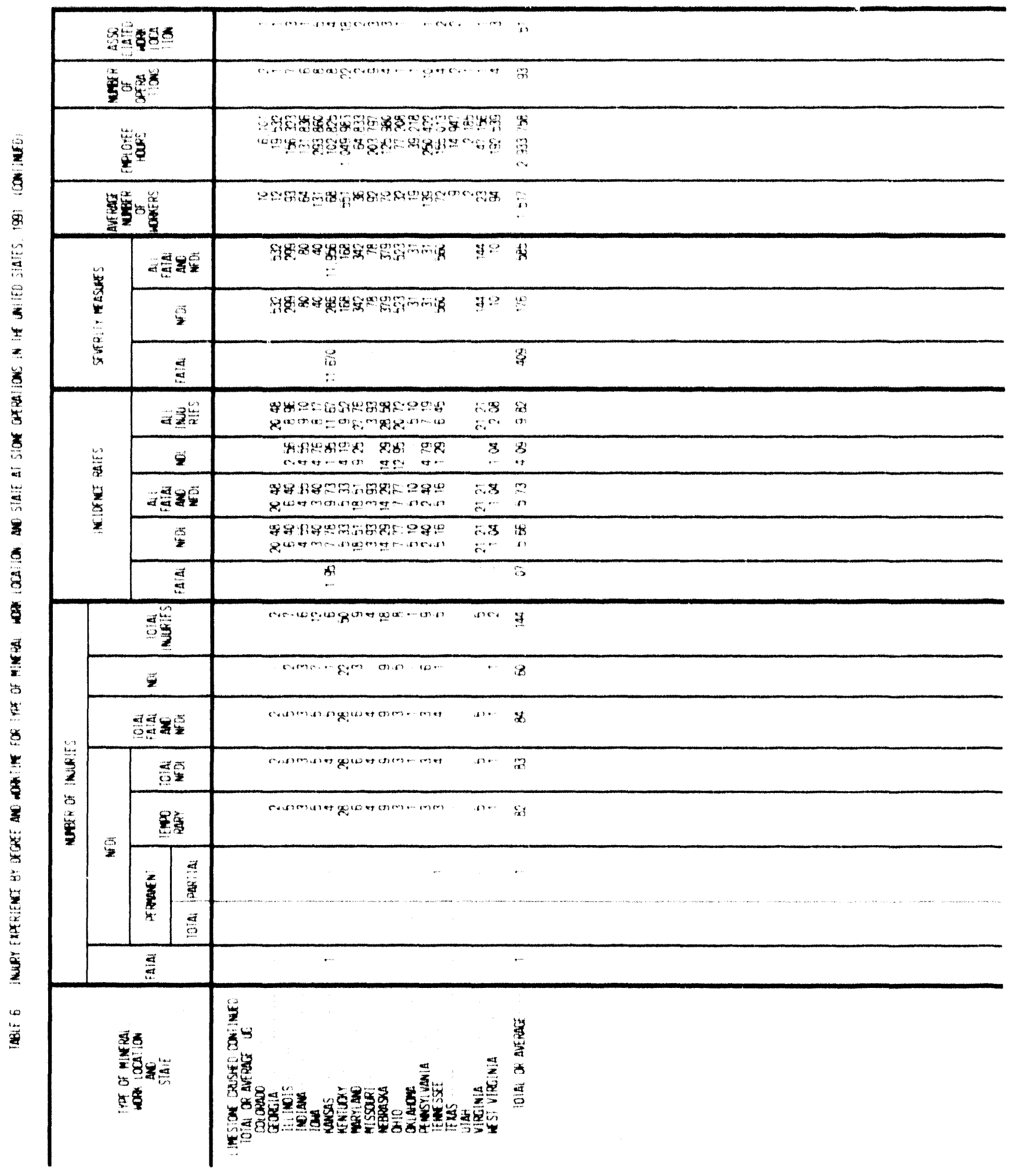




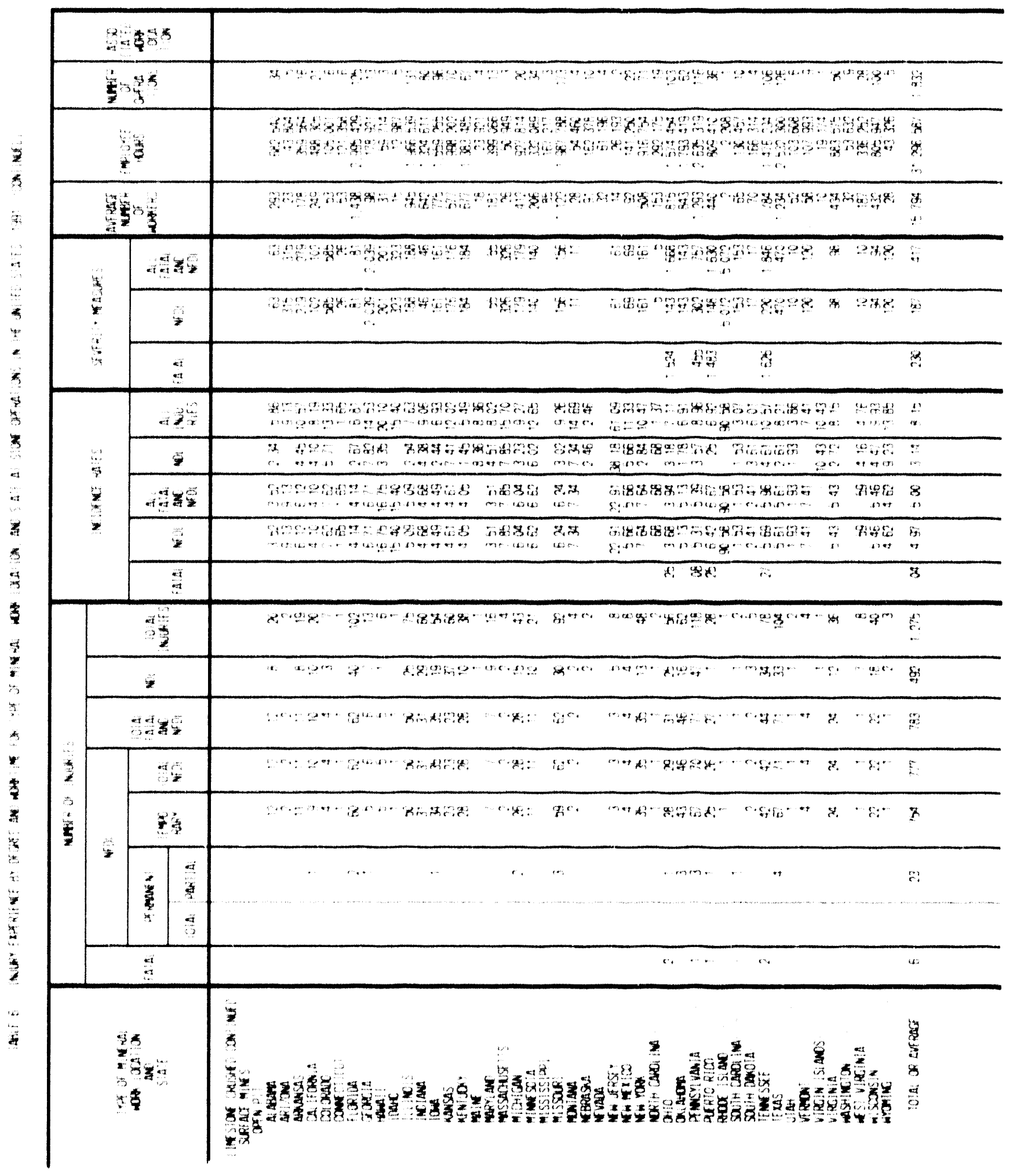




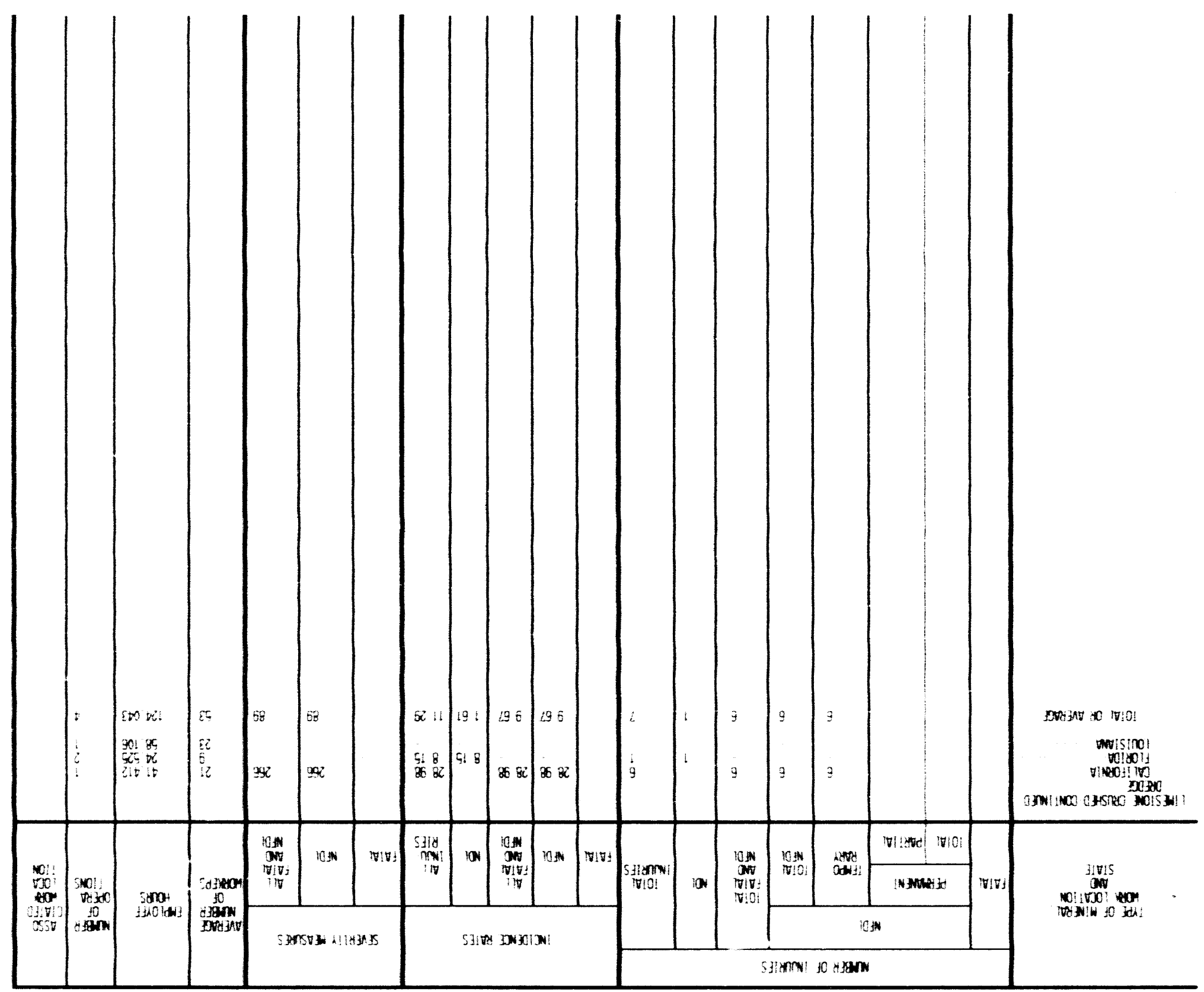

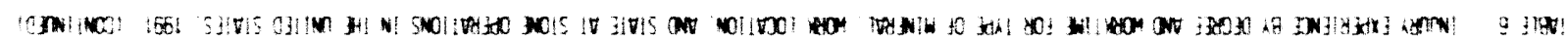




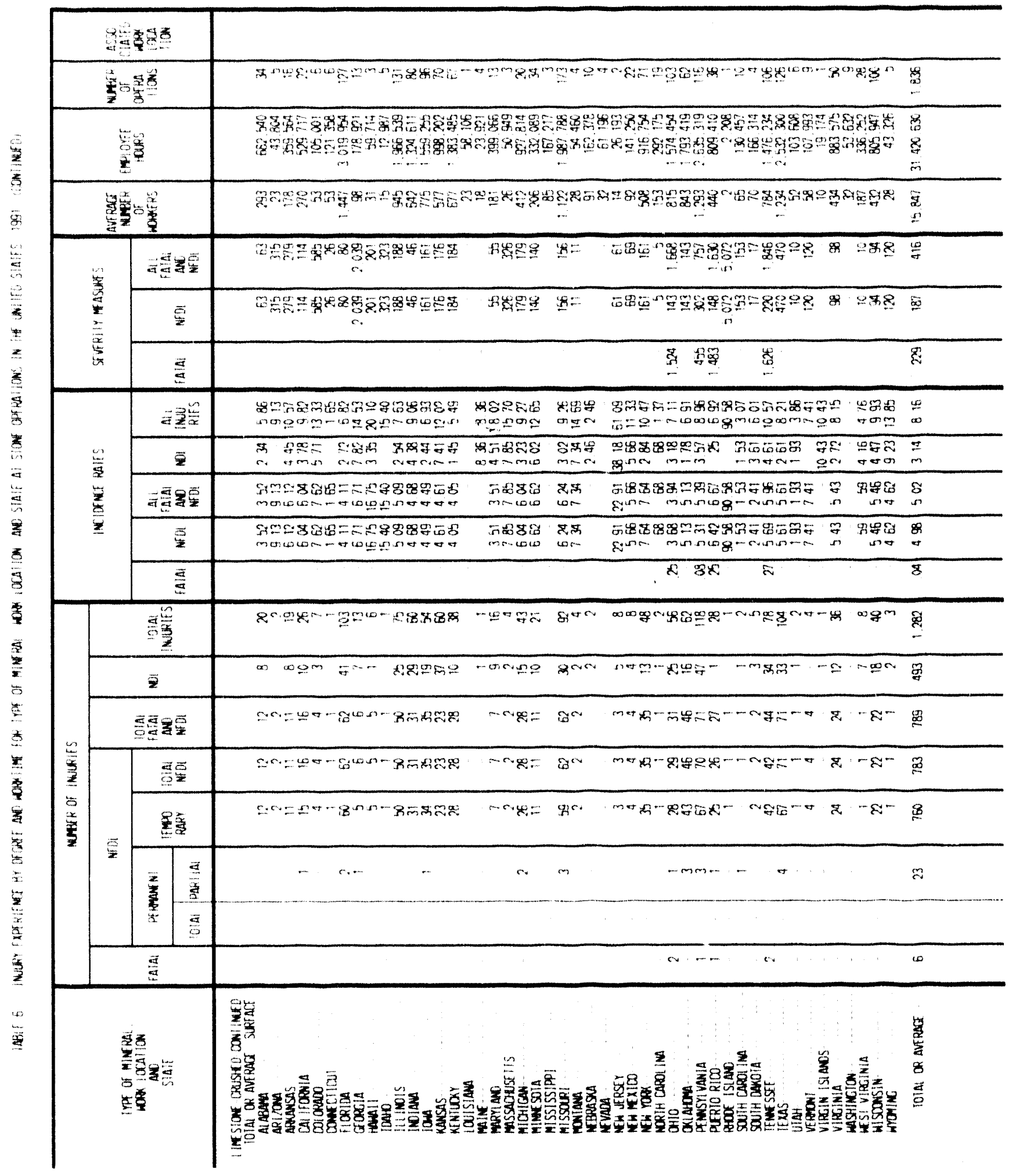




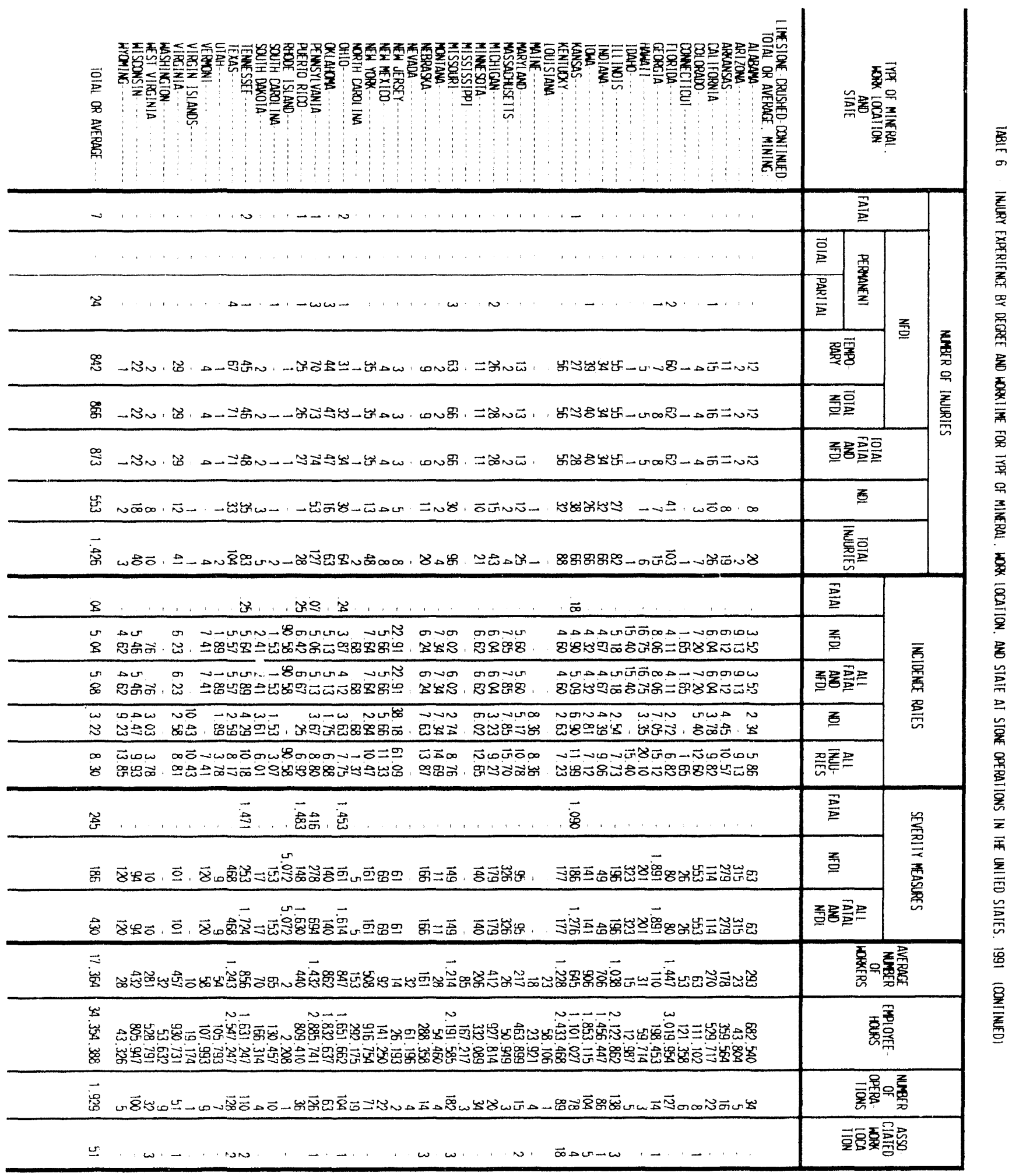




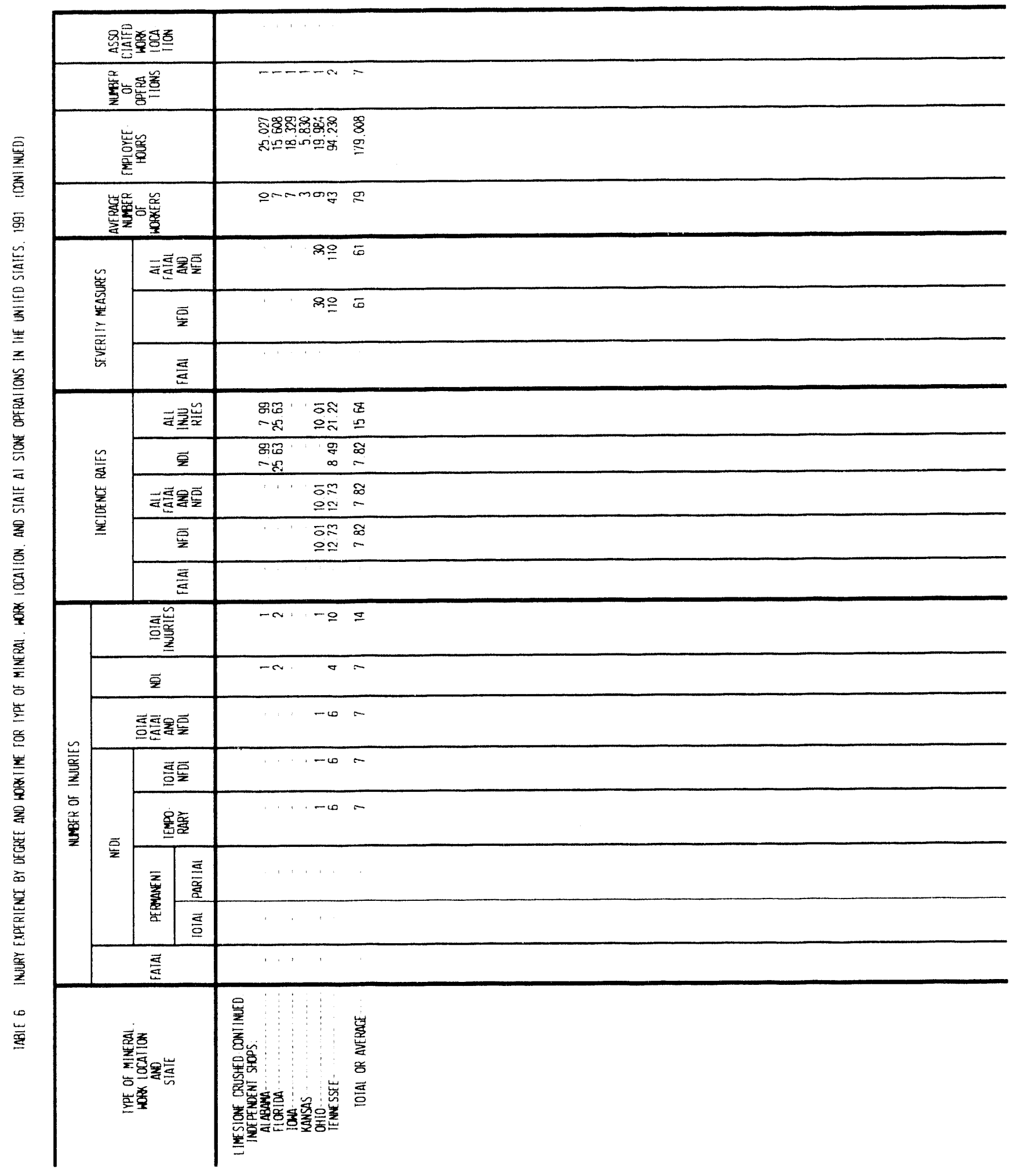




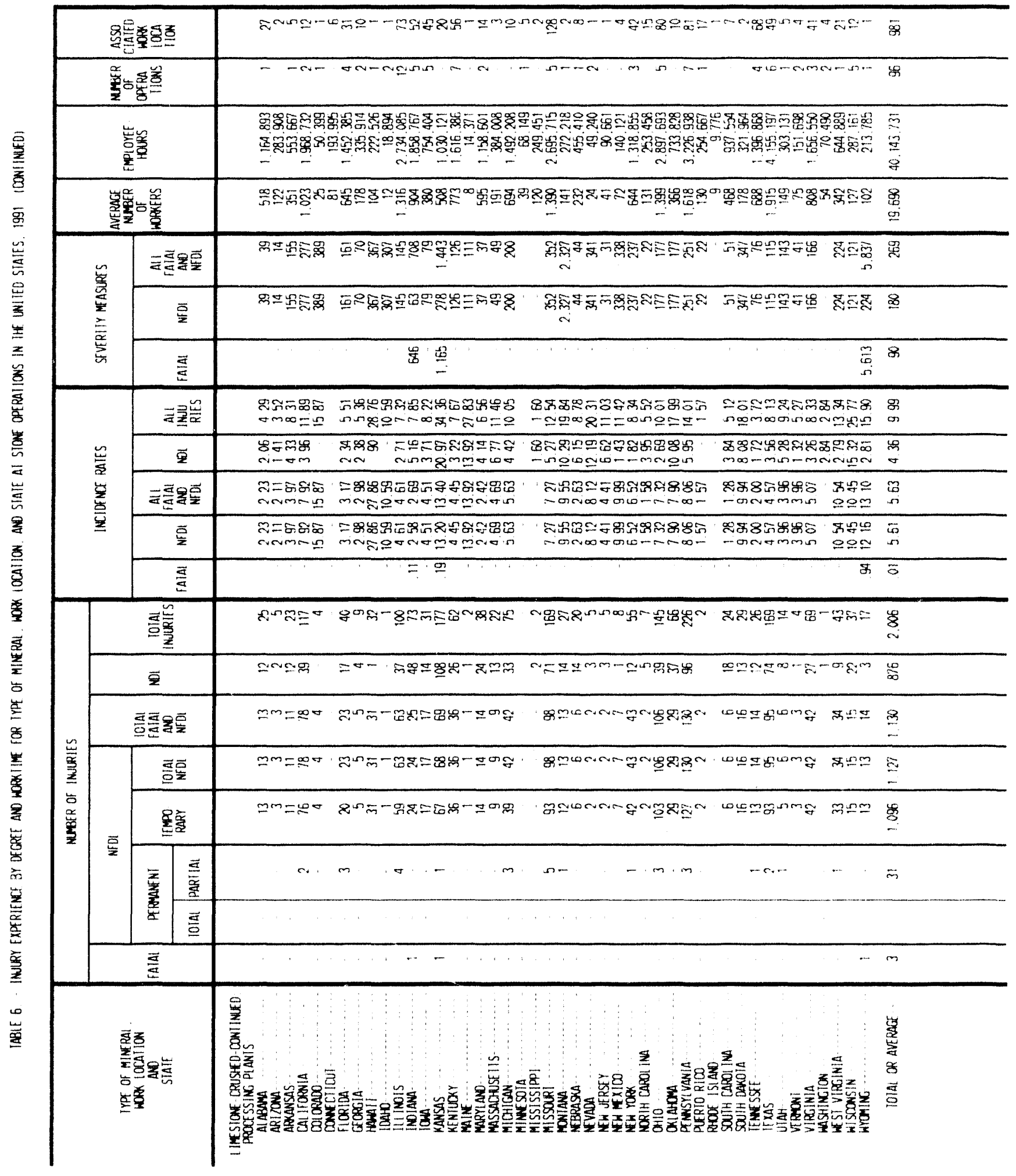




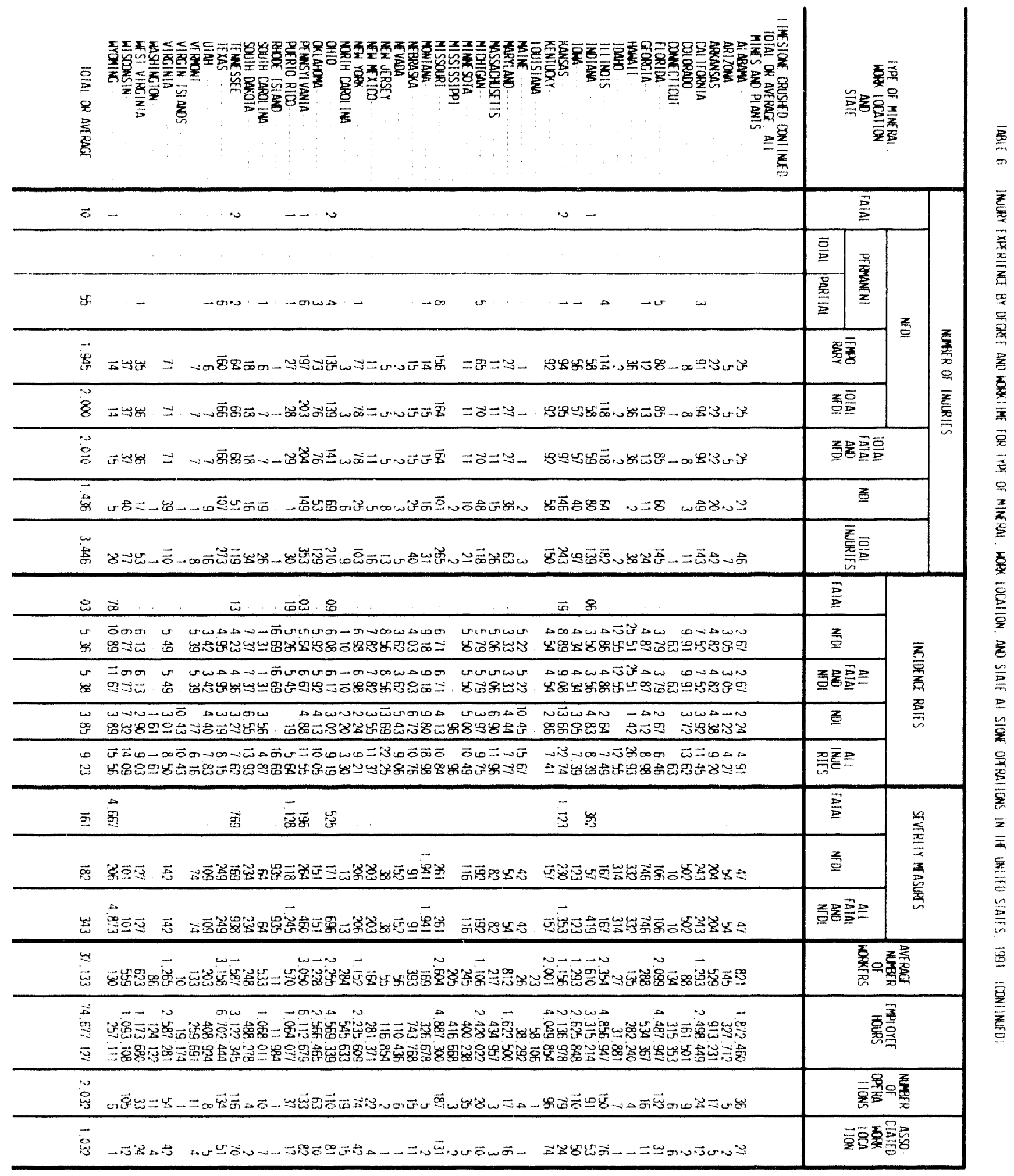




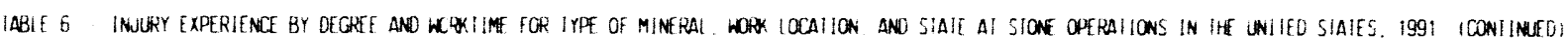

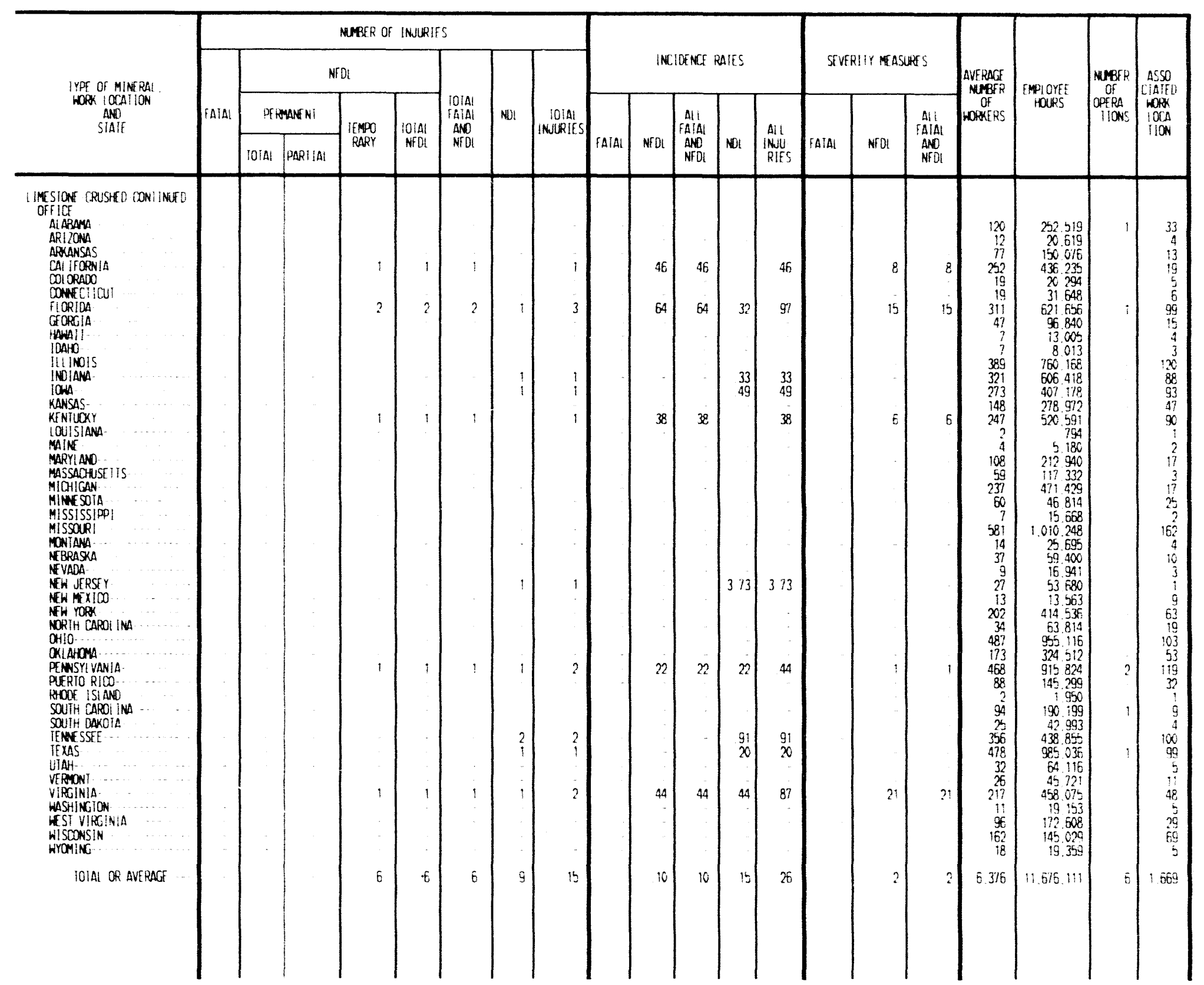




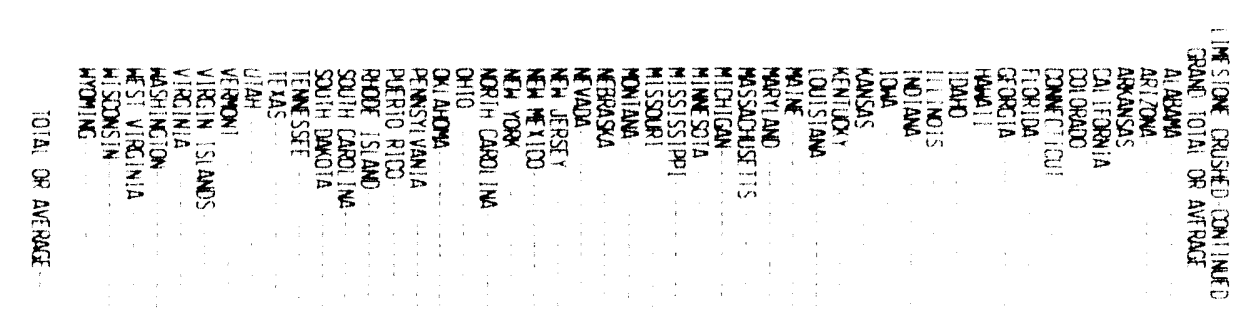

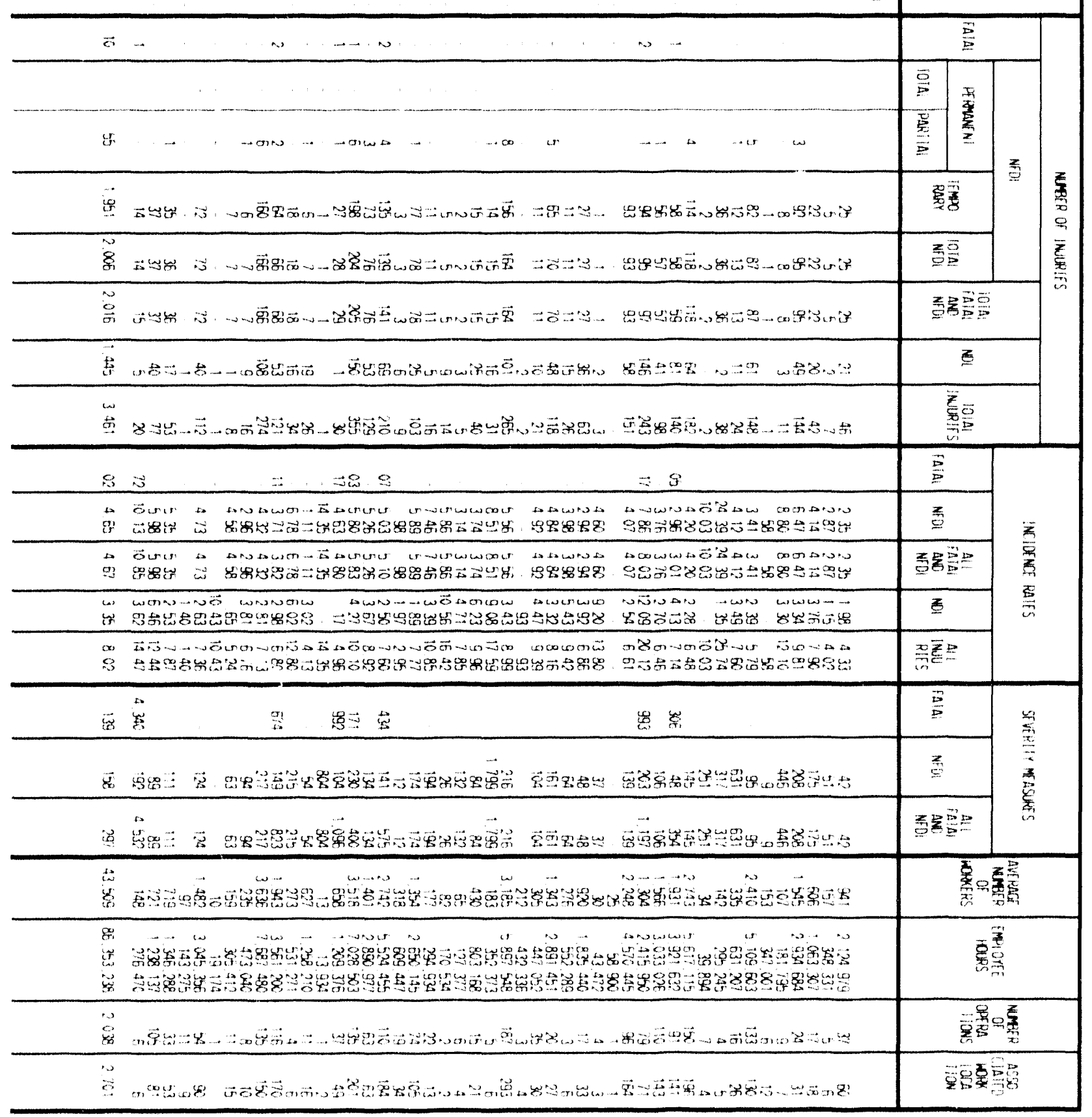




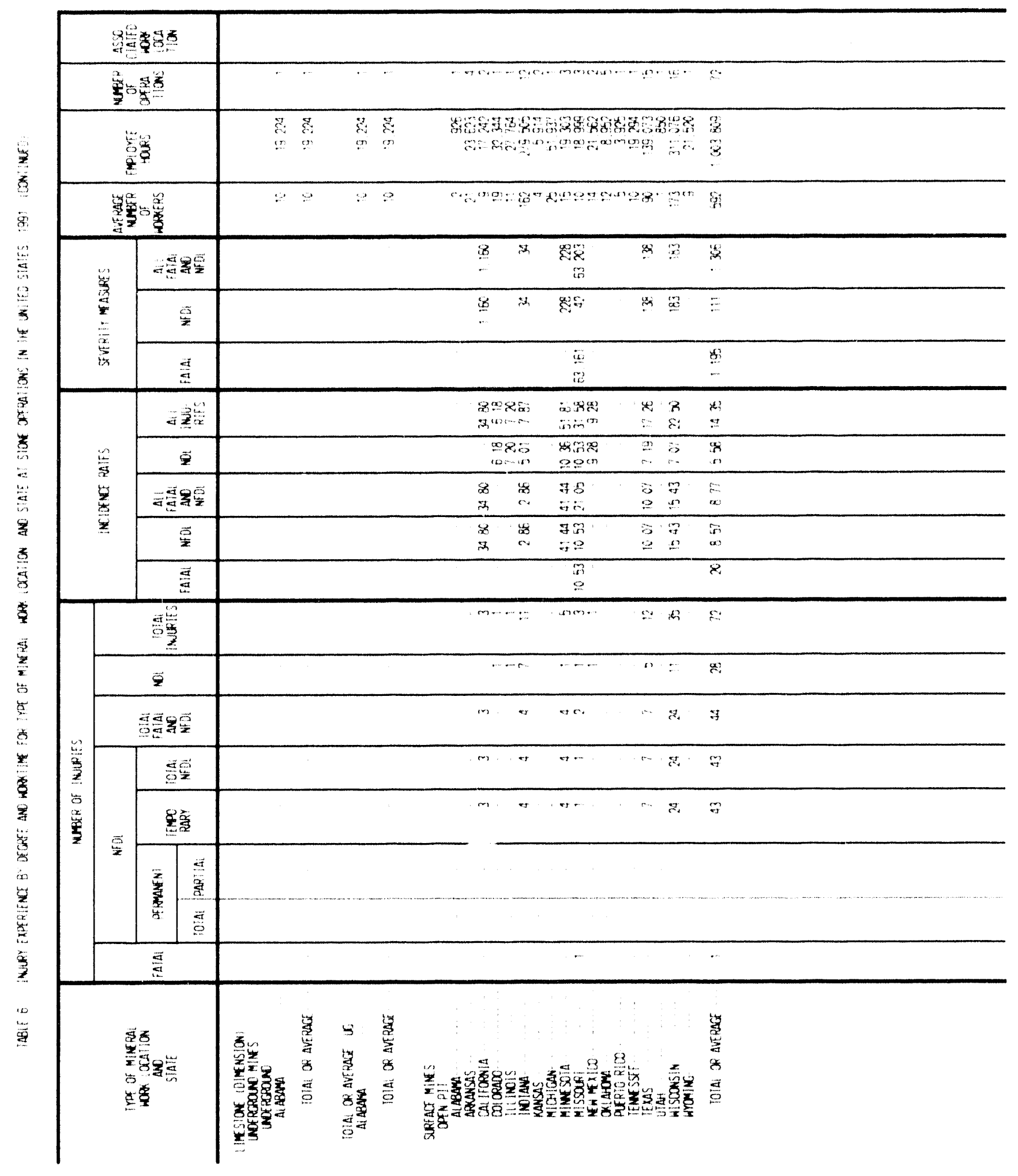




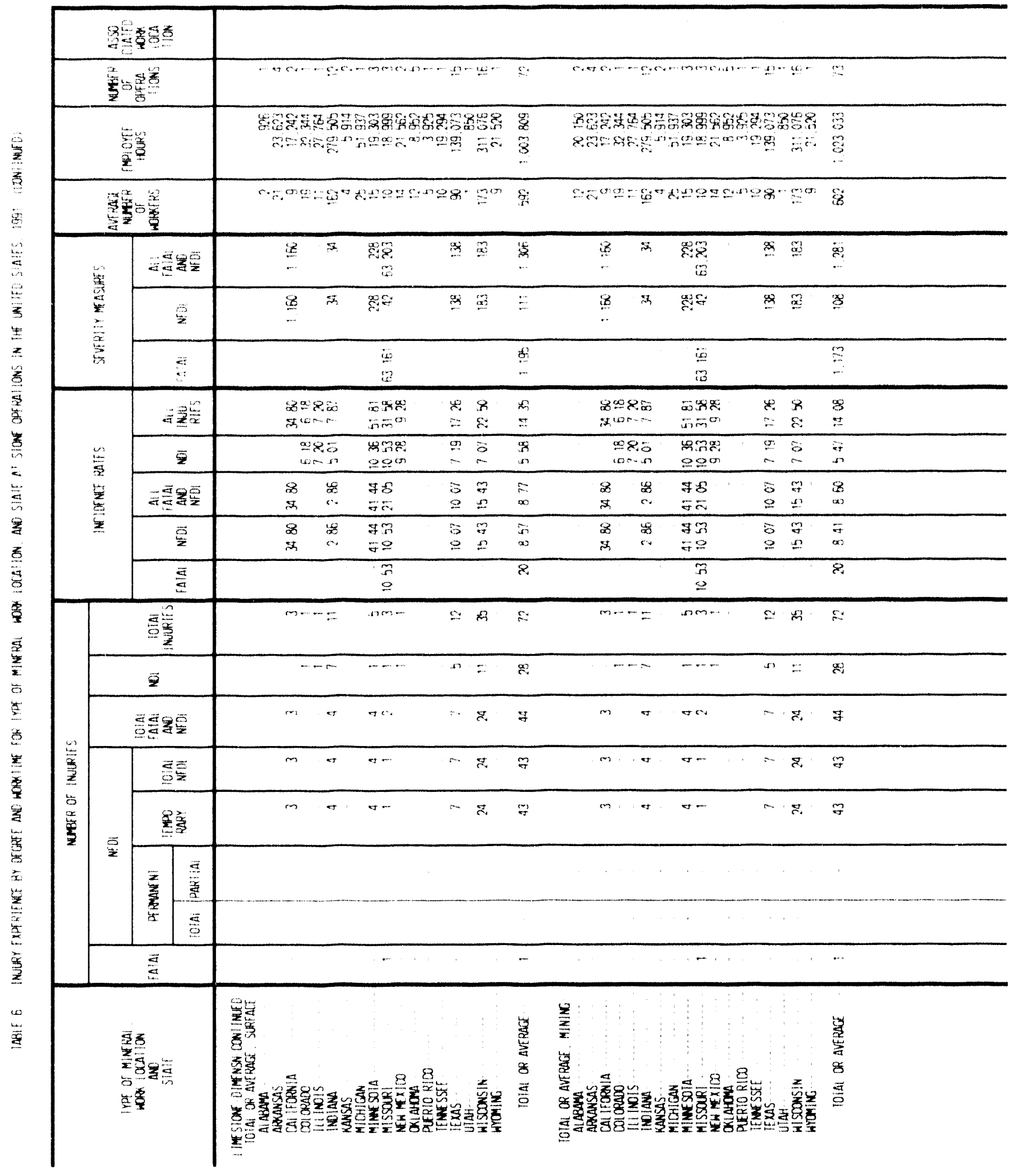




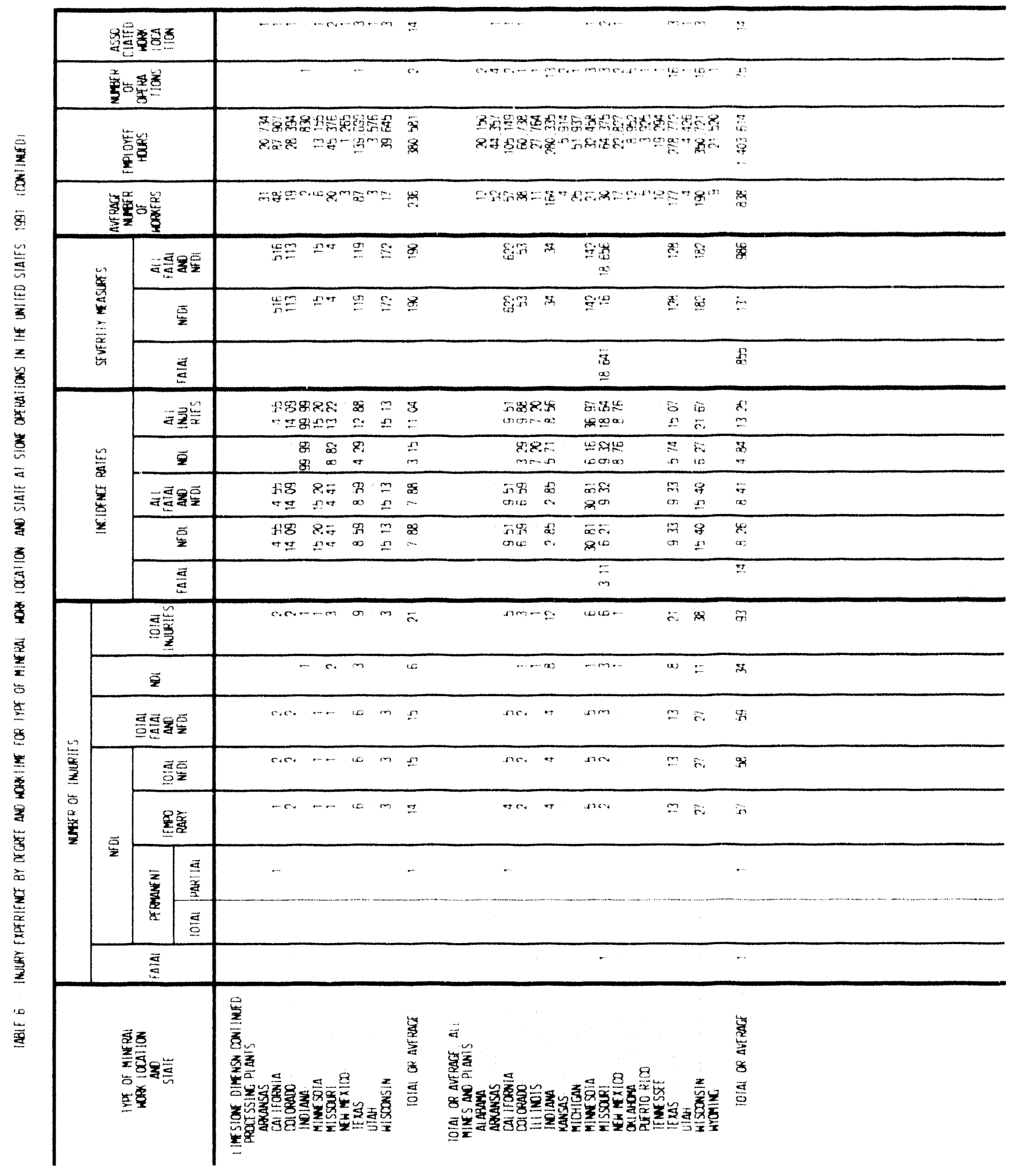




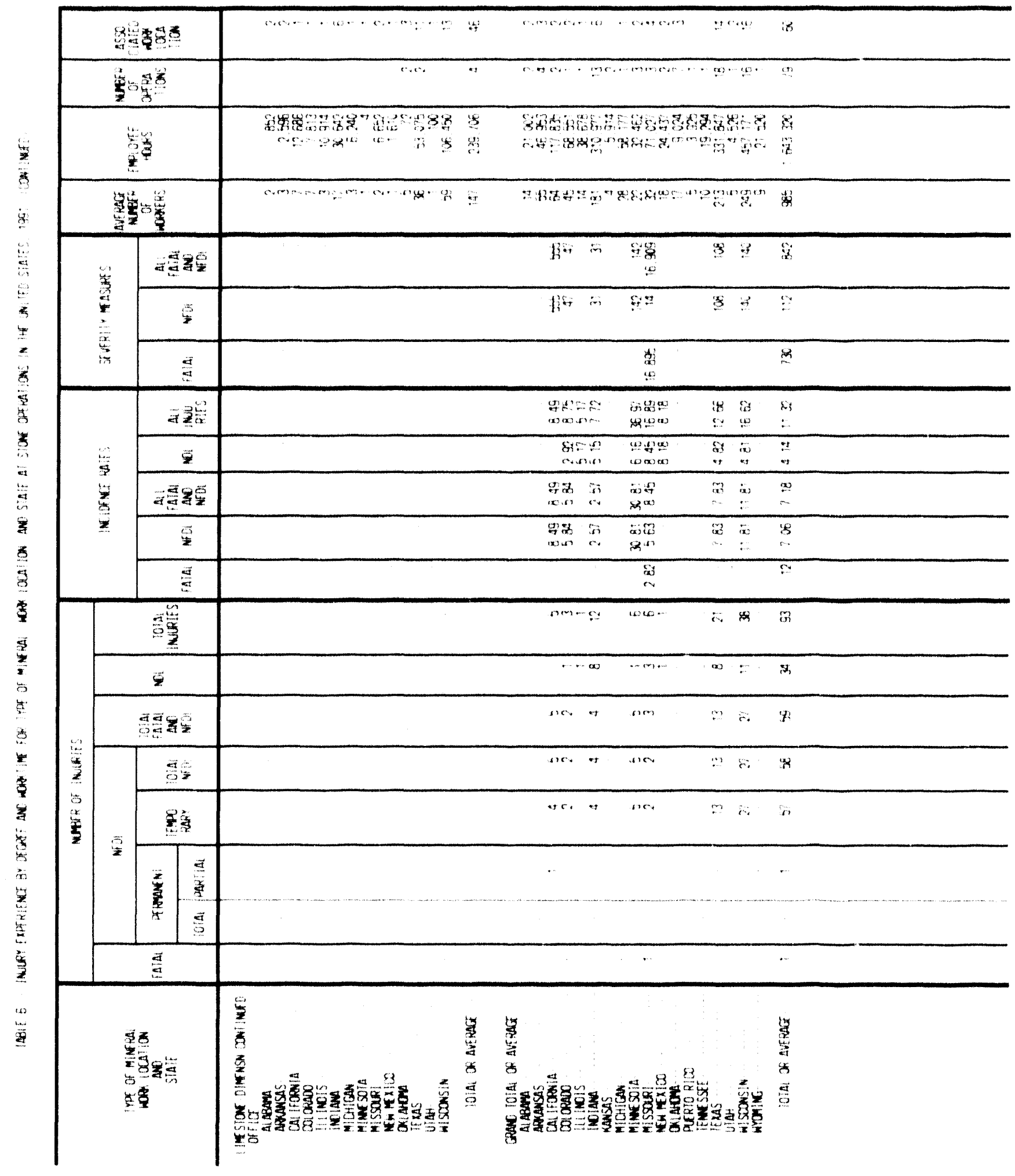




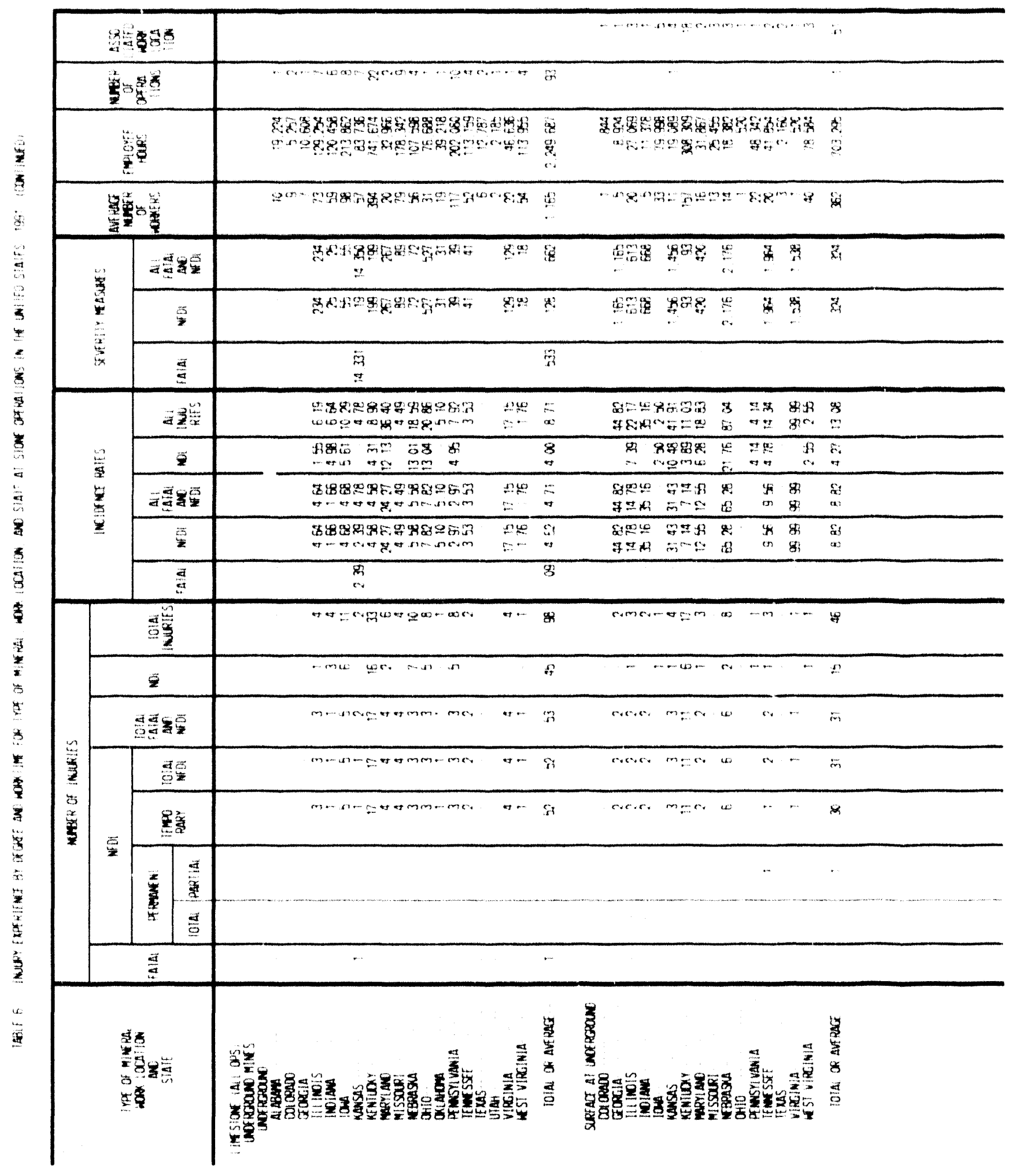




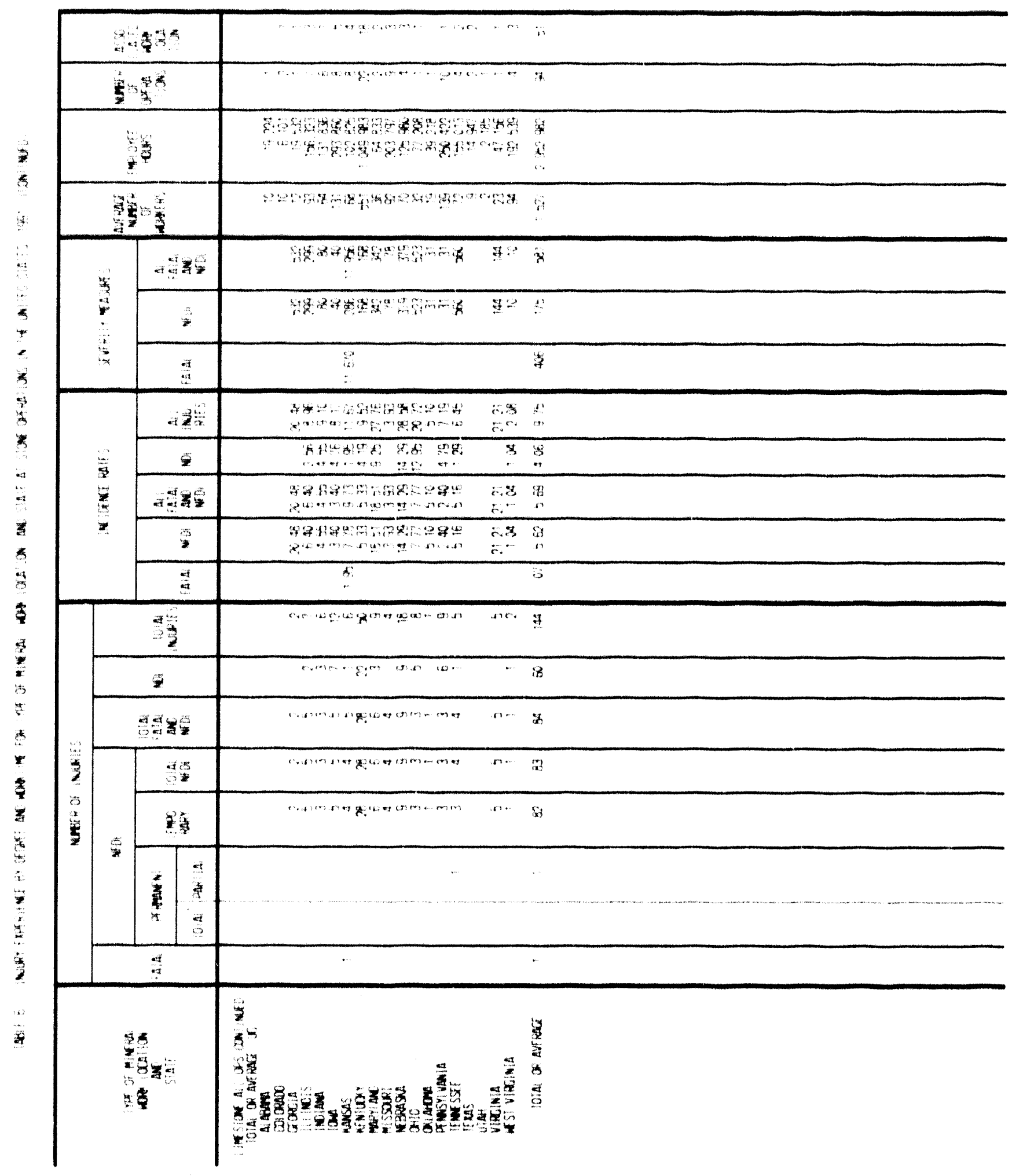



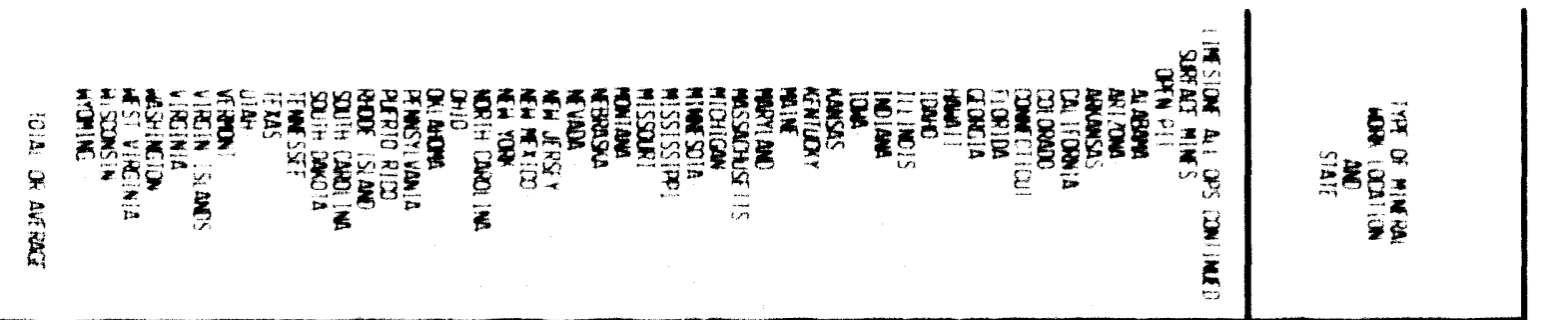

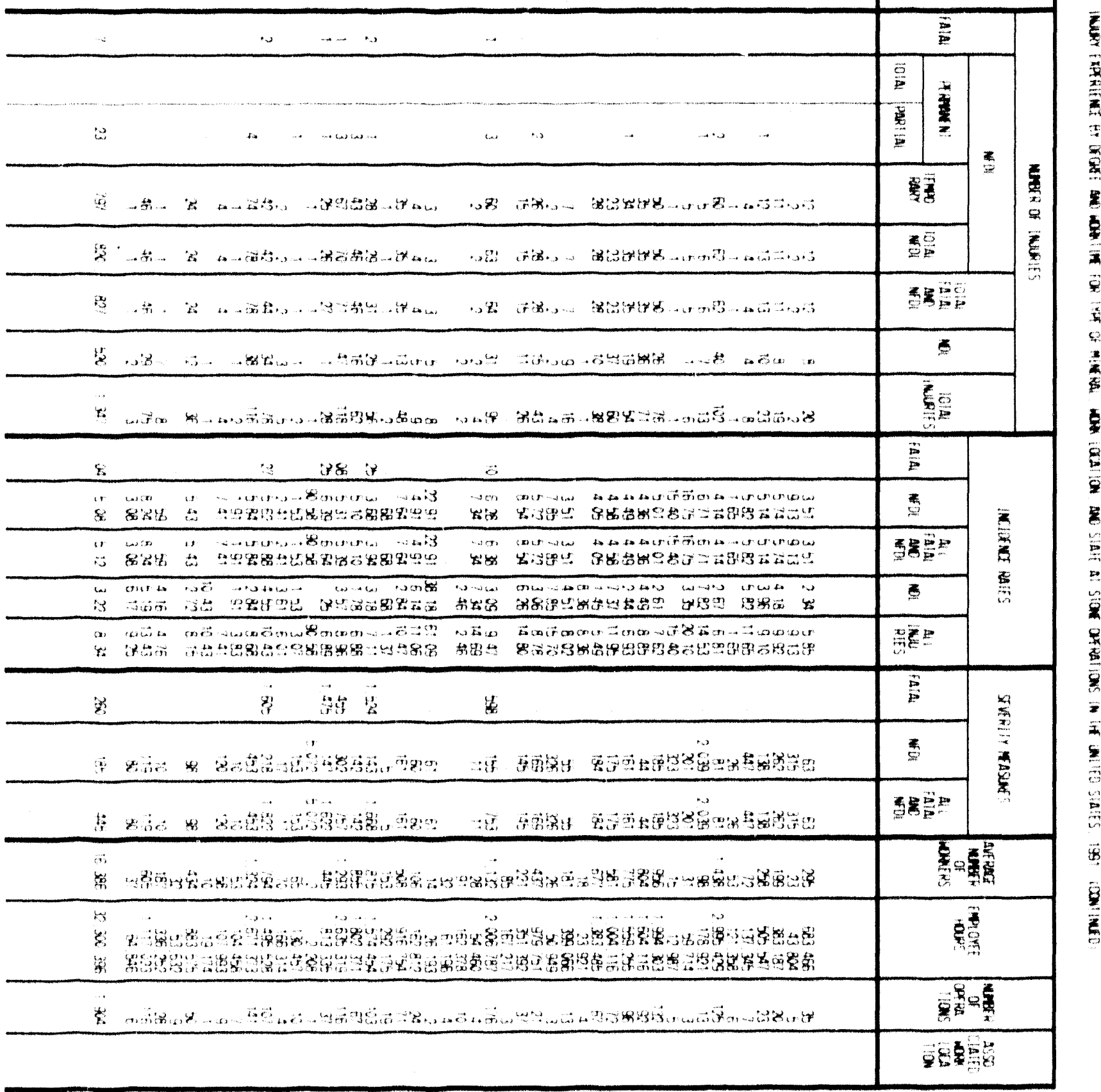




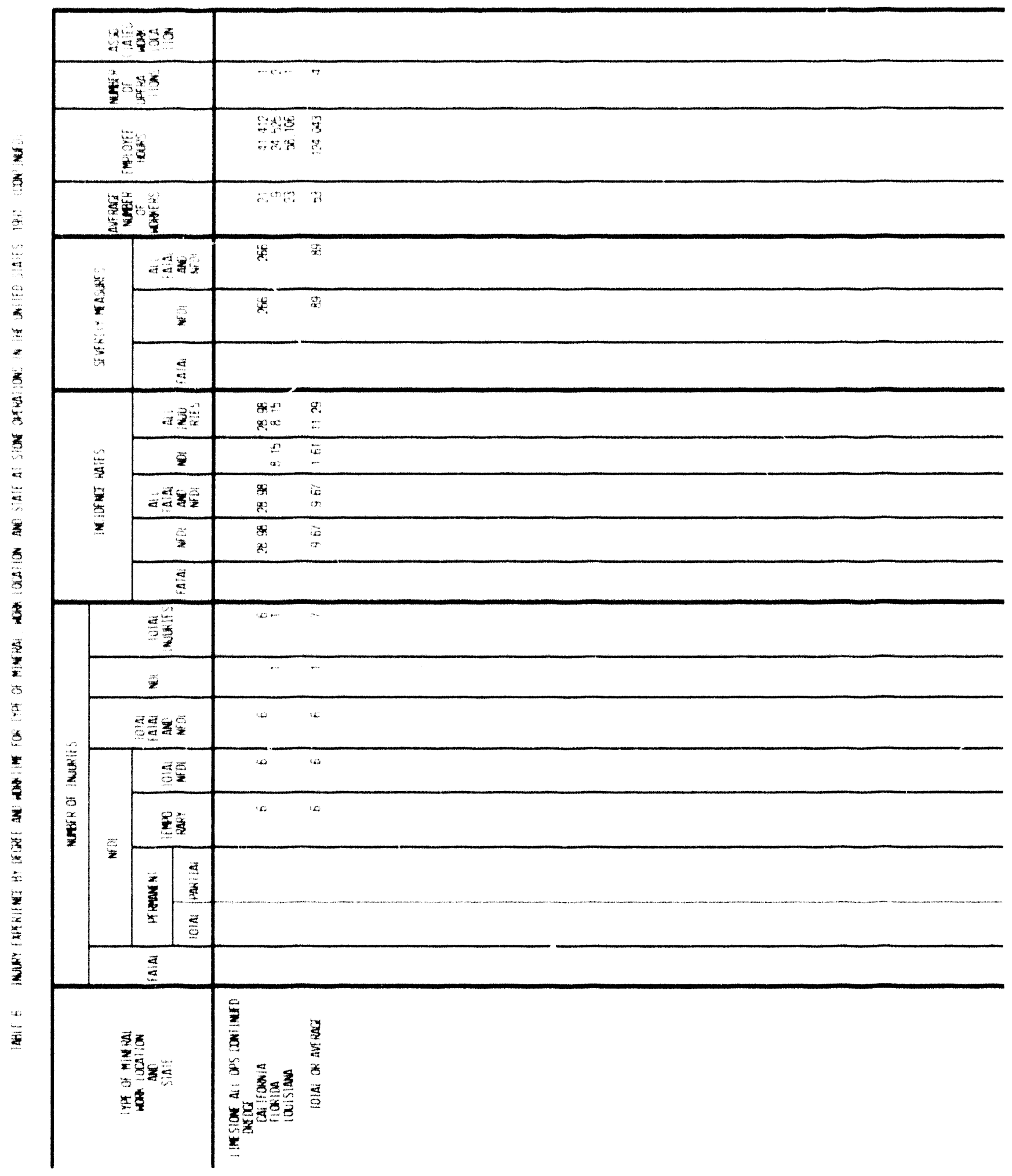




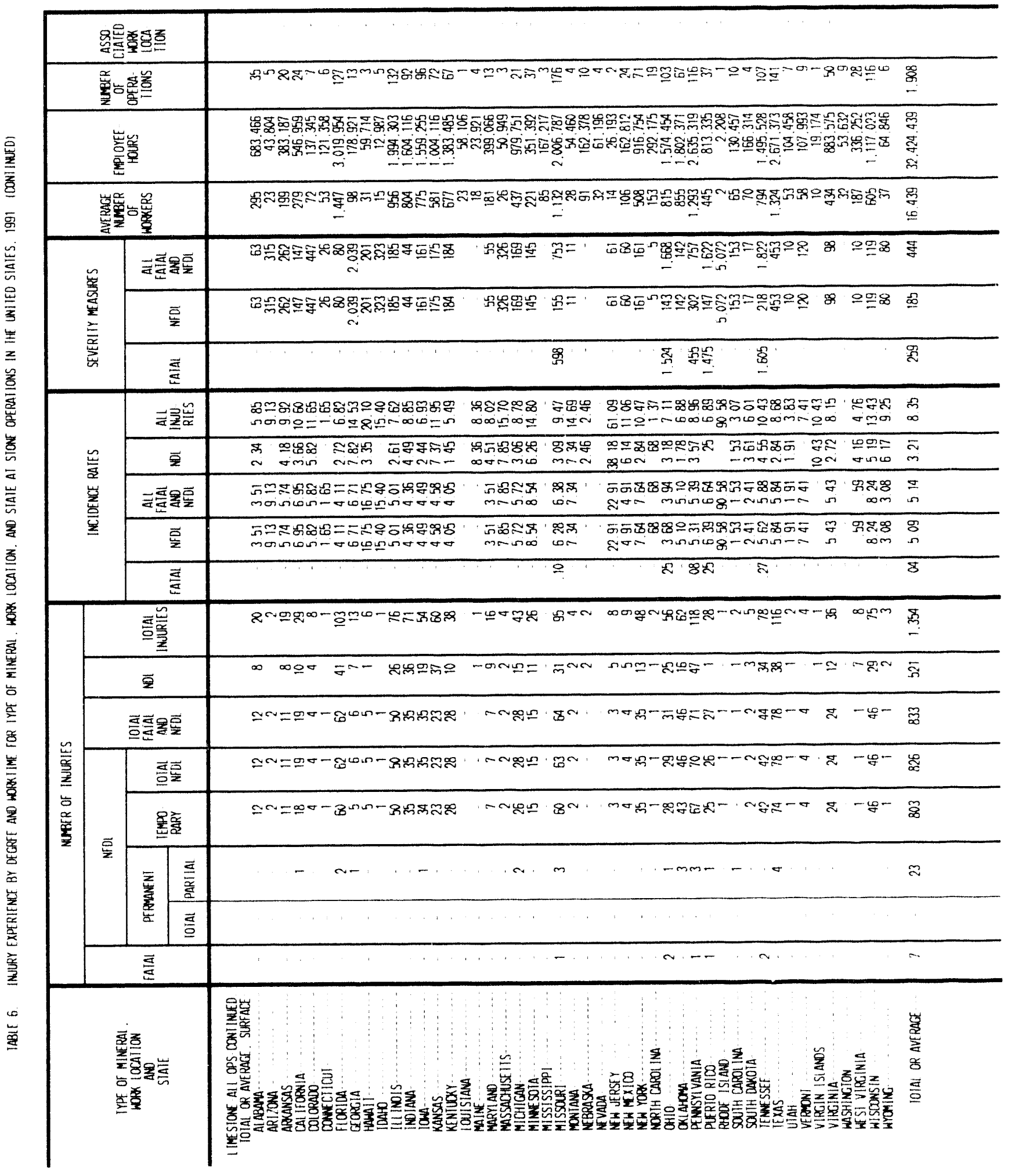




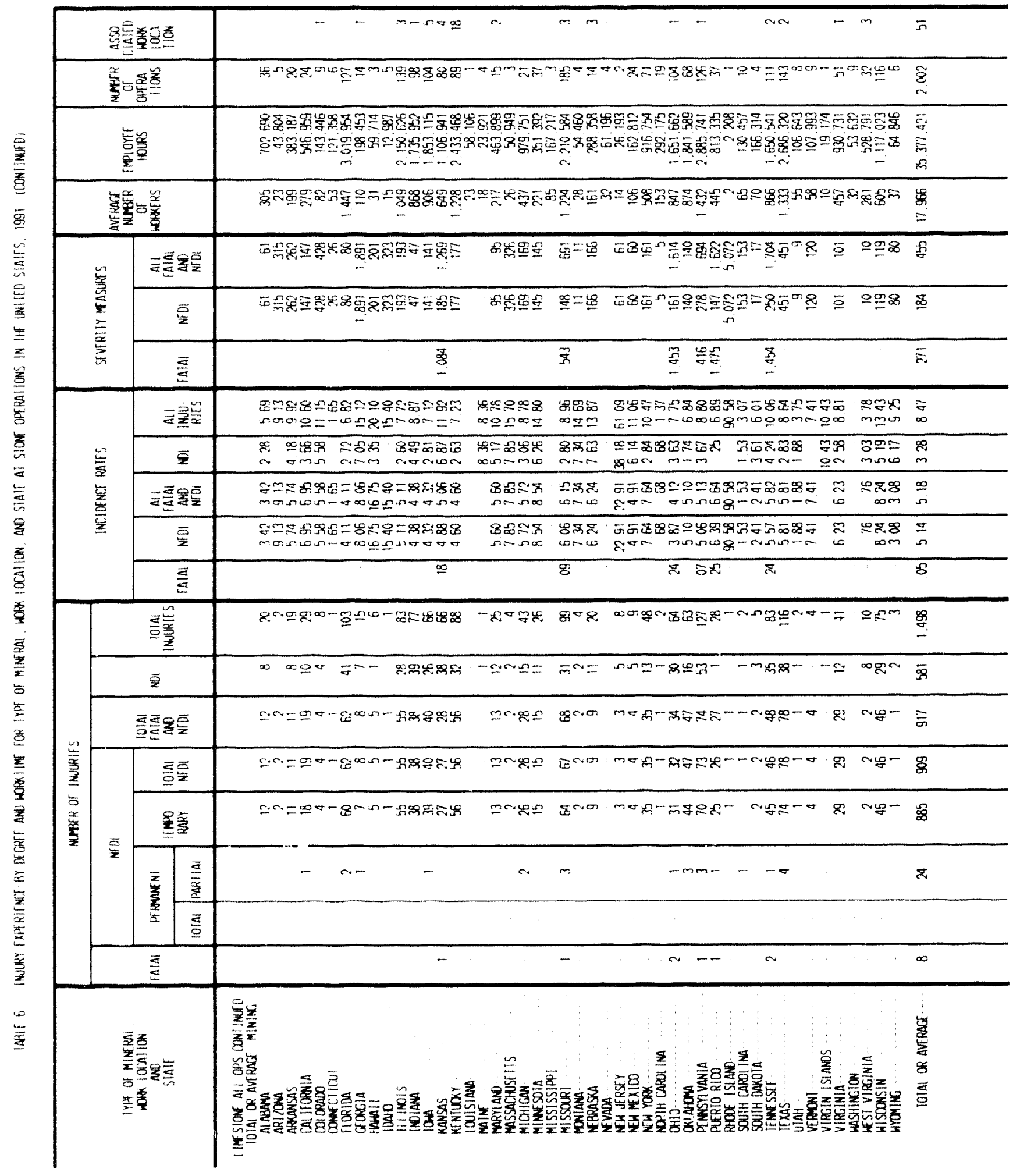




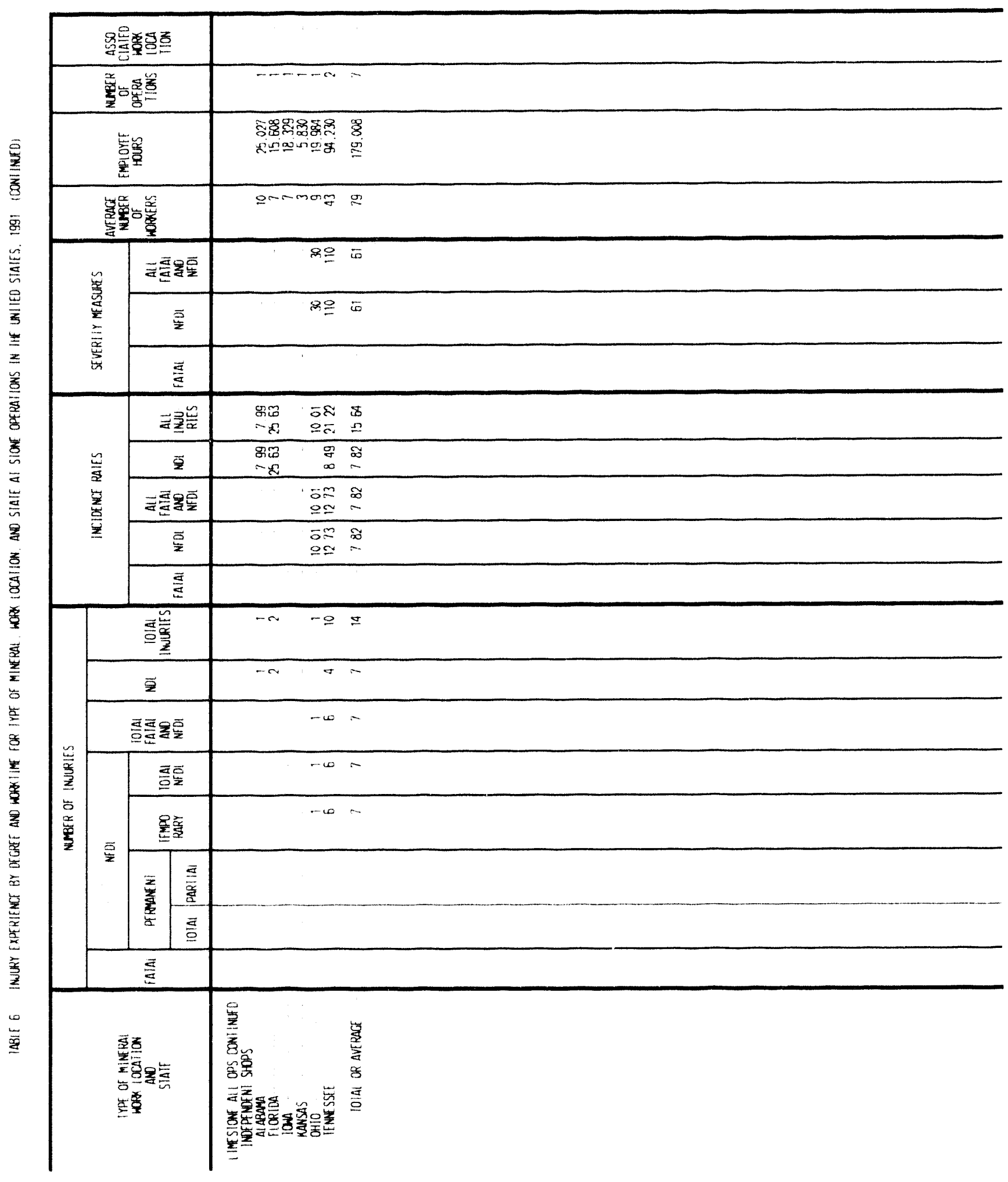




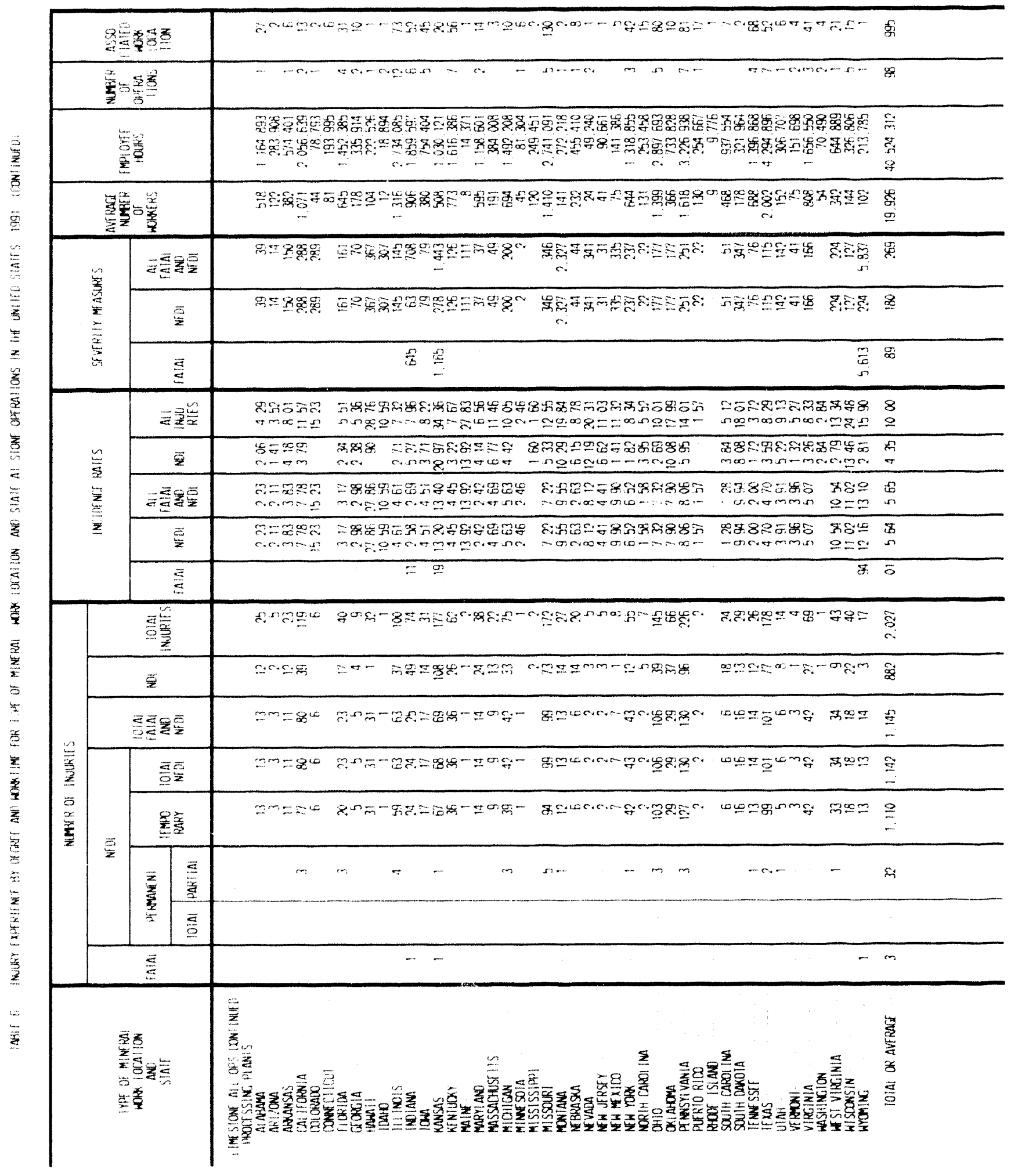




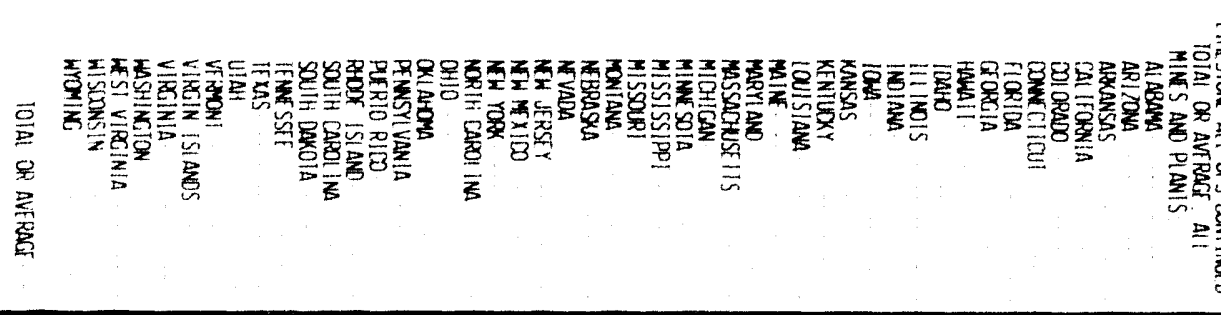

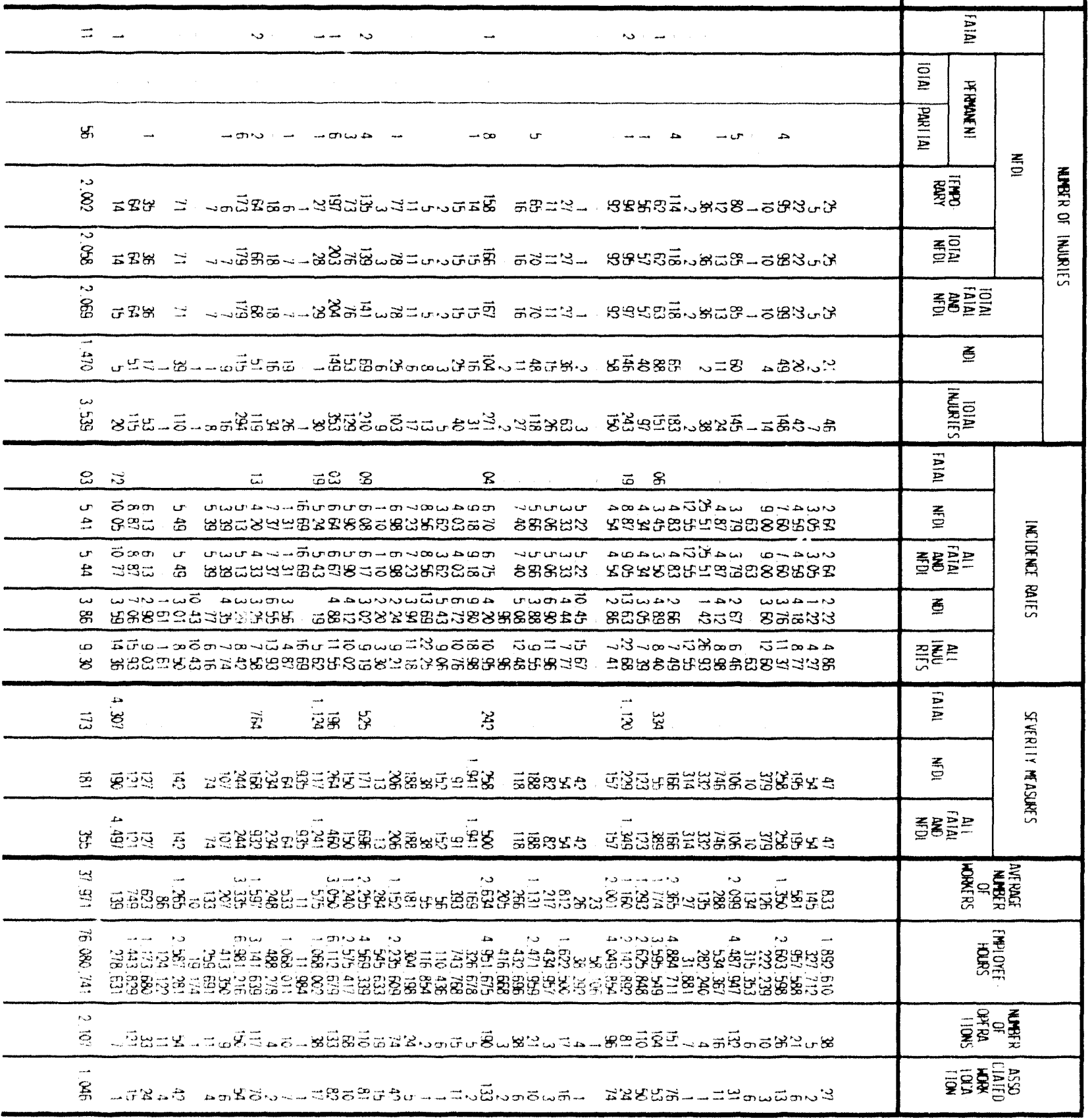




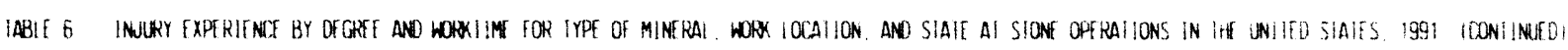

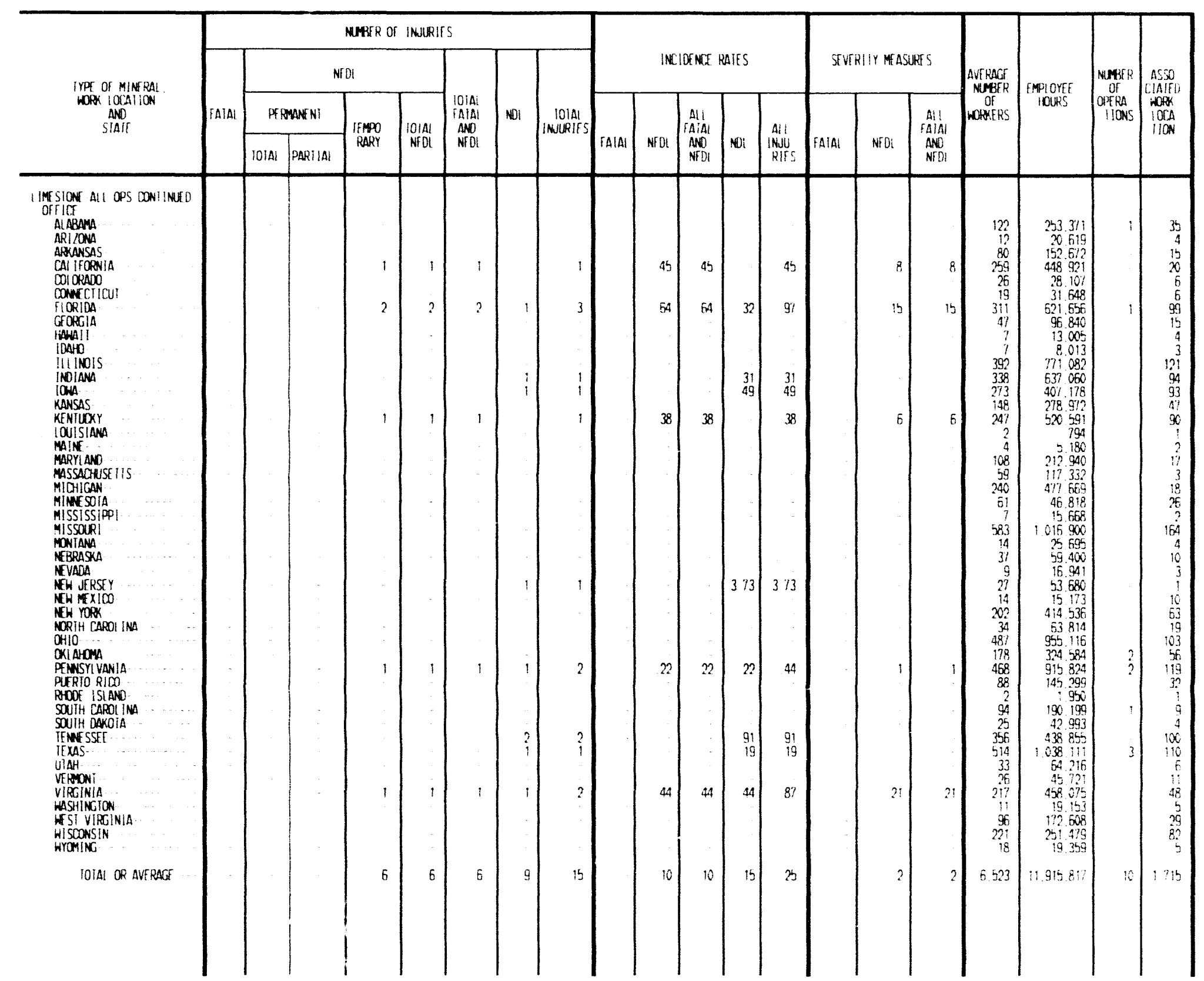




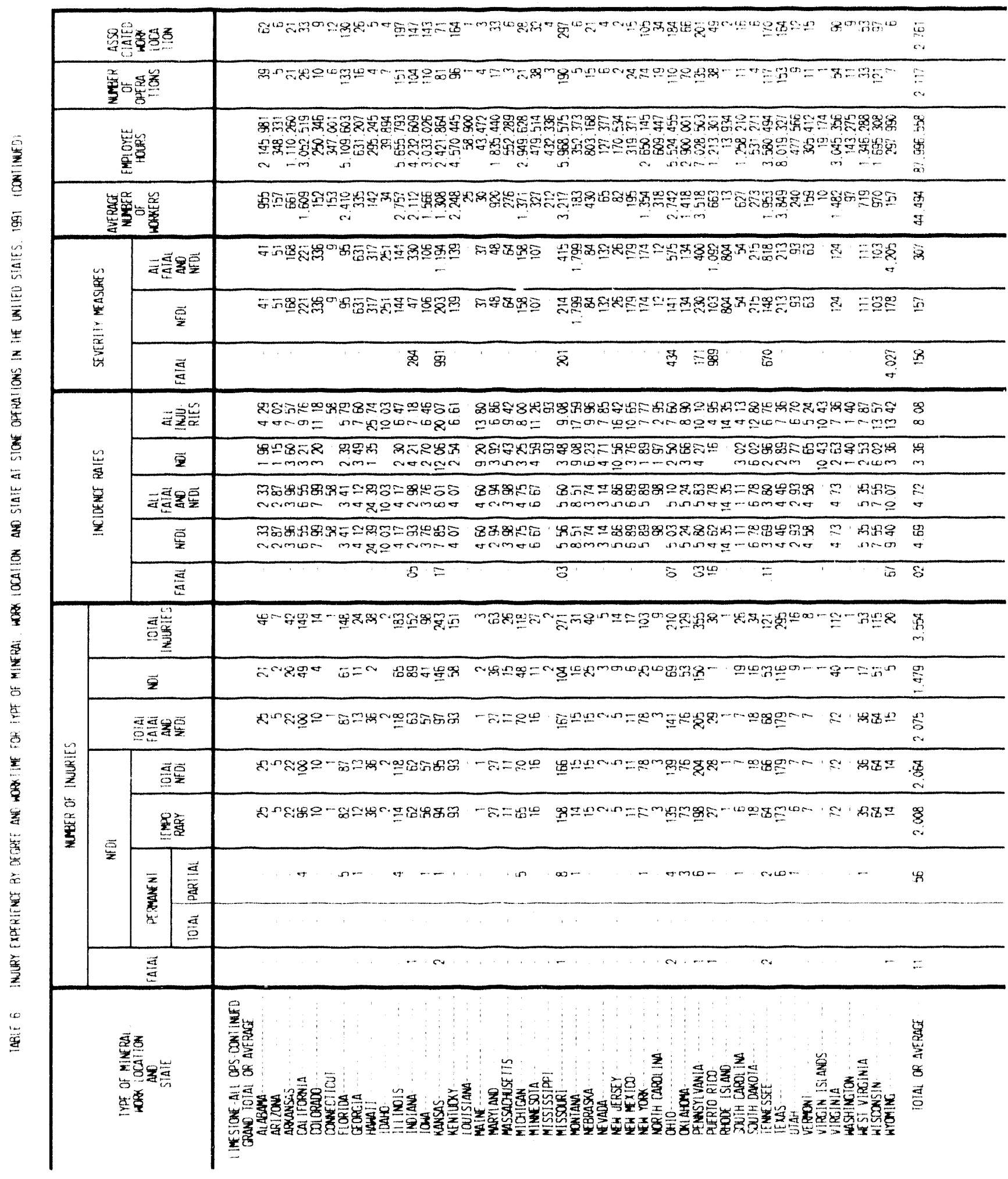



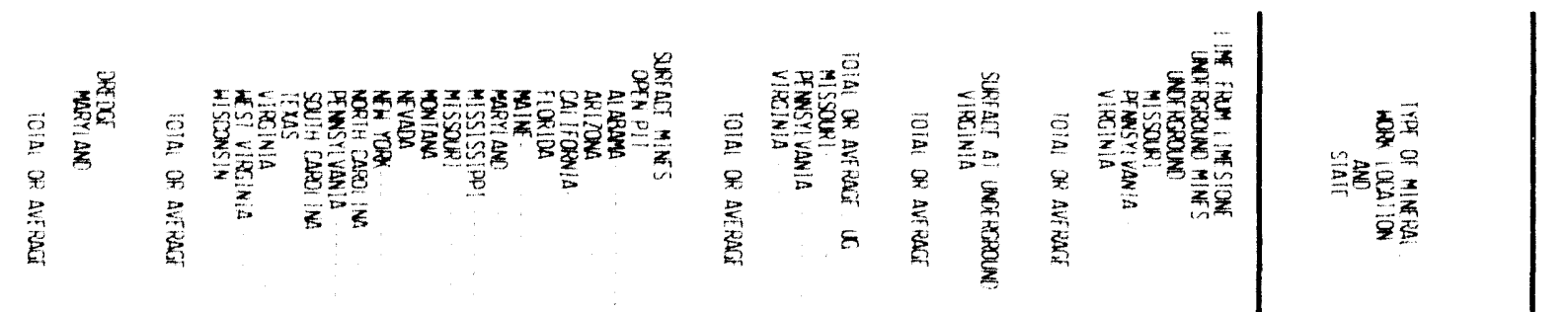

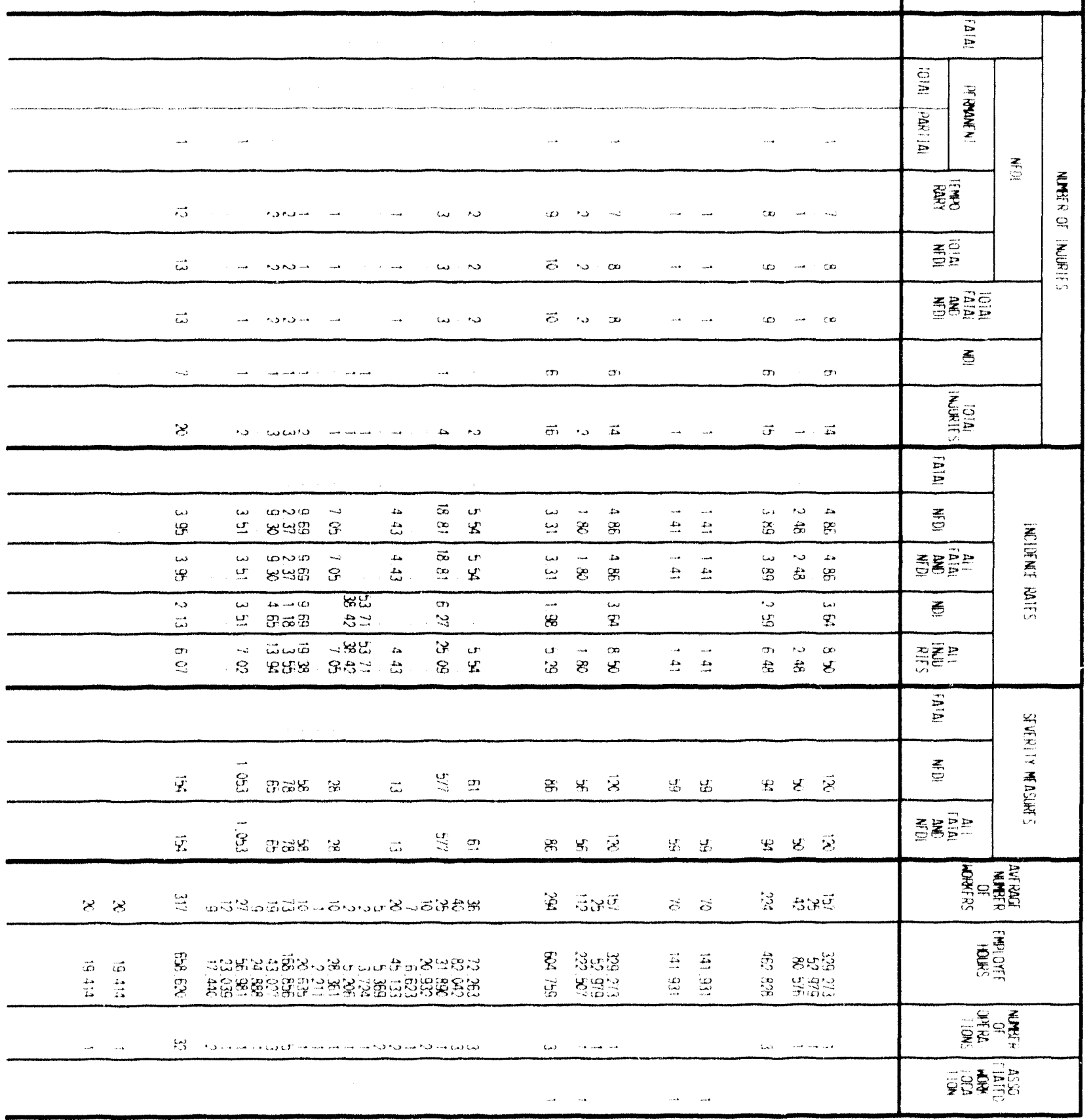



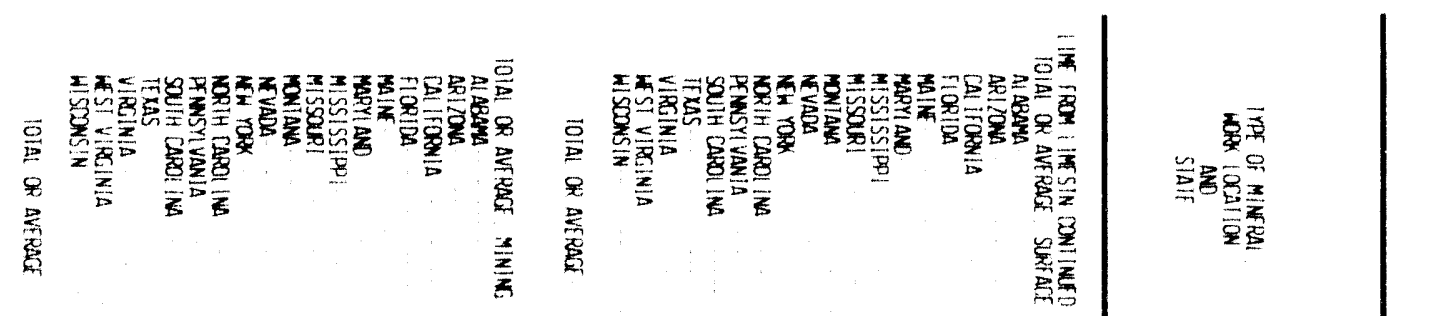

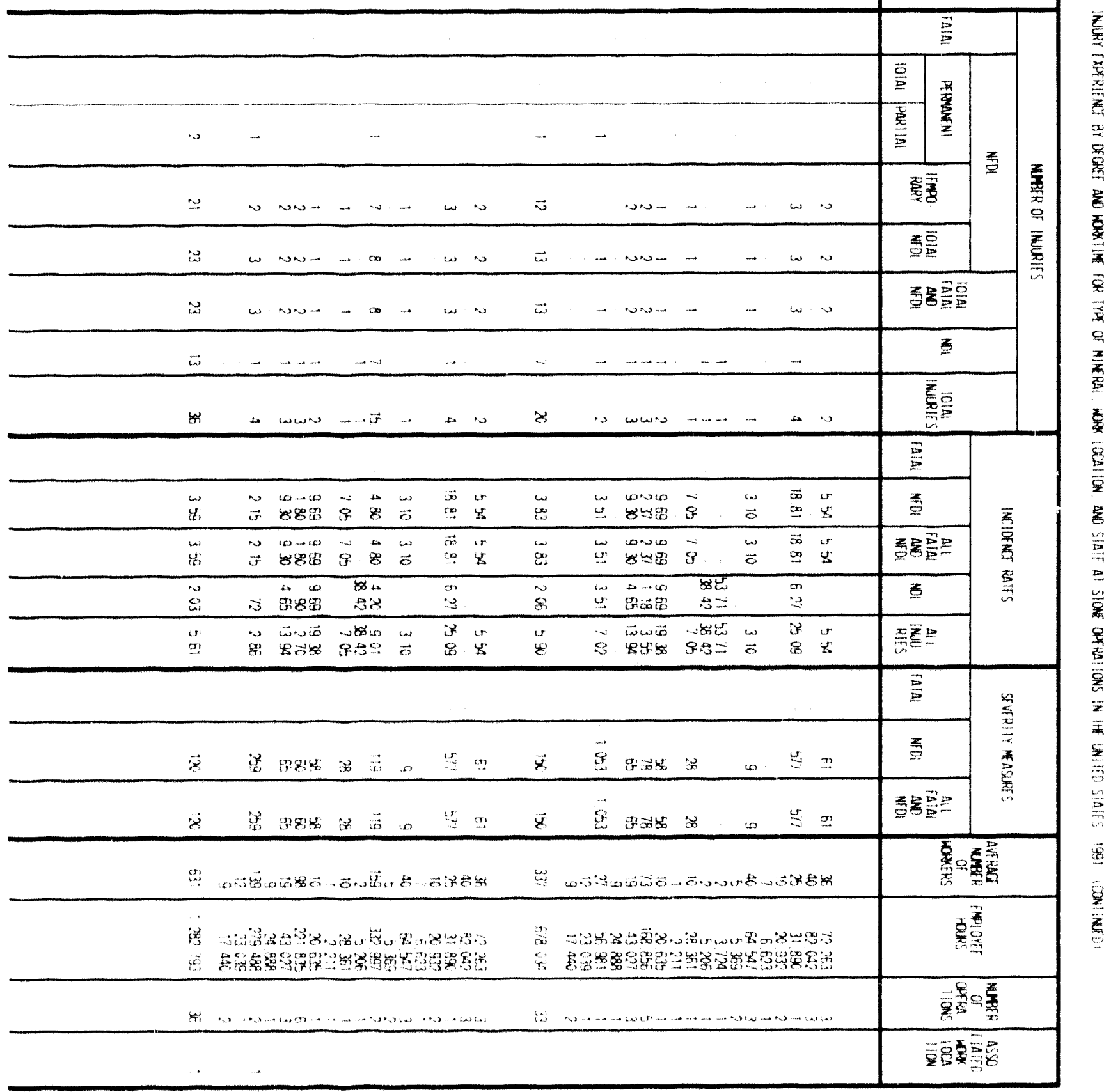




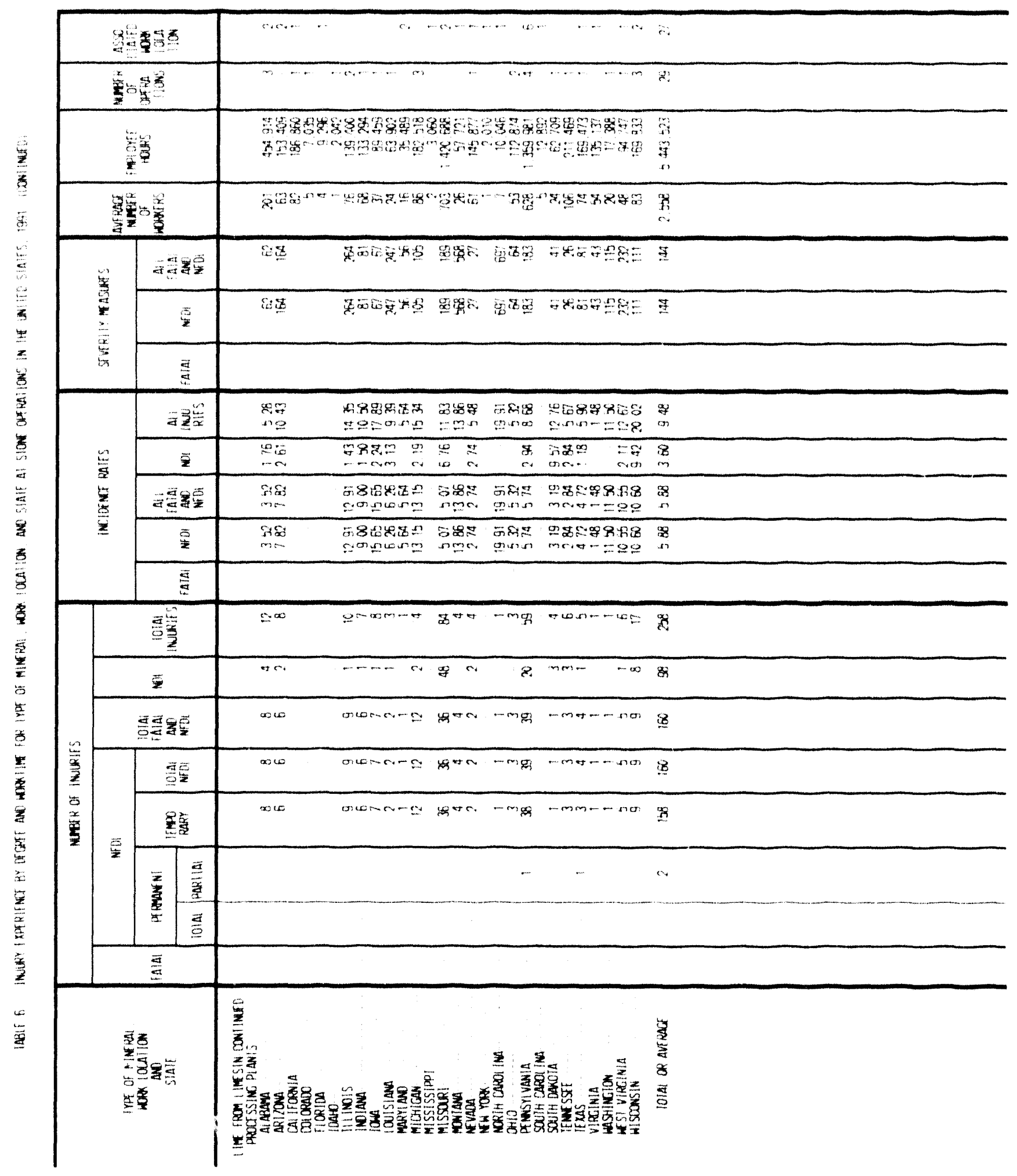




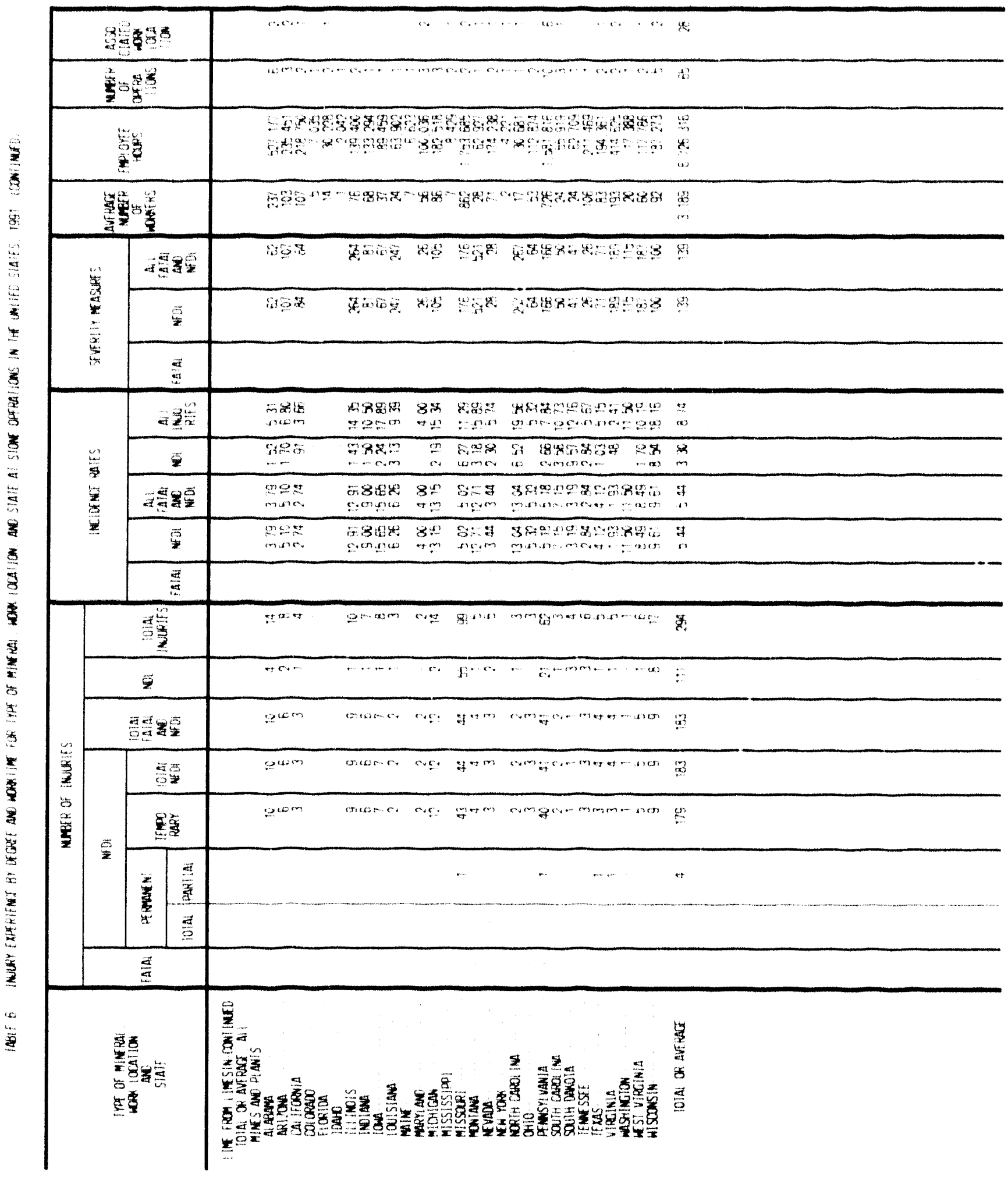




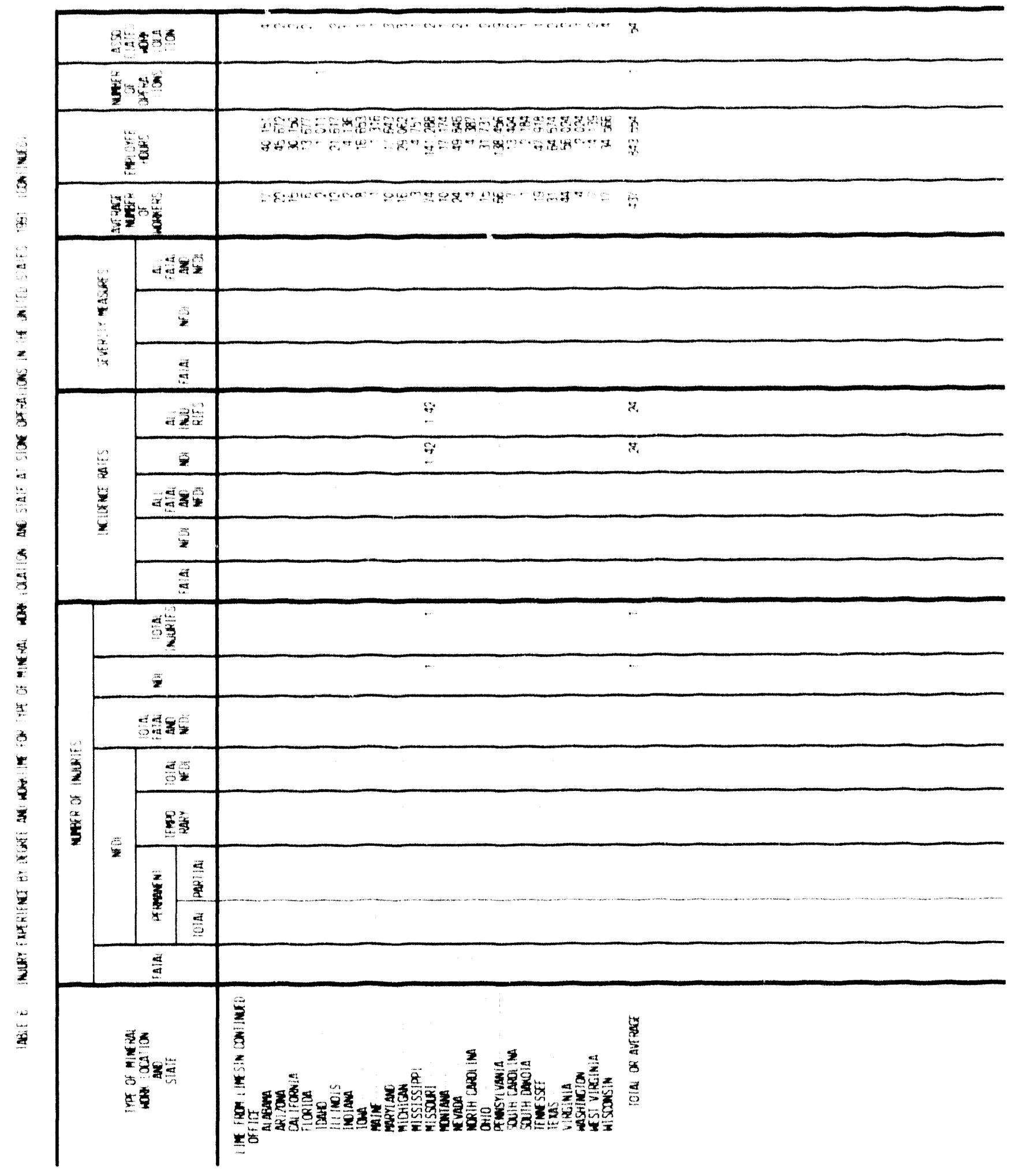




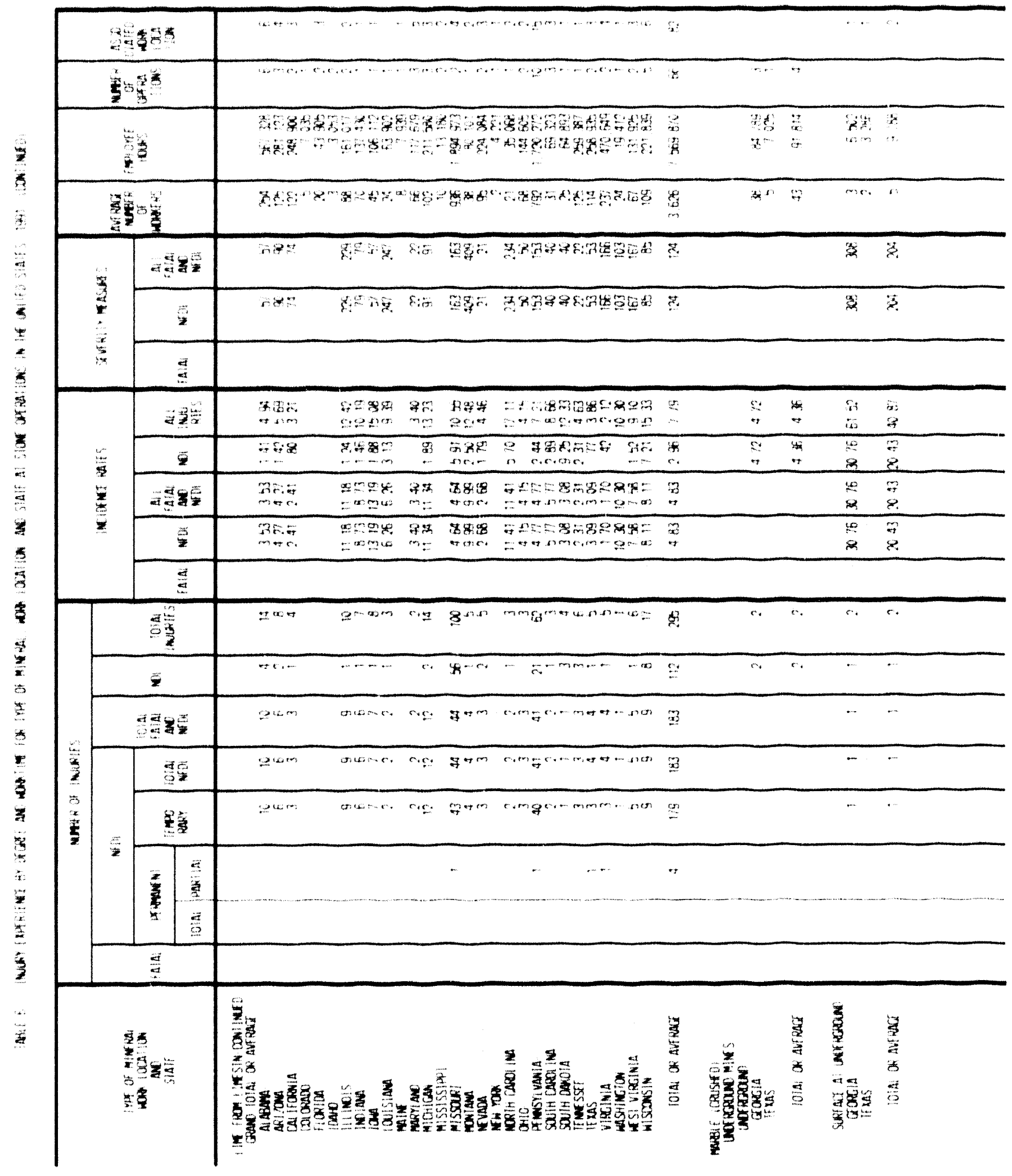




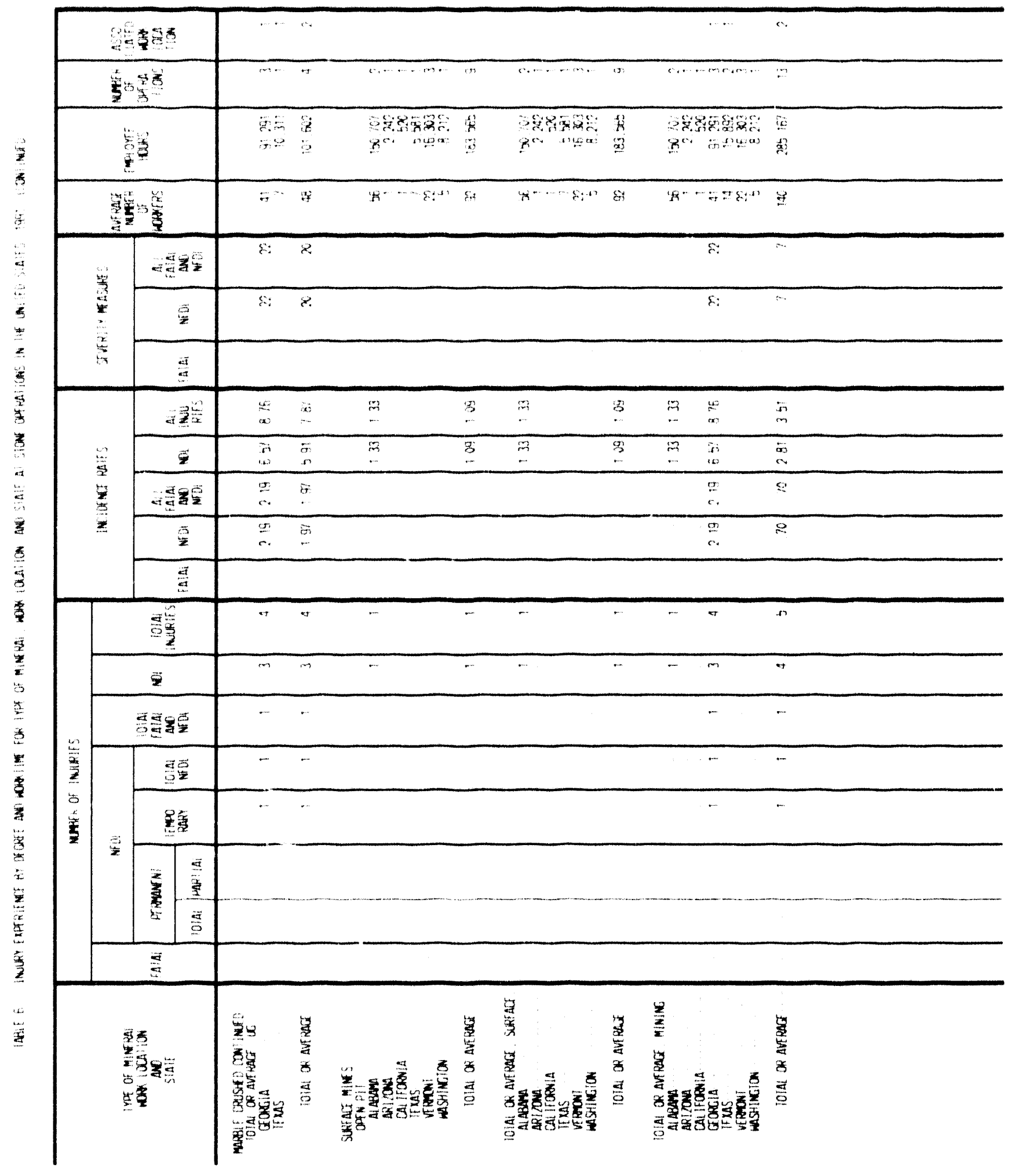




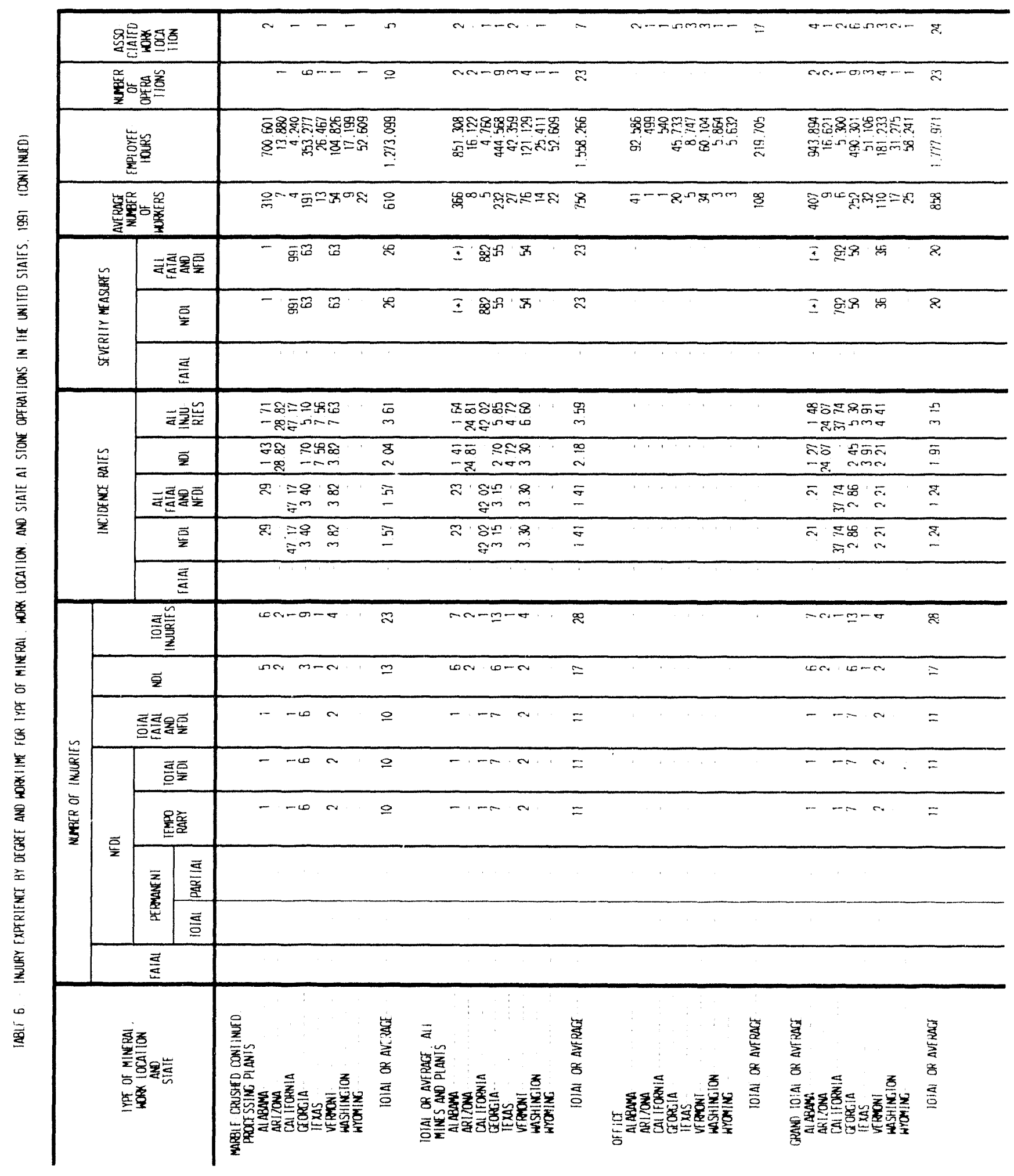




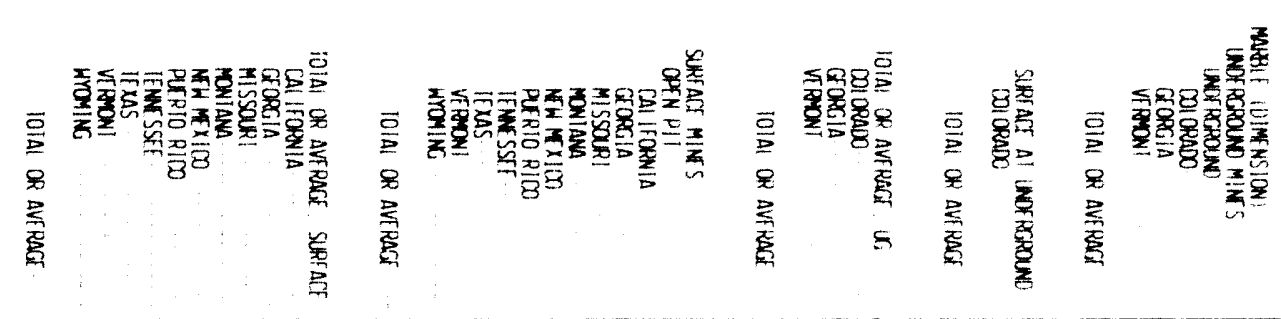

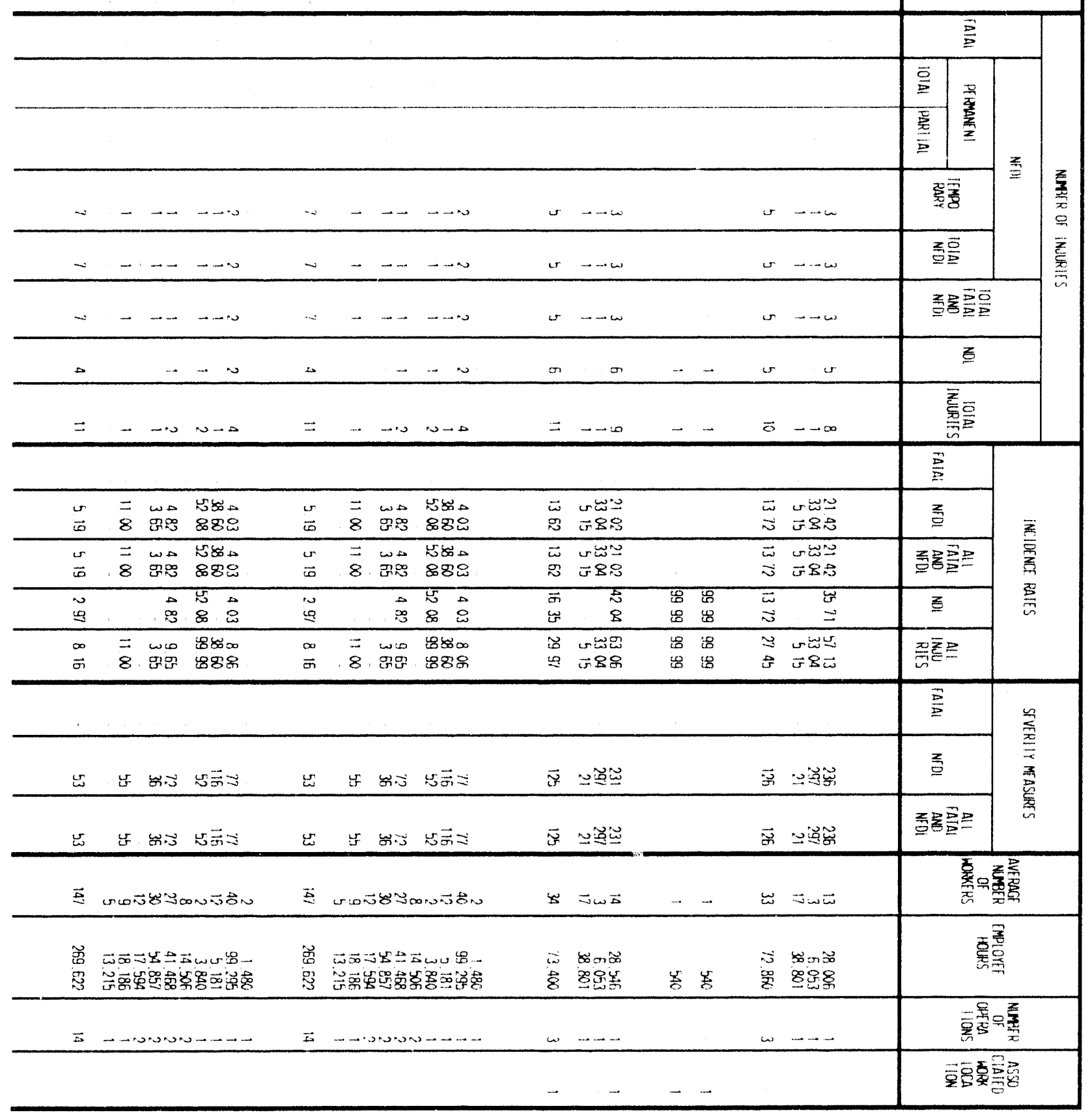




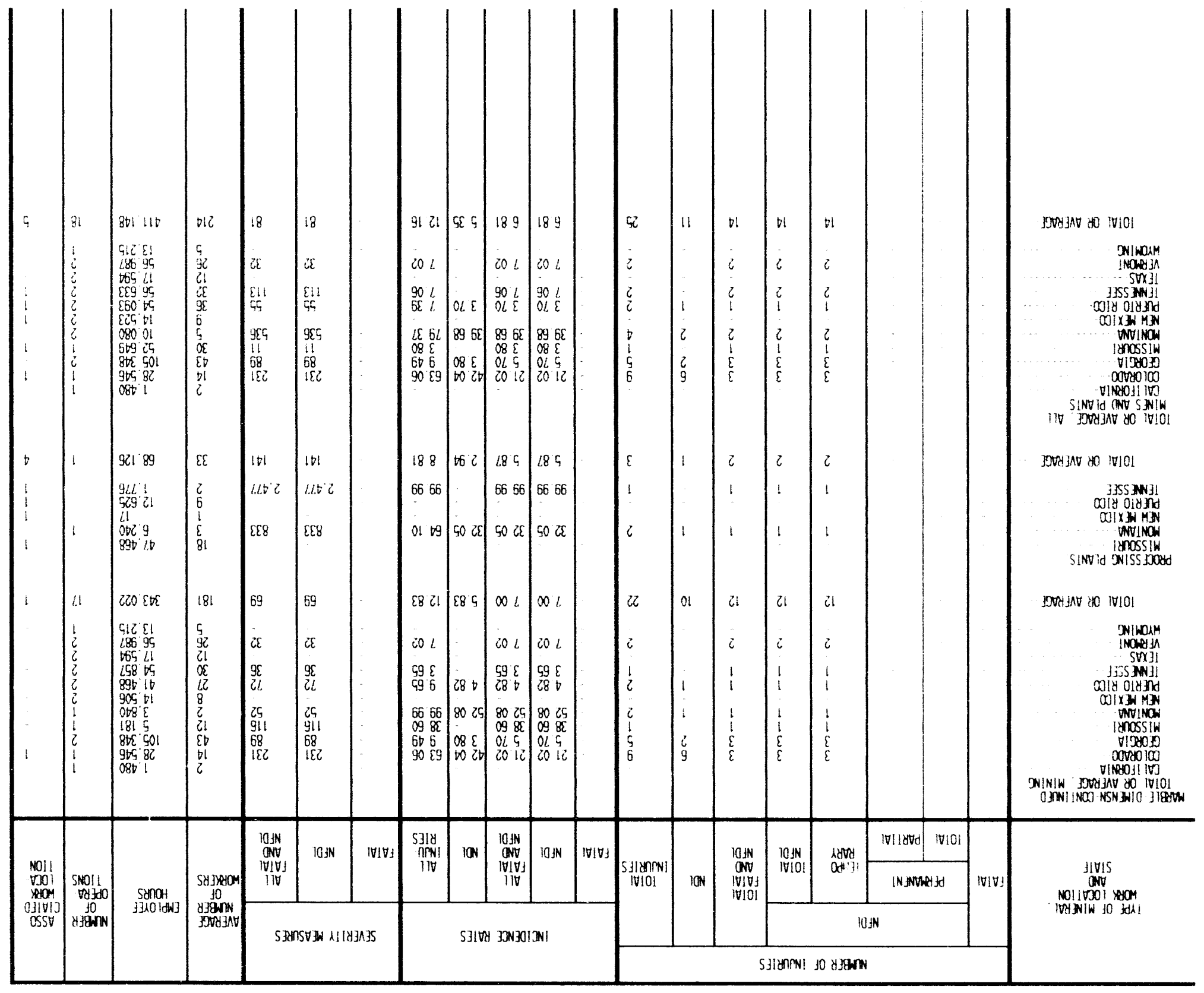

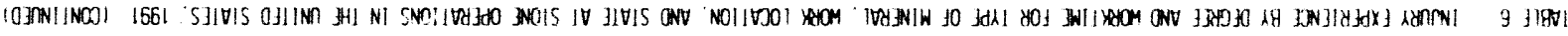




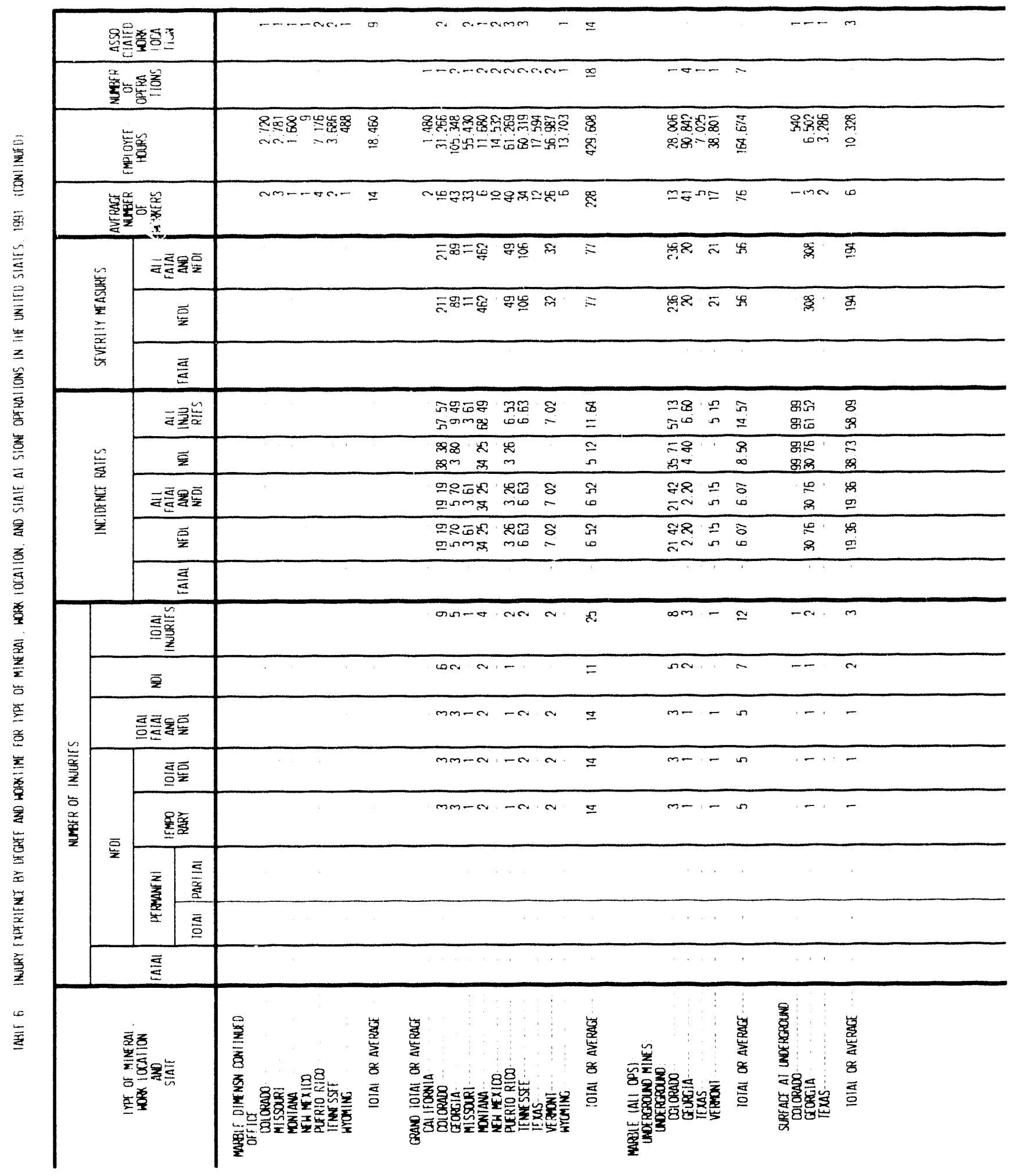




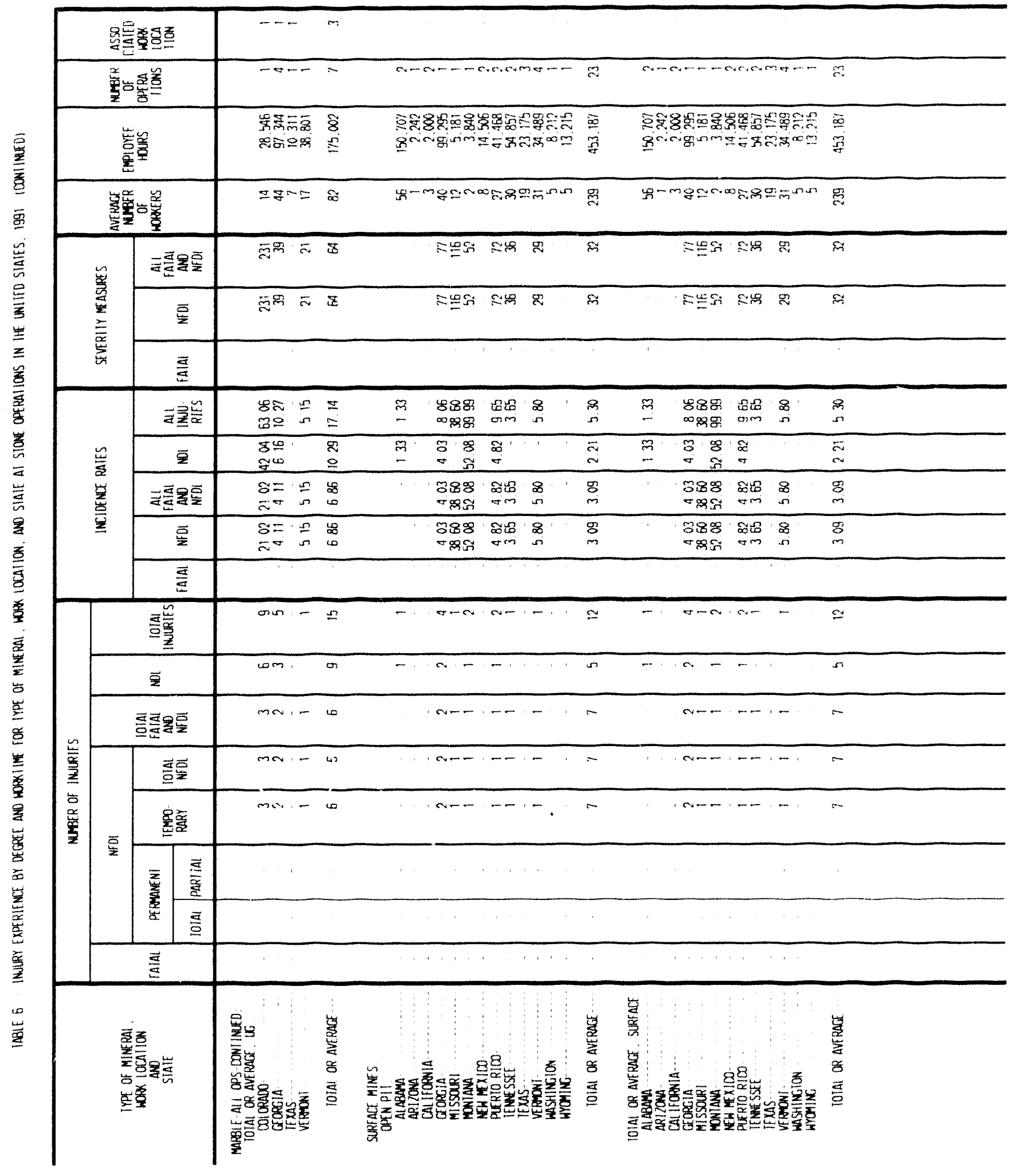




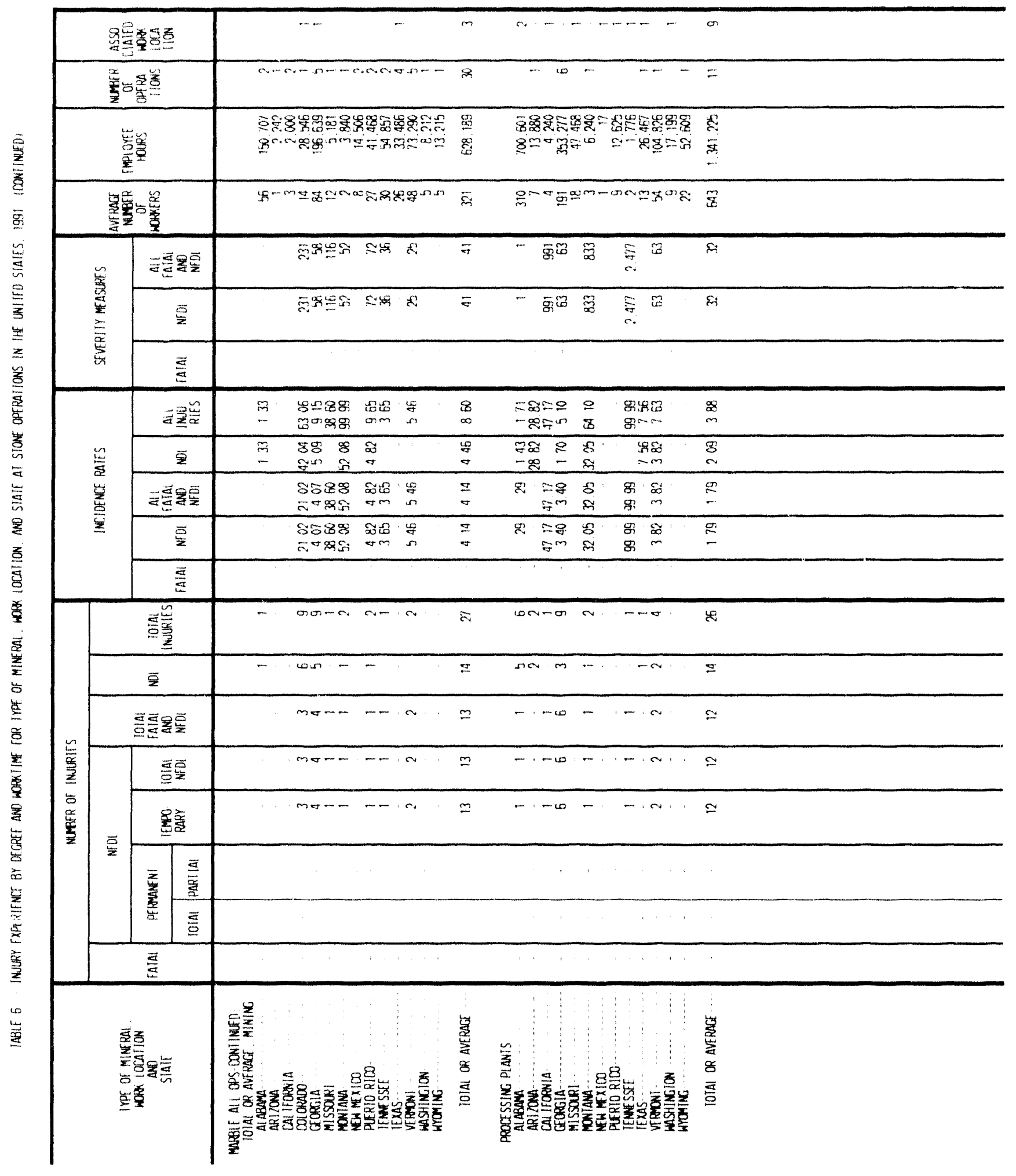



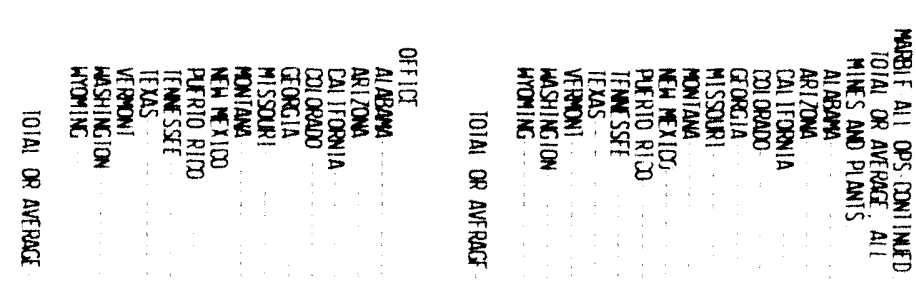

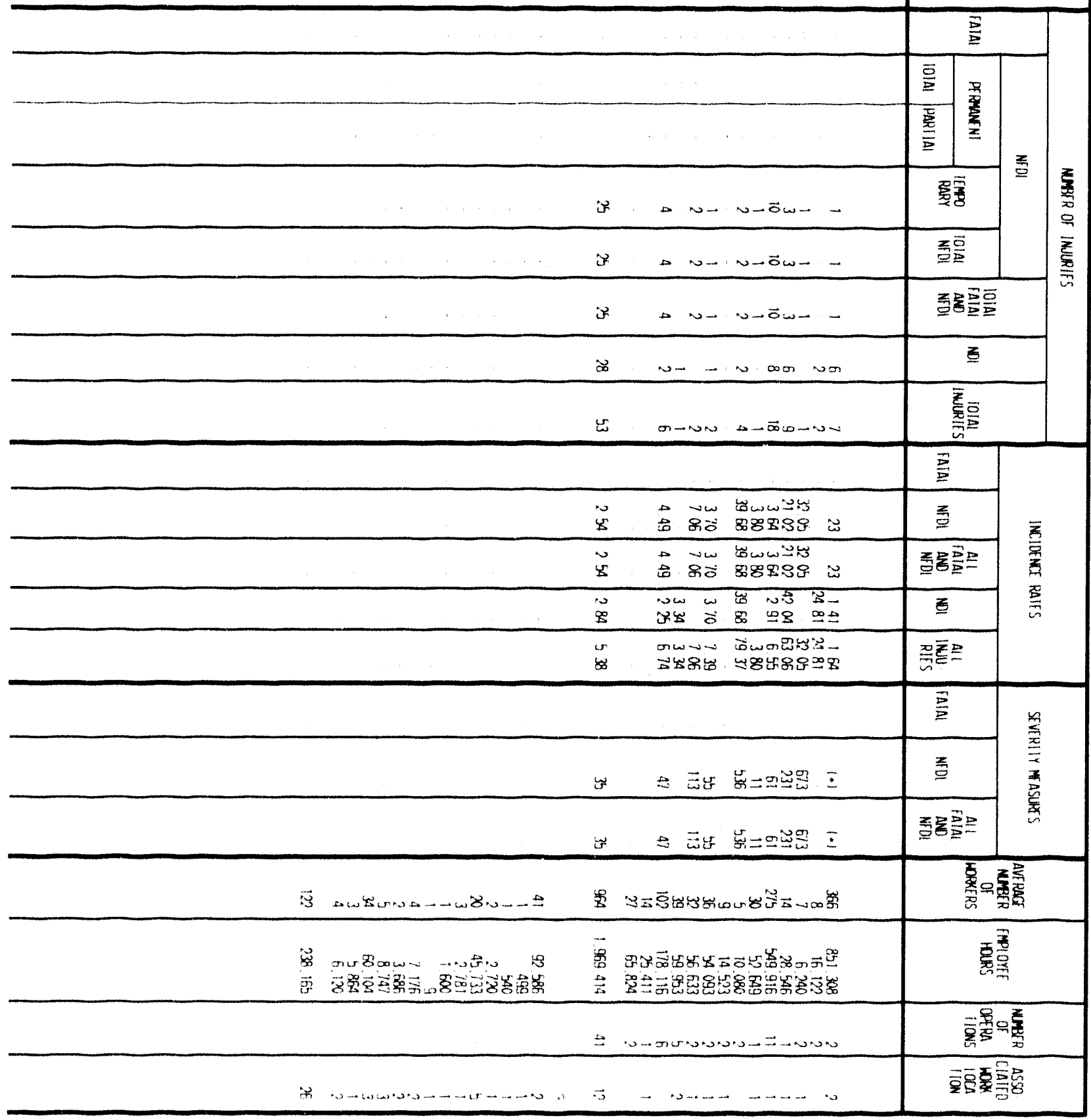




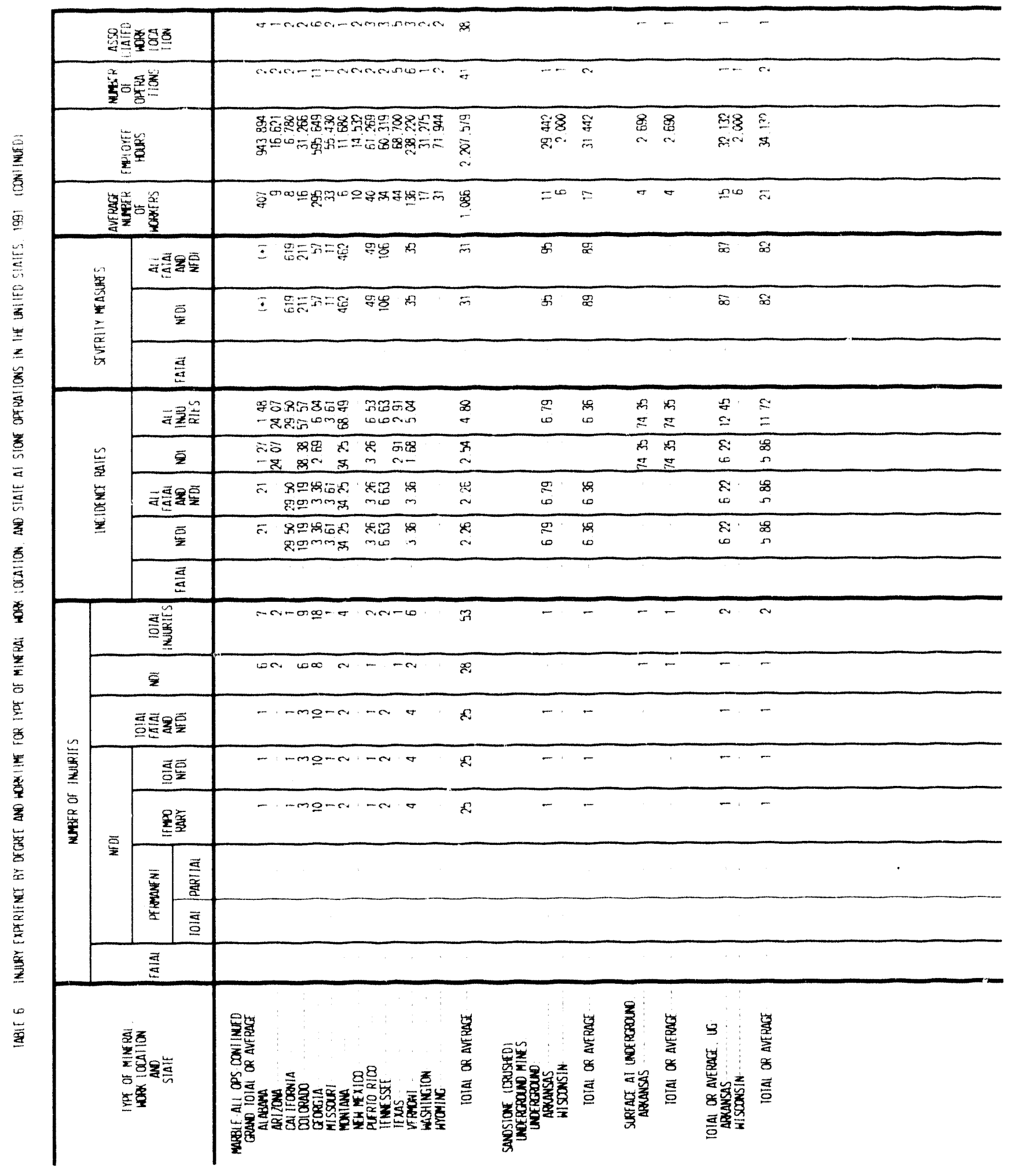




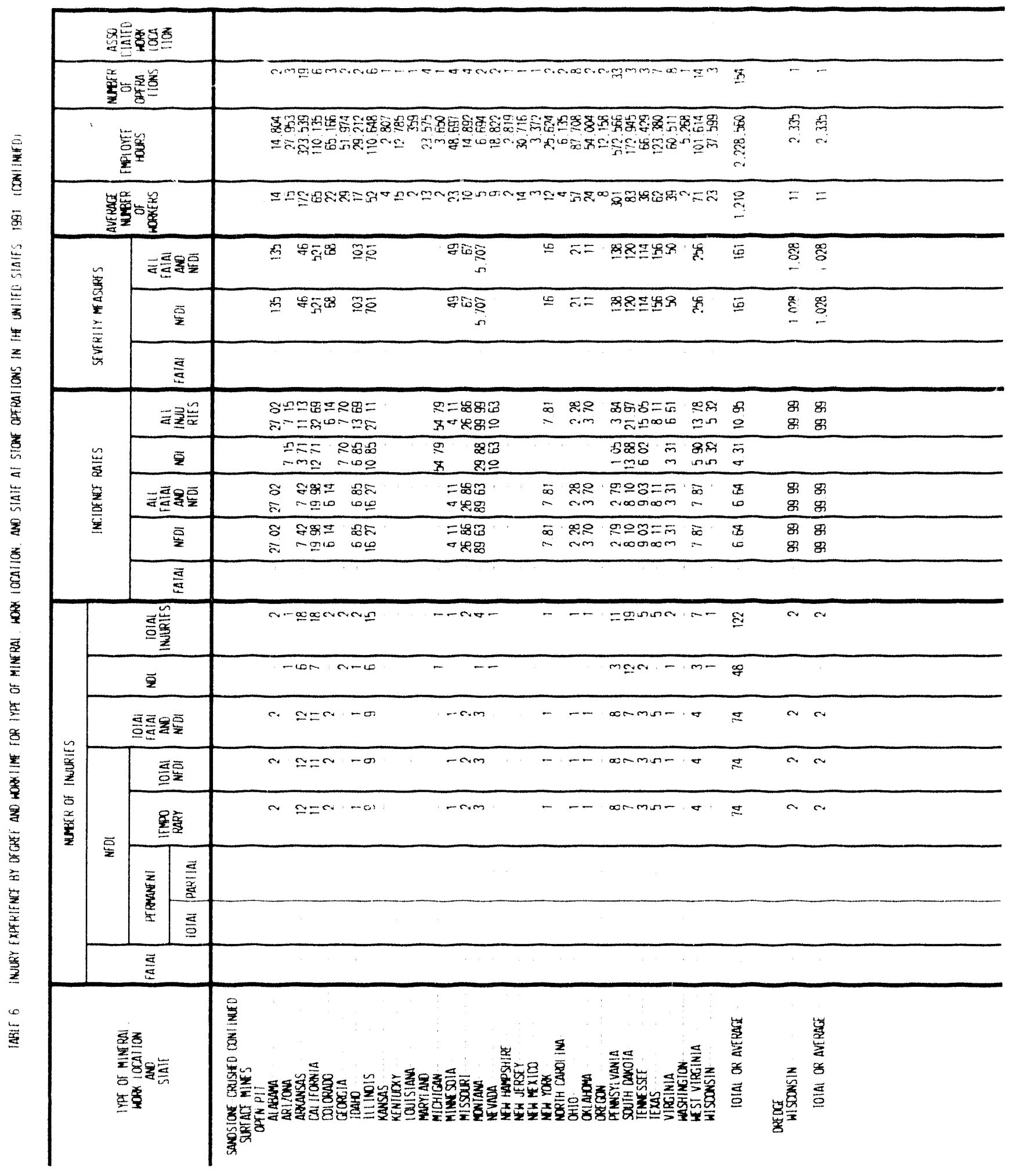




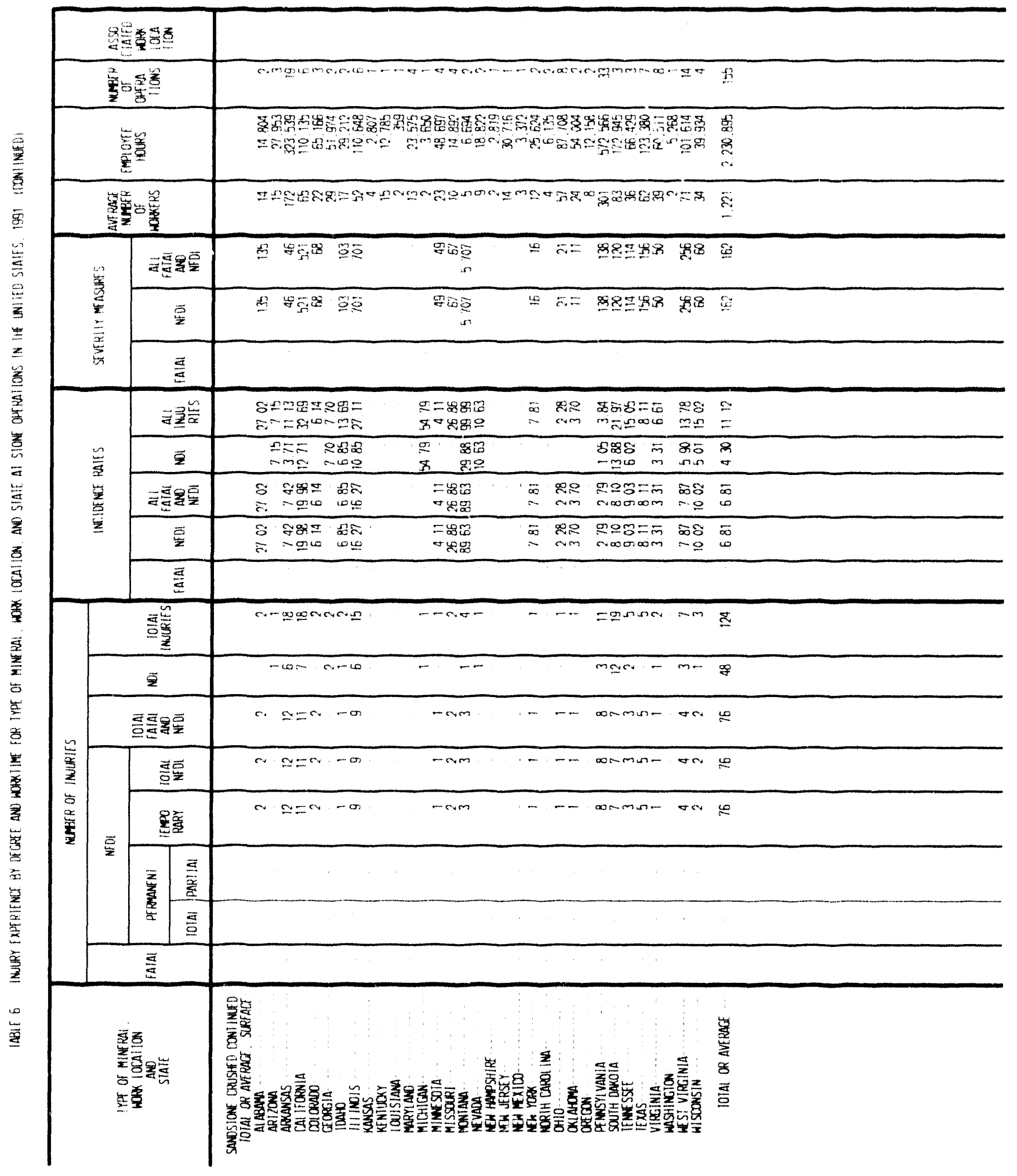



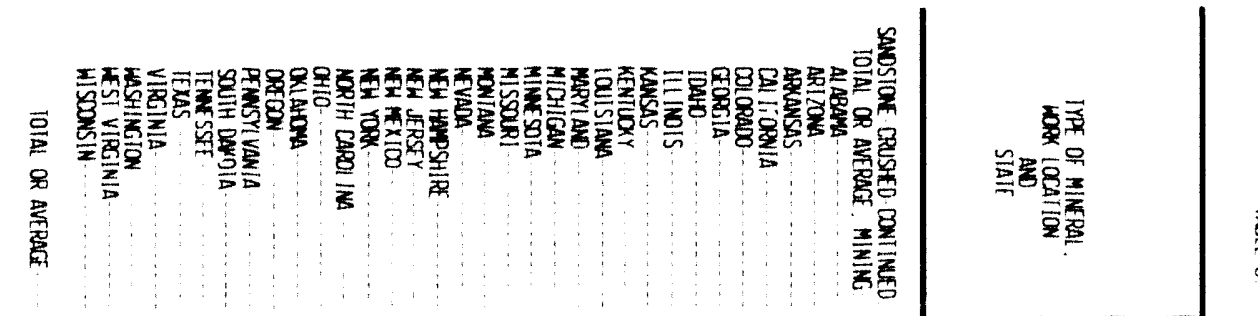

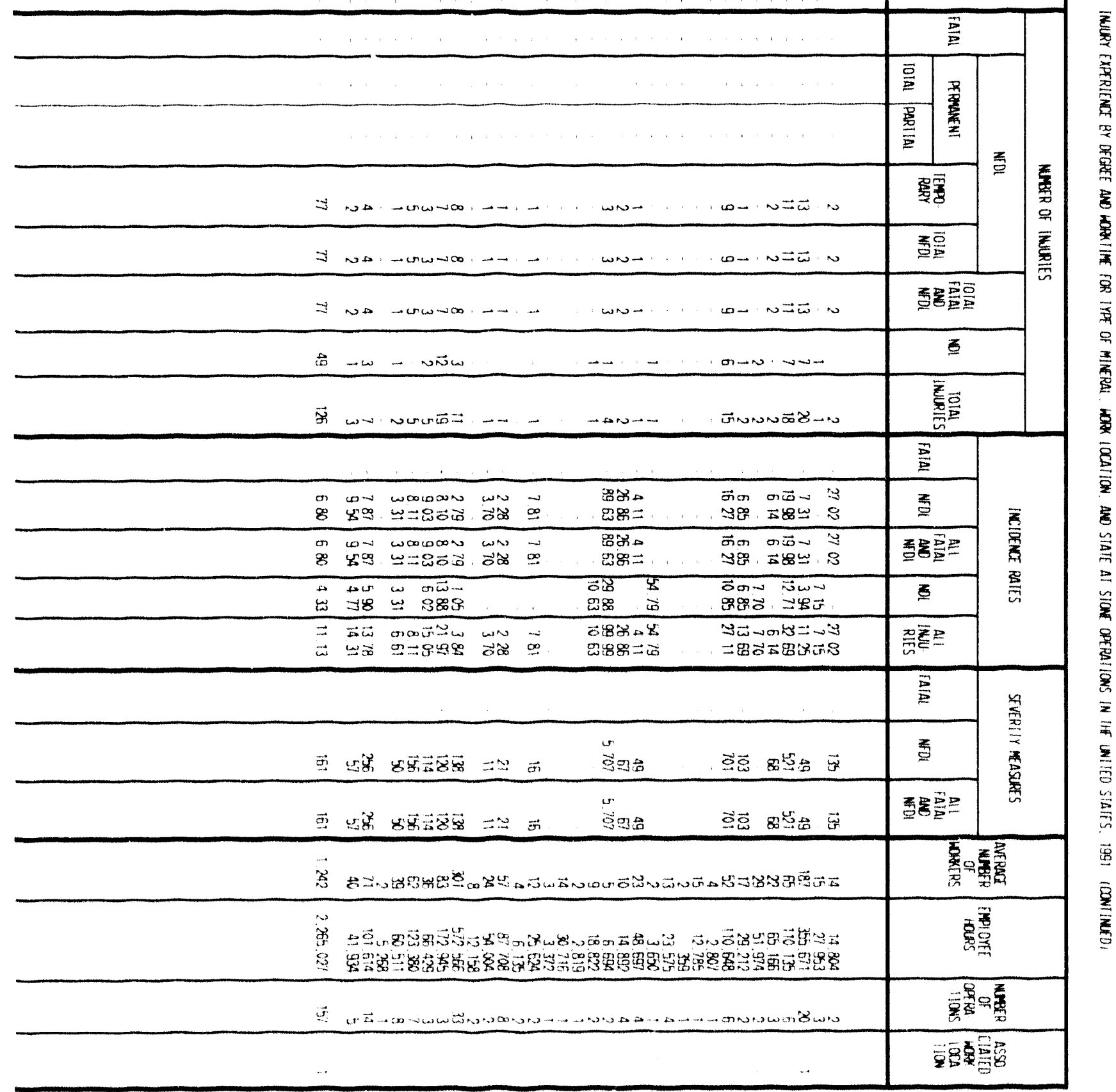




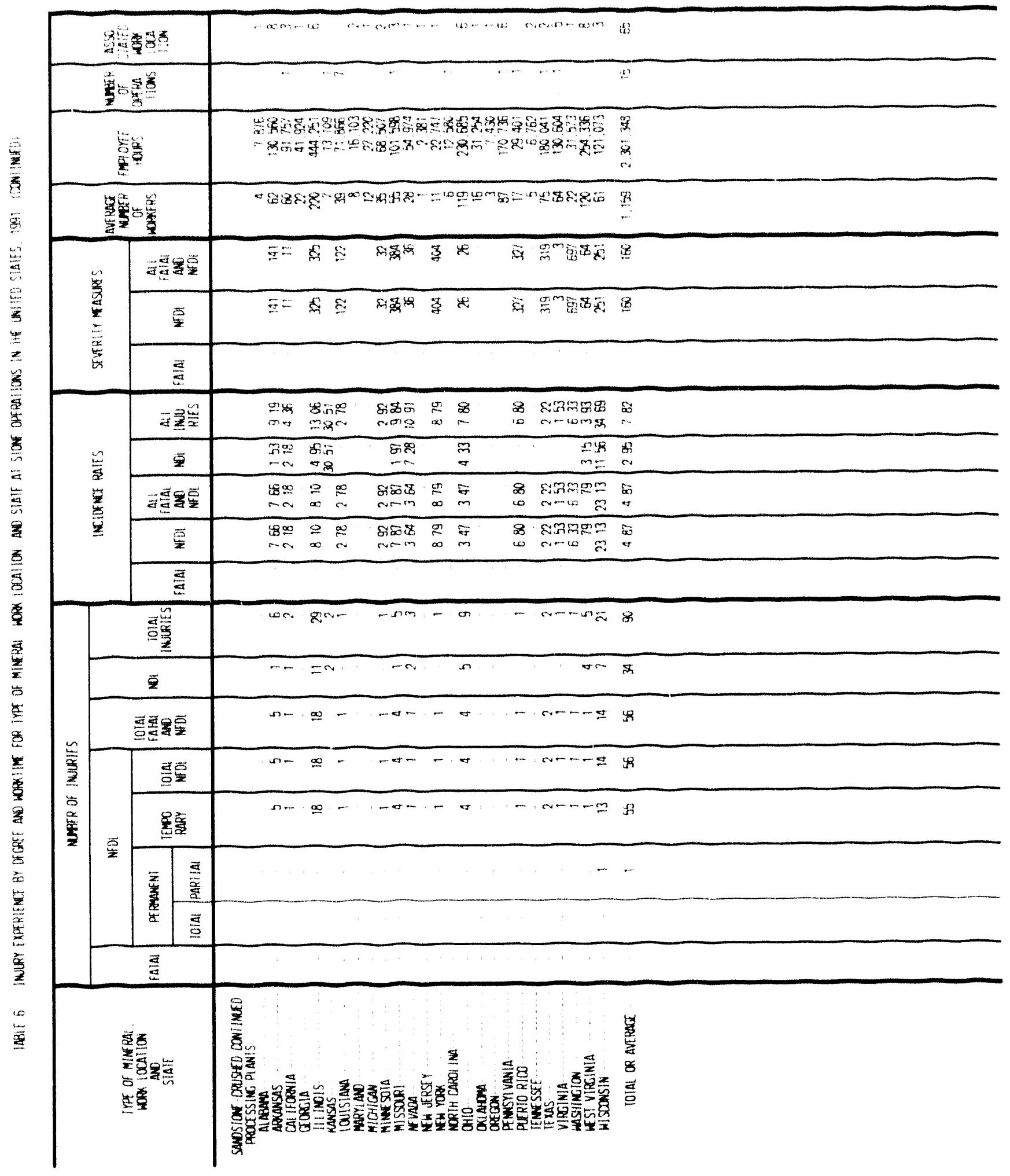




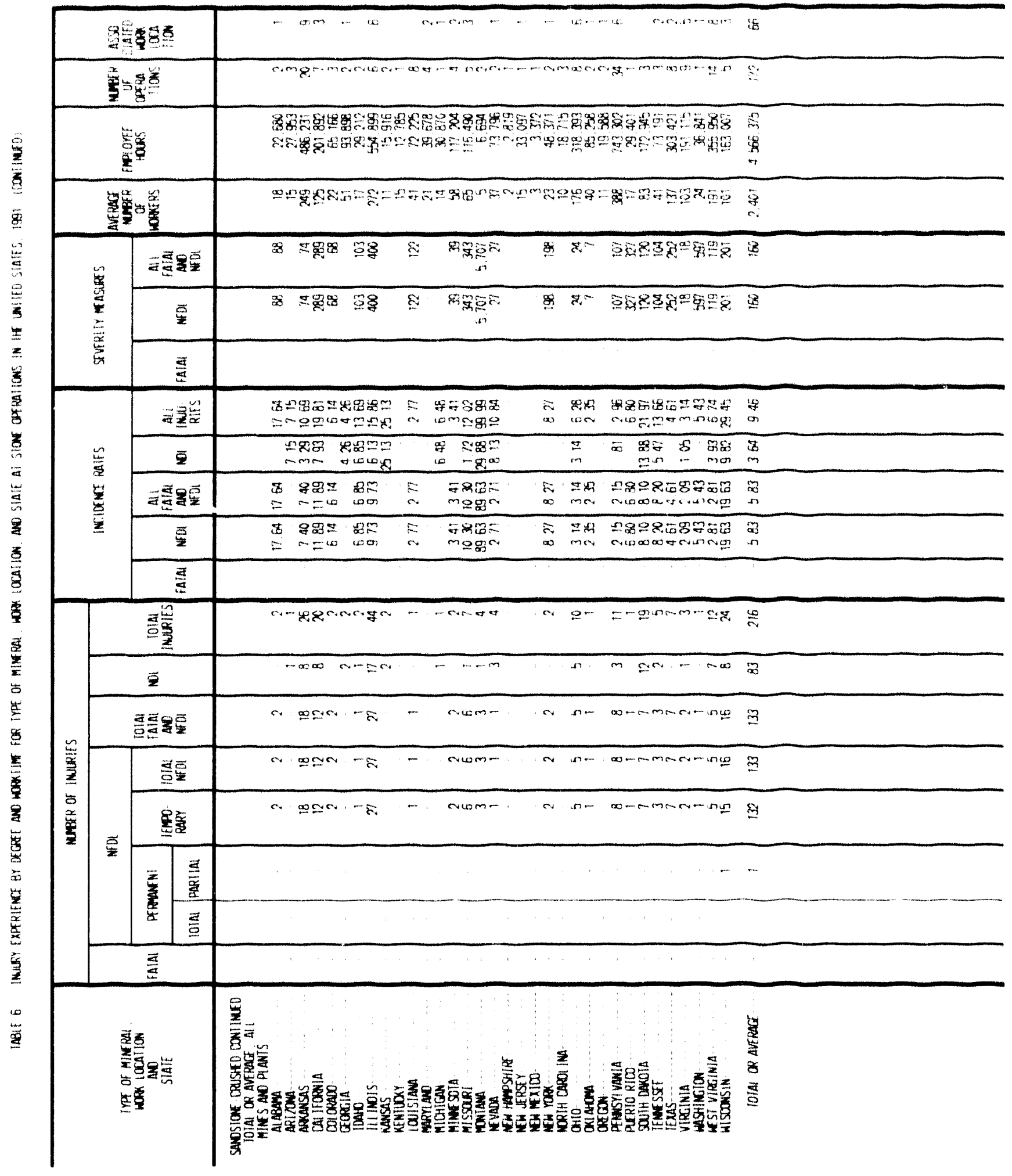




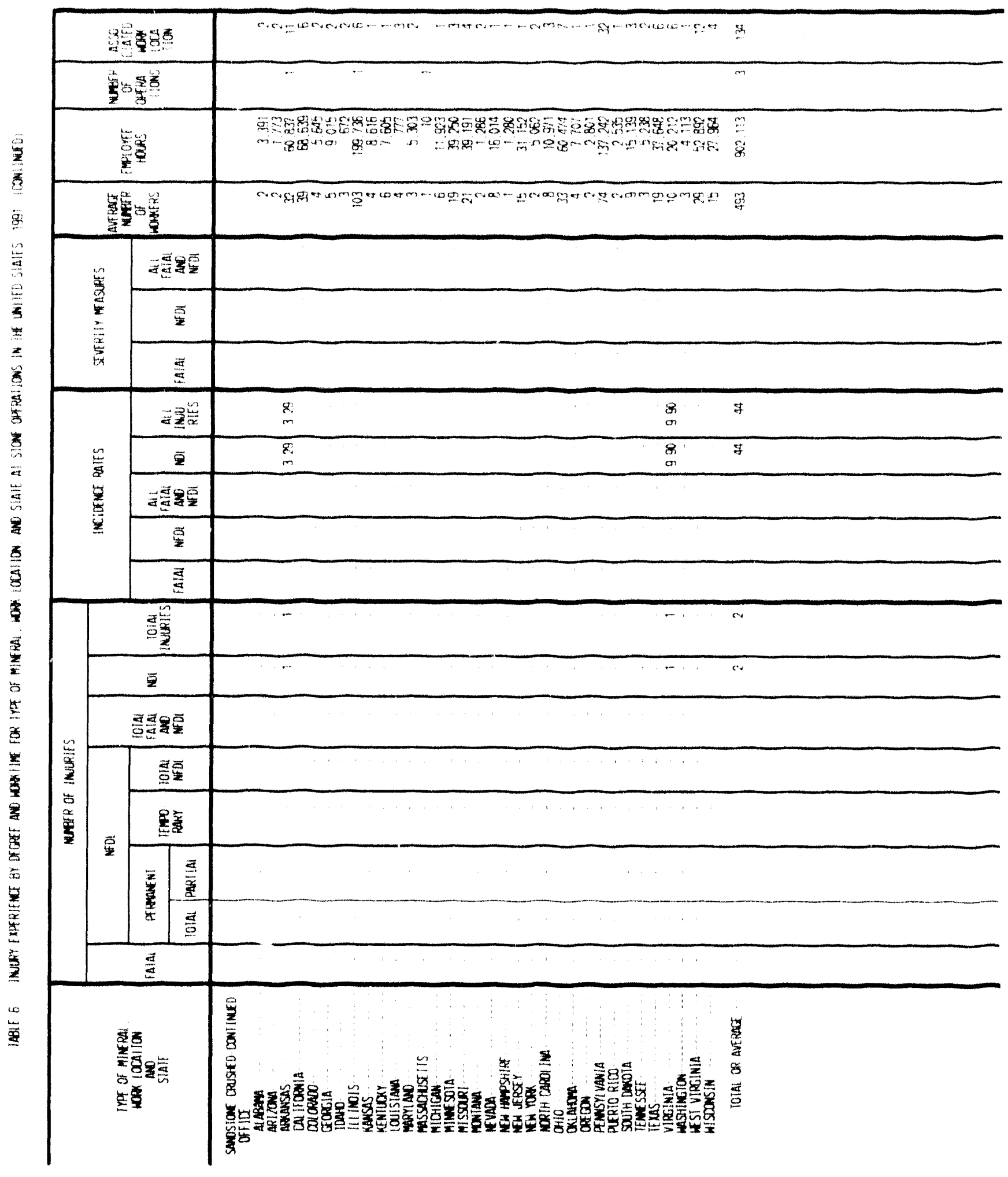



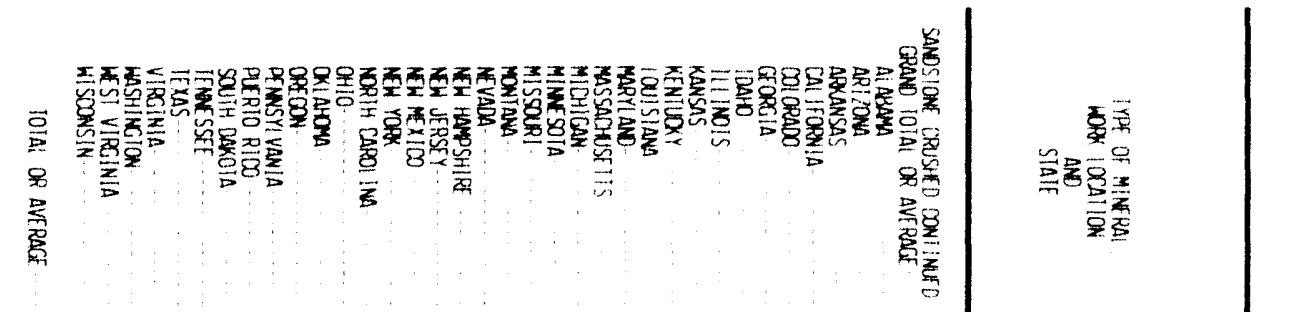

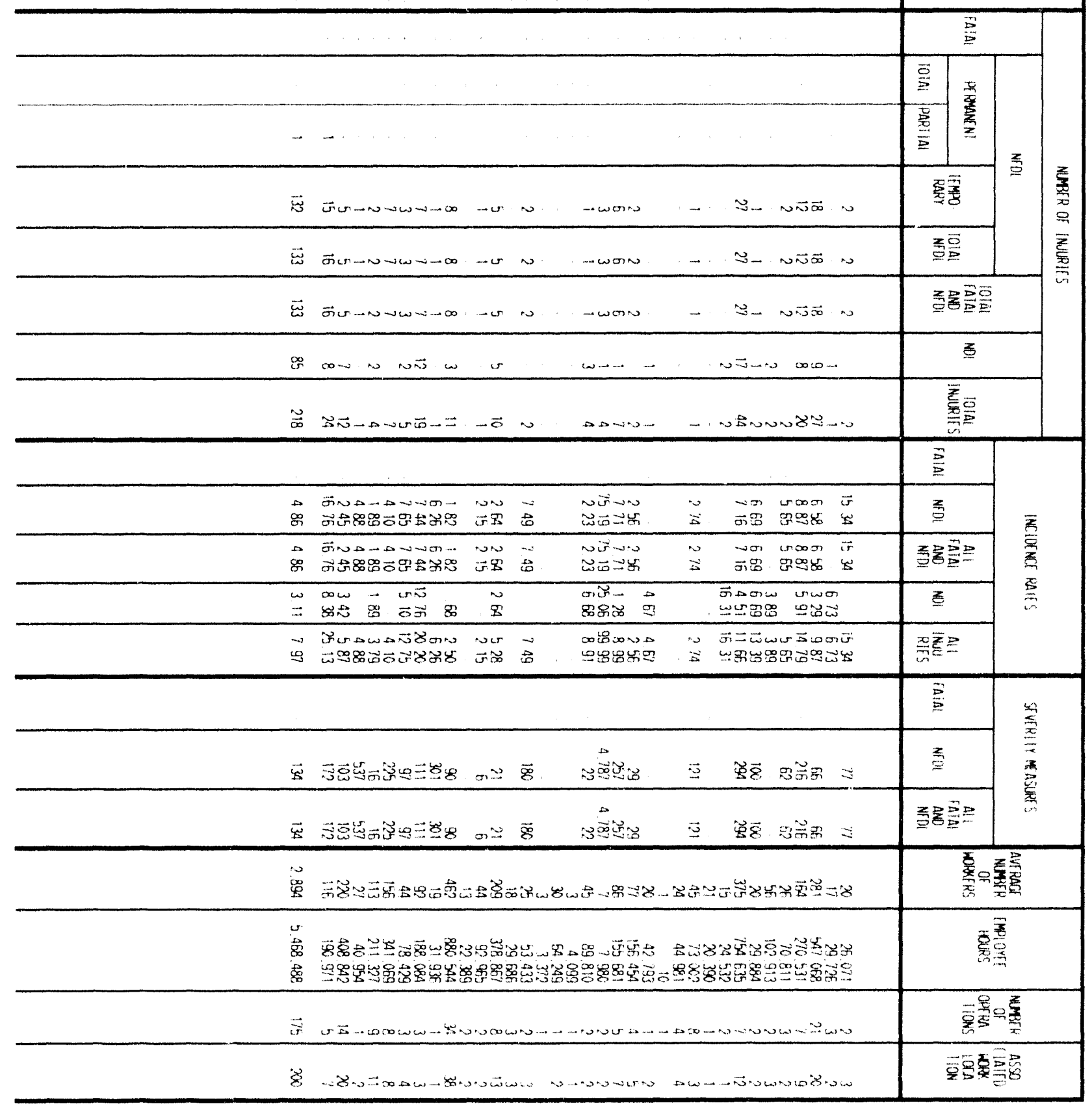




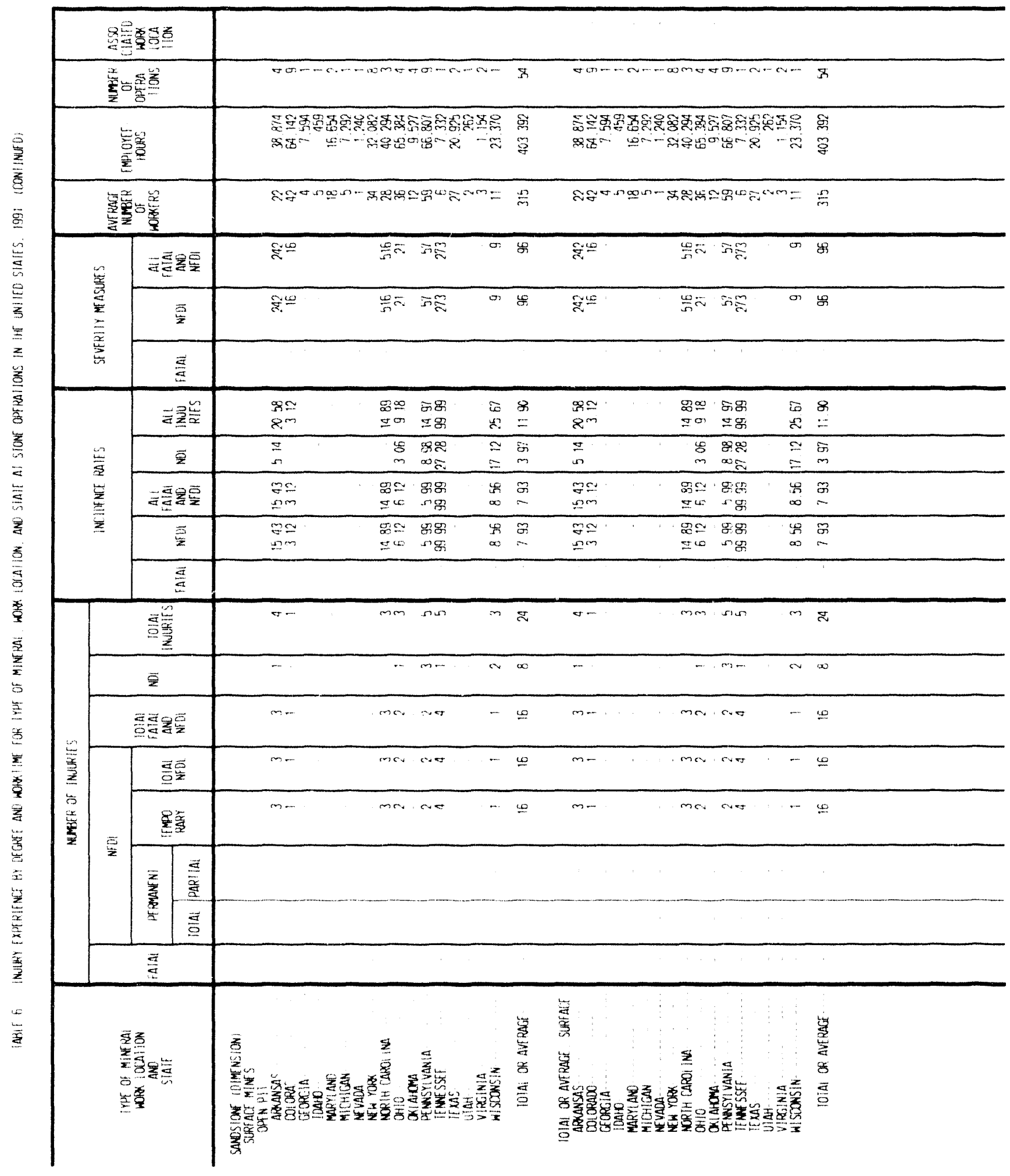




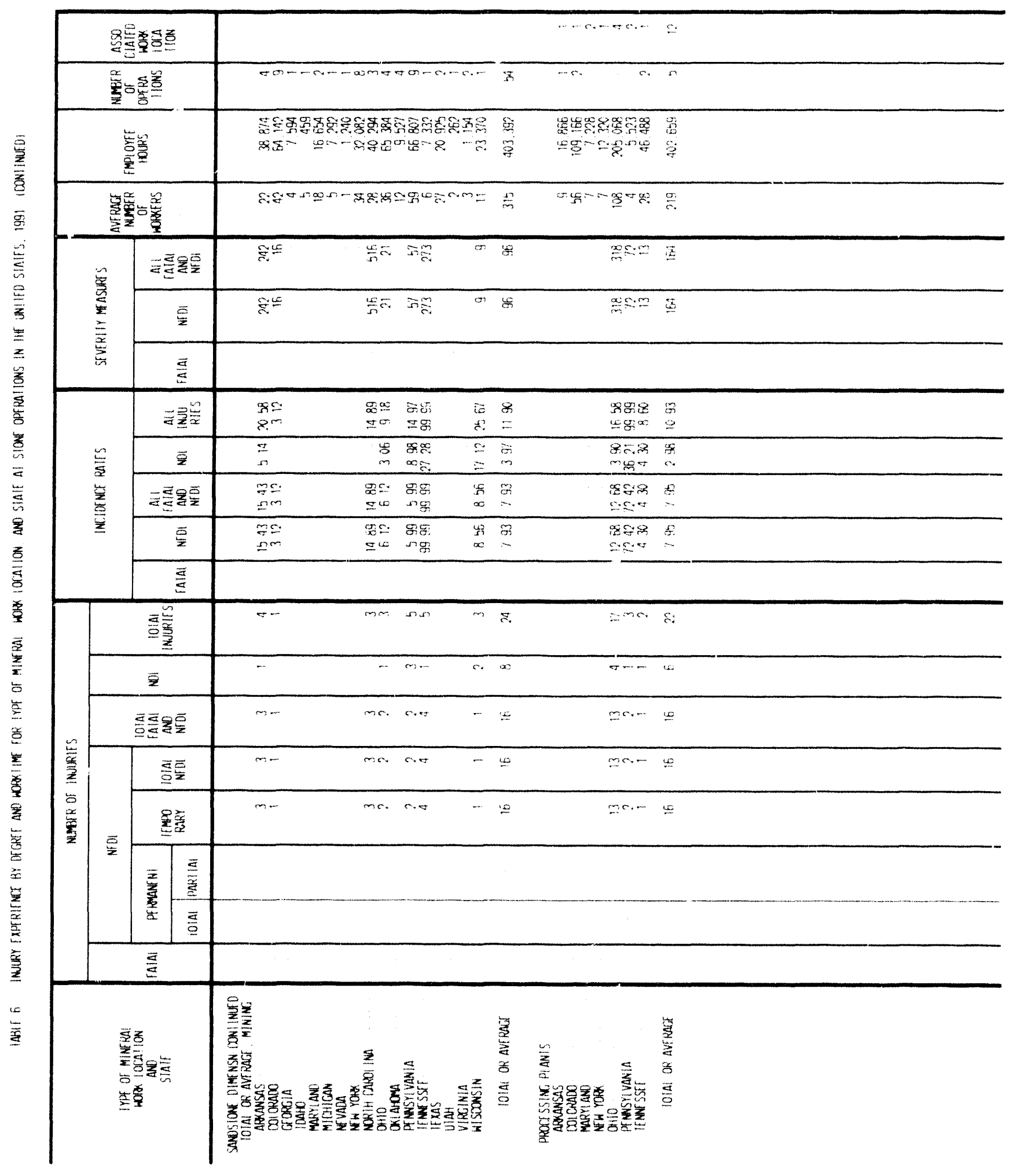



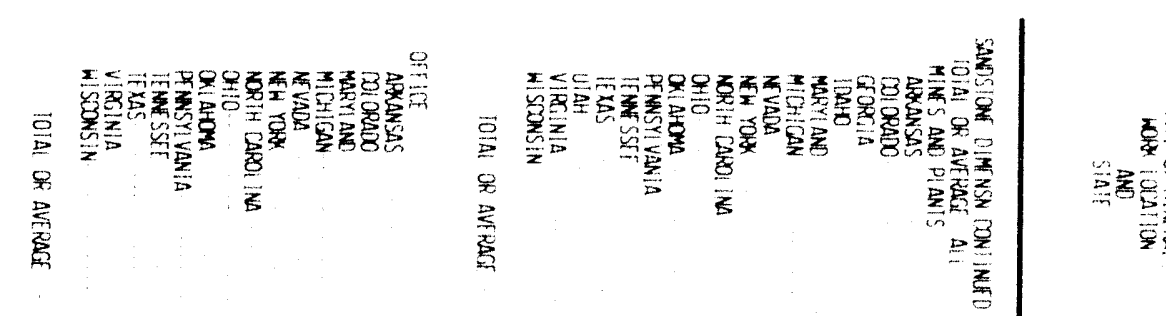

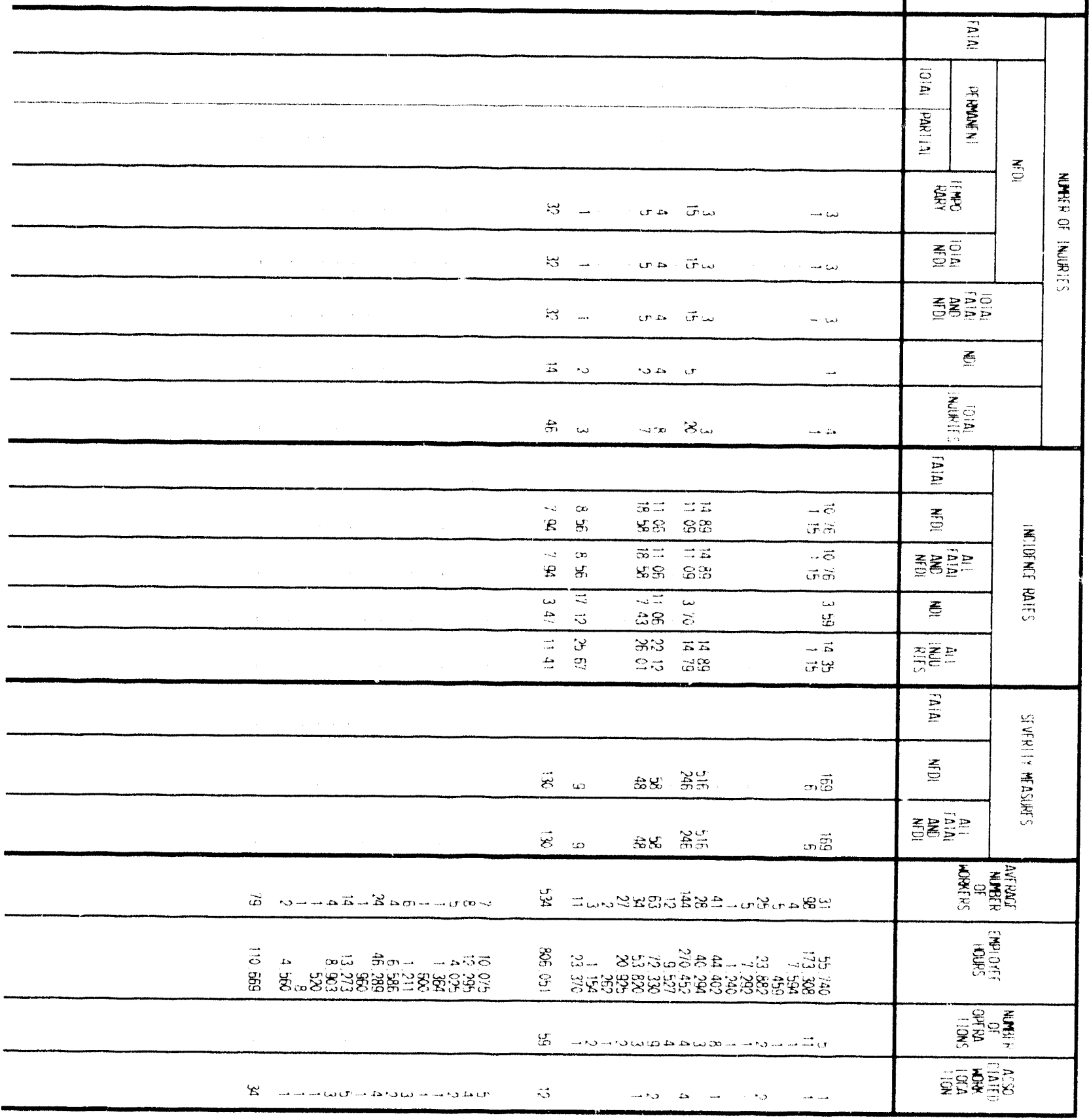



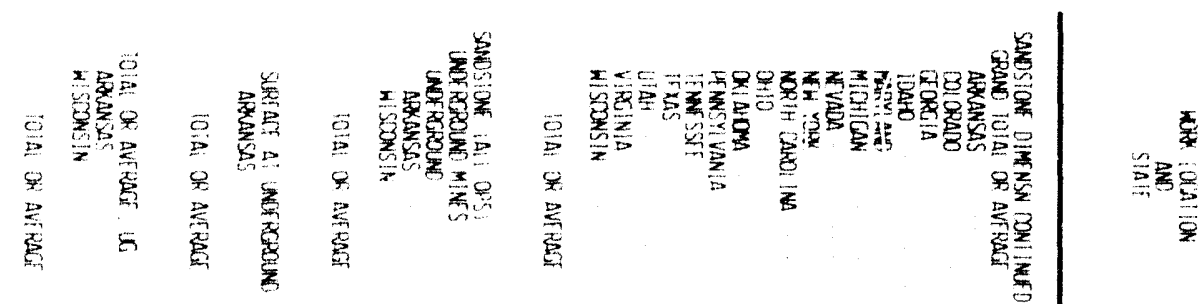

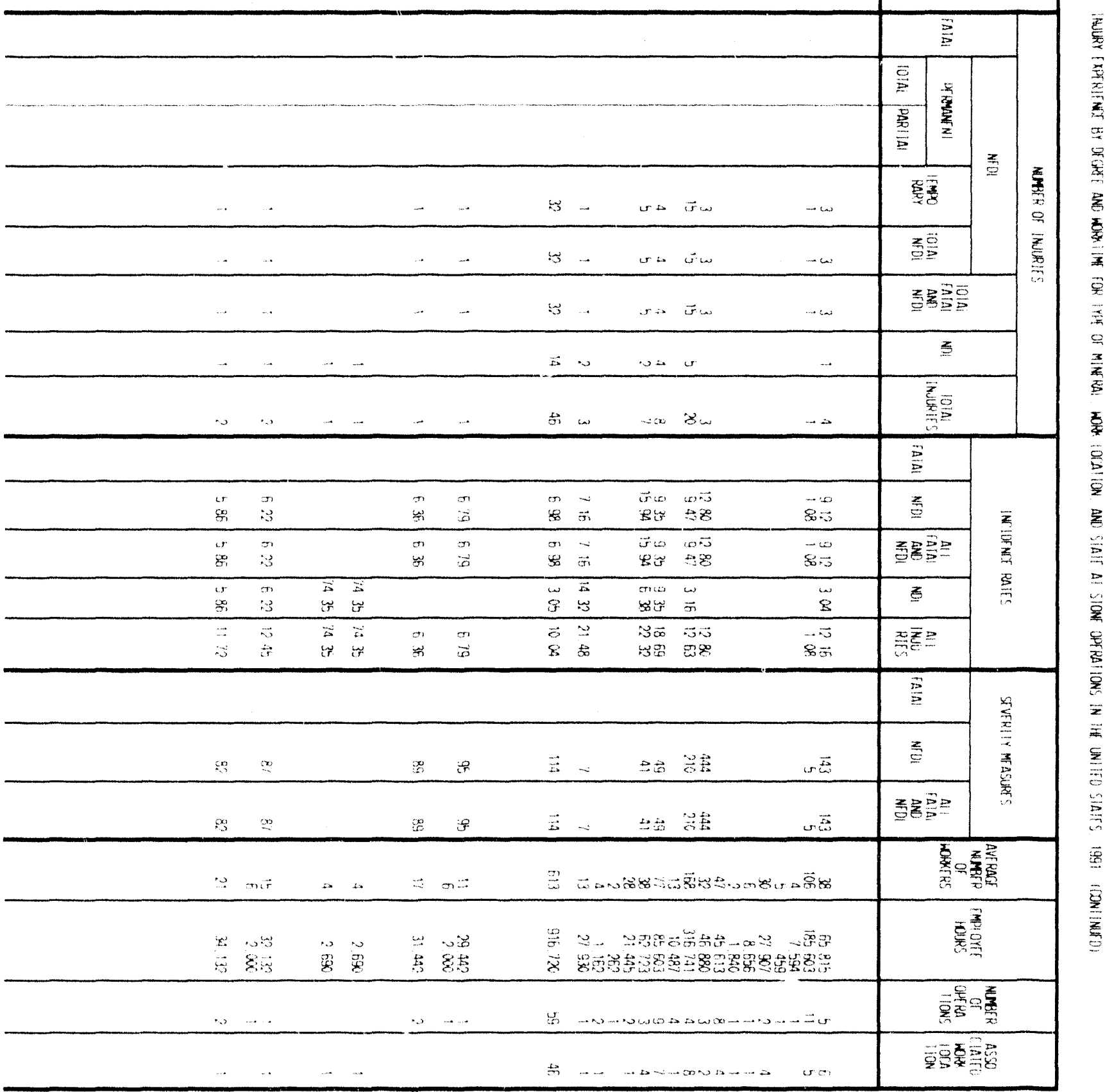




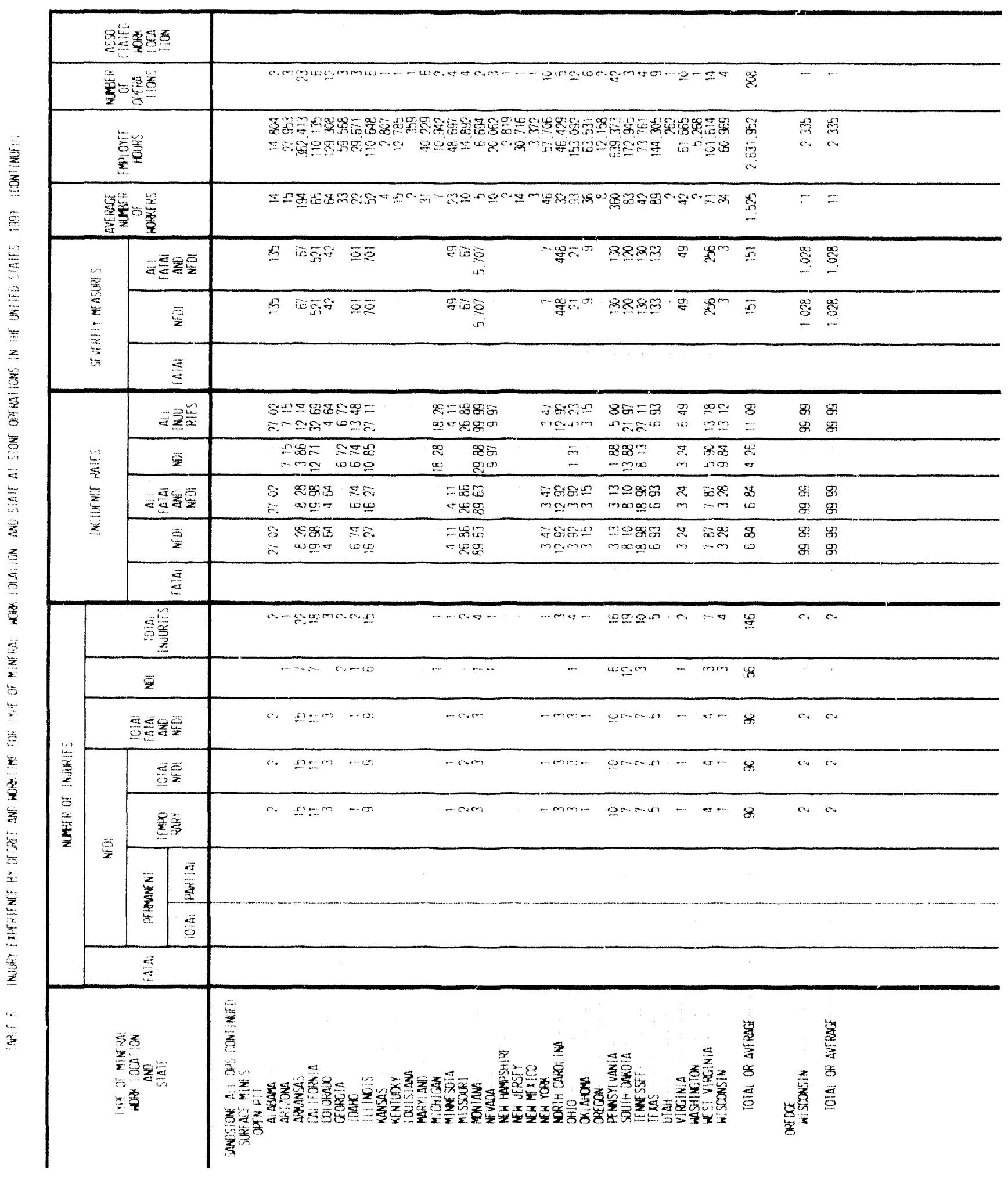



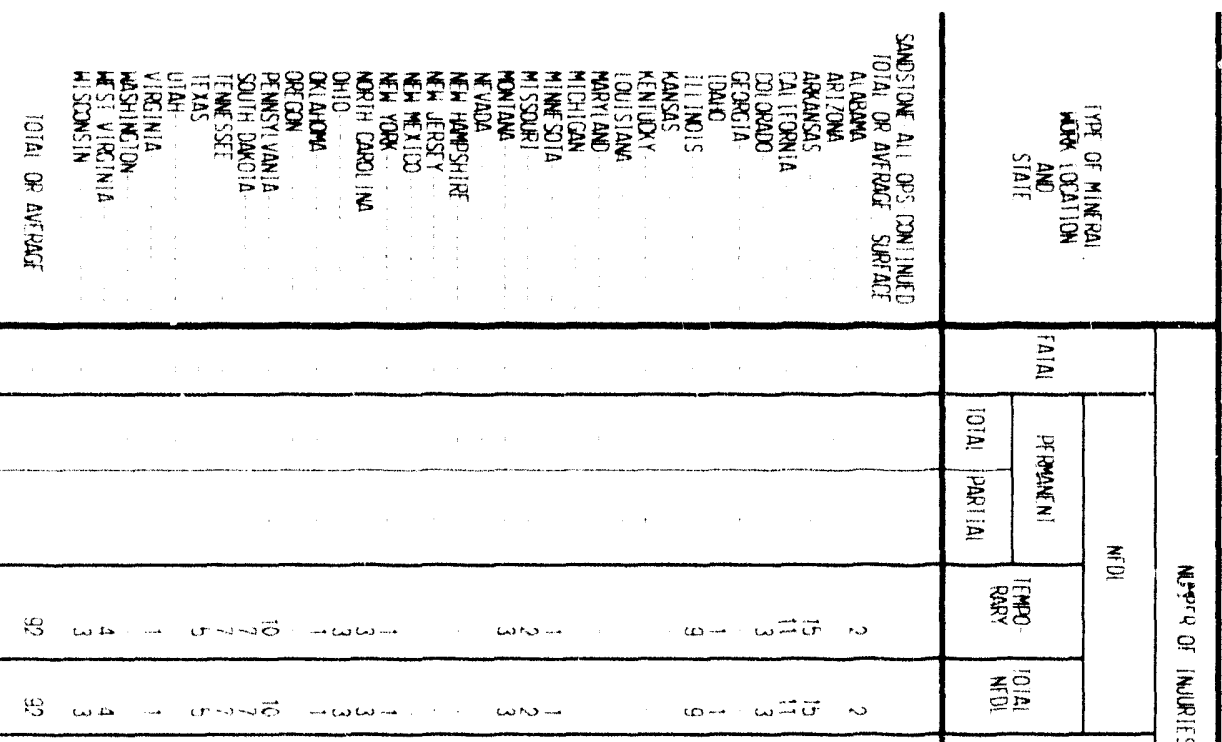

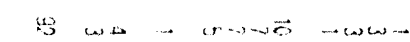

wN-

$\omega-\omega=\overrightarrow{5} \quad \ldots$

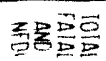

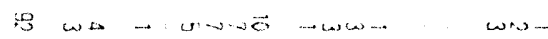

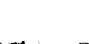

$\omega \rightarrow \omega,=\vec{\sigma} \omega$

St tos $\rightarrow$ wosion

$\vec{\sigma} \vec{\sigma} \vec{\pi}$

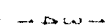

$\infty, \infty \ldots$

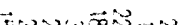

3

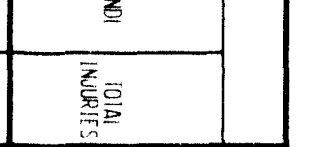

z

का

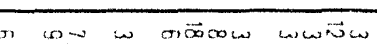

S 500 .

गु.

Cotis

䛧:

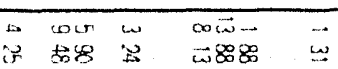

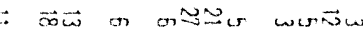

点

$0, \overline{0}$

000
480

wist

980002

$\vec{\infty}$




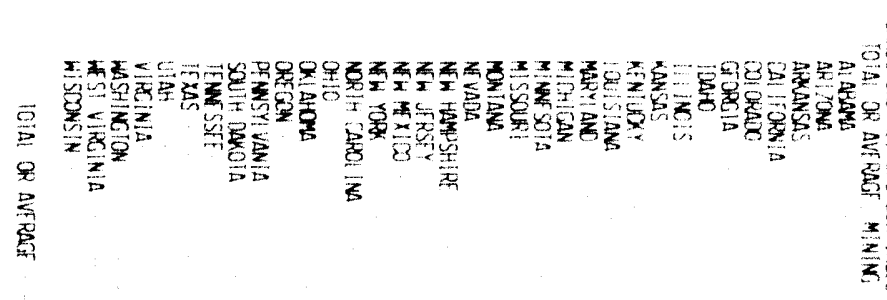

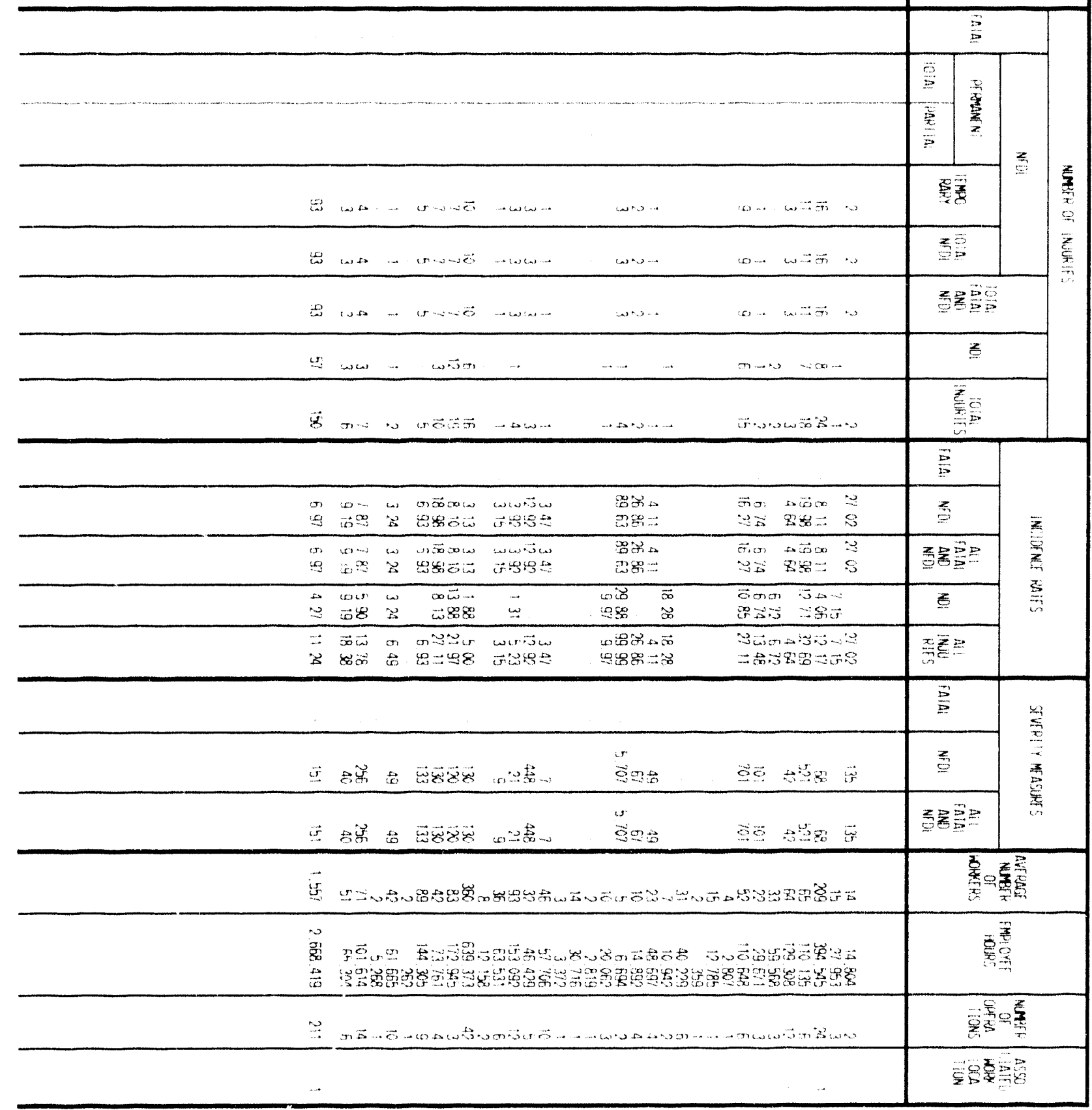




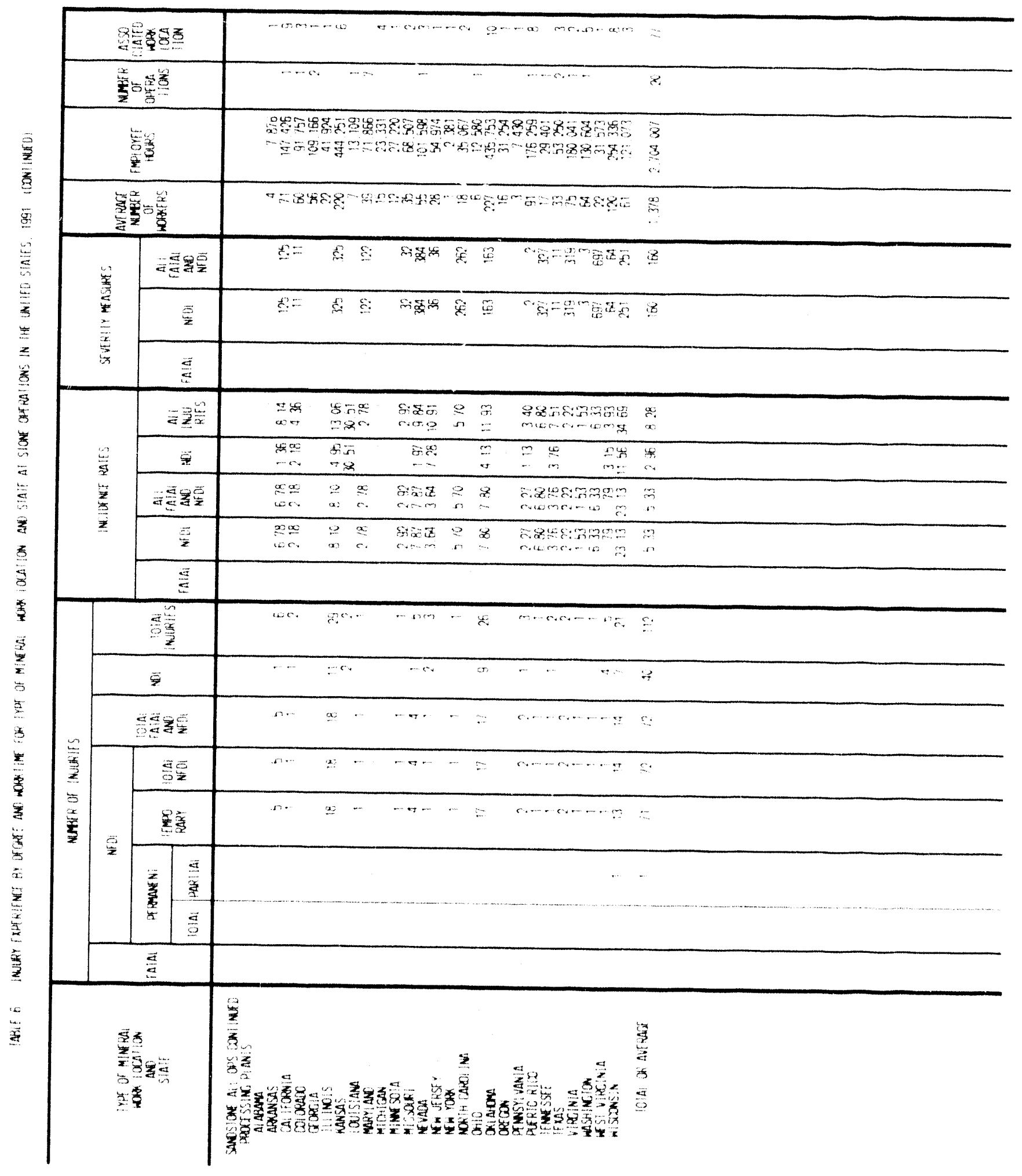




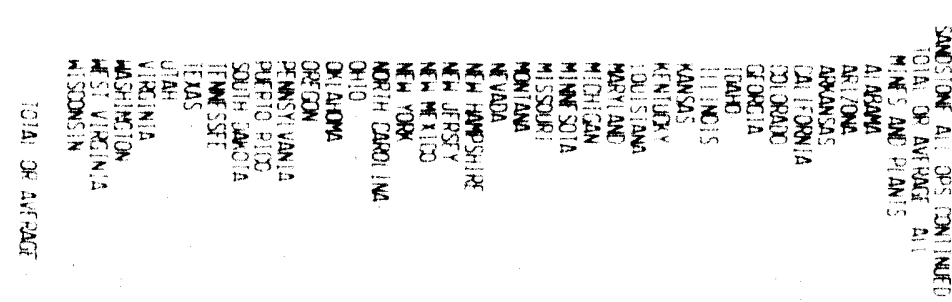

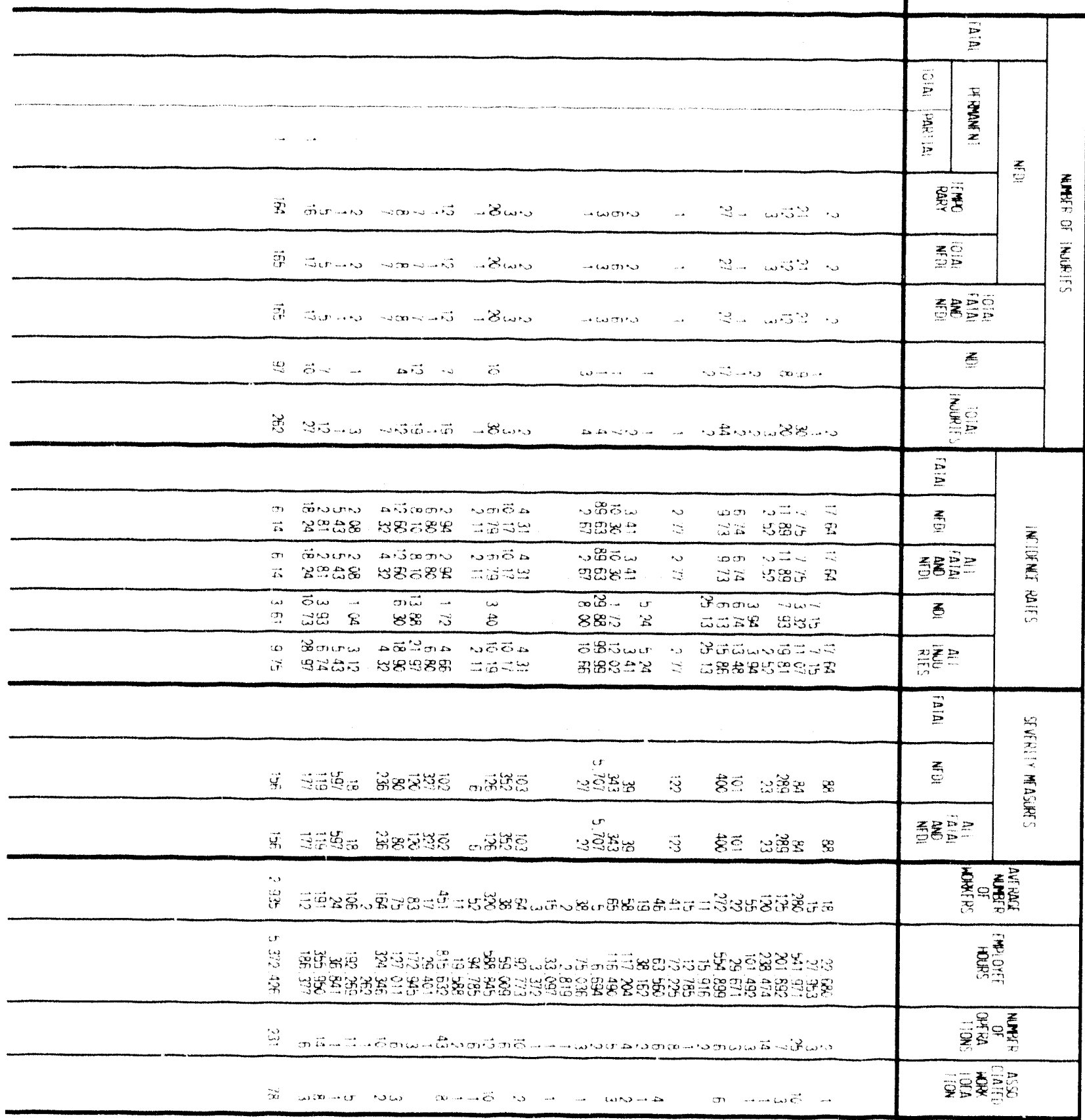




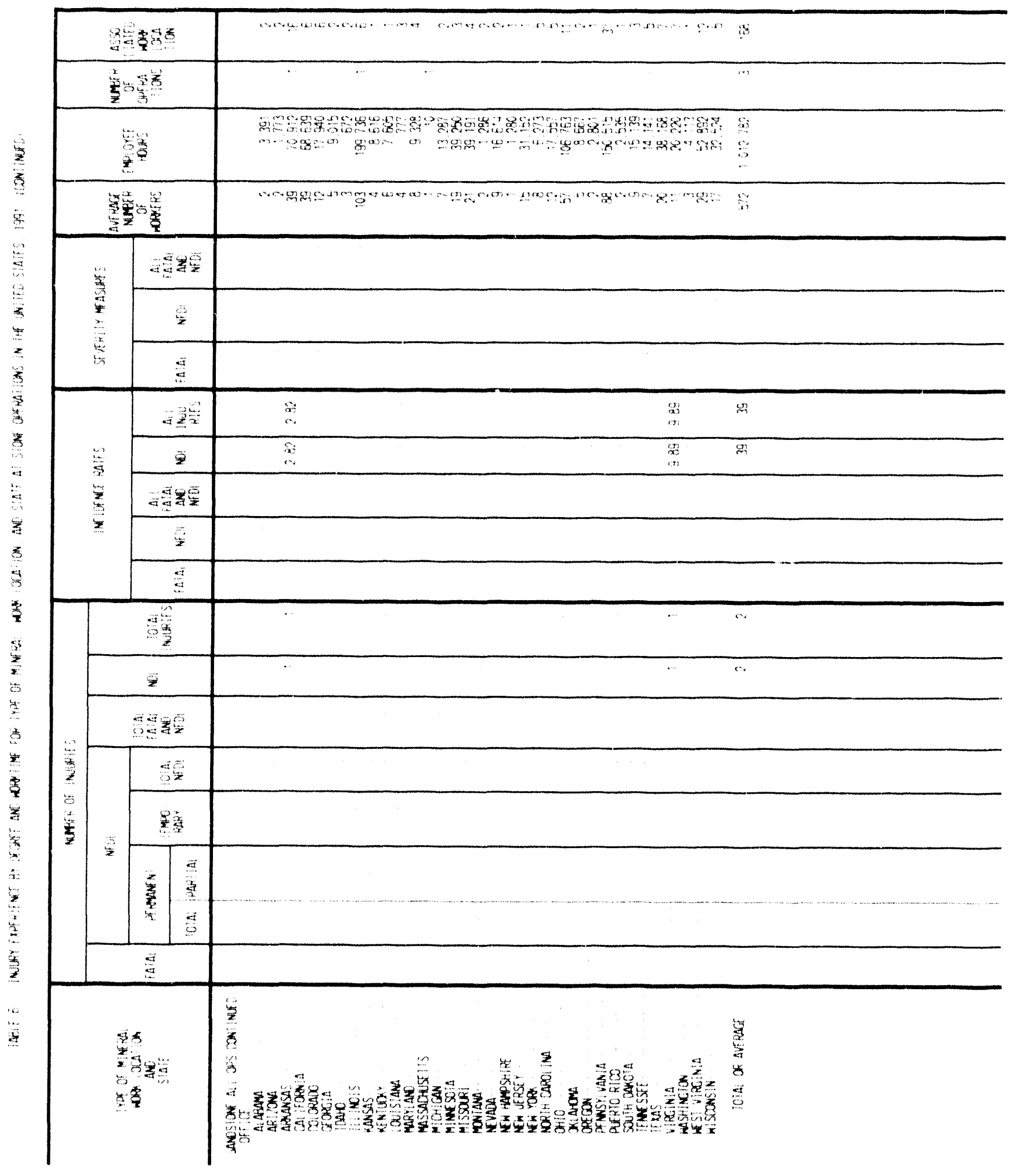




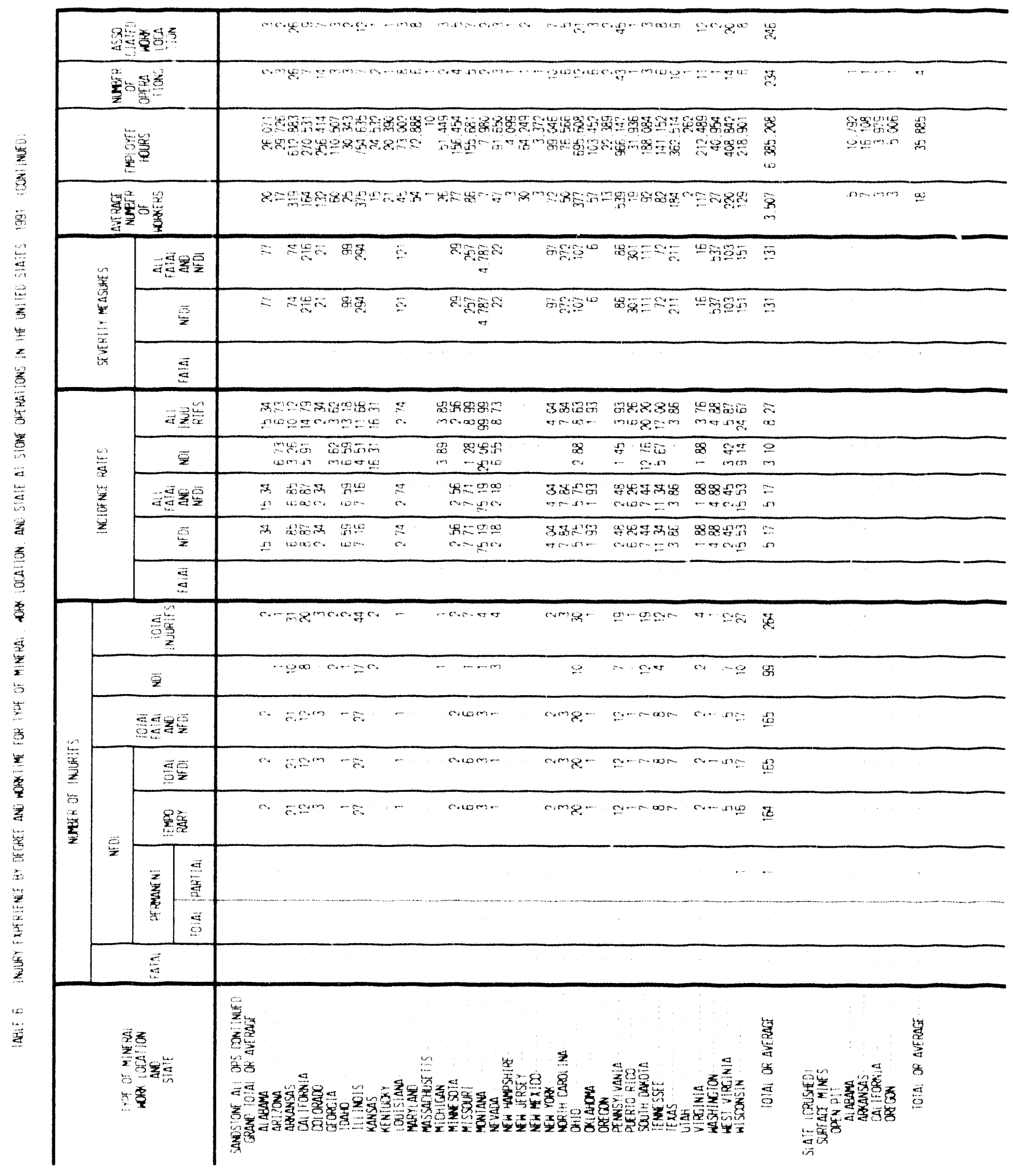




\begin{tabular}{|c|c|c|c|c|c|c|c|c|c|c|c|c|}
\hline & \multicolumn{2}{|c|}{ 움은홍ㅎㅇㅎㅡ } & & & & & - & - & - & - & $m-\cdots$ & - \\
\hline \multicolumn{3}{|c|}{ 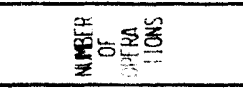 } & $\cdots$ & & $\cdots \cdots$ & & $\cdots-$ & 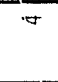 & $--m--\cdots$ & $\infty$ & & \\
\hline \multicolumn{3}{|c|}{ 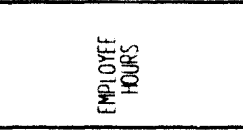 } & 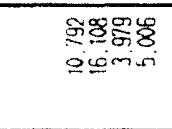 & & 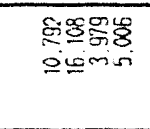 & & 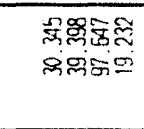 & $\begin{array}{l}\mathbb{G} \\
\substack{0 \\
0 \\
0}\end{array}$ & 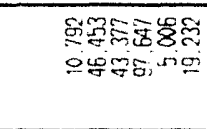 & 窎 & 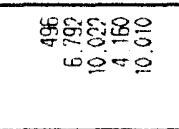 & $\frac{8}{\dot{s}}$ \\
\hline \multicolumn{3}{|c|}{ 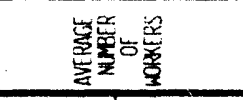 } & nimm & $\cong$ & simm & $\cong$ & $\pm \cong \bar{n}$ & $\mathscr{8}$ & 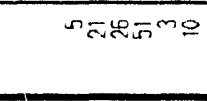 & $\underline{=}$ & terman & $\Phi$ \\
\hline \multirow{3}{*}{ 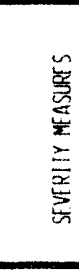 } & 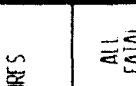 & 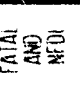 & & & & & $\Phi^{\sim} \overline{-}$ & $\infty$ & $\mathscr{Q}^{\infty} \bar{\sim}$ & $\bar{m}$ & & \\
\hline & 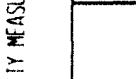 & 产 & & & & & $\Phi^{N}$ & 9 & $\underset{q}{q} \bar{\Sigma}$ & $\bar{m}$ & & \\
\hline & 总 & $\frac{a}{E}$ & & & & & & & & & & \\
\hline & & $=\frac{\tilde{a}}{a}$ & & & & & $\begin{array}{l}\text { oㅛㅇㅛ } \\
m 800\end{array}$ & $\stackrel{g}{=}$ & 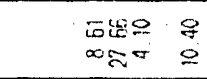 & $\begin{array}{l}\infty \\
0 \\
0 \\
0\end{array}$ & & \\
\hline & 與 & 豆 & & & & & $\begin{array}{l}\text { pogg } \\
\text { mina } \\
\end{array}$ & $\begin{array}{l}q \\
\sigma \\
\end{array}$ & 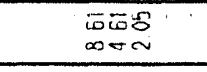 & $\begin{array}{l}8 \\
m \\
\end{array}$ & & \\
\hline & 崖 & 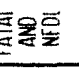 & & & & & $\begin{array}{l}\text { phis? } \\
\text { rino } \\
\end{array}$ & $\stackrel{8}{2}$ & 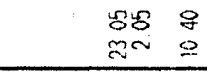 & $\begin{array}{l}9 \\
0 \\
0\end{array}$ & & \\
\hline & & 홀 & & & & & $\begin{array}{l}\text { pogs } \\
\text { pive }\end{array}$ & $\stackrel{8}{2}$ & 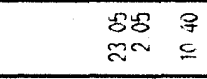 & $\begin{array}{l}9 \\
0 \\
0\end{array}$ & & \\
\hline & & $\underline{\underline{a}}$ & & & & & & & & & & \\
\hline \multirow{8}{*}{ 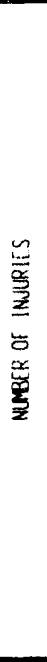 } & 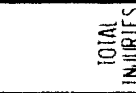 & & & & & & reon- & $=$ & ven ... & $=$ & & \\
\hline & 豆 & & & . & & & $n-$ & $\sigma$ & $n$ & $\sigma$ & & \\
\hline & 票哥舟 & & & & & & nor & - & $n-$ & - & & \\
\hline & \multirow{4}{*}{$\overline{\bar{E}}$} & 흥홀 & & v & & & $n-$ & - & $n-\cdots$ & - & & \\
\hline & & 塞裹 & & & & & nn..- & - & s. & - & & \\
\hline & & \begin{tabular}{|l} 
亲 \\
童 \\
\end{tabular} & & . & & & & 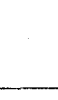 & . & & & \\
\hline & & $\underline{3}$ & & & & & & . & , & & & \\
\hline & \multicolumn{2}{|l|}{ 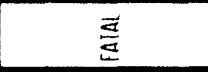 } & & & & & & . & ' & & & \\
\hline \multicolumn{3}{|c|}{ 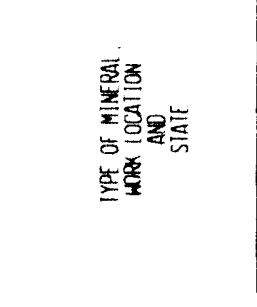 } & 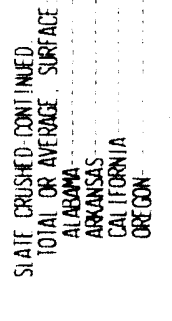 & 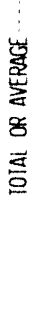 & 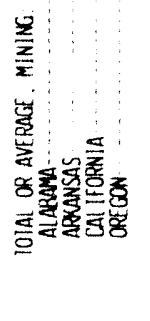 & 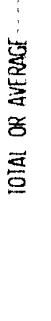 & 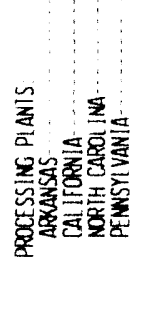 & 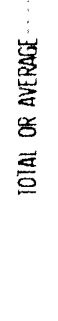 & 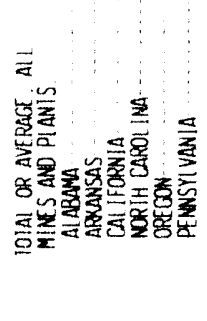 & 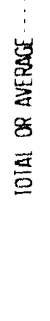 & 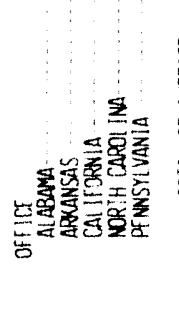 & 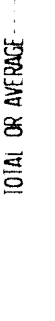 \\
\hline
\end{tabular}




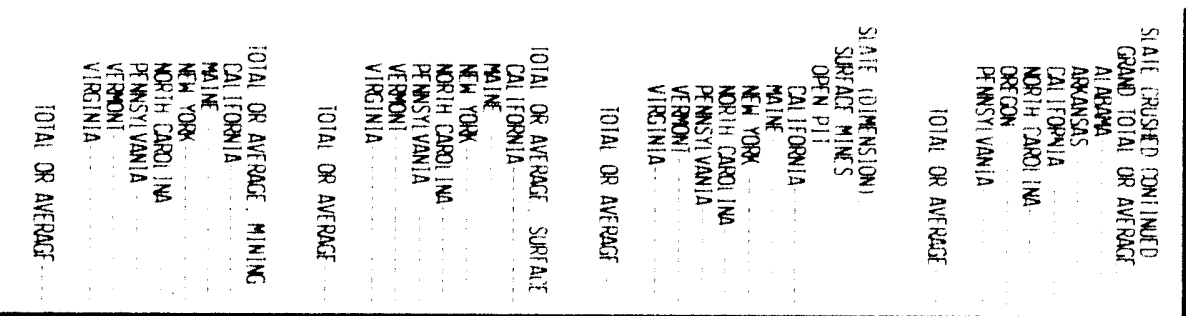

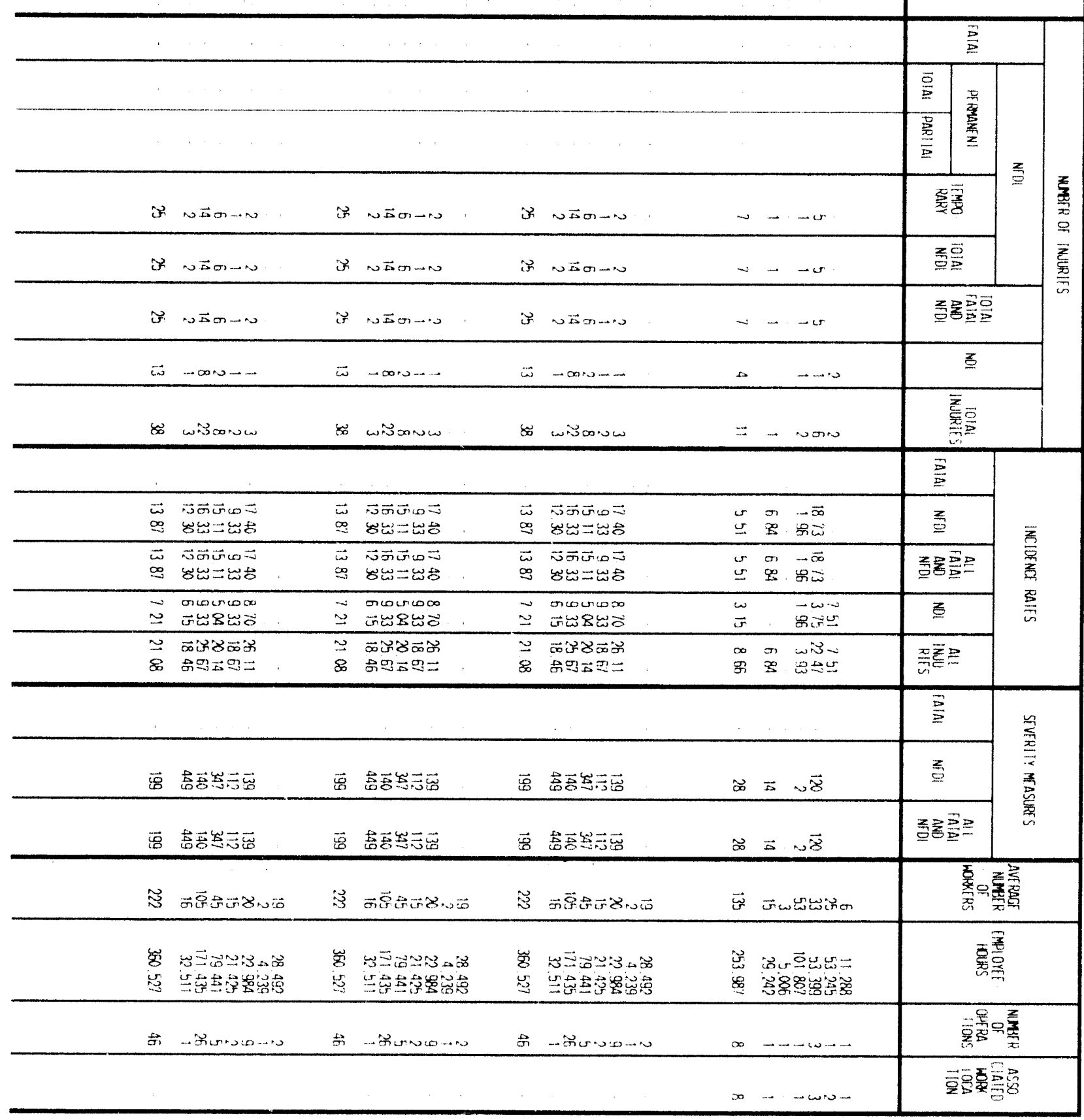




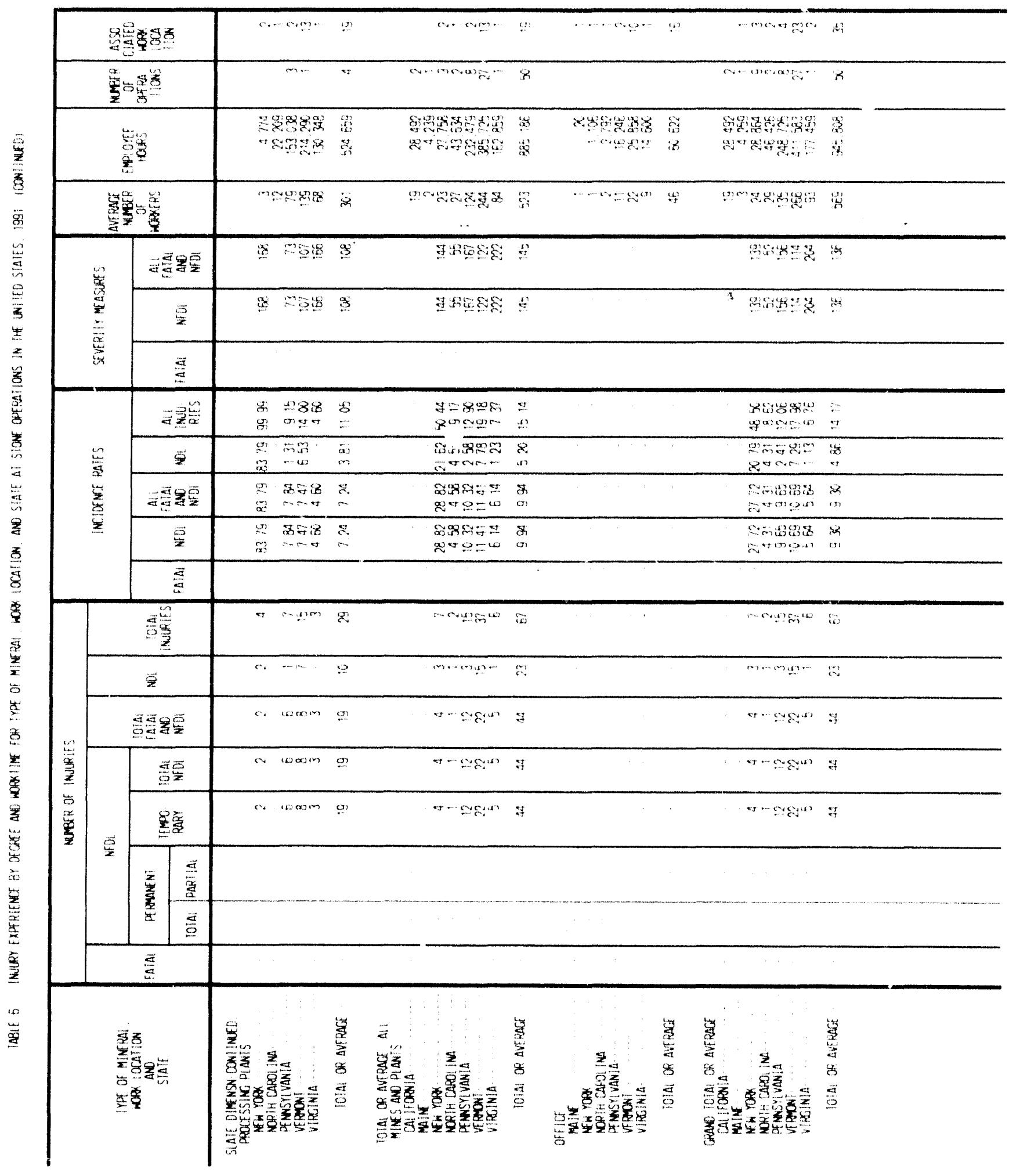




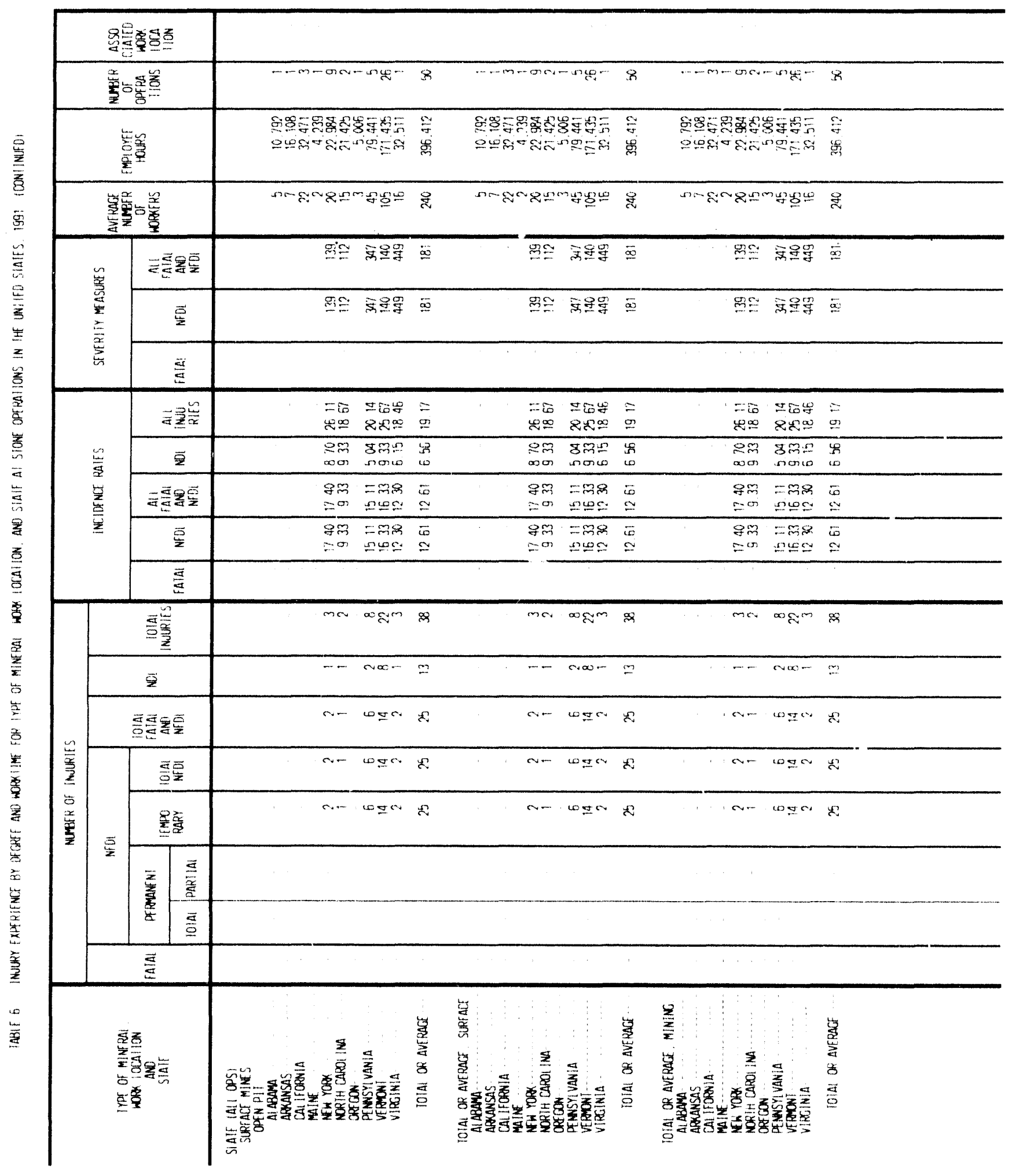



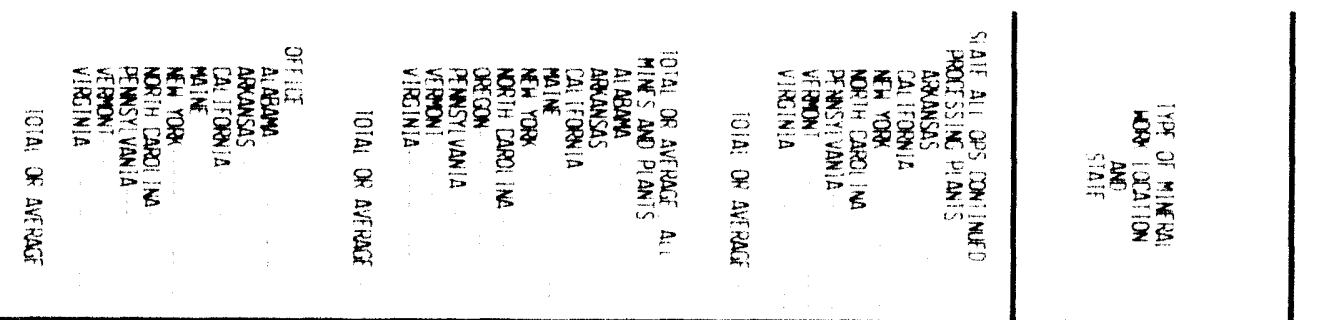

政

य)

b wrotatom

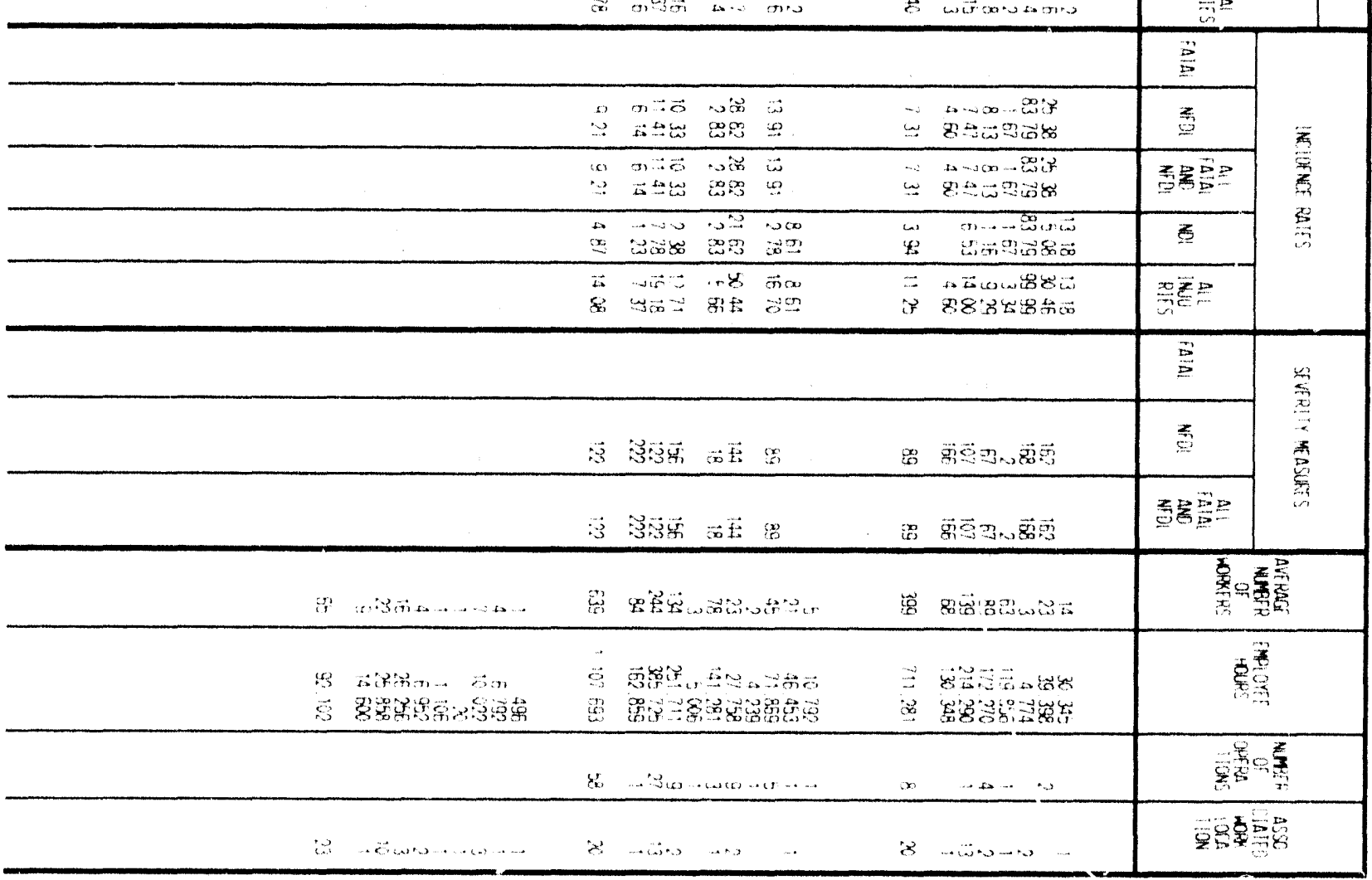



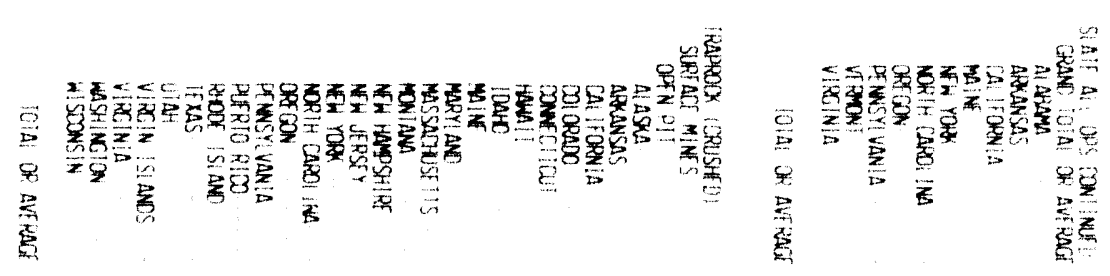

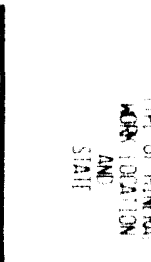

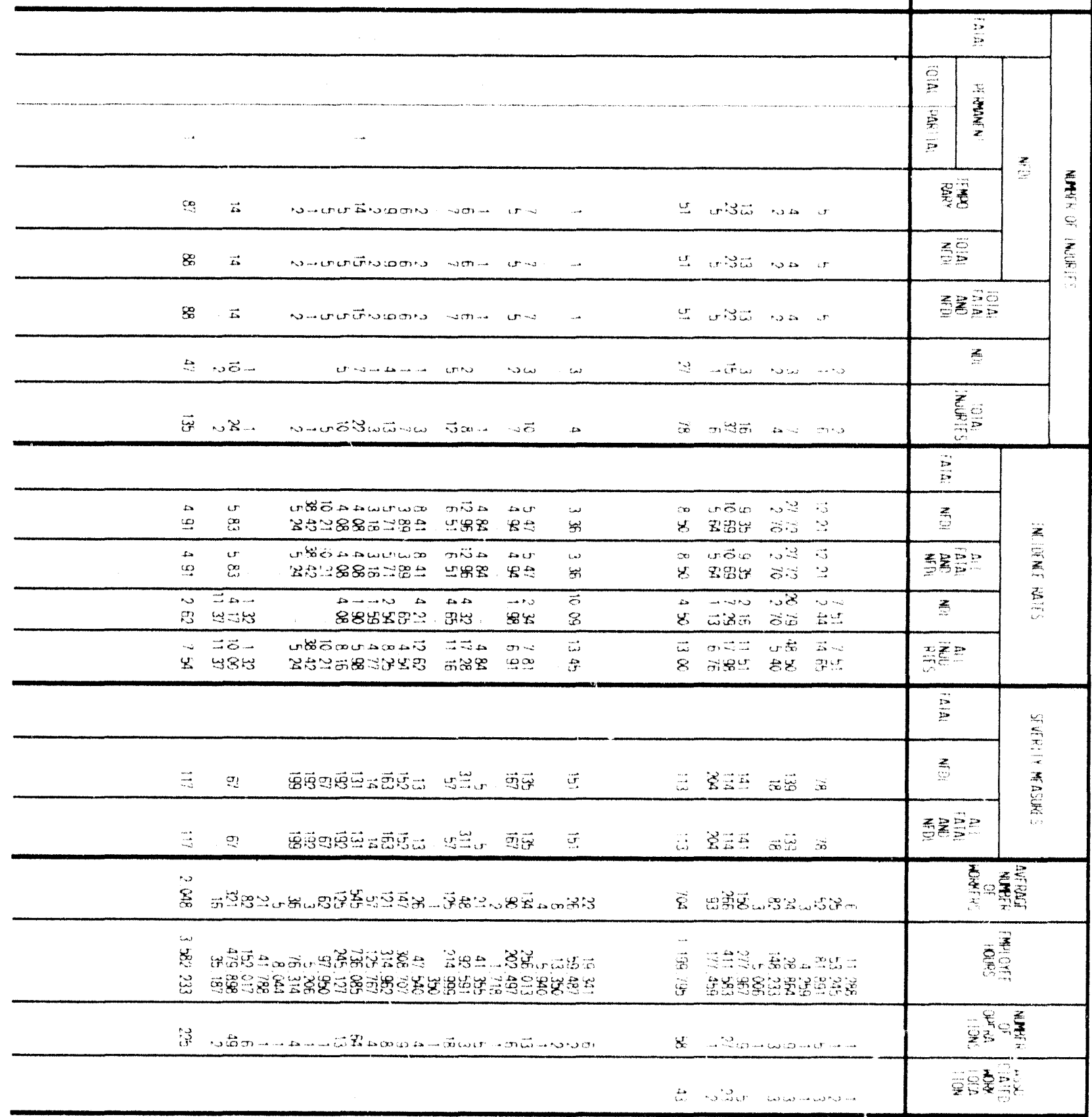




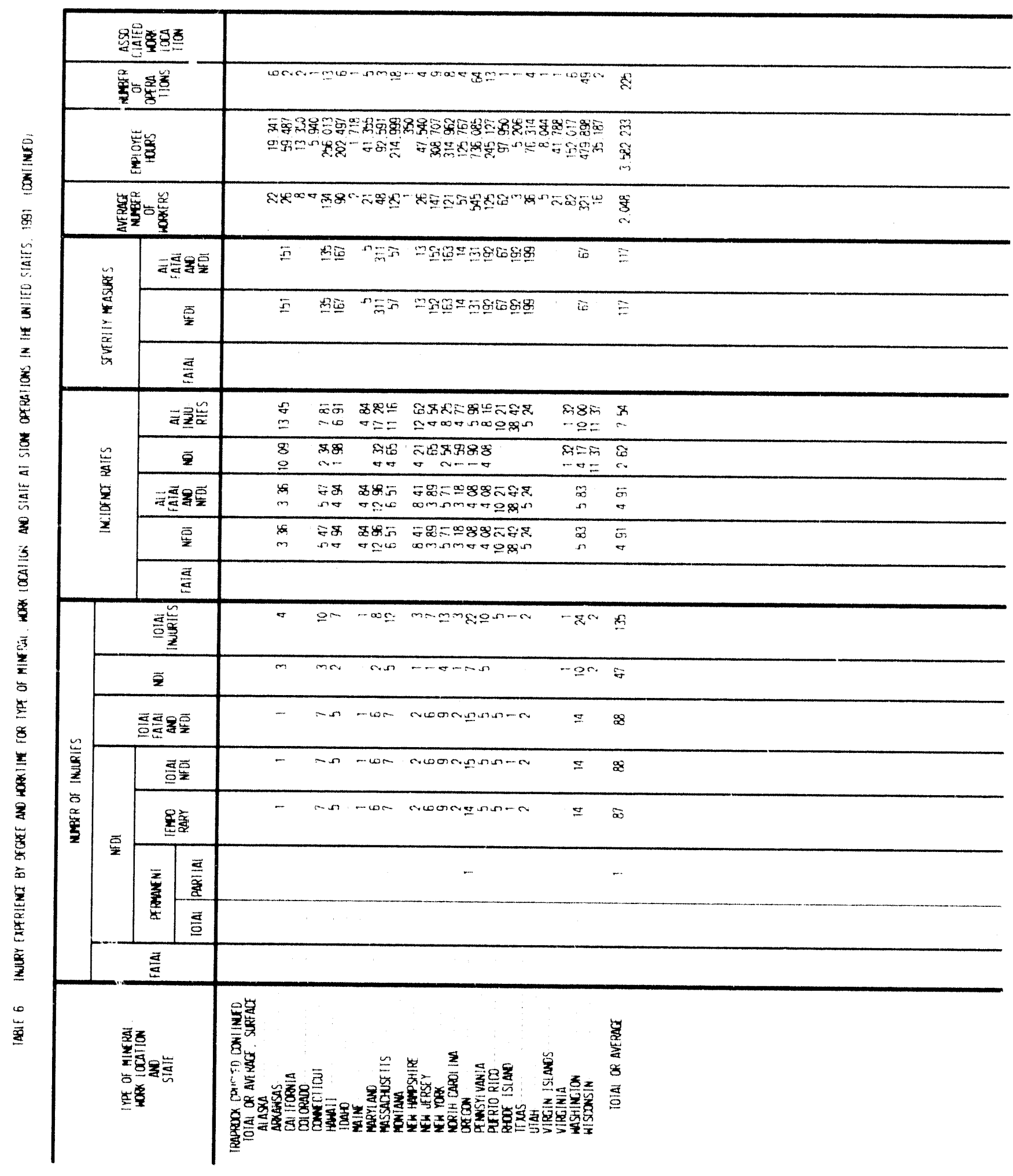



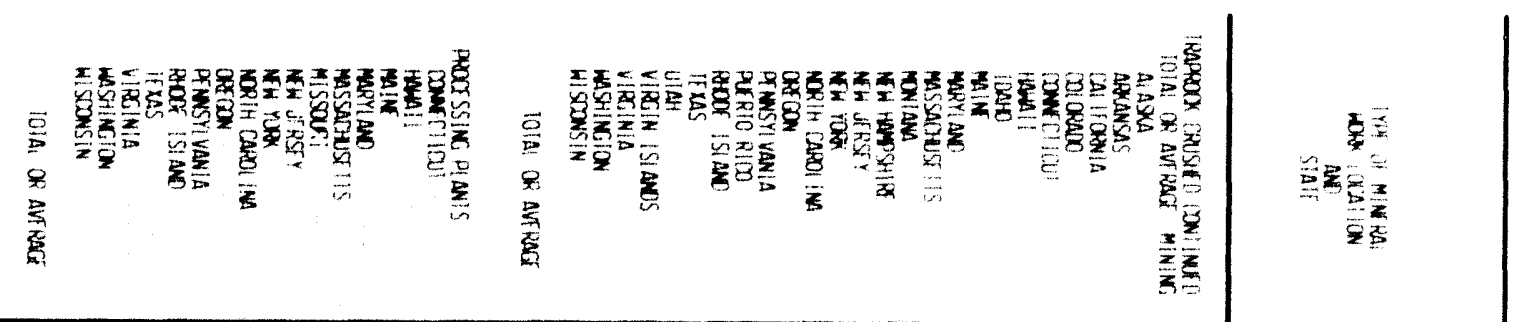

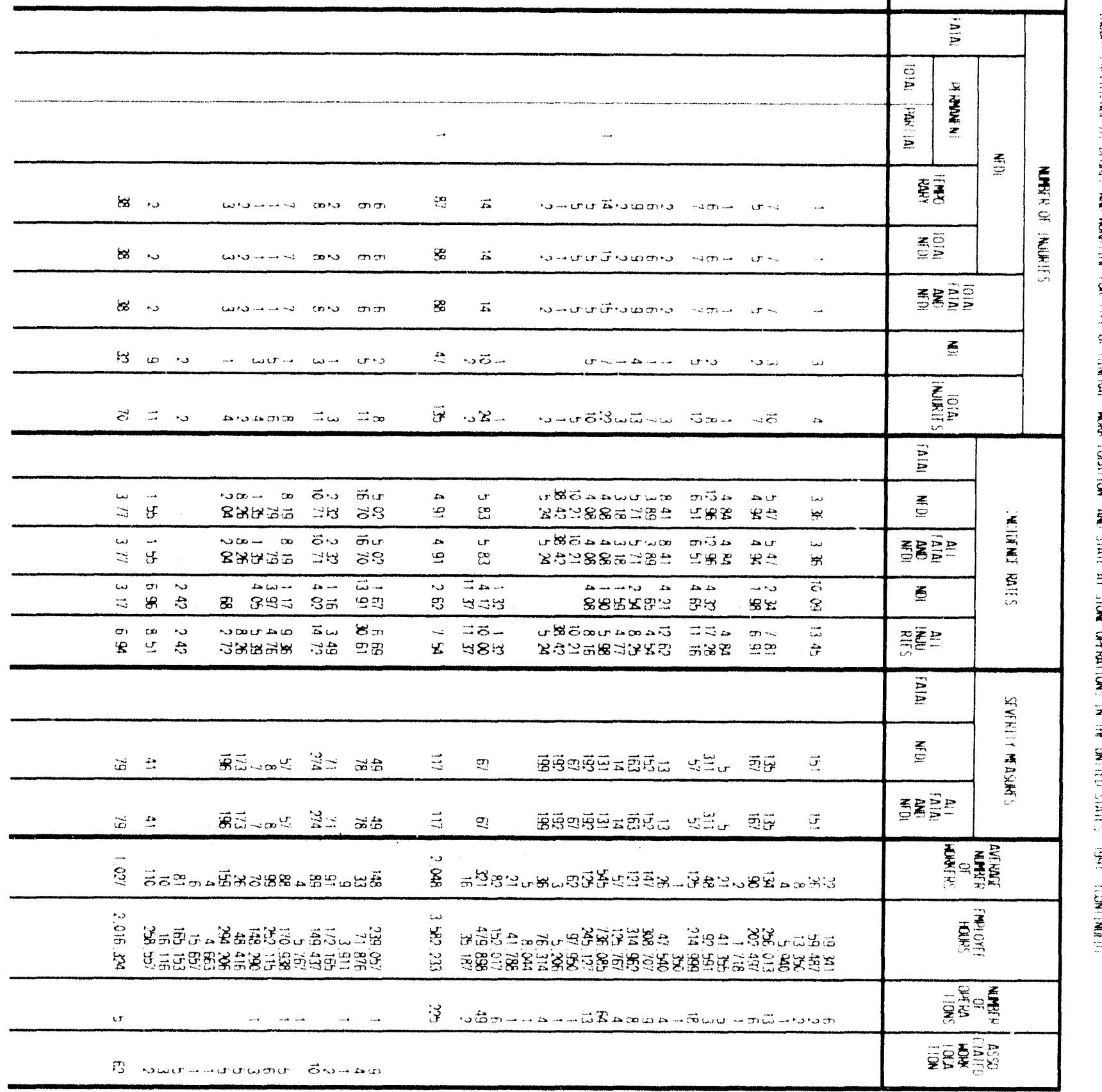




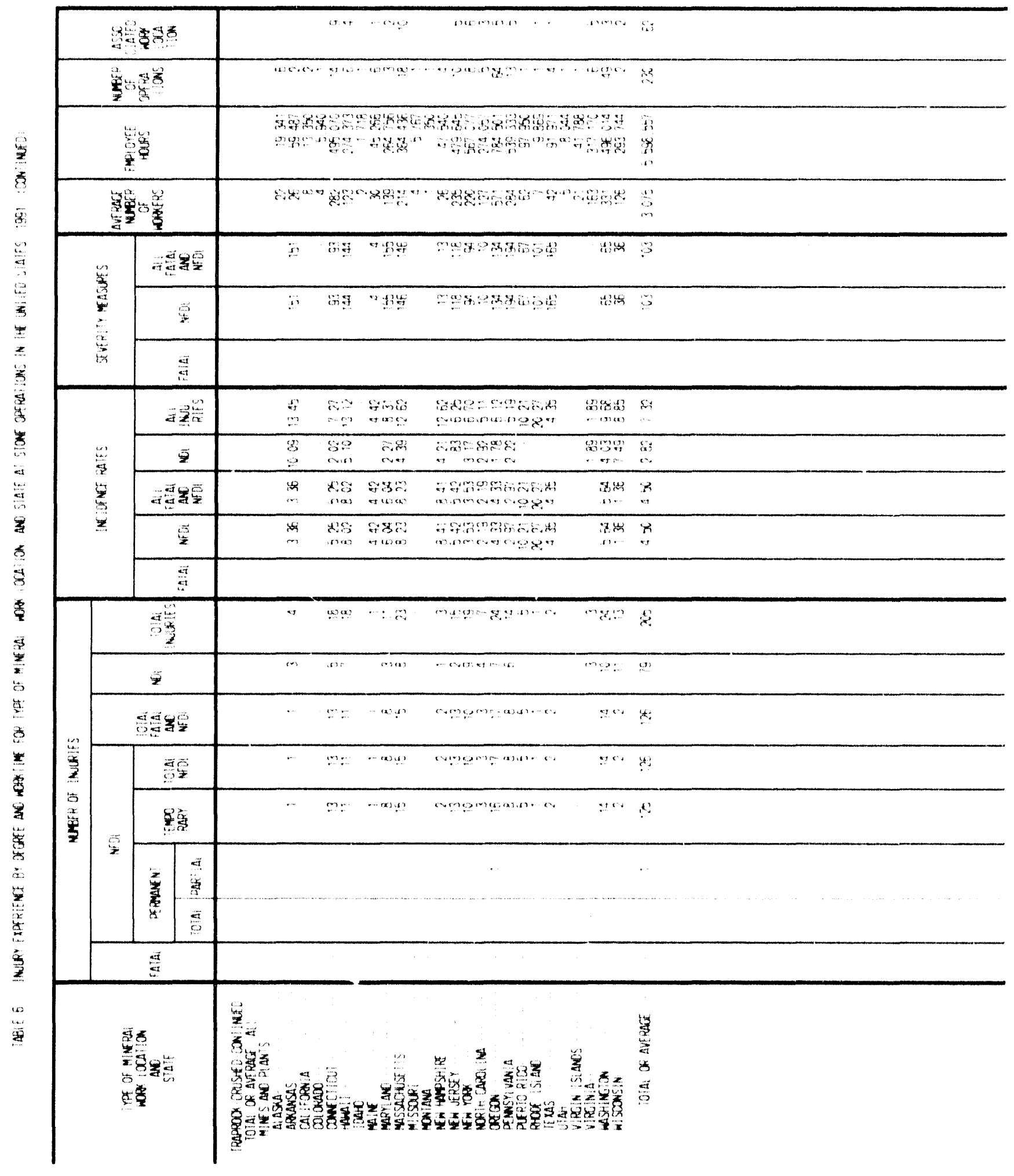




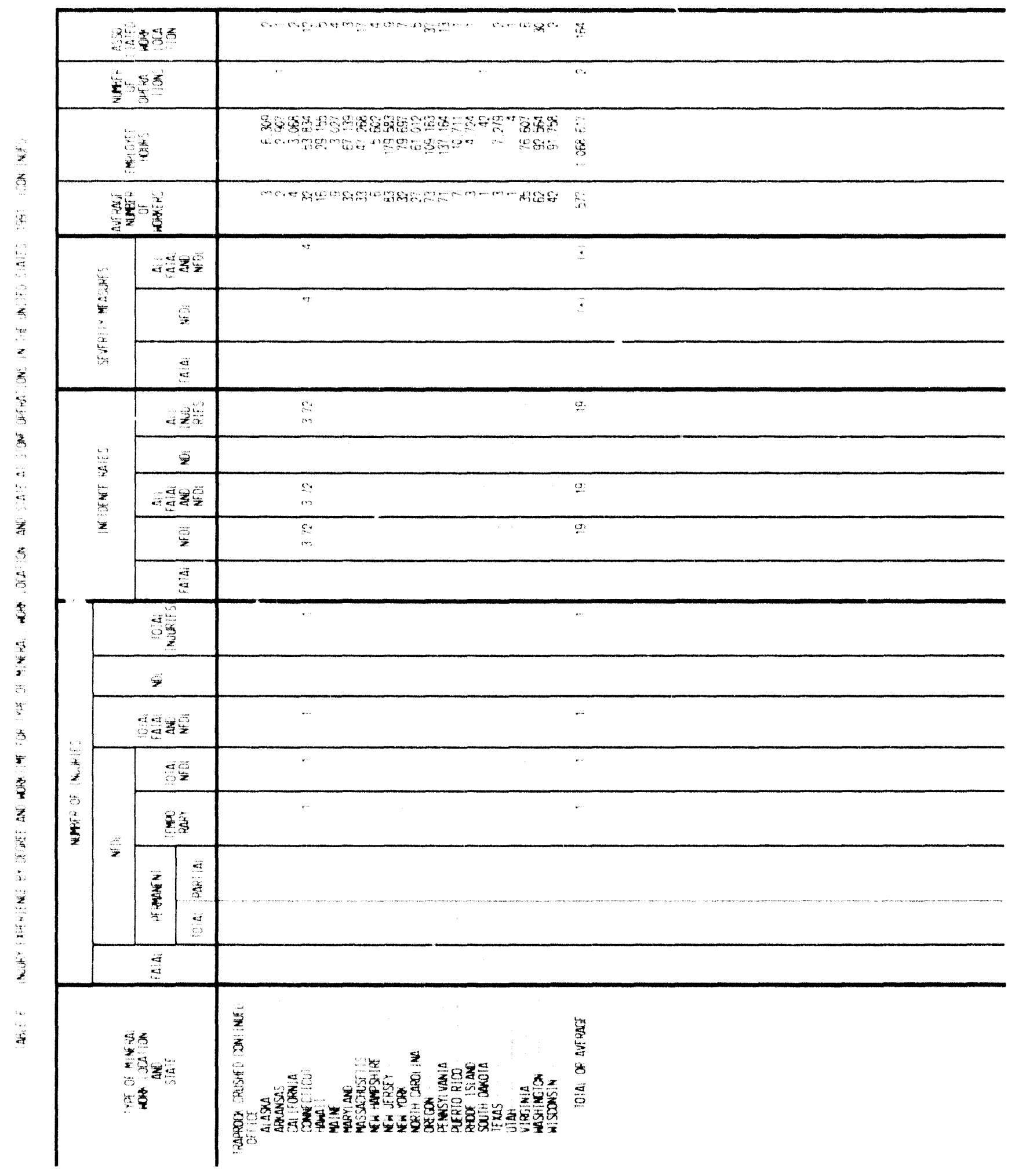




\begin{tabular}{|c|c|c|c|c|c|c|c|c|}
\hline \multicolumn{3}{|c|}{ 影数㐘 } & $\cdots \cdot$ & $\therefore a n$ & $" \theta \infty \alpha+\infty$ & 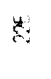 & & \\
\hline \multicolumn{3}{|c|}{ 案和管 } & $16 \mathrm{~m}$ & 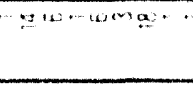 & 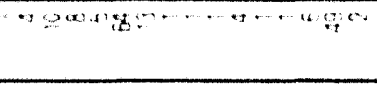 & 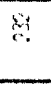 & $\ldots \ldots$ & $:$ \\
\hline \multicolumn{3}{|c|}{ 落 } & 数 & 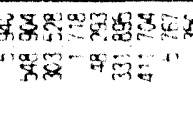 & 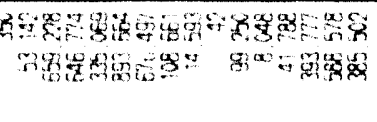 & $\begin{array}{l}5 \\
0 \\
0\end{array}$ & 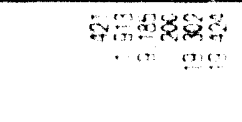 & $\begin{array}{l}3 \\
3 \\
8\end{array}$ \\
\hline \multicolumn{3}{|c|}{ 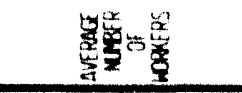 } & x些 & "g영 & 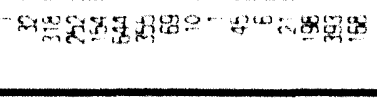 & : & andere & $\Rightarrow$ \\
\hline \multirow{3}{*}{\multicolumn{2}{|c|}{ 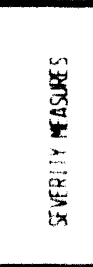 }} & 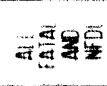 & 是 & $\frac{1}{2}+48$ & 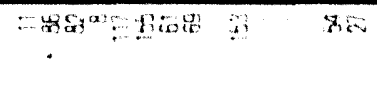 & (i) & $\therefore$ & $"$ \\
\hline & & 要 & 景 & $4: 8$ " & 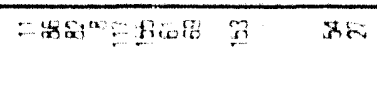 & $\overline{a j}$ & $\because$ & " \\
\hline & & $\frac{a}{a}$ & & & & & & \\
\hline & & 逢重 & 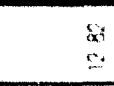 & 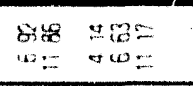 & 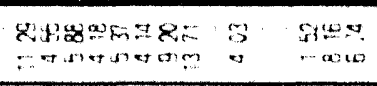 & $\stackrel{9}{\square}$ & 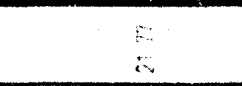 & $\stackrel{4}{*}$ \\
\hline & & $\underline{\sigma}$ & $\begin{array}{c}\text { at } \\
\text { an } \\
\end{array}$ & 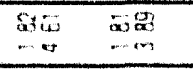 & 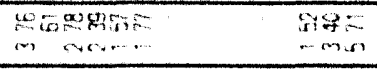 & in & & \\
\hline & 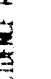 & 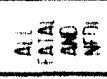 & $\therefore$ & \begin{tabular}{ll} 
ef \\
\hdashline
\end{tabular} & 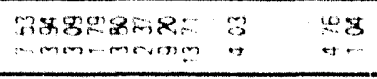 & 然 & $\therefore$ & 事 \\
\hline & & $\ddot{\ddot{z}}$ & $\because$ & 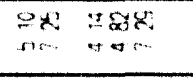 & 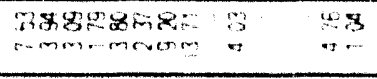 & a & $\therefore$ & 蛋 \\
\hline & & 童 & & & & & & \\
\hline \multirow{8}{*}{ 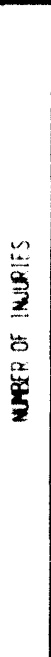 } & & 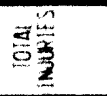 & $\cdot$ & 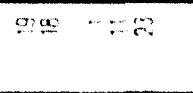 & $m$ meng & 岁 & $\gamma$ & . \\
\hline & & $\bar{q}$ & $m$ & she & $-\infty \cos _{1}-\infty \omega$ & ! & & \\
\hline & & 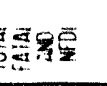 & .- & $y:=\cdots \infty$ & 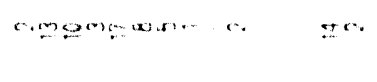 & 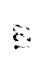 & .. & $\cdots$ \\
\hline & \multirow{4}{*}{ 虽 } & 苨豆 & $\cdots$ & $\pm \ldots-\infty$ & 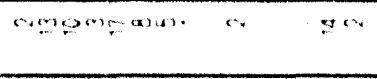 & 8 & $r$ & ? \\
\hline & & 謇 & & $\pm:-\infty$ & 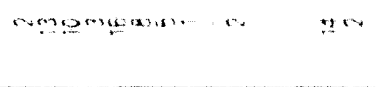 & $\stackrel{w}{\underline{2}}$ & $\cdot$ & $\cdots$ \\
\hline & & \multirow{2}{*}{$\frac{z}{z}$} & & & ... & .. & & \\
\hline & & & & & & & & \\
\hline & \multicolumn{2}{|r|}{ 这 } & & & & & & \\
\hline & & & 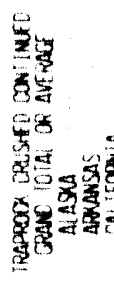 & 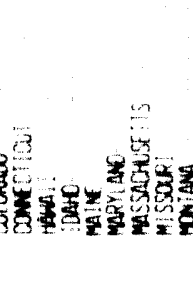 & 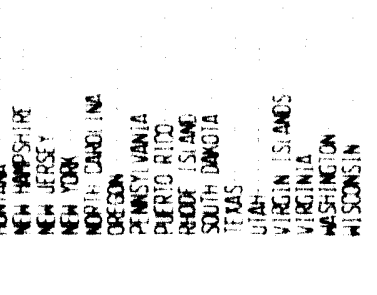 & 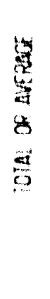 & 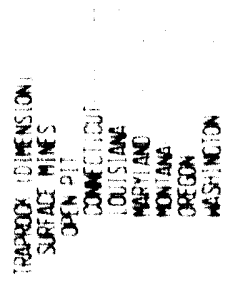 & $\begin{array}{l}3 \\
3 \\
3 \\
3 \\
3 \\
0 \\
4\end{array}$ \\
\hline
\end{tabular}




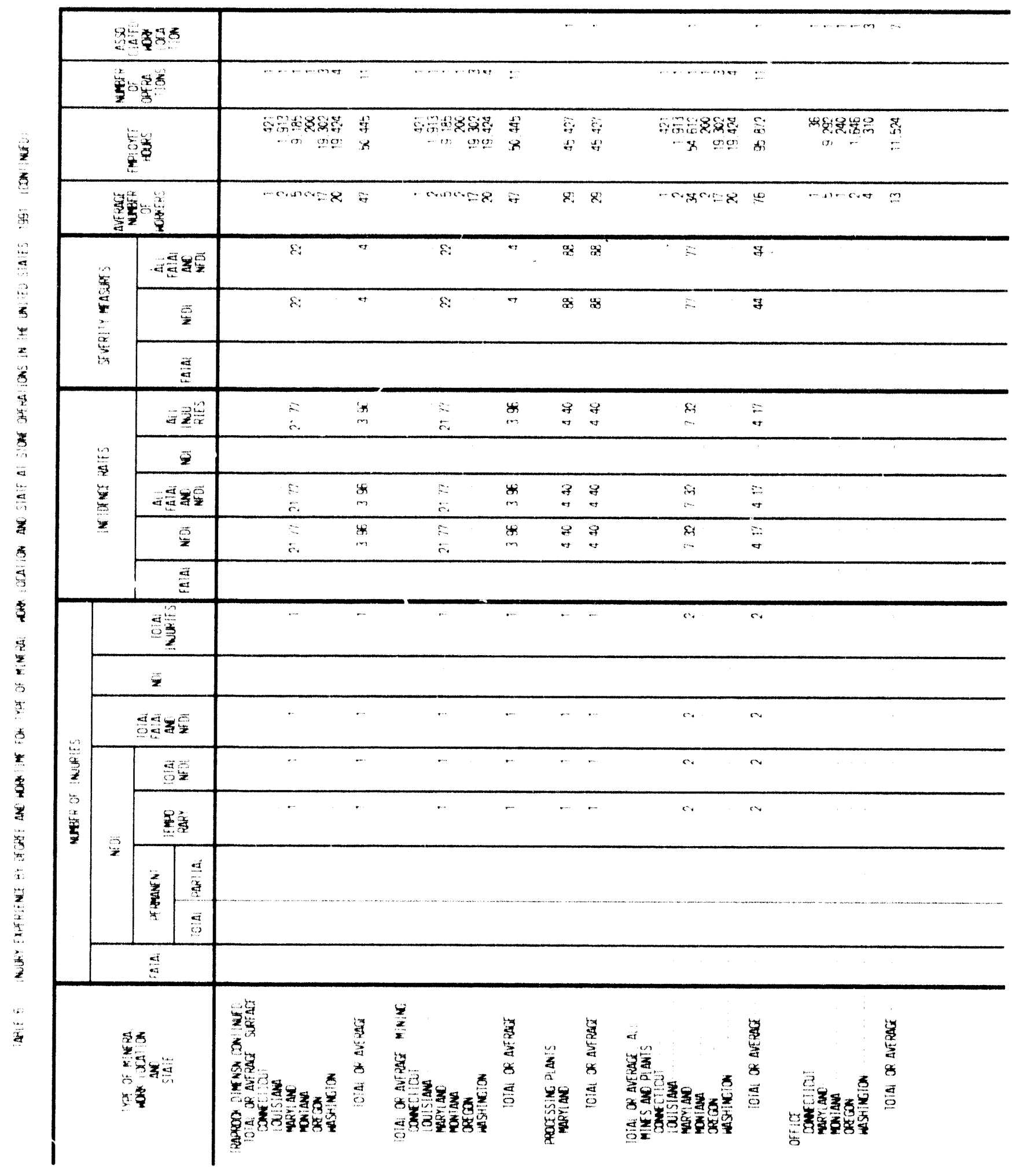




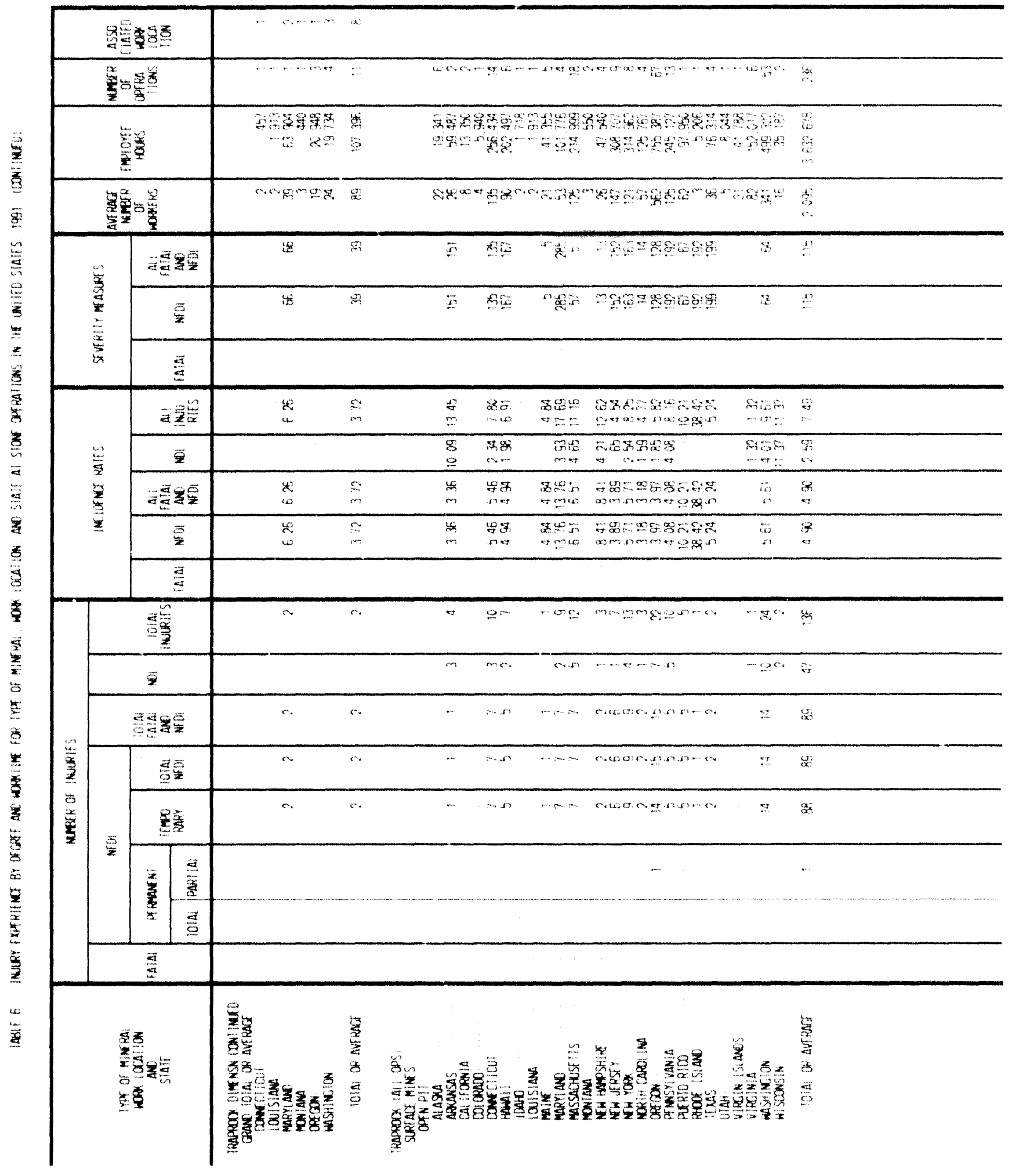




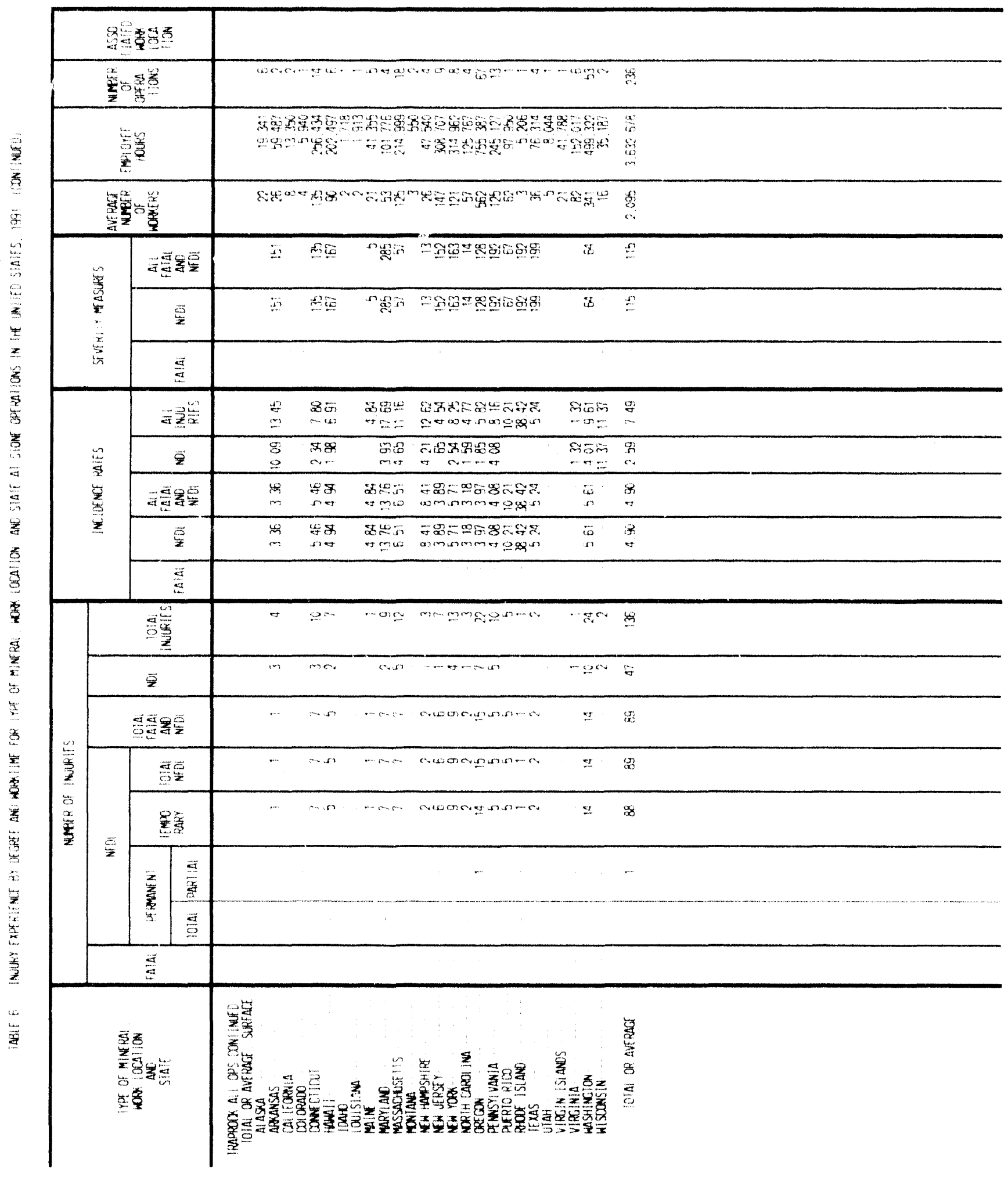




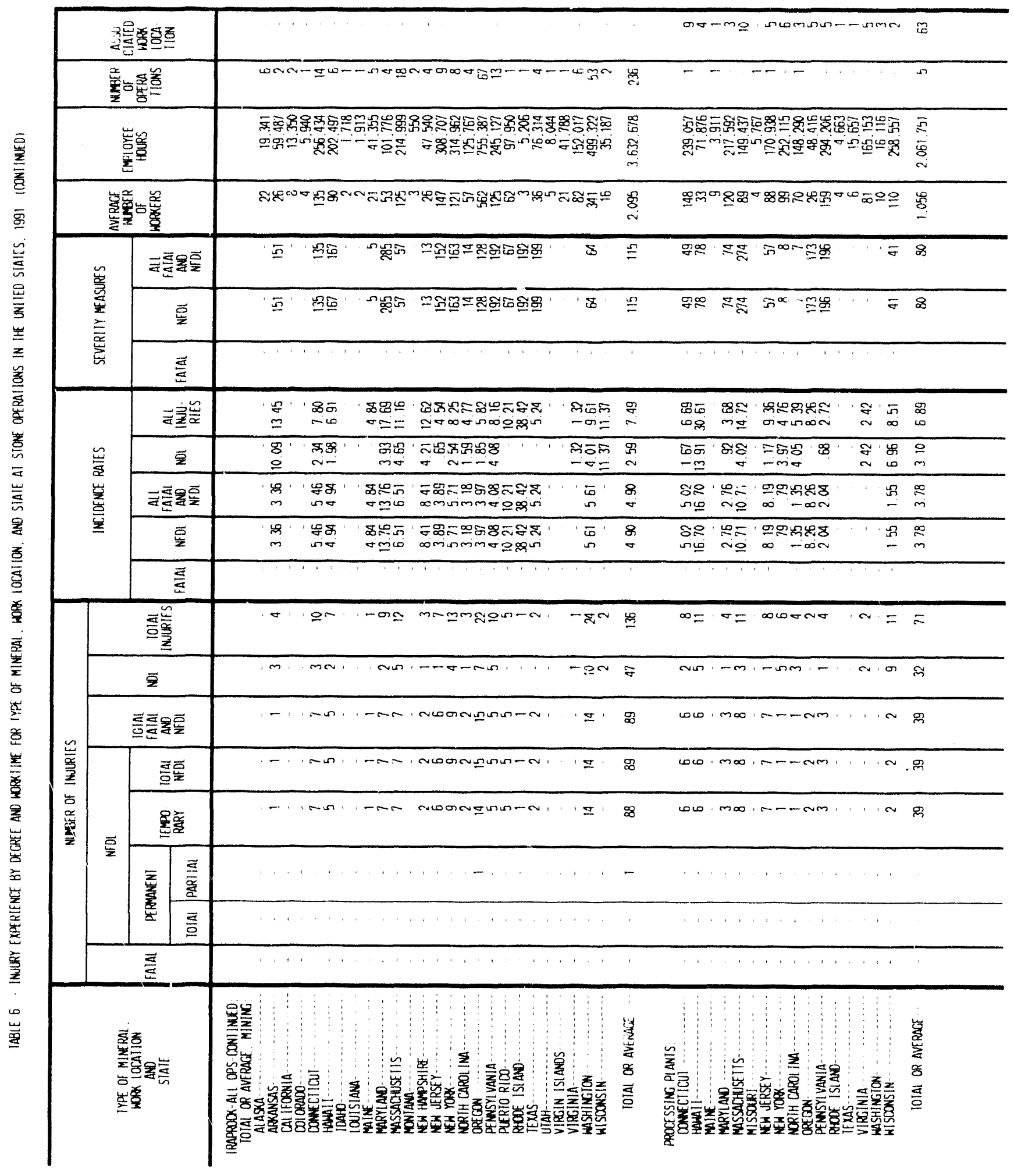




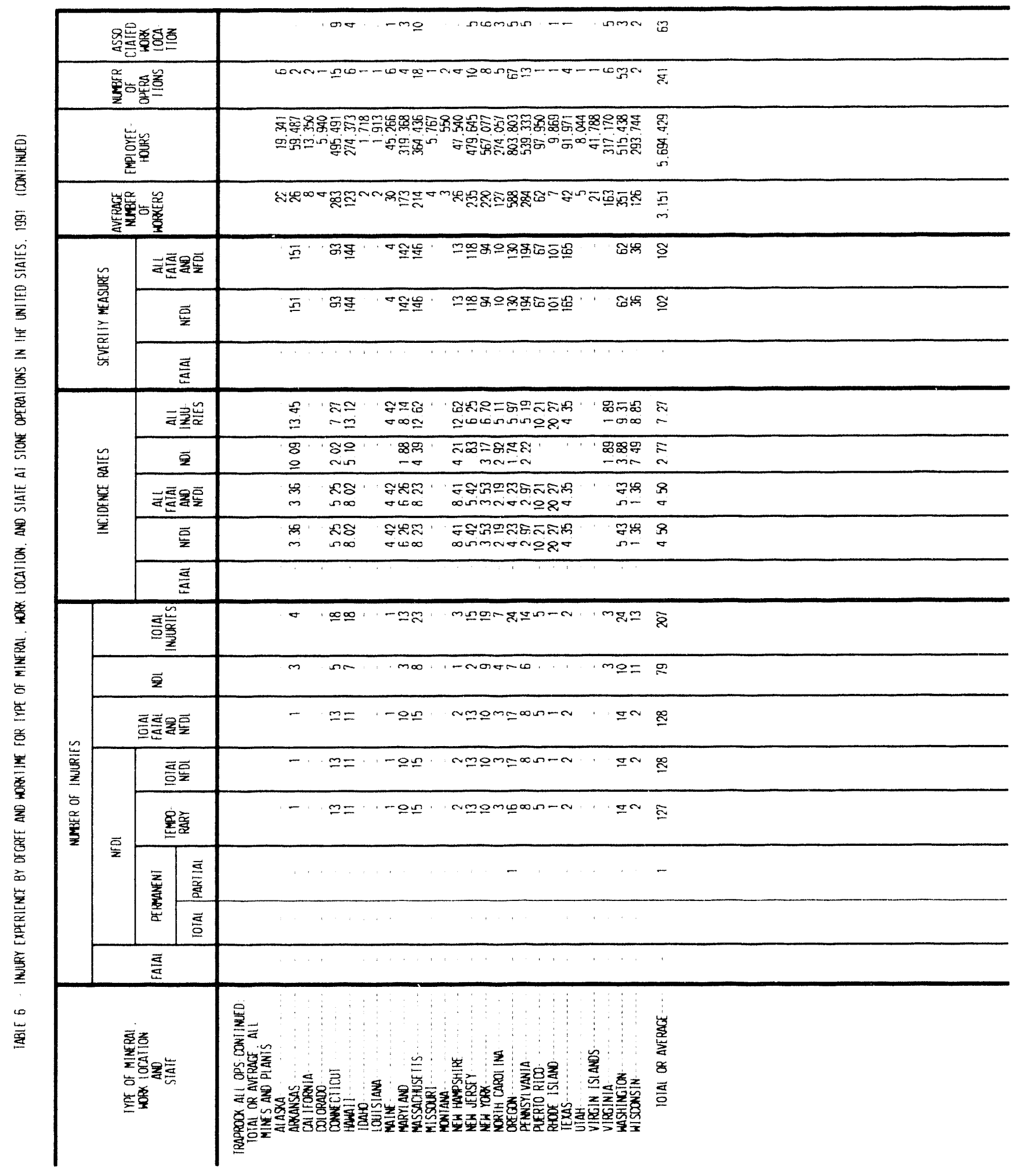




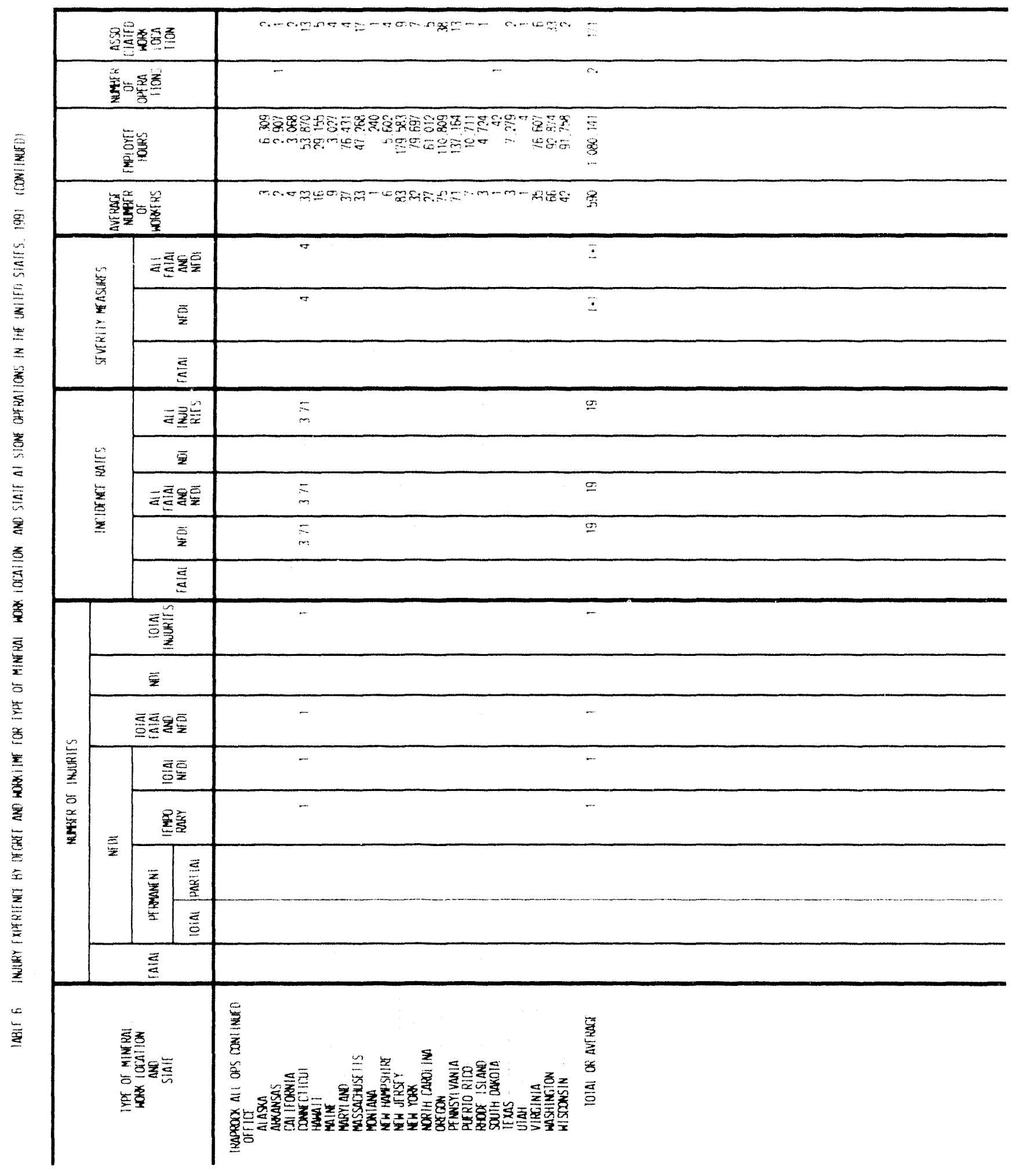




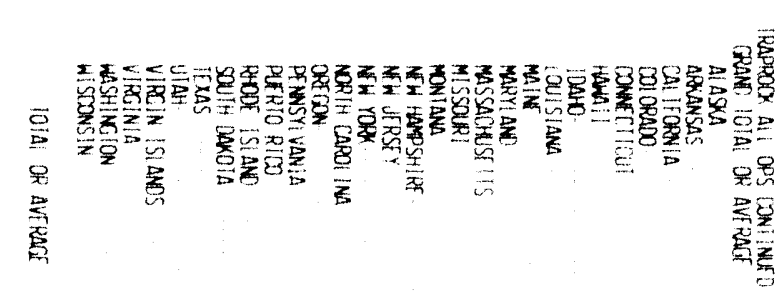

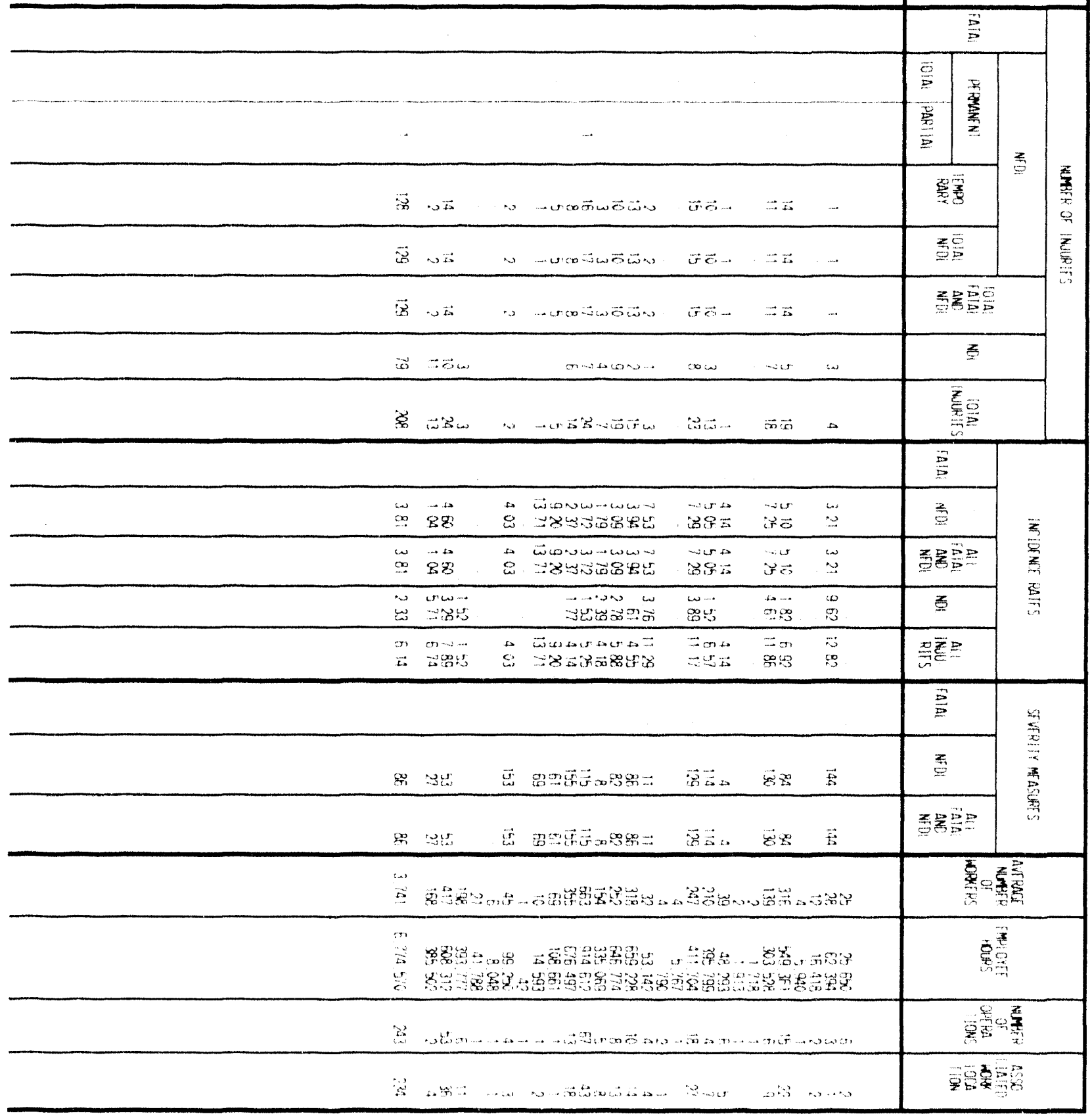



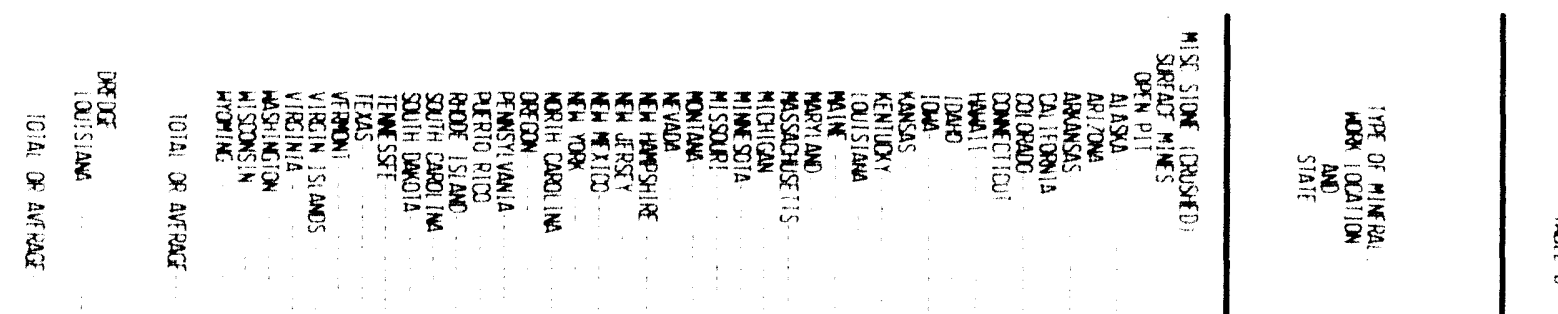

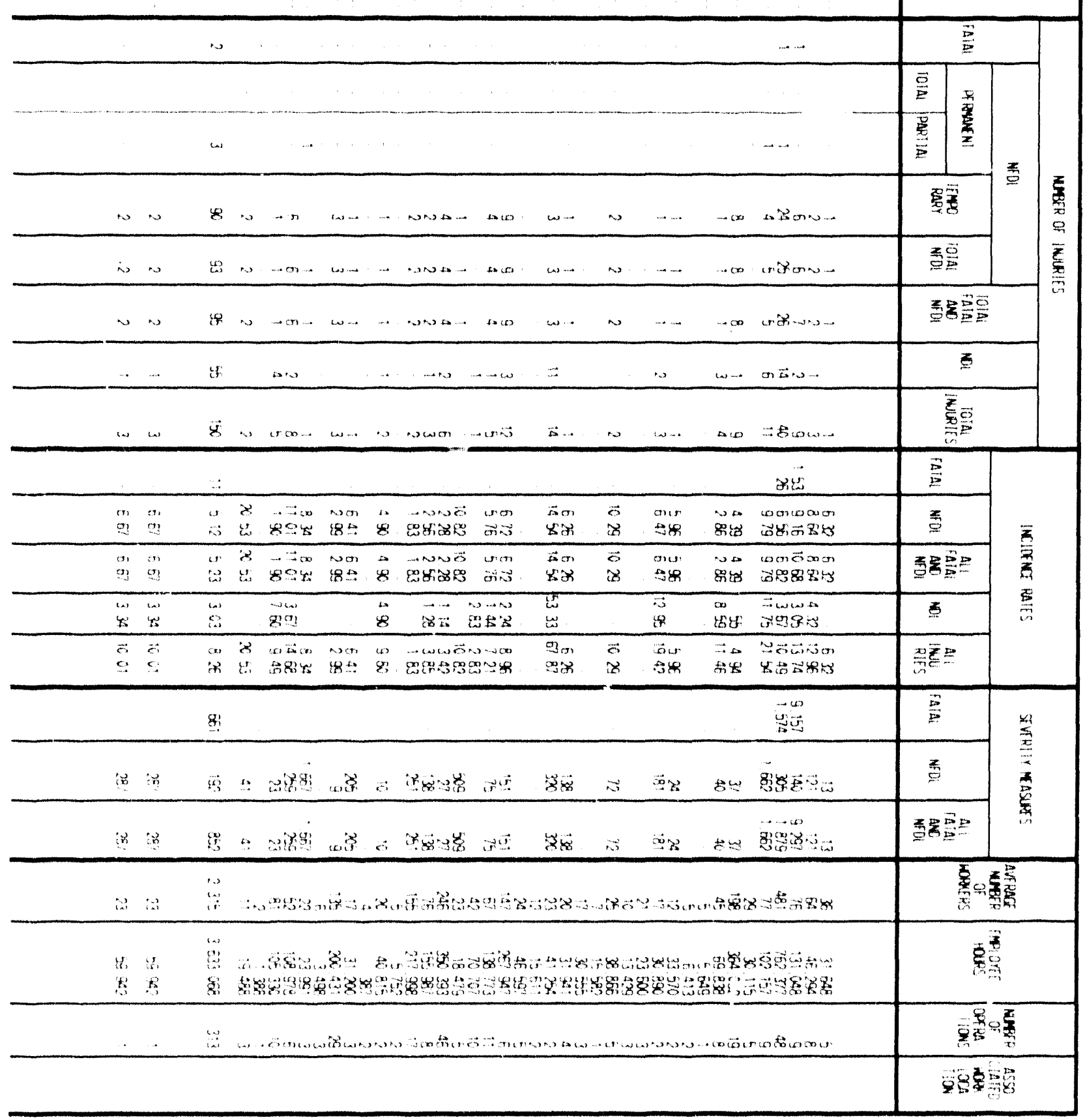




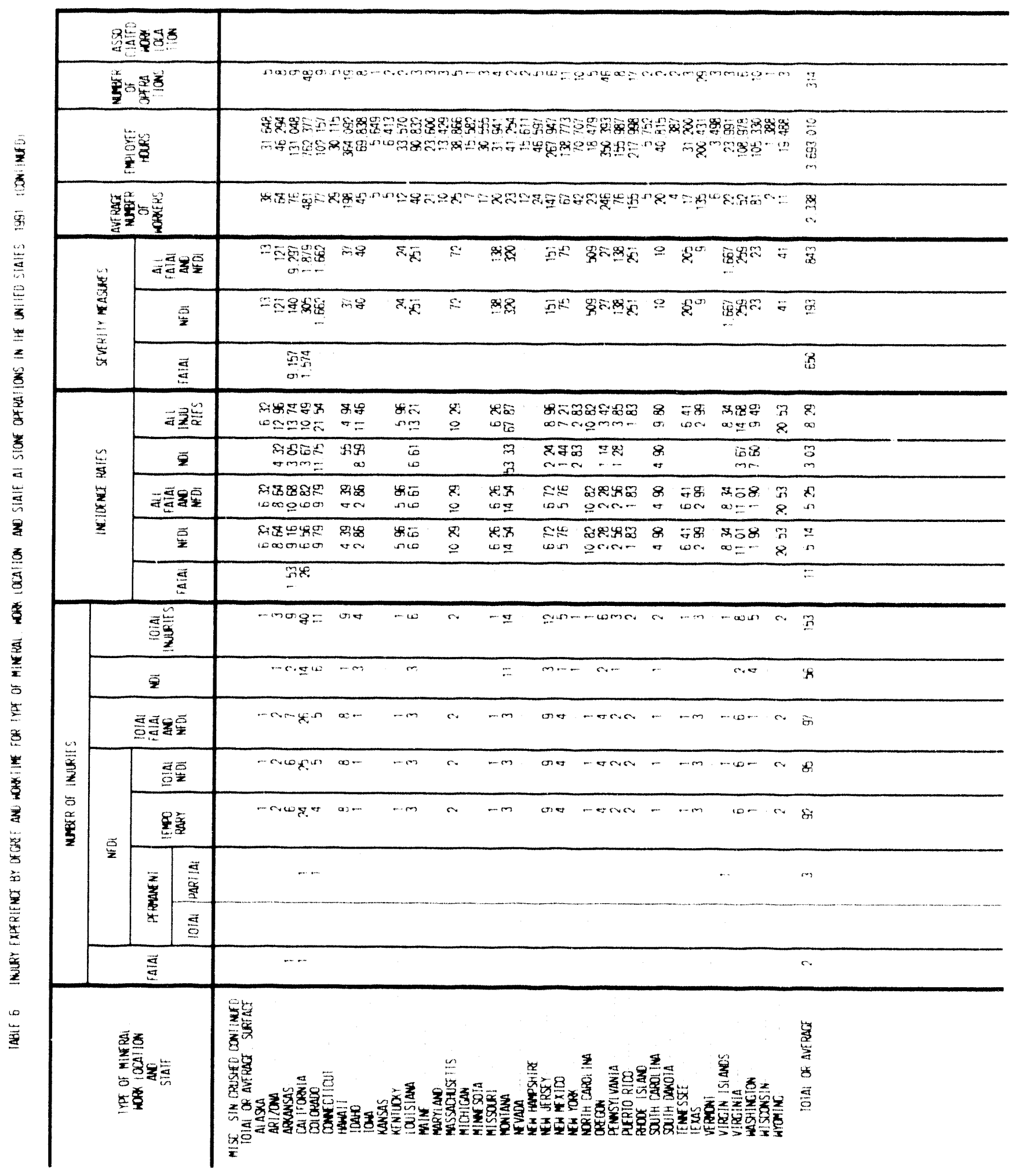




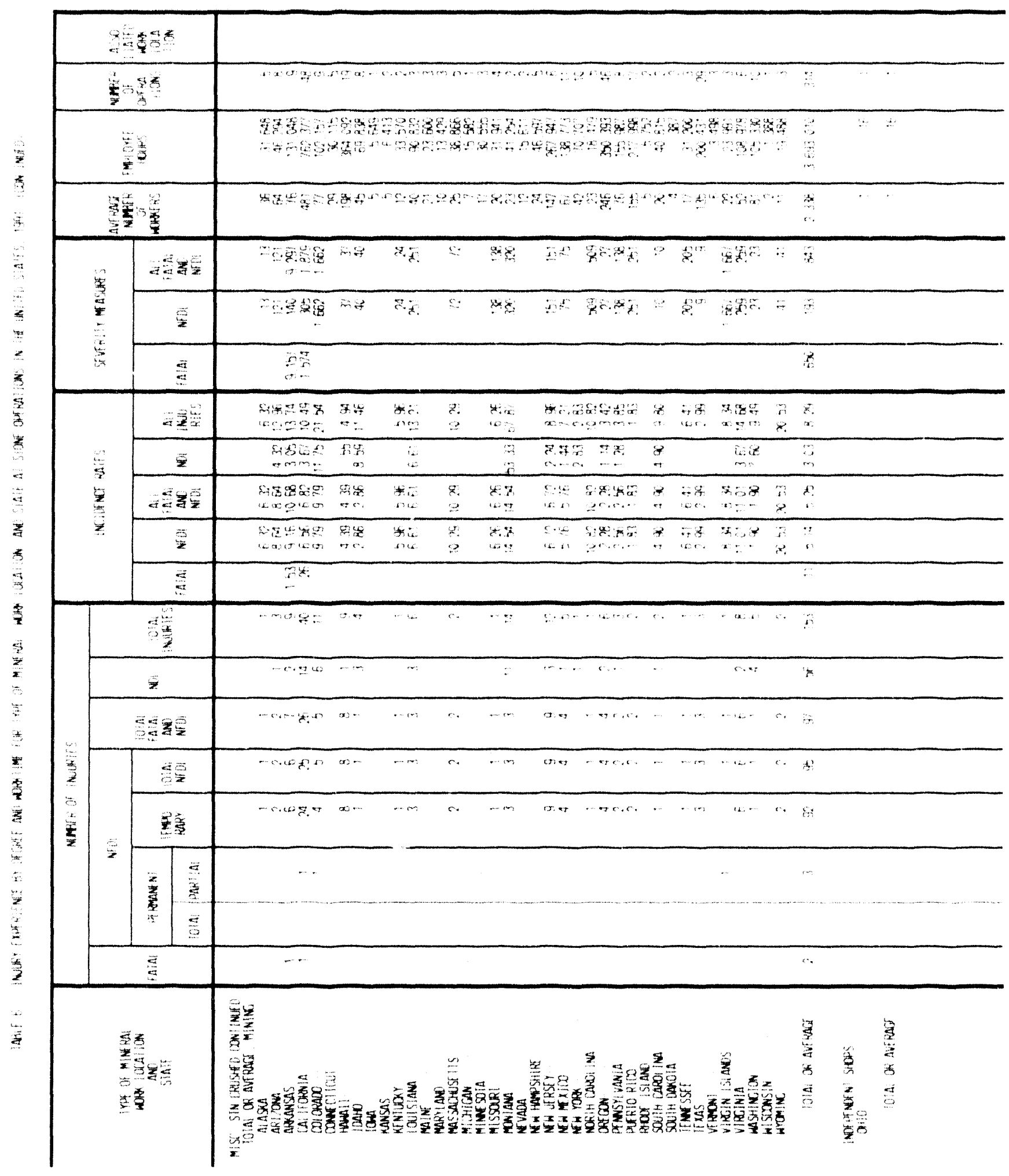




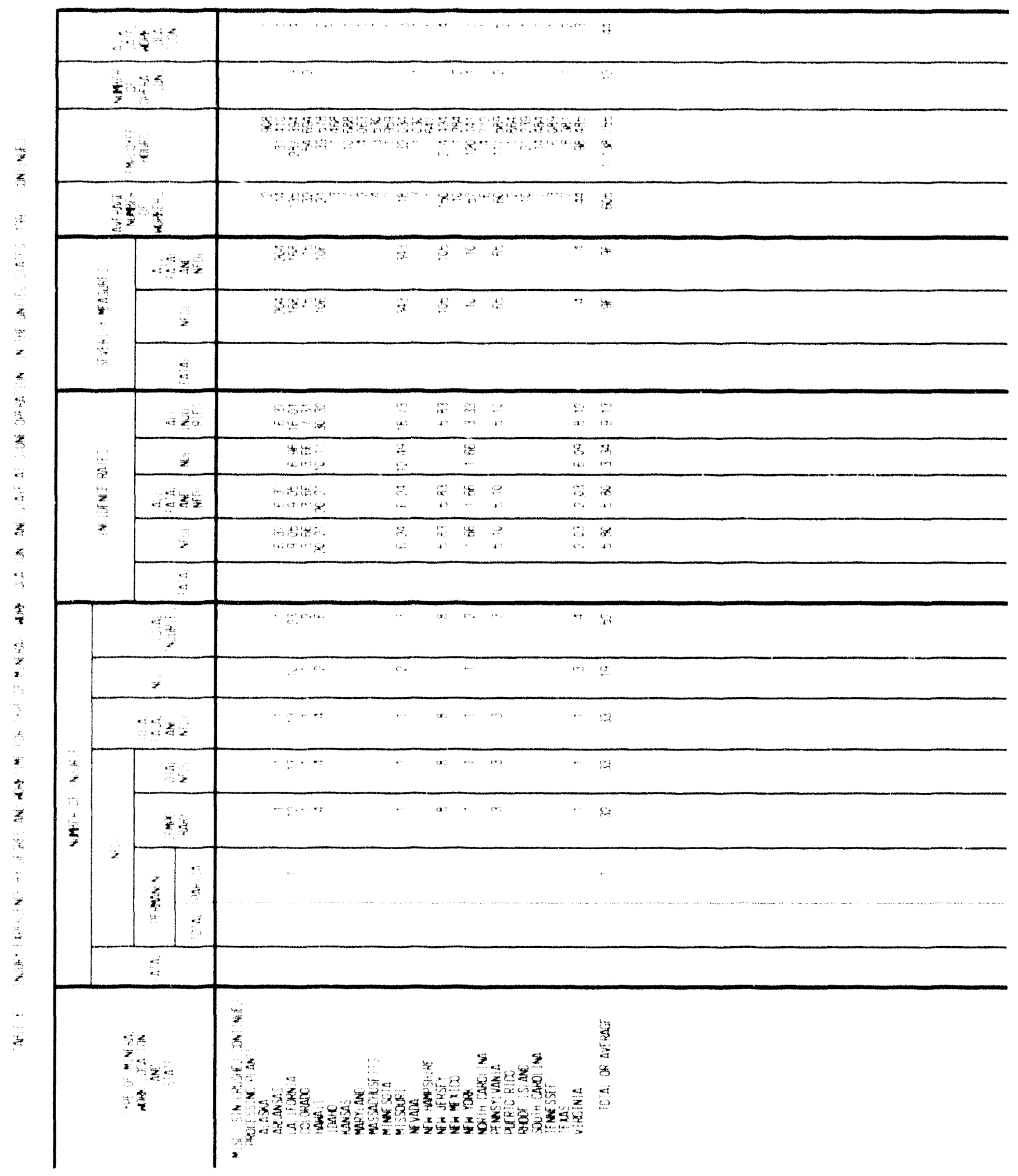




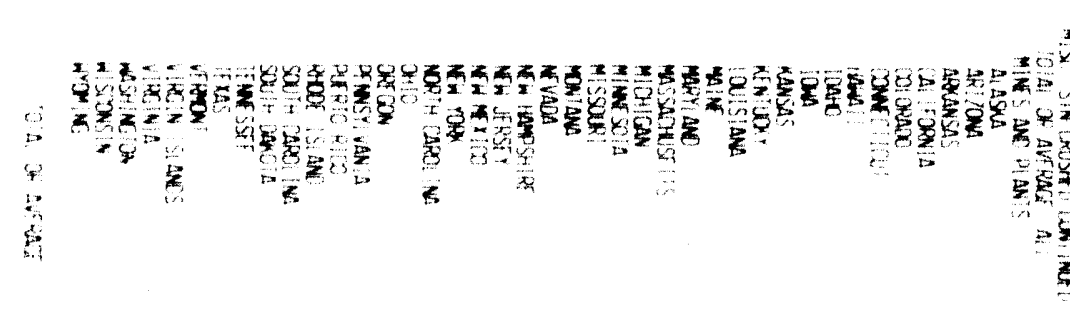

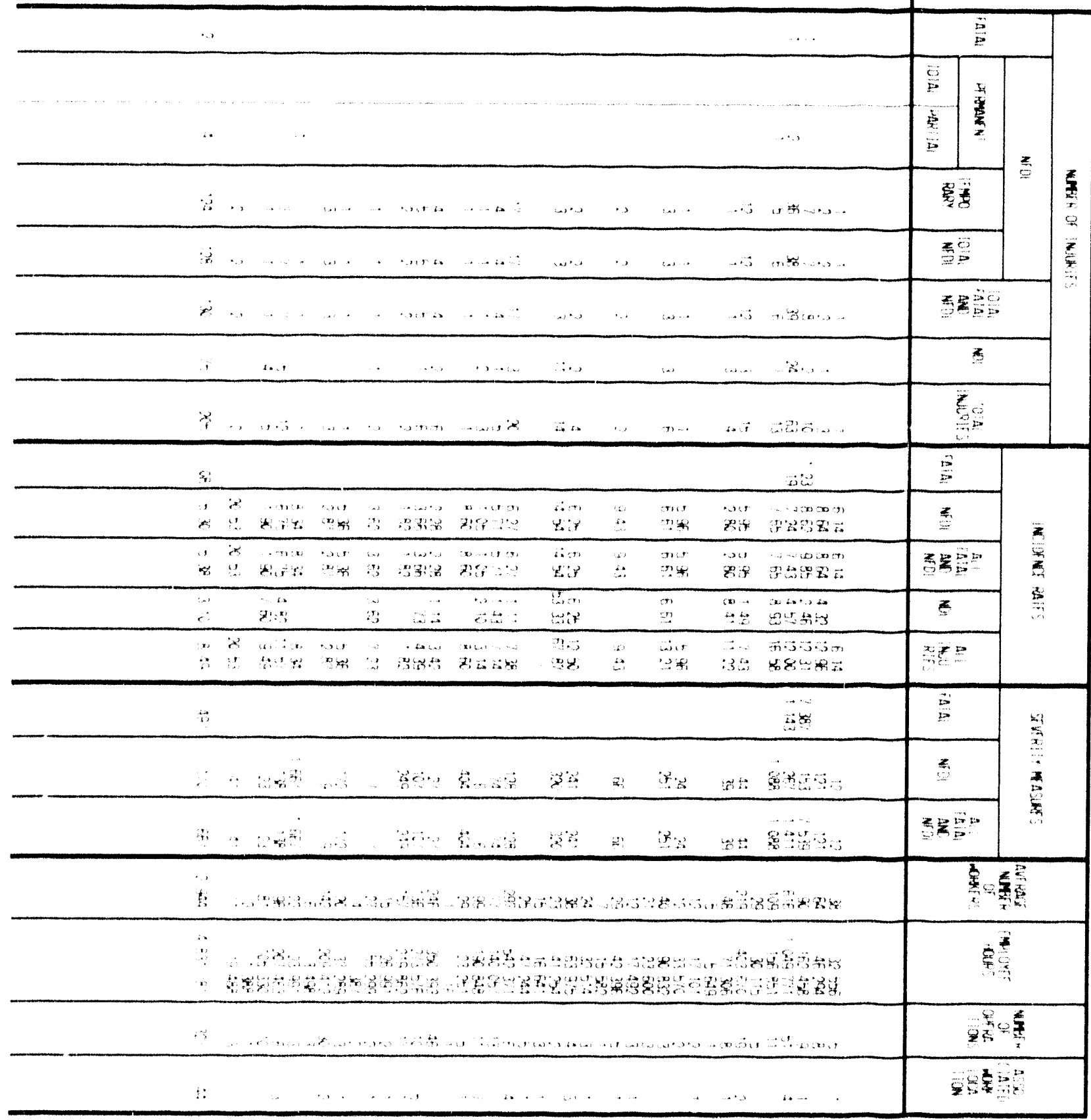




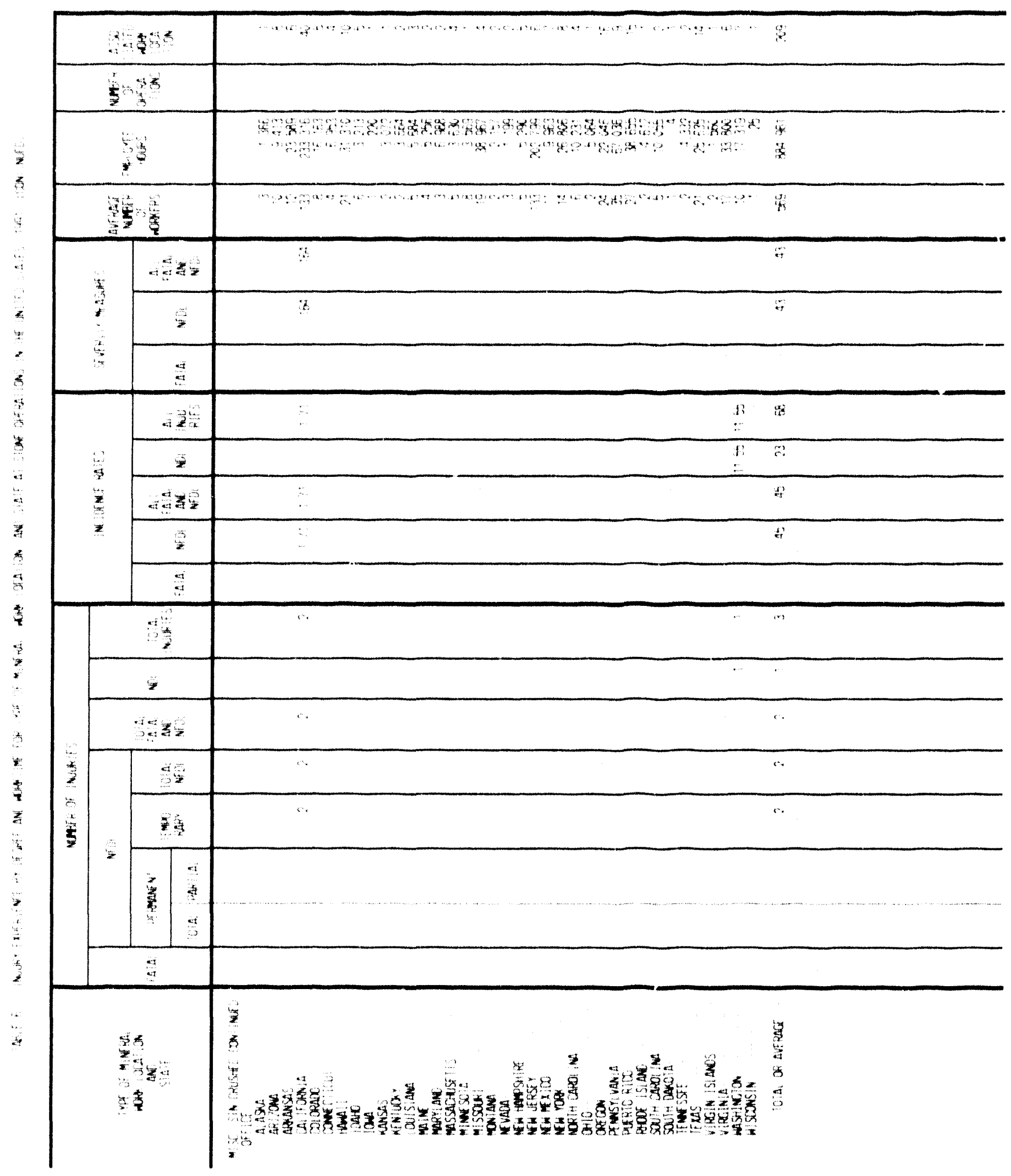




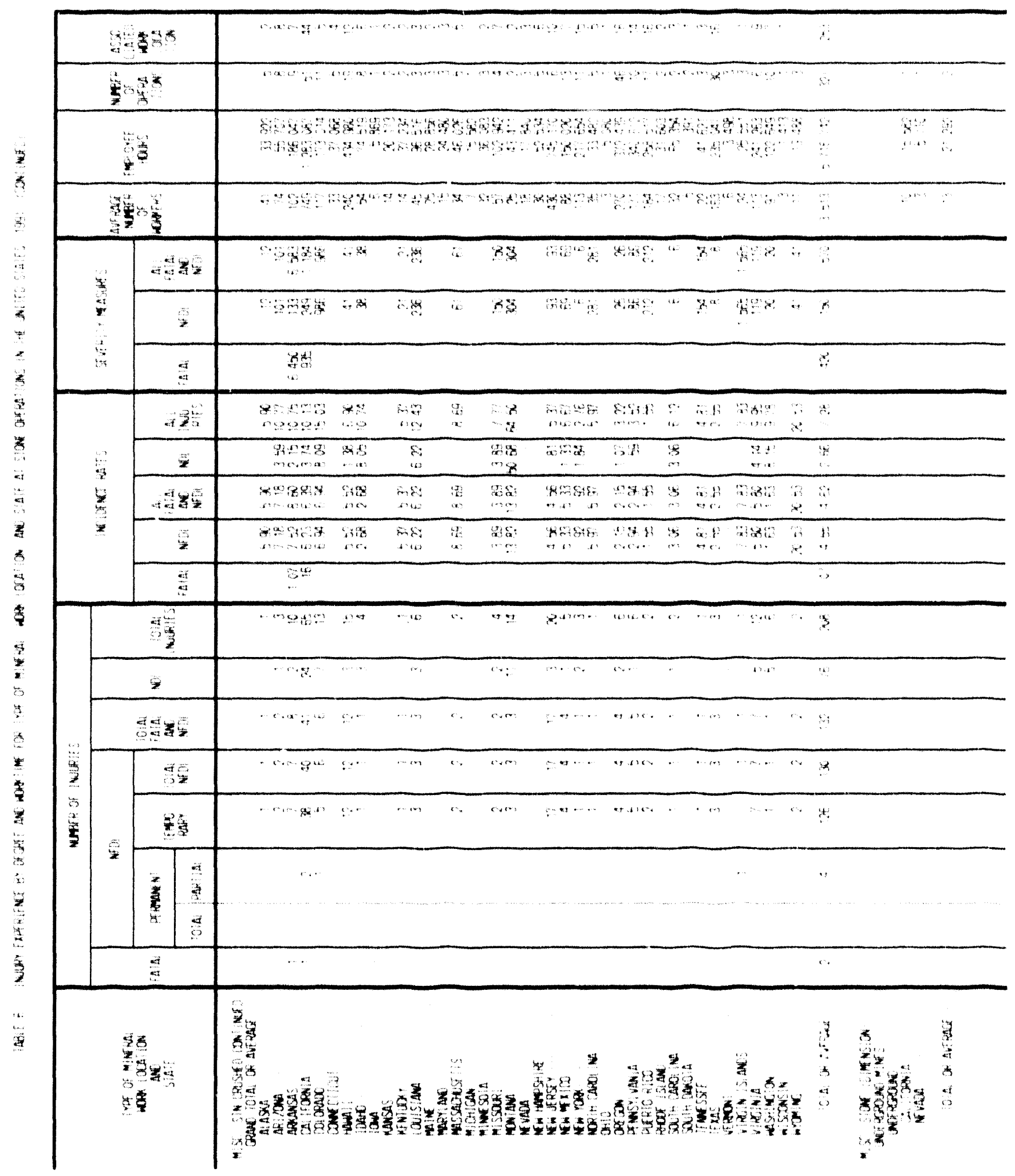




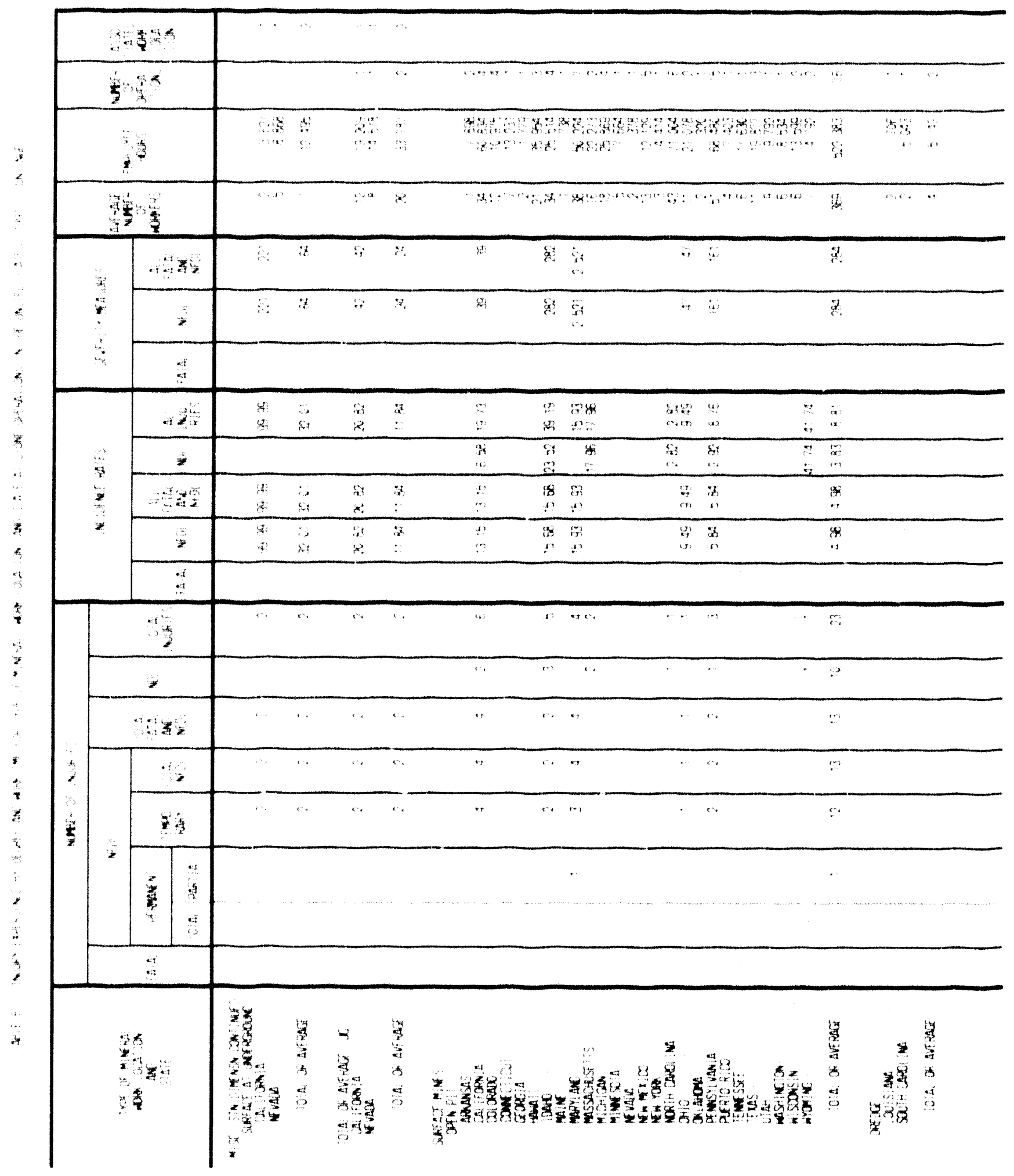




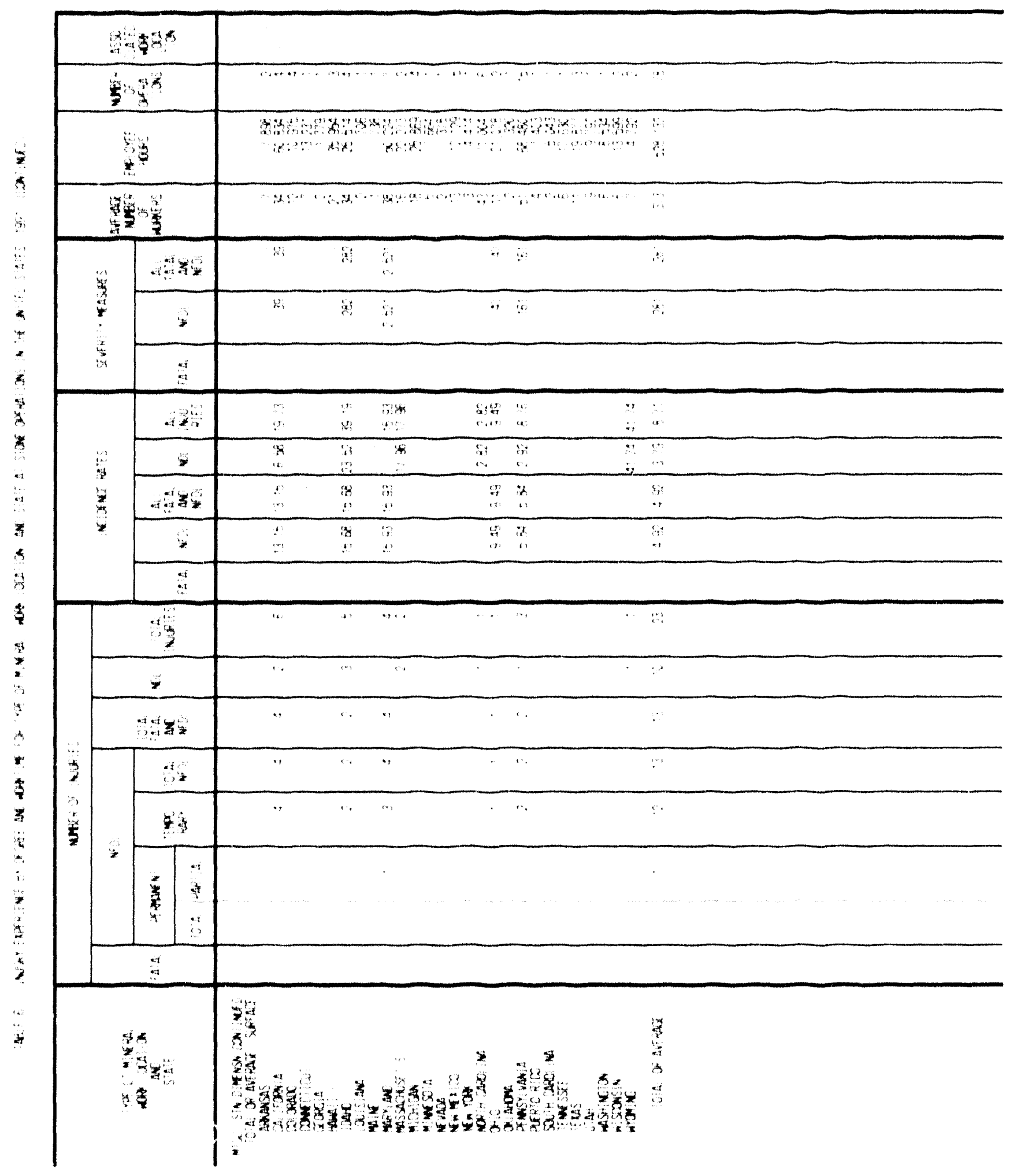




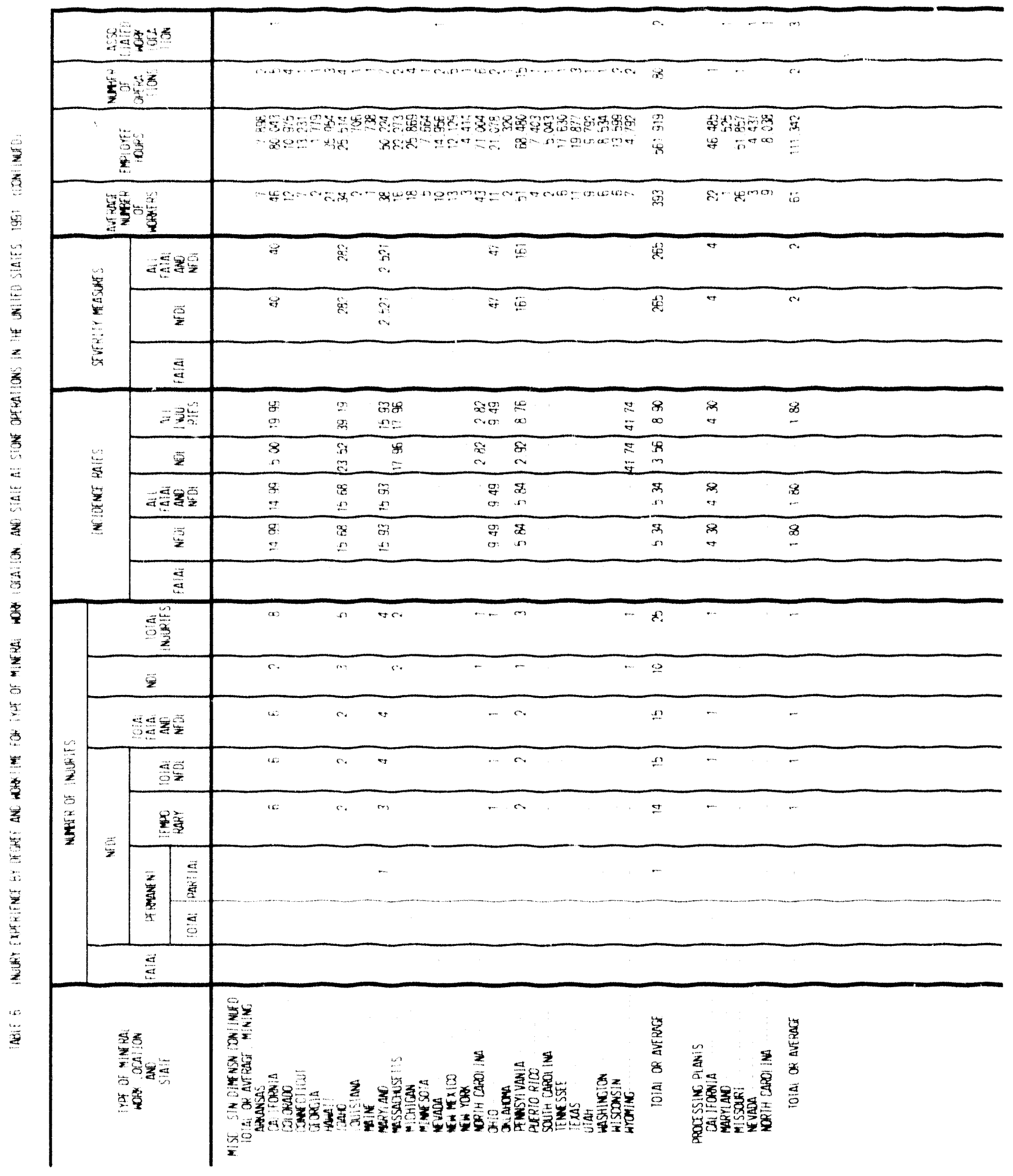




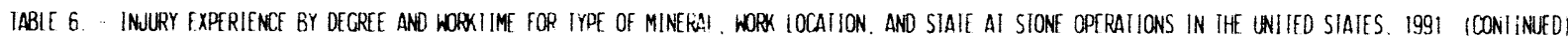

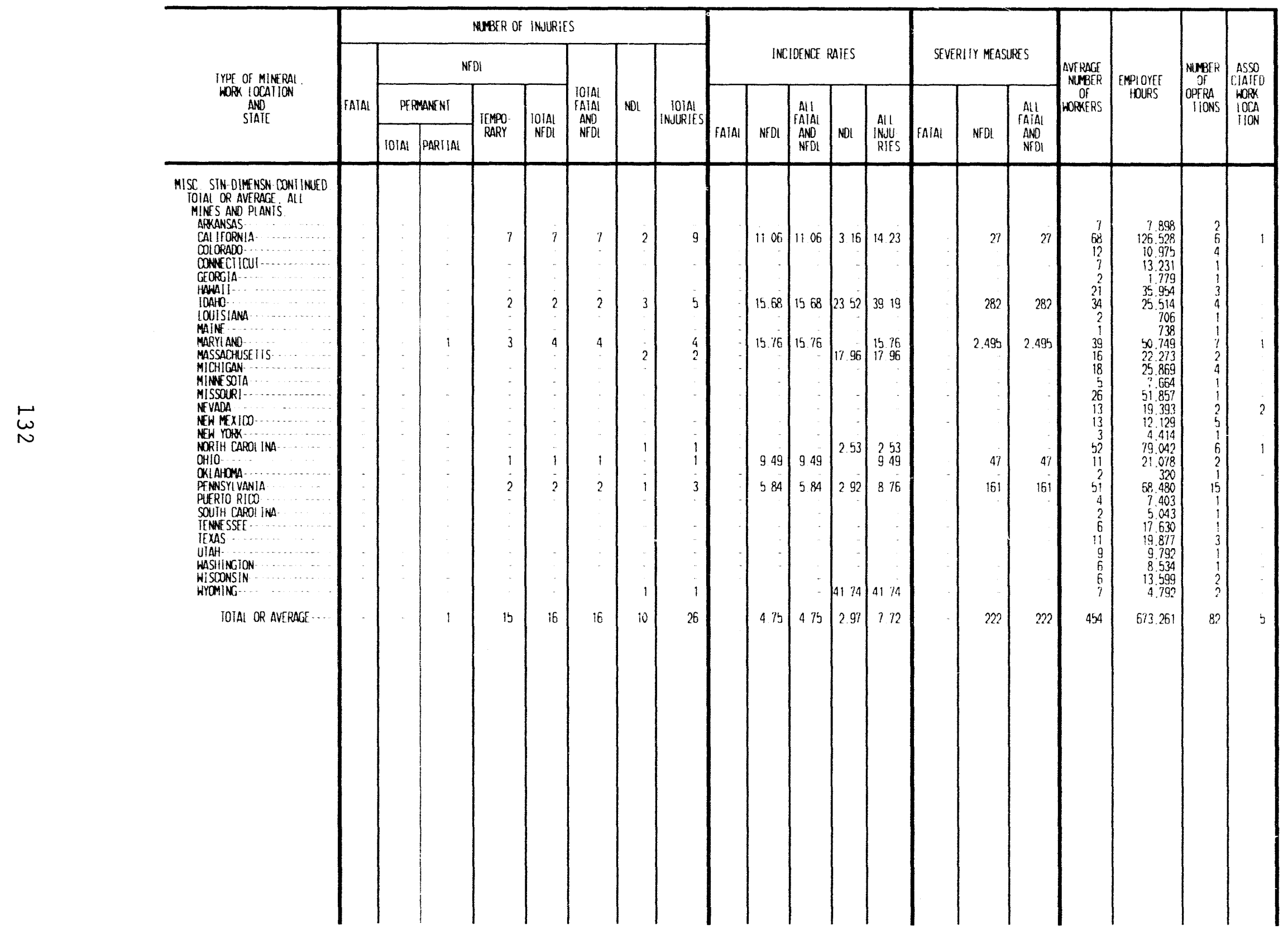




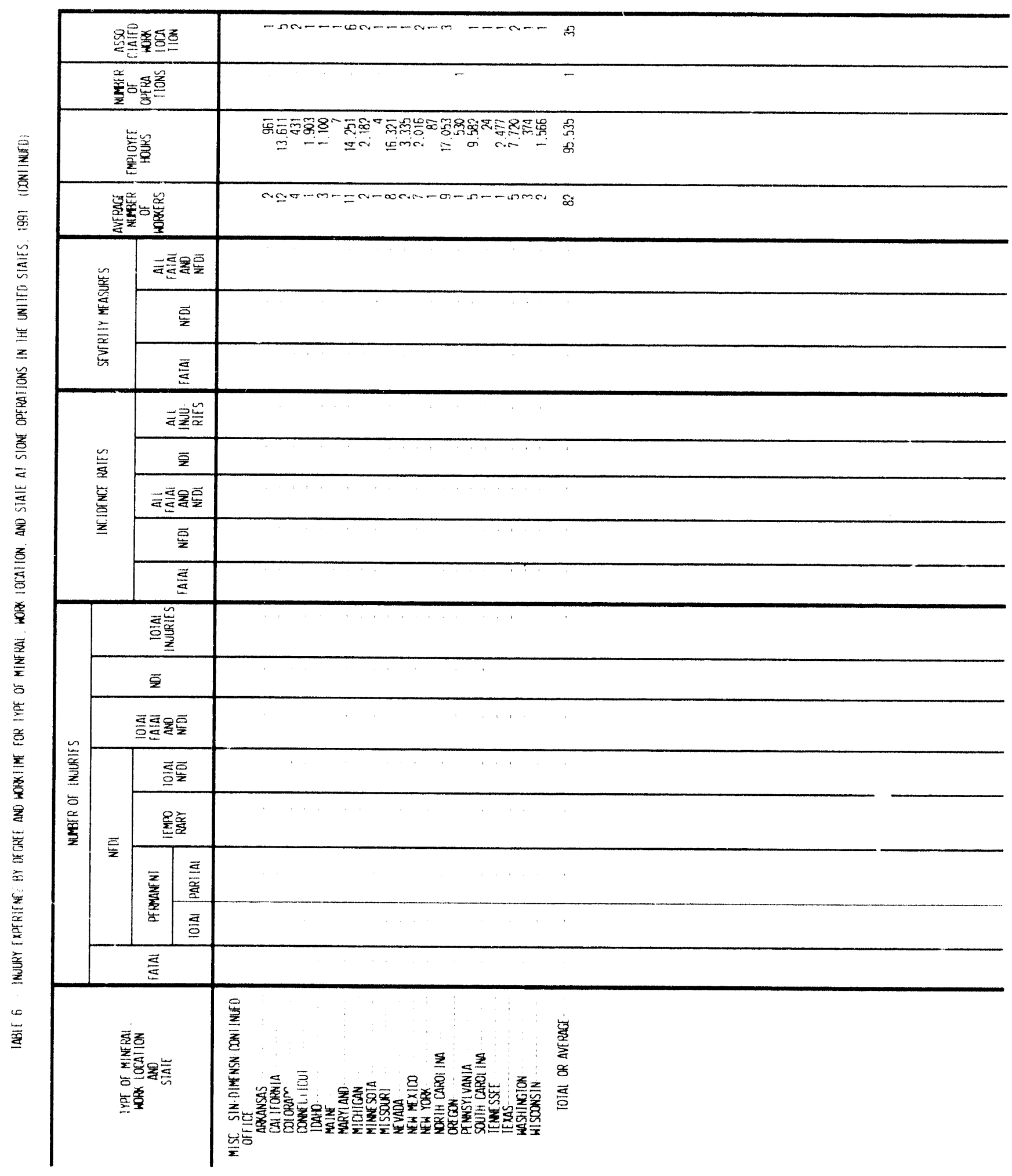




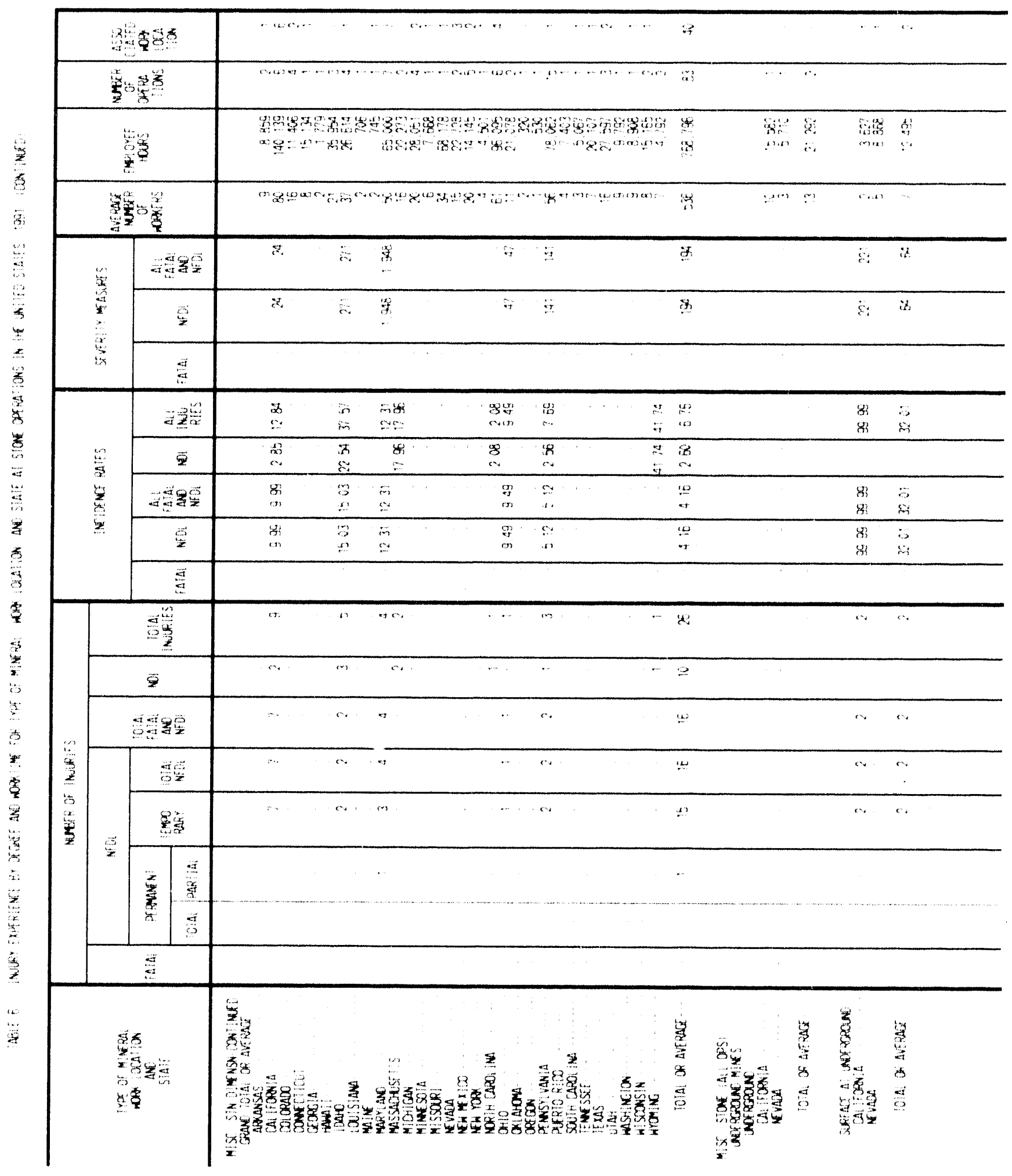




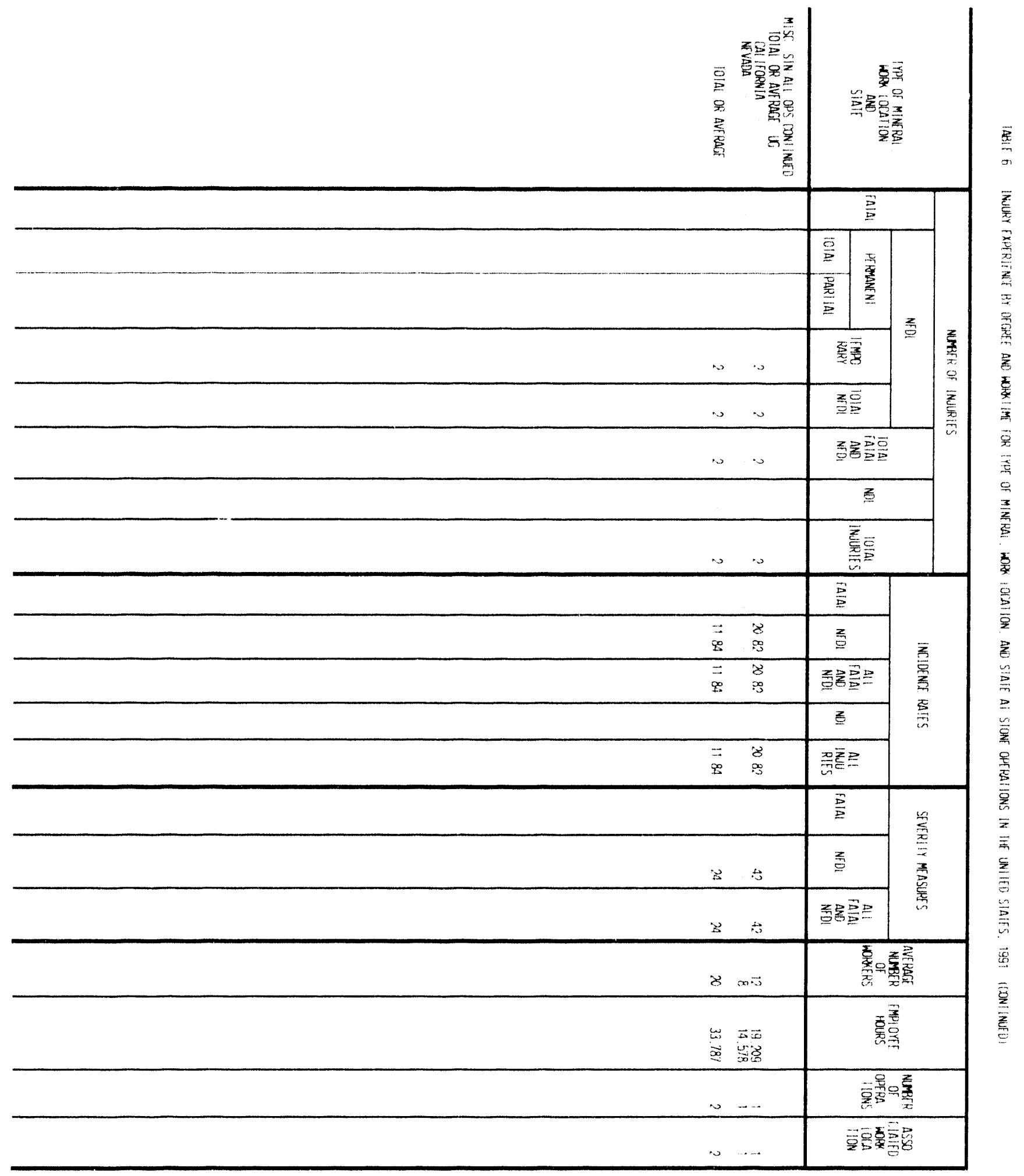




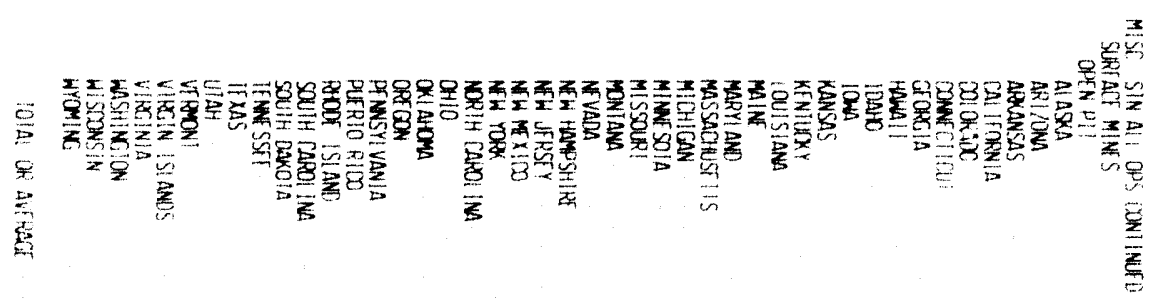

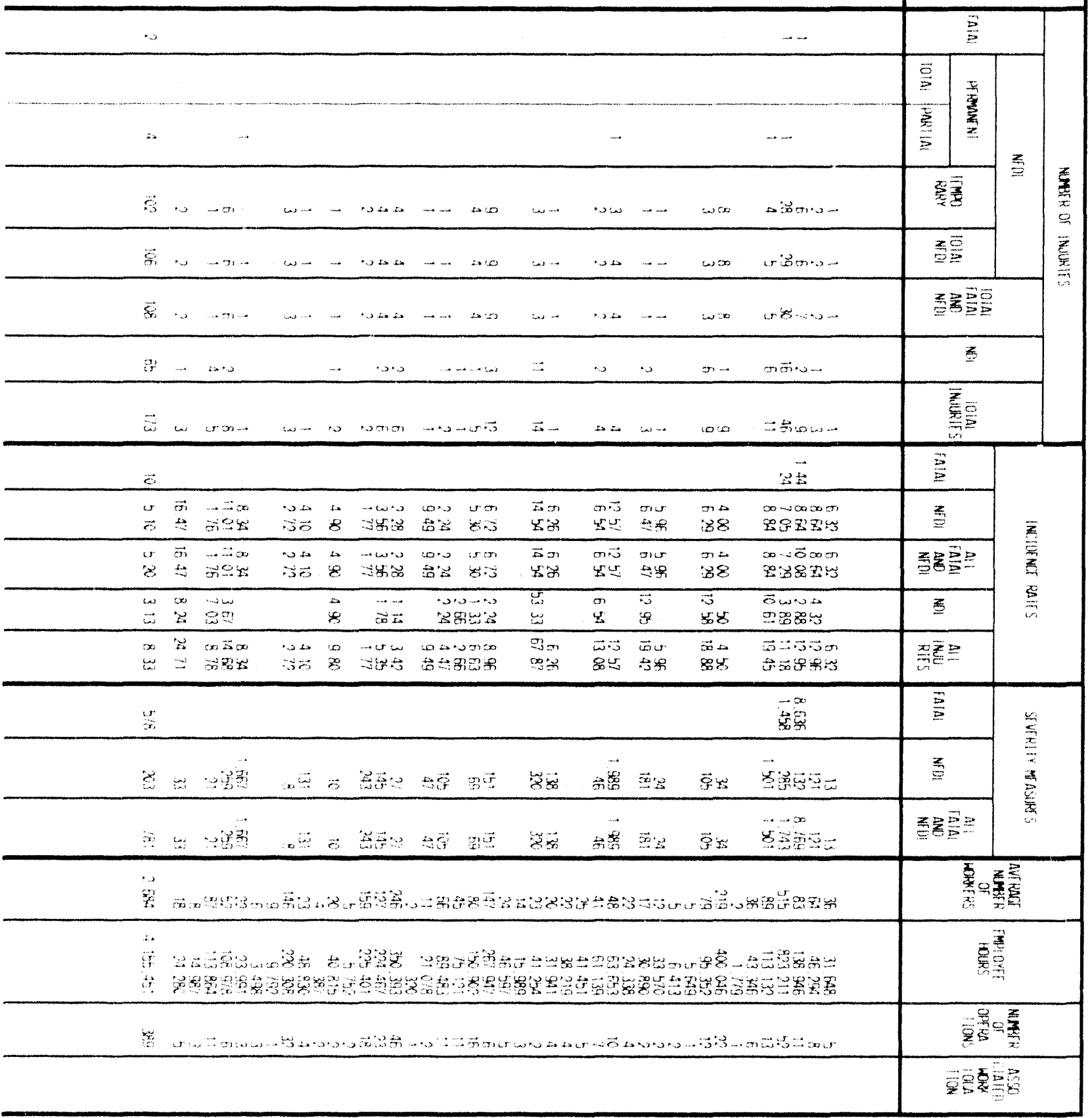




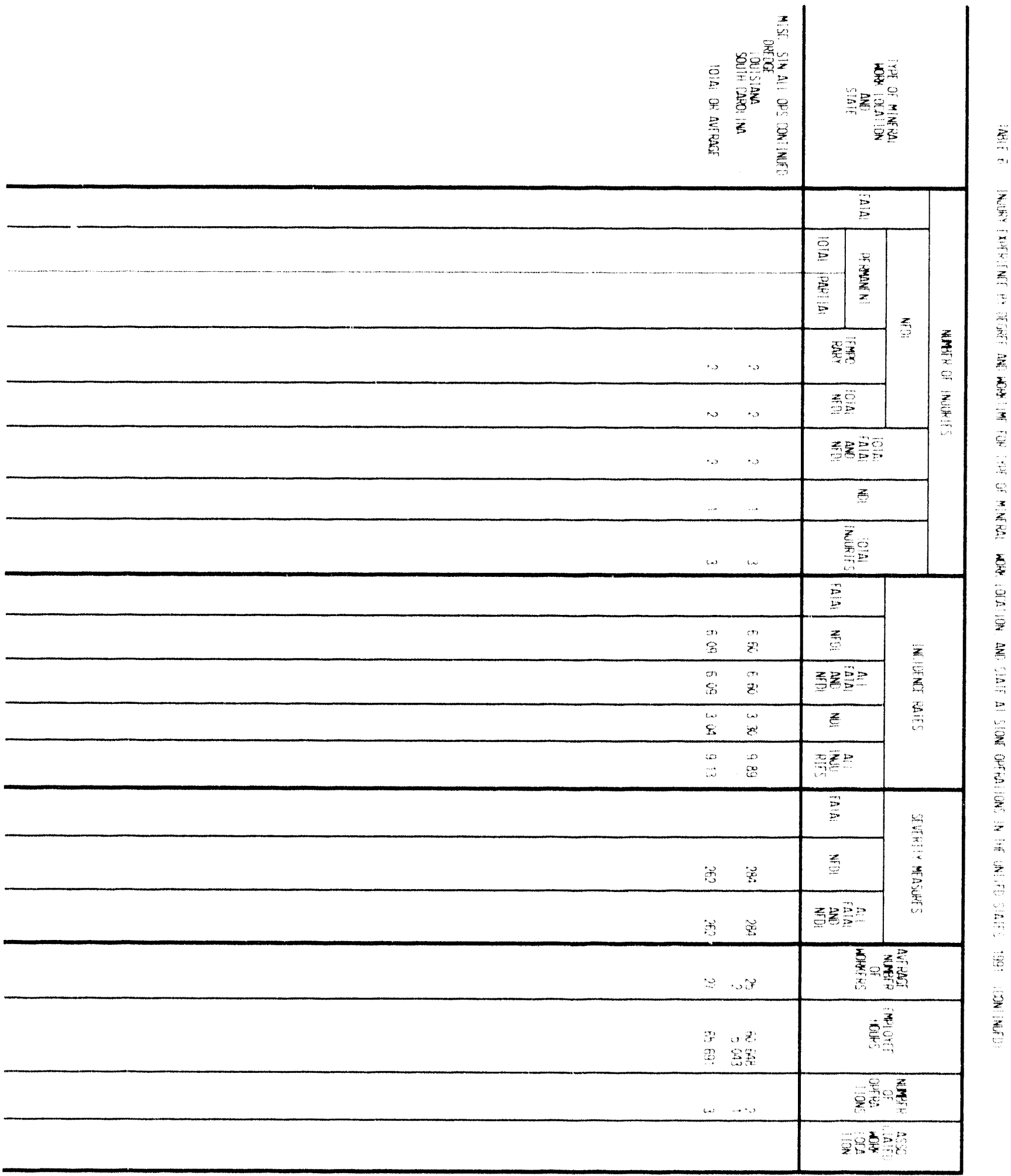




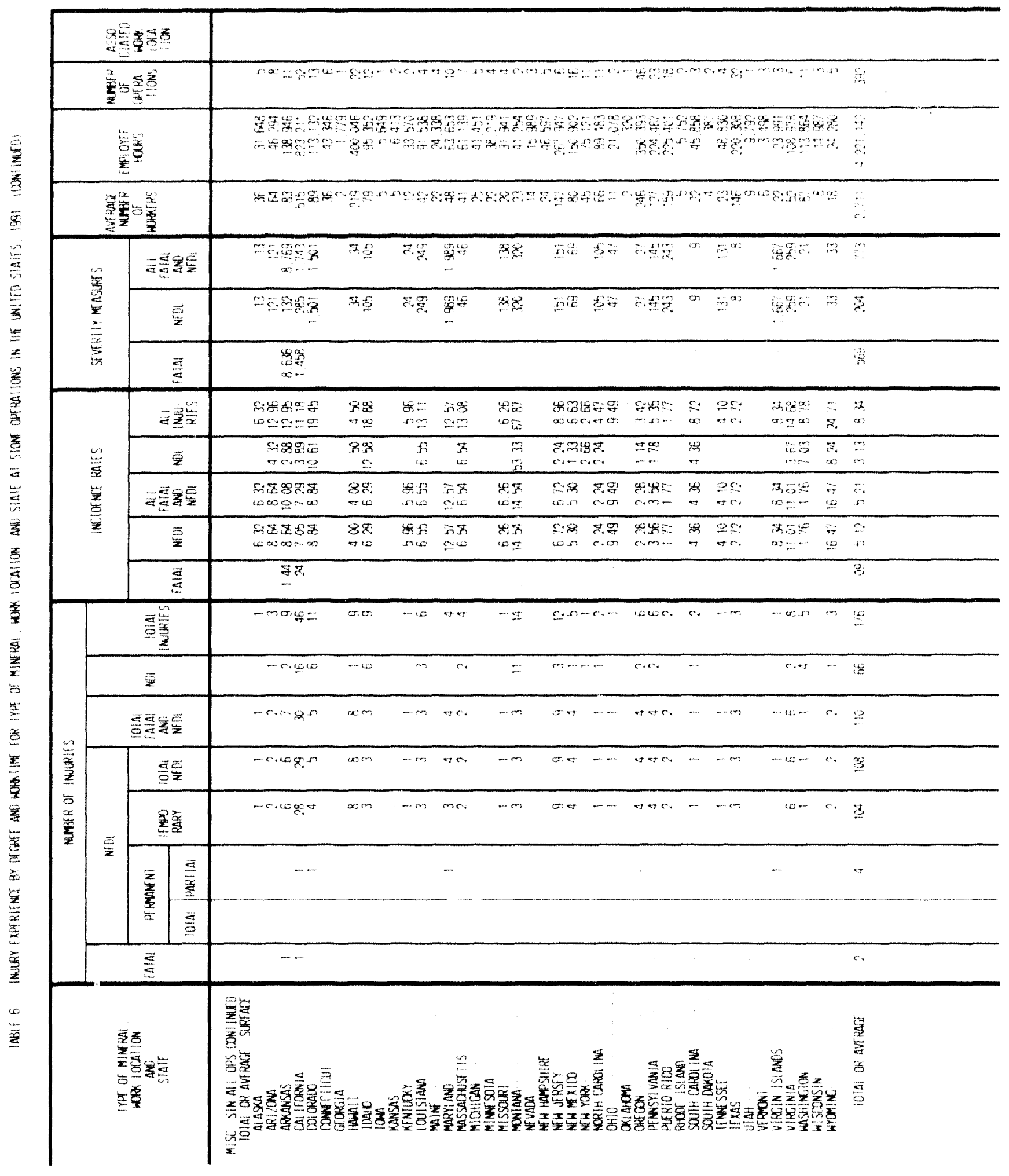




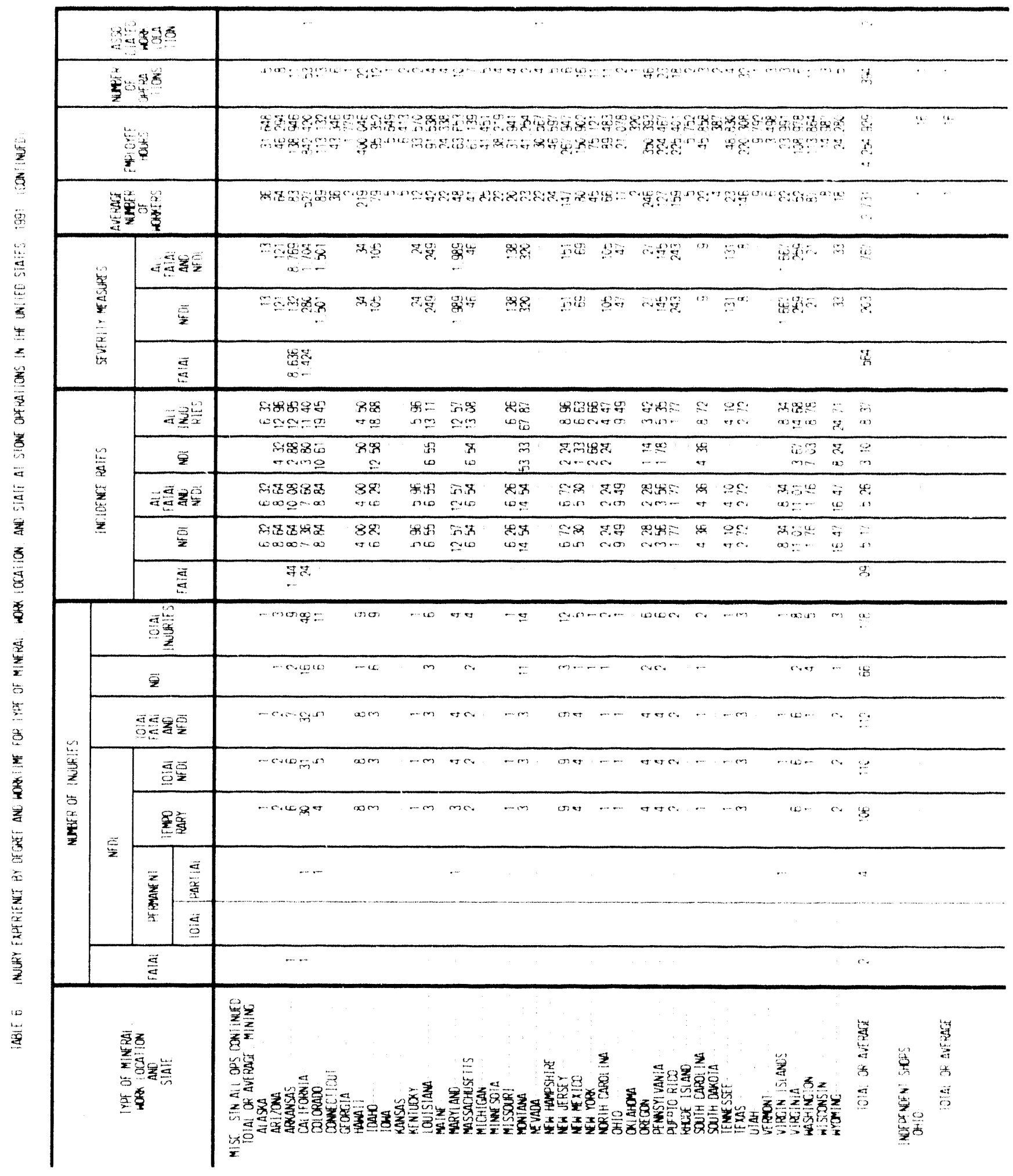




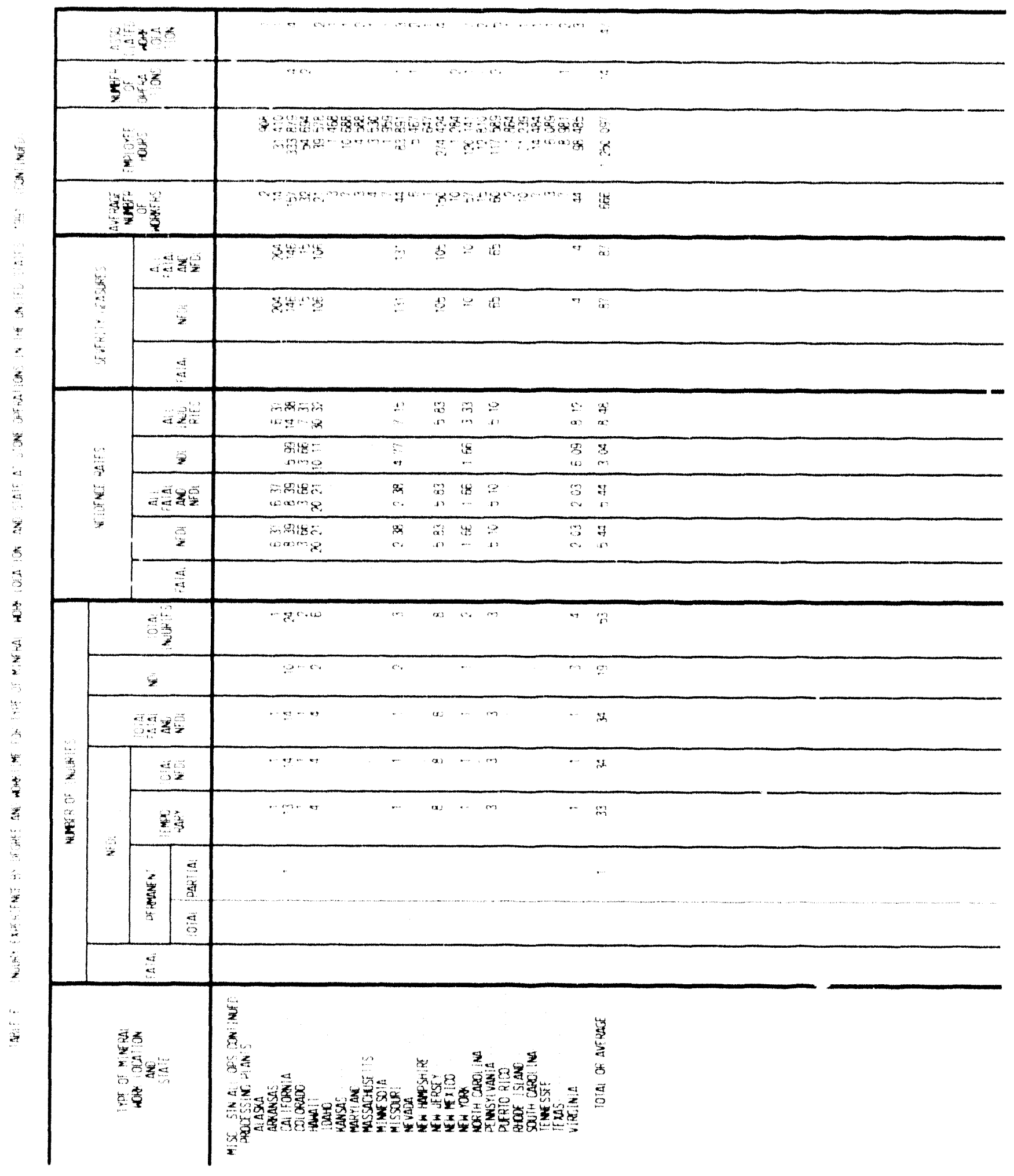




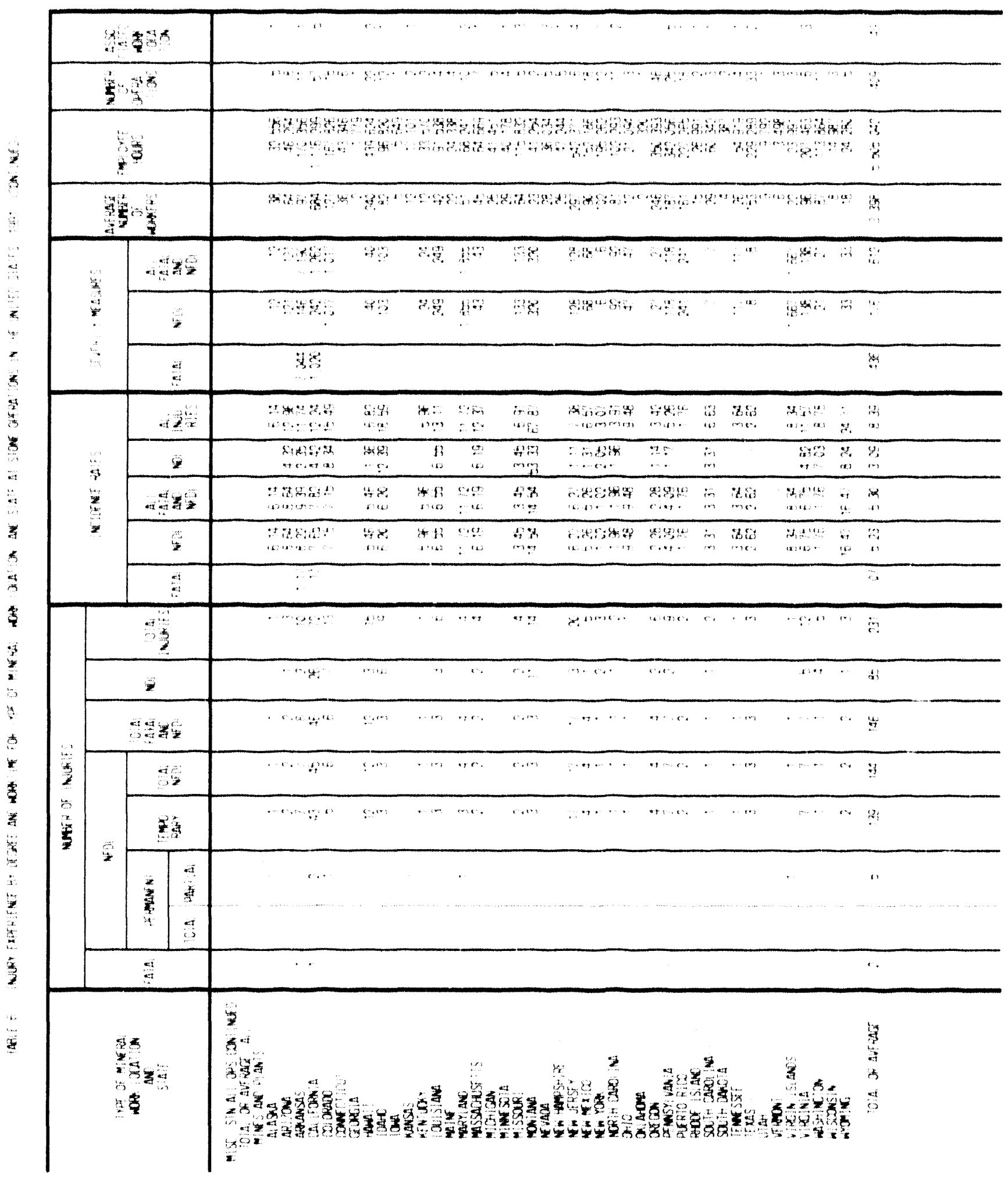



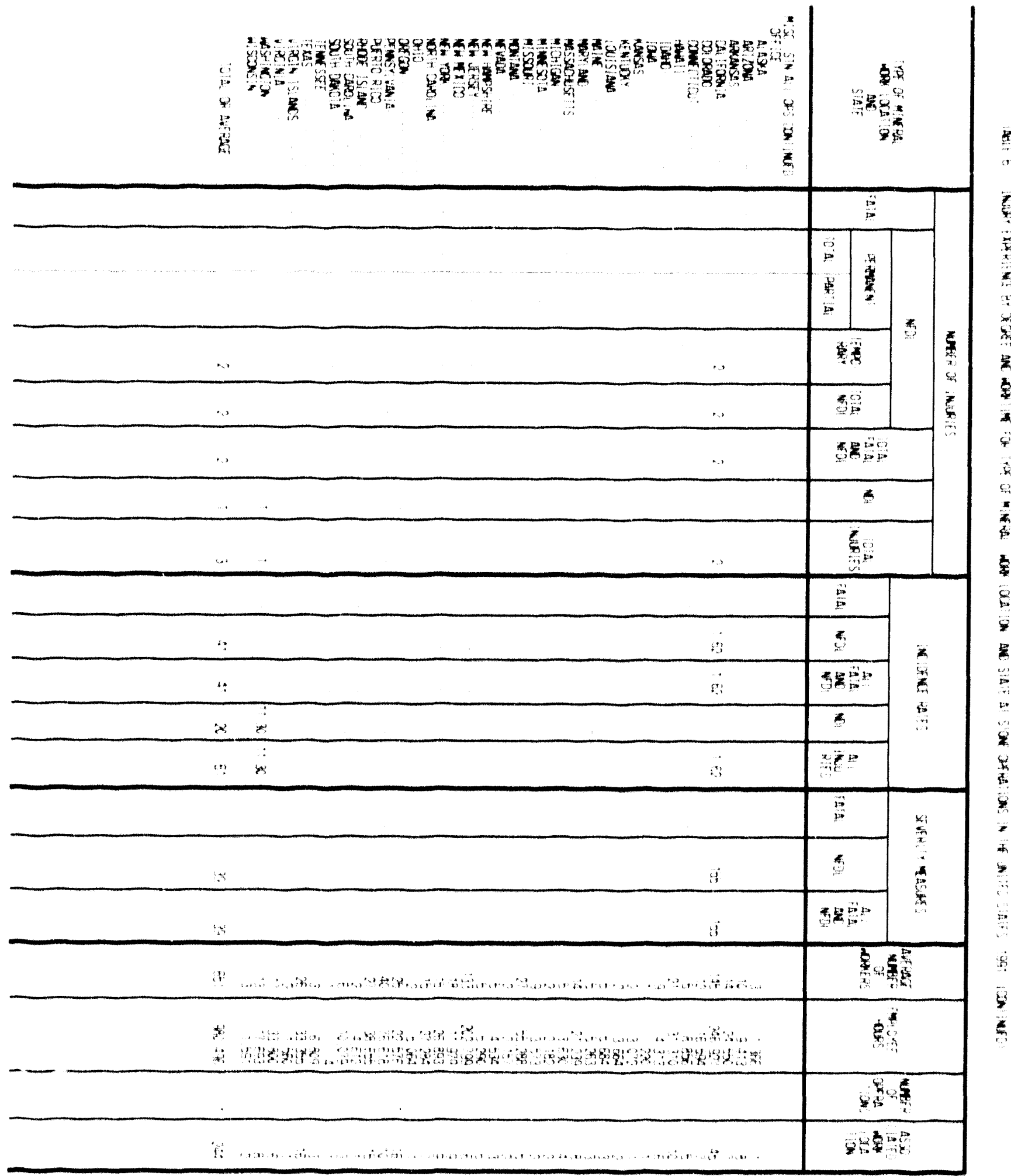


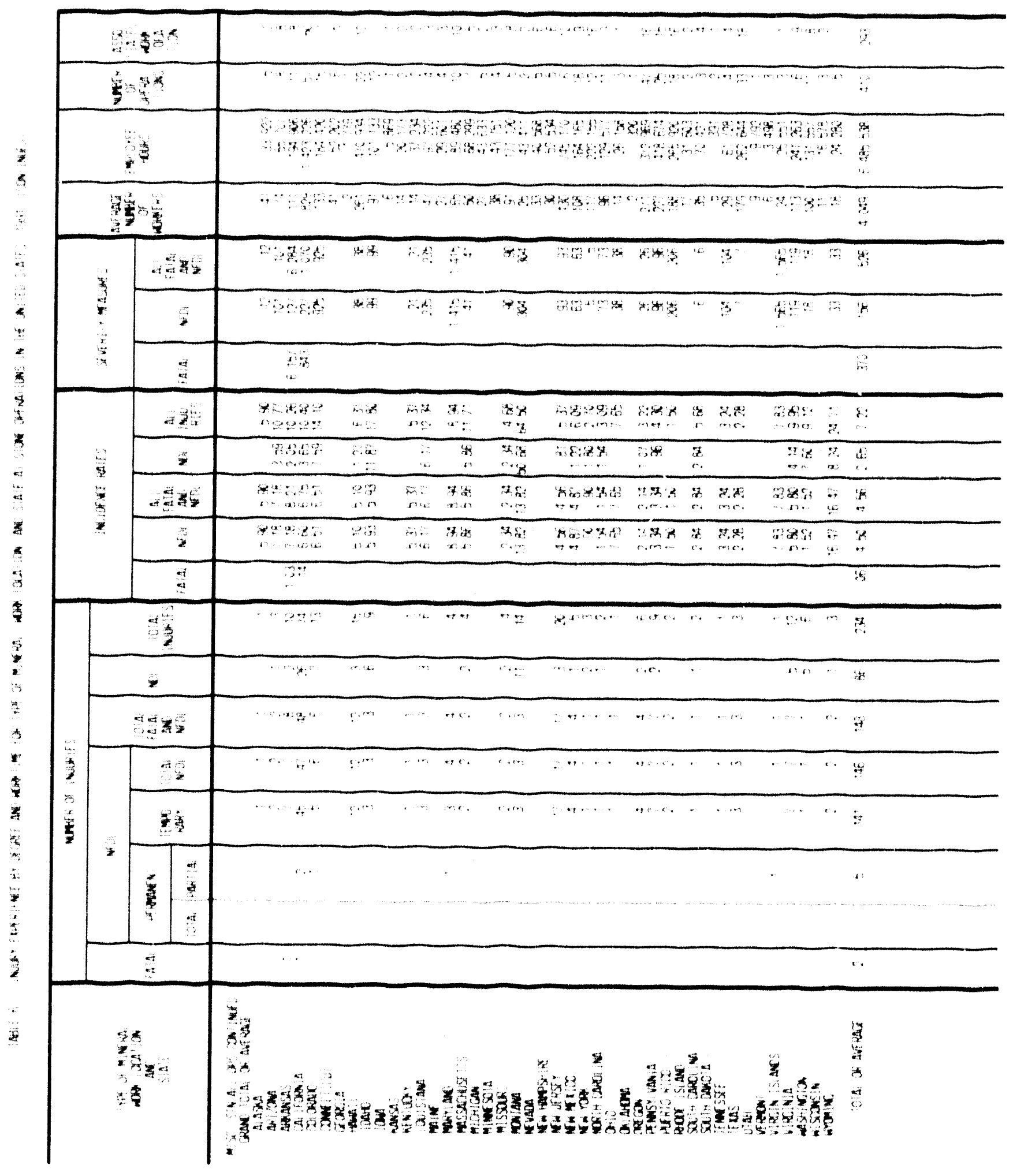




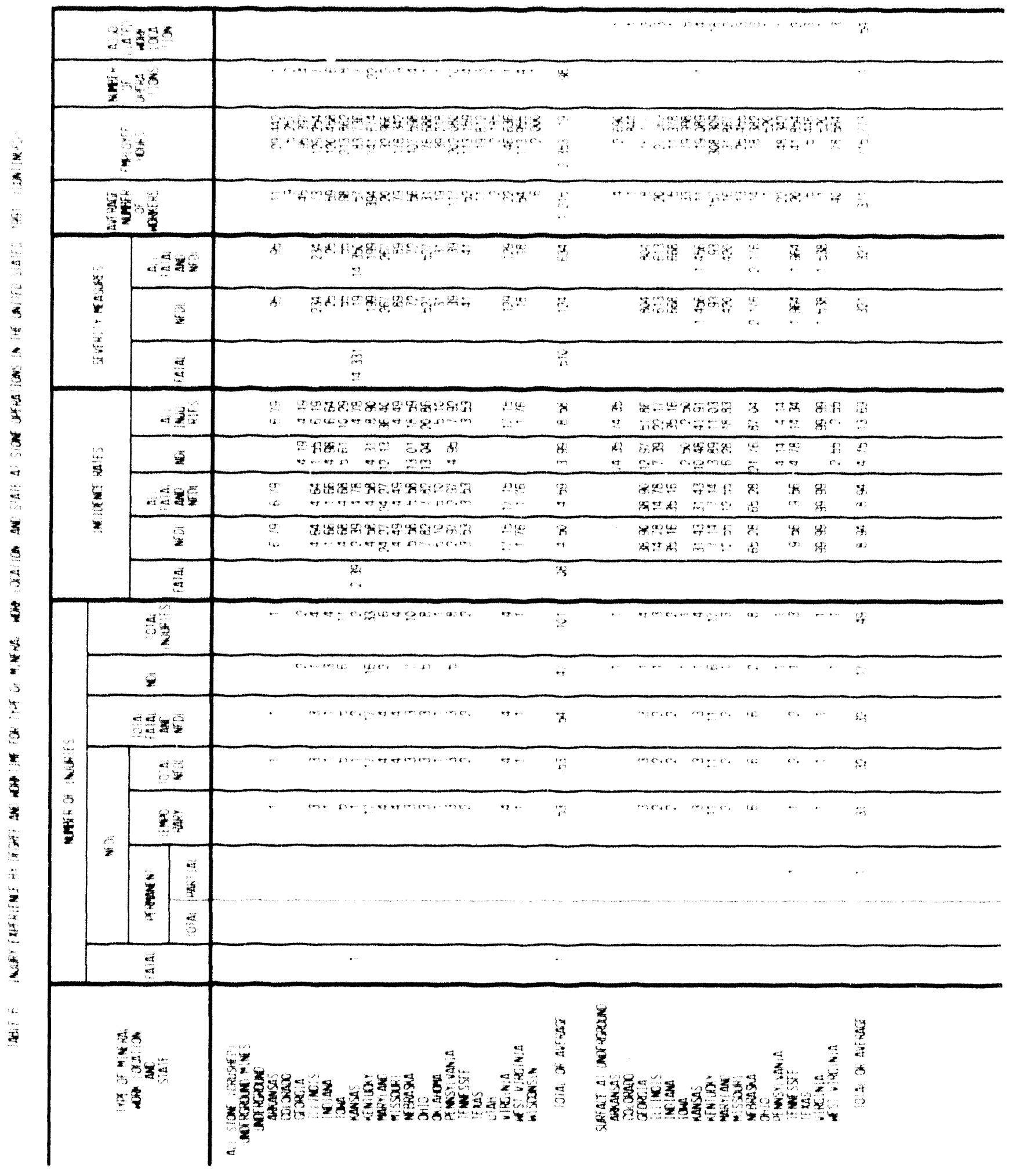




\begin{tabular}{|c|c|c|c|c|}
\hline & \multicolumn{2}{|c|}{235} & 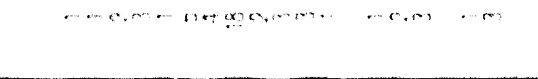 & 3 \\
\hline \multicolumn{3}{|c|}{ 管 } & \multicolumn{2}{|l|}{ 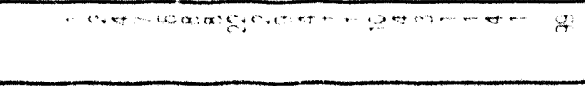 } \\
\hline \multicolumn{3}{|c|}{$\frac{5}{5}$} & \multicolumn{2}{|l|}{ 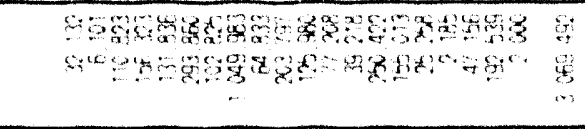 } \\
\hline \multicolumn{3}{|c|}{$\frac{2}{3}$} & \multicolumn{2}{|l|}{ 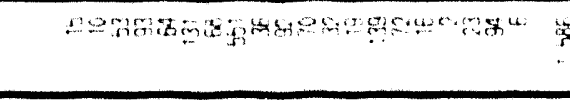 } \\
\hline \multirow{3}{*}{\multicolumn{2}{|c|}{ 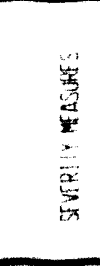 }} & 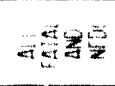 & 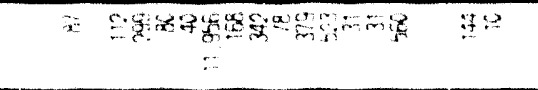 & is \\
\hline & & $\vec{z}$ & a chpor & 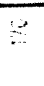 \\
\hline & & $\frac{a}{a}$ & 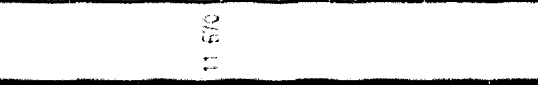 & 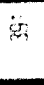 \\
\hline \multirow{5}{*}{\multicolumn{2}{|c|}{ 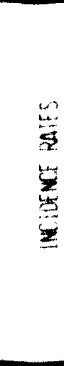 }} & $-2 \frac{25}{2}$ & 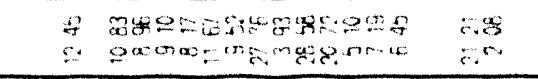 & 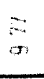 \\
\hline & 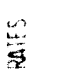 & 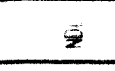 & 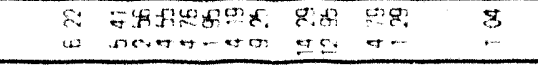 & 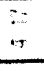 \\
\hline & & 运车事 & 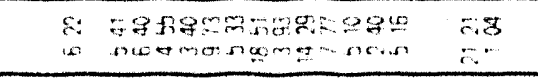 & s \\
\hline & & $\overline{2}$ & 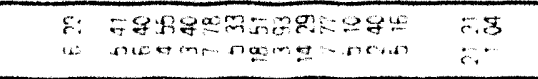 & $\begin{array}{ll}7 \\
3\end{array}$ \\
\hline & & $\frac{a}{a}$ & f) & 8 \\
\hline \multirow{8}{*}{ 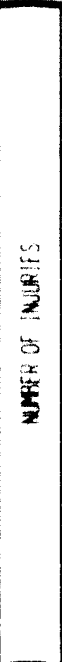 } & \multicolumn{2}{|c|}{ 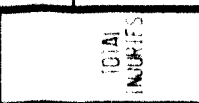 } & 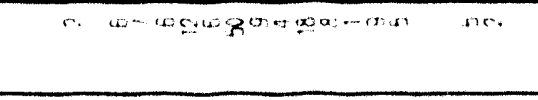 & 8 \\
\hline & \multicolumn{2}{|r|}{$\dot{z}$} & 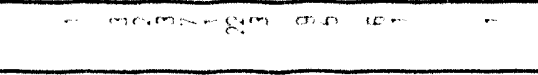 & $i$ \\
\hline & \multicolumn{2}{|c|}{ 可高象 } & 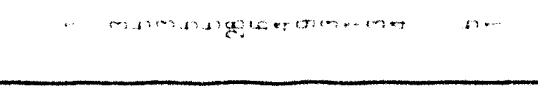 & 要 \\
\hline & \multirow{4}{*}{ 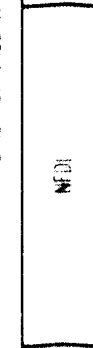 } & 5 & 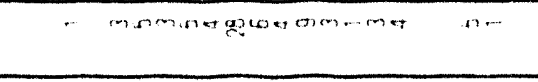 & $a^{2}$ \\
\hline & & 憘 & 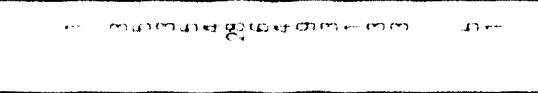 & 3 \\
\hline & & \multirow{2}{*}{$\underset{2}{z}$} & - & - \\
\hline & & & & \\
\hline & \multicolumn{2}{|r|}{$\sum_{i}^{a t}$} & . & $\cdots$ \\
\hline \multicolumn{3}{|c|}{ 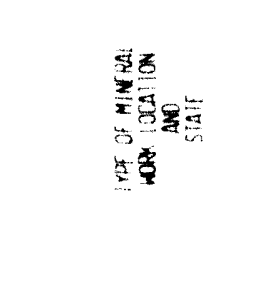 } & 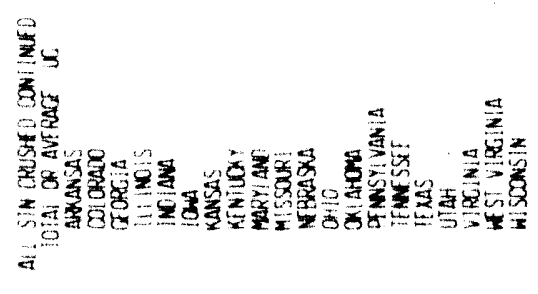 & 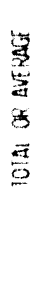 \\
\hline
\end{tabular}




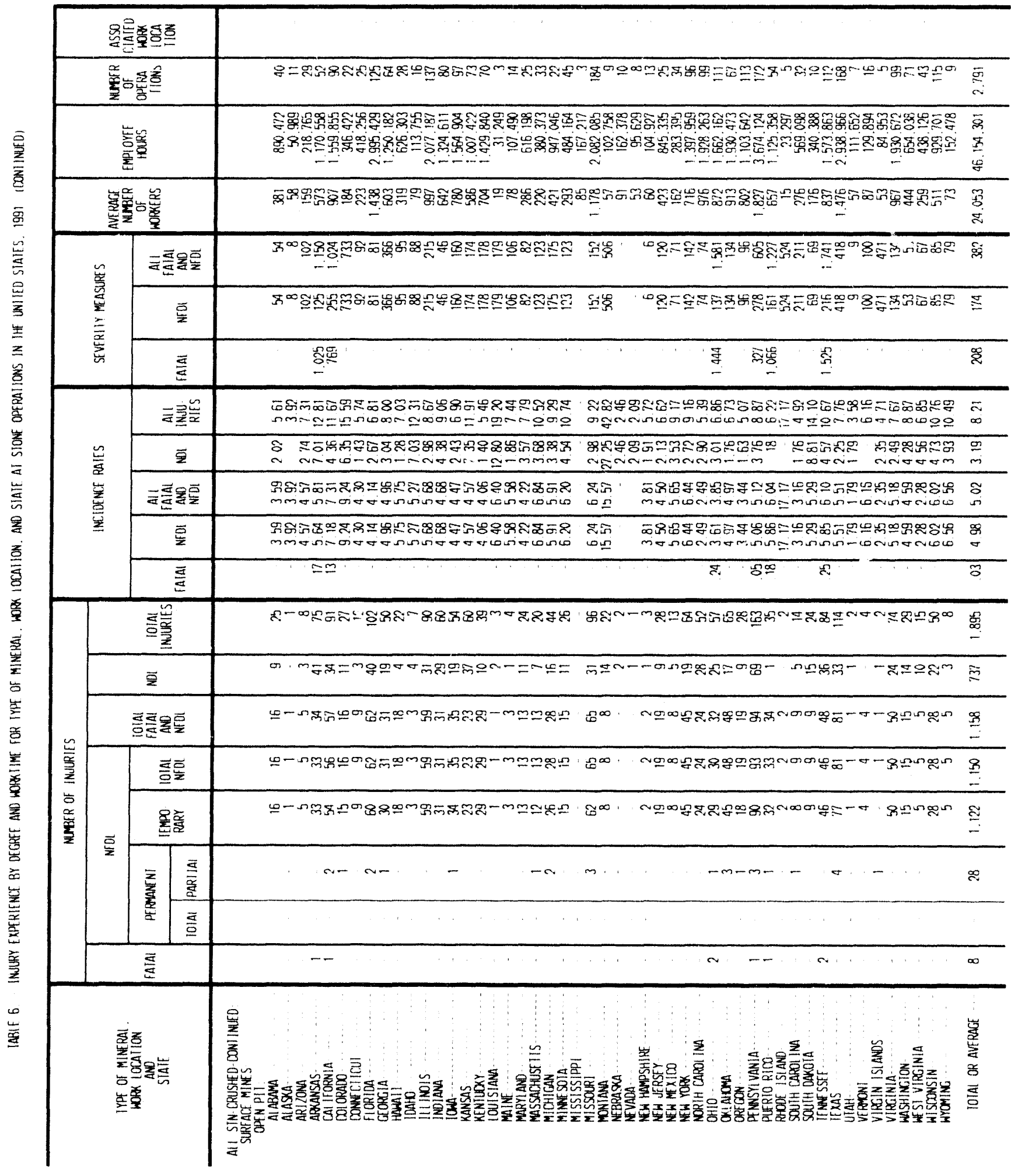




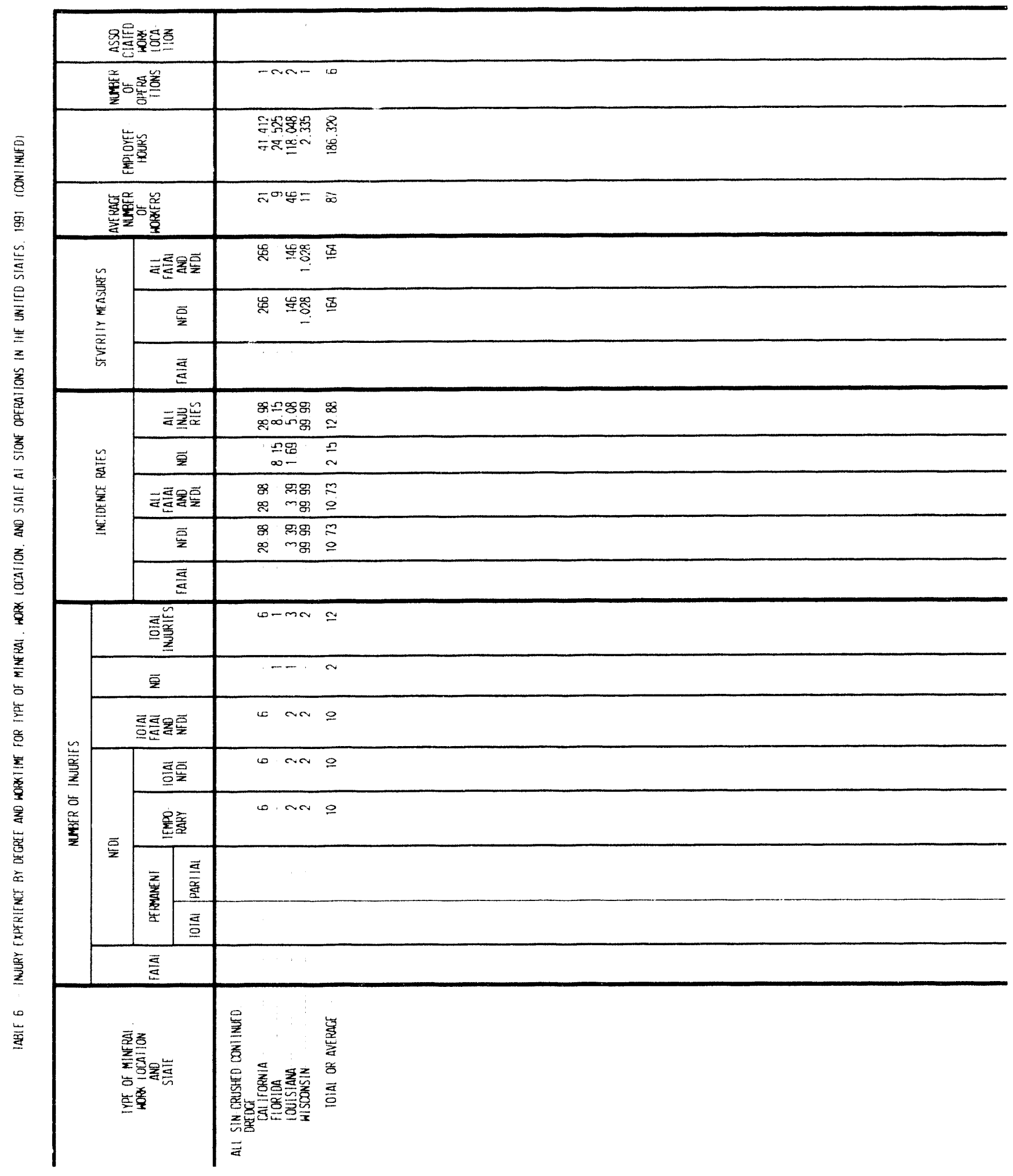




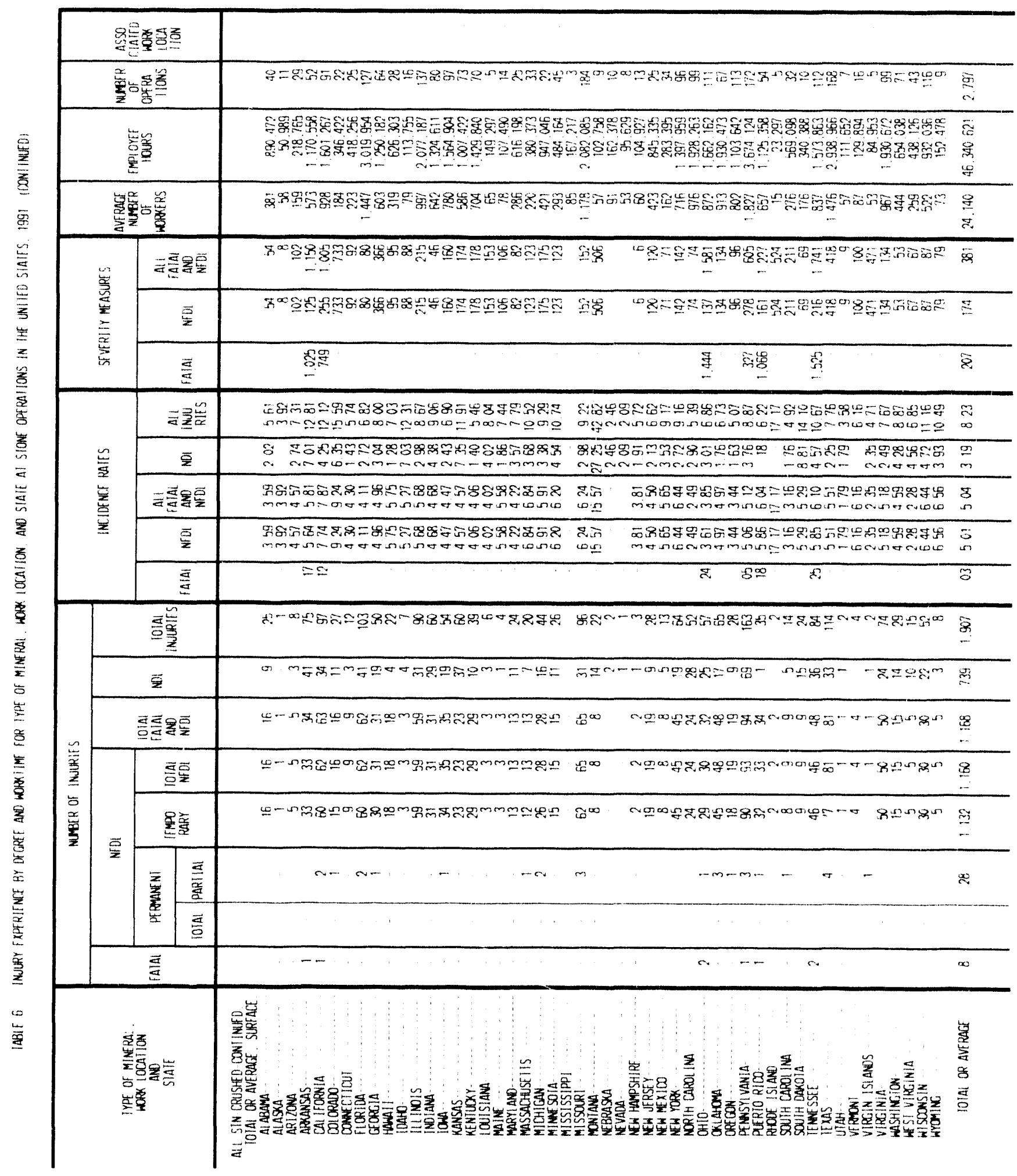




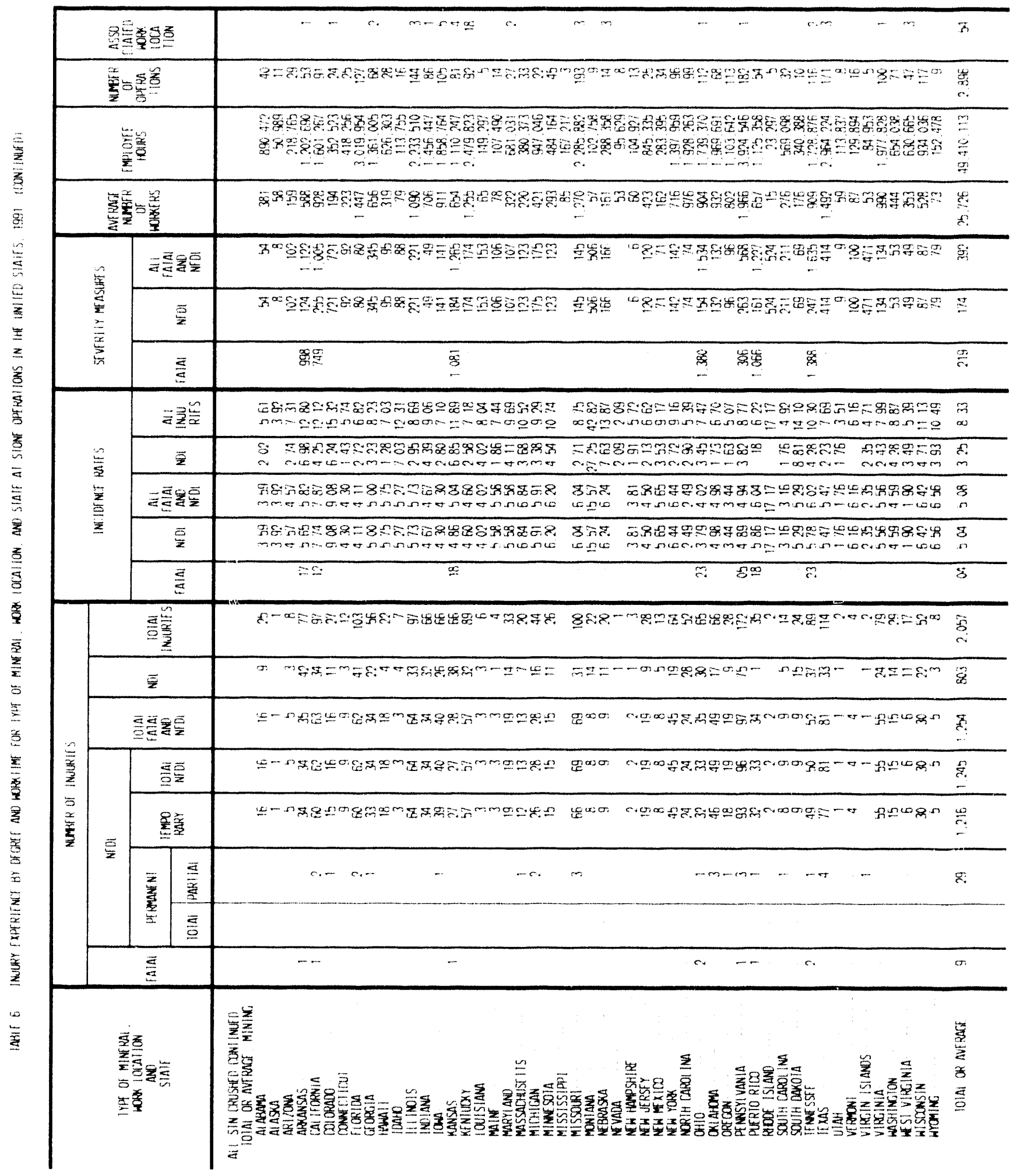




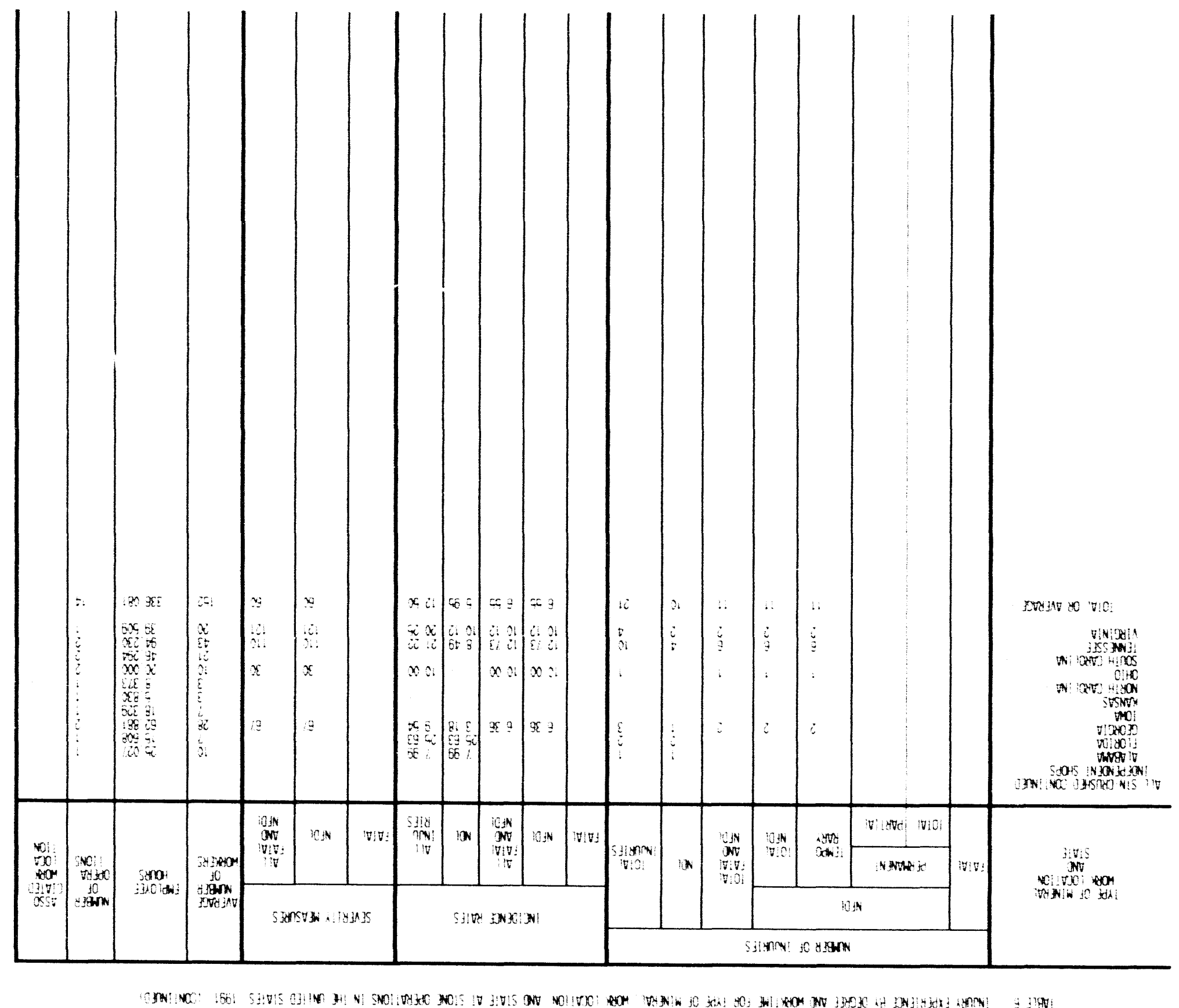




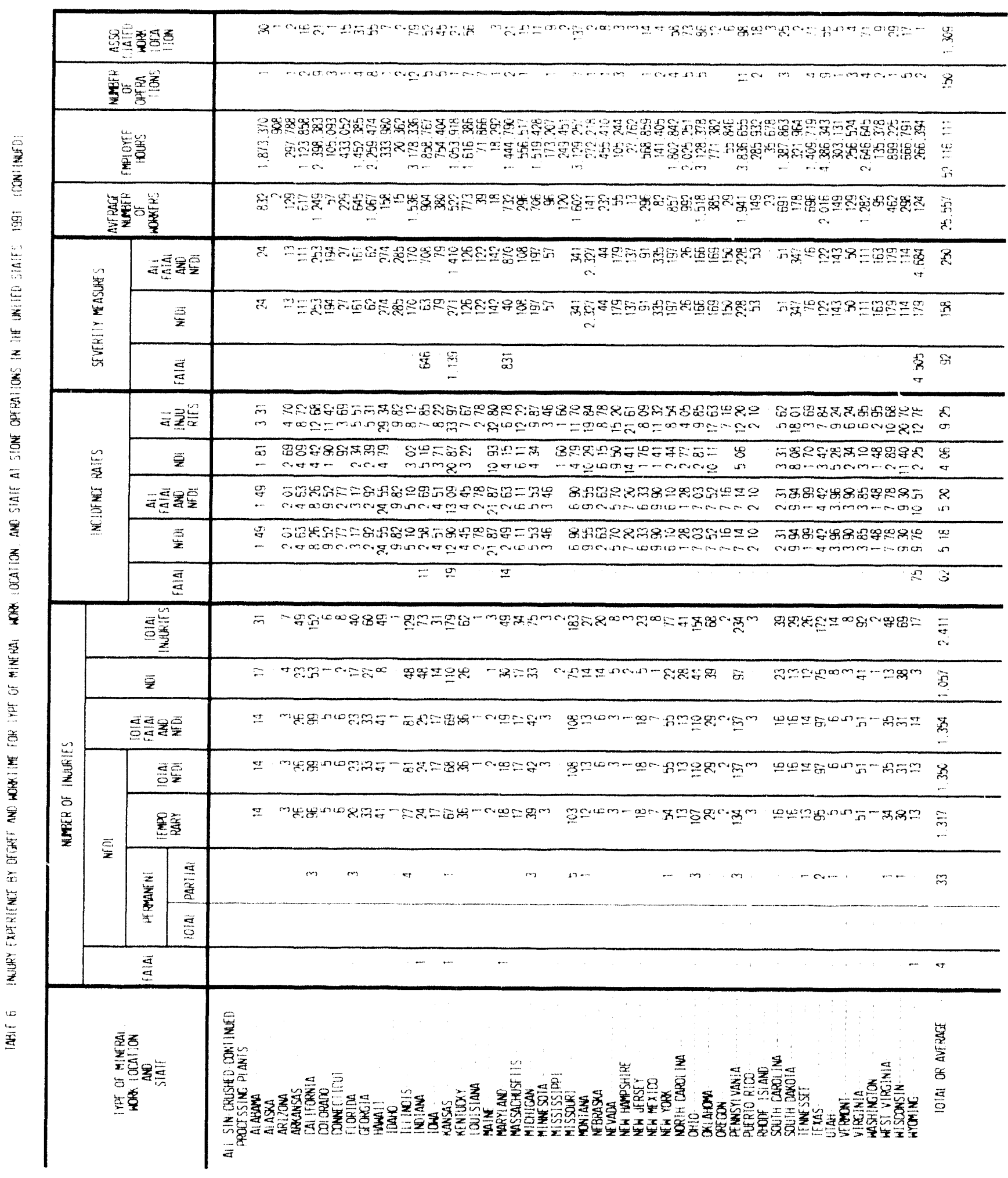




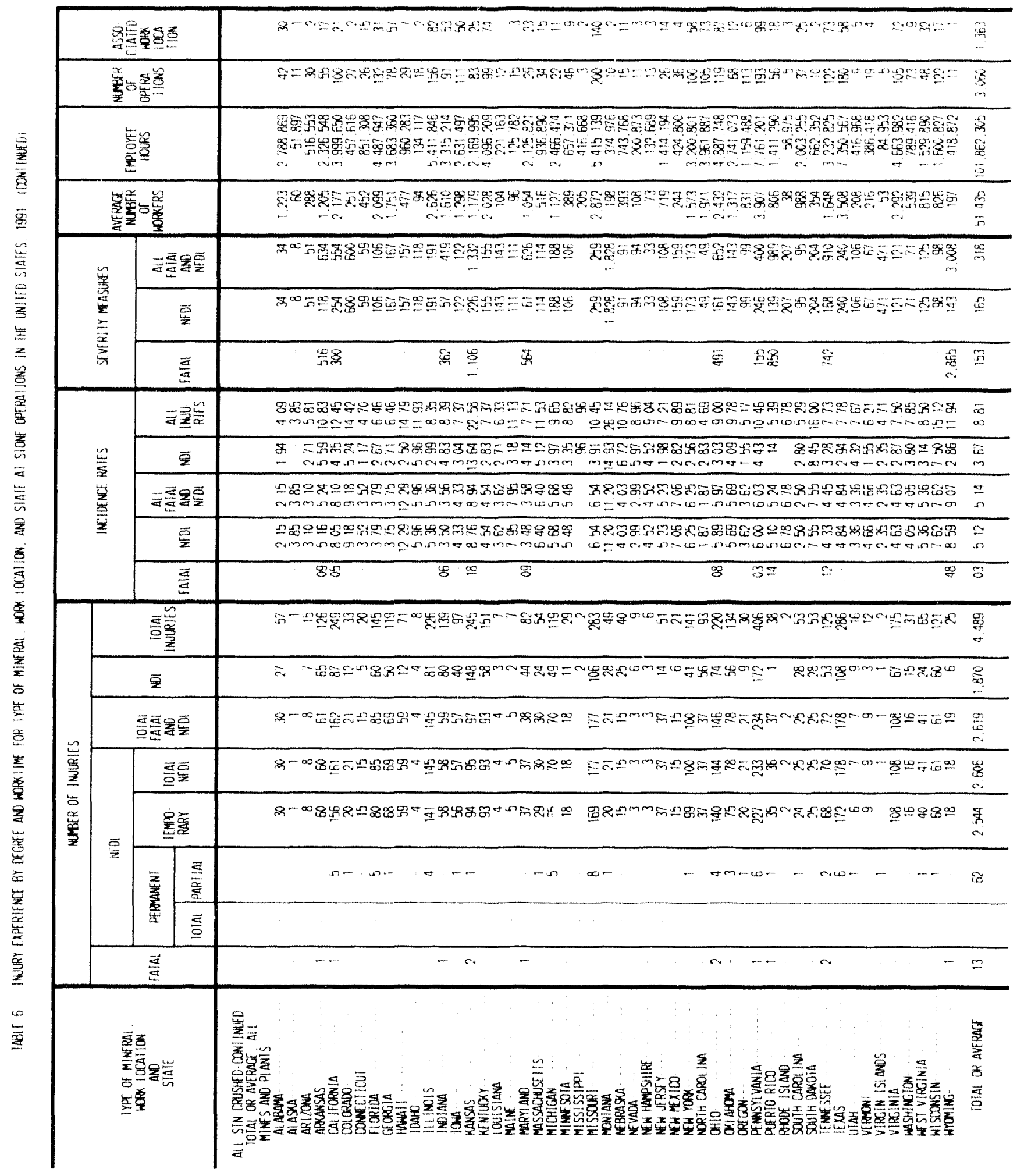




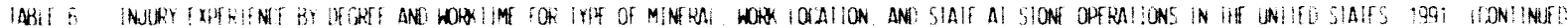

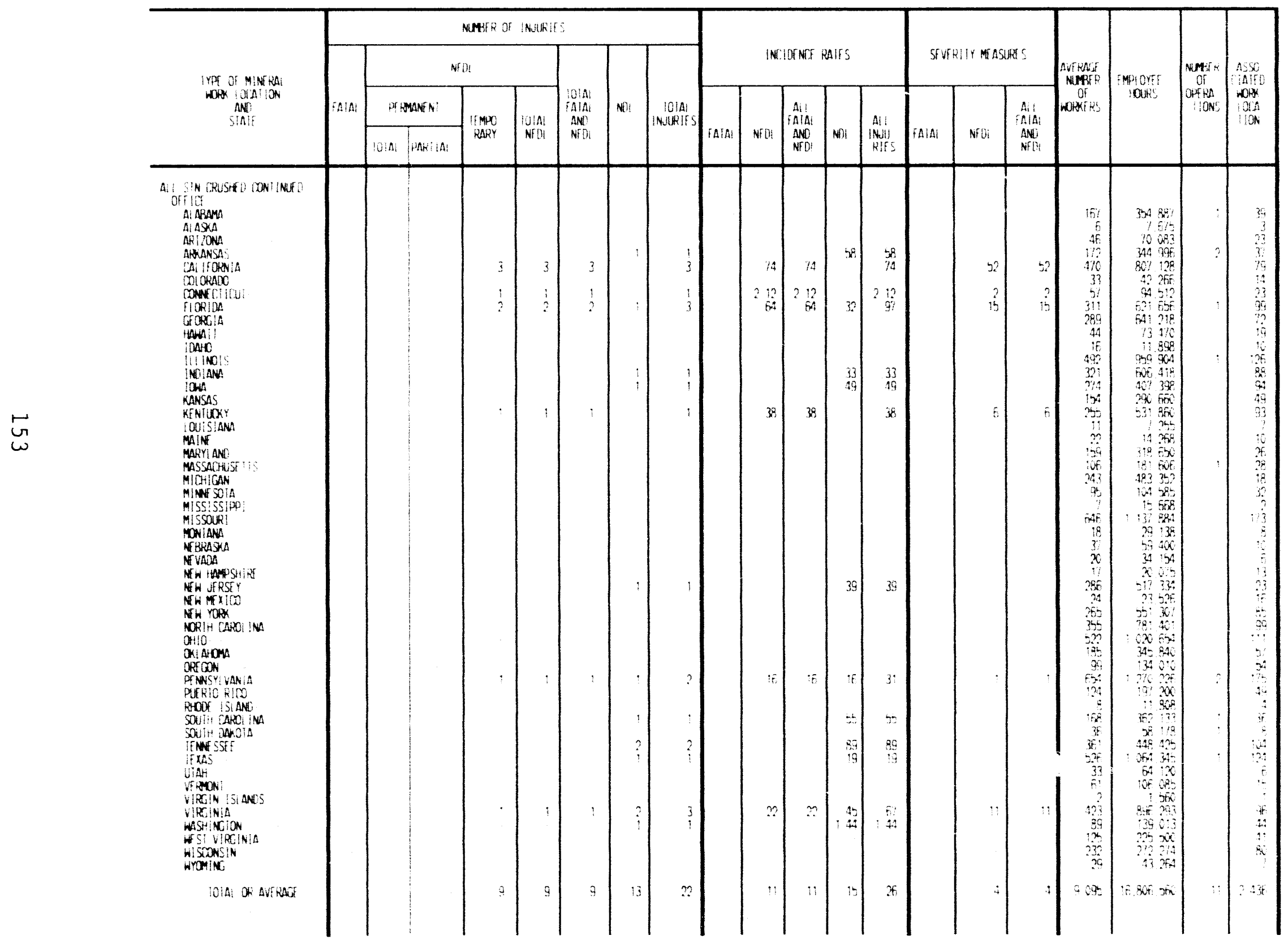




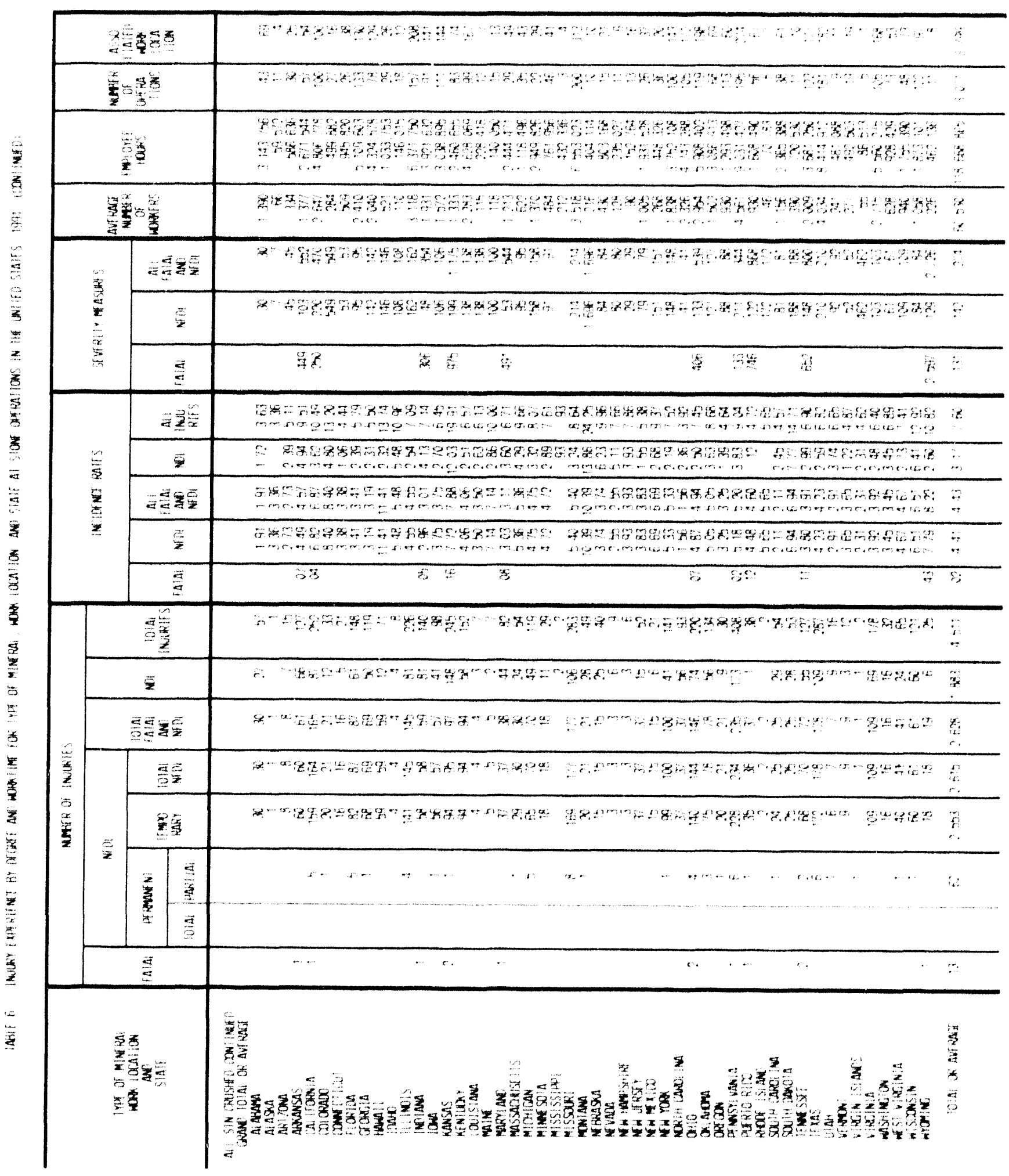




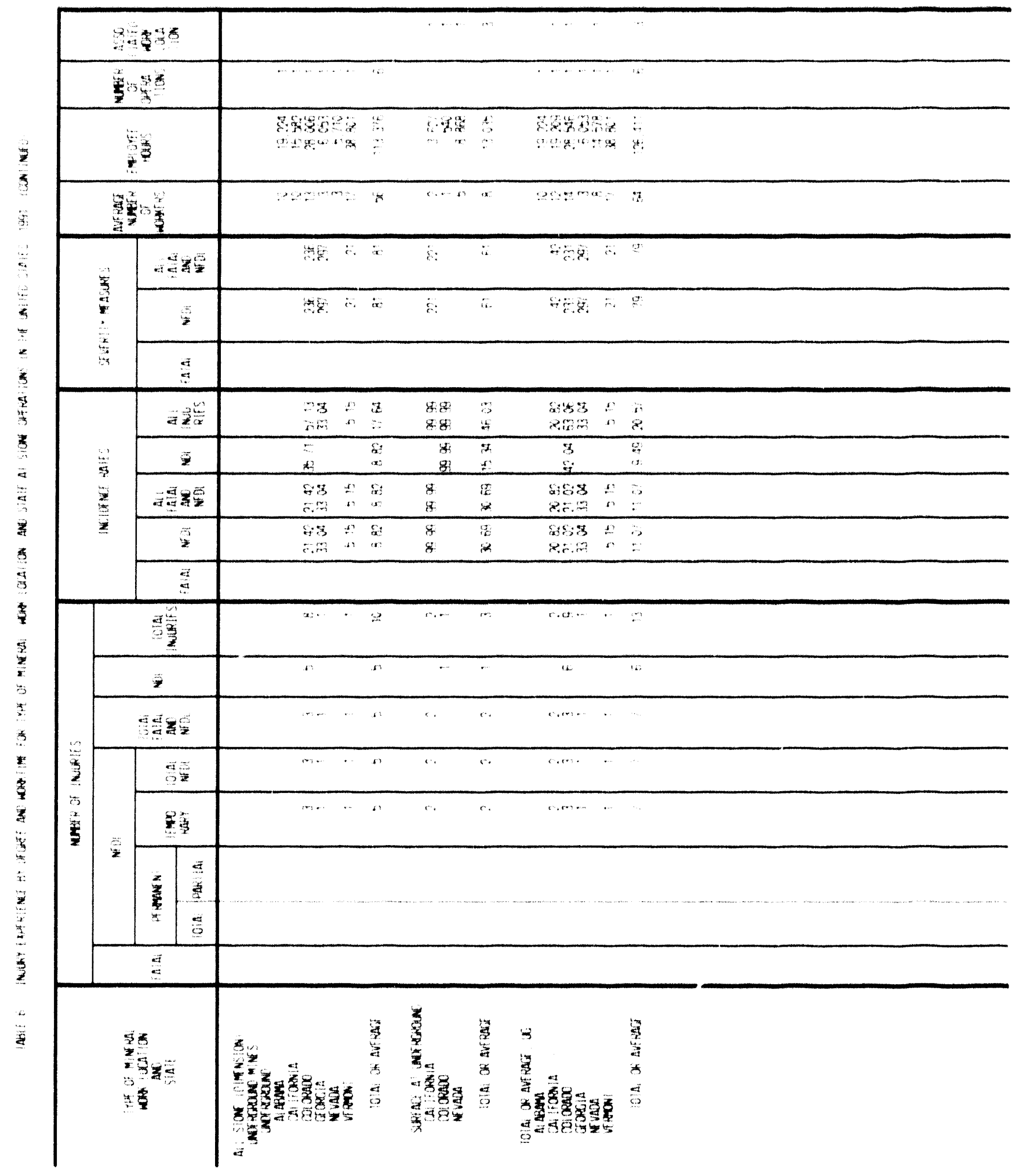




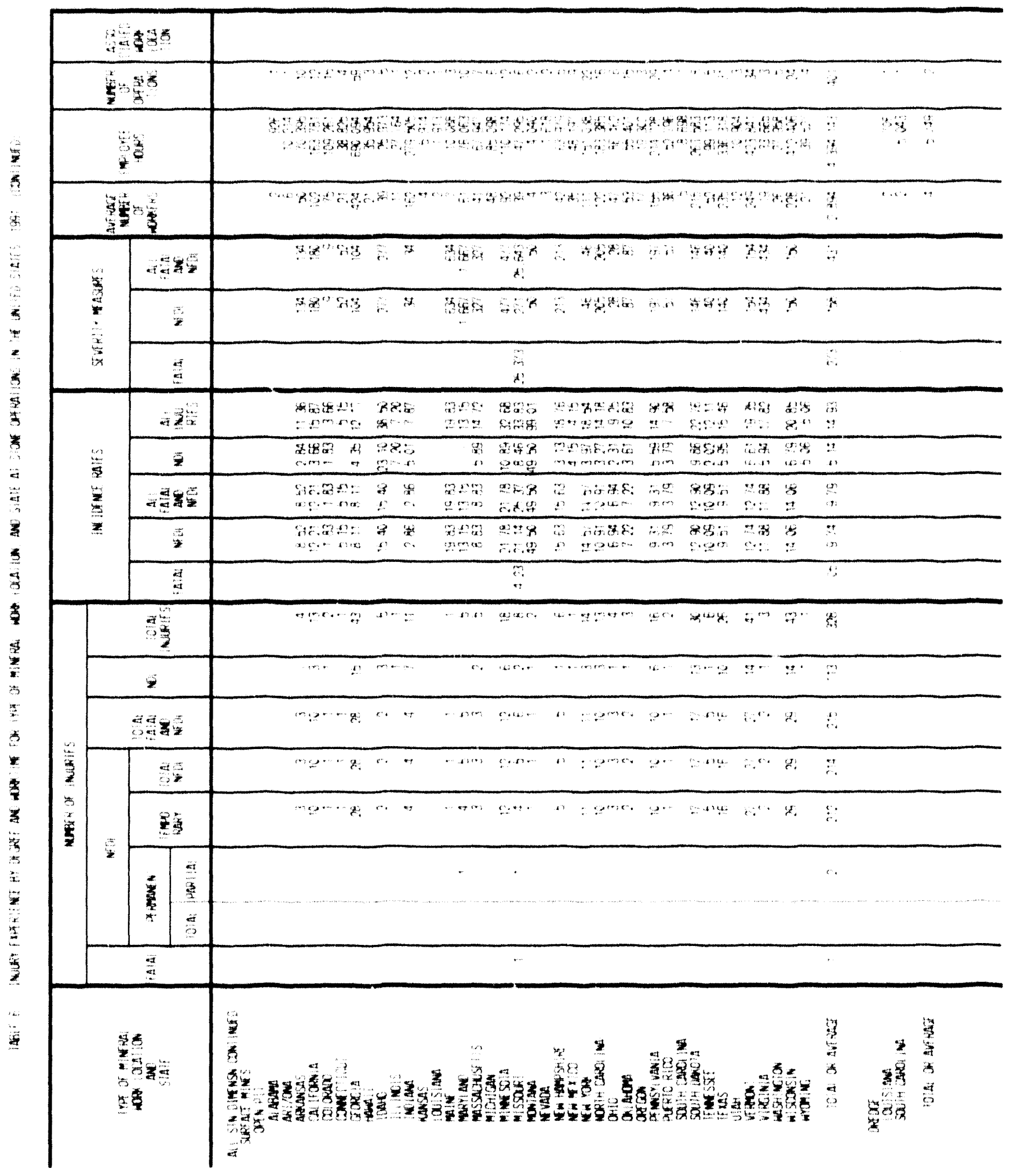




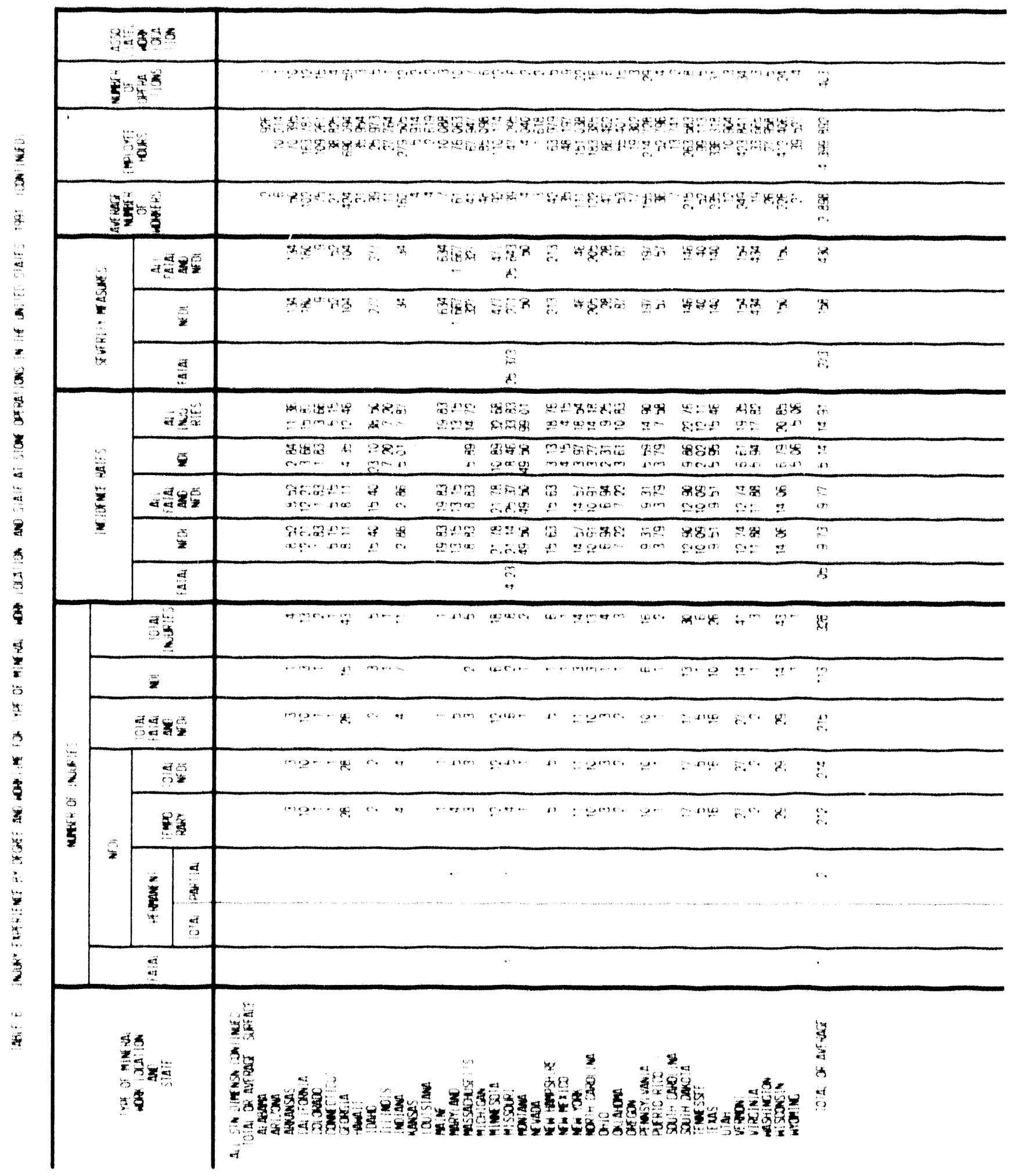




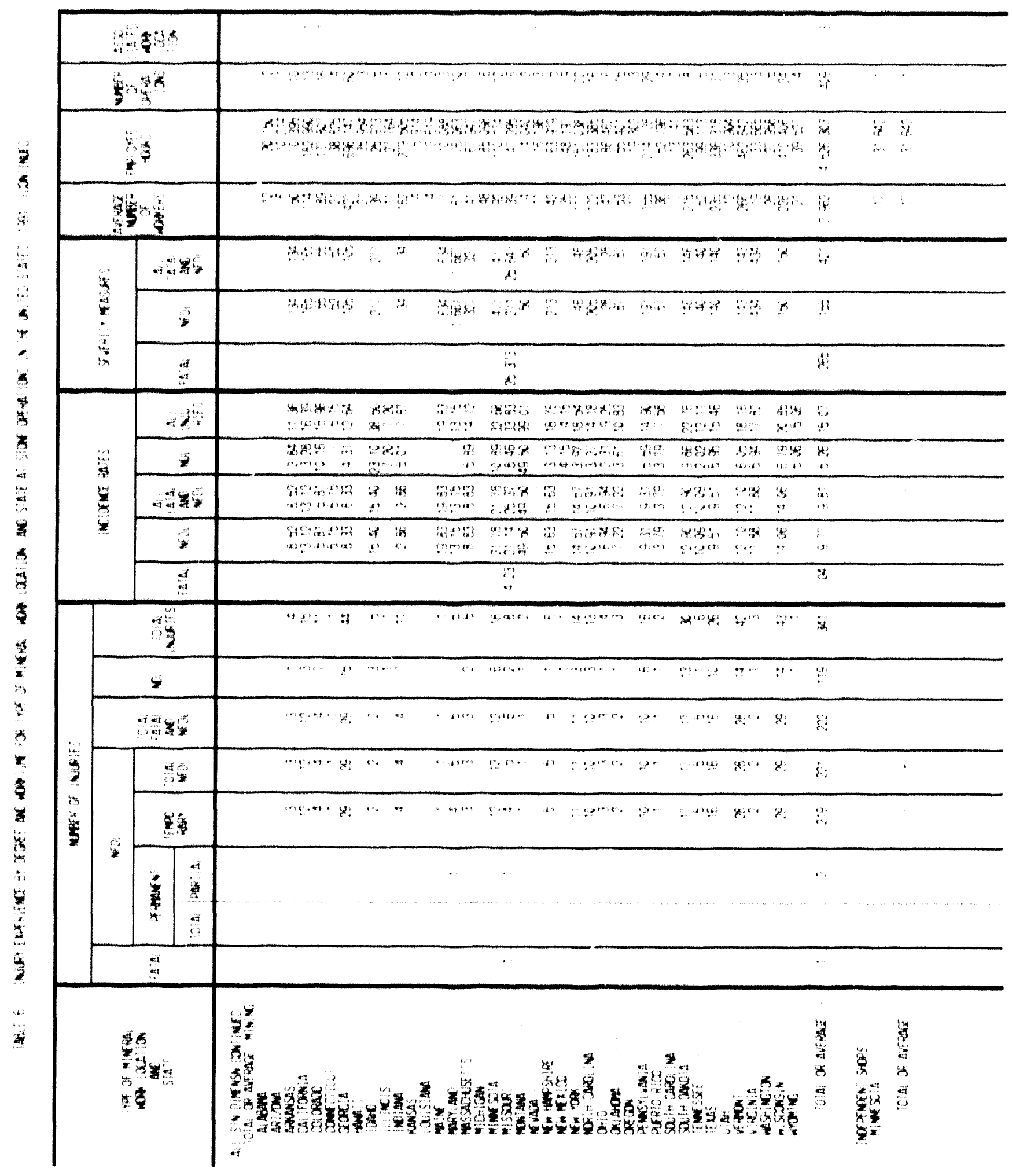




\begin{tabular}{|c|c|c|c|c|c|}
\hline & \multicolumn{2}{|c|}{ 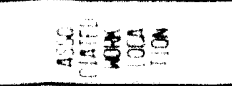 } & 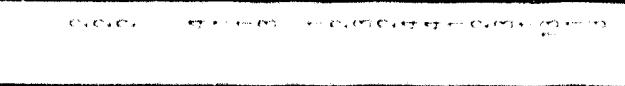 & $\cdots$ & \\
\hline & \multicolumn{2}{|c|}{ 施素 } & & 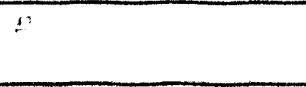 & \\
\hline \multicolumn{3}{|c|}{$\frac{5}{2}$} & 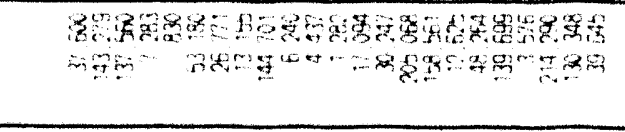 & $\frac{* 3}{3}$ & \\
\hline \multicolumn{3}{|c|}{ 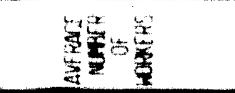 } & 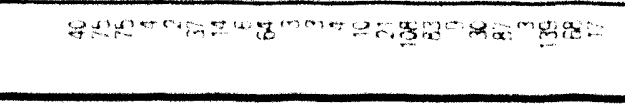 & 8 & \\
\hline \multirow{3}{*}{\multicolumn{2}{|c|}{ 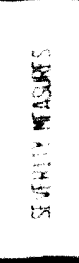 }} & 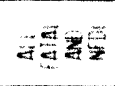 & 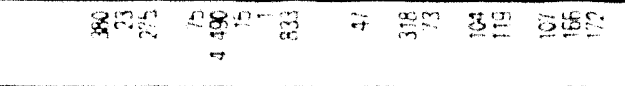 & 器 & \\
\hline & & 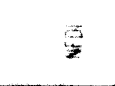 & 船 & 壱 & \\
\hline & & 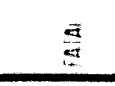 & & & \\
\hline & \multirow{5}{*}{$\begin{array}{l} \\
z \\
z \\
z \\
z\end{array}$} & 这要 & 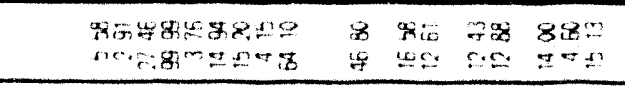 & $\stackrel{g}{\prime \prime}$ & \\
\hline & & $\bar{z}$ & 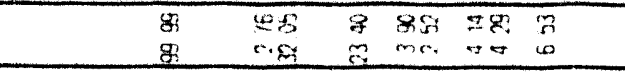 & $\stackrel{8}{\circ}$ & \\
\hline & & 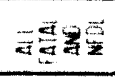 & 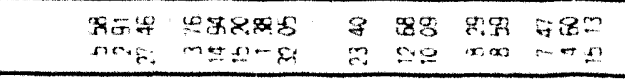 & $\cdots$ & \\
\hline & & $\overline{3}$ & 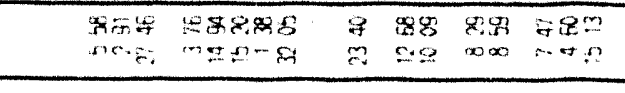 & 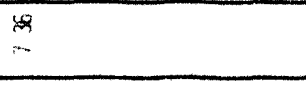 & \\
\hline & & $\stackrel{z}{s}$ & & & \\
\hline \multirow{8}{*}{$\begin{array}{l}\frac{3}{3} \\
\underline{z} \\
\vdots \\
z \\
z \\
z \\
z\end{array}$} & \multicolumn{2}{|c|}{$\begin{array}{l}5 \\
\\
\end{array}$} & 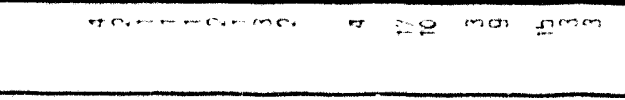 & $\bar{\infty}$ & \\
\hline & \multicolumn{2}{|r|}{ 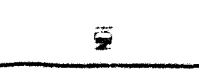 } & 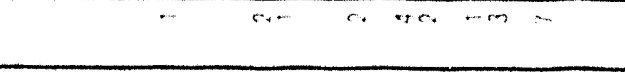 & $\ddot{m}$ & \\
\hline & \multicolumn{2}{|c|}{ 送事事 } & 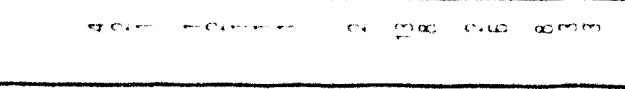 & $\infty$ & \\
\hline & \multicolumn{2}{|r|}{ 范 } & 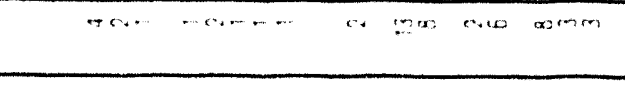 & 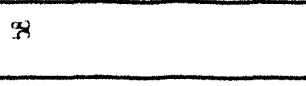 & \\
\hline & 5 & 寒 & 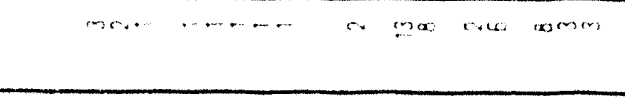 & s & \\
\hline & 7 & 喜 & .. & a & \\
\hline & & $\frac{a}{3}$ & & & \\
\hline & & $\frac{a}{3}$ & & & \\
\hline & & & 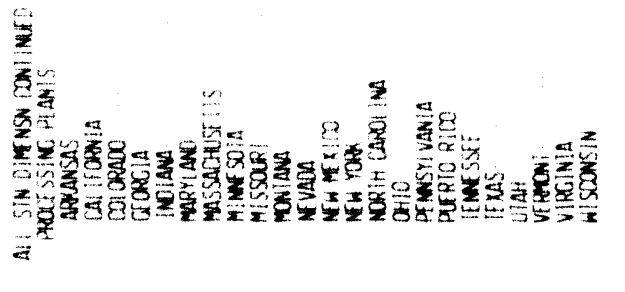 & 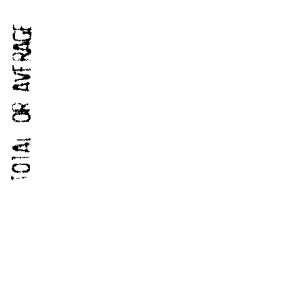 & \\
\hline
\end{tabular}




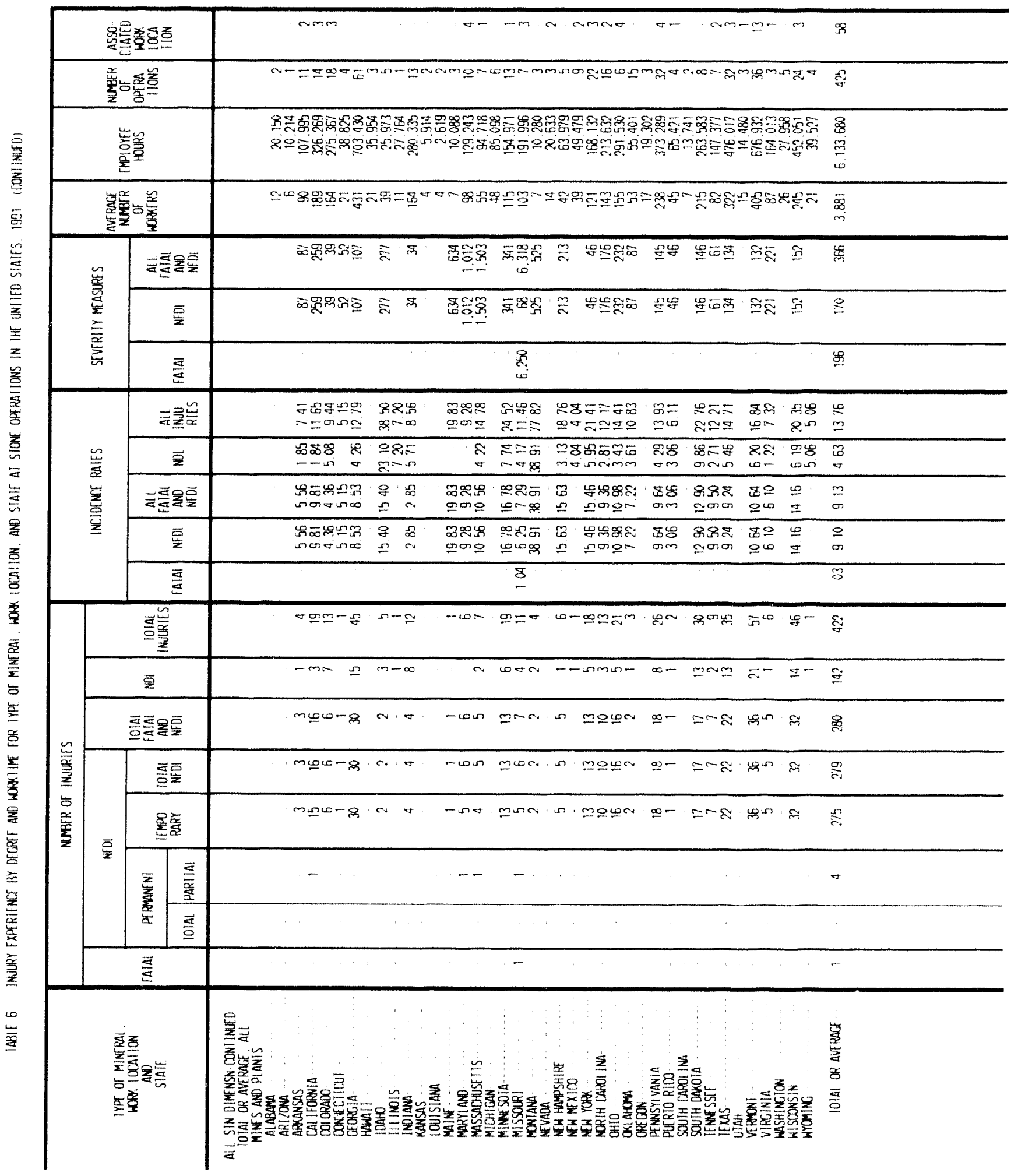




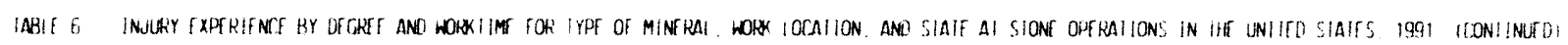

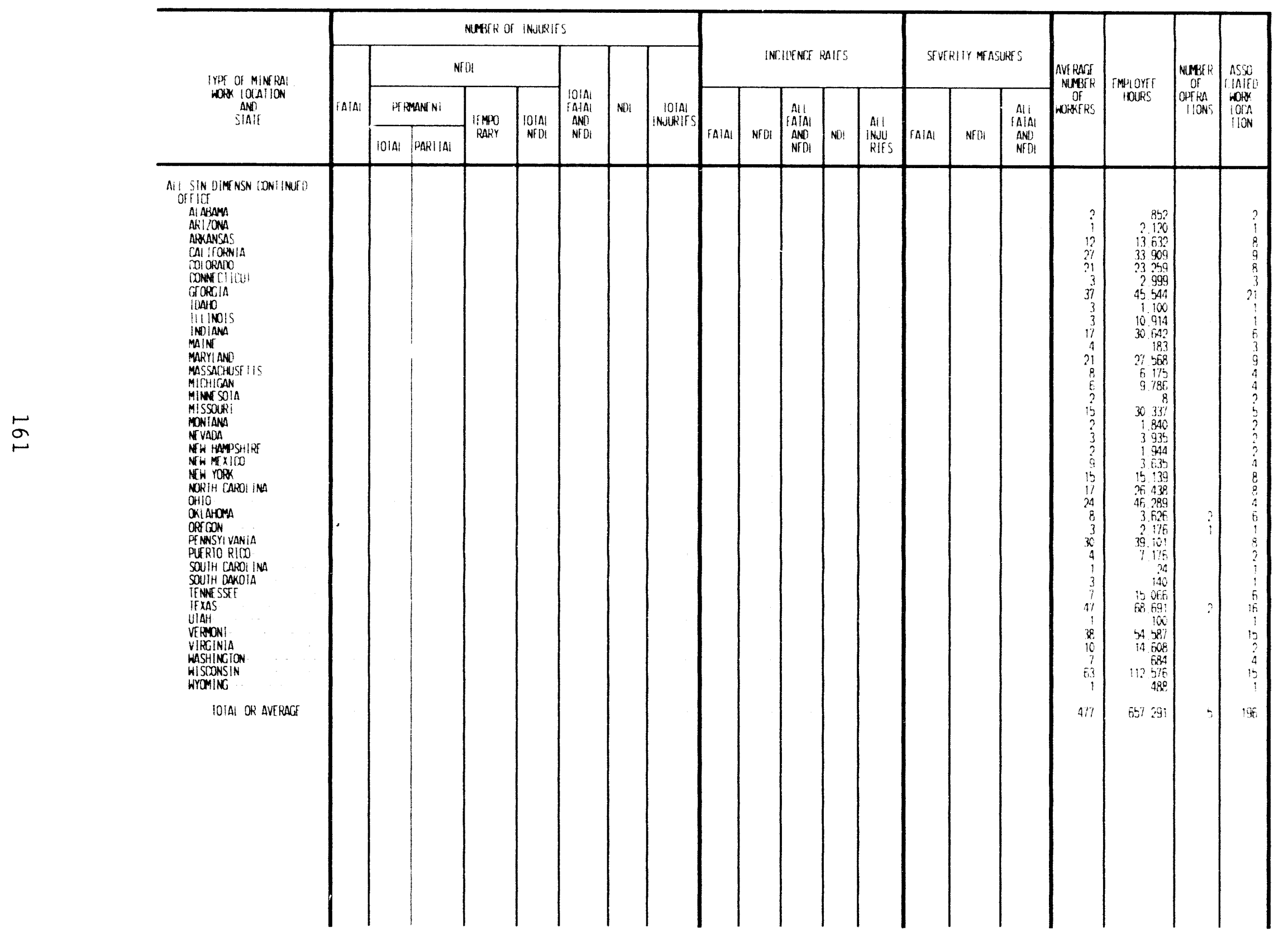




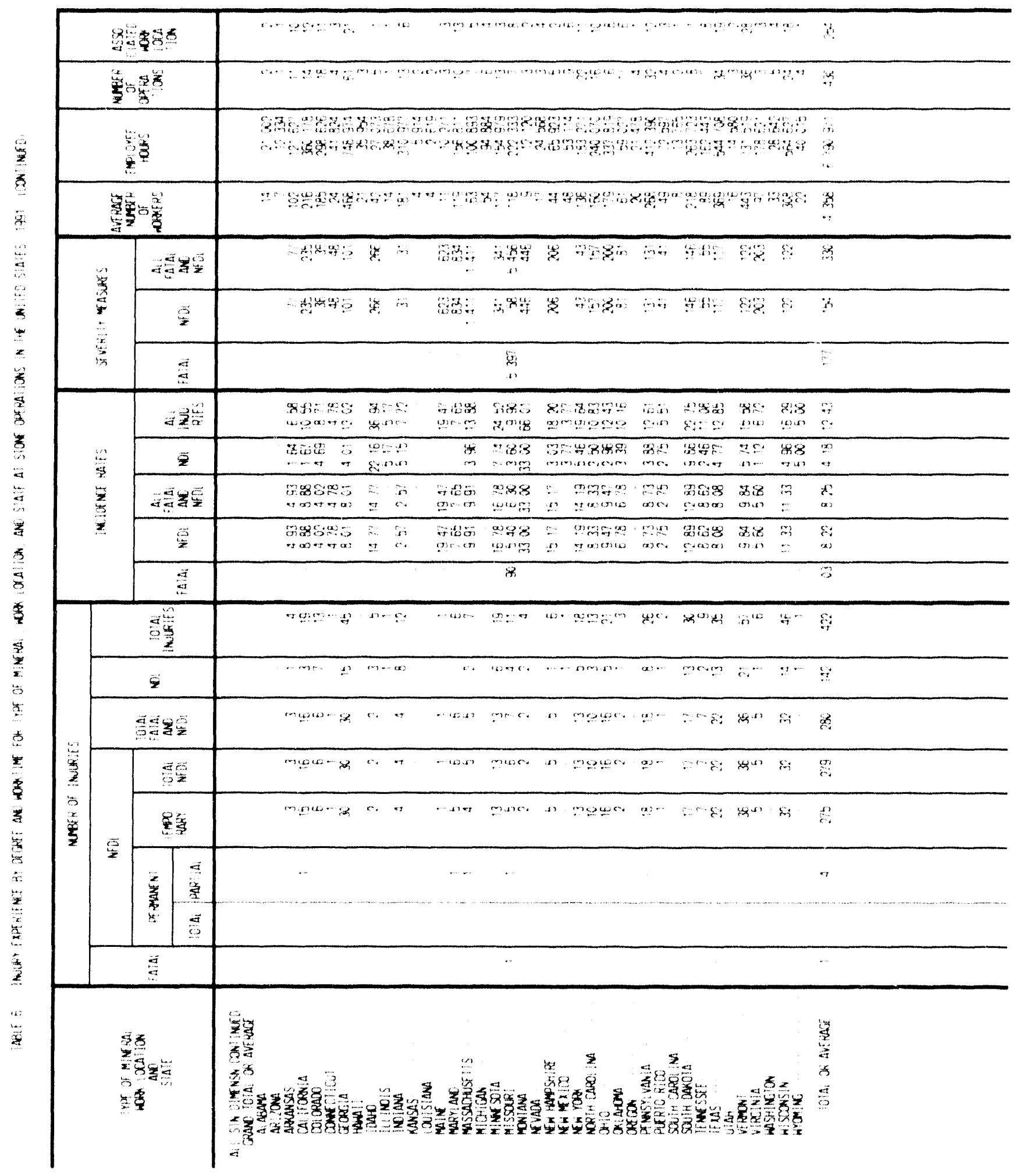



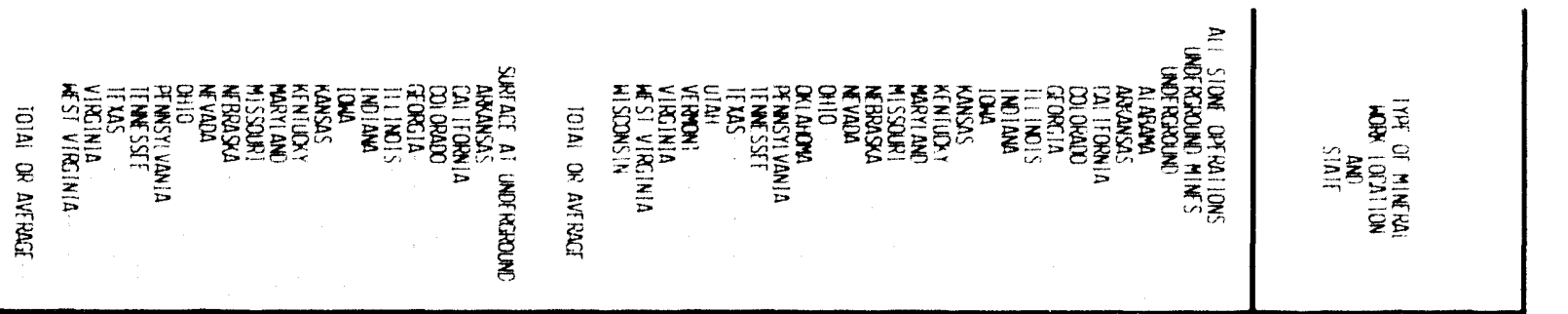

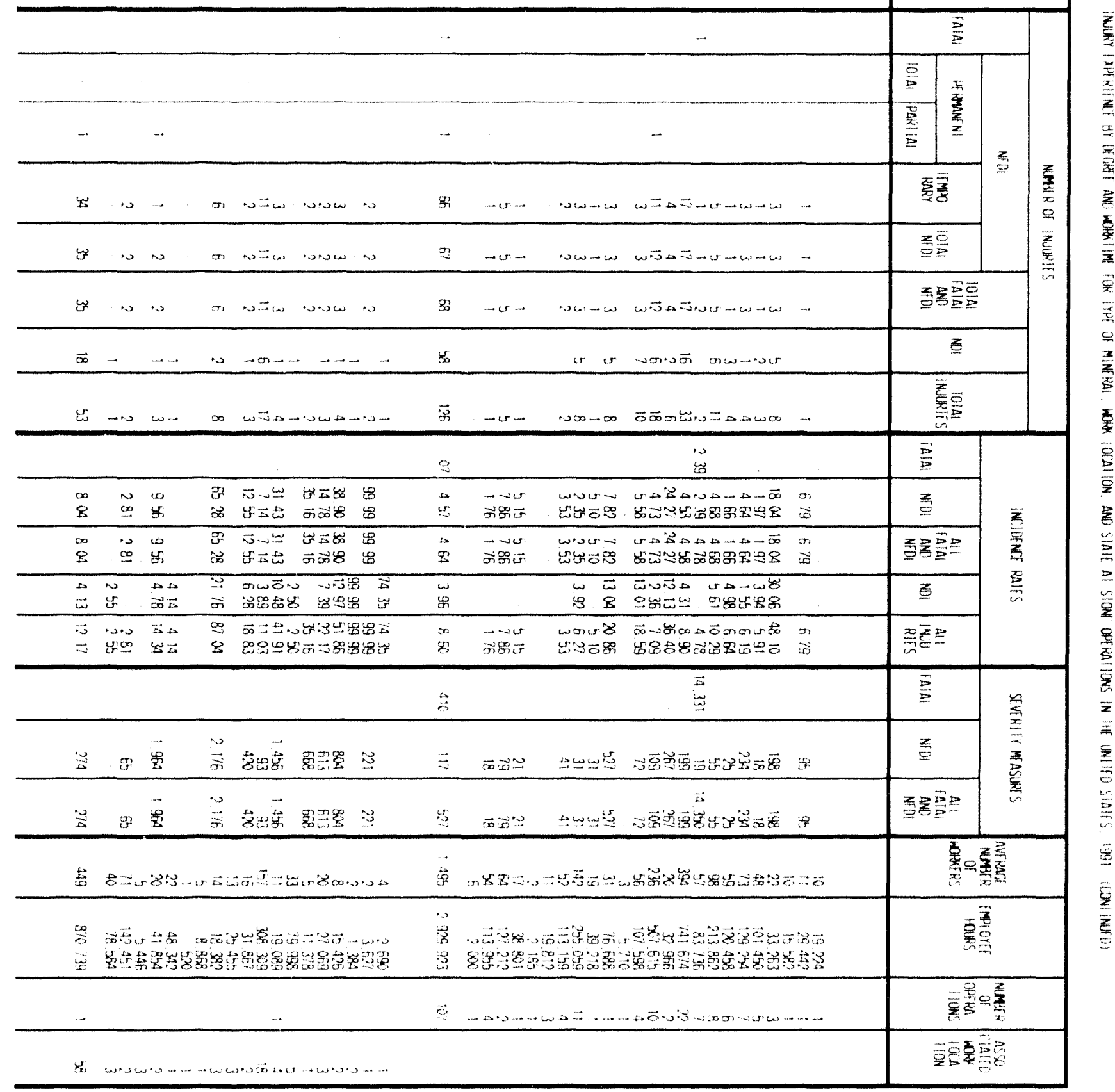




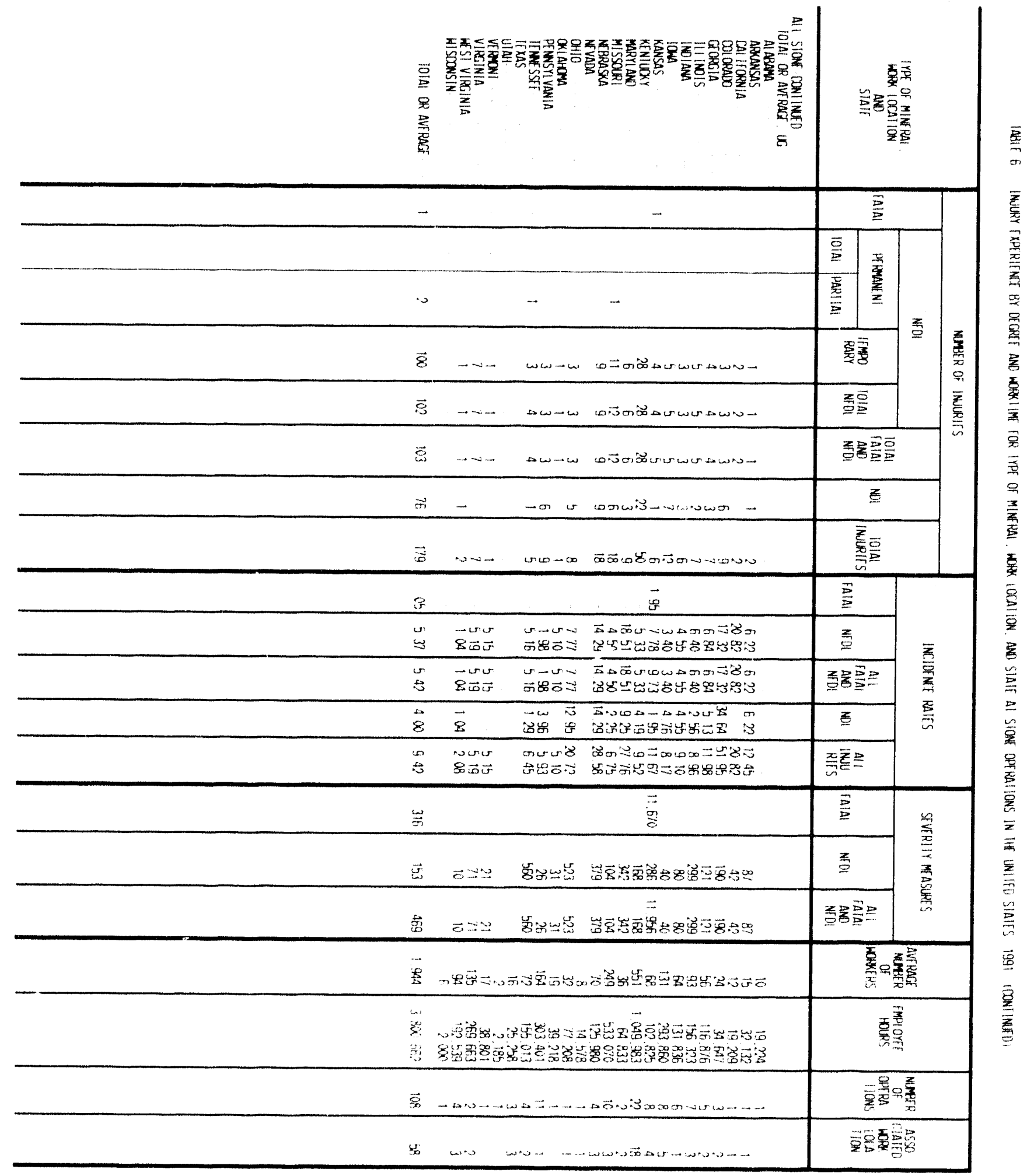




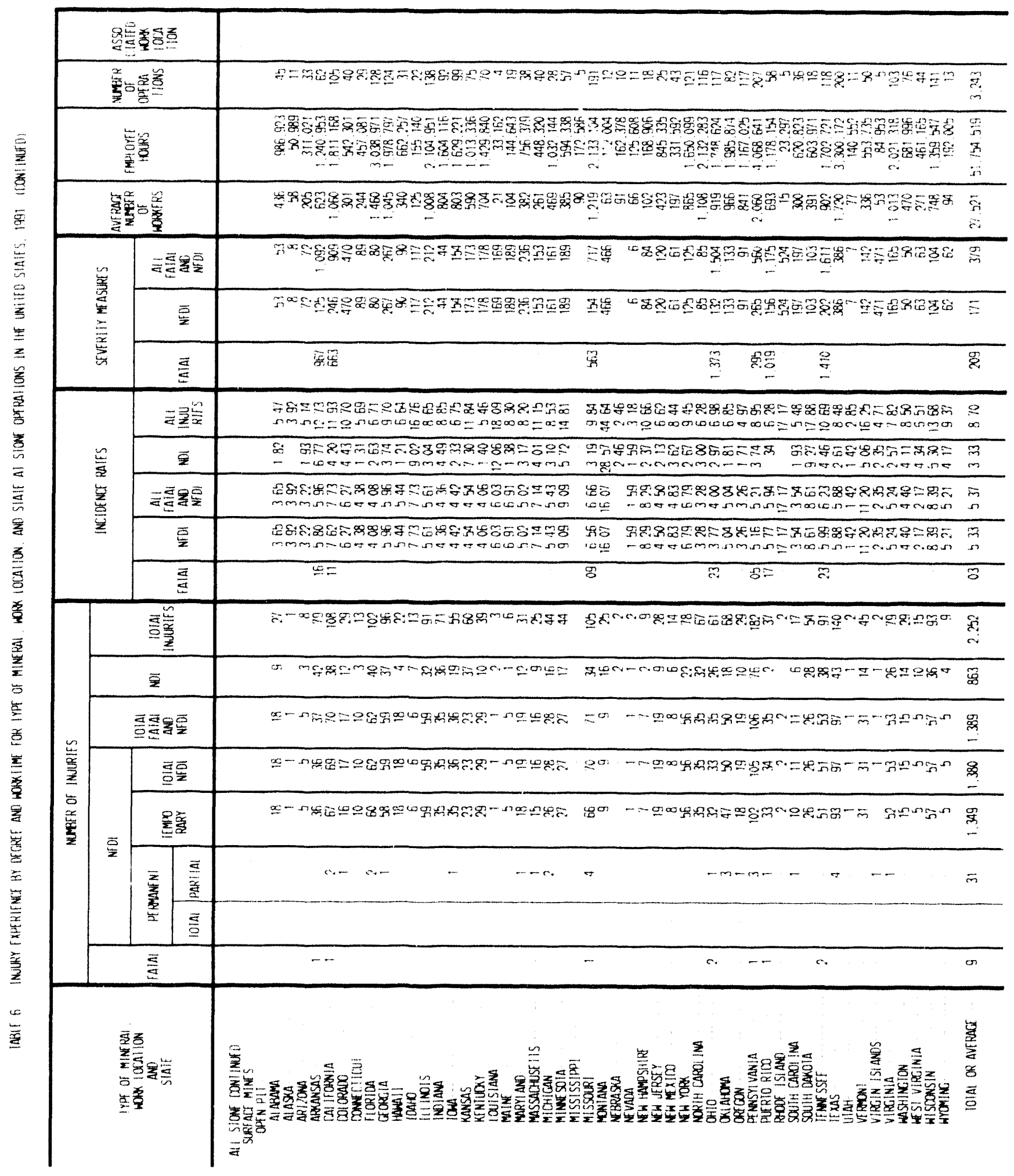




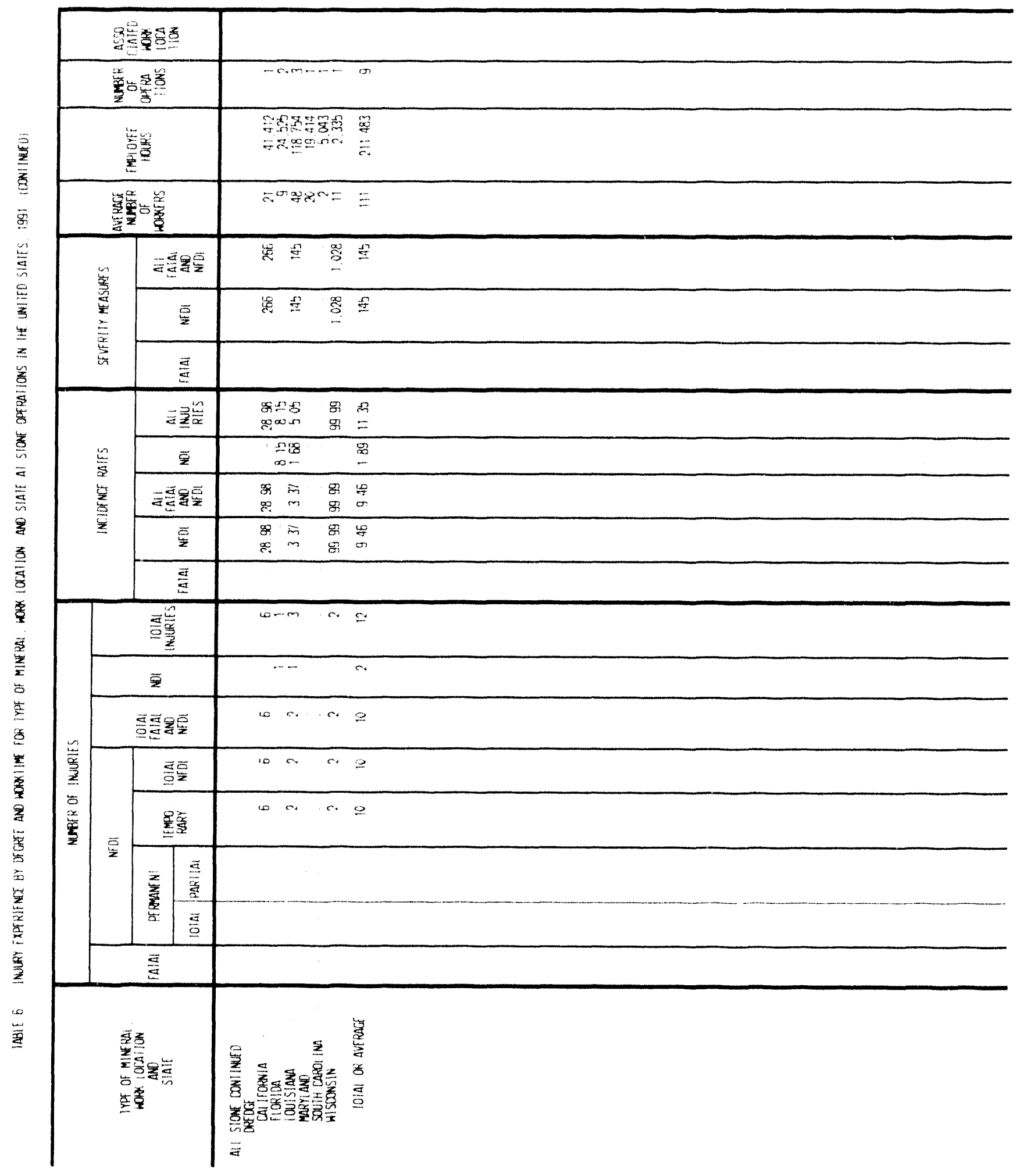




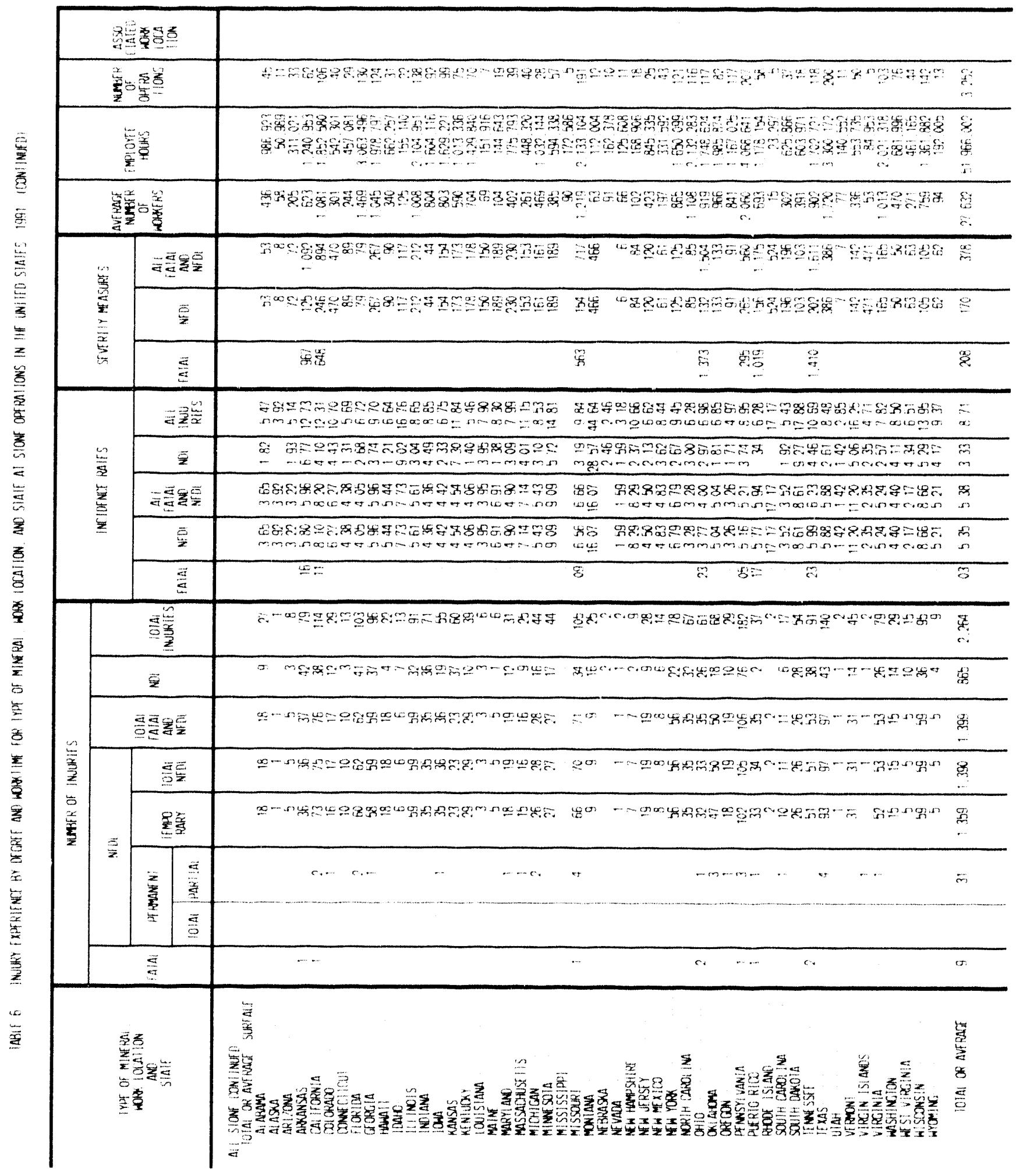




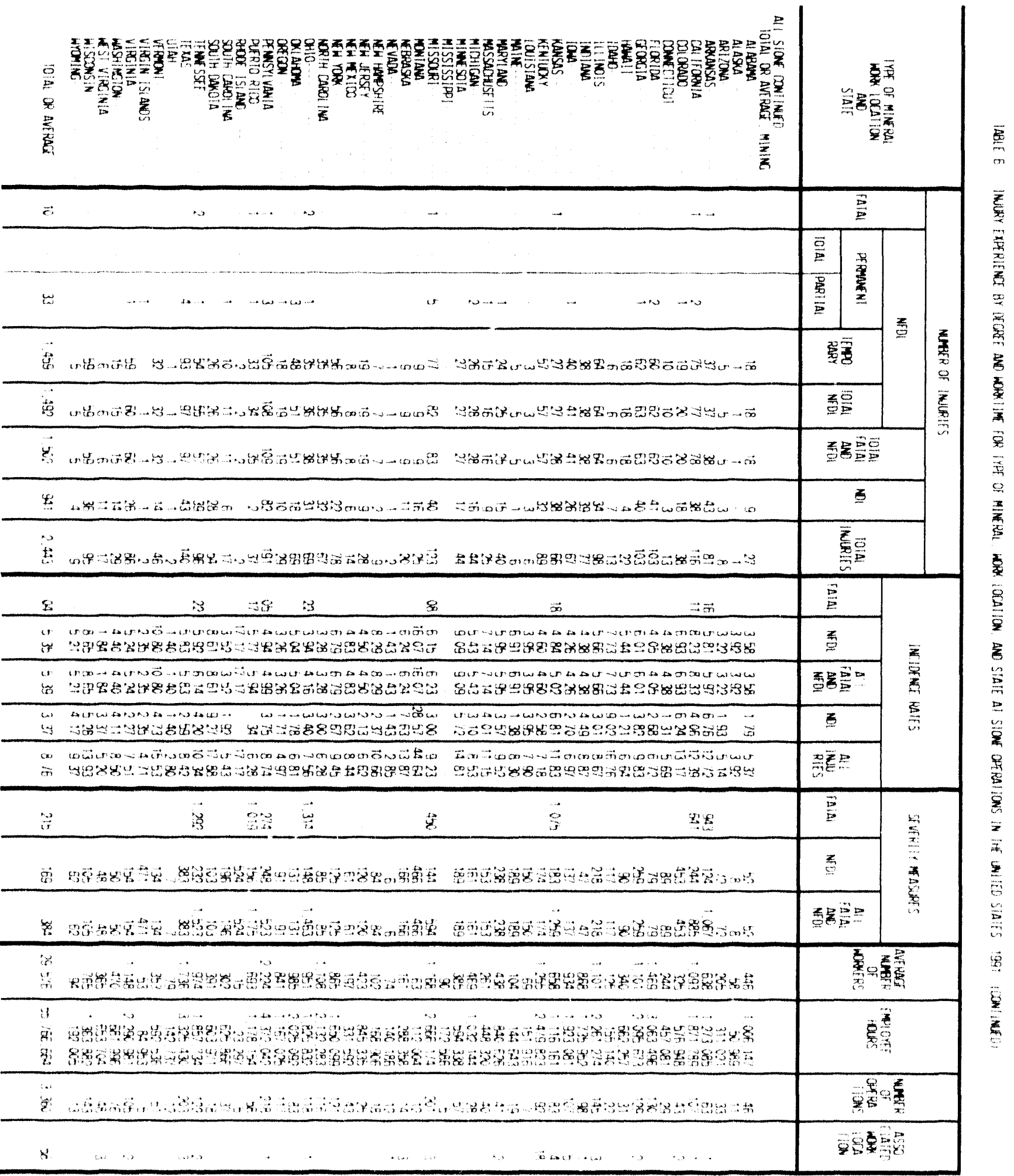




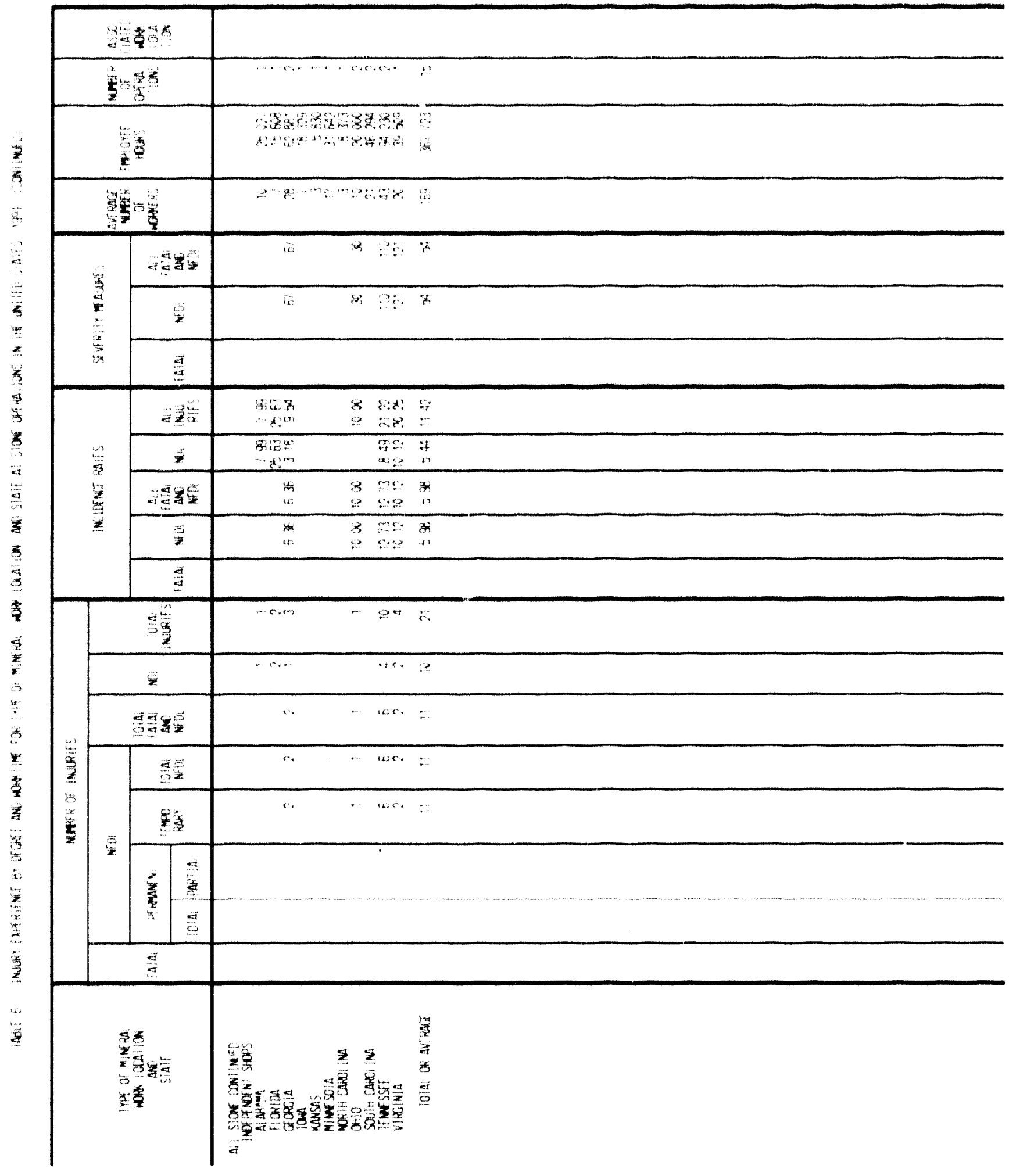




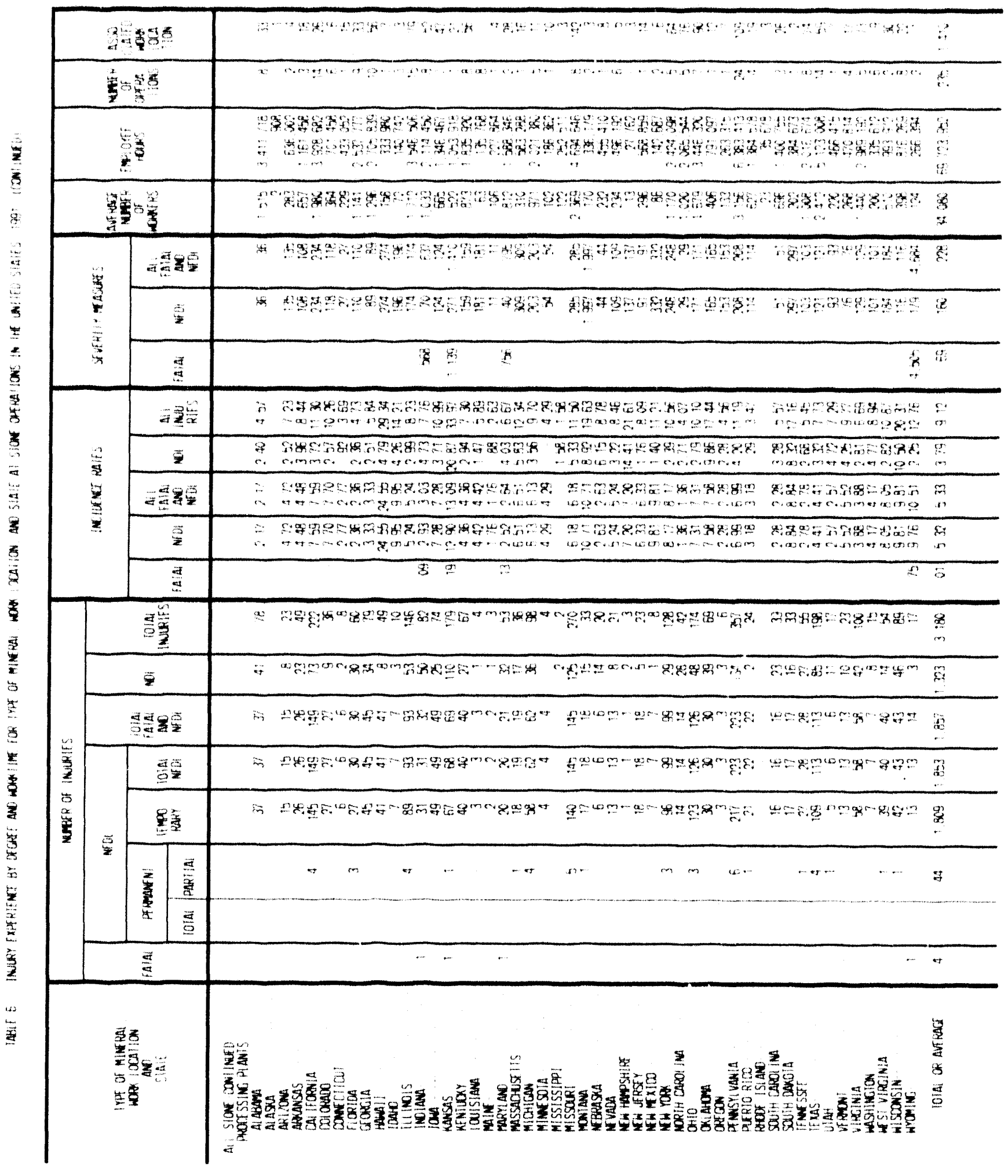




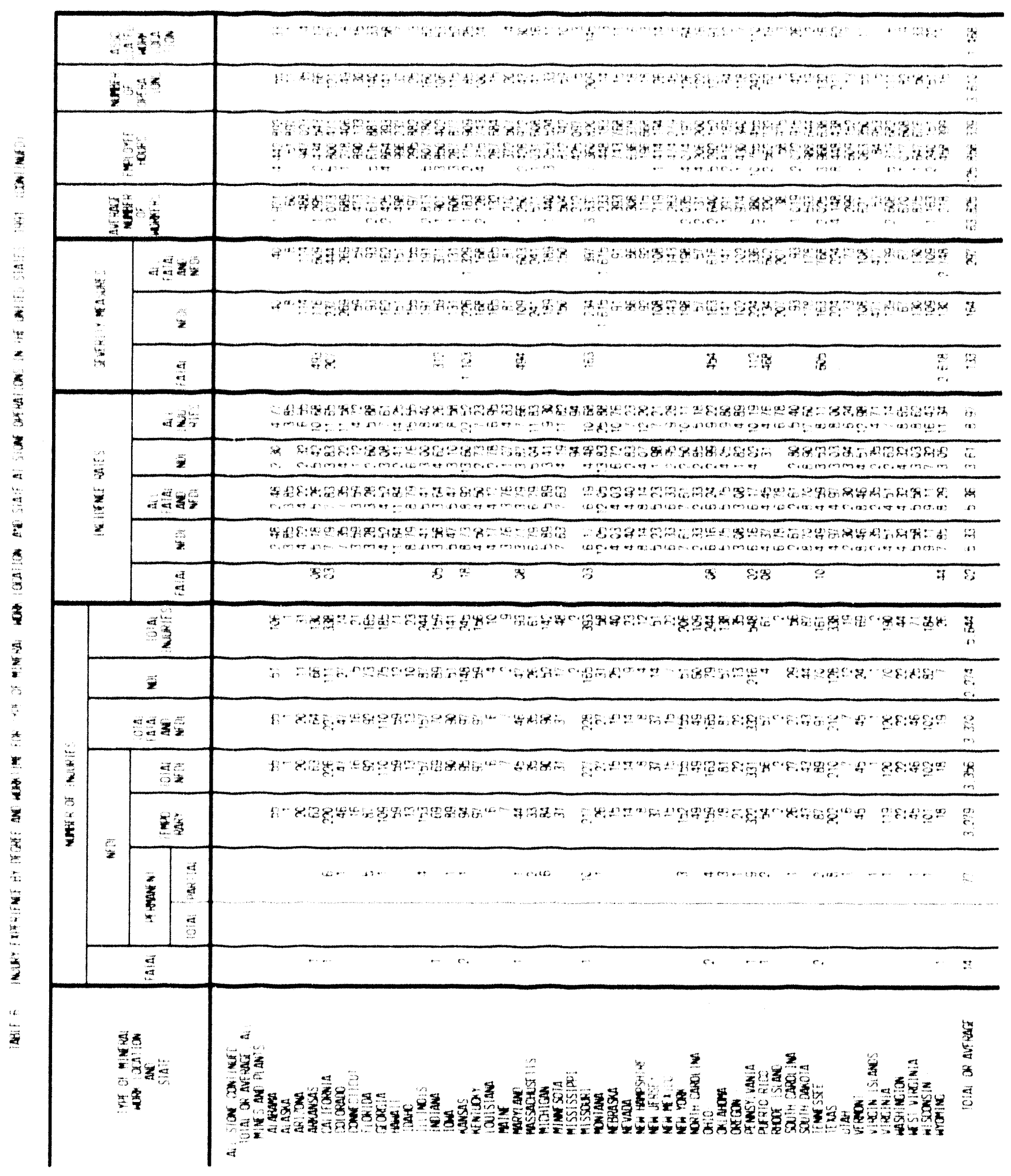




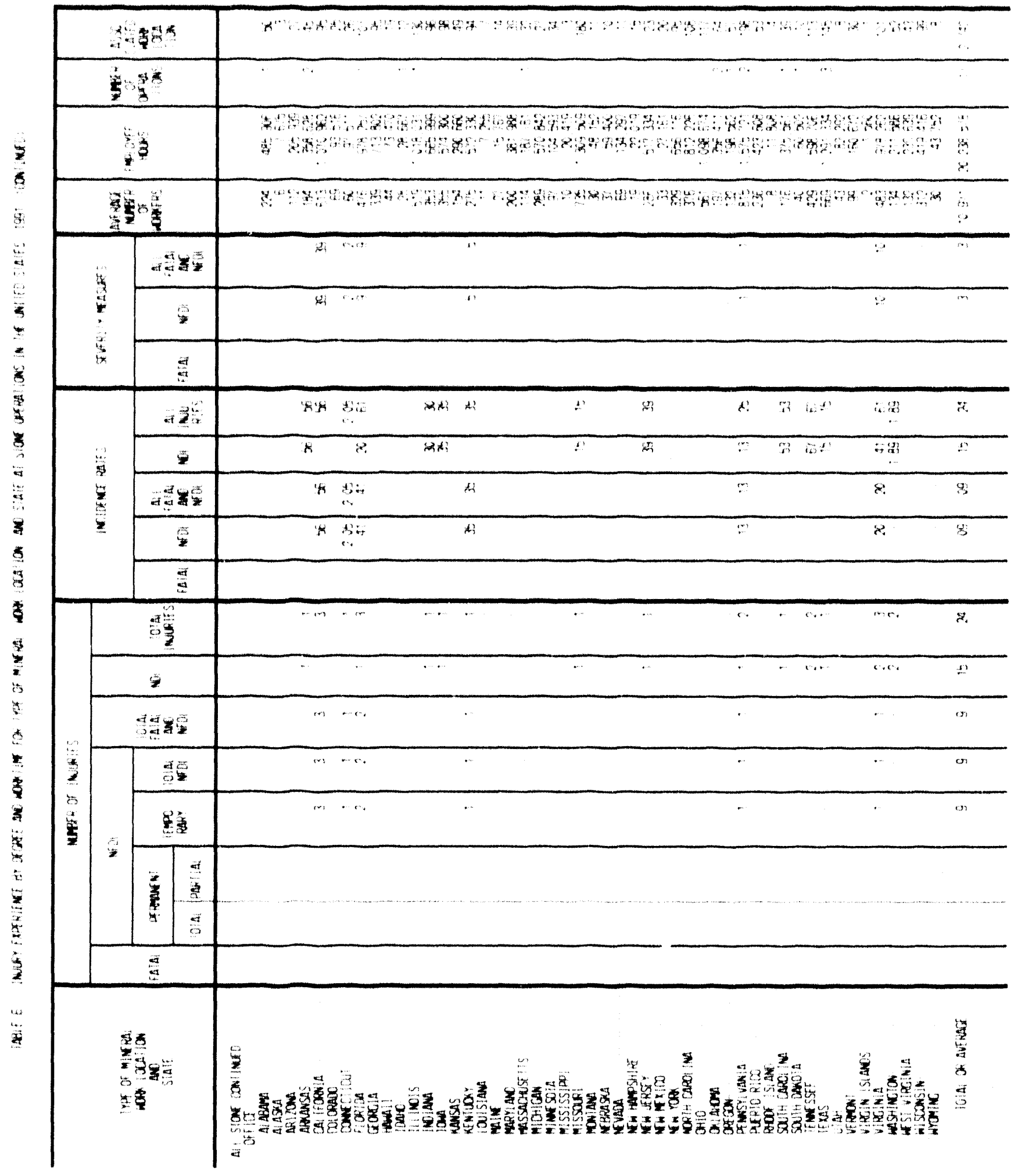




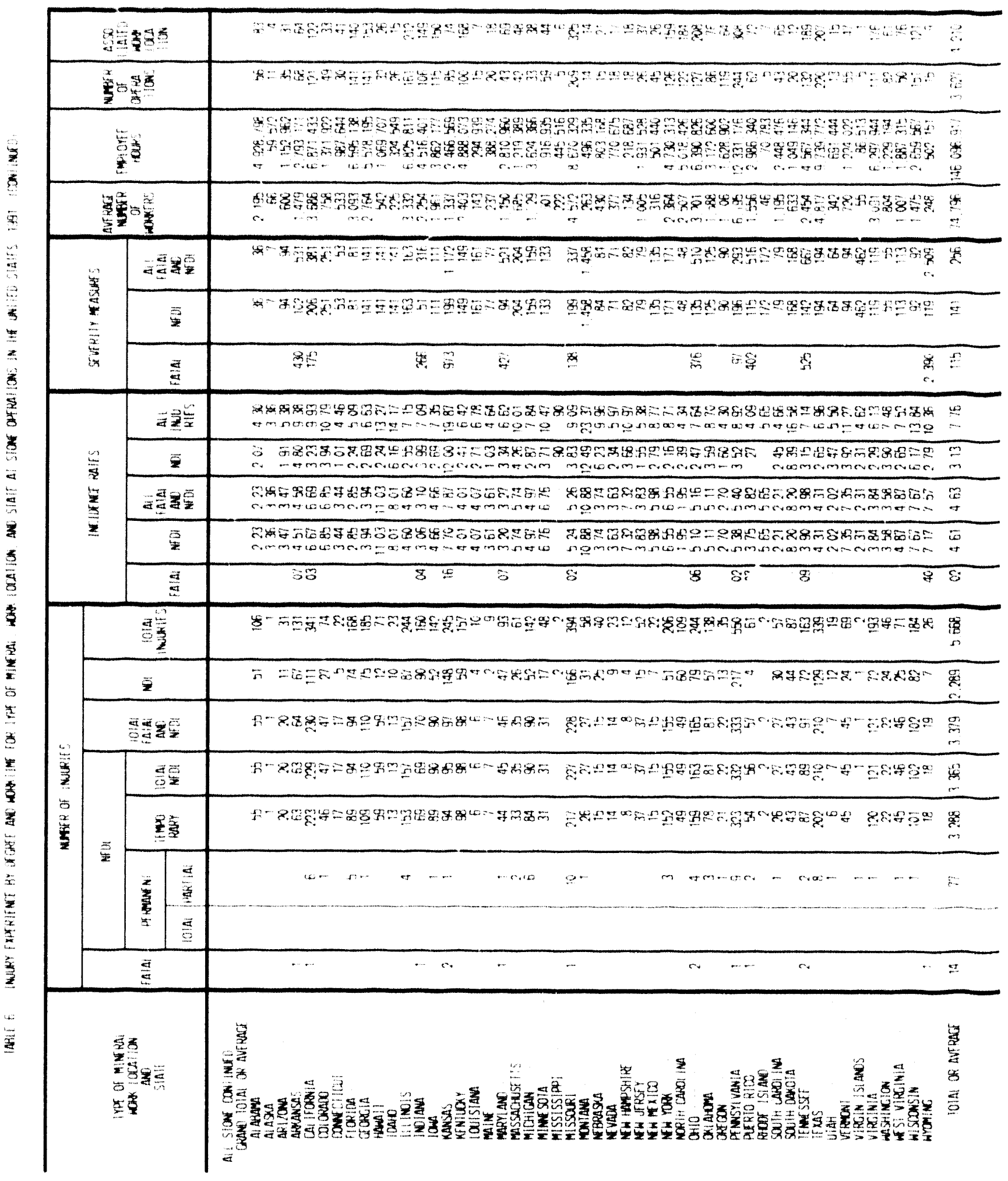




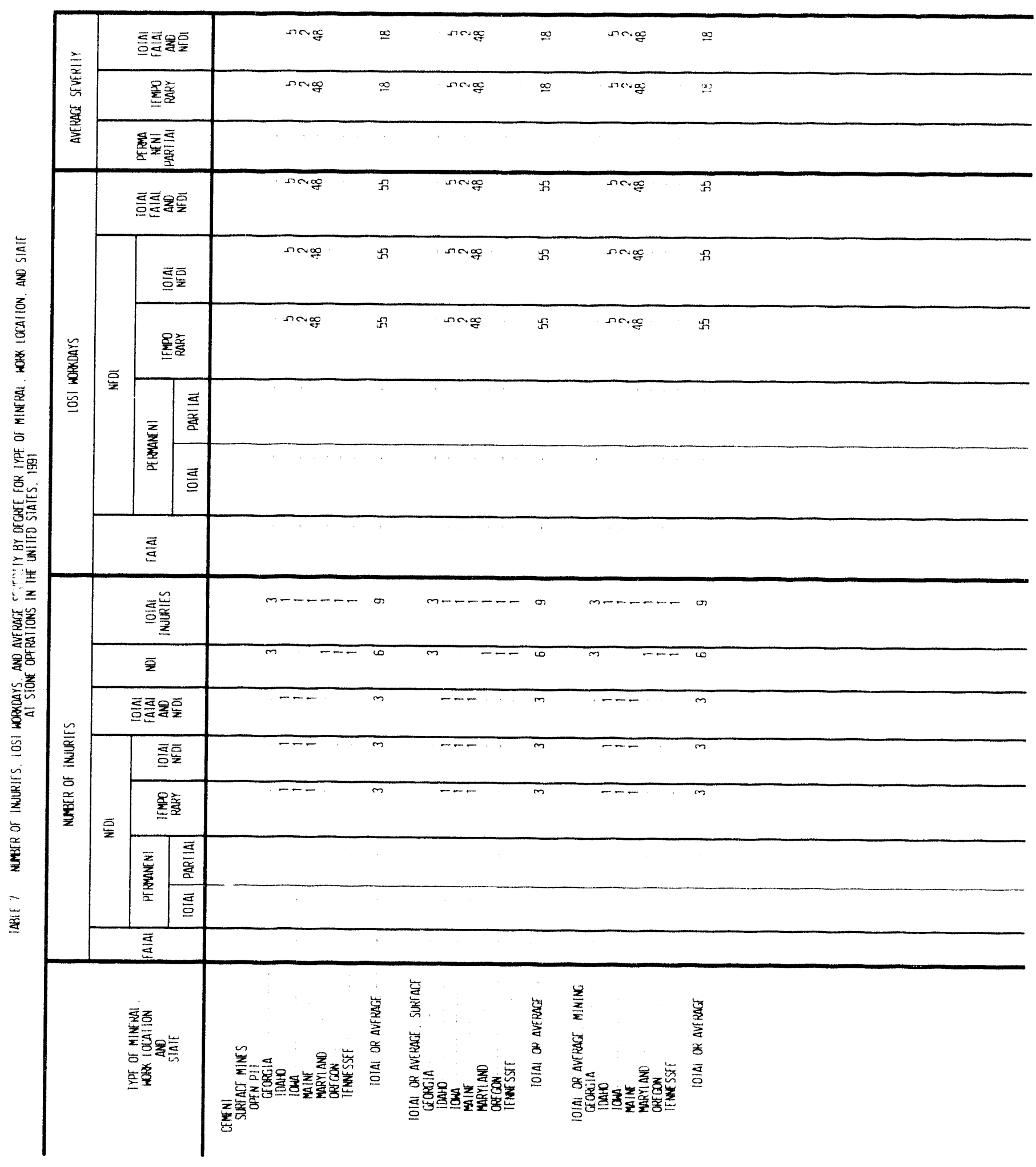




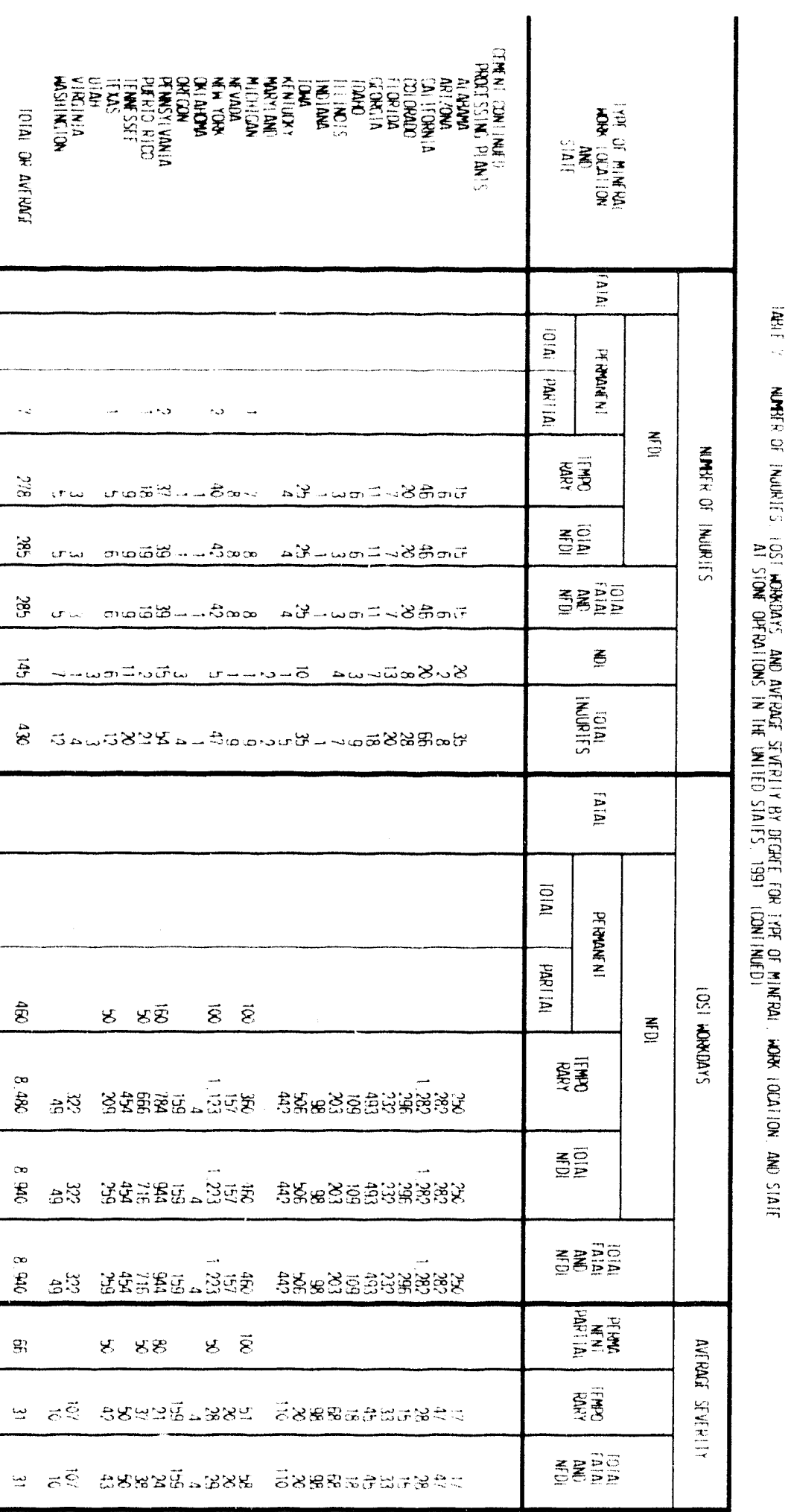




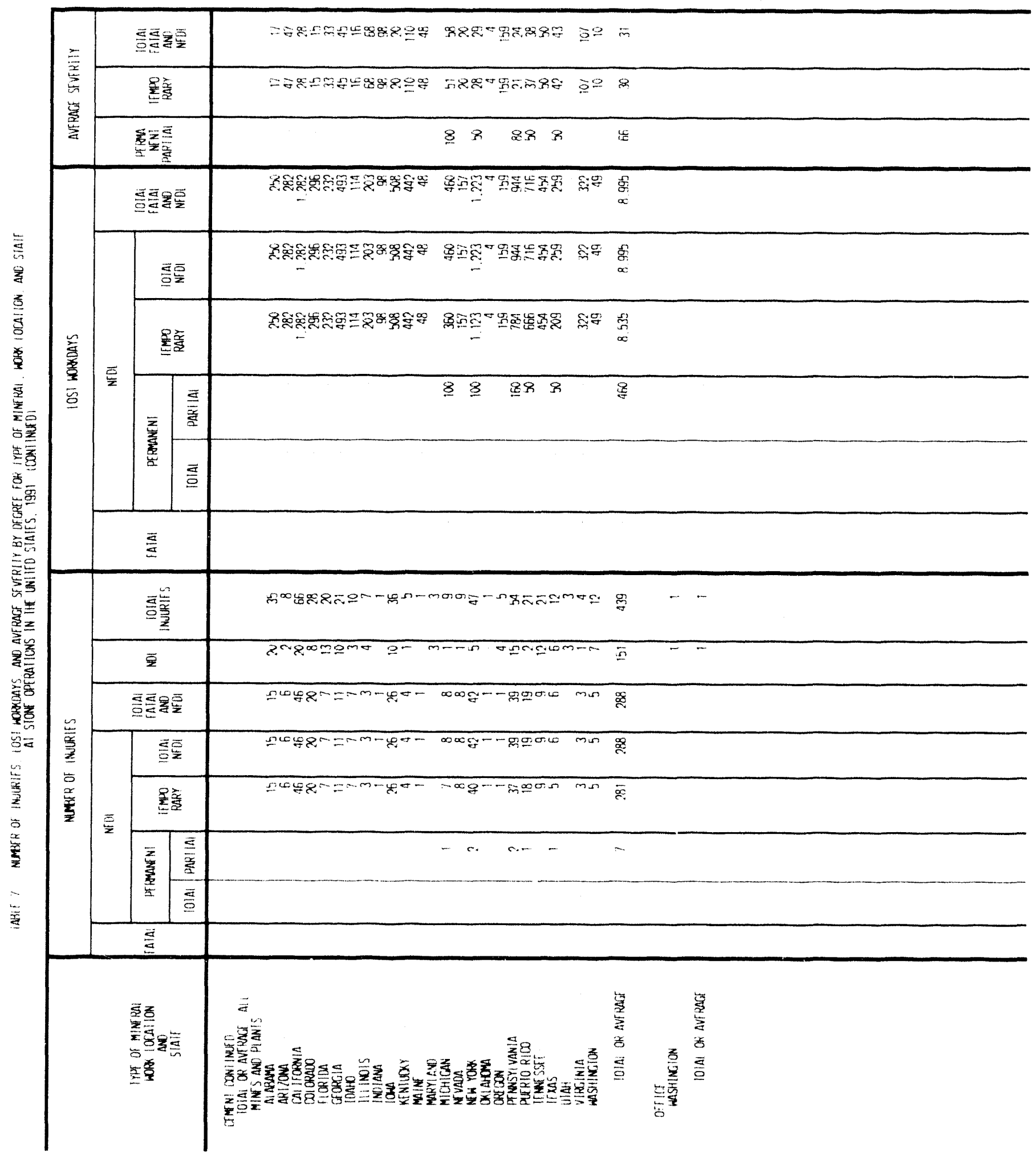




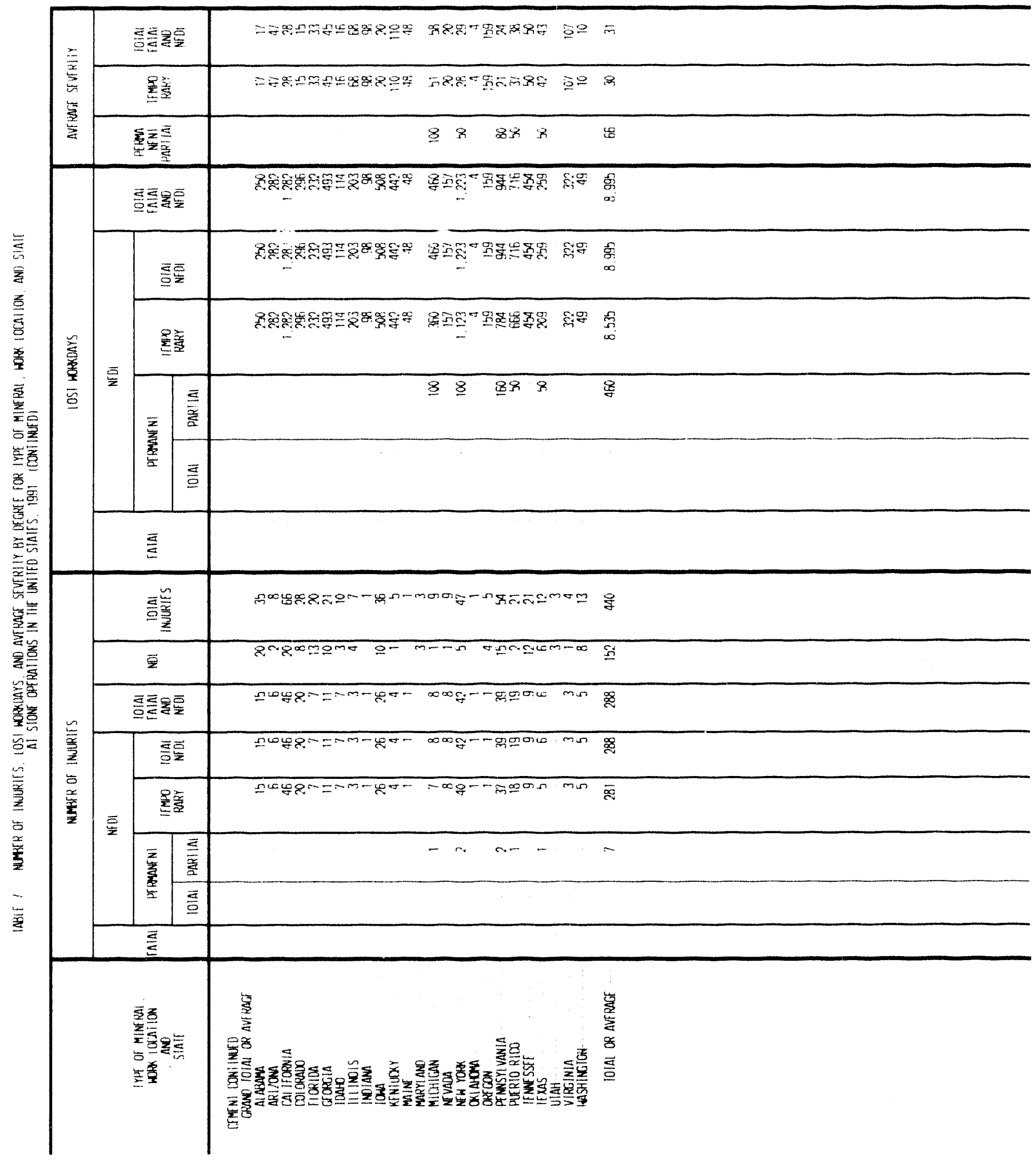




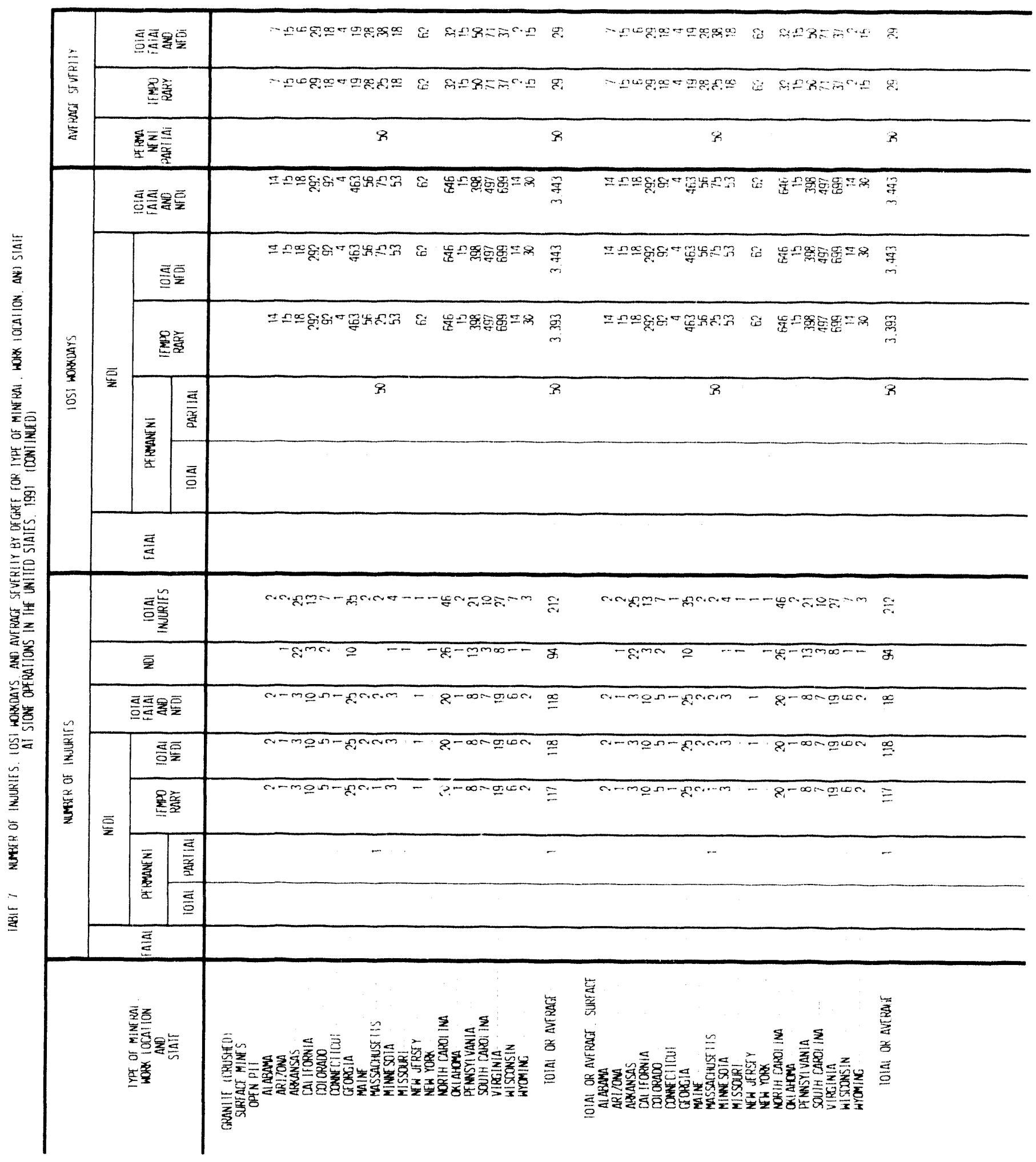



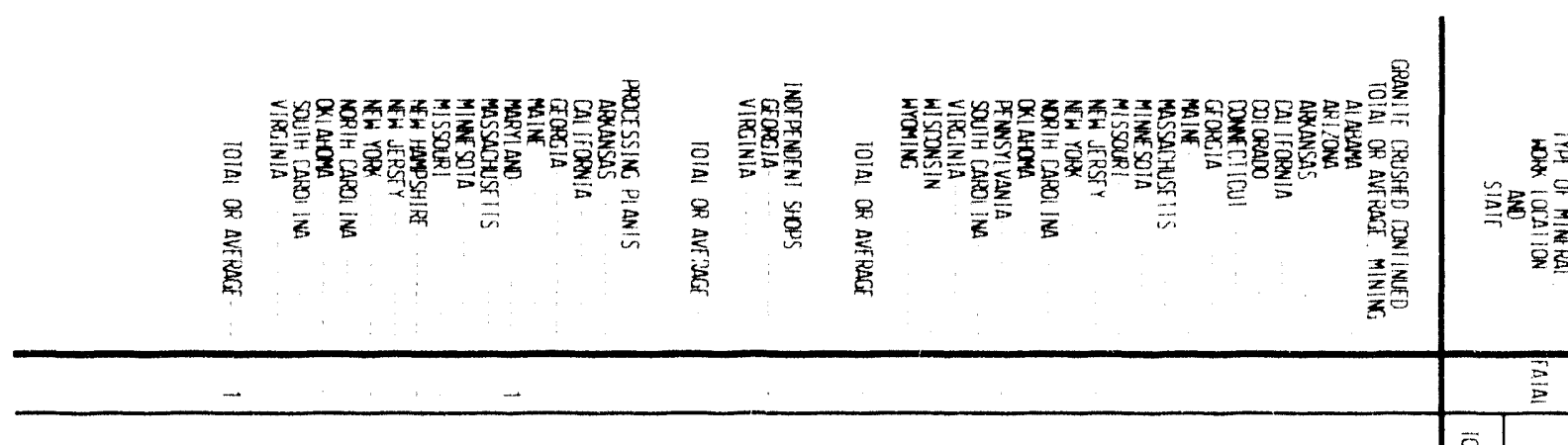

(20)

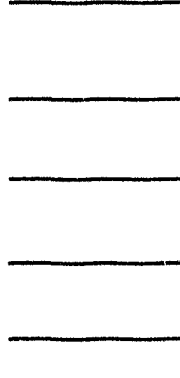

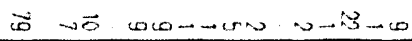

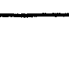

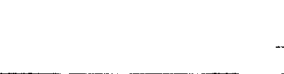

$-\quad \mid$

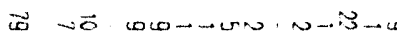

$\rightarrow+3$

झ

$\triangle$ WN

$\overrightarrow{\vec{\infty}}$ Nenta $\overrightarrow{0} \sim \infty$

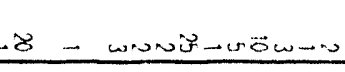

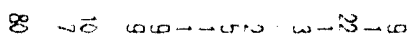

$D . . \infty$

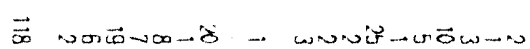

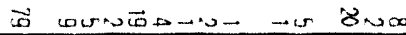

$\mathscr{A}+\ldots$ ond

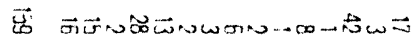

$\sim \infty \omega$

$\vdots$

w

$\stackrel{n}{8}$

8

¿:

s.

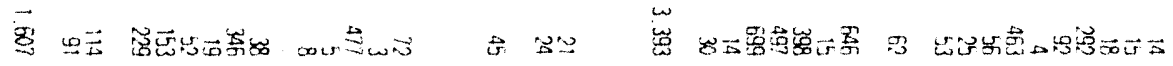

露要

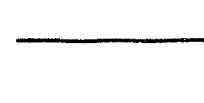

$\overrightarrow{8}$

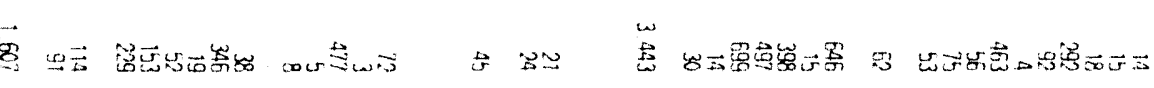

象

종흠

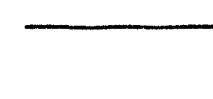

(2)

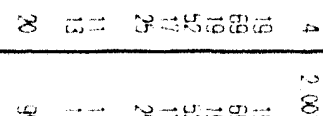

(4) 世

$\because \overrightarrow{\nabla \vec{c}}$

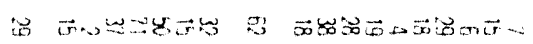

y $\quad y$

즐호흐를

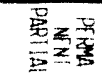

翼变

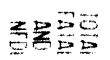




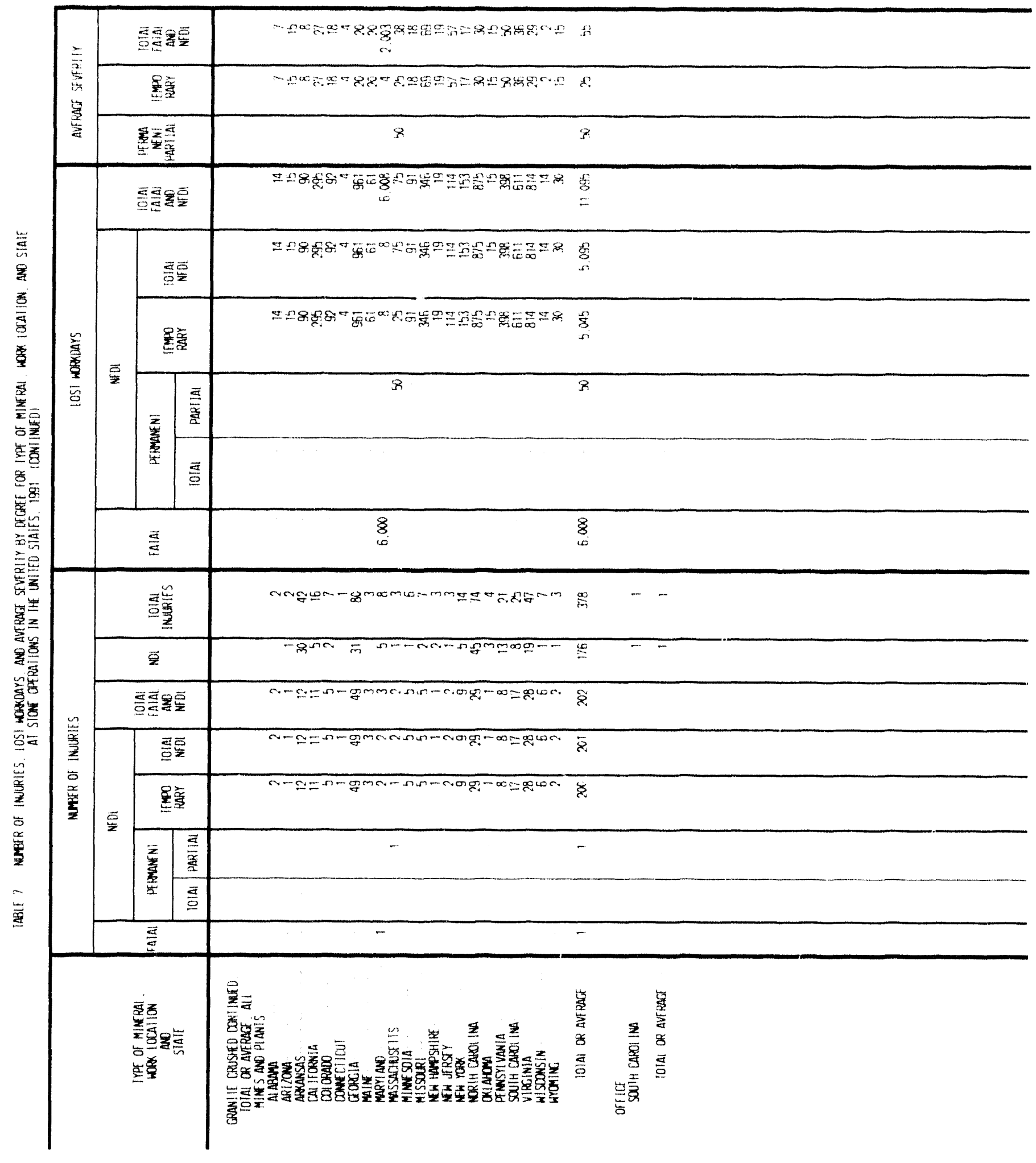




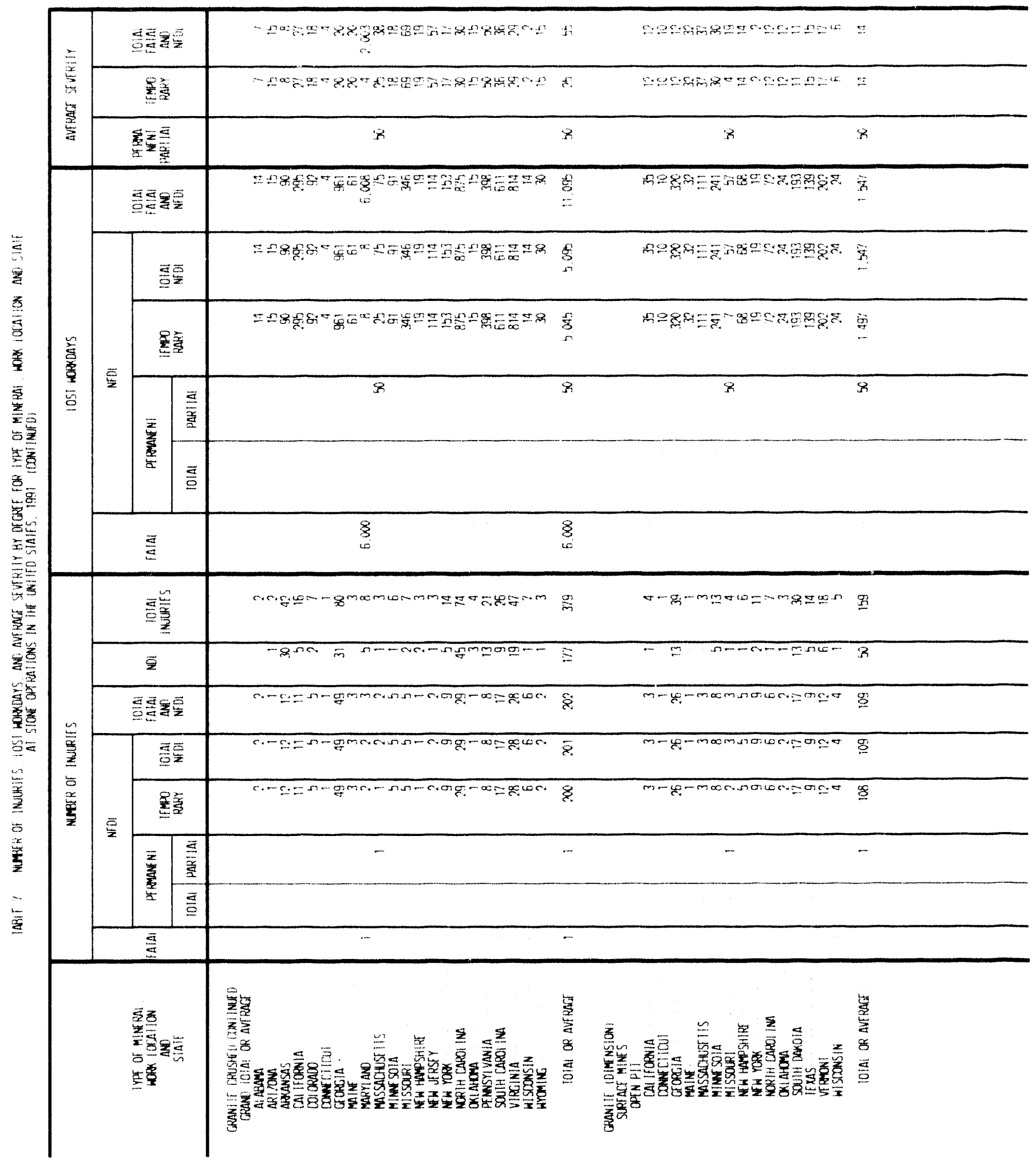



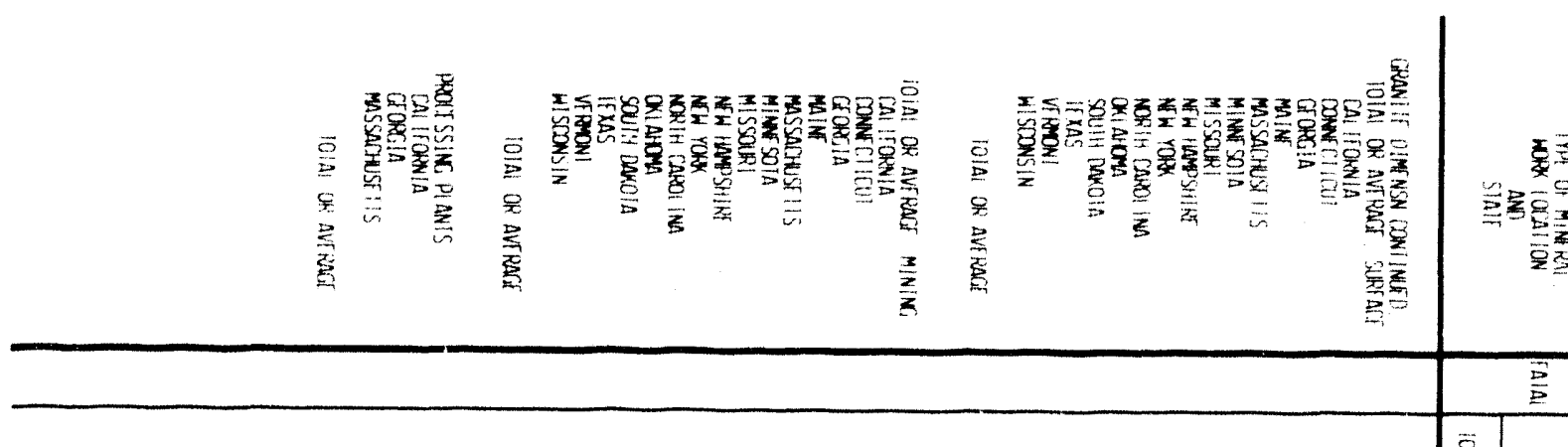

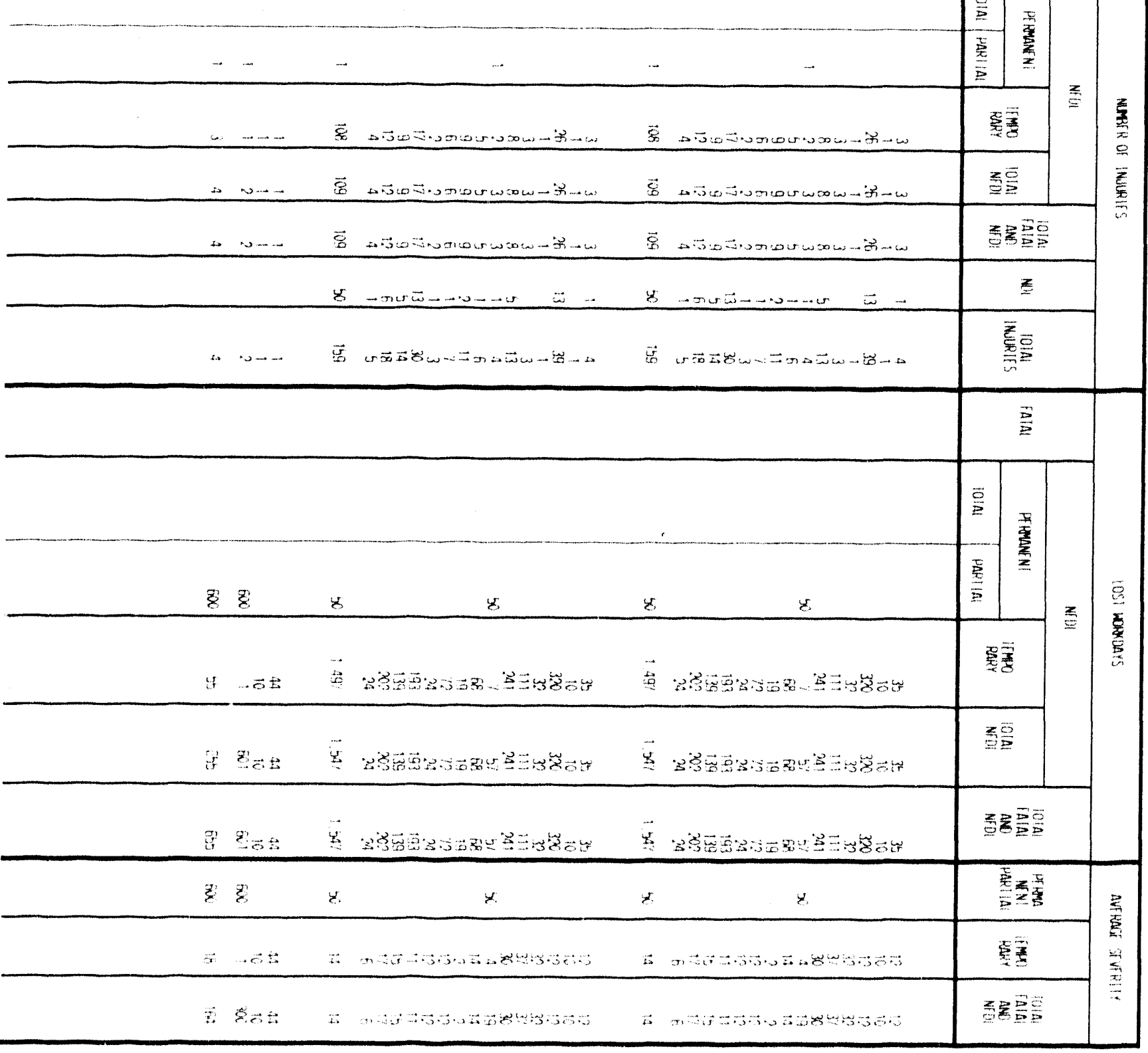




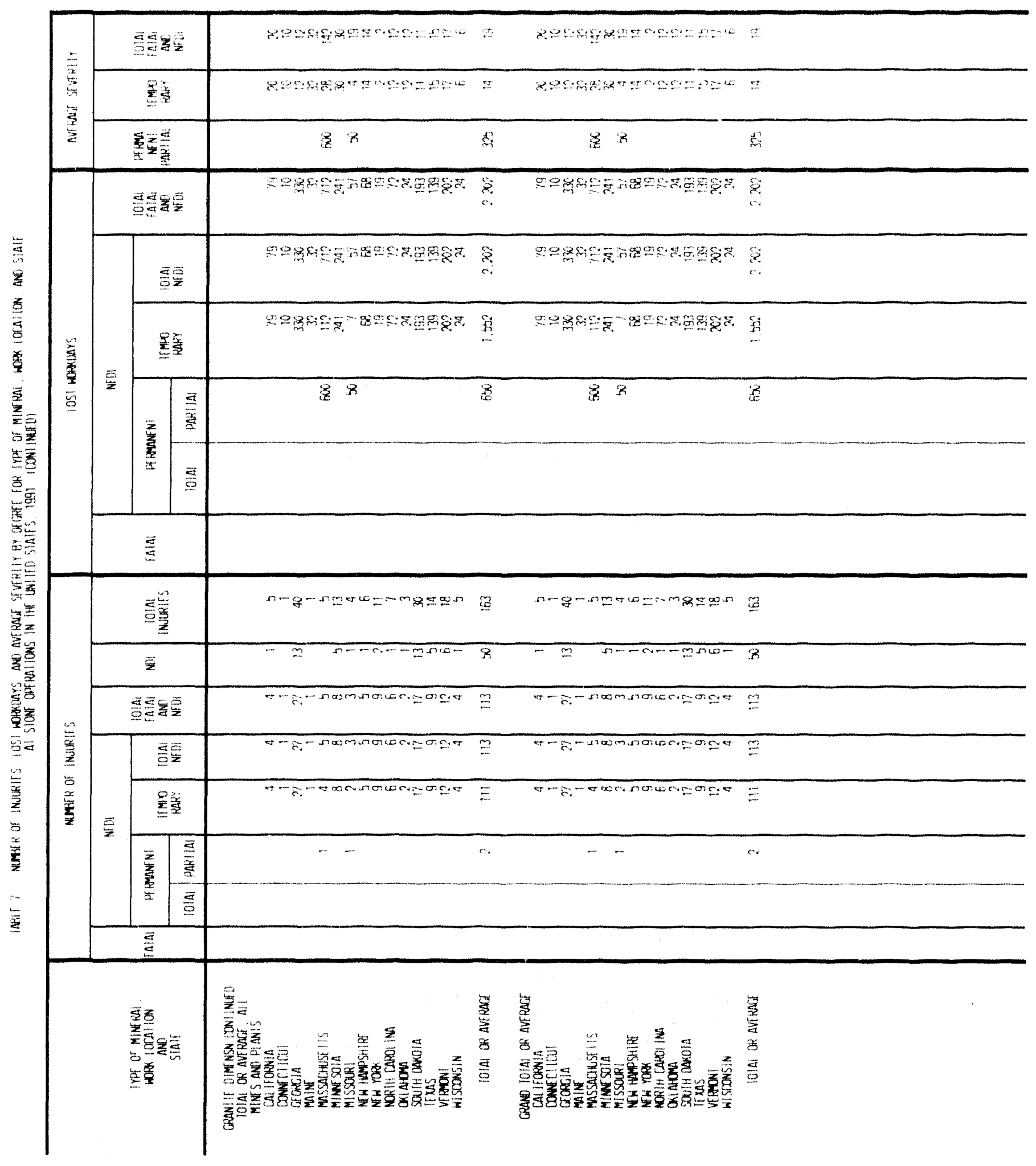




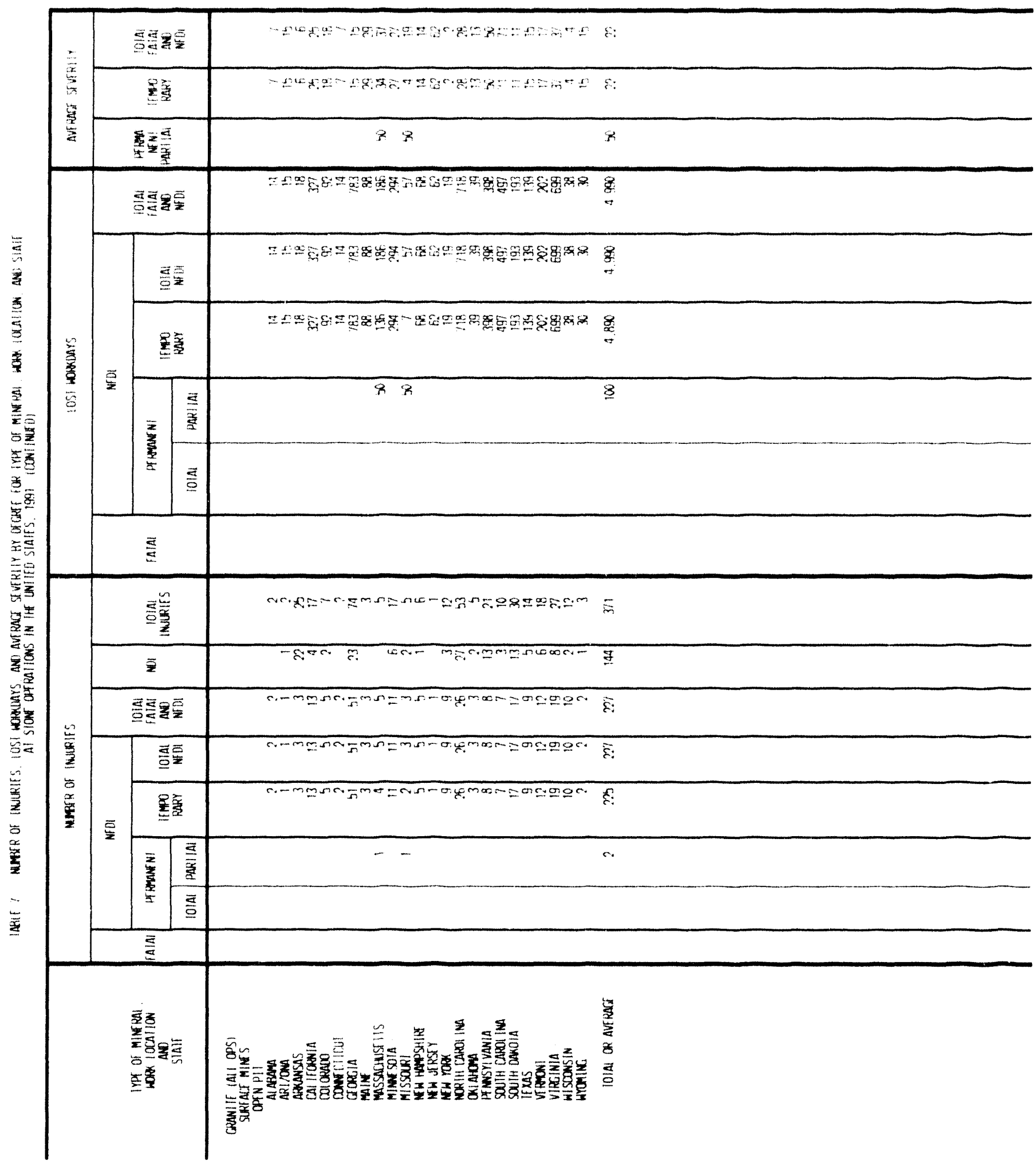




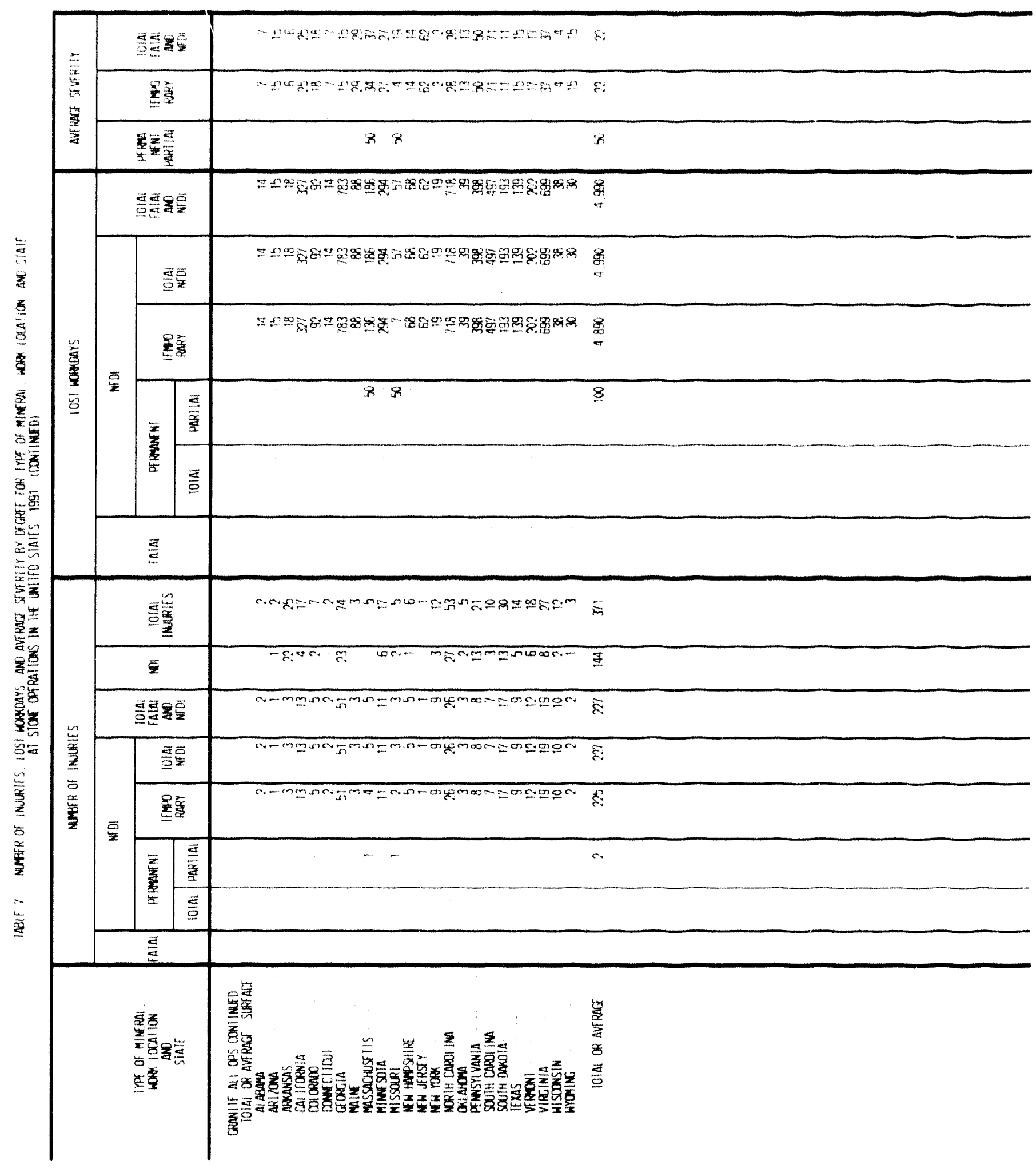




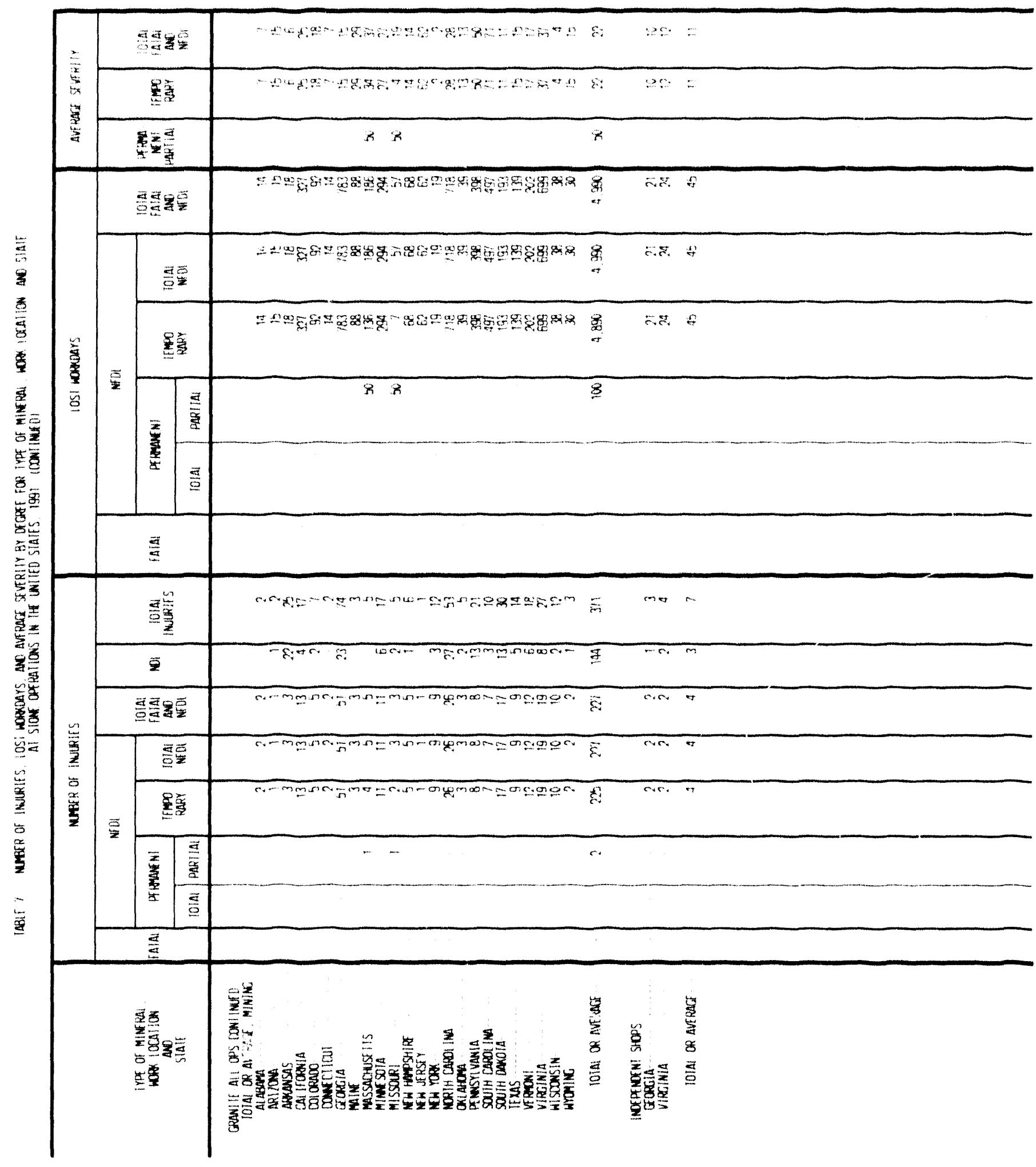




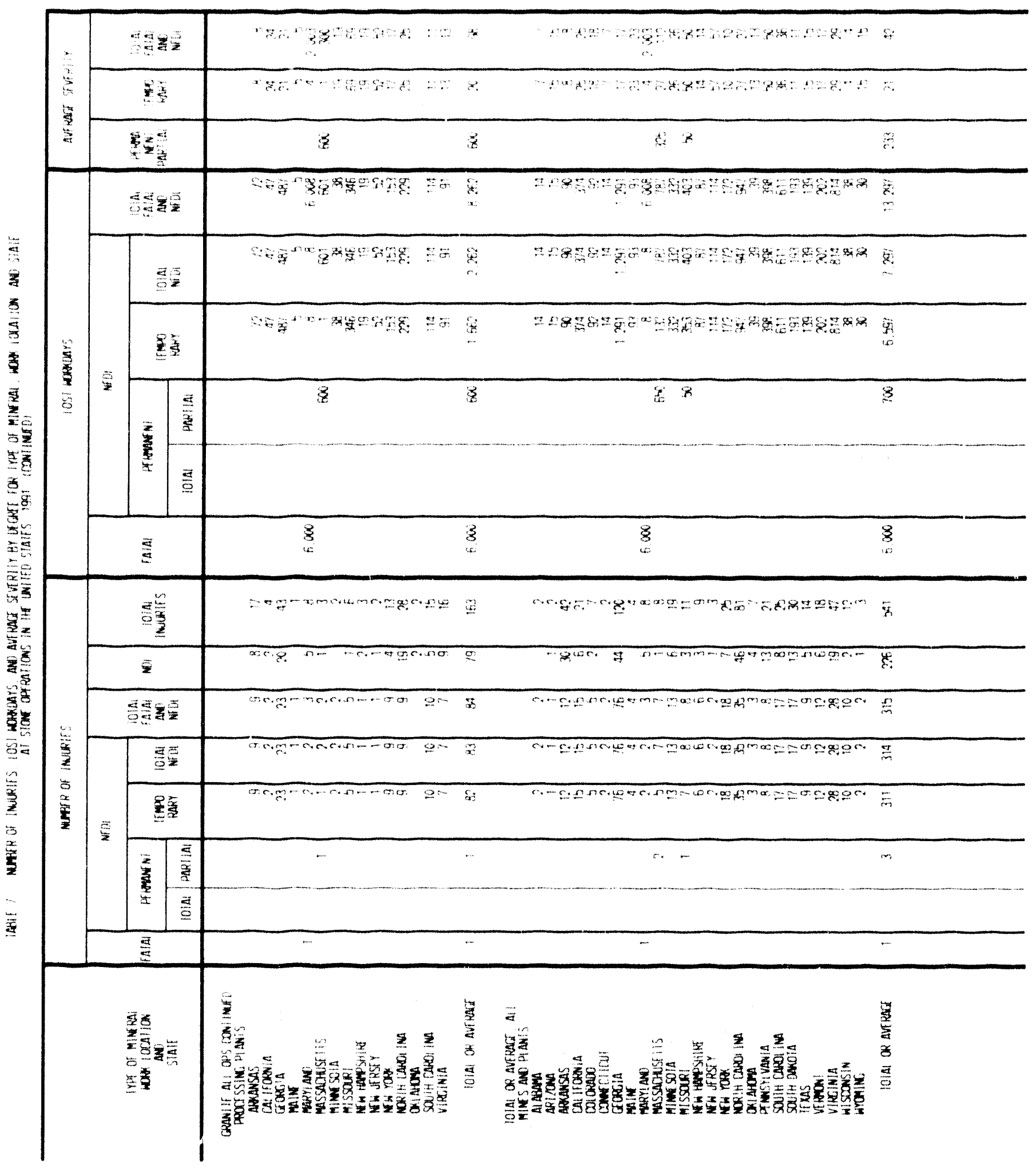




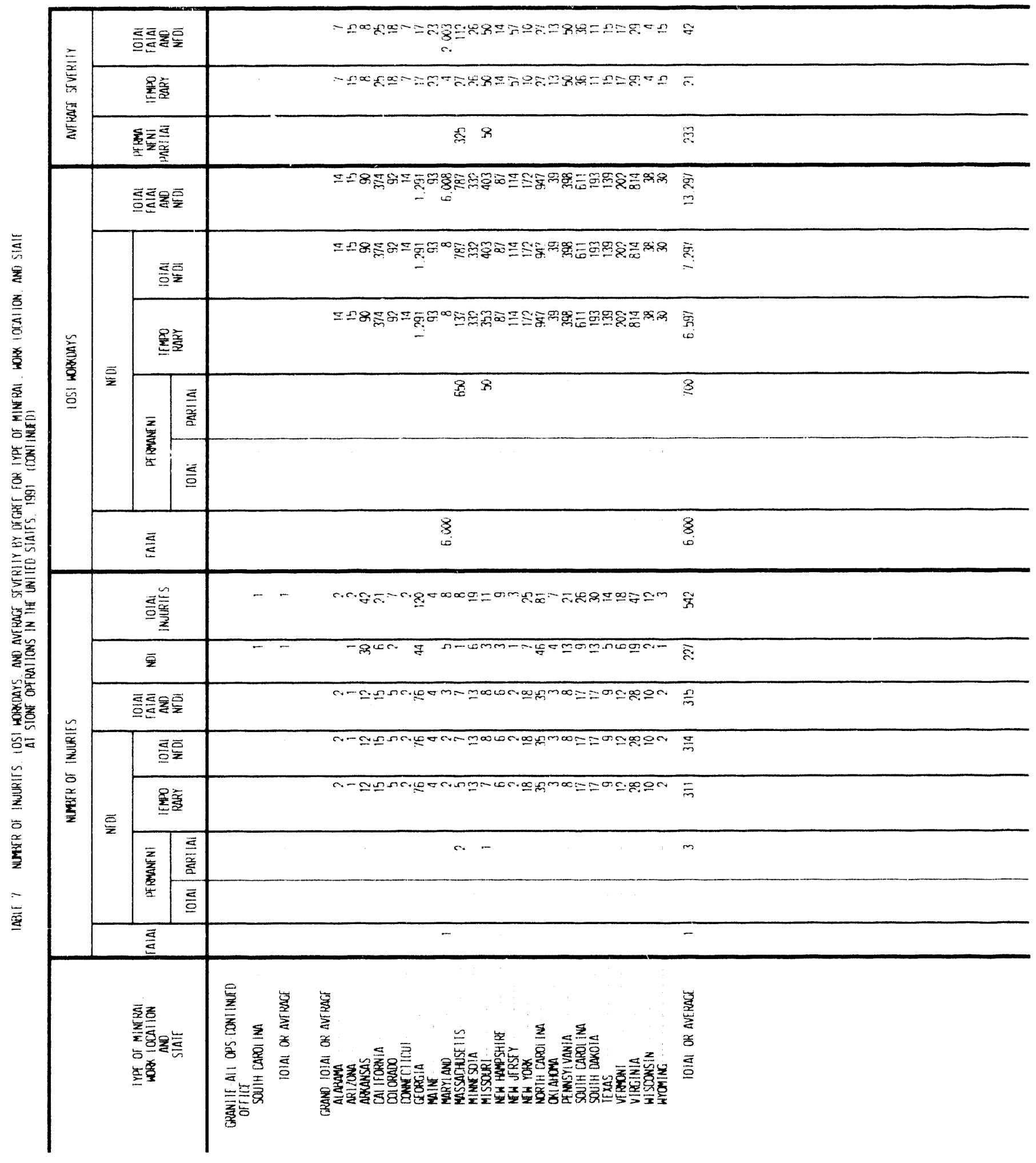




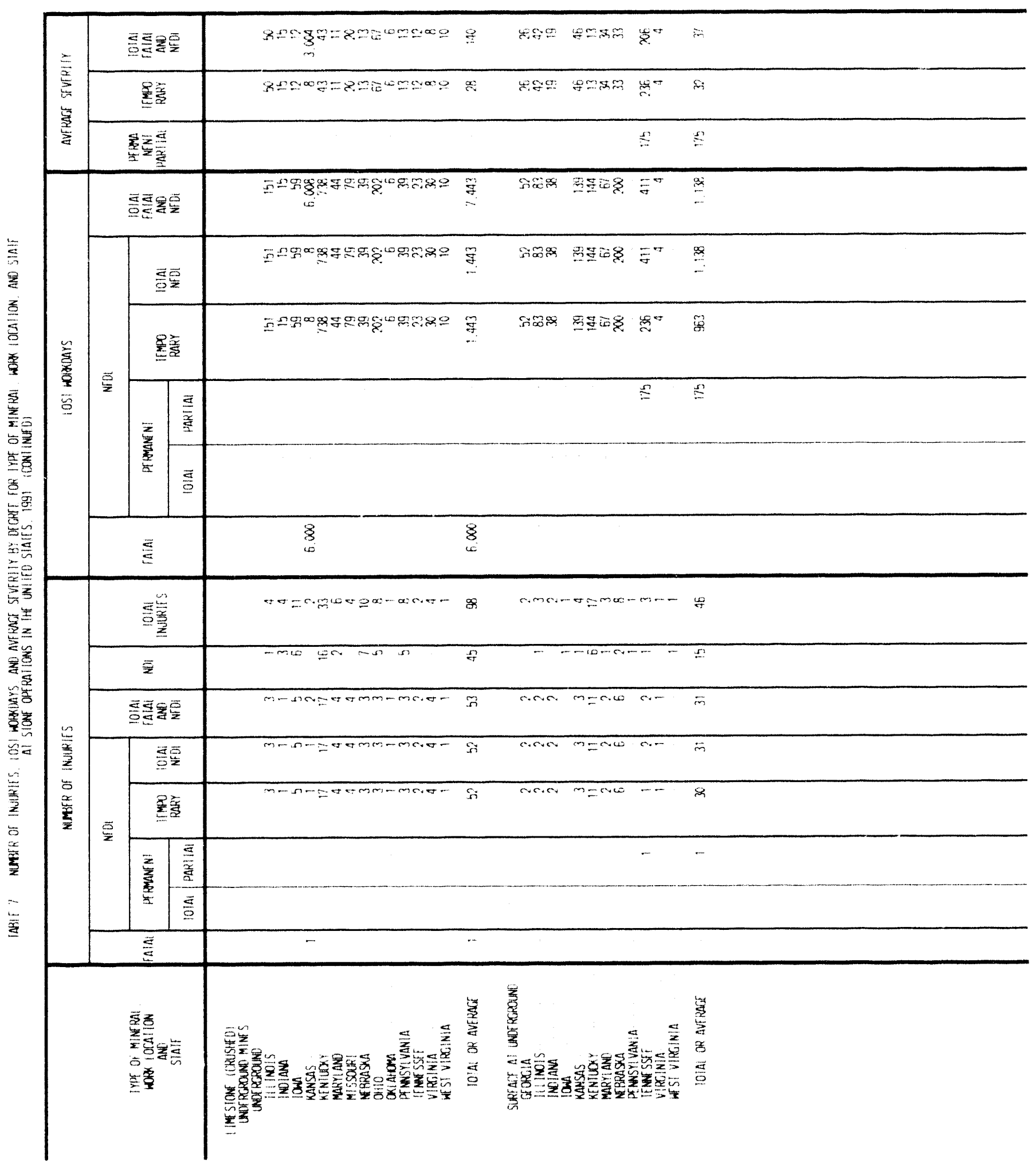




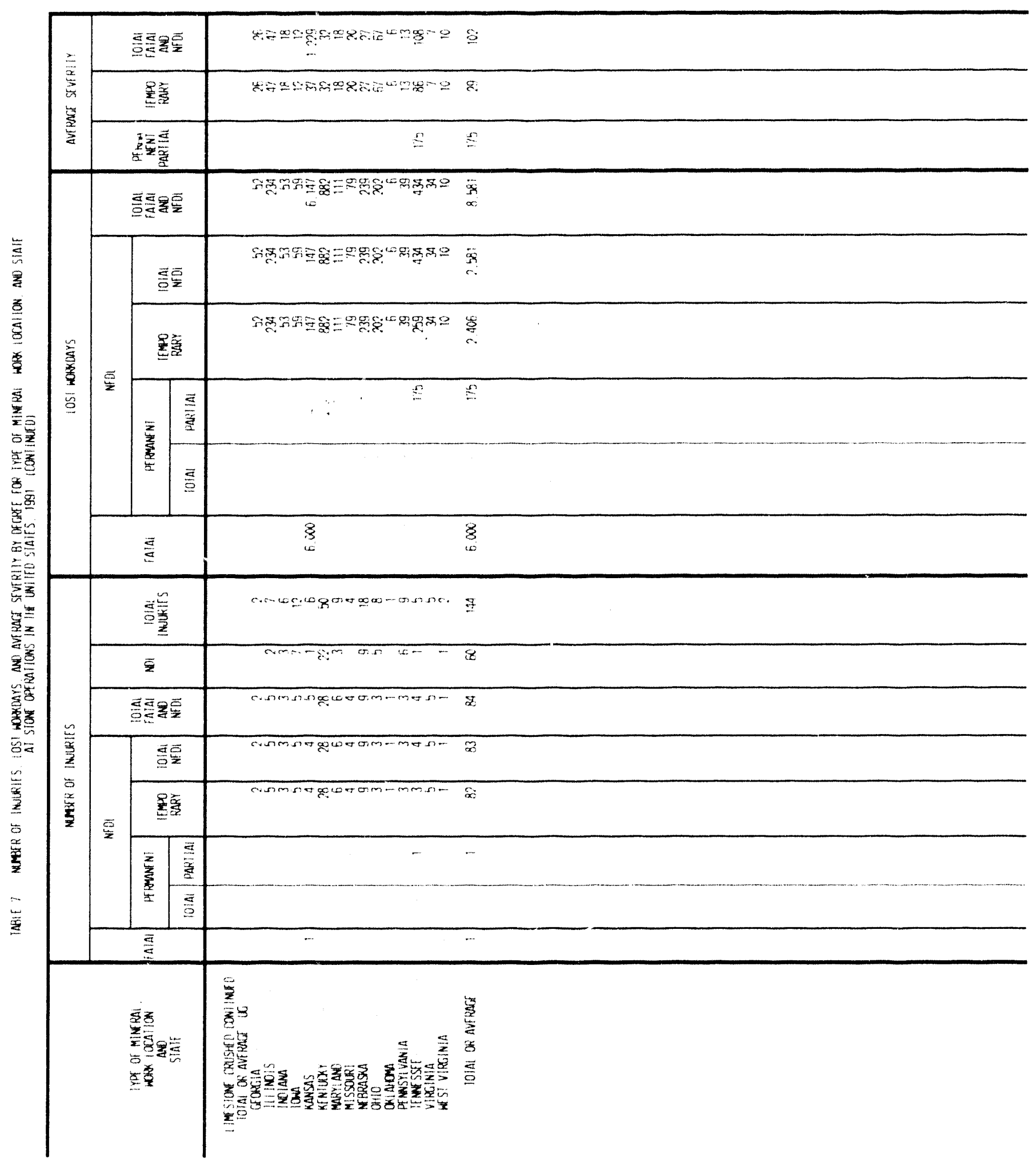




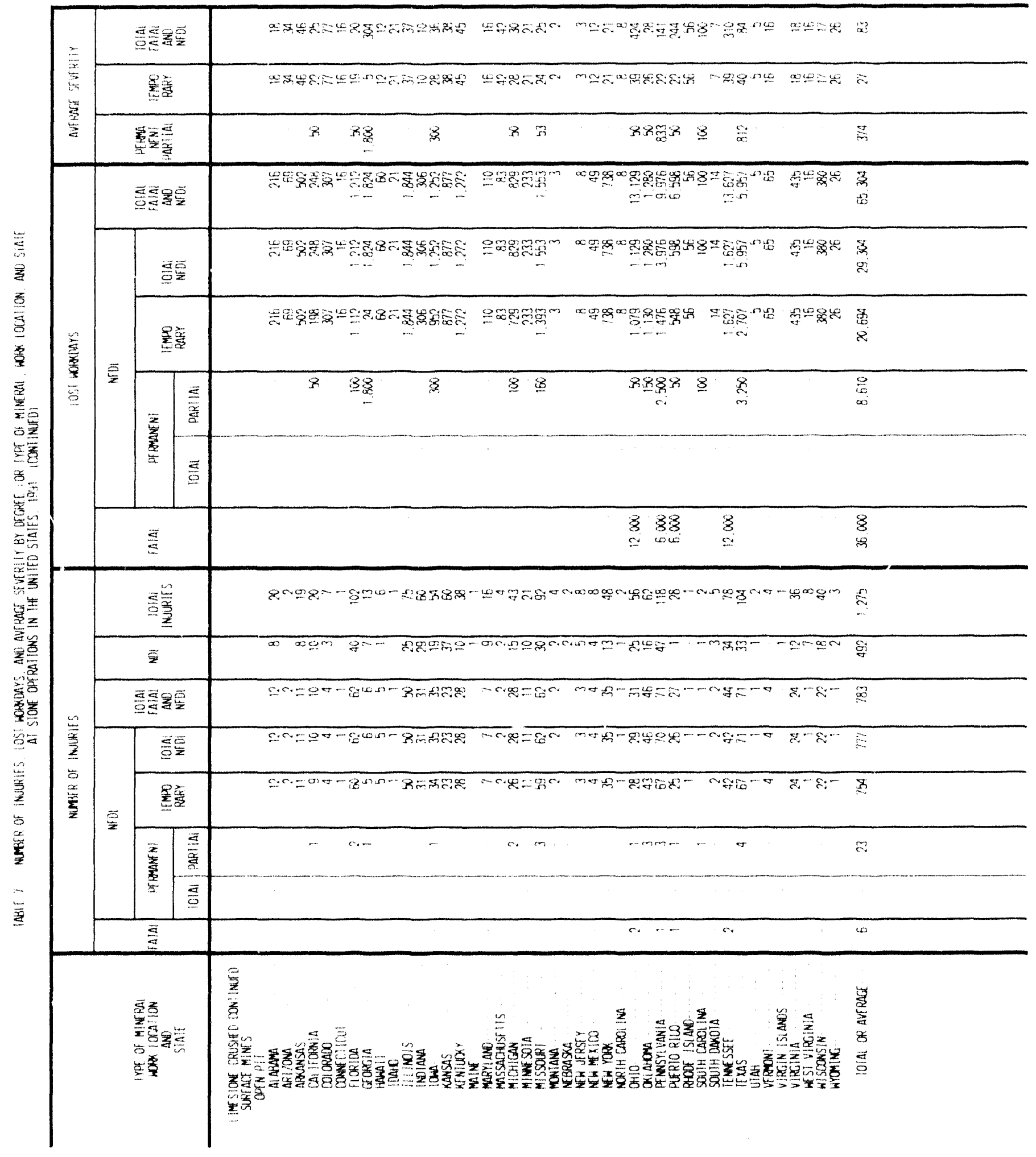




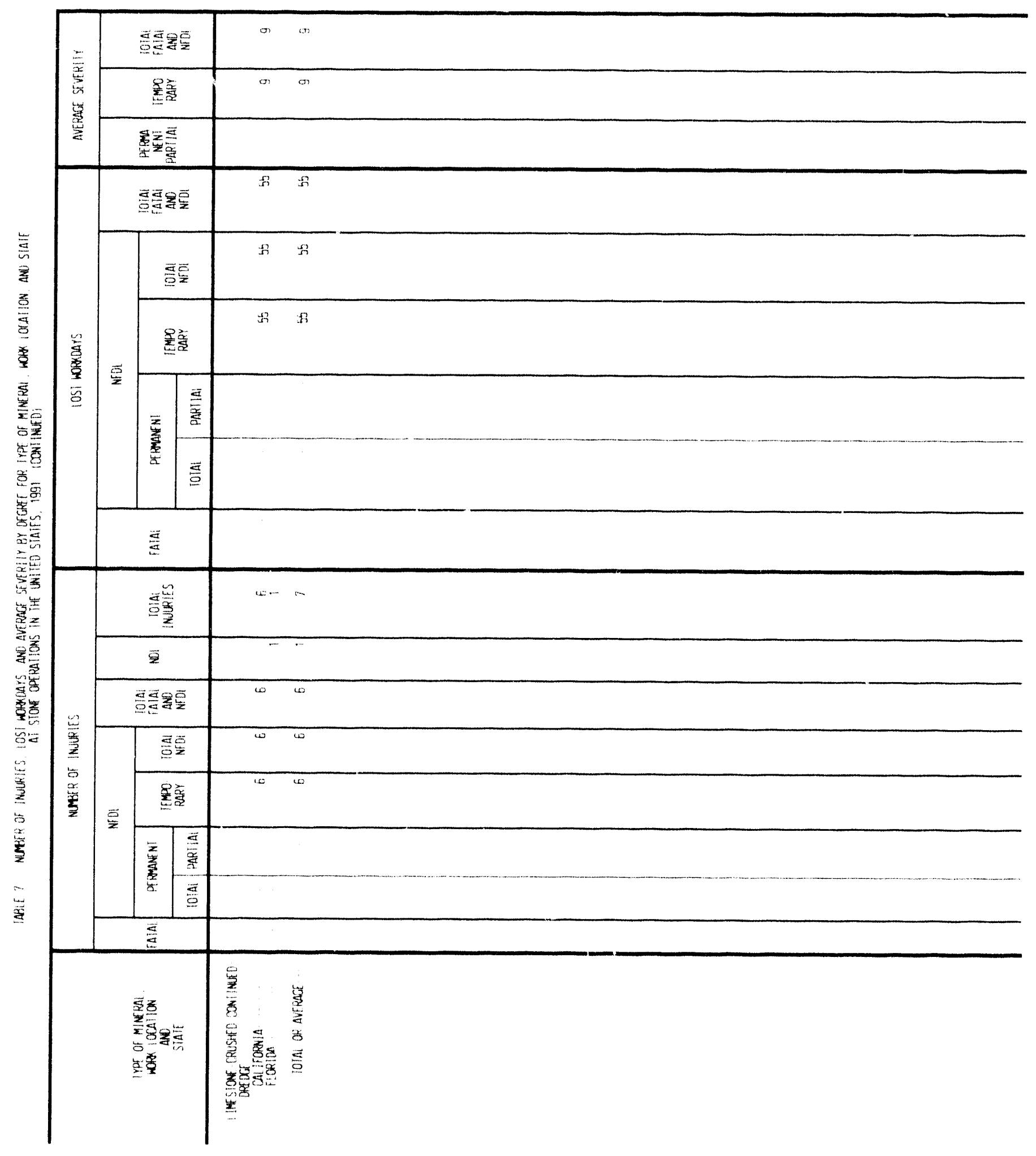




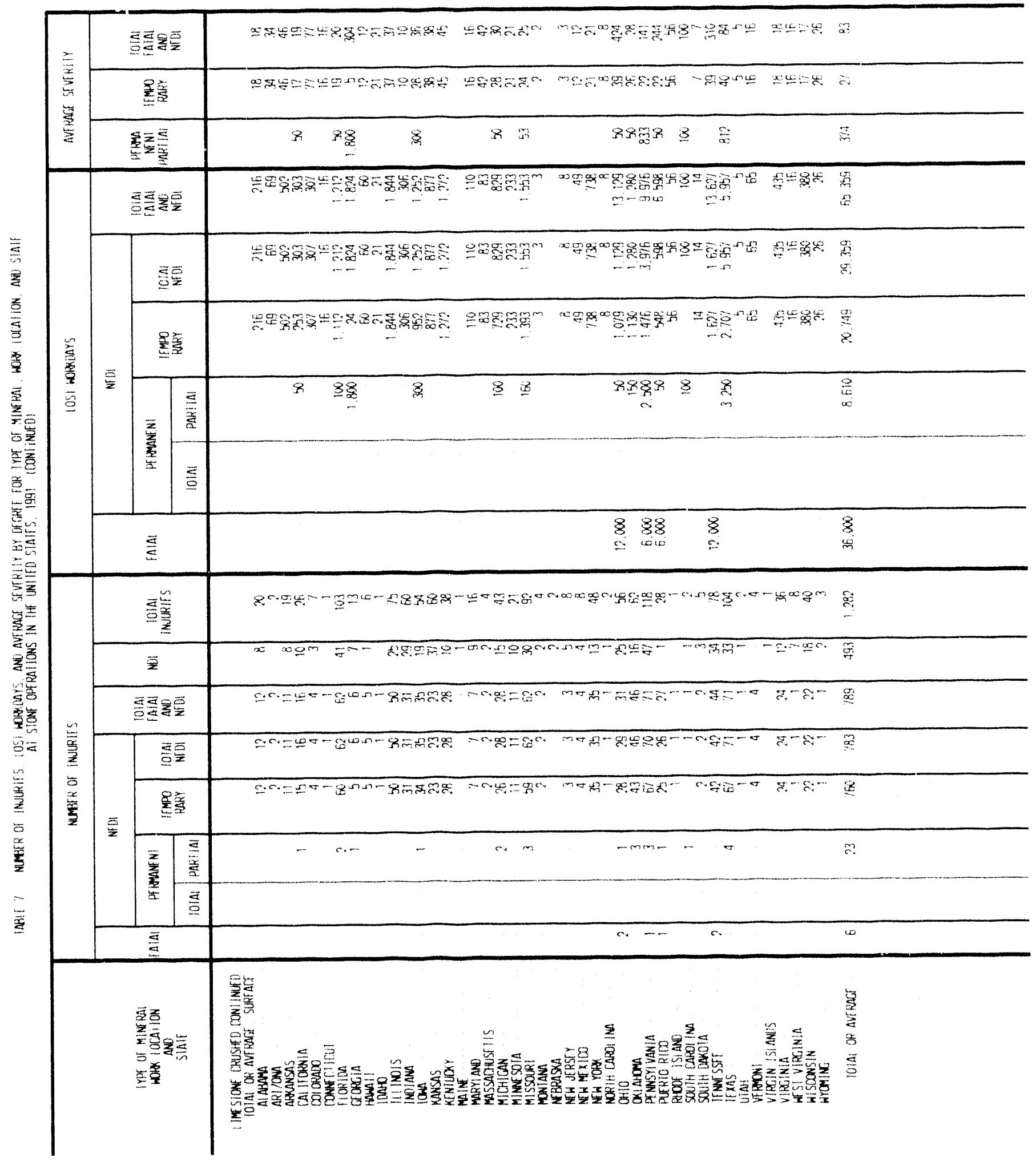




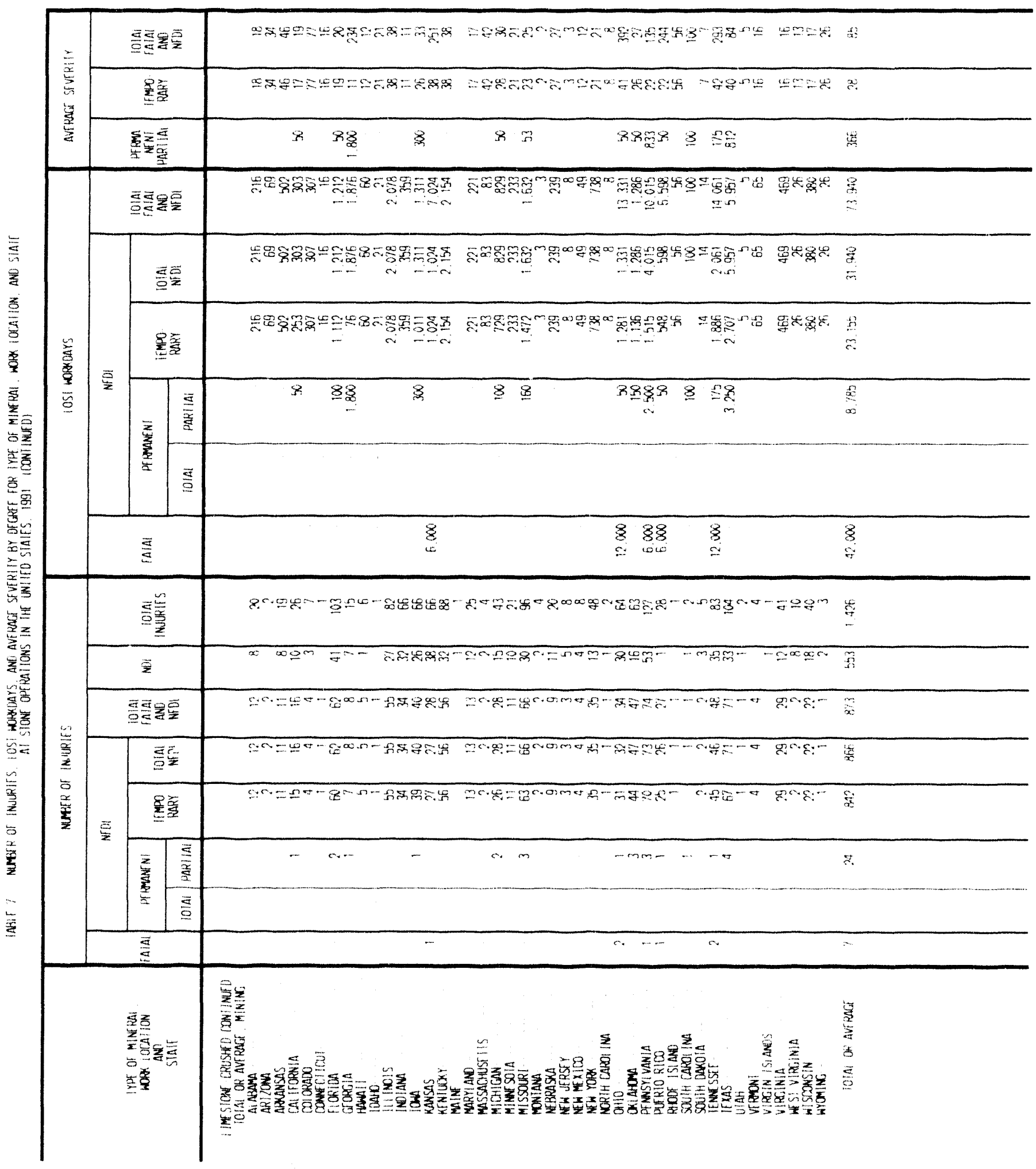




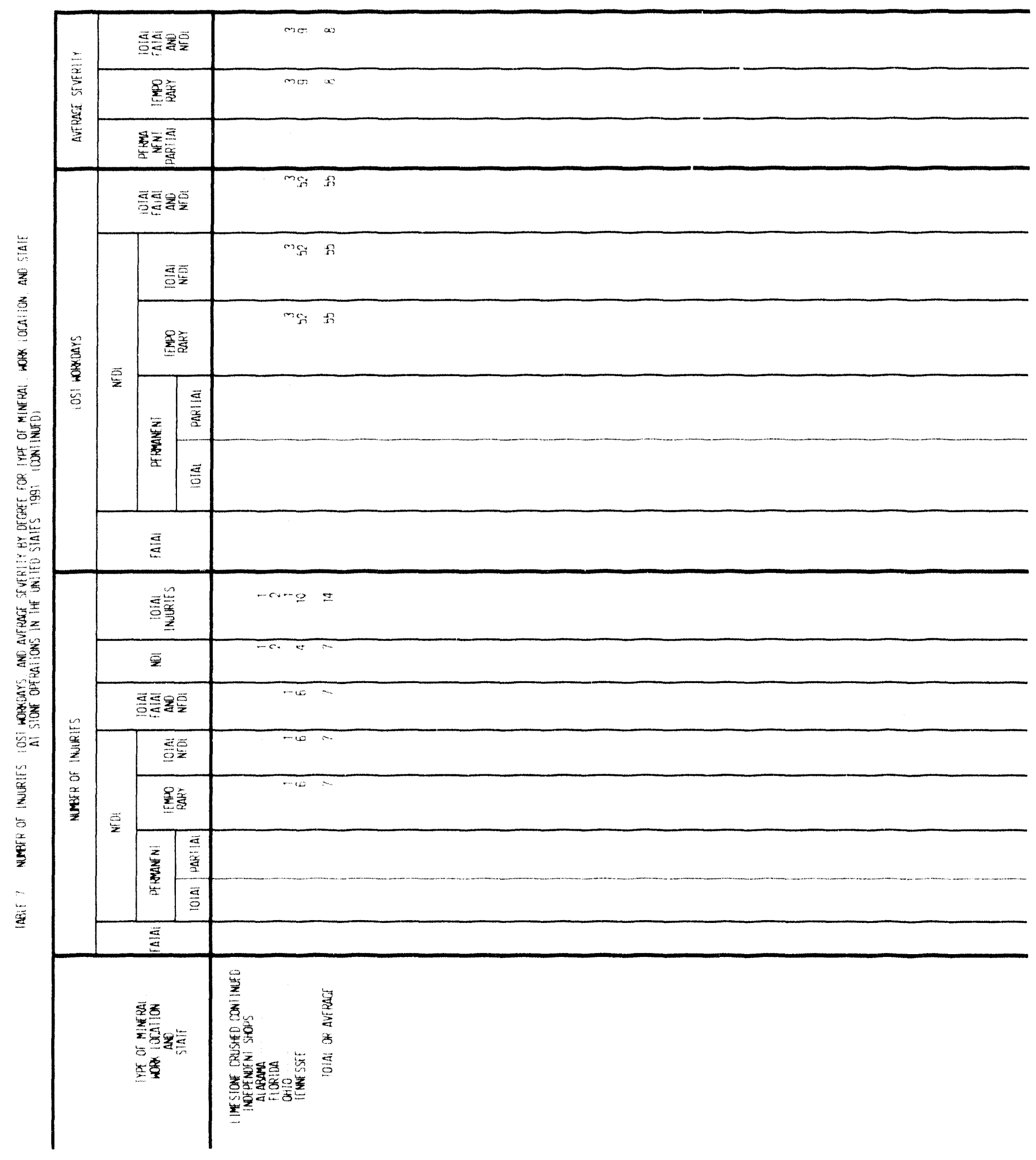




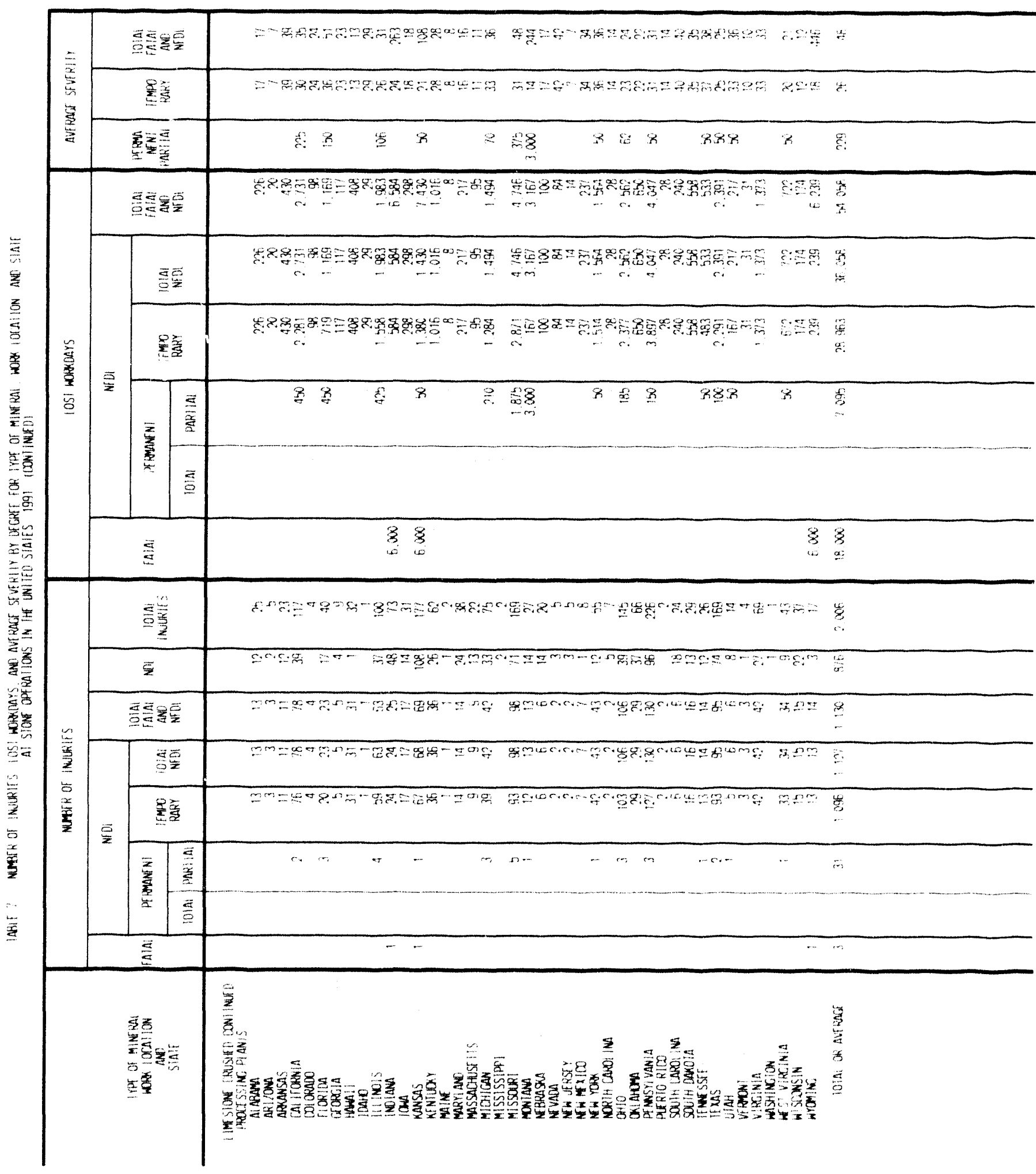




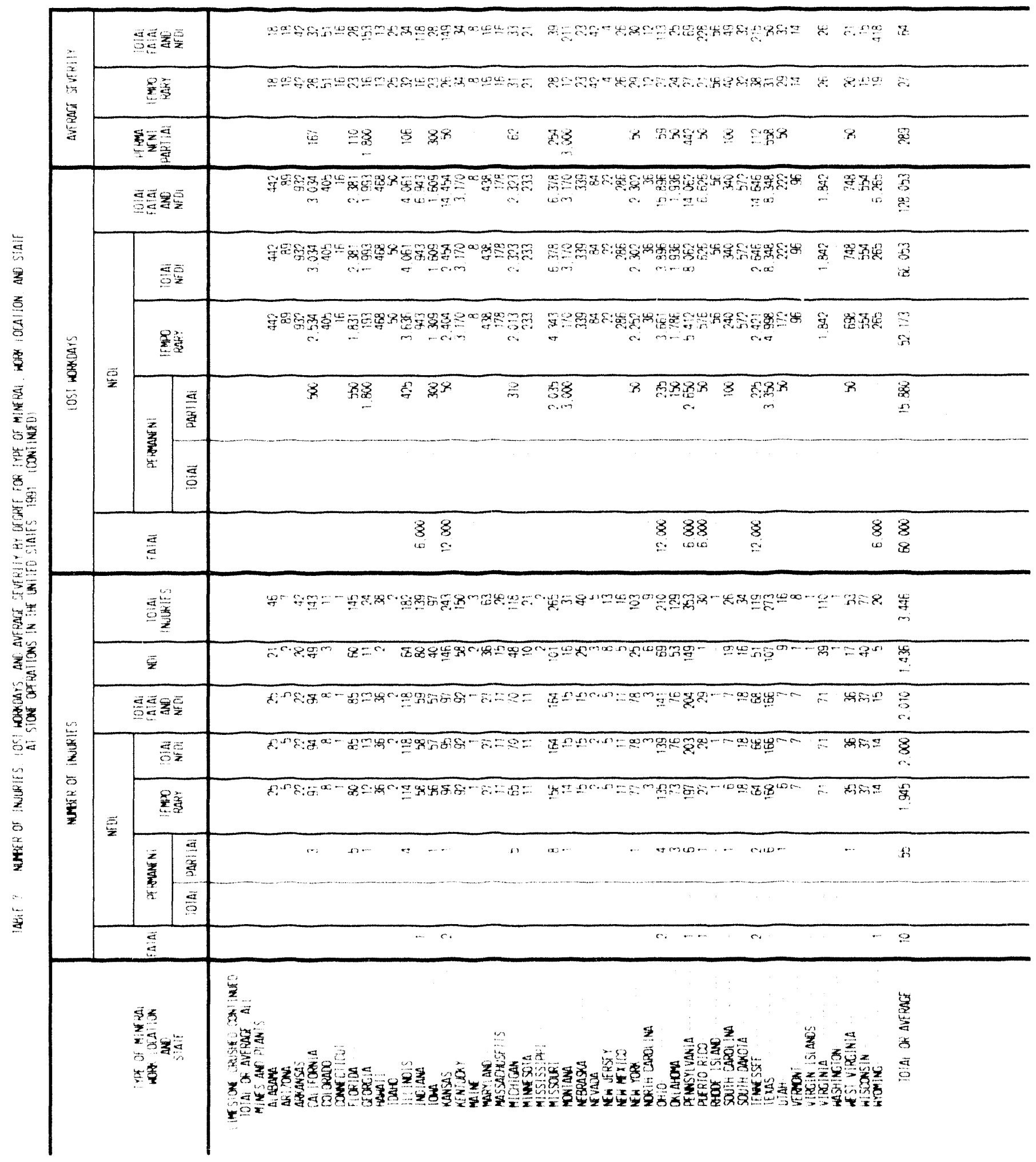




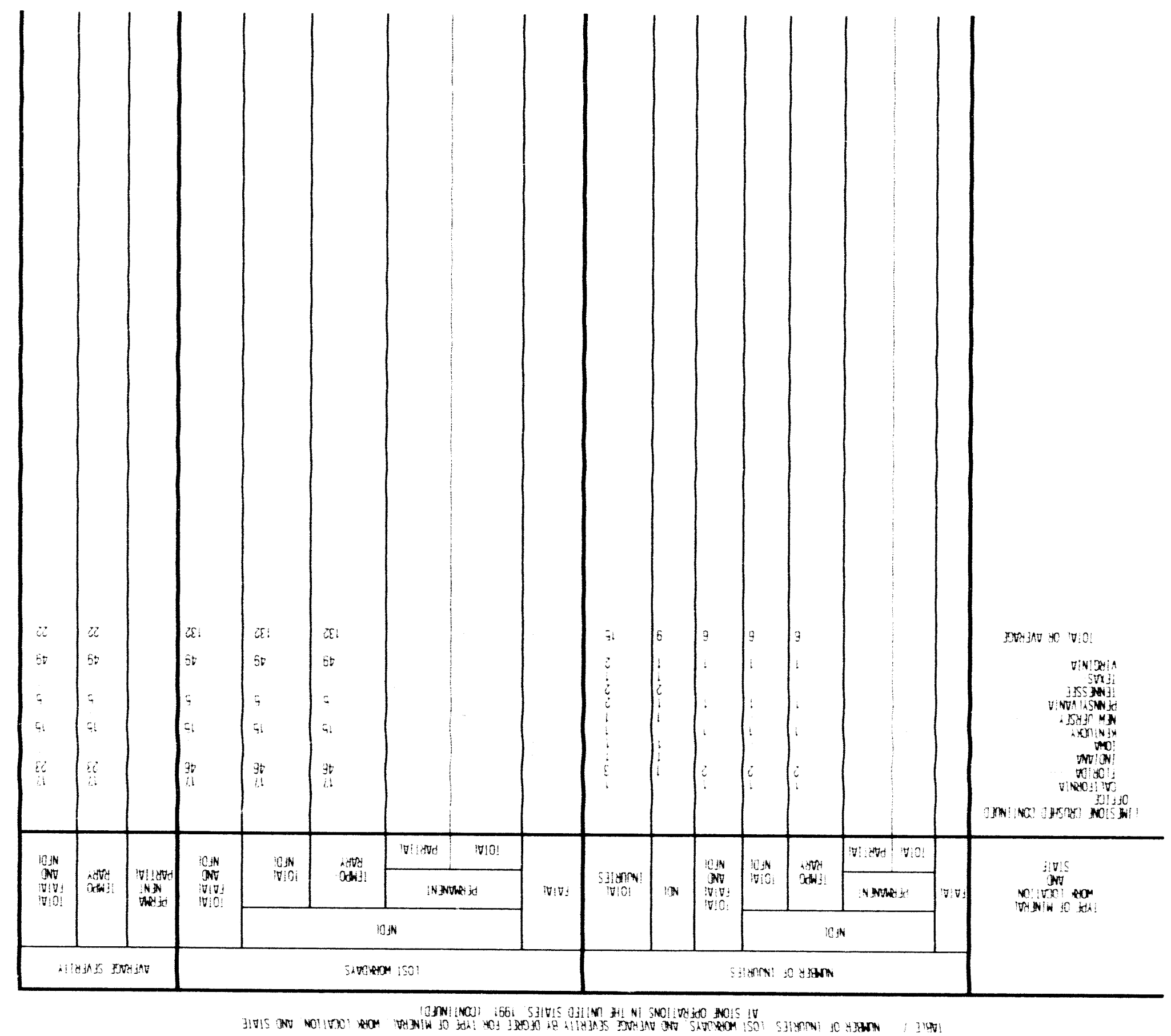




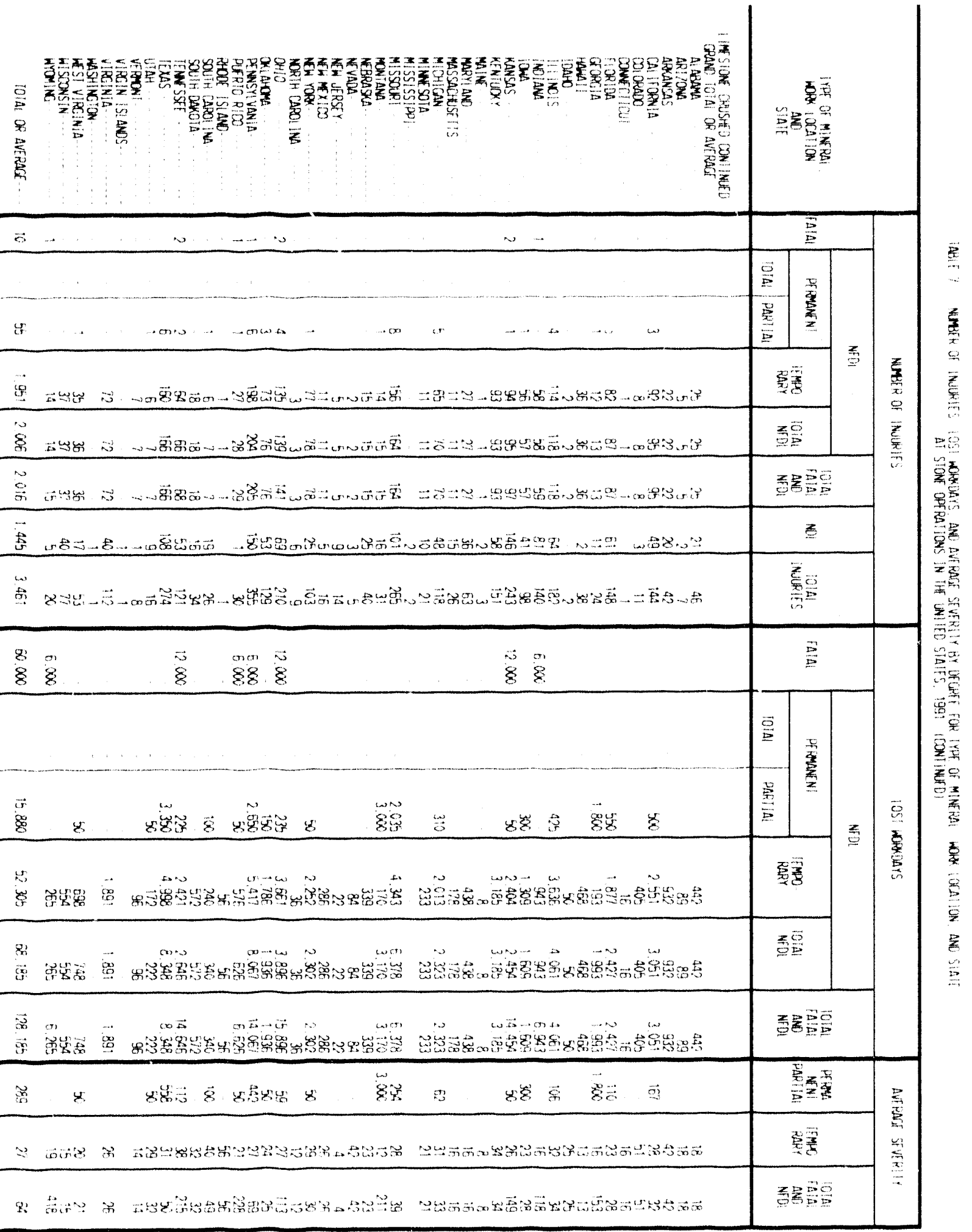




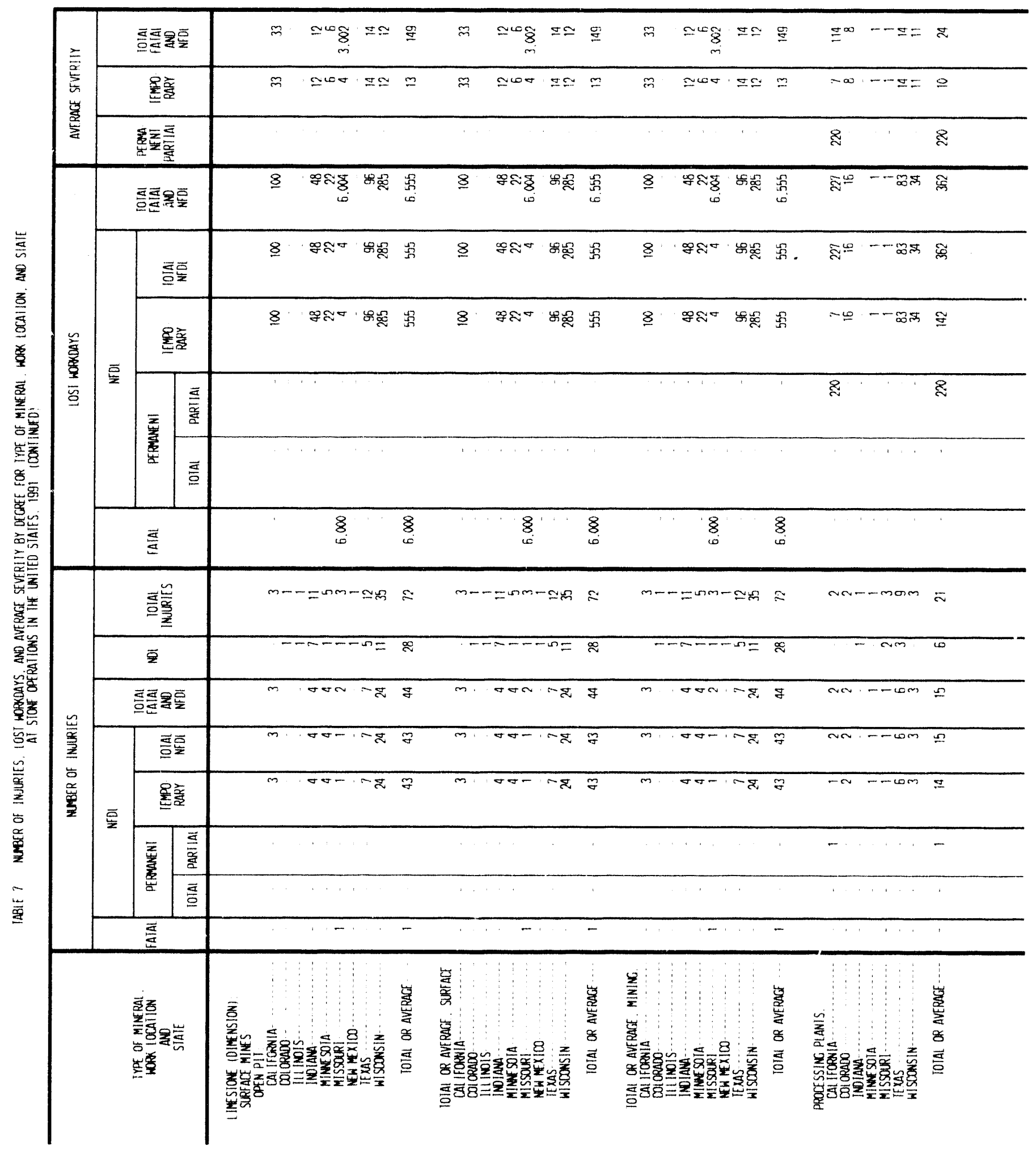




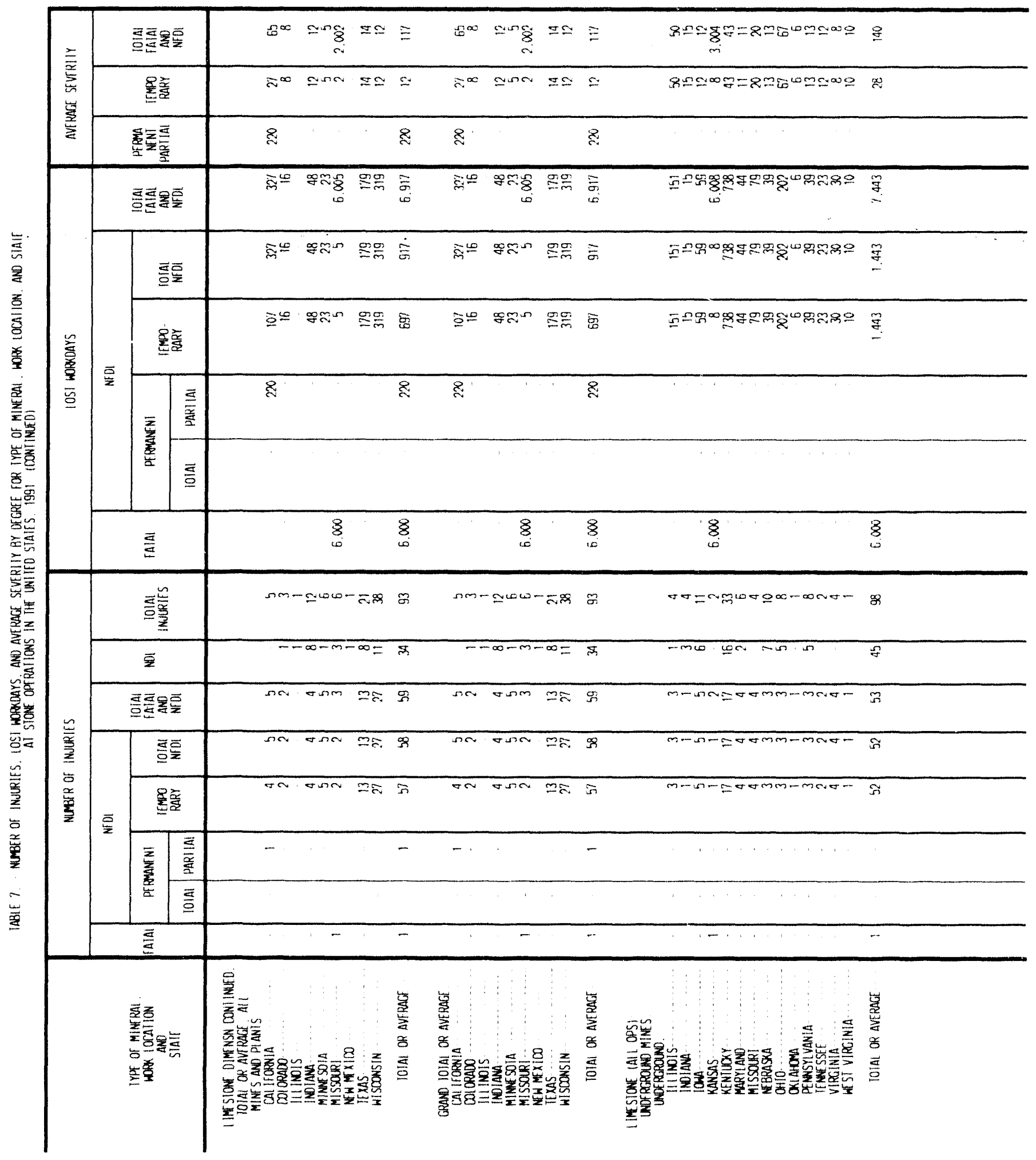




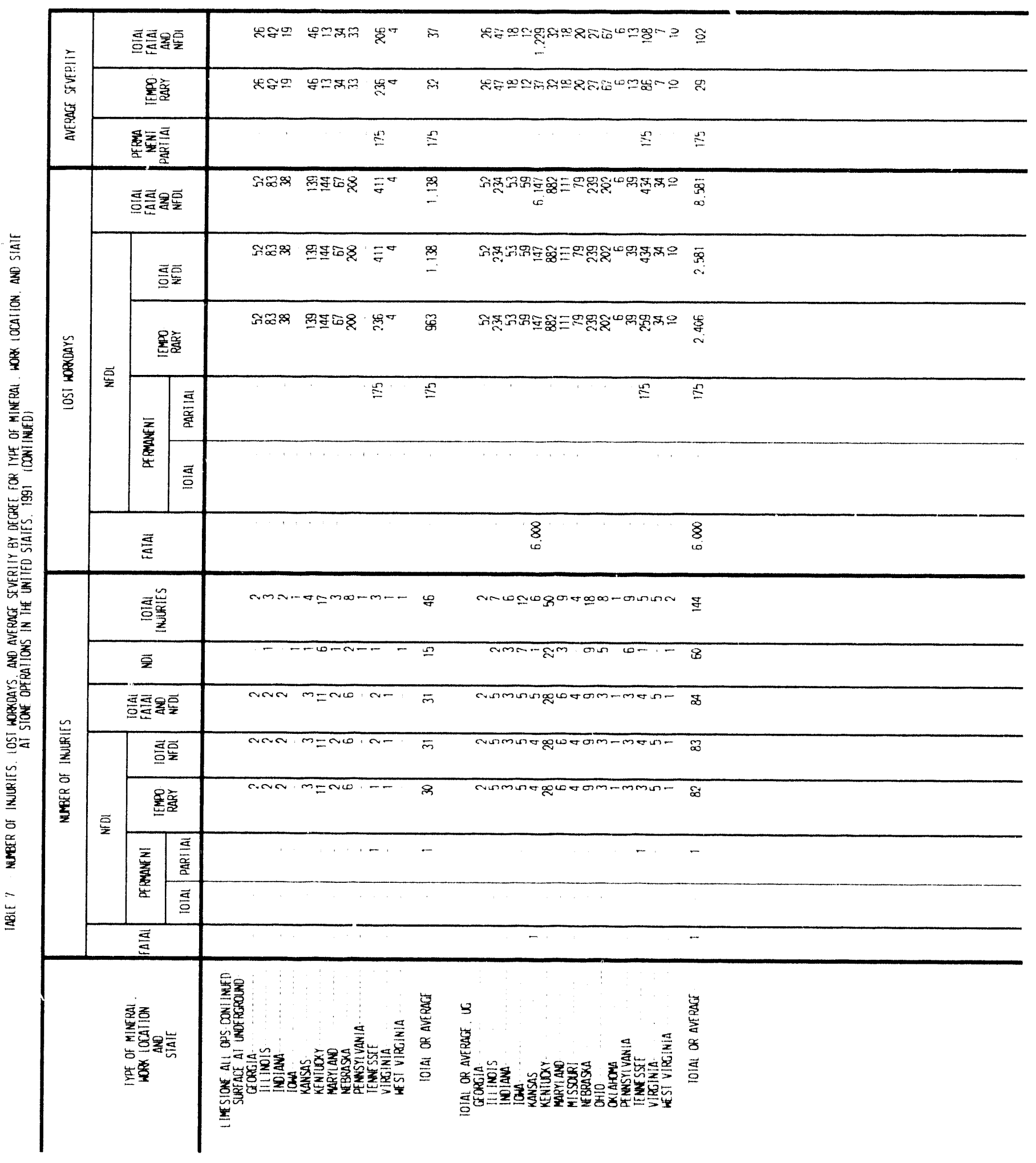




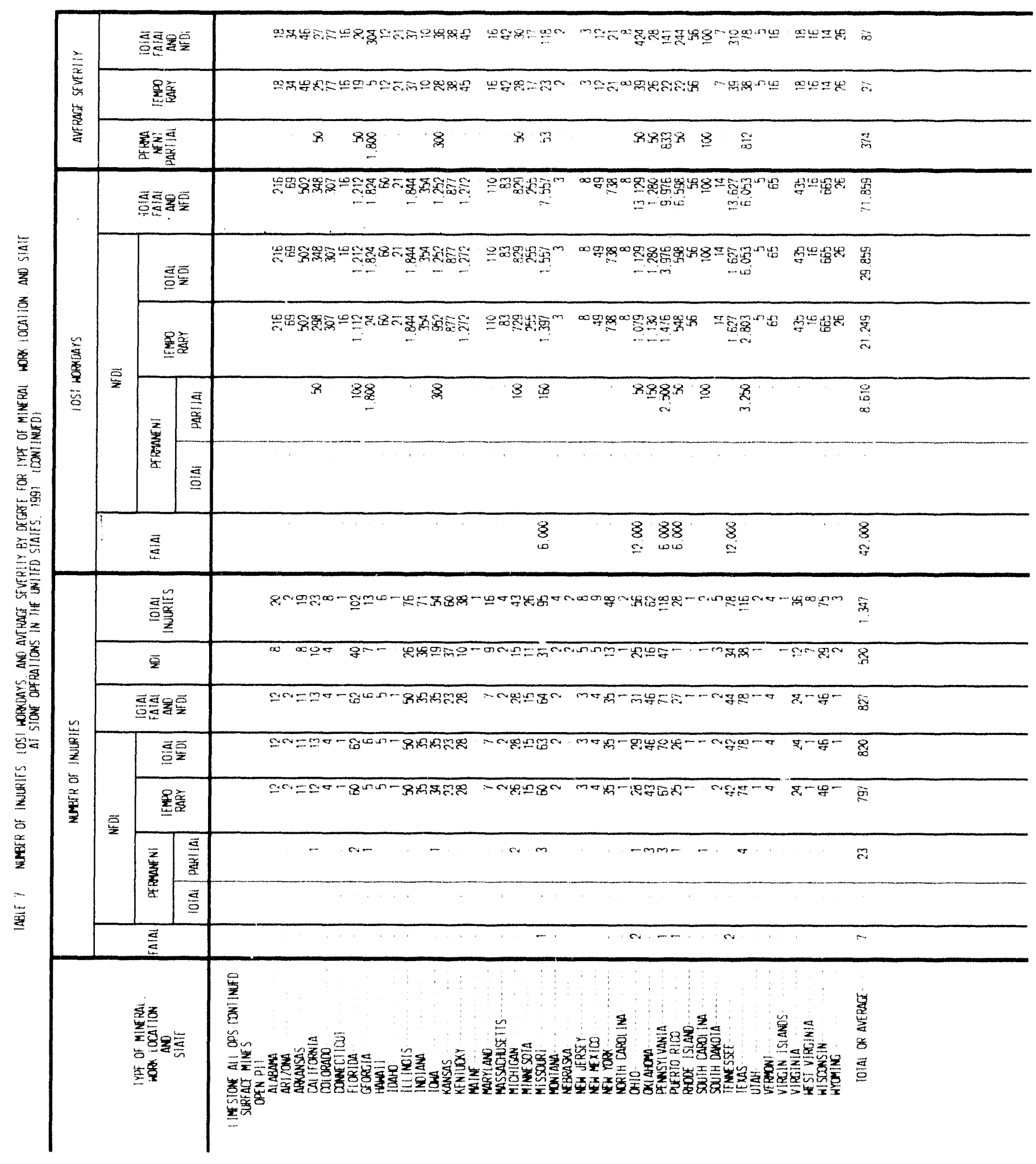




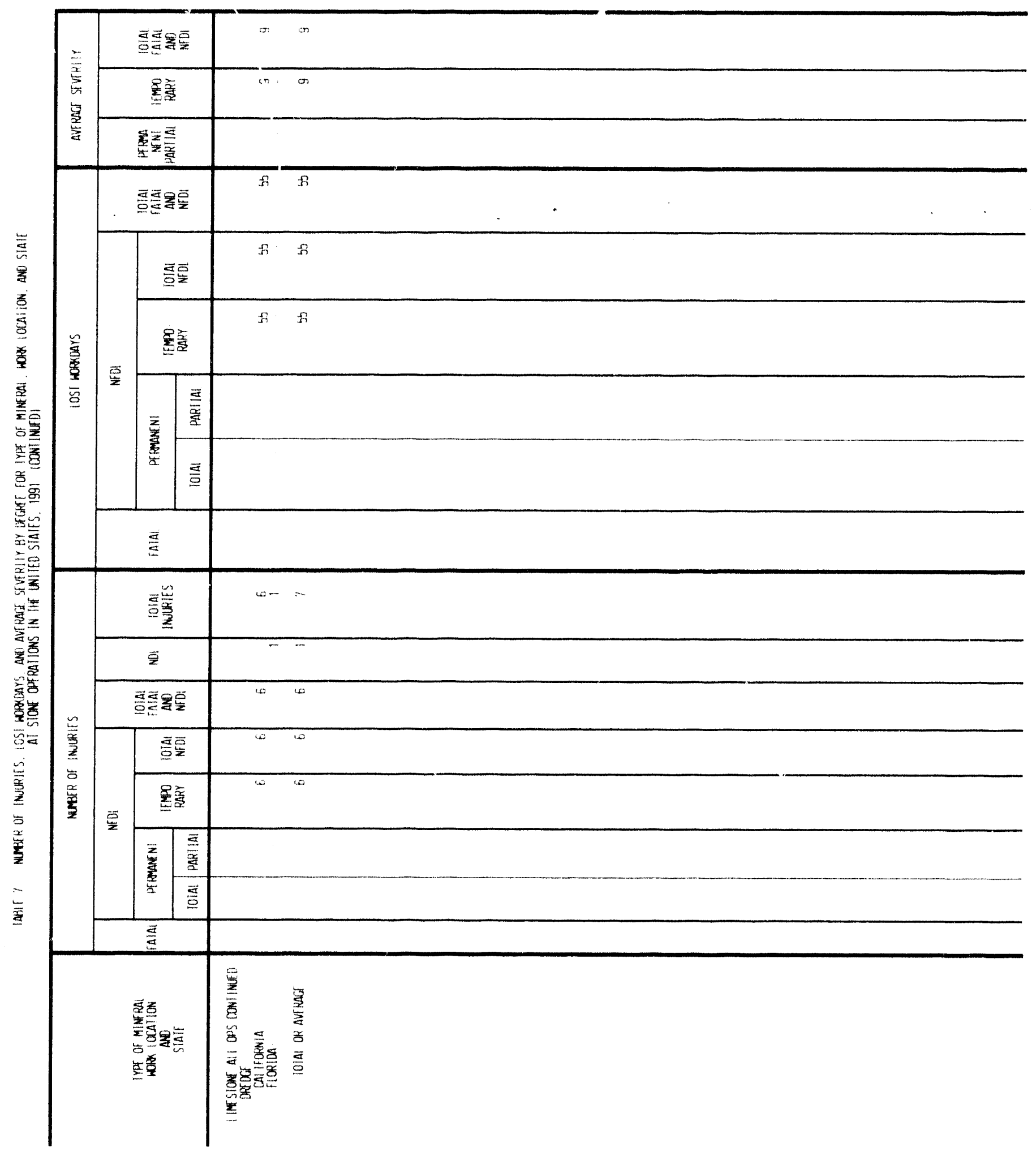




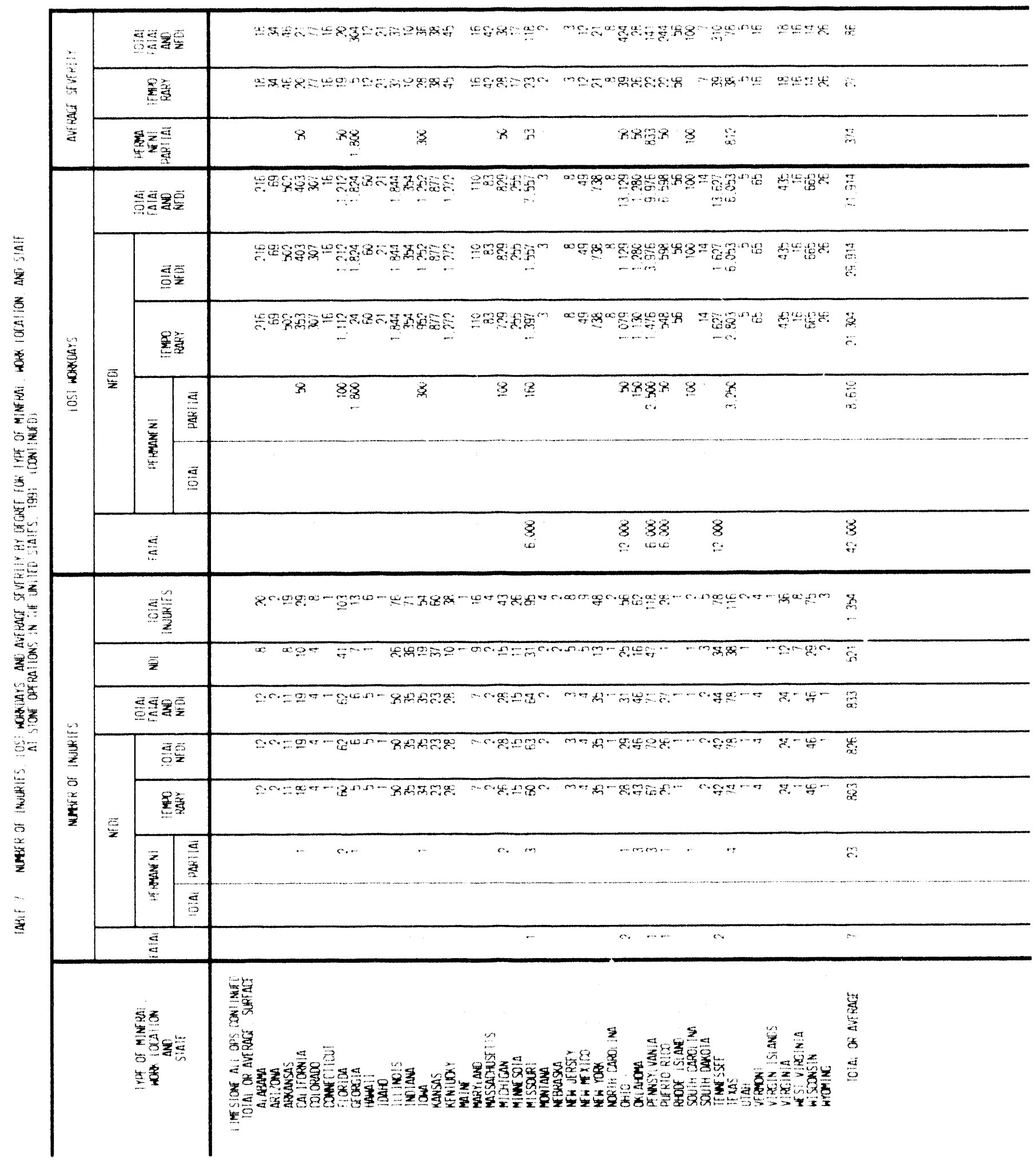




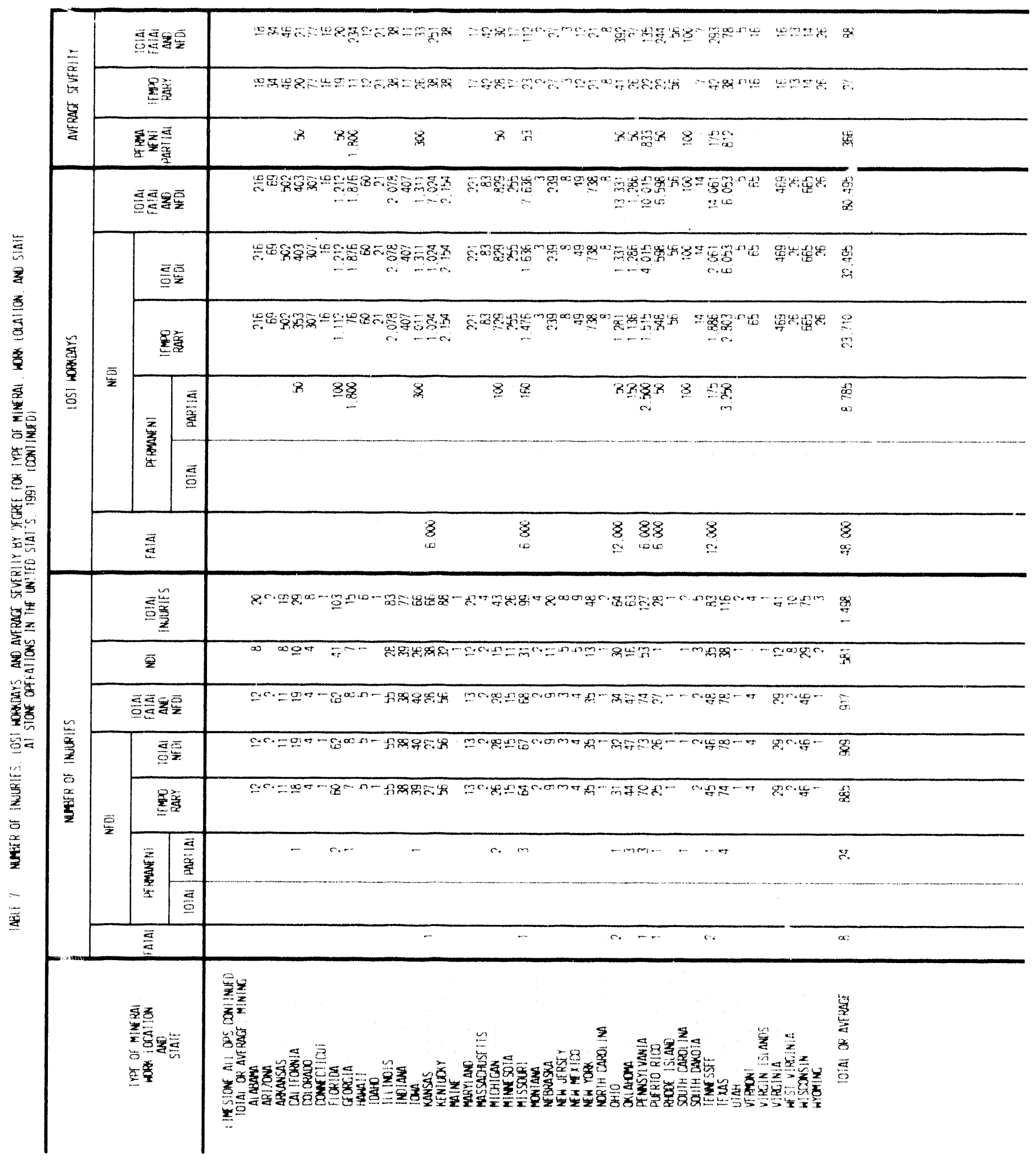




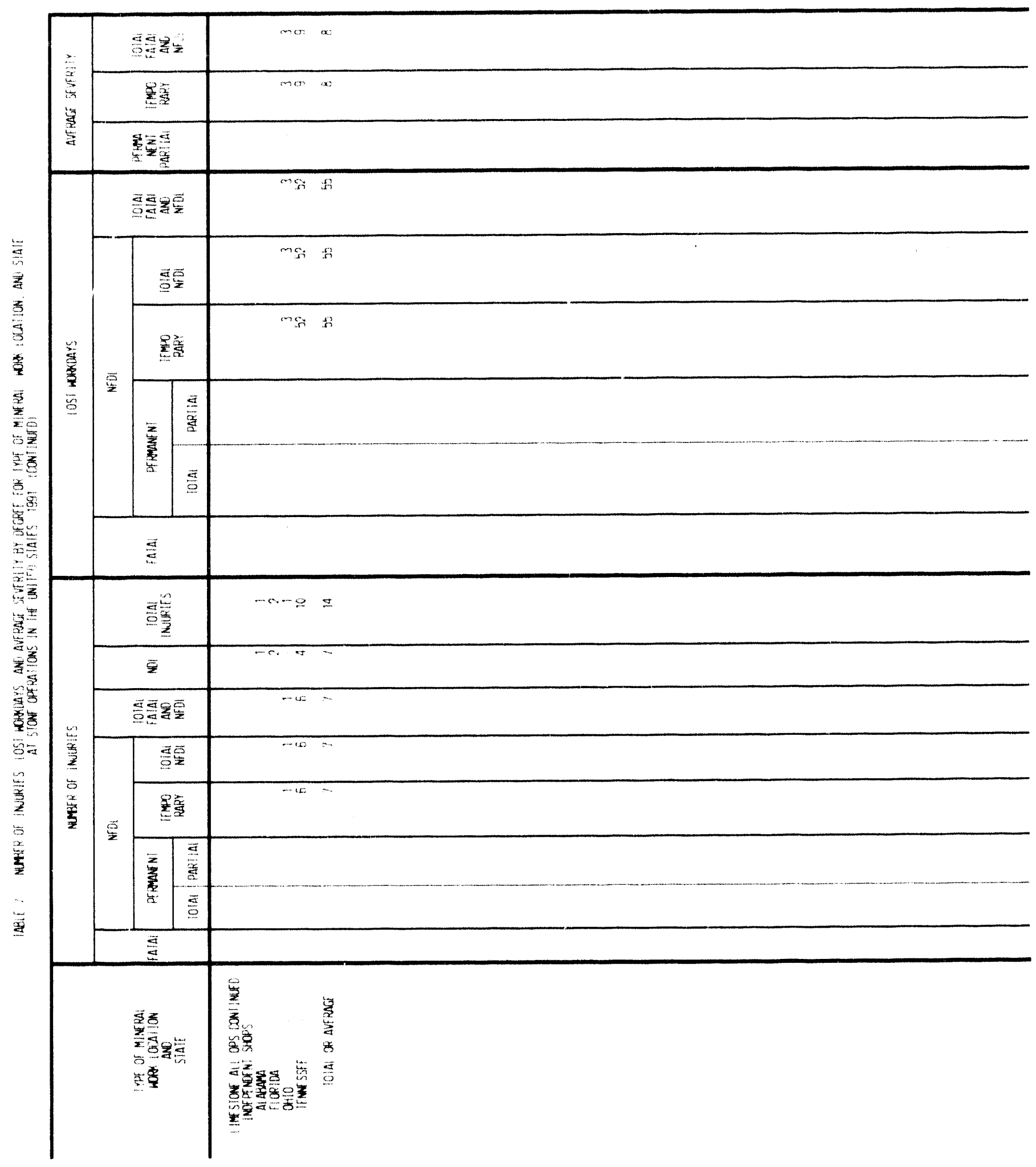




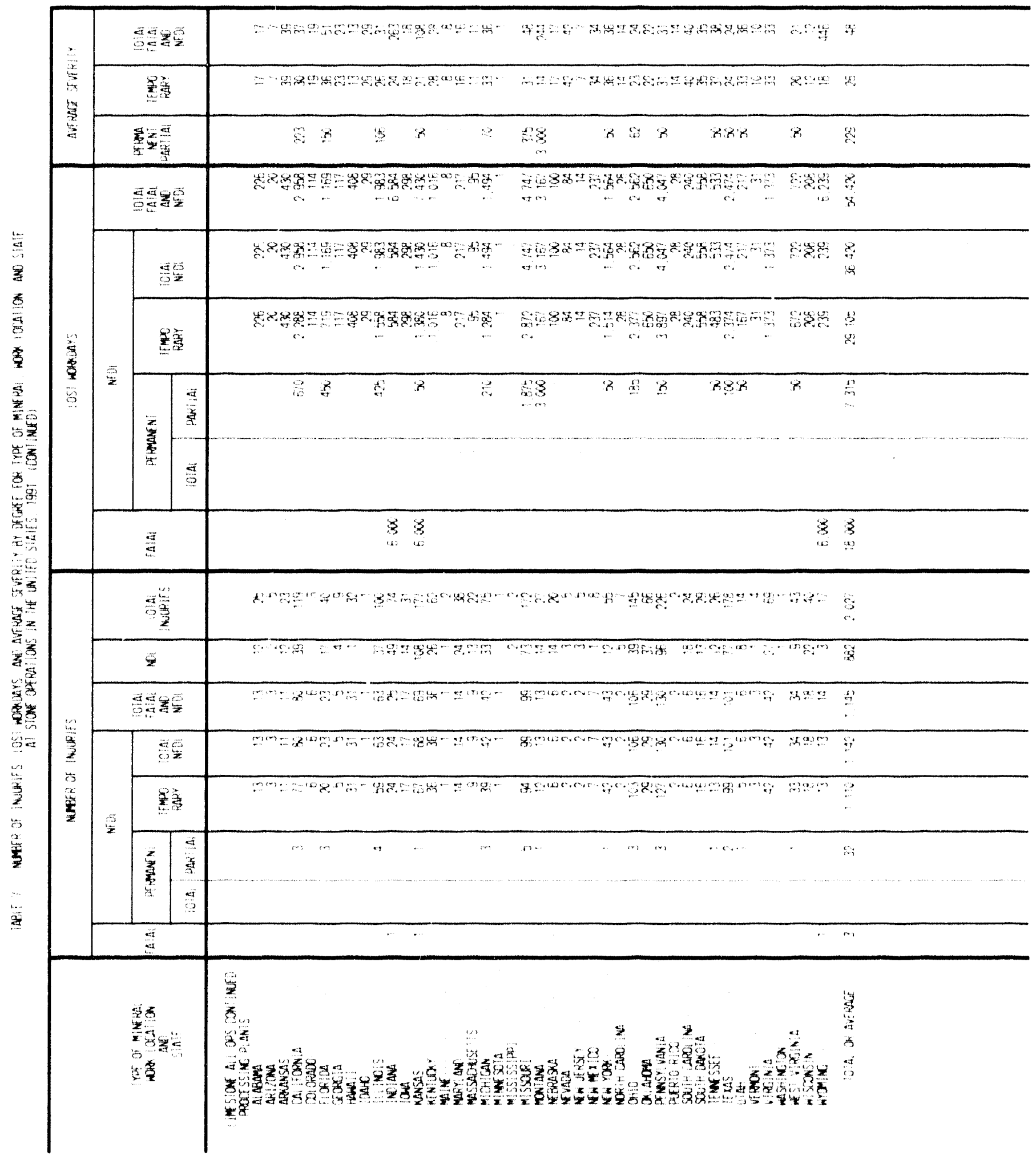



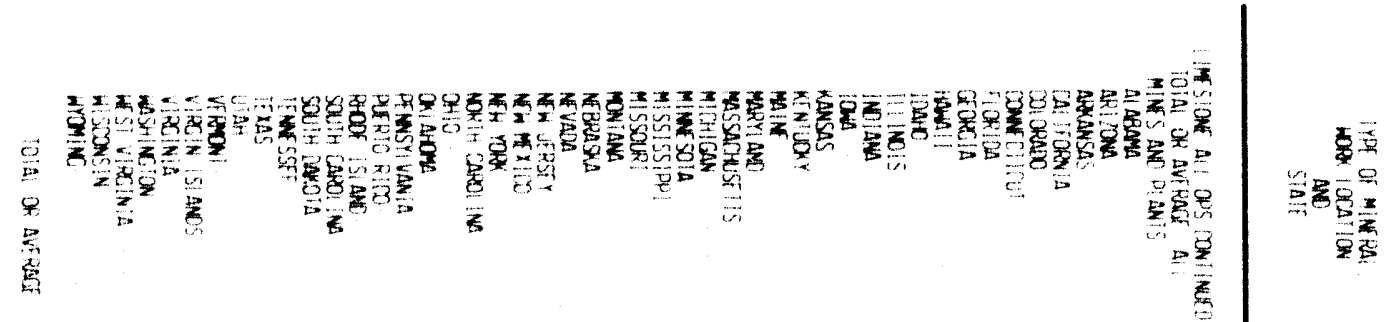

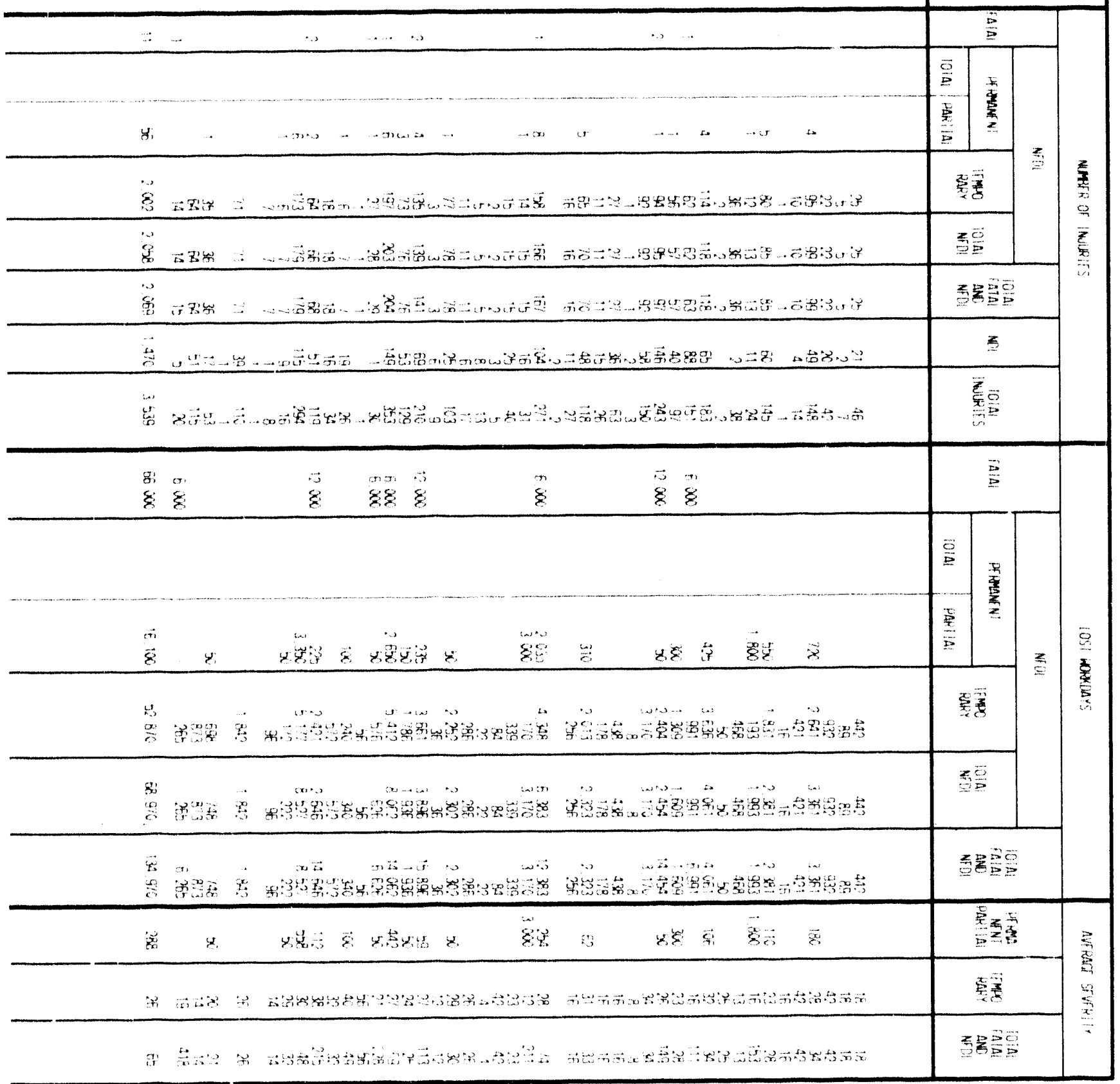




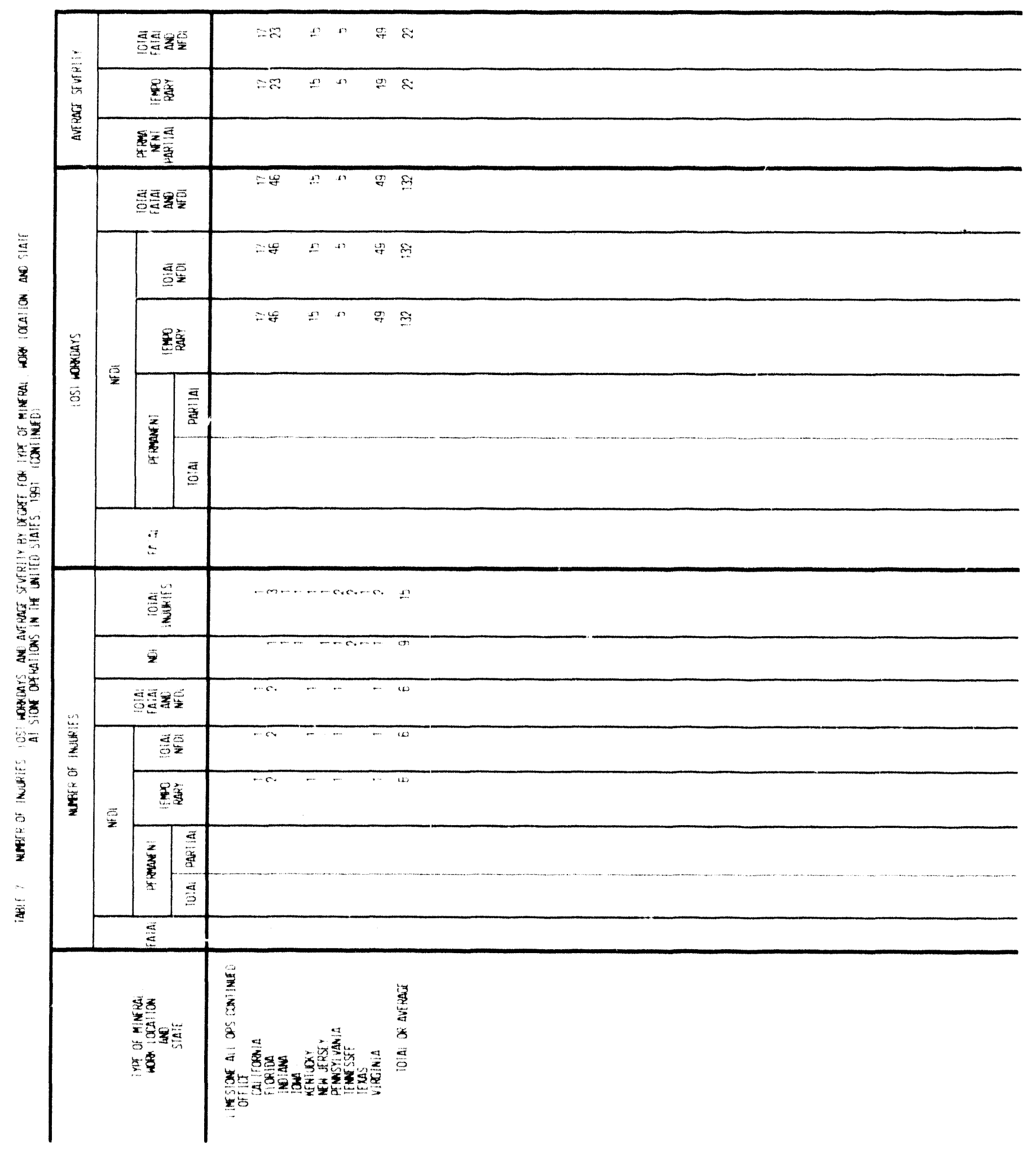




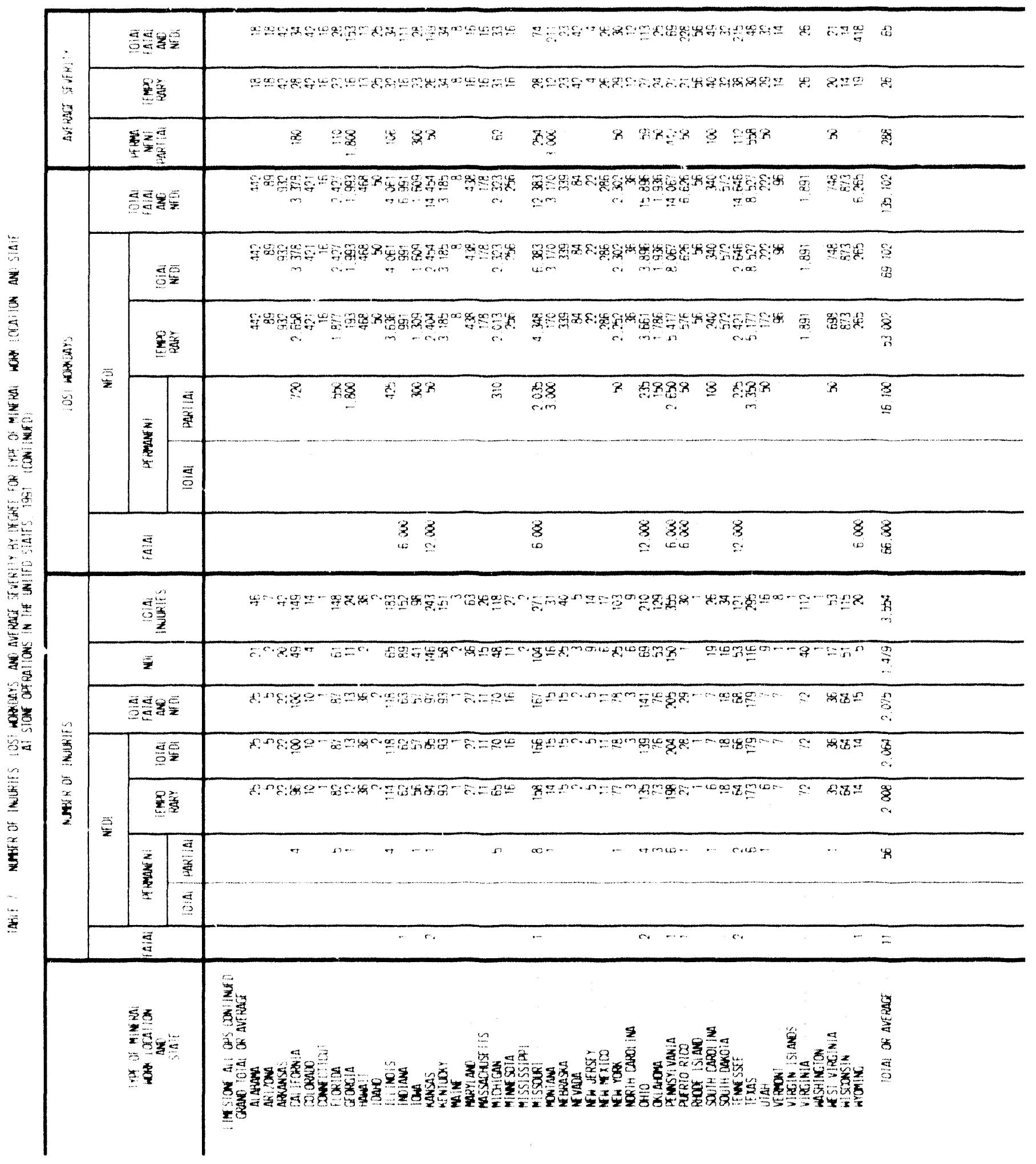




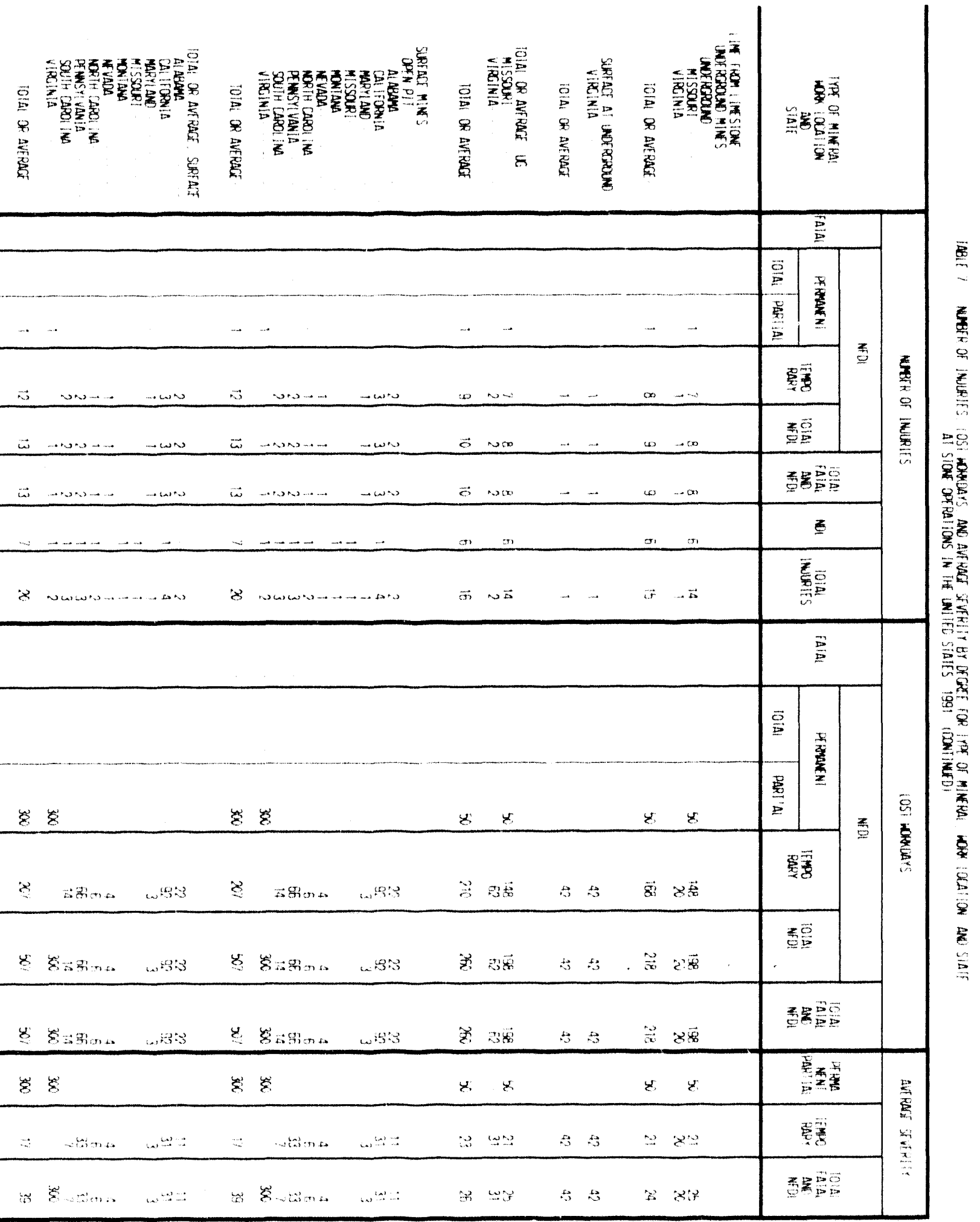




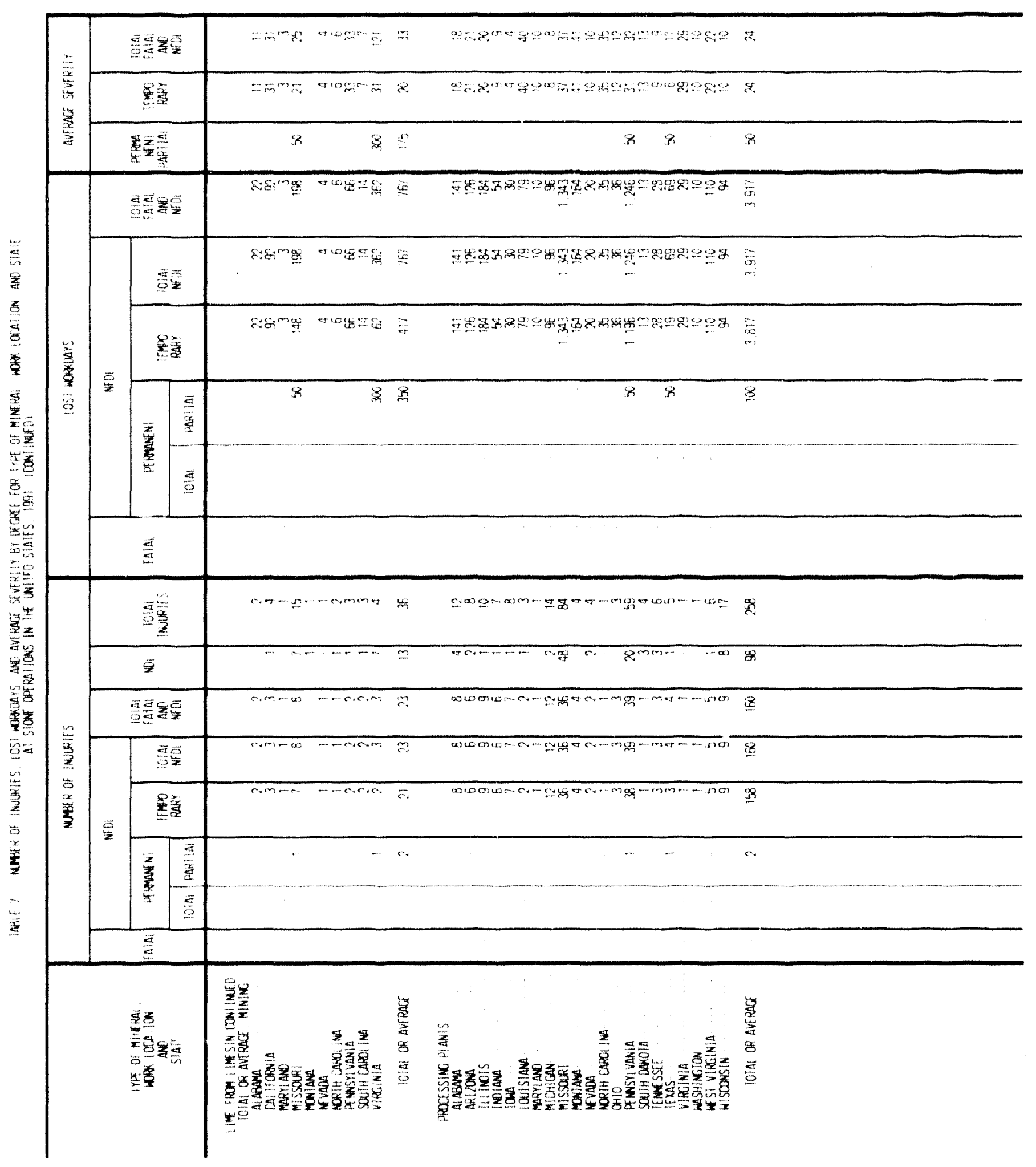




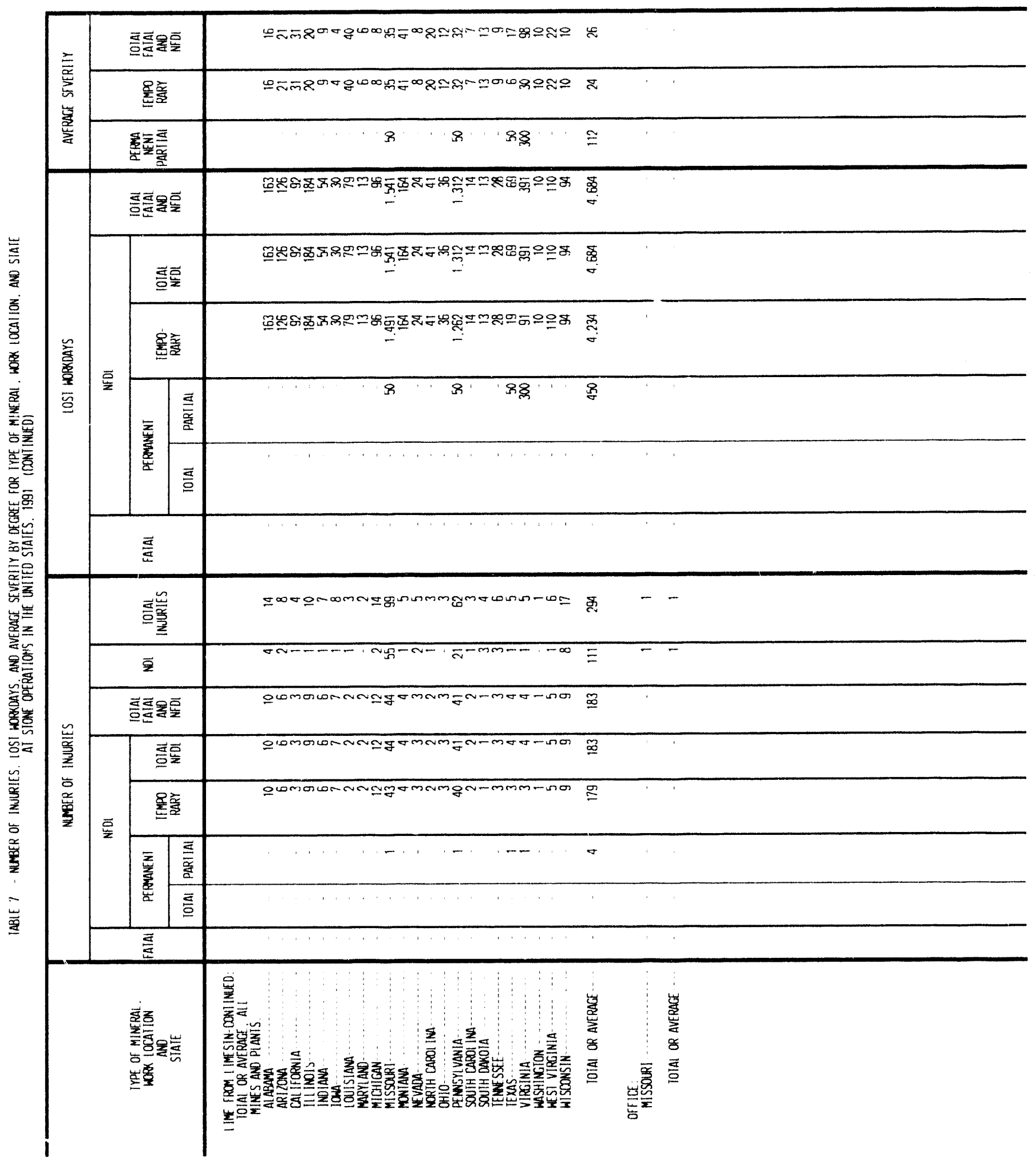




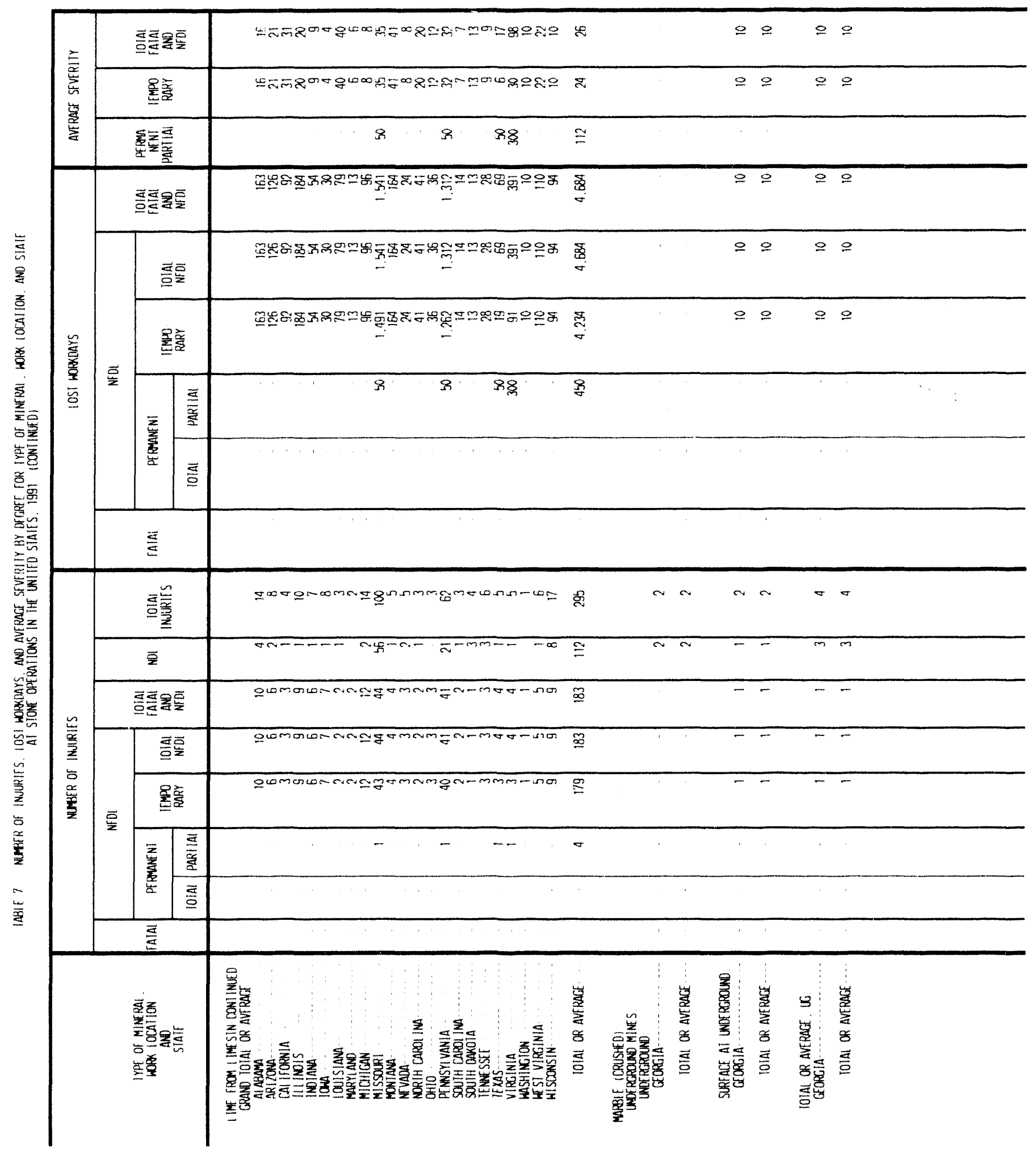




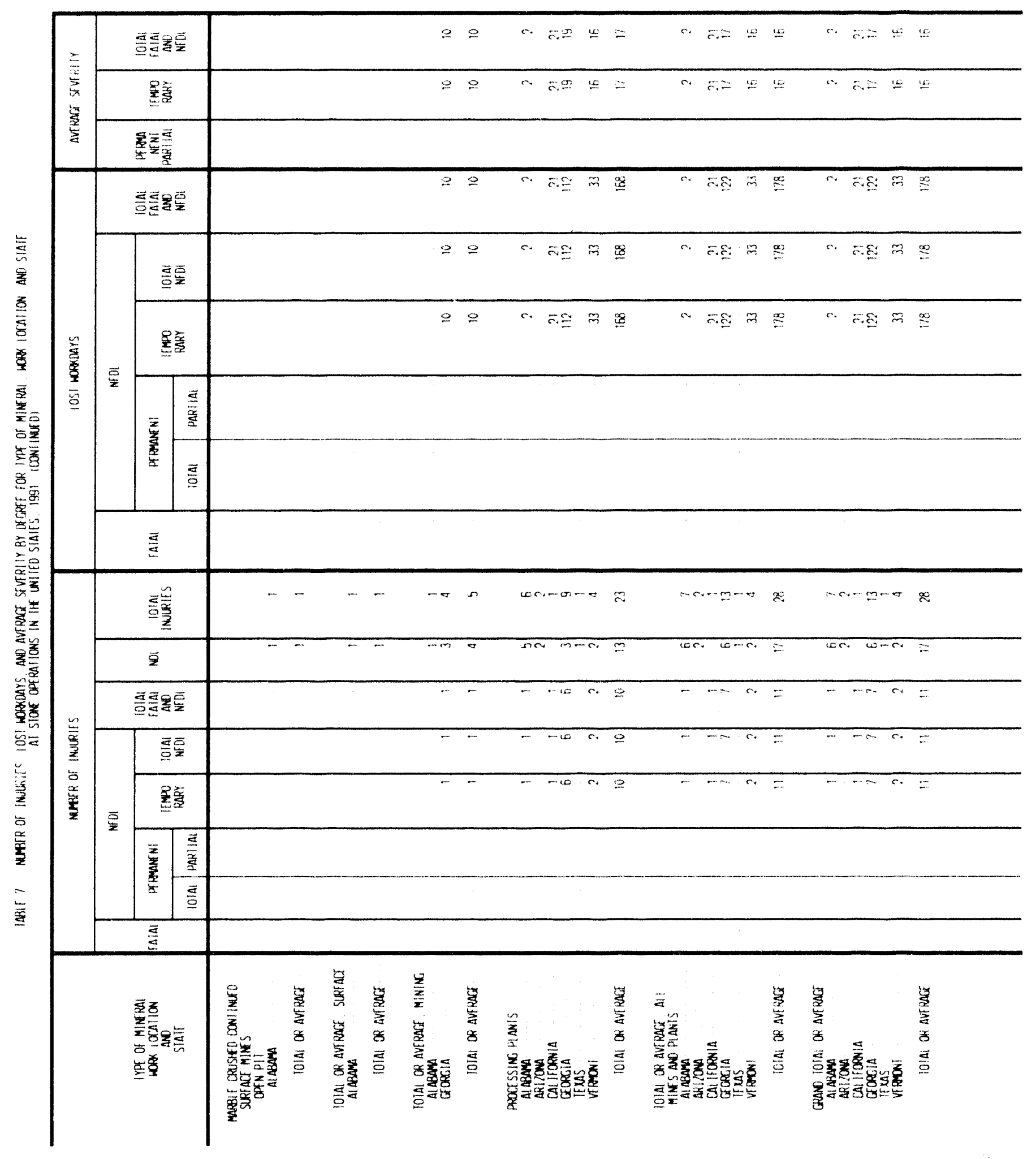



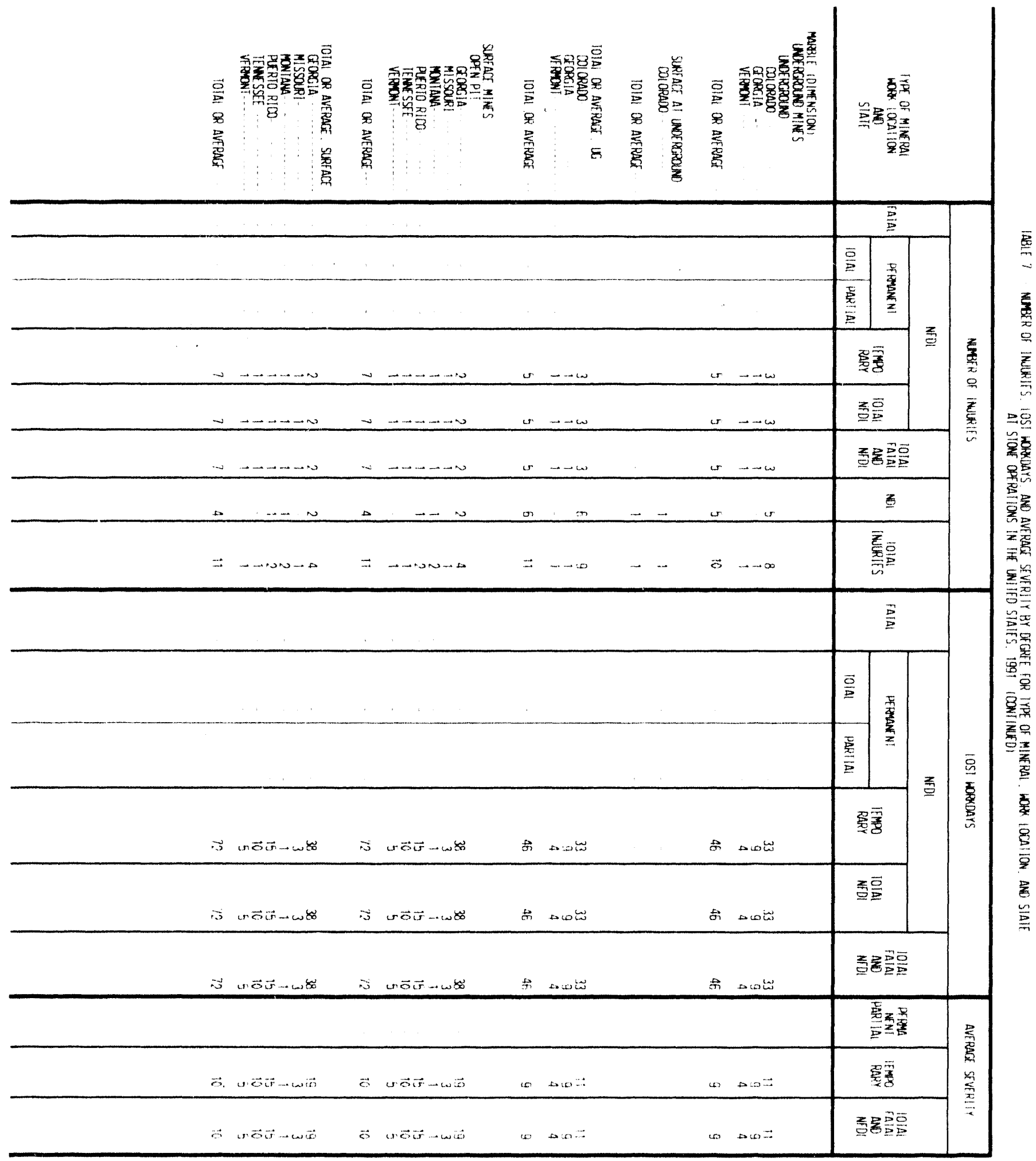


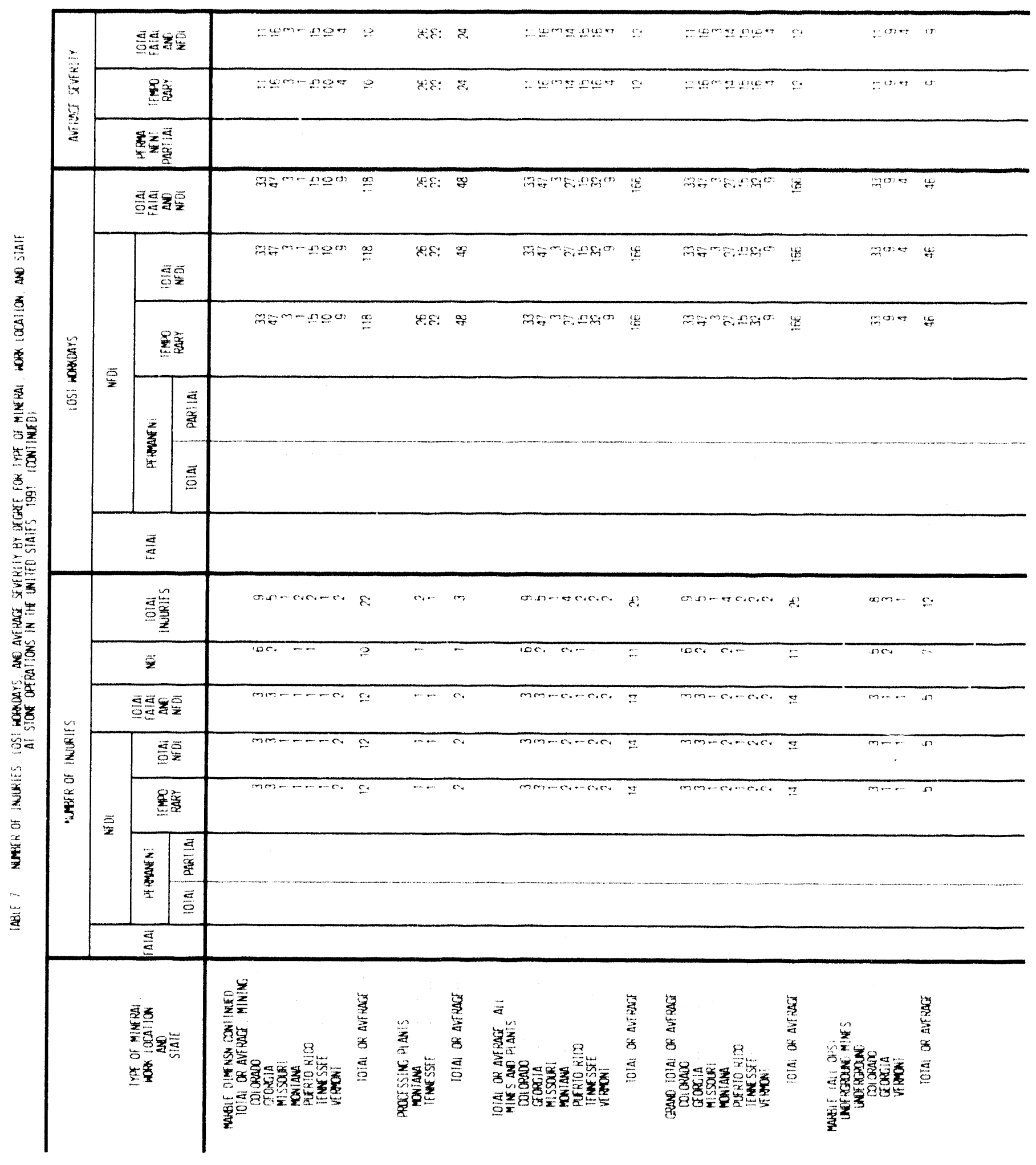



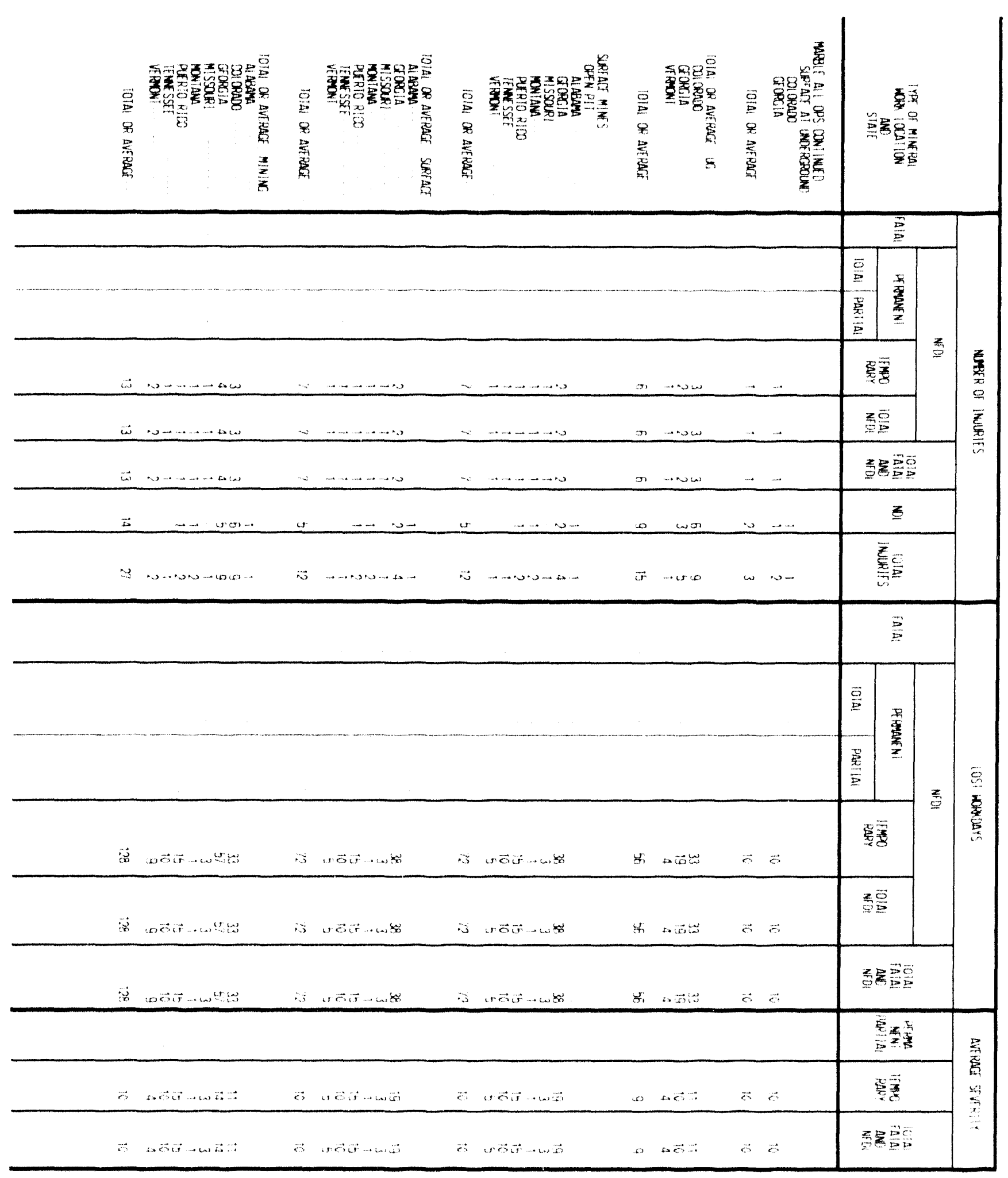


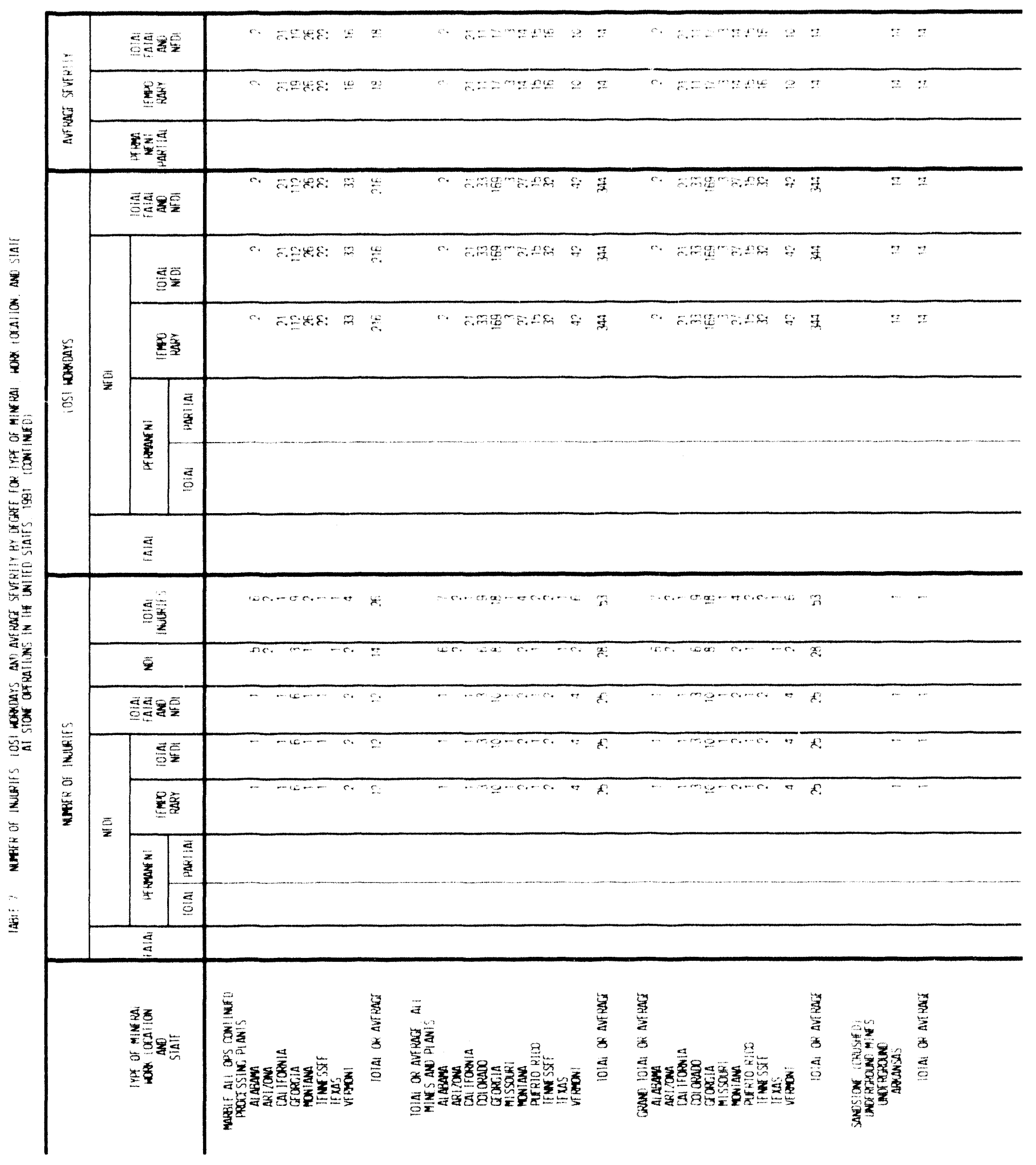




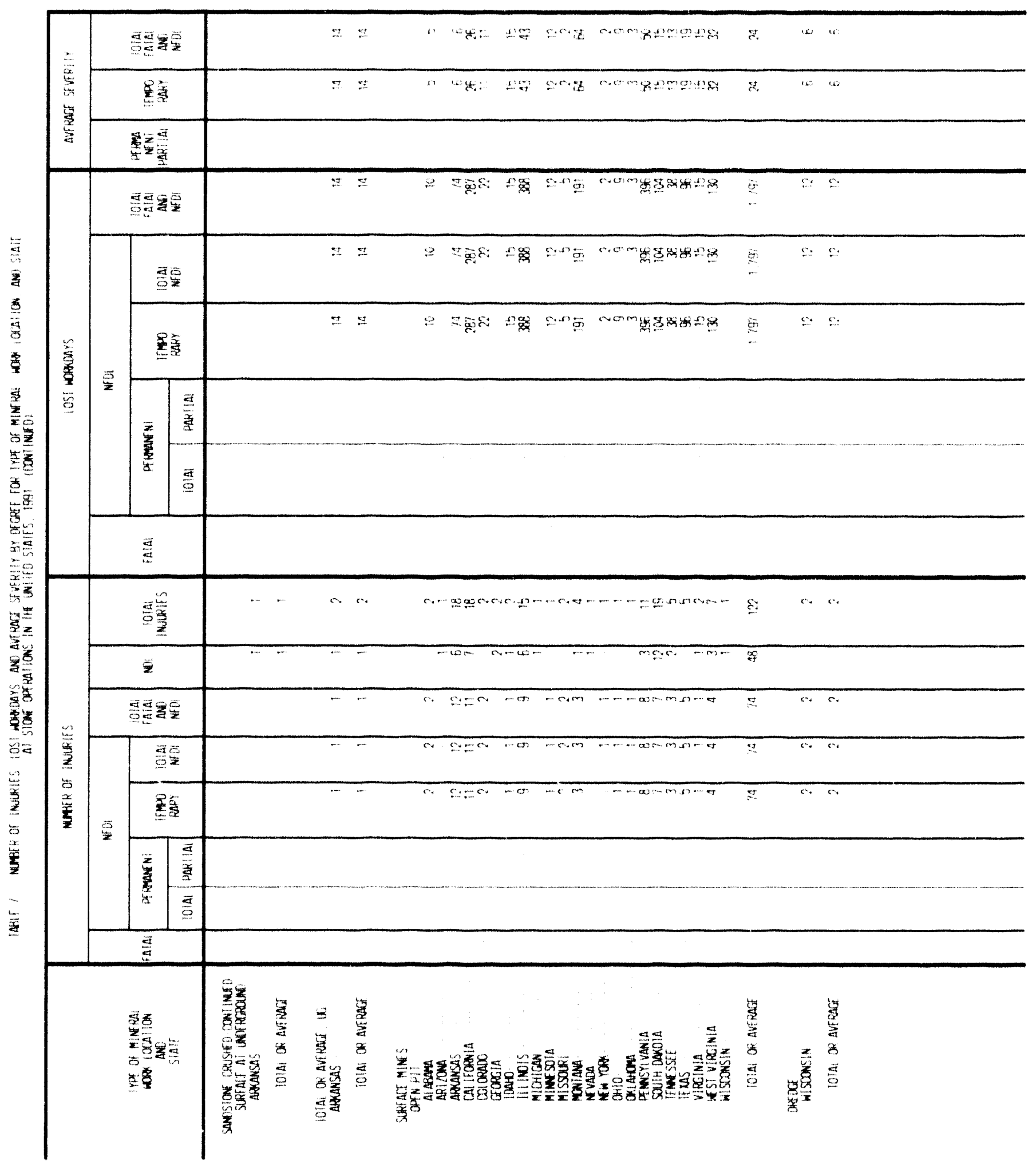




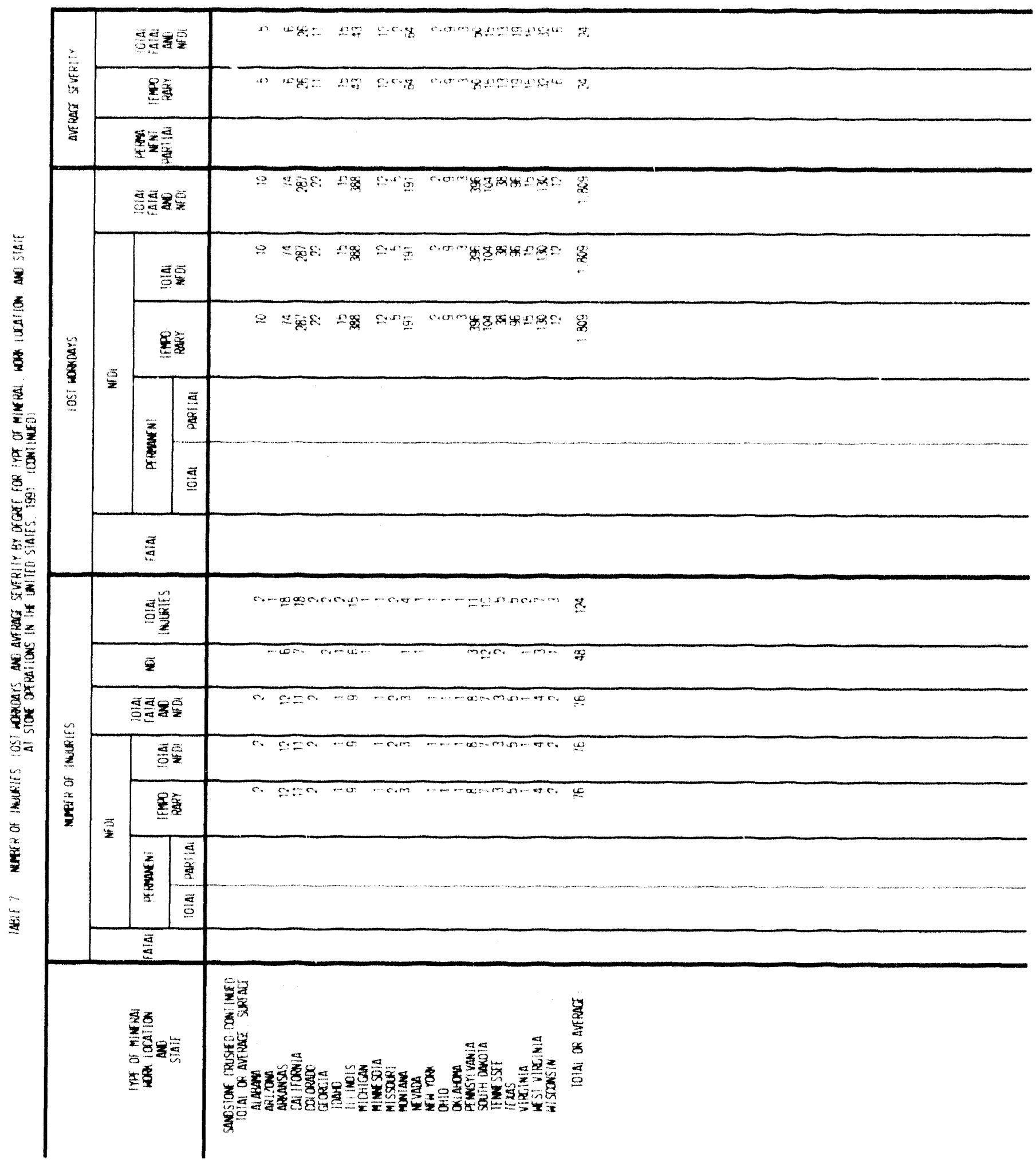




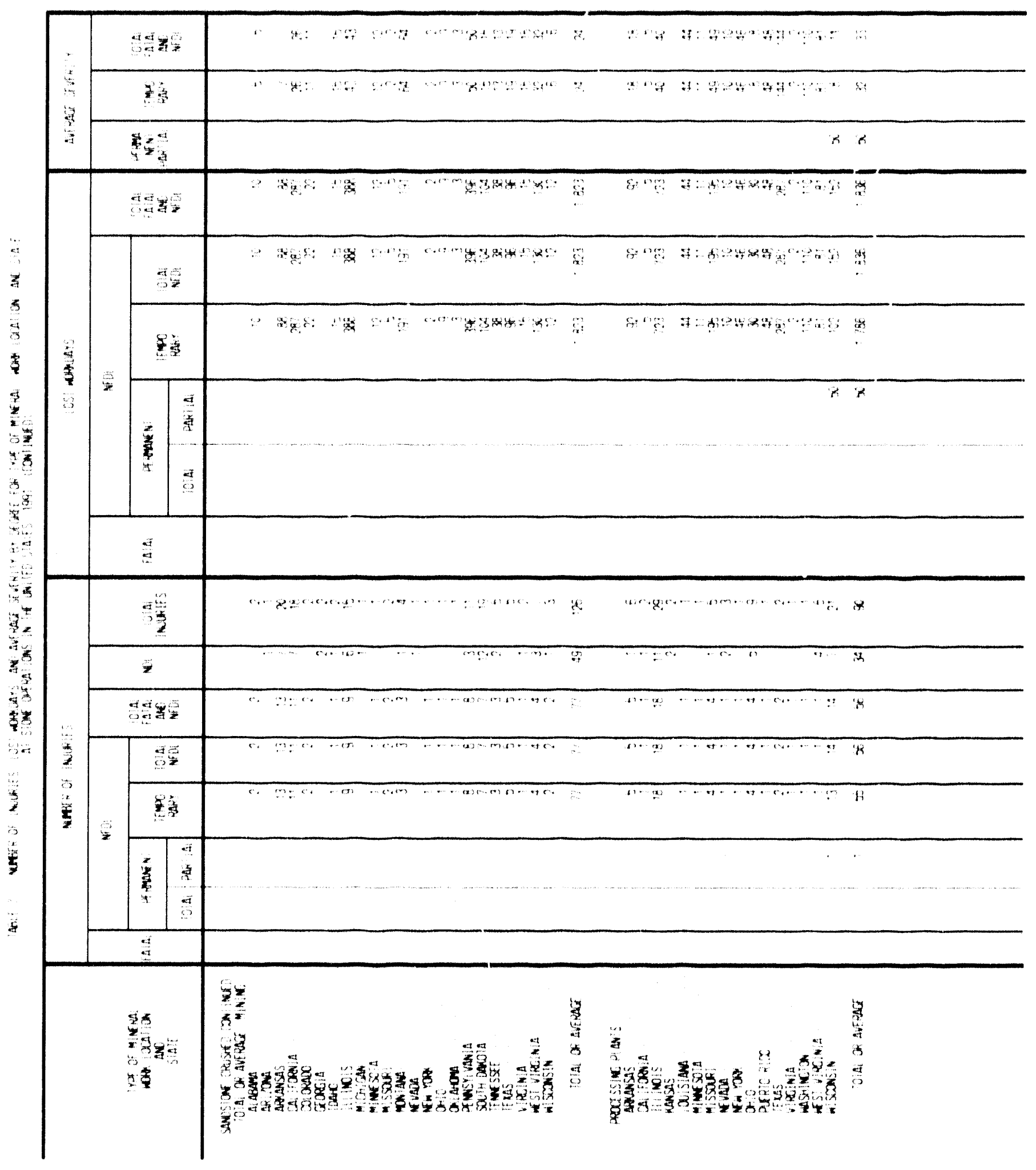




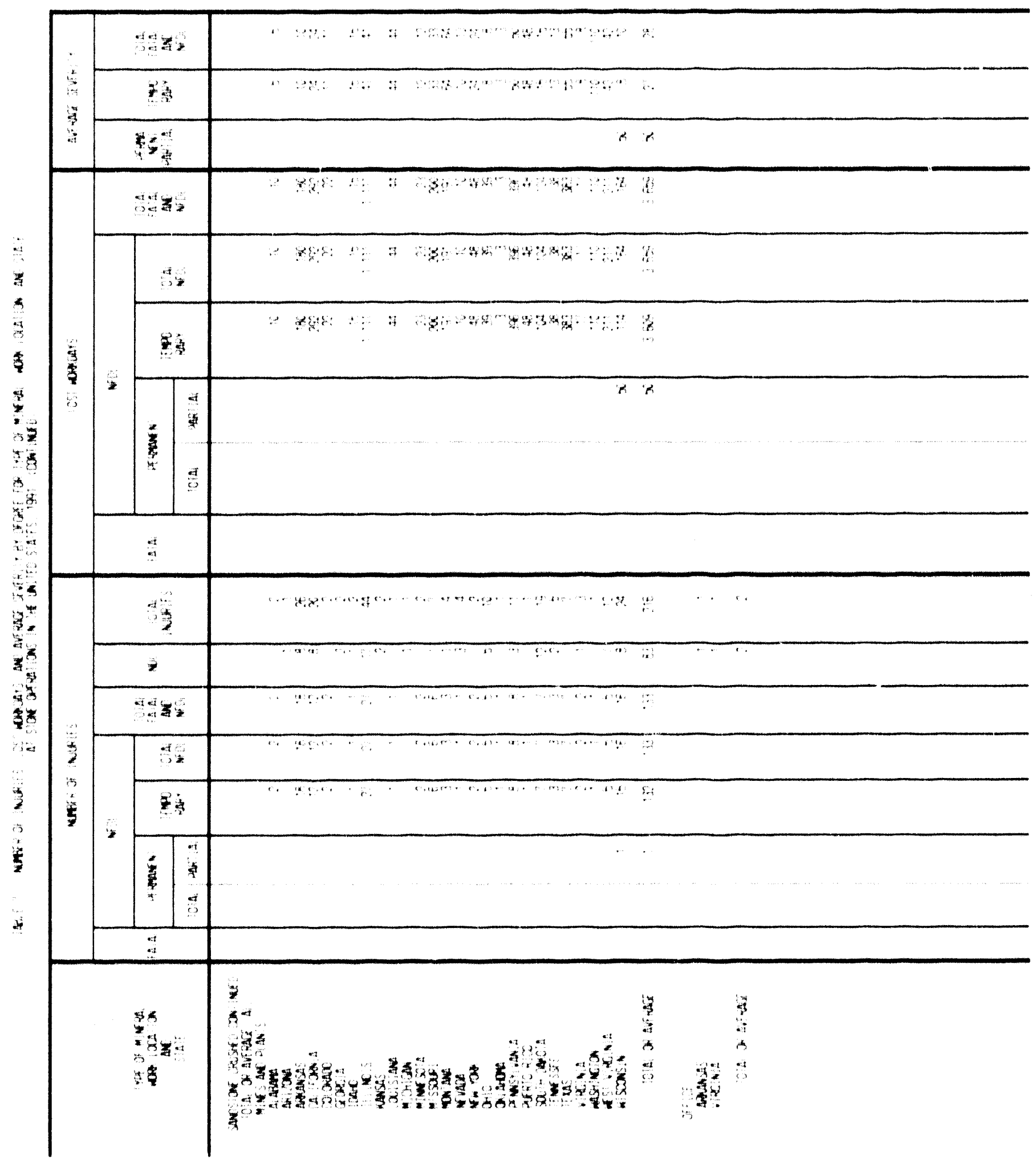




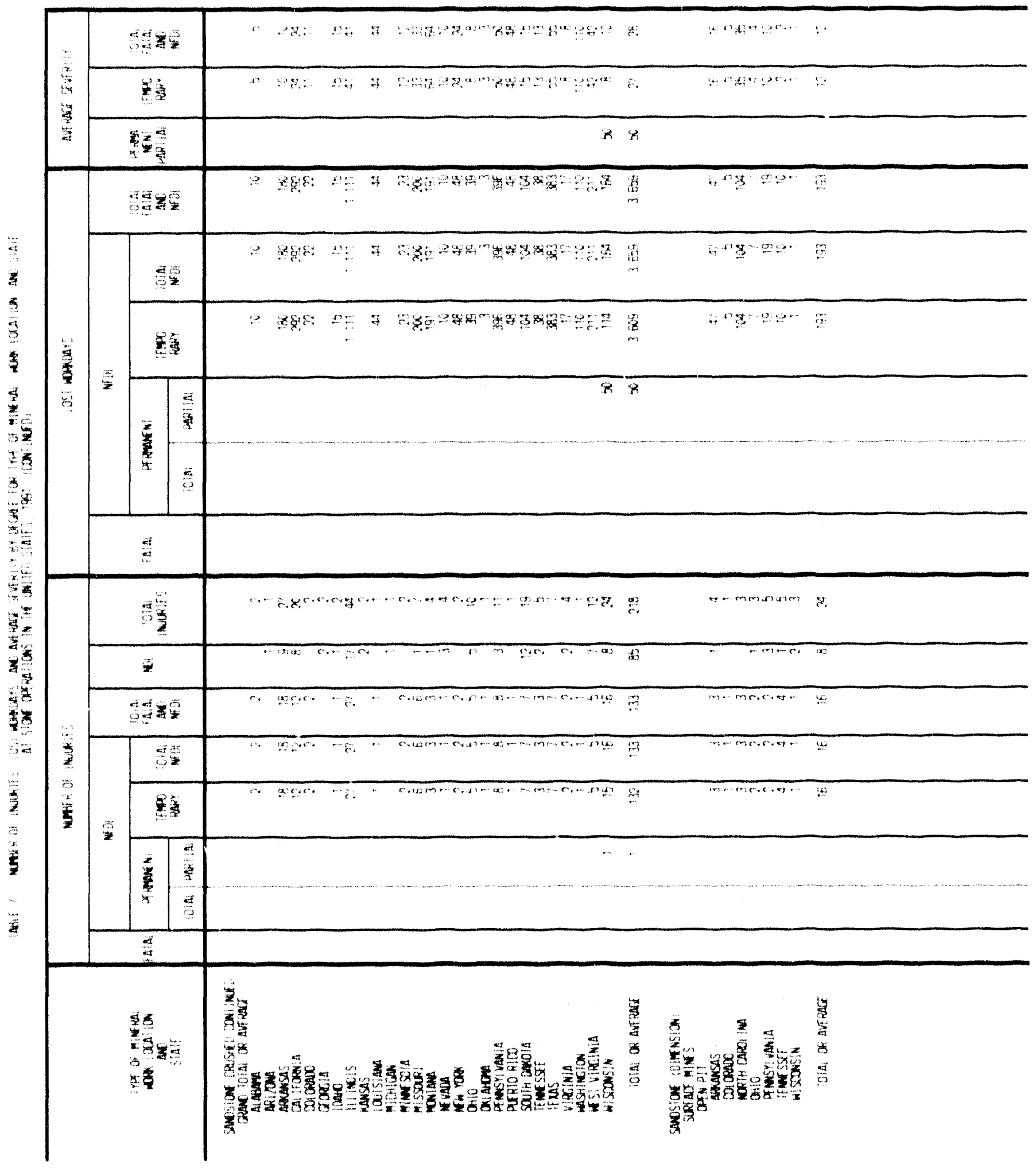




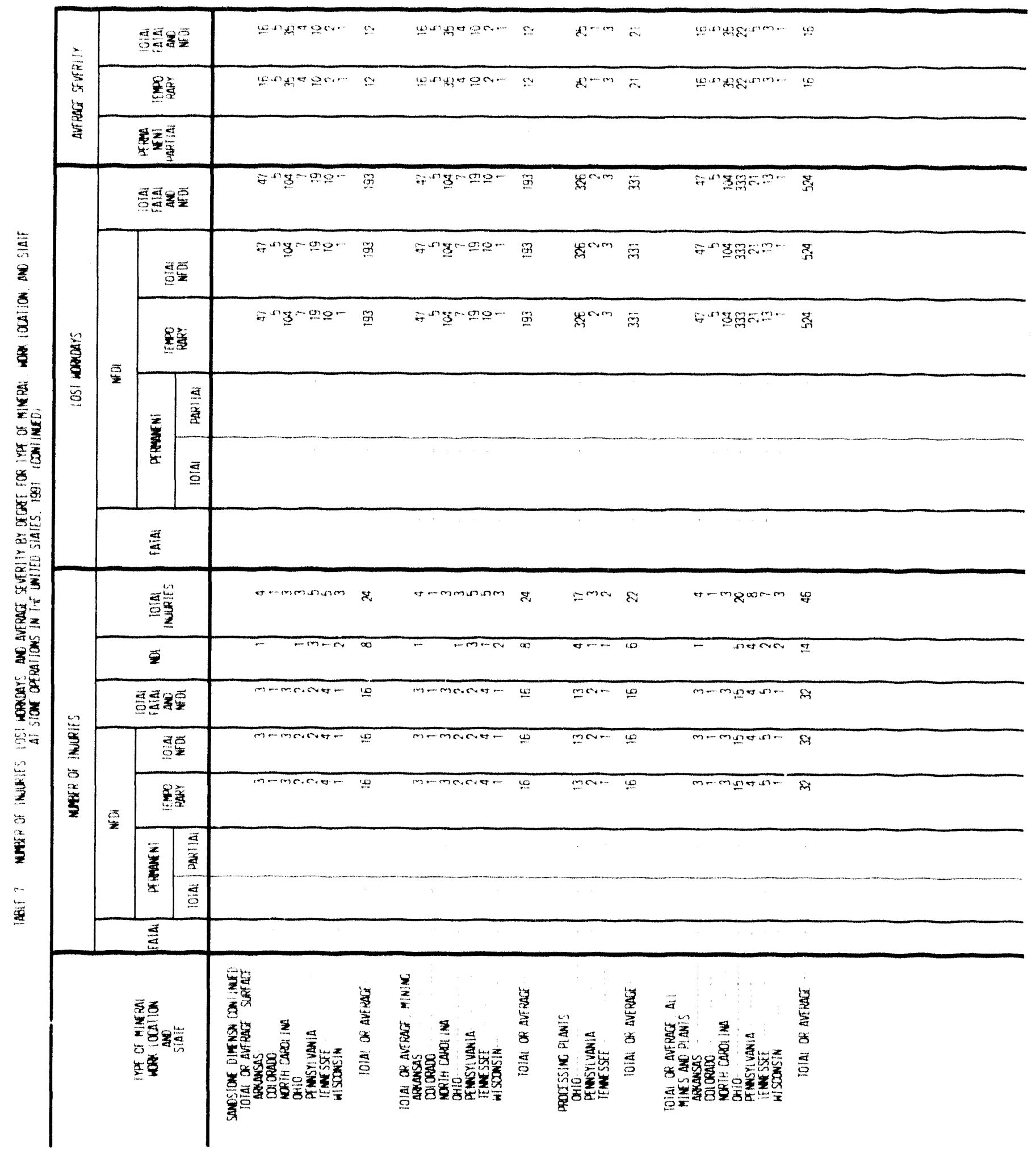



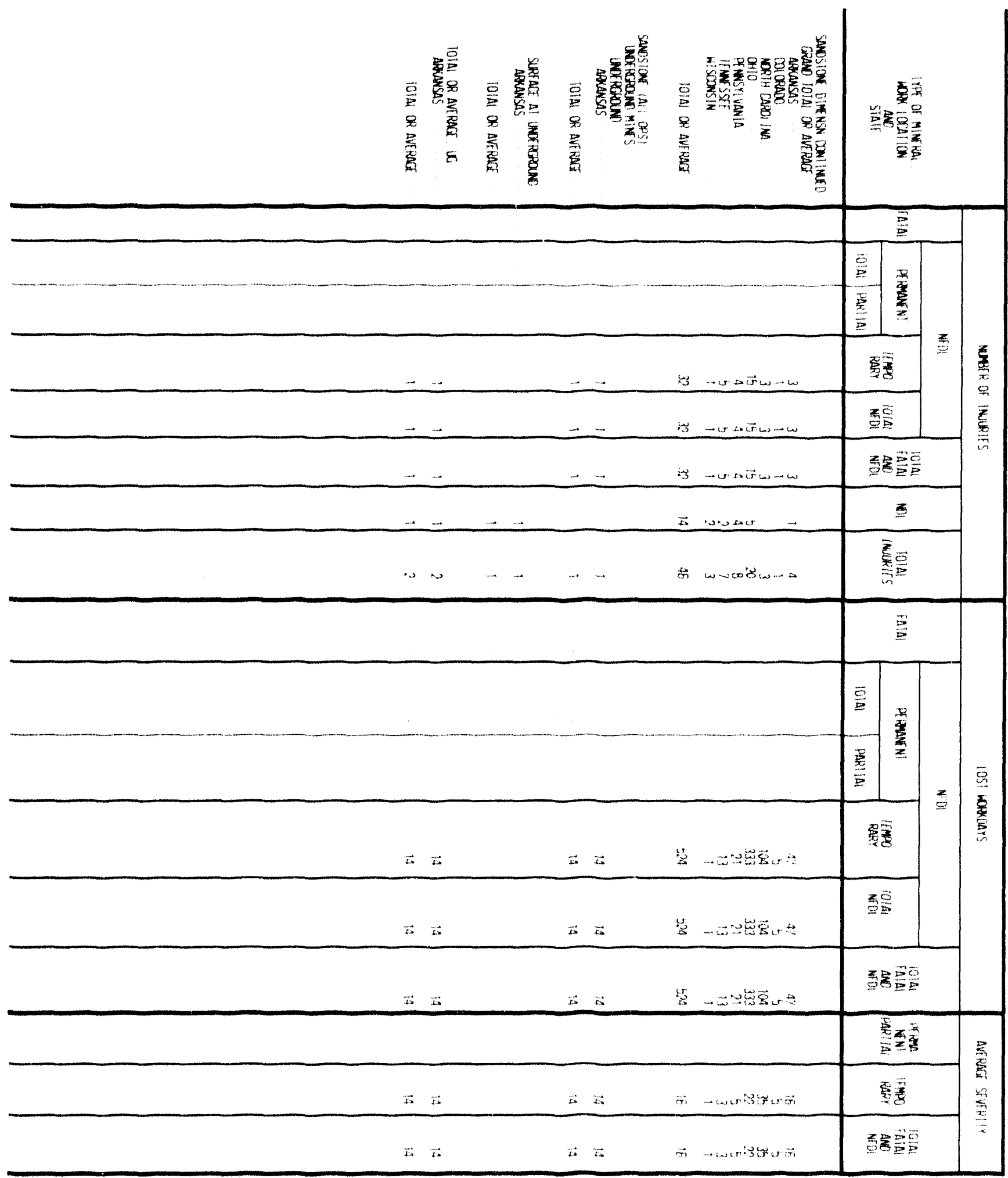


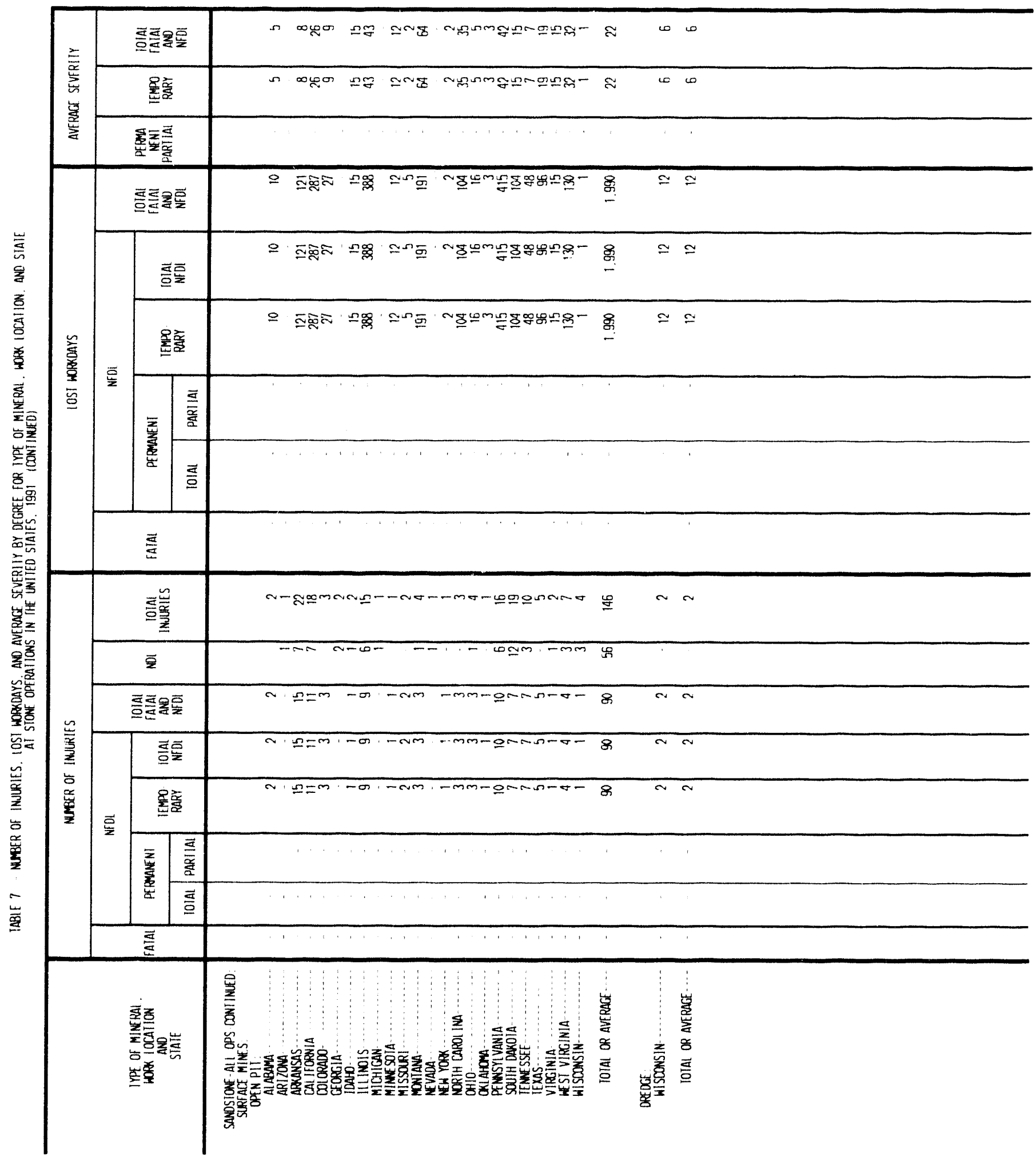




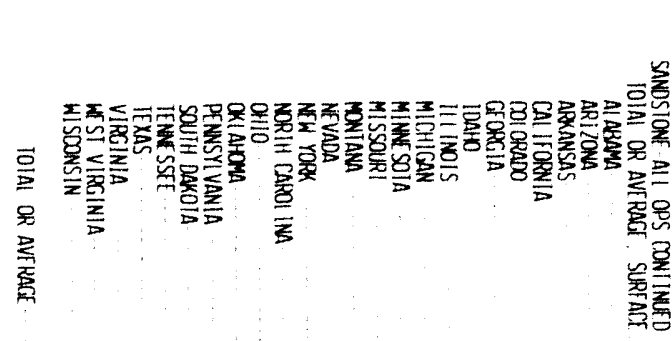

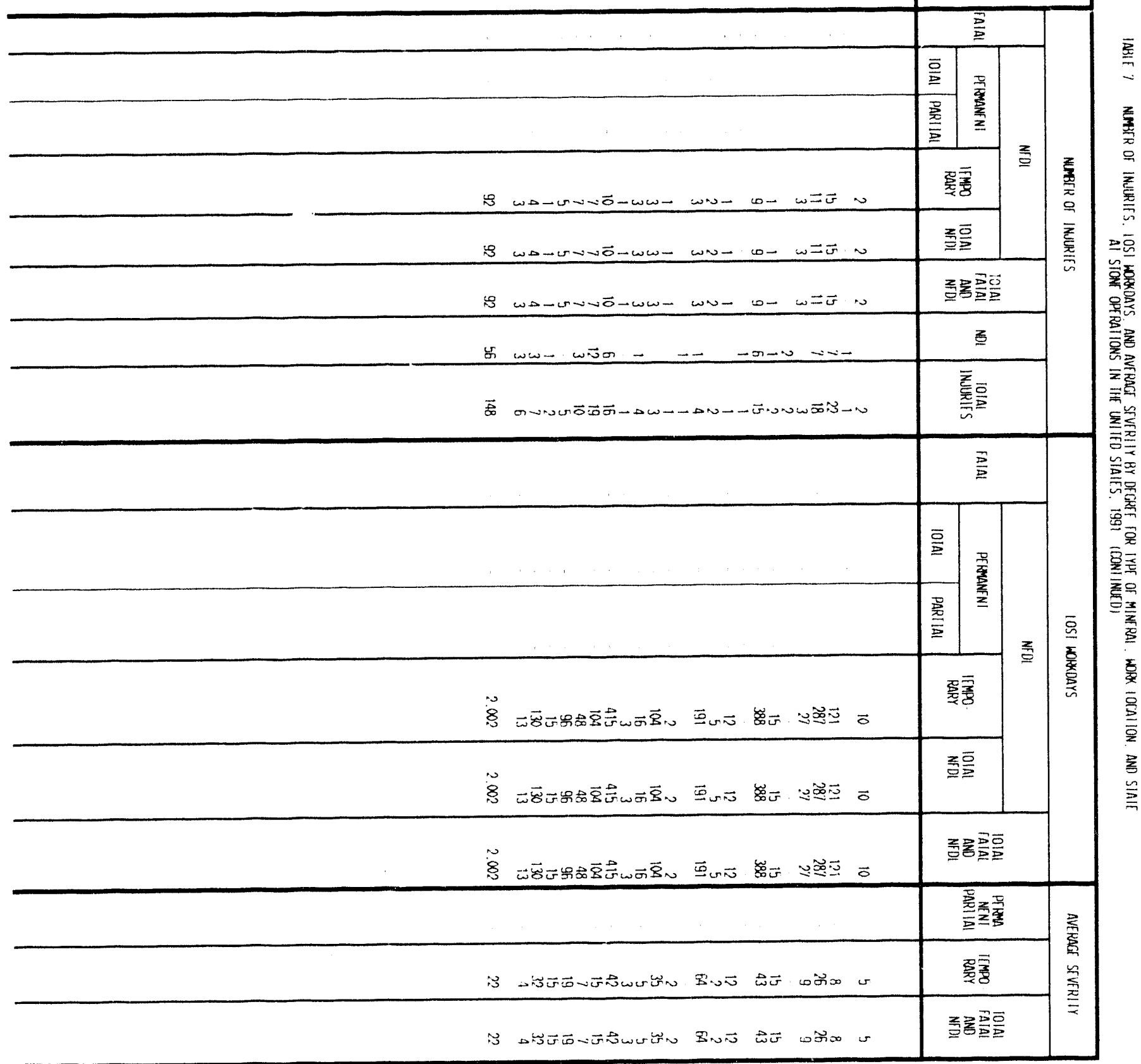




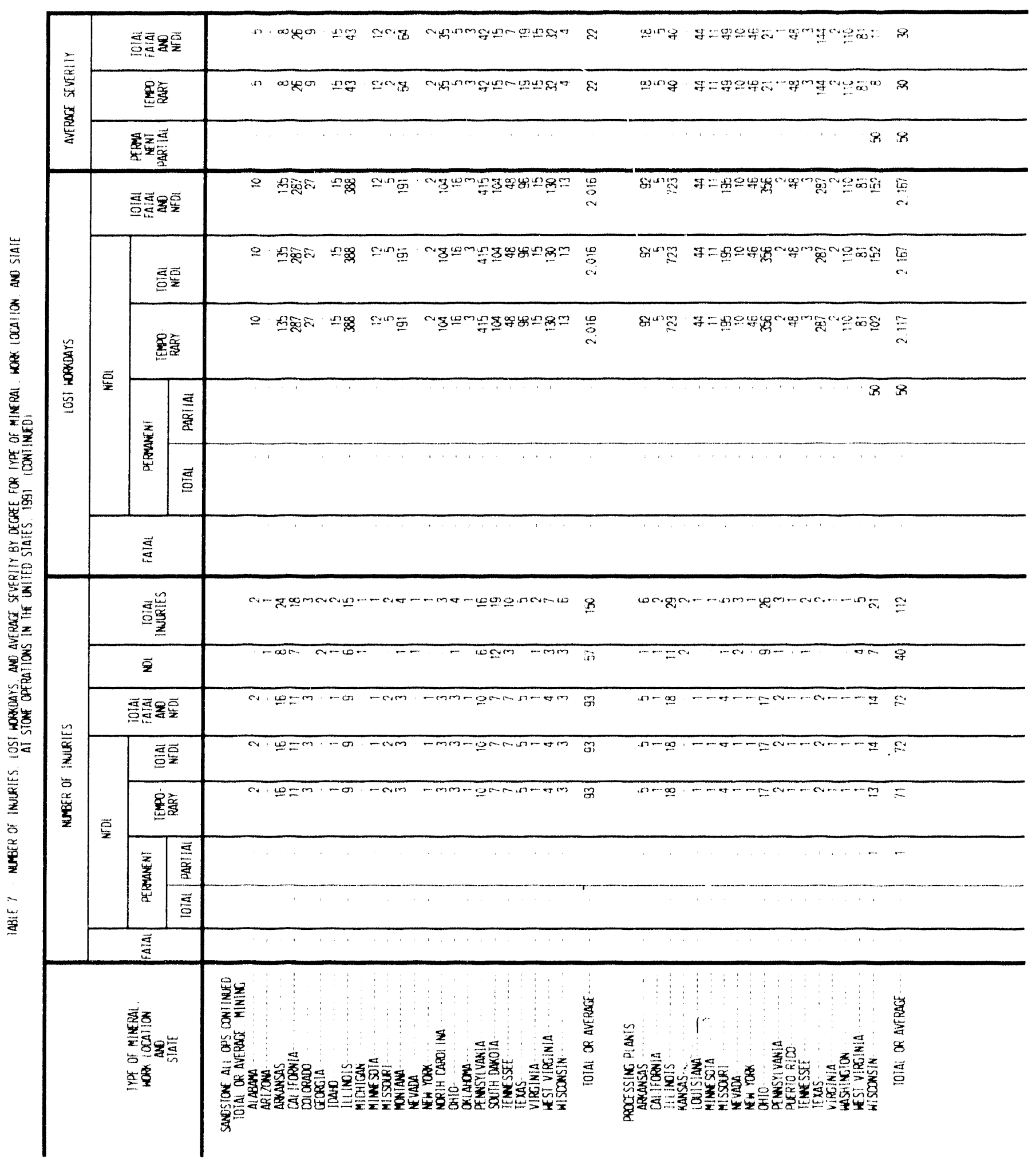




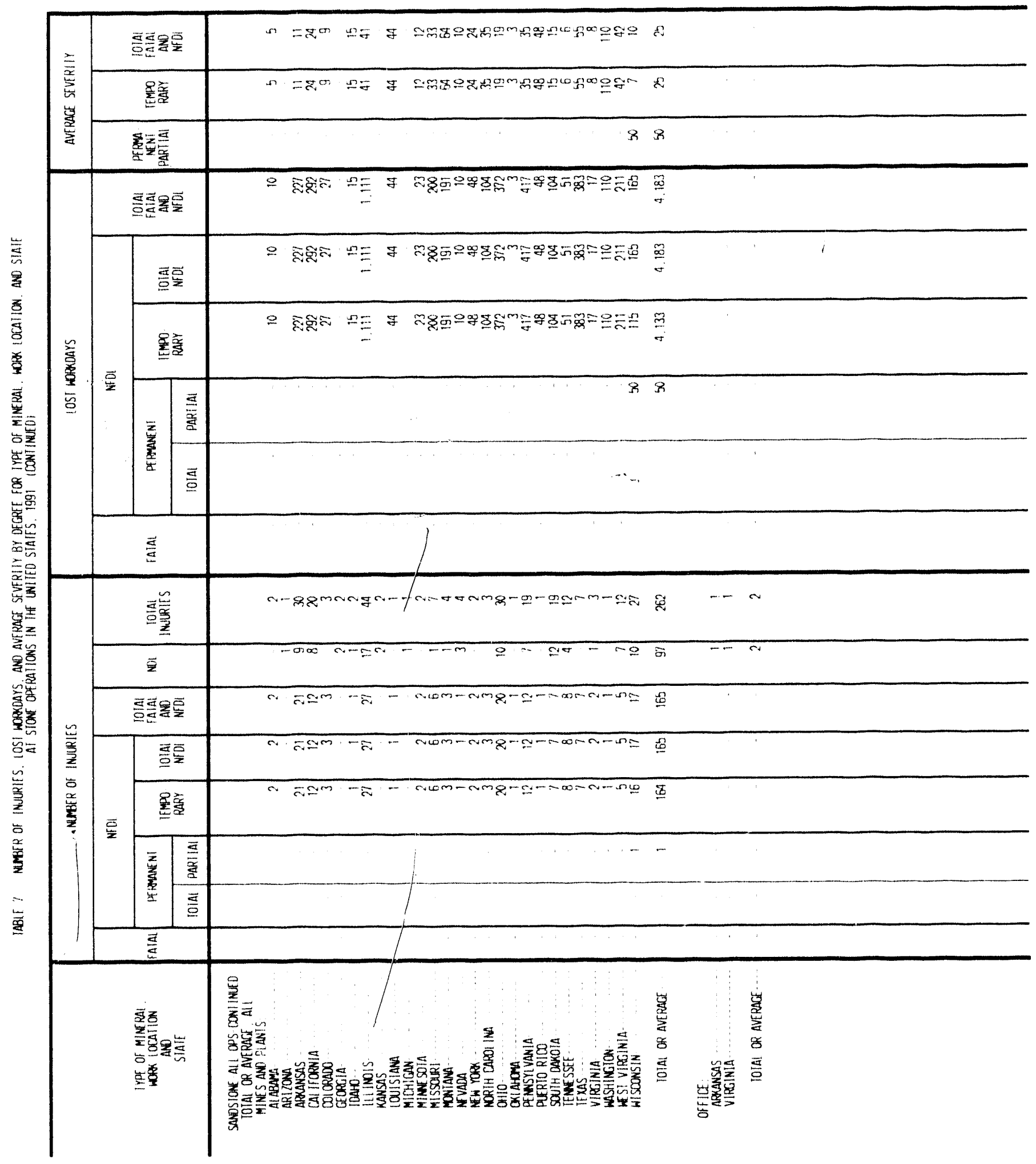




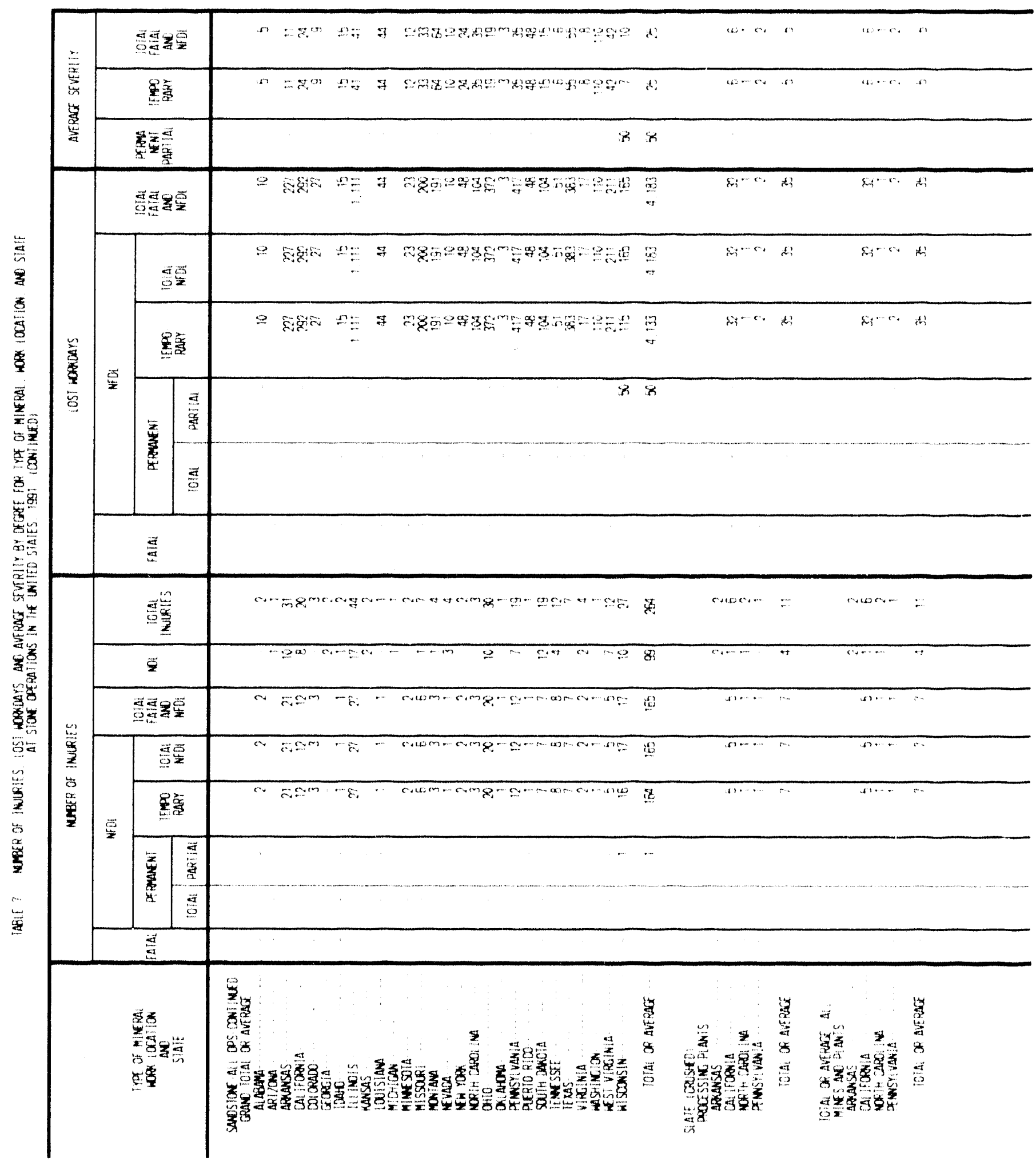




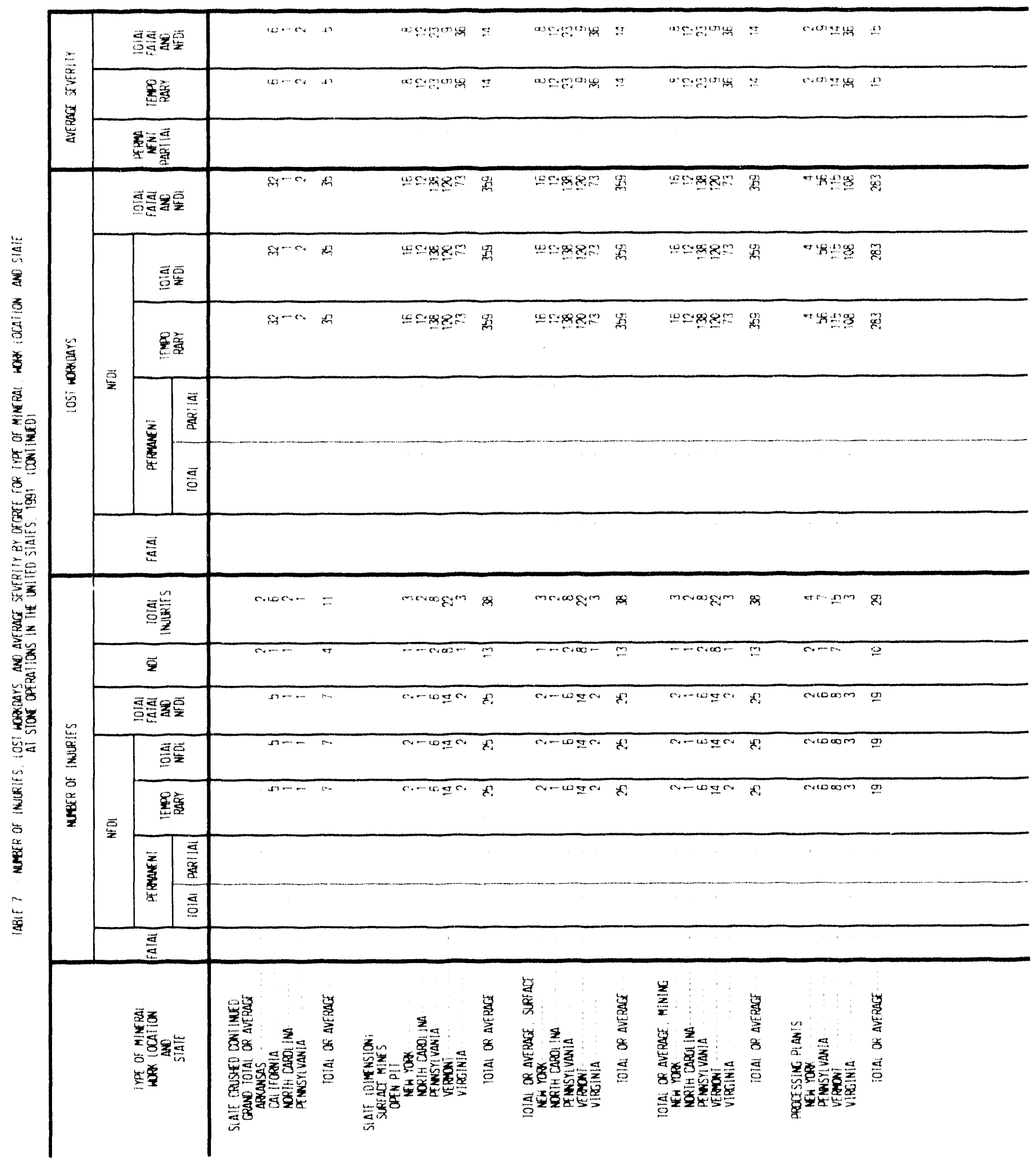




\begin{tabular}{|c|c|c|c|c|c|c|c|c|c|c|c|c|c|}
\hline$\geq$ & \multicolumn{3}{|c|}{ 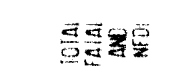 } & 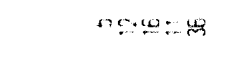 & $s$ & astex & $\therefore$ & acomong & $\because$ & $a \cos (1)$ b & $:$ & 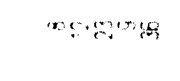 & $:$ \\
\hline$\sum_{i=n}^{n}$ & \multicolumn{3}{|c|}{ 影 } & $\operatorname{scos}=2$ & $s$ & foves:- & 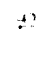 & aromos dis & $=$ & congen & $:$ & a) & $:$ \\
\hline 䒬 & \multicolumn{3}{|c|}{ 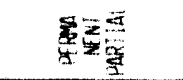 } & & & & & & & & & & \\
\hline \multirow{6}{*}{ 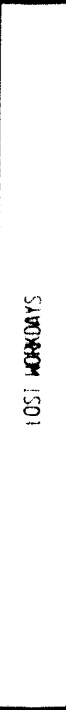 } & \multicolumn{3}{|c|}{ 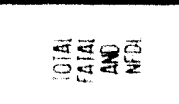 } & 859800 & F & 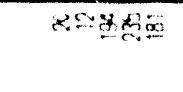 & 总 & 40.08 & 9 & $\subseteq a_{x}+\cdots$ & 4 & $45 \mathrm{gem}$ & 点 \\
\hline & \multirow{4}{*}{$\bar{E}$} & \multicolumn{2}{|c|}{ 至方 } & 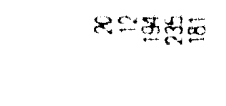 & 8 & 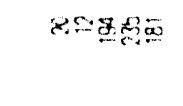 & 臹 & wos & 虽 & 5008 & 9 & 40.88 & 哭 \\
\hline & & \multicolumn{2}{|c|}{ 害害 } & 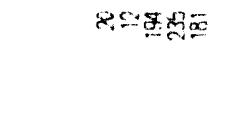 & 㖓 & 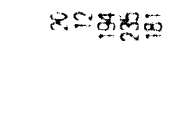 & 8 & $4=828$ & 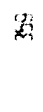 & $40 \% 8$ & 票 & $5092 m$ & 彎 \\
\hline & & \multirow{2}{*}{ 䈉 } & 吾 & & & & & & & & & & \\
\hline & & & $\stackrel{a}{\underline{a}}$ & & & & & & & & & & \\
\hline & \multicolumn{3}{|c|}{$\underset{\mathrm{a}}{\mathrm{a}}$} & & & & & & & & & & \\
\hline \multirow{8}{*}{ 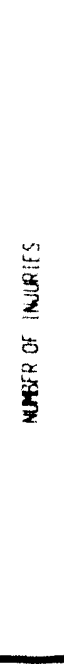 } & \multicolumn{3}{|c|}{ 琶愛 } & $\therefore a n$ & $i$ & 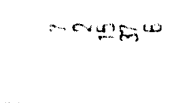 & 6 & $m\left(\cdots, y^{m}\right.$ & $x$ & morosgin & $\infty$ & $m[+\infty) g_{i}$ & $m$ \\
\hline & \multicolumn{3}{|c|}{$\ddot{2}$} & $m-m s$ & 8 & $m+m n$ & $\pi$ & $--(x)$ & 9 & reto & $m$ & & 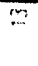 \\
\hline & \multicolumn{3}{|c|}{ 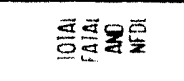 } & $+\cdots z^{\prime \prime}$ & $\because$ & $x-58$ & * & $\cdots+\cos 00$ & ? & $\cdots+b \geq 0$ & $f$ & $\alpha, \omega)$ & ह? \\
\hline & \multicolumn{3}{|c|}{ 可宸 } & $7-x y^{\prime \prime}$ & 7 & $7-5845$ & 7 & $\cdots-\omega \pm 0$ & E. & 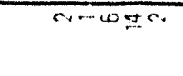 & 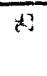 & $\alpha+1+9 n$ & 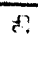 \\
\hline & है & \multicolumn{2}{|c|}{ 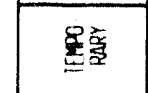 } & $+\cdots \cdot g_{i n}^{n}$ & g & $-5-58$ & 7 & $\alpha-10 \geq 0$ & 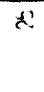 & $\alpha+\infty \pm$ & A & 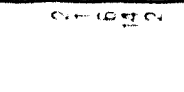 & \&? \\
\hline & & \multirow{2}{*}{ 惫 } & $\vec{a}$ & & & & & & & & & & \\
\hline & & & $\frac{\pi}{0}$ & & & & & & & & & & \\
\hline & \multicolumn{3}{|c|}{$\frac{E}{3}$} & & & & & & & & & & \\
\hline & & 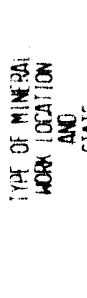 & & 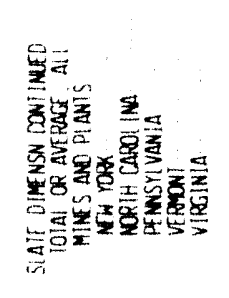 & 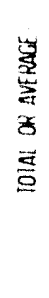 & 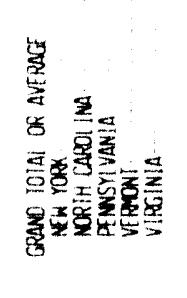 & 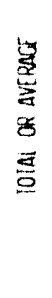 & 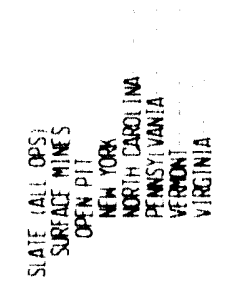 & 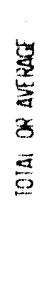 & 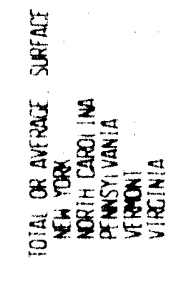 & 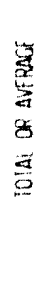 & 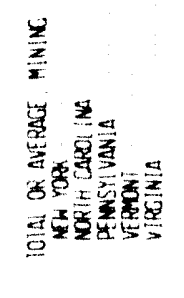 & $\begin{array}{l}3 \\
\frac{3}{2} \\
0 \\
a \\
a \\
0\end{array}$ \\
\hline
\end{tabular}




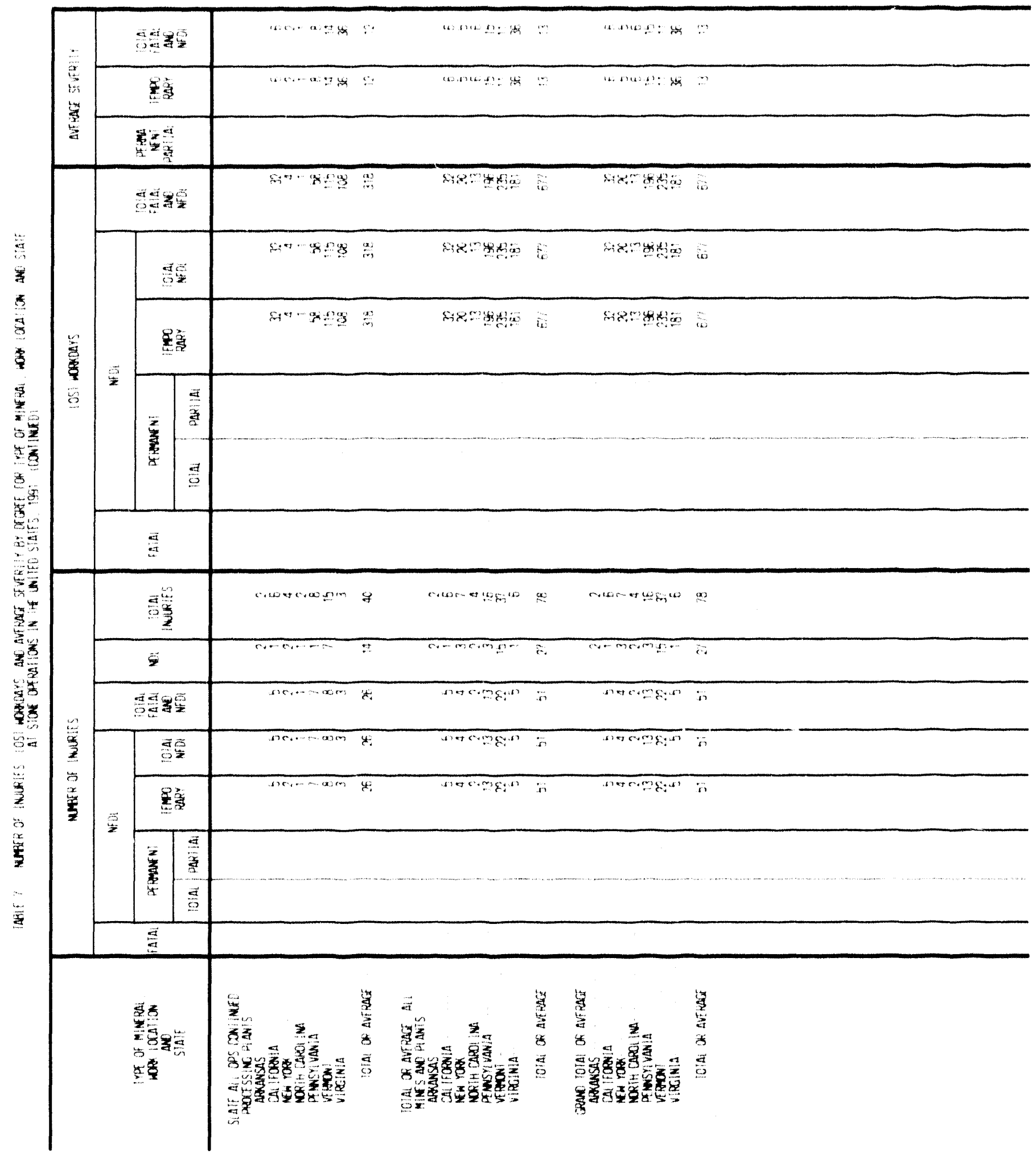




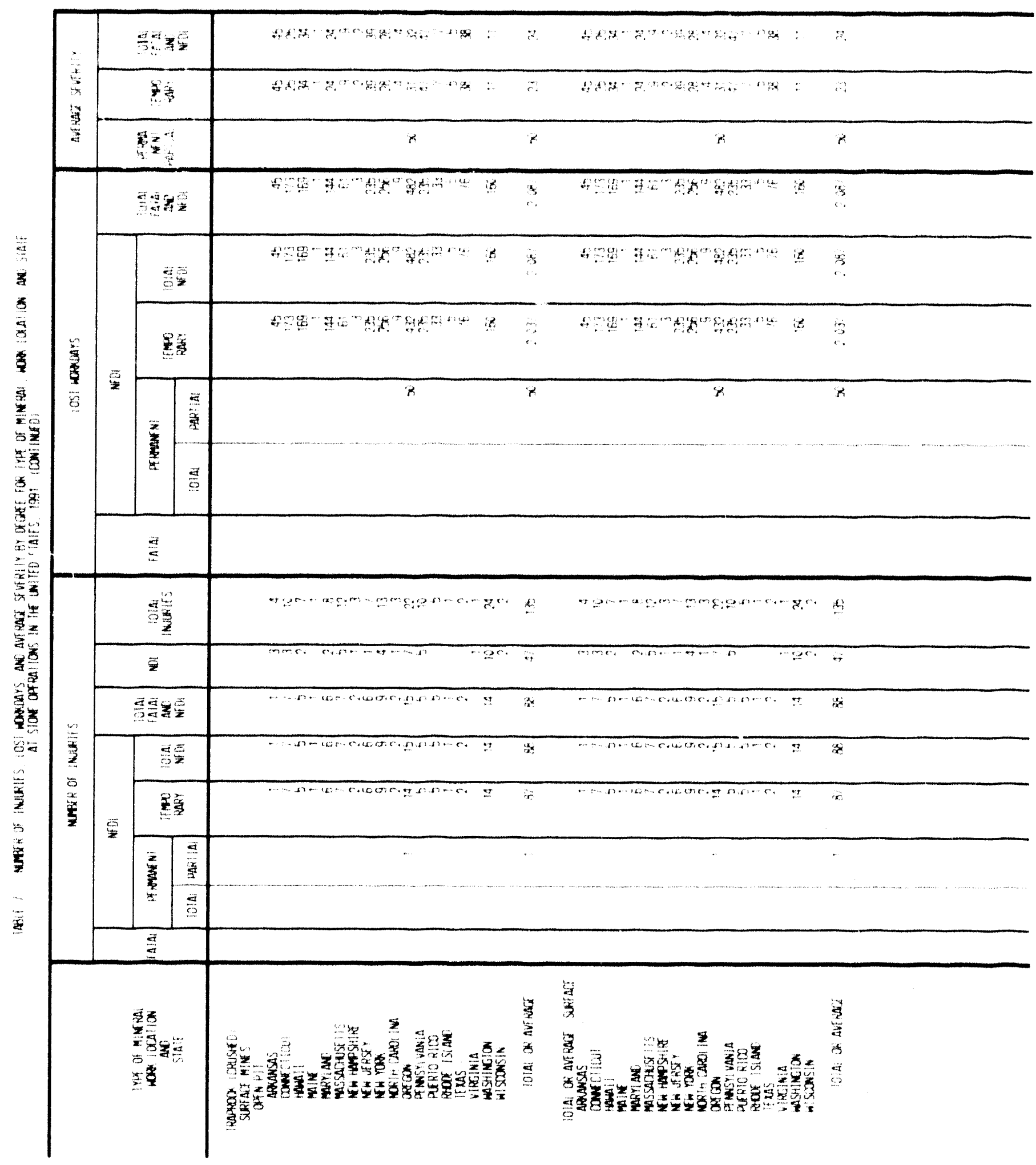




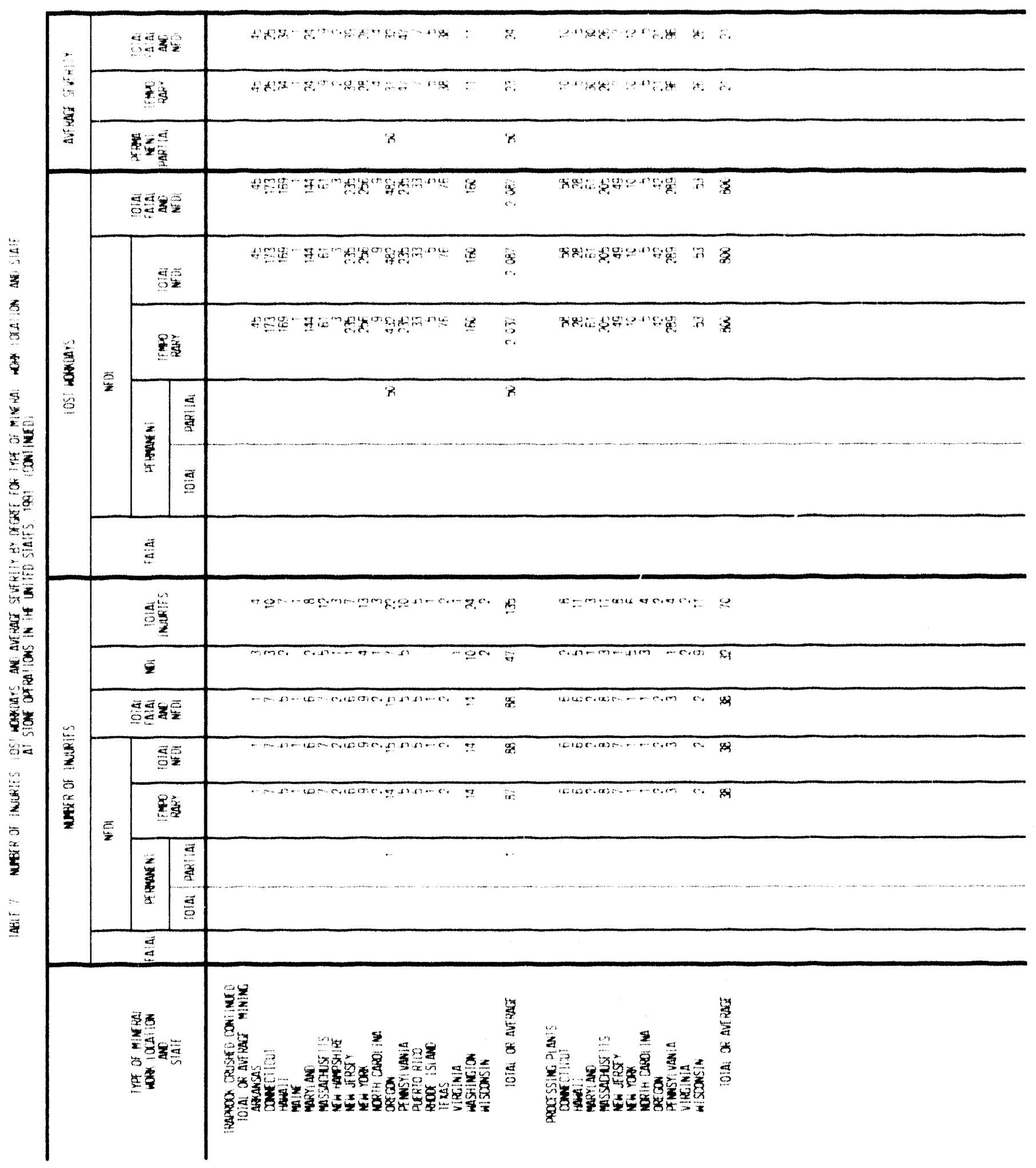




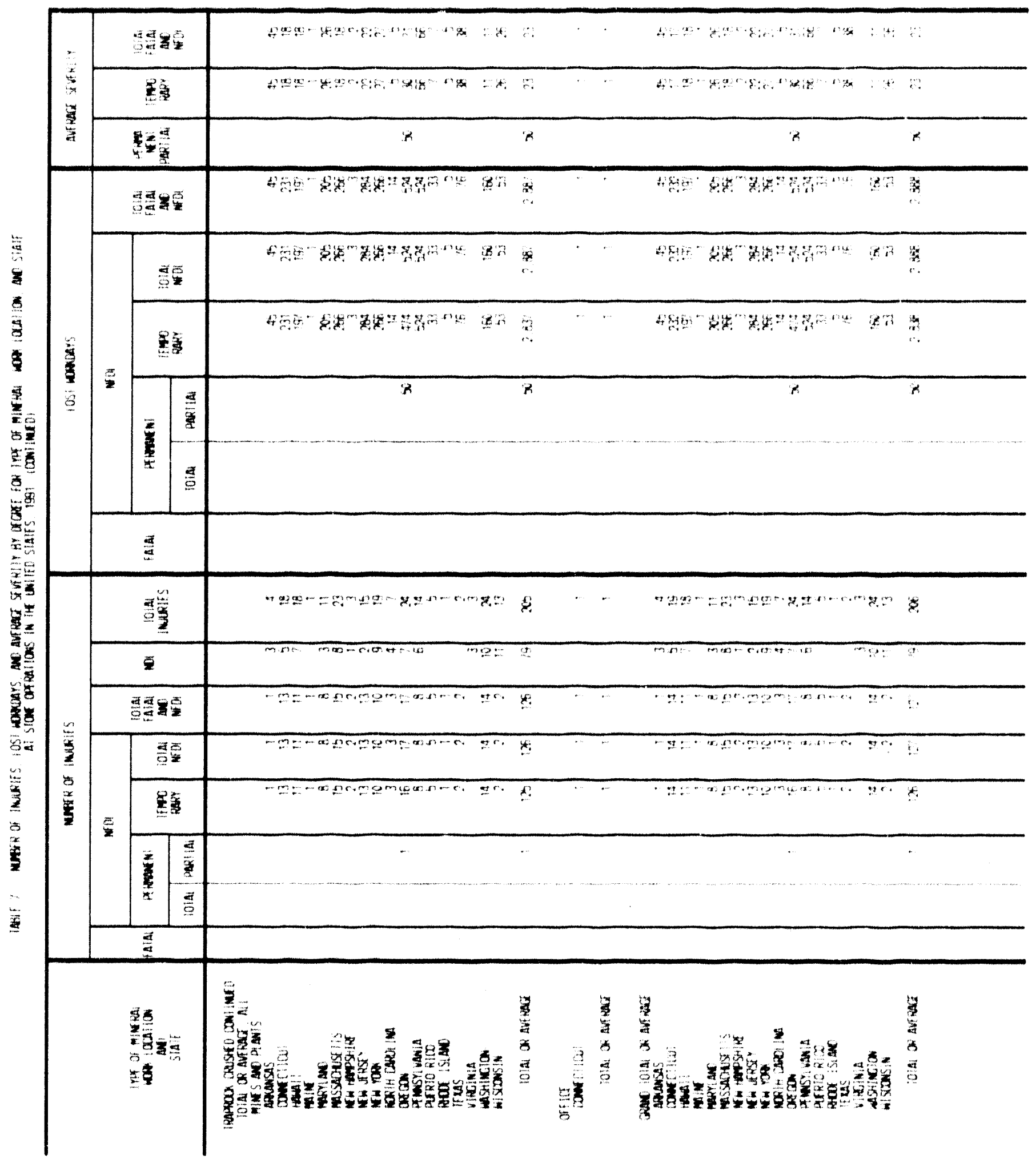




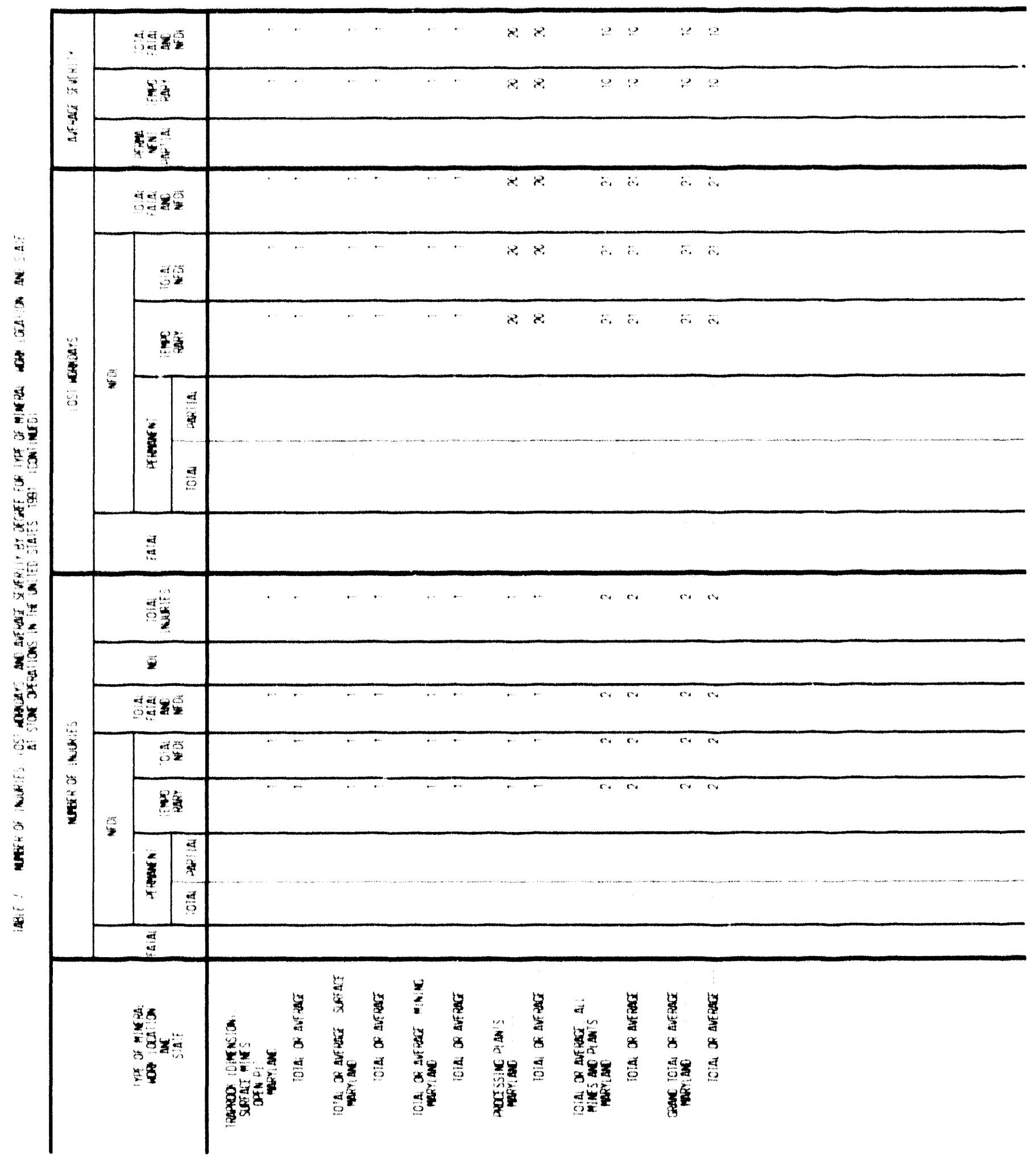




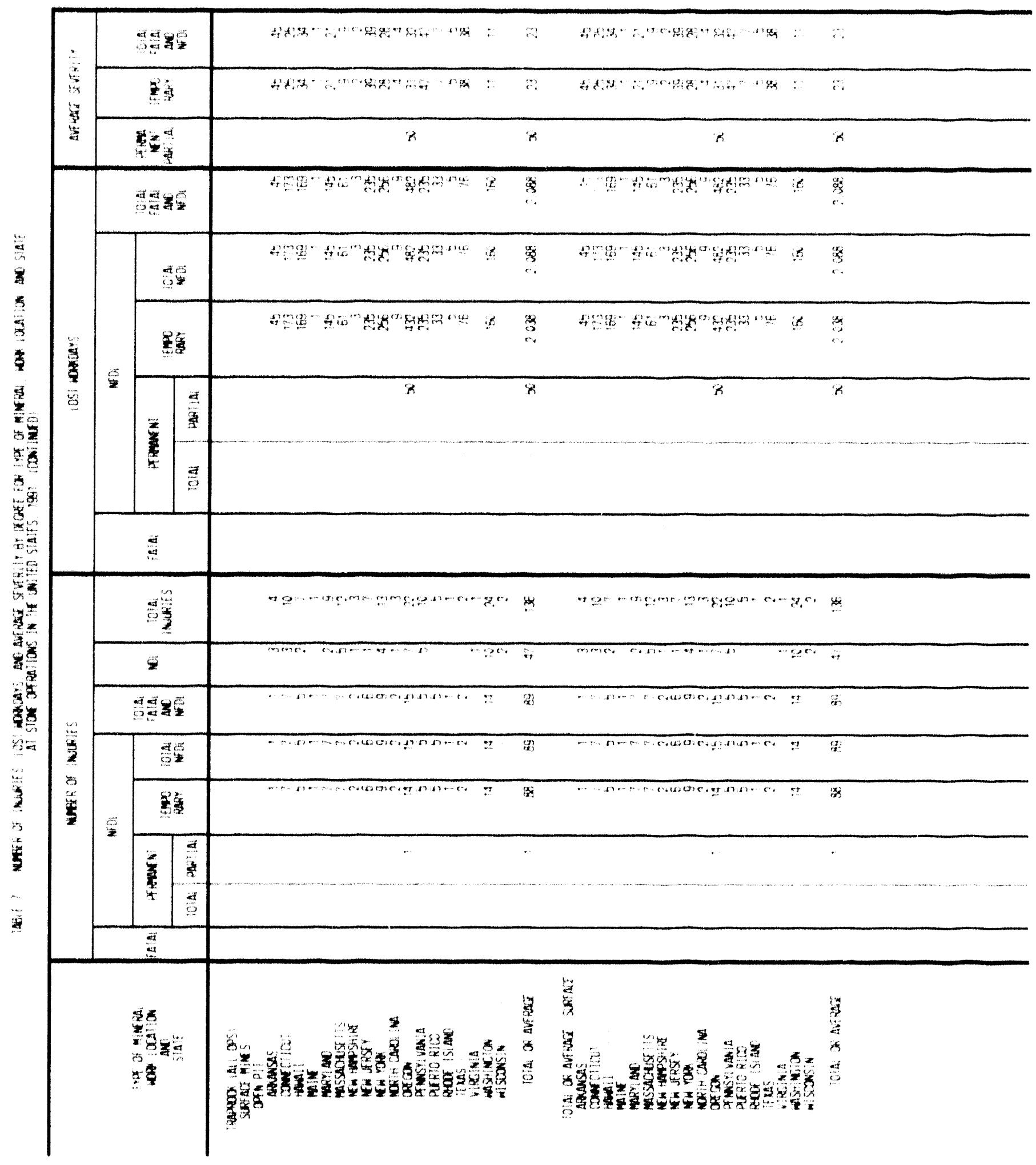




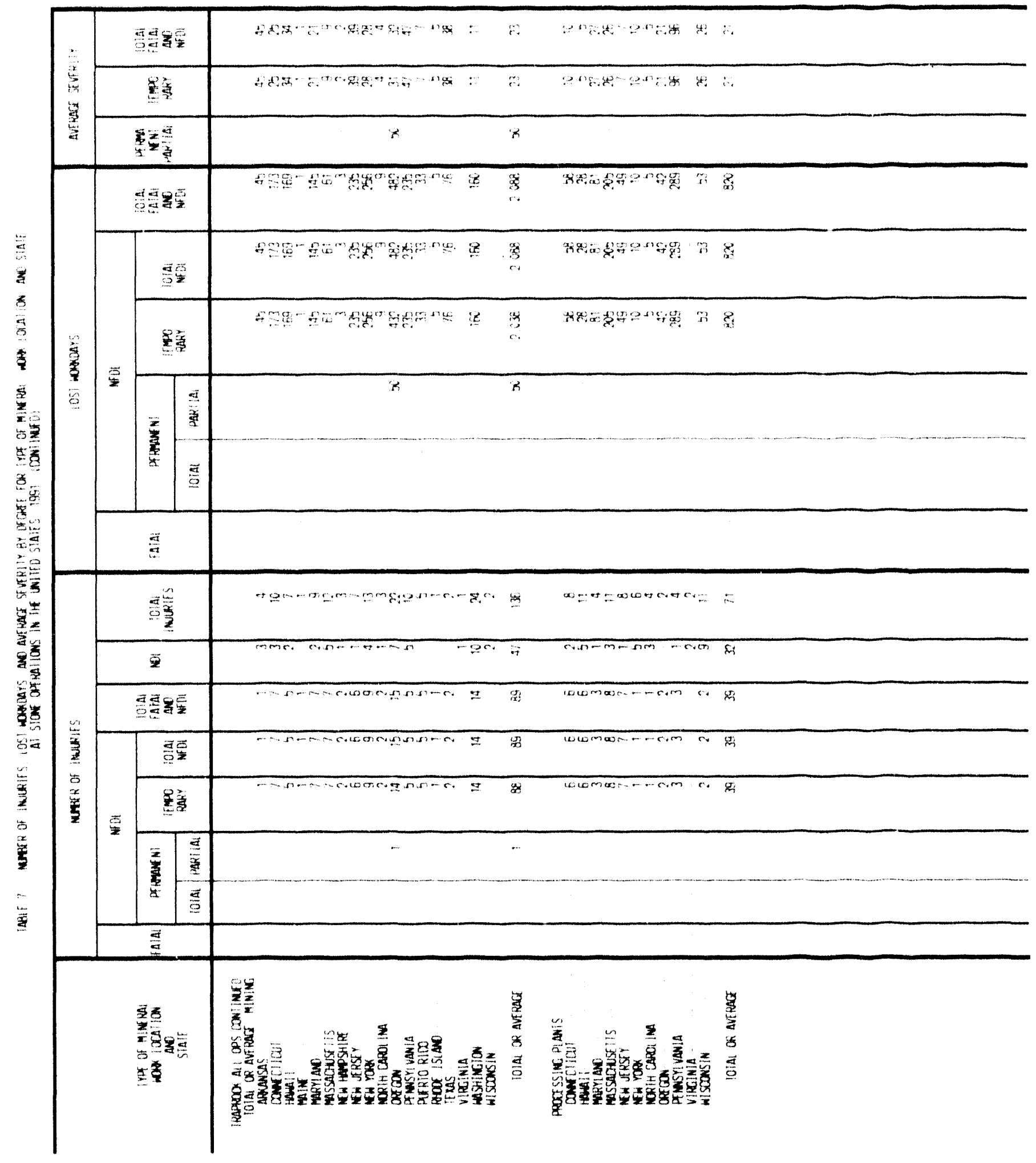




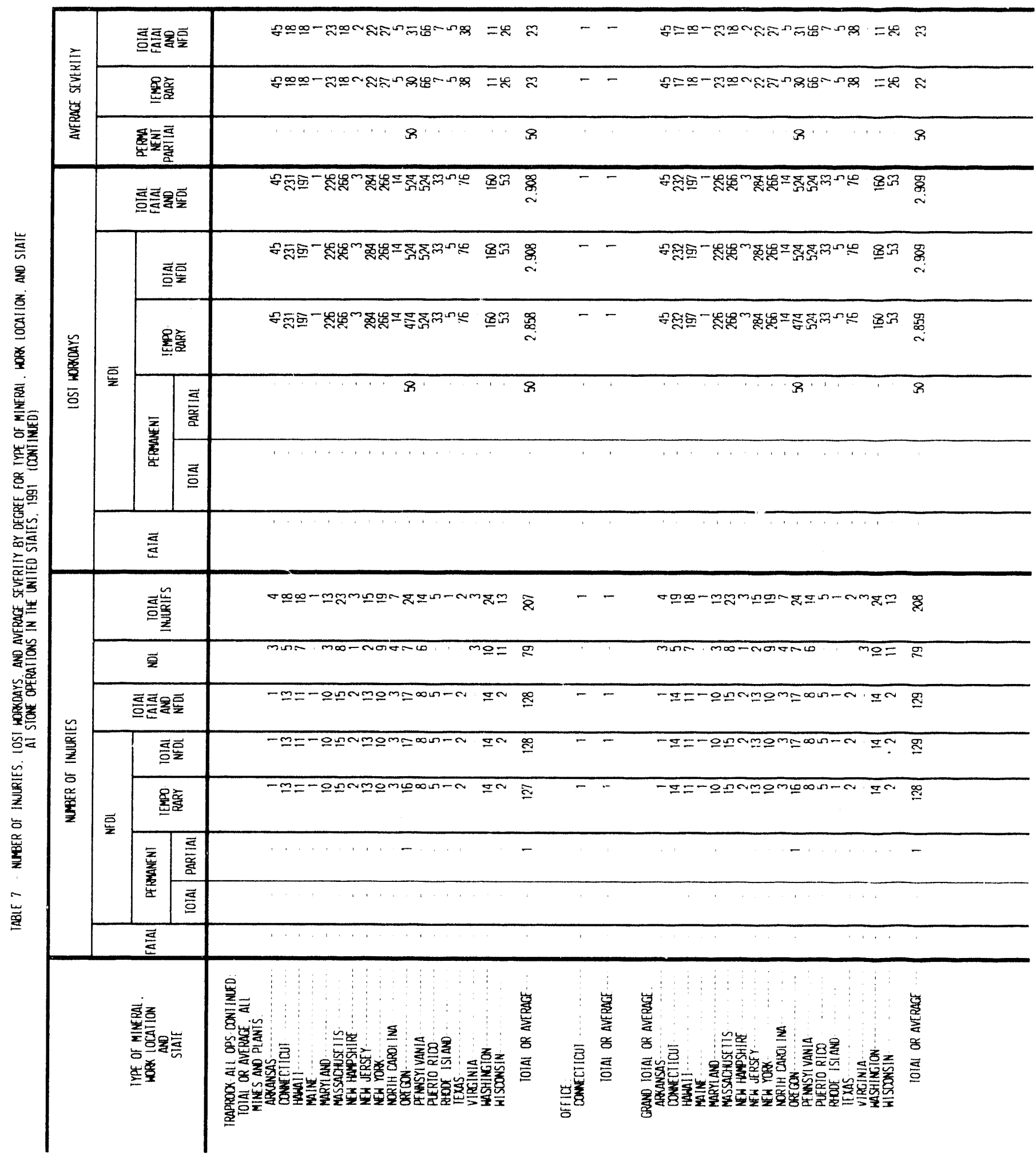




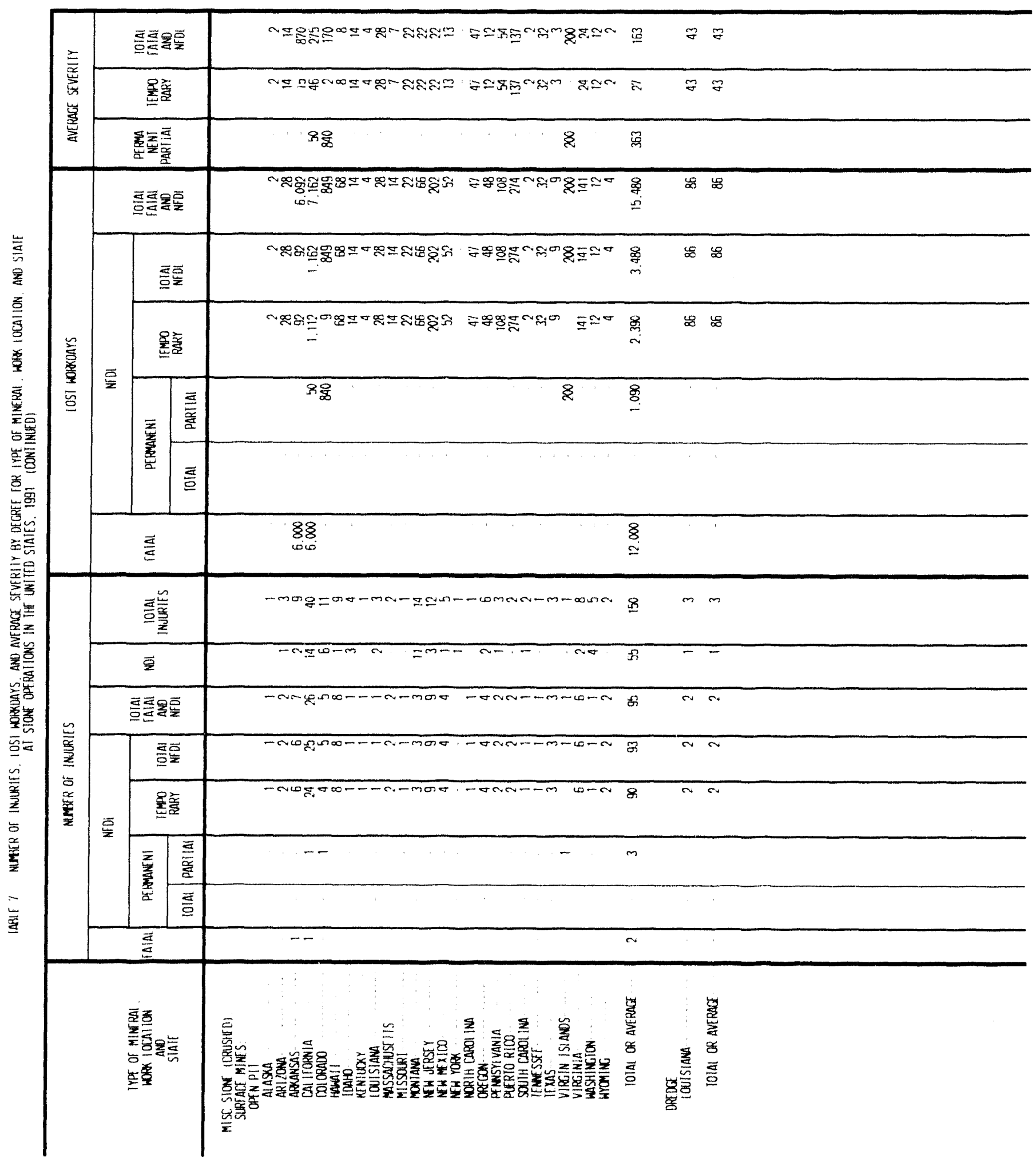




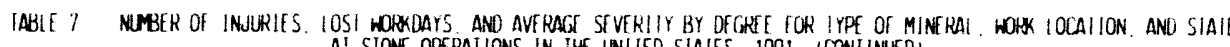

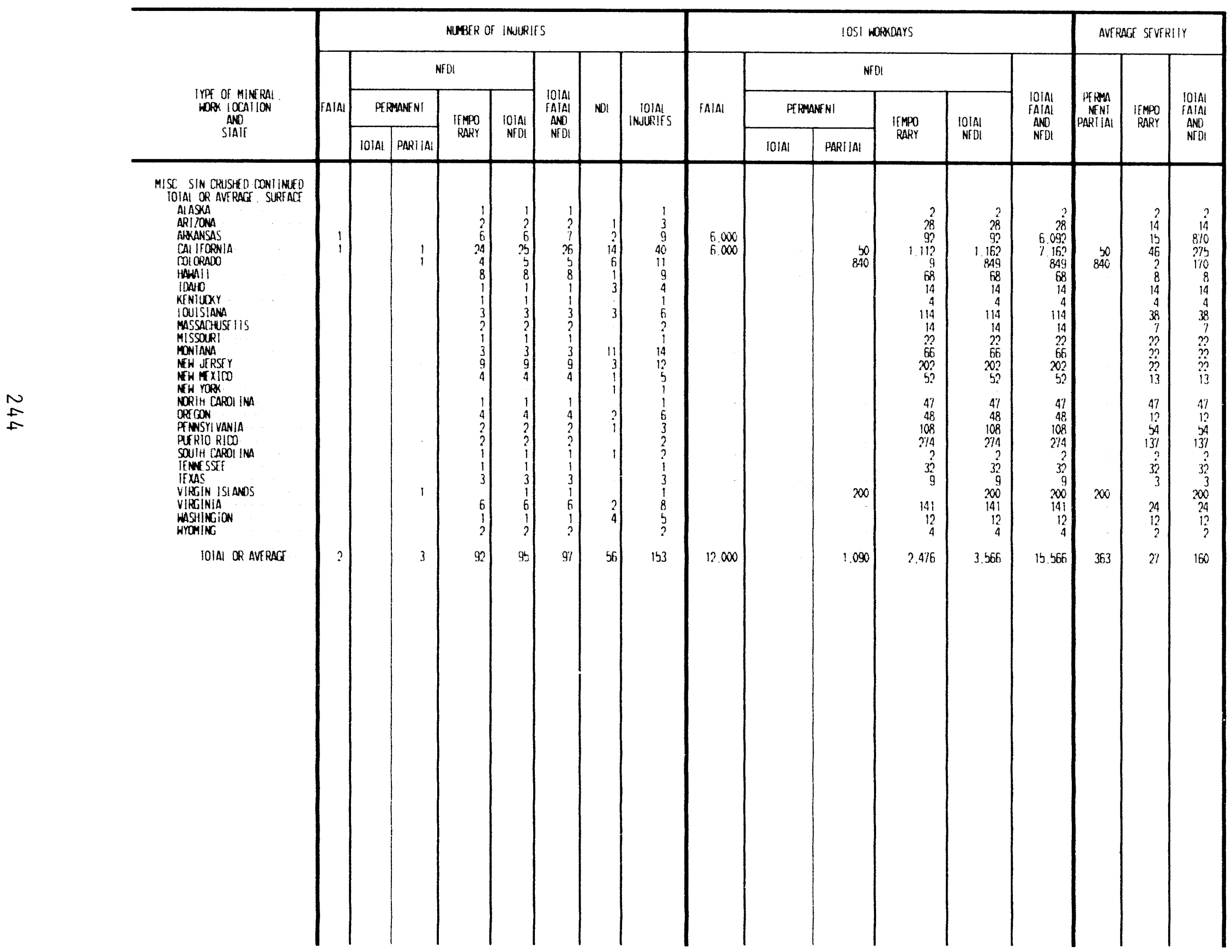




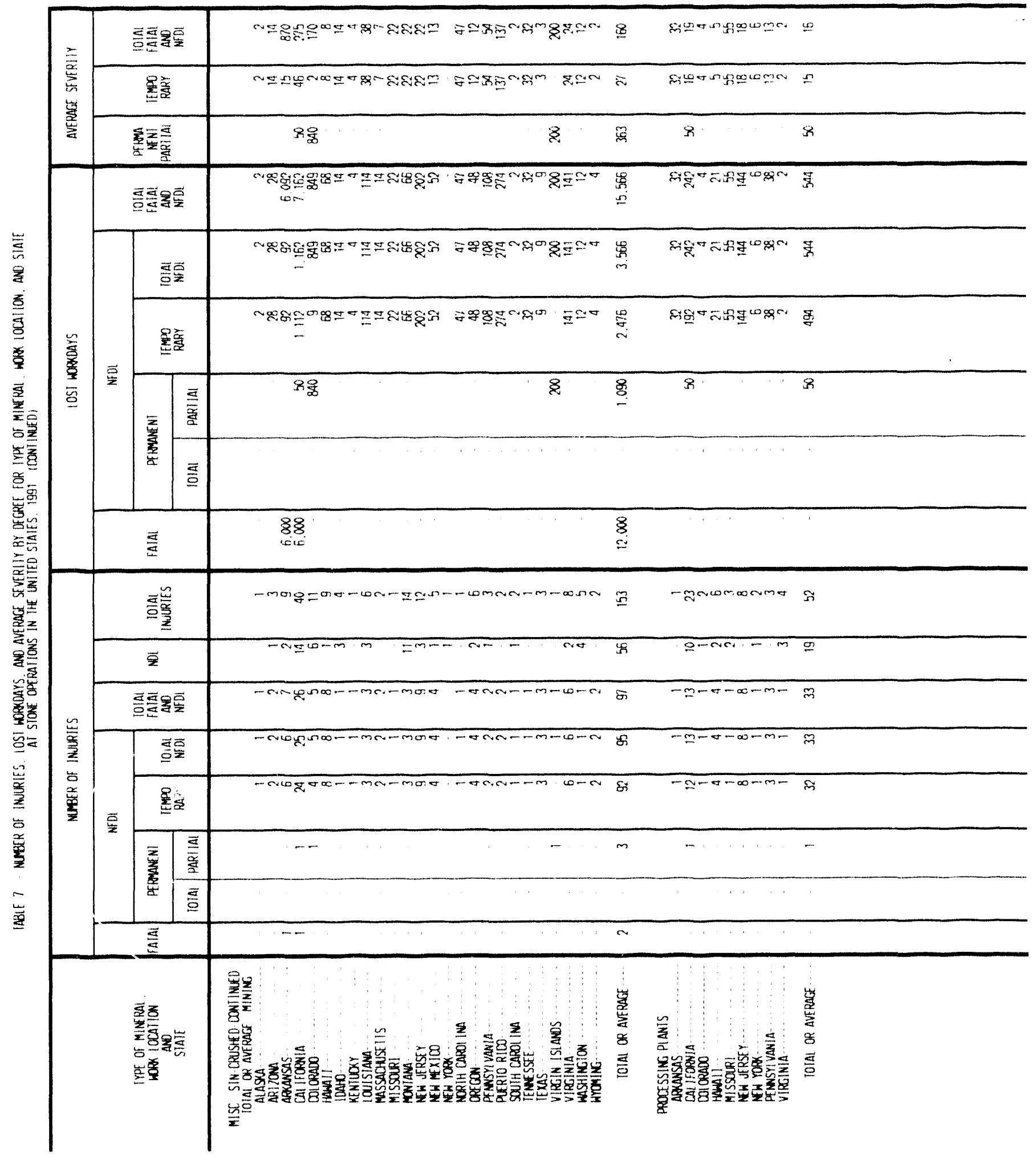




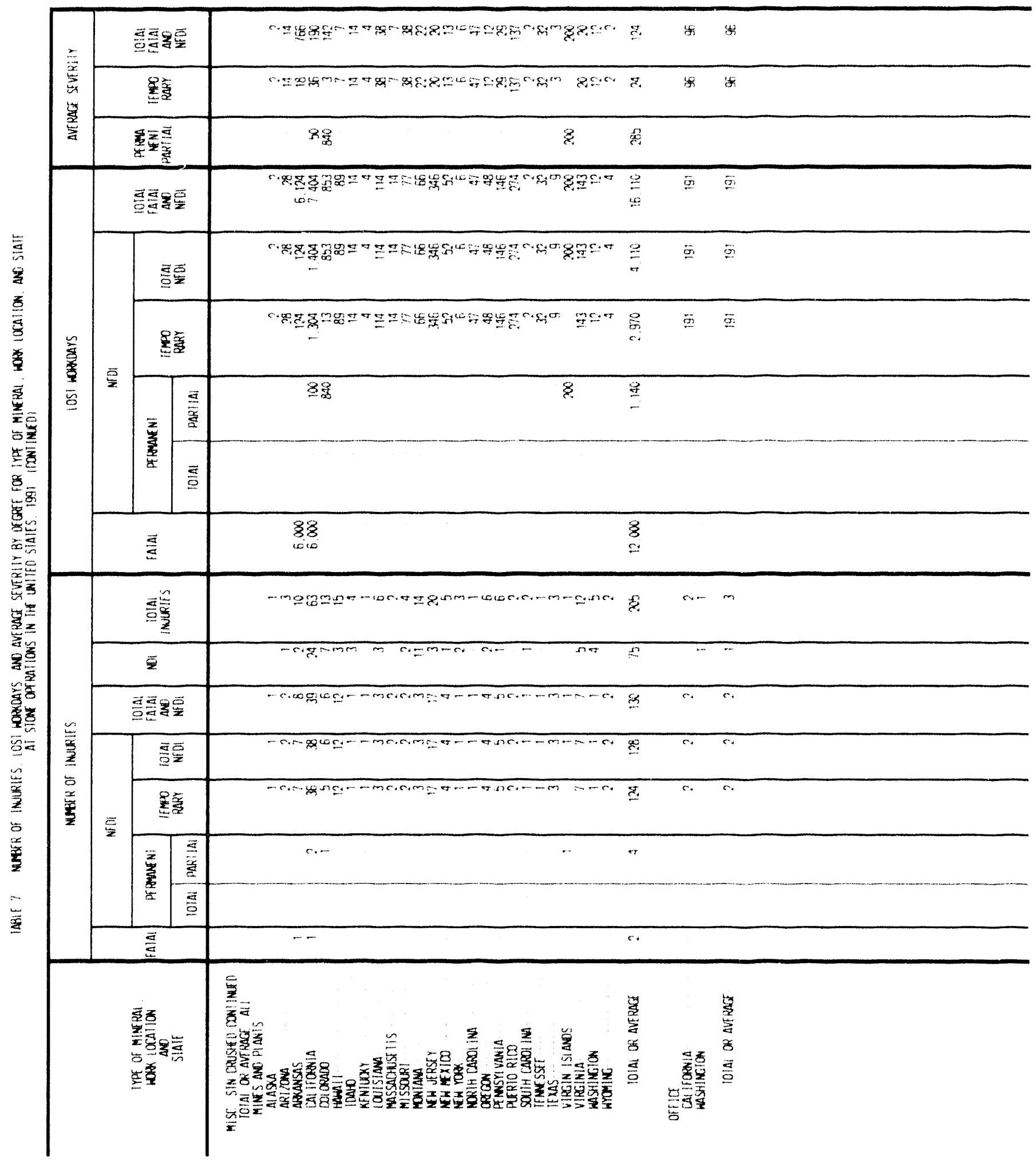




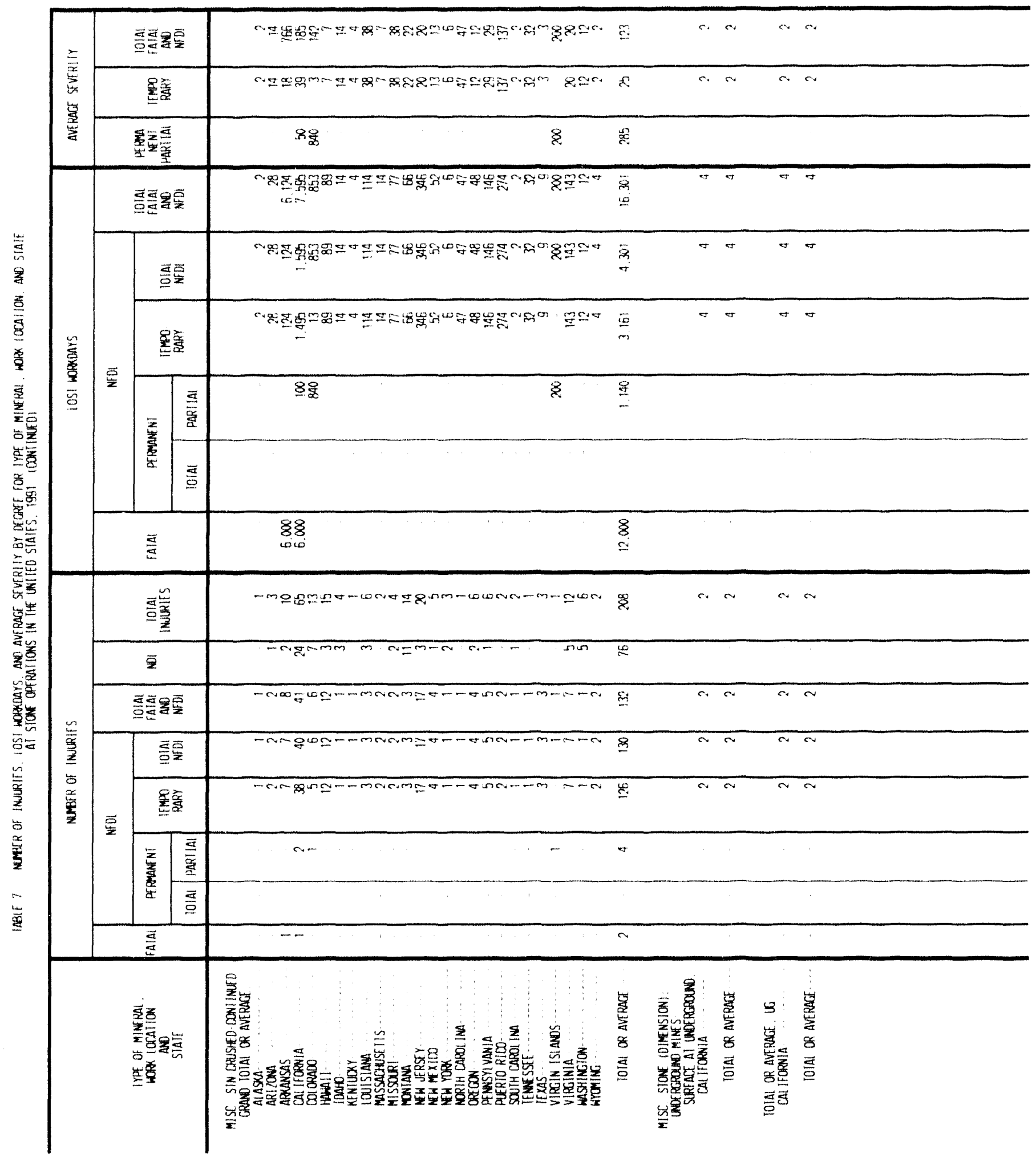




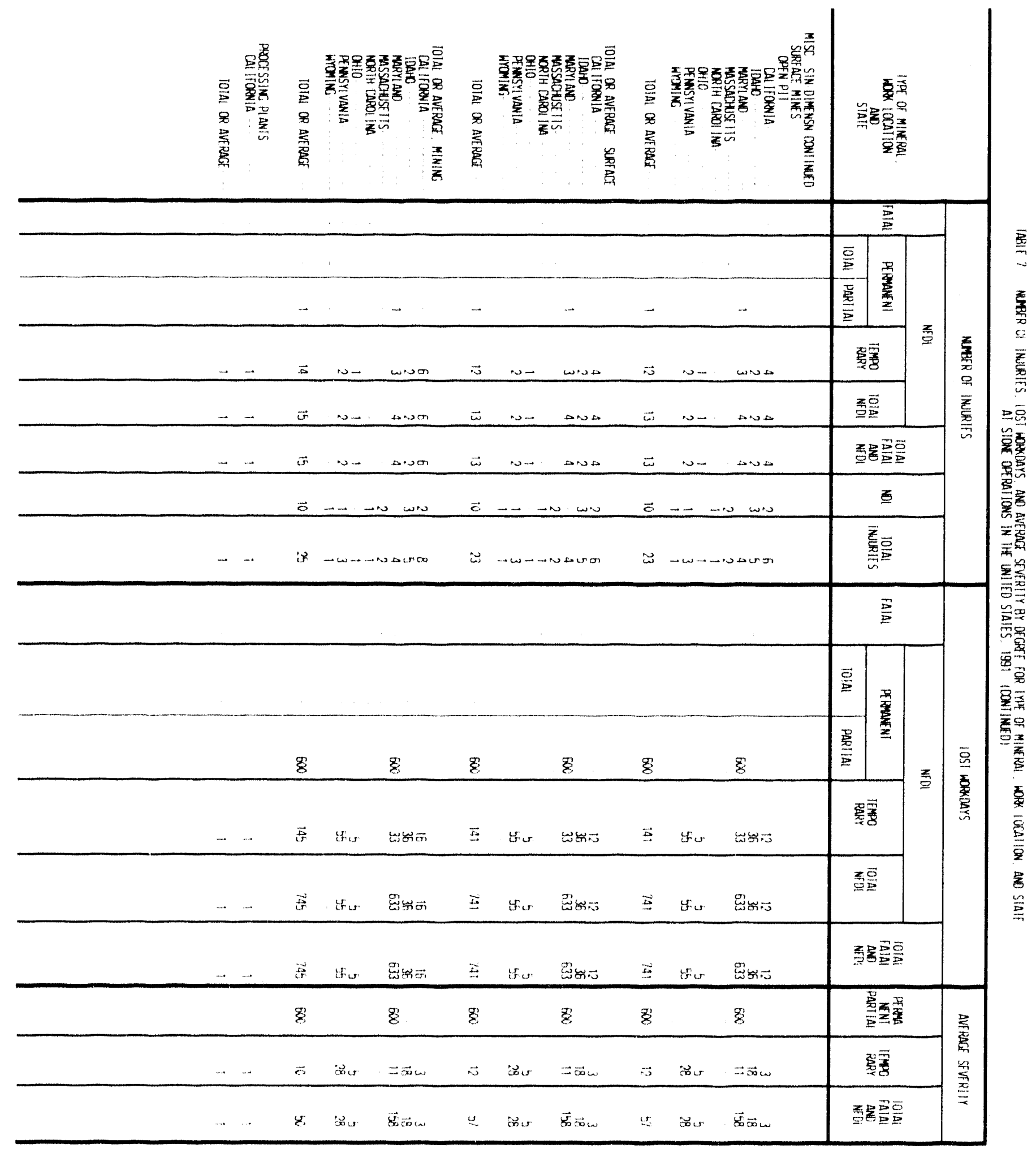




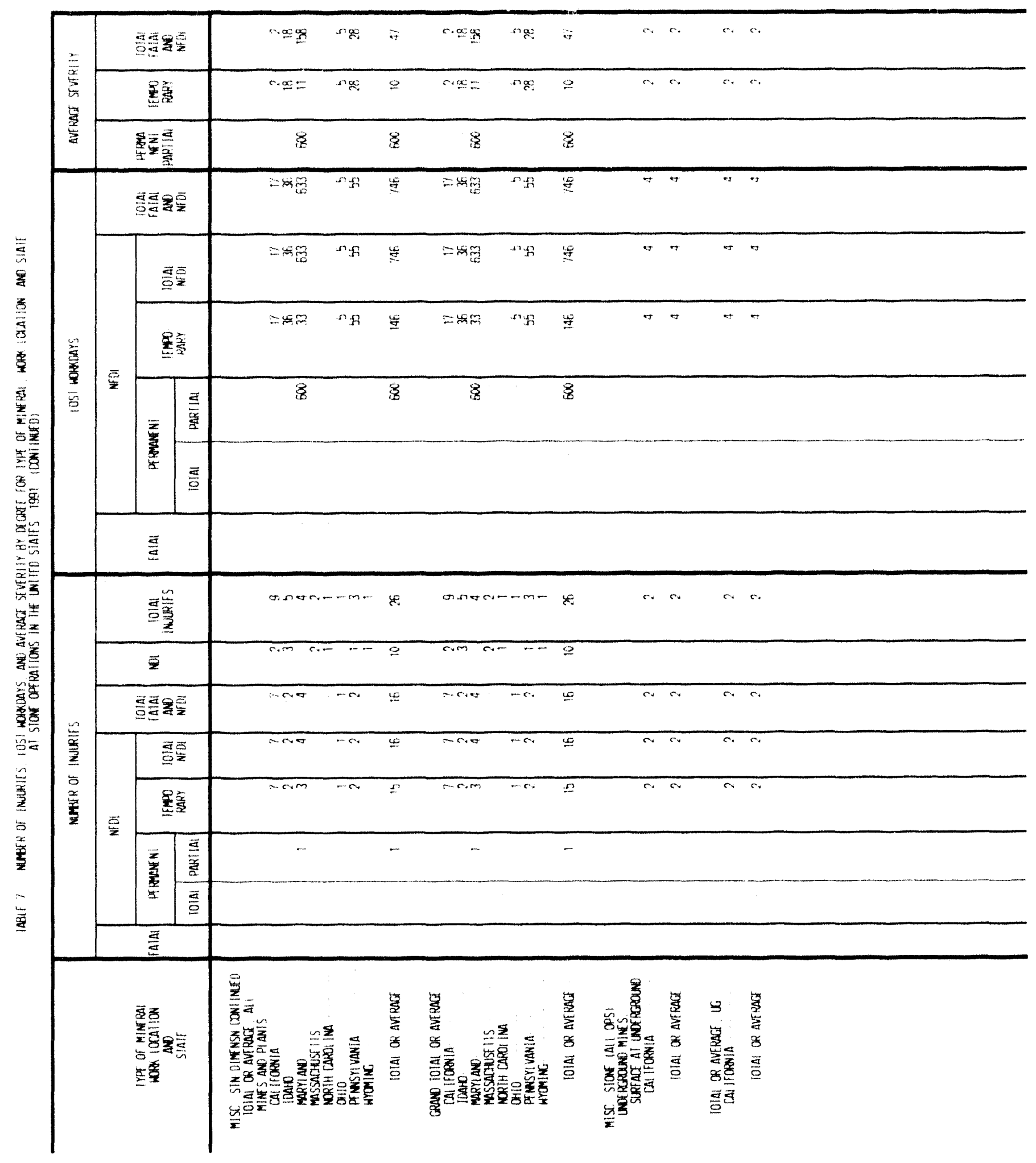




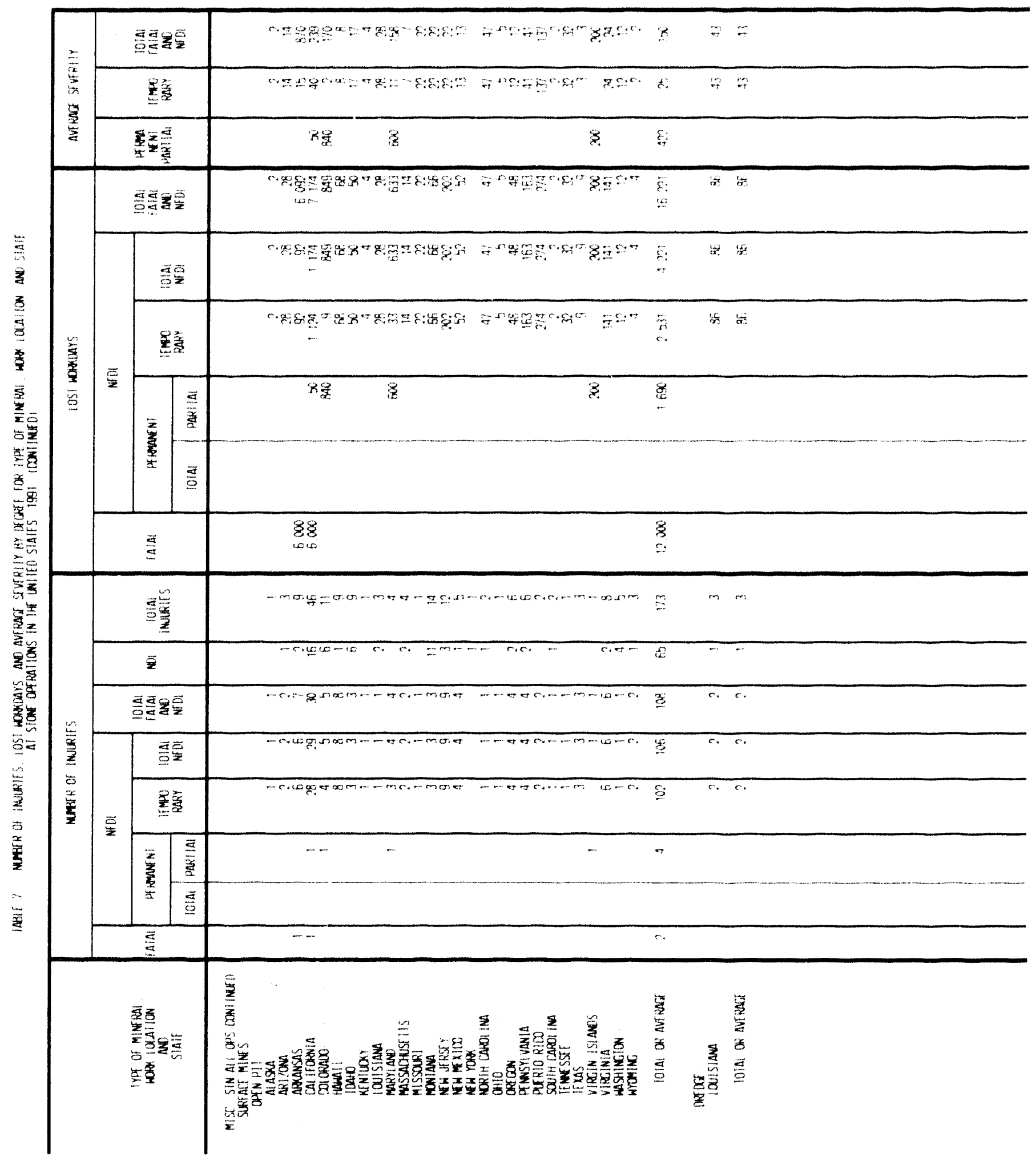




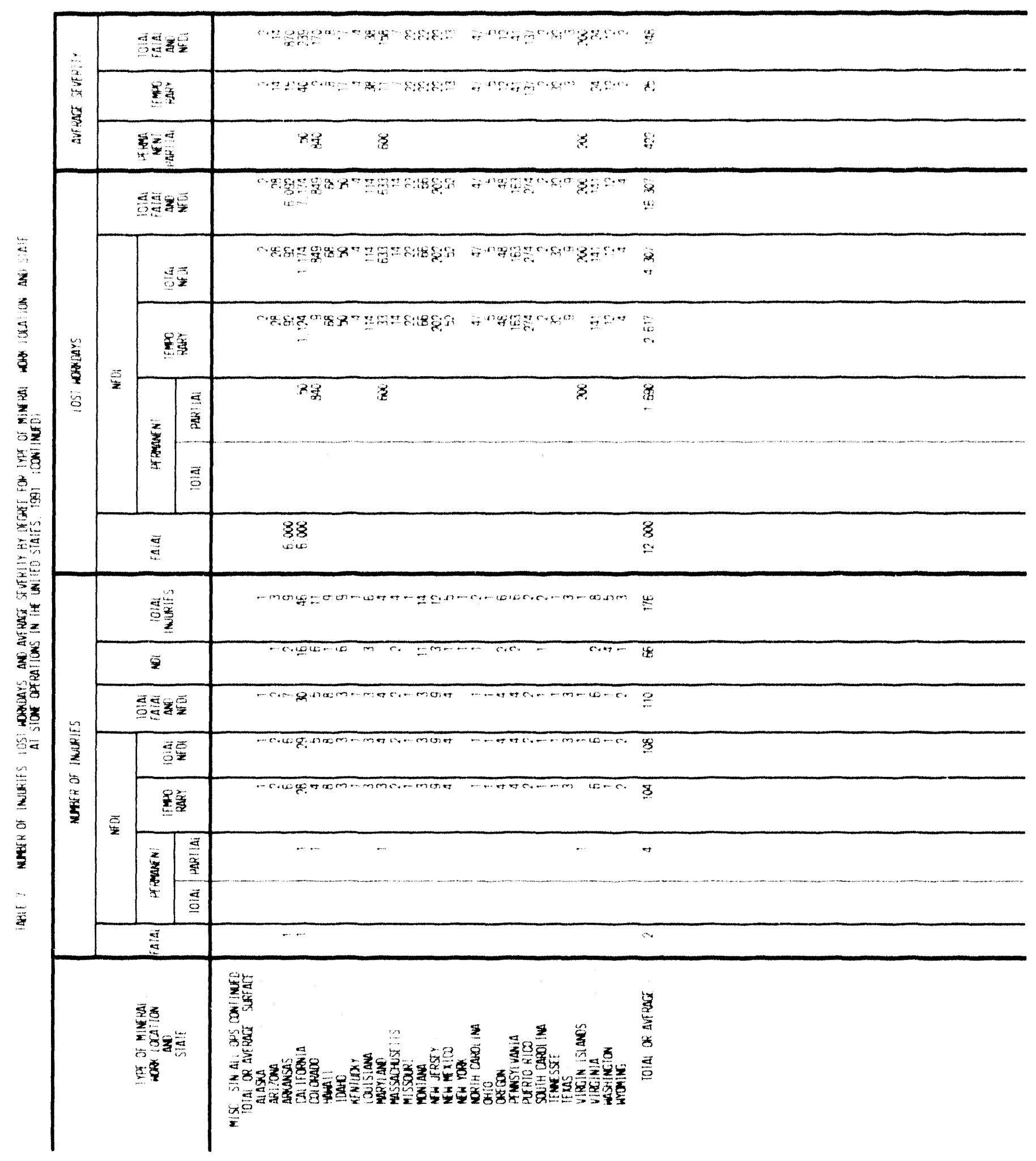




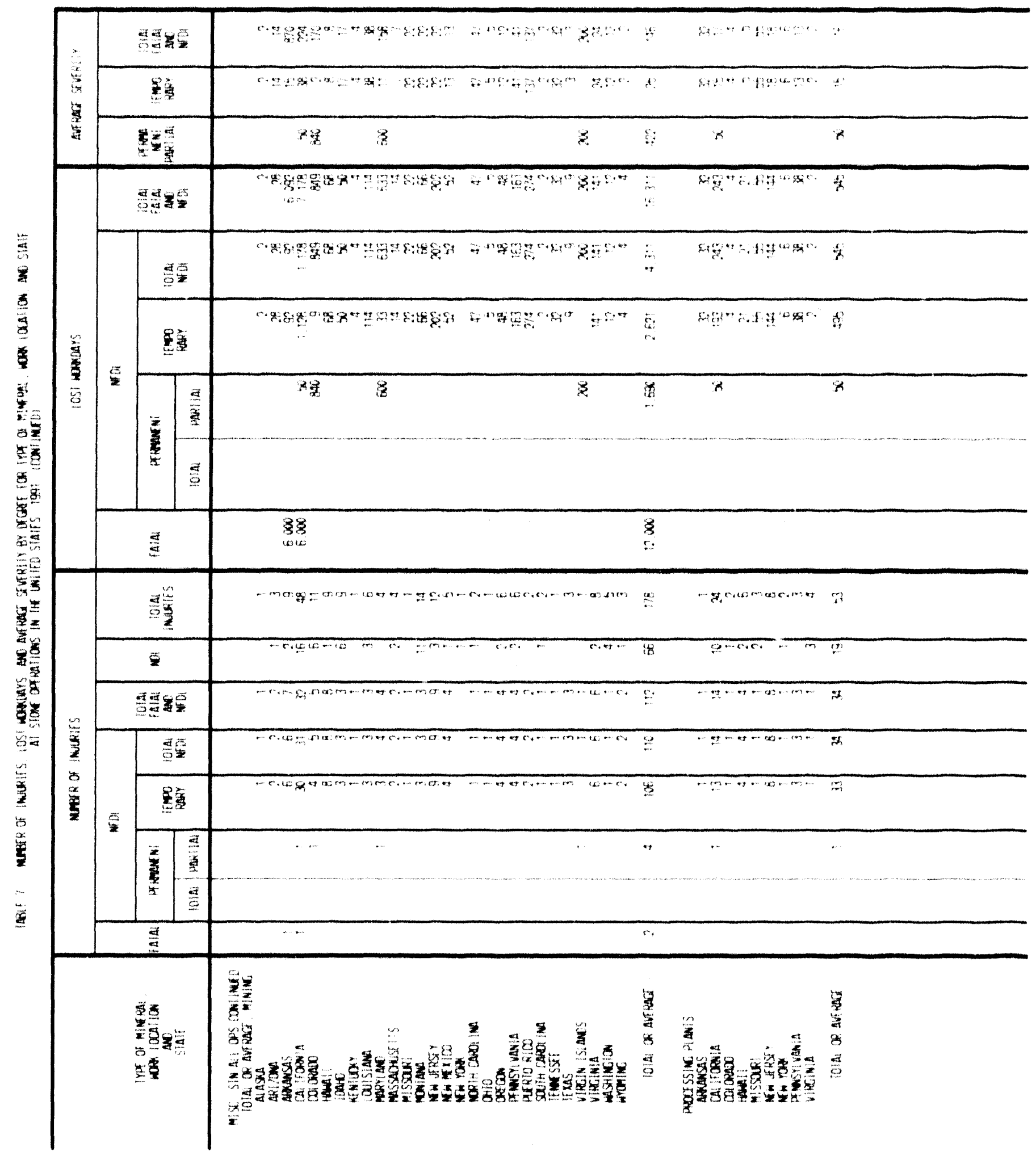




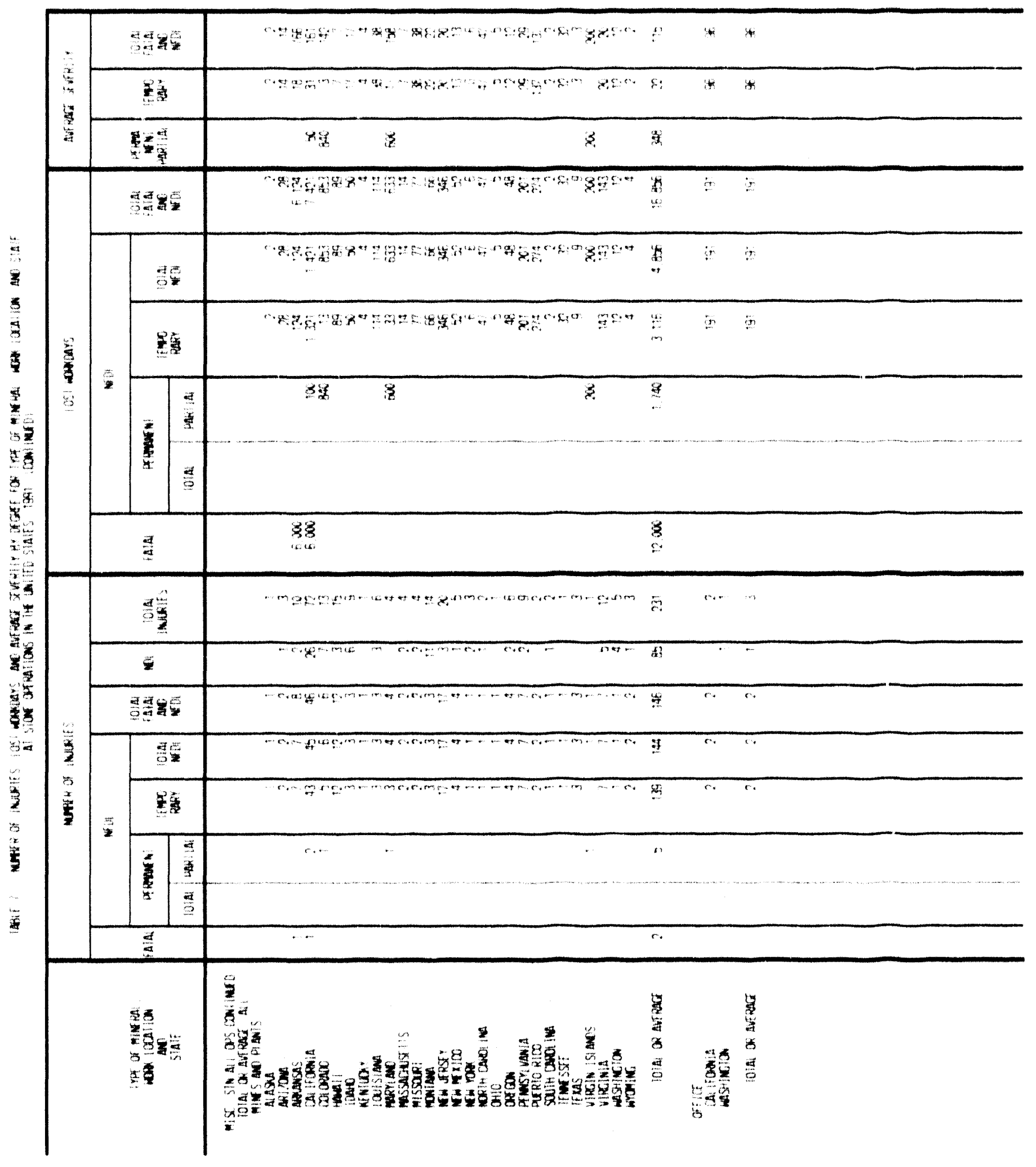




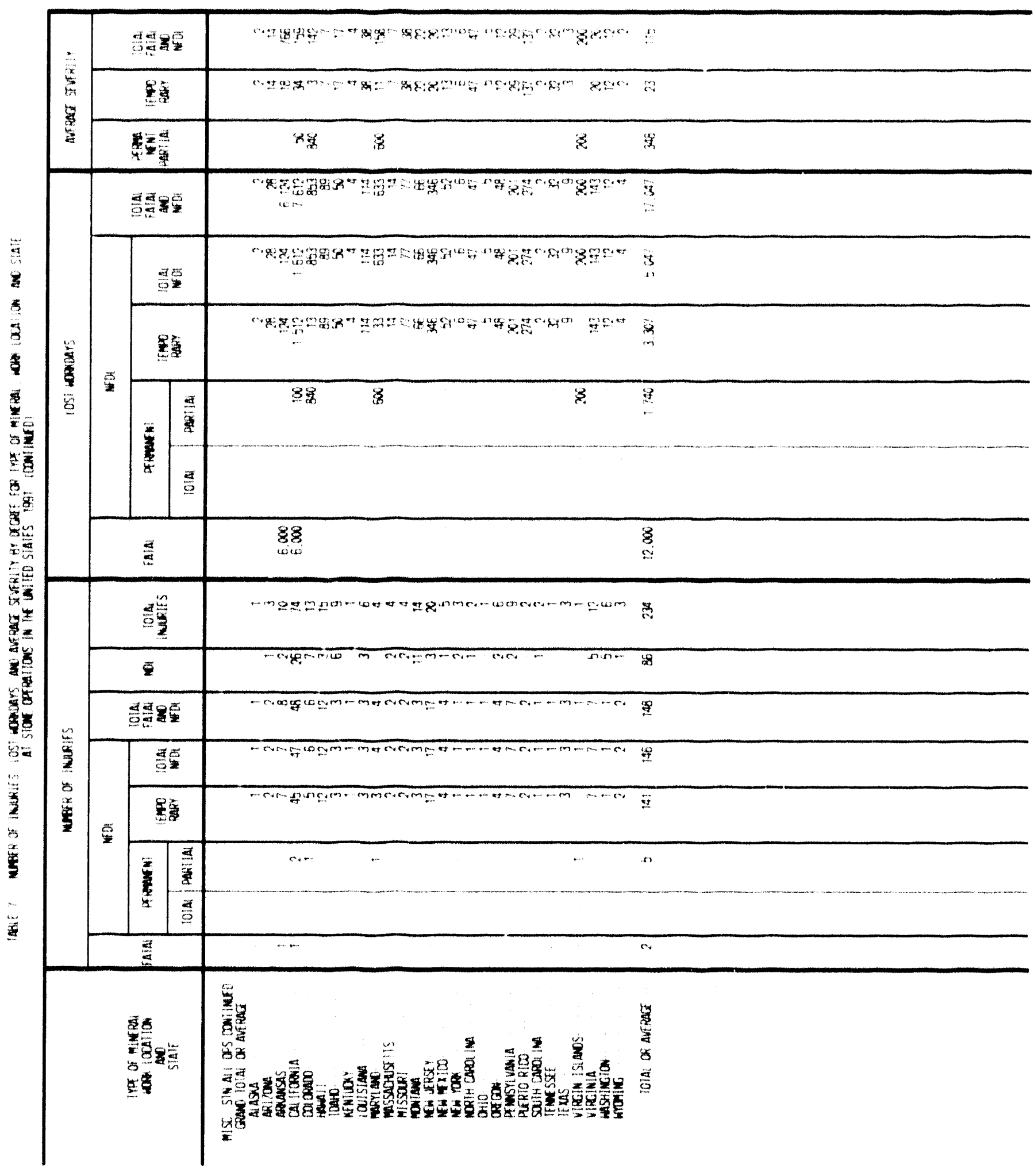




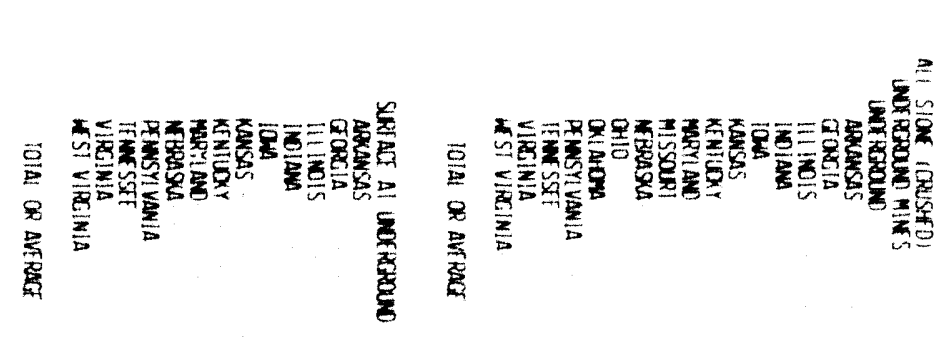

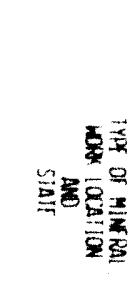

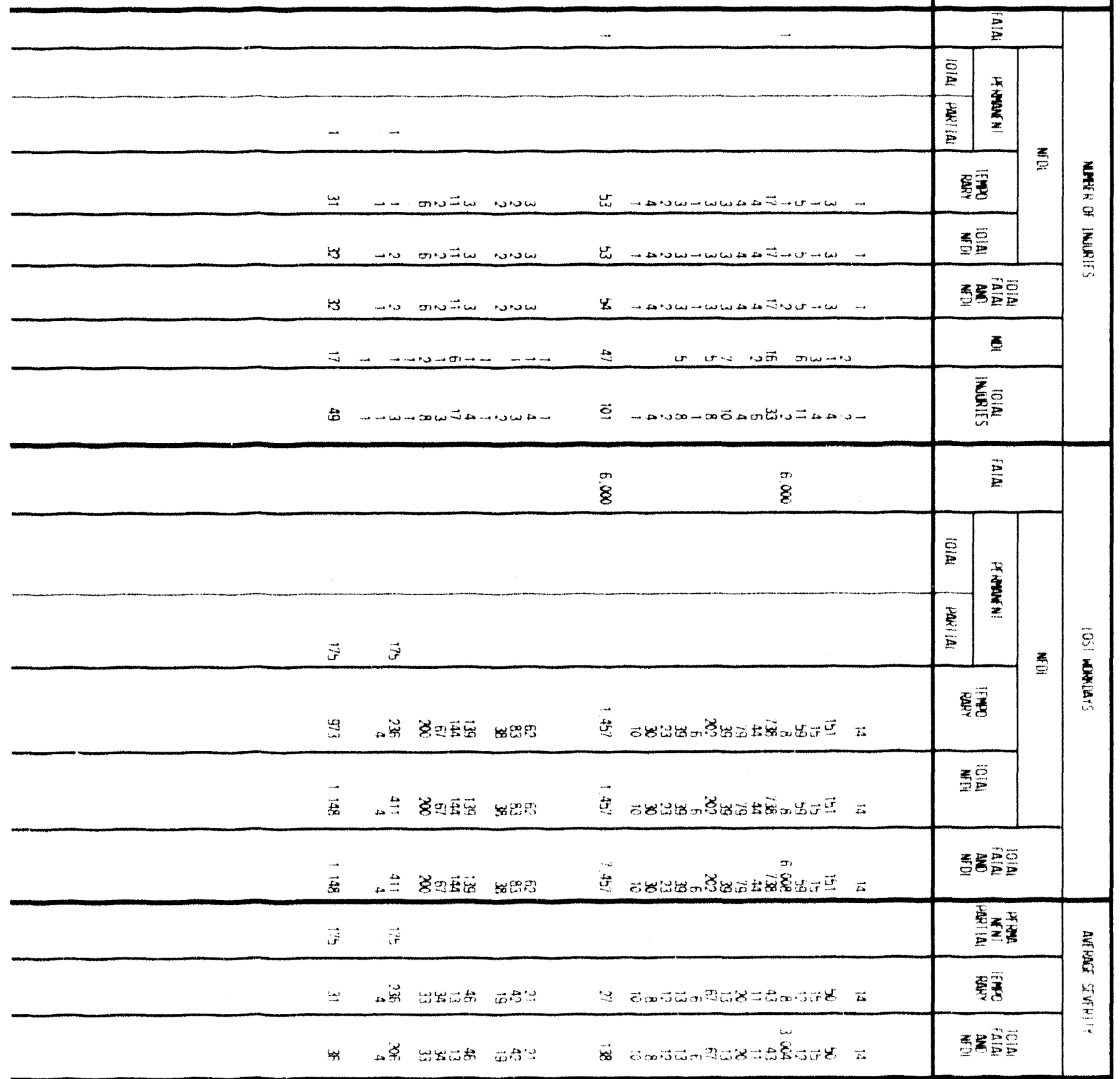




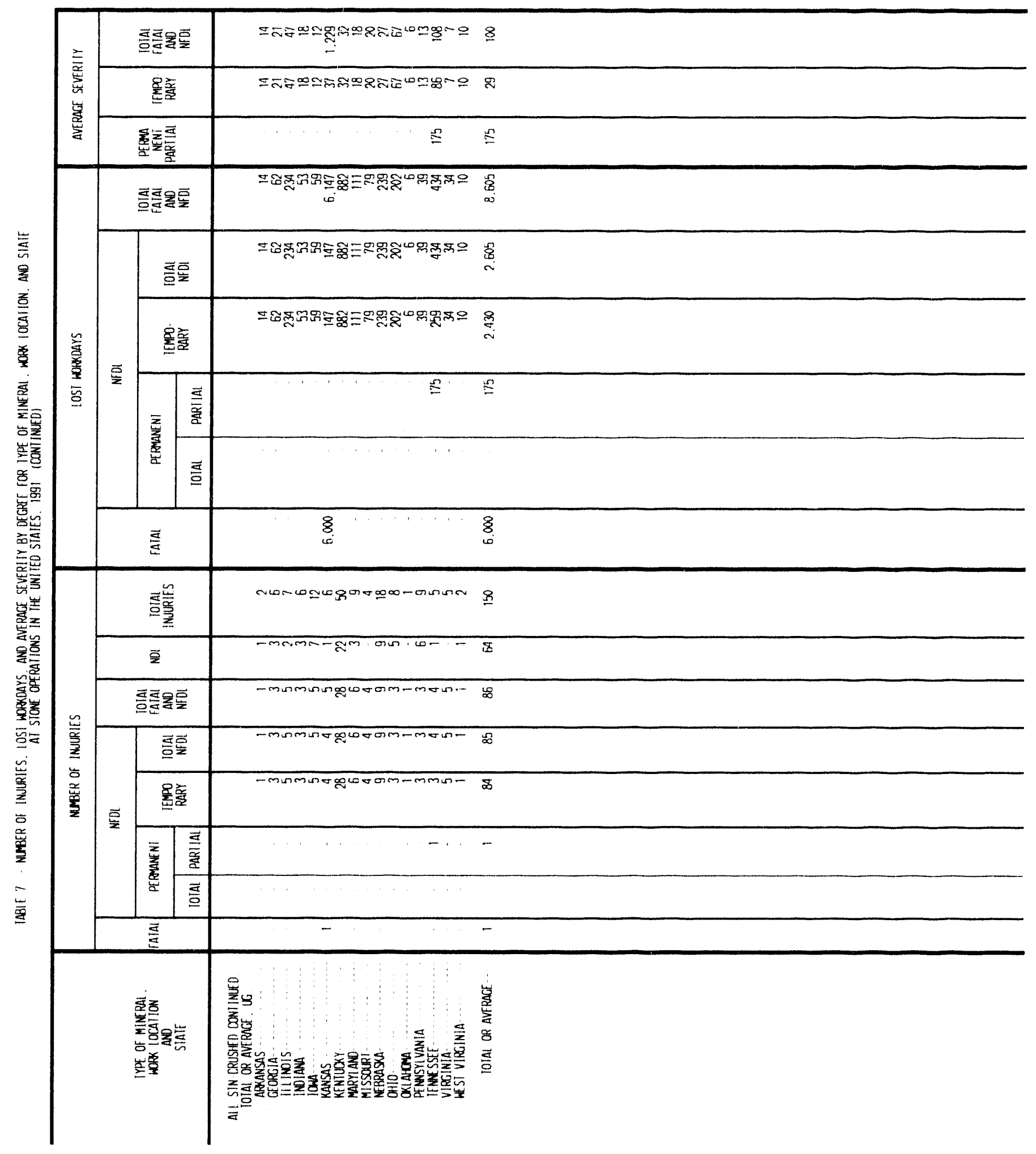




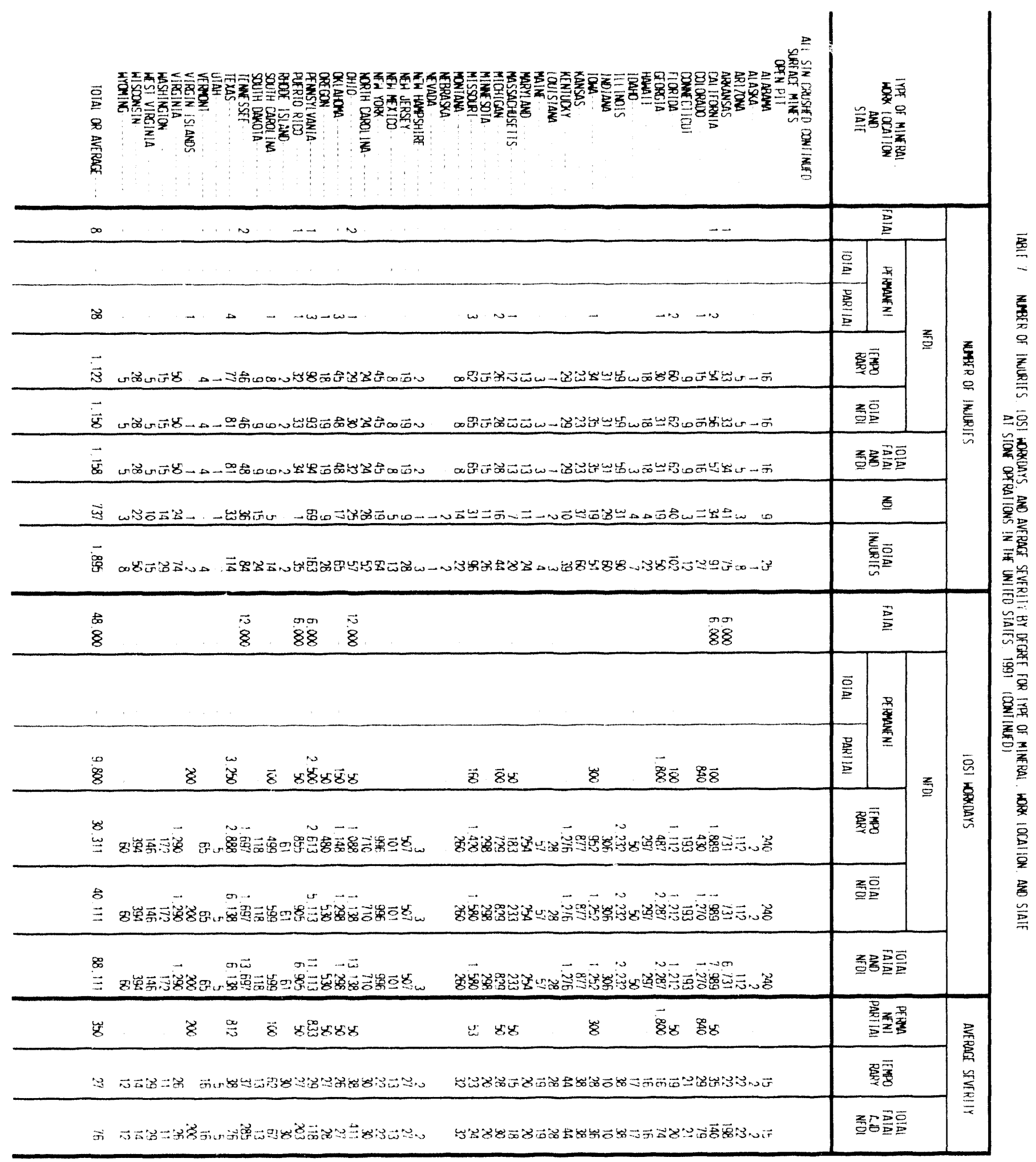




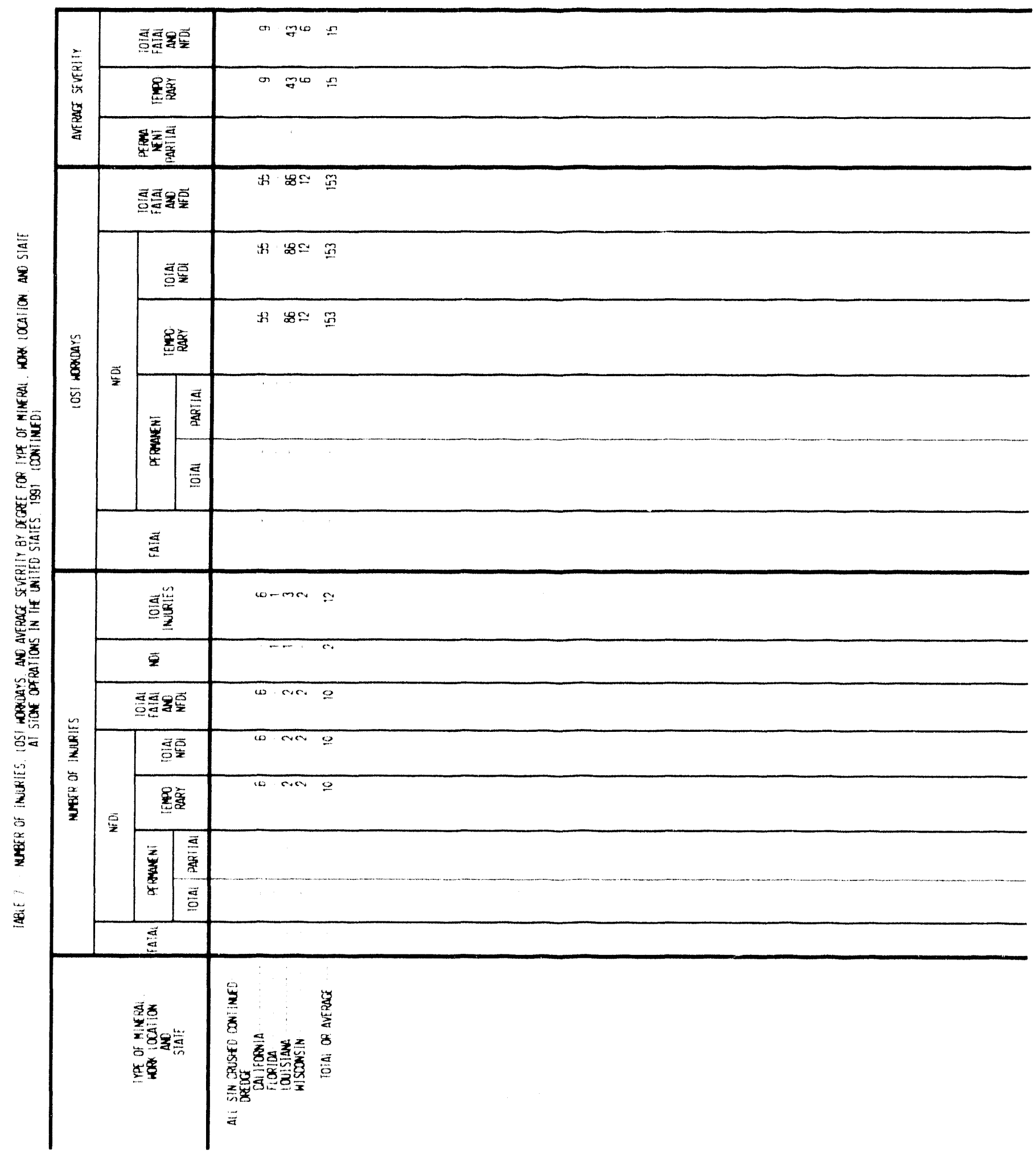




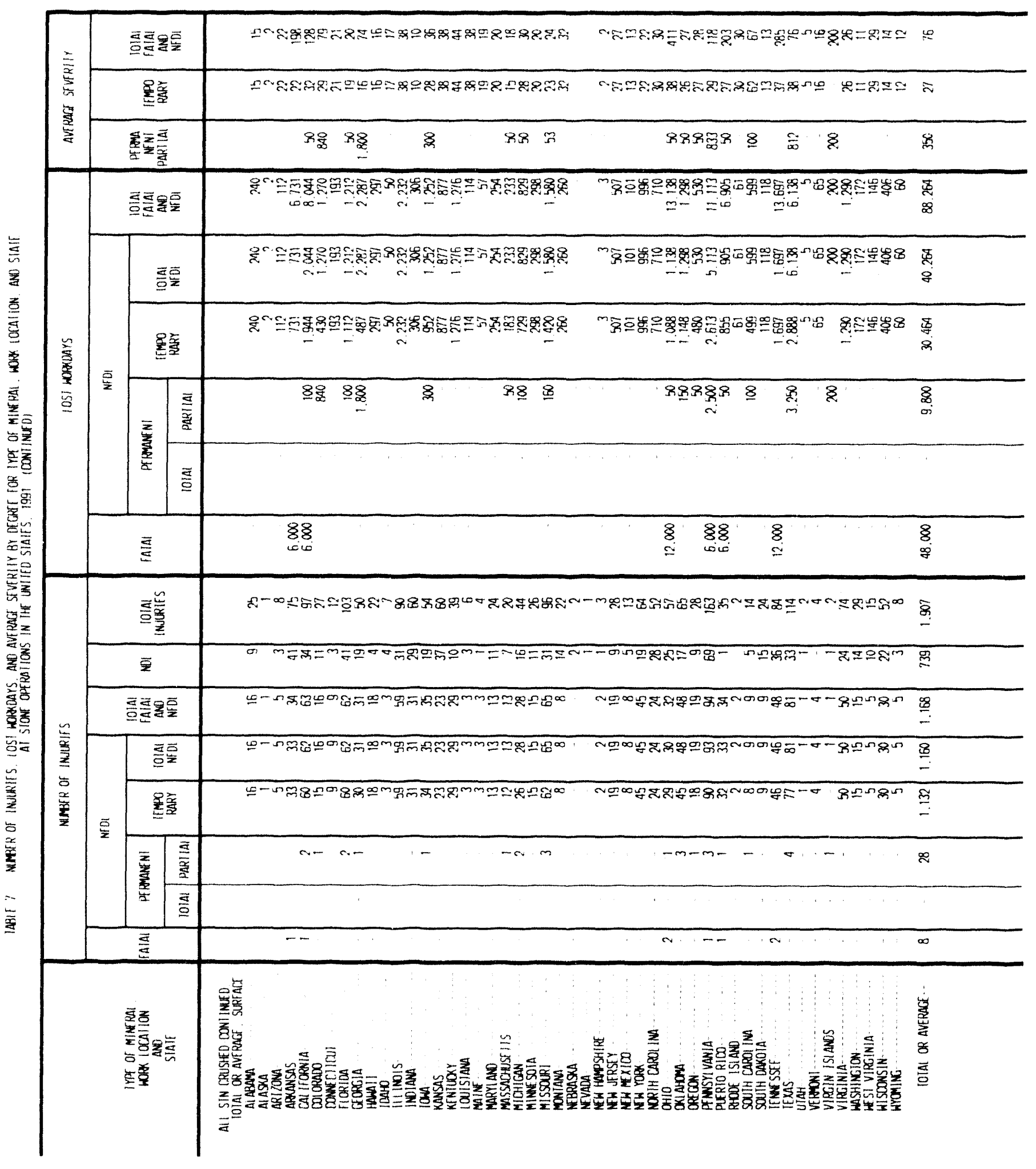




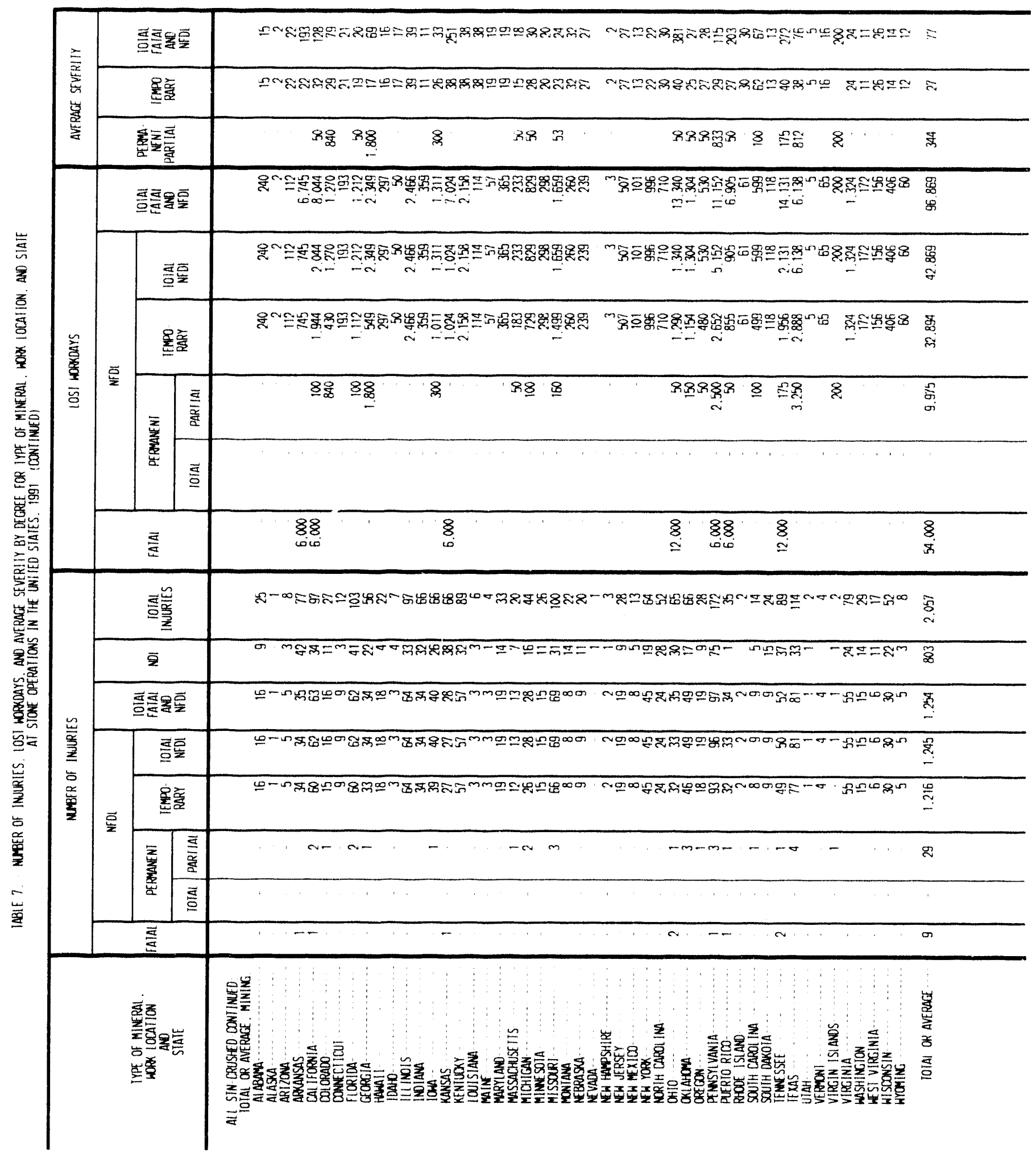




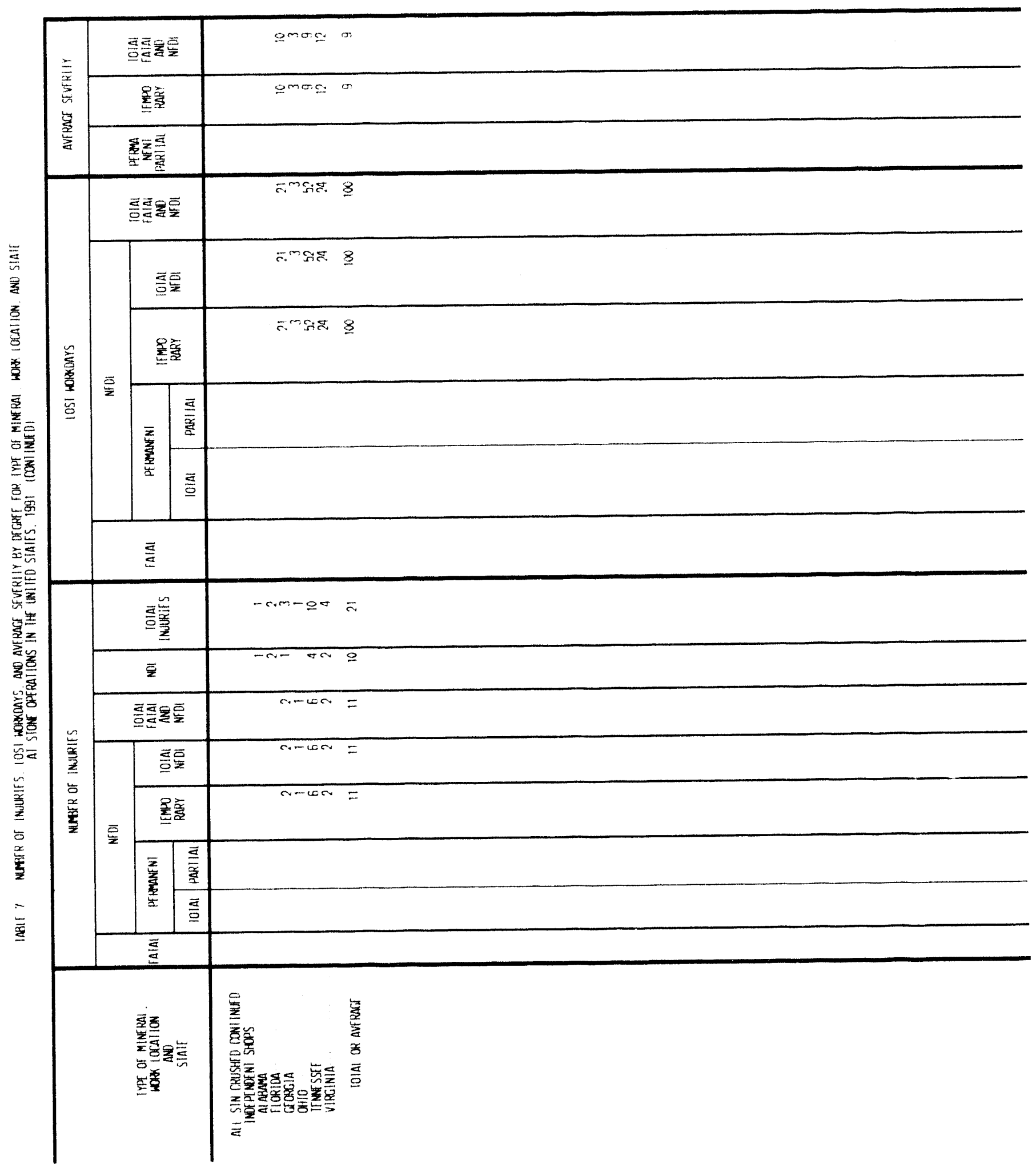




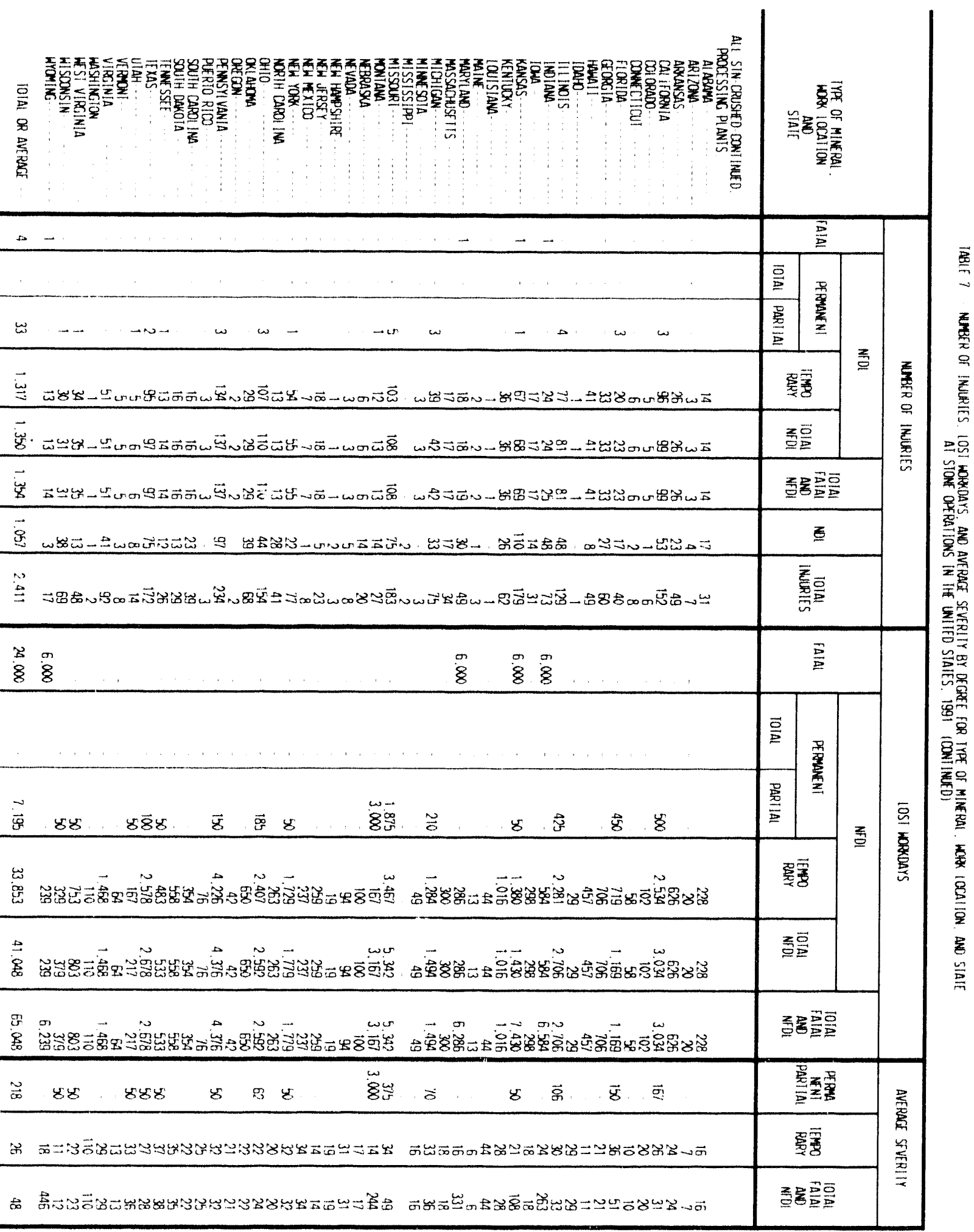




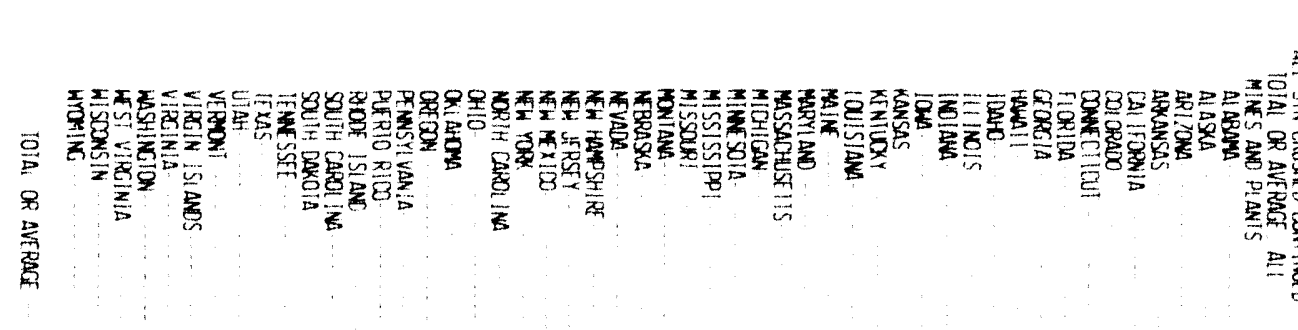

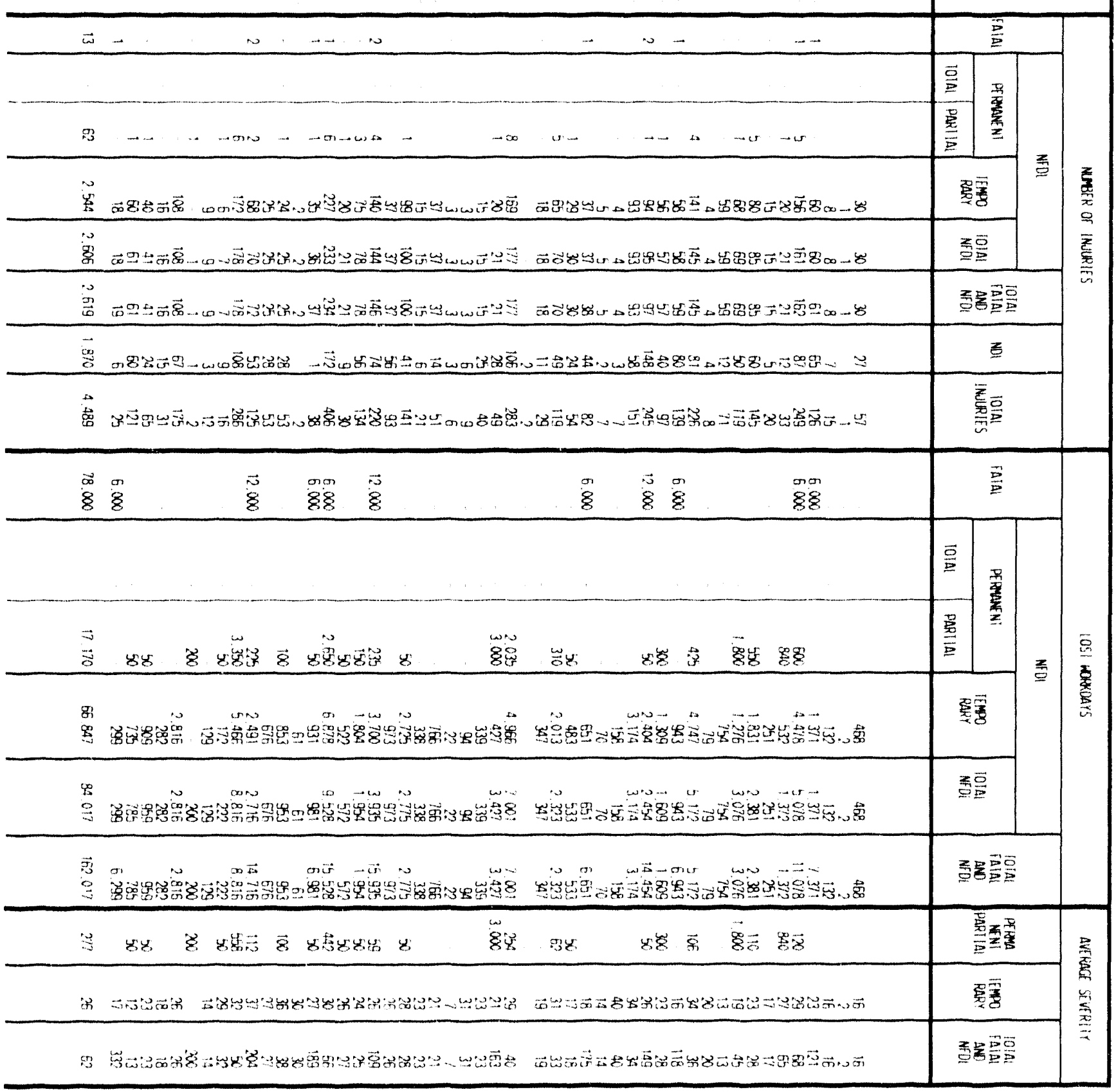




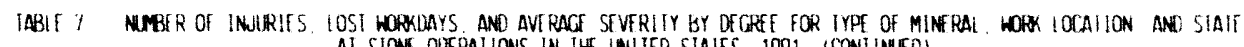

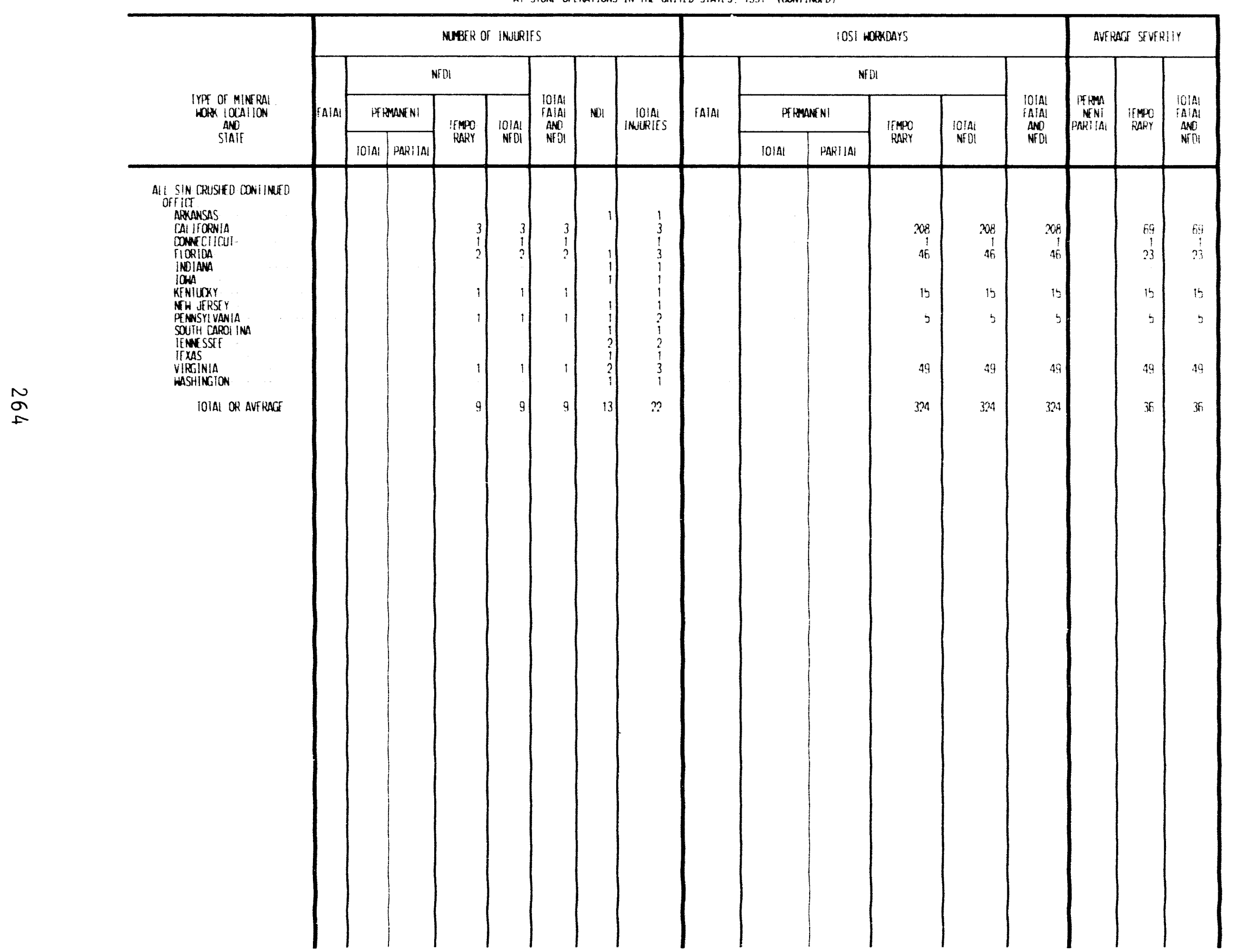



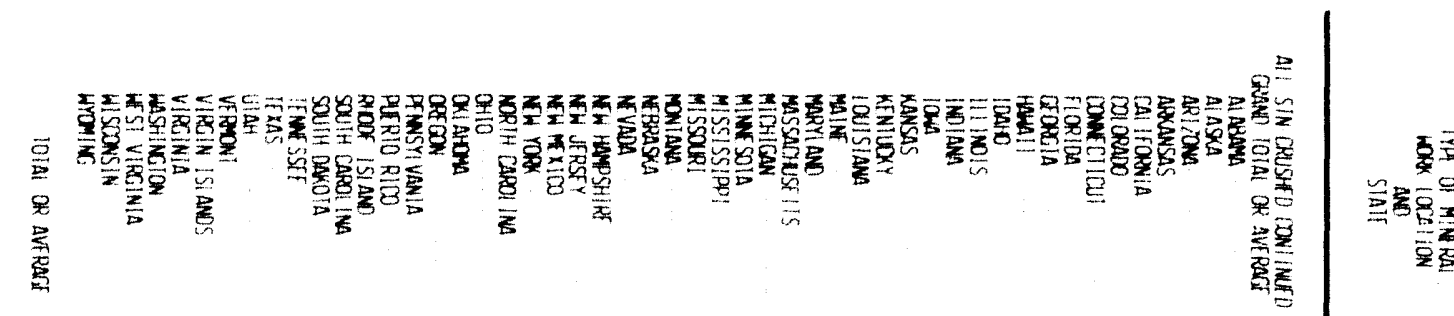

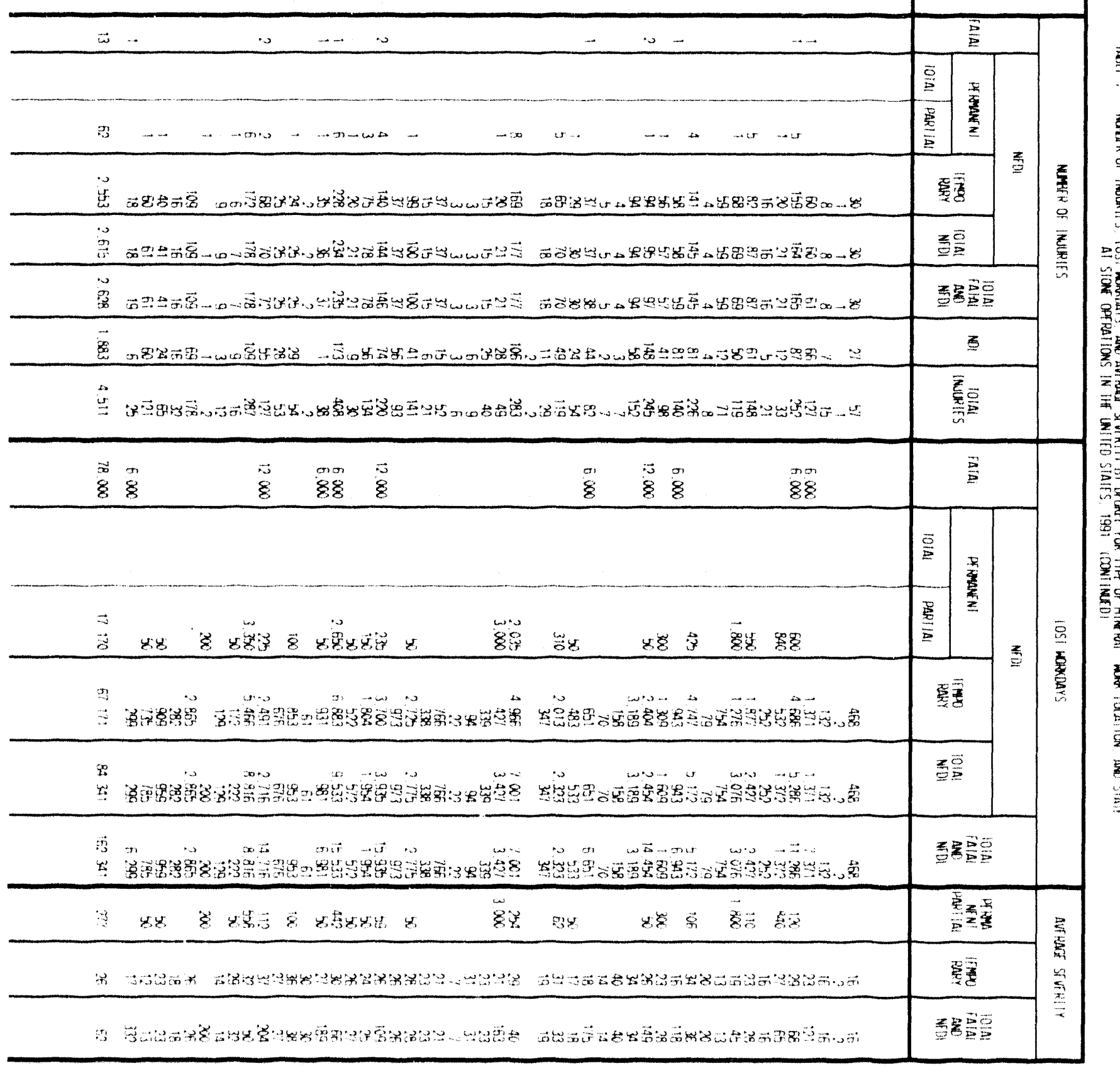




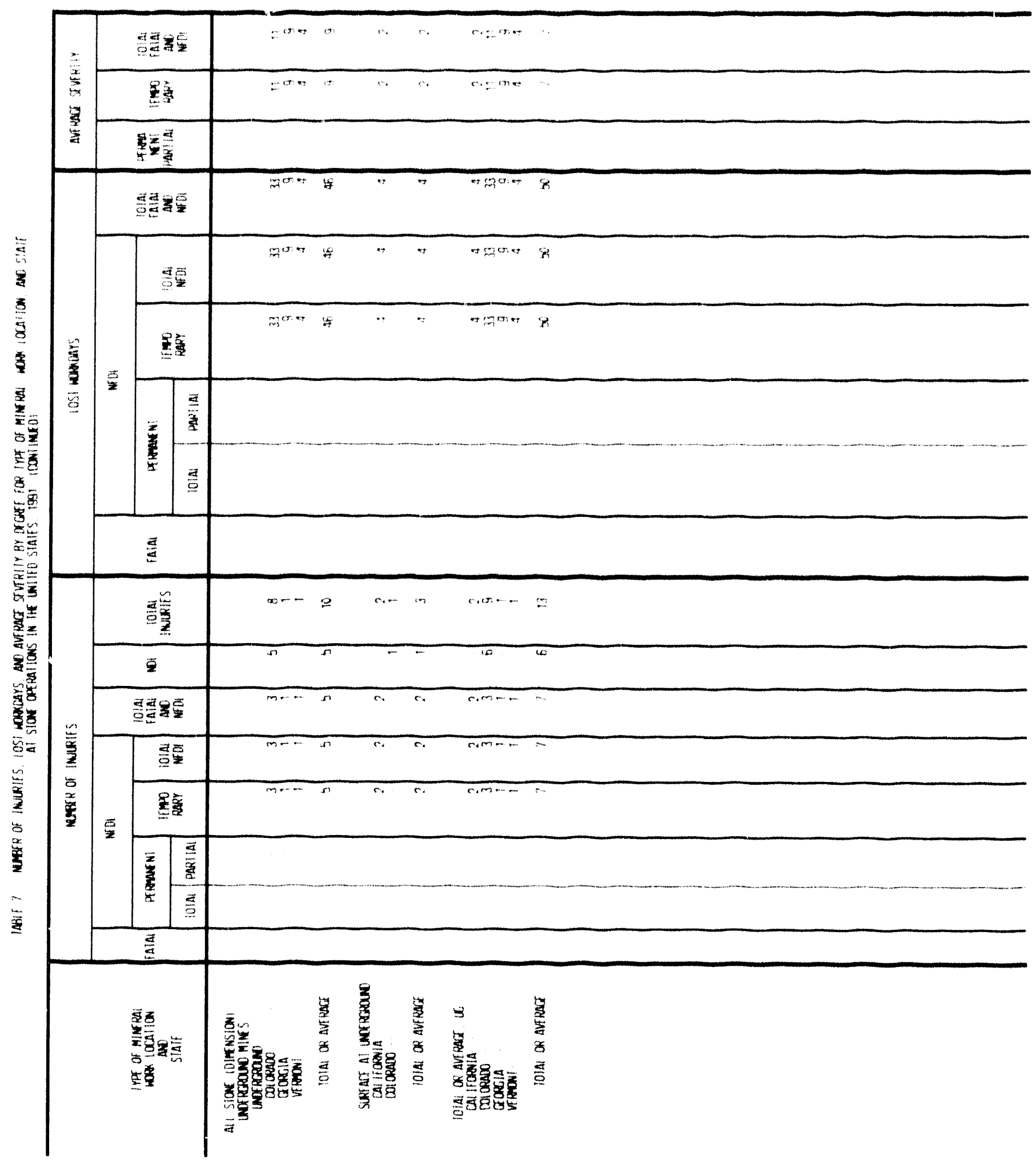




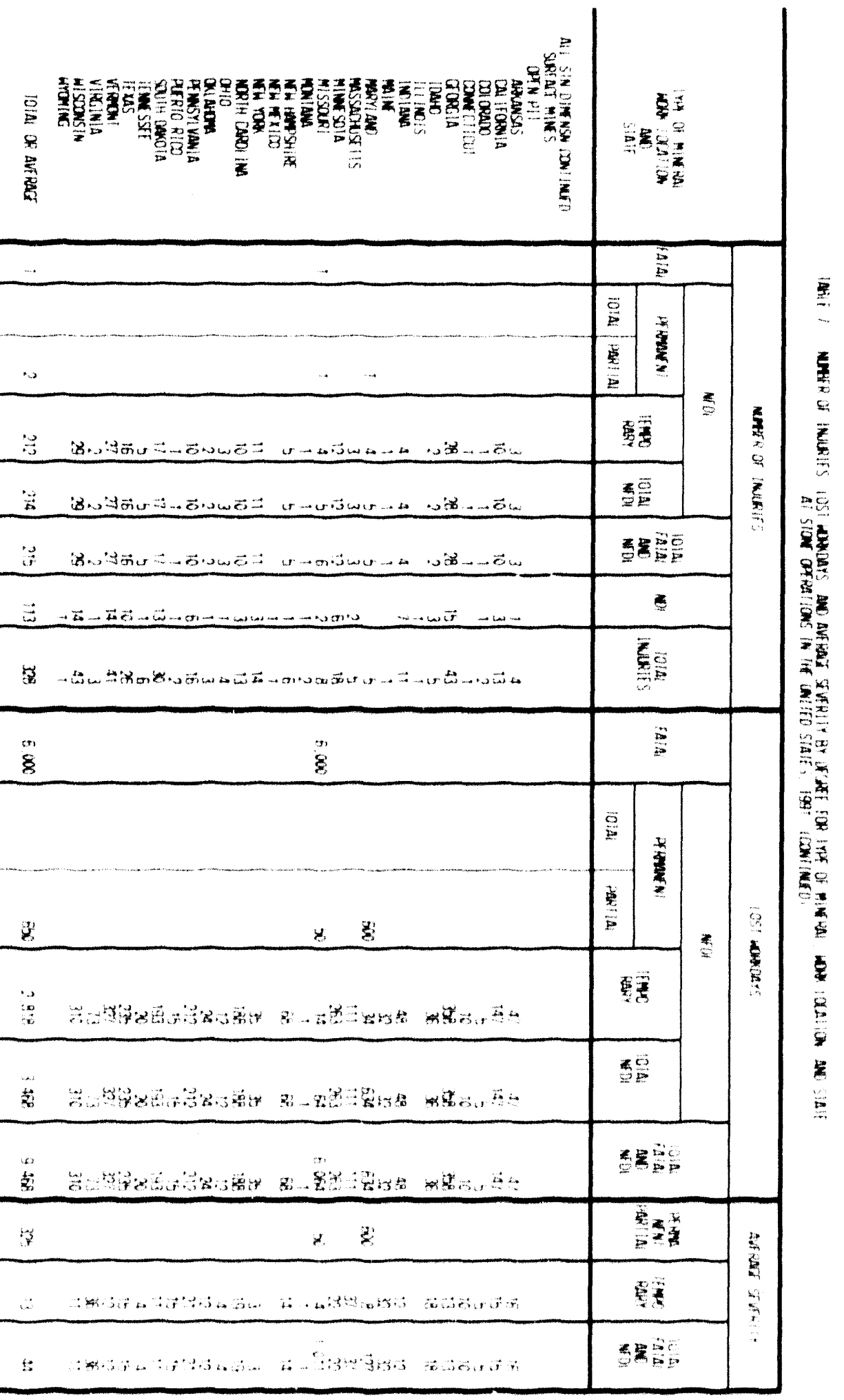




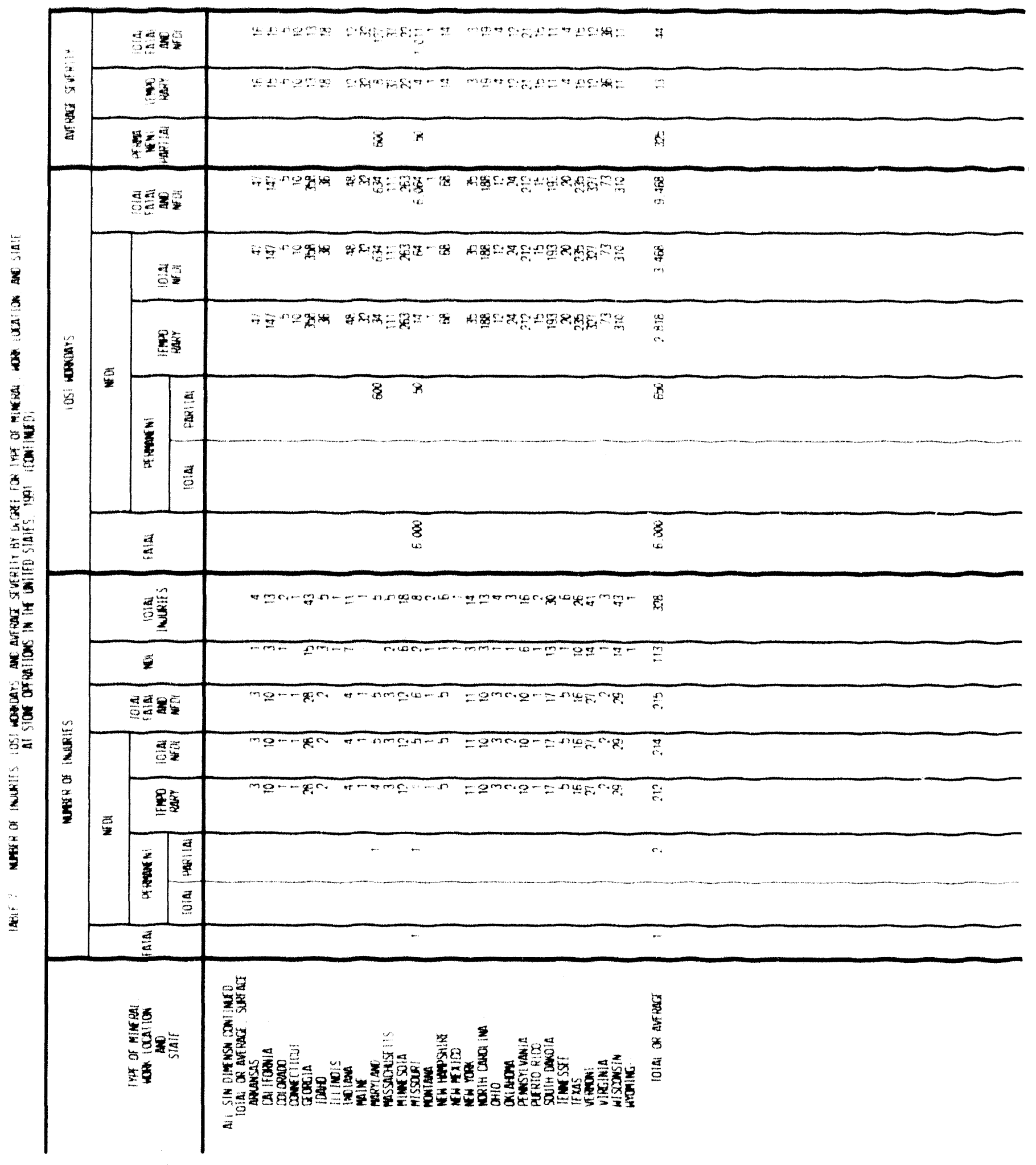




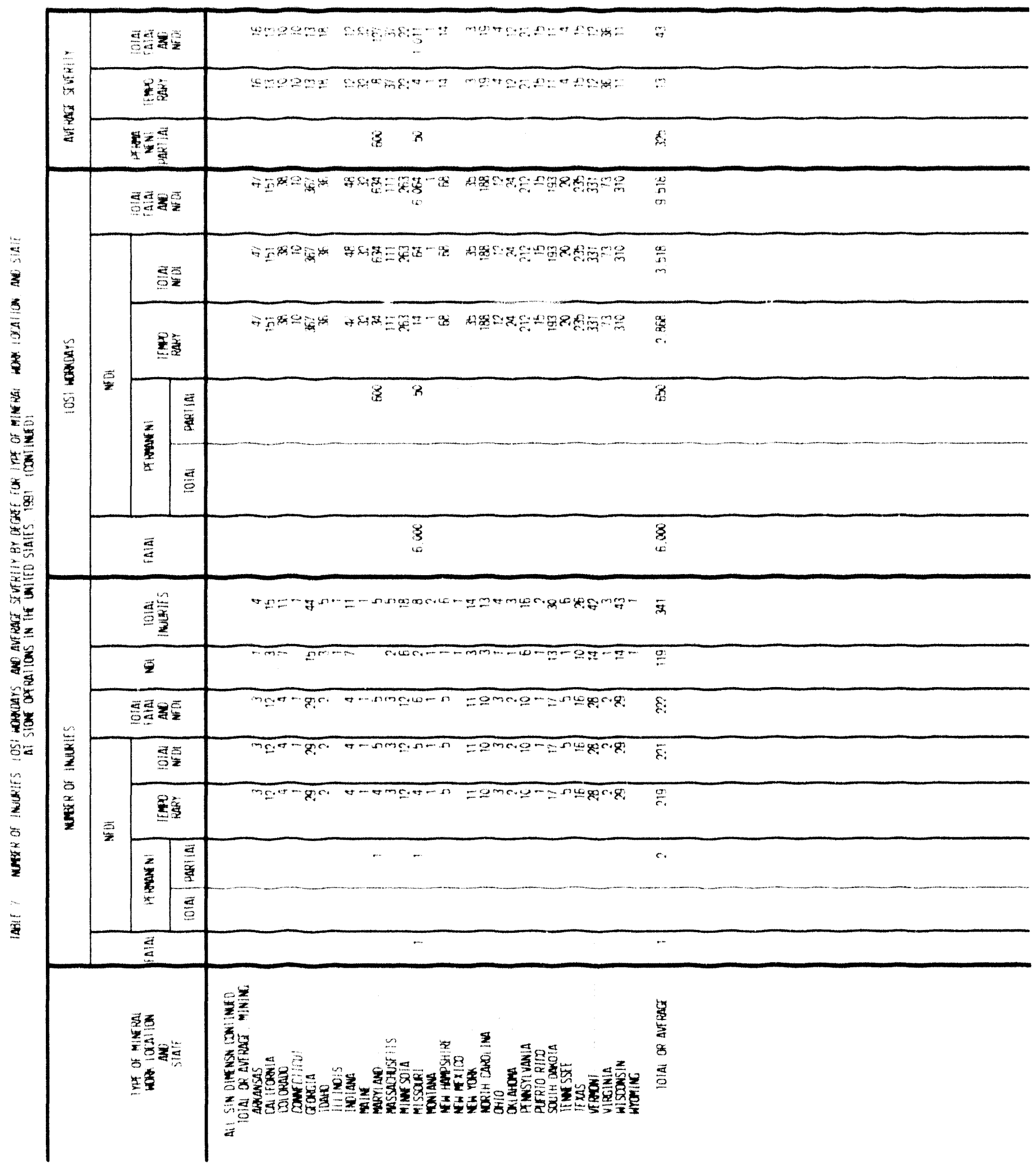




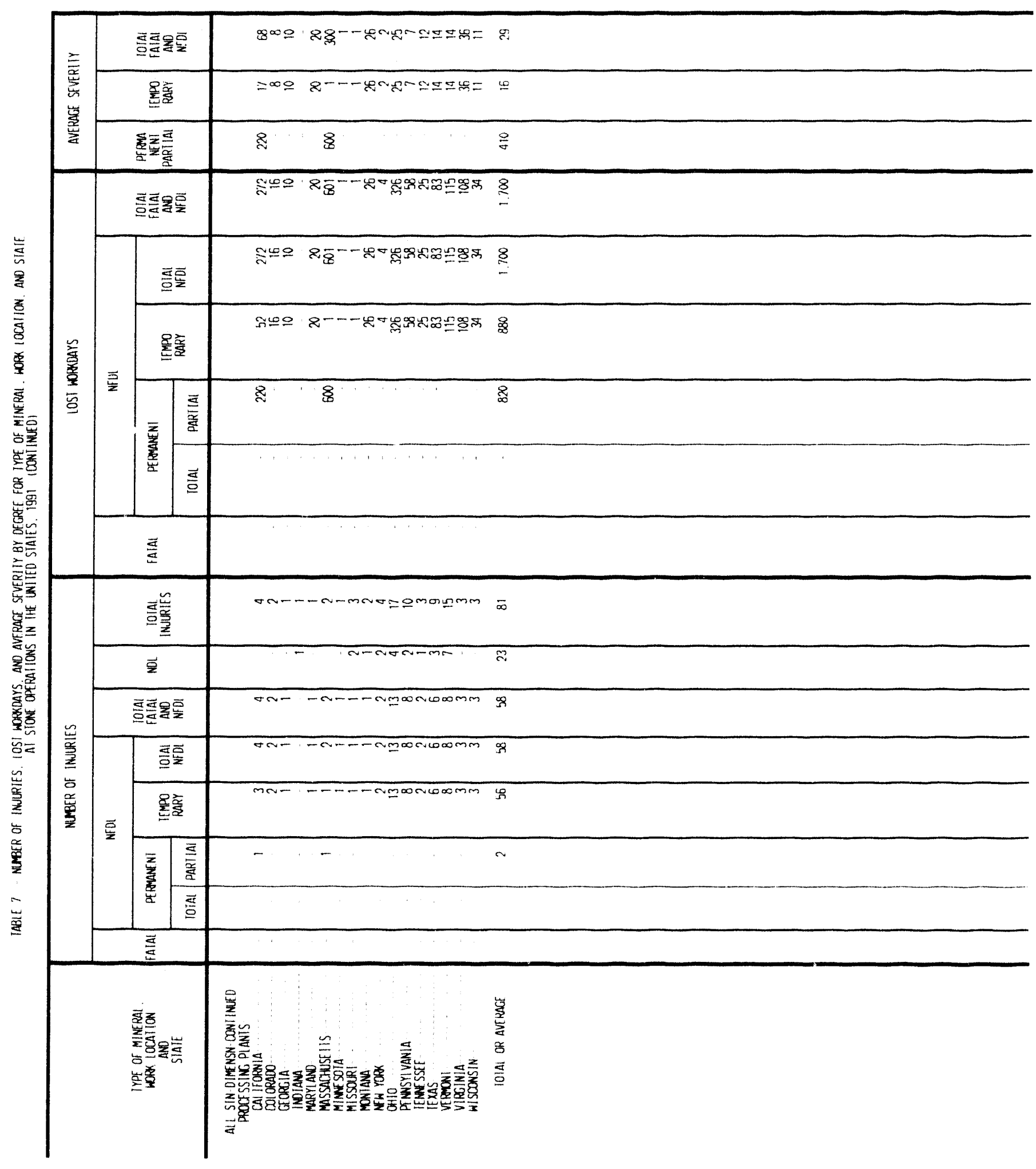




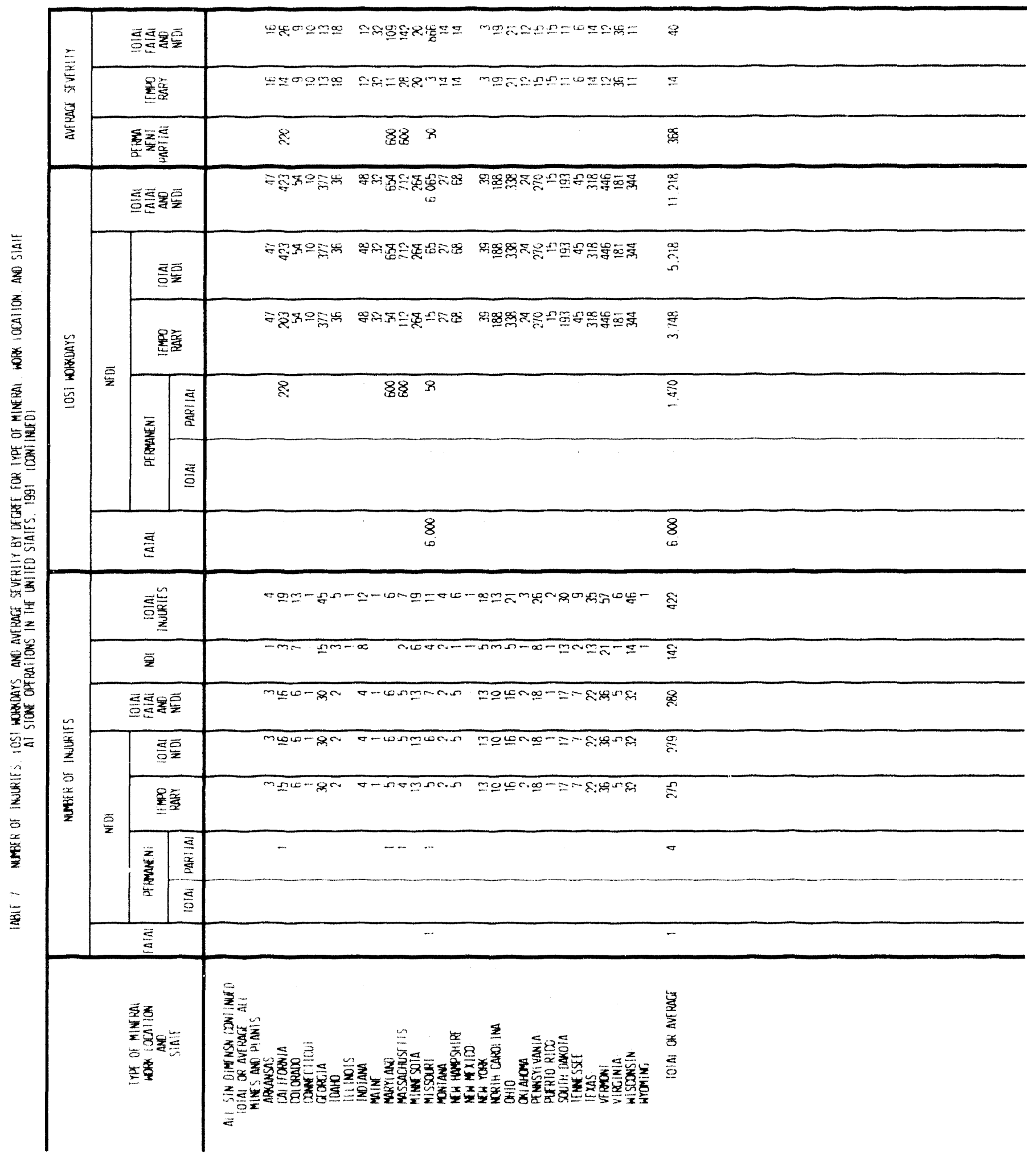




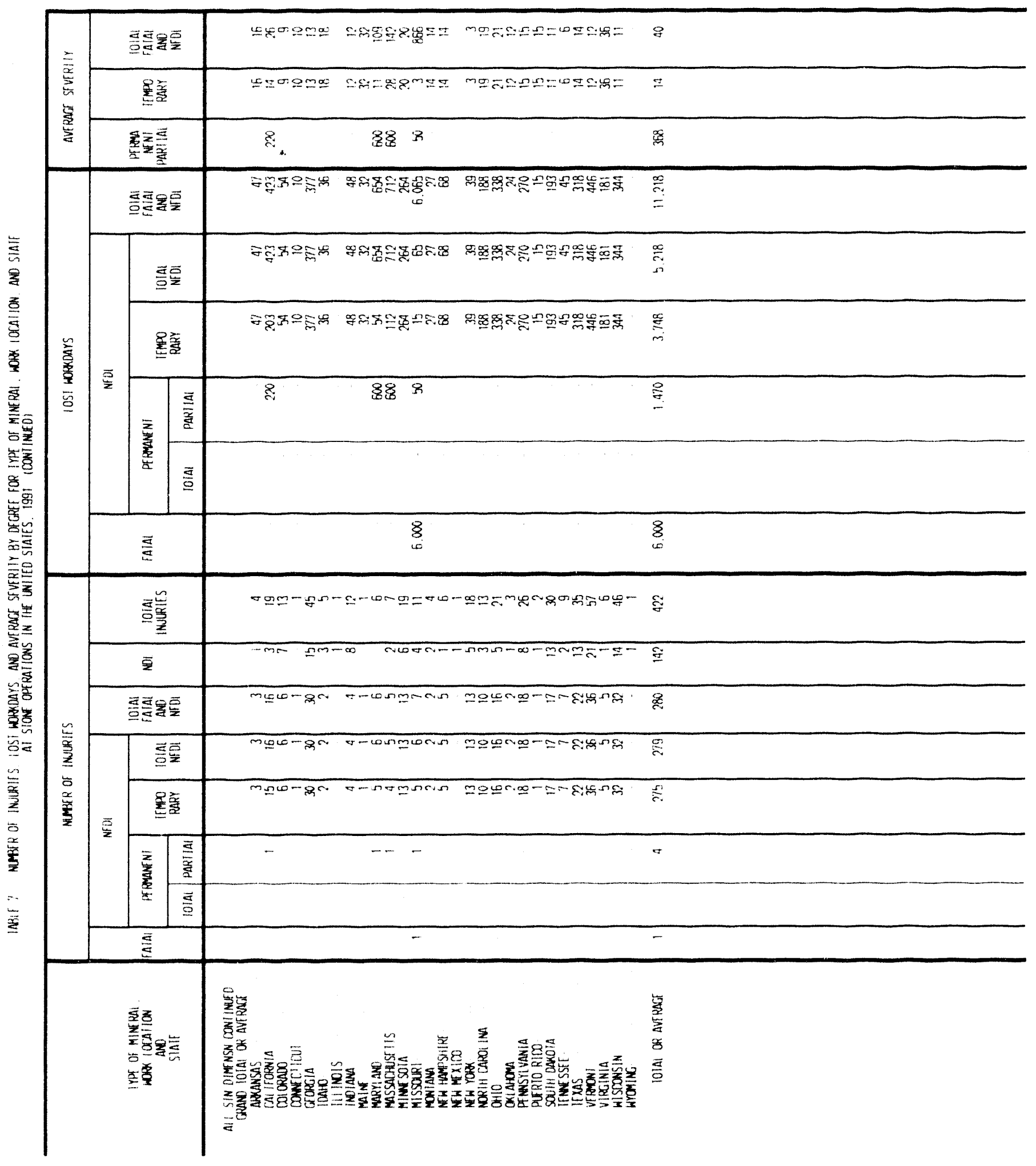




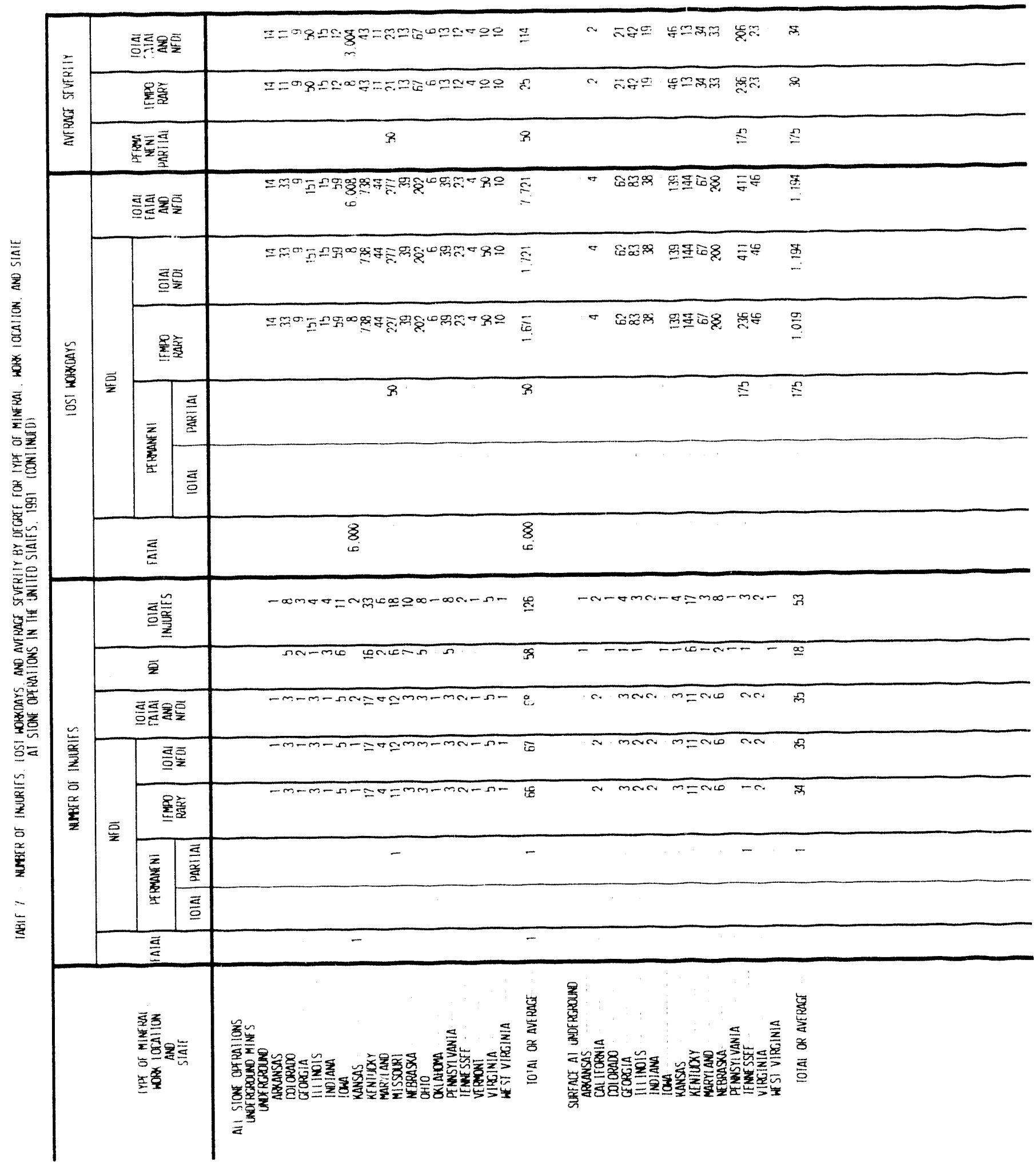




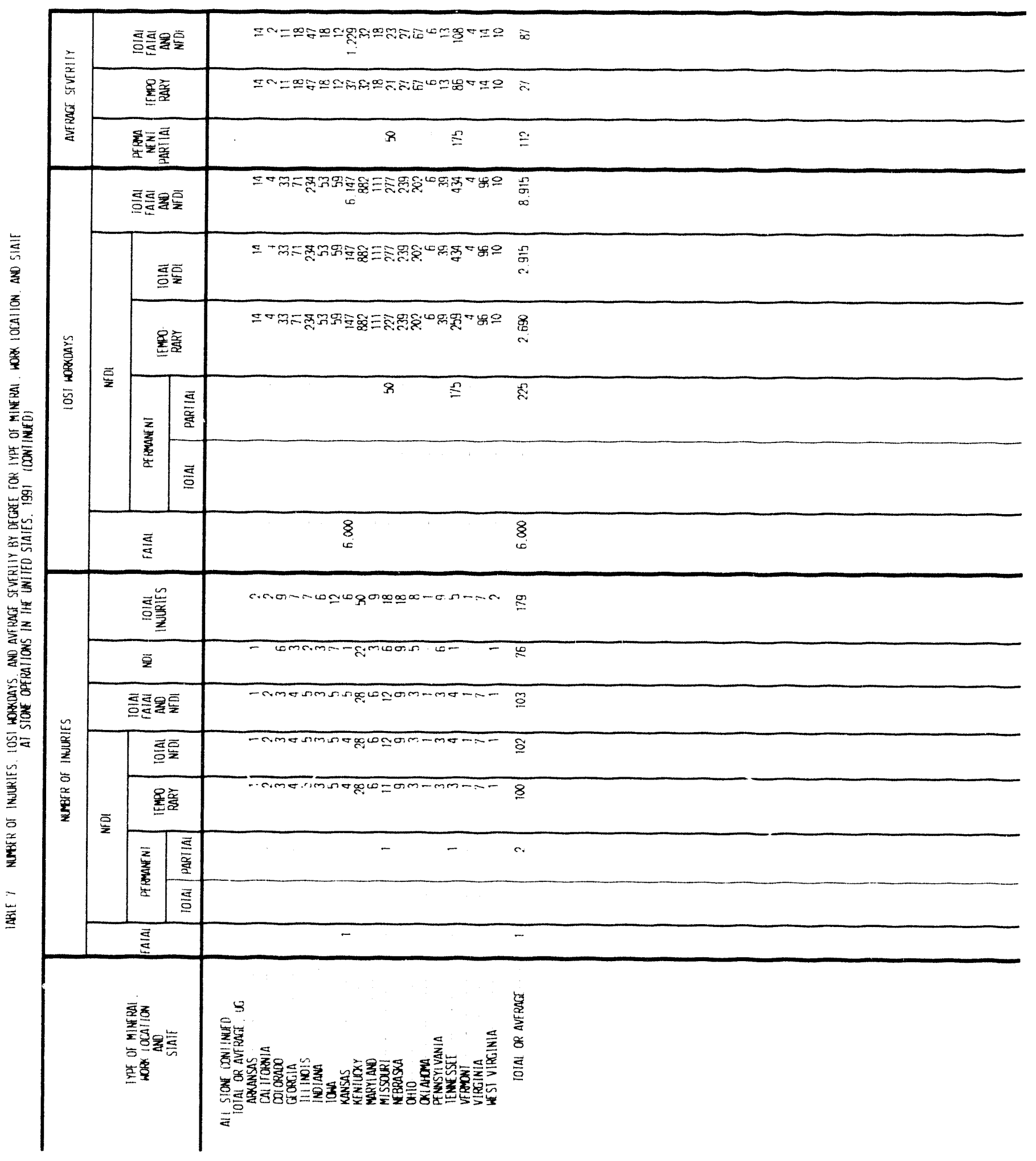




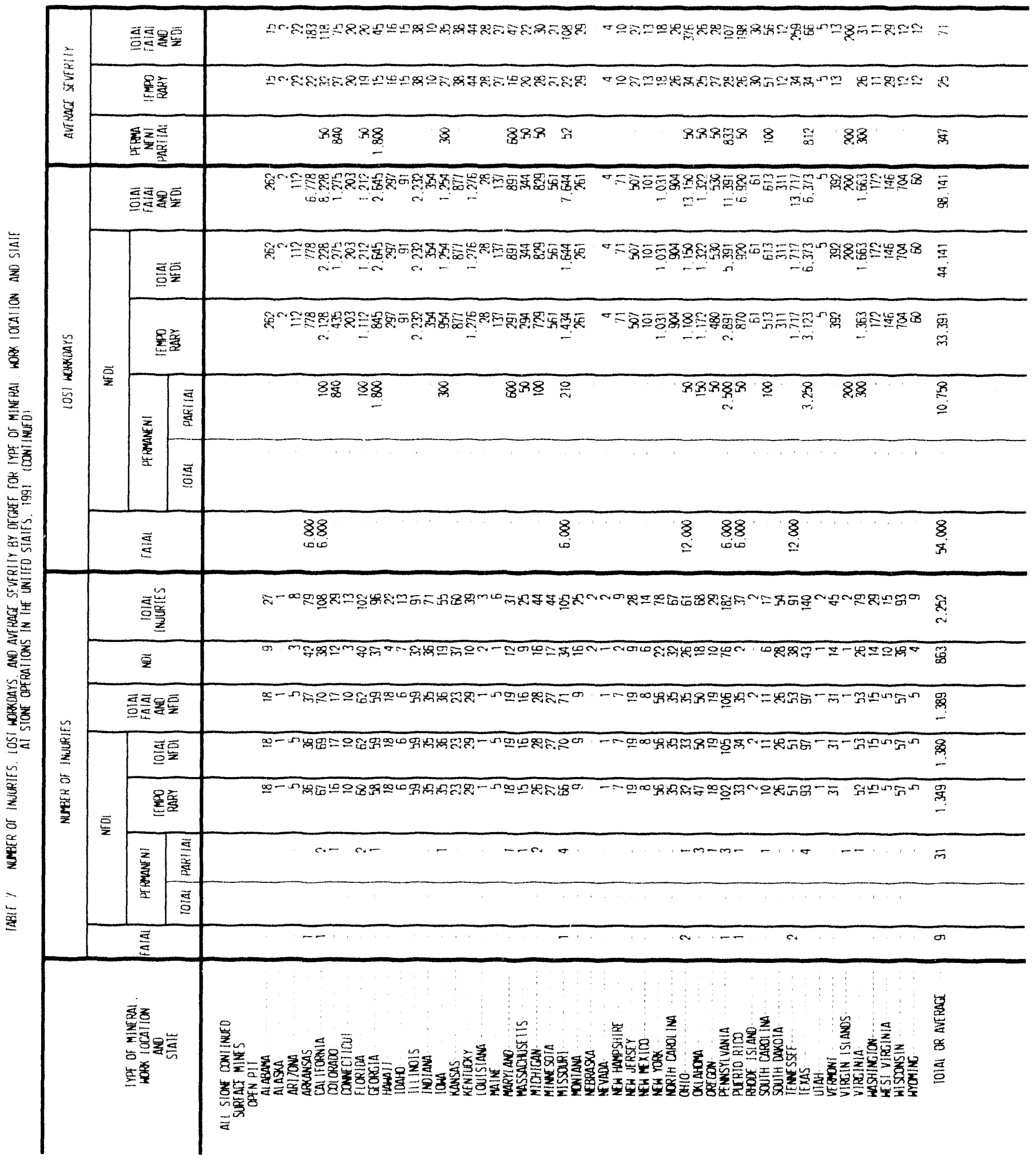




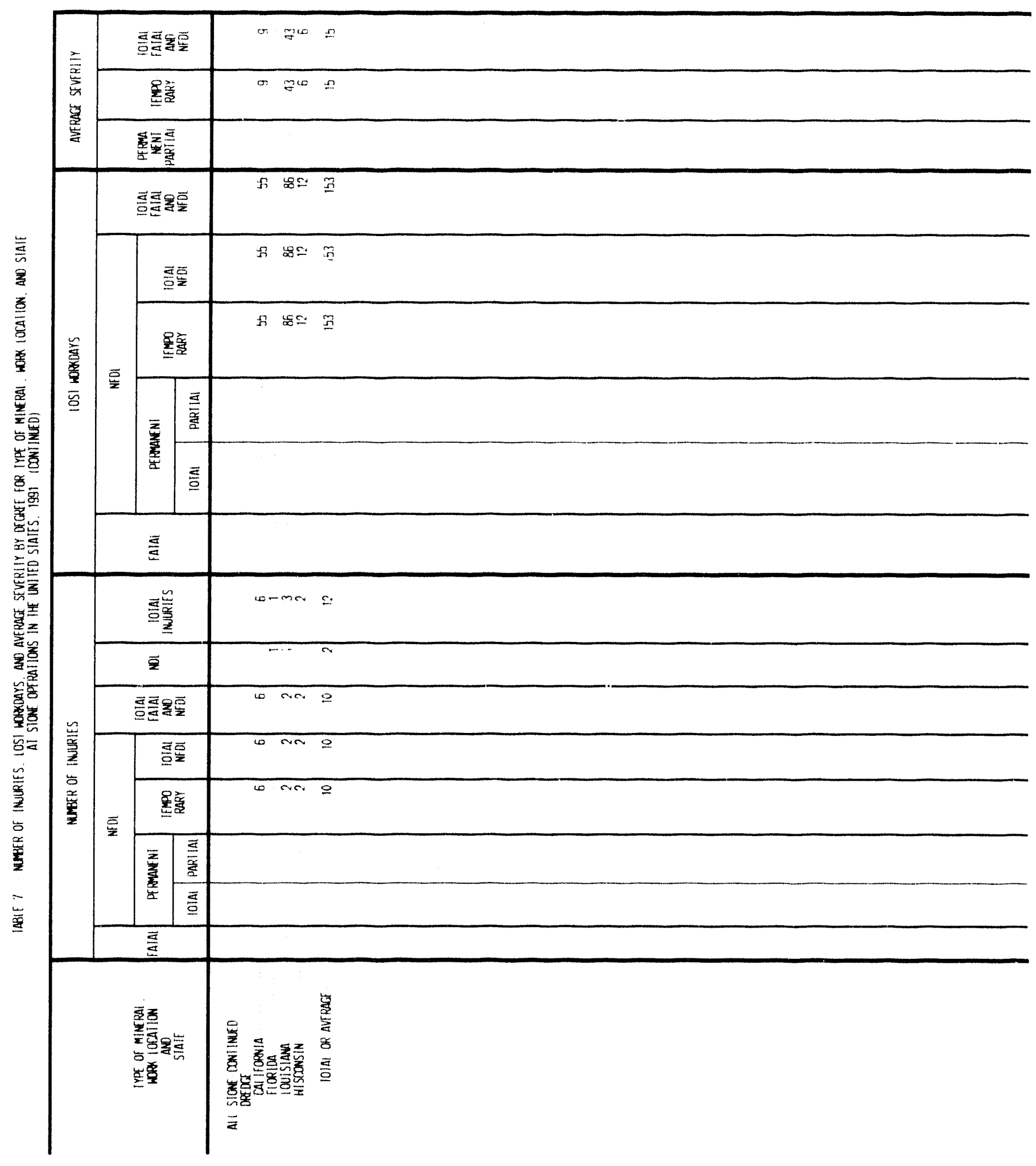




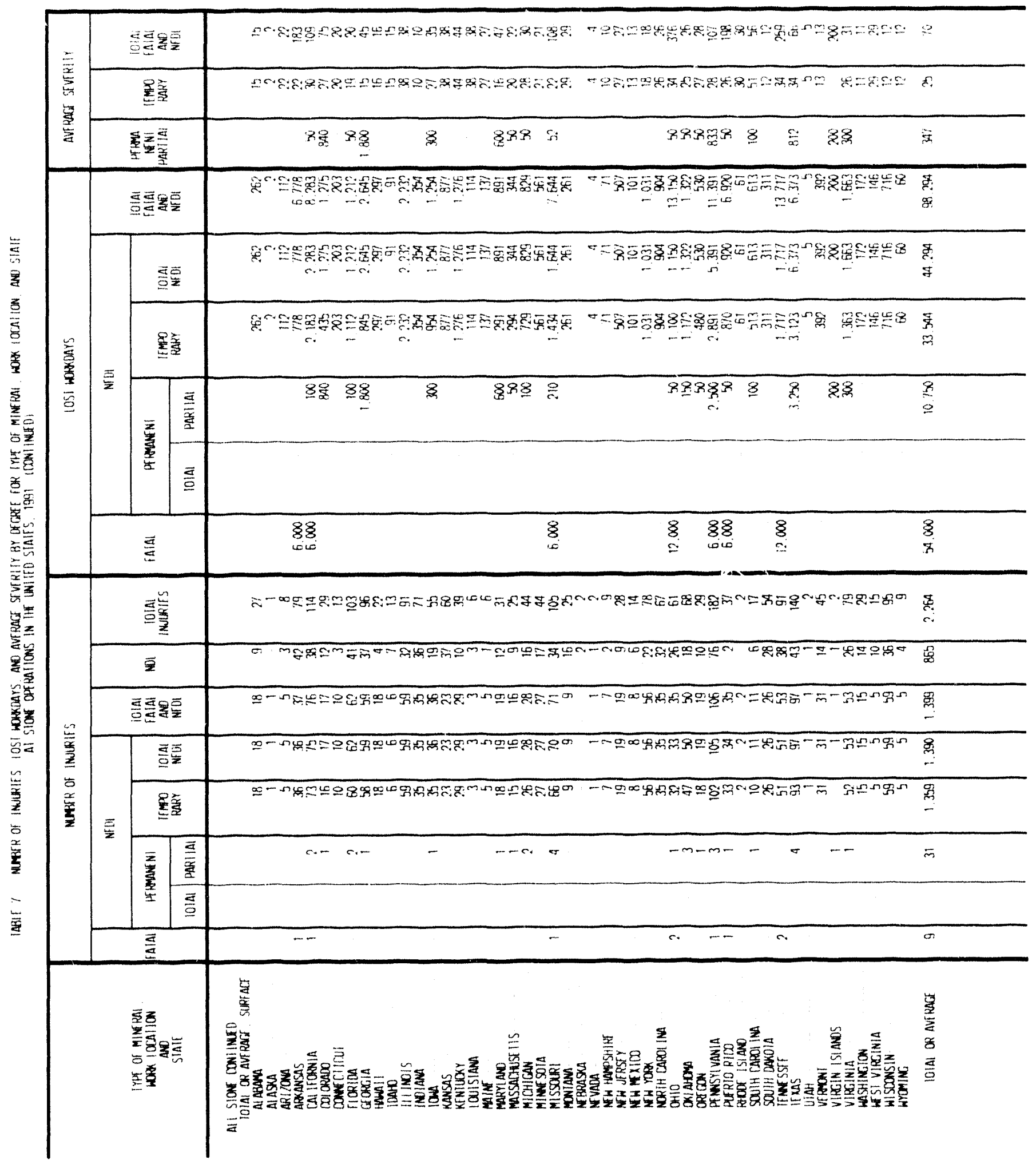




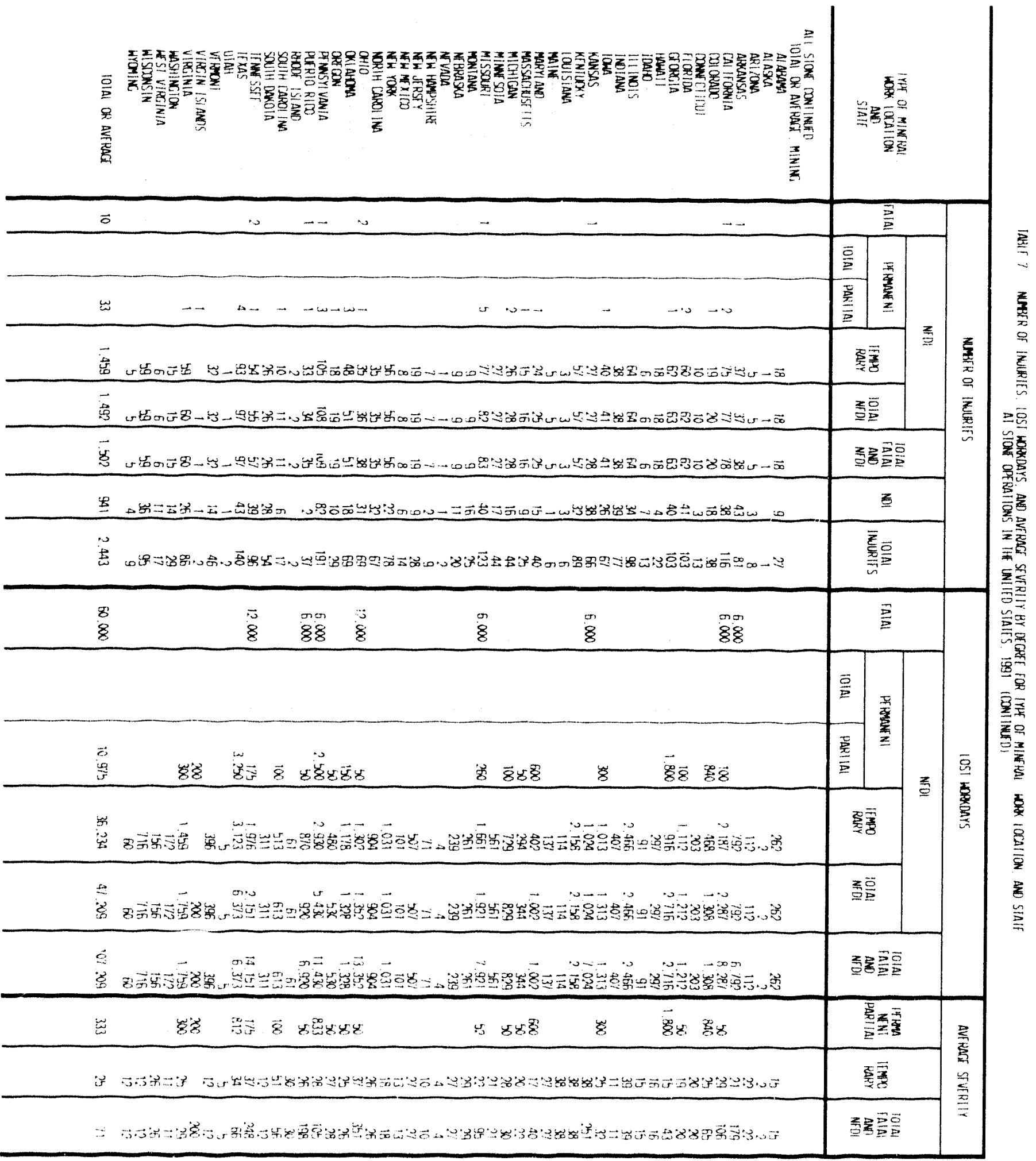




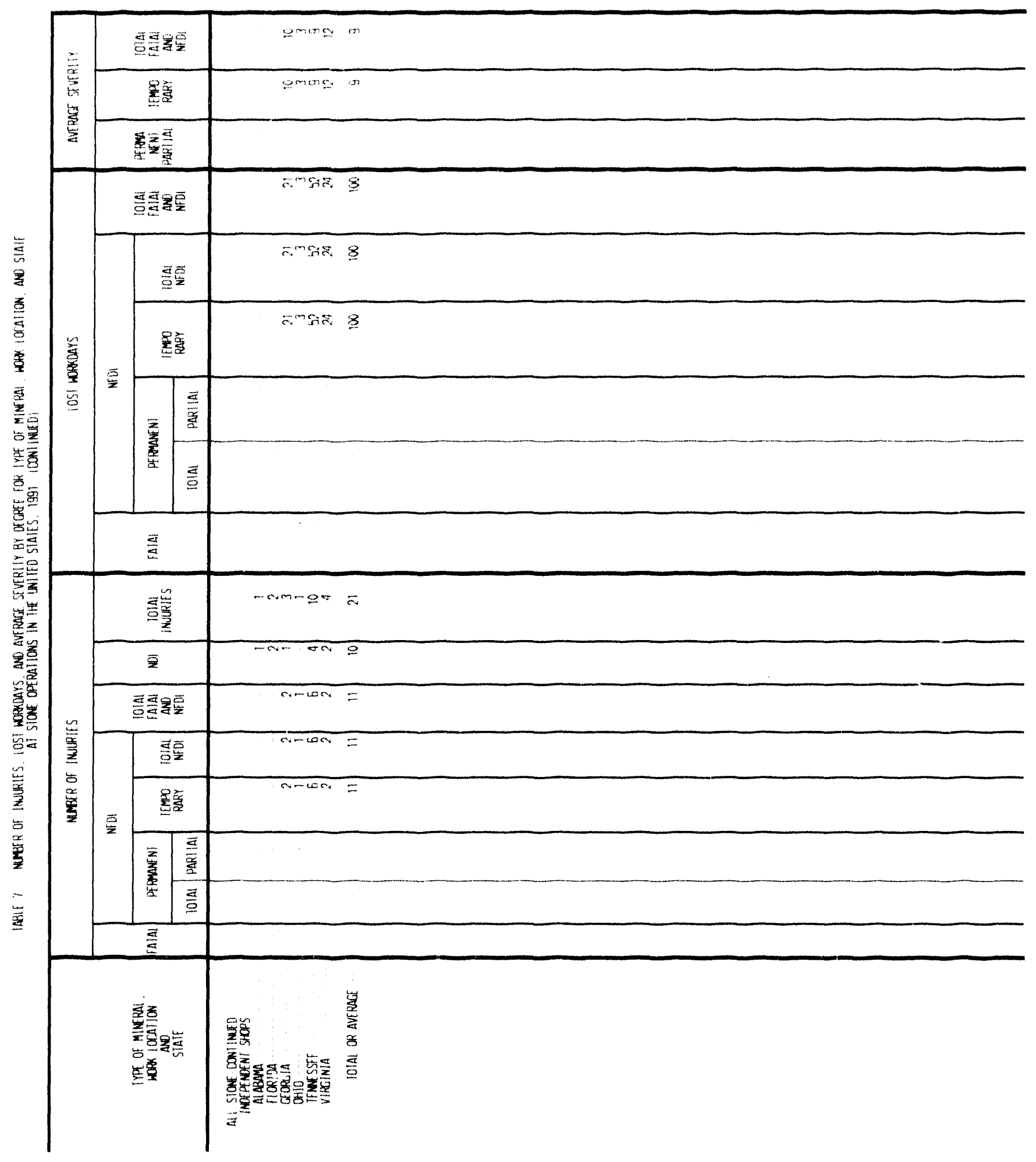




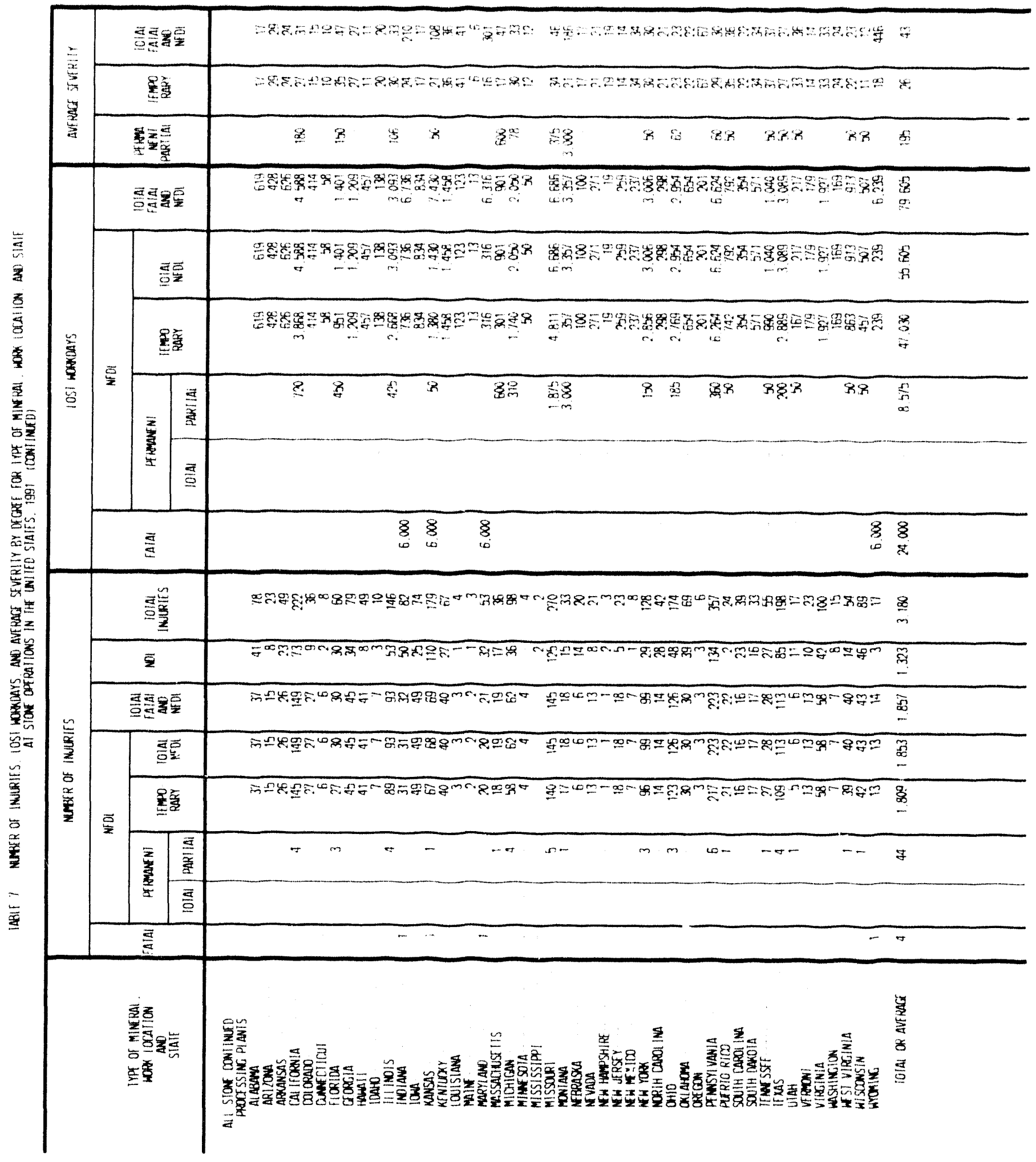




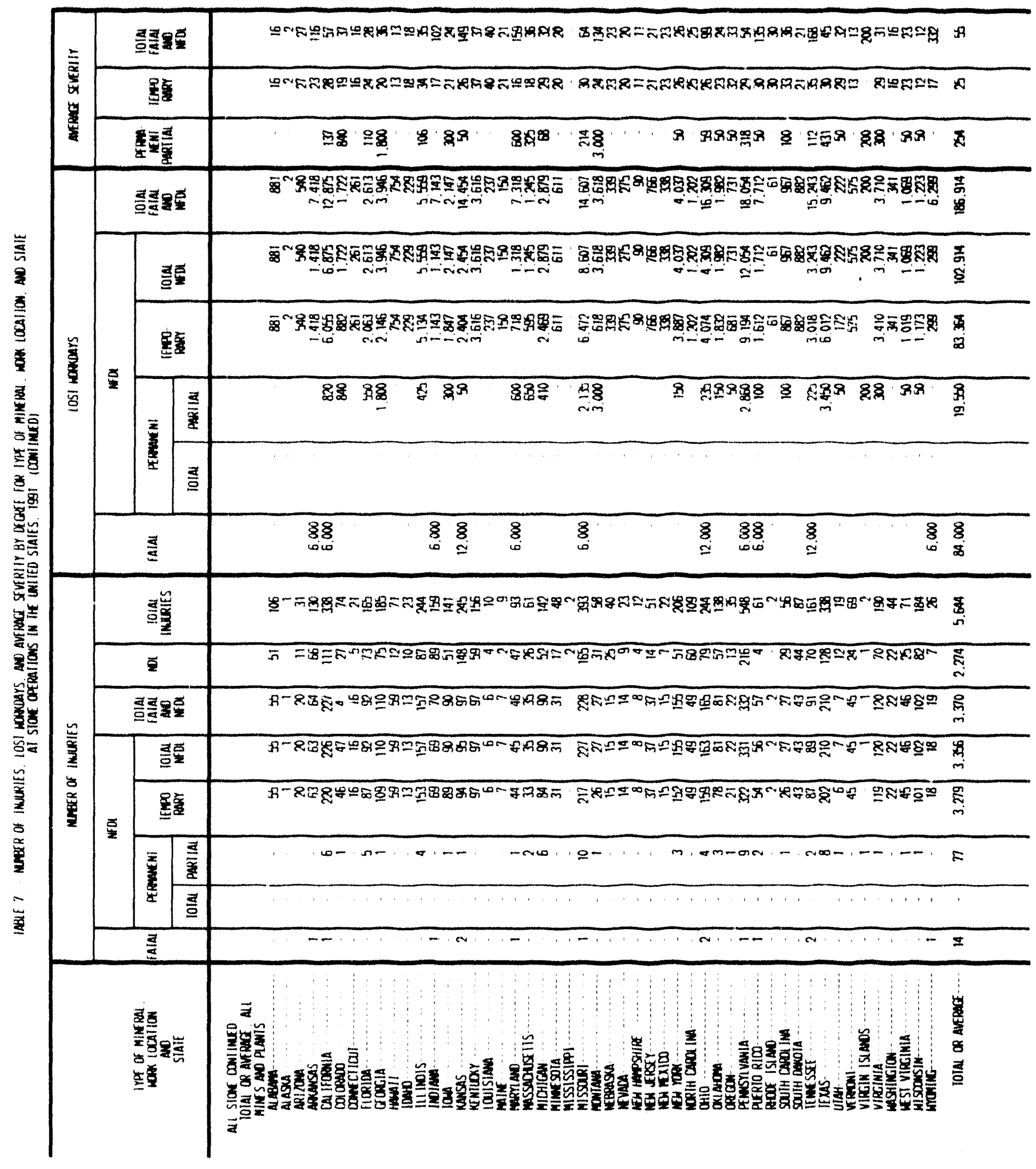




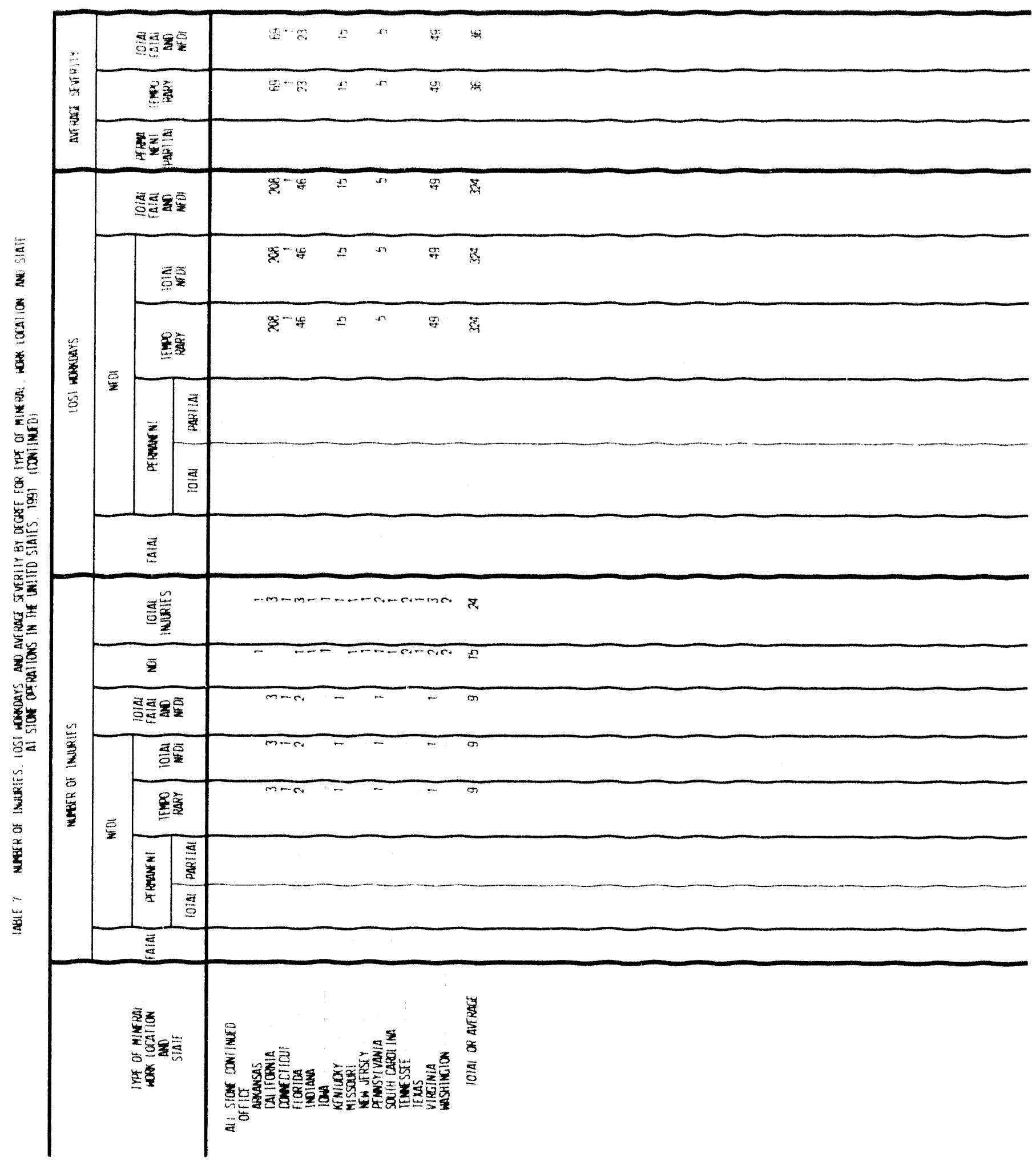




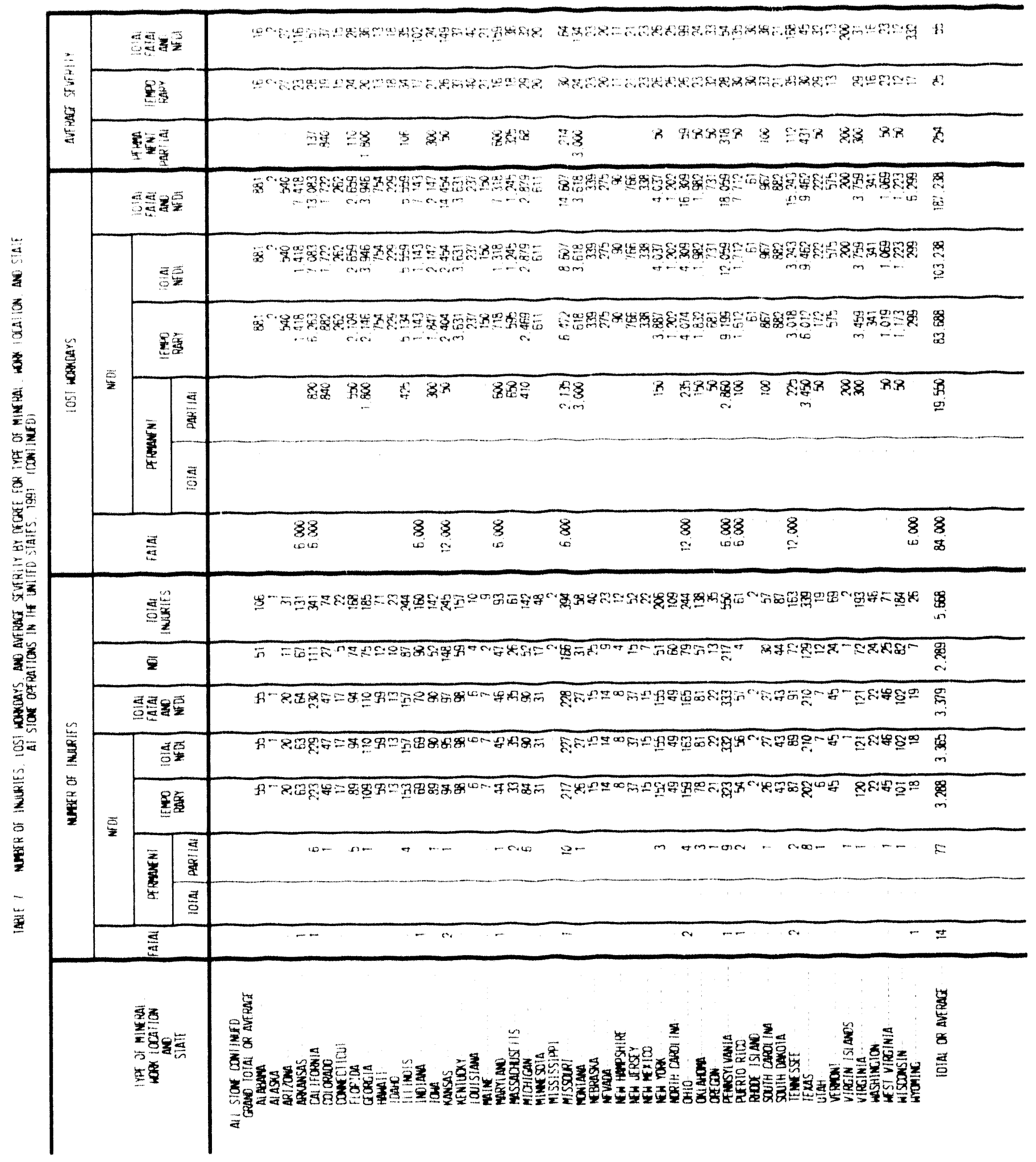




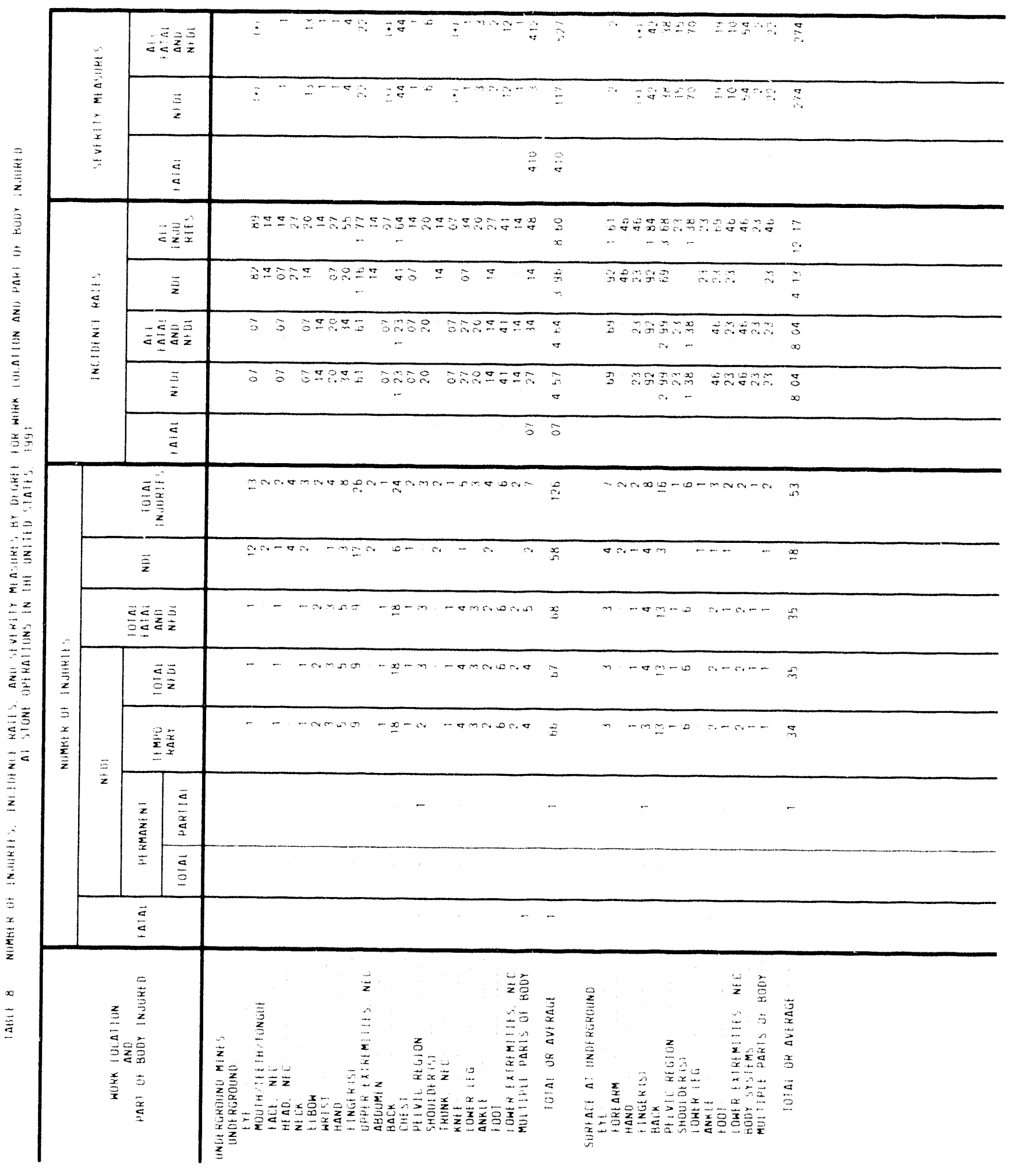




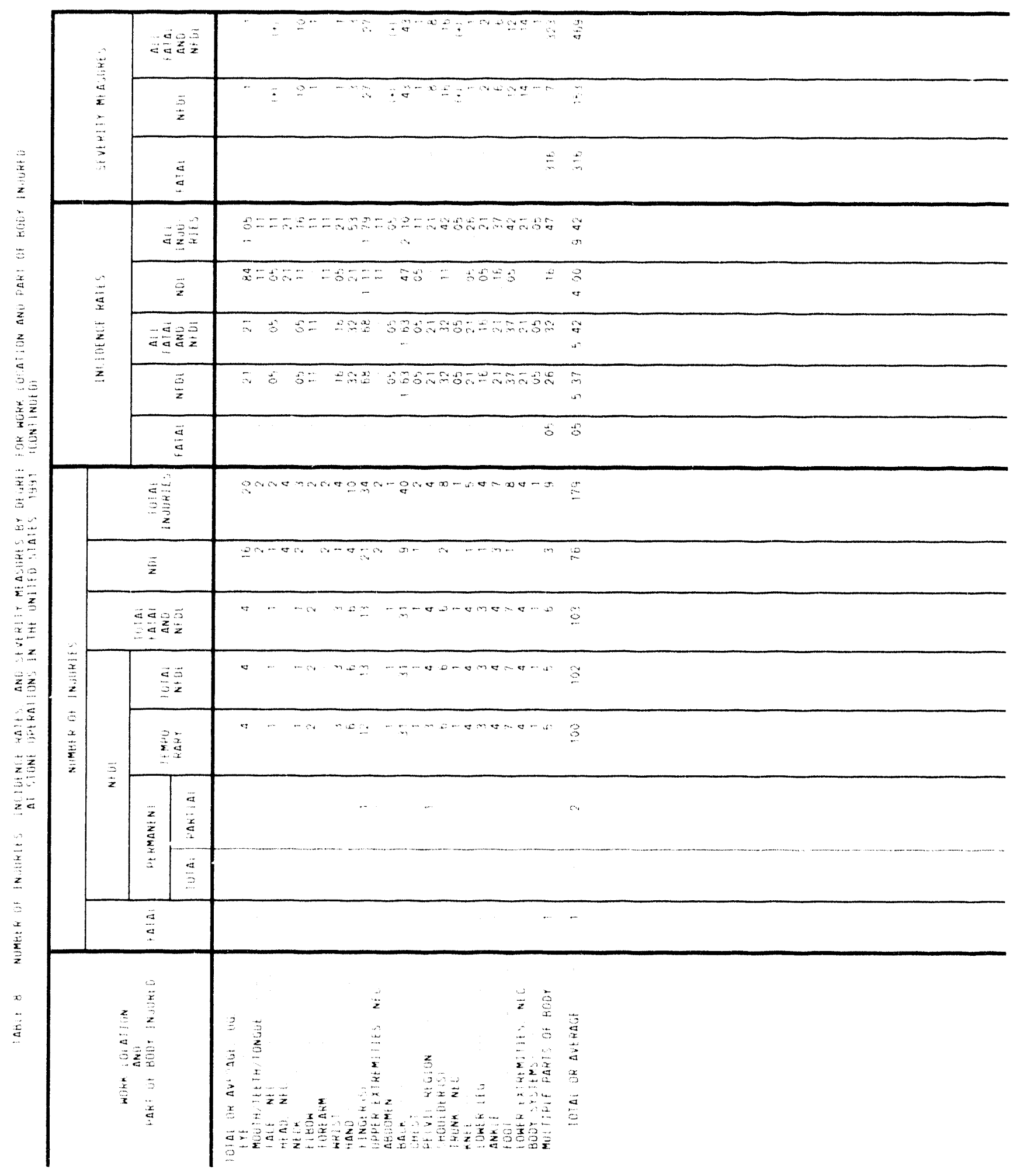




$$
\text { III }
$$




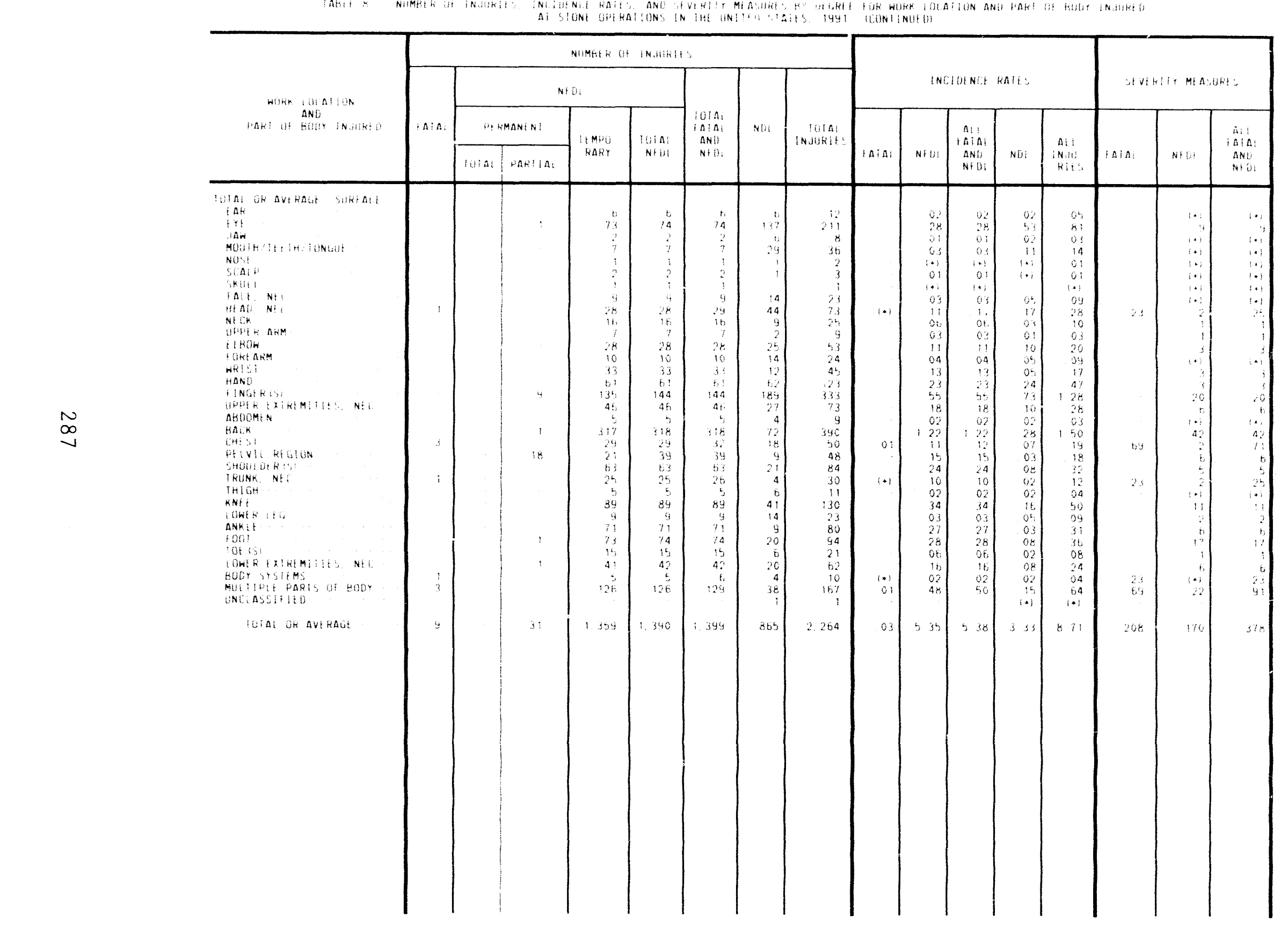




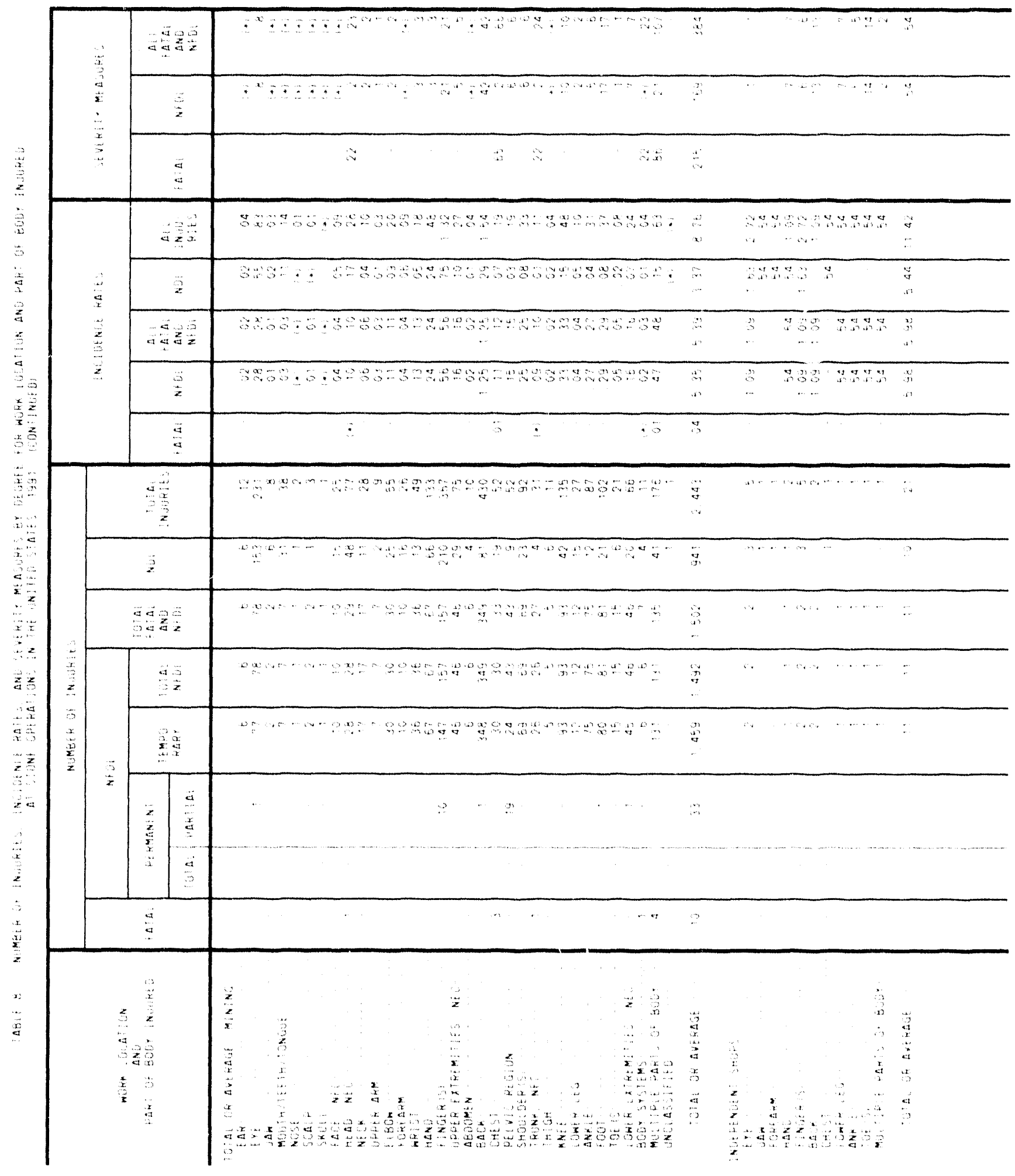




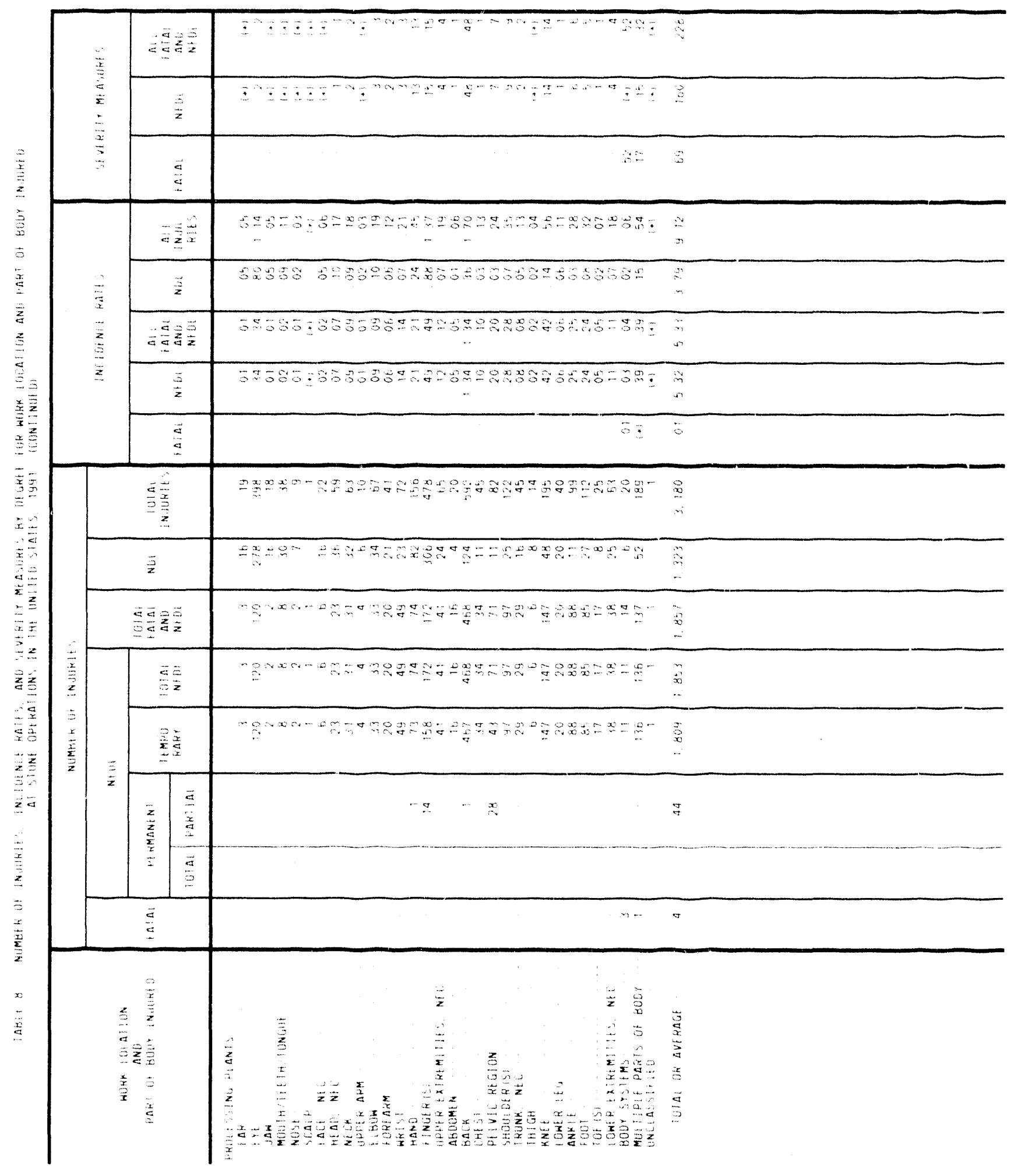




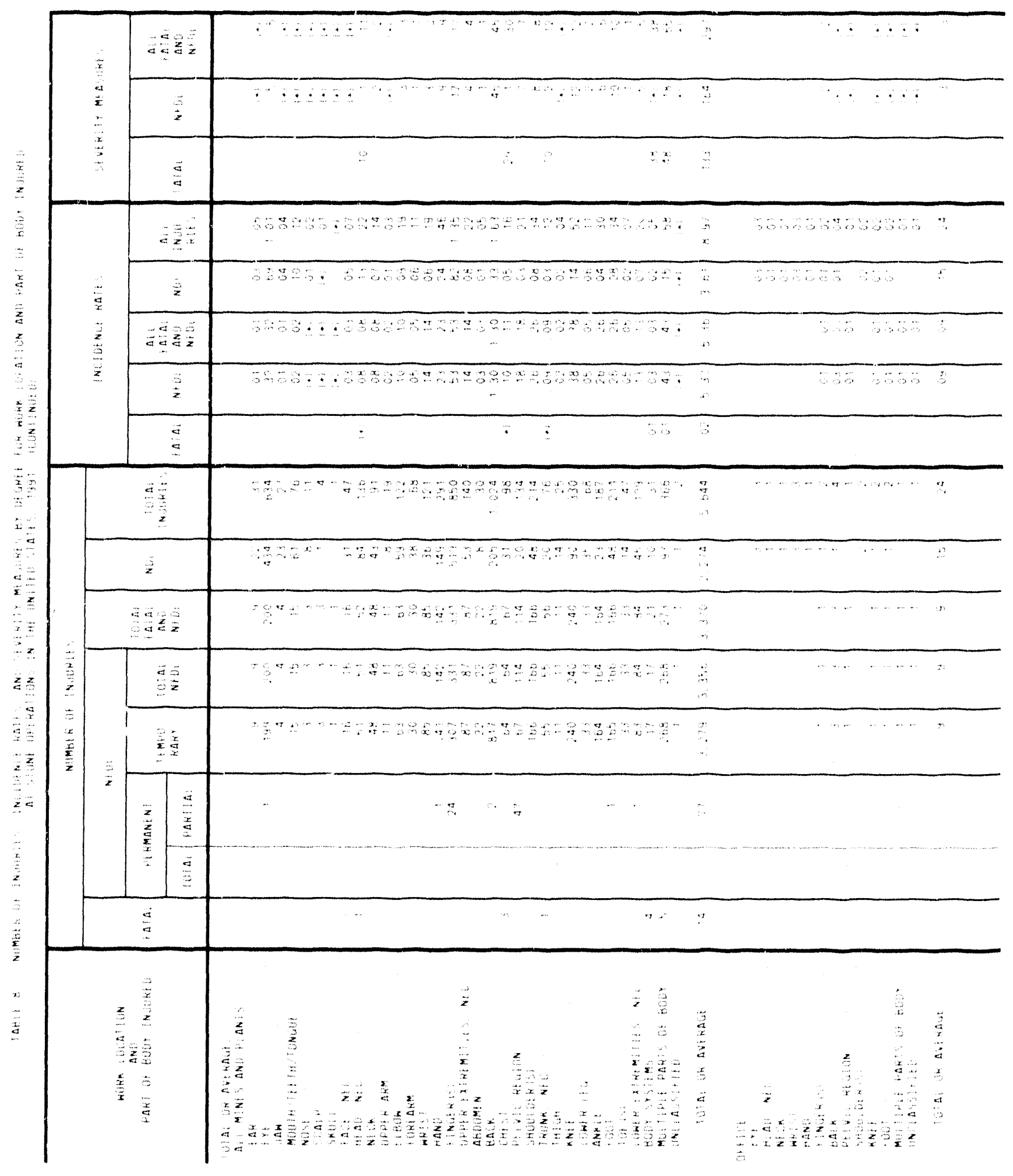




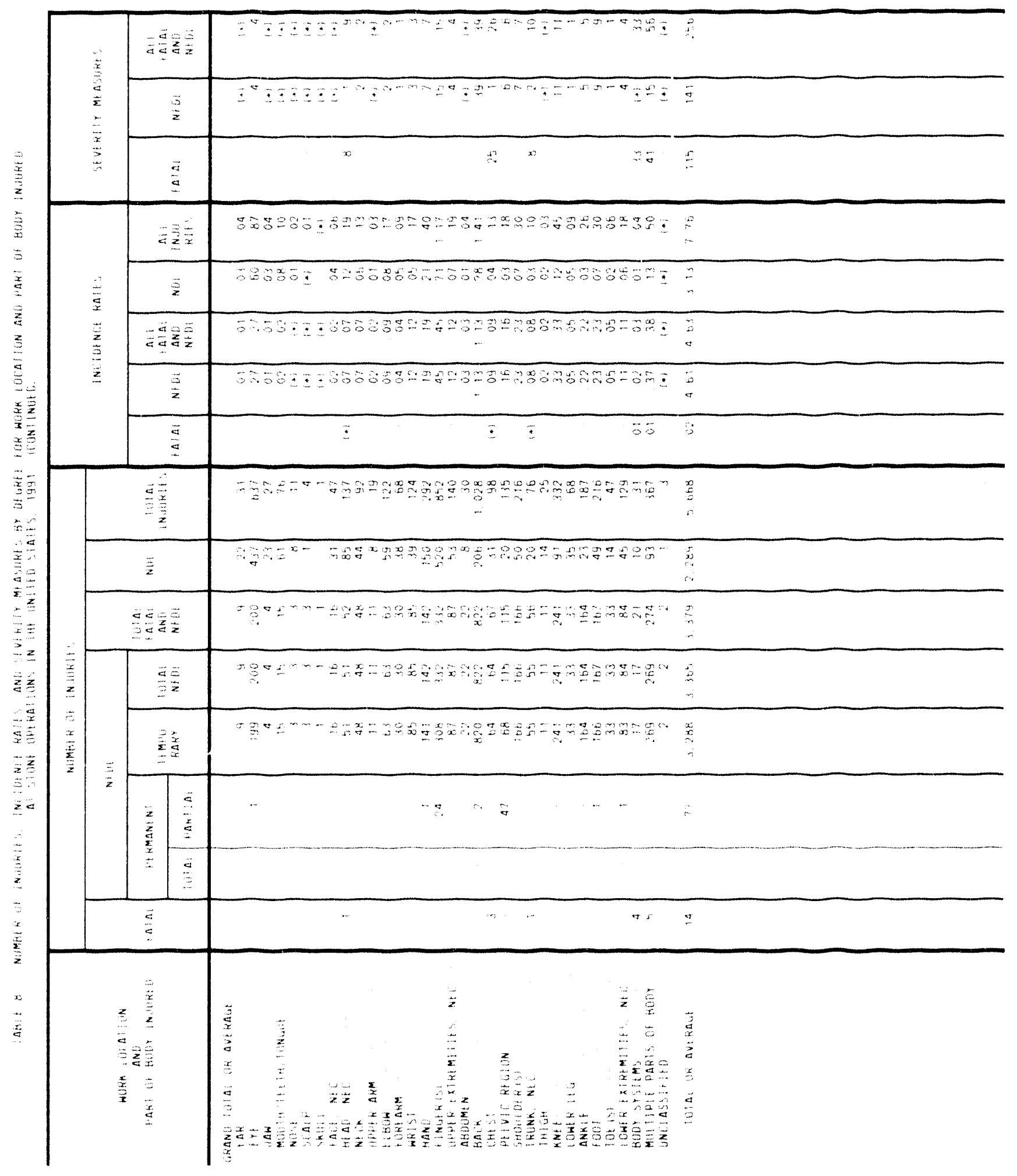




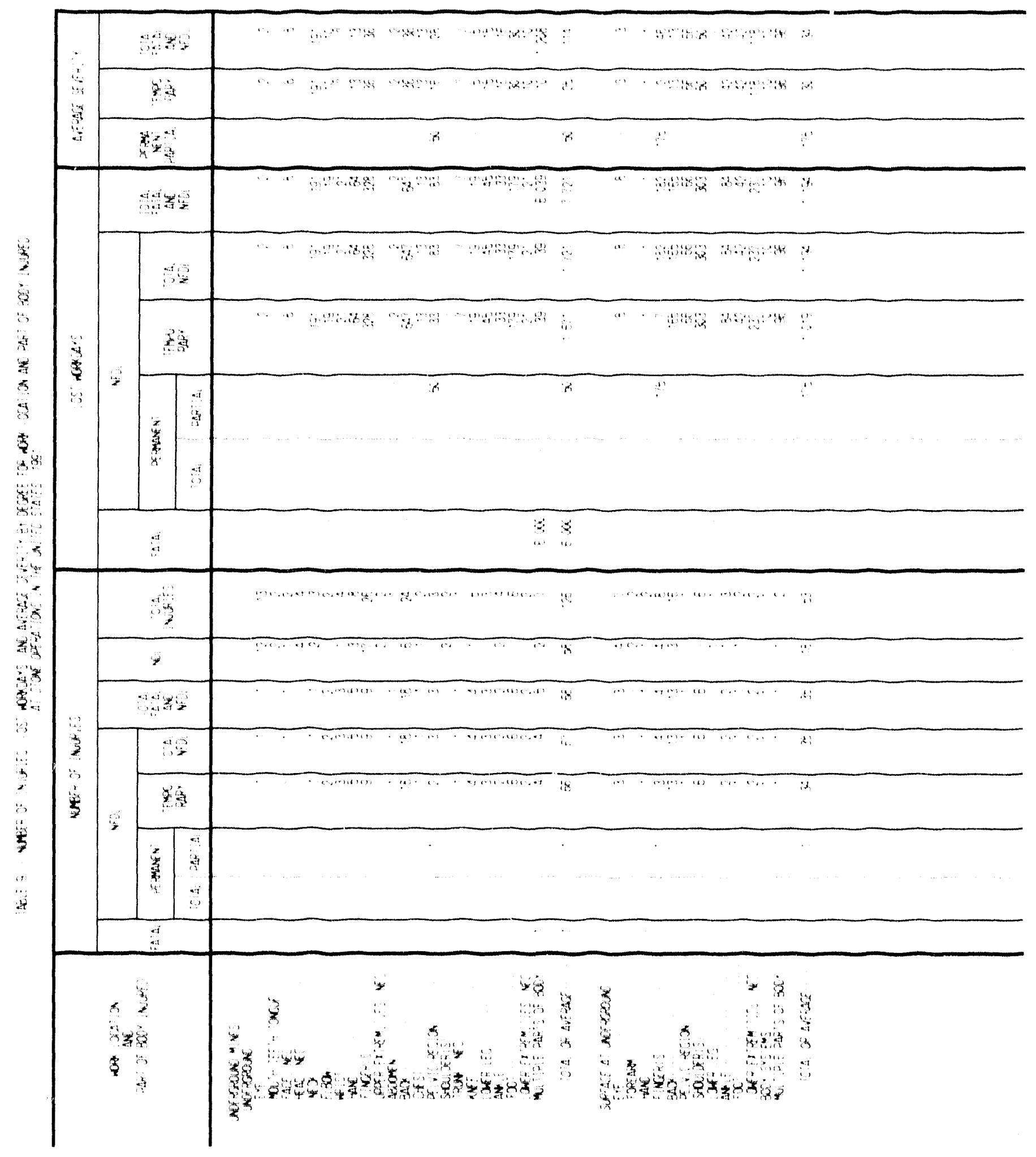




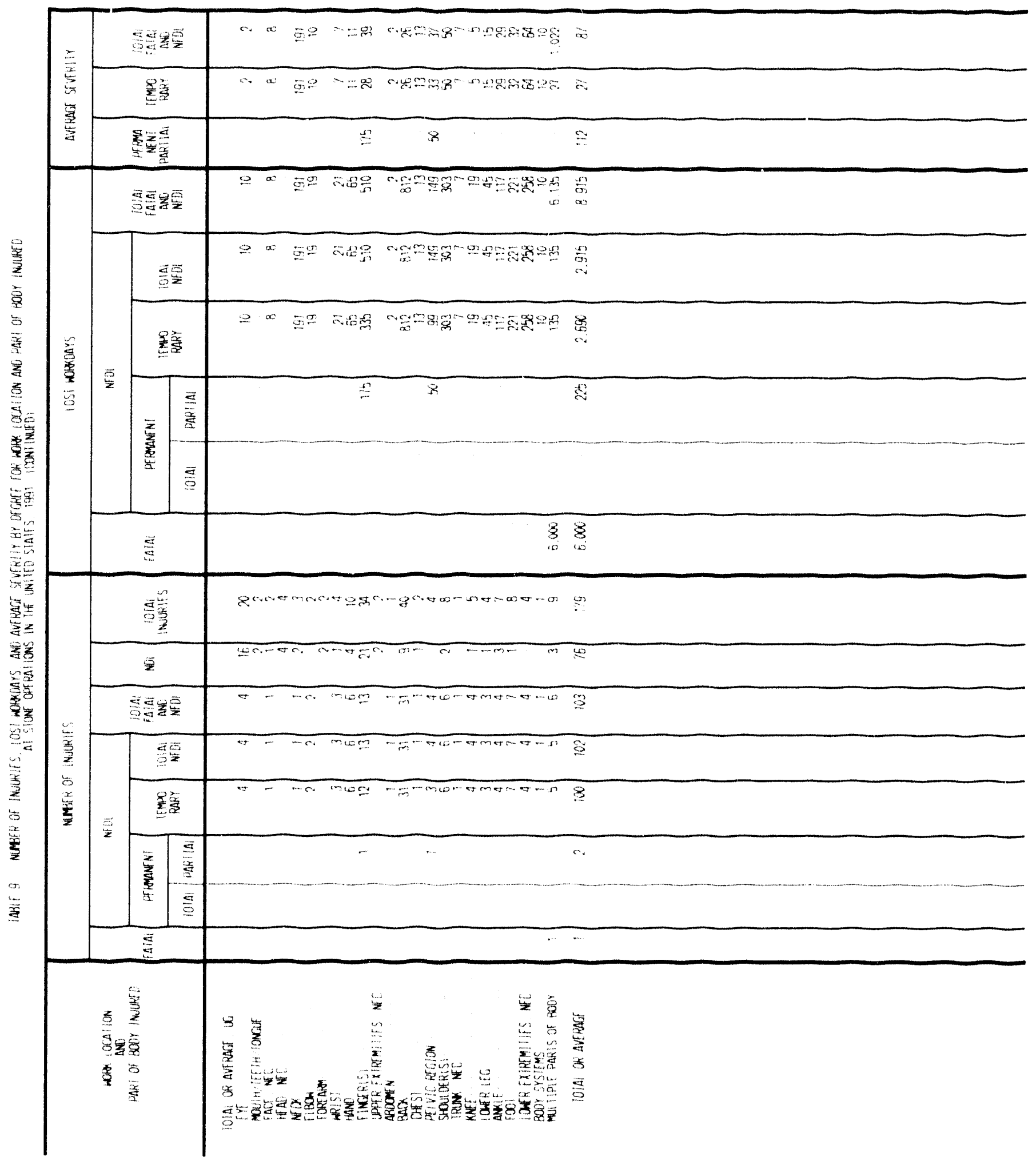




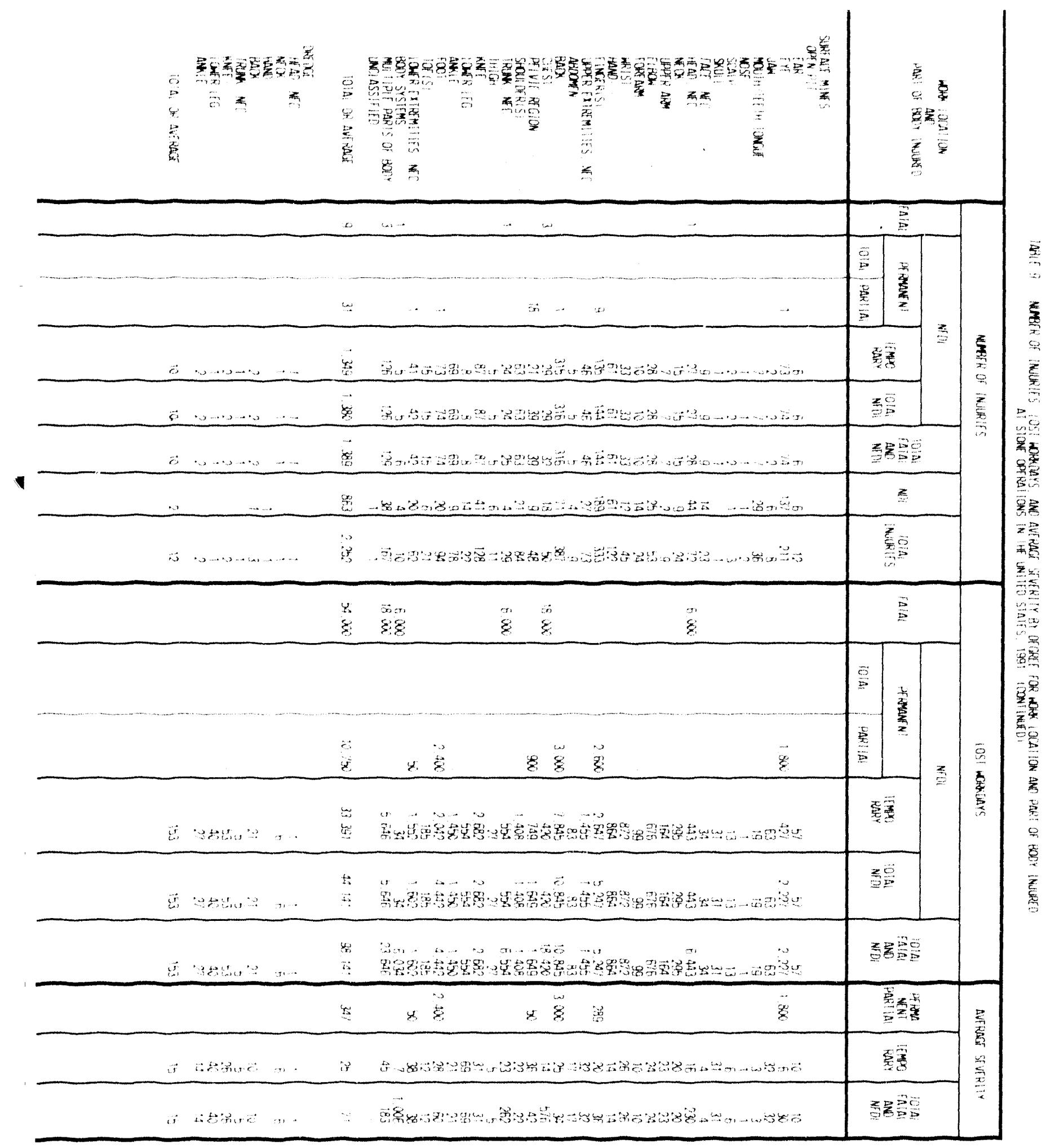




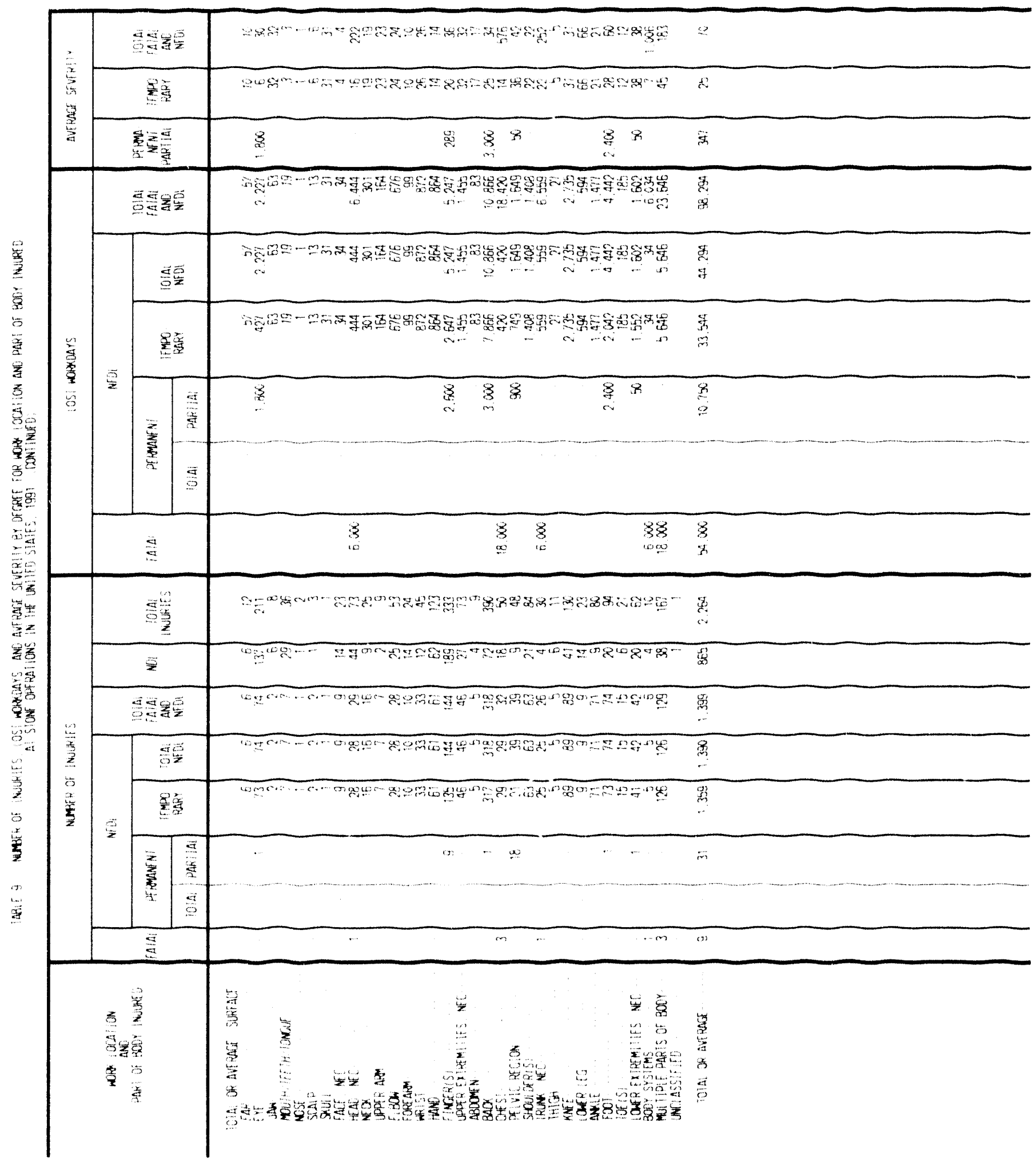




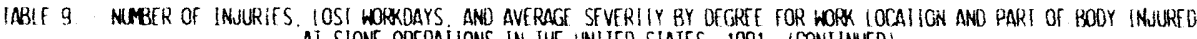
AI SIONE OPERAIIONS IN IHE UNIITD SIATES, 1991 IODNINIED

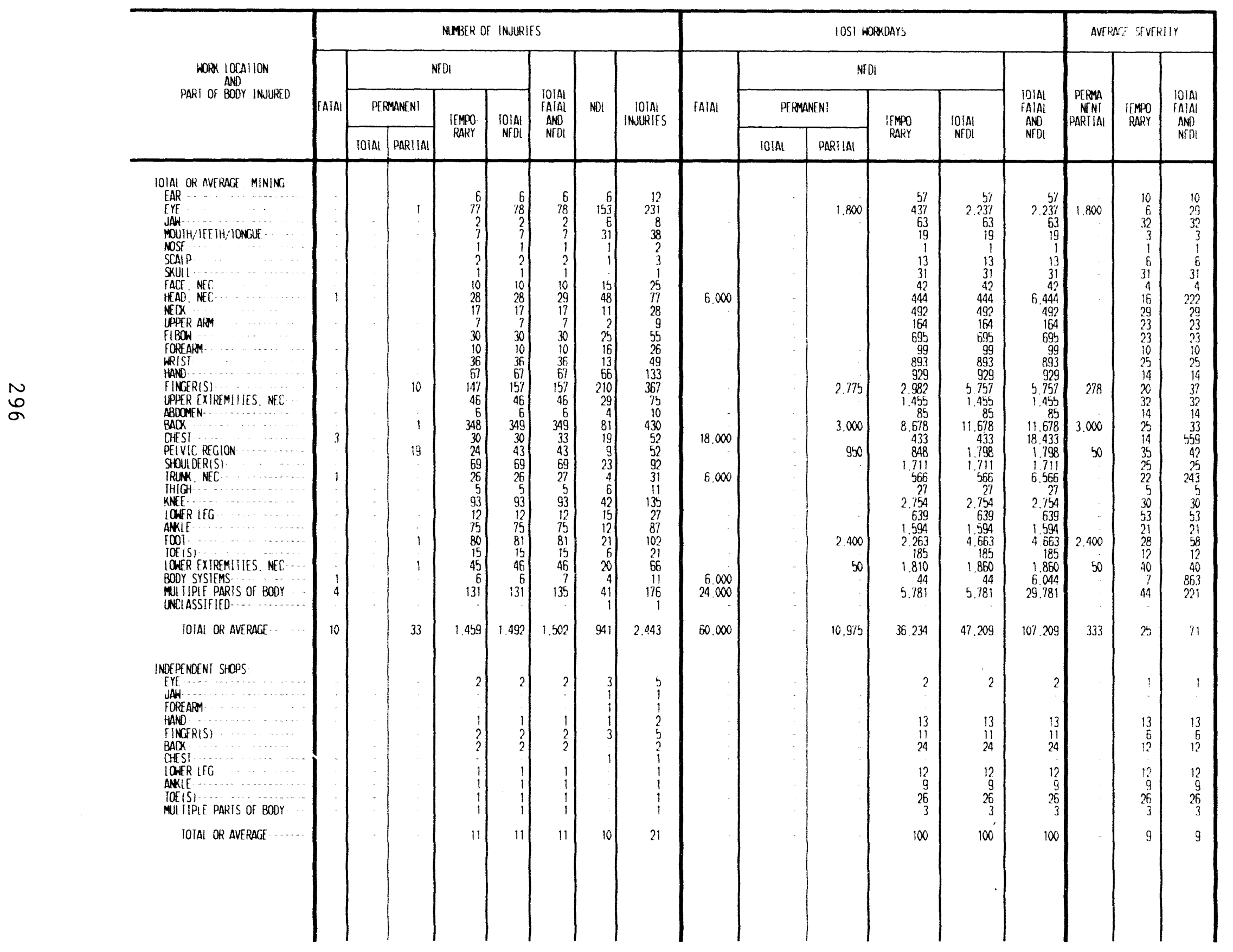




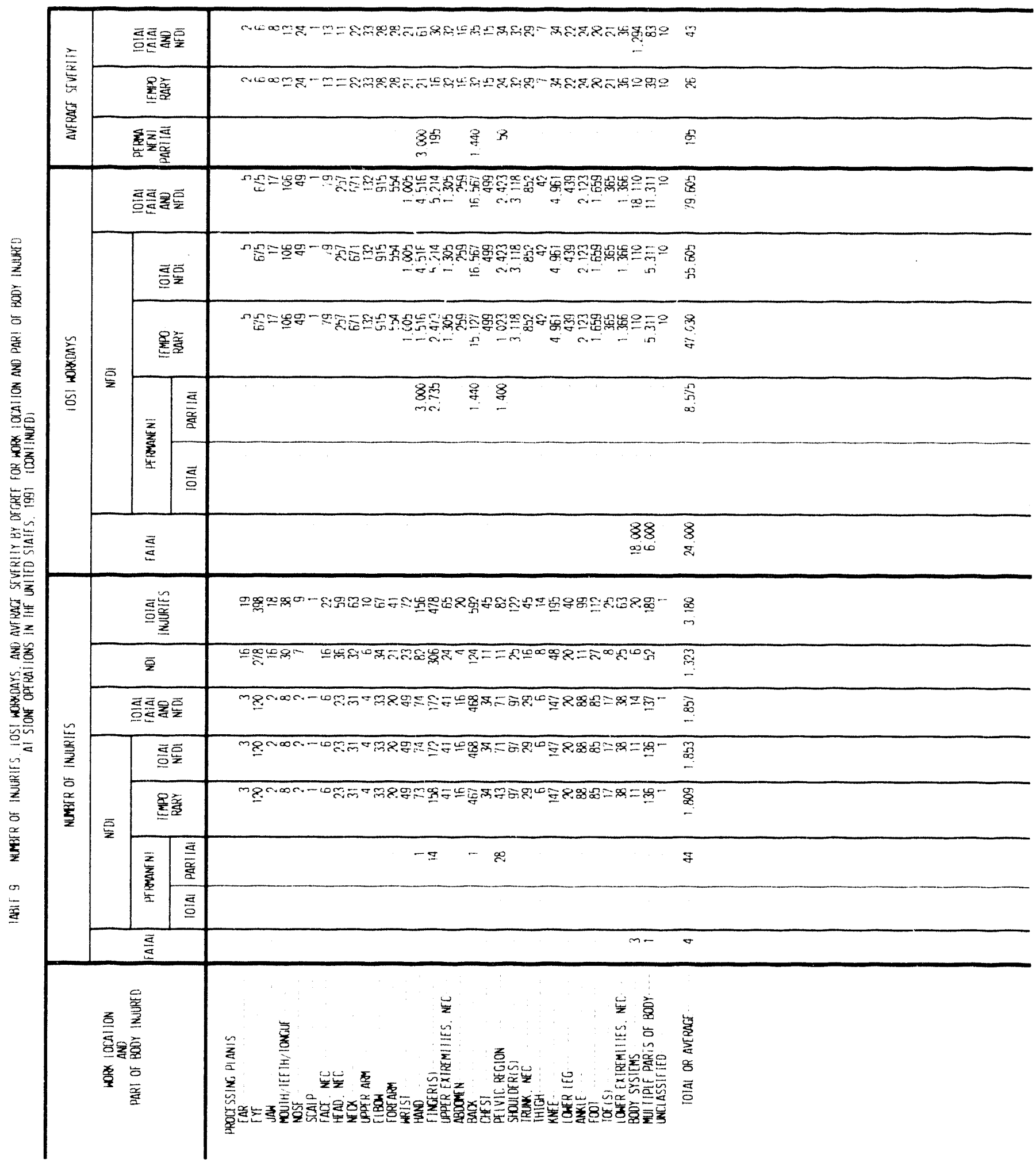




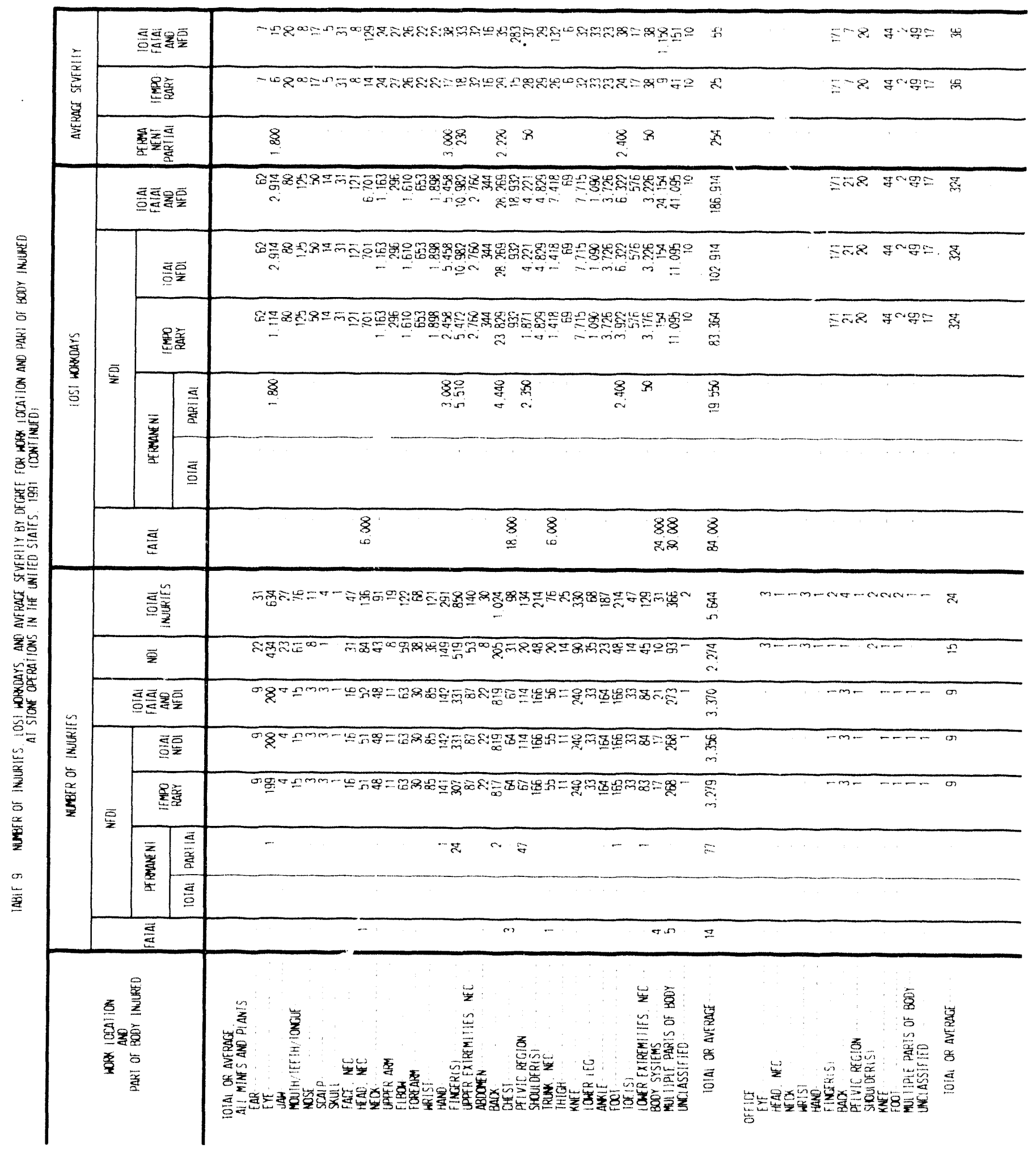




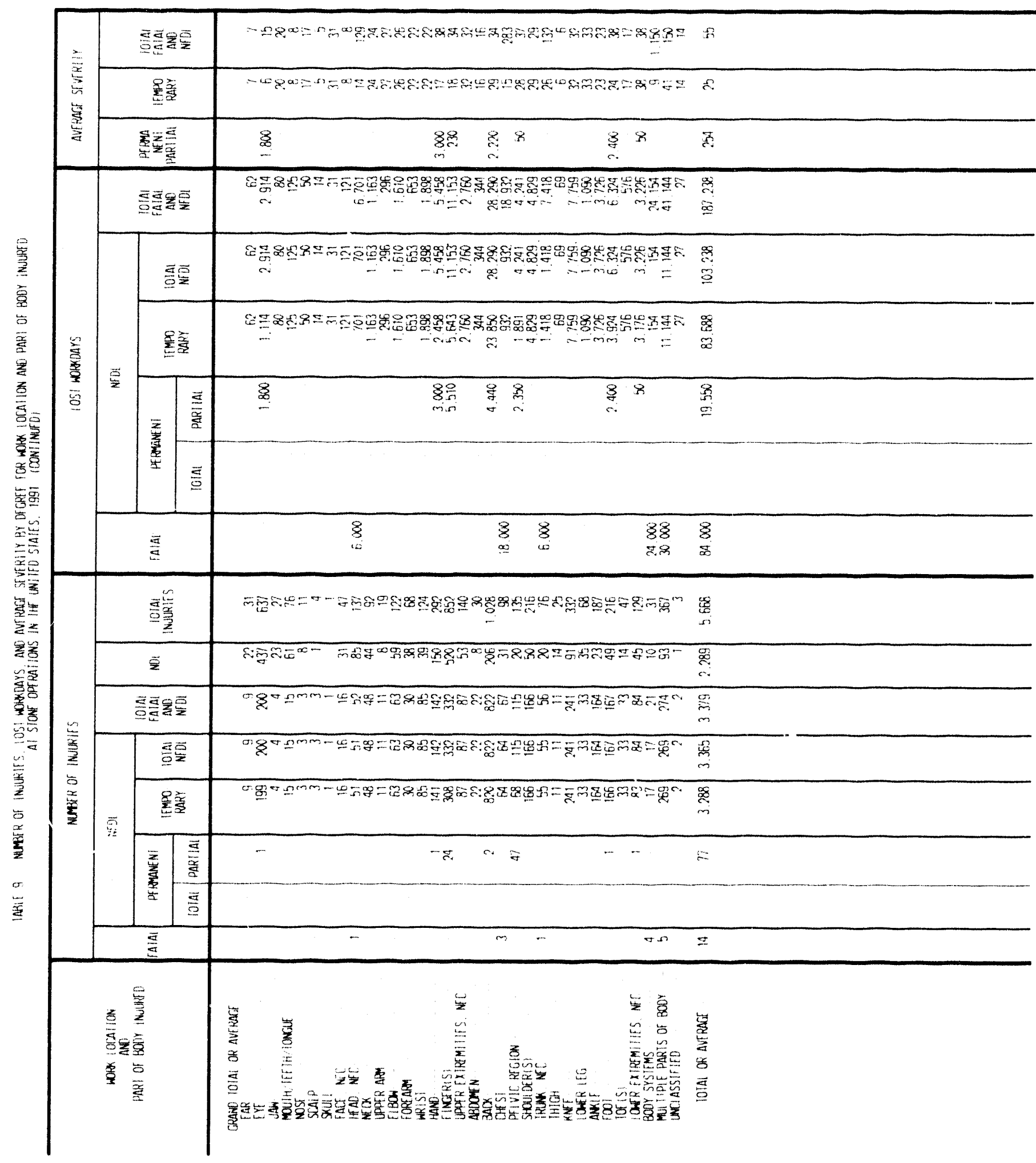




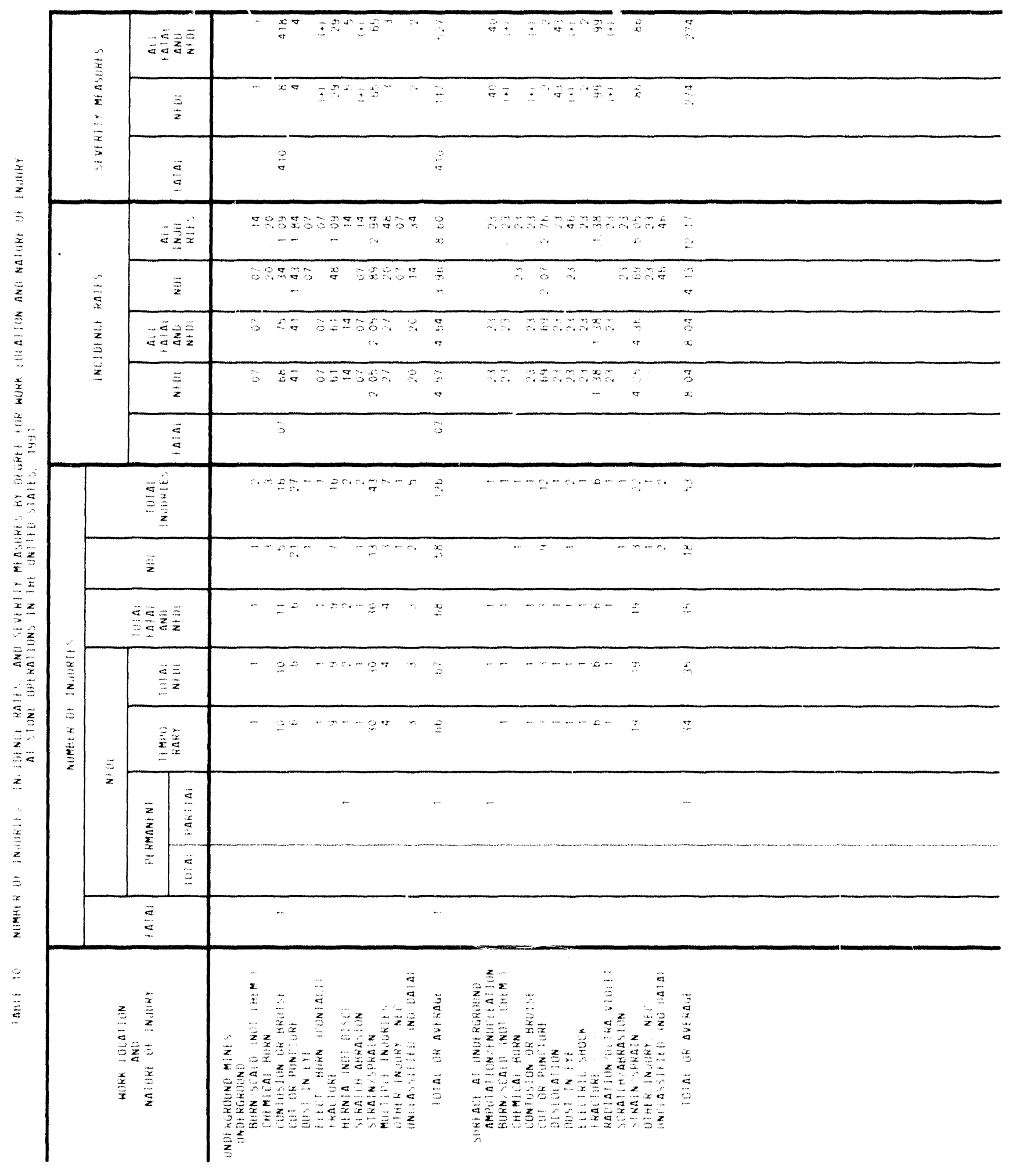




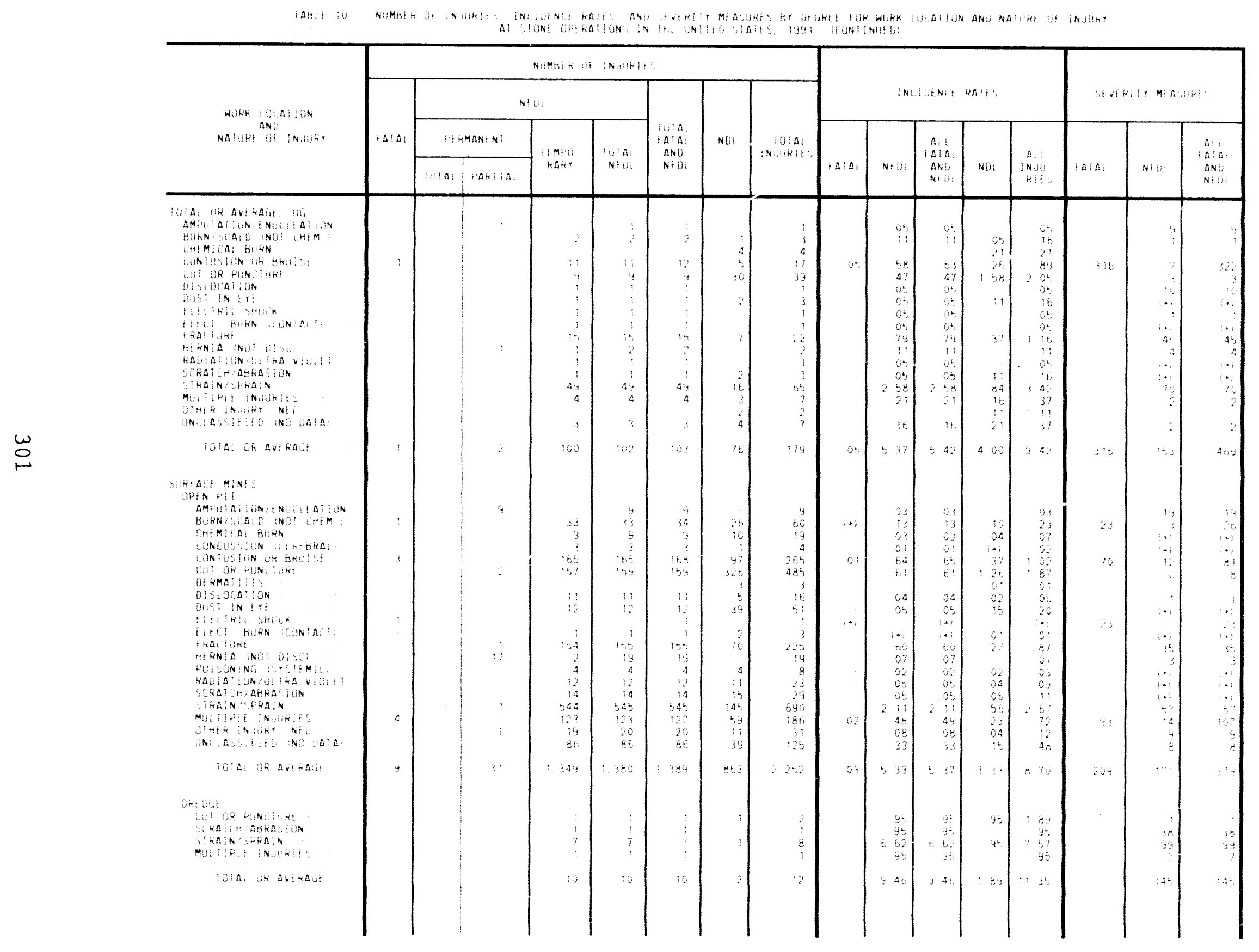




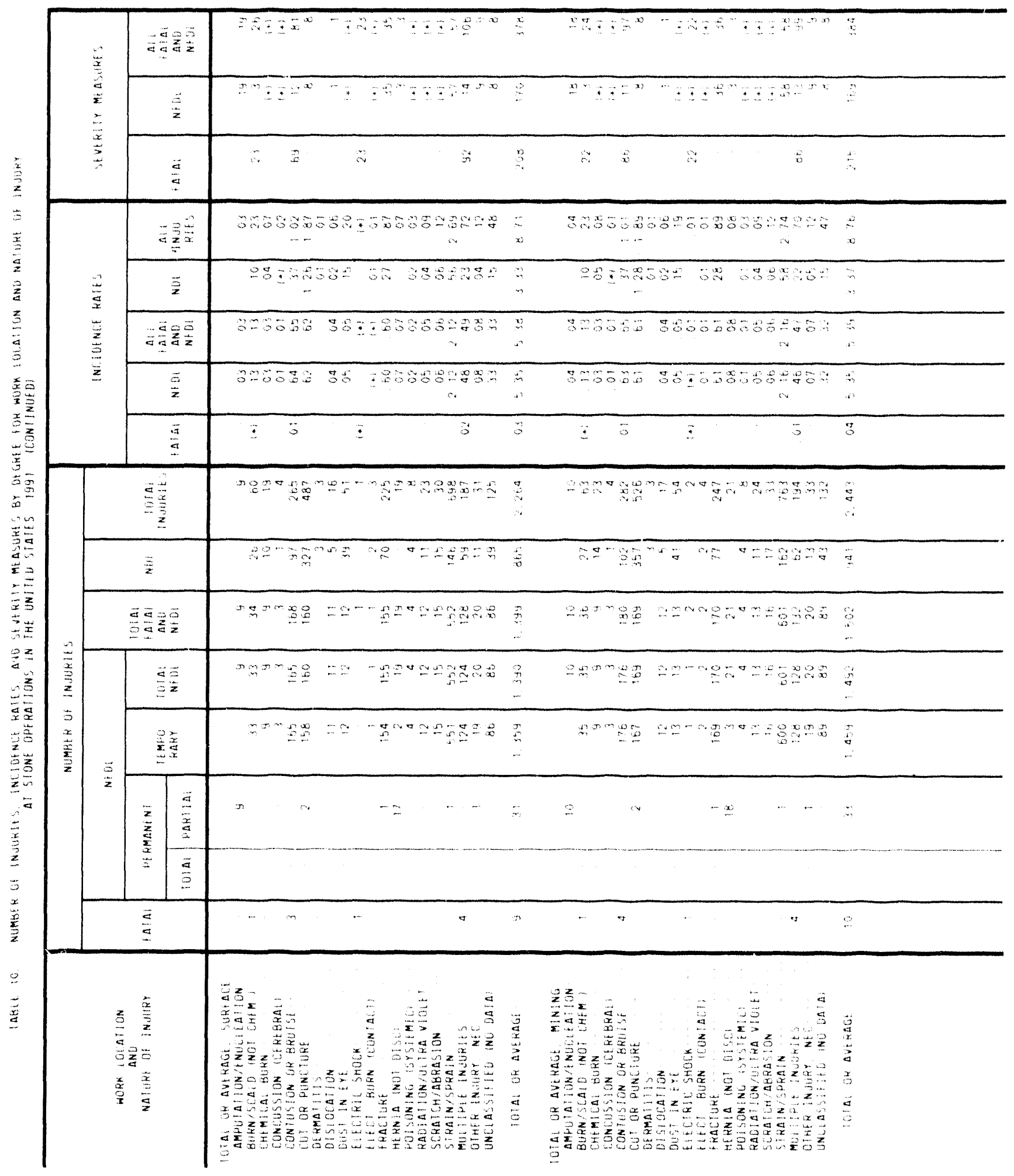




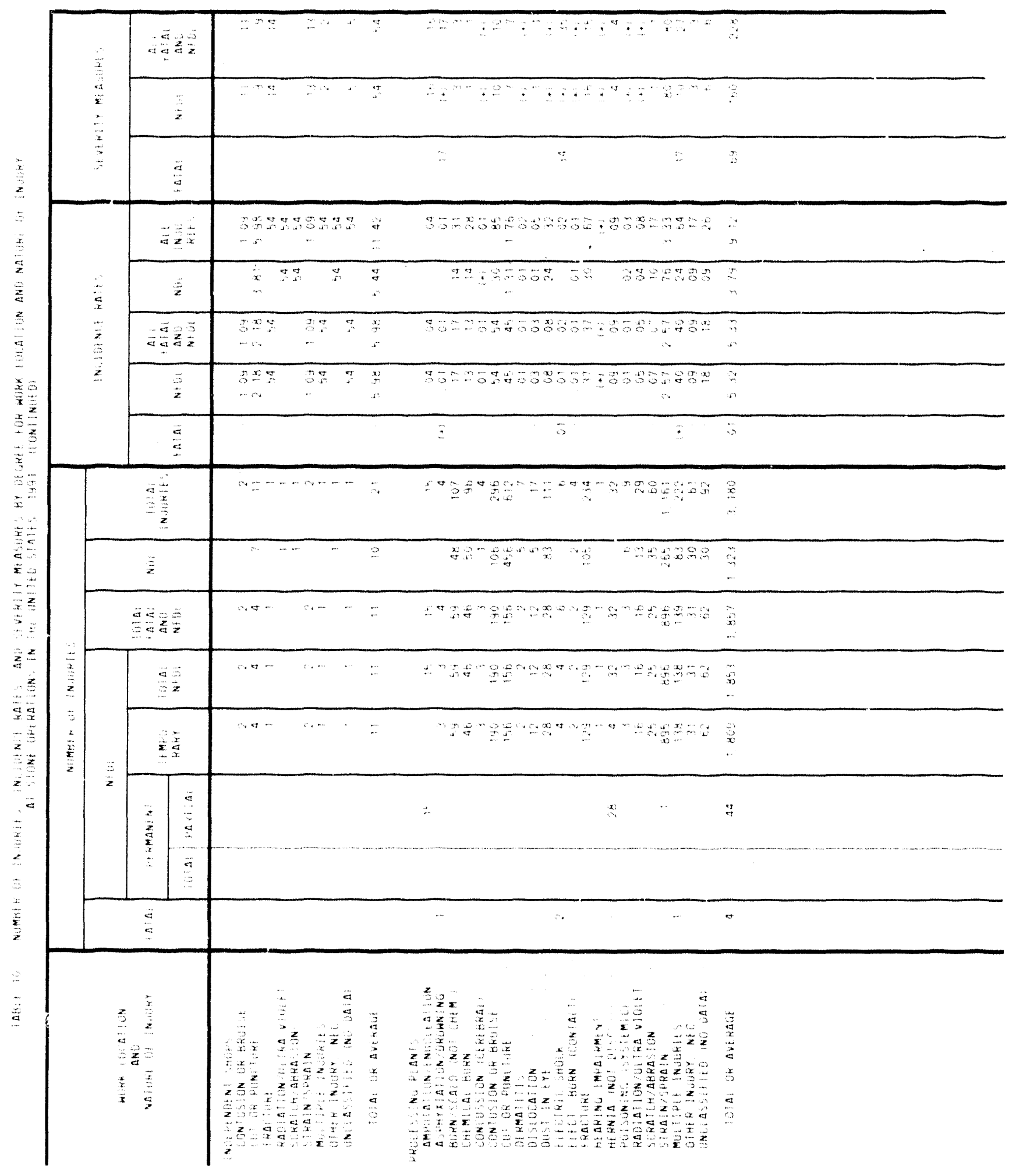




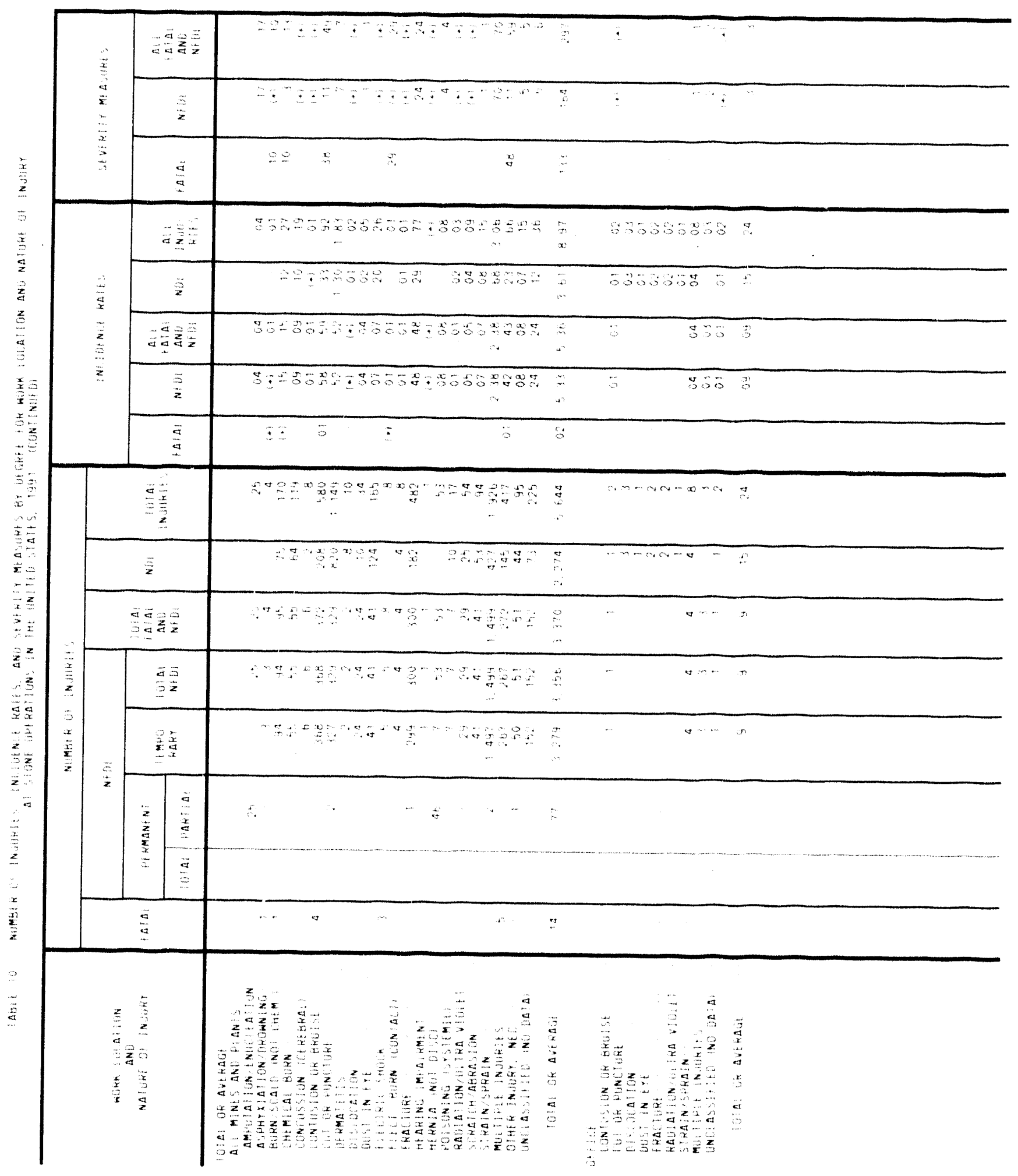




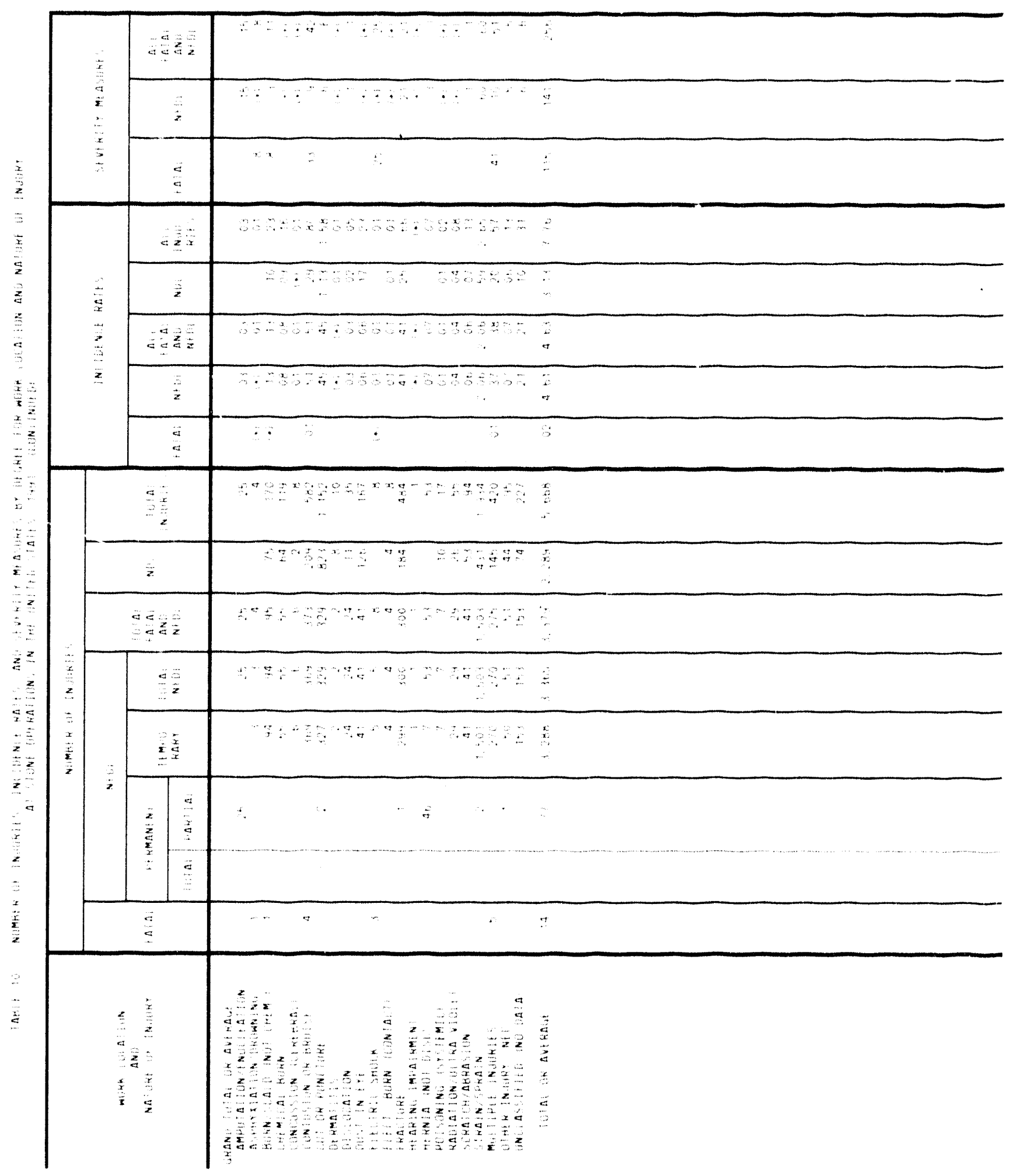




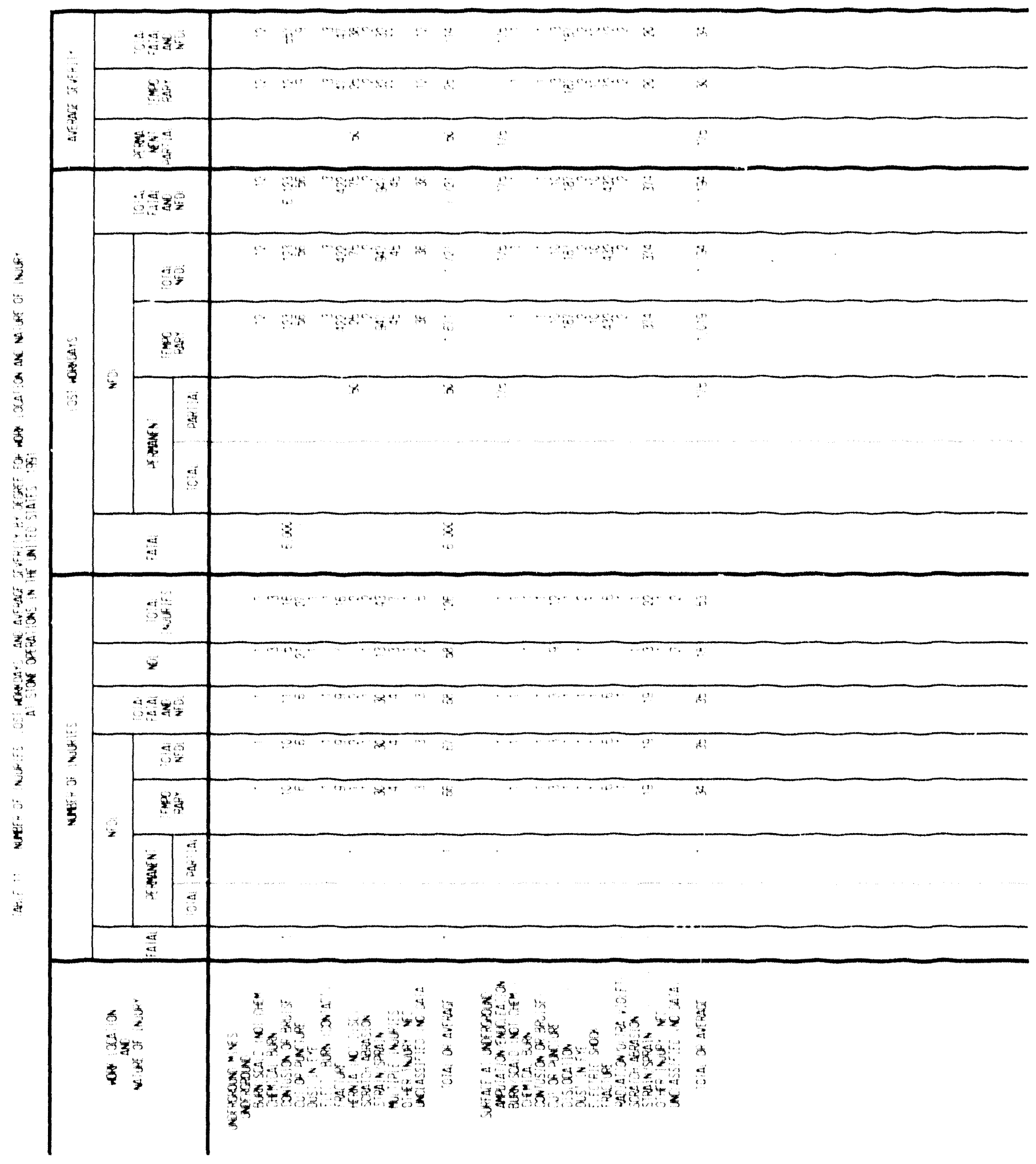



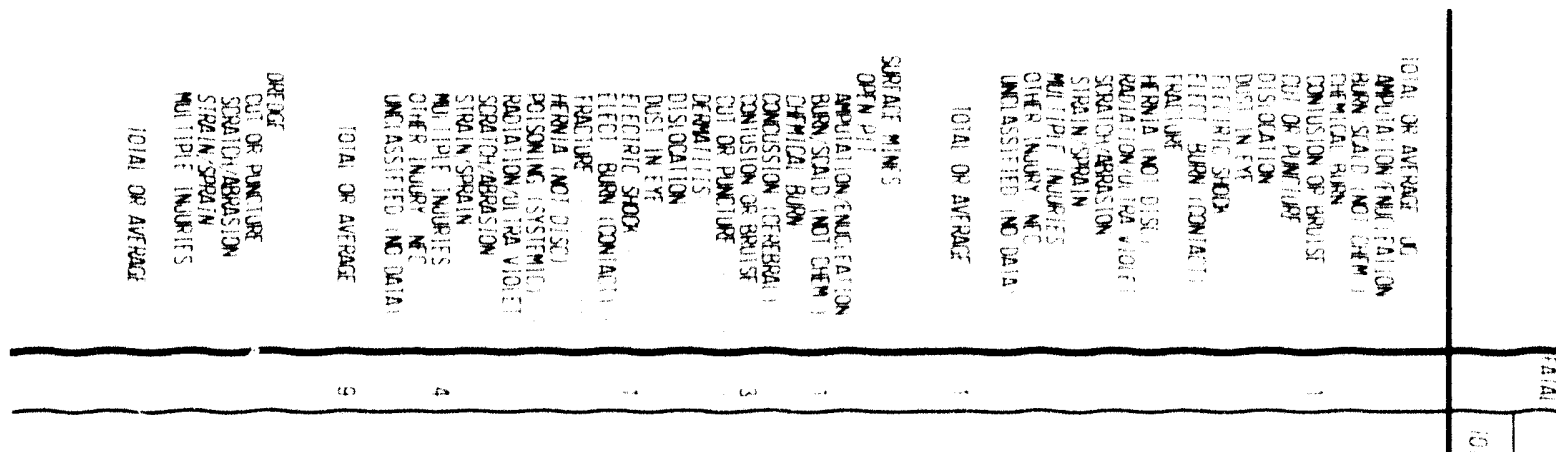

$\begin{array}{lllllllll} & \ldots & \ldots & \ldots & \ldots & \ldots & \ldots & \ldots & \ldots\end{array}$

\begin{tabular}{|c|c|c|c|}
\hline $\overrightarrow{8}$ & $\ldots \ldots$ & 蜜 & 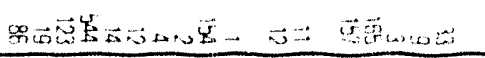 \\
\hline$\therefore$ & $\ldots+\ldots$ & 笶 & 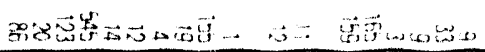 \\
\hline$\vec{b}$ & $-\ldots \ldots$ & $\overrightarrow{.4}$ & 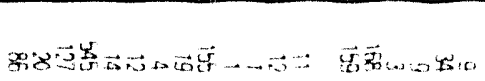 \\
\hline is & $\ldots$ & 总 & 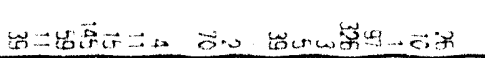 \\
\hline$\sigma$ & $\ldots+\infty \ldots$ & 13 & 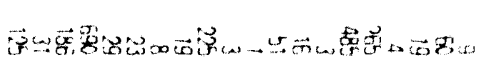 \\
\hline
\end{tabular}

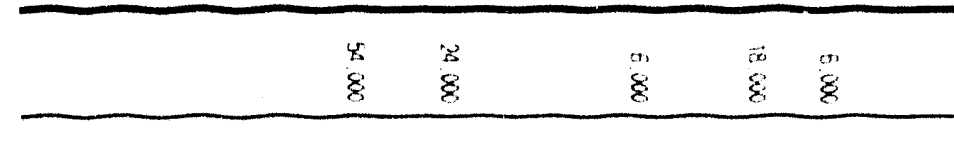

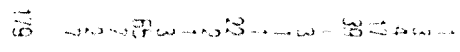

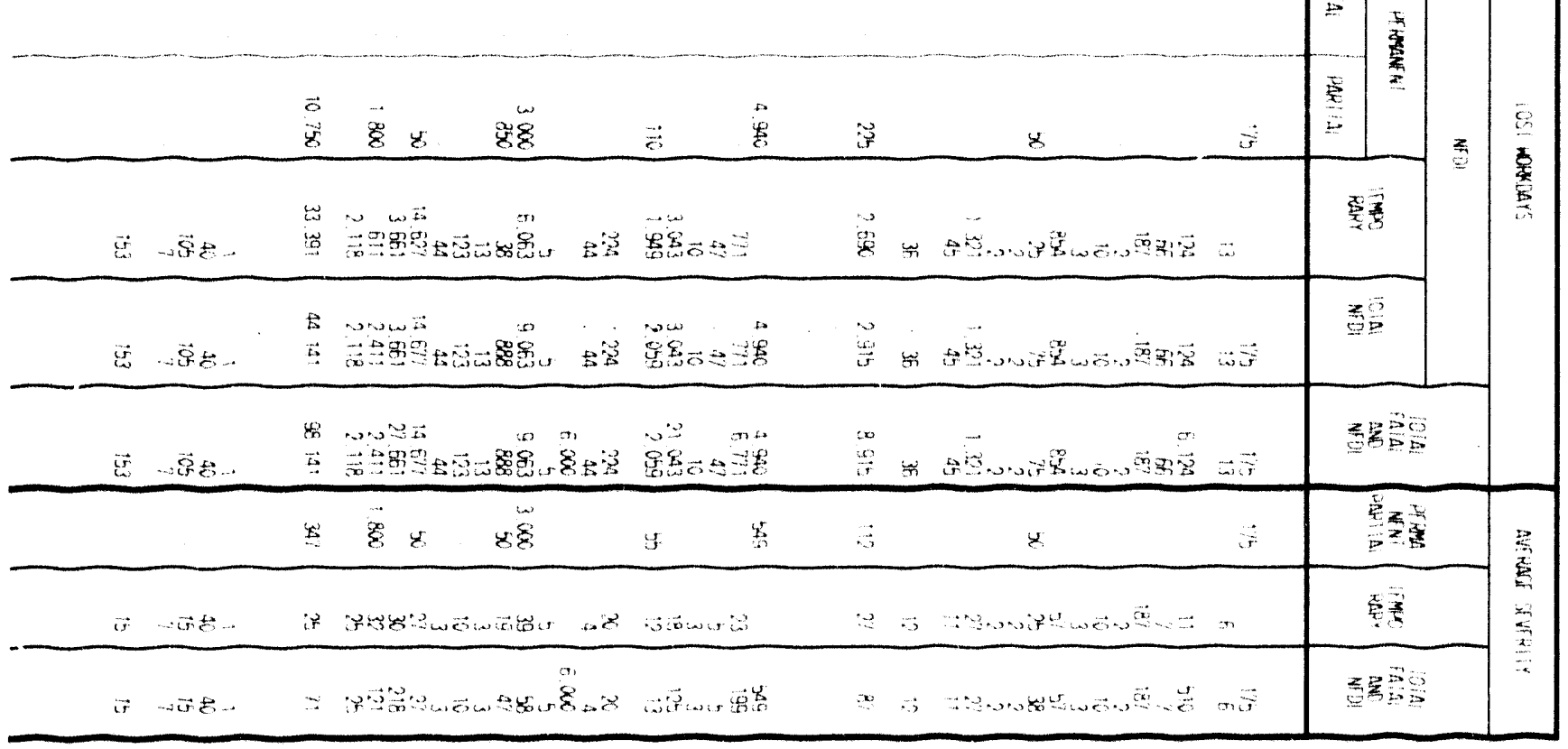



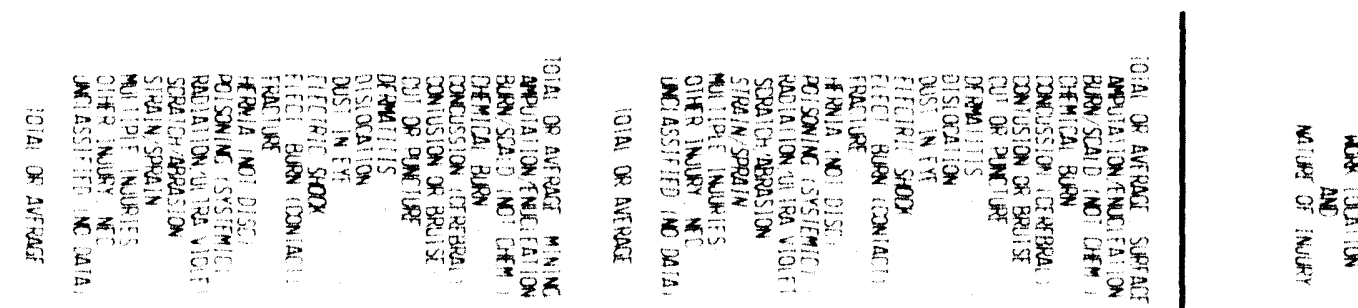

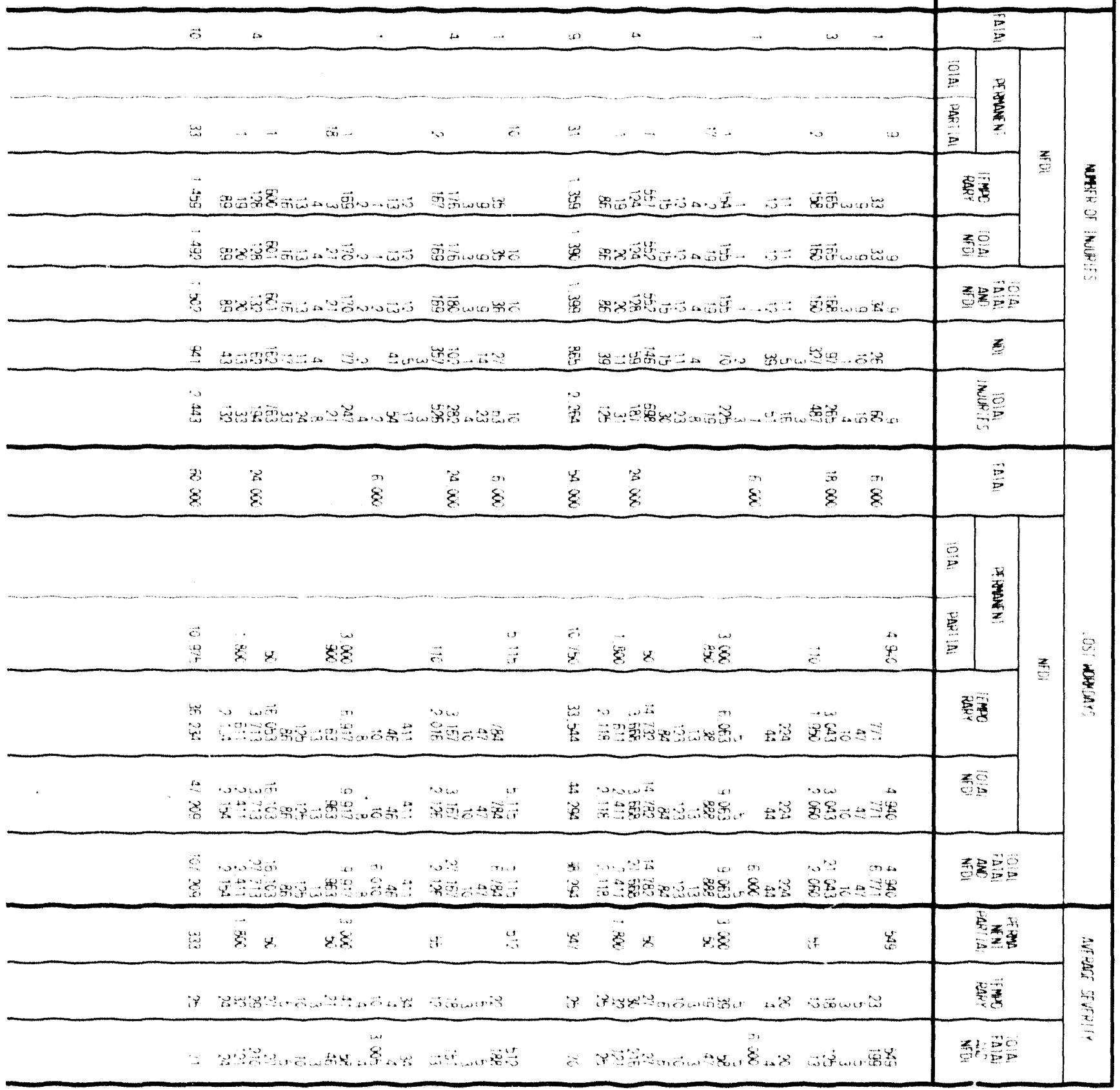




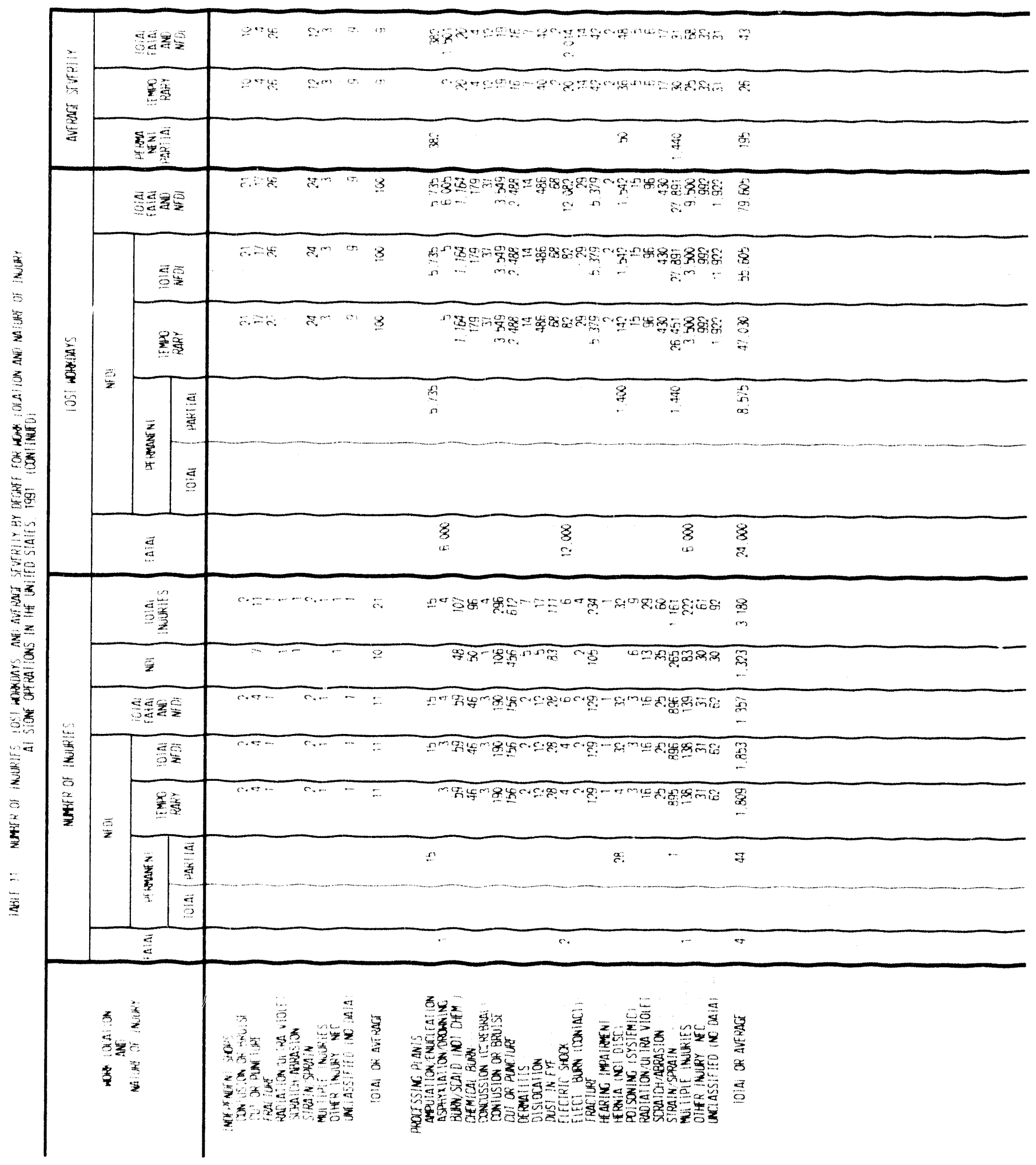




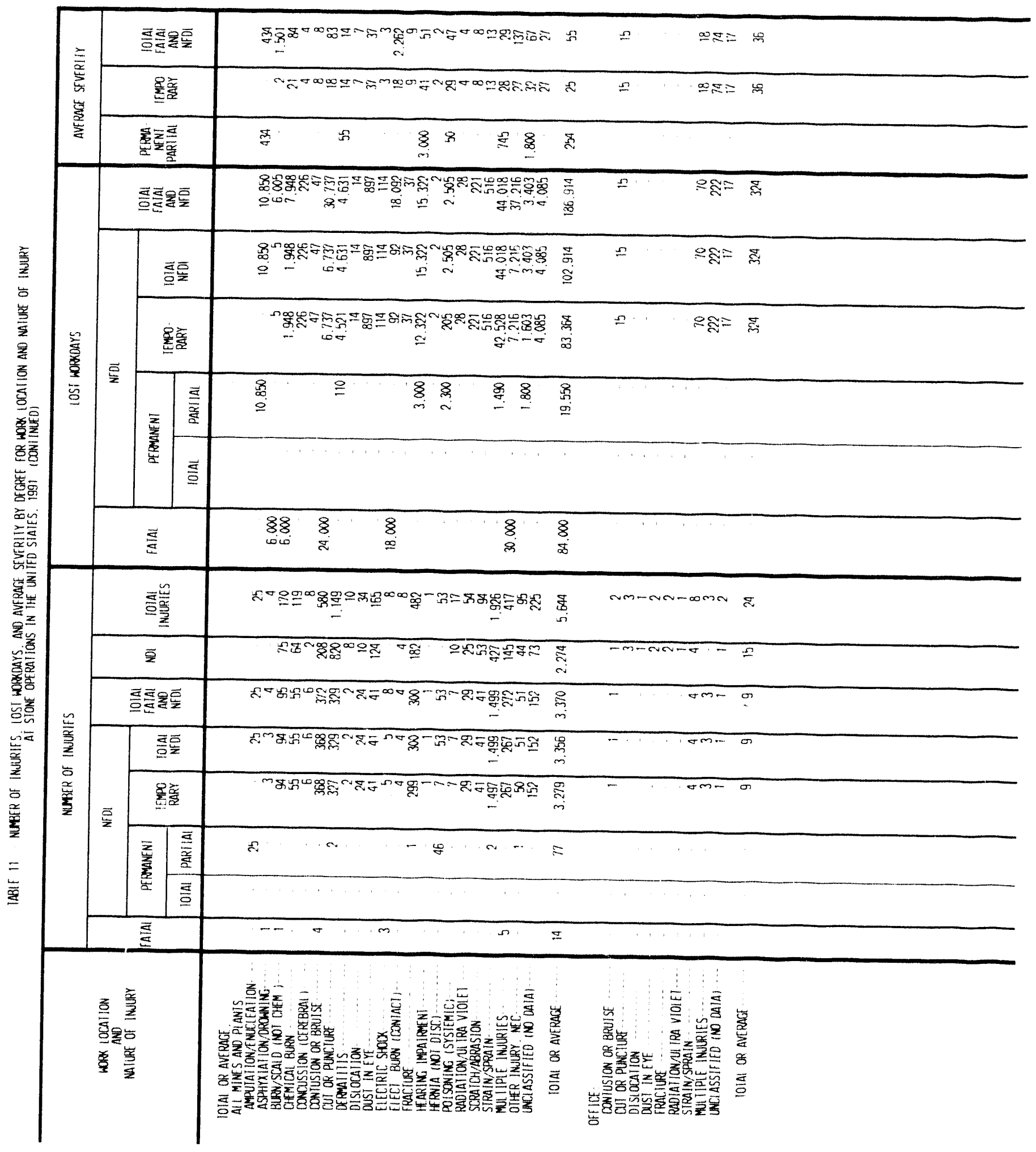




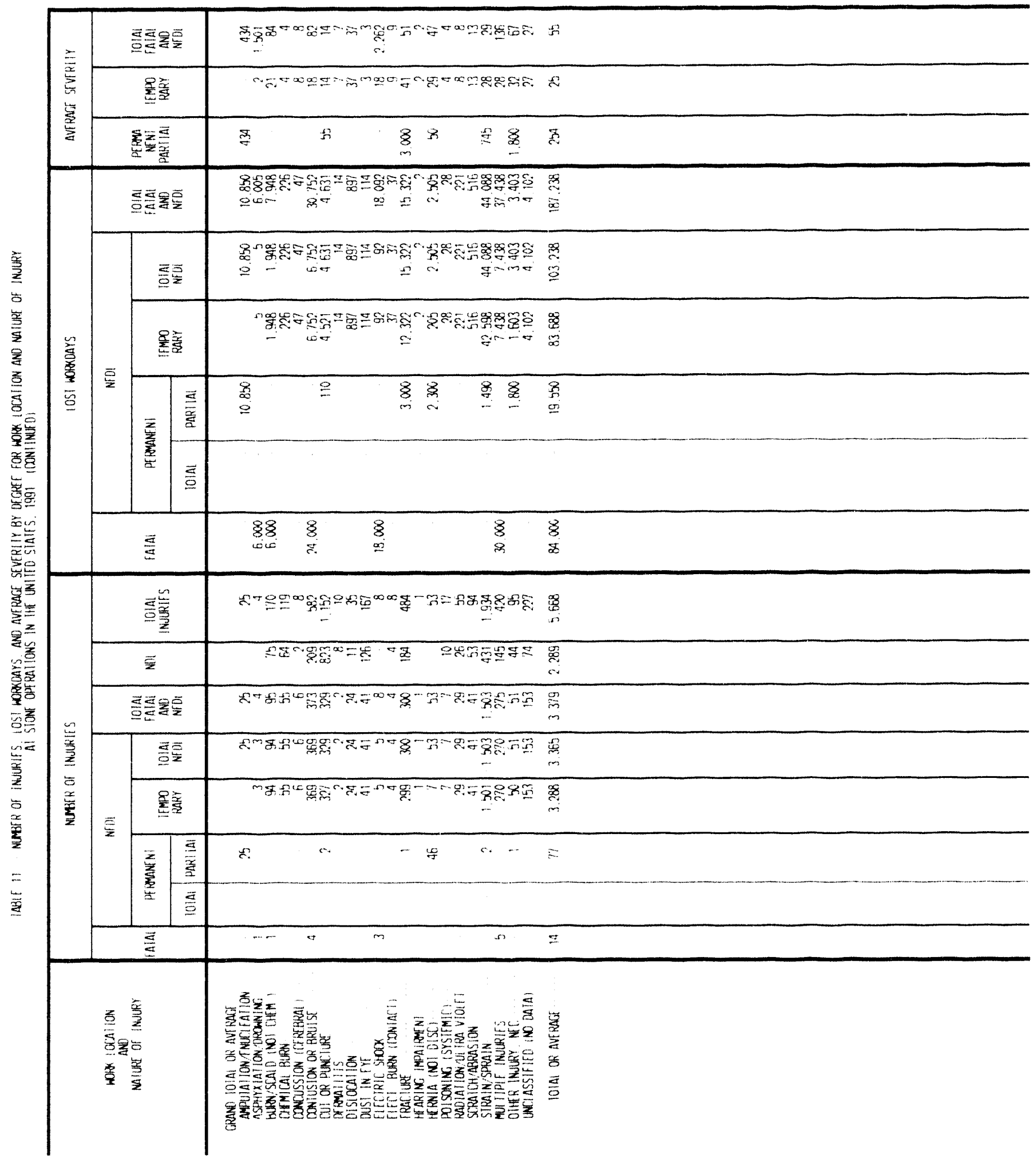




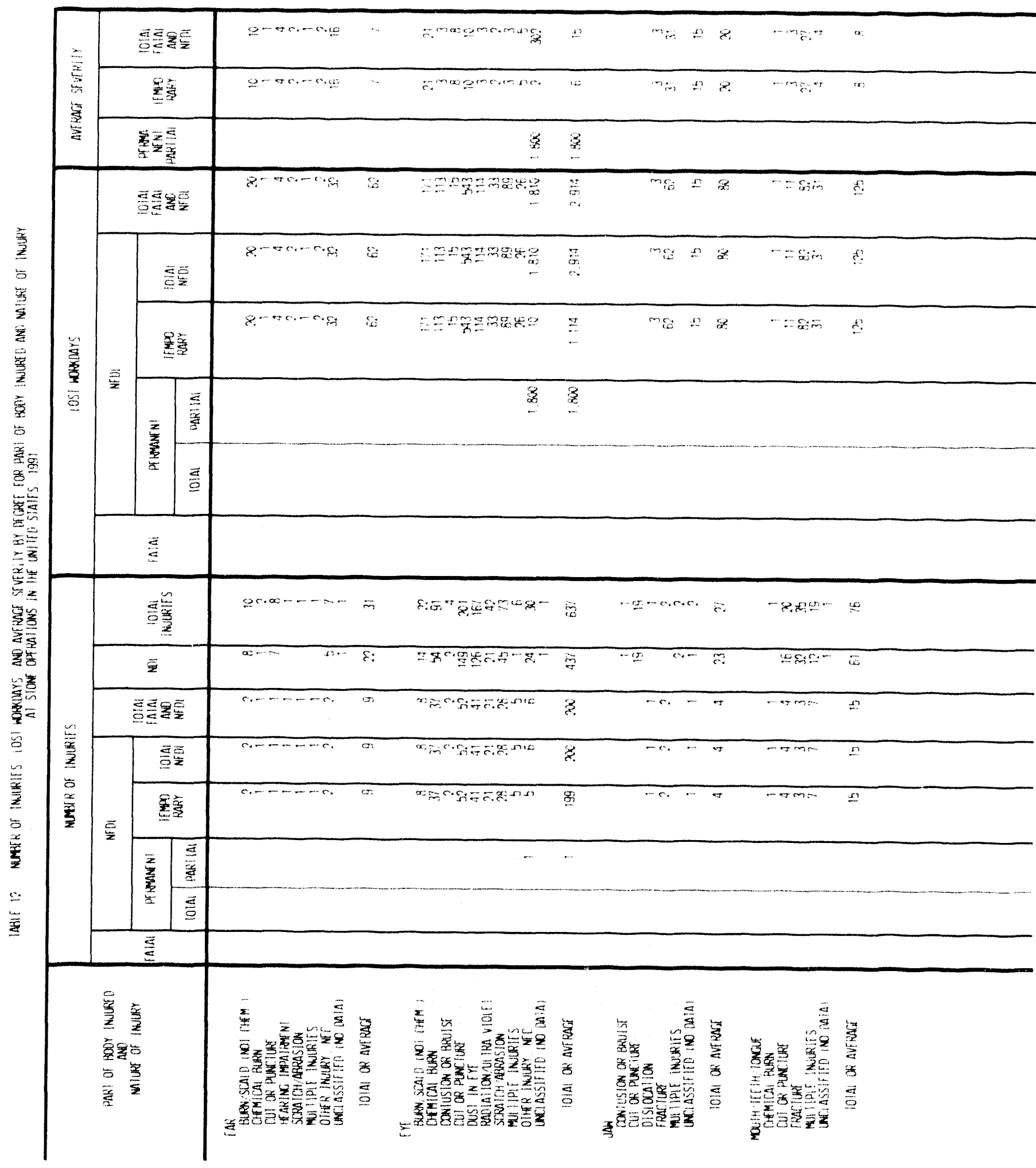




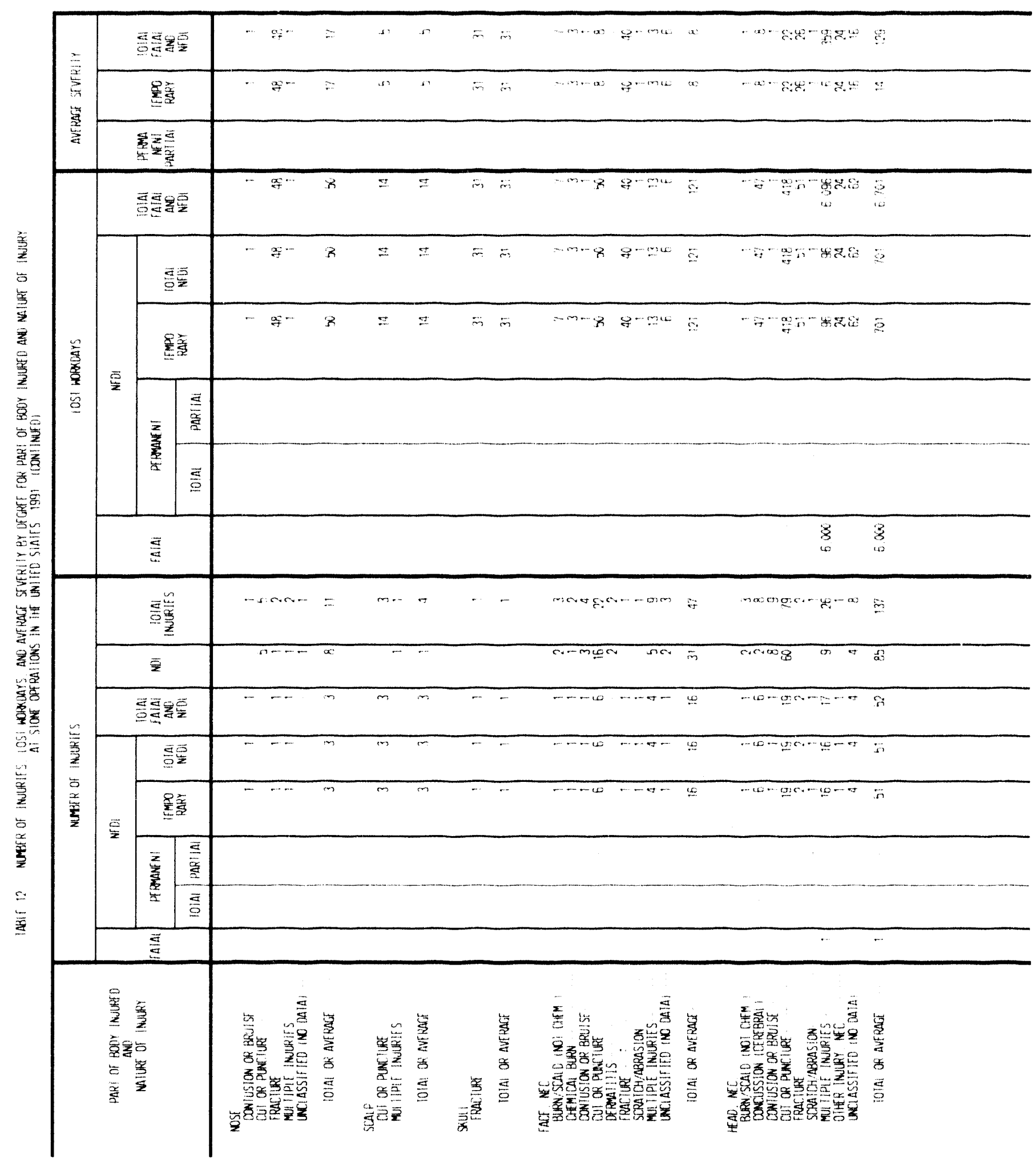




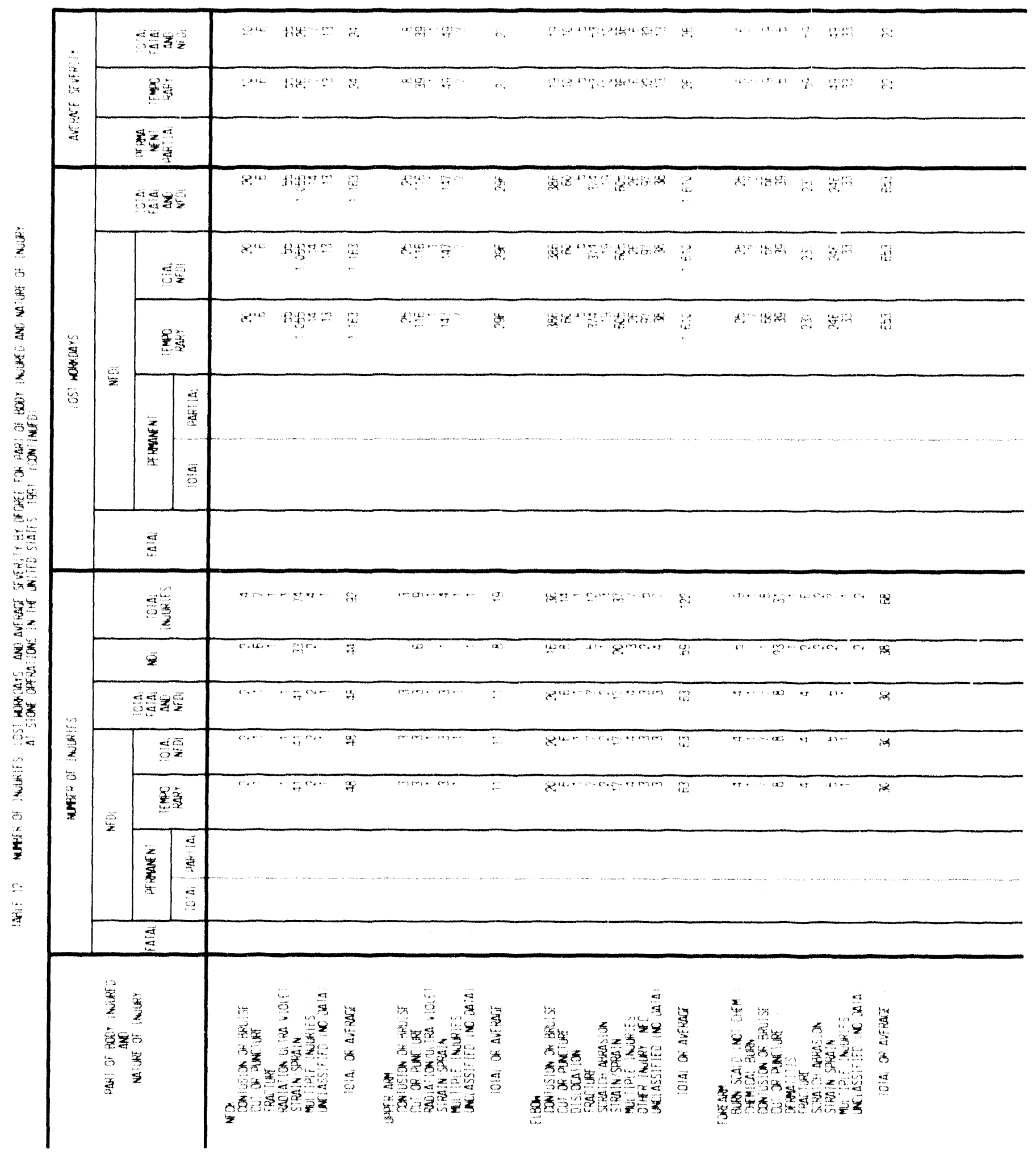




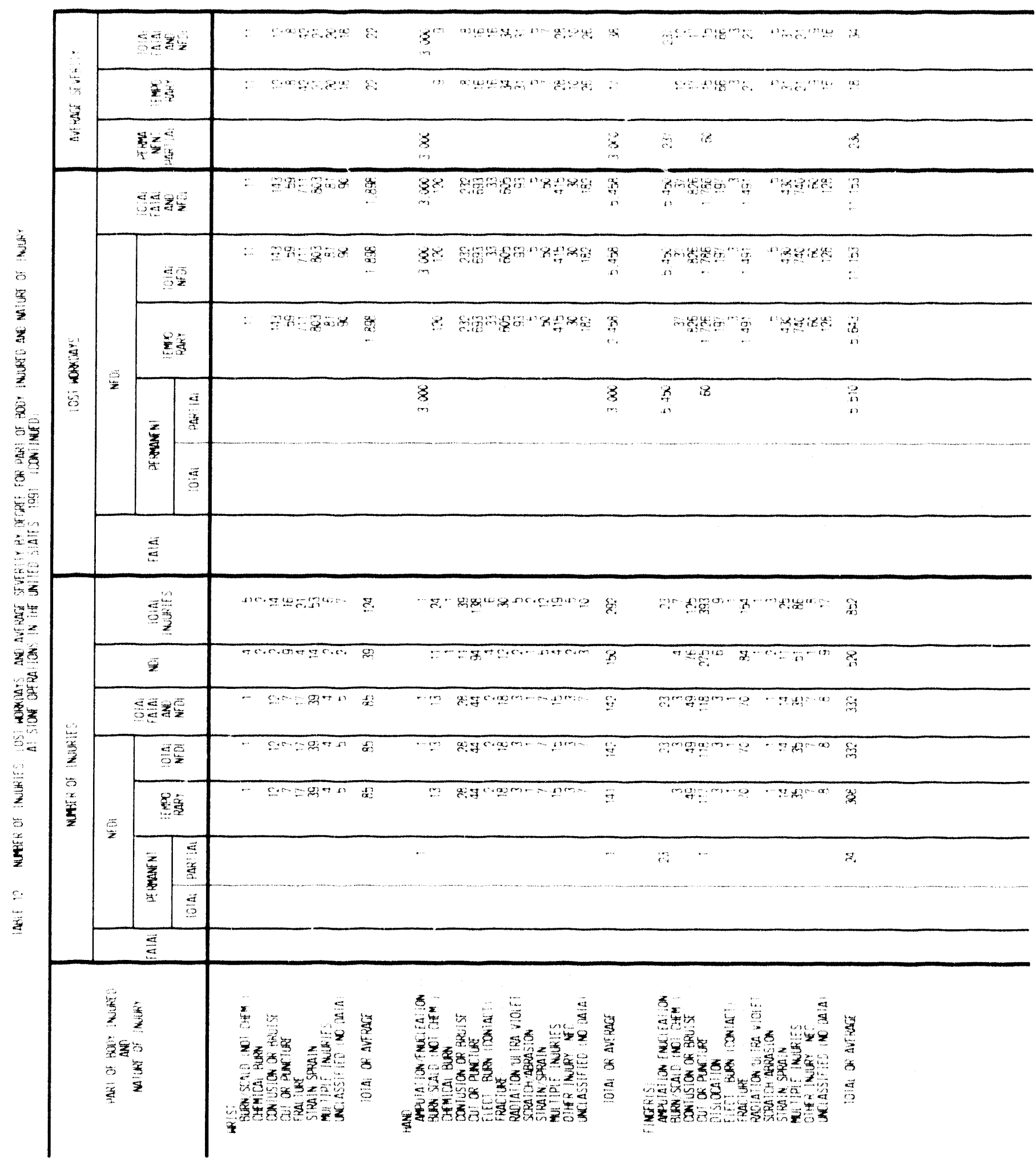




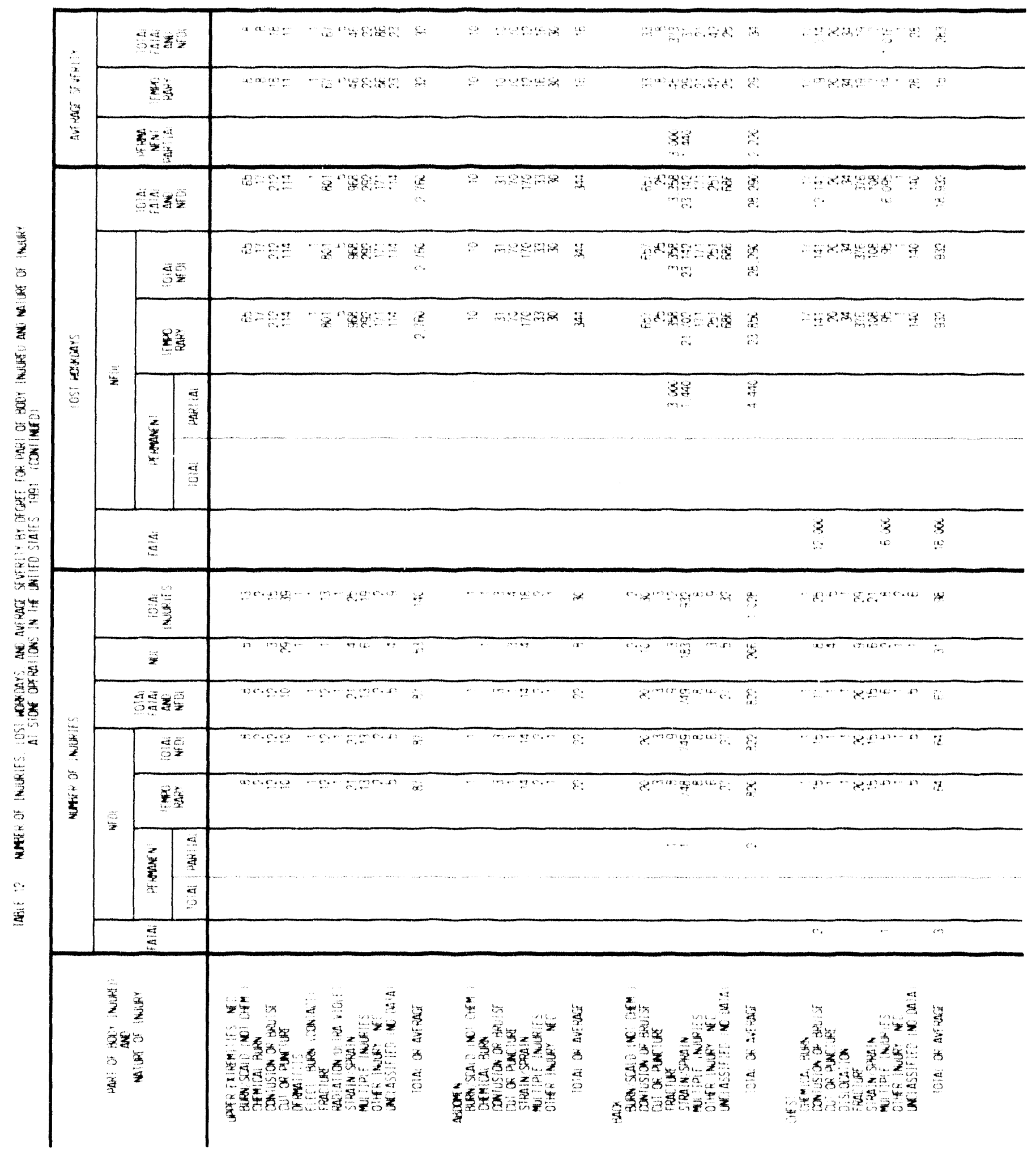




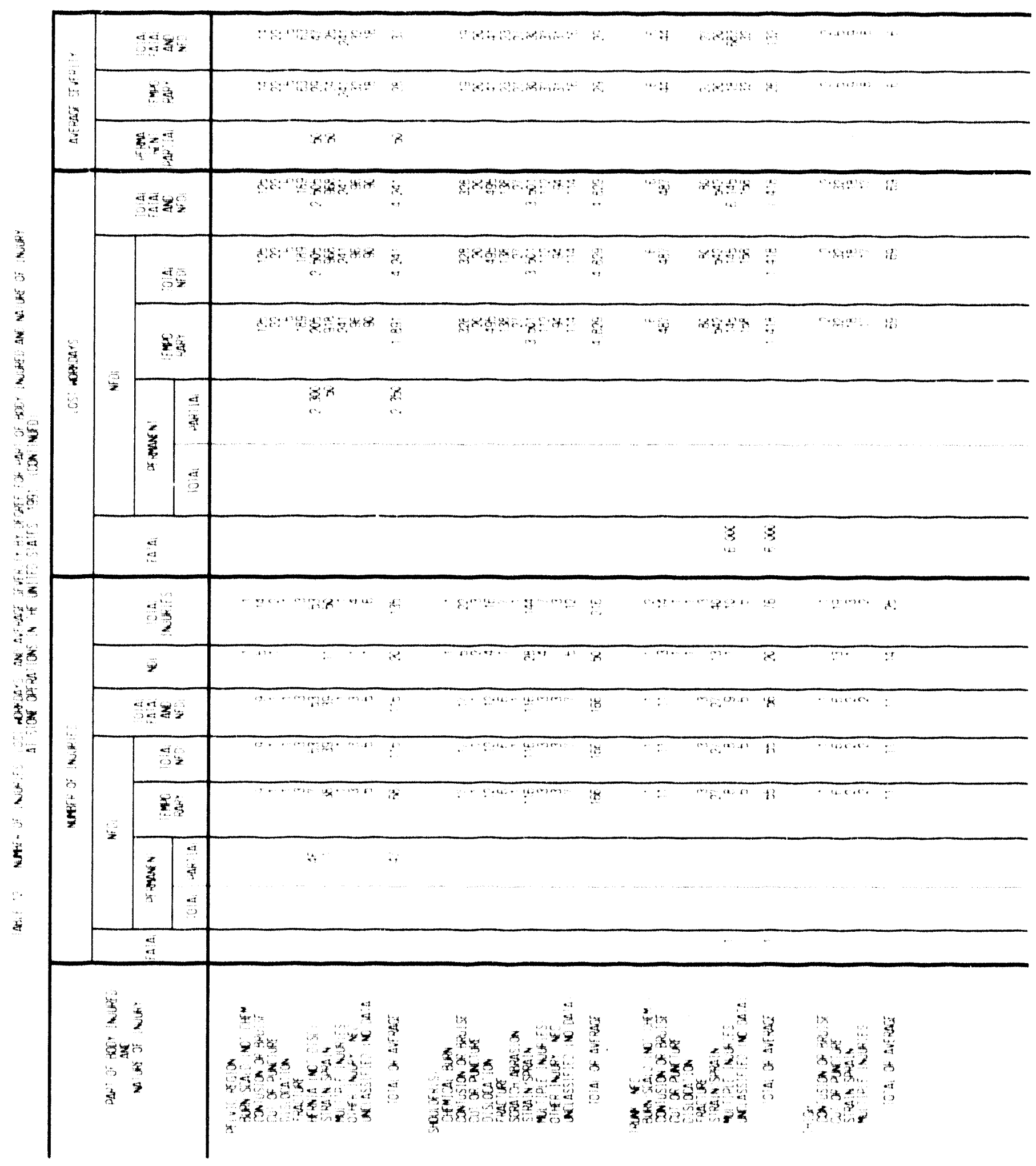




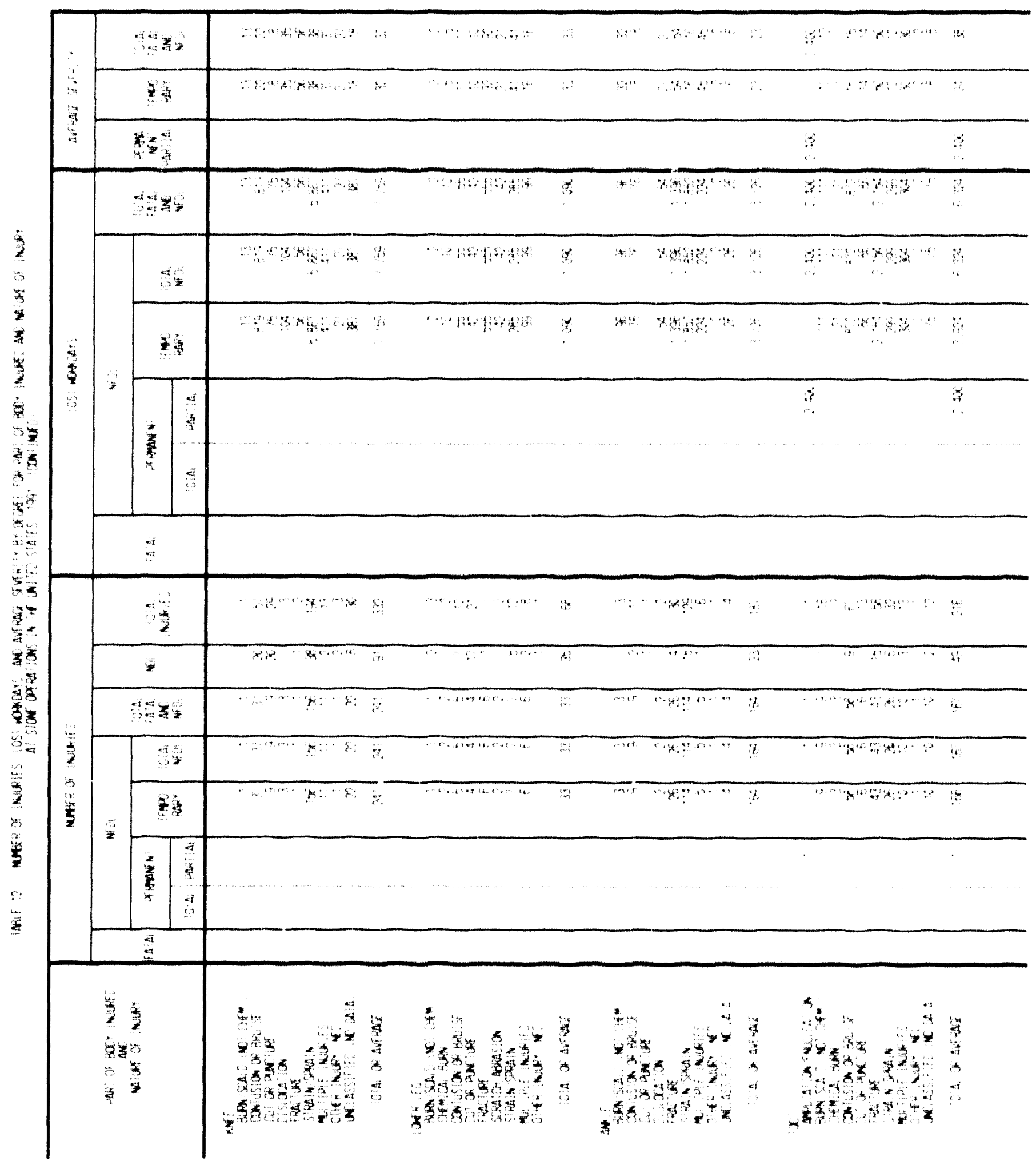




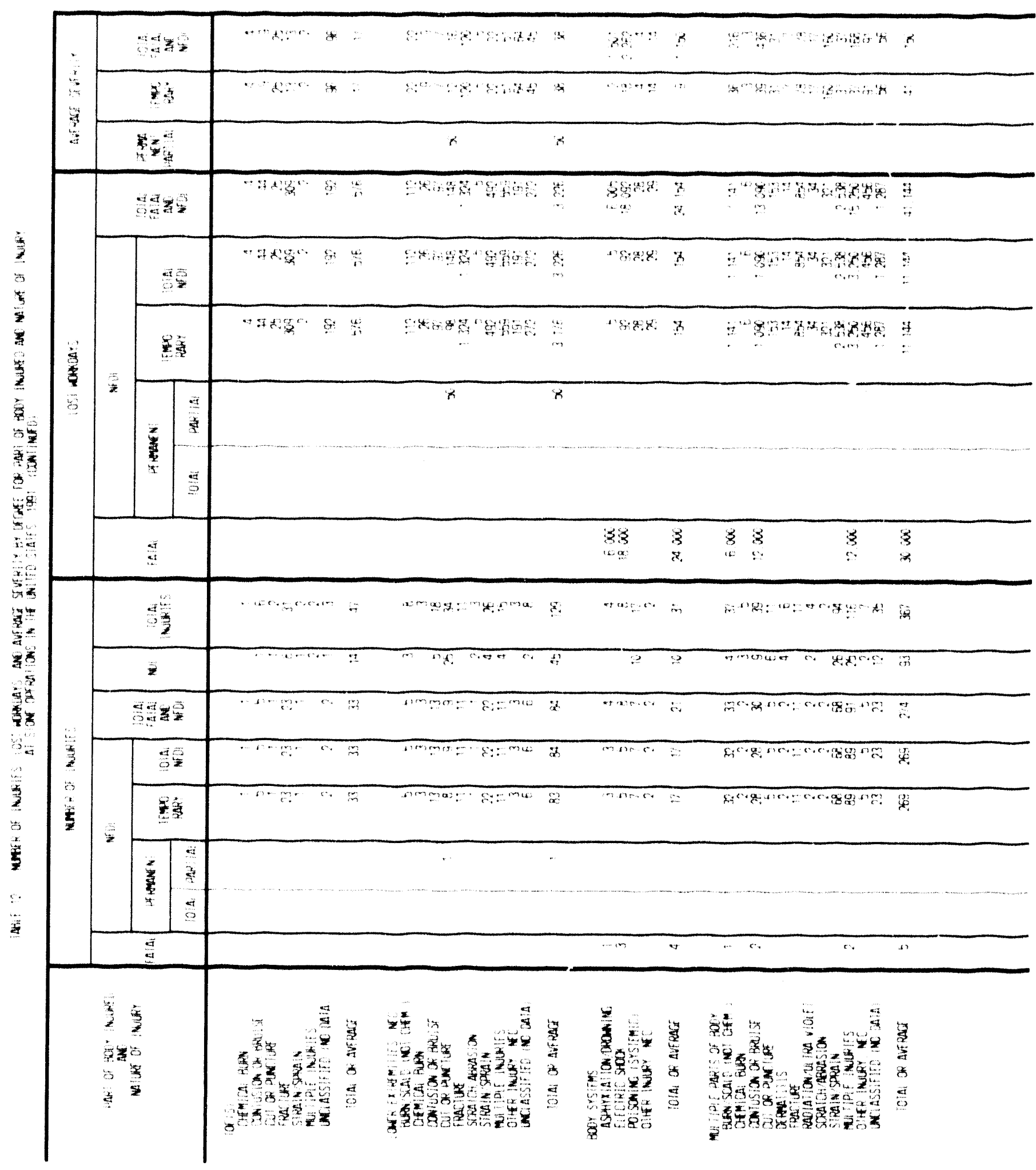




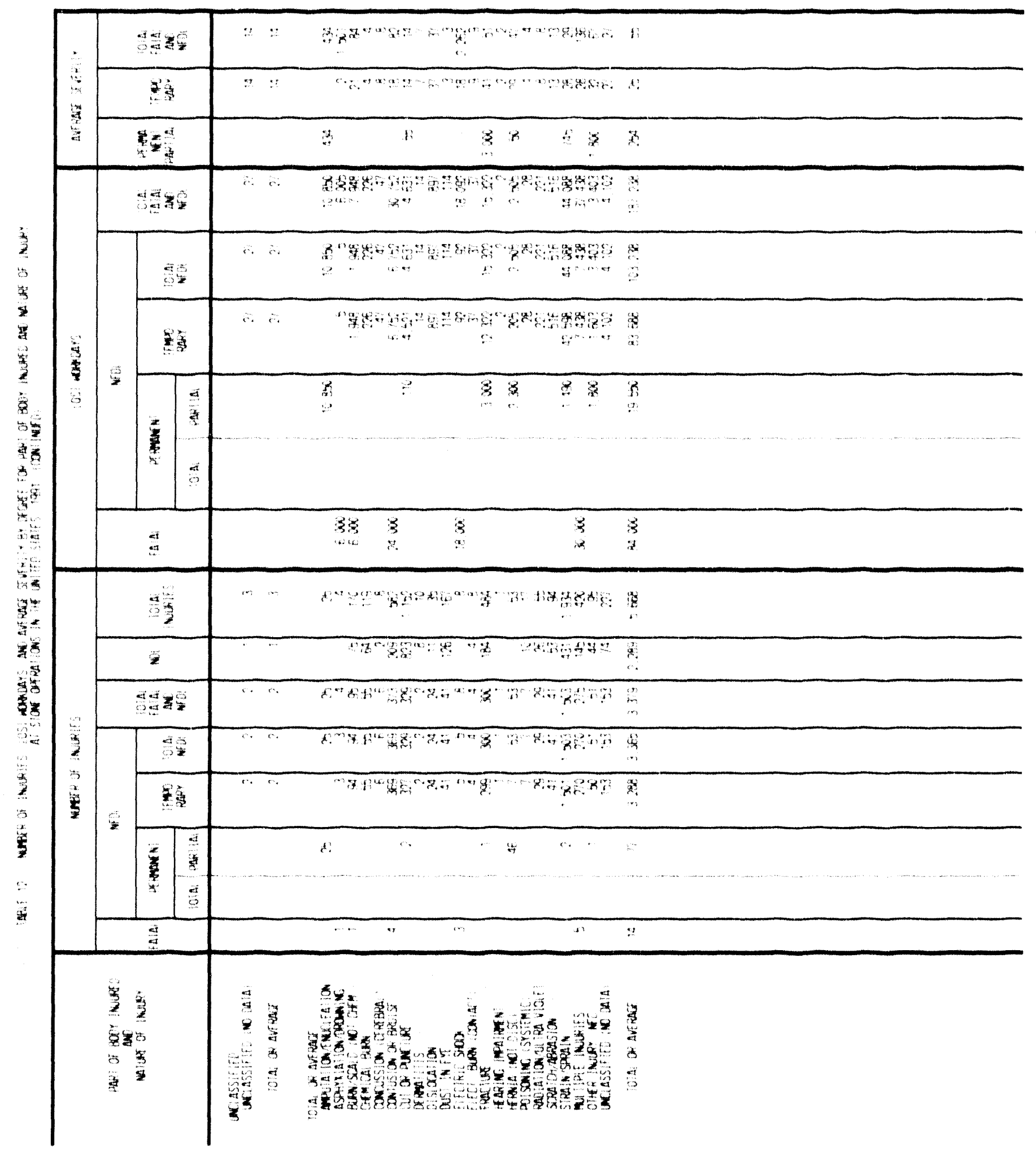




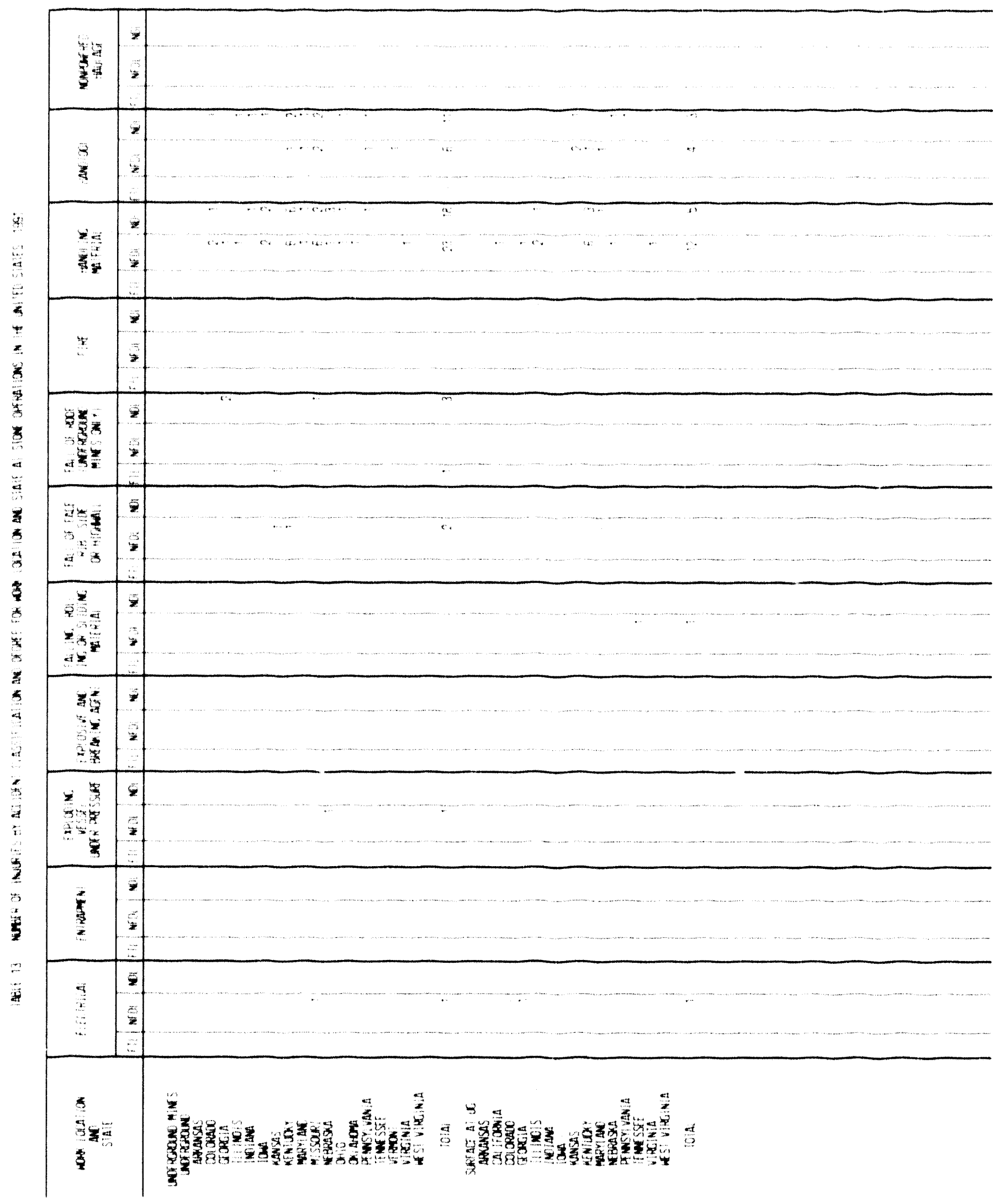




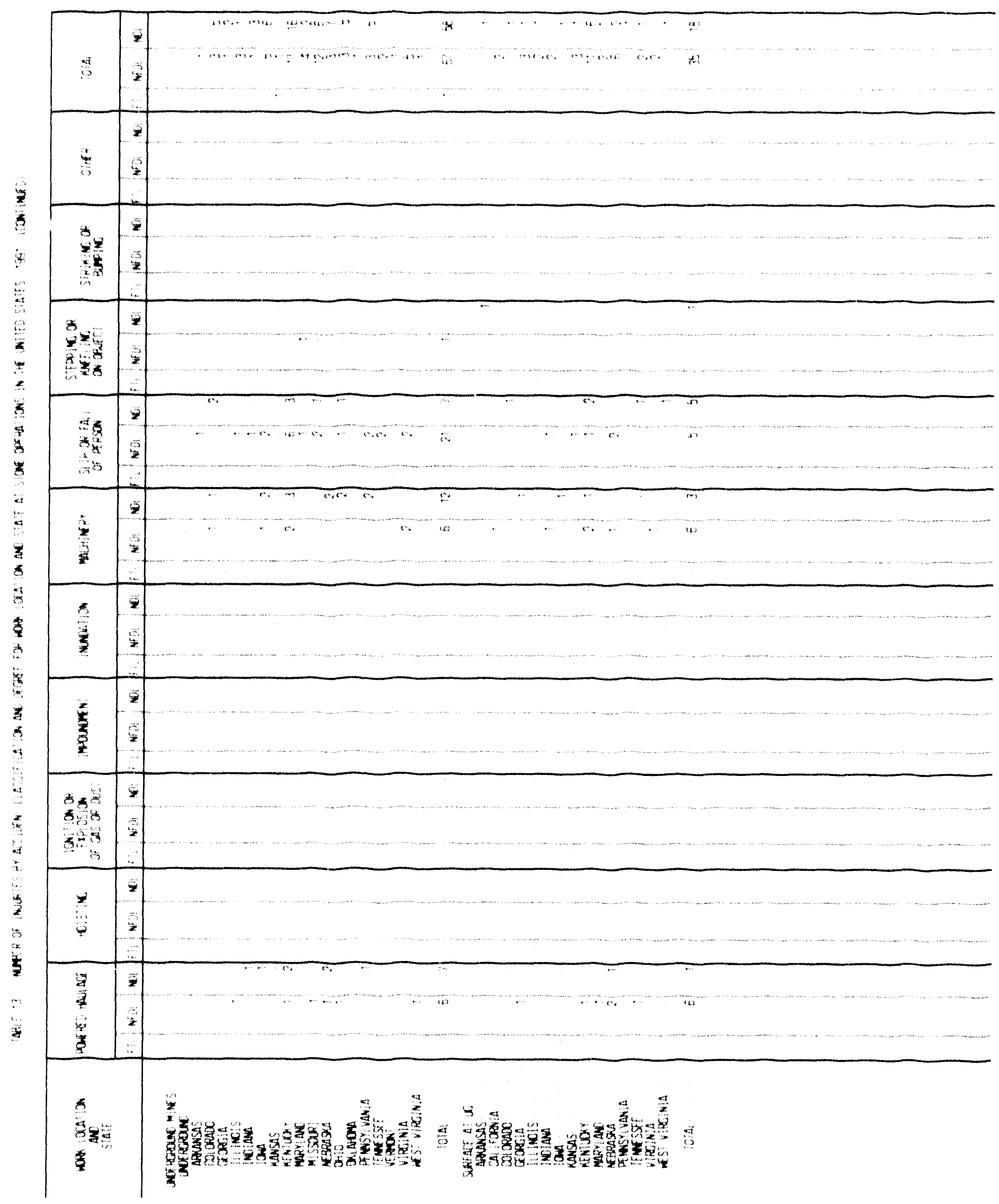




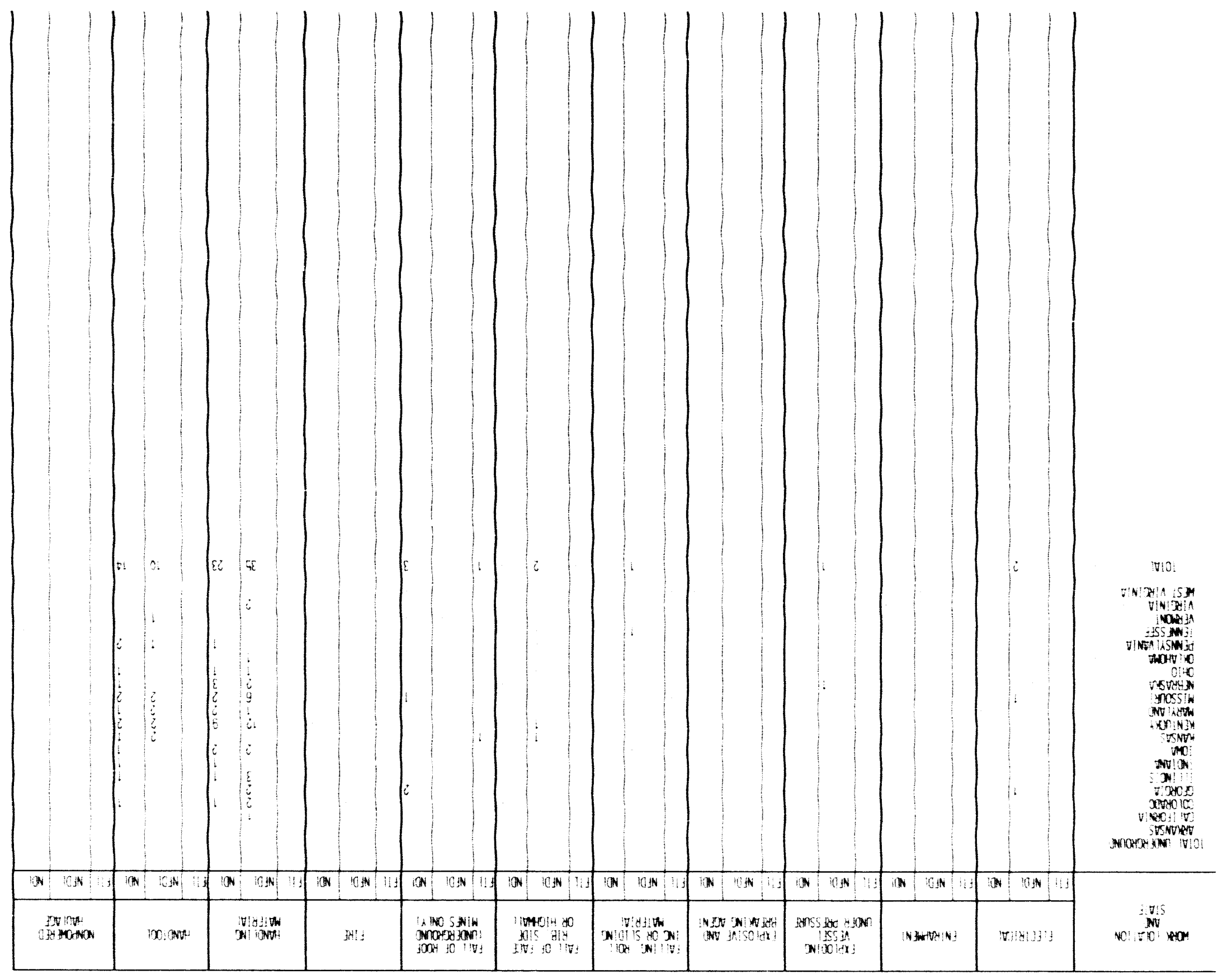

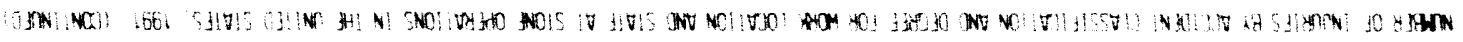

(1) IM 


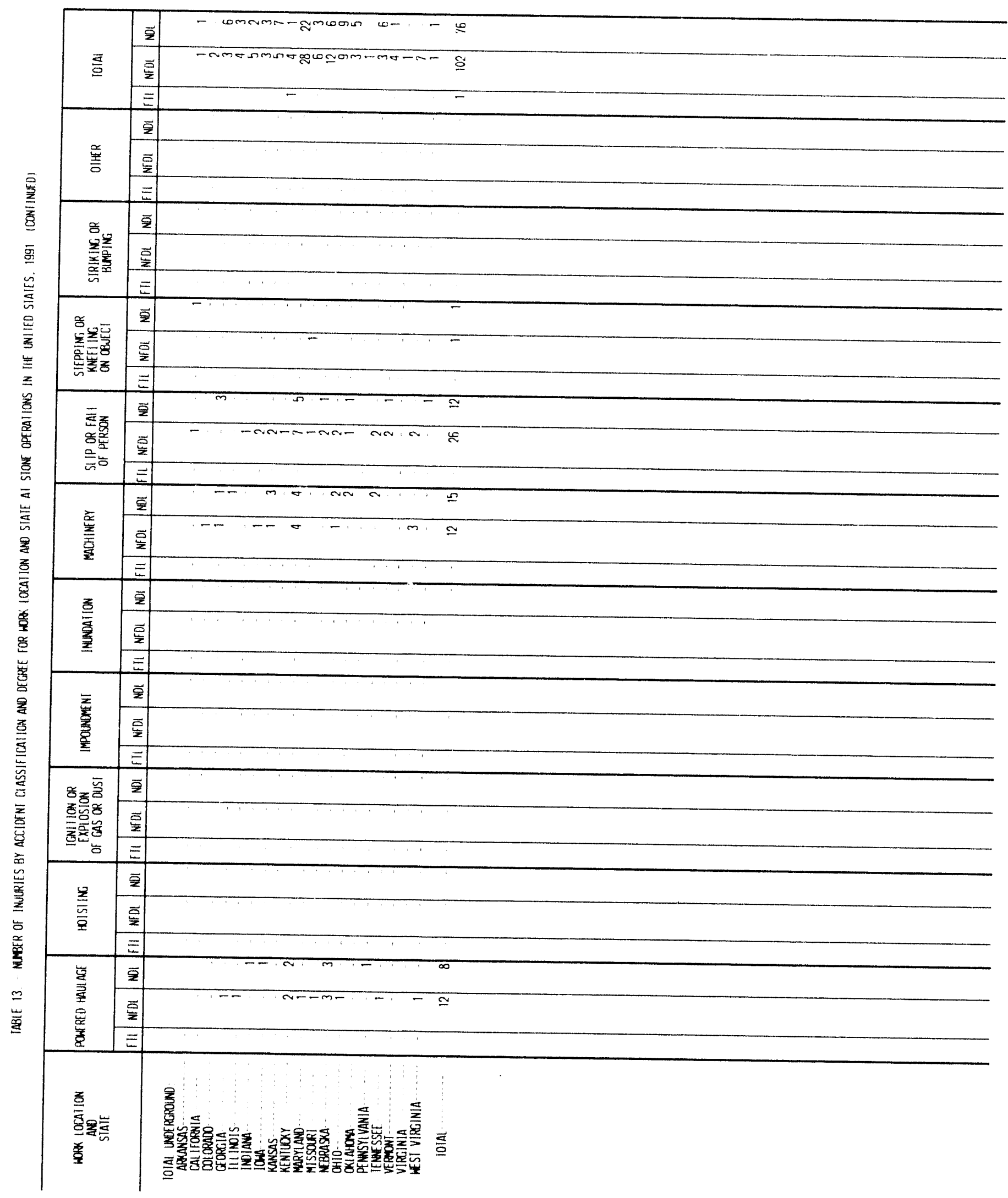




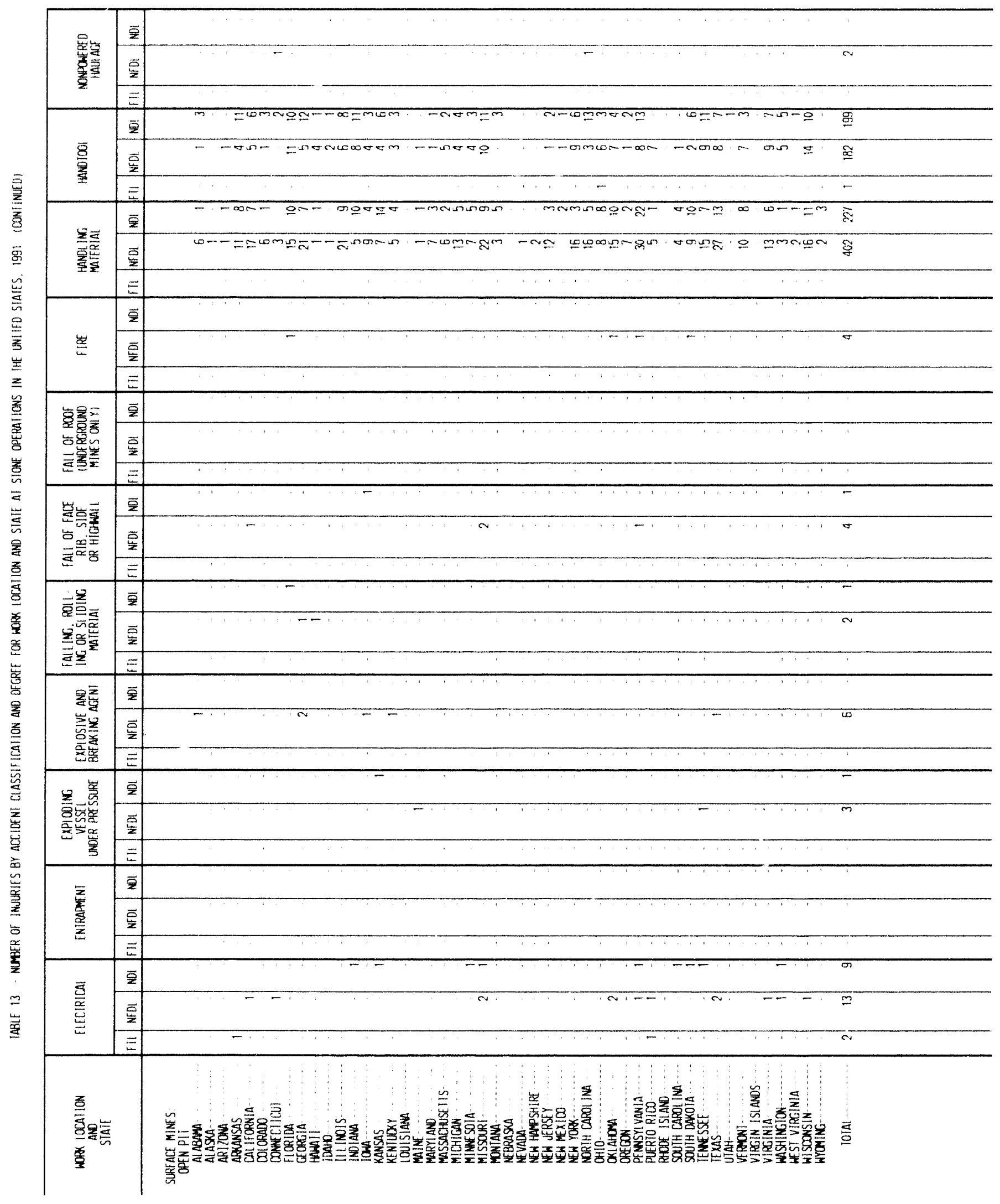




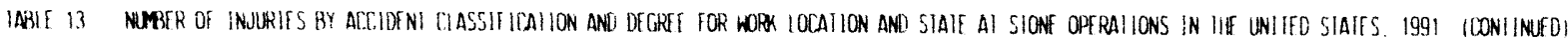

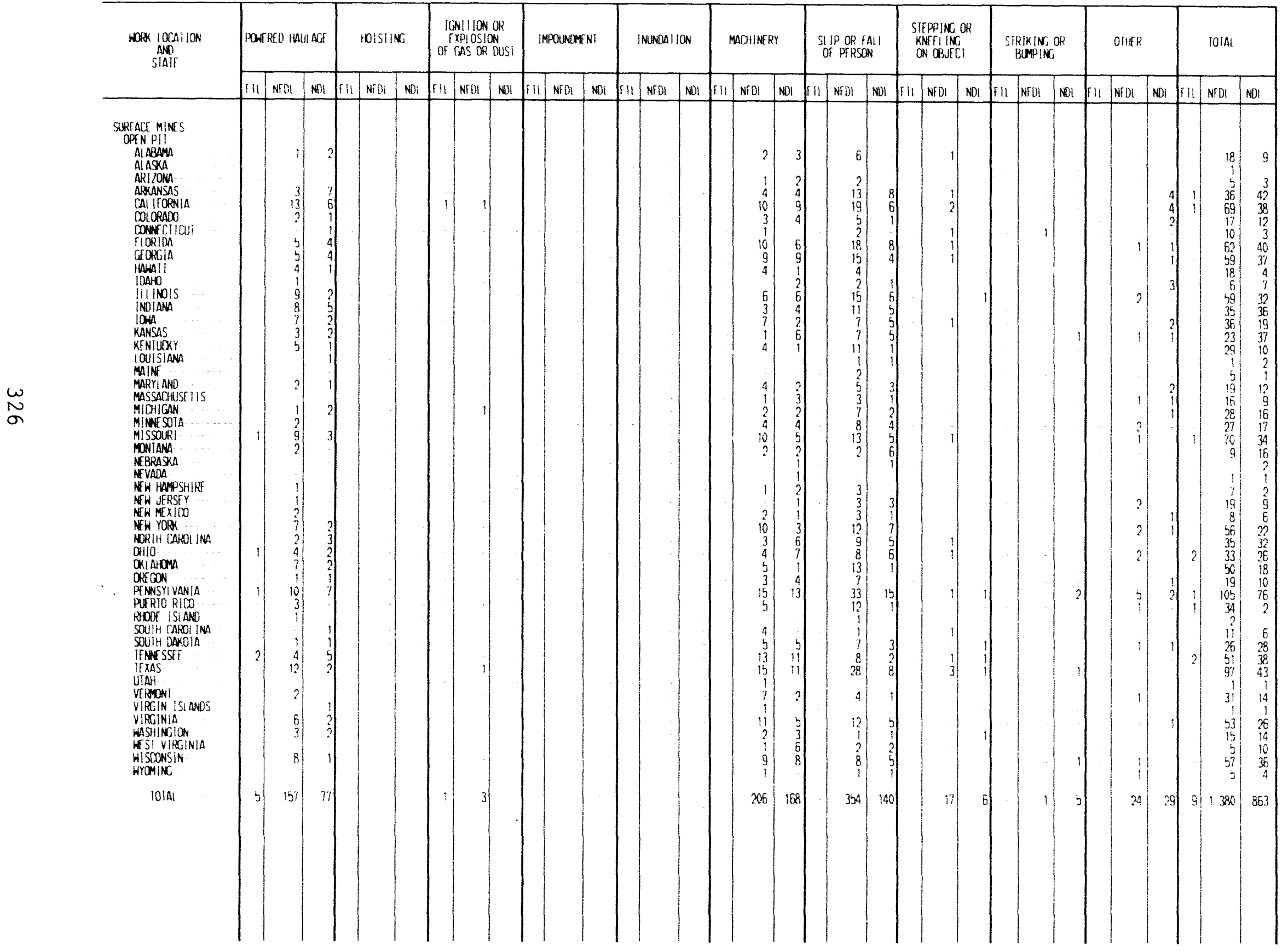




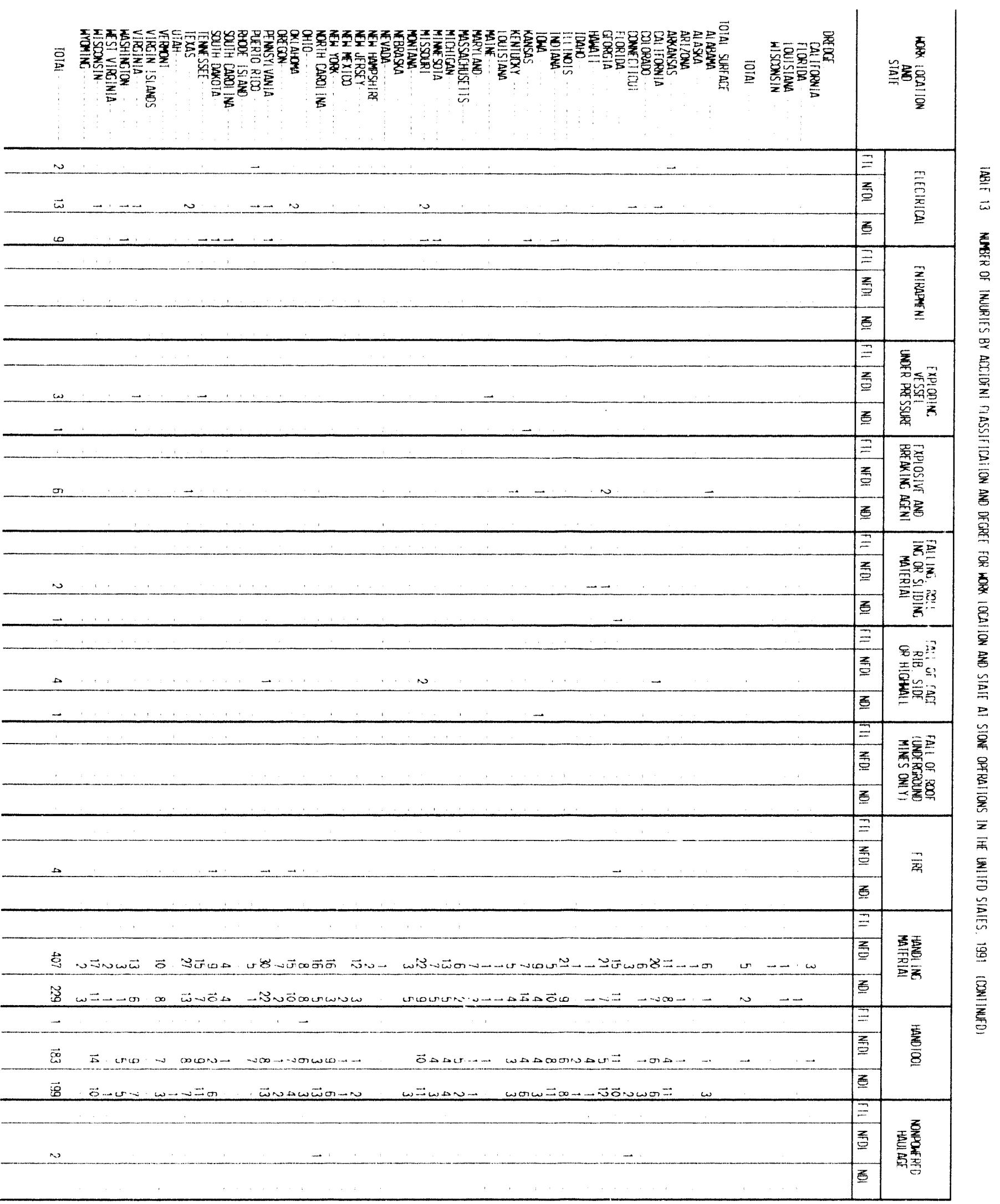




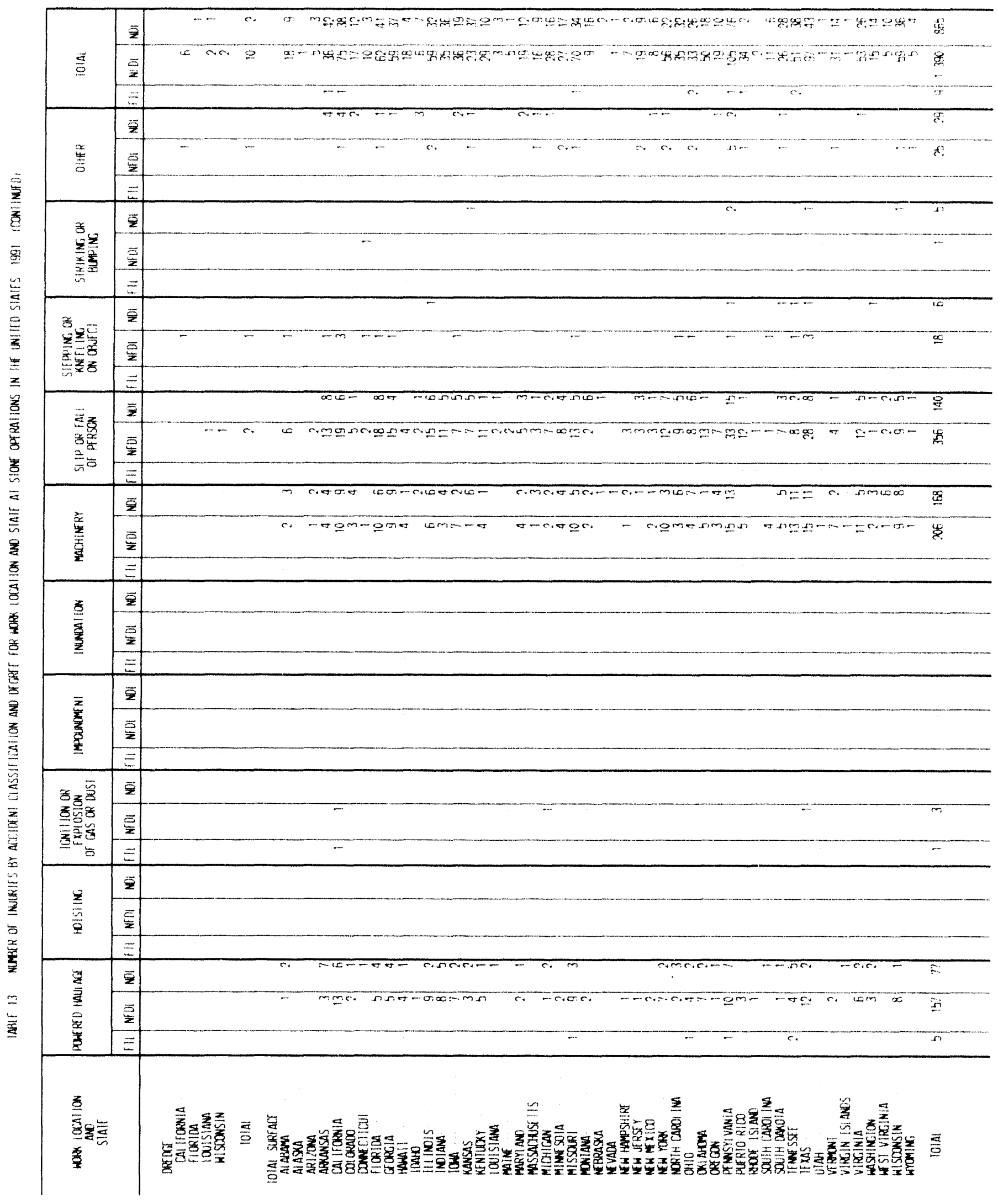




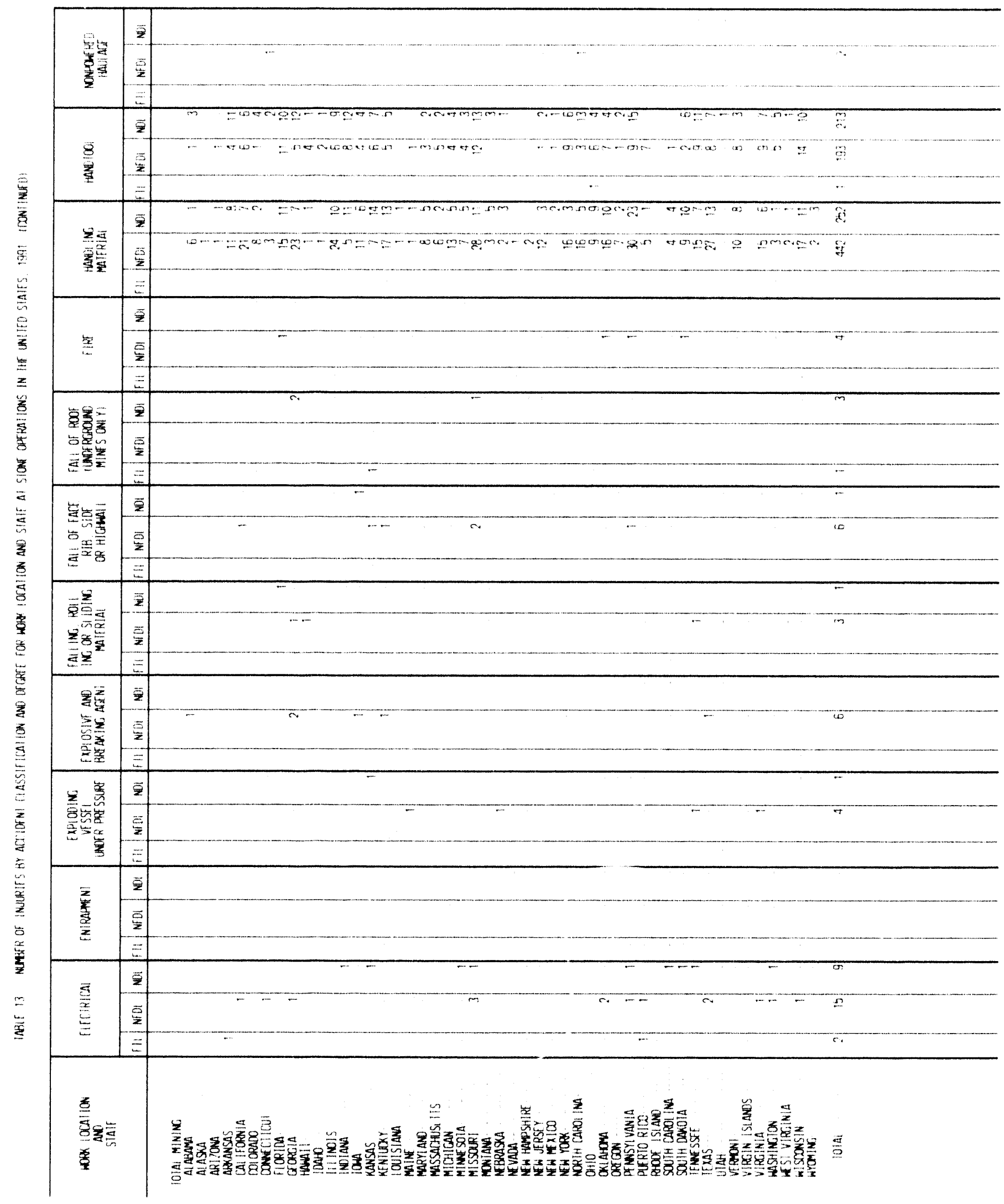




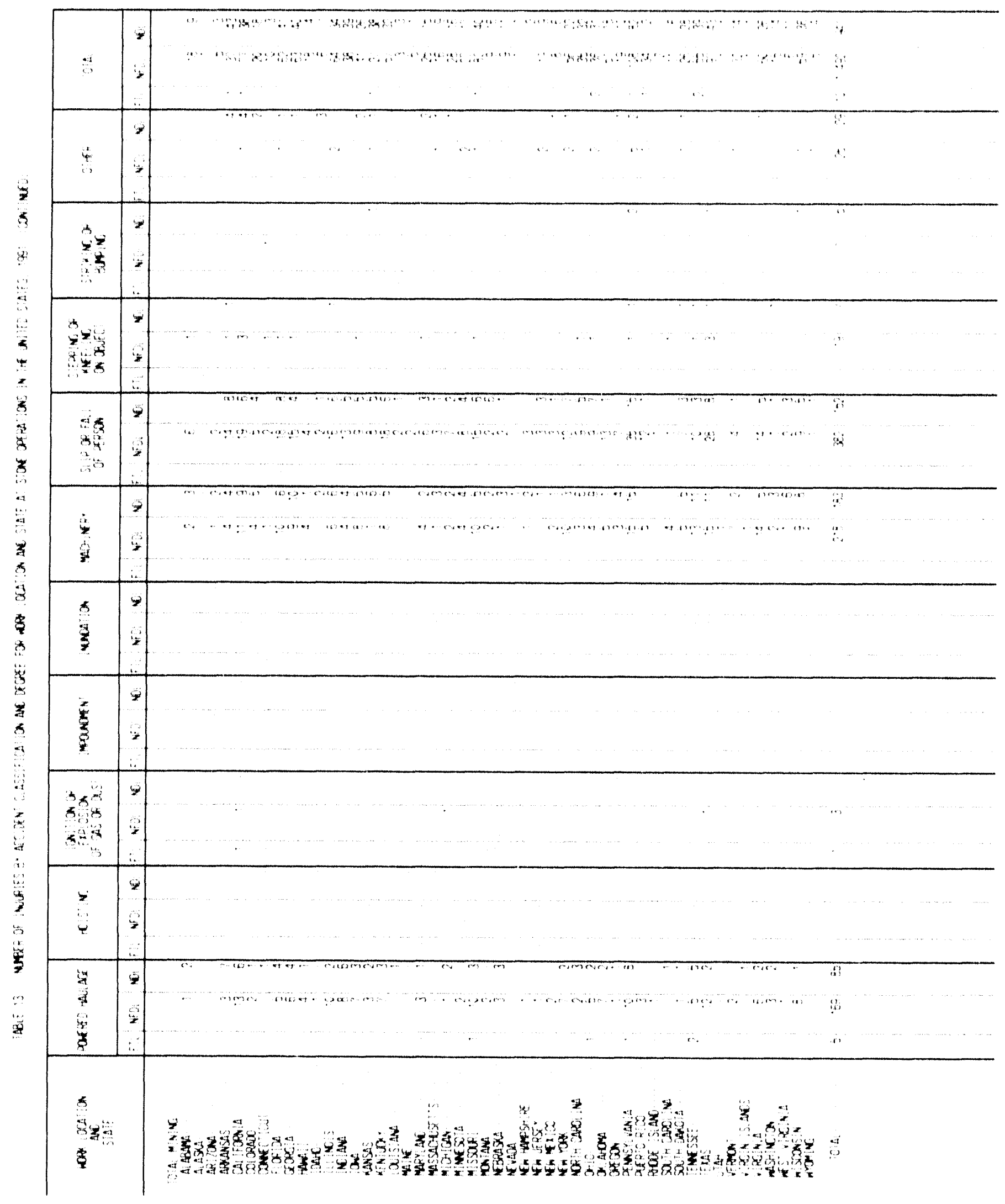




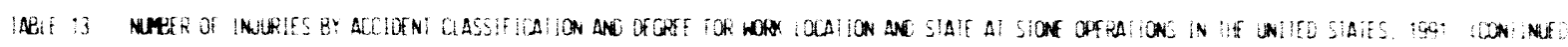

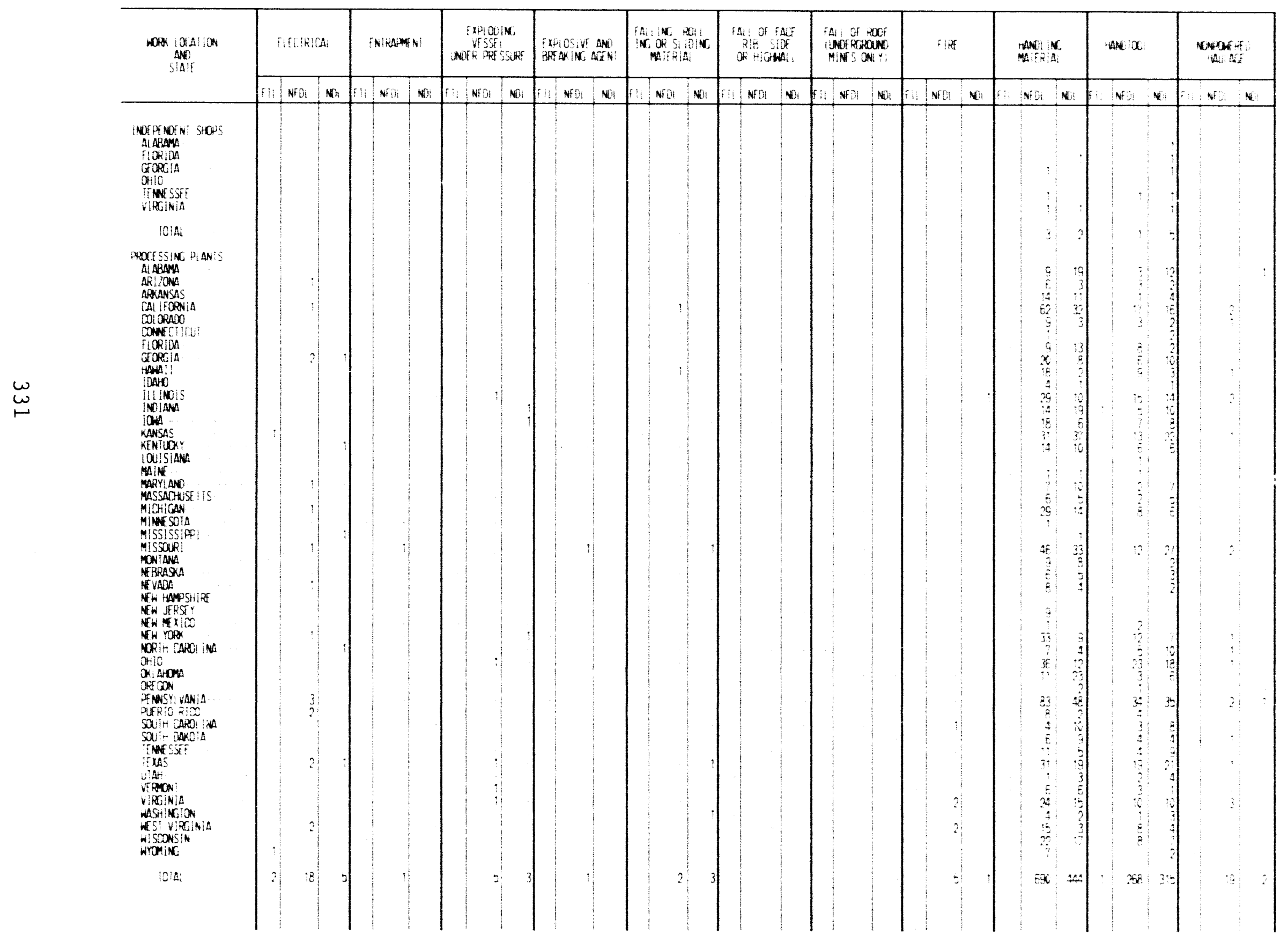




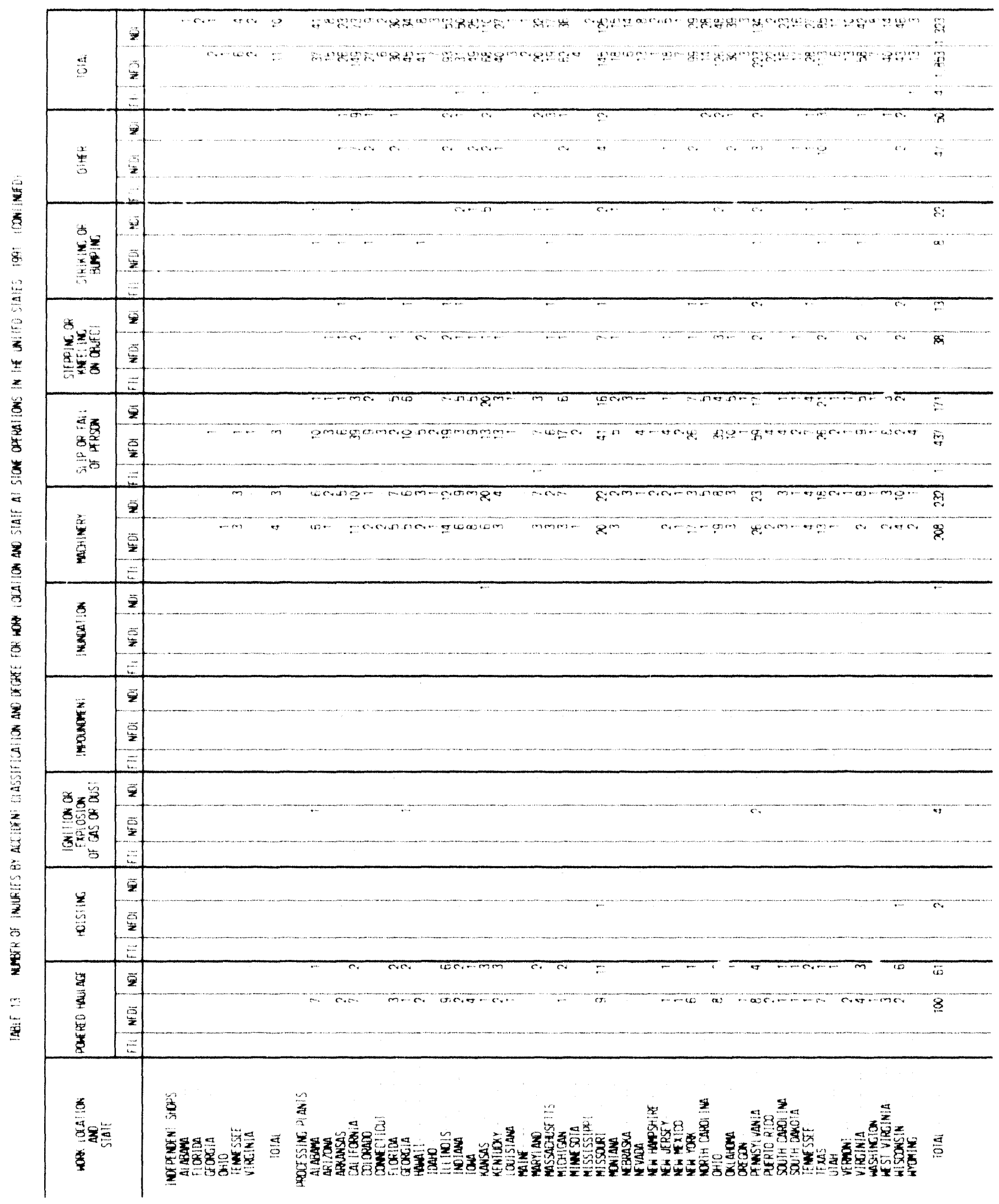




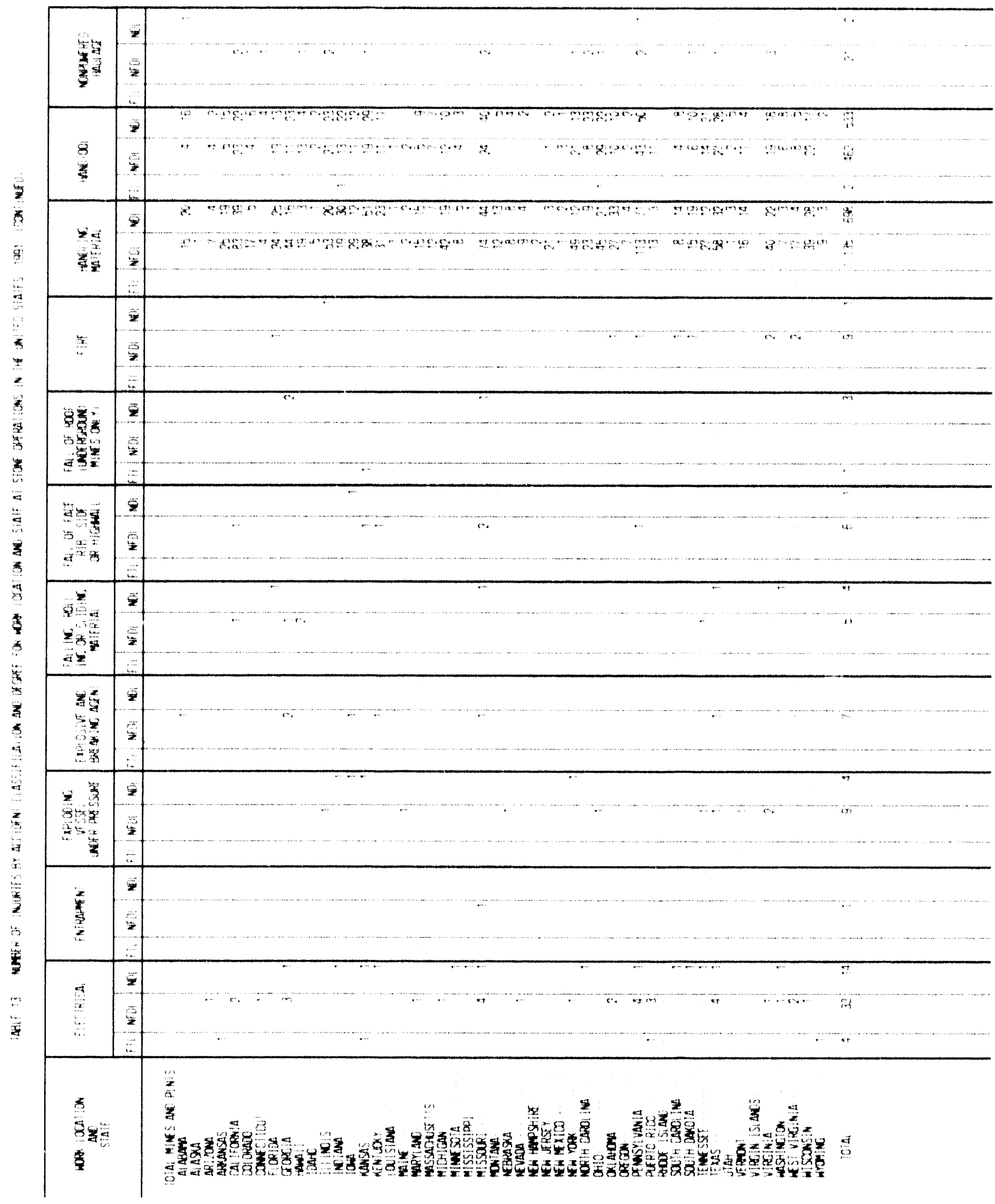




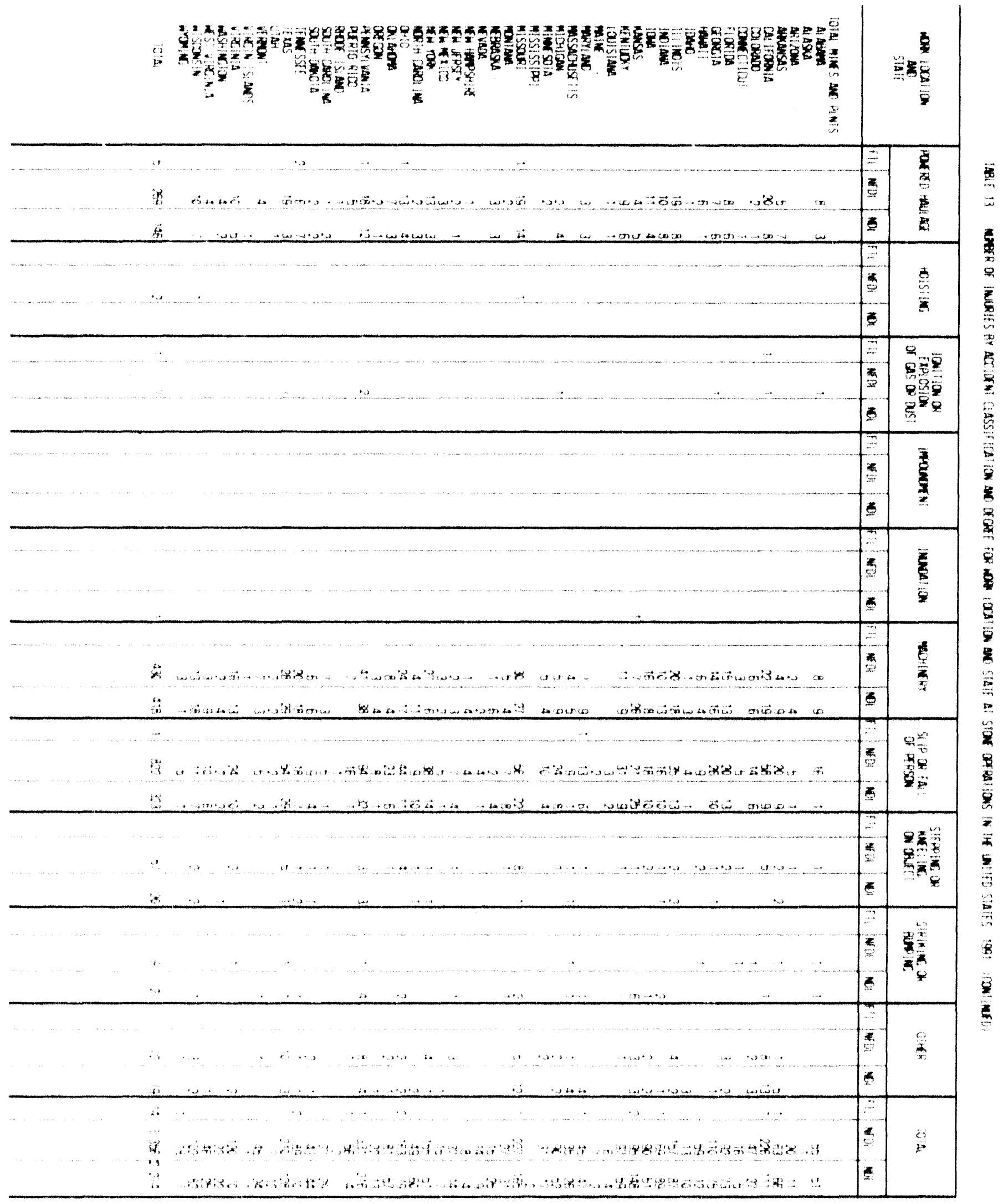




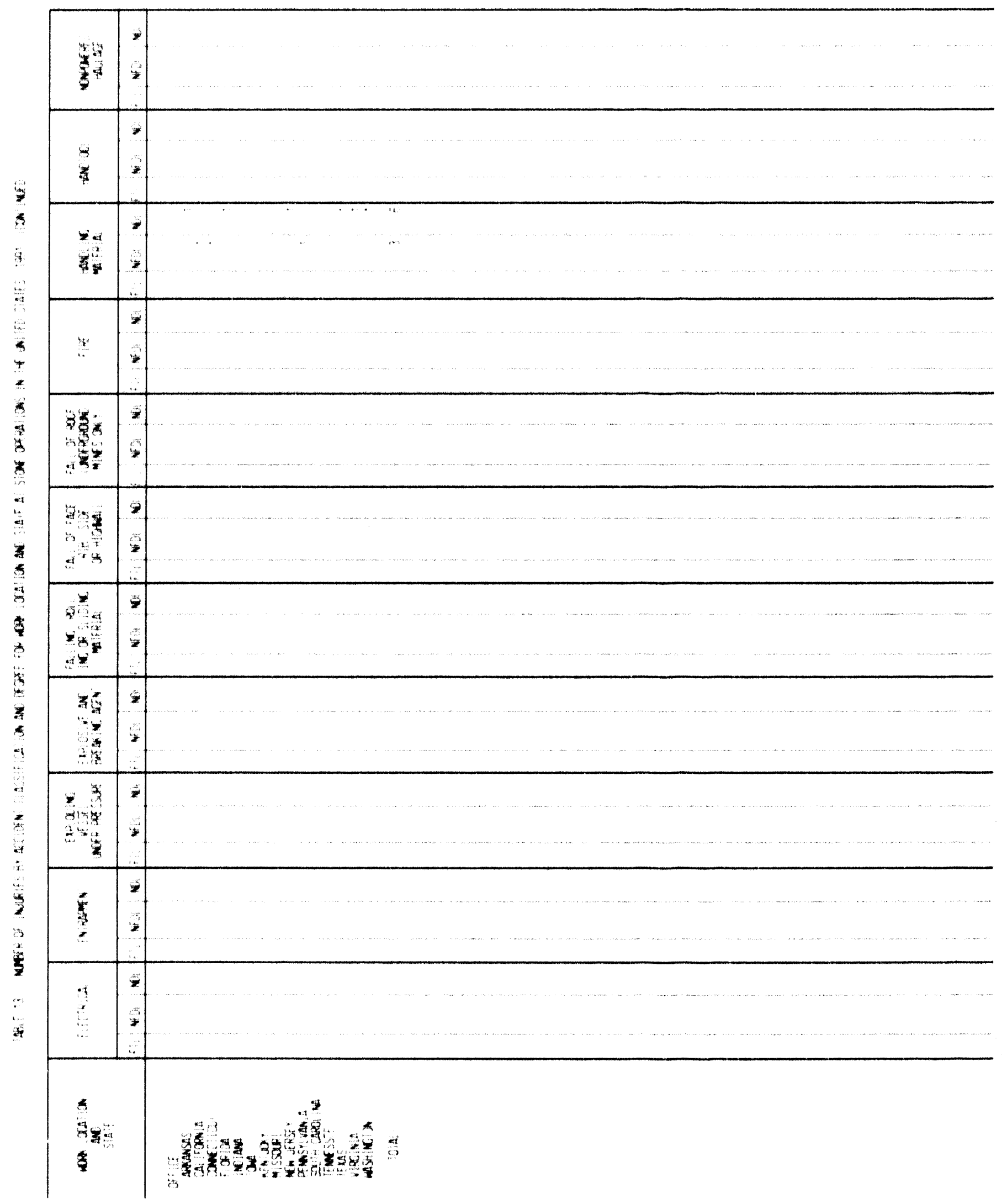

335 


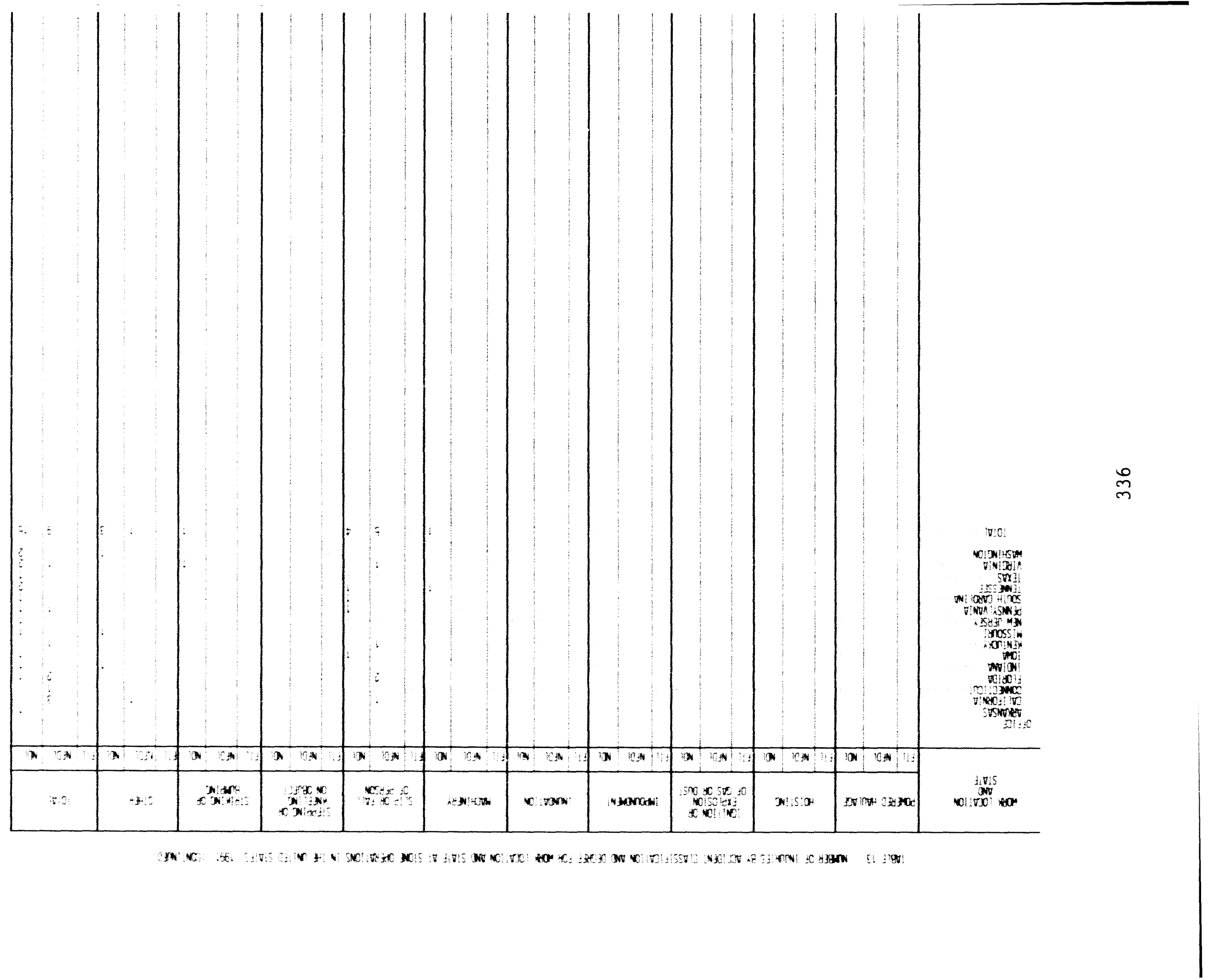




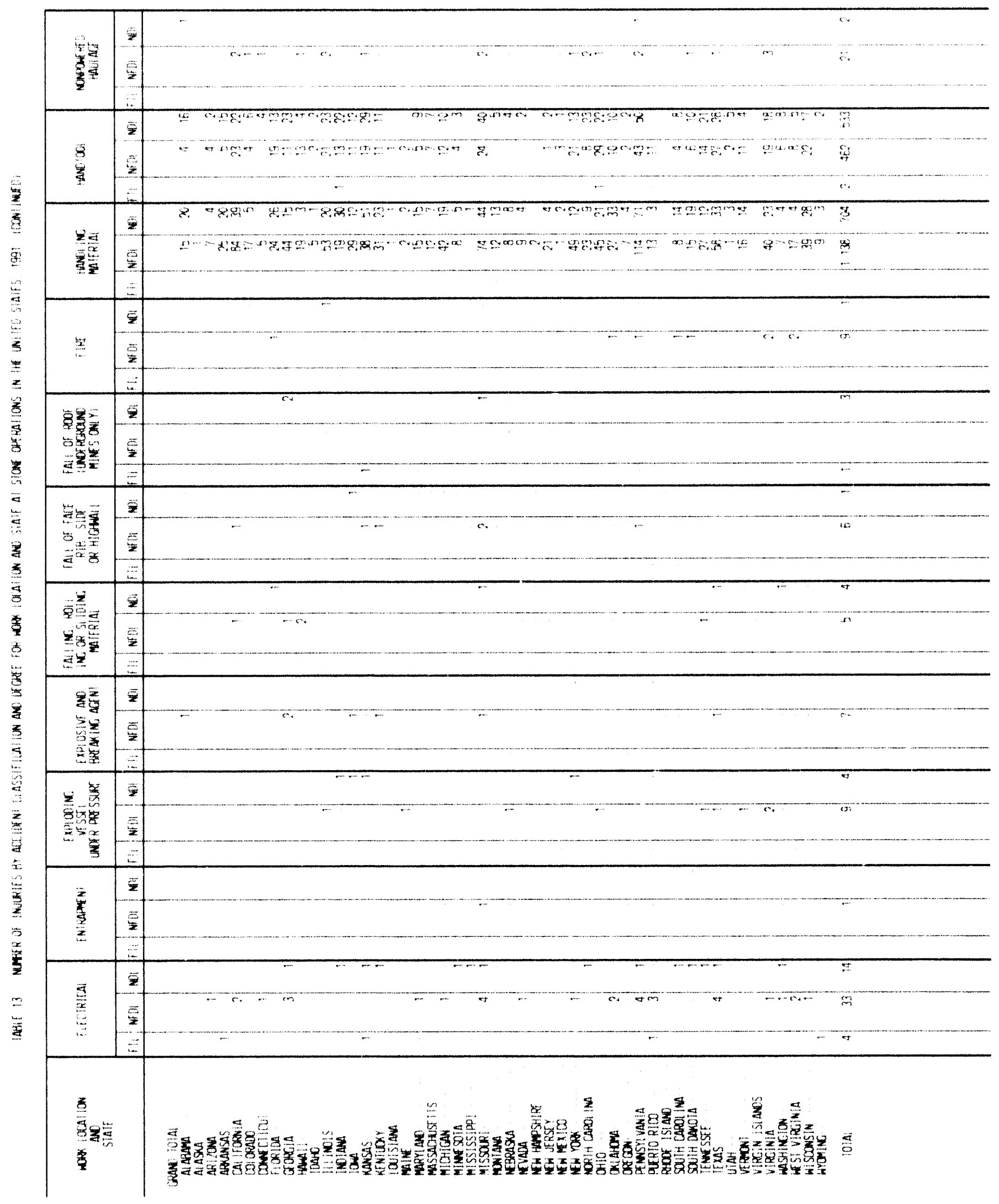




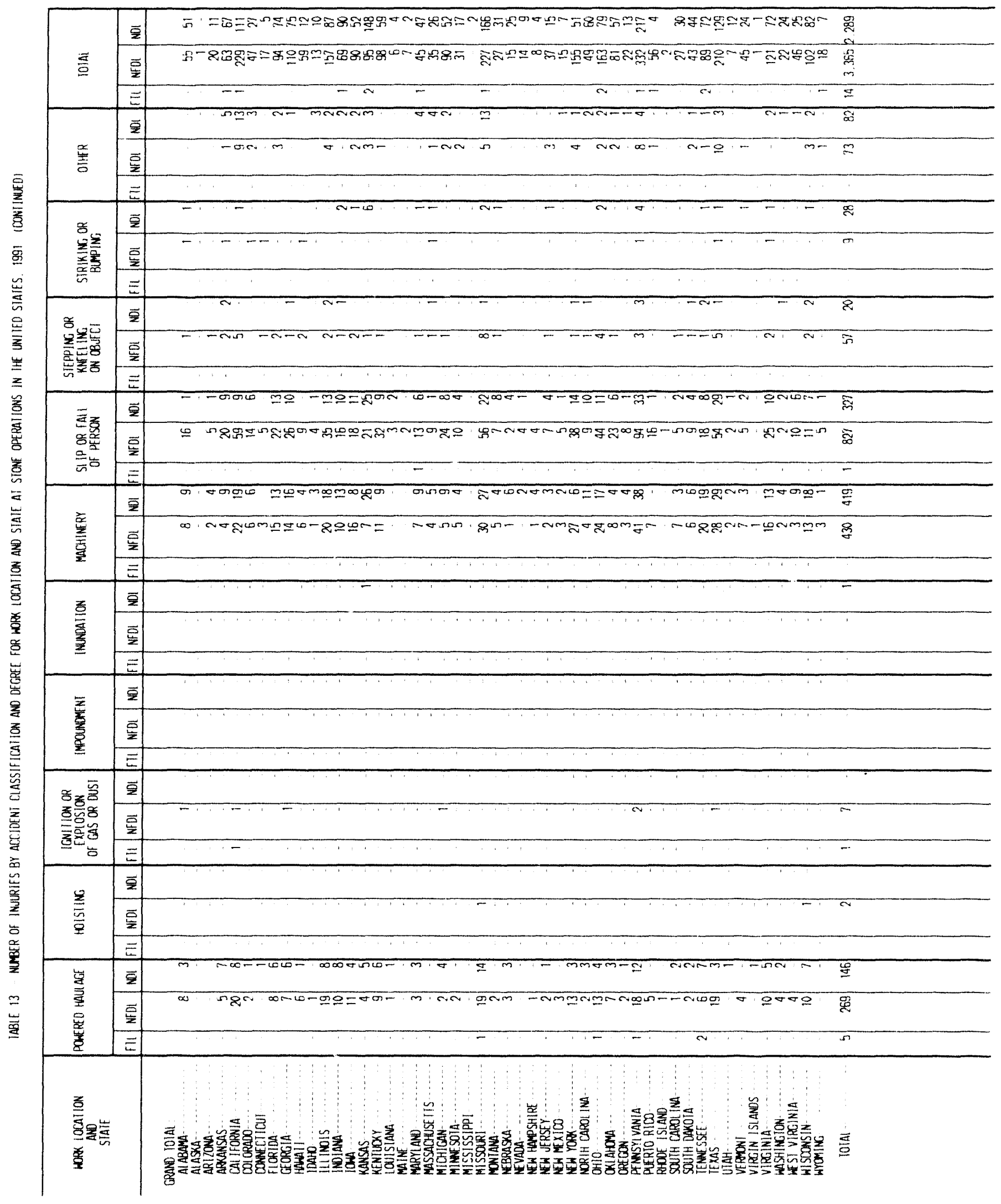




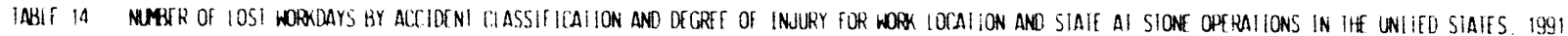

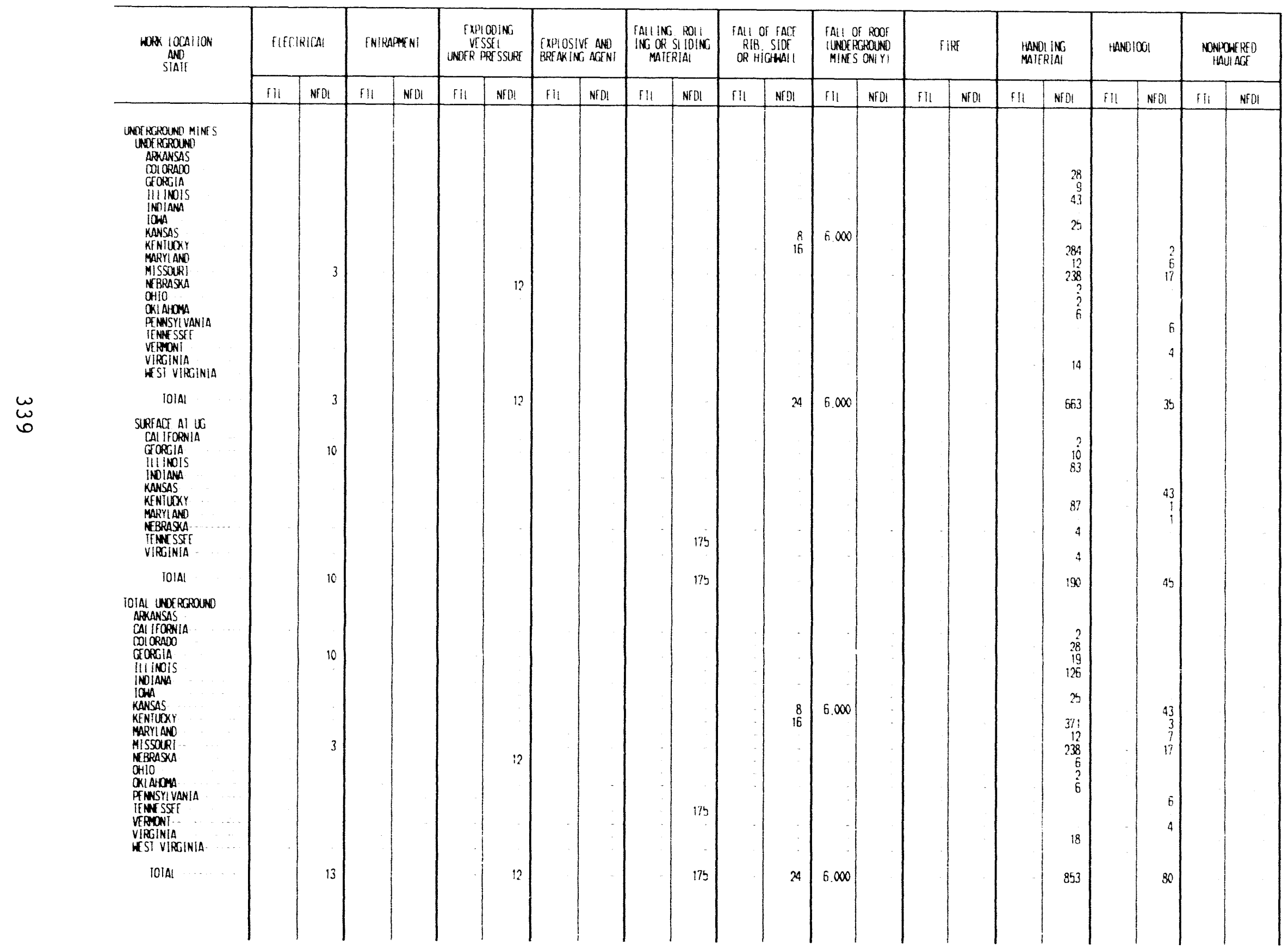




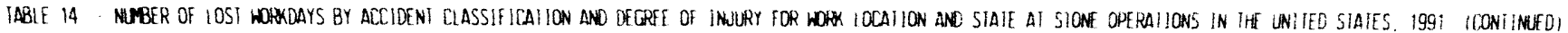

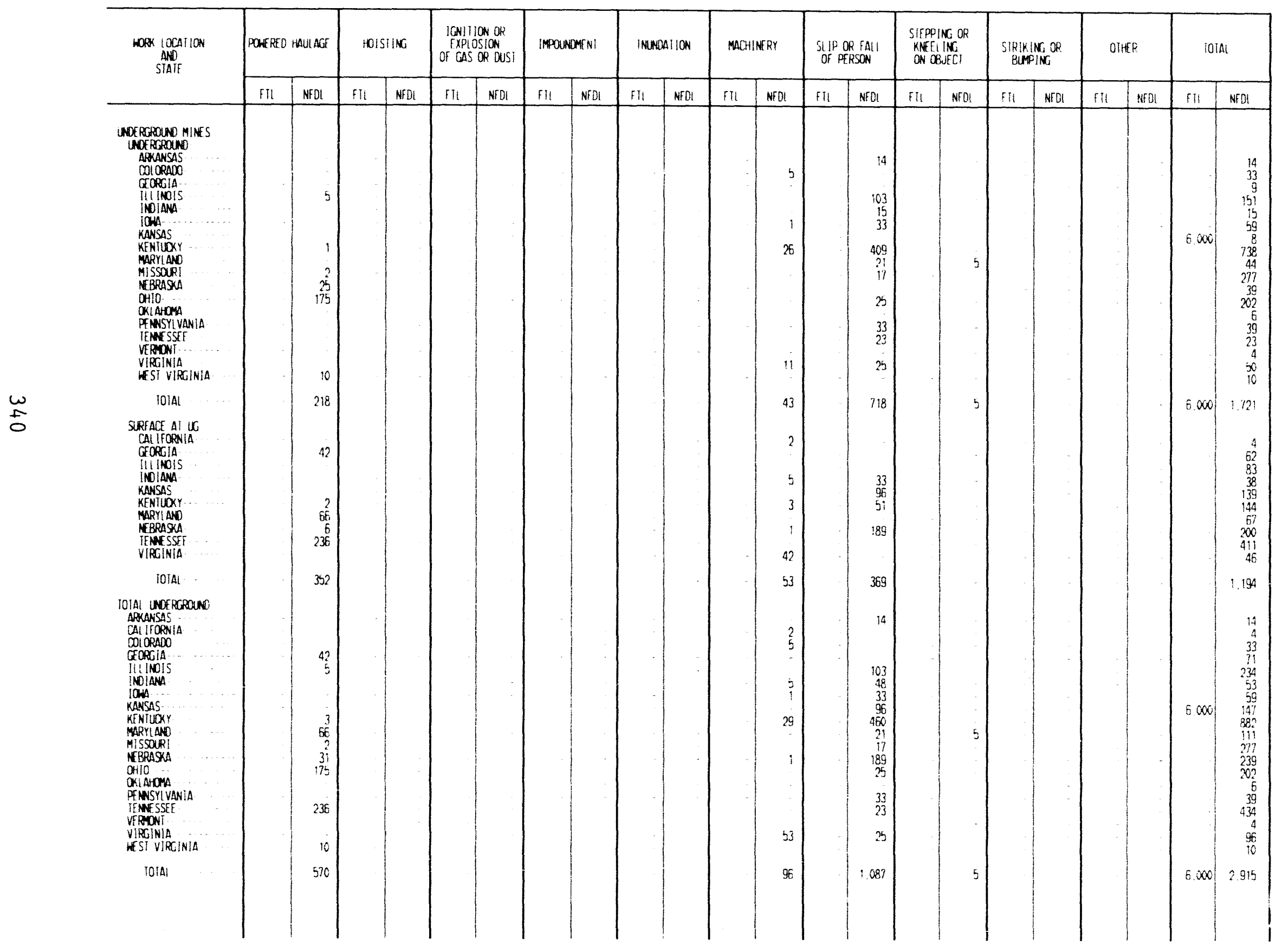




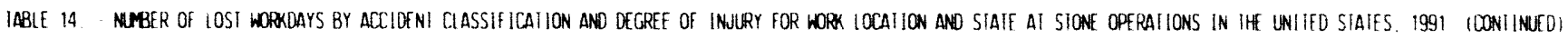

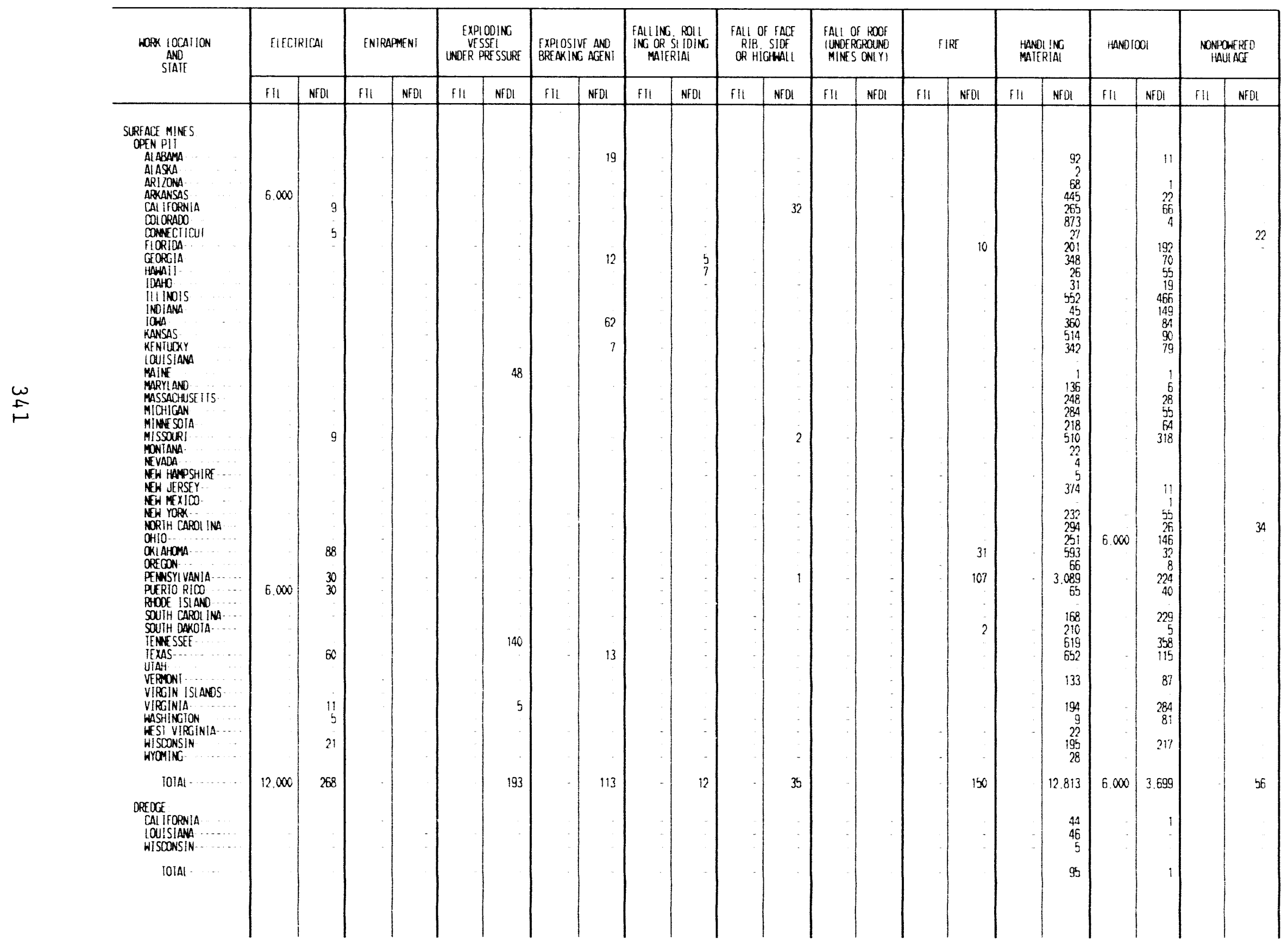




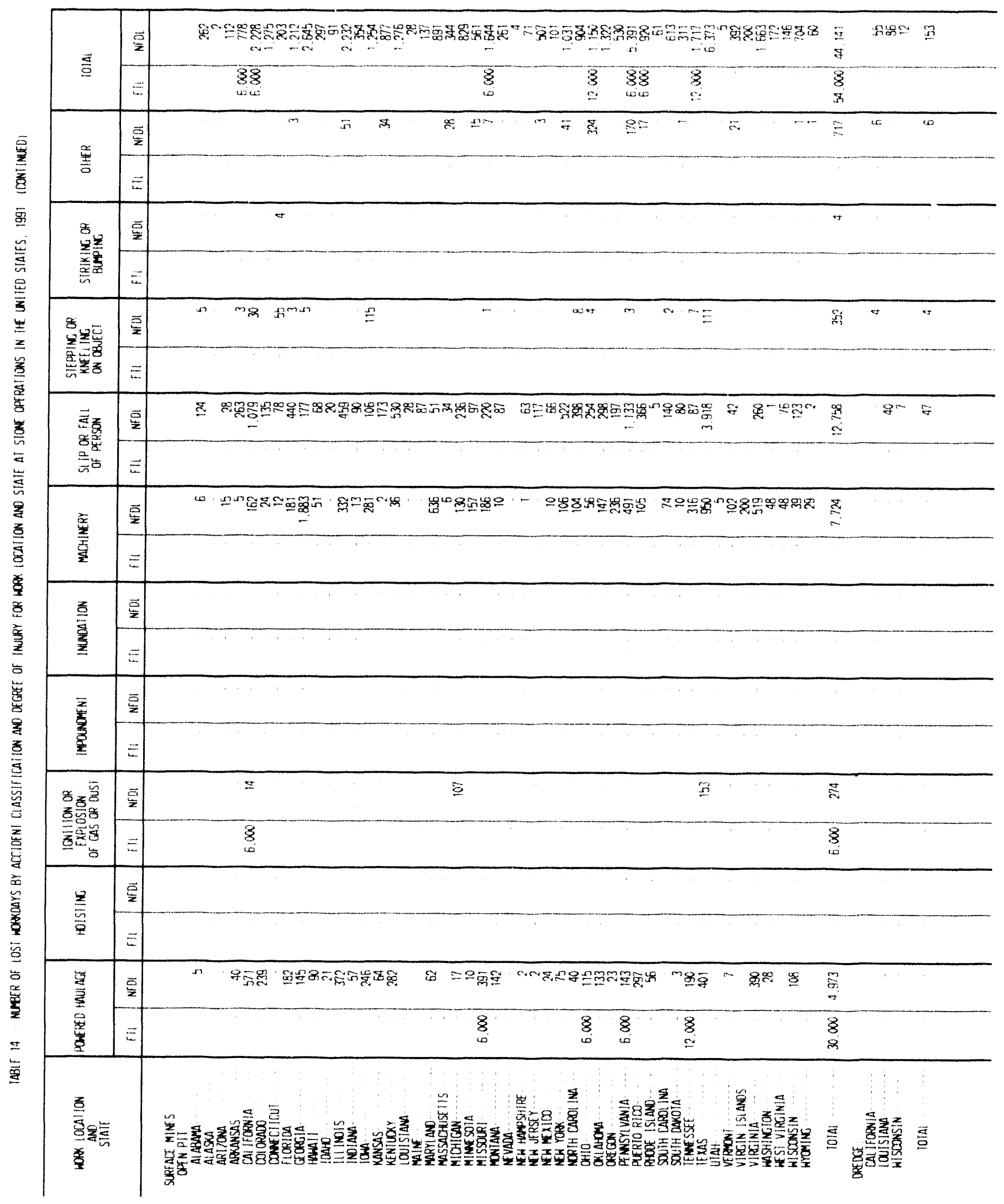




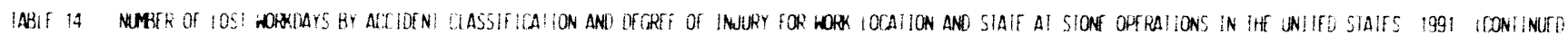

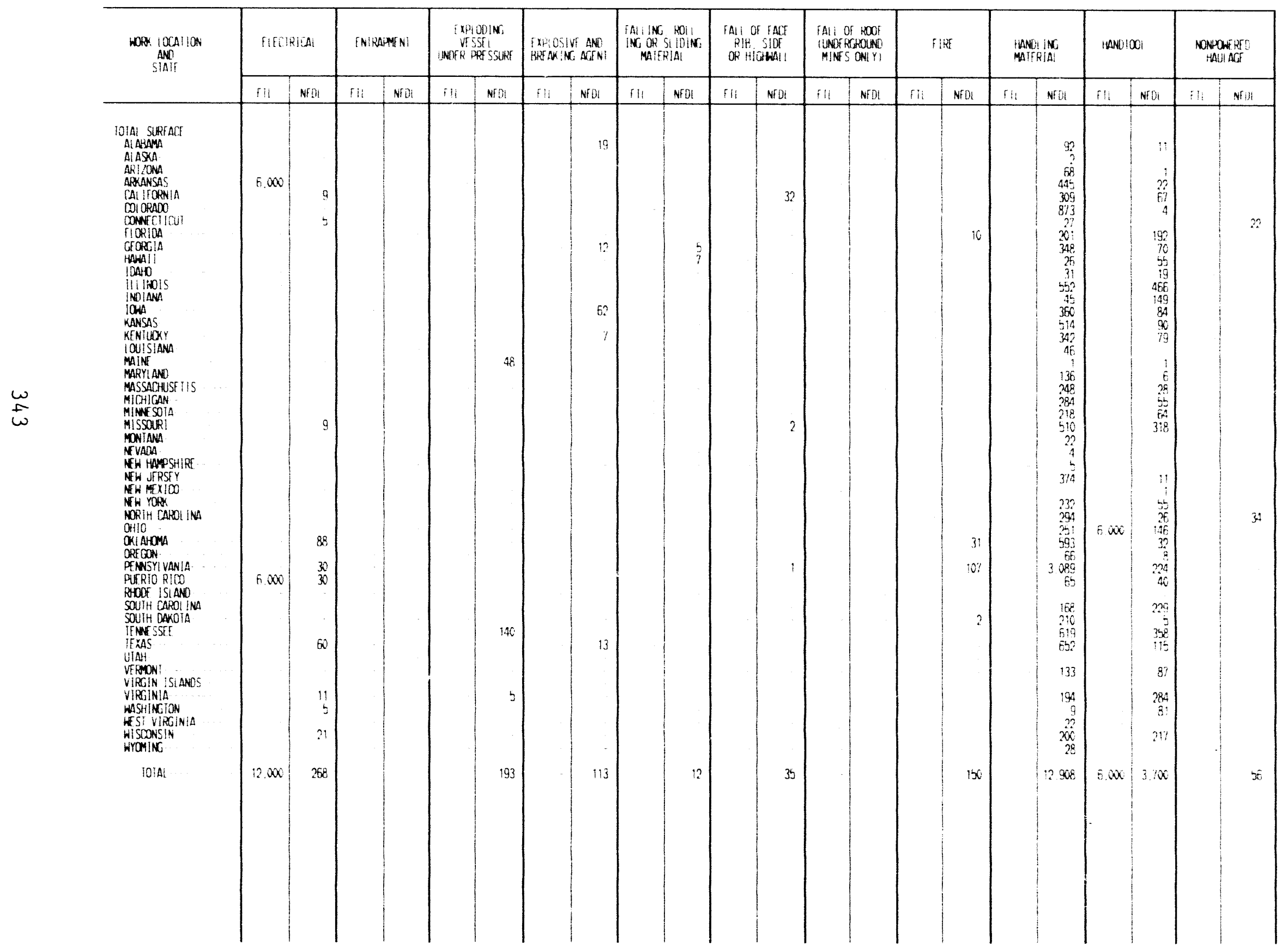




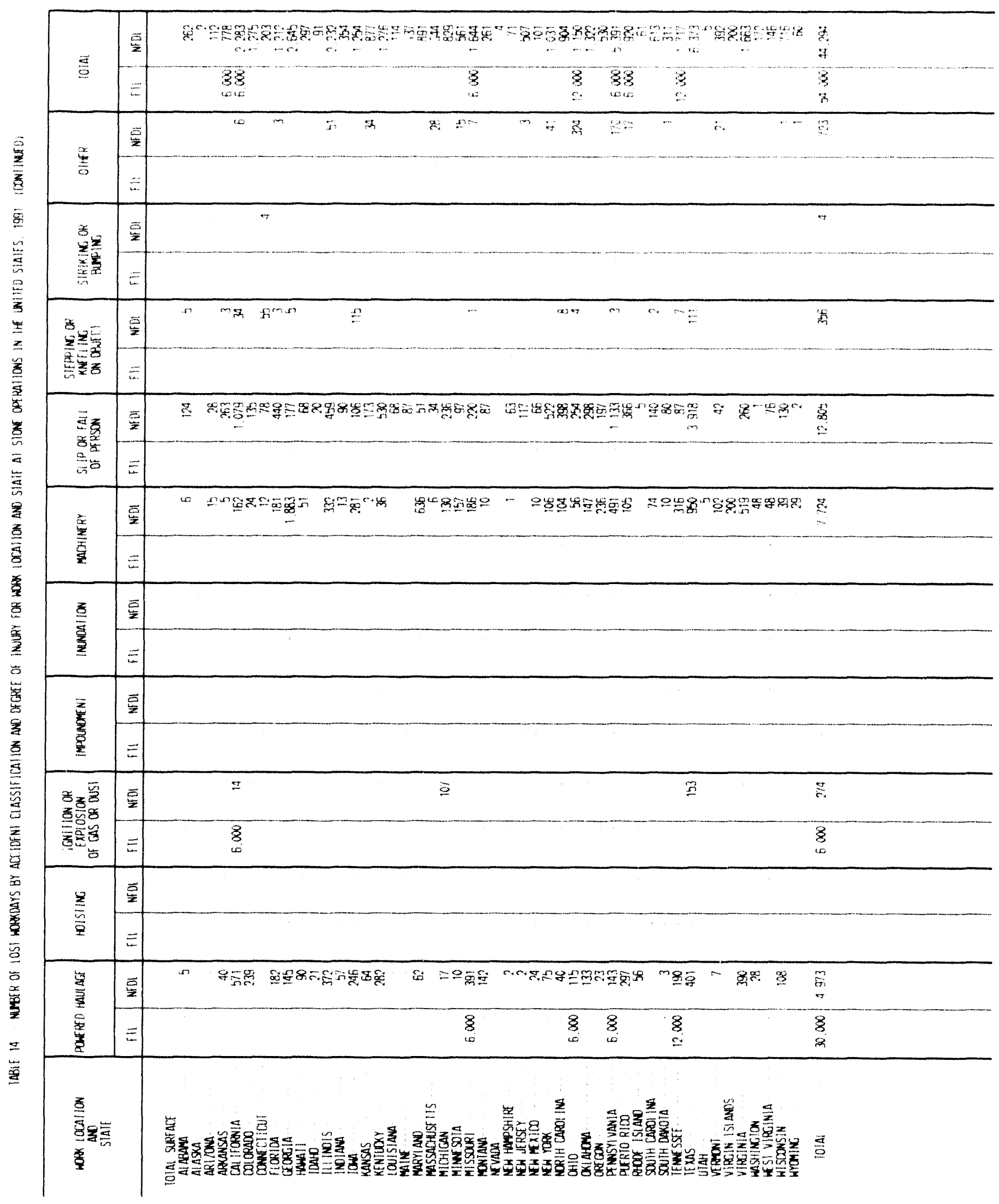




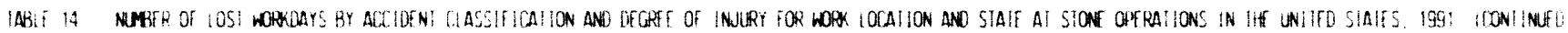

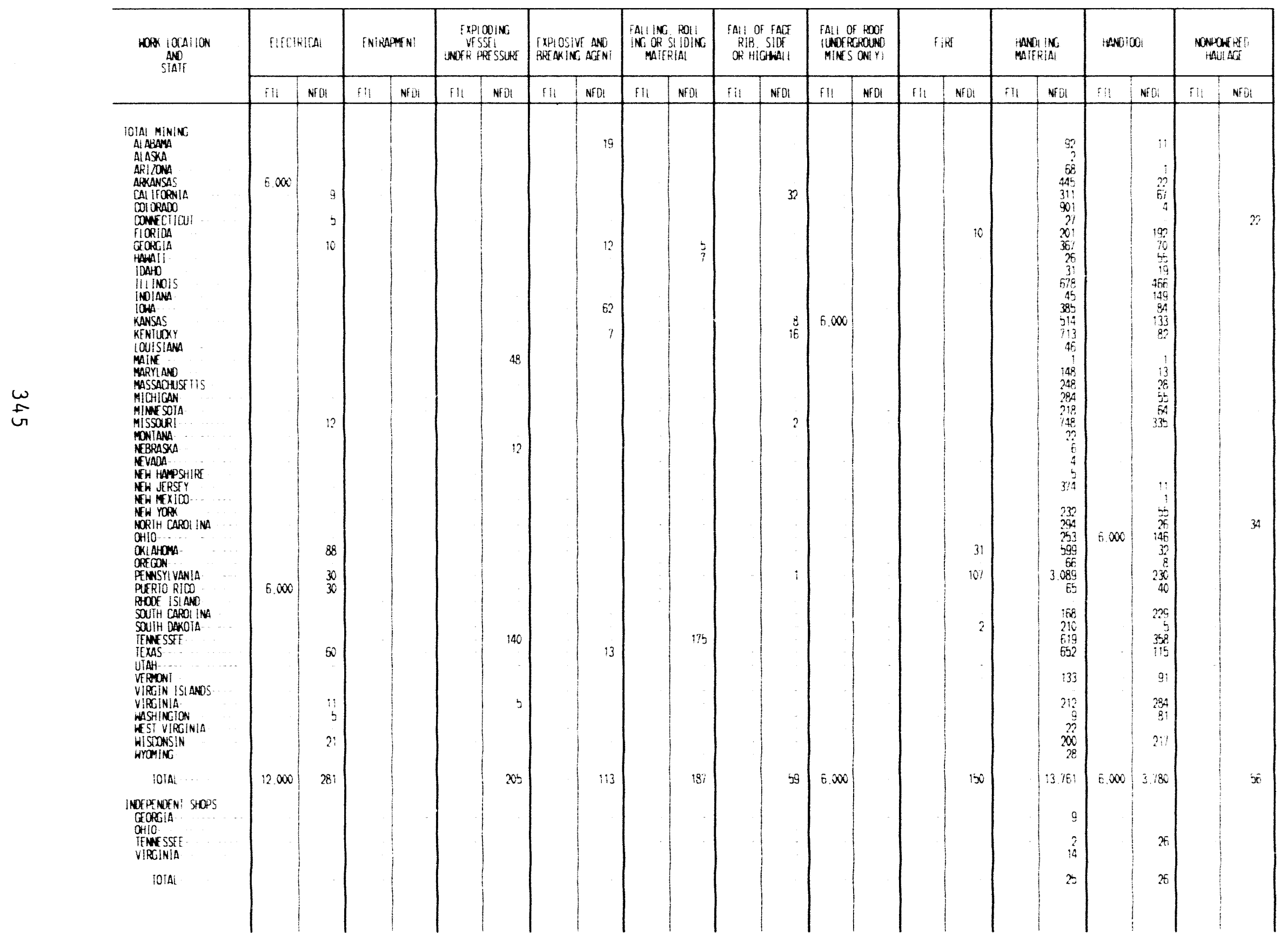




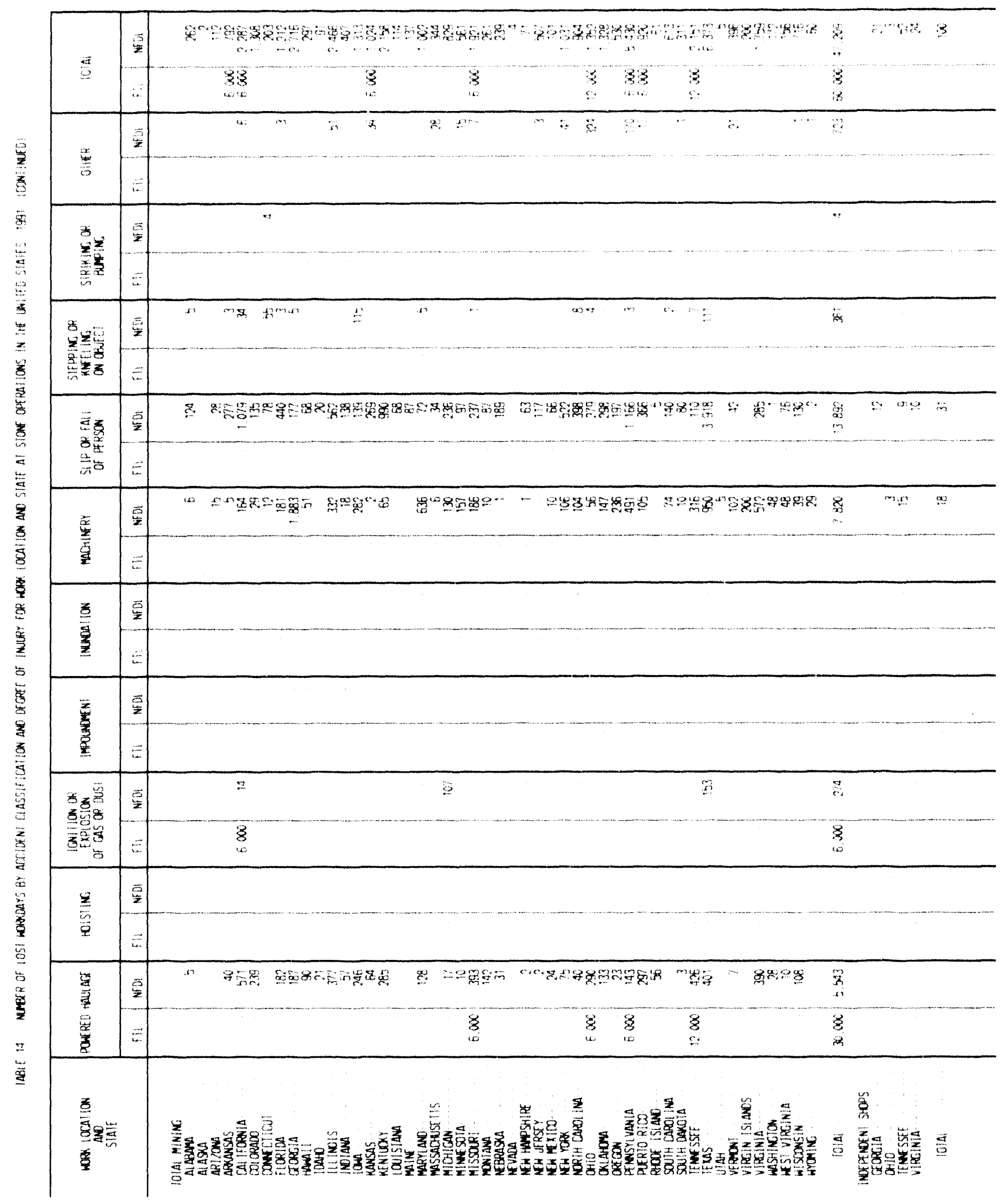




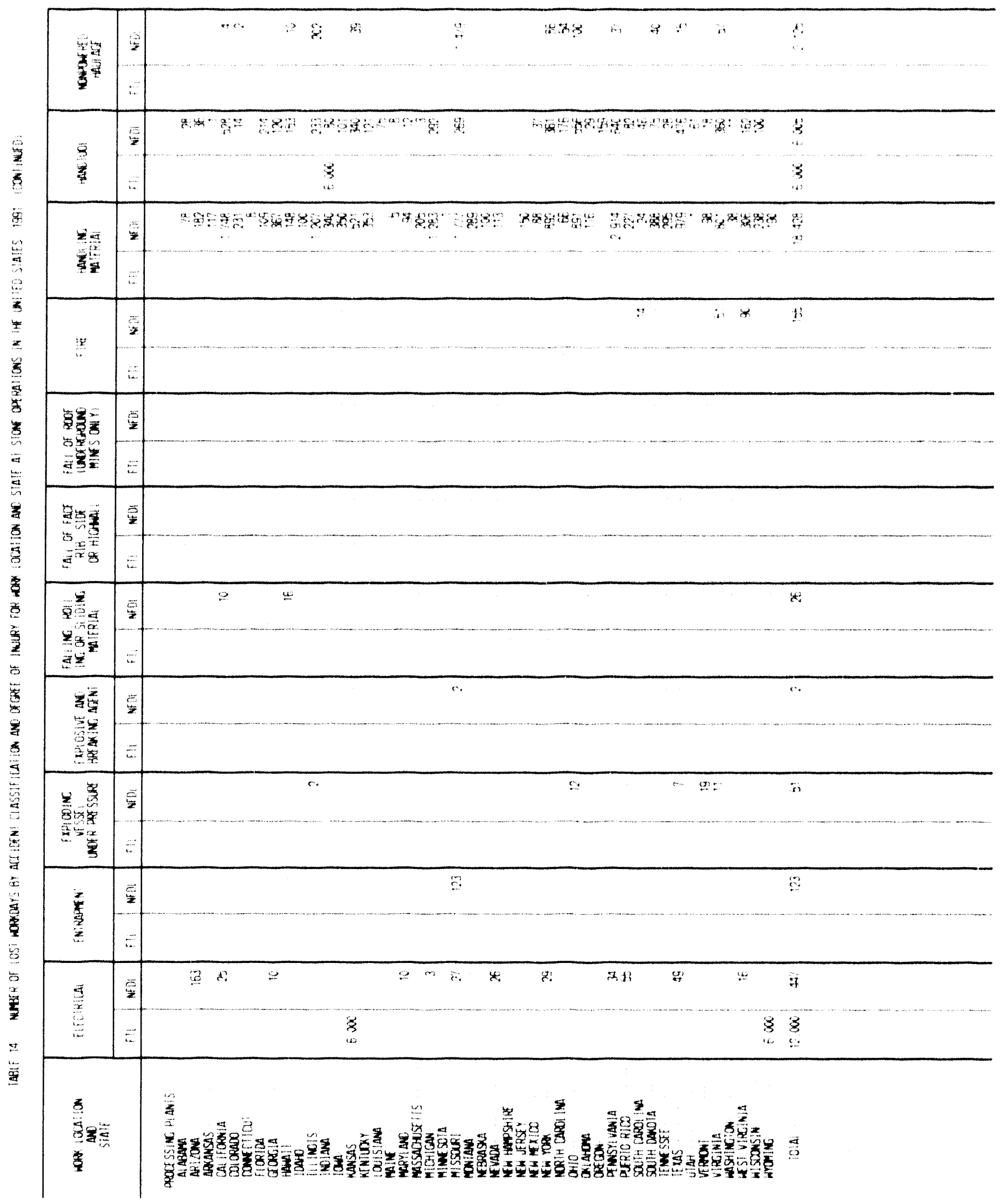




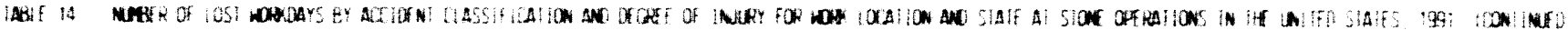

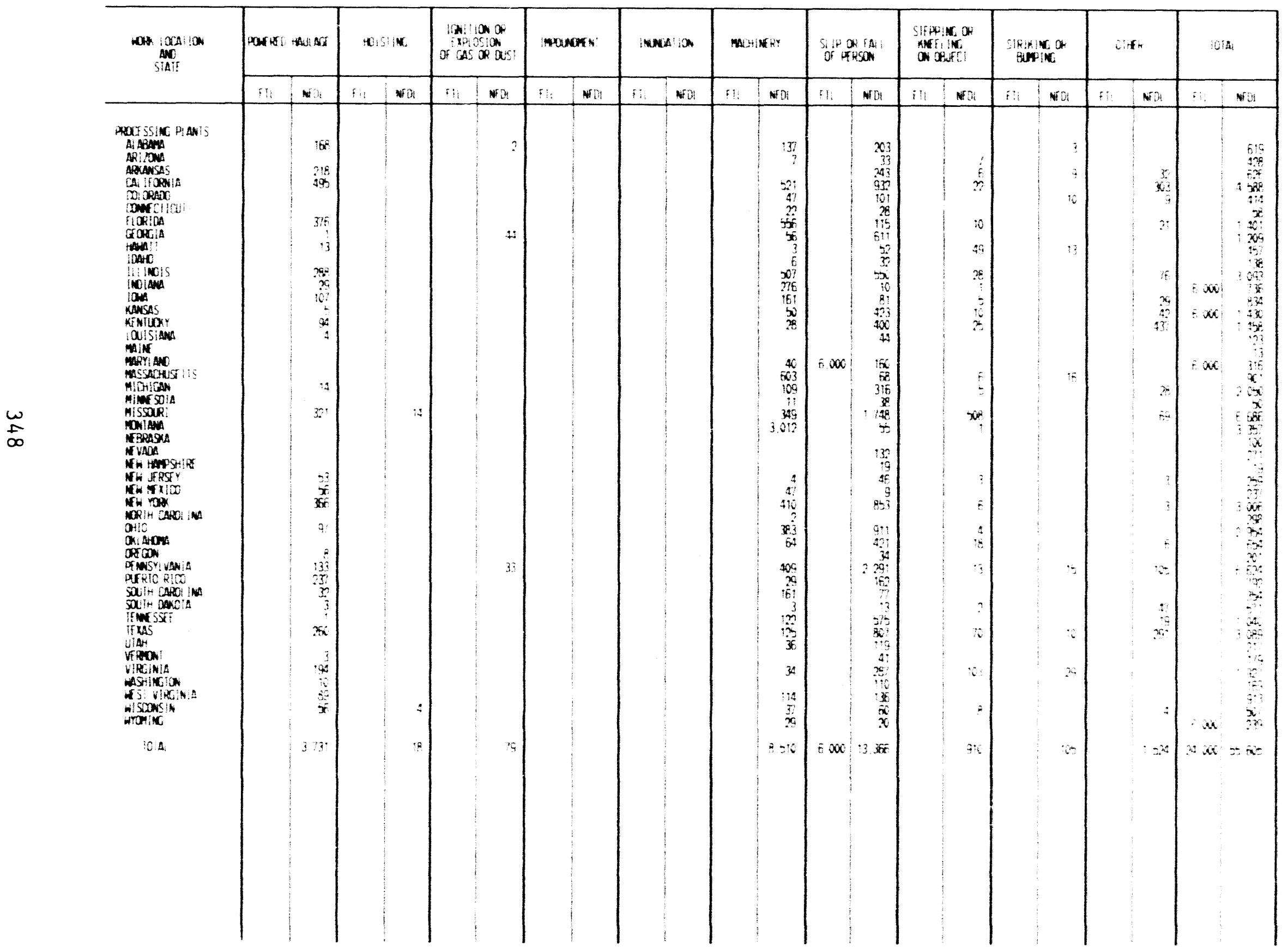




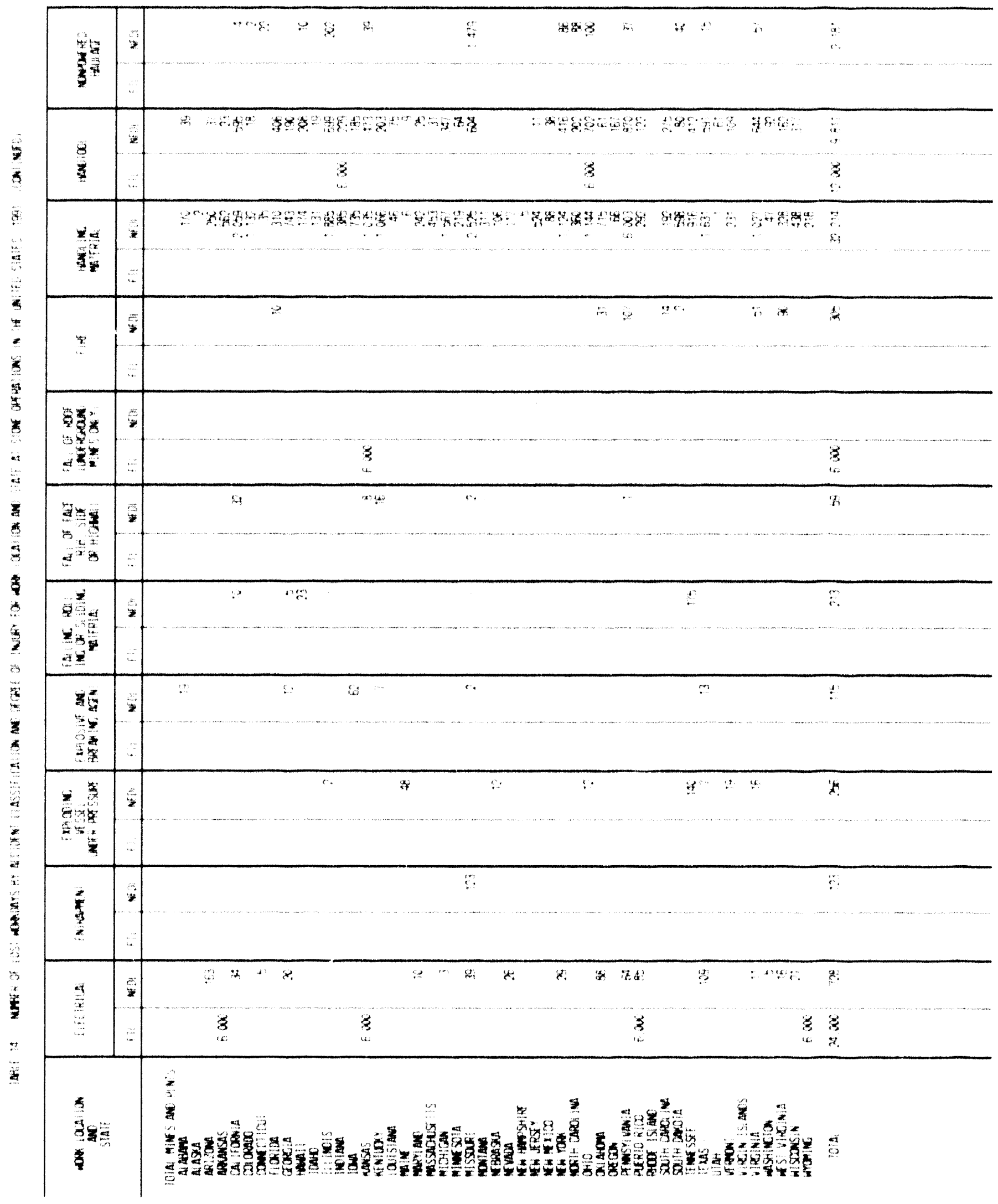




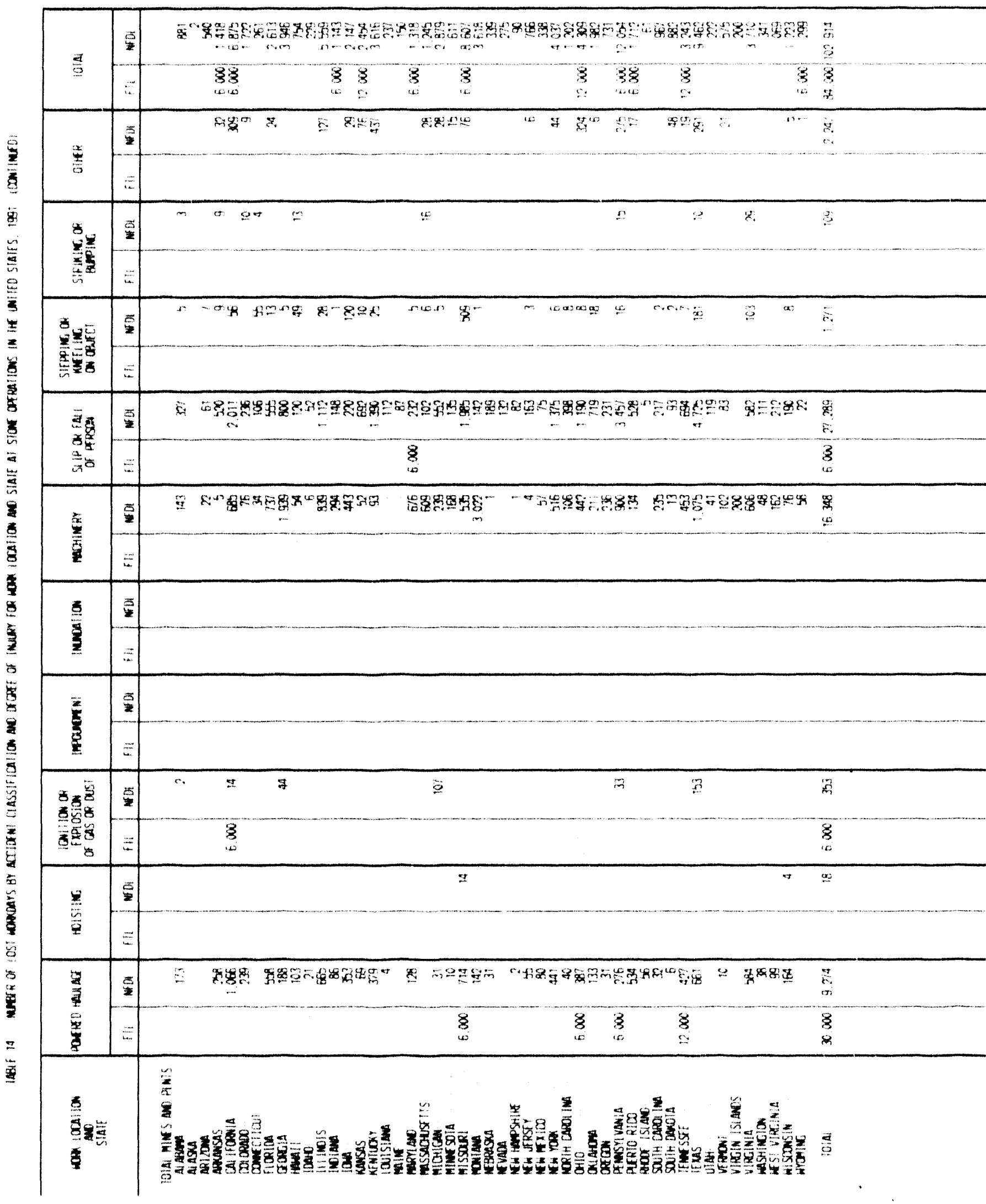




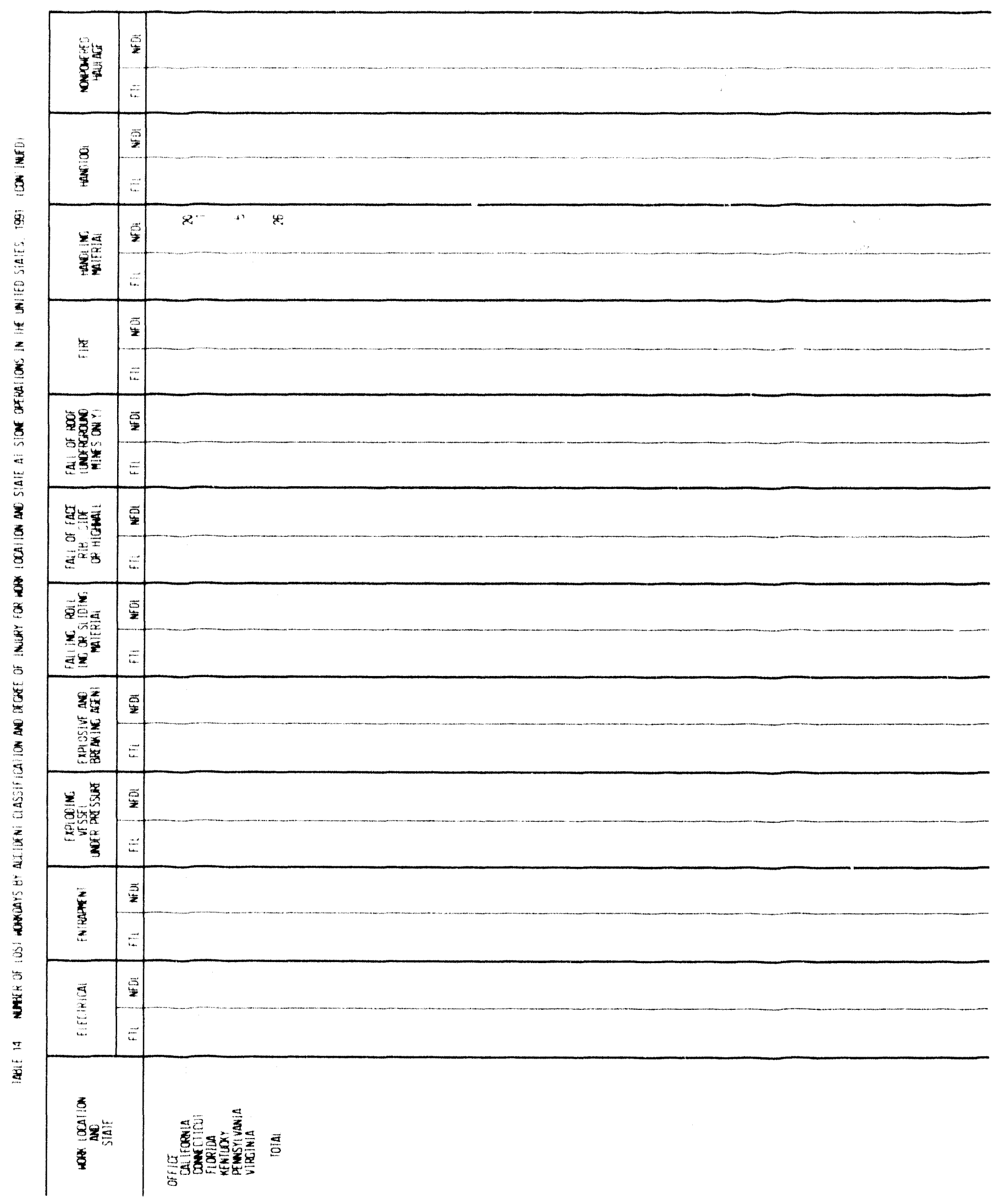




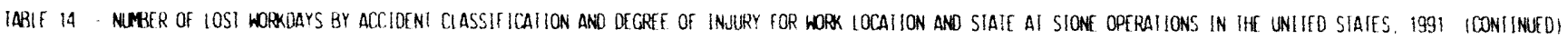

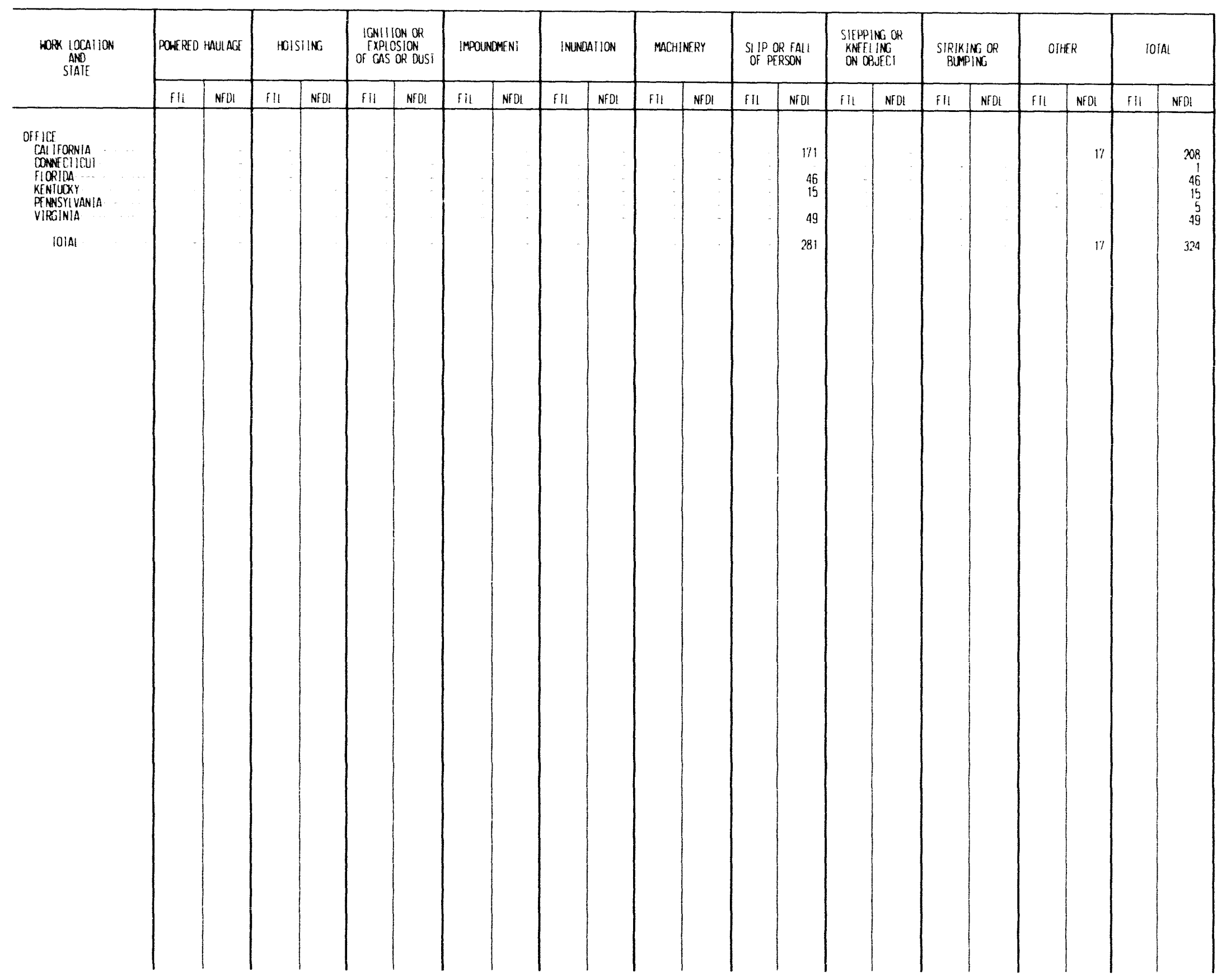




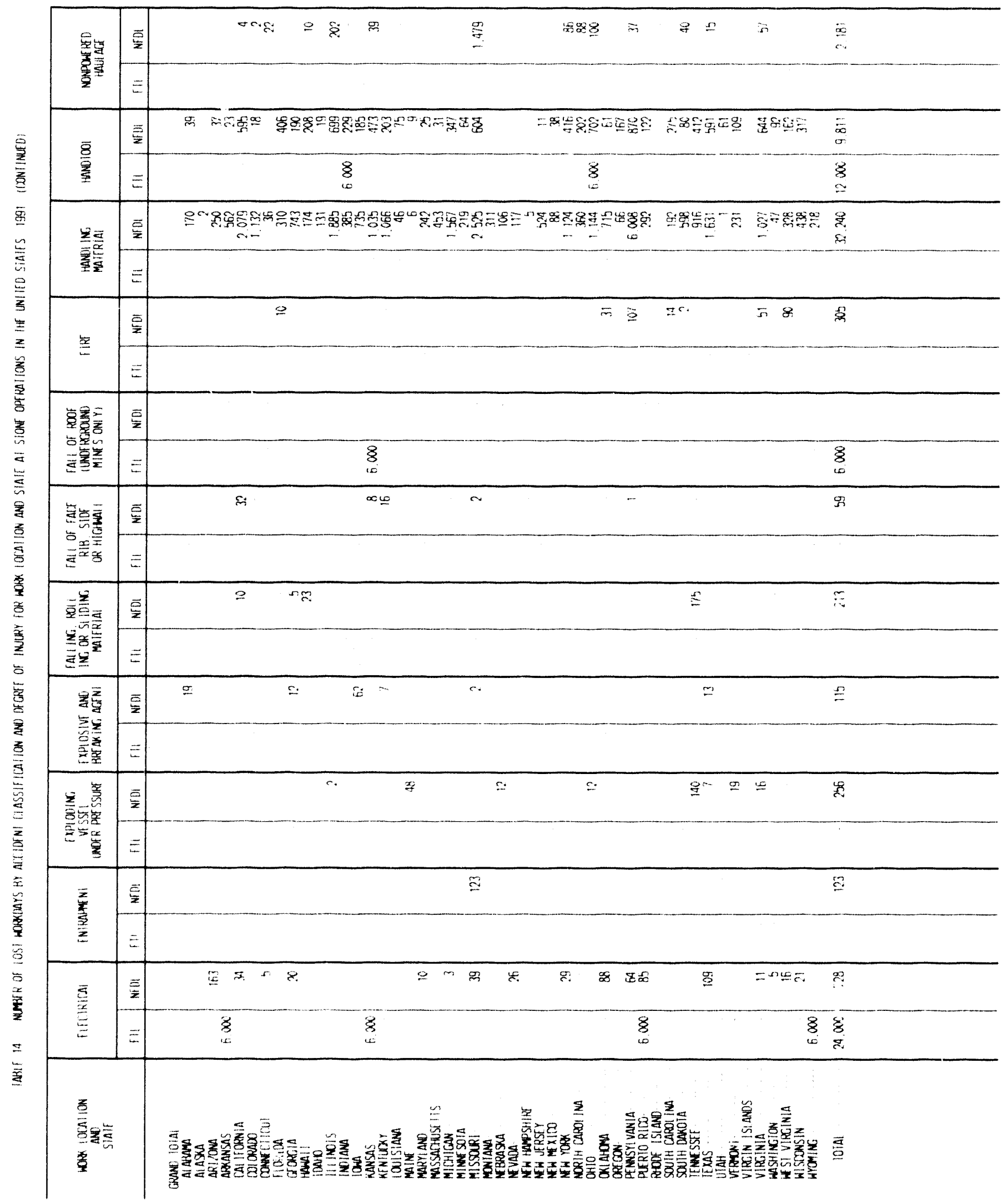




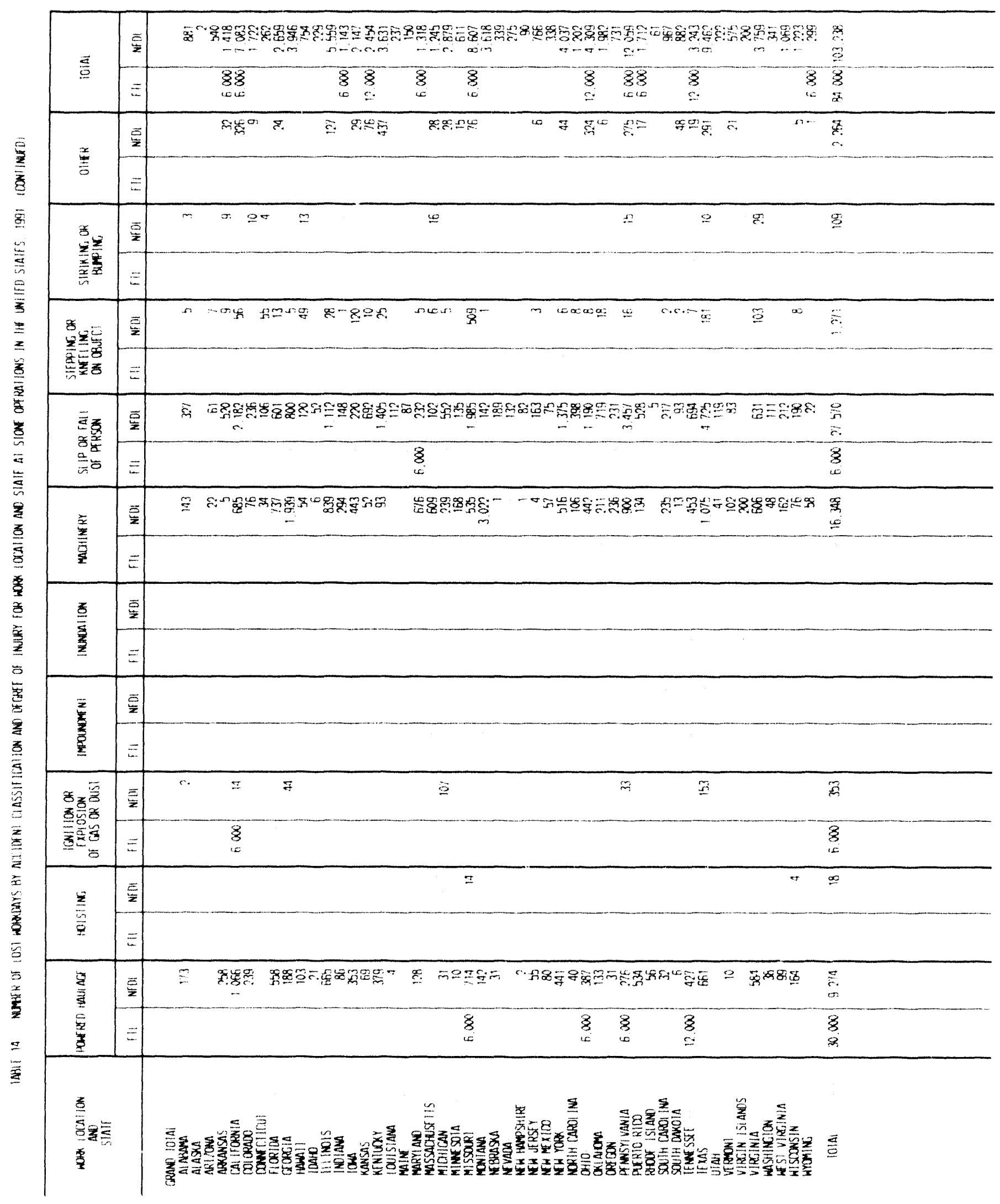




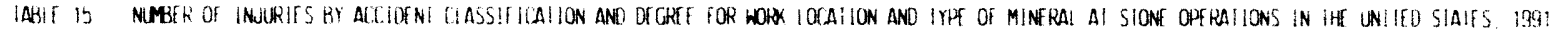

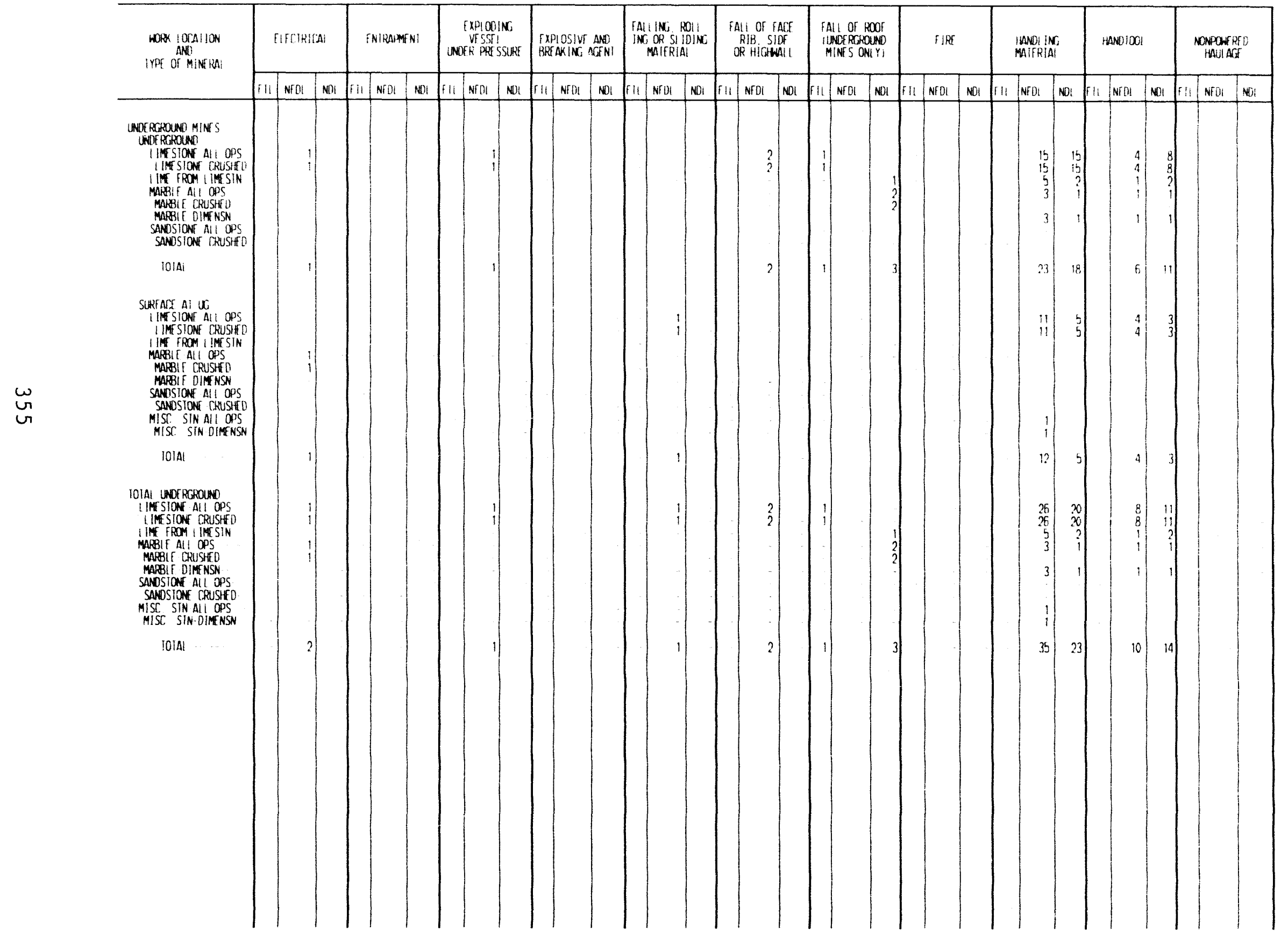




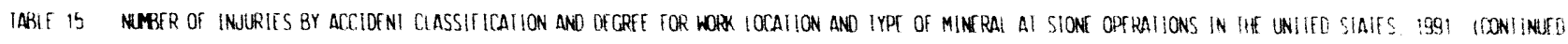

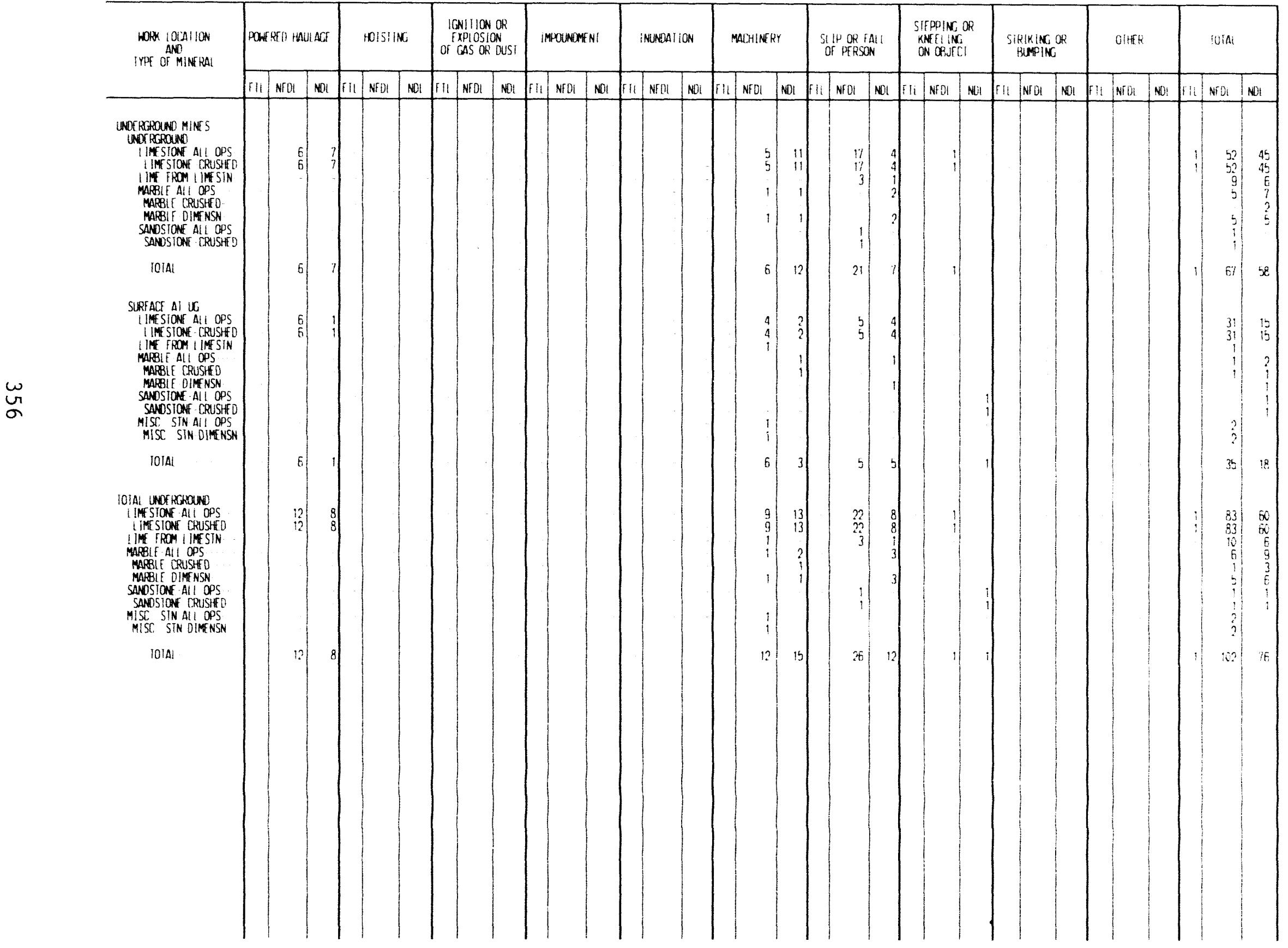




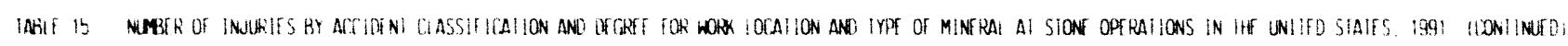

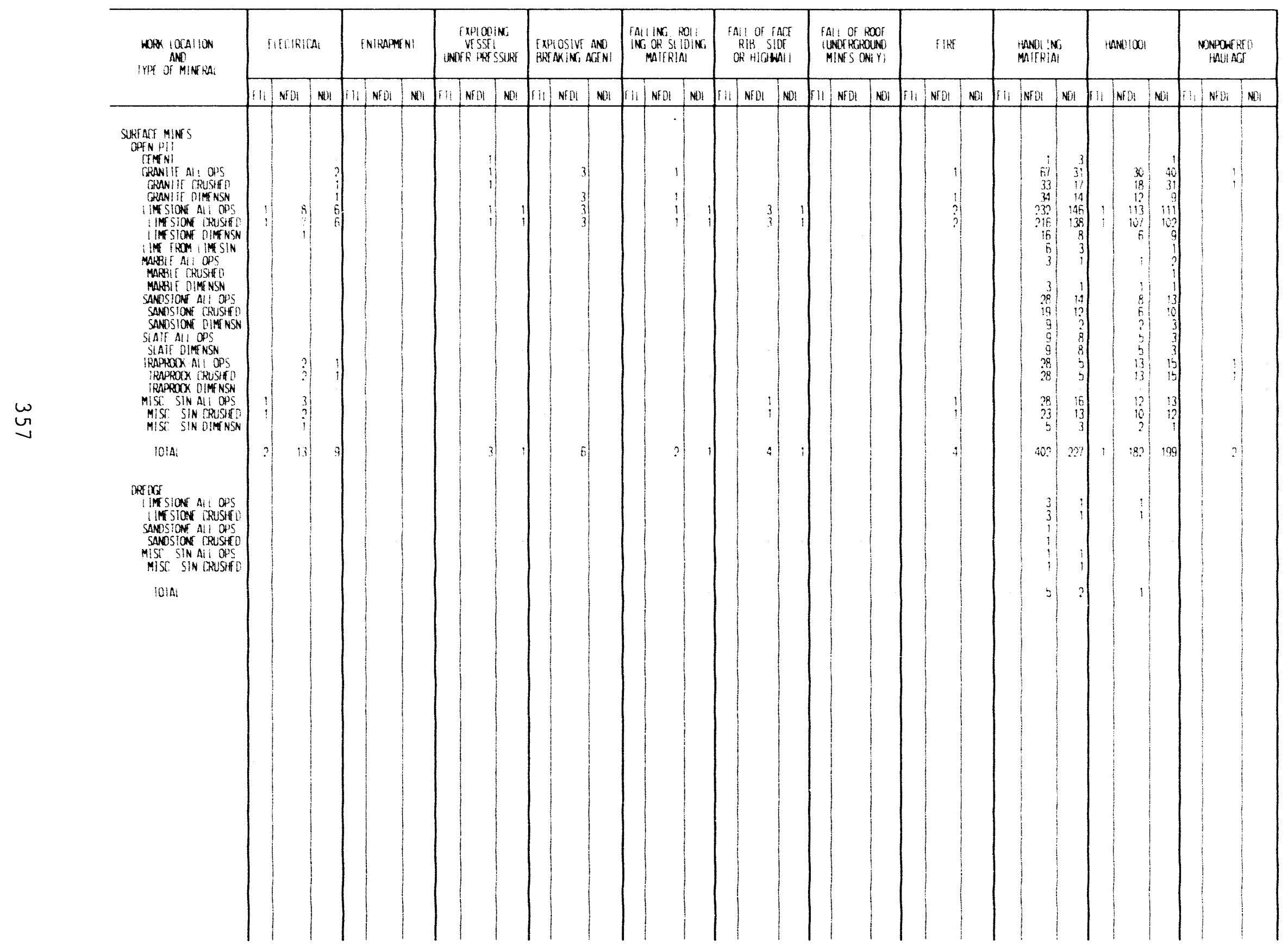




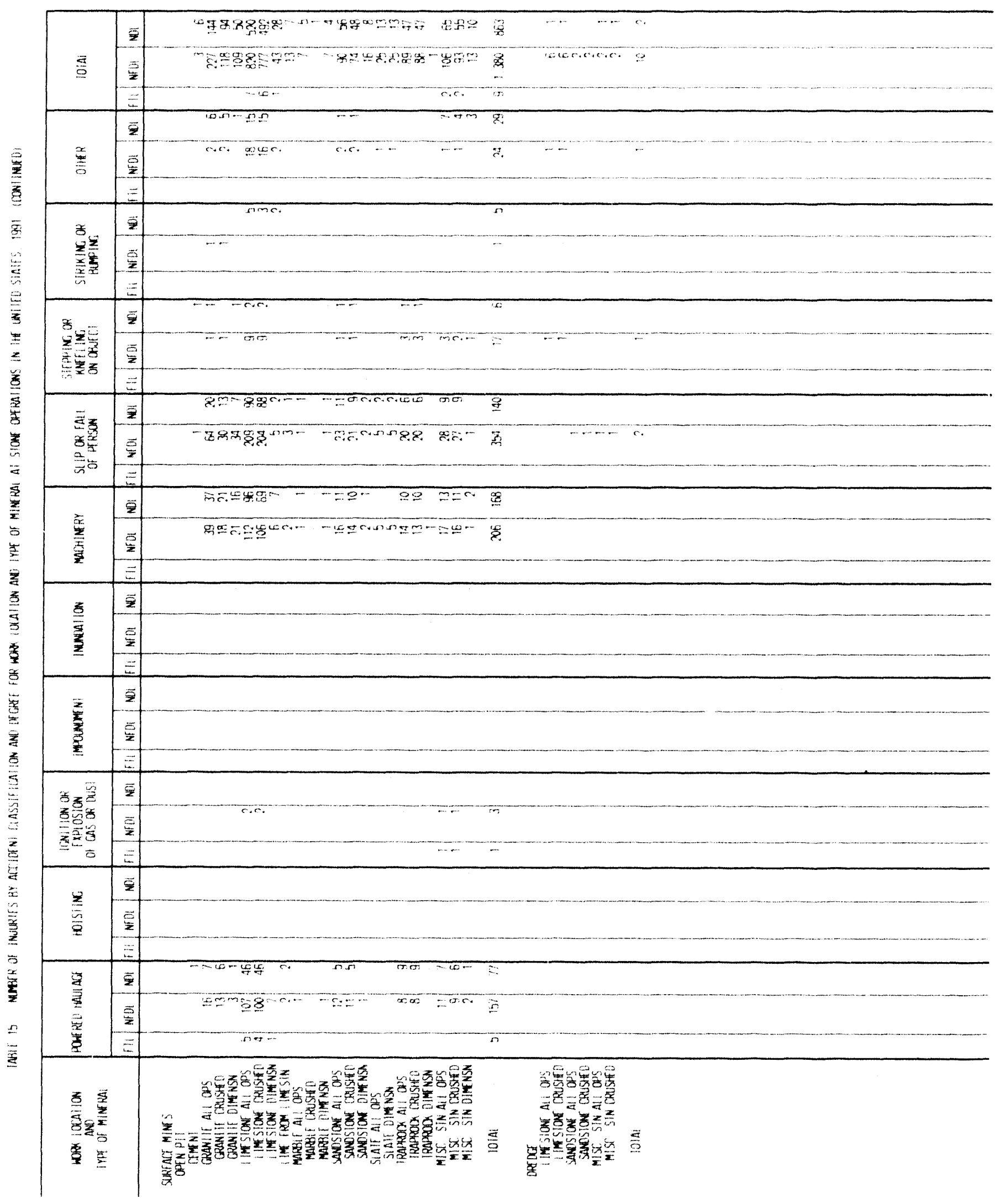




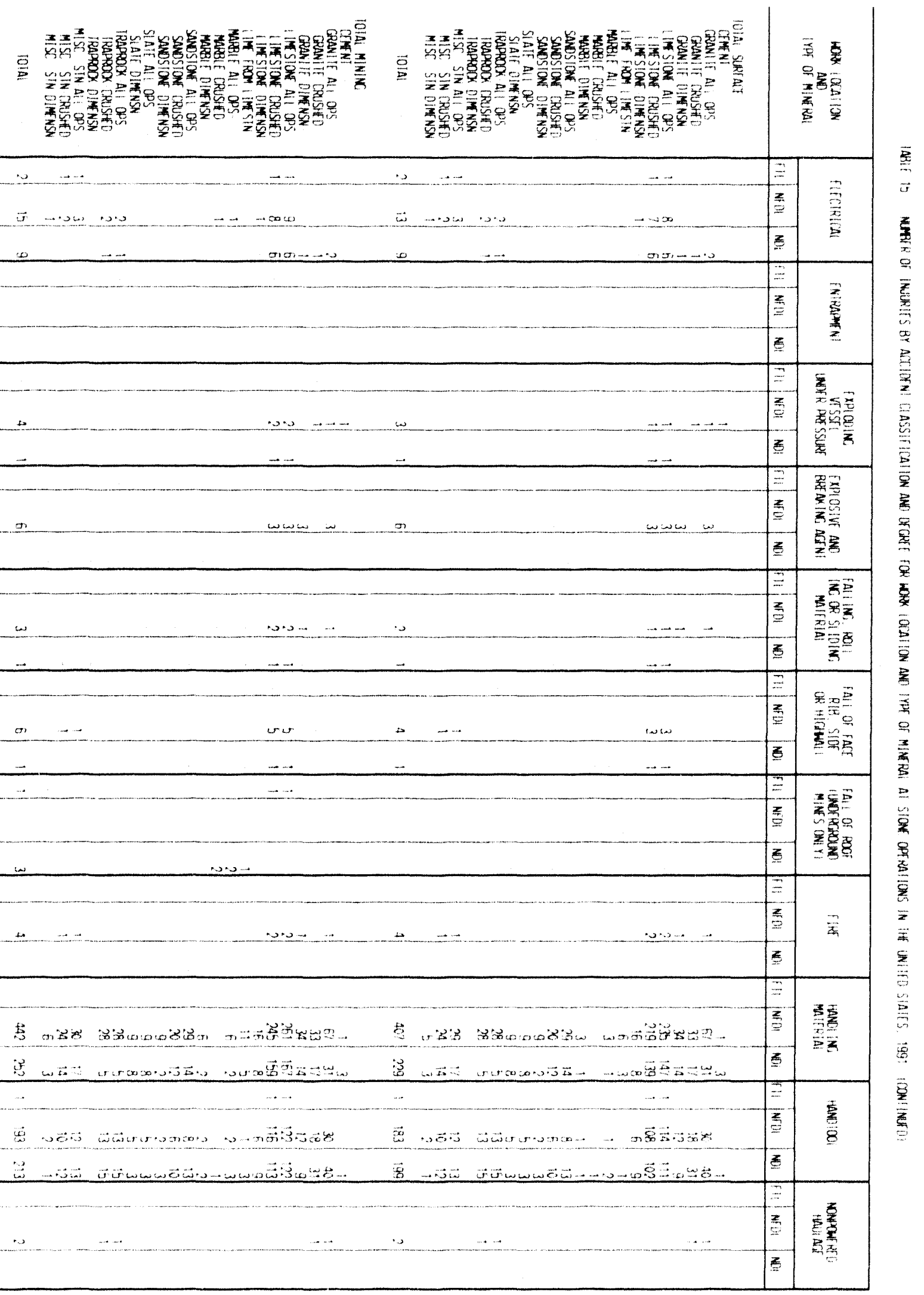



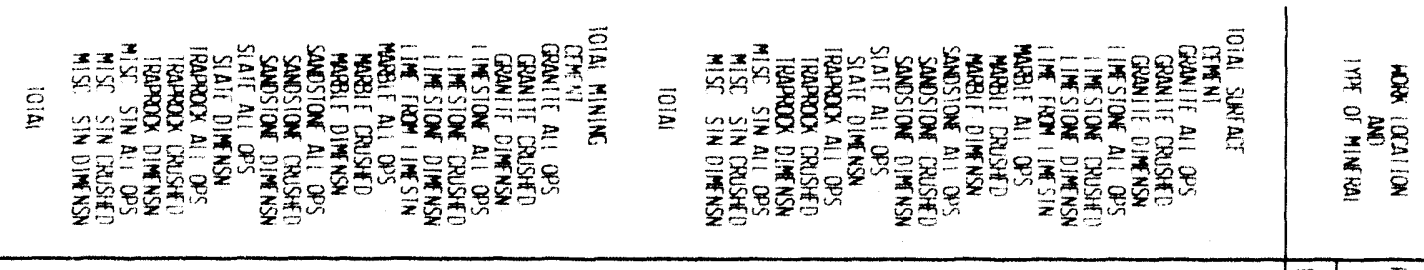

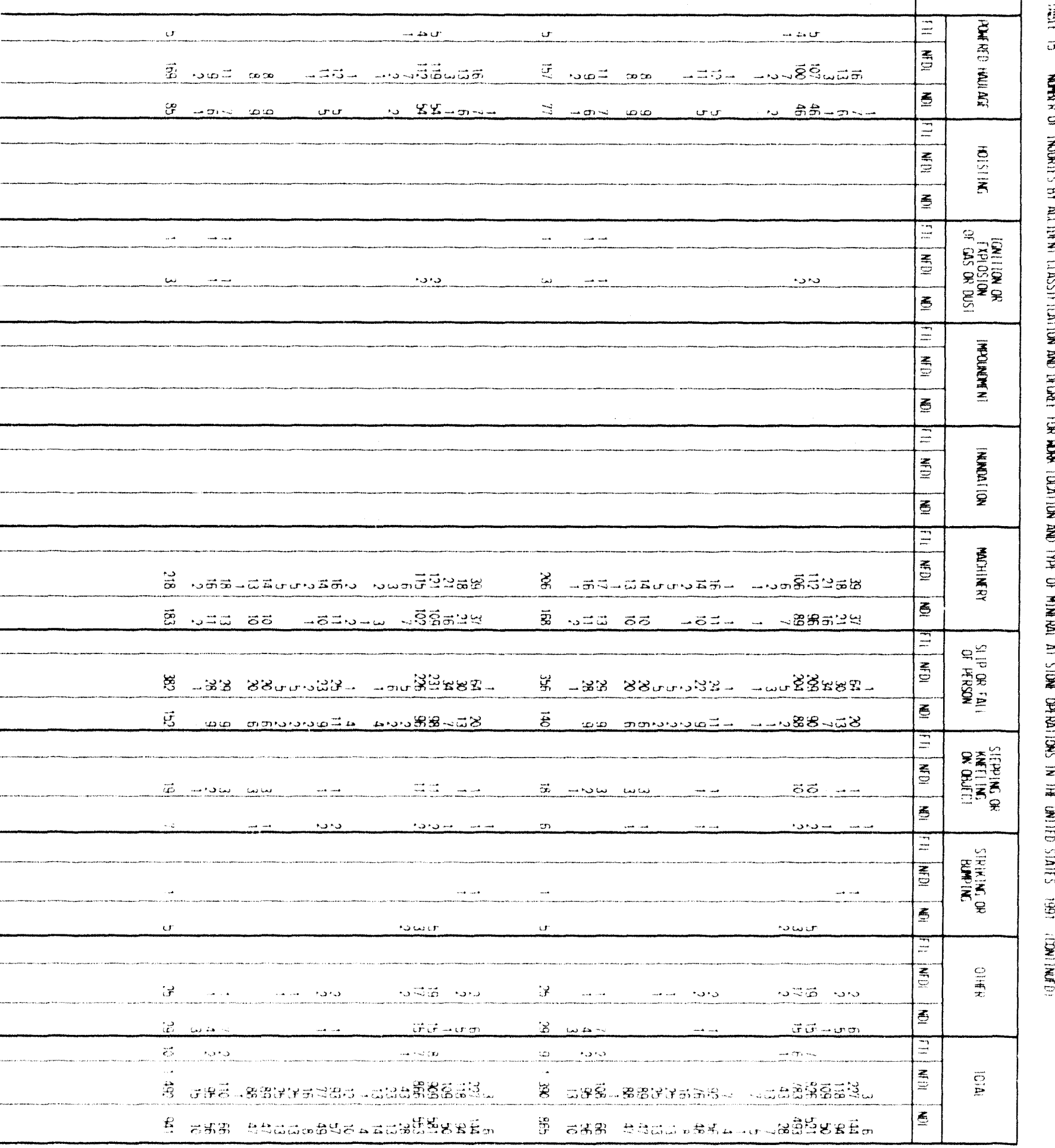




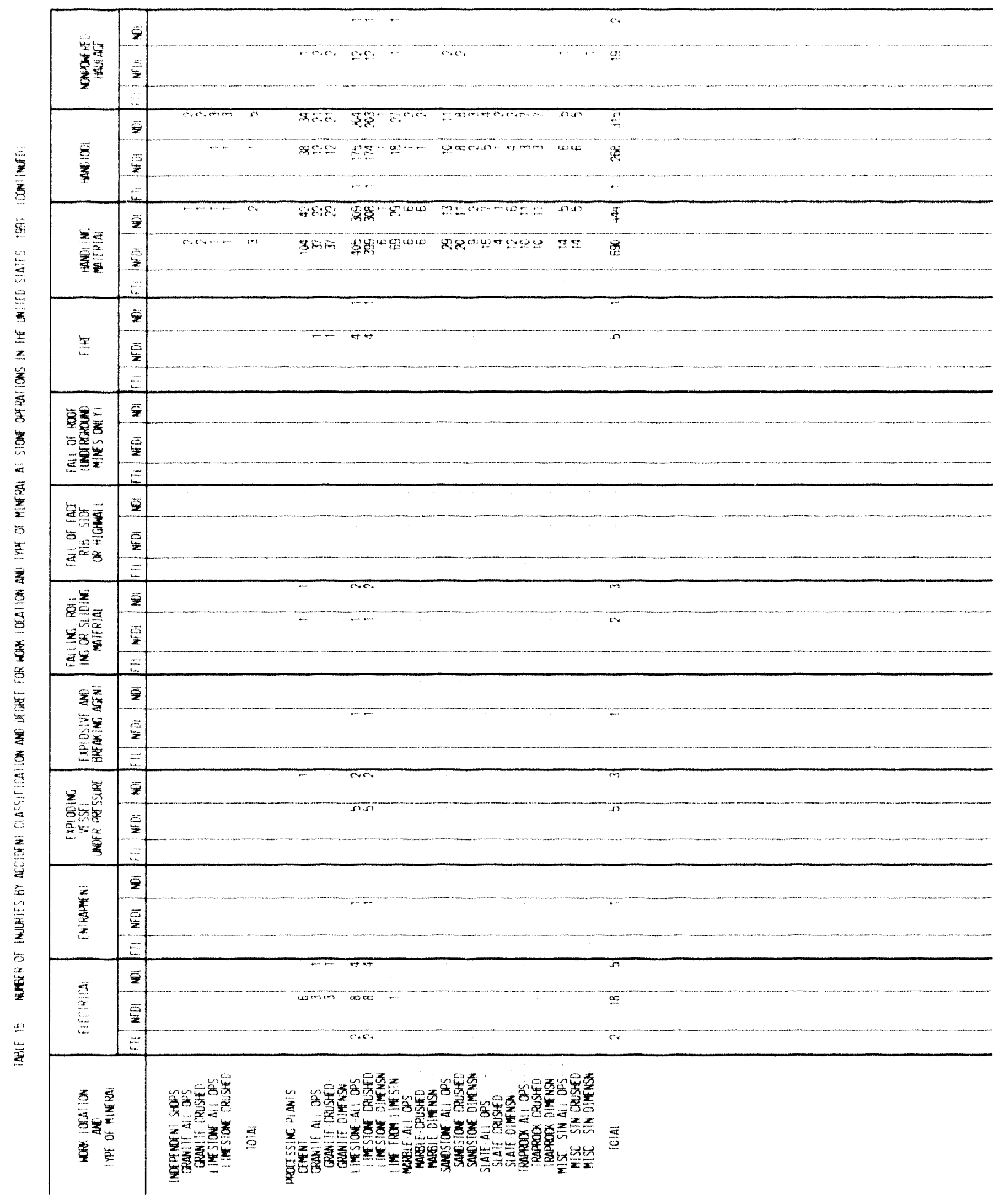




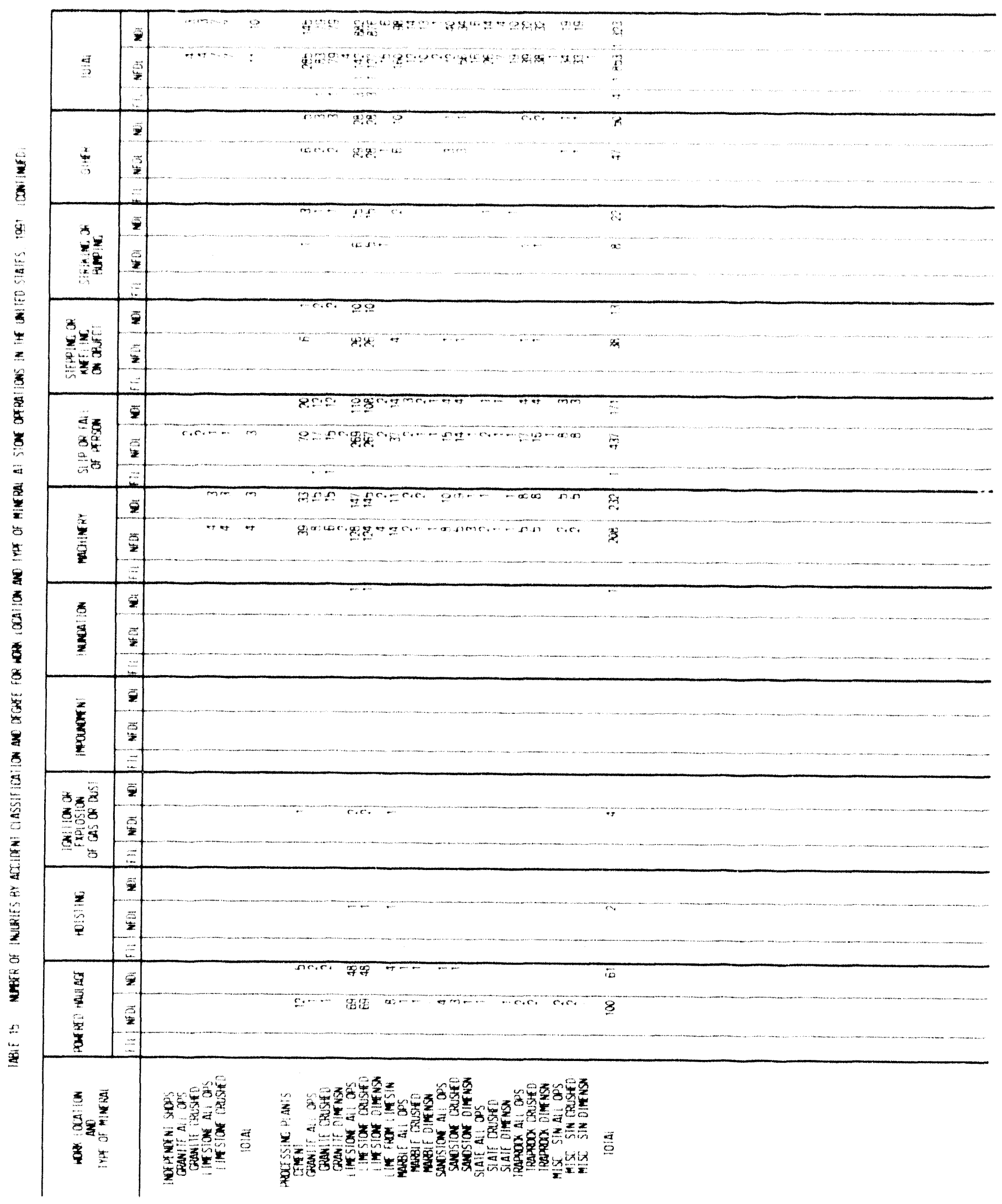




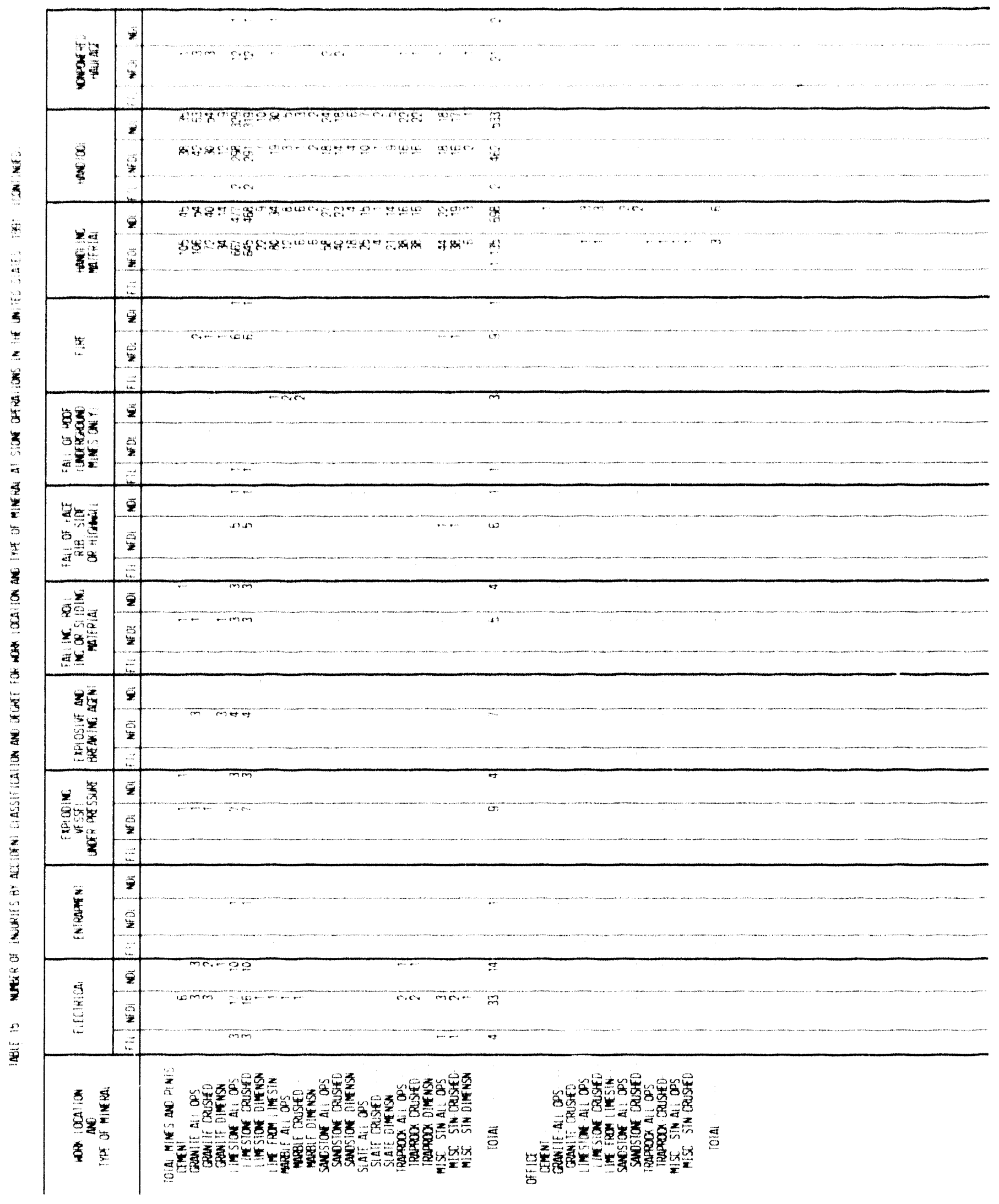




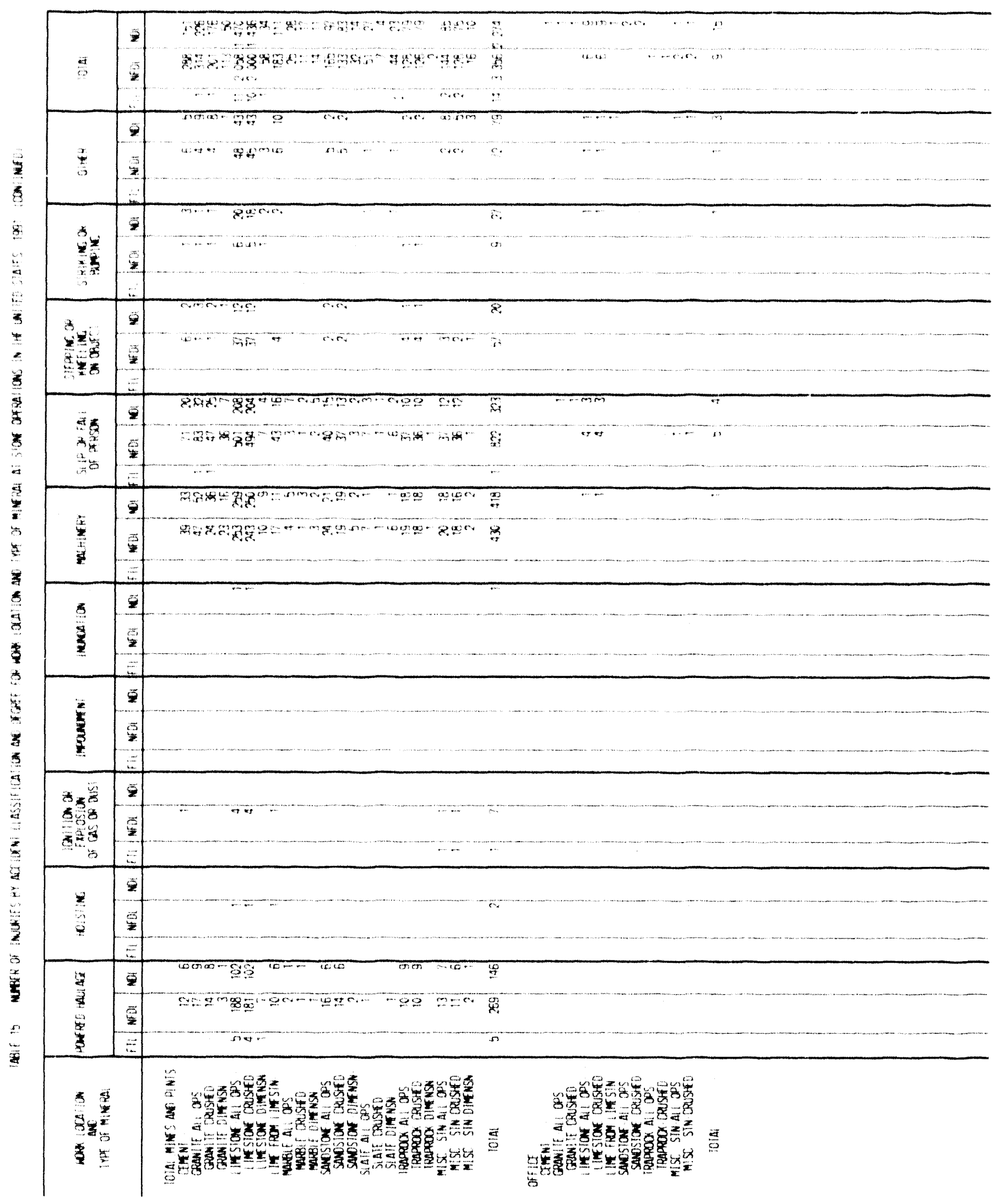




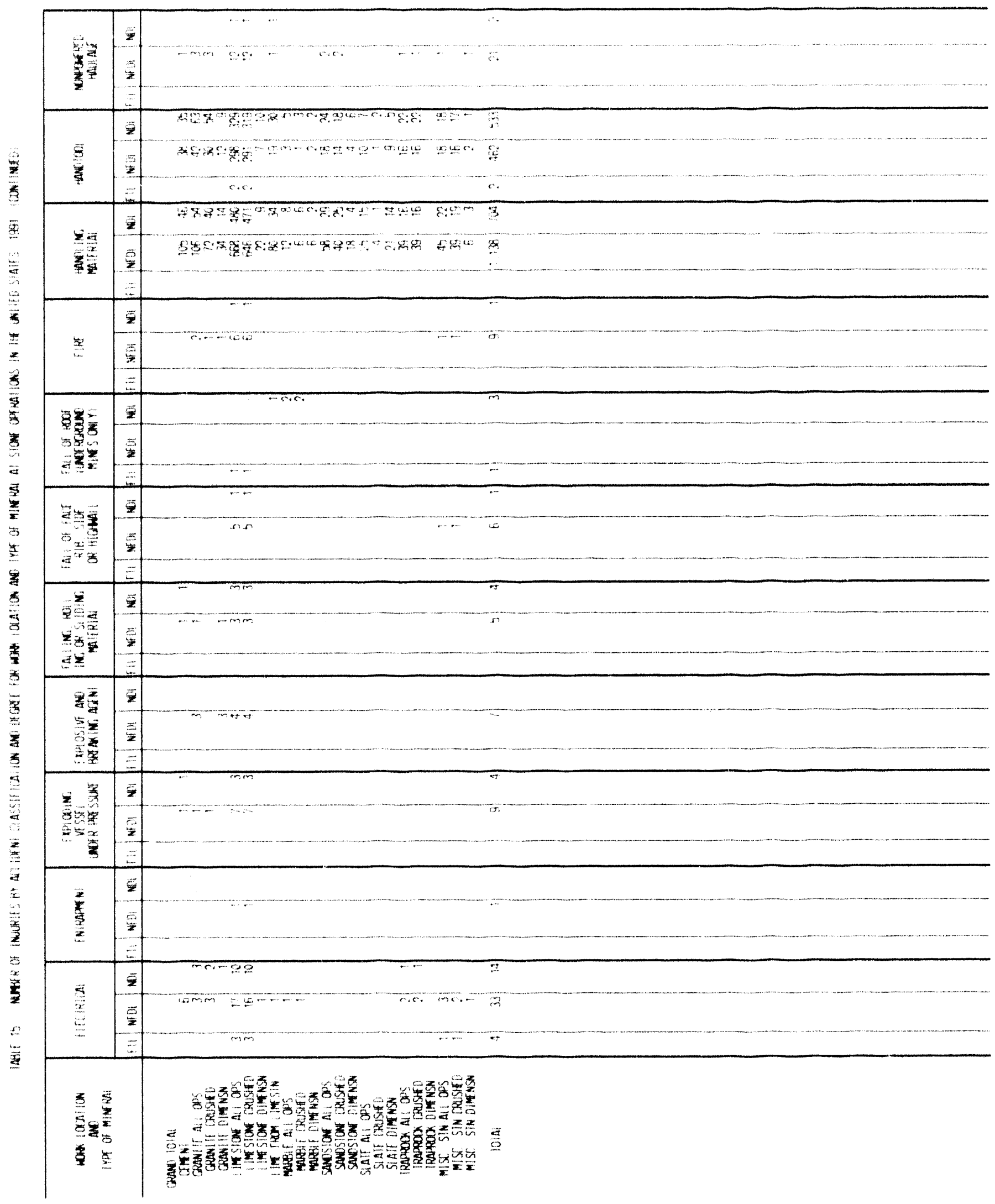




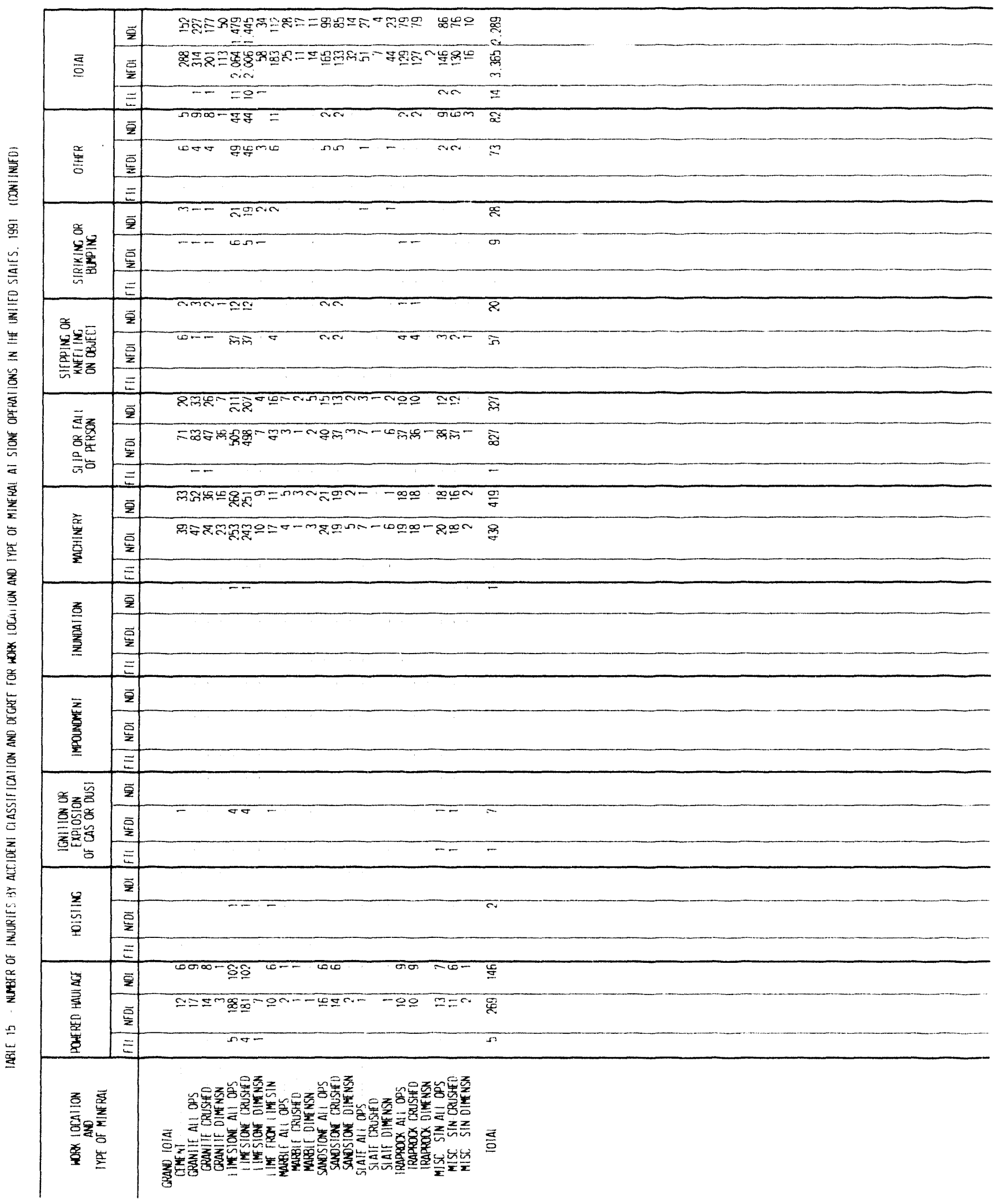




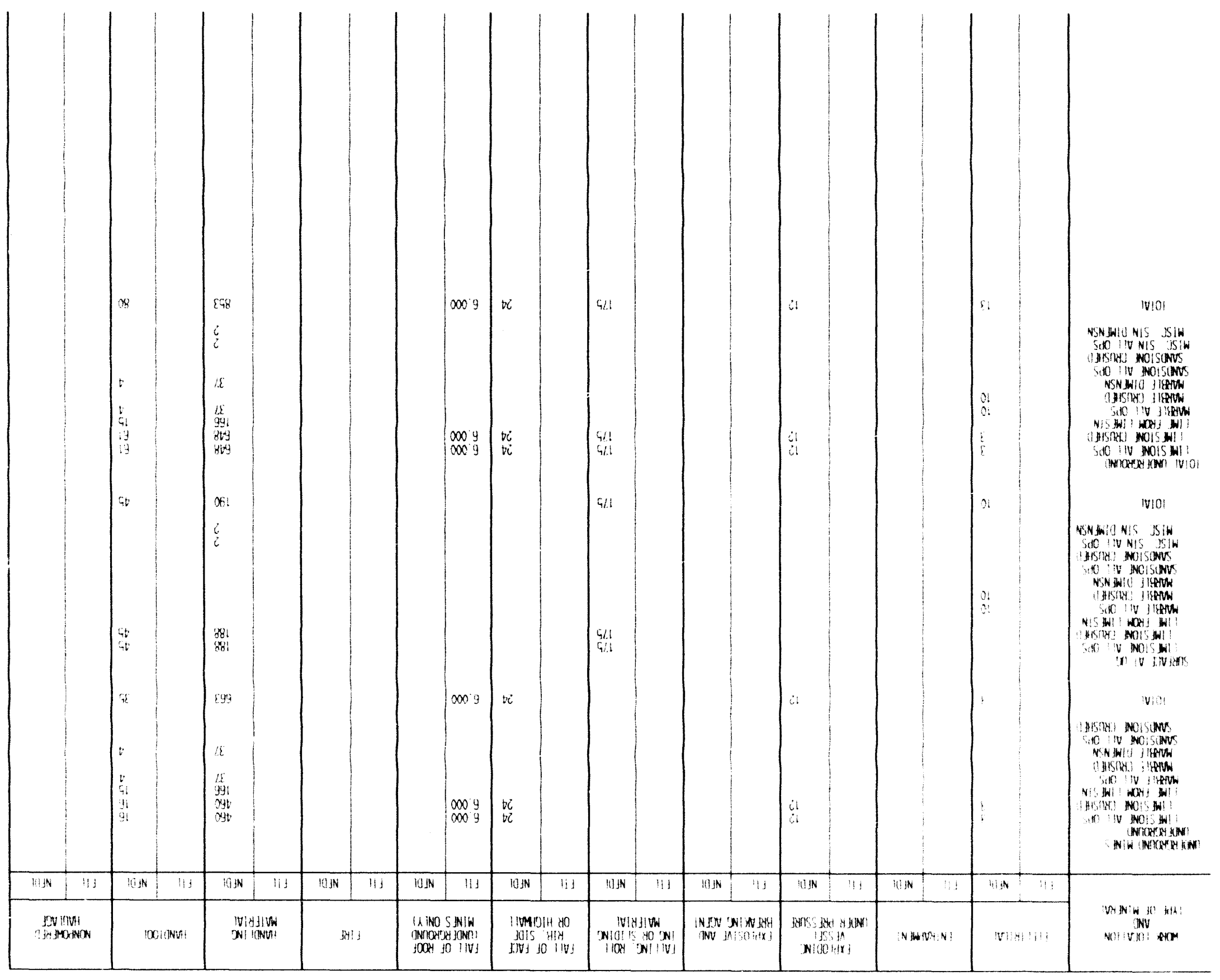

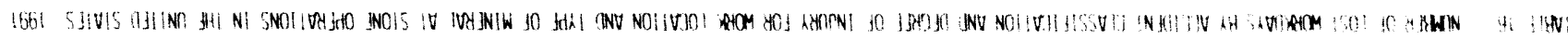




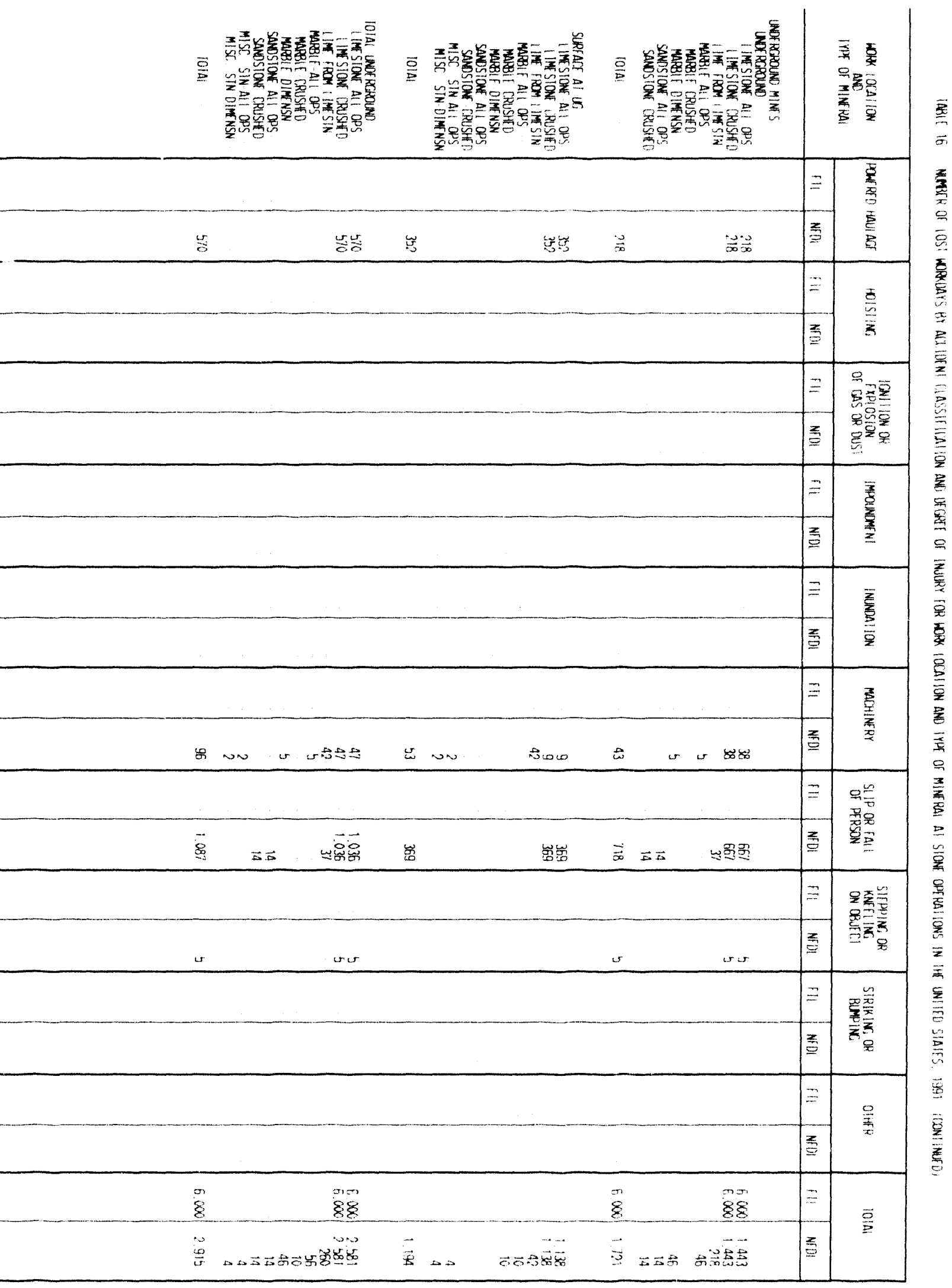




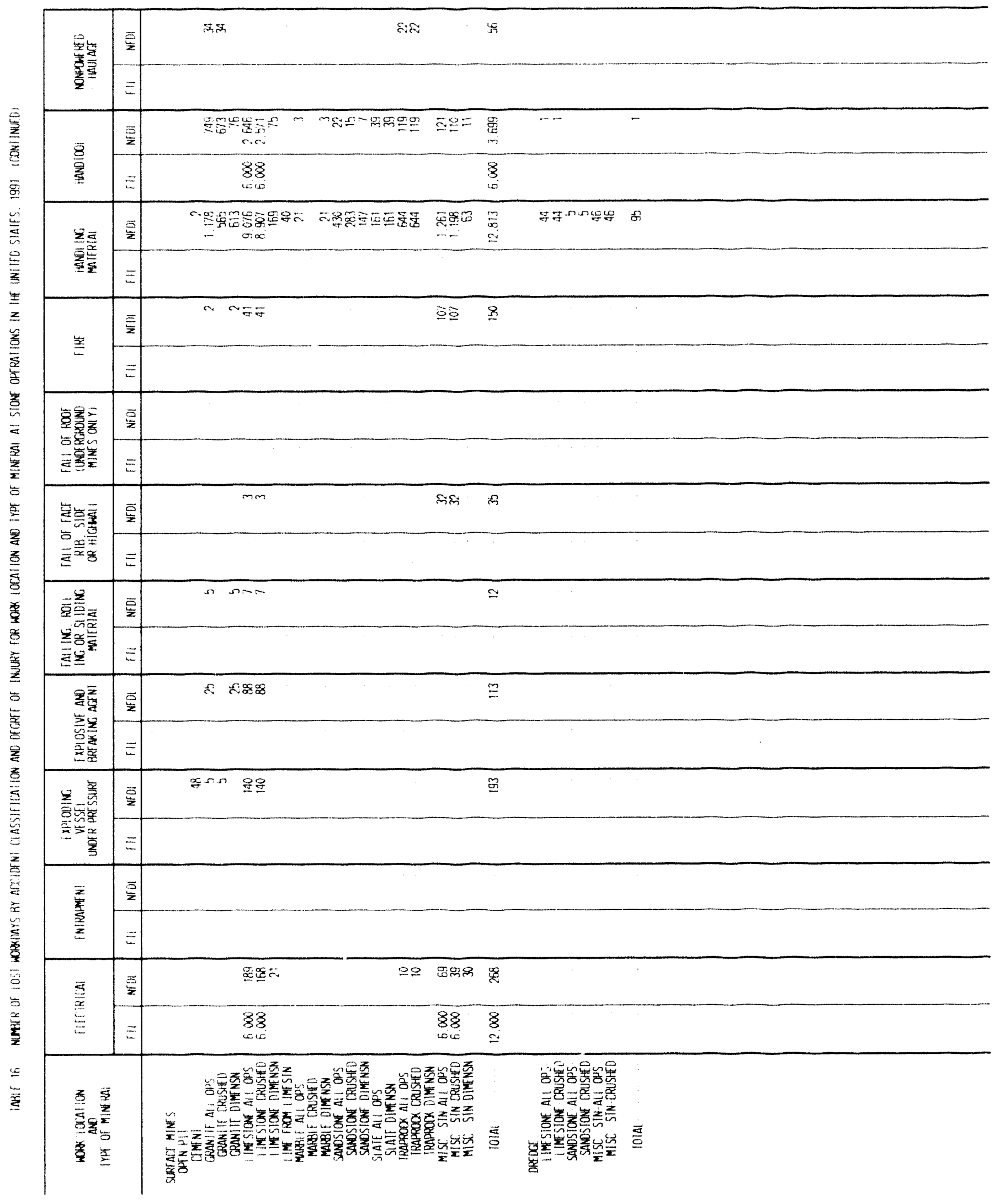




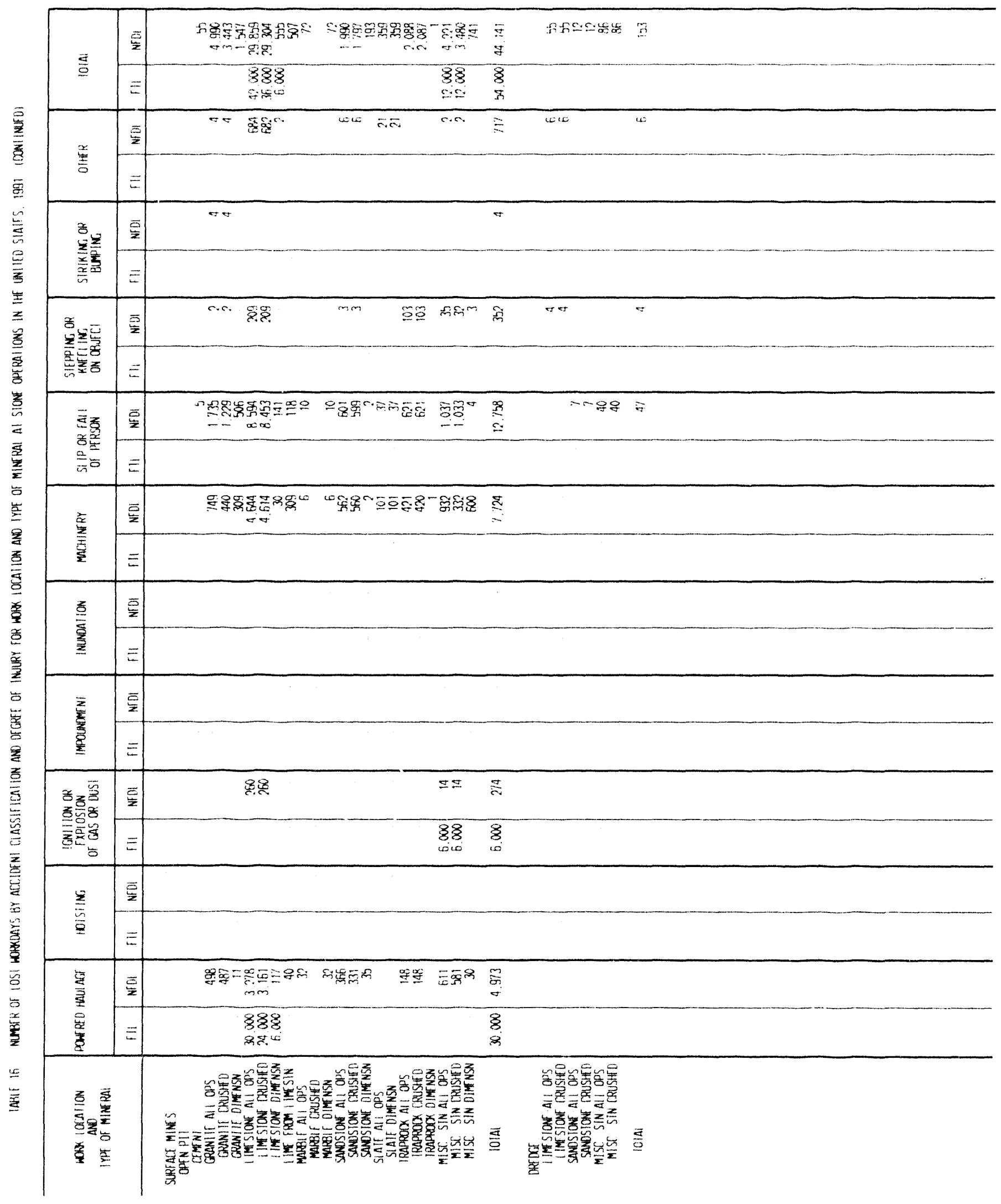




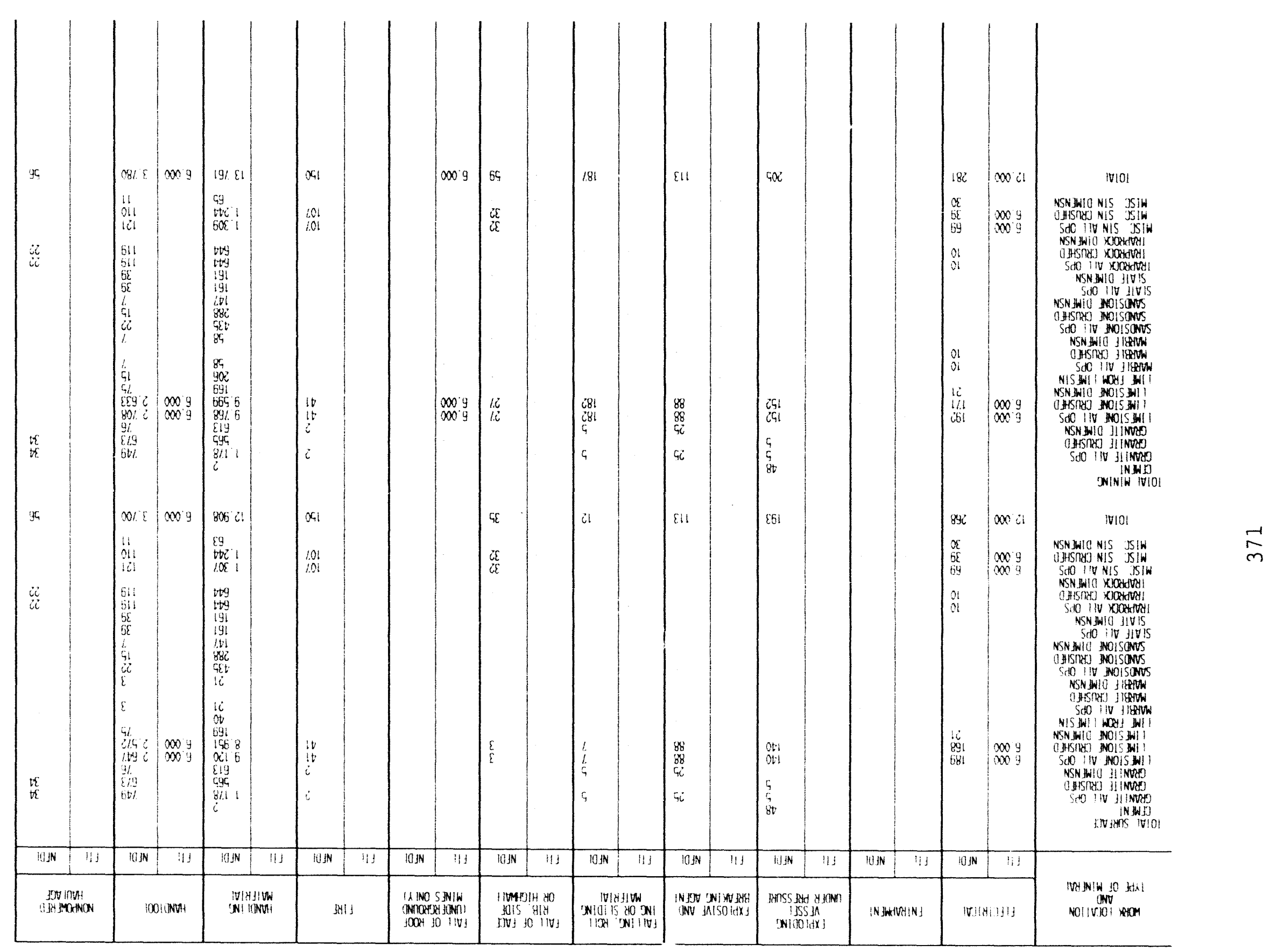

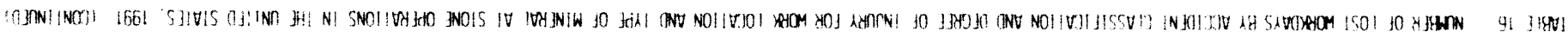




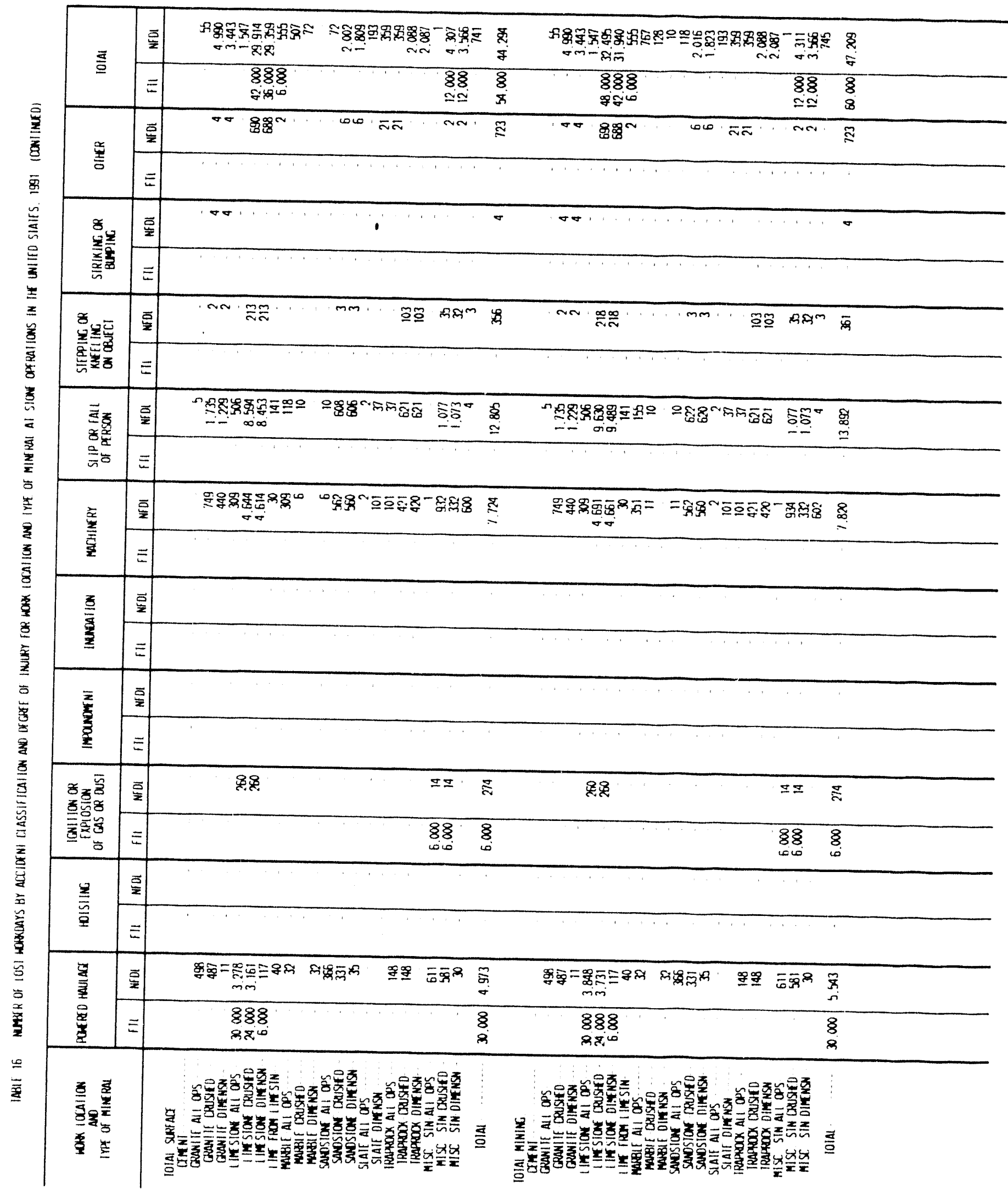




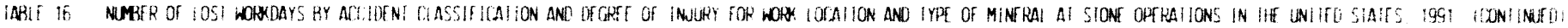

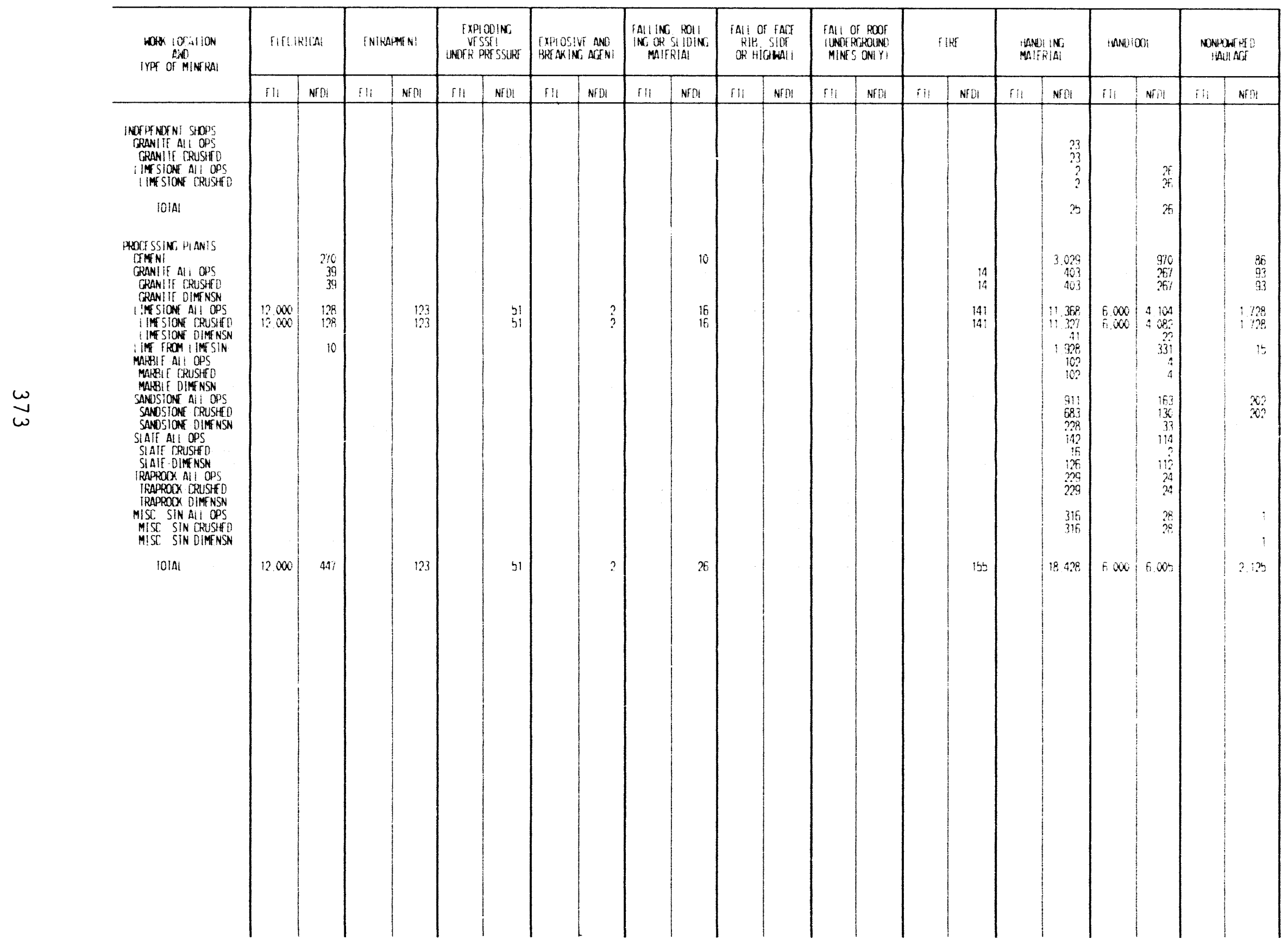




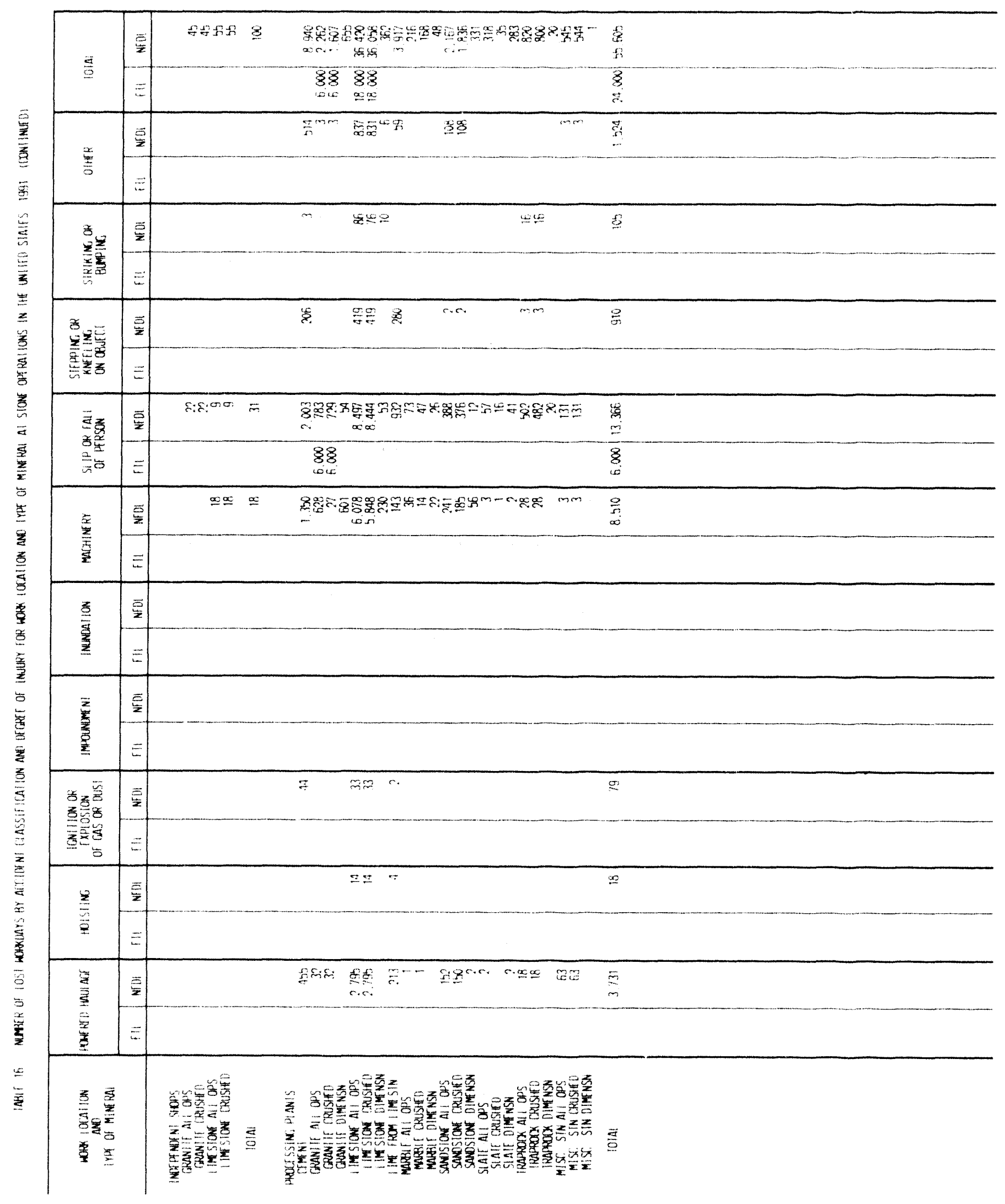




\begin{tabular}{|c|c|c|c|c|}
\hline \multirow{2}{*}{ 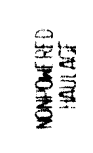 } & 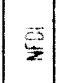 & 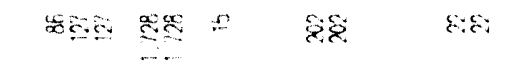 & $\cdots-$ & $\begin{array}{l}x \\
a \\
c\end{array}$ \\
\hline & $=$ & & & \\
\hline \multirow{2}{*}{$\frac{\overline{8}}{9}$} & 产 & 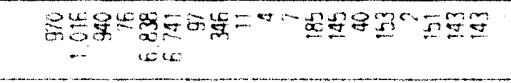 & 声器:= & $\underset{c}{\bar{c}}$ \\
\hline & 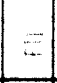 & $\begin{array}{c}38 \\
: 5 \\
\end{array}$ & & $\begin{array}{l}8 \\
8 \\
5\end{array}$ \\
\hline \multirow{2}{*}{ 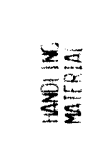 } & 至 & 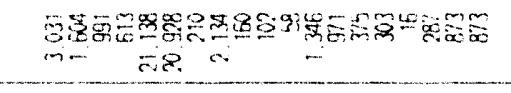 & $f_{0} \varepsilon_{0}$ & $\begin{array}{lll}D_{0} & n & \end{array}$ \\
\hline & $\equiv$ & & & \\
\hline \multirow{2}{*}{ 军 } & 政 & 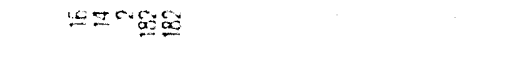 & 25 & 点 \\
\hline & $=$ & & & \\
\hline \multirow{2}{*}{ 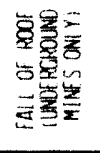 } & $\frac{\dot{\rho}}{2}$ & & & \\
\hline & $E$ & $\begin{array}{l}88 \\
100\end{array}$ & & $\begin{array}{l}8 \\
4\end{array}$ \\
\hline \multirow{2}{*}{ 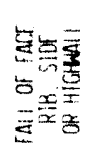 } & $\overline{\bar{s}}$ & $\bar{\pi}$ & Eहल & $g$ \\
\hline & $\equiv$ & & & \\
\hline \multirow{2}{*}{ 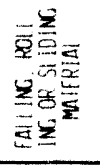 } & $\overline{\frac{5}{2}}$ & es sgg & & $\stackrel{m}{c}$ \\
\hline & $E$ & & & \\
\hline \multirow{2}{*}{ 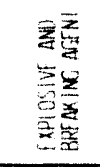 } & $\overline{\underline{z}}$ & f. 588 & & $\cong$ \\
\hline & $\Xi$ & & & \\
\hline \multirow{2}{*}{ 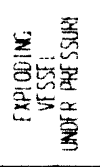 } & 斻 & पूan है. & & s. \\
\hline & $\equiv$ & & & \\
\hline \multirow{2}{*}{$\begin{array}{l}\text { 妾 } \\
\bar{z} \\
\bar{z}\end{array}$} & 할 & $\Xi$ & & $\cong$ \\
\hline & $E$ & & & \\
\hline \multirow{2}{*}{$\begin{array}{l}\stackrel{\Xi}{\underline{x}} \\
\underline{E}\end{array}$} & 产 & 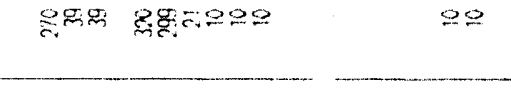 & 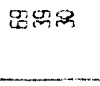 & $\stackrel{x}{\stackrel{x}{:}}$ \\
\hline & $\equiv$ & $\begin{array}{l}88 \\
08 \\
0\end{array}$ & $\begin{array}{l}88 \\
0.060\end{array}$ & $\frac{9}{8}$ \\
\hline 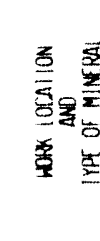 & & 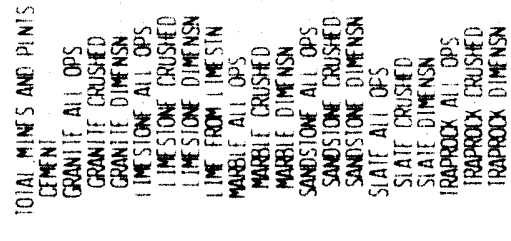 & 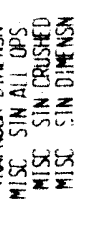 & 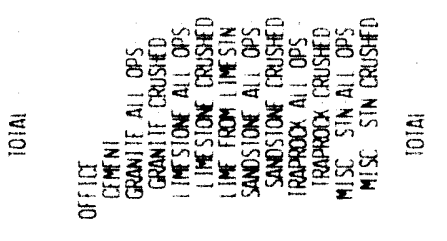 \\
\hline
\end{tabular}




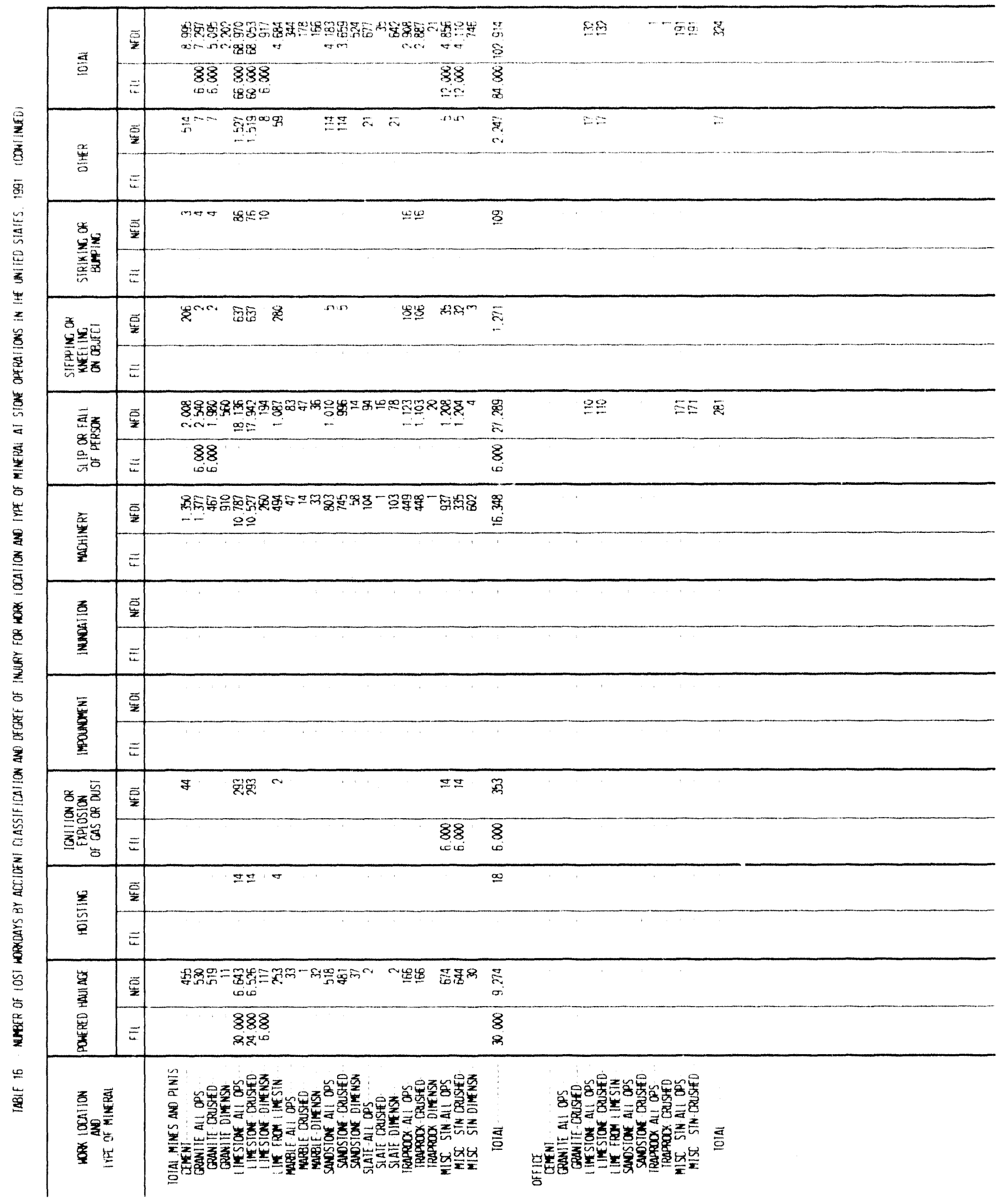




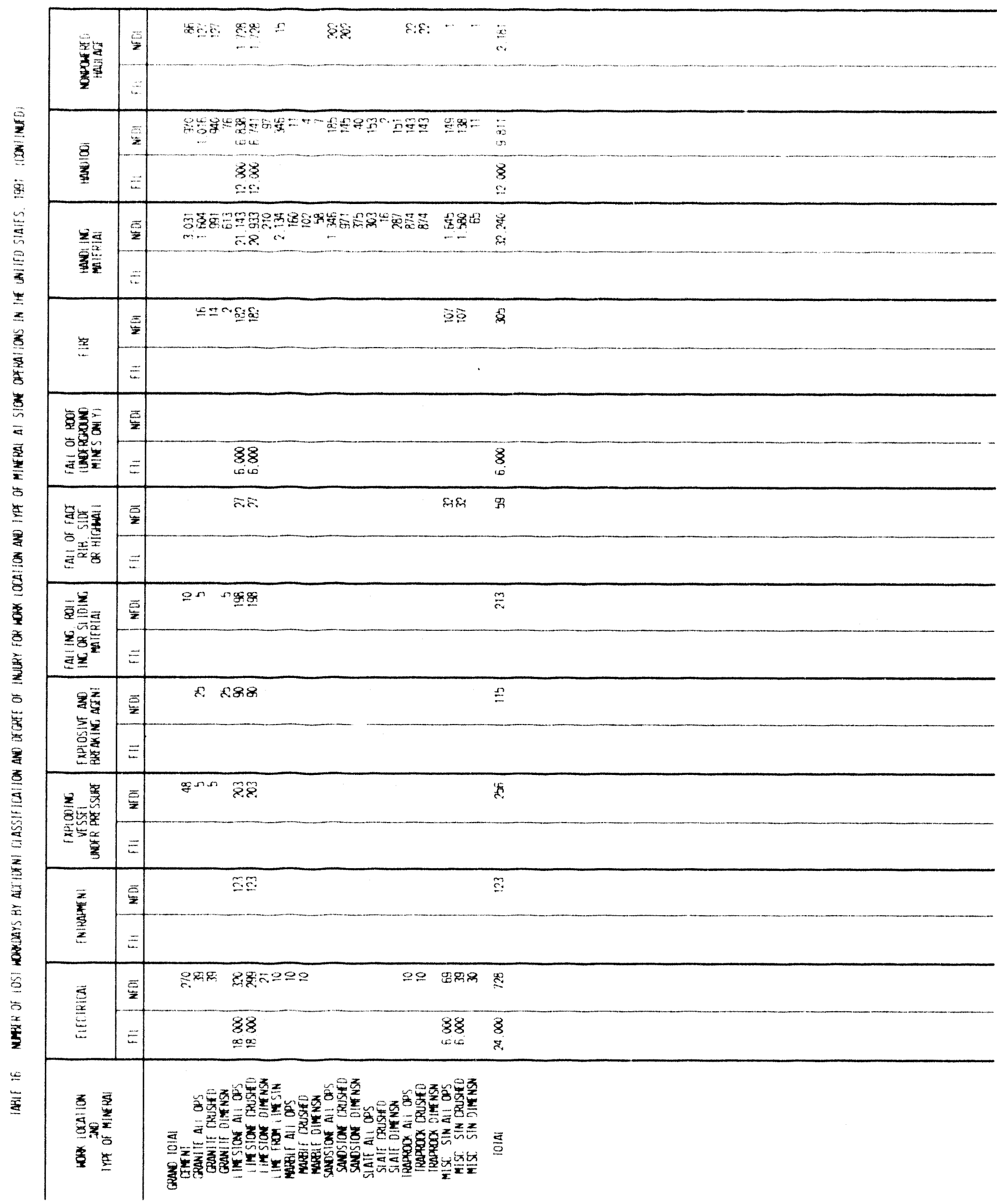




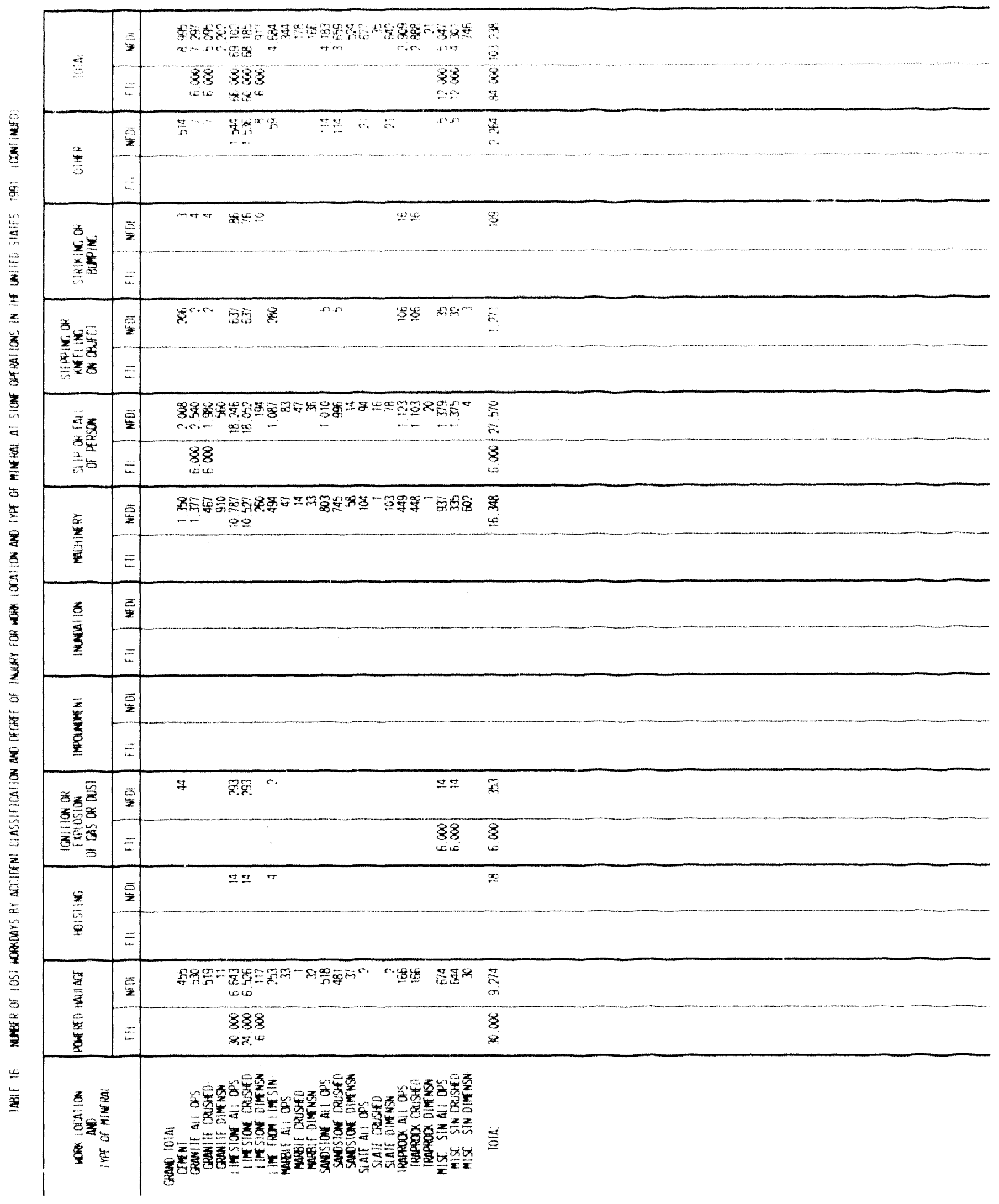




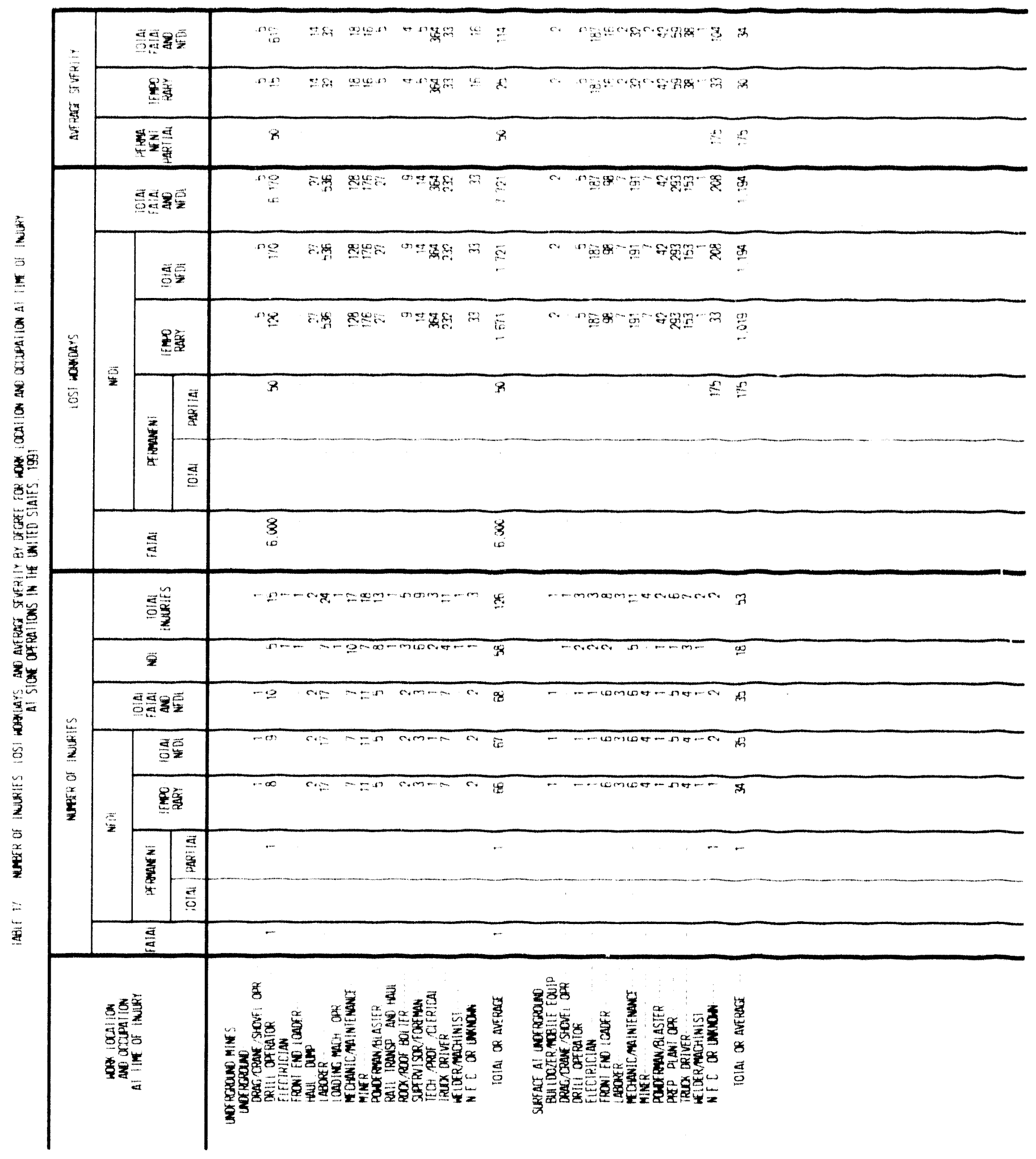



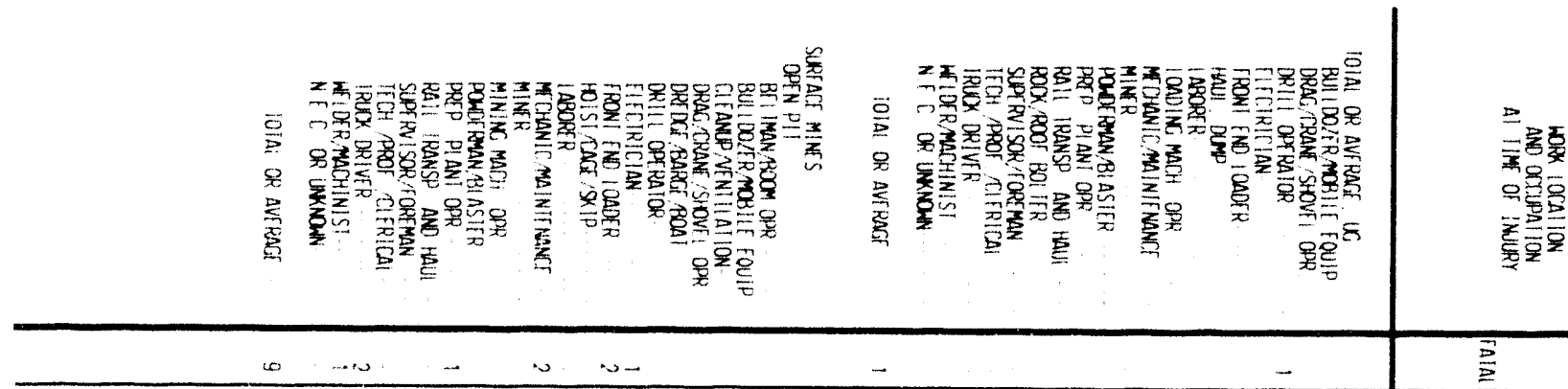

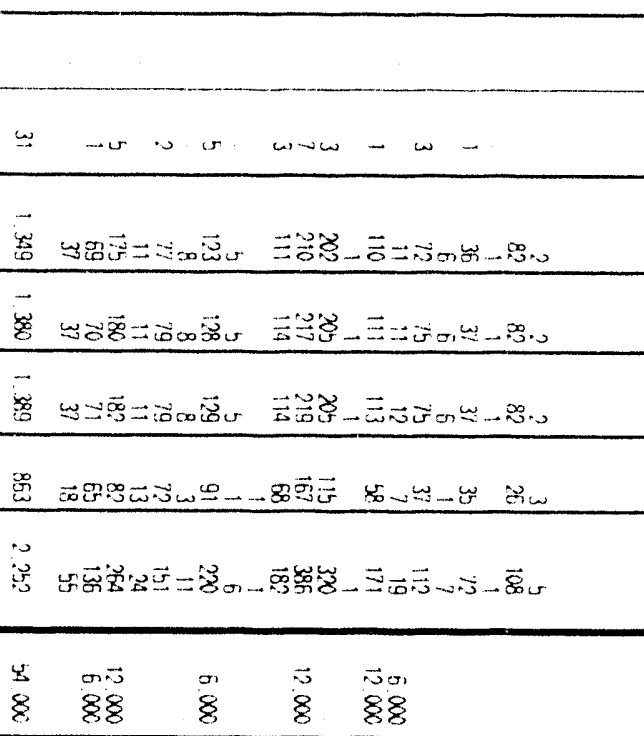

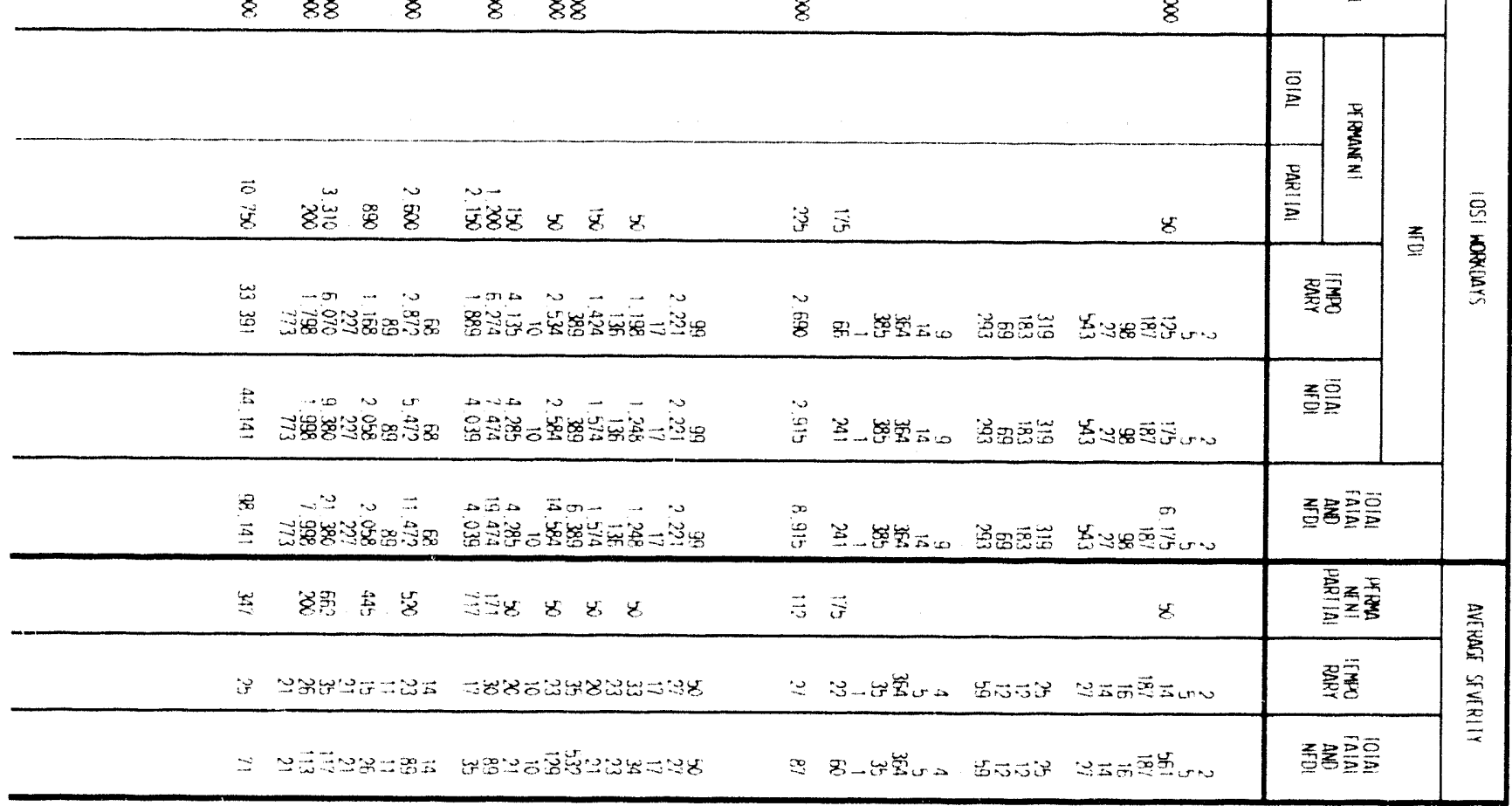




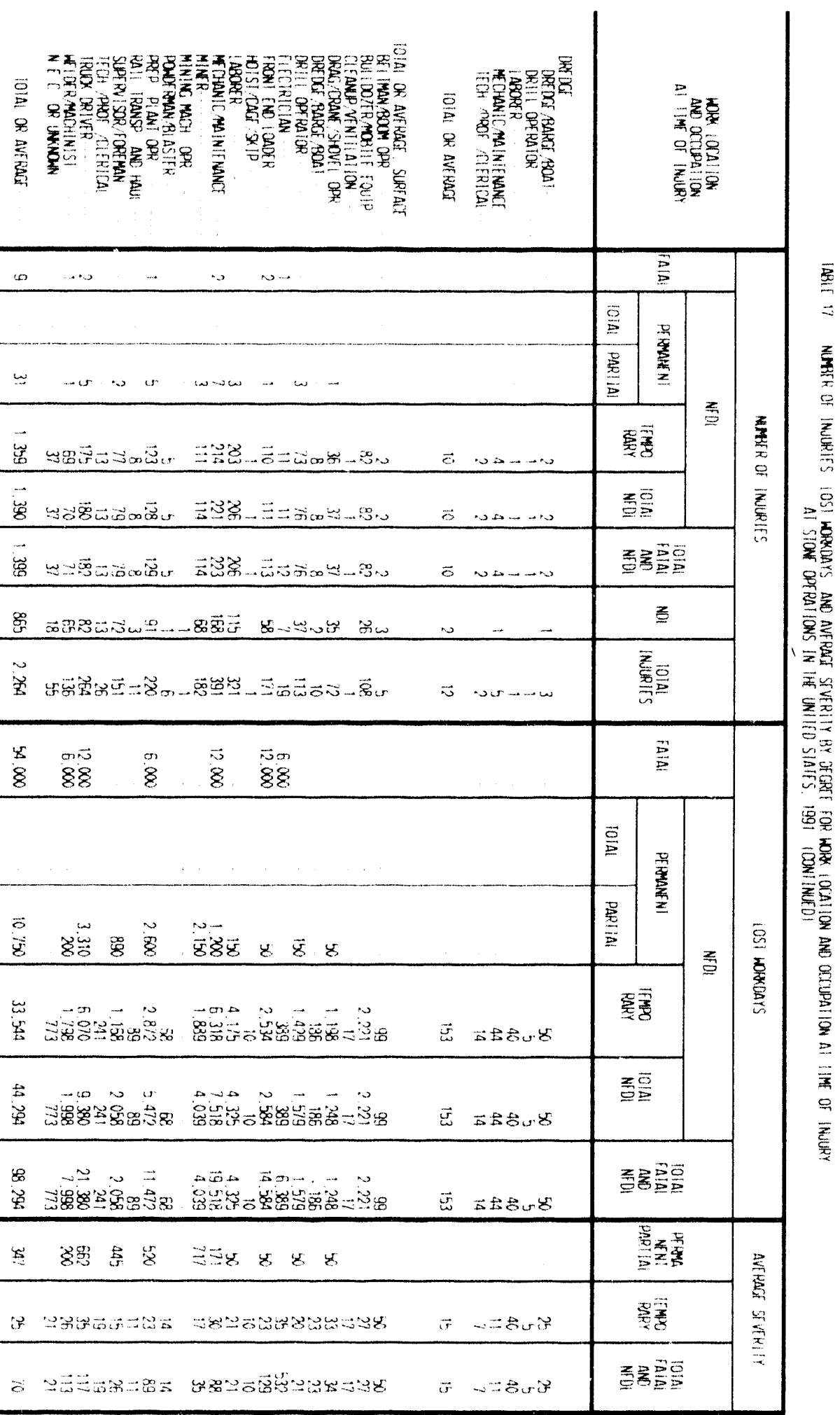




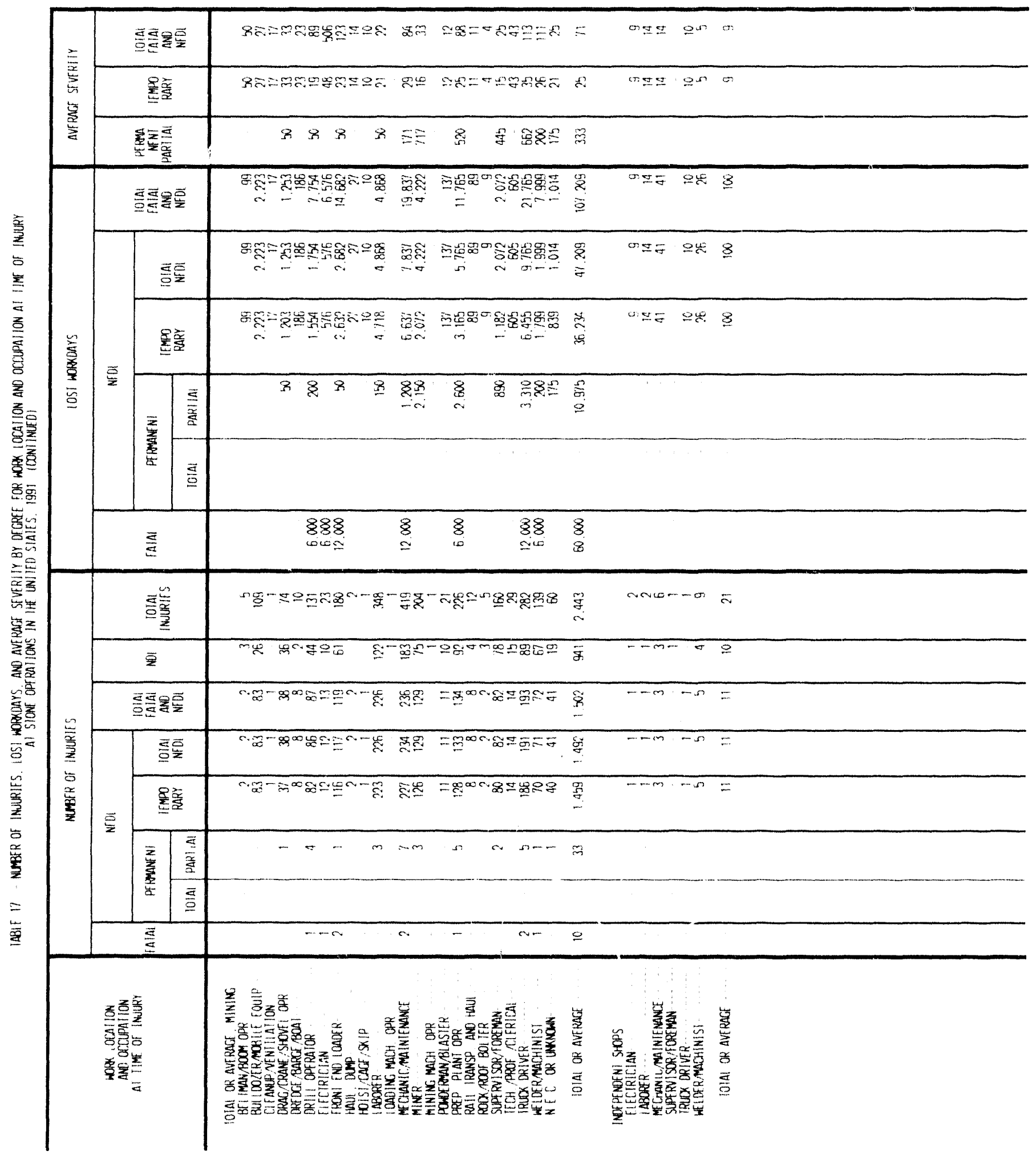




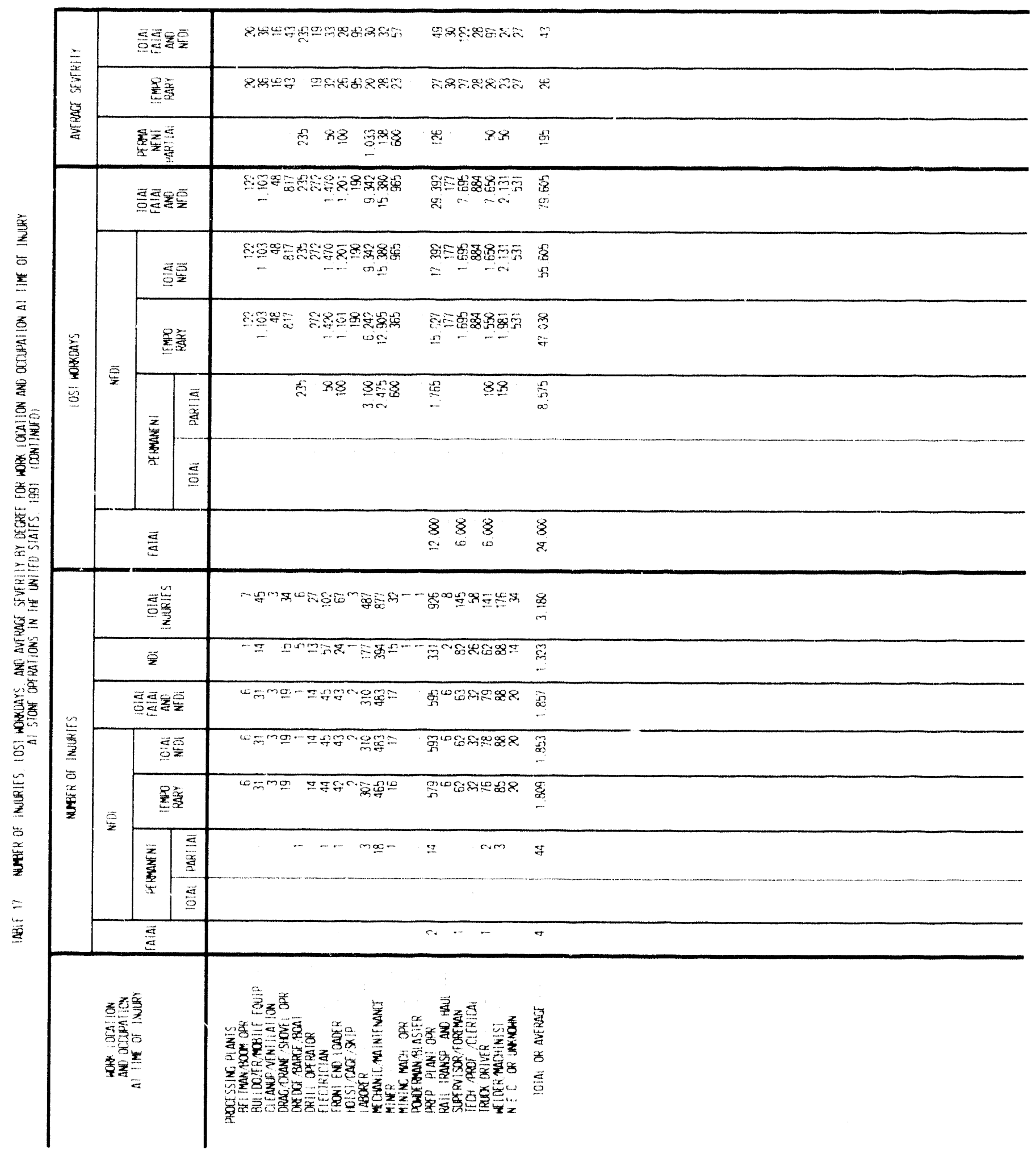




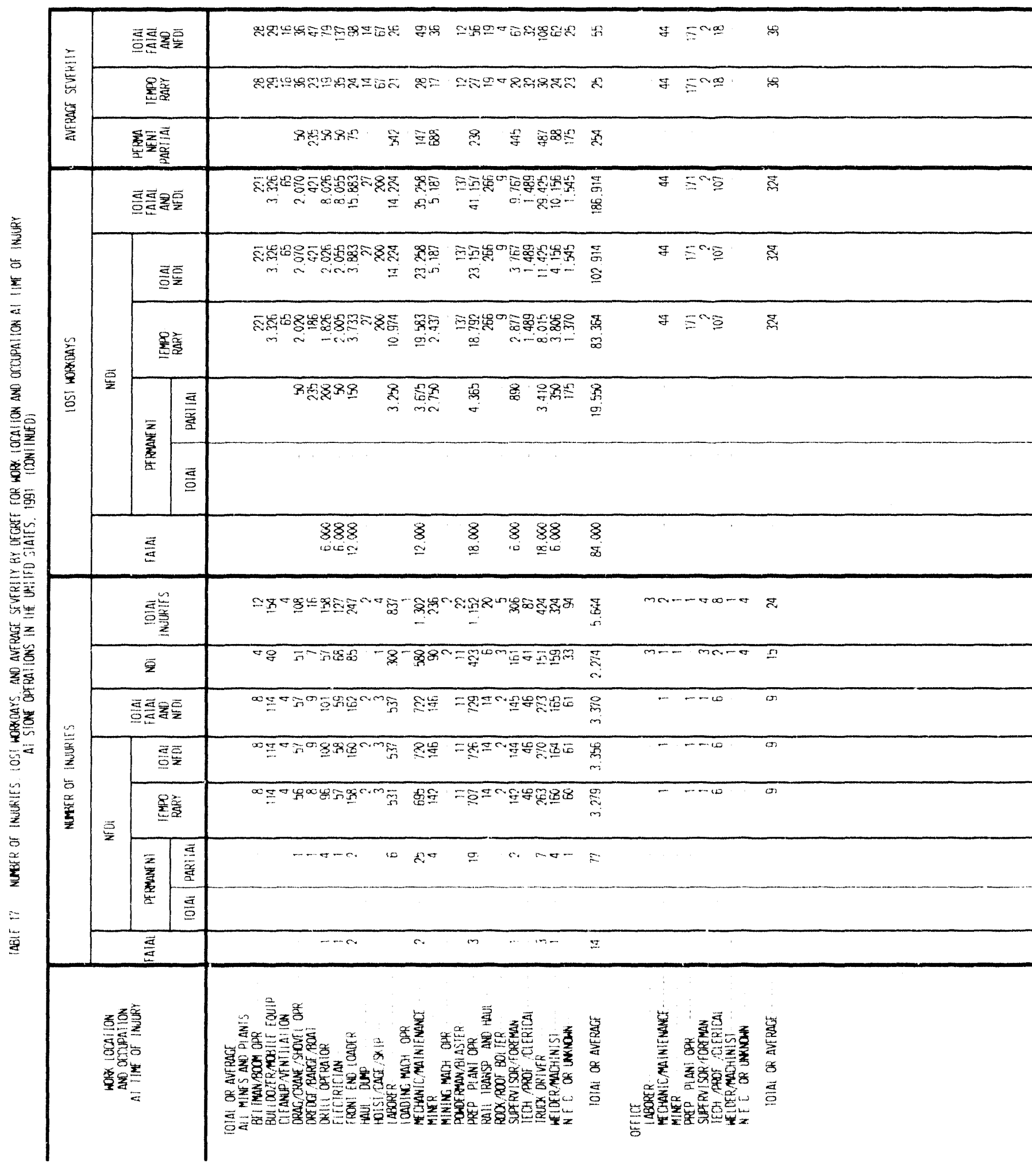




\begin{tabular}{|c|c|c|c|c|}
\hline \multirow{3}{*}{ 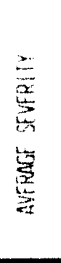 } & \multicolumn{2}{|c|}{ 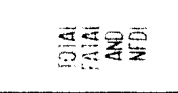 } & 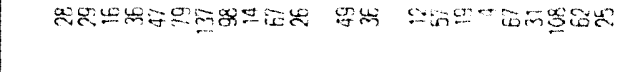 & $f$ \\
\hline & \multicolumn{2}{|c|}{ 倳 } & 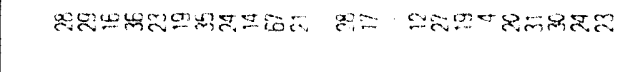 & \\
\hline & \multicolumn{2}{|c|}{ 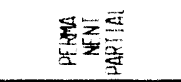 } & 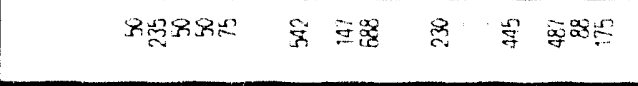 & 续 \\
\hline \multirow{6}{*}{ 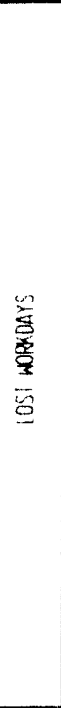 } & \multicolumn{2}{|c|}{ 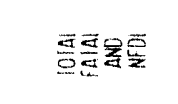 } & 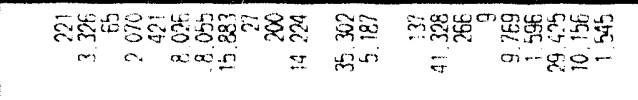 & te \\
\hline & \multicolumn{2}{|c|}{ 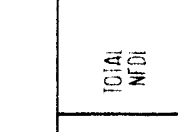 } & 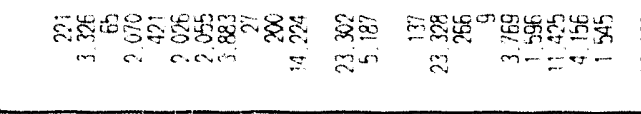 & 这 \\
\hline & \multicolumn{2}{|c|}{ 薏变 } & 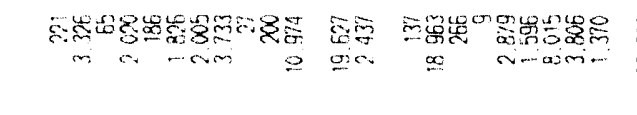 & 8 \\
\hline & \multirow{2}{*}{$\overline{\bar{I}}$} & \multirow{2}{*}{ 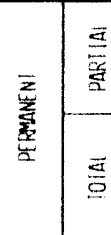 } & 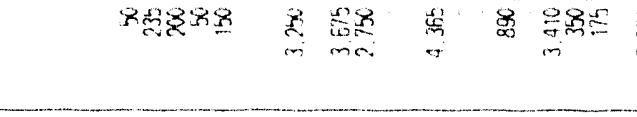 & \\
\hline & & & & \\
\hline & \multicolumn{2}{|l|}{$\stackrel{\ddot{z}}{E}$} & 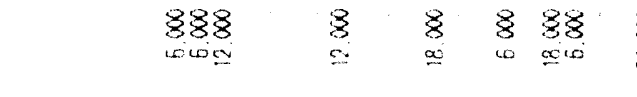 & 草 \\
\hline \multirow{8}{*}{ 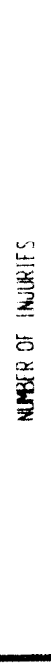 } & \multicolumn{2}{|c|}{ 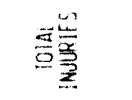 } & 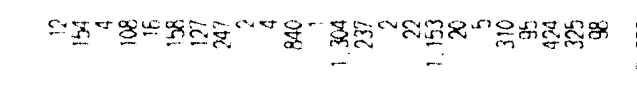 & to \\
\hline & \multicolumn{2}{|l|}{$\overrightarrow{\mathrm{z}}$} & 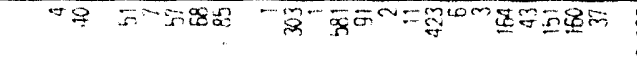 & Sa \\
\hline & \multicolumn{2}{|c|}{ 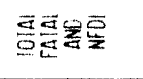 } & 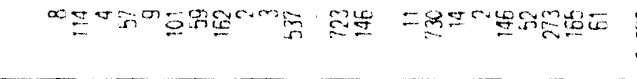 & 5 \\
\hline & \multirow{3}{*}{ 产 } & इ5 & 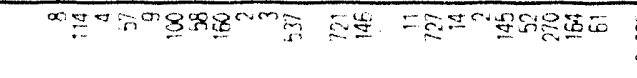 & s. \\
\hline & & 笘童 & 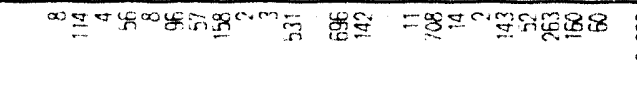 & 8 \\
\hline & & 焉 & 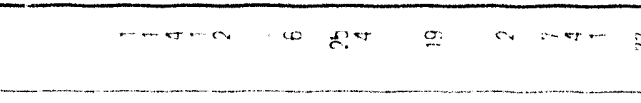 & $=$ \\
\hline & & $\mid \frac{1}{2}$ & & \\
\hline & \multicolumn{2}{|l|}{ 吾 } & $m-$ & 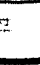 \\
\hline & 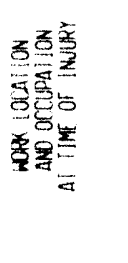 & & 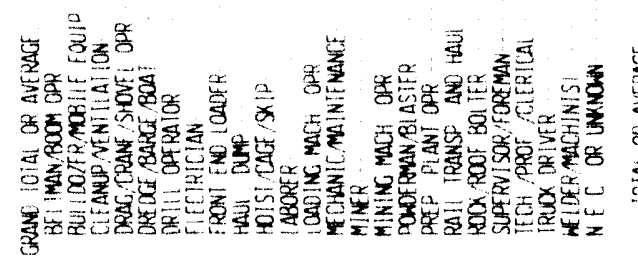 & $\begin{array}{l}\frac{5}{5} \\
\frac{5}{5} \\
\text { है } \\
\frac{5}{5}\end{array}$ \\
\hline
\end{tabular}



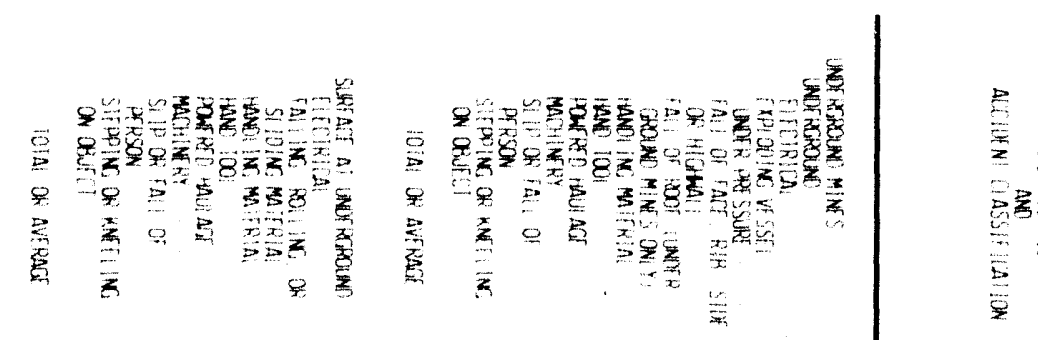

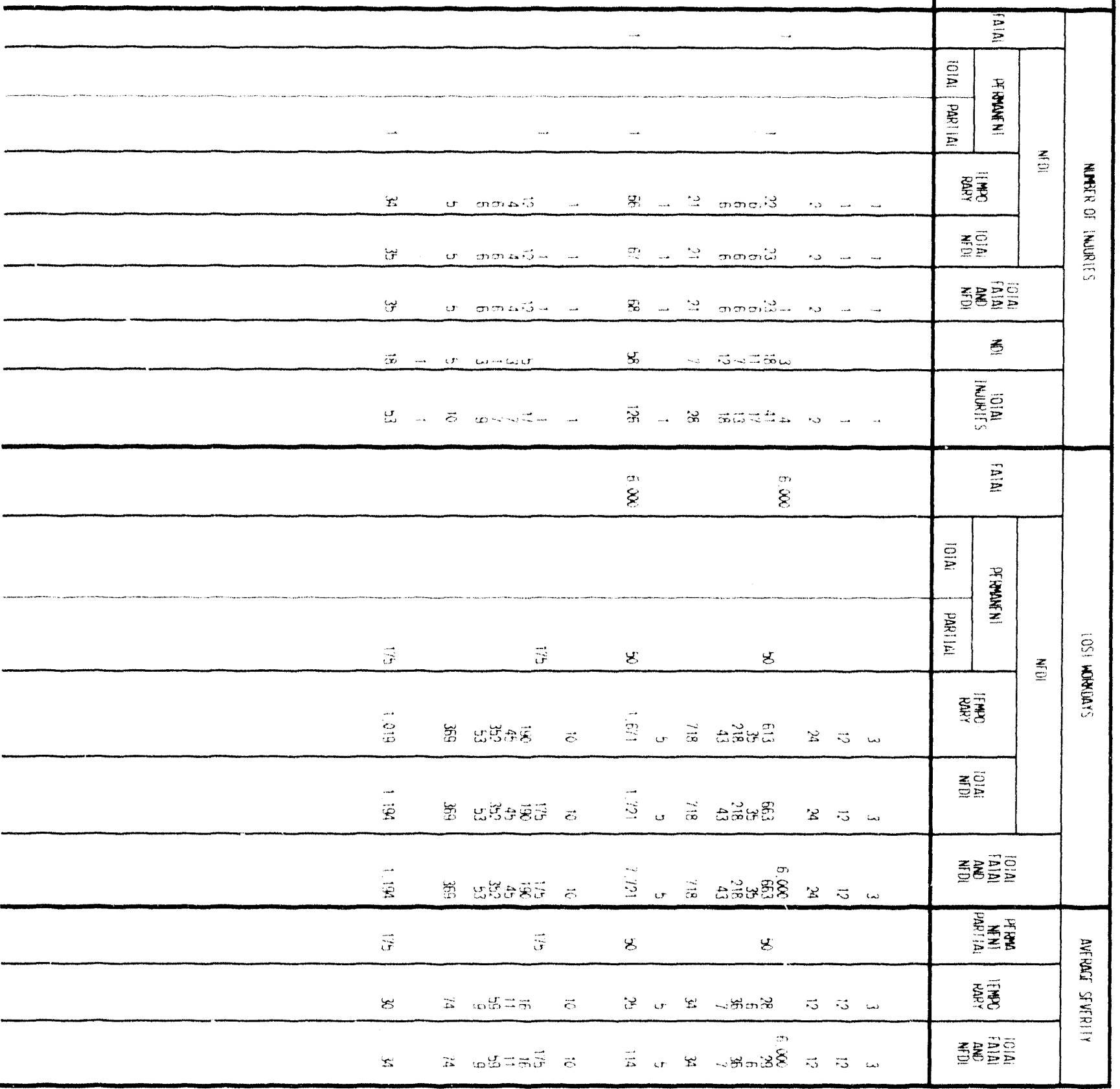



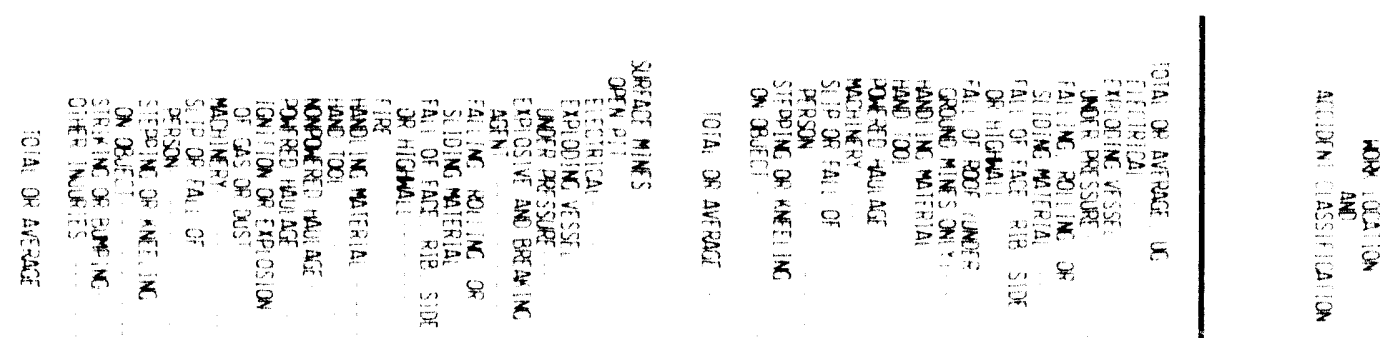

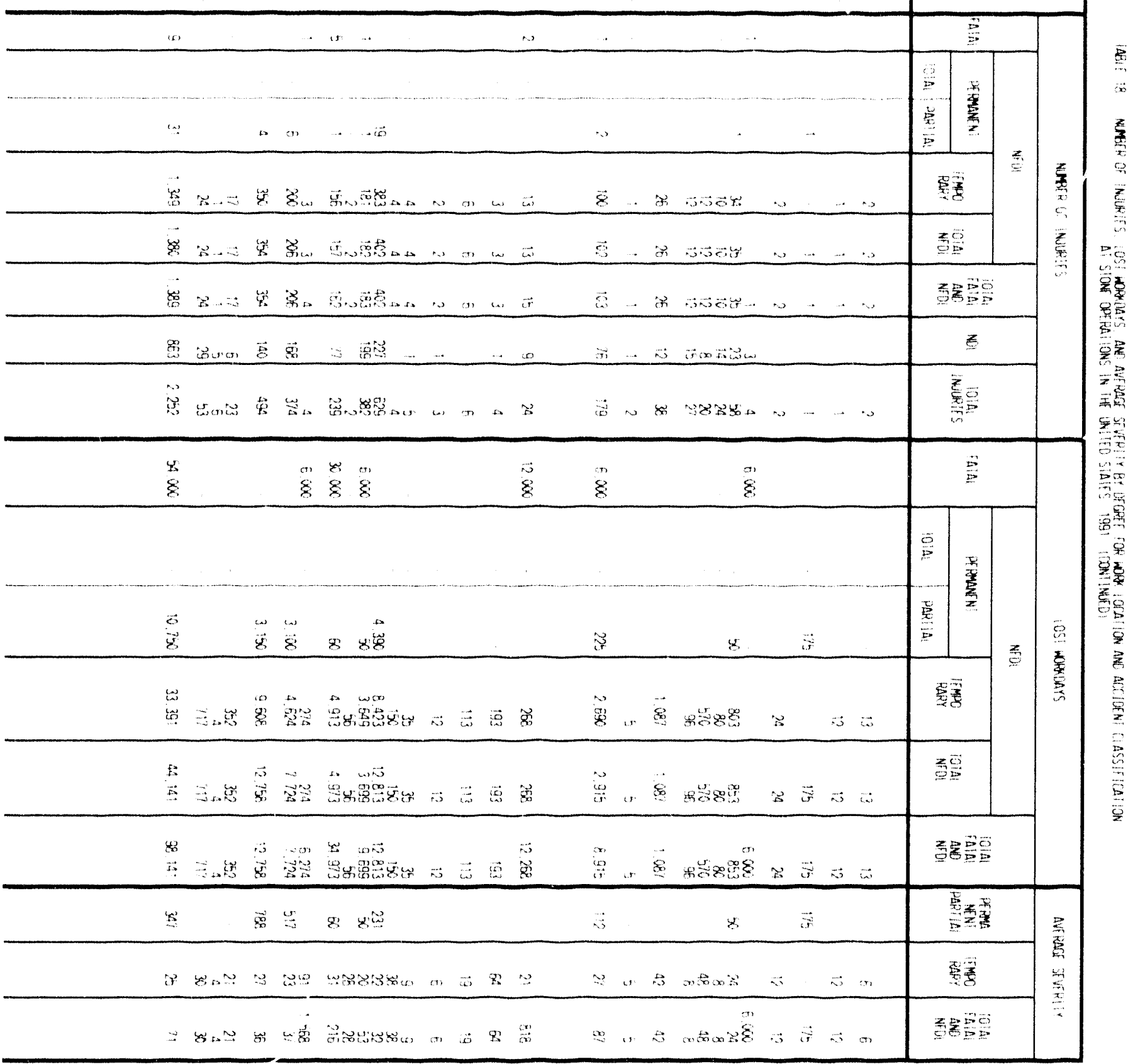




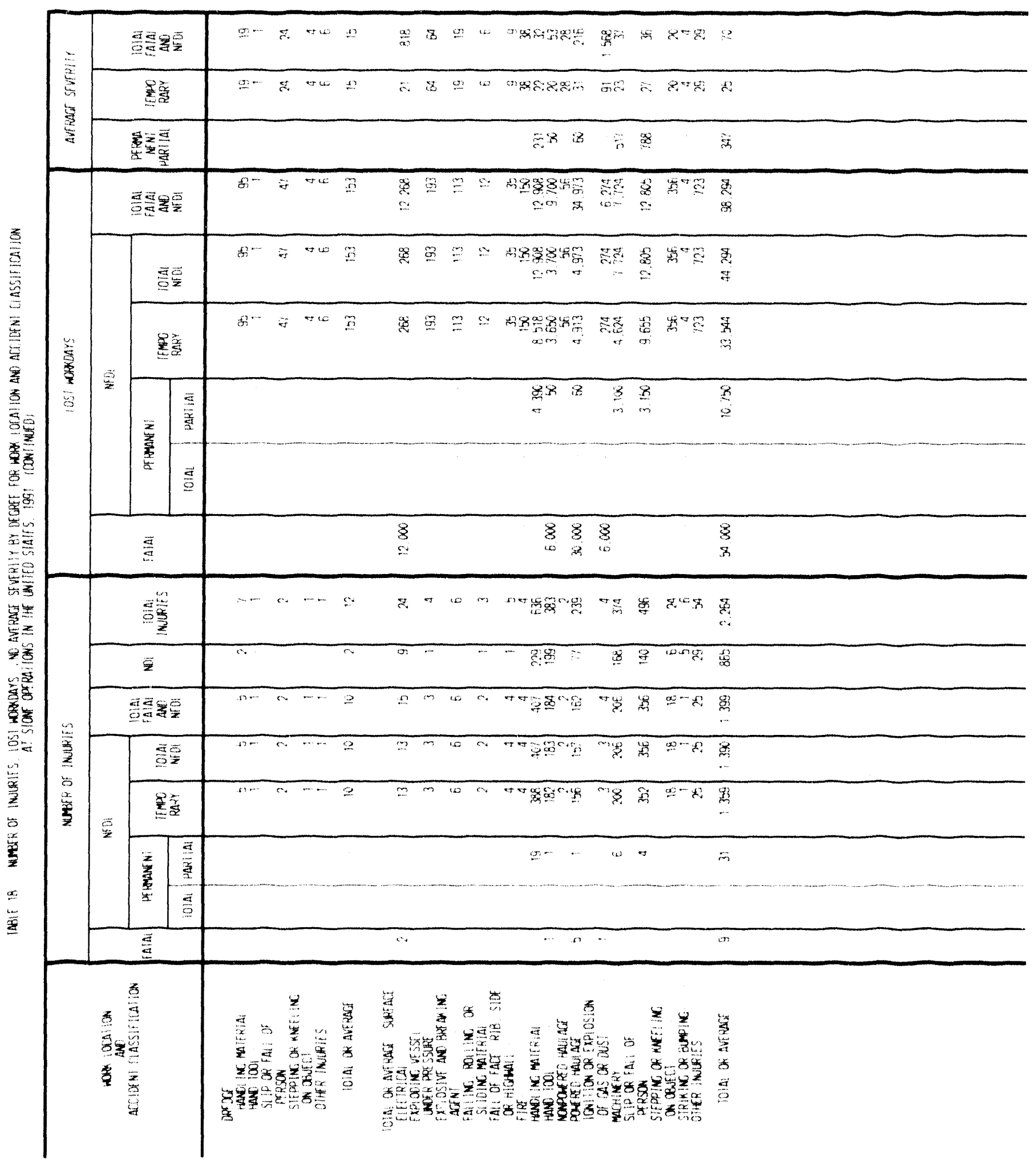




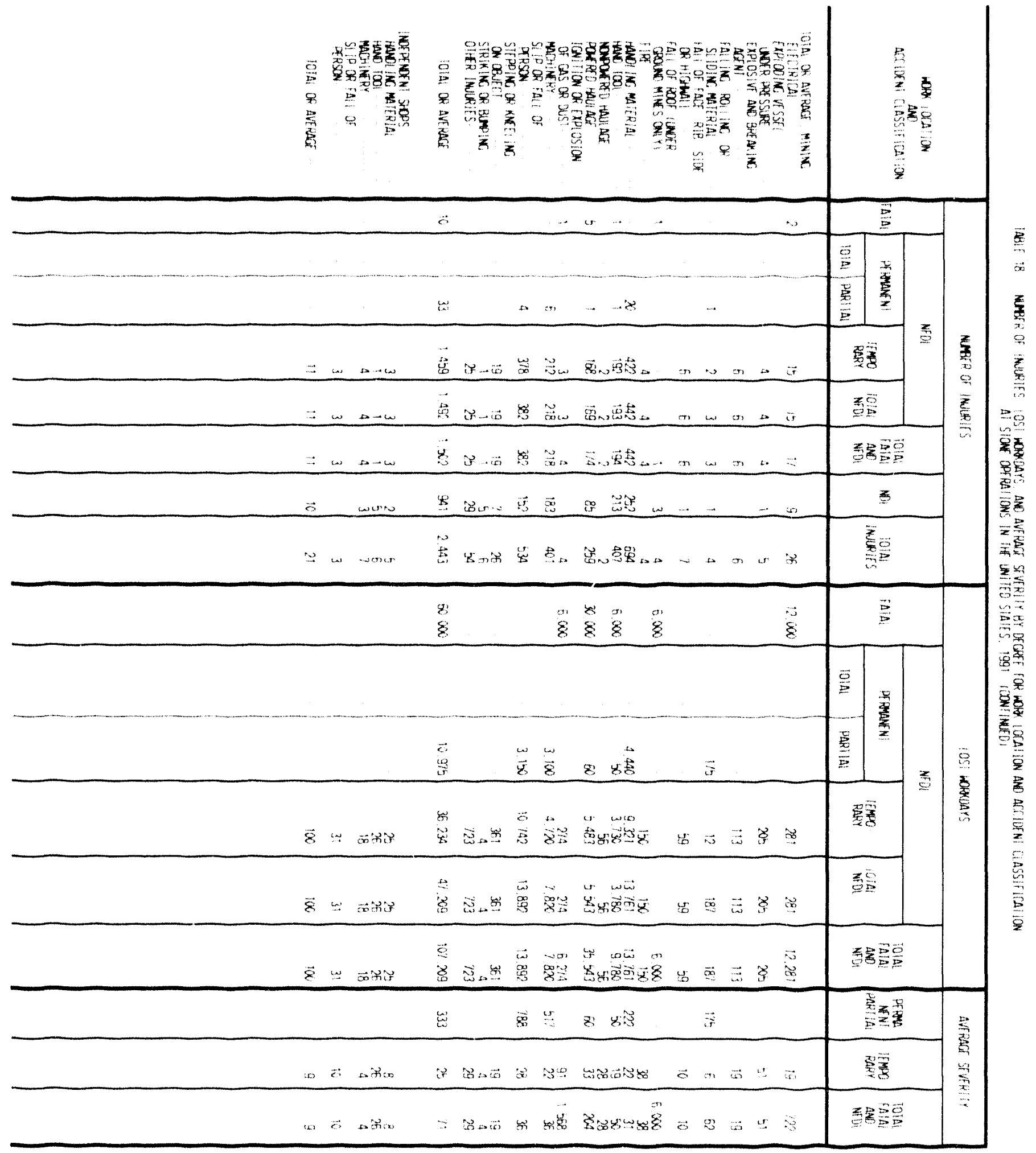




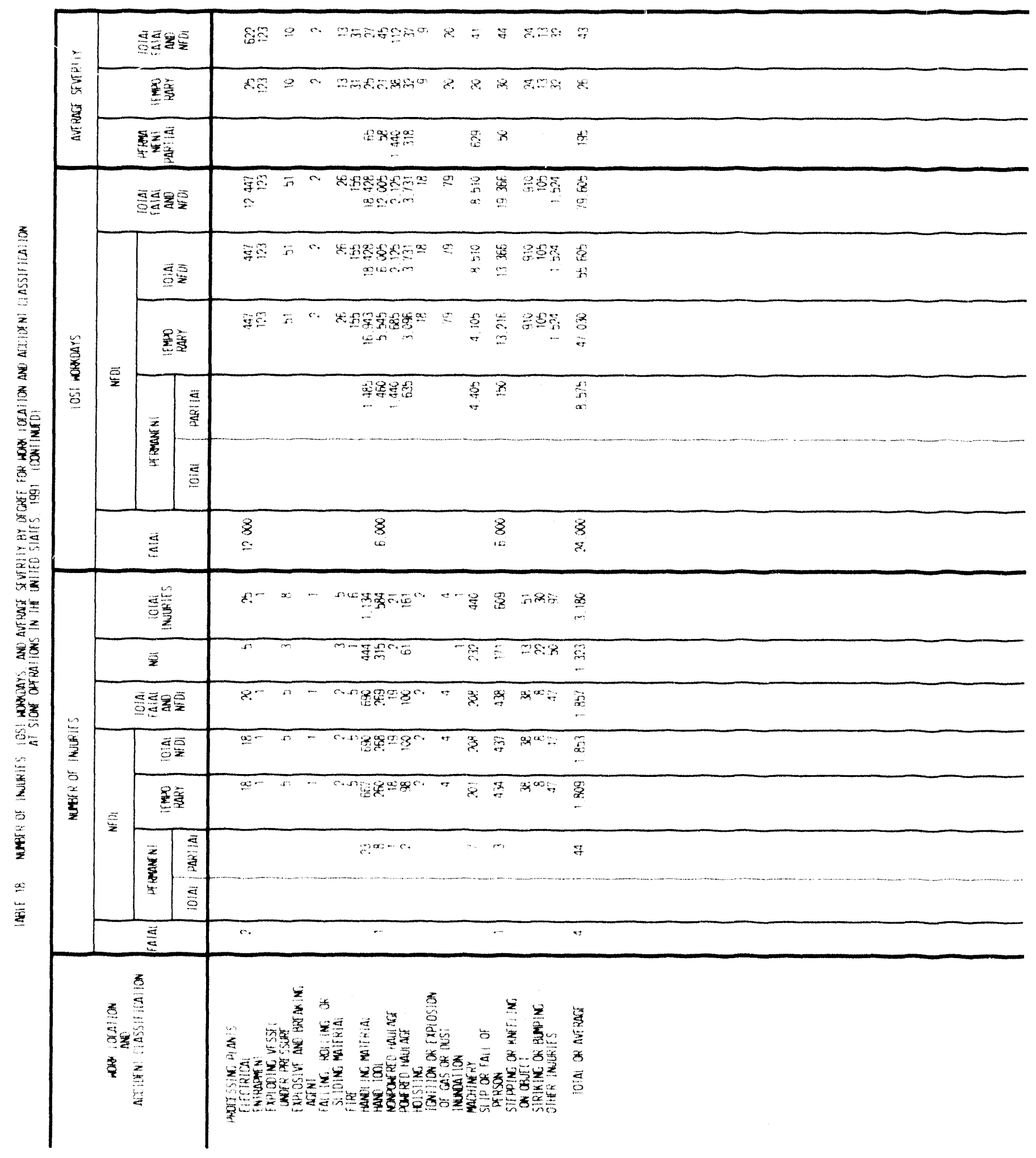




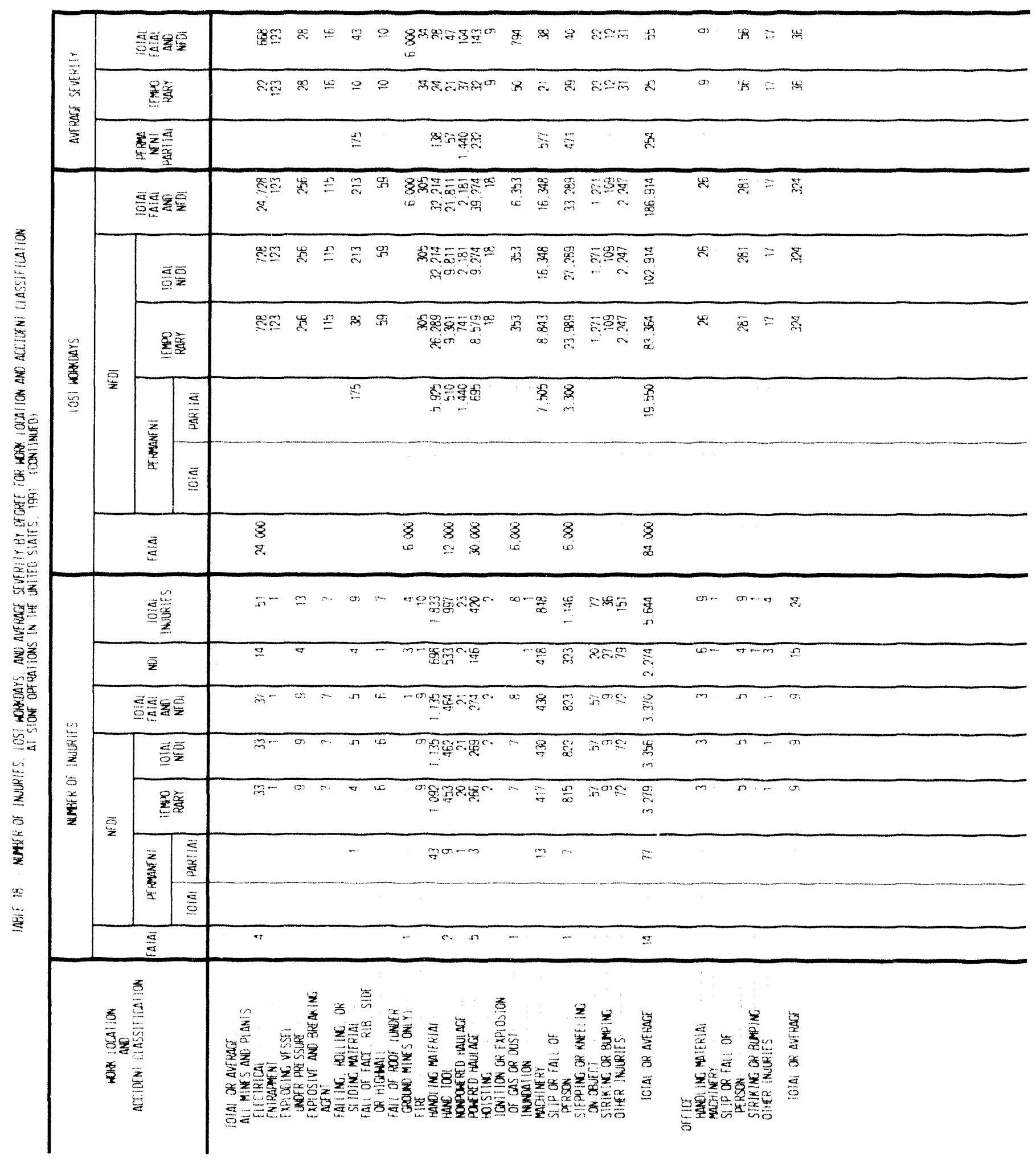




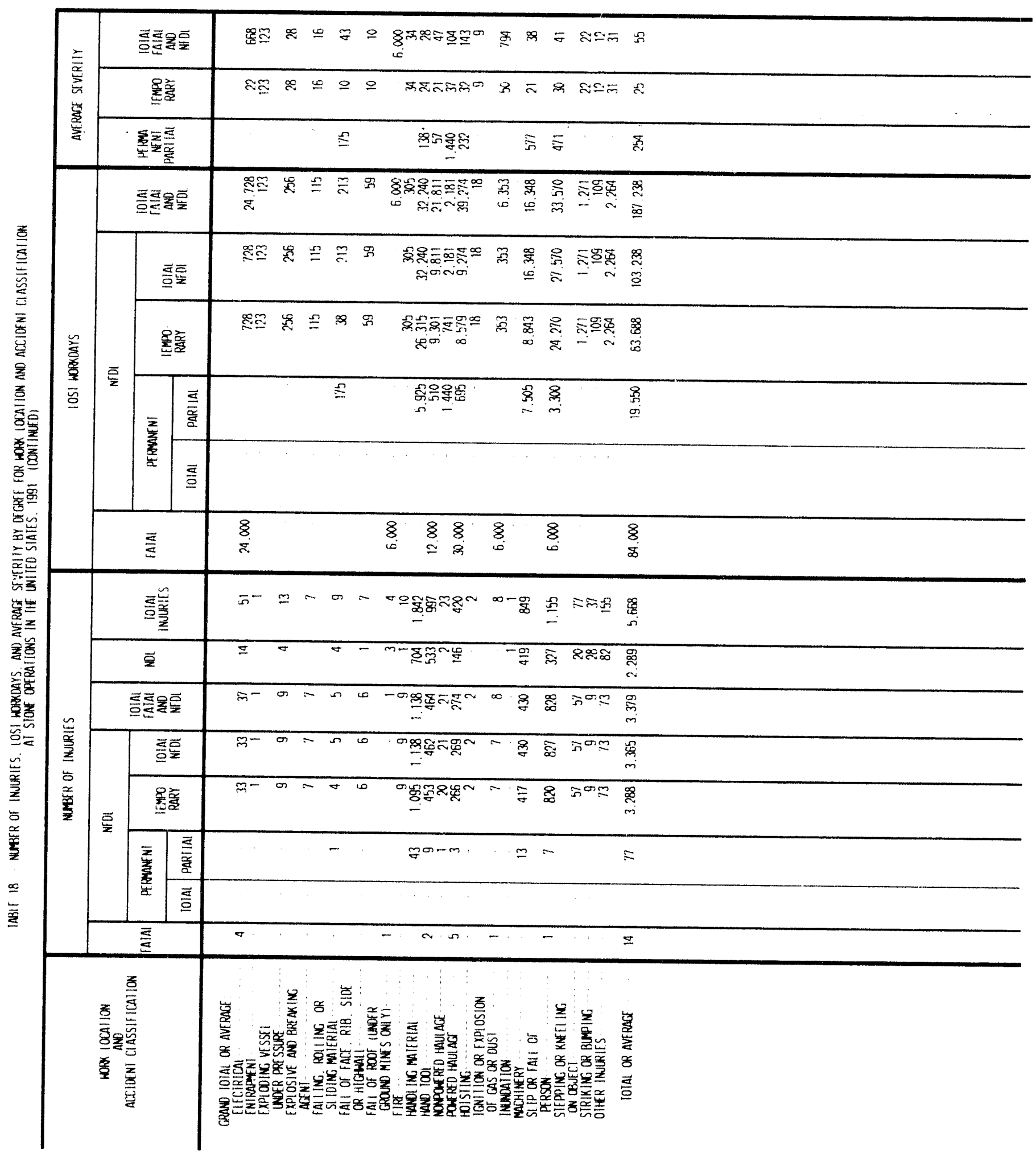




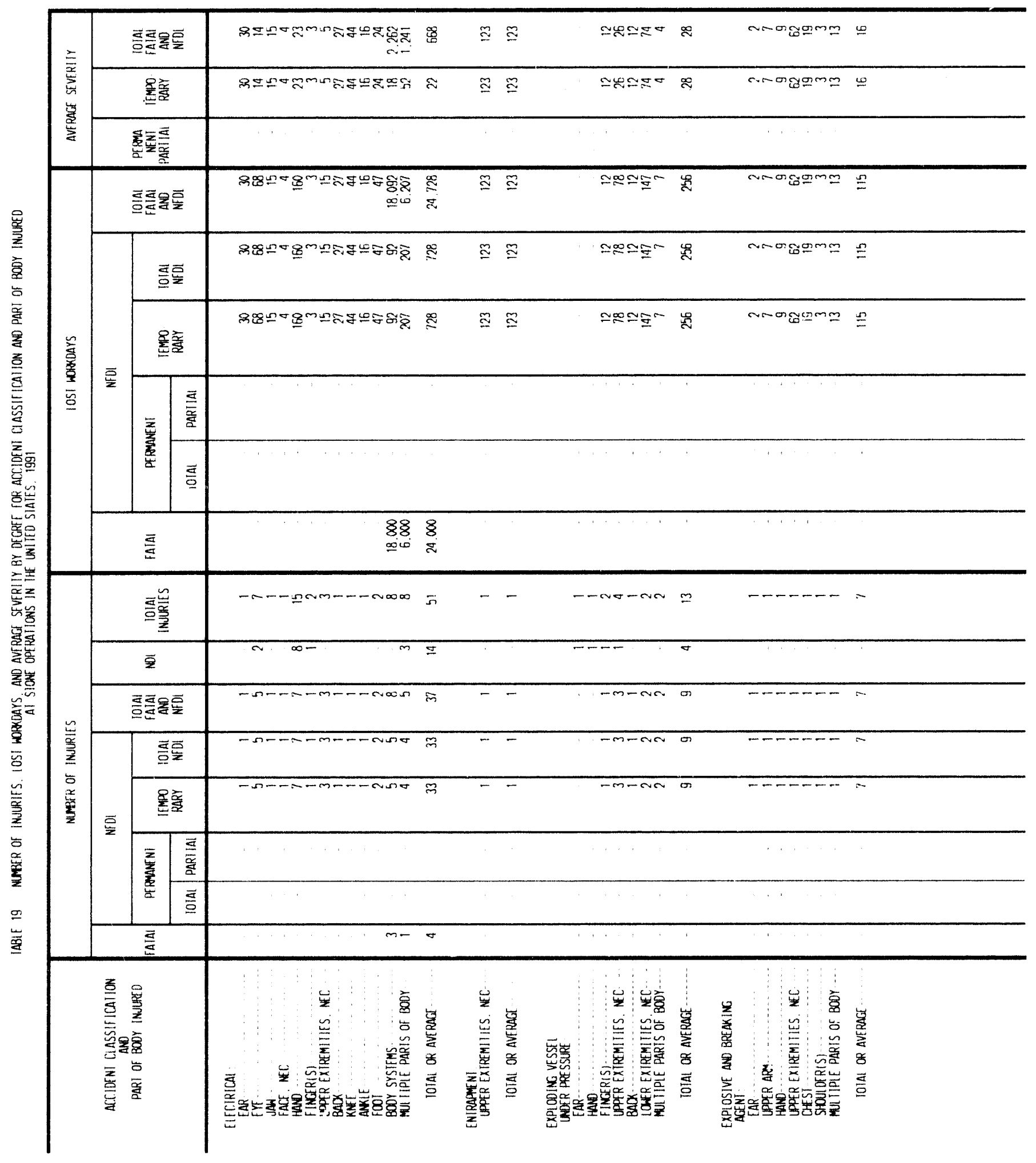




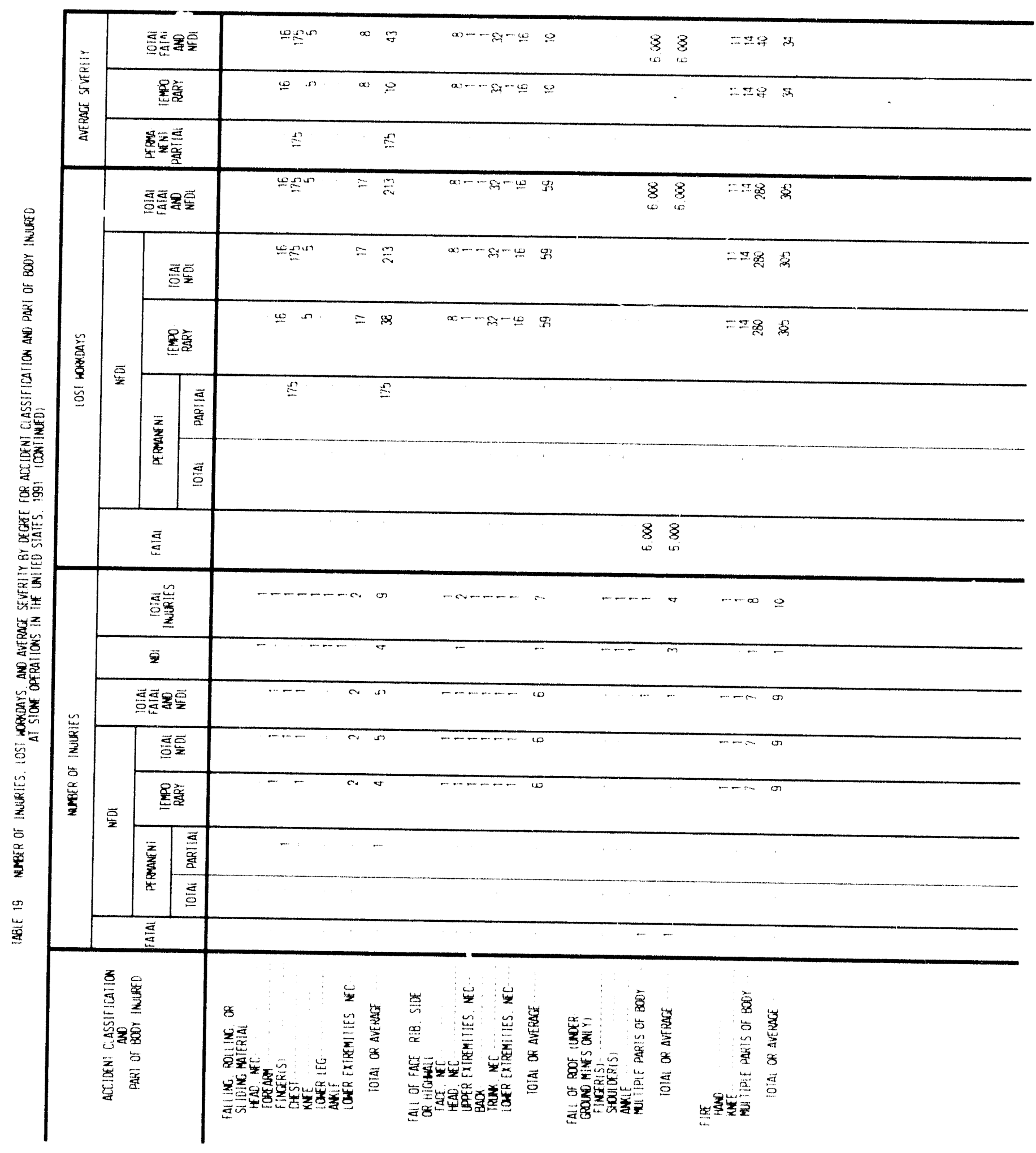




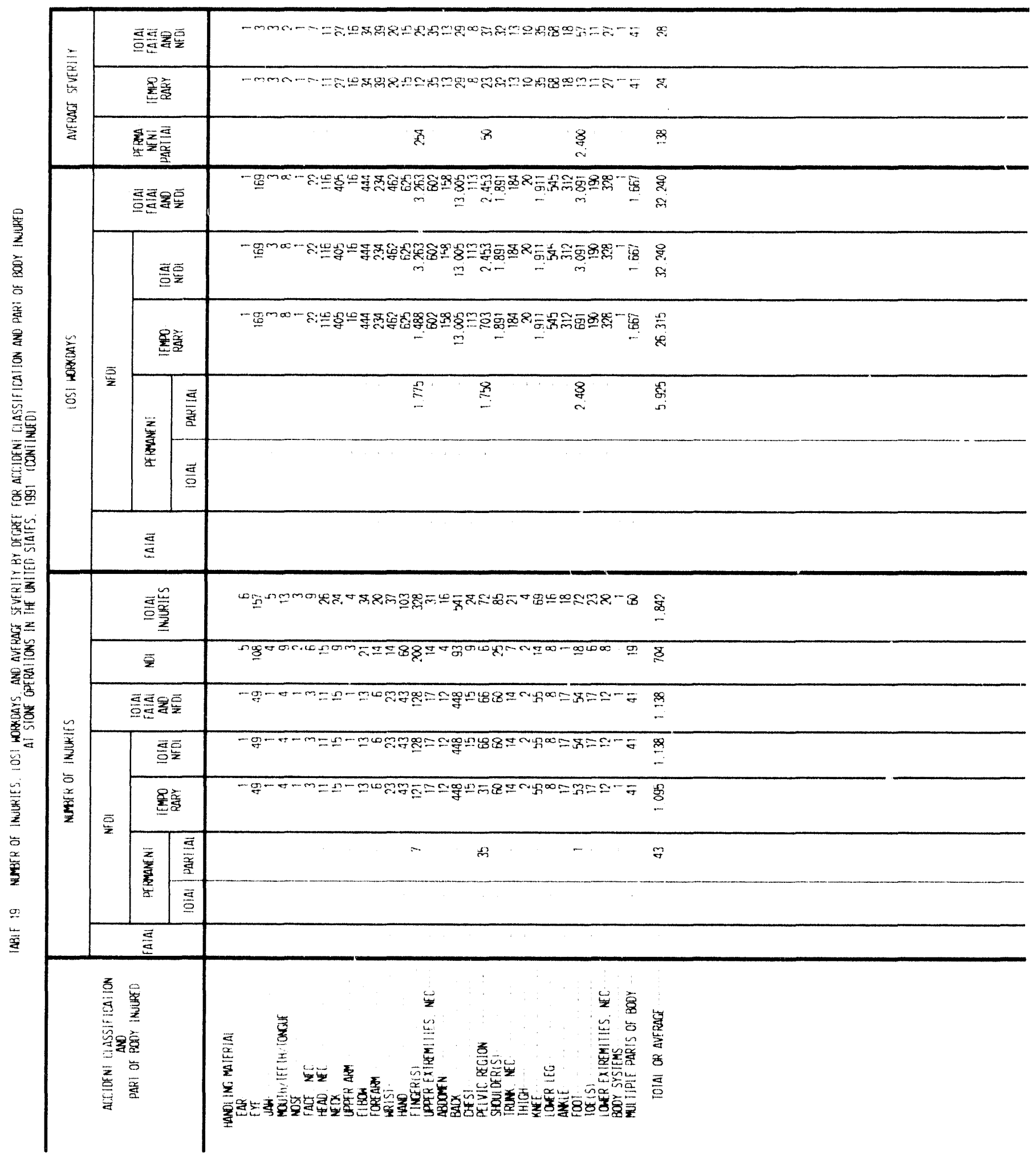




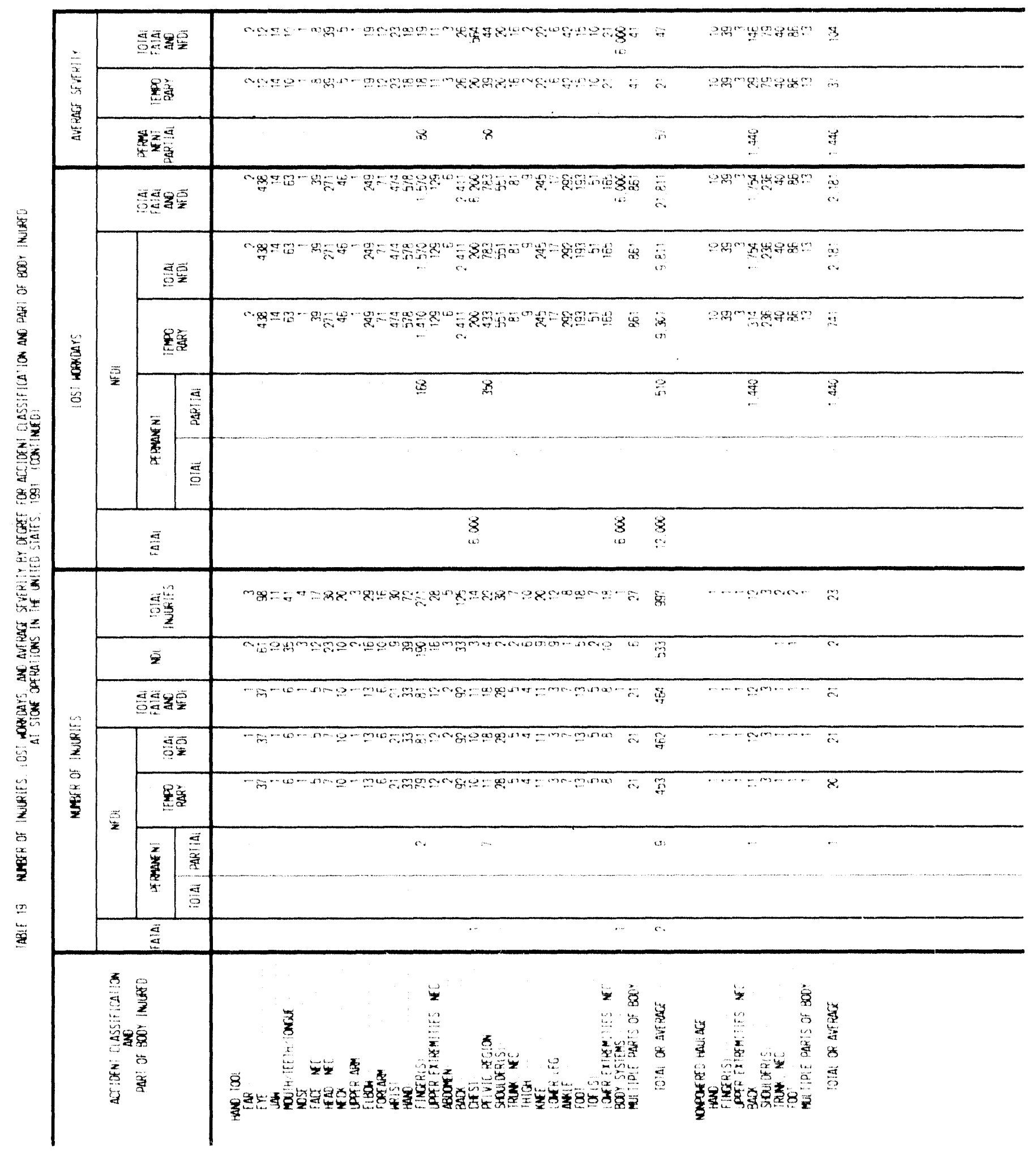




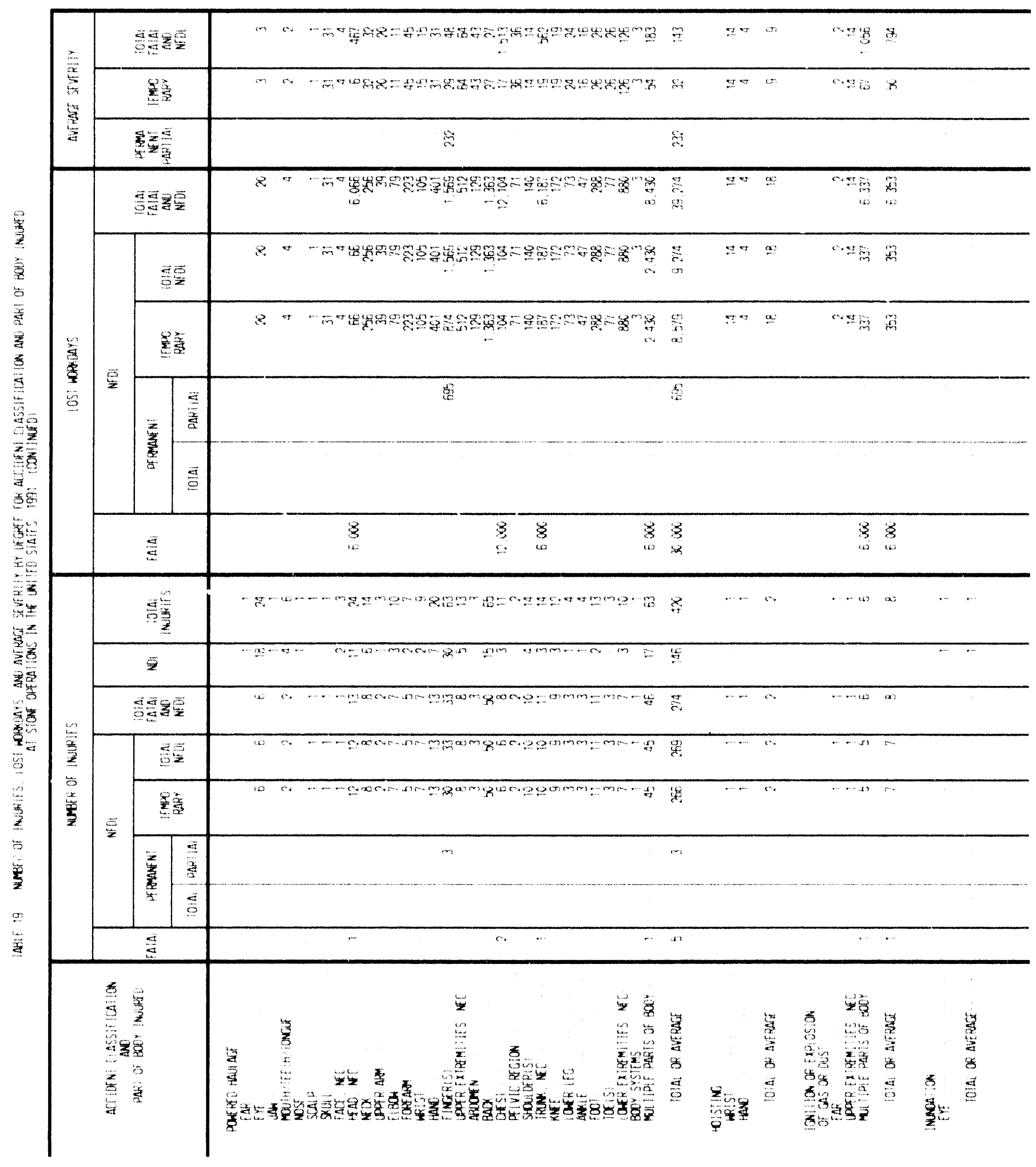




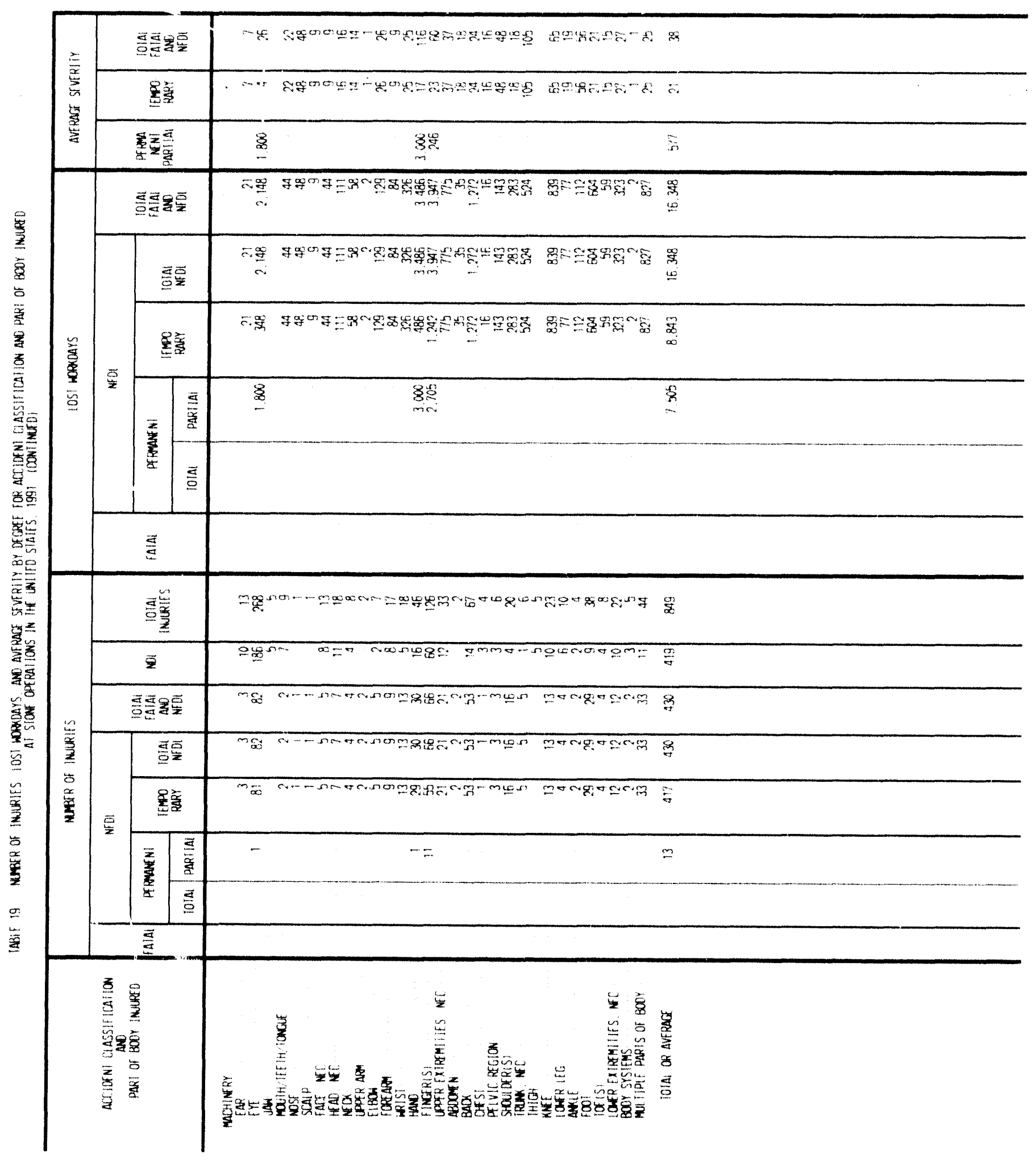




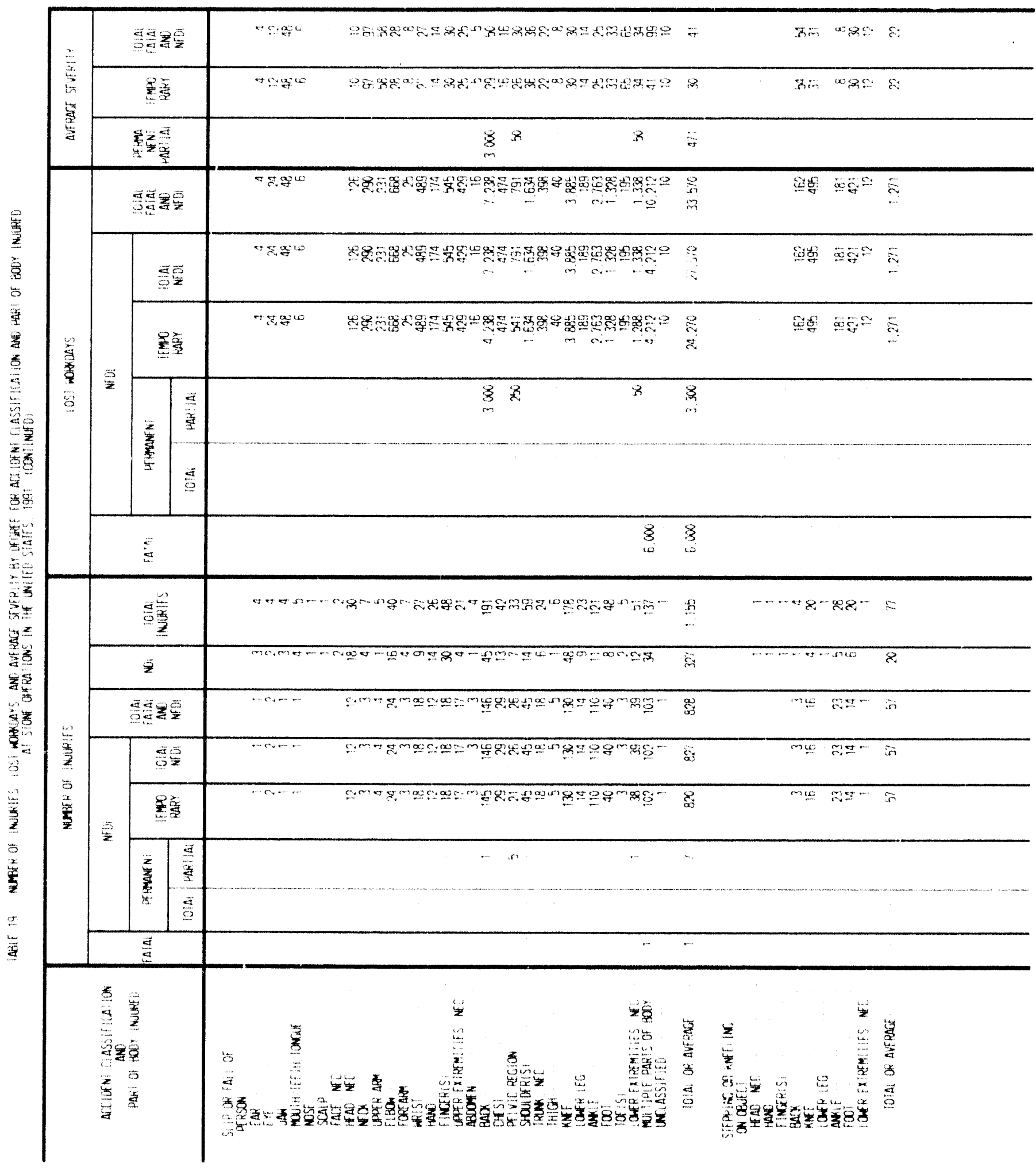




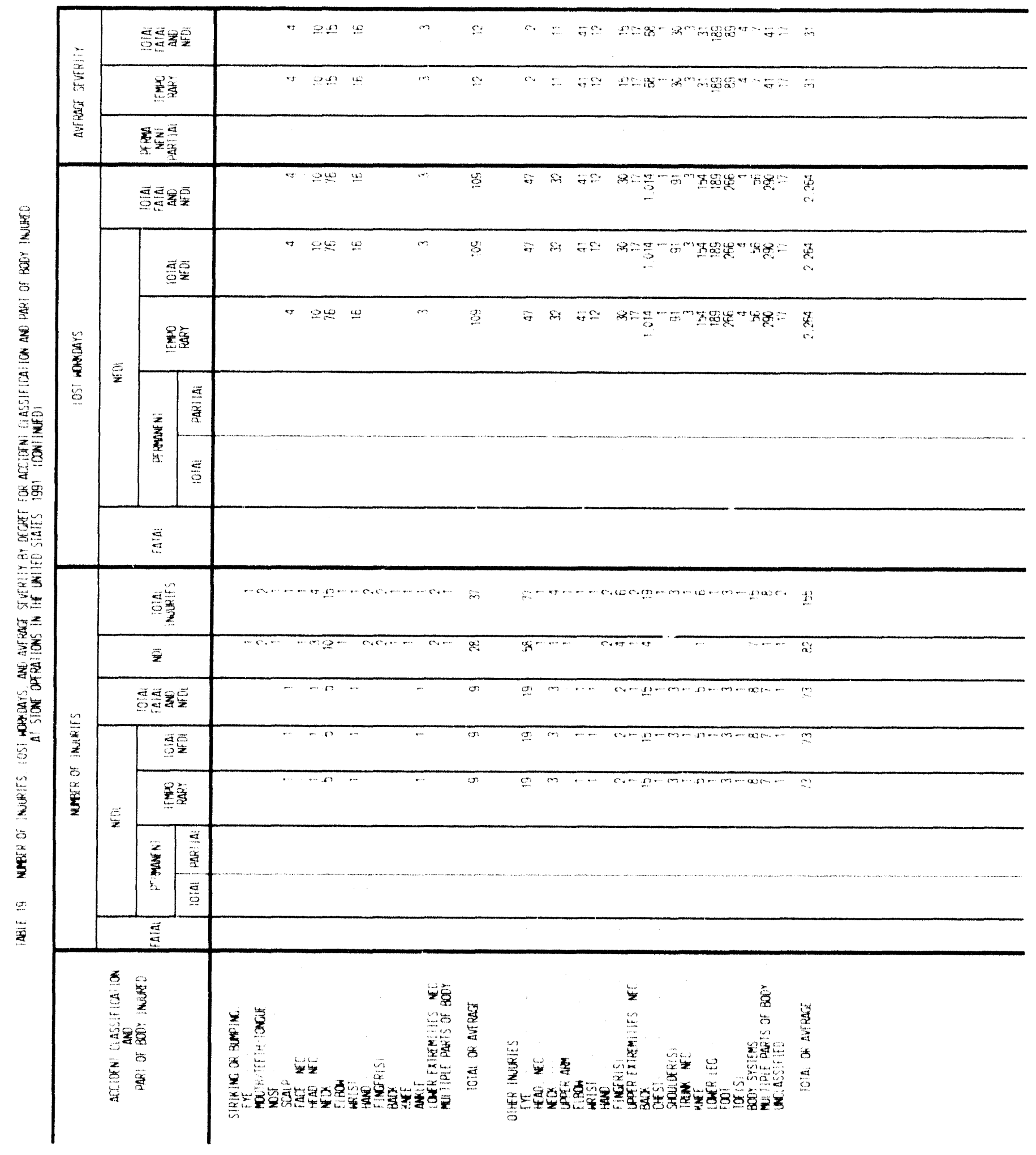




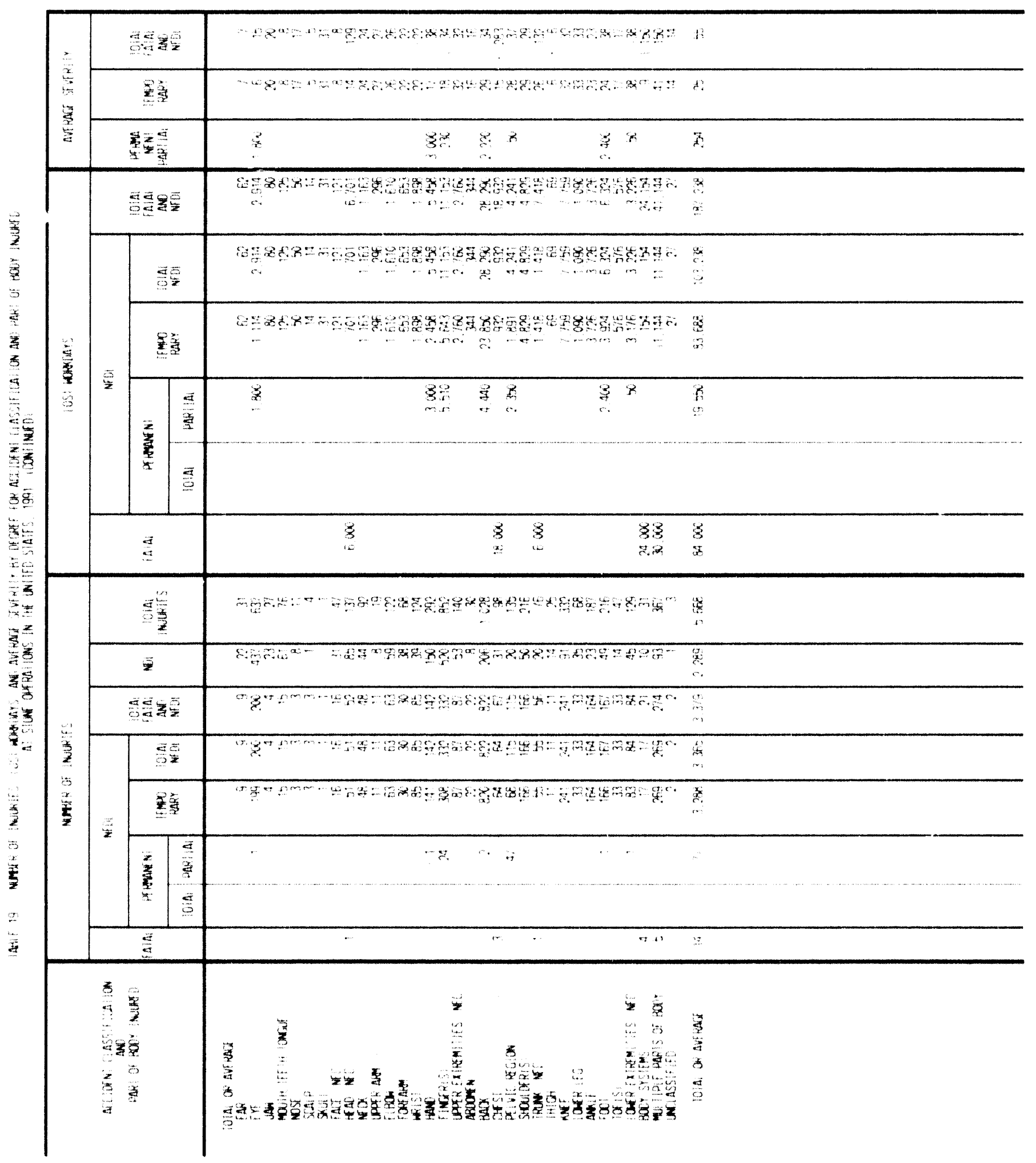




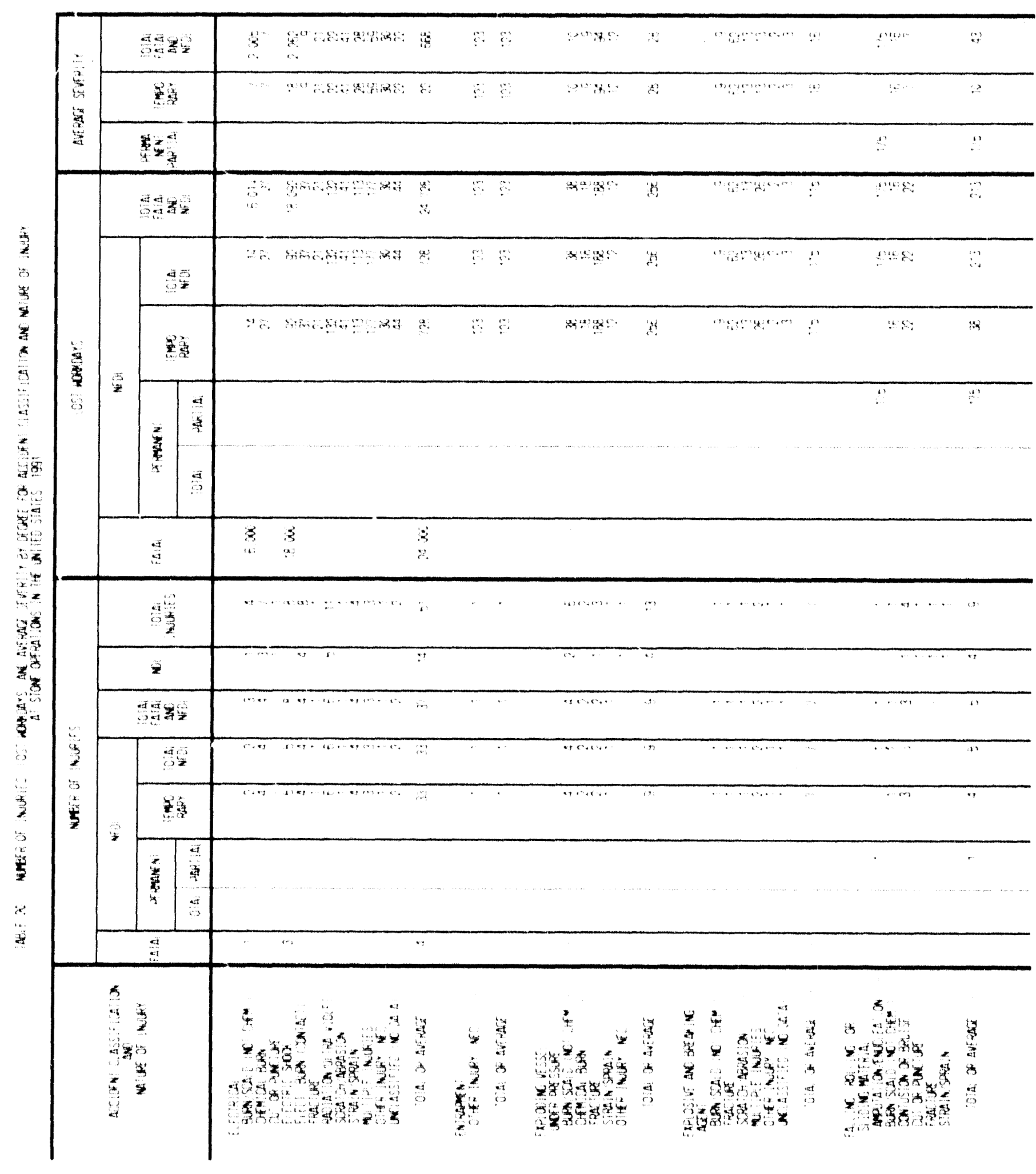




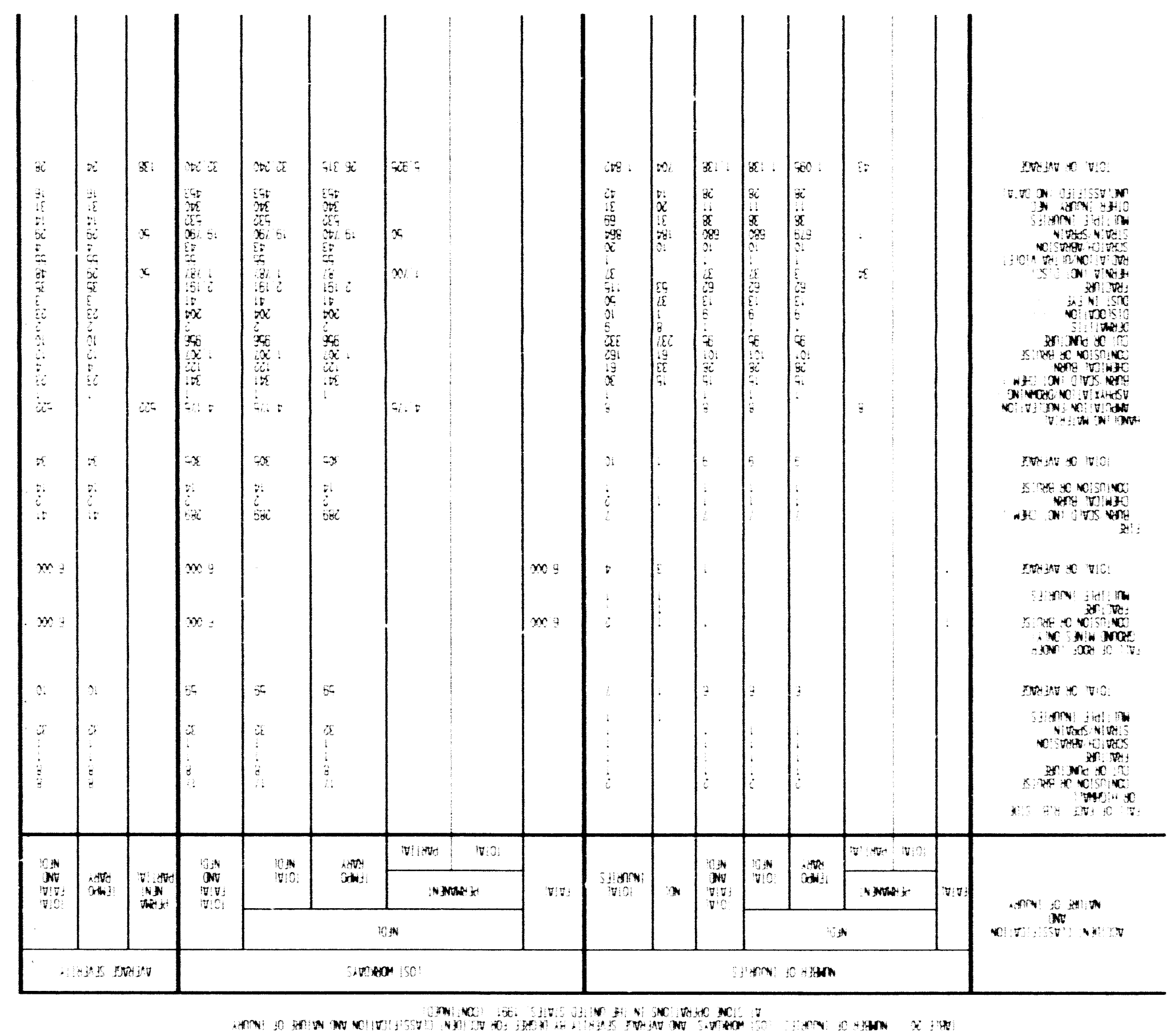




\begin{tabular}{|c|c|c|c|c|c|c|c|c|c|c|c|}
\hline \multirow{3}{*}{$\begin{array}{l}x \\
5 \\
5 \\
5 \\
5 \\
5 \\
3 \\
3\end{array}$} & \multicolumn{2}{|c|}{ 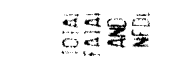 } & 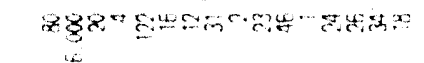 & $\Rightarrow$ & W & $2 \pm$ & 3 & 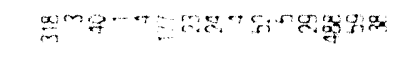 & 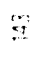 & $\pi z$ & $n$ \\
\hline & \multicolumn{2}{|c|}{ 鼓 } & 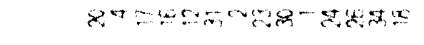 & $\therefore$ & $\therefore y_{n}$ & 4 & 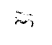 & 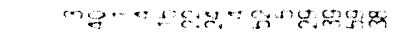 & $s_{i}$ & $\because \pm$ & $\omega$ \\
\hline & \multicolumn{2}{|c|}{ 基迹焉 } & 8 & 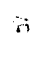 & & $\stackrel{9}{7}$ & $\frac{7}{7}$ & $a$ & $\therefore$ & & \\
\hline \multirow{5}{*}{$\frac{5}{8}$} & \multicolumn{2}{|c|}{ 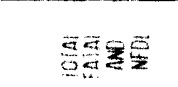 } & 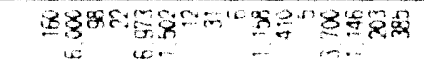 & $\therefore$ & $\cdots$ & $g^{2}$ & $\begin{array}{l}\cdots \\
\cdots \\
\cdots\end{array}$ & 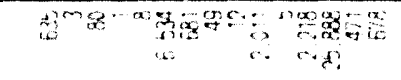 & gi & $\pi 2$ & 4 \\
\hline & \multirow{3}{*}{$\underline{E}$} & 3 & 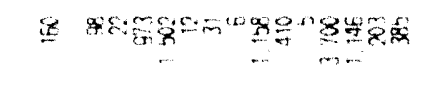 & 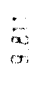 & $\cdots$ & $5^{4 x}$ & 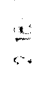 & 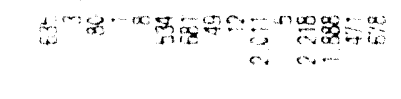 & sis & 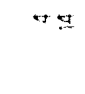 & $\infty ?$ \\
\hline & & 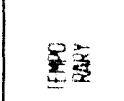 & 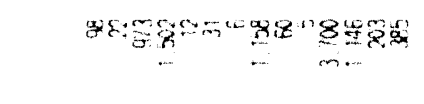 & 9 & $m$ & $m_{i s}$ & $\underline{\underline{z}}$ & 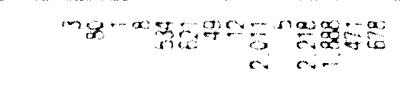 & is & $\because \leq$ & $\infty$ \\
\hline & & $\frac{a}{\vec{x}}$ & 8 & 27 & & 导 & $\frac{9}{2}$ & 8 & 综 & & \\
\hline & \multicolumn{2}{|l|}{$\frac{a}{a}$} & $\begin{array}{l}3 \\
3\end{array}$ & 8 & & & & $\begin{array}{ll}3 & 3 \\
15 & 8\end{array}$ & 3 & & \\
\hline \multirow{8}{*}{$\begin{array}{l}w \\
\underline{z} \\
z \\
y \\
z \\
z \\
z\end{array}$} & \multicolumn{2}{|c|}{$\frac{2}{5}$} & 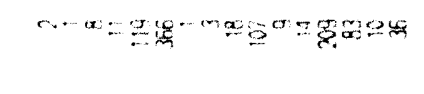 & $\bar{\sharp}$ & $\cdots+\cdots$ & $9 . \cdots$ & $\Leftrightarrow$ & 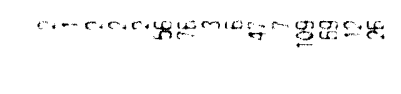 & \& & $\cdots$ & $\cdots$ \\
\hline & \multicolumn{2}{|l|}{3} & mnge r. & 9 & & $\cdots$ & $\because$ & 9. & $\underline{\underline{Z}}$ & & \\
\hline & \multicolumn{2}{|c|}{ 迋要这 } & a. sub & 涤 & $\cdots$ & $x-$ & $\therefore$ & 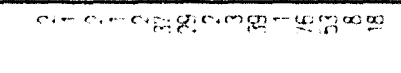 & $\ddot{8}$ & $\cdots \cdots$ & 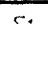 \\
\hline & \multicolumn{2}{|c|}{ 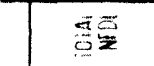 } & $\therefore$ nLigy & $\sum_{x}^{2}$ & $\cdots \cdots$ & $\nsubseteq \cdots$ & $\approx$ & 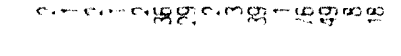 & 昰 & $\cdots$ & $\cdots$ \\
\hline & \multicolumn{2}{|c|}{$\frac{2}{z}$} & 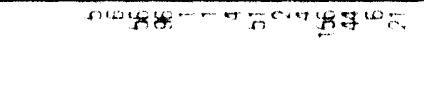 & 9 & $\cdots \cdot$ & $\ldots-\cdots$ & 8 & 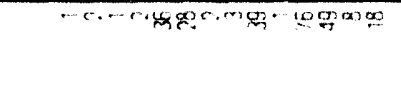 & $\$$ & $\cdots$ & $r$ \\
\hline & \multirow{2}{*}{ 焉 } & $\frac{\underline{\underline{z}}}{\underline{\underline{z}}}$ & $\cdots$ & $s$ & & 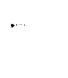 & $\ldots$ & $\therefore$ & $m$ & & \\
\hline & & $\frac{j}{0}$ & & & & & & & & & \\
\hline & \multicolumn{2}{|l|}{$\underline{3}$} & $\cdots$ & c. & & & 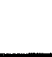 & $\cdots$ & $n$ & & \\
\hline & 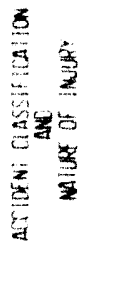 & & 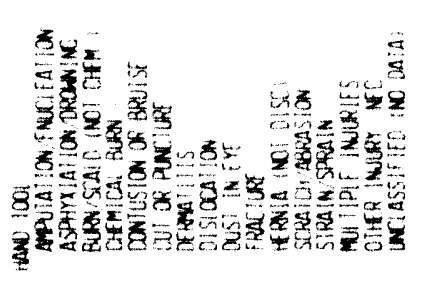 & 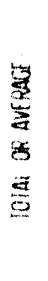 & 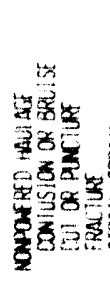 & 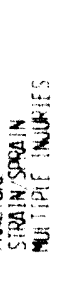 & 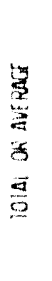 & 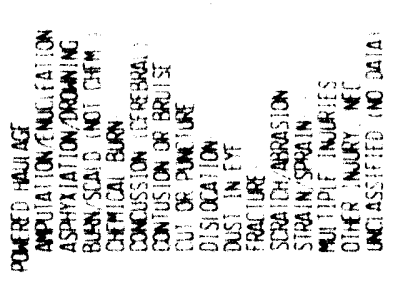 & 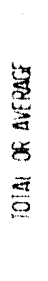 & 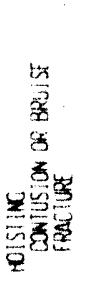 & 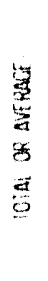 \\
\hline
\end{tabular}




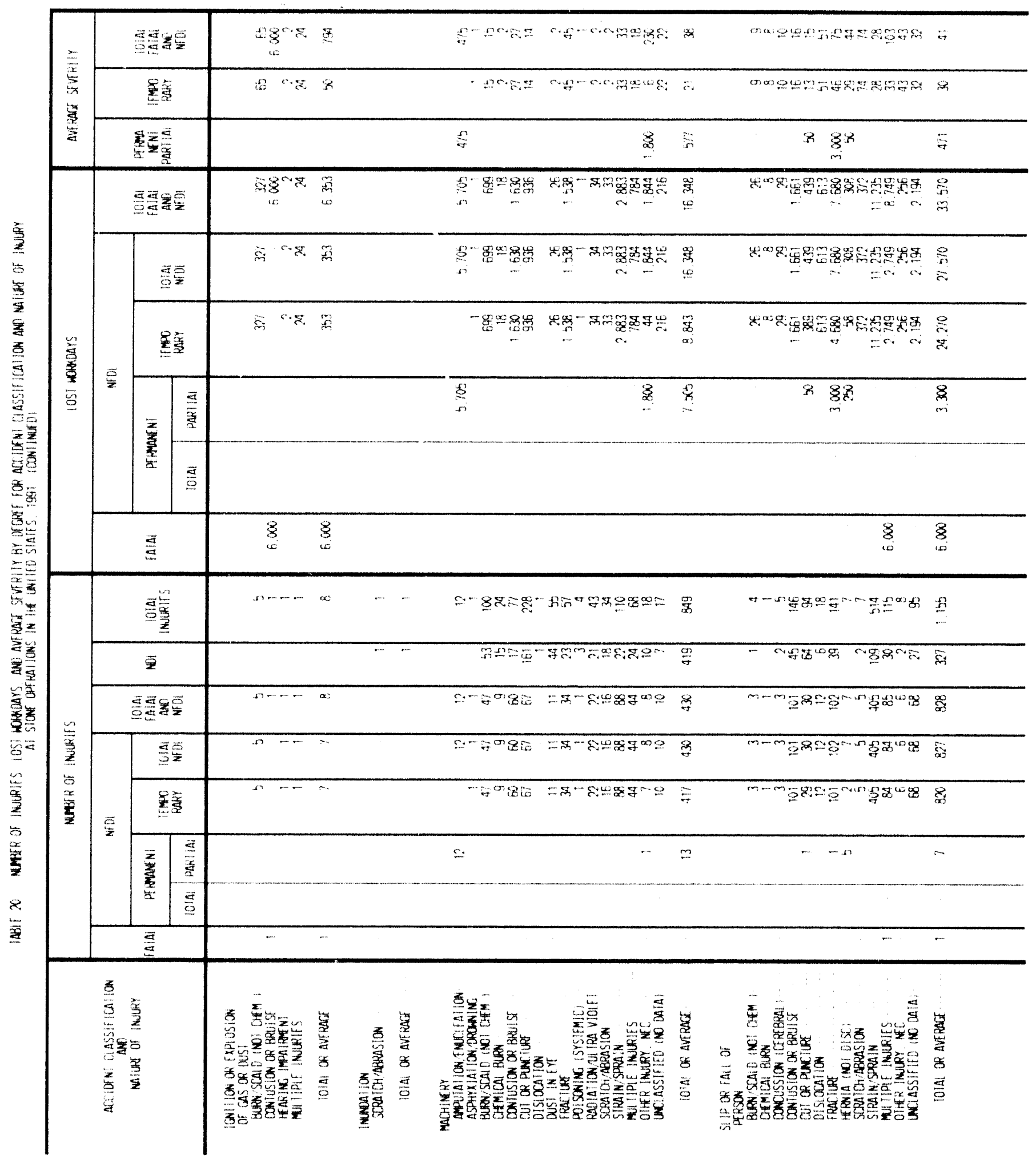




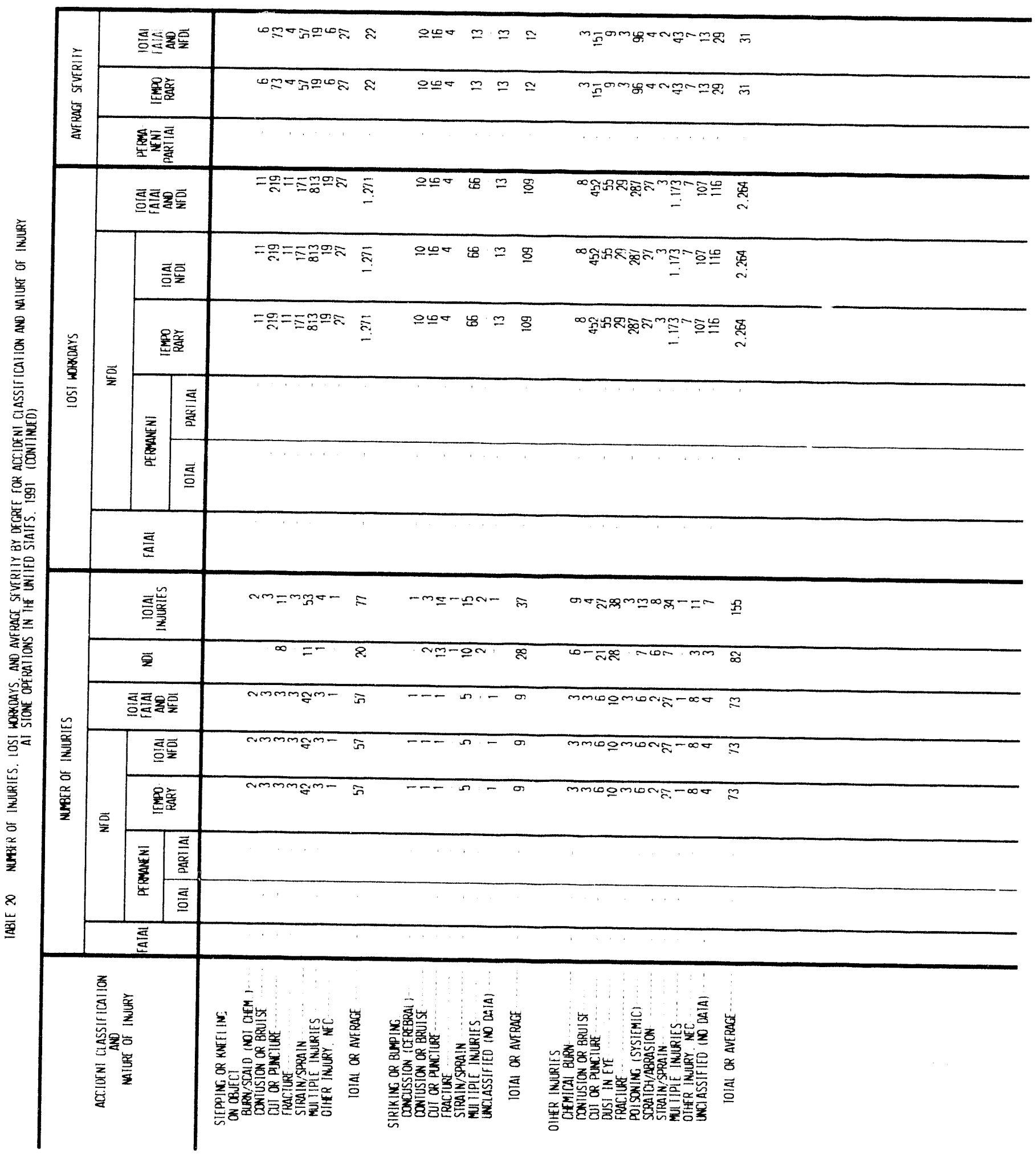




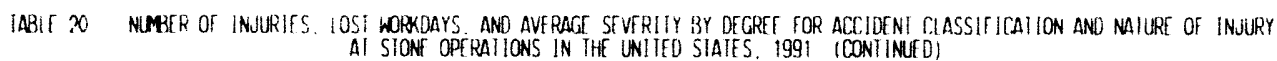

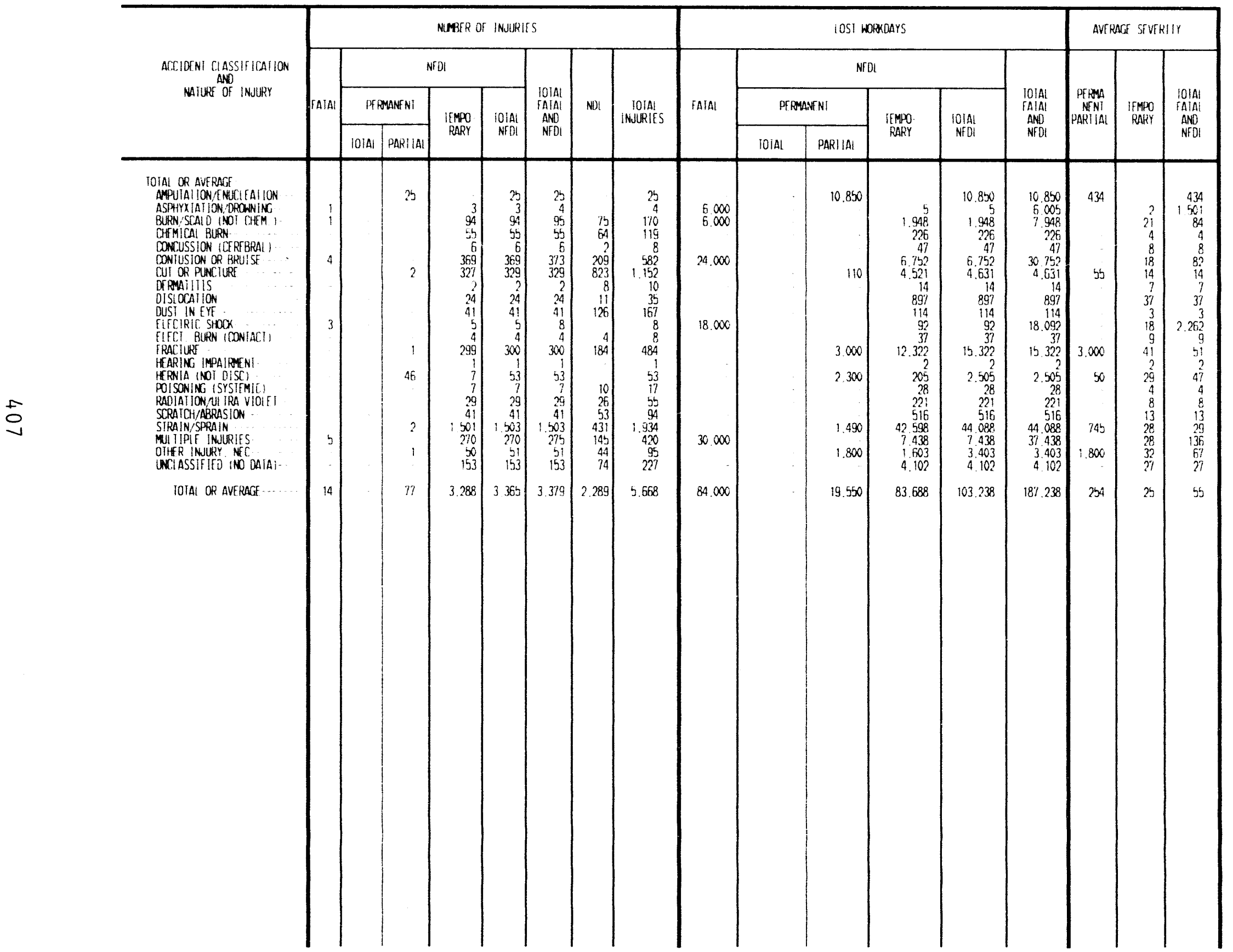




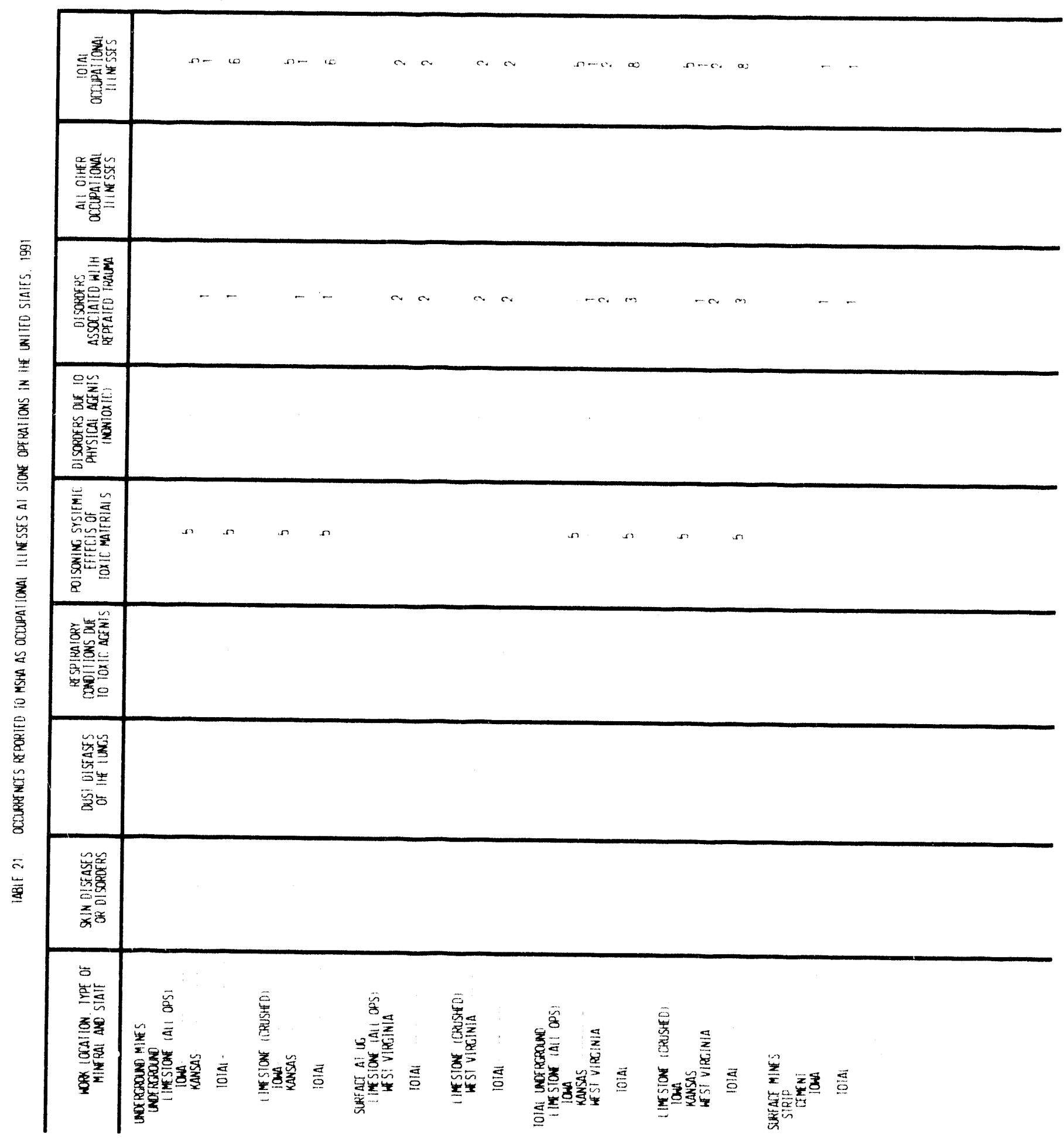




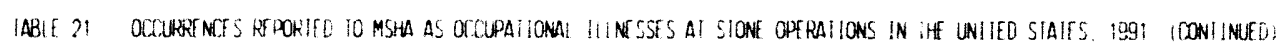

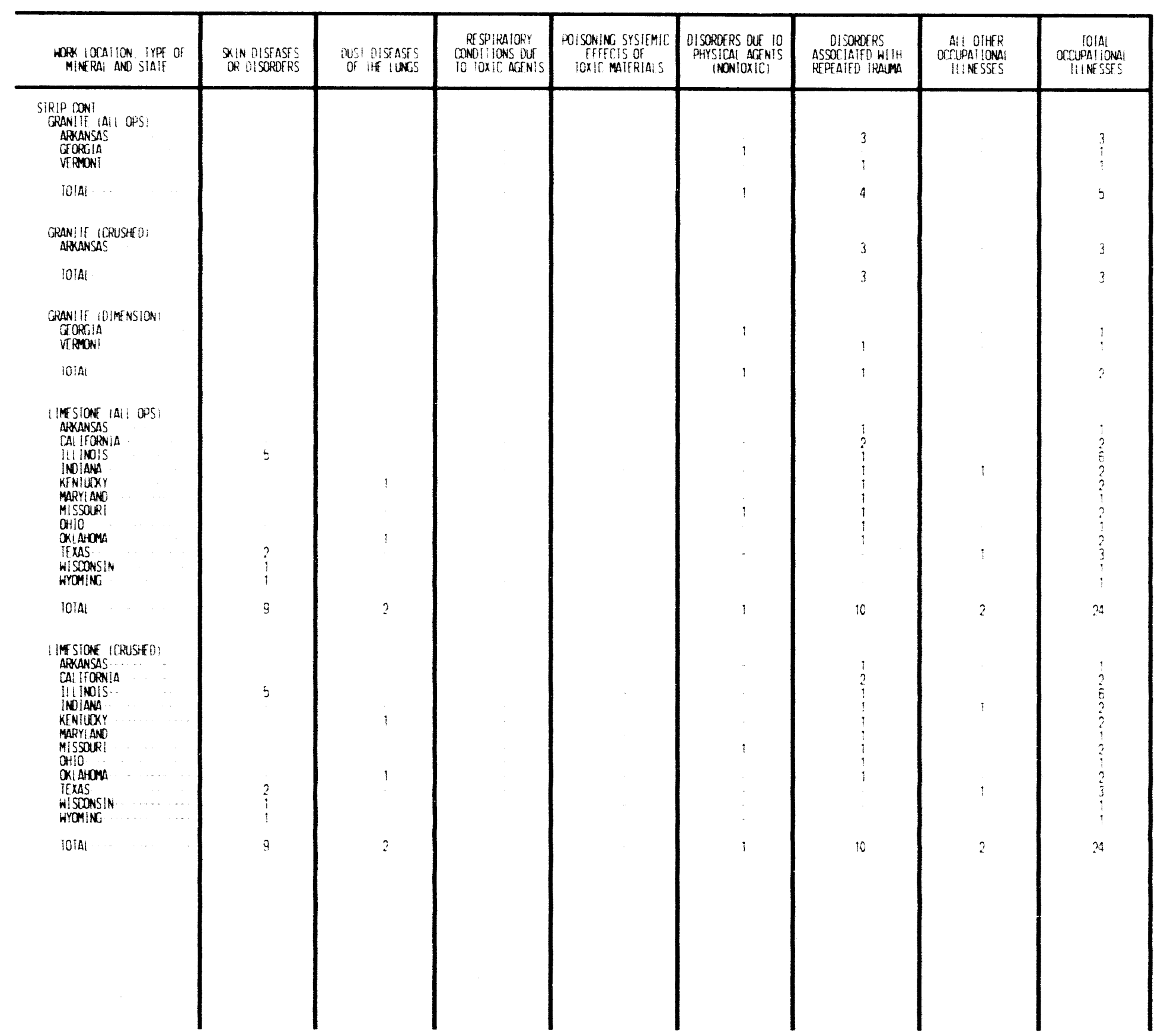




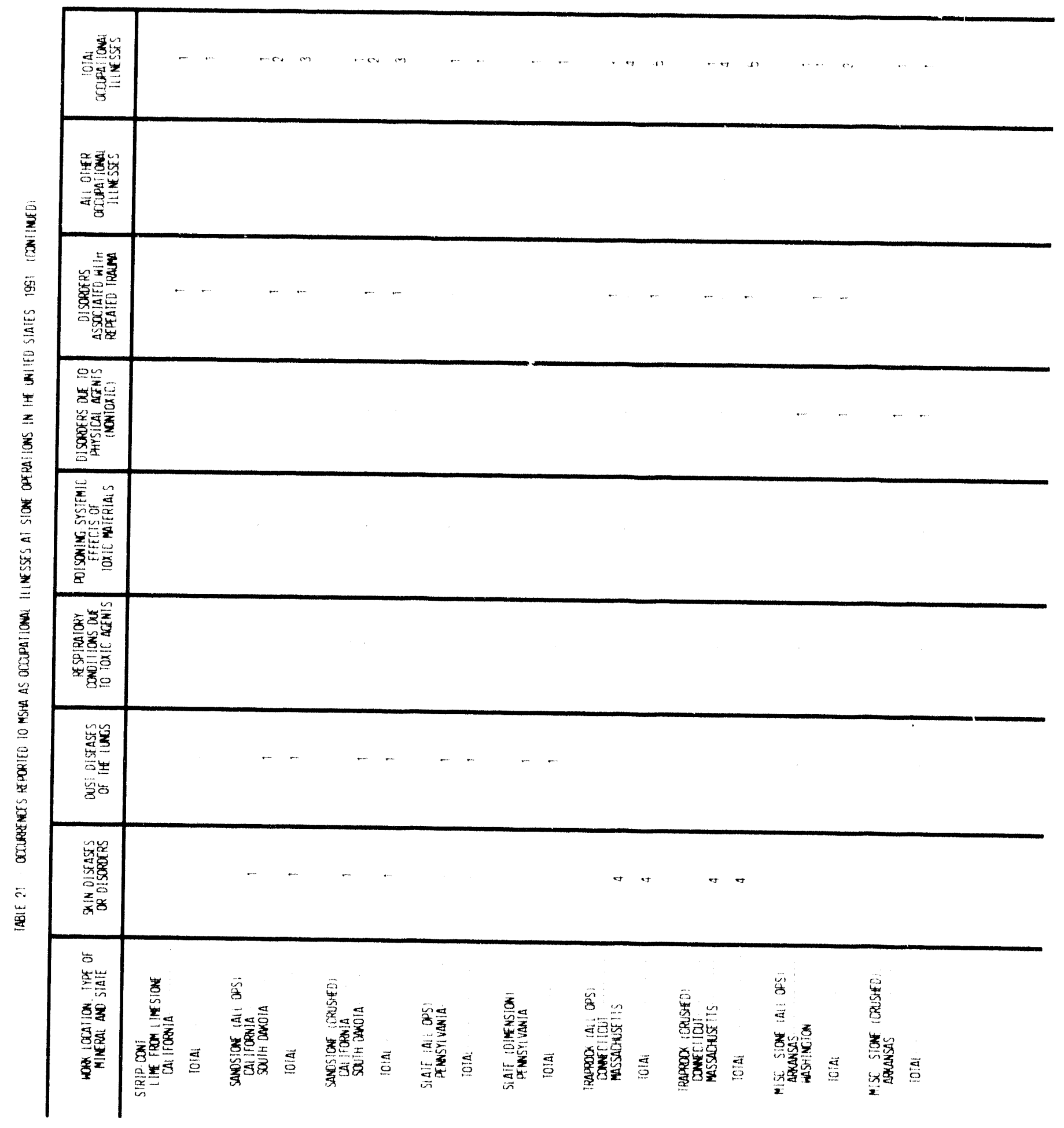




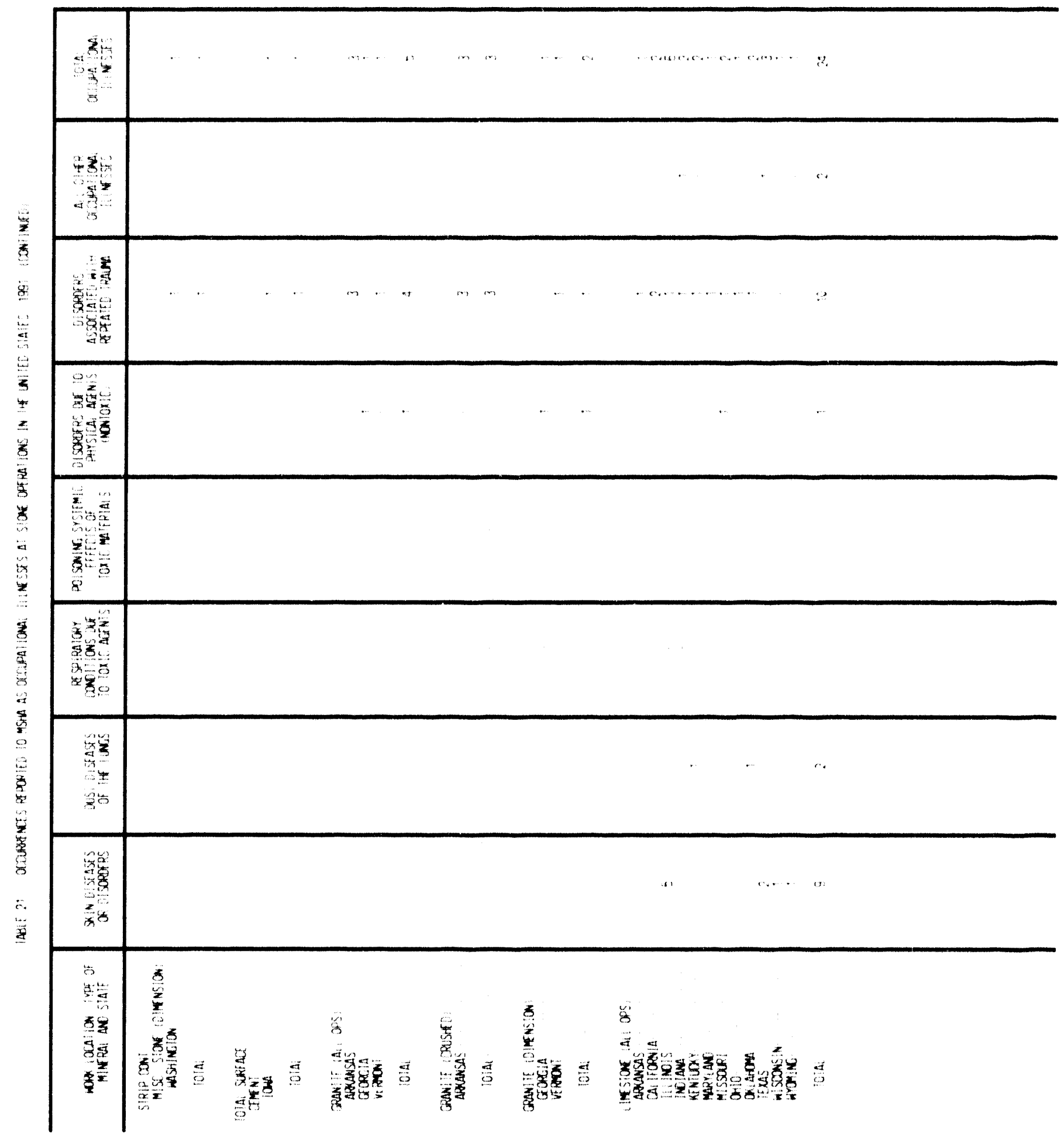




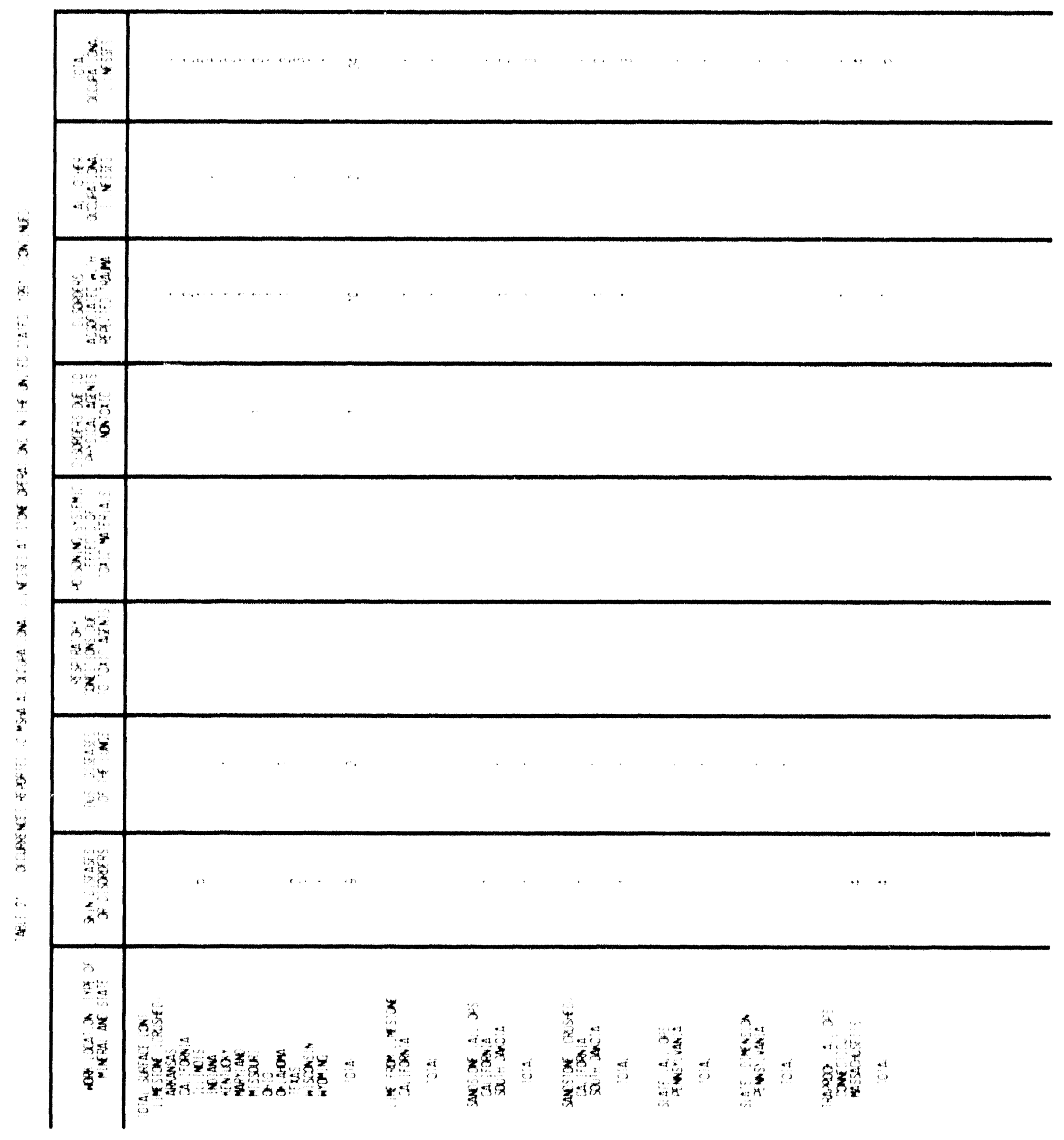




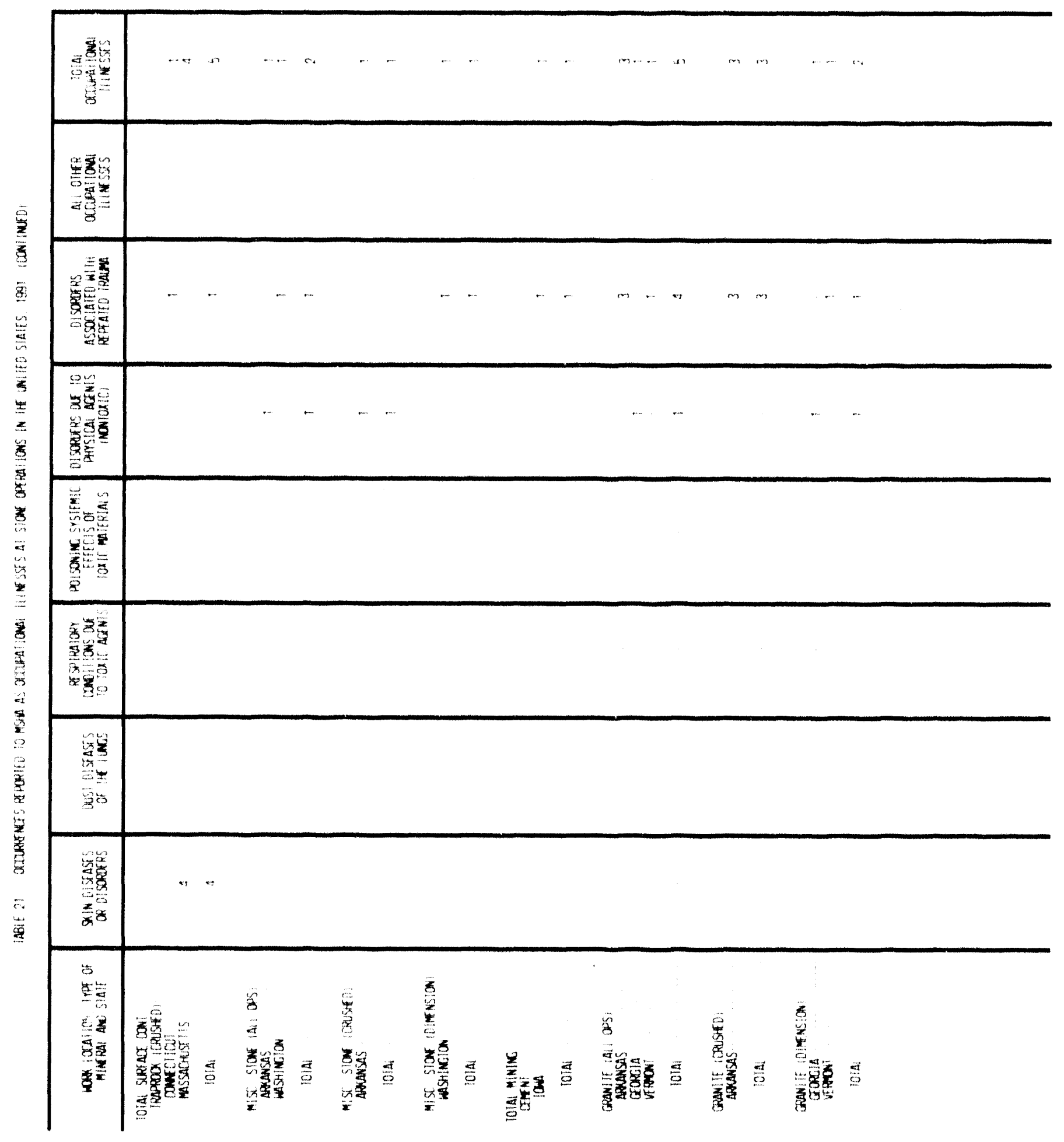




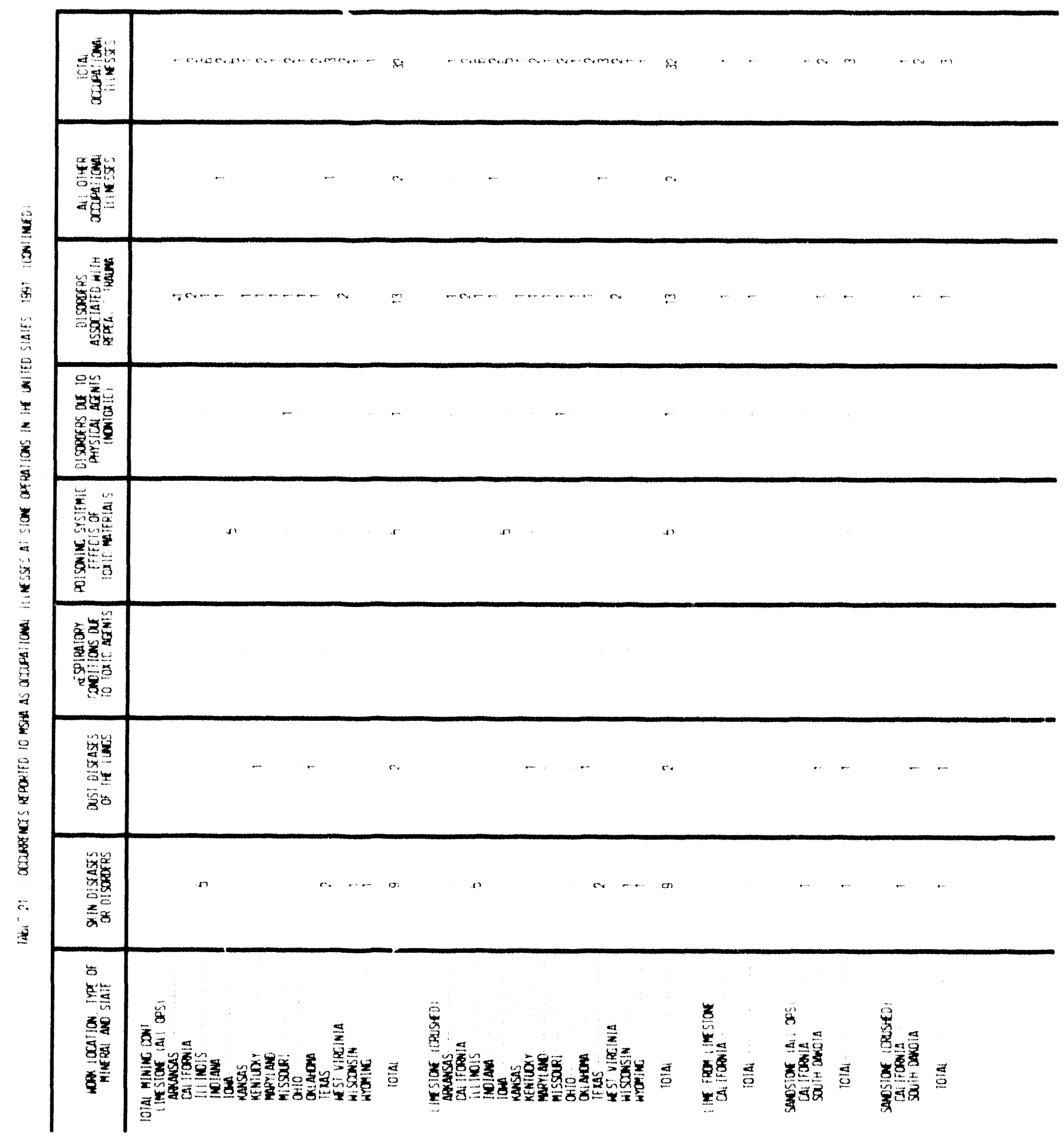




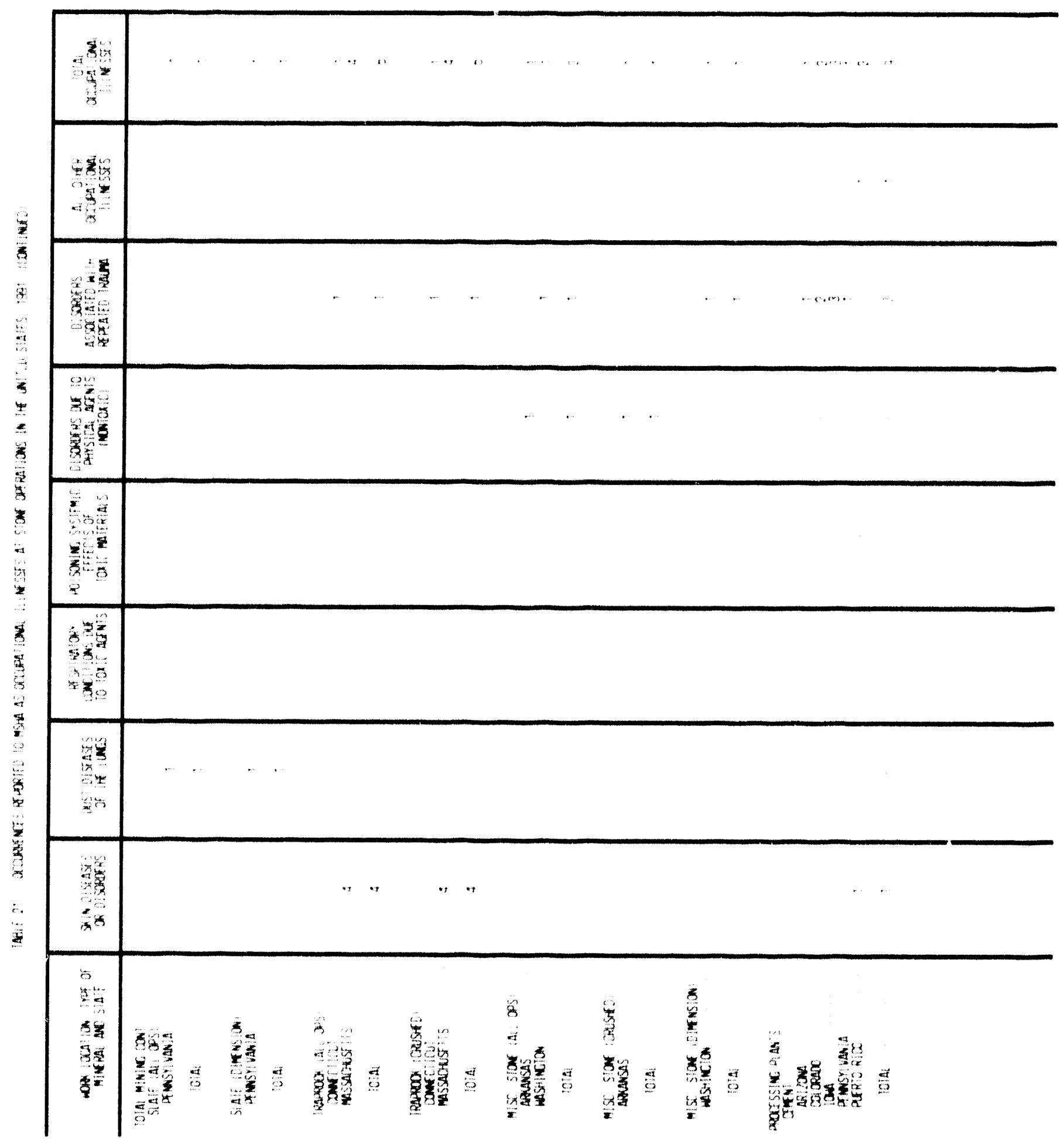




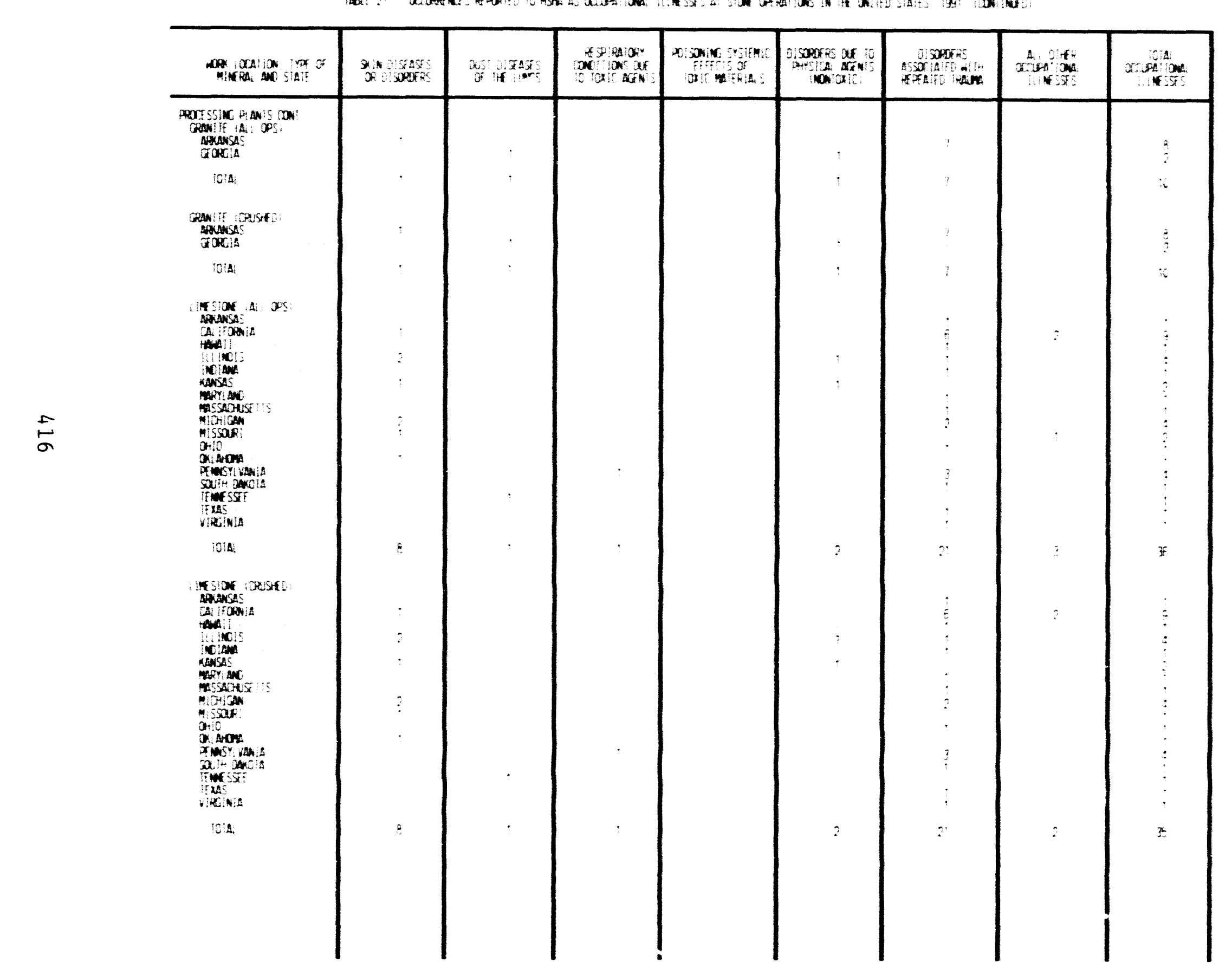




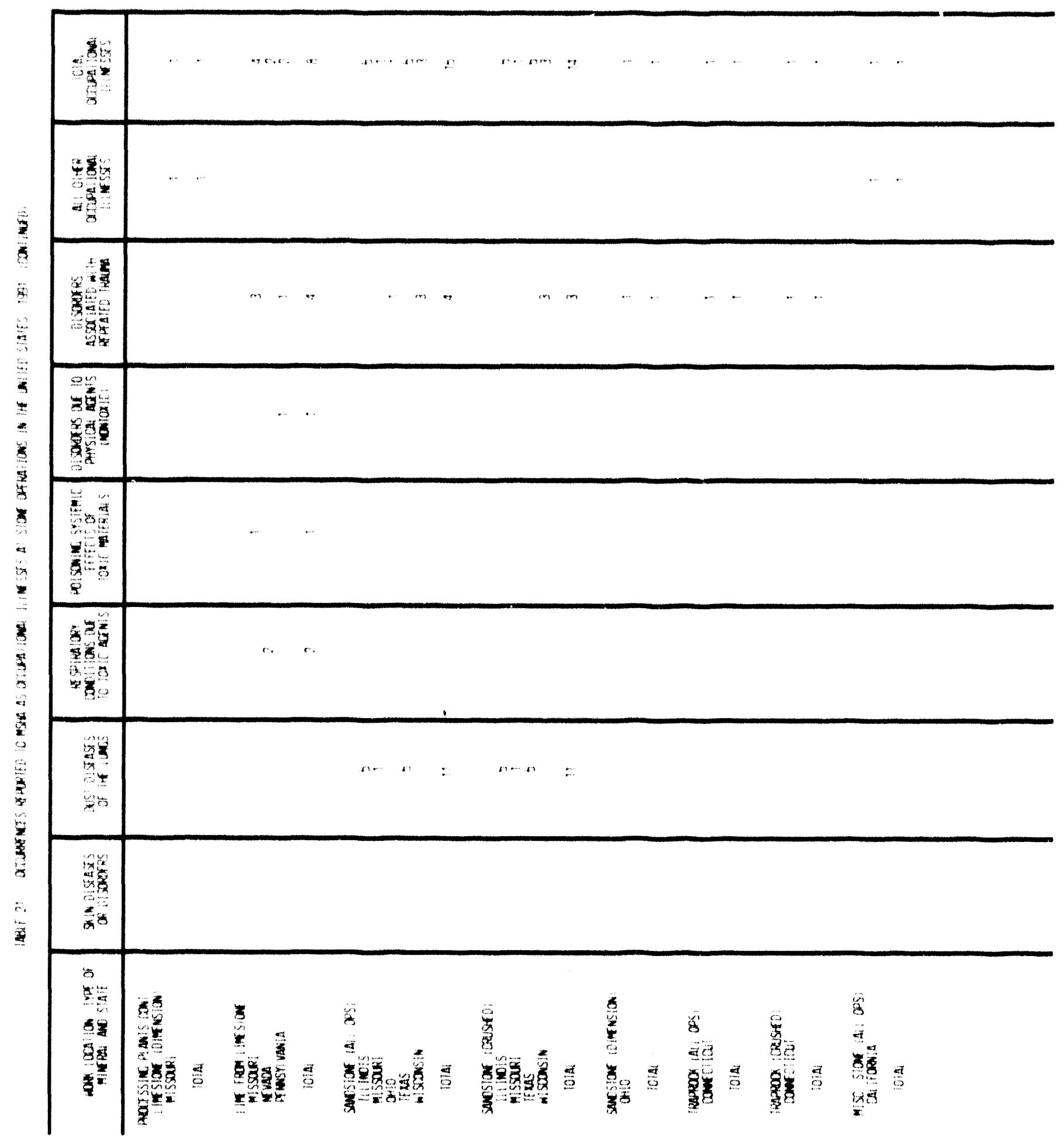




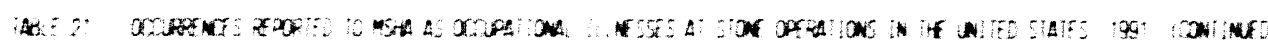

$\stackrel{5}{\infty}$

\begin{tabular}{|c|c|c|c|c|c|c|c|c|}
\hline 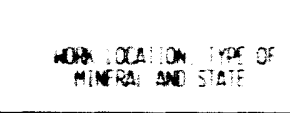 & 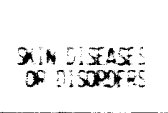 & 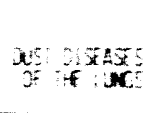 & and & 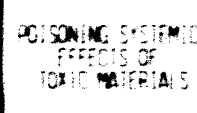 & 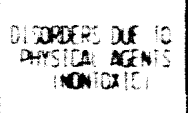 & 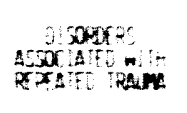 & 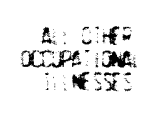 & $\sin ^{2}$ \\
\hline 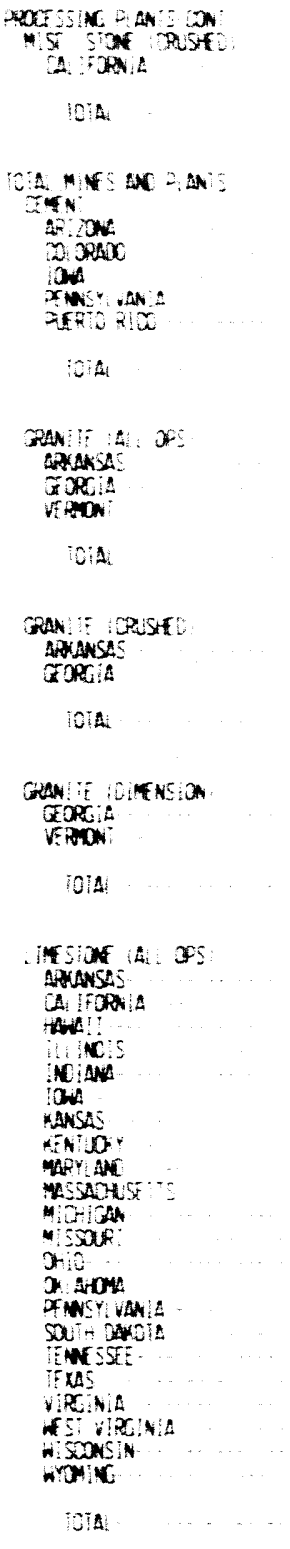 & $\begin{array}{l}\vdots \\
\vdots \\
\vdots \\
? \\
\vdots \\
\vdots \\
\vdots \\
\vdots \\
\vdots\end{array}$ & $\begin{array}{l} \\
\vdots \\
\vdots \\
\vdots \\
\vdots \\
\vdots\end{array}$ & $\therefore$ & 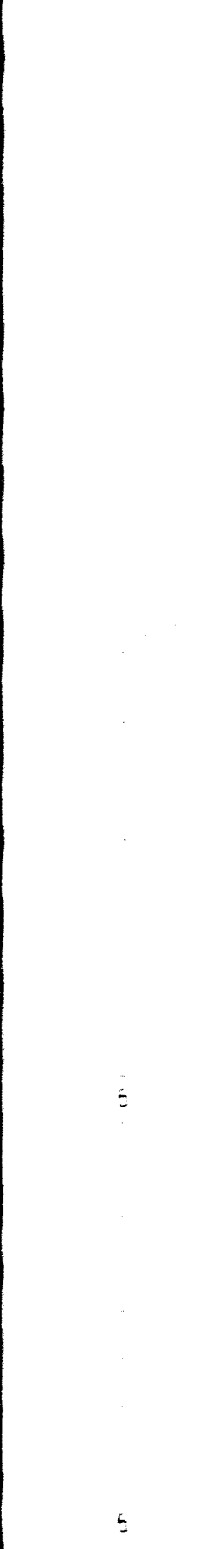 & $\begin{array}{l}: \\
\vdots \\
\vdots \\
3\end{array}$ & 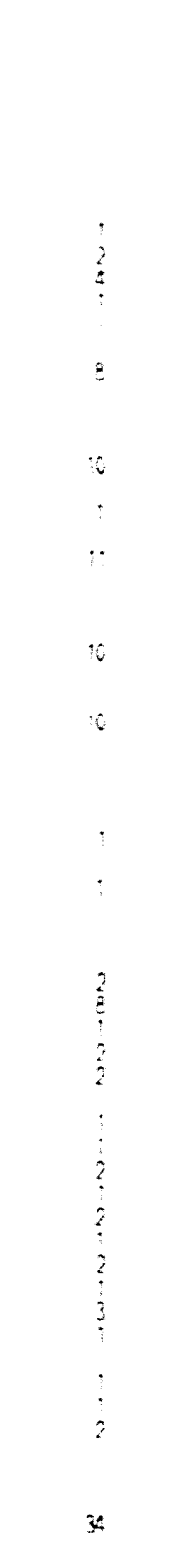 & $\begin{array}{l}2 \\
\vdots \\
\vdots \\
\\
=\end{array}$ & $\begin{array}{c}\vdots \\
\vdots \\
\ddots \\
\vdots \\
\vdots \\
\vdots \\
\vdots \\
\vdots \\
\vdots \\
\vdots \\
\vdots \\
\vdots \\
\vdots \\
\vdots \\
\vdots \\
\vdots \\
\vdots \\
\vdots \\
\vdots \\
\vdots\end{array}$ \\
\hline
\end{tabular}




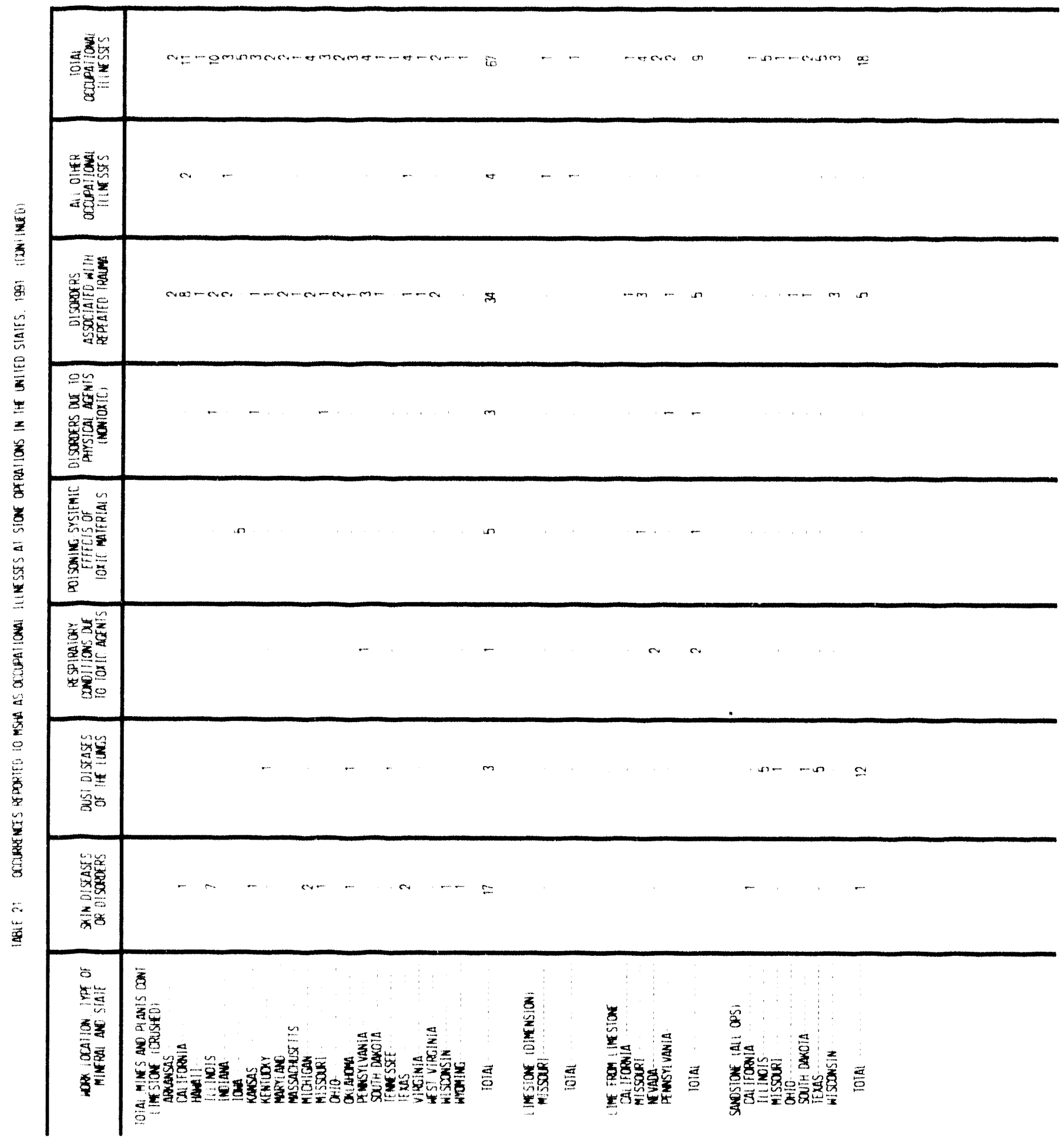




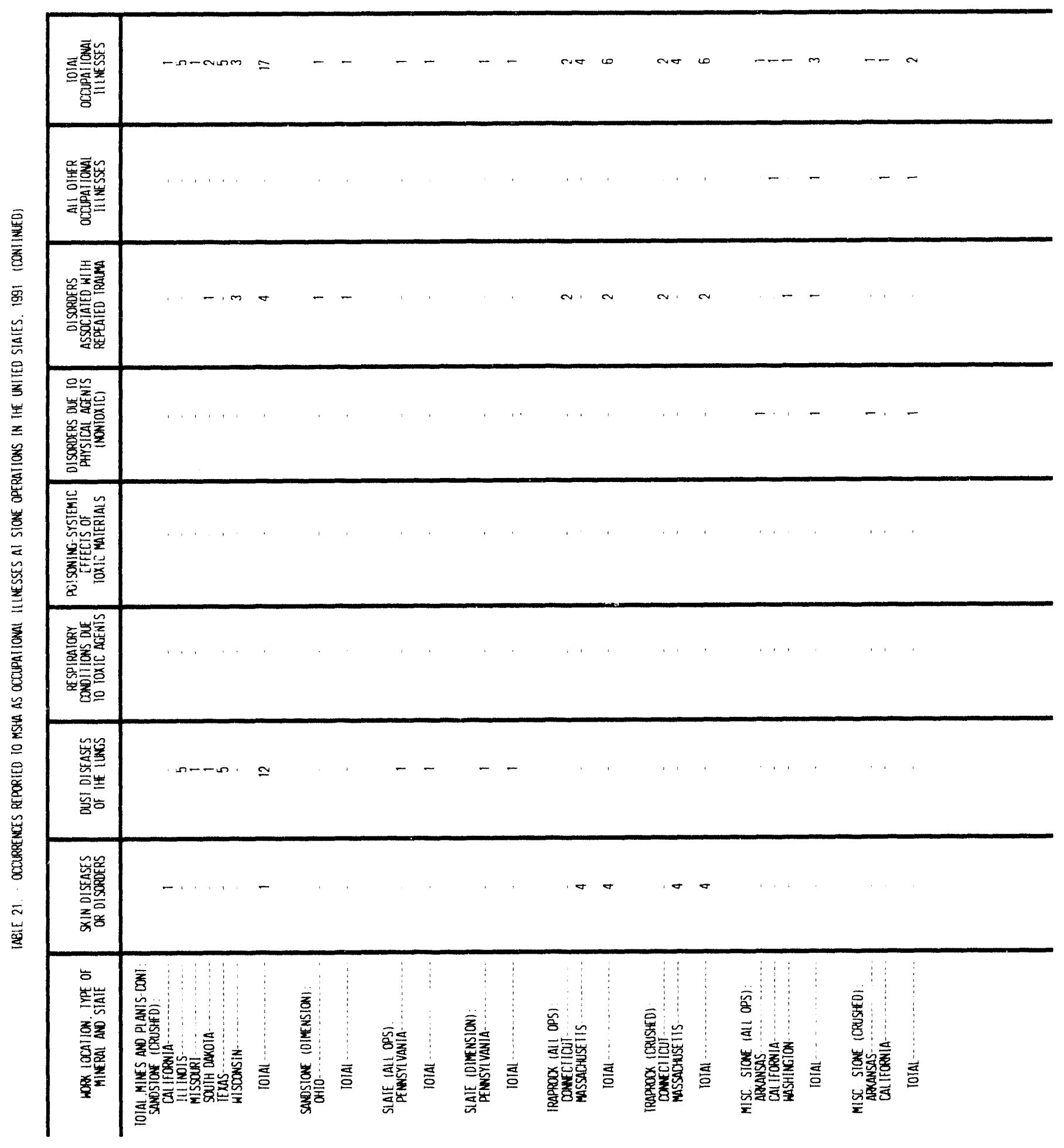




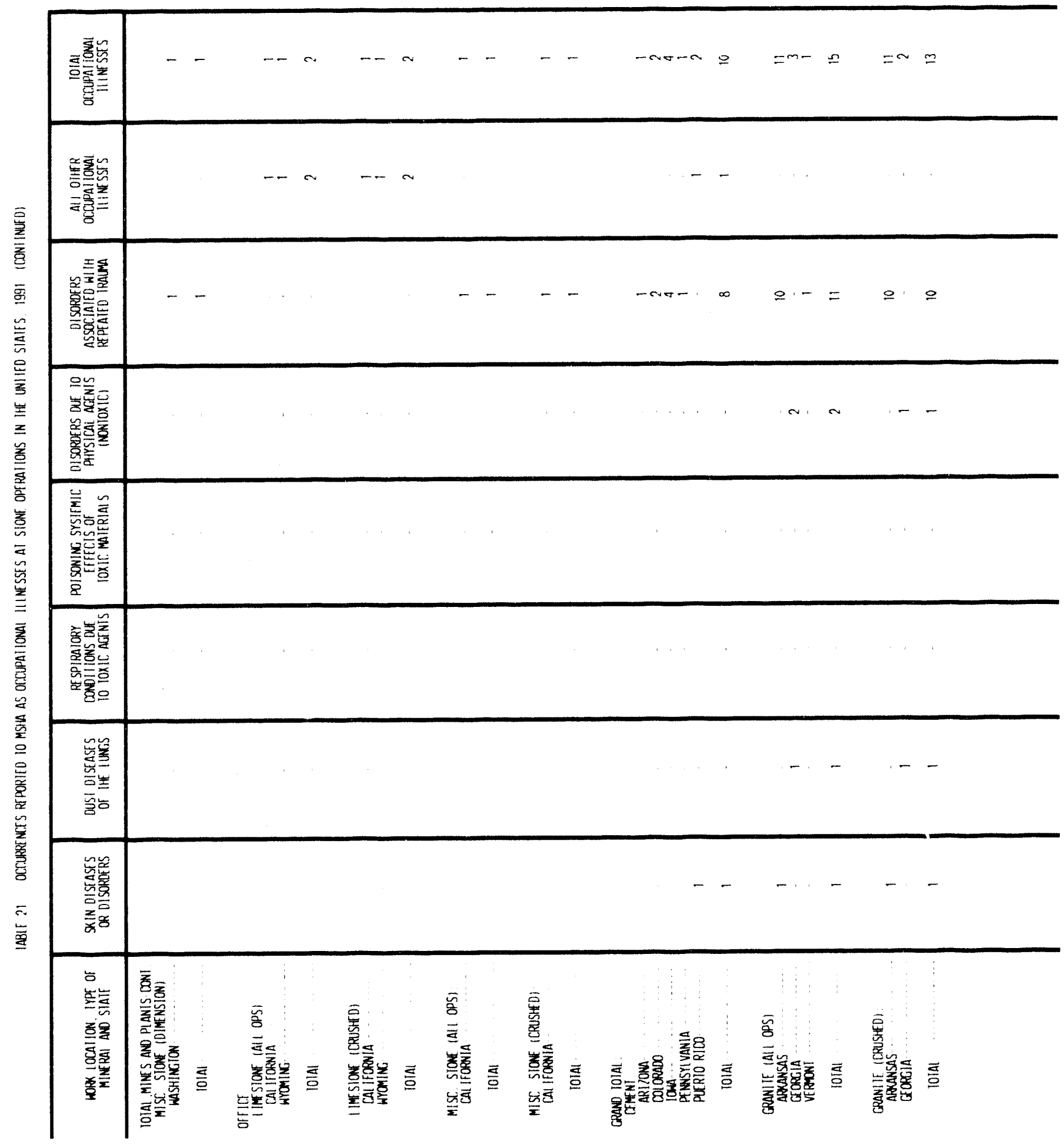




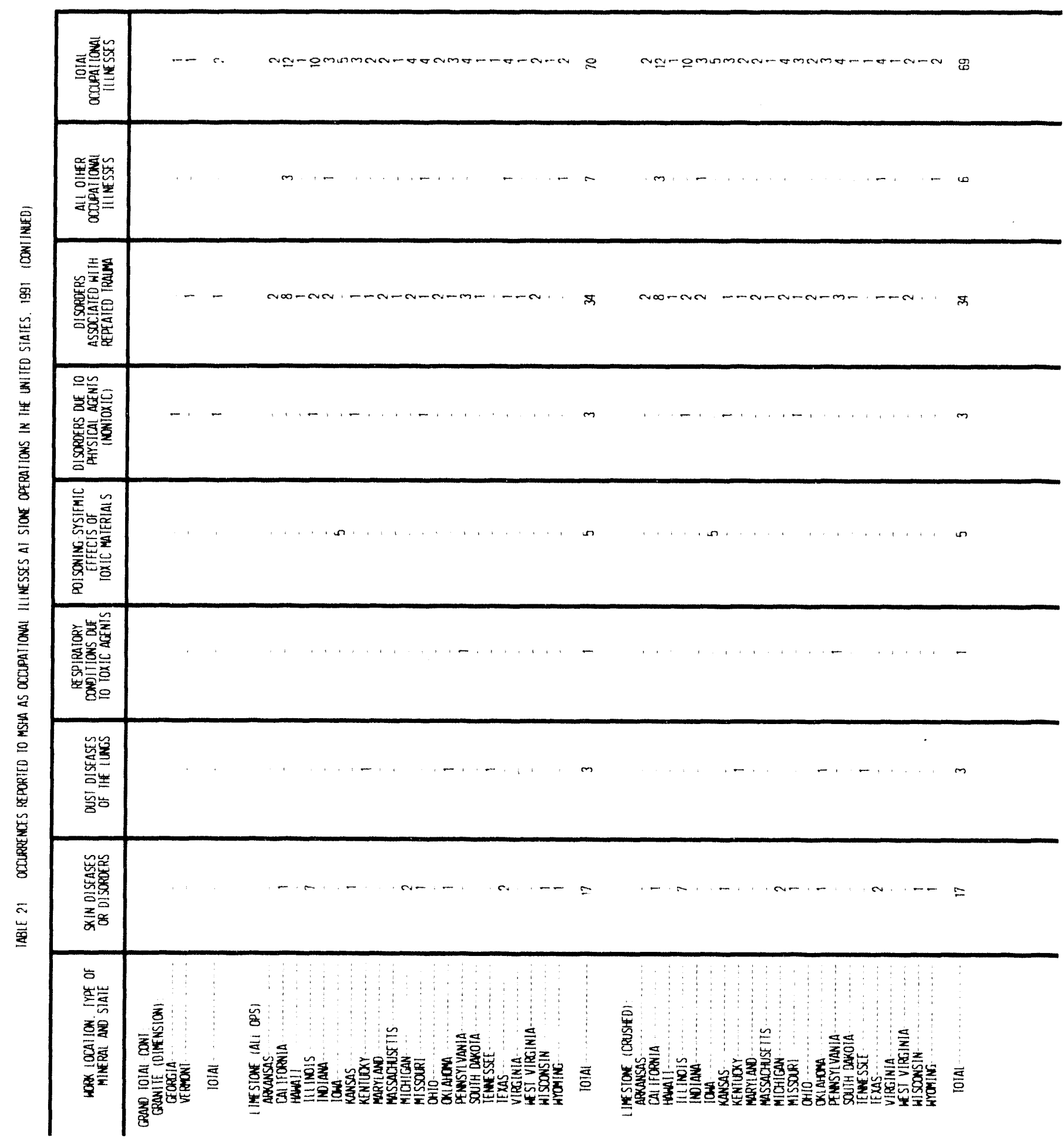




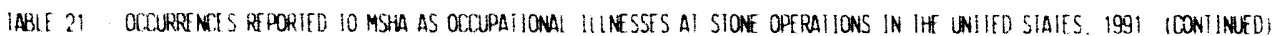

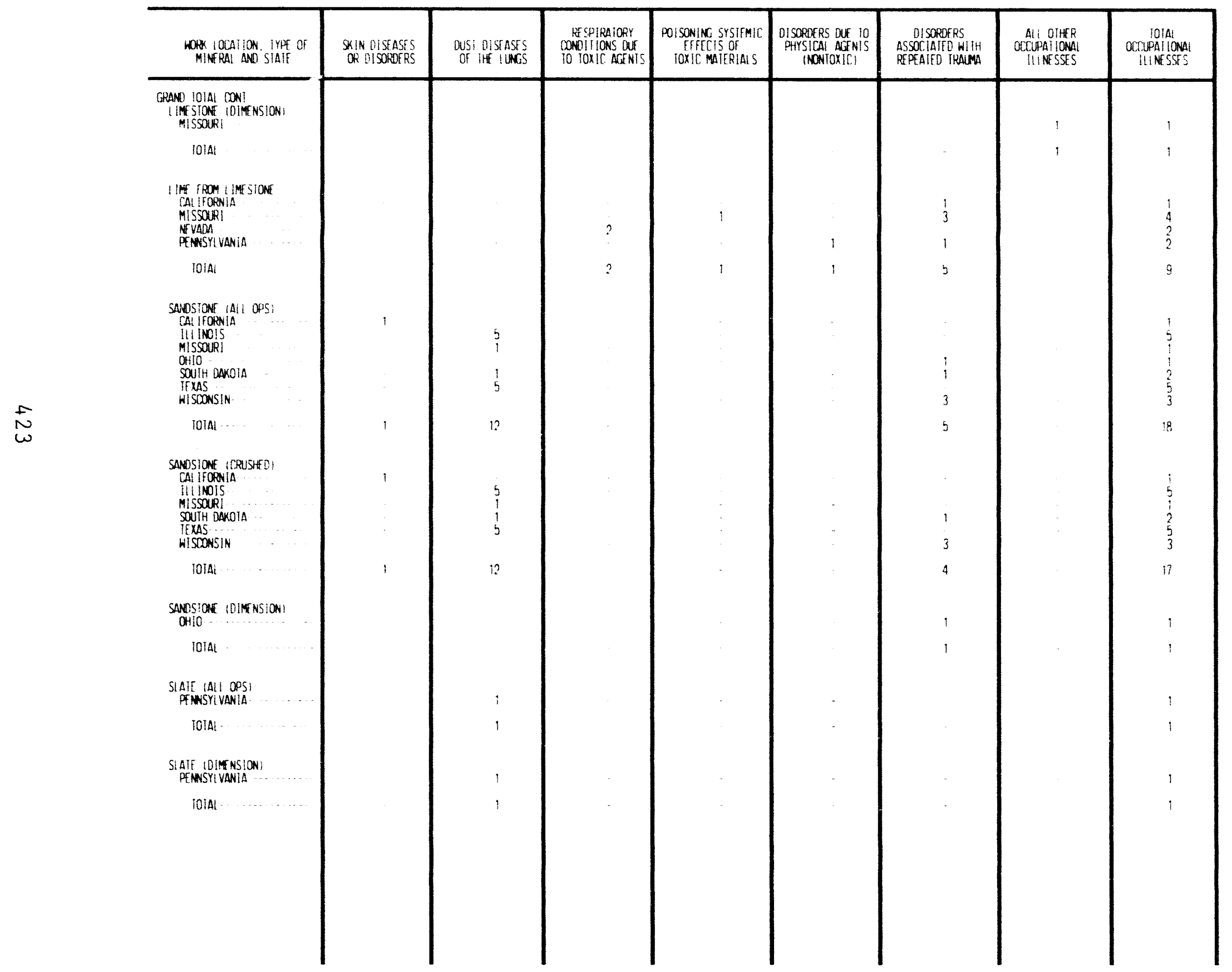




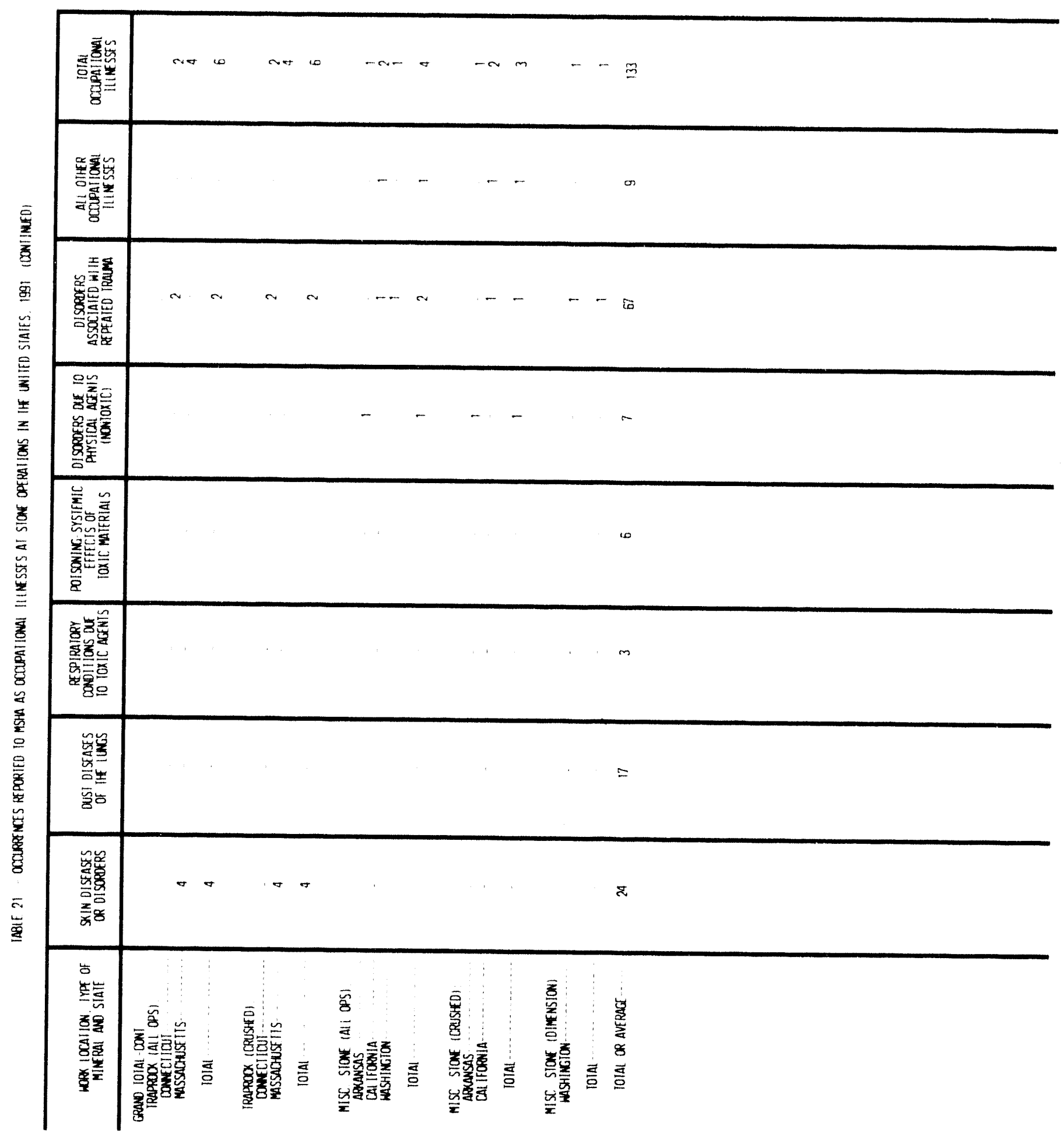


TABLE 22 - RELATED NONCOAL MINING SUMMARY REFERENCE TABULATION. NUMBER OF INJURIES. INCIDENCE RATES, AVERAGE NUMBER OF EMPLOYEES, AND EMPLOYEE-HOURS BY MINERAL INDUSTRY AND WORK LOCATION, (OPERATOR DATA)

\begin{tabular}{|c|c|c|c|c|c|c|c|c|c|c|}
\hline $\begin{array}{l}\text { WORK LOCATION } \\
\text { AND } \\
\text { MINERAL INDUSTRY }\end{array}$ & $|A| A \mid$ & $\begin{array}{c}\text { IAIAL } \\
\text { INCiGENCE } \\
\text { RAIf }\end{array}$ & NF U: & $\begin{array}{c}\text { NIDL } \\
\text { INLIDENCE } \\
\text { RATE }\end{array}$ & Noi & $\begin{array}{l}\text { NOL } \\
\text { INCIDENCE } \\
\text { PAIE }\end{array}$ & $\begin{array}{c}\text { Ali } \\
\text { OCCURPENC: }\end{array}$ & $\begin{array}{c}\text { A!I } \\
\text { INCIDENCE } \\
\text { PAI? }\end{array}$ & 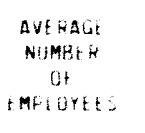 & 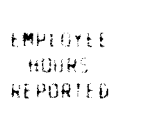 \\
\hline 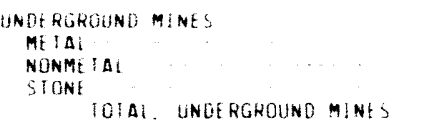 & $\begin{array}{c}8 \\
1 \\
1 \\
10\end{array}$ & $\begin{array}{l}10 \\
02 \\
02 \\
07\end{array}$ & $\begin{array}{l}531 \\
223 \\
102 \\
850\end{array}$ & $\begin{array}{ll}6 & 33 \\
5 & 16 \\
5 & 37 \\
5 & 86\end{array}$ & $\begin{array}{r}441 \\
98 \\
76 \\
6: 5\end{array}$ & $\begin{array}{ll}\begin{array}{l}5 \\
2\end{array} 26 \\
2 & 27 \\
4 & 00 \\
4 & 21\end{array}$ & $\begin{array}{r}980 \\
322 \\
178 \\
1.48\end{array}$ & $\begin{array}{rr}1 & t y \\
7 & 46 \\
9 & 4 \\
10 & 14\end{array}$ & $\begin{array}{r}8.543 \\
4.281 \\
1.944 \\
14.878\end{array}$ & $\begin{array}{r}1 ., 44.748 \\
8.36 .464 \\
3.800 .562 \\
25.201 .868\end{array}$ \\
\hline 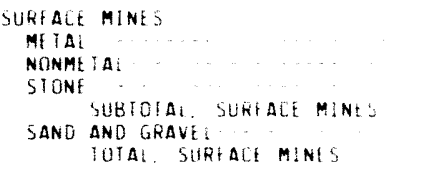 & $\begin{array}{r}5 \\
1 \\
9 \\
15 \\
11 \\
26\end{array}$ & $\begin{array}{l}03 \\
02 \\
03 \\
03 \\
04 \\
03\end{array}$ & $\begin{array}{r}663 \\
187 \\
1.396 \\
2.240 \\
1.267 \\
3.307\end{array}$ & $\begin{array}{ll}3 & 70 \\
3 & 03 \\
5 & 35 \\
4 & 47 \\
4 & 57 \\
4 & 51\end{array}$ & $\begin{array}{r}342 \\
130 \\
865 \\
1.337 \\
801 \\
2.138\end{array}$ & $\begin{array}{ll}3 & 91 \\
2 & 10 \\
3 & 33 \\
2 & 6 ? \\
2 & 89 \\
2 & 75\end{array}$ & $\begin{array}{r}1.010 \\
318 \\
2.254 \\
3549 \\
2.079 \\
5.67 ?\end{array}$ & $\begin{array}{ll}5 & 64 \\
5 & 15 \\
5 & 7 \\
3 & 17 \\
3 & 50 \\
7 & 29\end{array}$ & $\begin{array}{r}17.315 \\
6.670 \\
27.632 \\
51.617 \\
34.335 \\
85.952\end{array}$ & 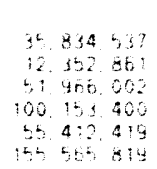 \\
\hline IOTAL. MINES & 36 & 04 & 4. 36.3 & 4. 72 & 2.753 & 298 & 7. 152 & 774 & 100.850 & $144.70 \% .607$ \\
\hline $\begin{array}{l}\text { MILIS } \\
\text { MEIAI } \\
\text { NOMETIAI } \\
\text { SIONE TOTAL MIISS }\end{array}$ & $\begin{array}{l}1 \\
4 \\
5\end{array}$ & $\begin{array}{l}0 ! \\
0 ! \\
0 !\end{array}$ & $\begin{array}{r}80 E \\
708 \\
1.853 \\
3.367\end{array}$ & $\begin{array}{ll}3 & 94 \\
4 & 14 \\
5 & 32 \\
4 & 65\end{array}$ & $\begin{array}{r}590 \\
387 \\
2323 \\
2300\end{array}$ & $\begin{array}{ll}2 & 88 \\
2 & 26 \\
3 & 79 \\
3 & 18\end{array}$ & $\begin{array}{l}1.346 \\
1.056 \\
3.180 \\
5.672\end{array}$ & $\begin{array}{ll}5 & 82 \\
5 & 4 \\
9 & 12 \\
7 & 83\end{array}$ & $\begin{array}{l}20.80: \\
16.921 \\
34.080 \\
70.868\end{array}$ & 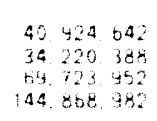 \\
\hline $\begin{array}{l}\text { INDE PENDEN SHOPS ANE TAGDS } \\
\text { MEIAL } \\
\text { NONMETAI } \\
\text { STONE TOTAI. SHOPS AND YAROS }\end{array}$ & & & $\begin{array}{l}: 1 \\
33\end{array}$ & $\begin{array}{l}581 \\
5.98 \\
587\end{array}$ & $\begin{array}{l}10 \\
22\end{array}$ & $\begin{array}{ll}3 & 17 \\
5 & 44 \\
3 & 9 !\end{array}$ & 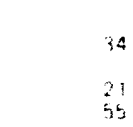 & $\begin{array}{cc}8 & 94 \\
11 & 42 \\
9 & 78\end{array}$ & $\begin{array}{l}36: \\
169 \\
551\end{array}$ & $\begin{array}{r}75+74 \\
36793 \\
124.464\end{array}$ \\
\hline 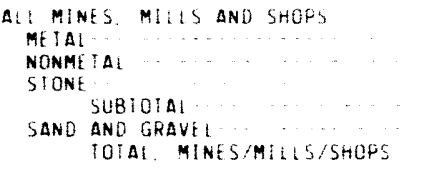 & $\begin{array}{r}13 \\
3 \\
14 \\
30 \\
11 \\
41\end{array}$ & $\begin{array}{l}03 \\
01 \\
02 \\
02 \\
04 \\
0.2\end{array}$ & $\begin{array}{l}2.022 \\
1.118 \\
3.356 \\
6496 \\
1.267 \\
7.763\end{array}$ & $\begin{array}{l}429 \\
405 \\
533 \\
472 \\
457 \\
4 \quad 69\end{array}$ & $\begin{array}{r}1.385 \\
615 \\
2.274 \\
4.274 \\
801 \\
5.075\end{array}$ & $\begin{array}{ll}2 & 94 \\
2 & 23 \\
3 & 5 \\
3 & 10 \\
2 & 89 \\
3 & 07\end{array}$ & $\begin{array}{r}3.420 \\
1.736 \\
5.644 \\
10.800 \\
2.079 \\
12.879\end{array}$ & $\begin{array}{ll}3 & 25 \\
6 & 29 \\
8 & 97 \\
7 & 84 \\
7 & 50 \\
7 & 79\end{array}$ & $\begin{array}{r}46.517 \\
27.472 \\
63.825 \\
137.914 \\
34.335 \\
172.249\end{array}$ & 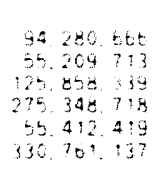 \\
\hline 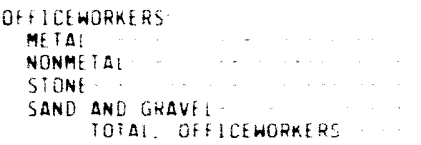 & & & $\begin{array}{r}8 \\
8 \\
9 \\
5 \\
30\end{array}$ & $\begin{array}{l}13 \\
19 \\
09 \\
10 \\
12\end{array}$ & $\begin{array}{r}14 \\
5 \\
15 \\
8 \\
42\end{array}$ & $\begin{array}{l}23 \\
12 \\
15 \\
17 \\
17\end{array}$ & $\begin{array}{l}22 \\
13 \\
24 \\
13 \\
72\end{array}$ & $\begin{array}{l}36 \\
30 \\
24 \\
27 \\
23\end{array}$ & $\begin{array}{r}6.25 \\
4.740 \\
10.97 \\
7.22 \\
29.18\end{array}$ & 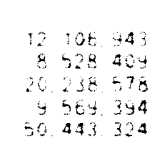 \\
\hline 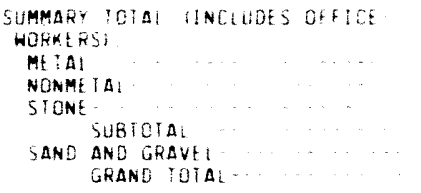 & $\begin{array}{r}13 \\
3 \\
14 \\
30 \\
1 ! \\
4 !\end{array}$ & $\begin{array}{l}02 \\
01 \\
02 \\
02 \\
63 \\
02\end{array}$ & $\begin{array}{l}2.030 \\
1.26 \\
3.365 \\
0.521 \\
1.272 \\
7.793\end{array}$ & $\begin{array}{ll}3 & 82 \\
3 & 53 \\
4 & 61 \\
4 & 12 \\
3 & 91 \\
4 & 09\end{array}$ & $\begin{array}{r}\text { 1. } 399 \\
\text { 2. } 520 \\
\text { 4. } 289 \\
808 \\
809 \\
\text { 5. } 117\end{array}$ & $\begin{array}{ll}2 & 63 \\
1 & 95 \\
3 & 13 \\
2 & 72 \\
2 & 49 \\
2 & 68\end{array}$ & $\begin{array}{r}3.442 \\
1.749 \\
5.668 \\
10.859 \\
2.092 \\
12.95\end{array}$ & $\begin{array}{ll}6 & 47 \\
5 & 49 \\
7 & 76 \\
2 & 87 \\
6 & 14 \\
0 & 79\end{array}$ & $\begin{array}{r}22.870 \\
32.29 \\
7496 \\
159878 \\
49.56 \\
201.434\end{array}$ & 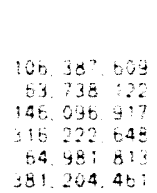 \\
\hline
\end{tabular}



TABLE 23 - RELATED COAL MINING SUMMARY REFERENCE TABULATION. NUMBER OF INUURIES. INCIDENCE RATES, AVERAGE
NUMBER OF EMPLOYEES. EMPLOYEE-HOUPS AND PRODUCTION BY TYPE OF COAL MINED AND WORK LOCATION. 1991 (OPERATOR DATA)

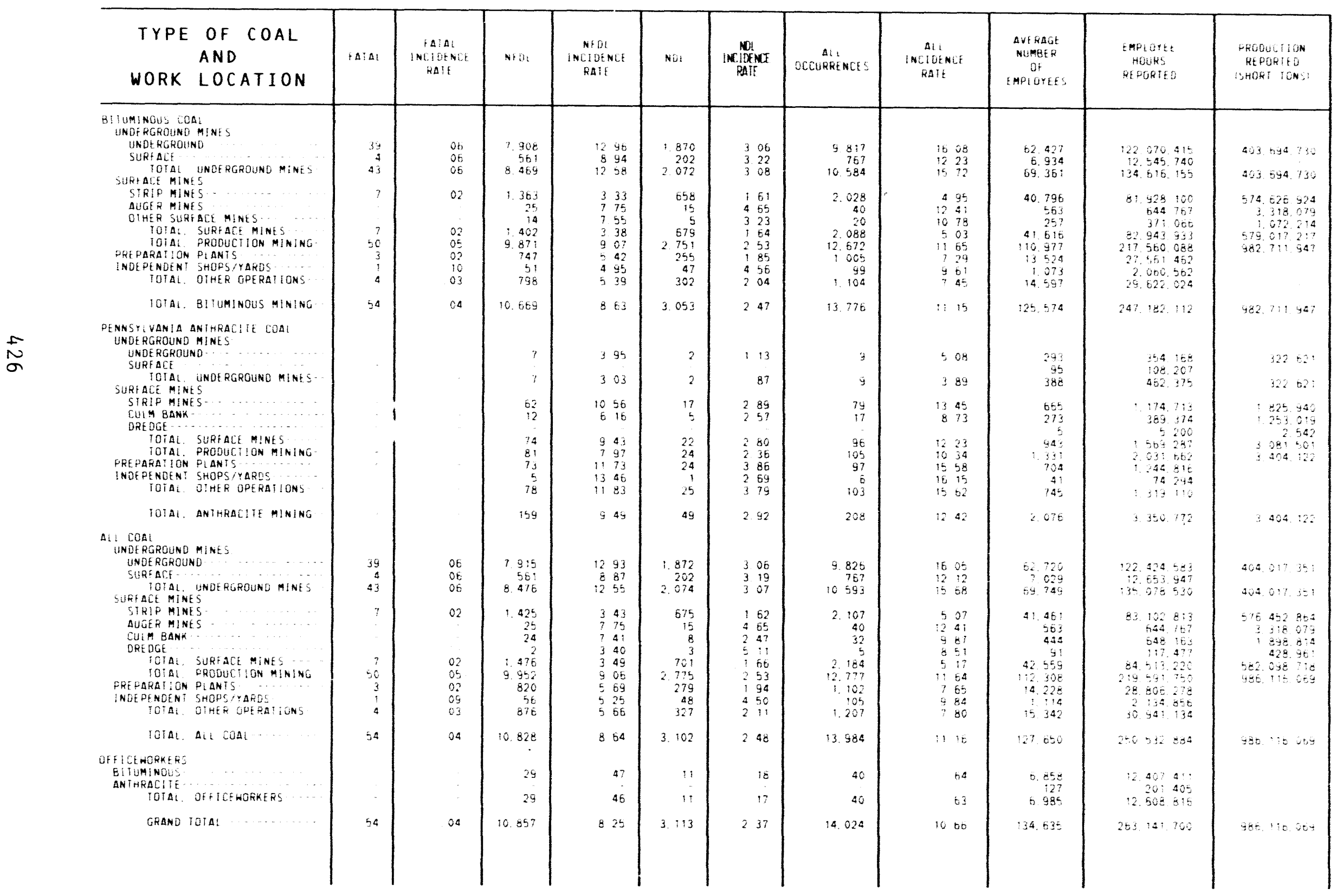


CONTRACTOR DATA 


\section{TABLE REFERENCE GUIDE (CONTRACTOR DATA)}

The following reference guide facilitates locating particular data elements contained in the annual statistical tables for contractors. The number appearing in each cell indicates the table number in which that particular element may be found. All elements listed under the stub headings appear as vertical headings in the tables. Elements listed under column headings appear as horizontal headings. The only exception to this is table 1 , in which the headings have been reversed.

To use this guide, read down or across the elements 1 isted in both the stub and column headings to determine which combinations are of interest. For example, lost workdays by work location appear in eight different tables, numbers $3,7,9,11$, $14,16,17$. and 18. However, only one of these, table 11, provides a further division by nature of injury as well. The occupation of the injured is found in table 17 and, as seen in the column headings, provides the number of injuries, lost workdays, and average severity.

Although injuries and illnesses occurring to contractor employees are identifiable as to state in which they occurred or commodity mined, matching exposure hours are not available. Incidence rates for such employees are possible only at the national level for the various subunits and only for the coal industry as a whole and the noncoal industry as a whole. Consequently, certain tables have been entirely eliminated from the contractor portion of this report. The remaining tables; are numbered to correspond with the comparable table in the operator portion of this report. 


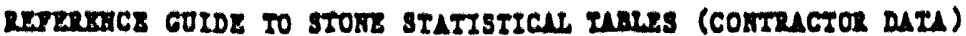

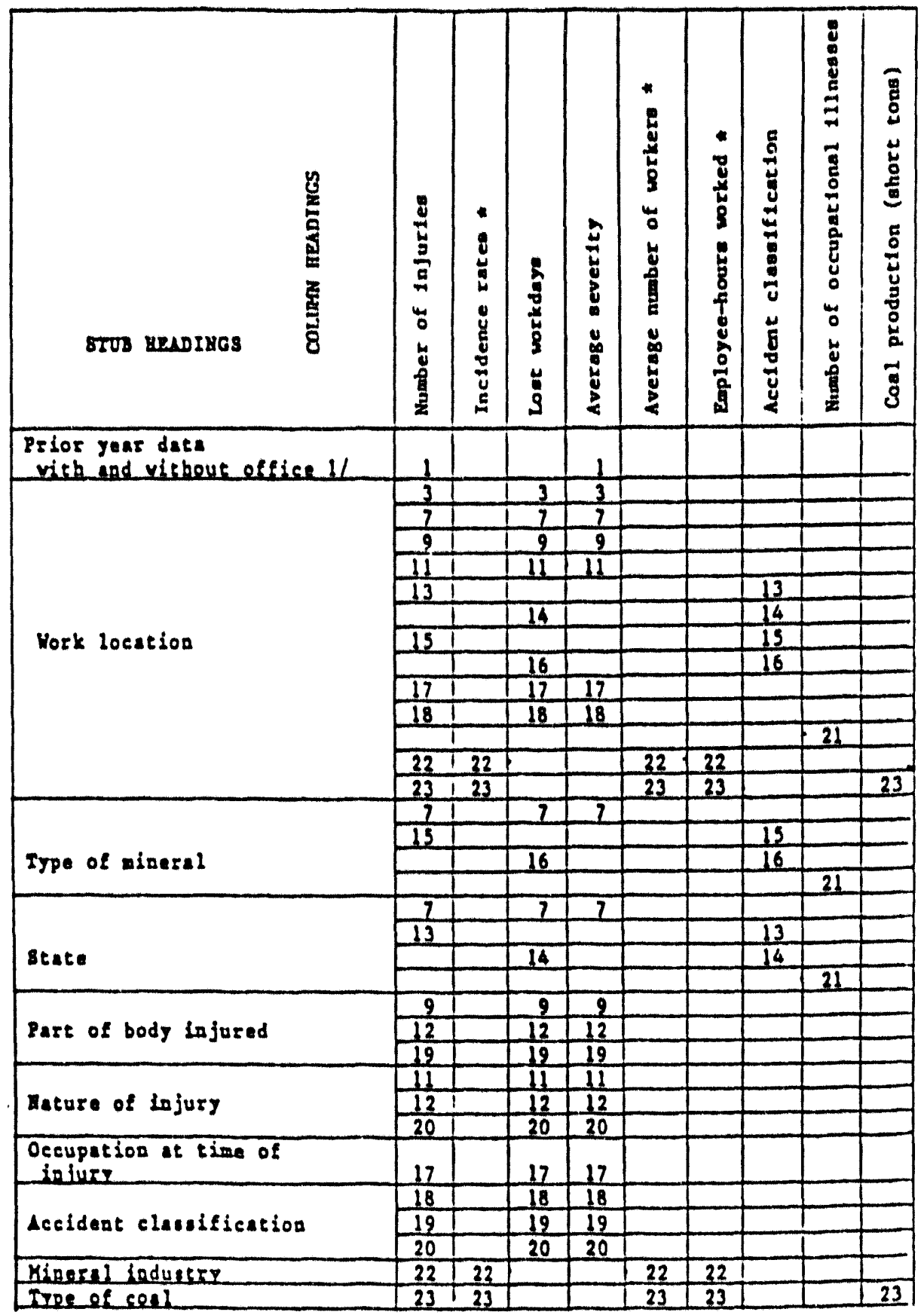

1/ Tor table I only, the heading bave been reversed to aimplify this guide; that is, the data elements Indicated in the columa besdings for this table actually appear as rertical beadings.

- Contractor daca appeariog in Iable 22 for the colum beadingu denoted with an aoteriak (*) La this guide are obtainable oply for the noocosl loduatry at atole and do not repreceat a particular alueral laduaty. 


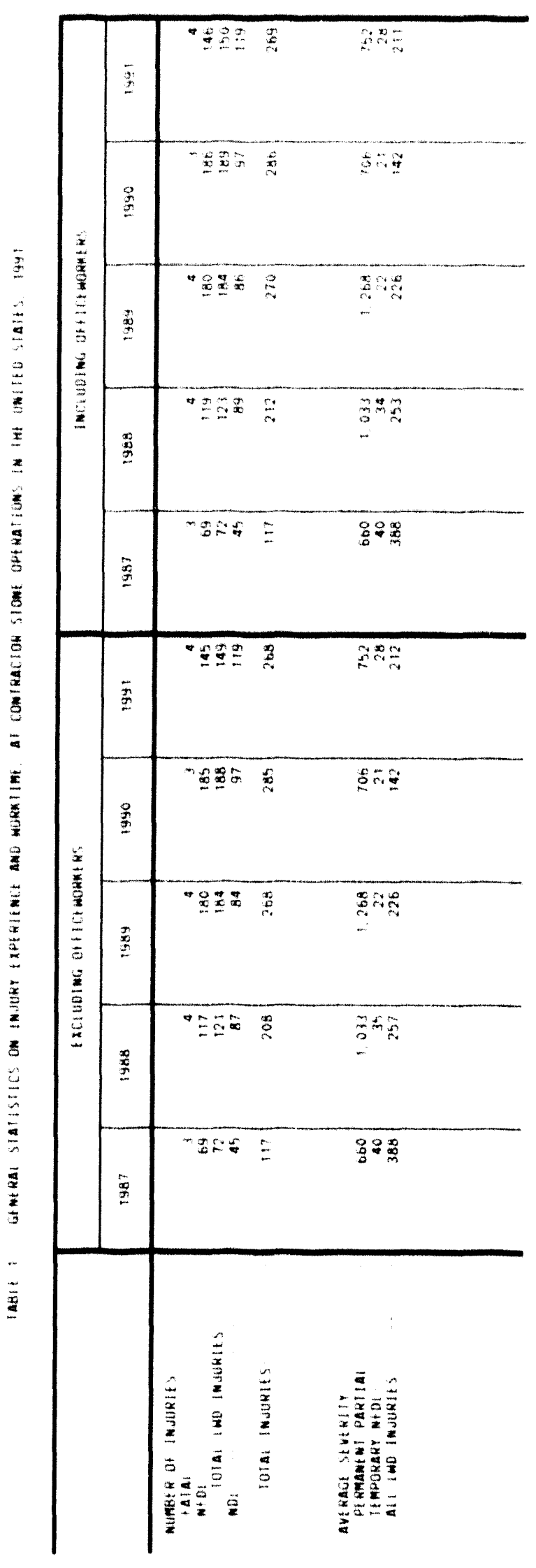




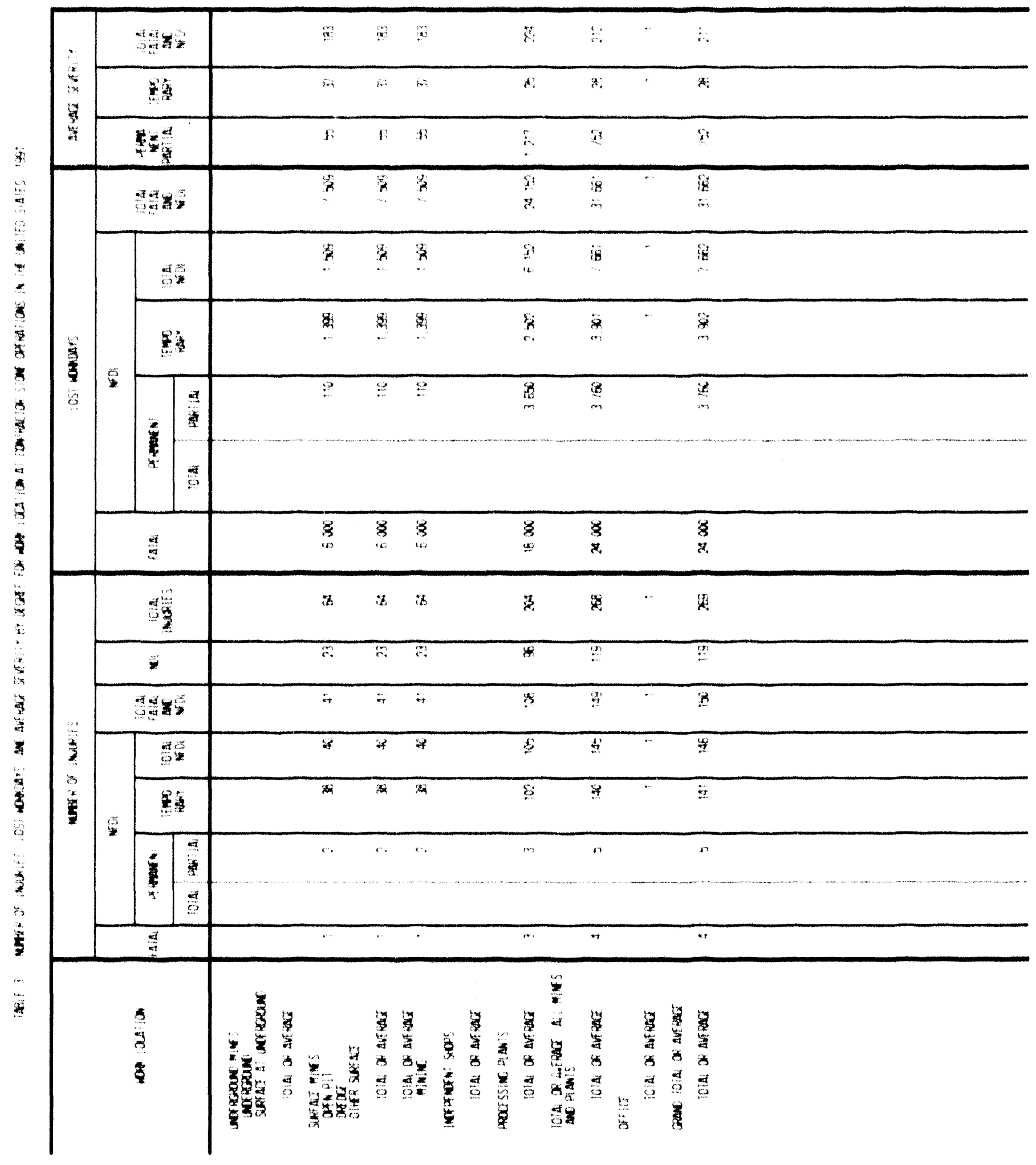




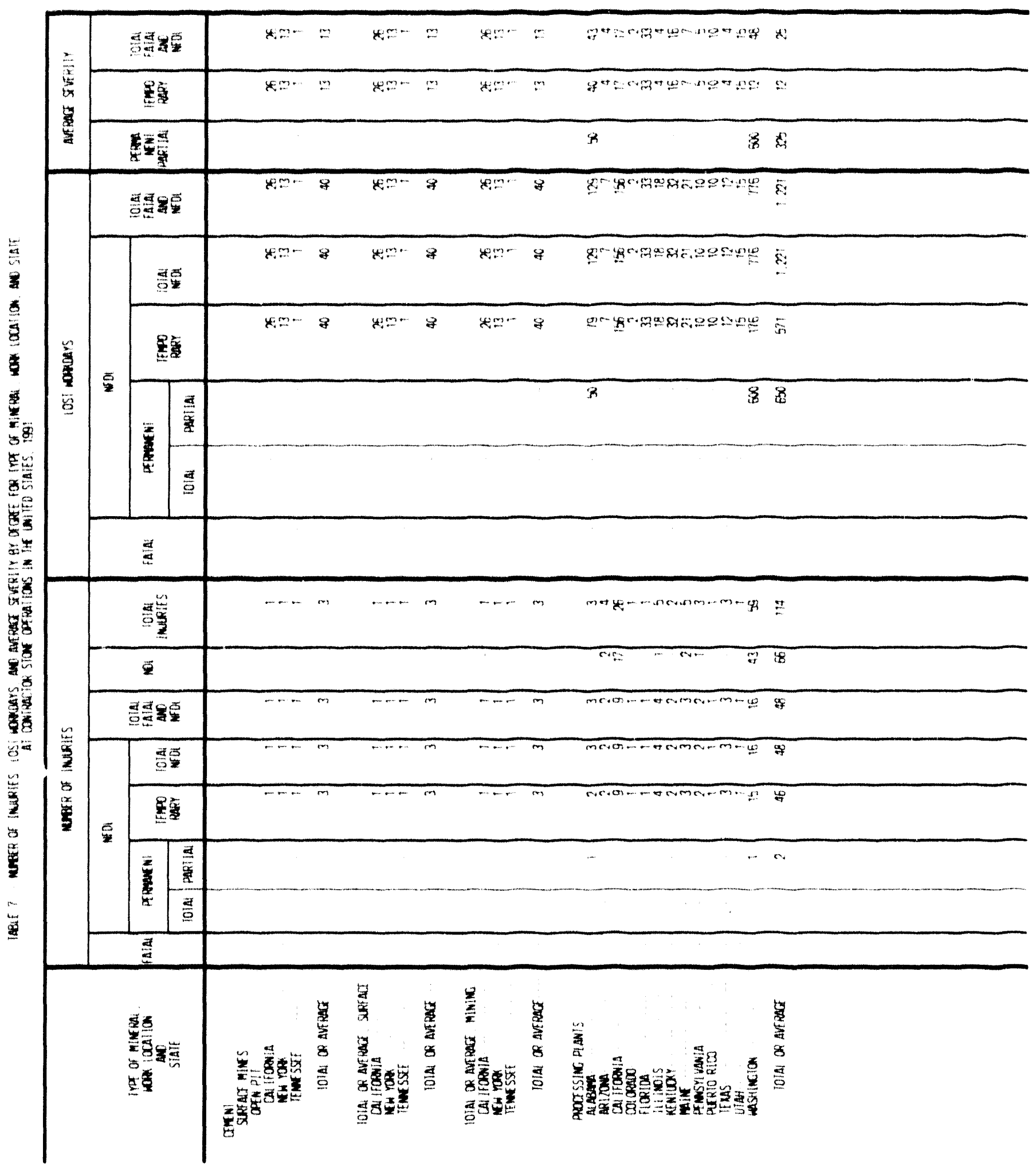




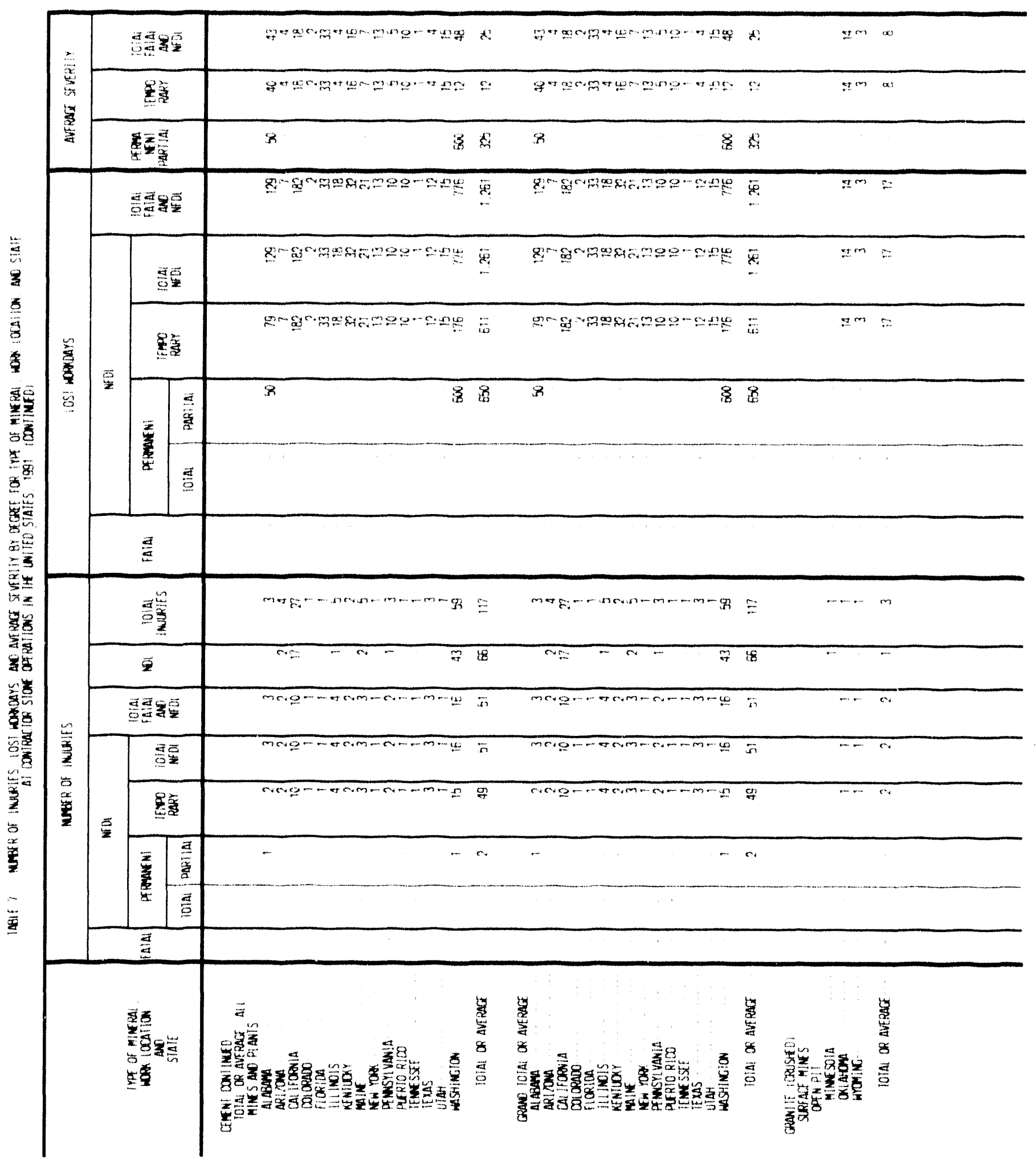




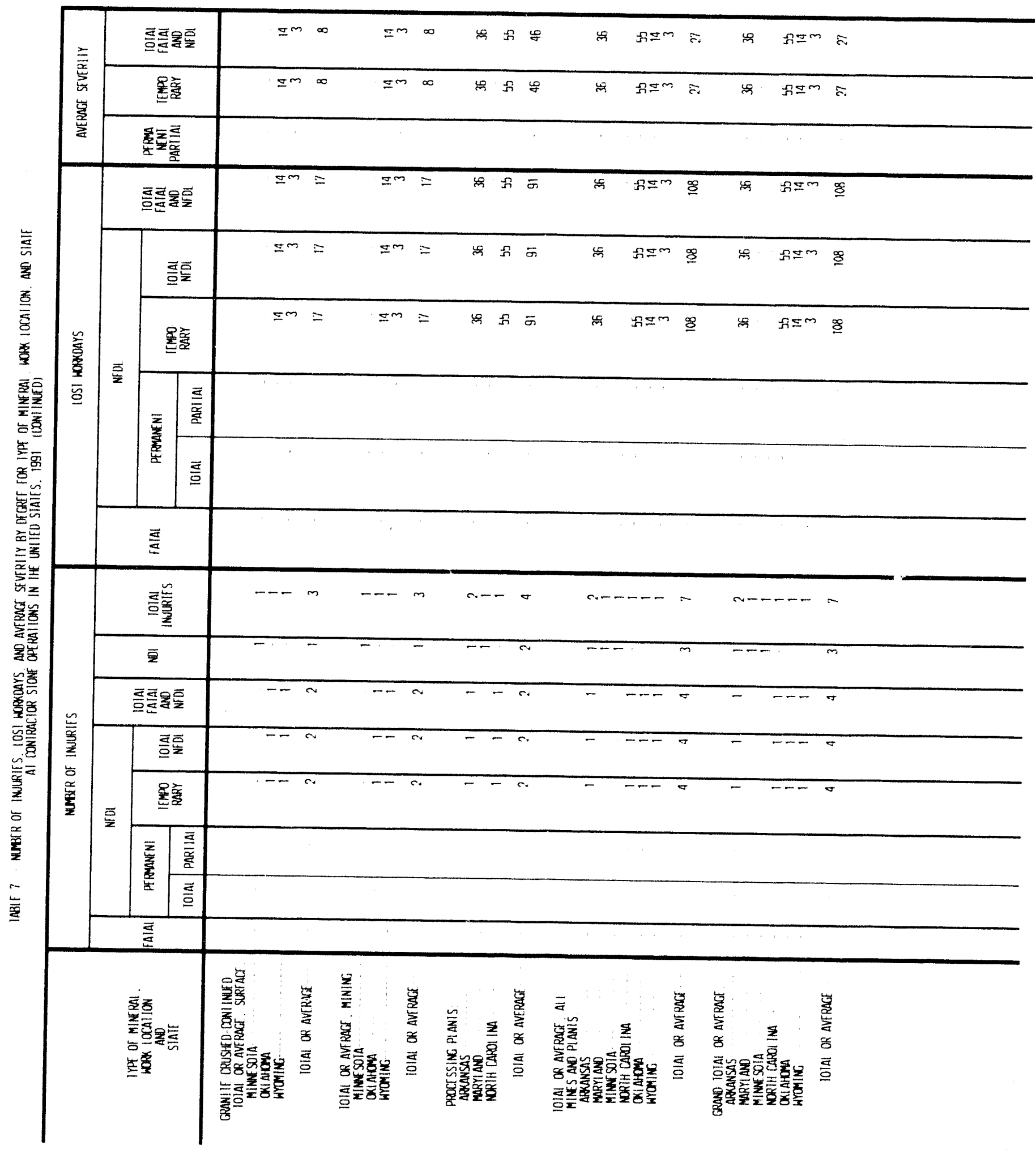




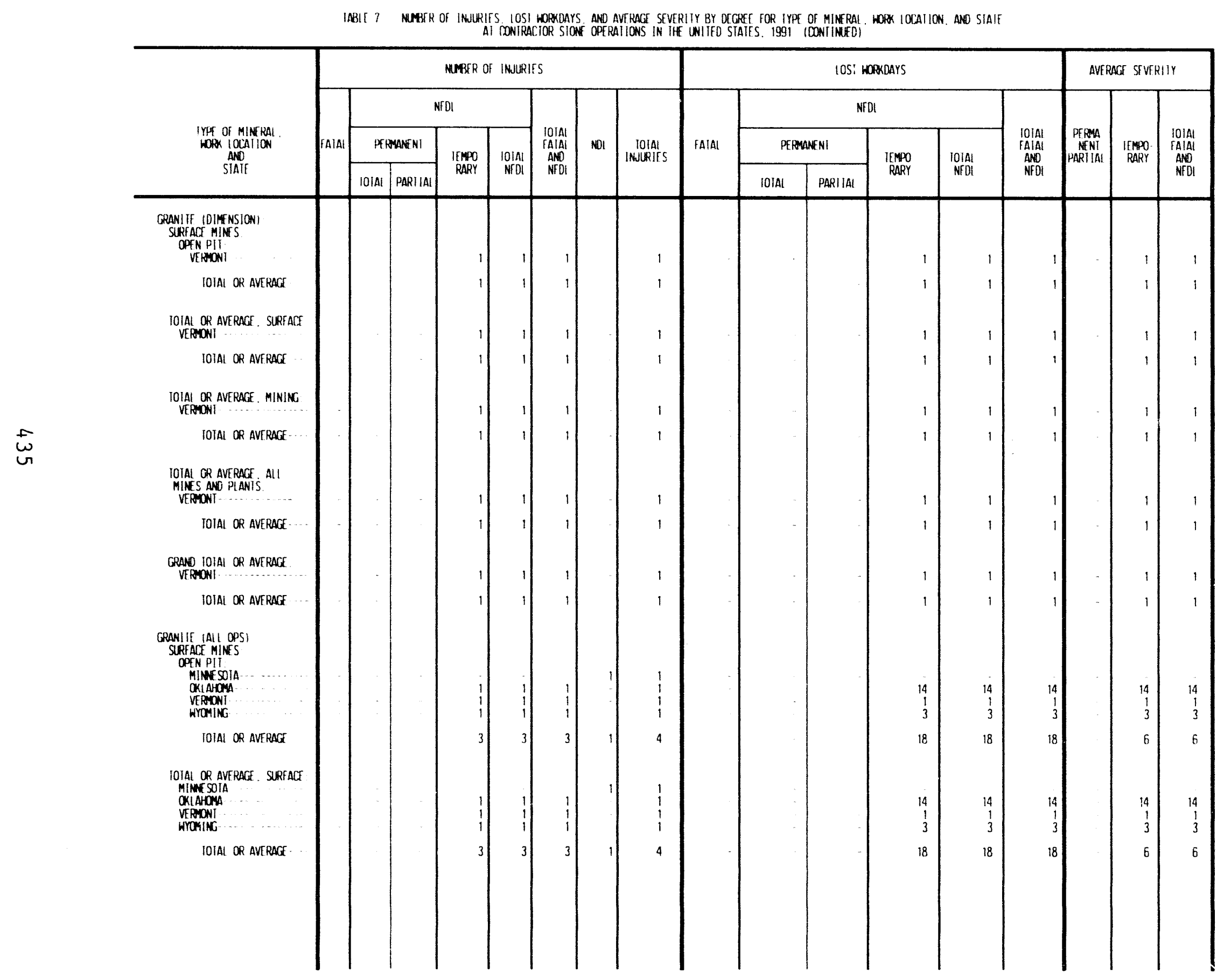




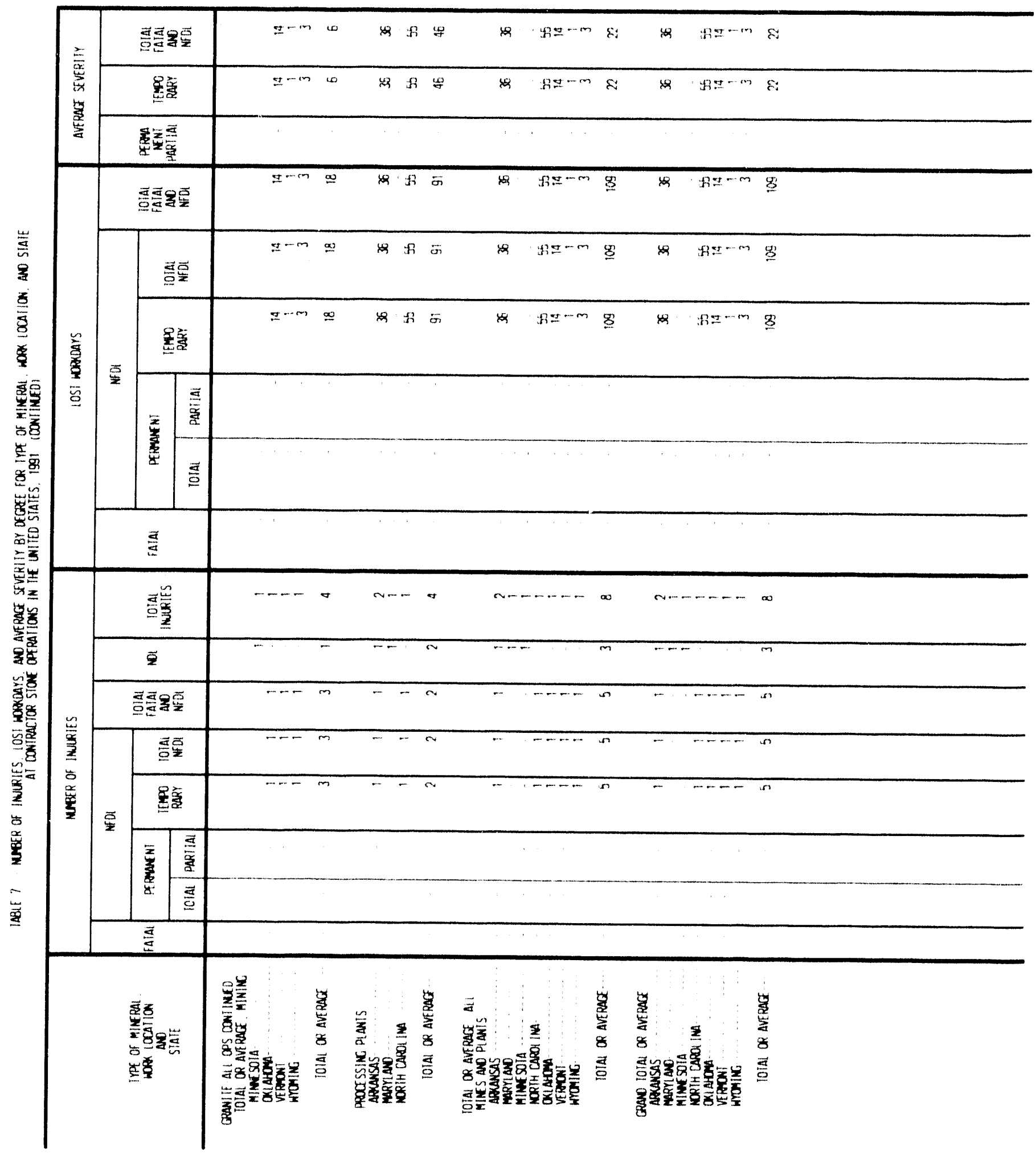




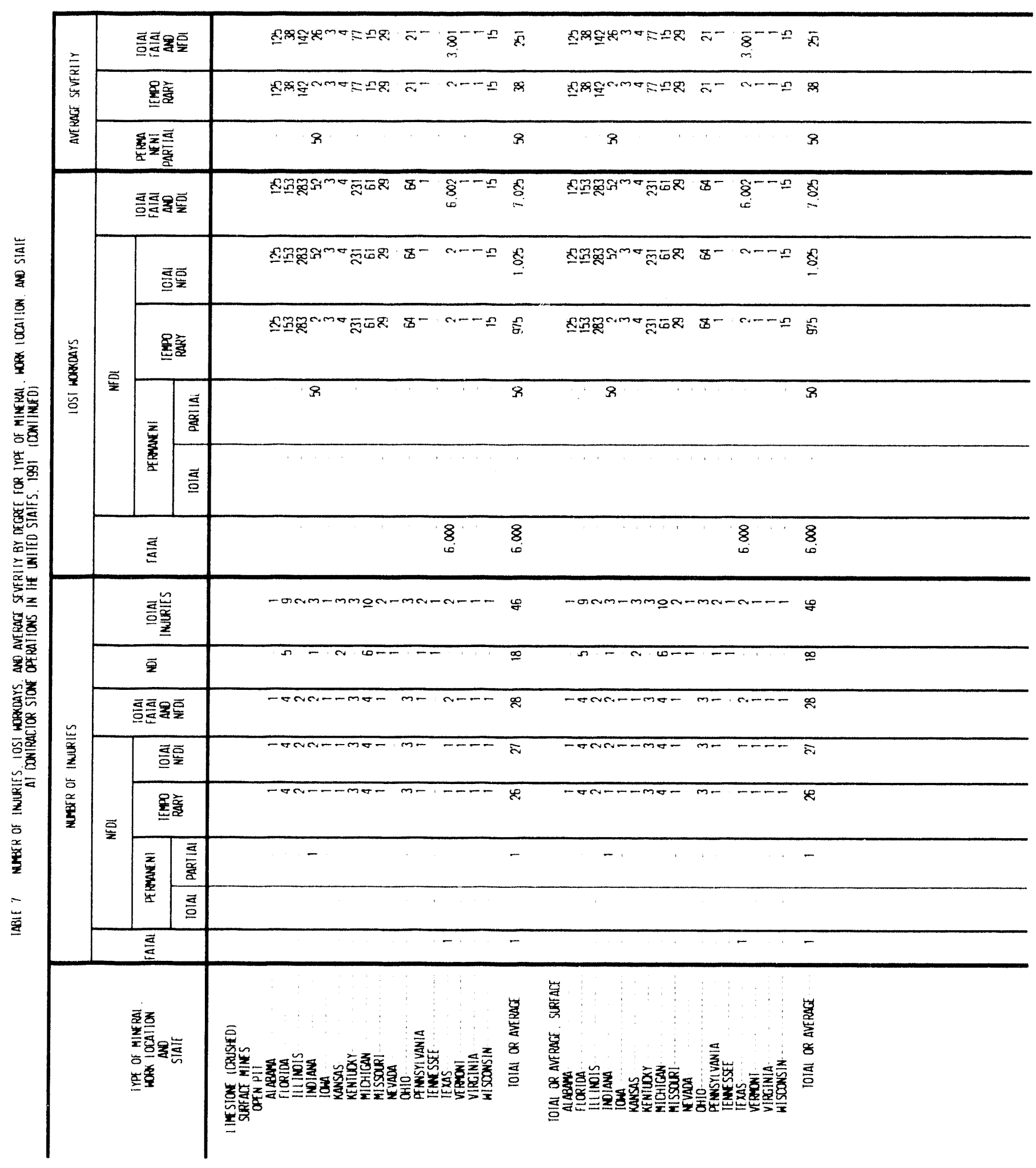




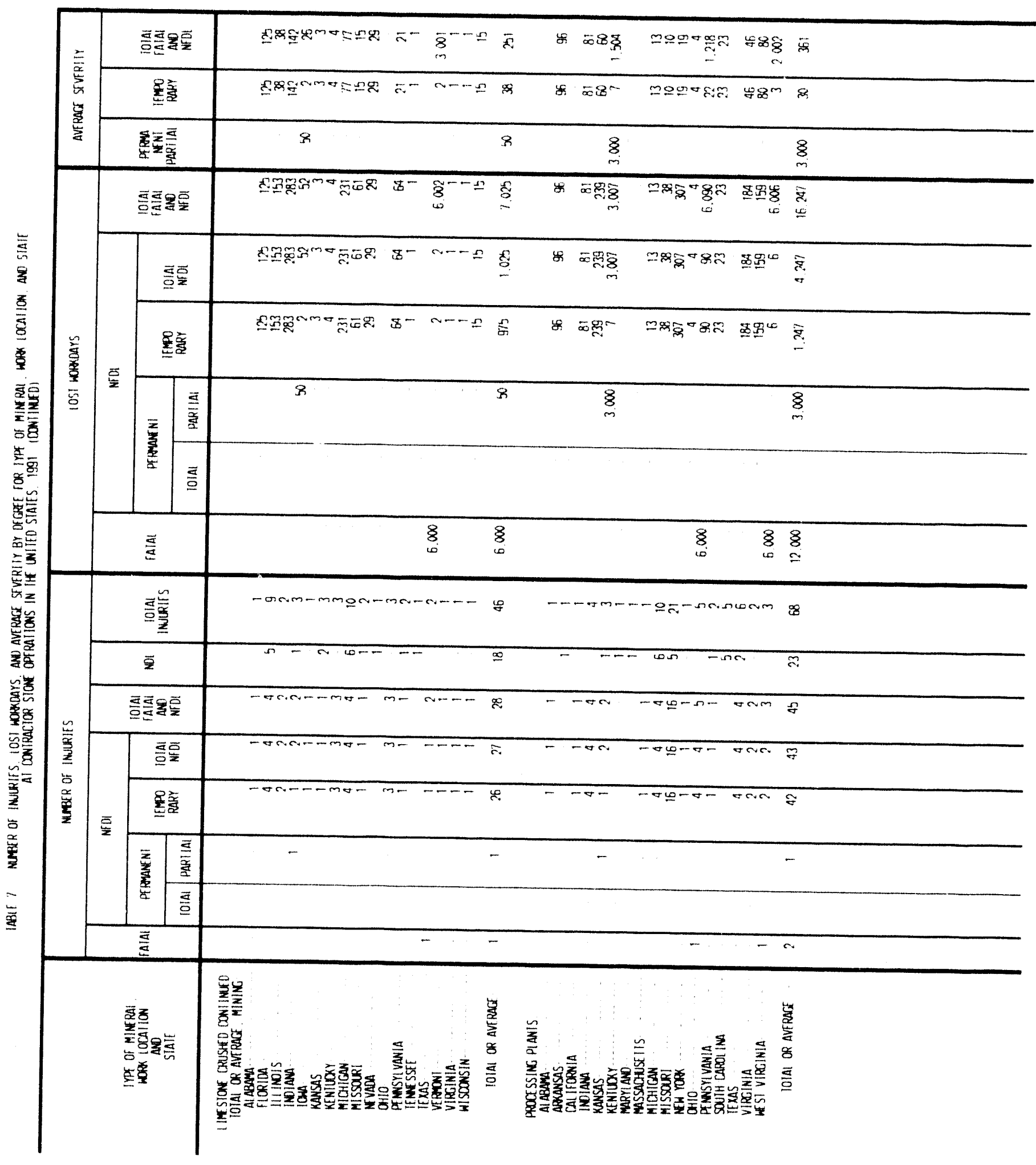




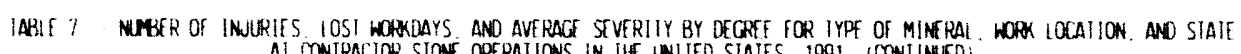

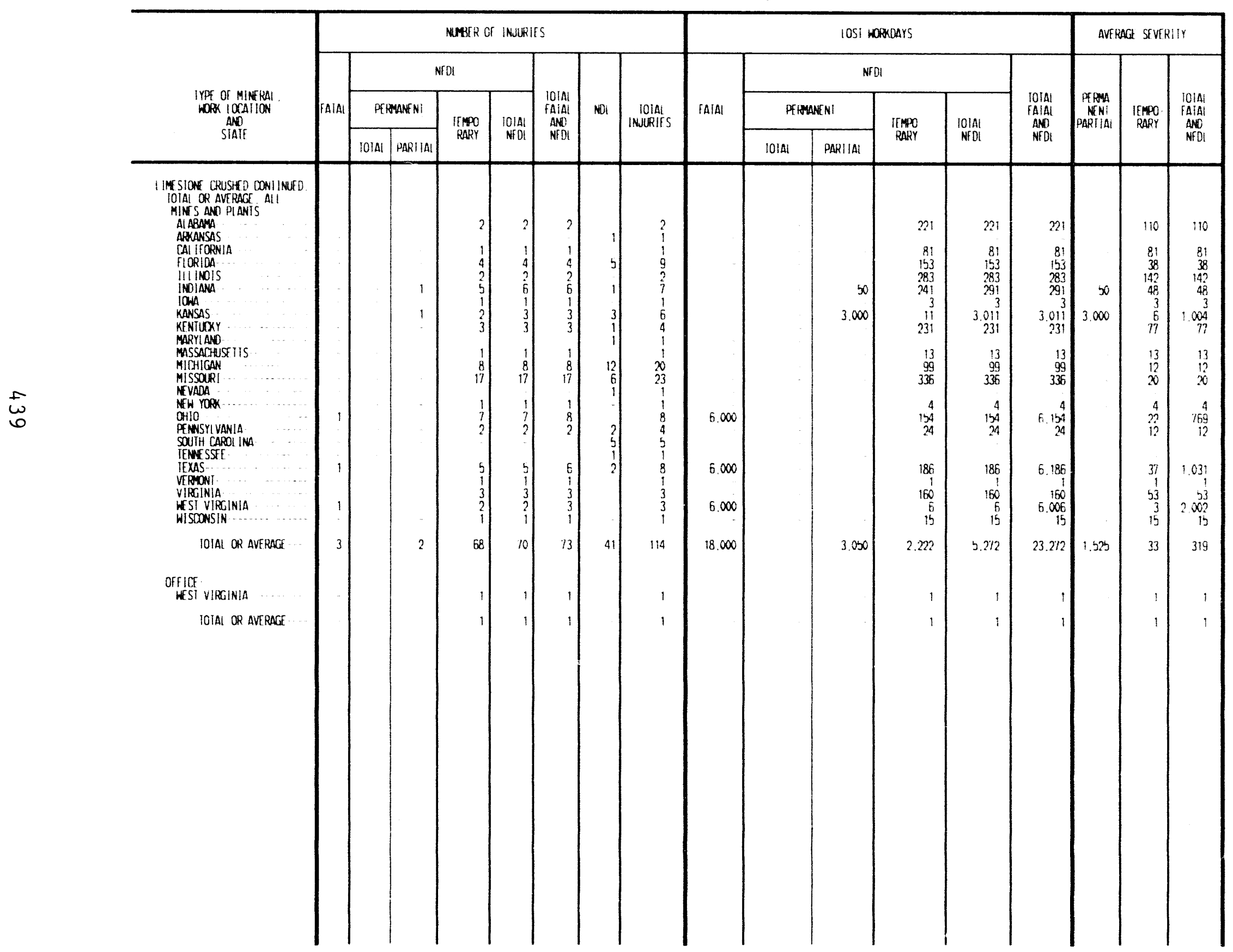




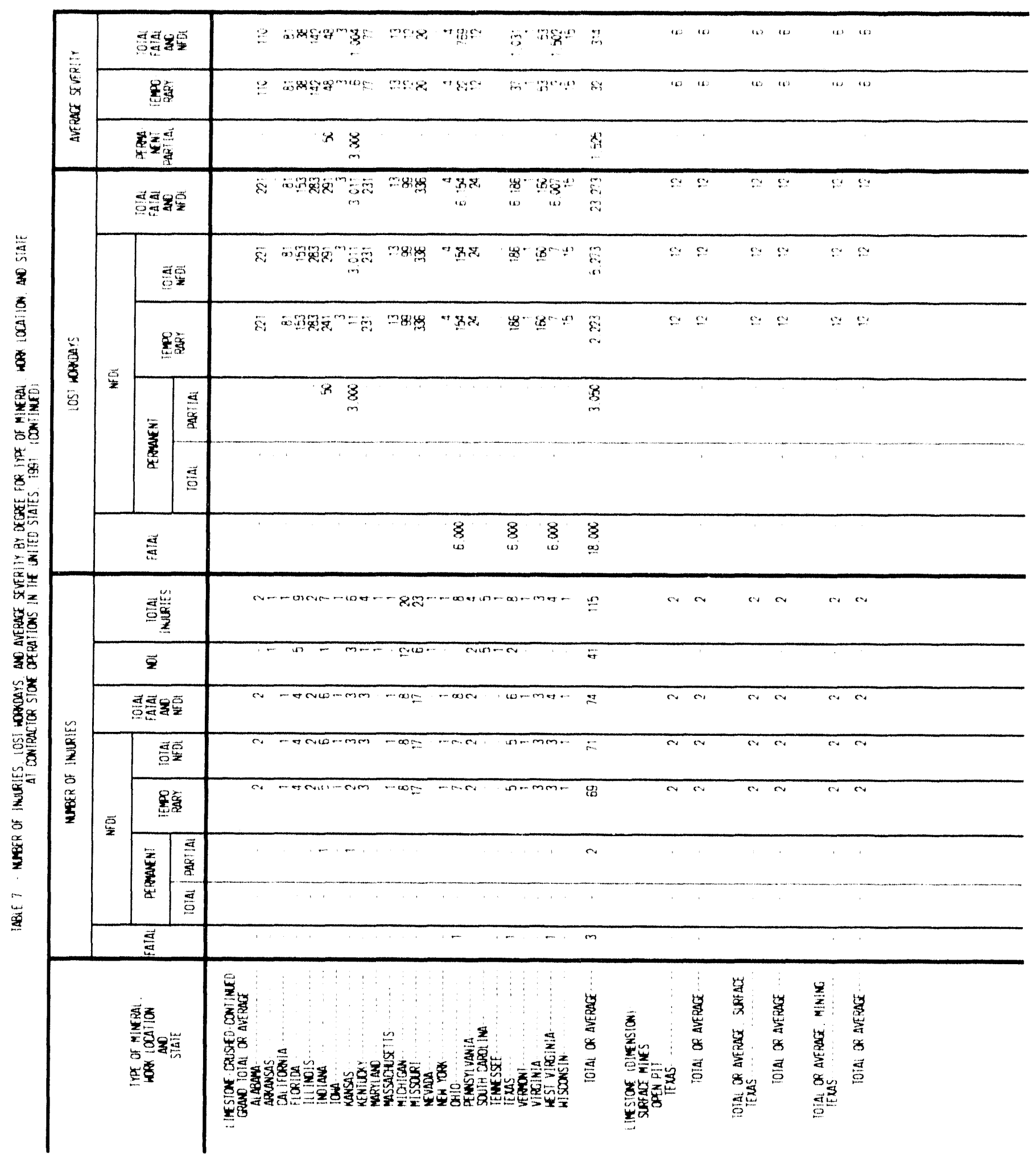




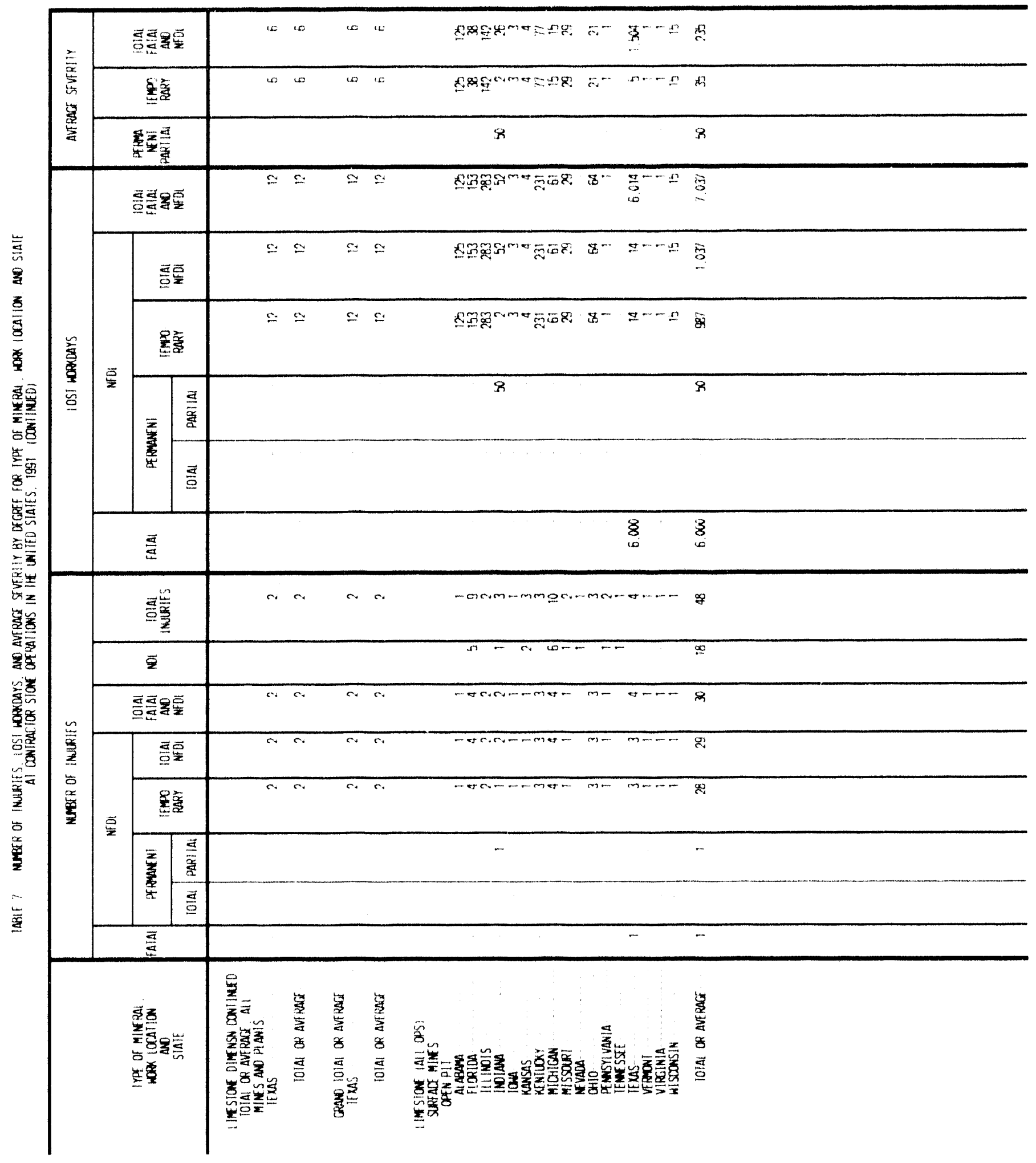




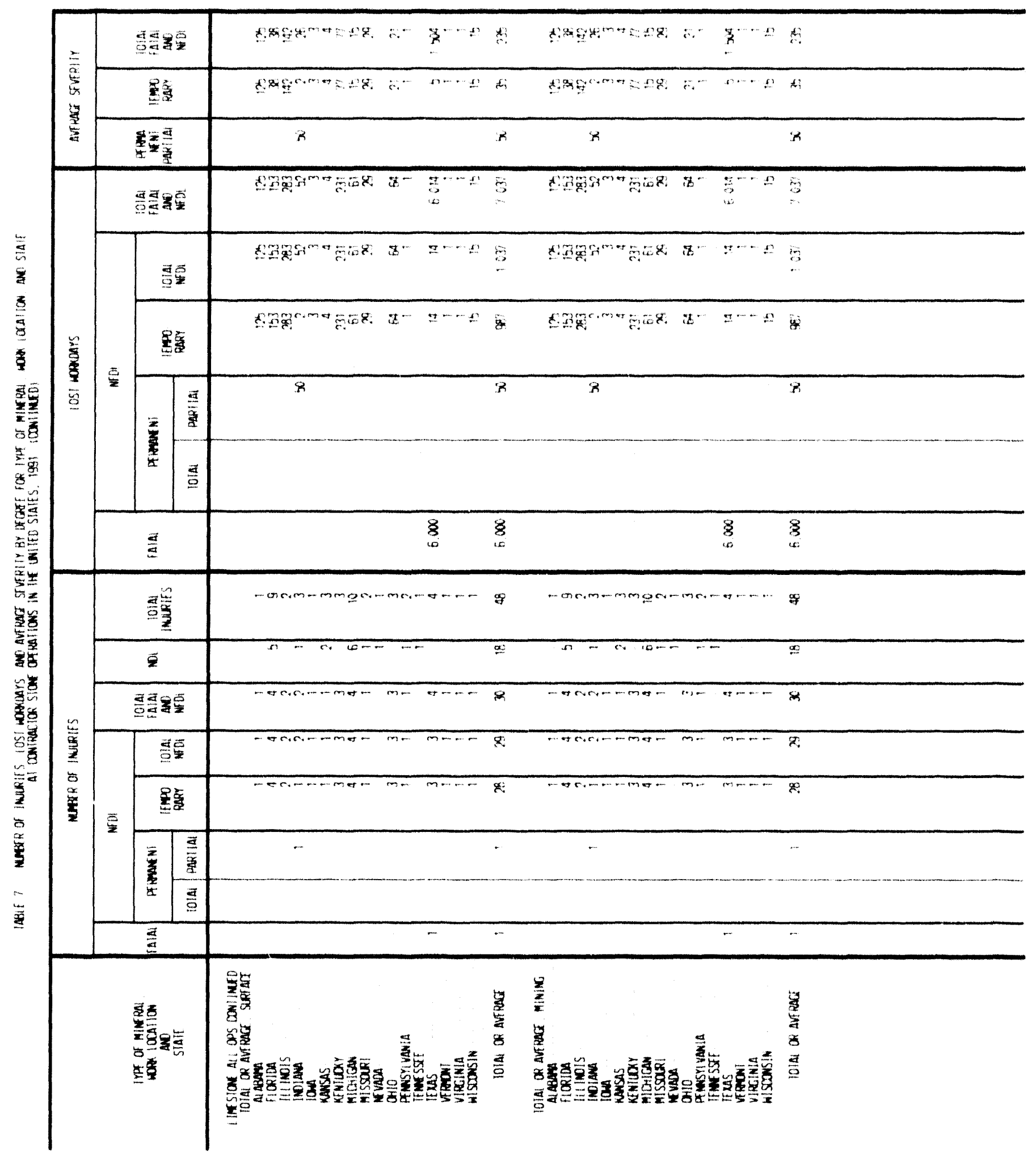




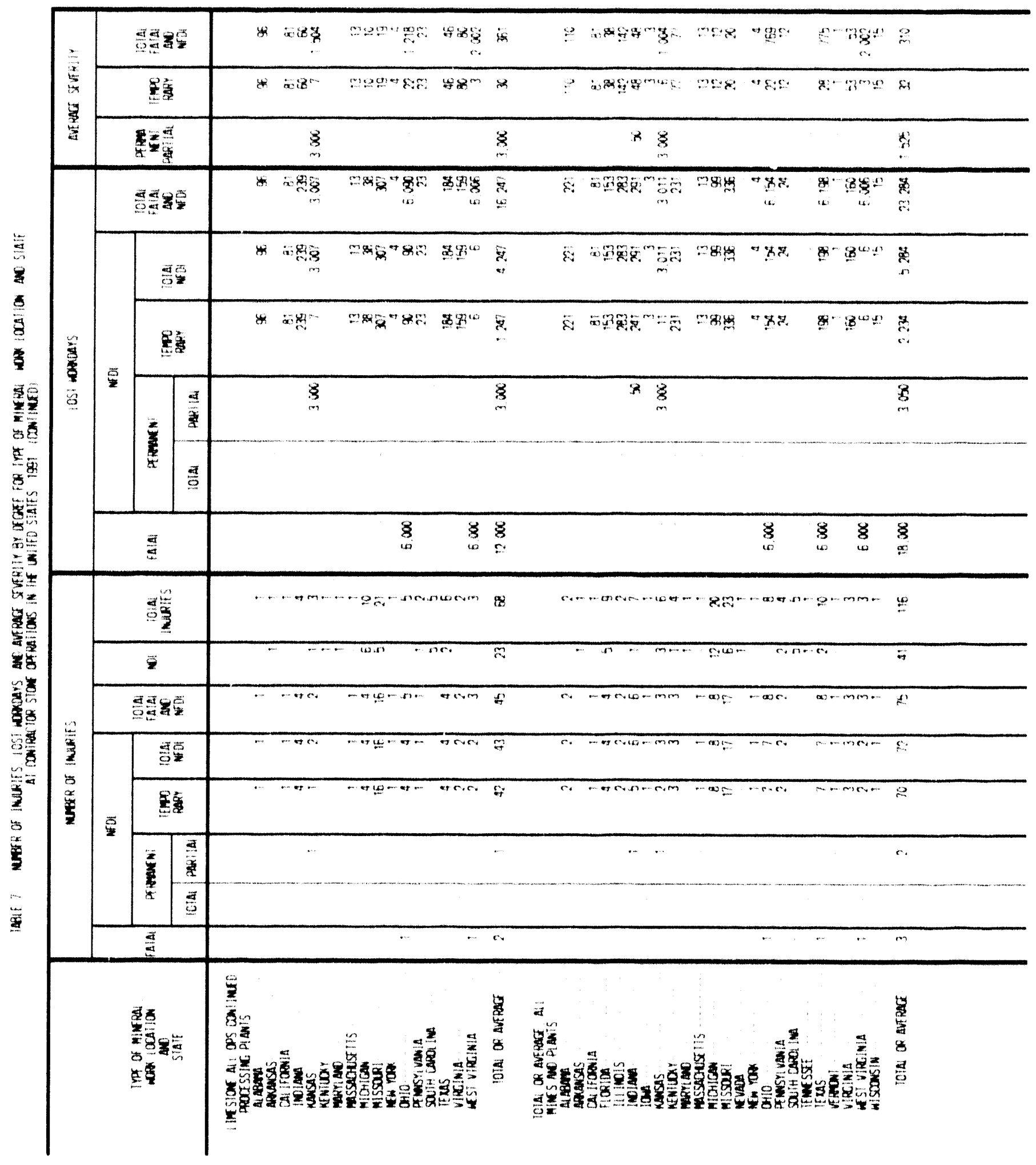




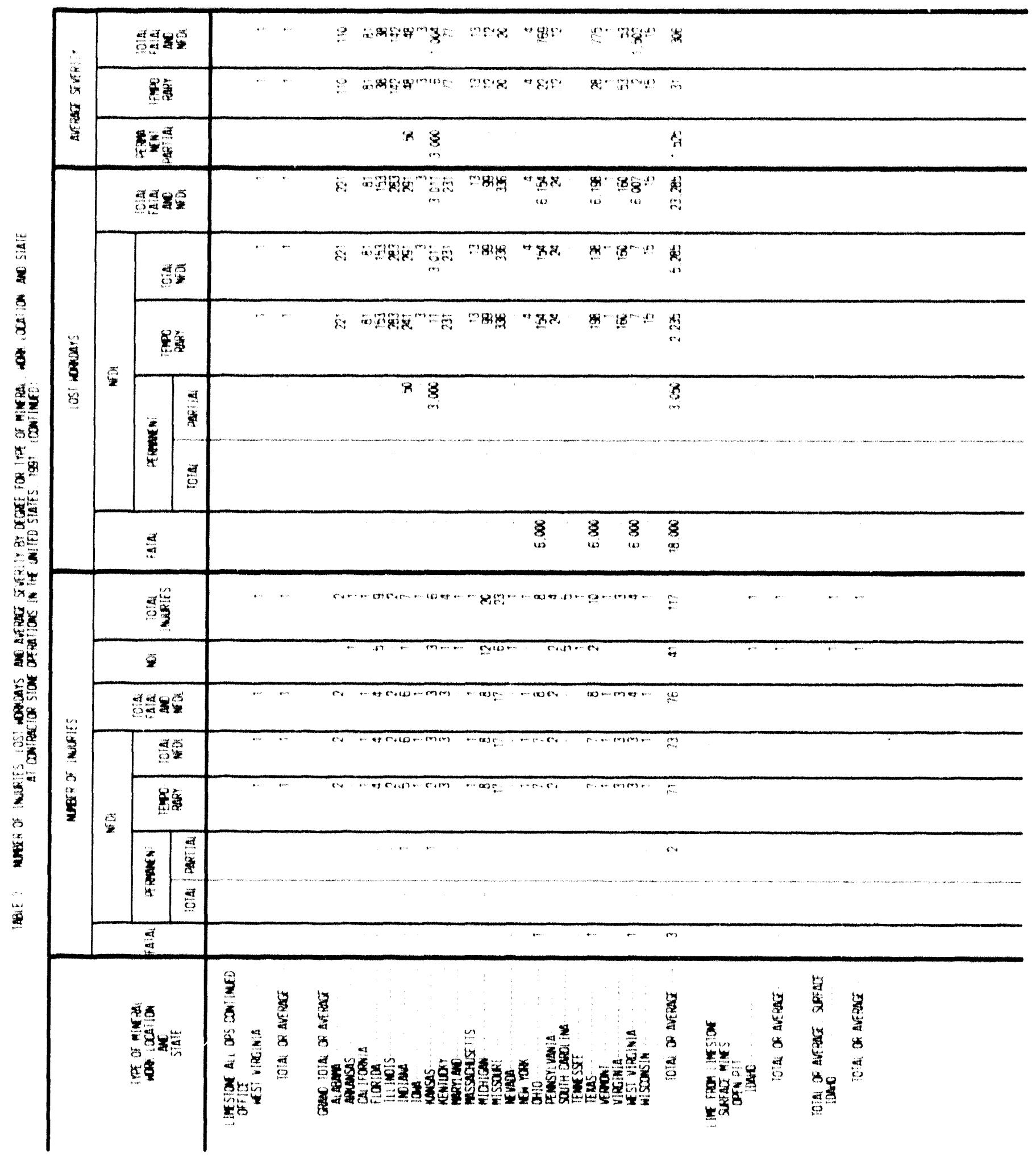




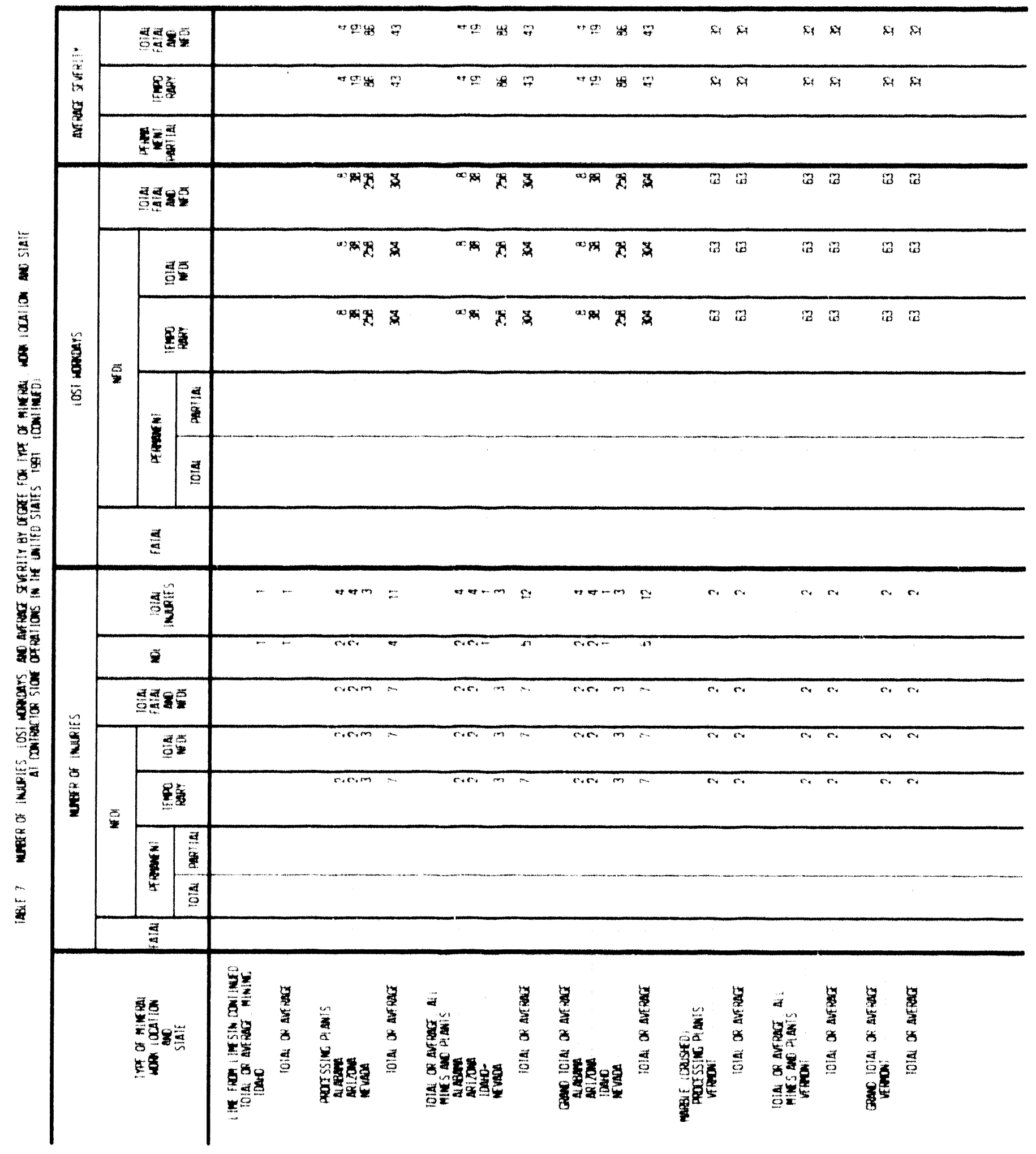




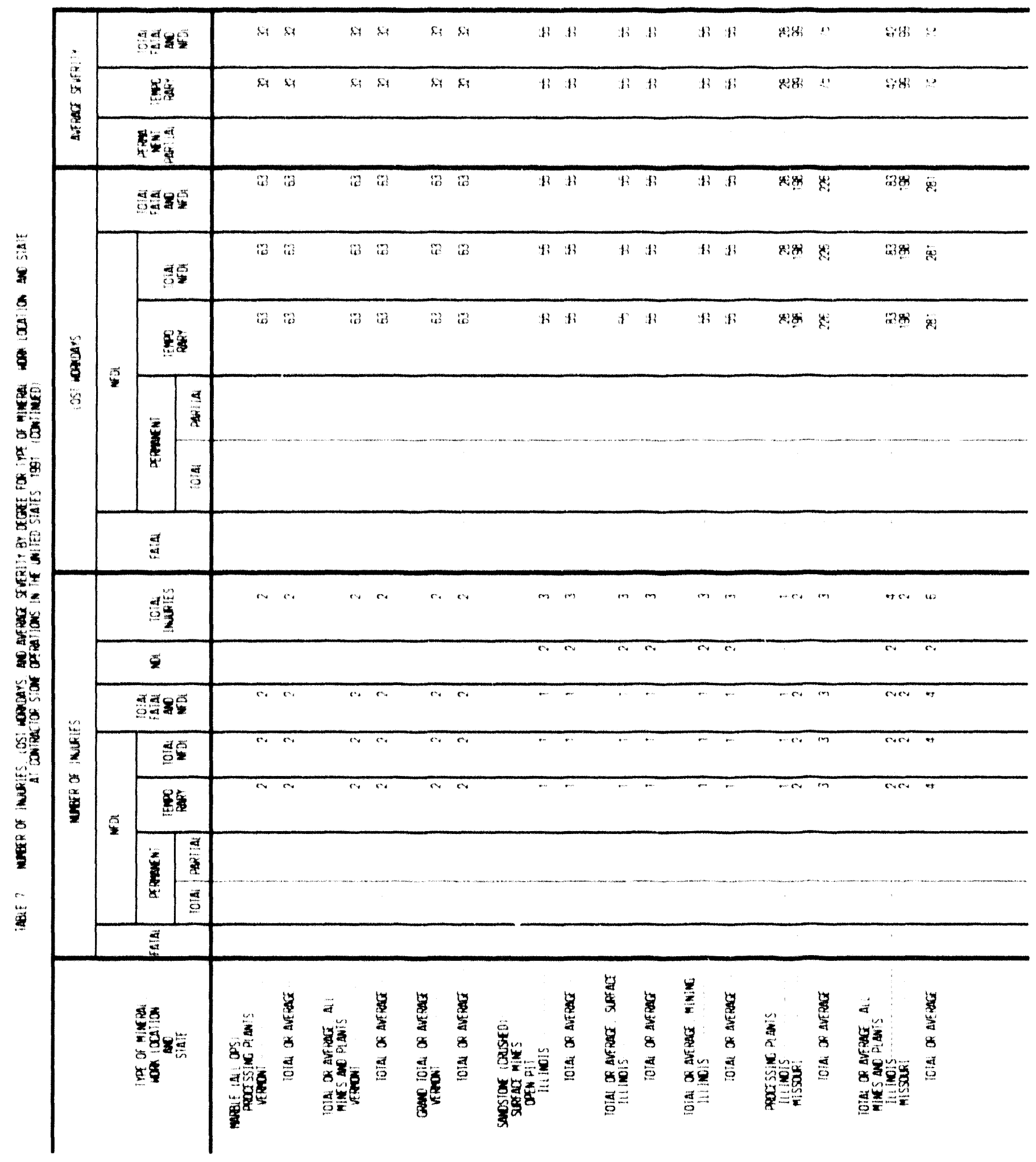




\begin{tabular}{|c|c|c|c|c|c|c|c|c|c|c|c|c|c|c|c|c|}
\hline \multirow{3}{*}{ 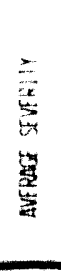 } & 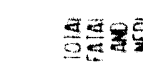 & & $\& \&$ & & h & $f f$ & 53 & $f$ & $n$ & f & gृ & 5 & 98 & 2 & sक & e \\
\hline & 语 & & $8 \$$ & $?$ & $f$ & 3 & $f ?$ & $f i$ & $n$ & f & 果名 & $?$ & इ & 2 & FE: & e \\
\hline & 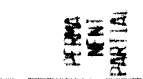 & & & & & & & & & & & & & & & \\
\hline \multirow{6}{*}{ 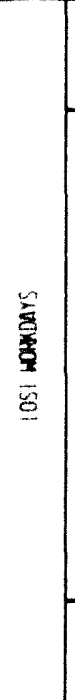 } & 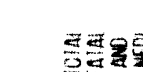 & & $\approx \%$ & $\bar{g}$ & $f$ & $f 3$ & 3 & 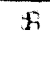 & $f$ & fR & 98 & है & $8 \%$ & $\bar{E}$ & $g$ & 可 \\
\hline & \multirow{4}{*}{$\bar{E}$} & 吾要 & $2 \%$ & $\ddot{x}$ & $f$ & $f ?$ & f & $\Re$ & $n$ & $\mathrm{~A}$ & g्यg & 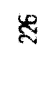 & $\infty \%$ & $\bar{g}$ & $9 \%$ & ק \\
\hline & & 是萻 & $x$ & $\overline{\mathrm{e}}$ & $f 3$ & ? $f$ & $f 2$ & H & $s$ & 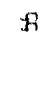 & $\%$ & تئّ & $\mathscr{\infty} \dot{z}$ & 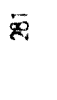 & 98 & 实 \\
\hline & & & & & & & & & & & & & & & & \\
\hline & & & & & & & & & & & & & & & & \\
\hline & \multicolumn{2}{|l|}{$\frac{\pi}{a}$} & & & & & & & & & & & & & & \\
\hline \multirow{8}{*}{ 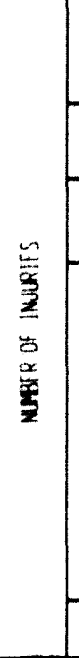 } & \multicolumn{2}{|l|}{ 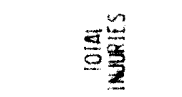 } & For & $\infty$ & $m$ & , $m$ & $m$ & $m$ & m & $m$ & $-\infty$ & $m$ & +a & $\infty$ & $+\infty$ & مـ \\
\hline & \multicolumn{2}{|l|}{$\vec{z}$} & 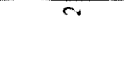 & $\sigma$ & 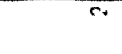 & 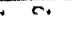 & 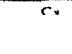 & $r$ & $r$ & 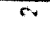 & & & $\pi$ & r. & $\sigma$ & \%. \\
\hline & \multicolumn{2}{|c|}{ 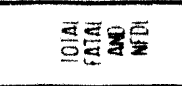 } & $a r$ & 7 & - & - & 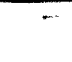 & - & $\cdots$ & - & $-\infty$ & $m$ & rase & $\sigma$ & cor. & 7 \\
\hline & \multicolumn{2}{|c|}{ 造垔 } & $\pi$ & 7 & $\cdots$ & - & $=$ & $=$ & - & - & $-r$ & $m$ & ron & 7 & ras & $\bar{\sigma}$ \\
\hline & \multicolumn{2}{|c|}{ 夆害 } & r.r. & $\overline{7}$ & - & - & 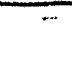 & - & - & - & $-r$ & $m$ & 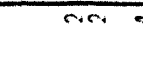 & $\bar{\sigma}$ & Cr. & $\overline{7}$ \\
\hline & \multirow{2}{*}{\multicolumn{2}{|c|}{ 尊 }} & & & & & & & & & & & & & & \\
\hline & & & & & & & & & & & & & & & & \\
\hline & \multicolumn{2}{|l|}{$\frac{a}{E}$} & & & & & & & & & & & & & & \\
\hline & 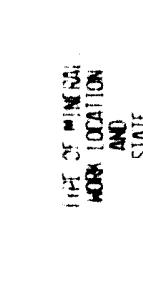 & & 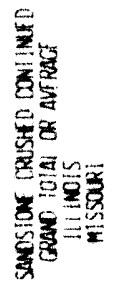 & 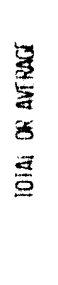 & 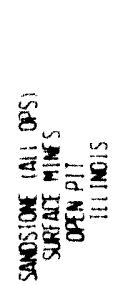 & 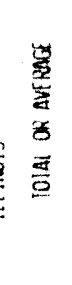 & 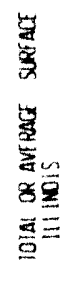 & 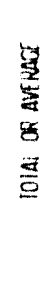 & 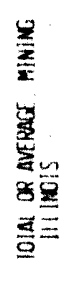 & 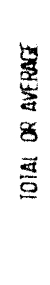 & 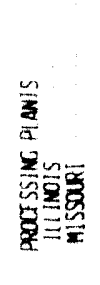 & 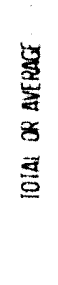 & 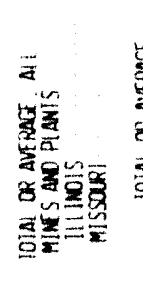 & 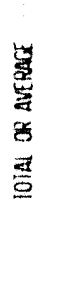 & 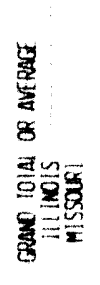 & 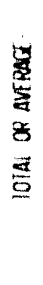 \\
\hline
\end{tabular}




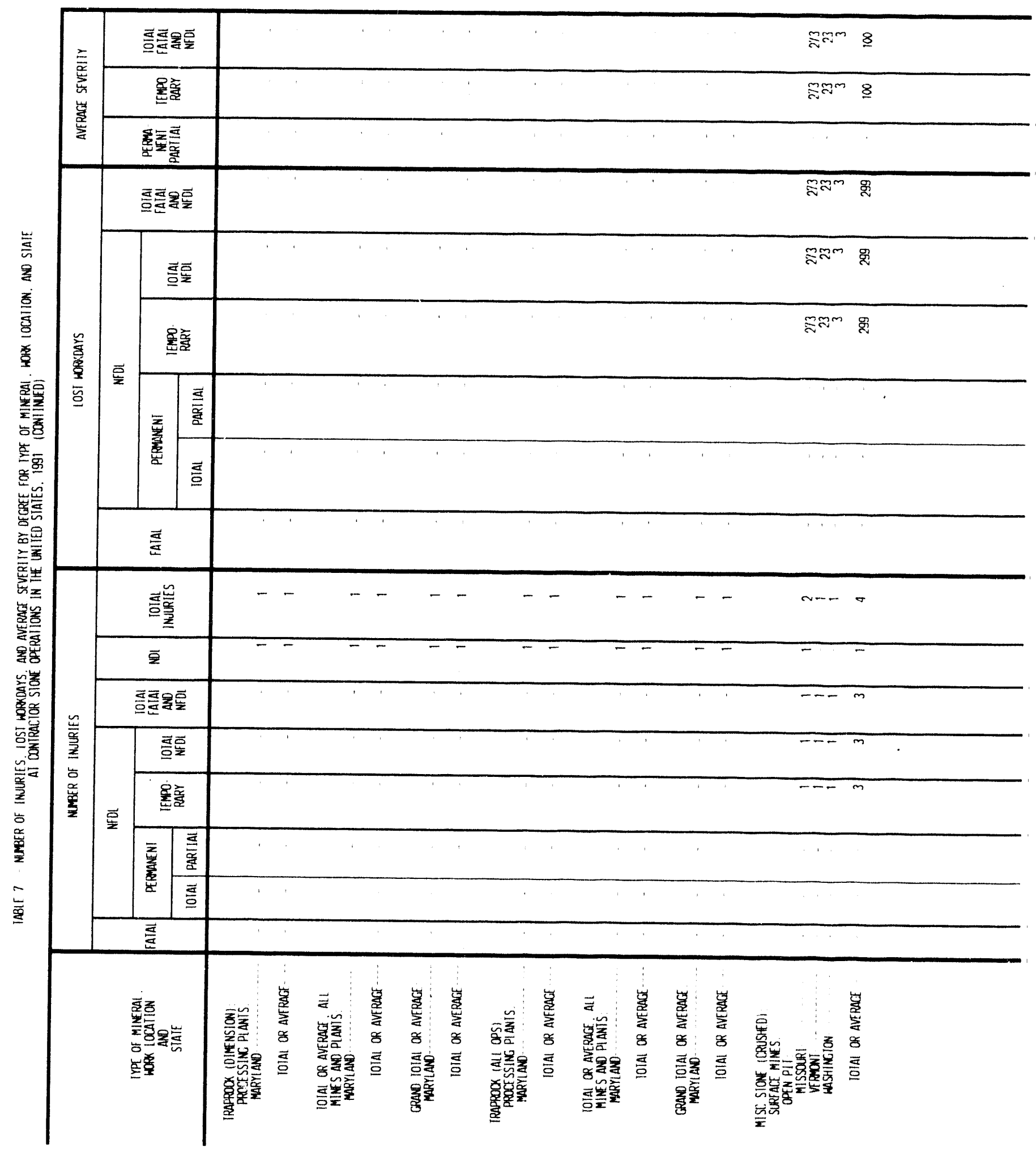




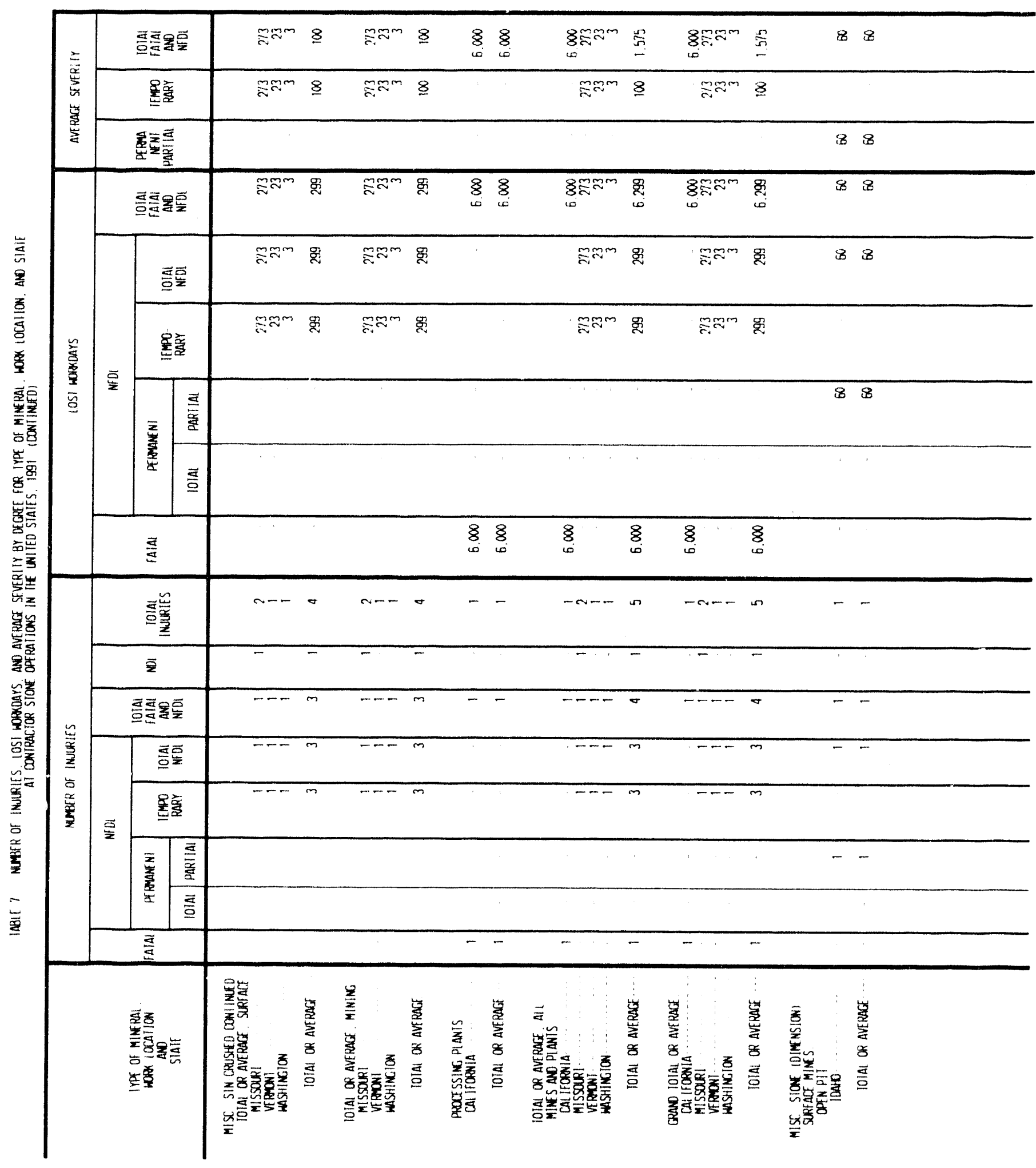




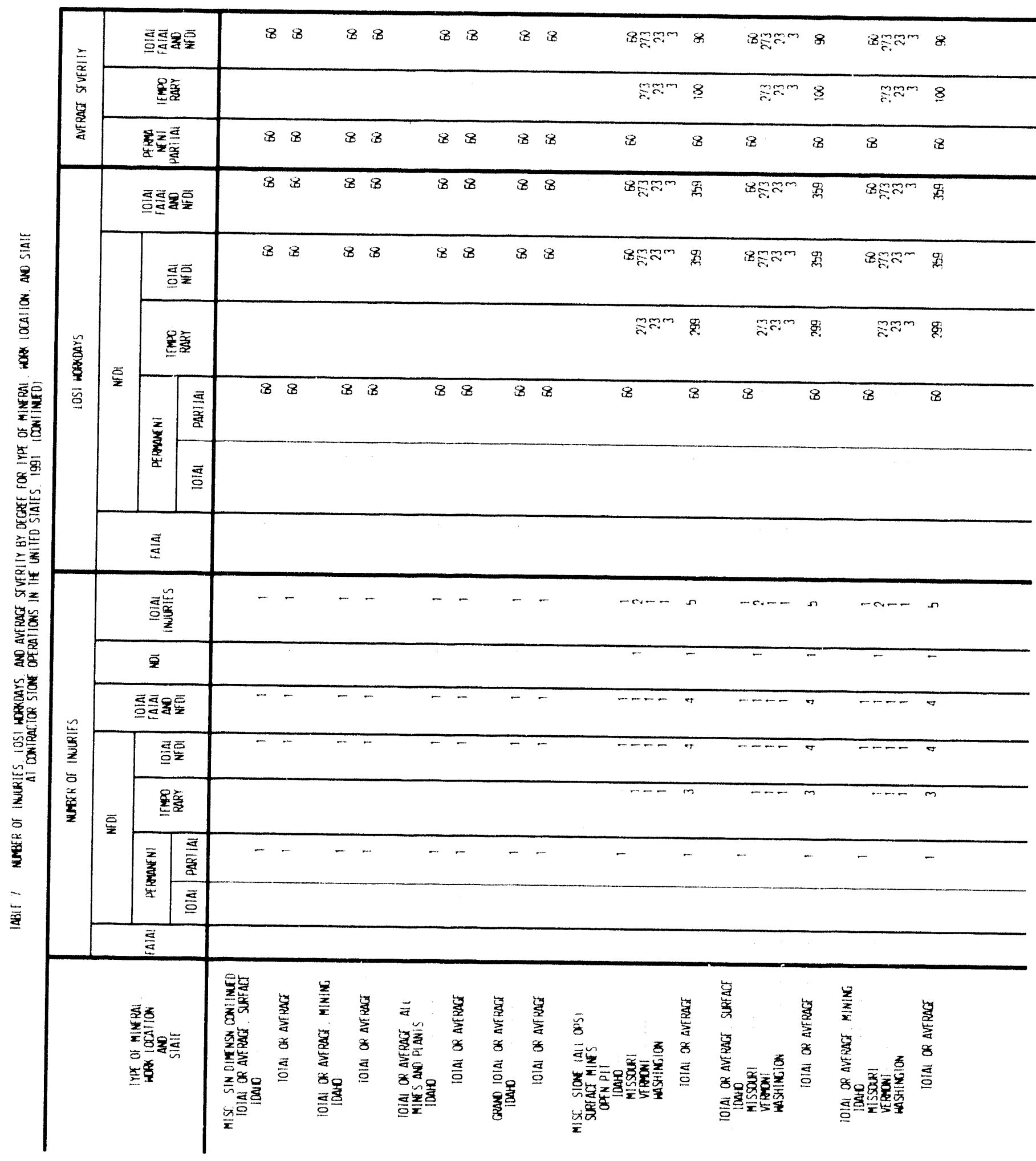




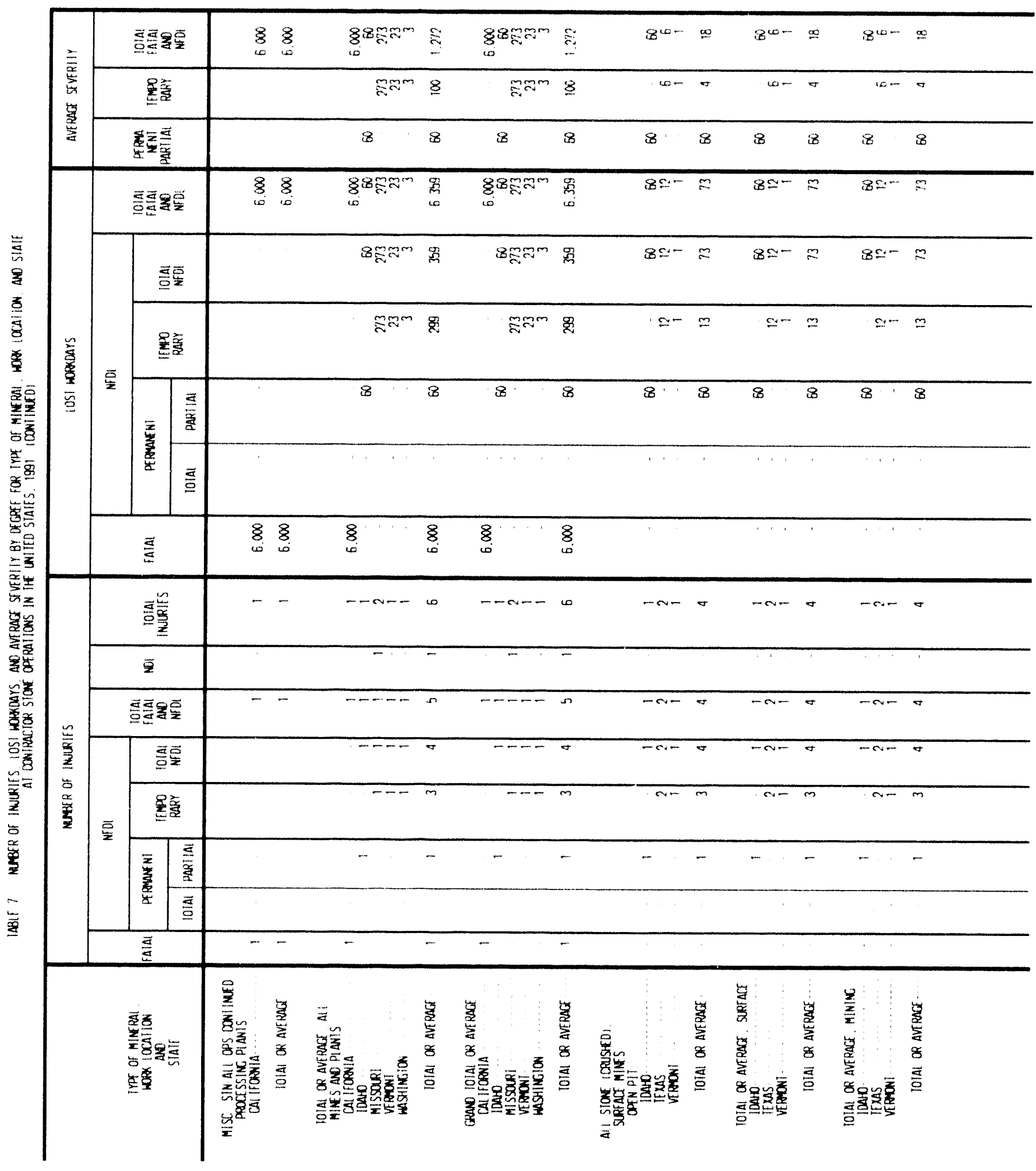




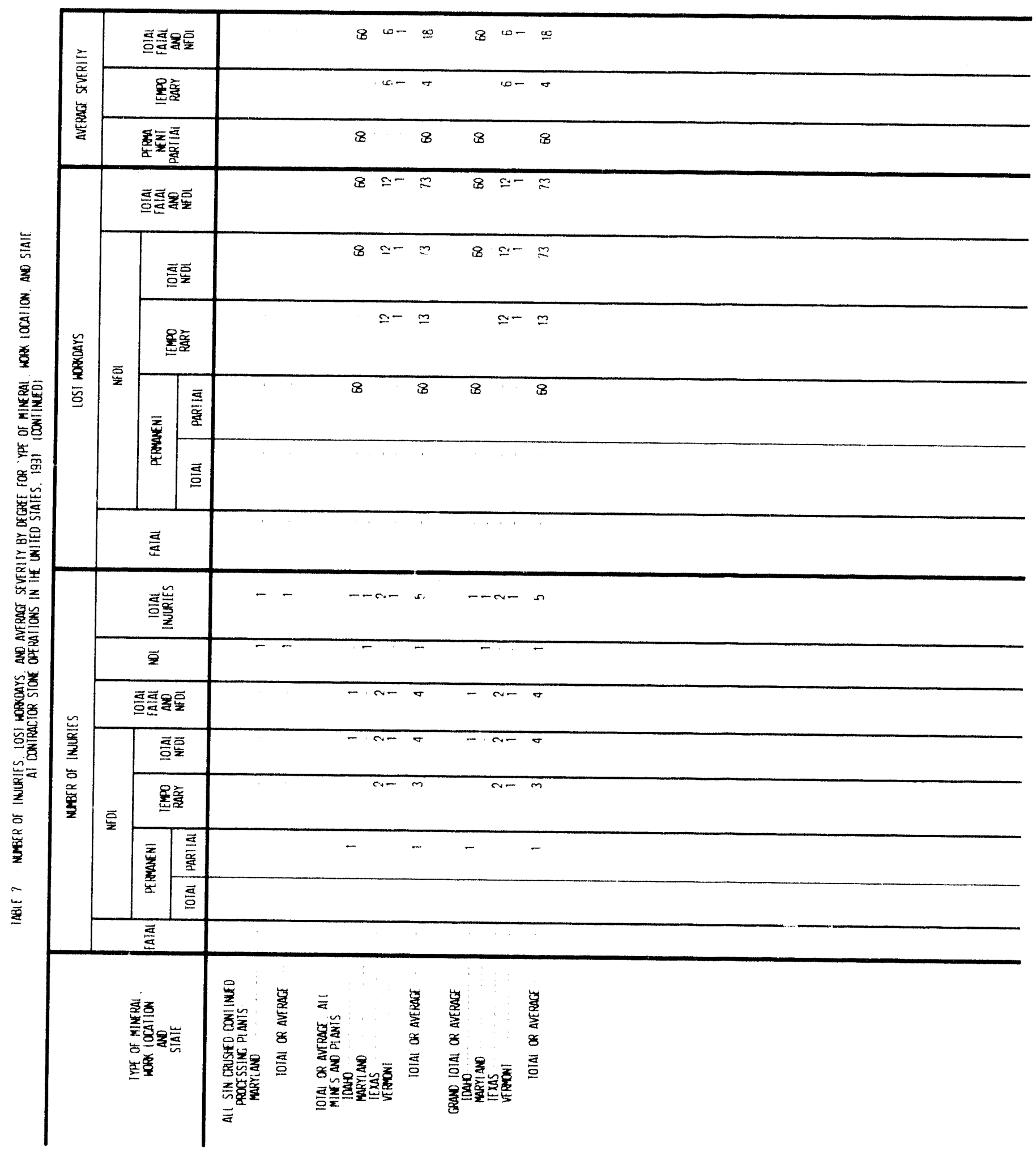




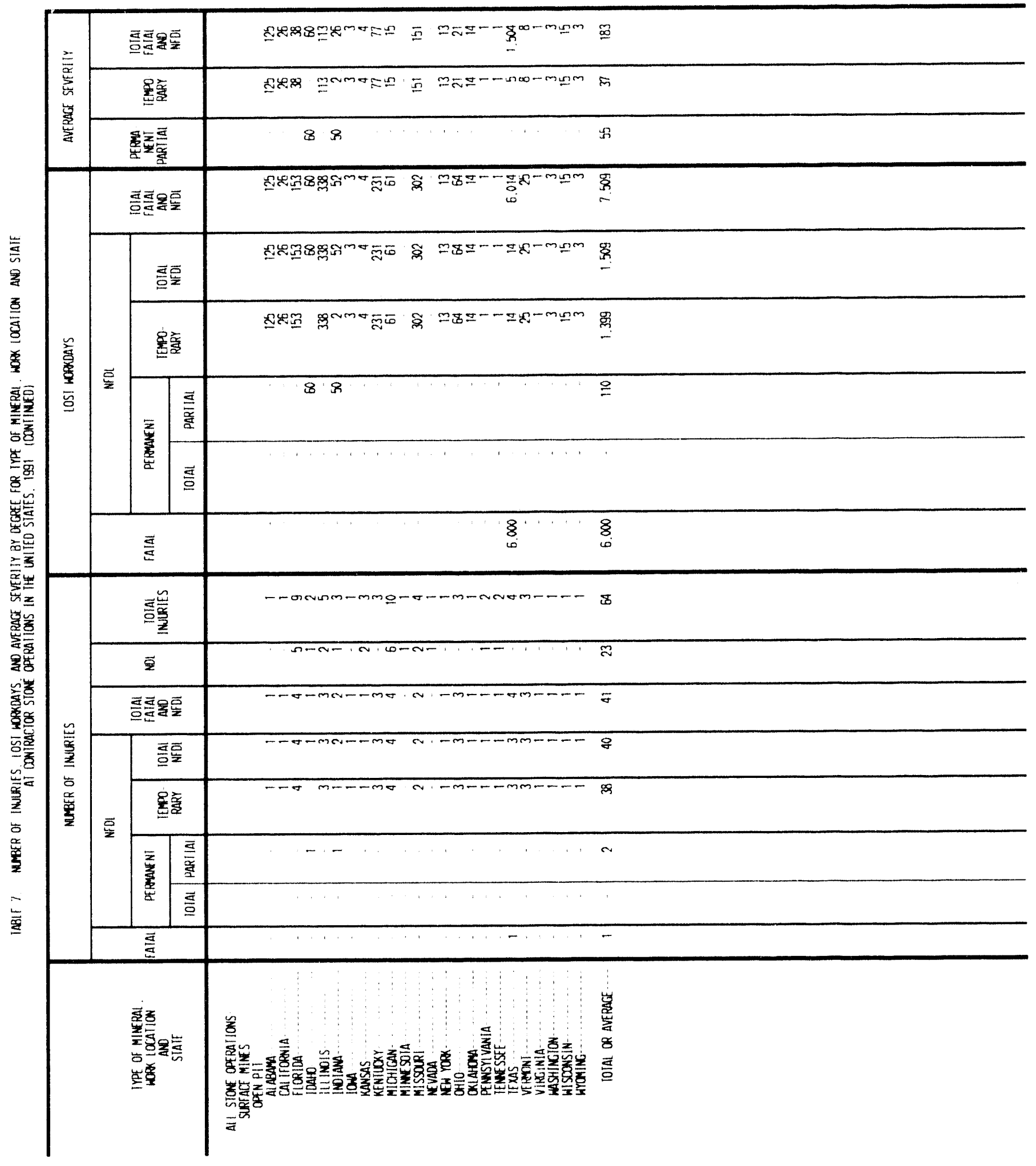




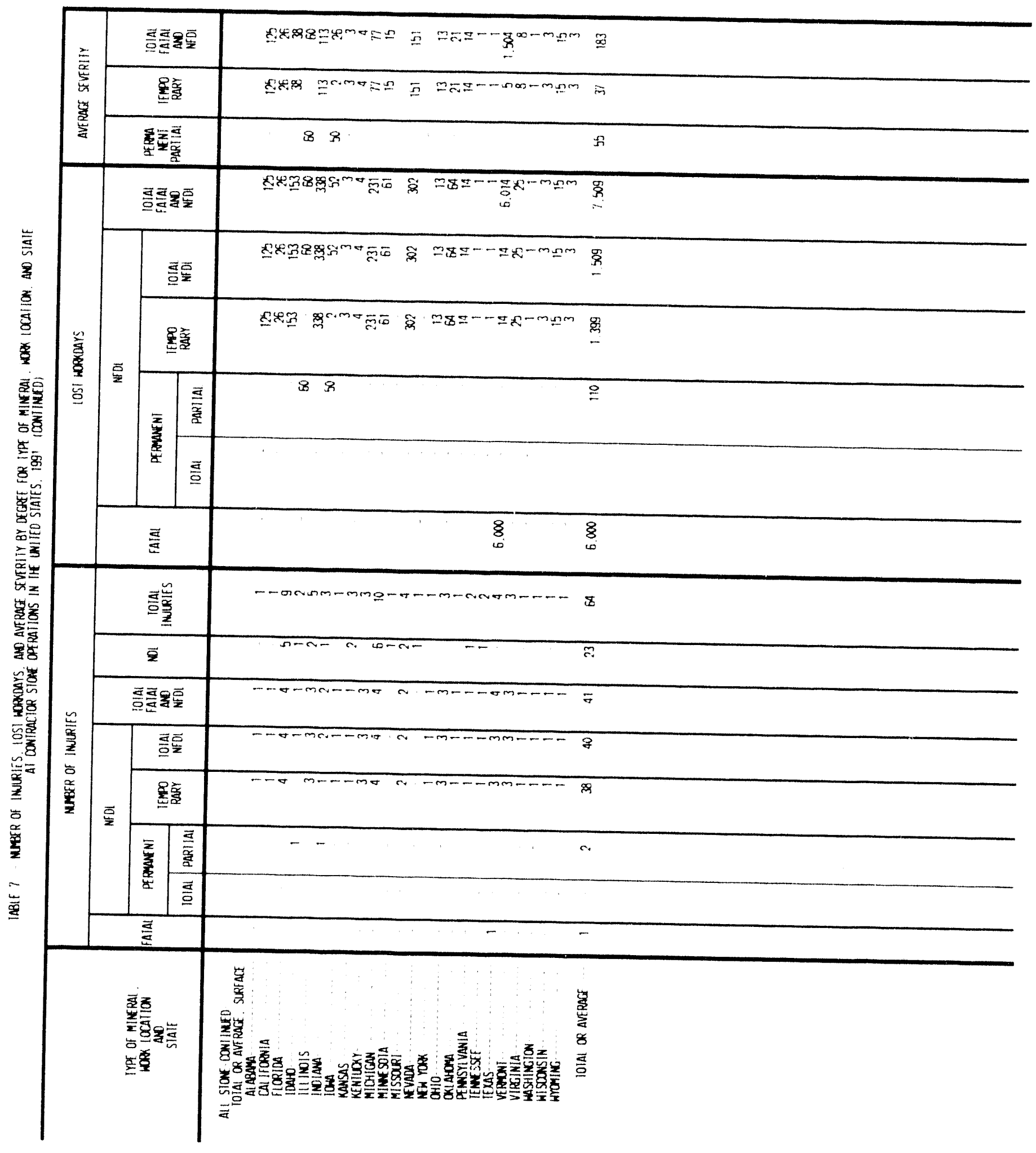




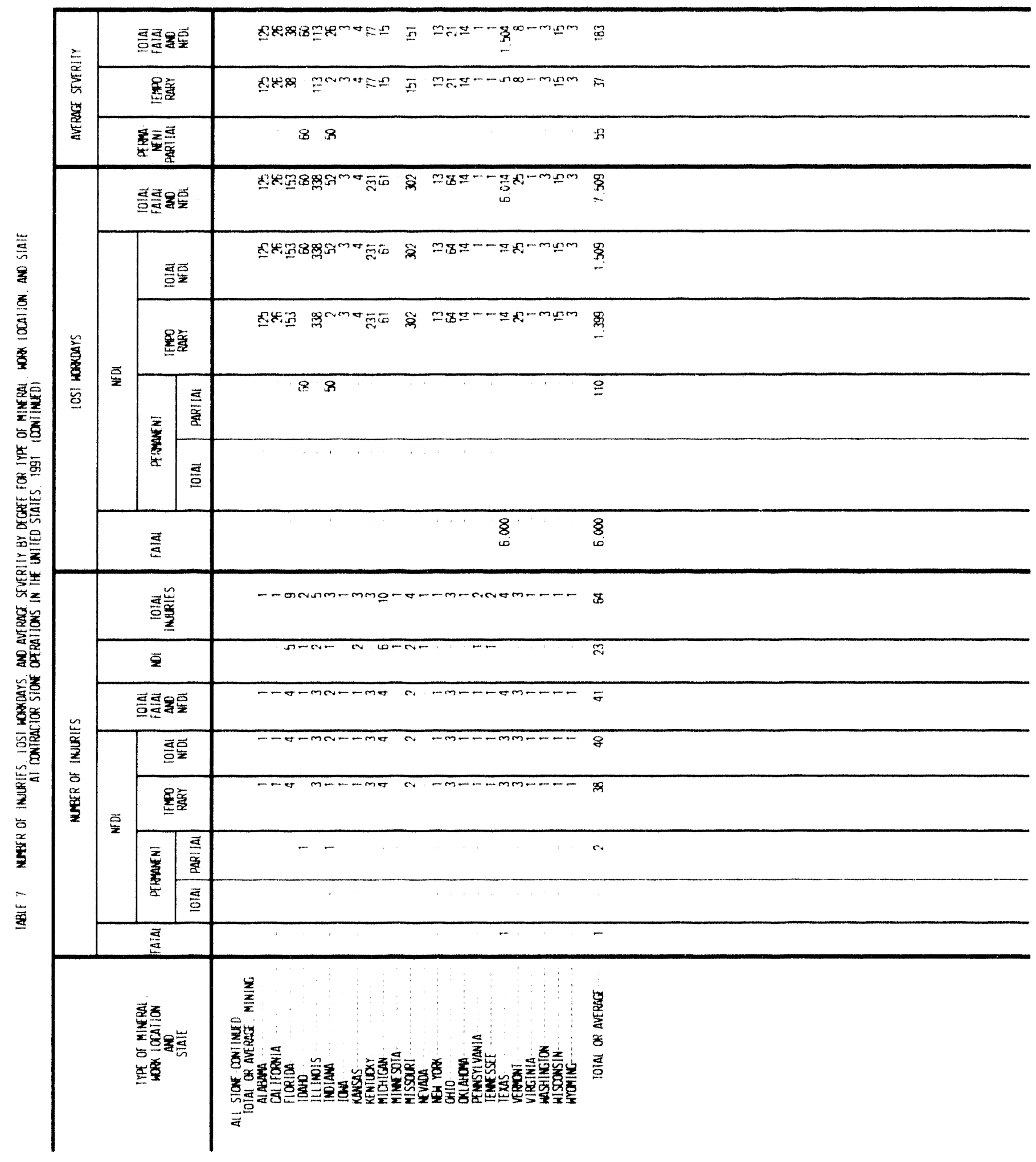




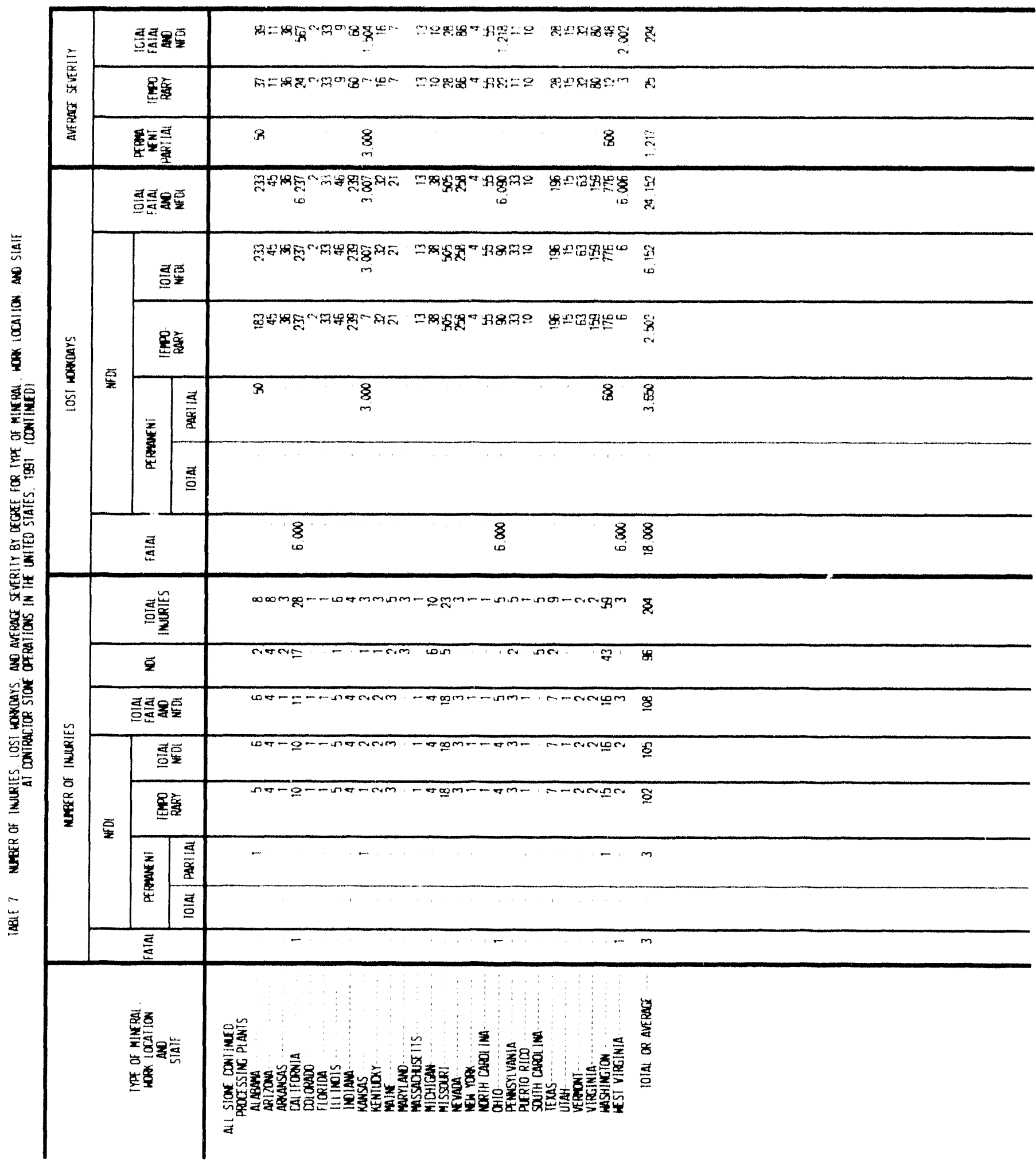




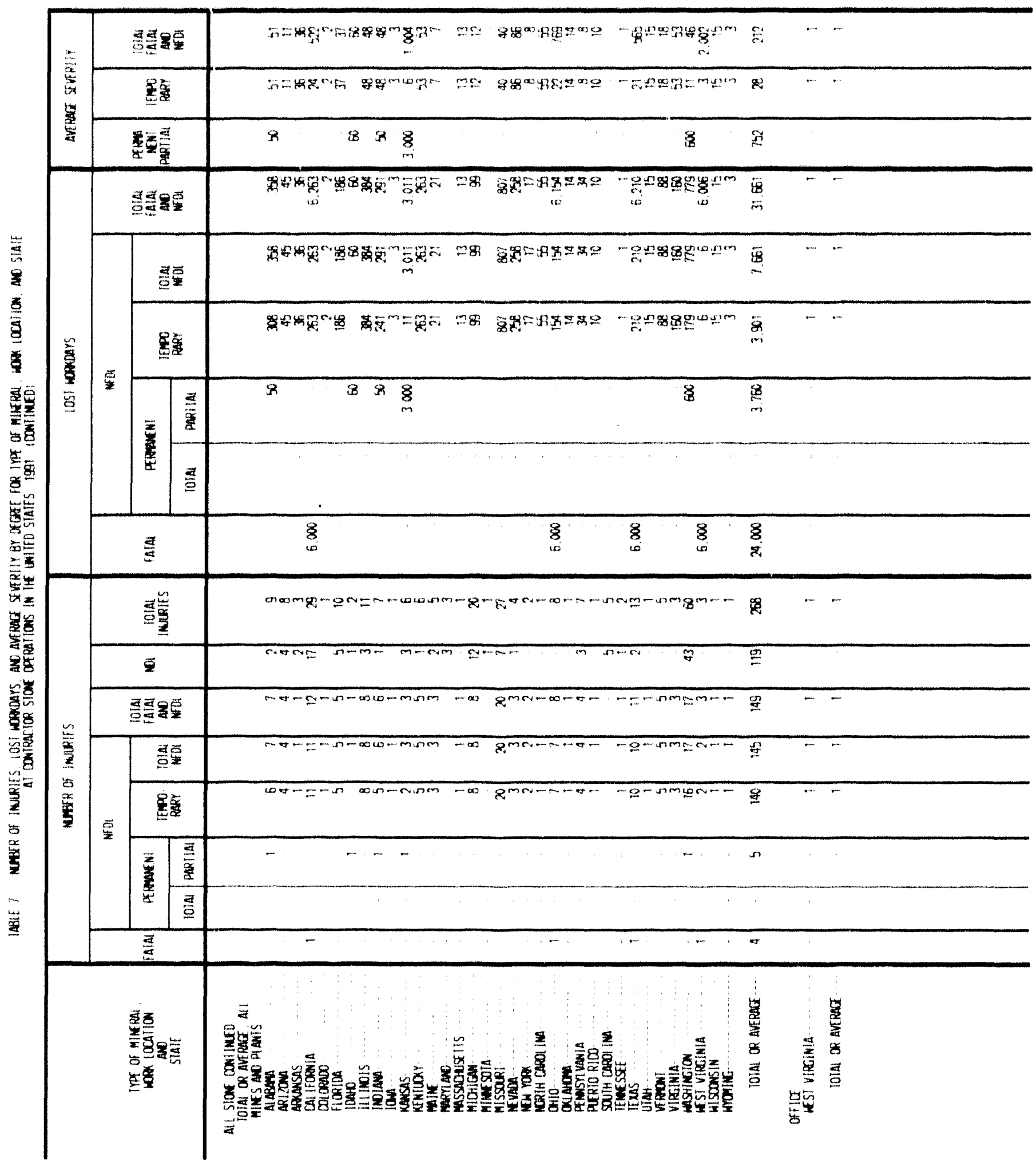




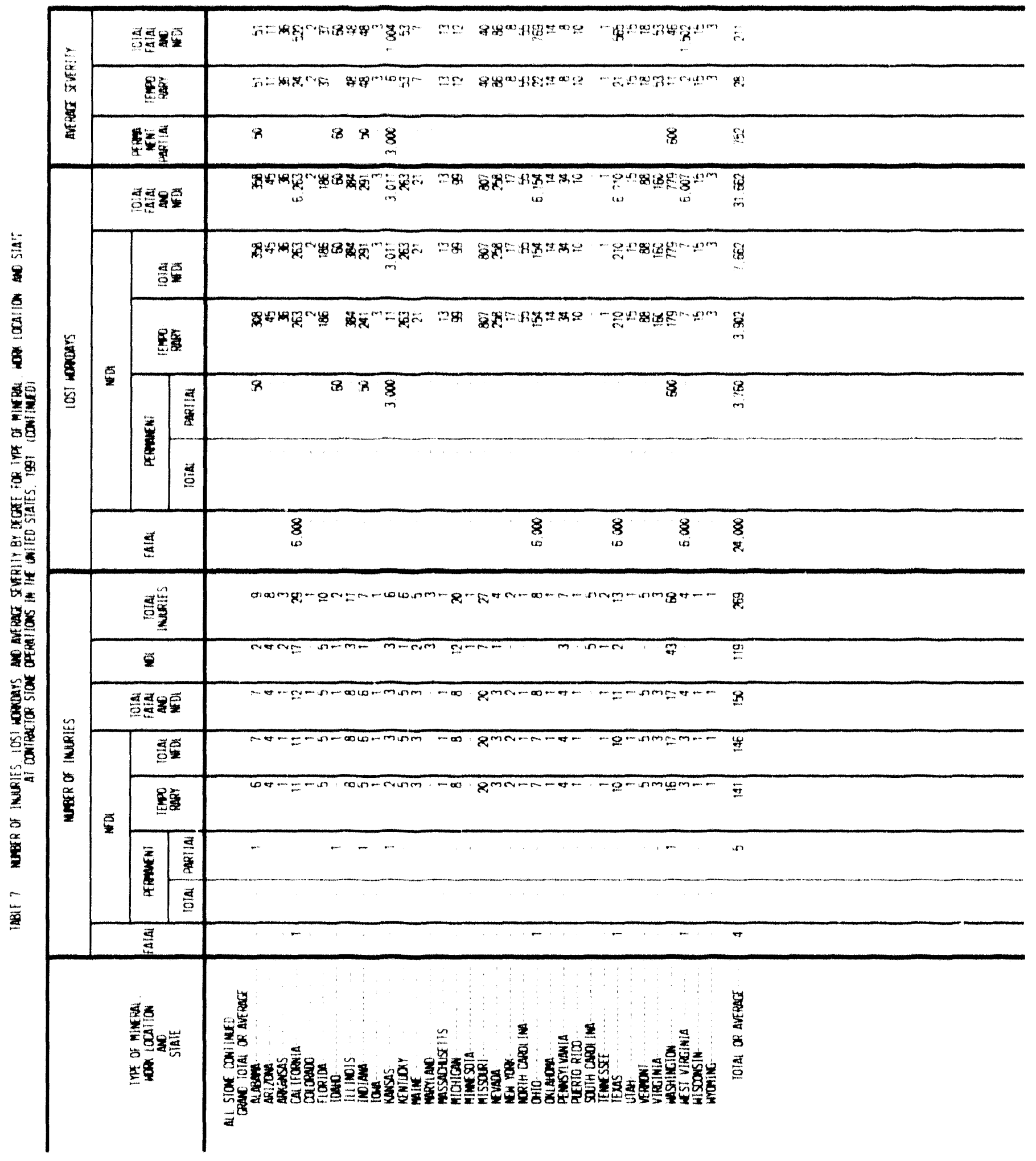




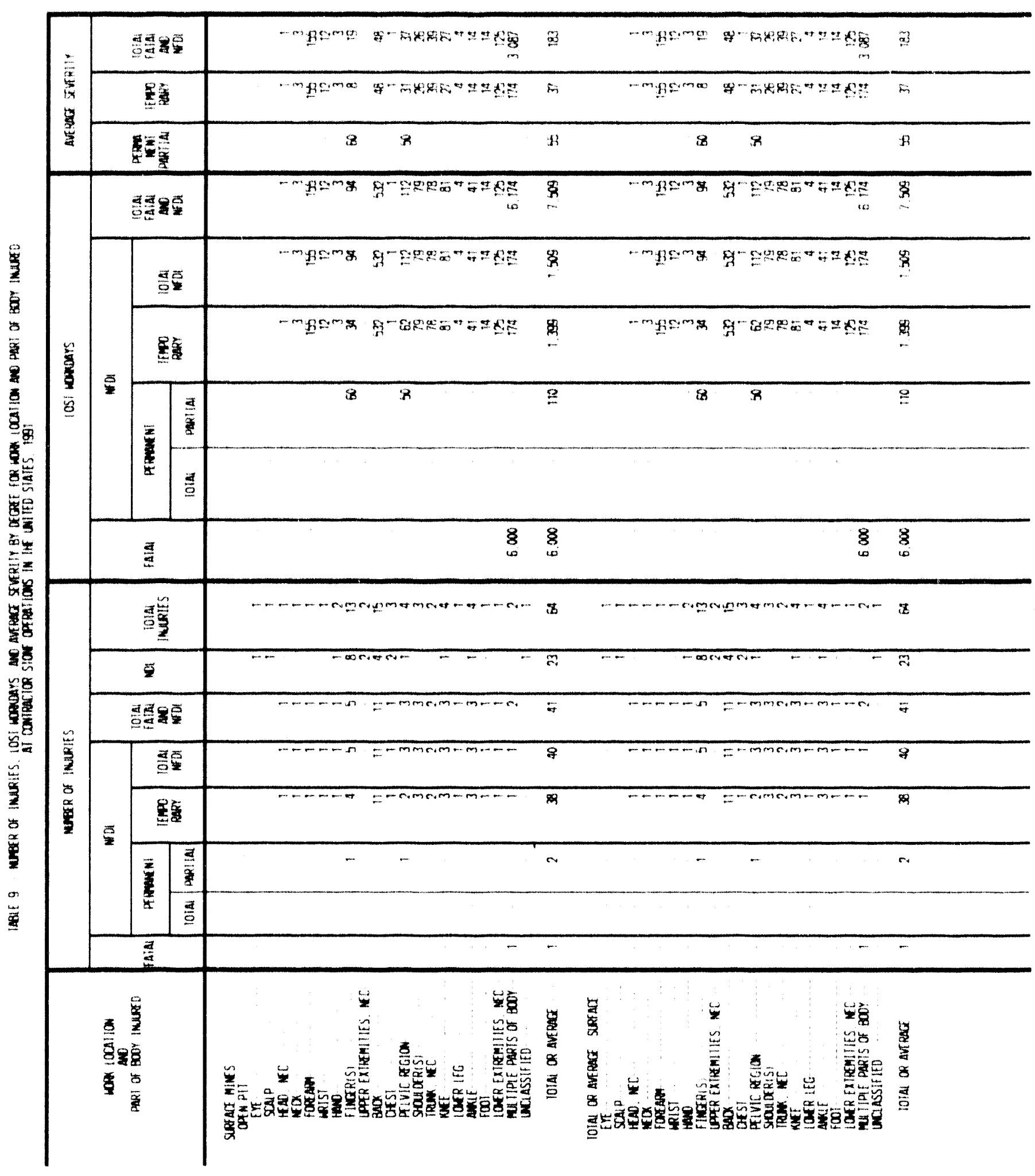




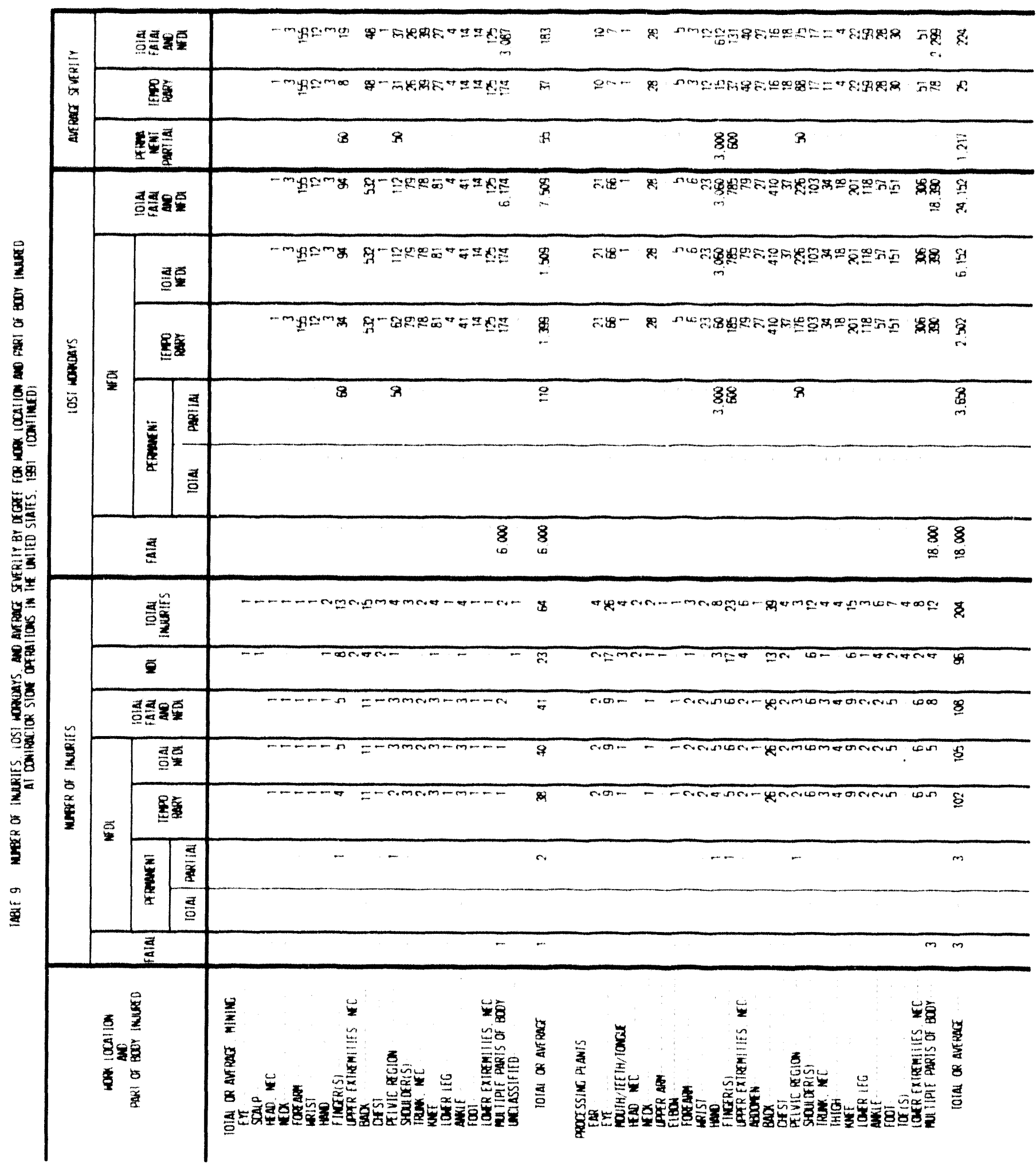




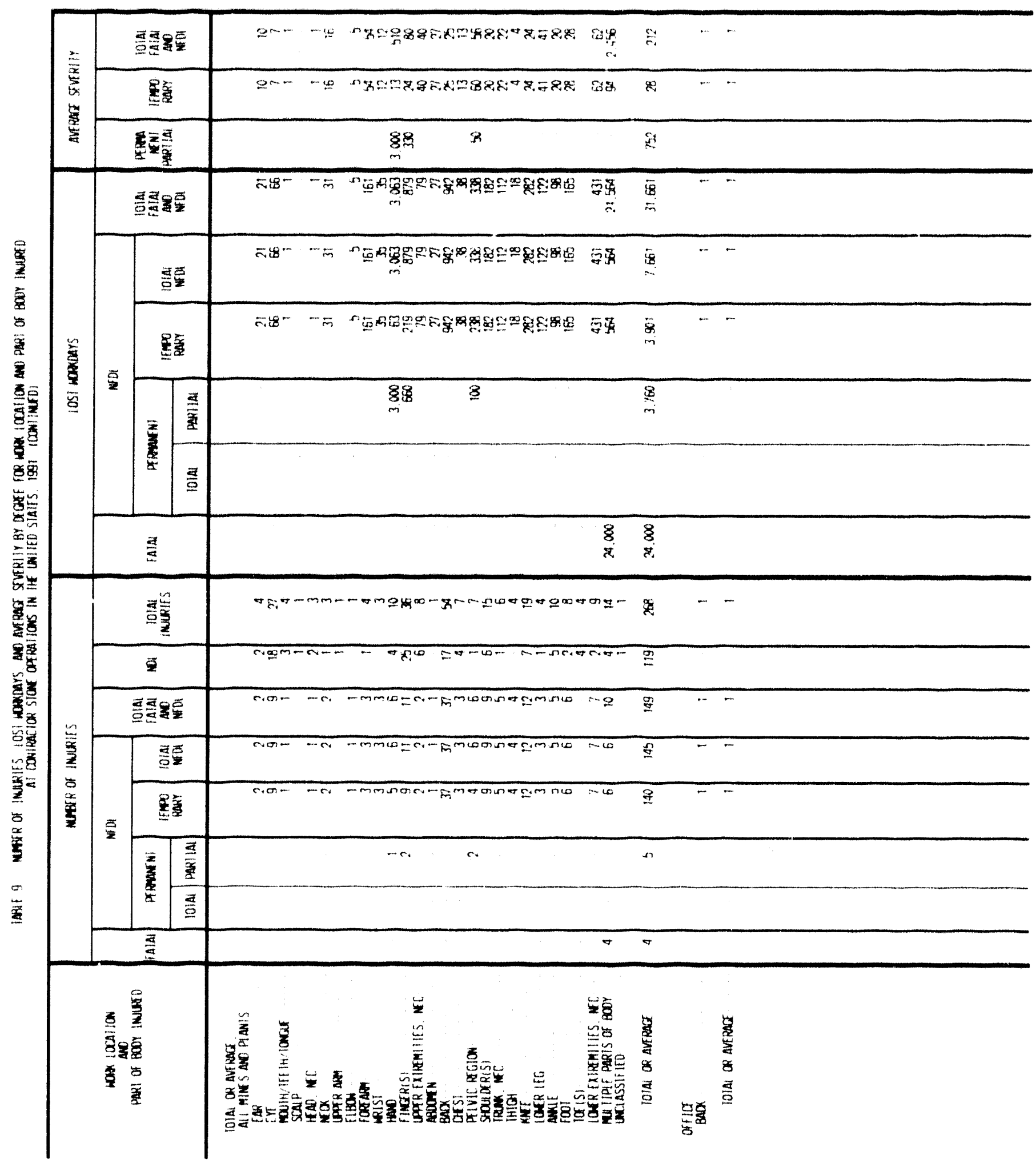




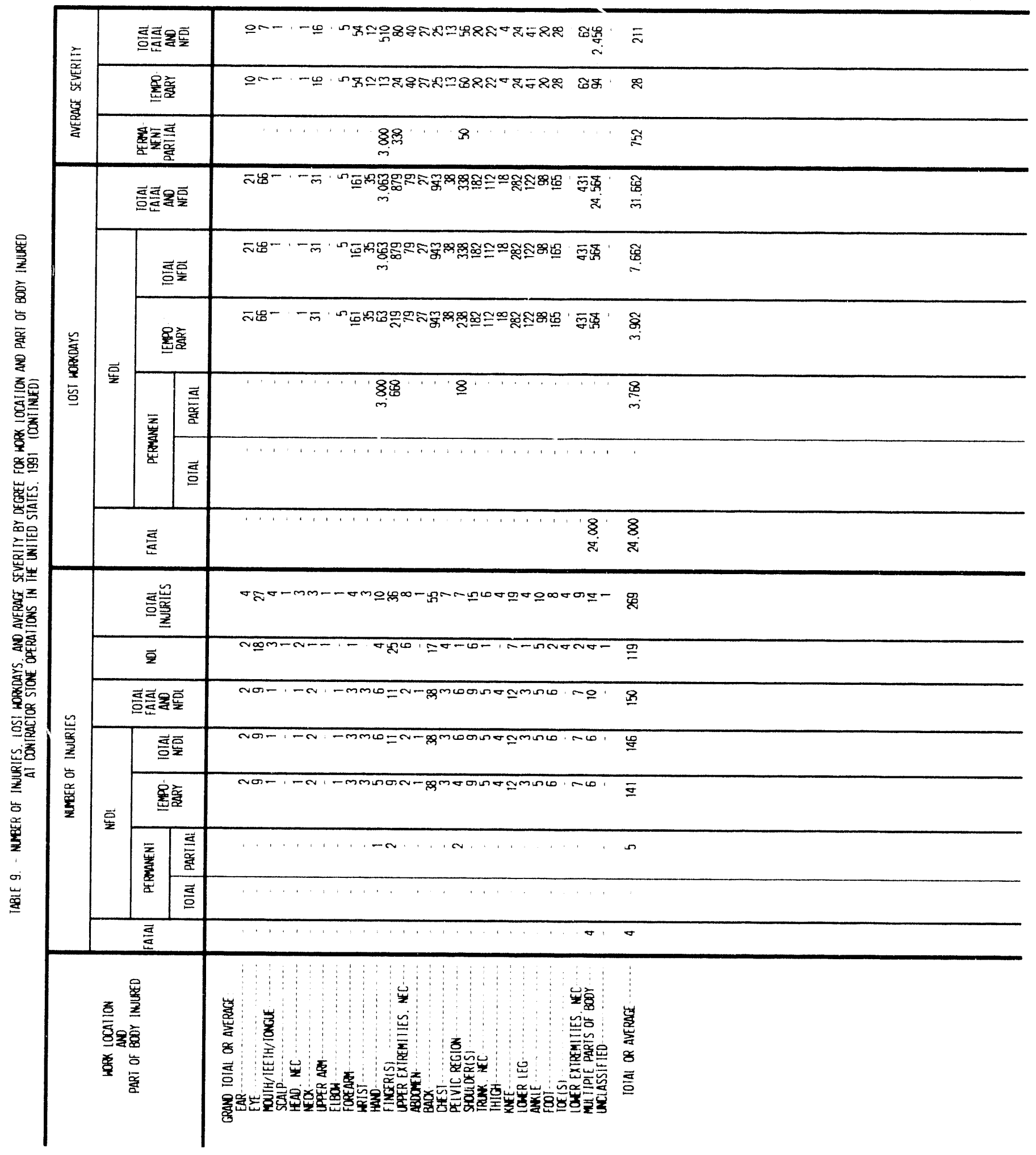



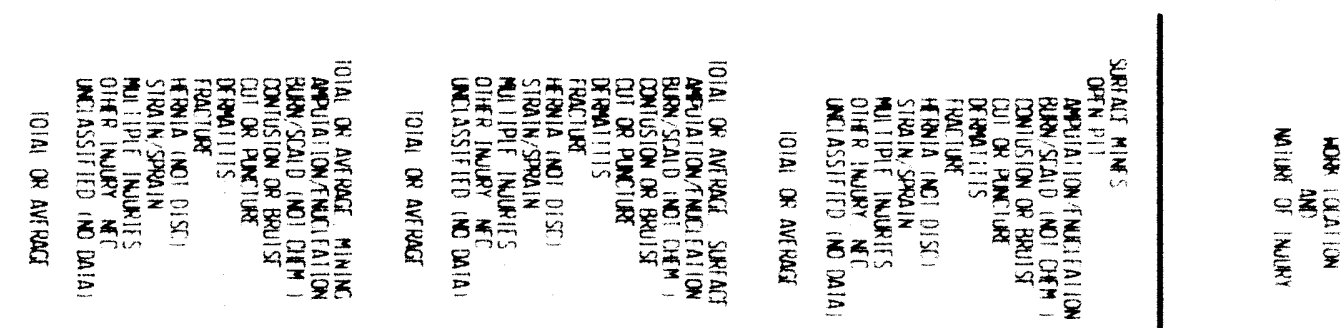

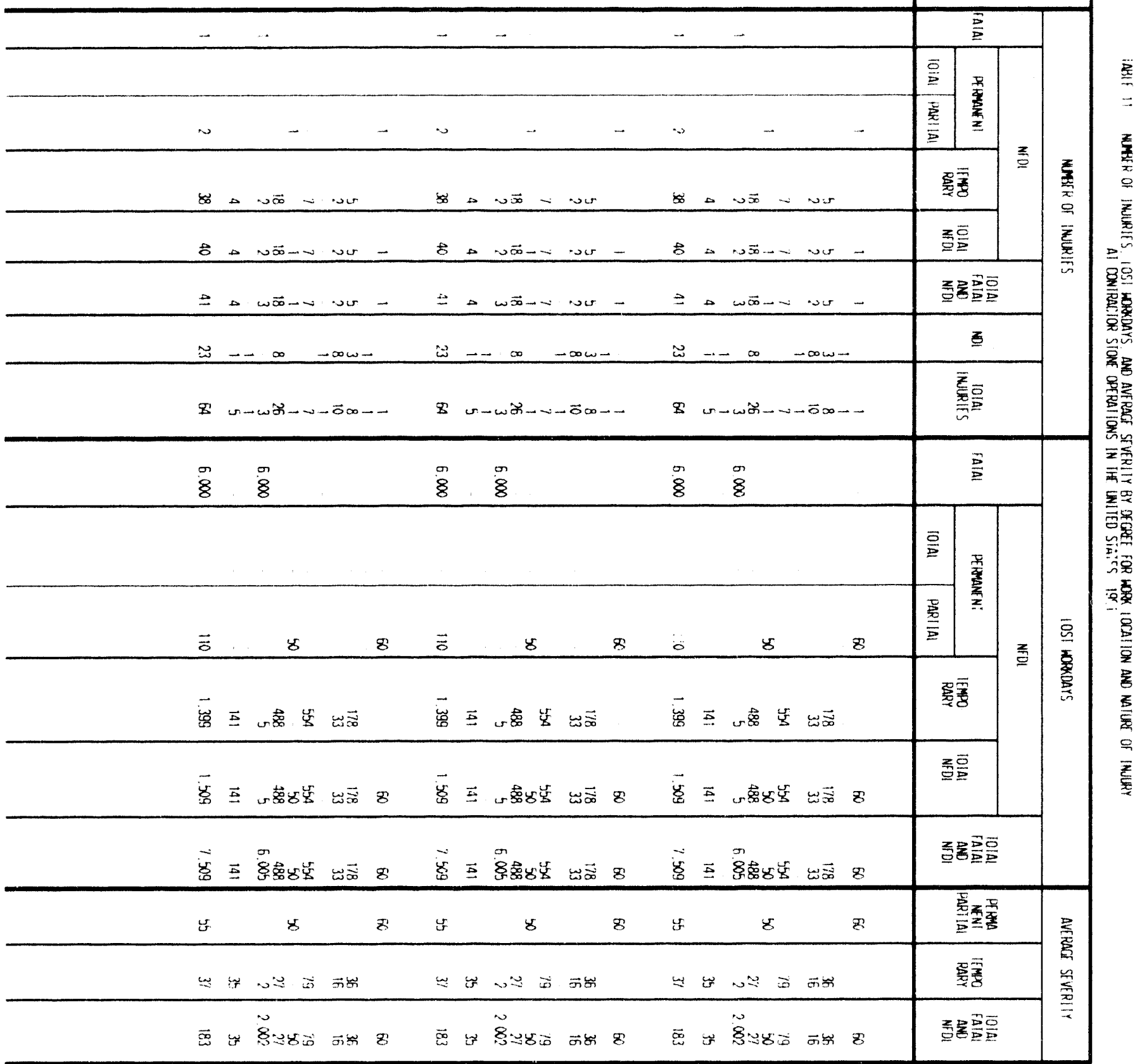




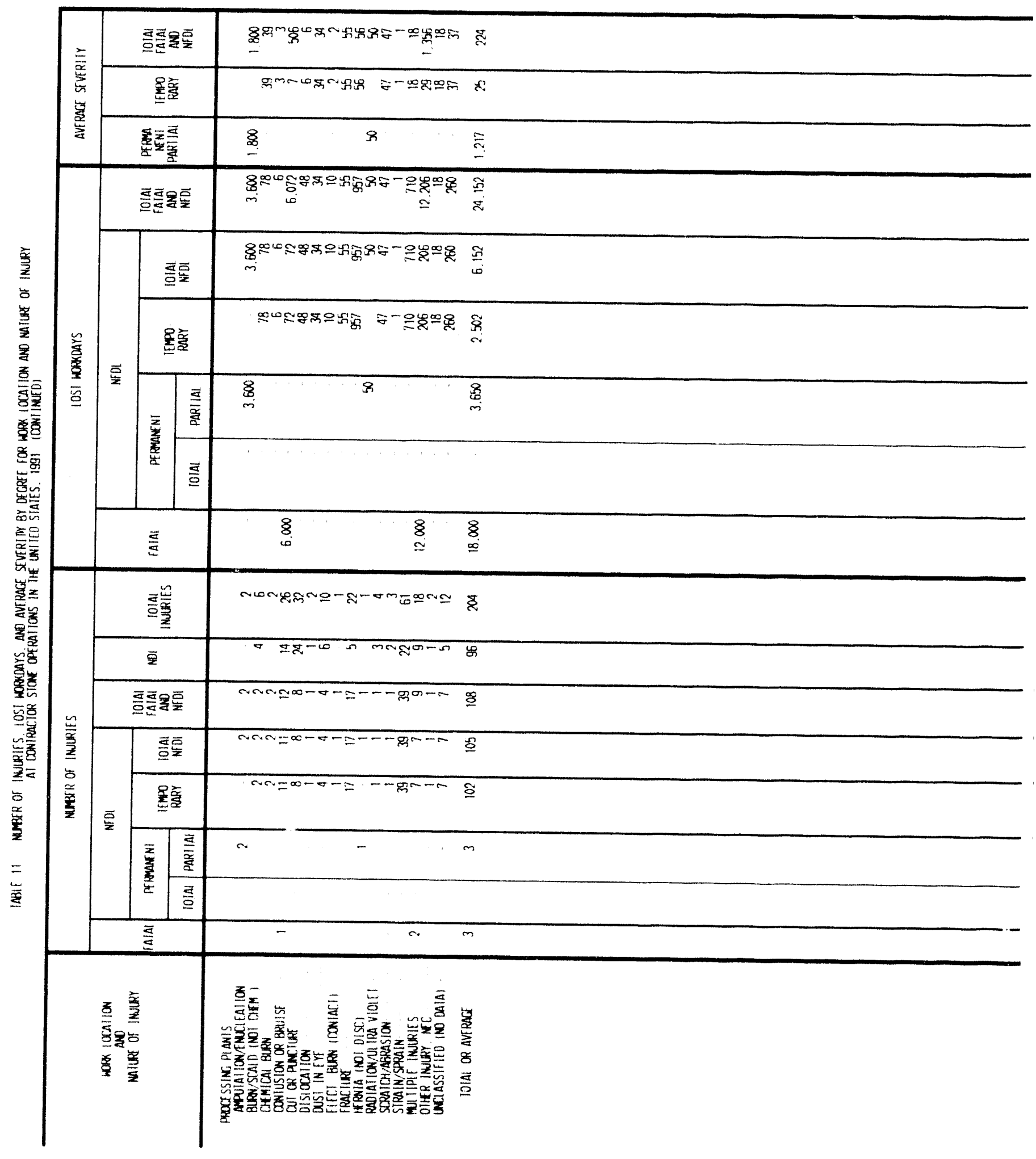




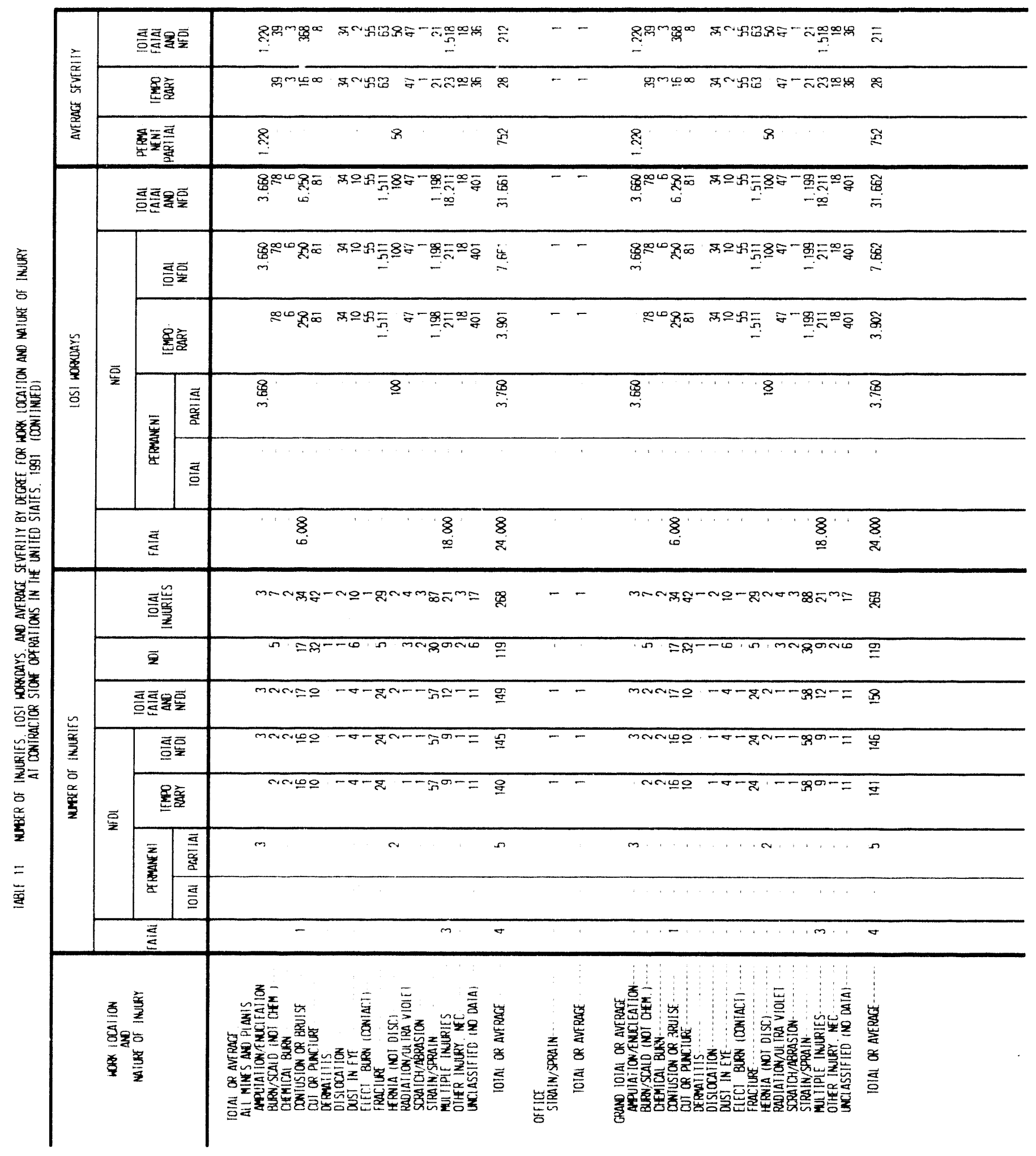




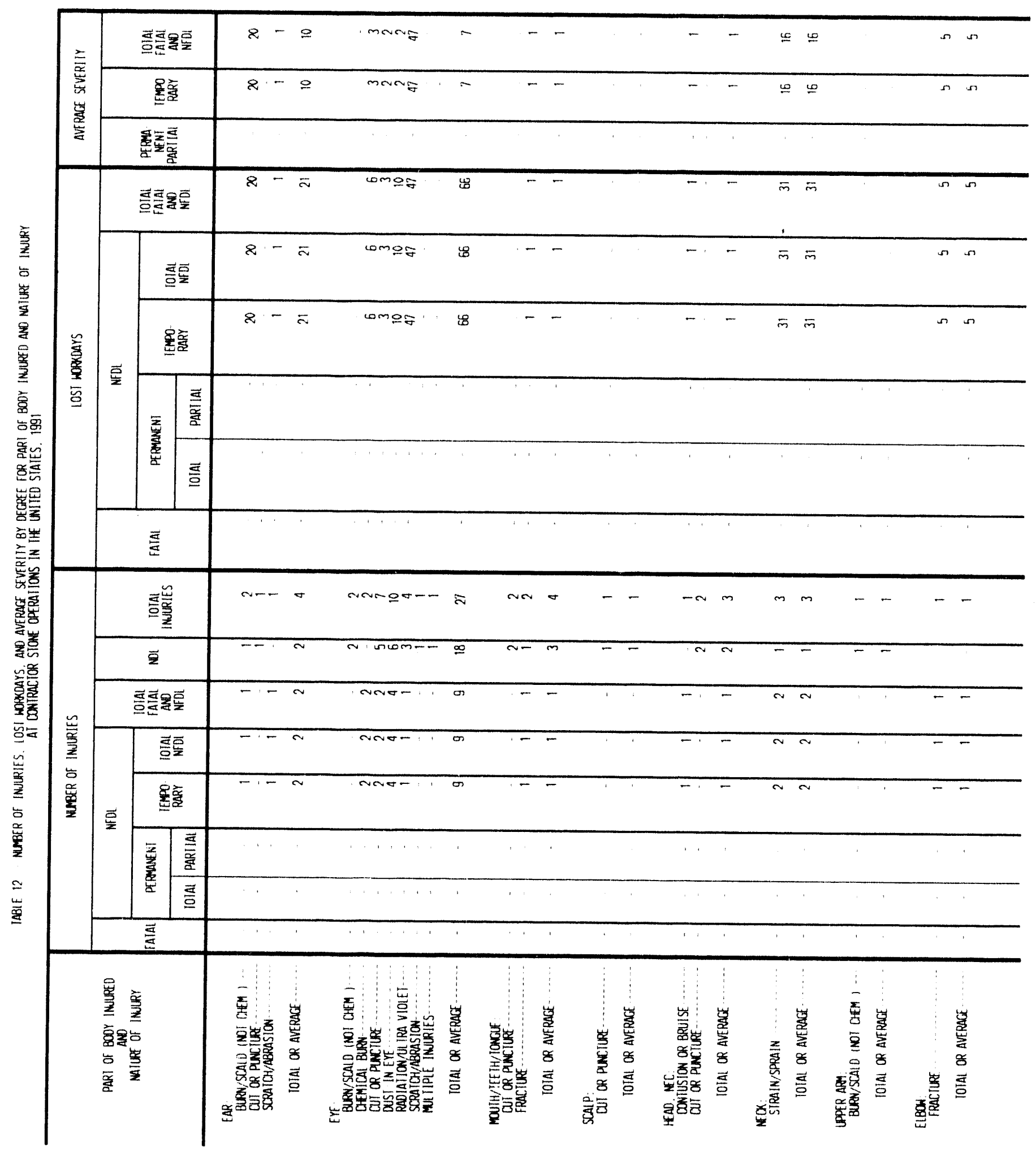




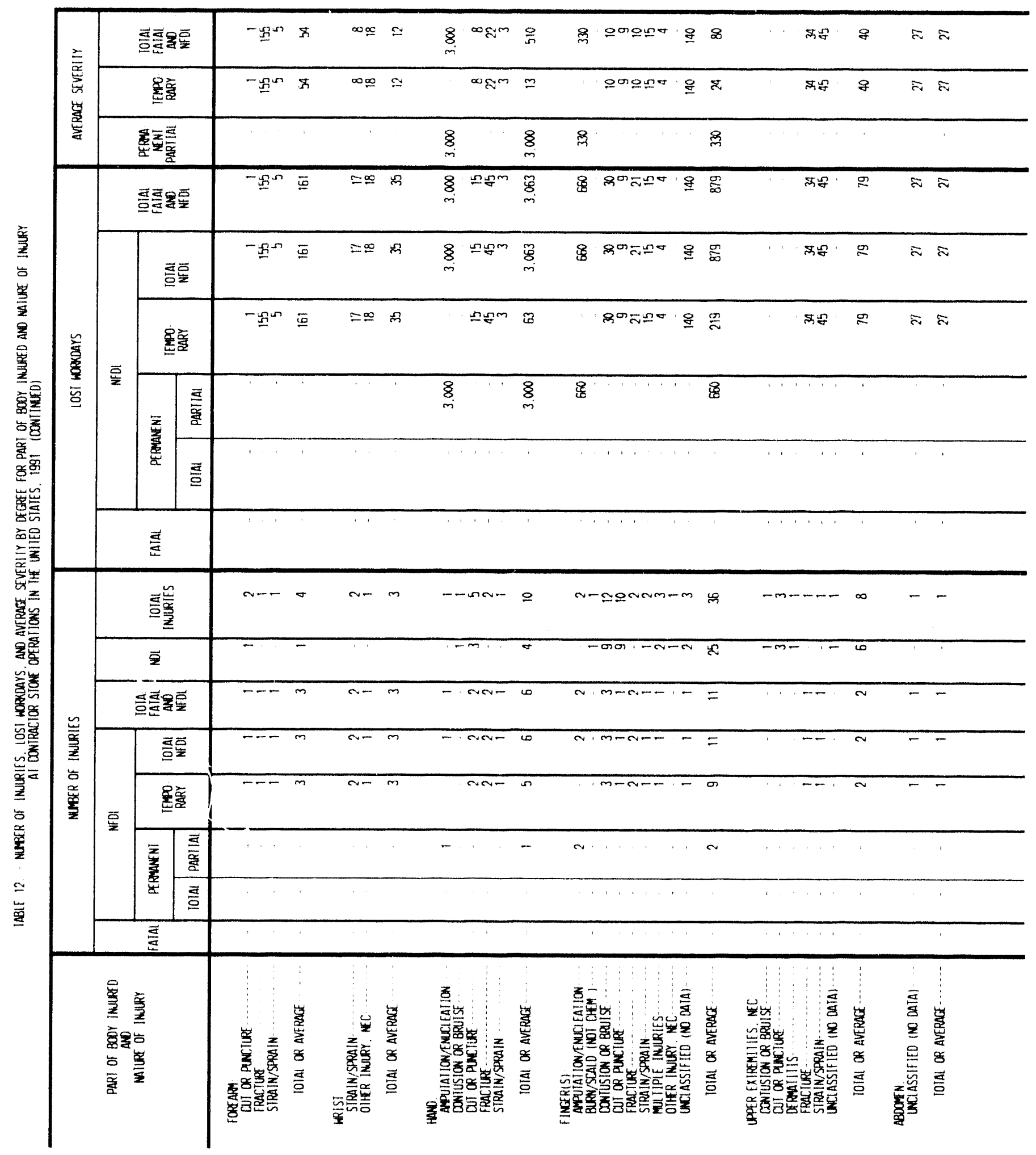




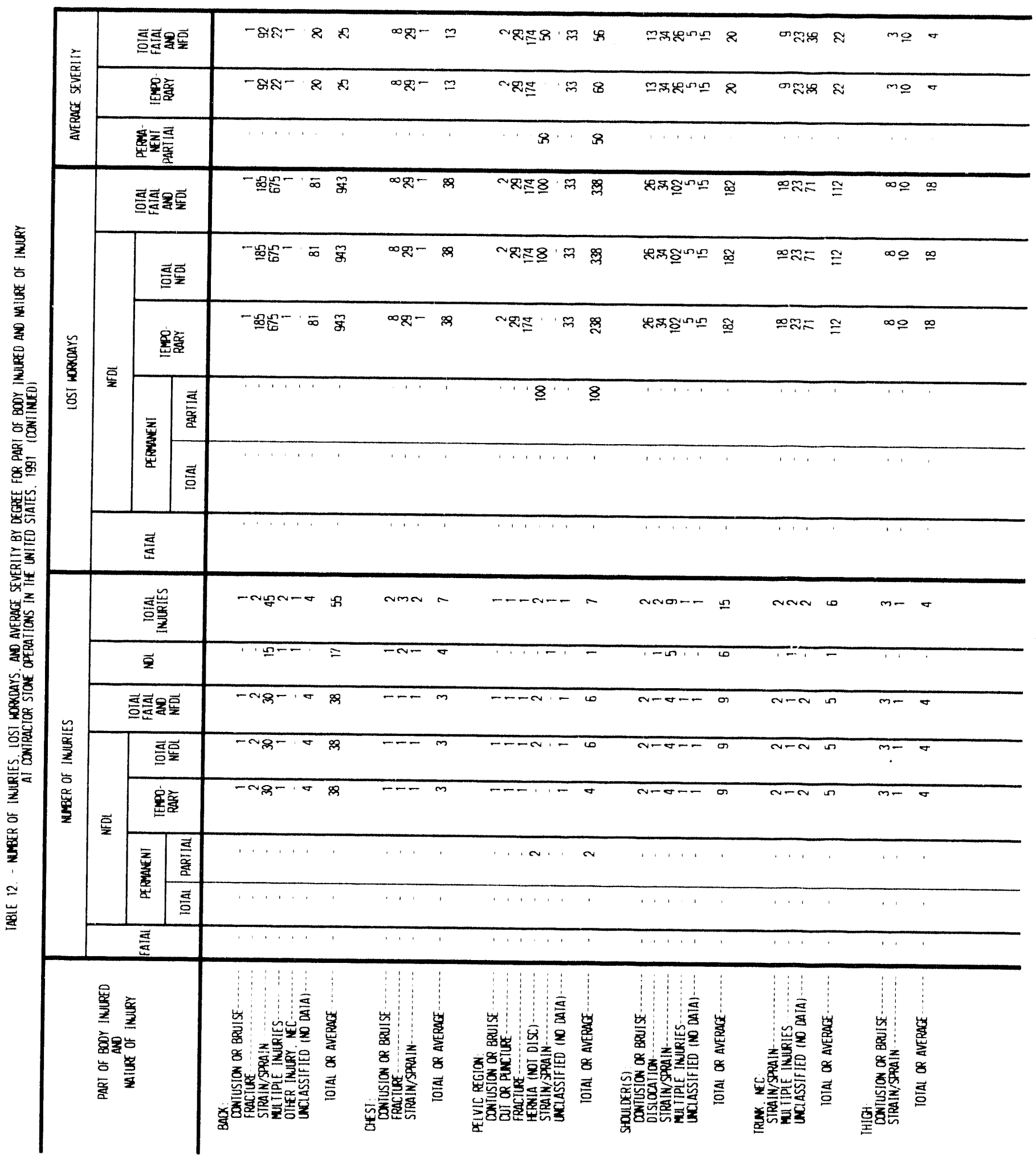



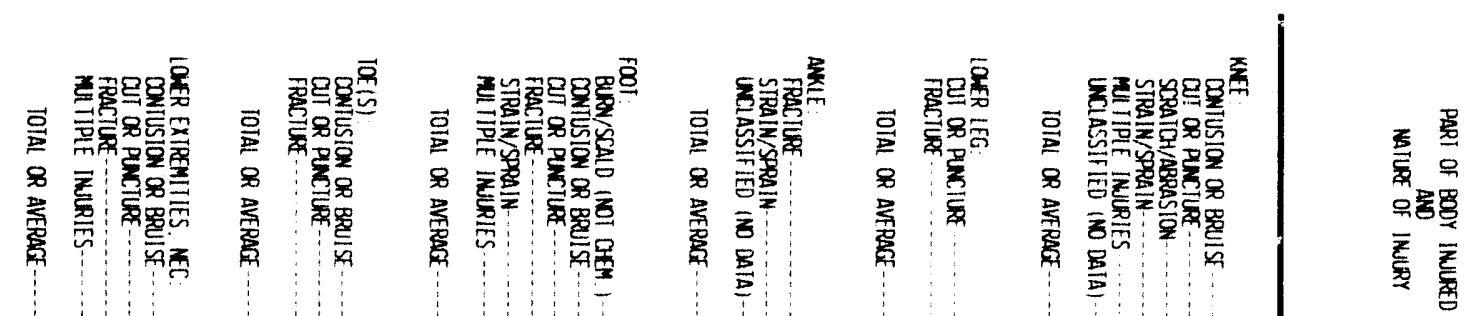

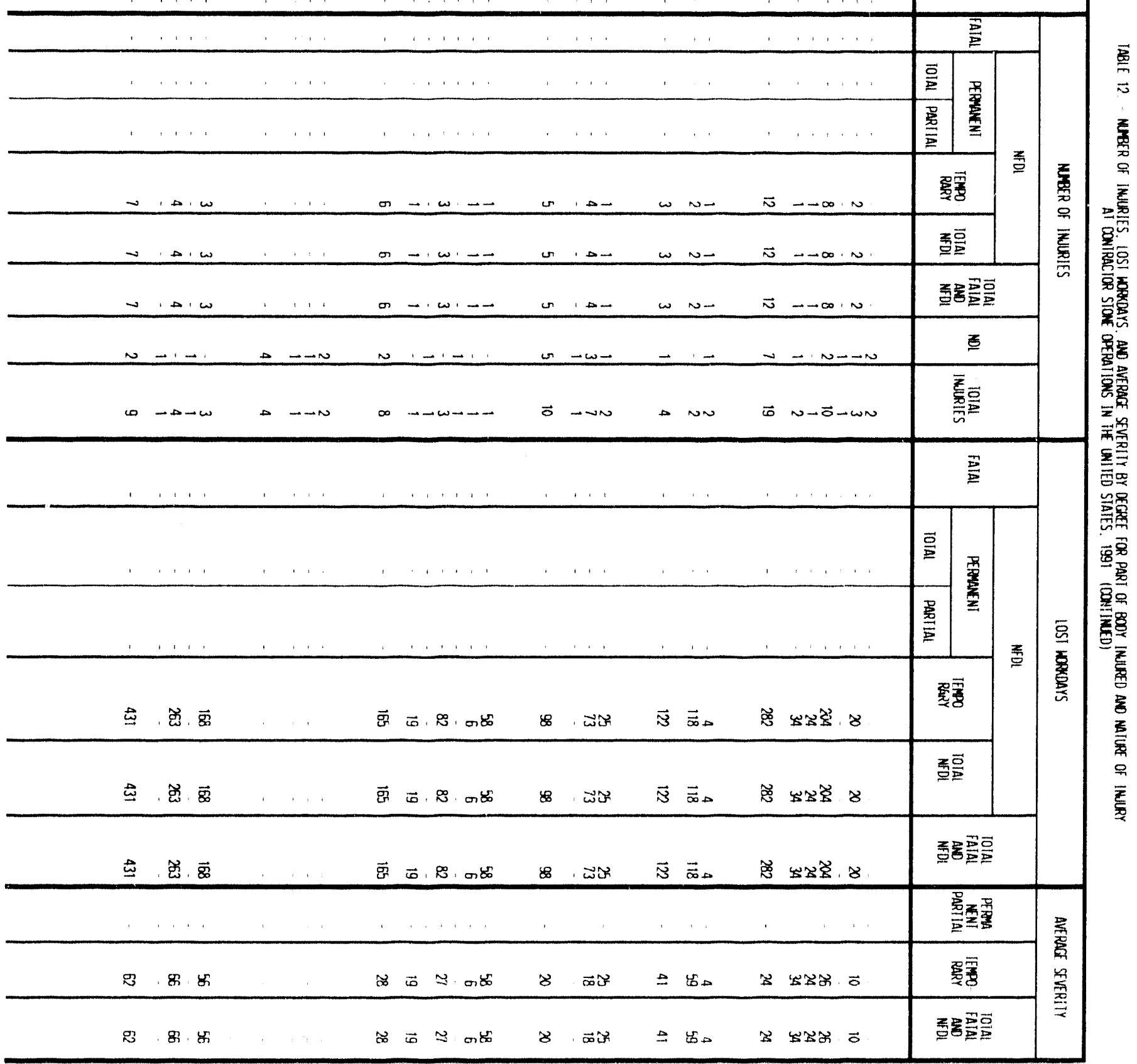




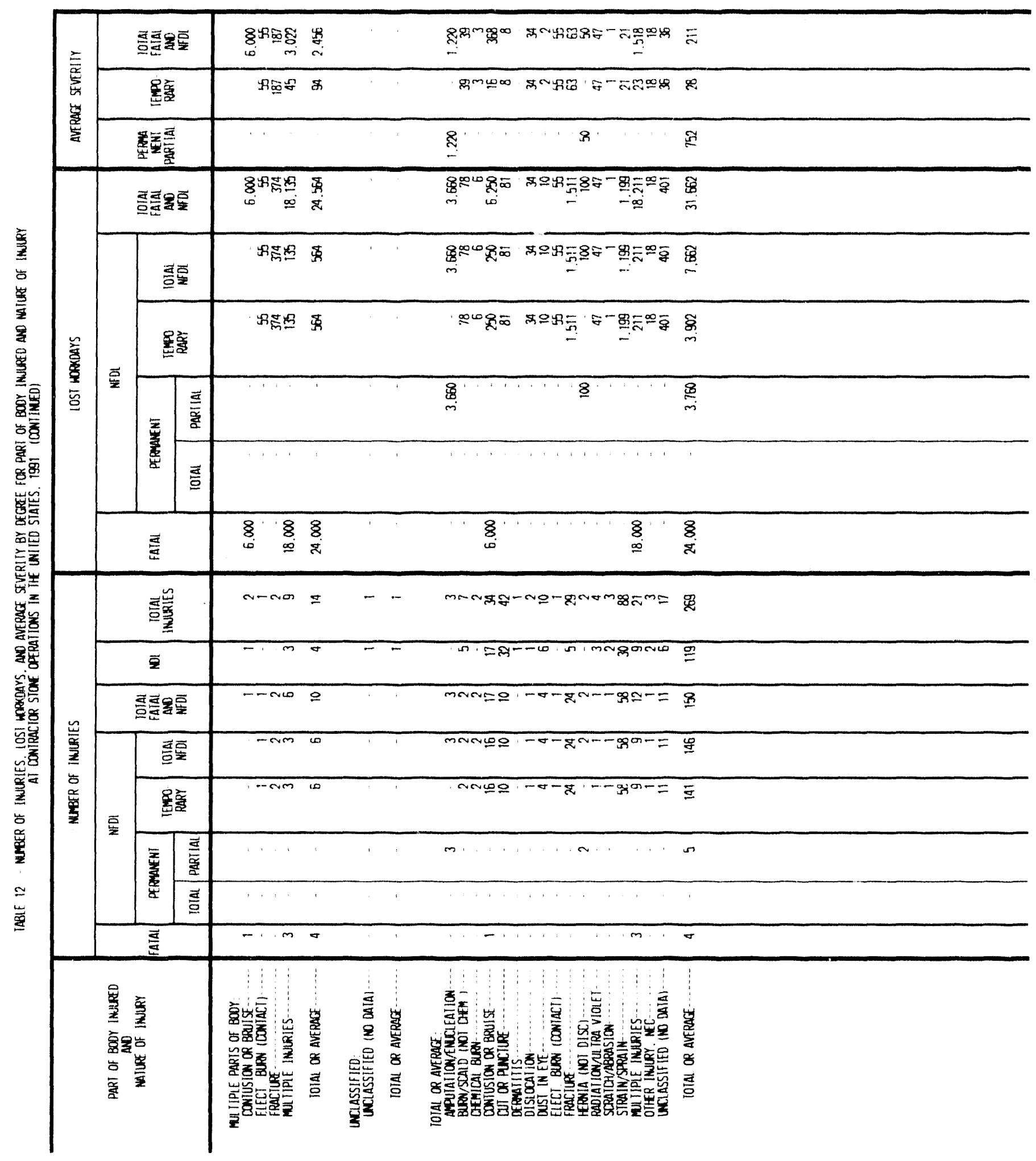




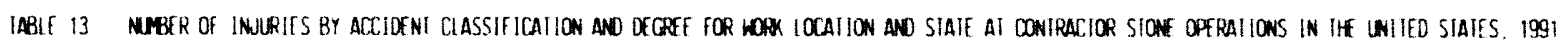

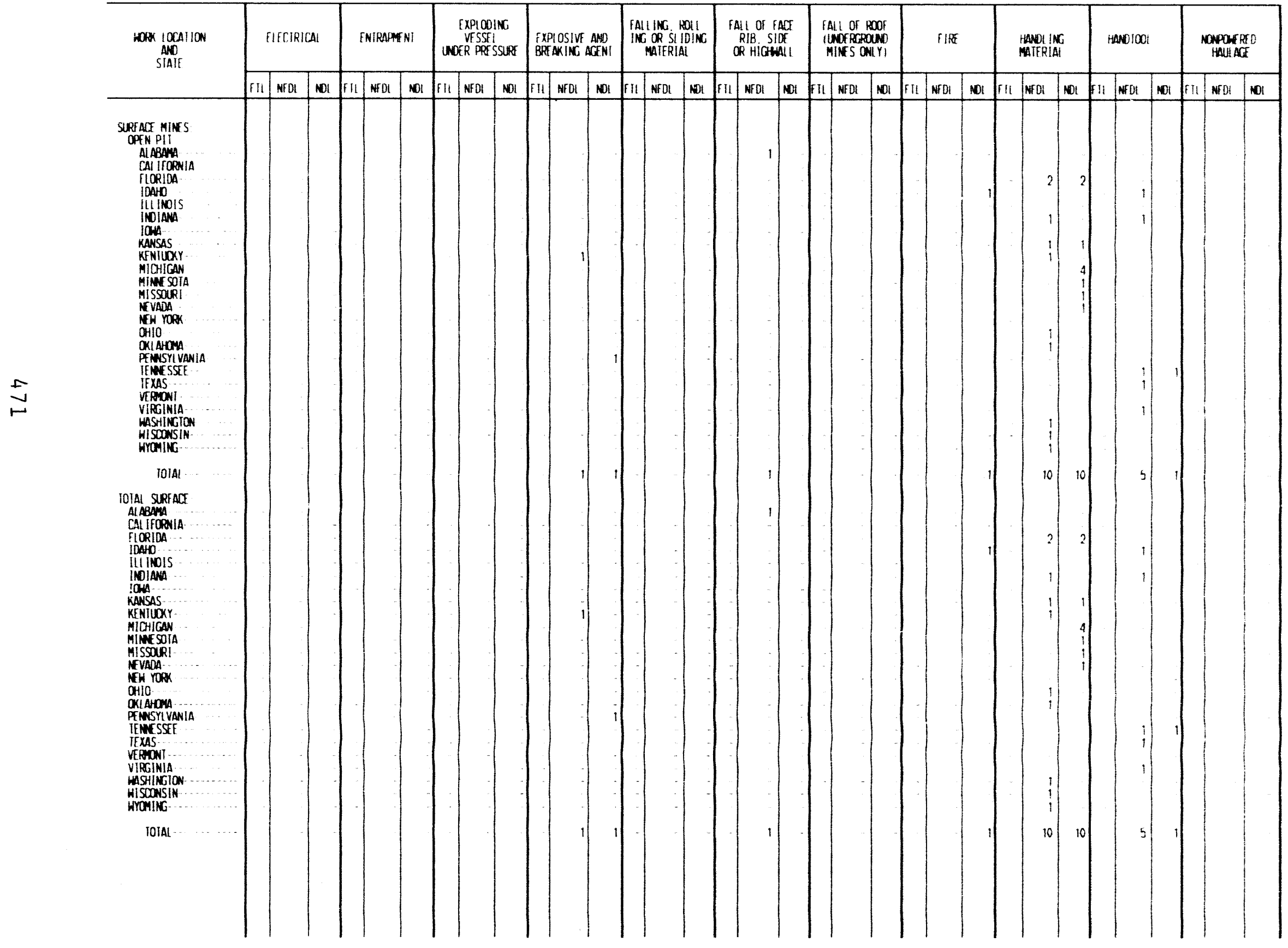




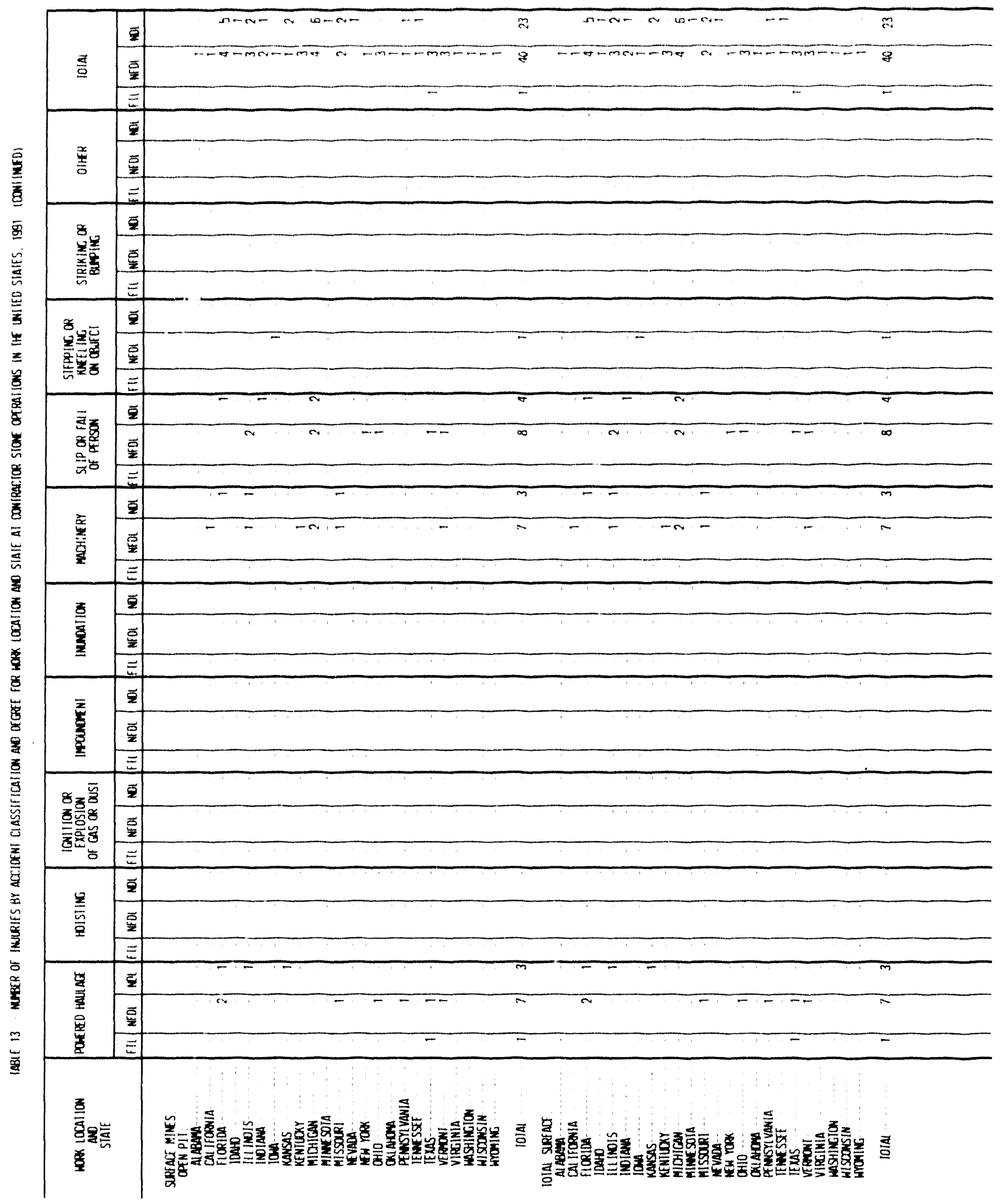




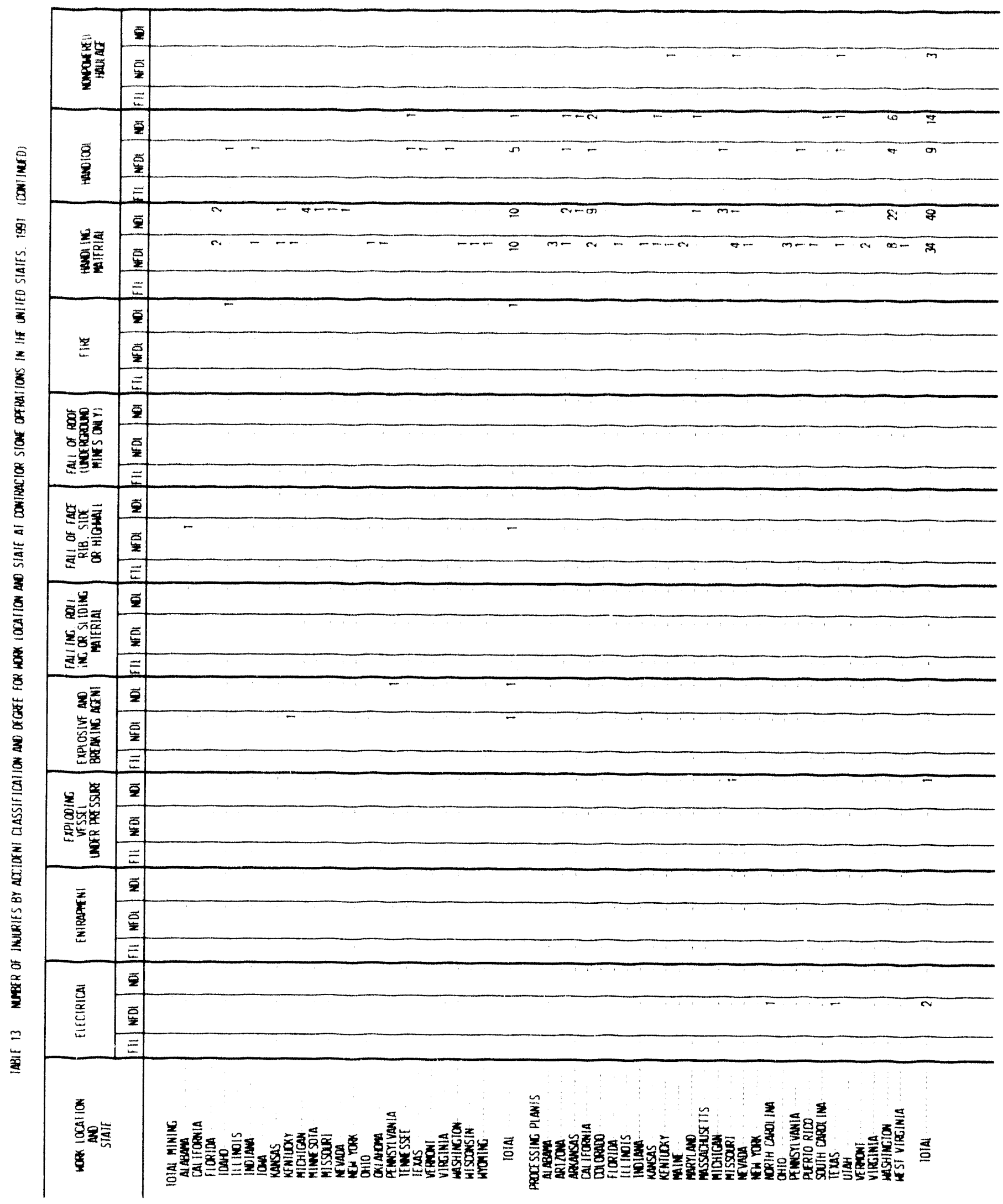




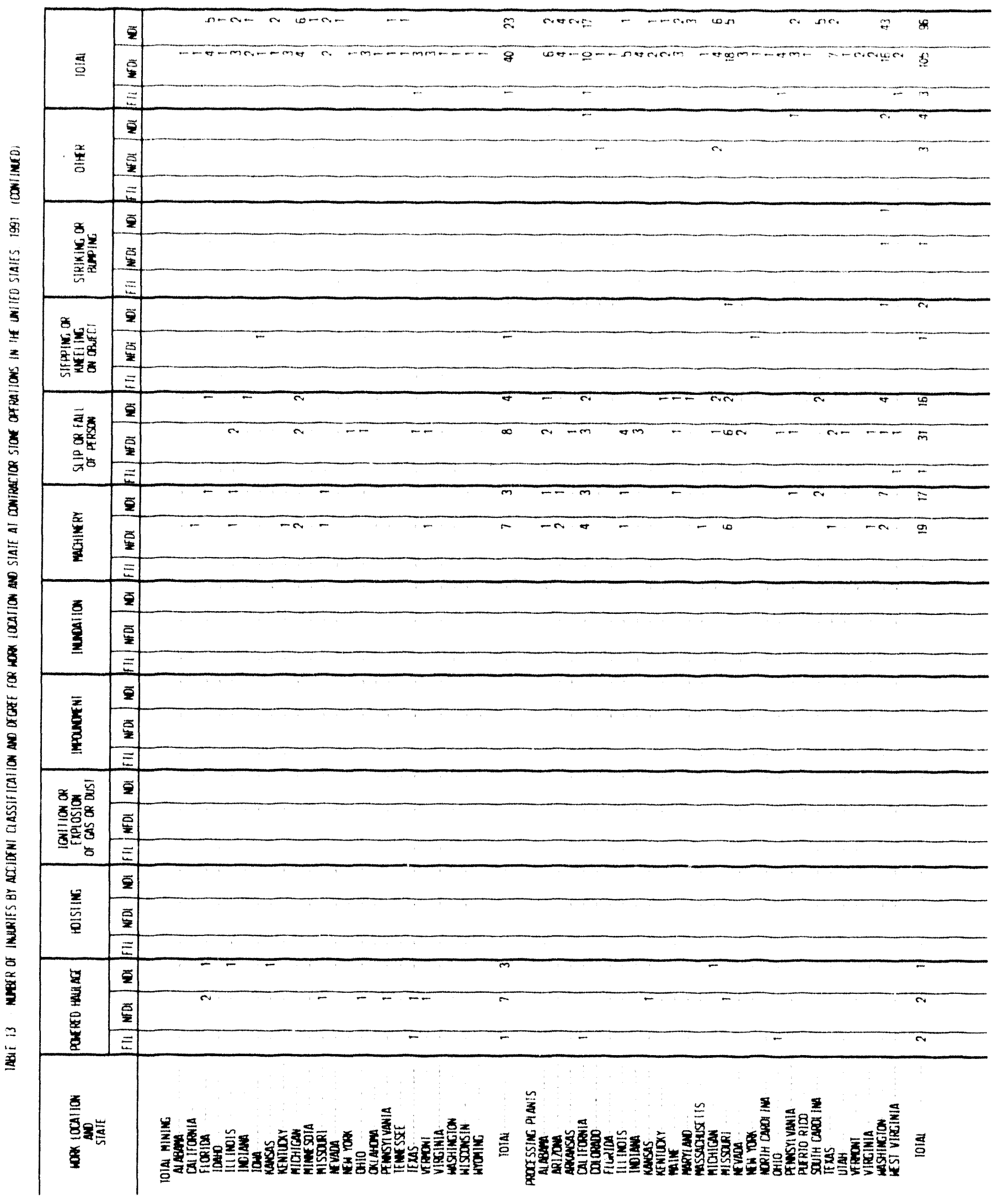




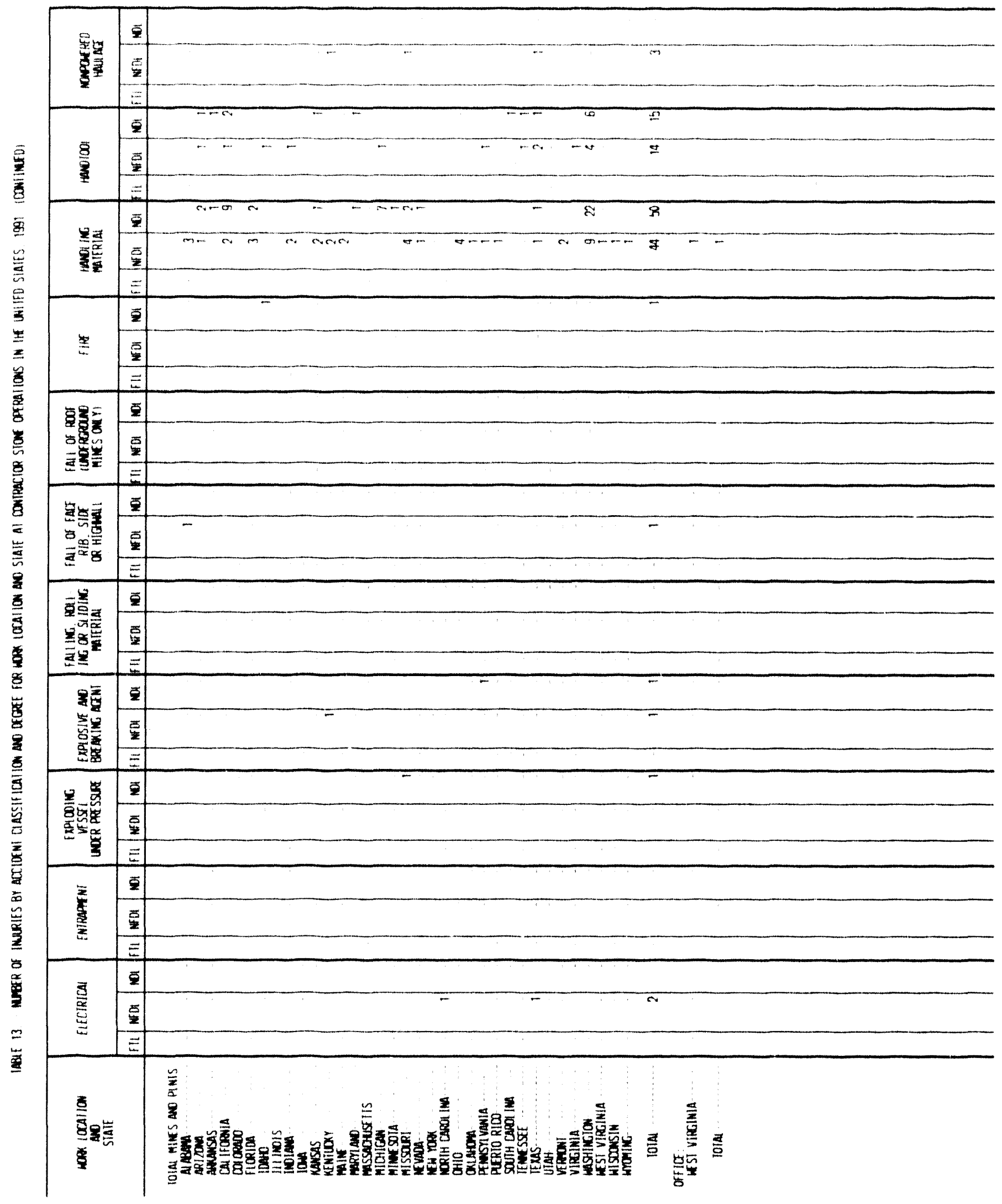




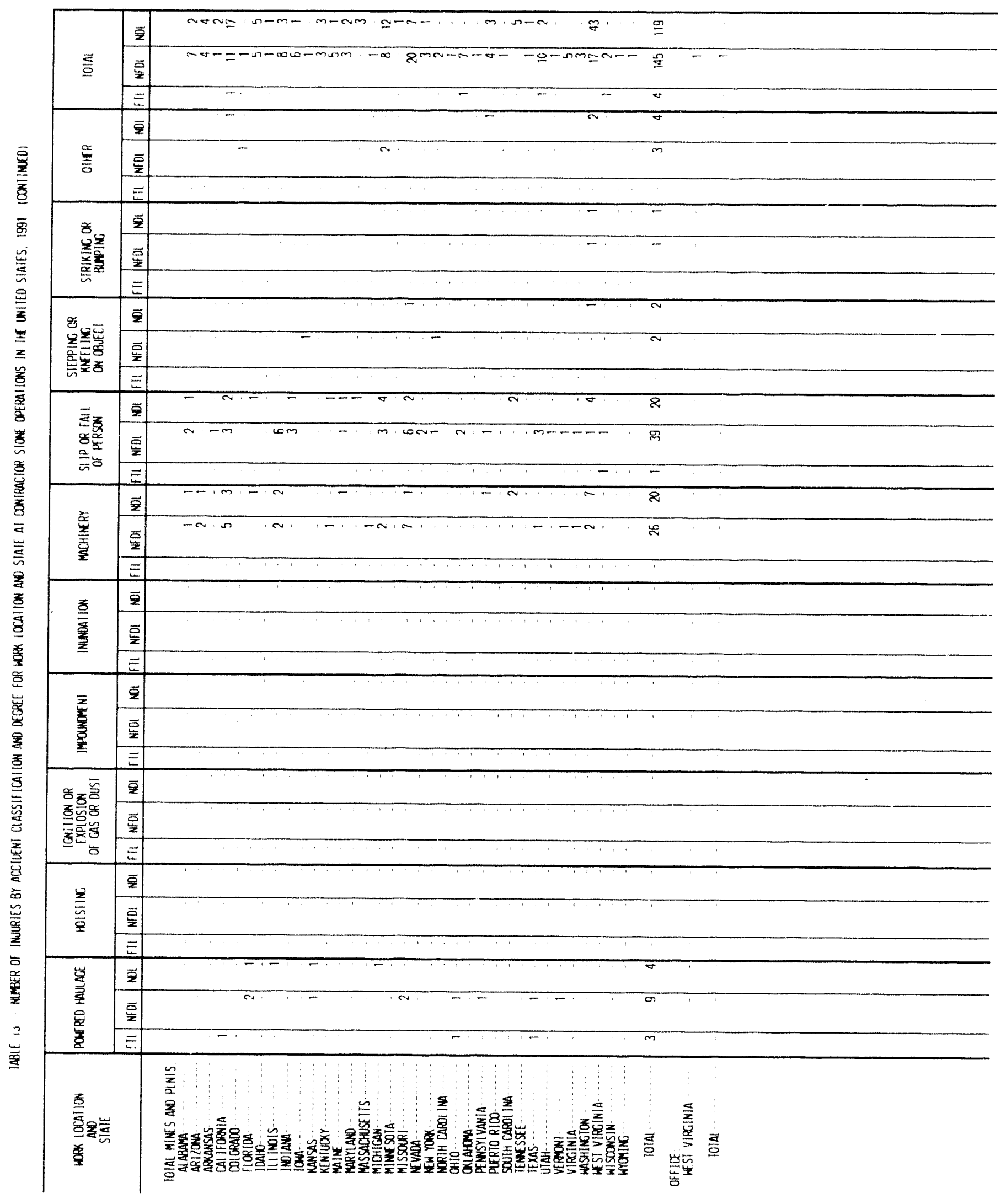




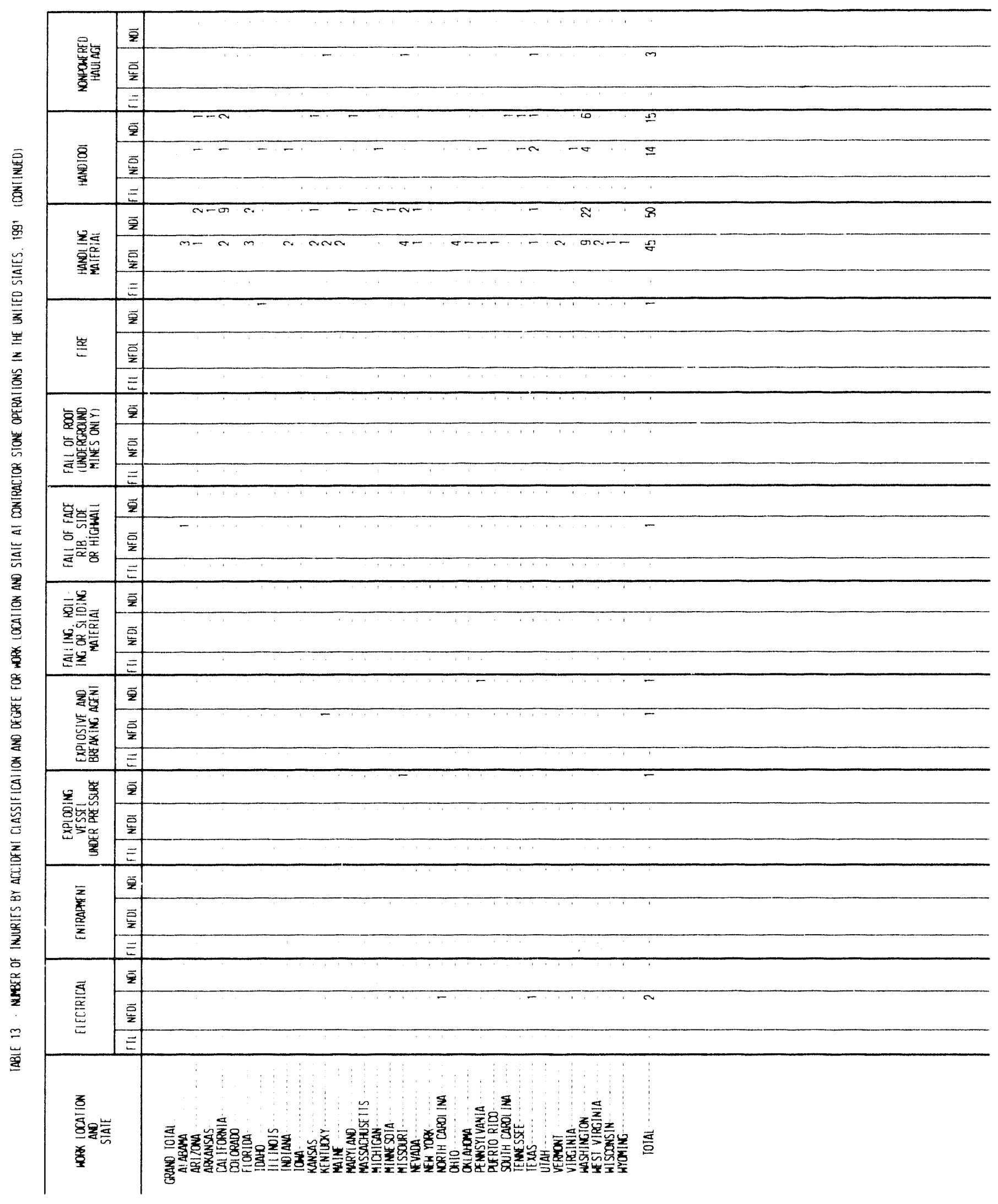




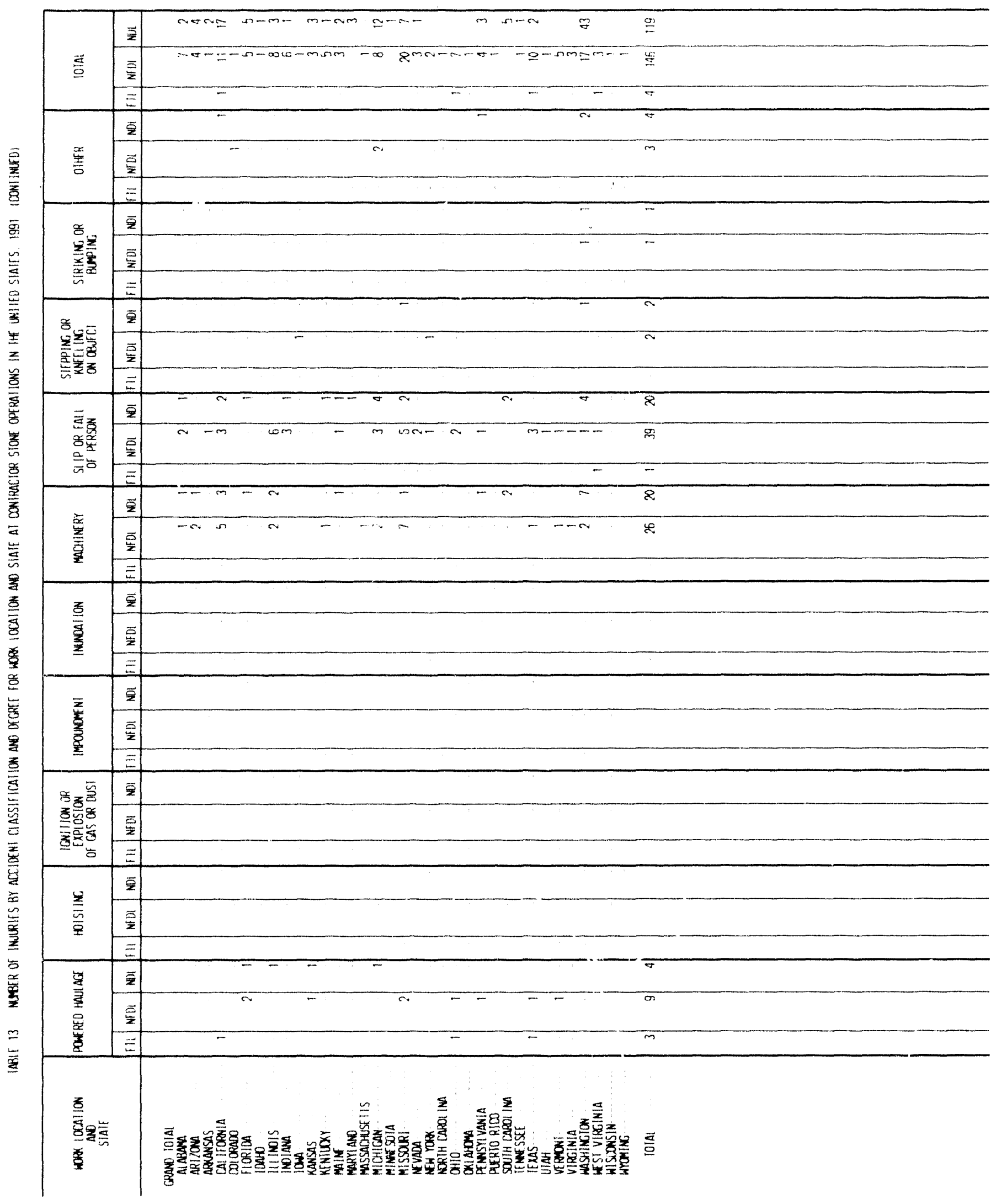




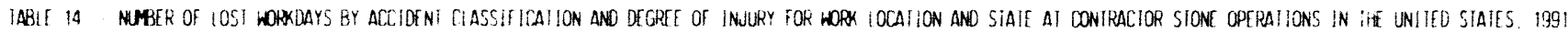

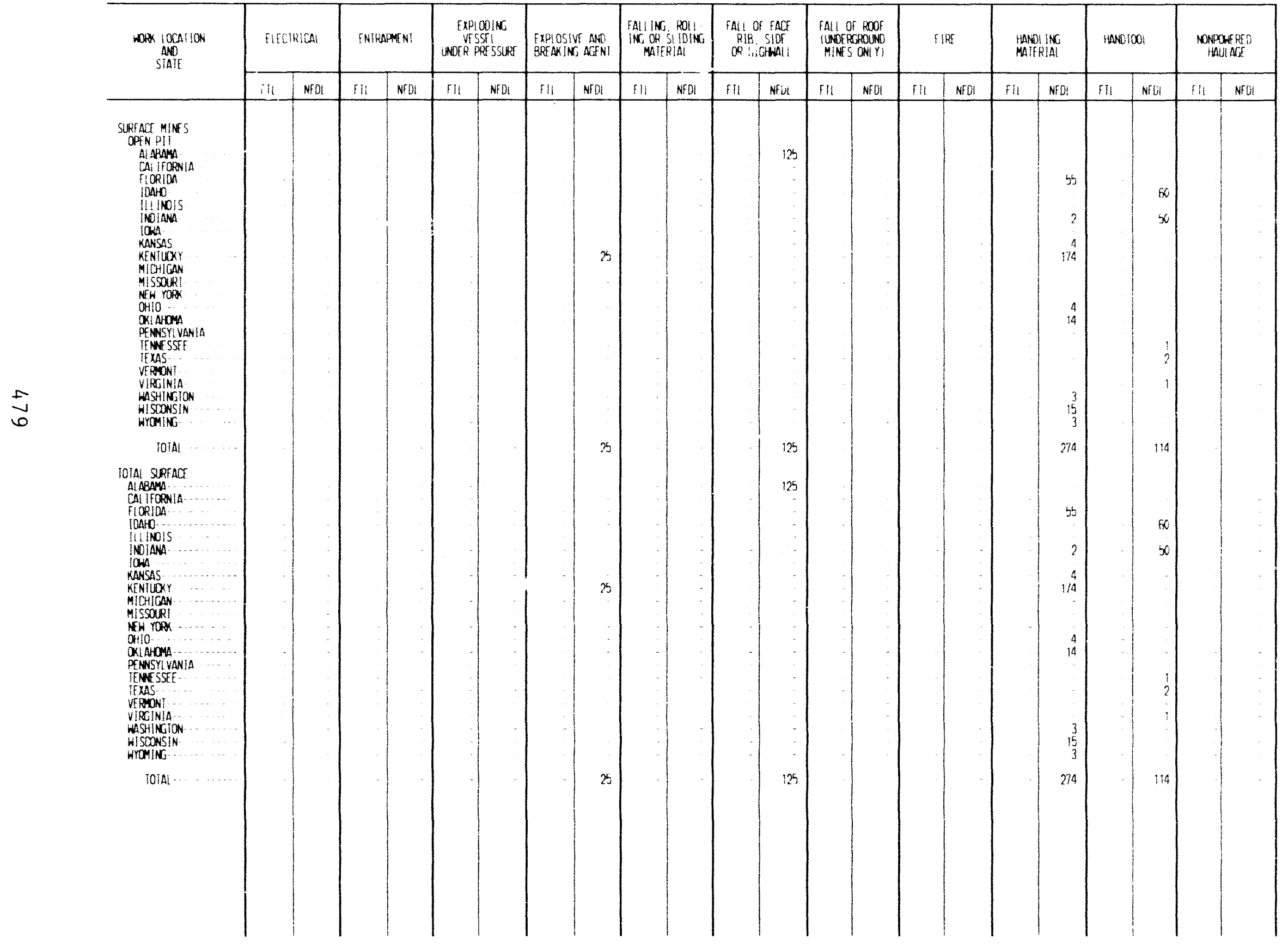




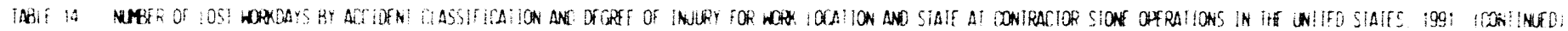

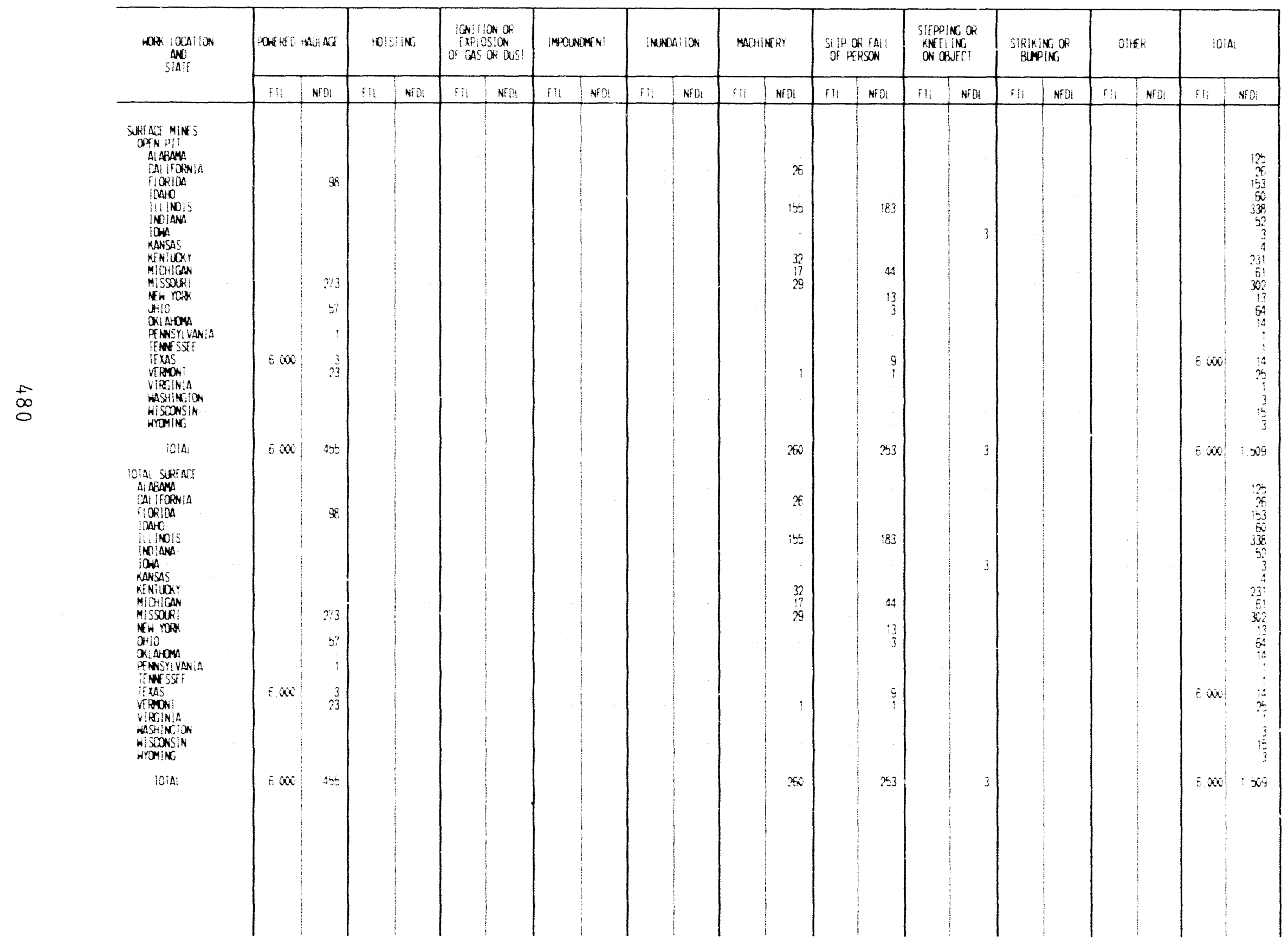




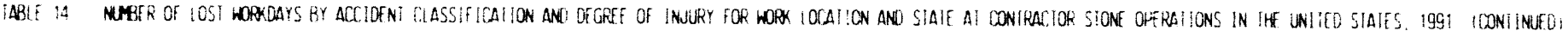

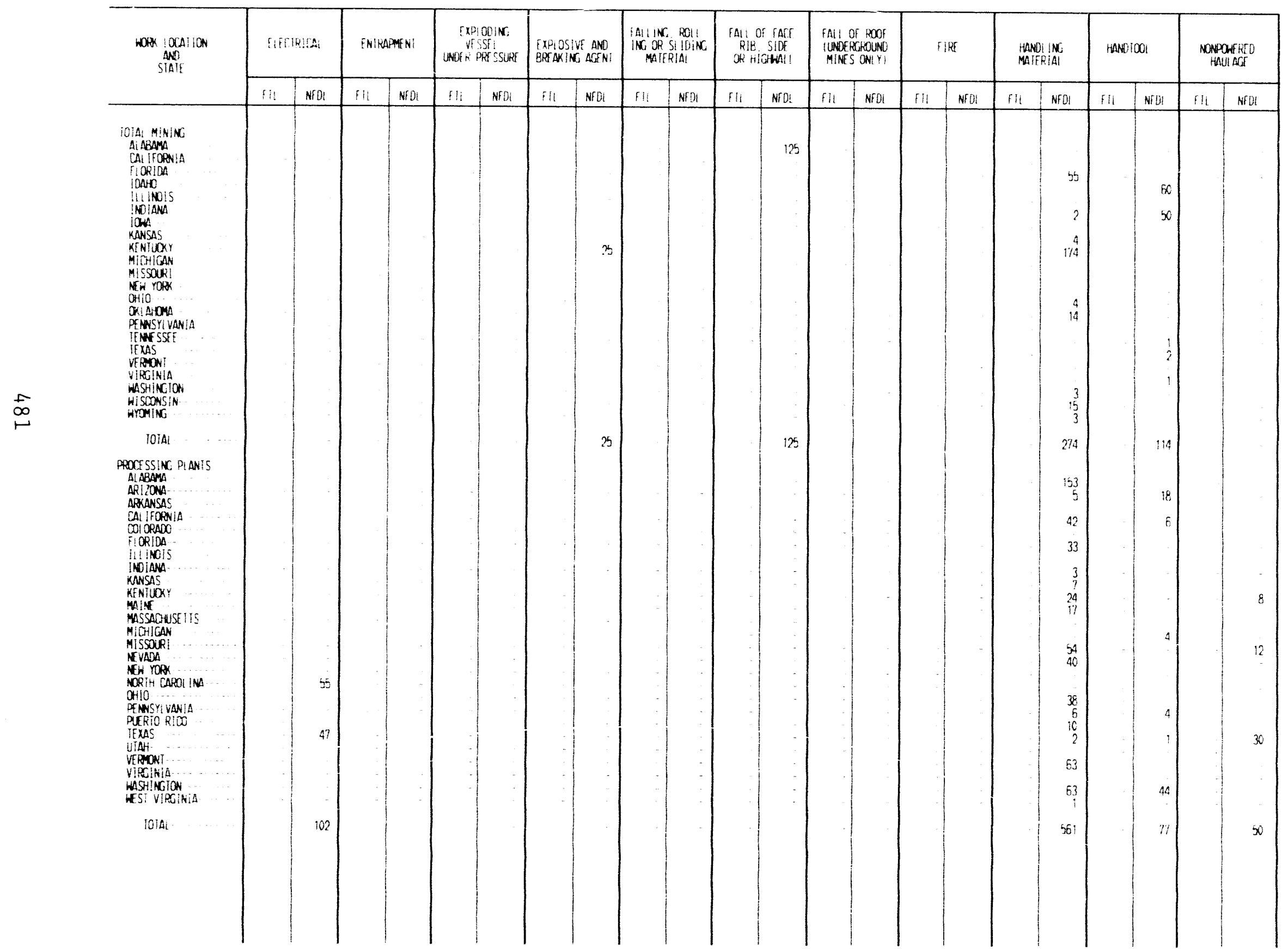




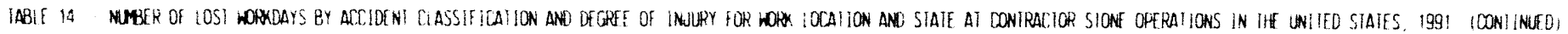

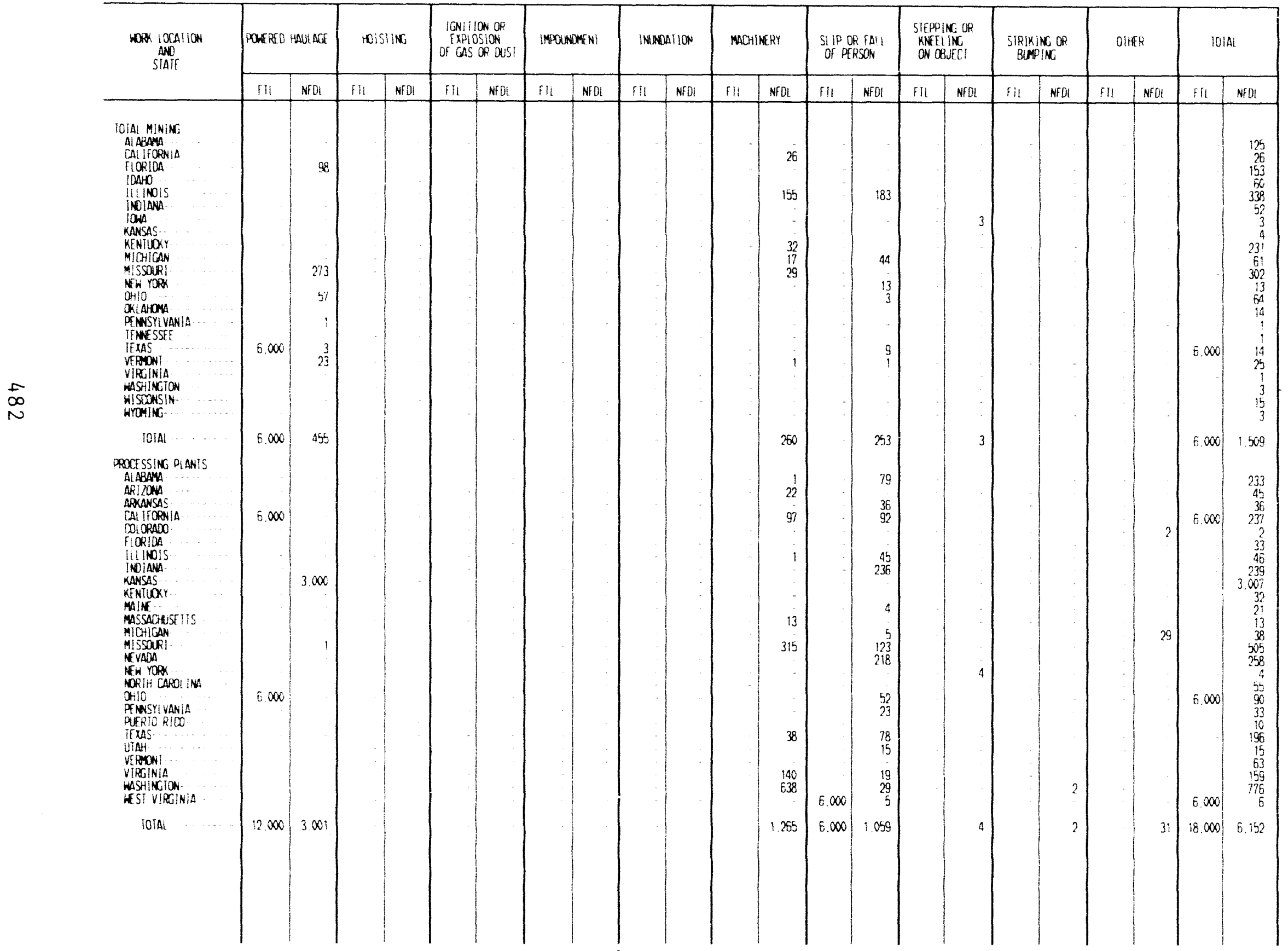




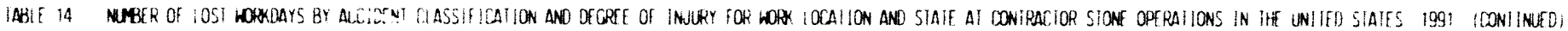

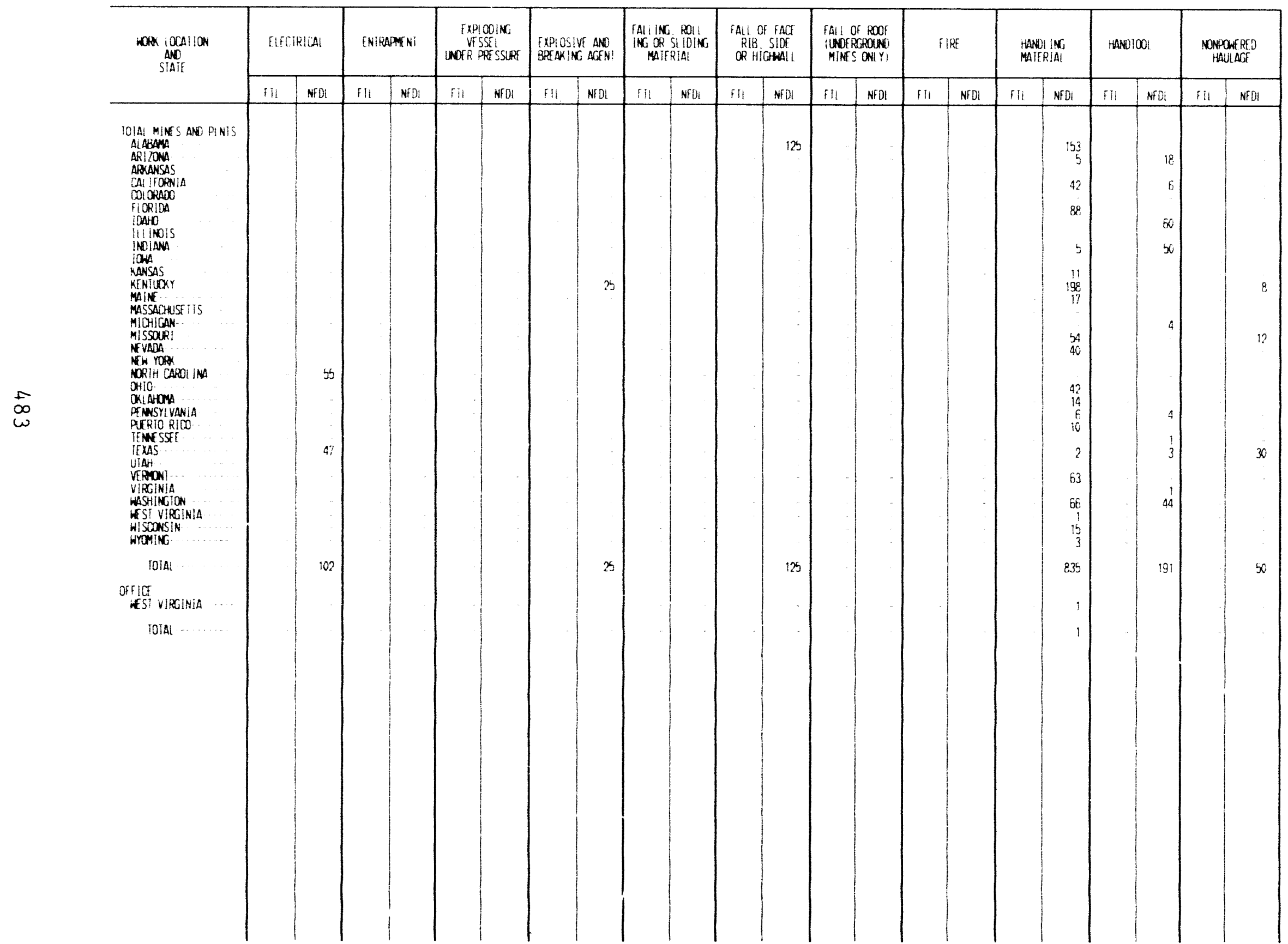




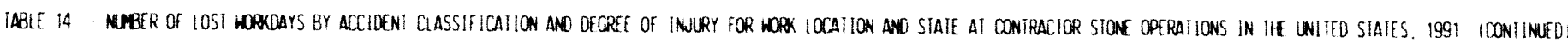

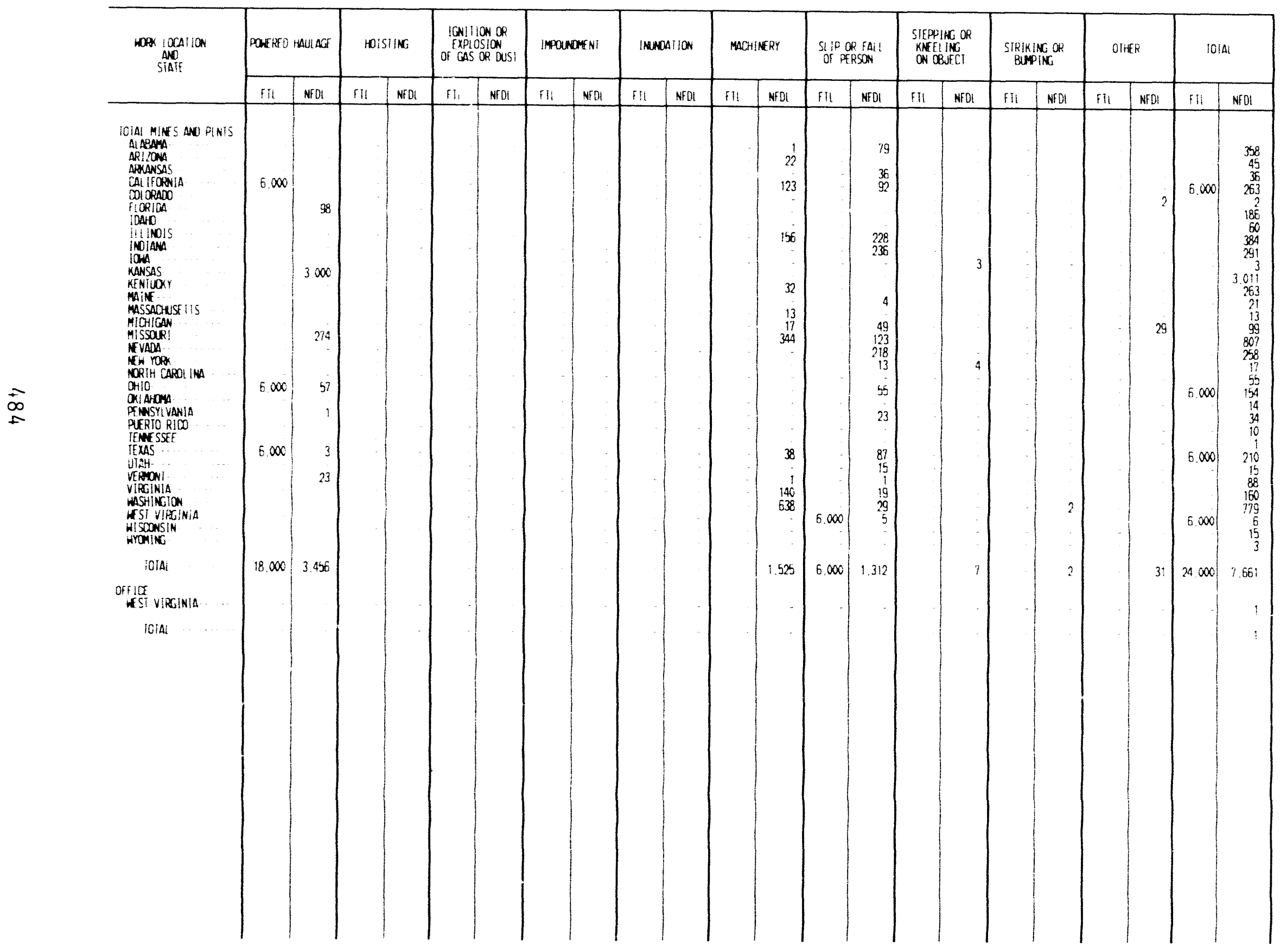




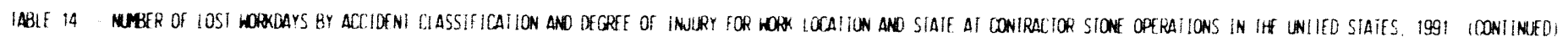

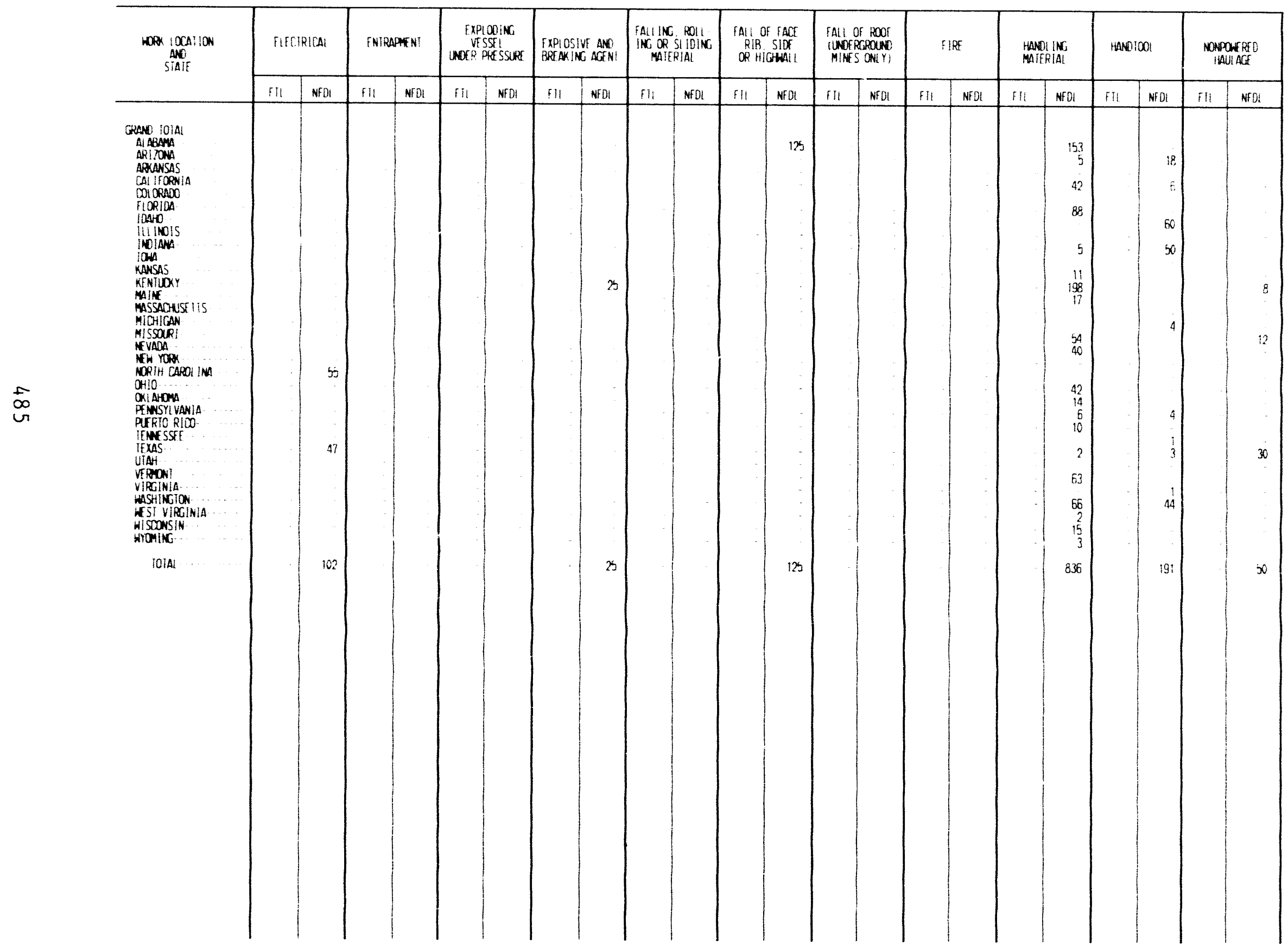



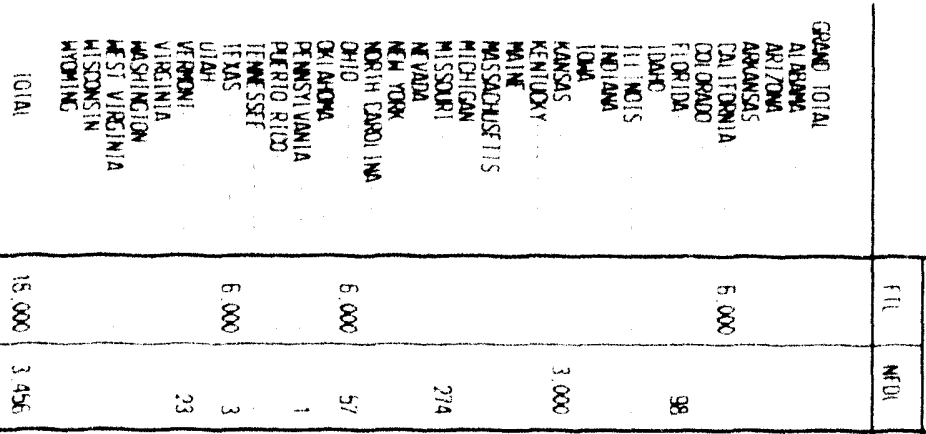

\begin{tabular}{|c|c|c|c|c|c|c|c|}
\hline $\begin{array}{l}\overline{\pi n} \\
\overbrace{}^{n}\end{array}$ & $\frac{\pi}{8}$ & \& & & & $\frac{\pi}{8}$ & $\equiv$ & 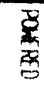 \\
\hline$\vec{z}$ & $\dot{\omega} \omega$ & ي & 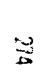 & 8 & $\$$ & 至 & $\frac{\frac{\xi}{2}}{2}$ \\
\hline
\end{tabular}

\begin{tabular}{r|l}
\hline & $=$ \\
\hline &
\end{tabular}

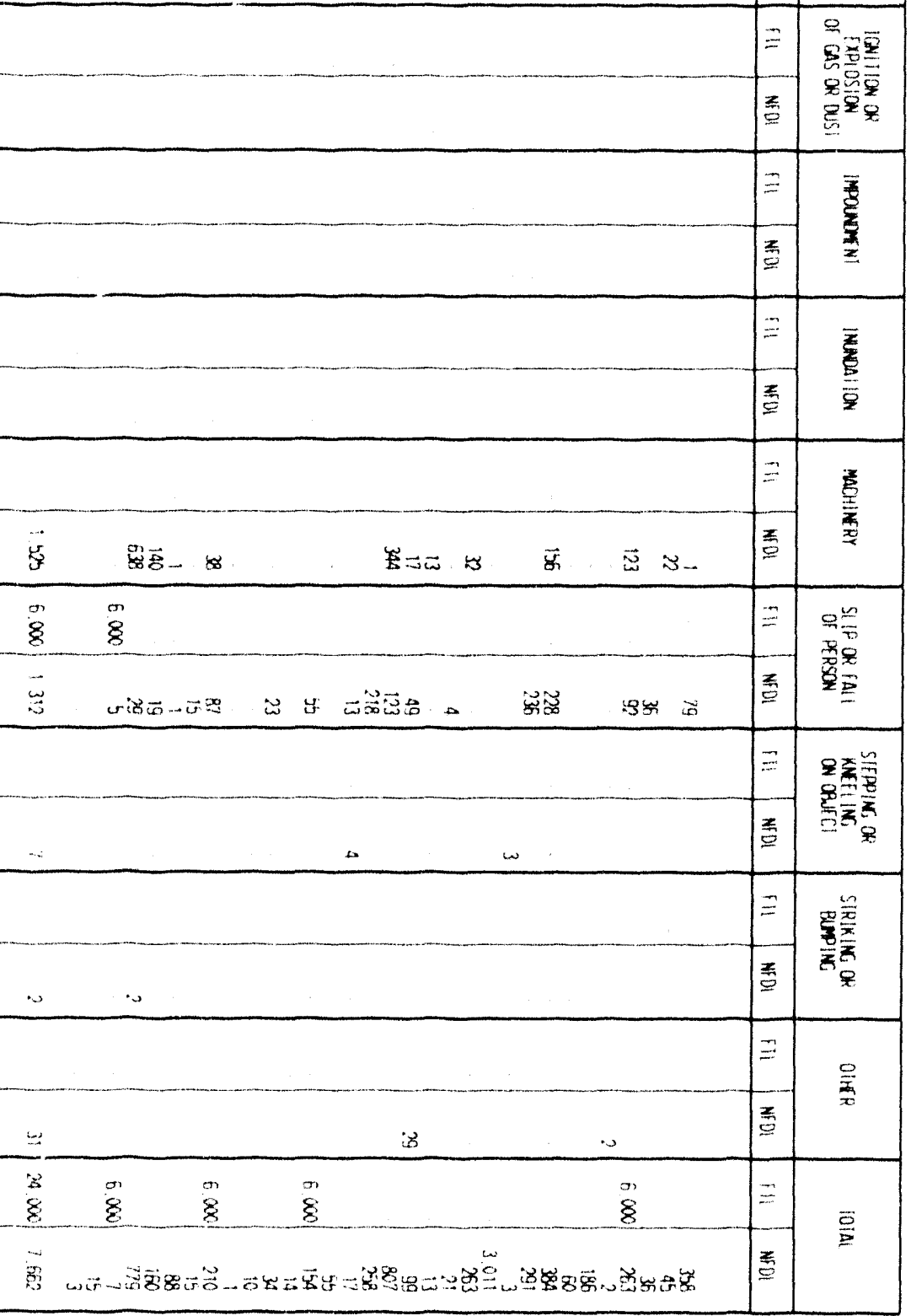




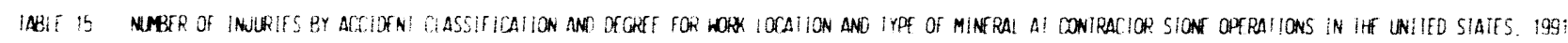

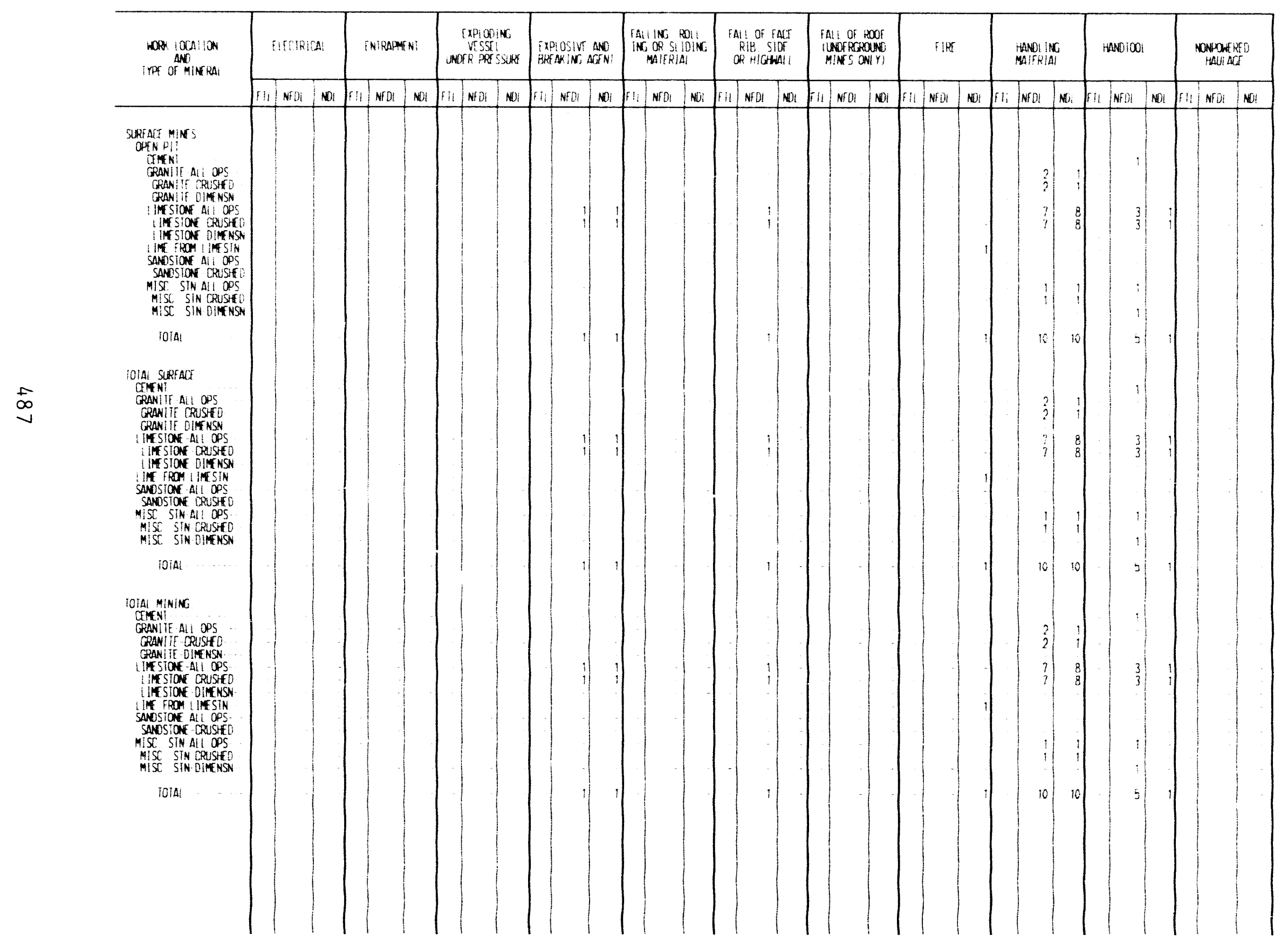




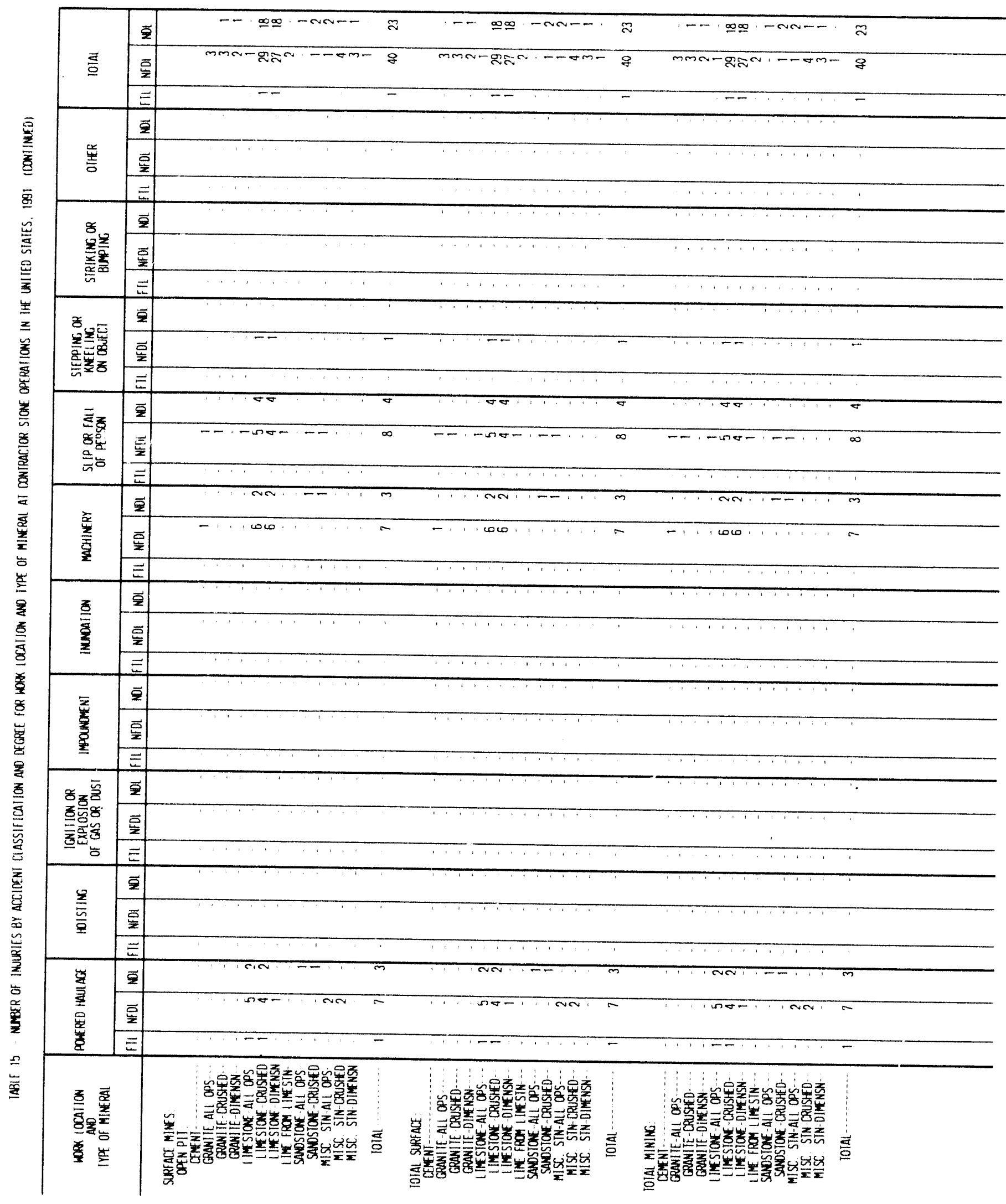




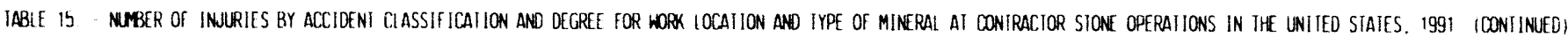

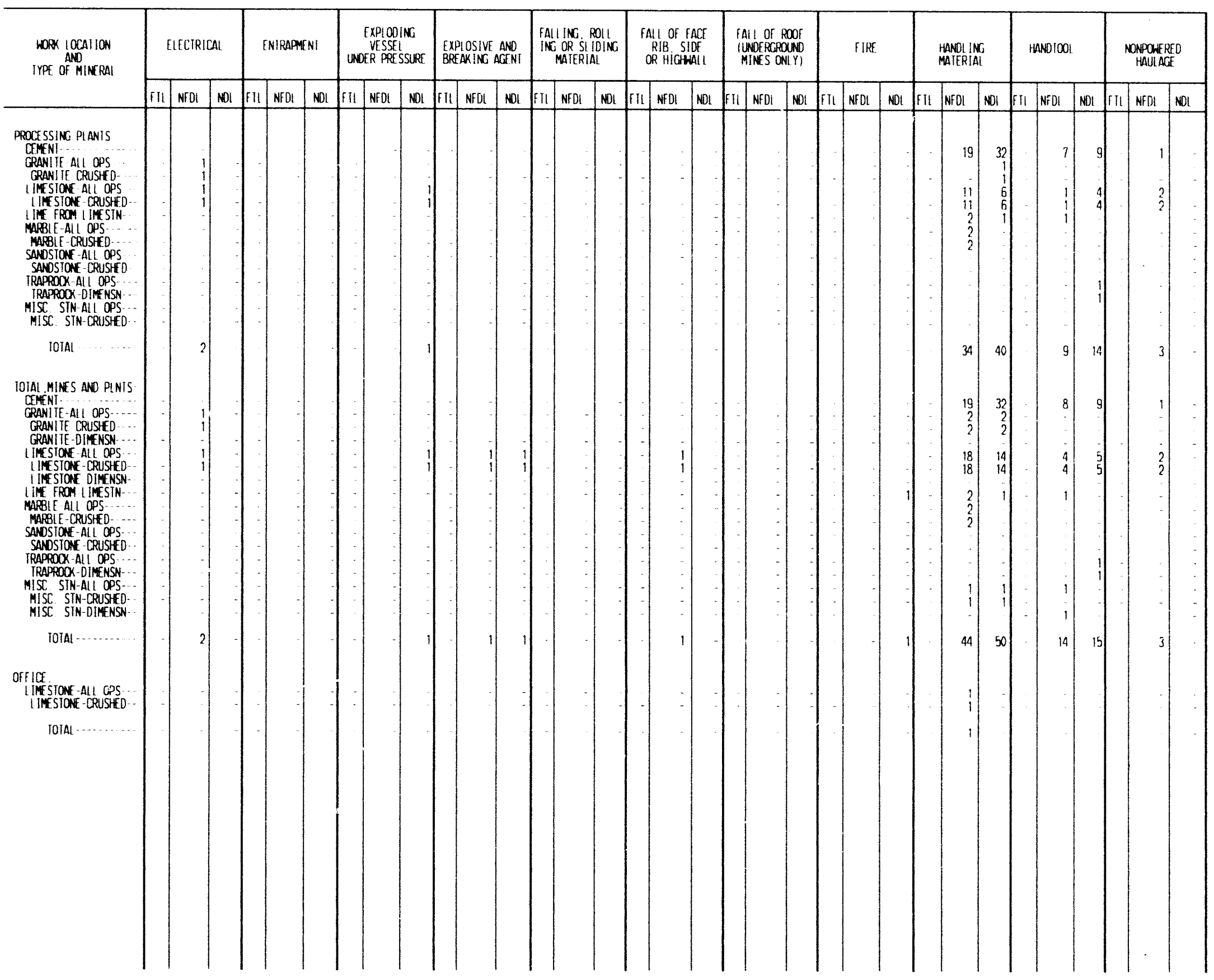




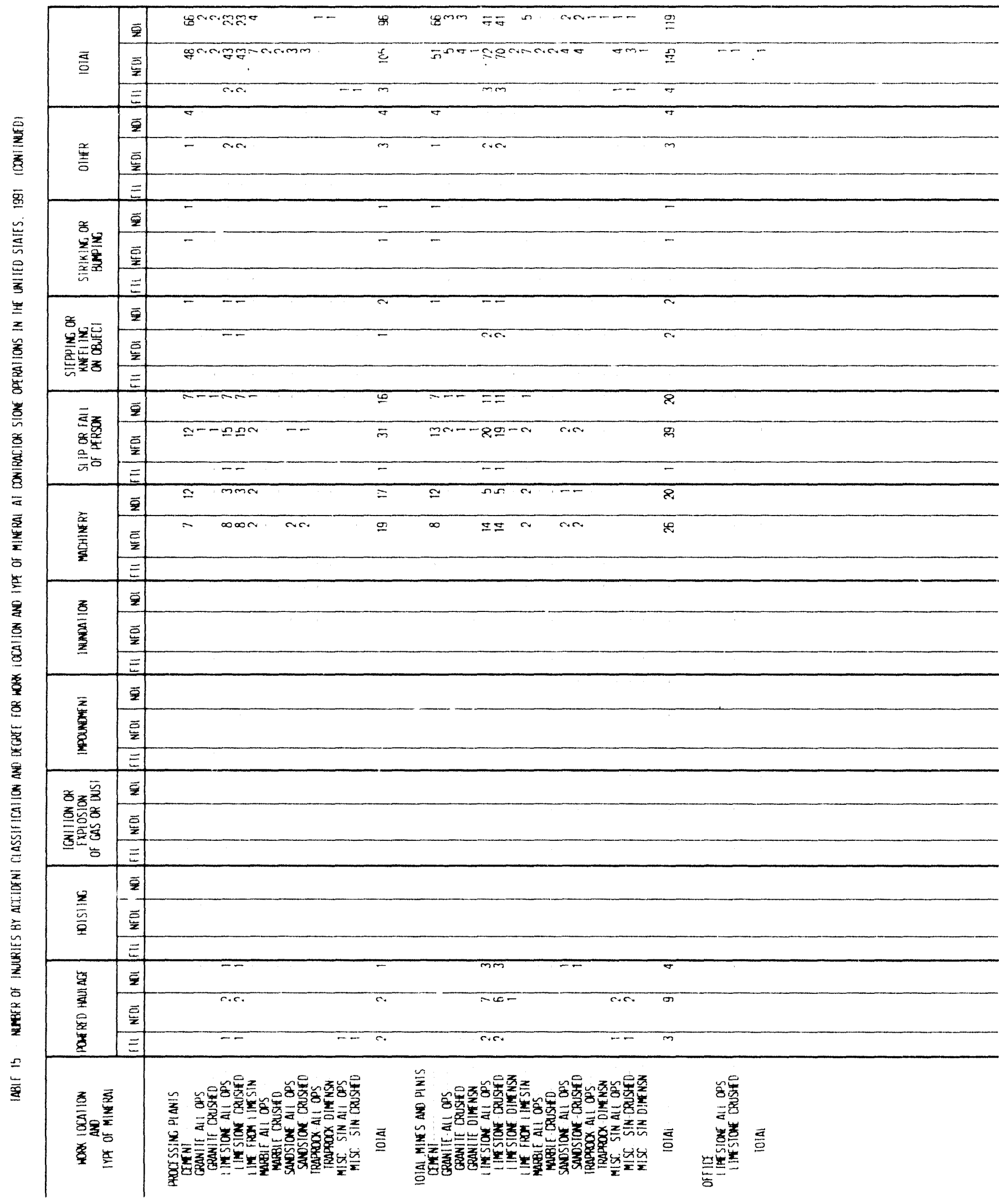




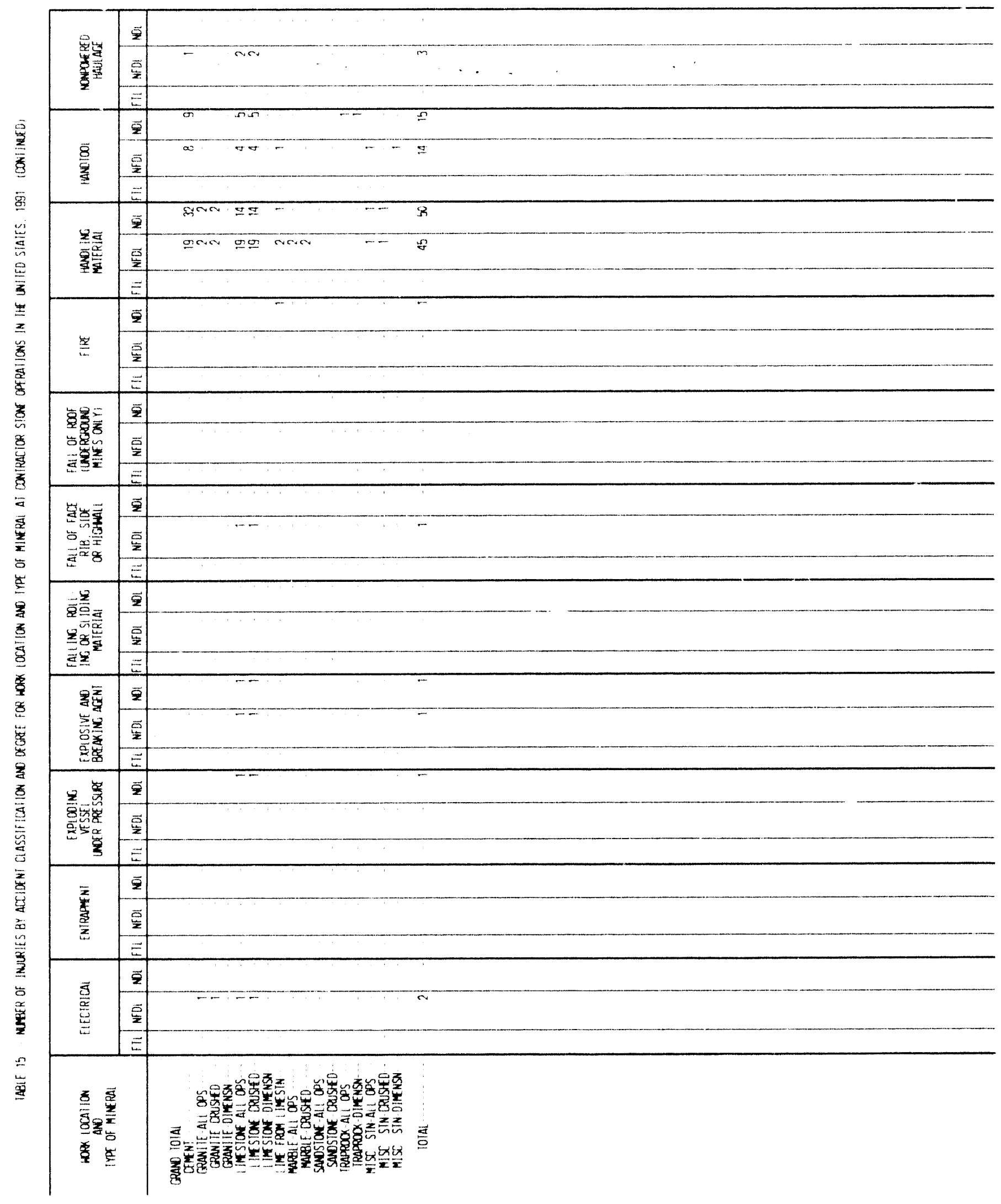




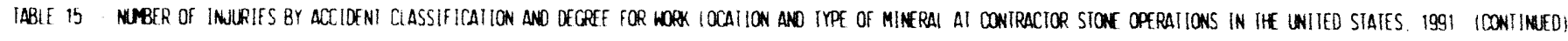

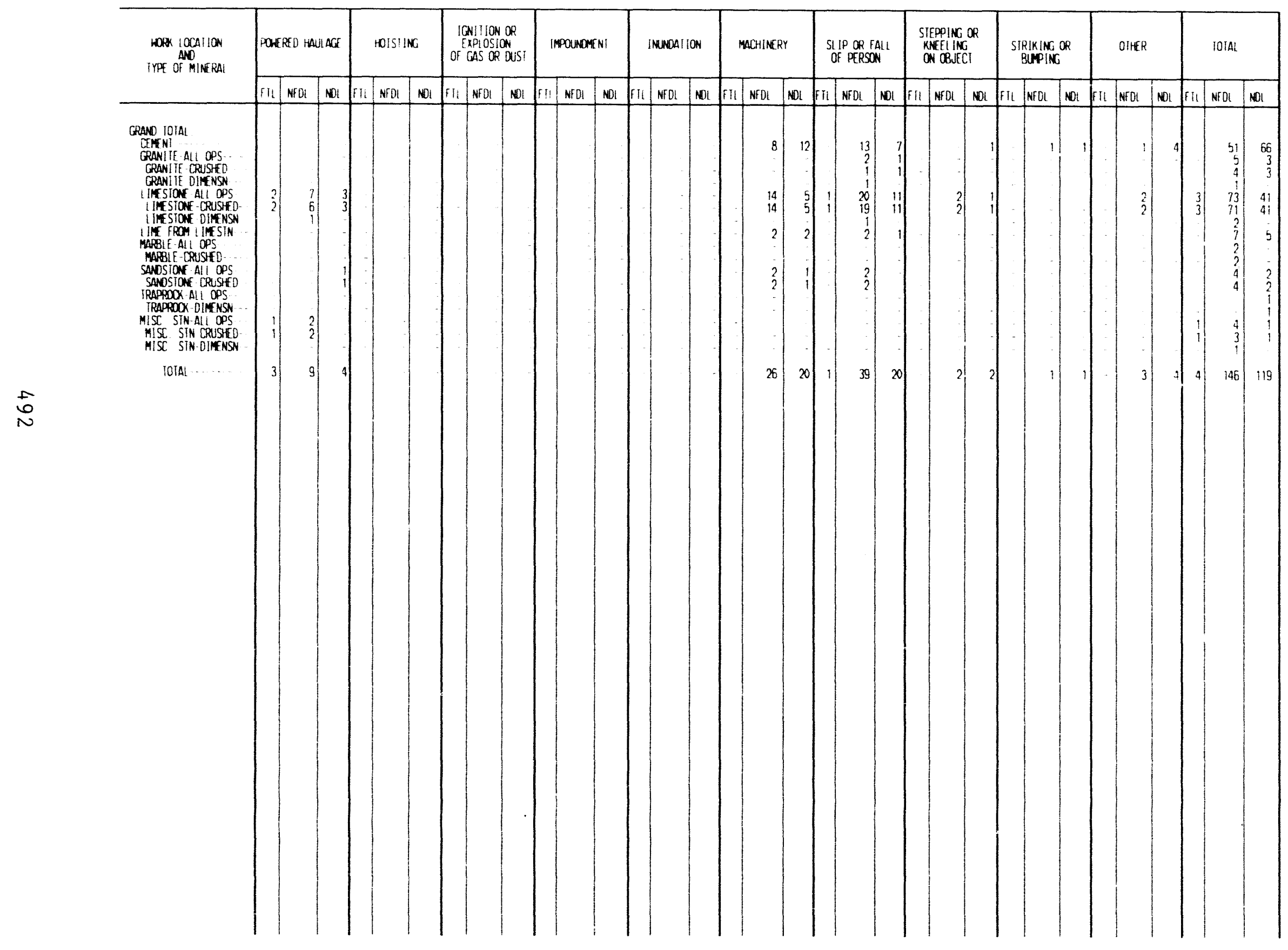




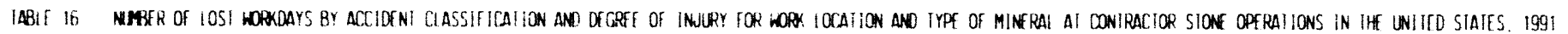

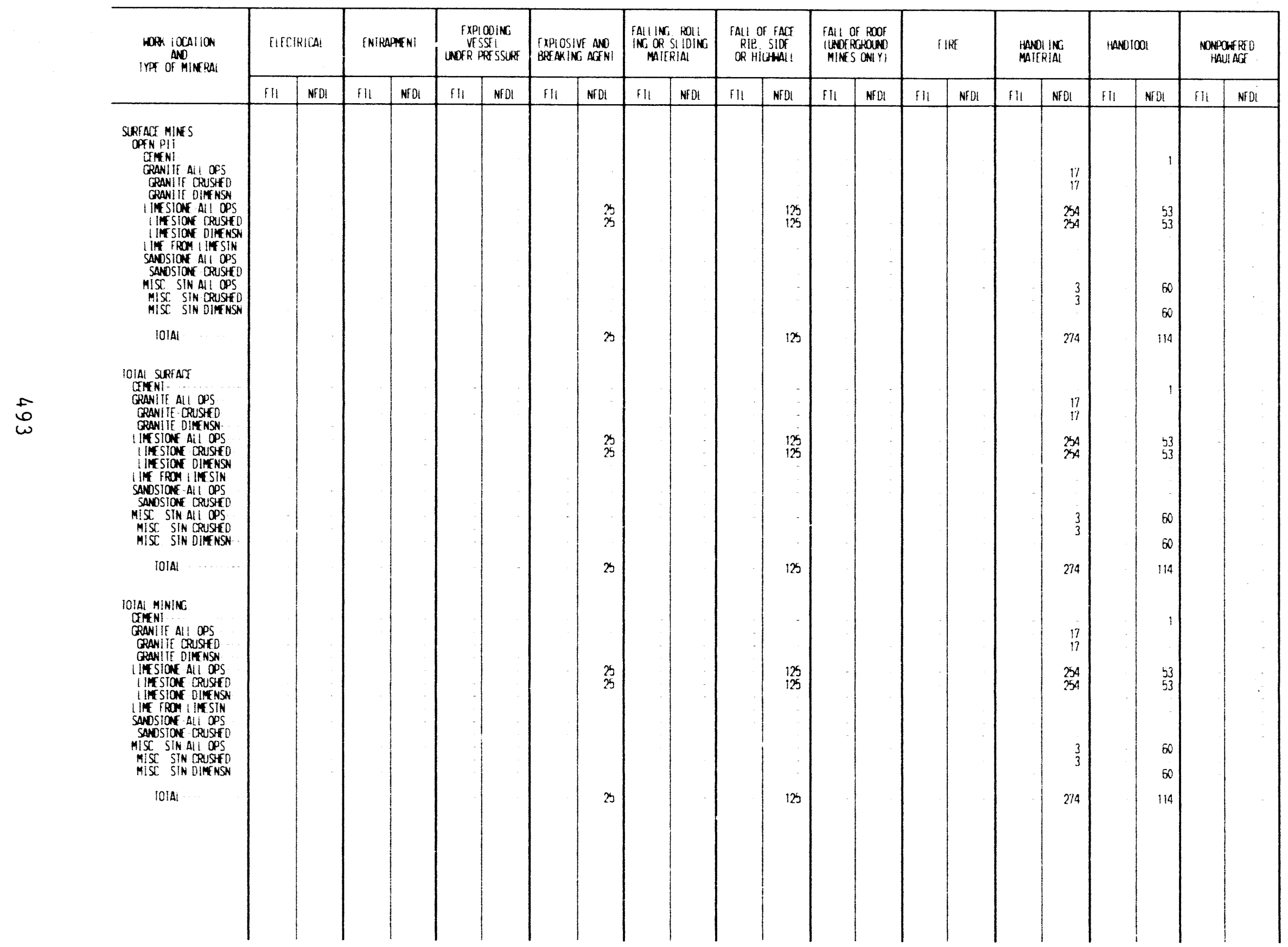




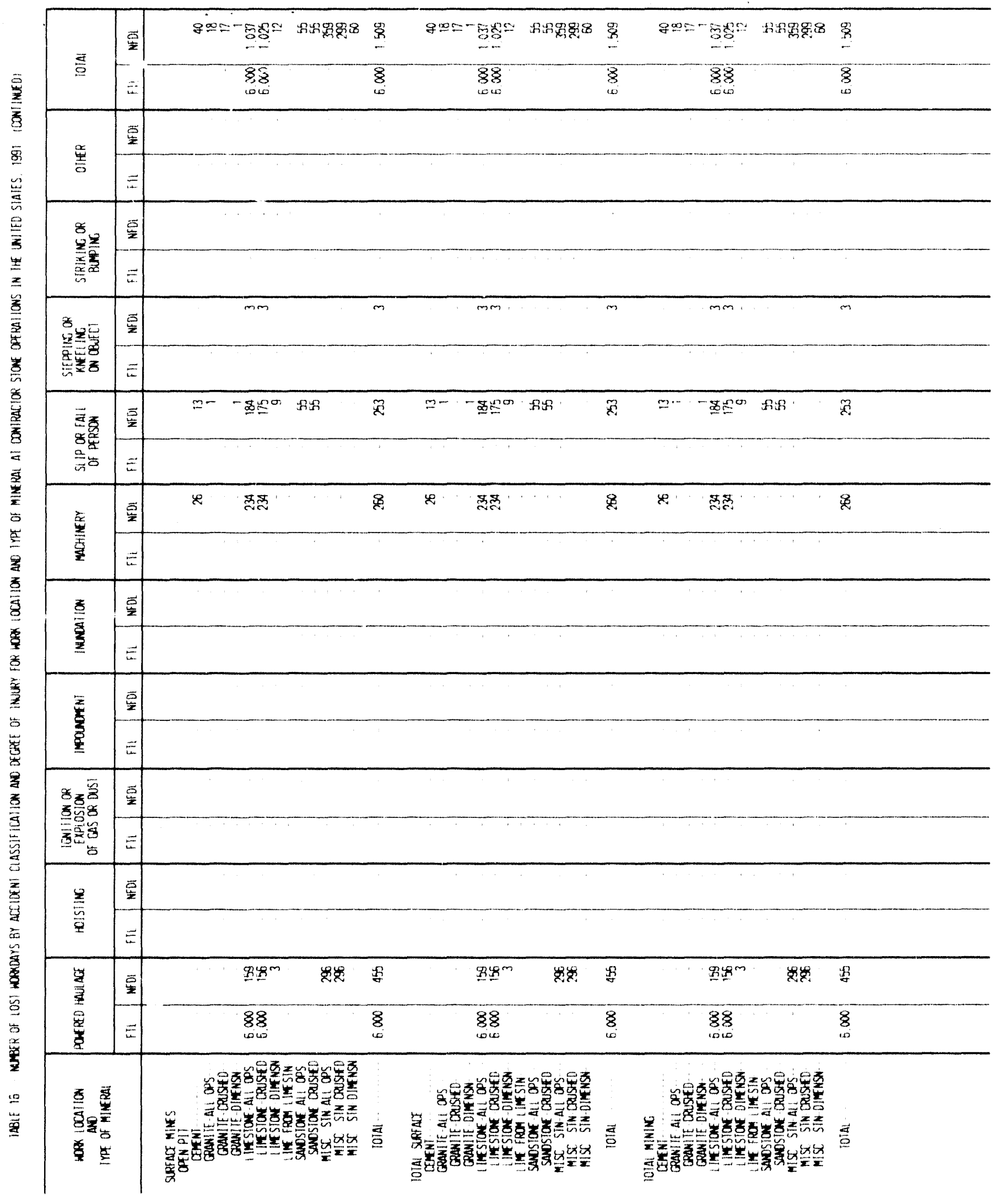




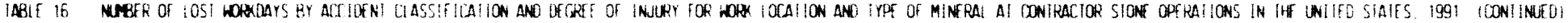

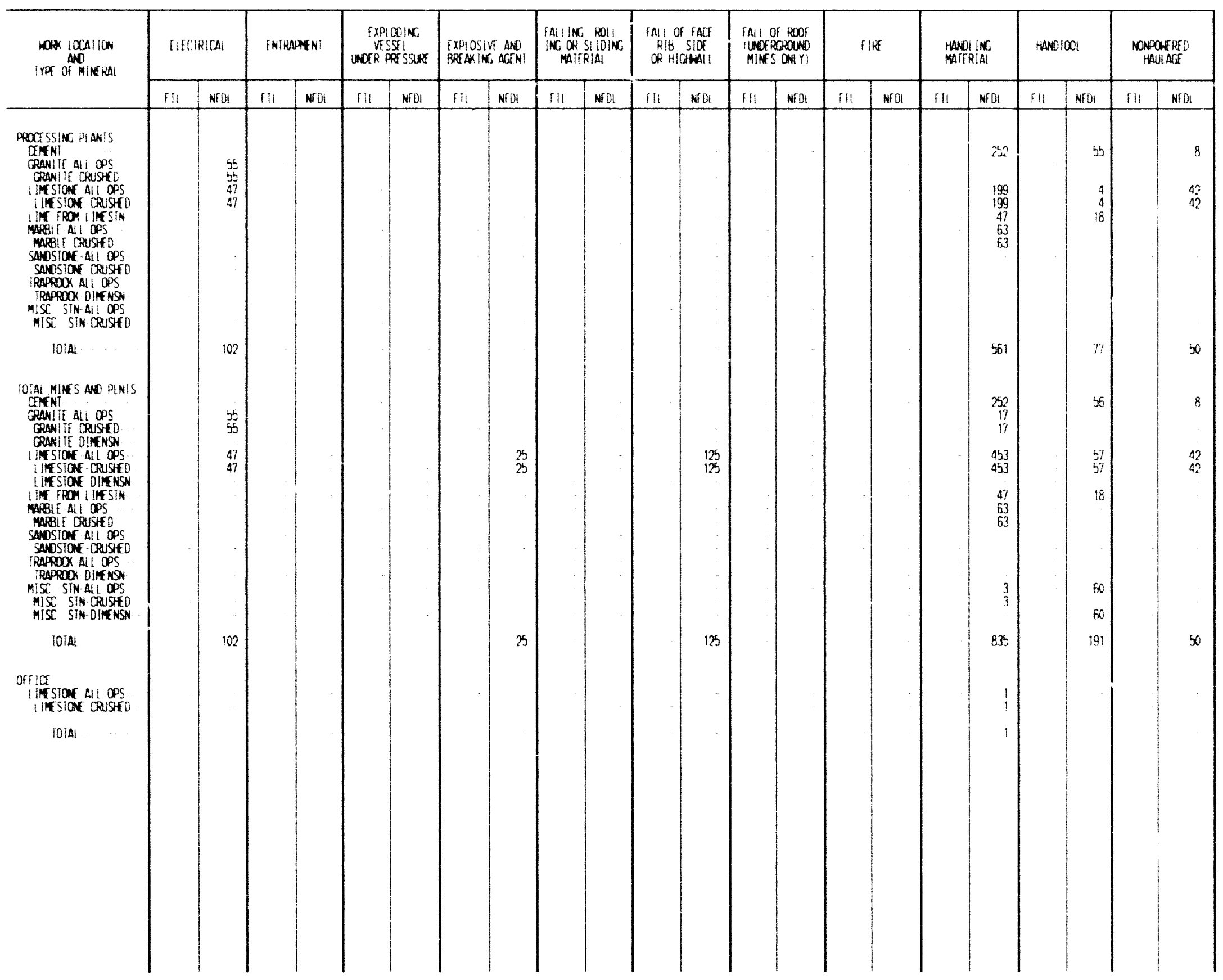




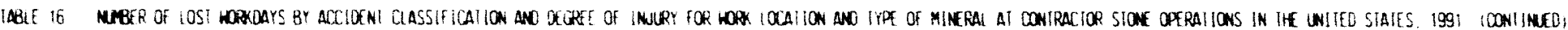

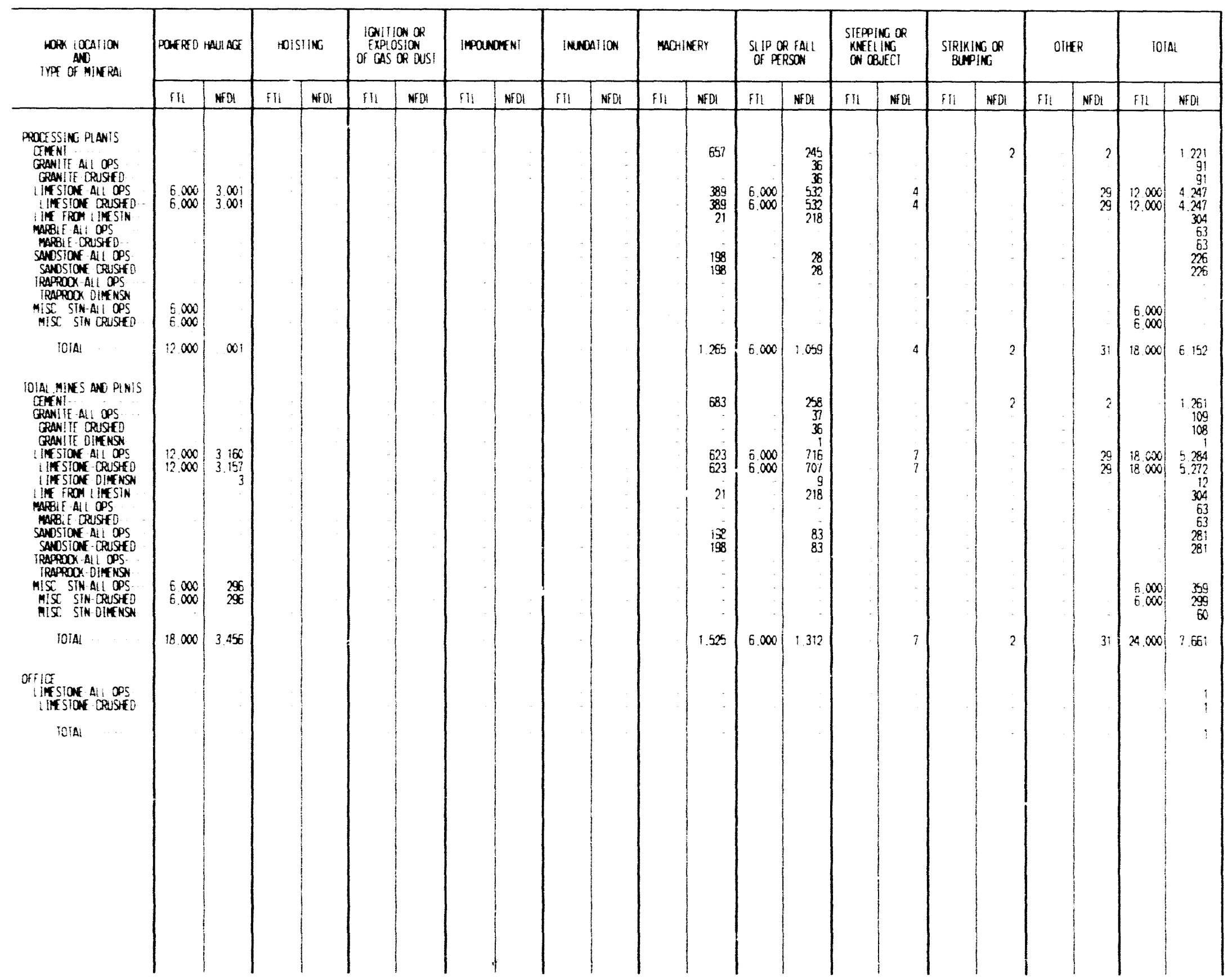




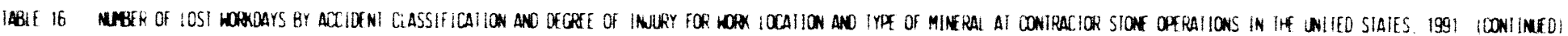

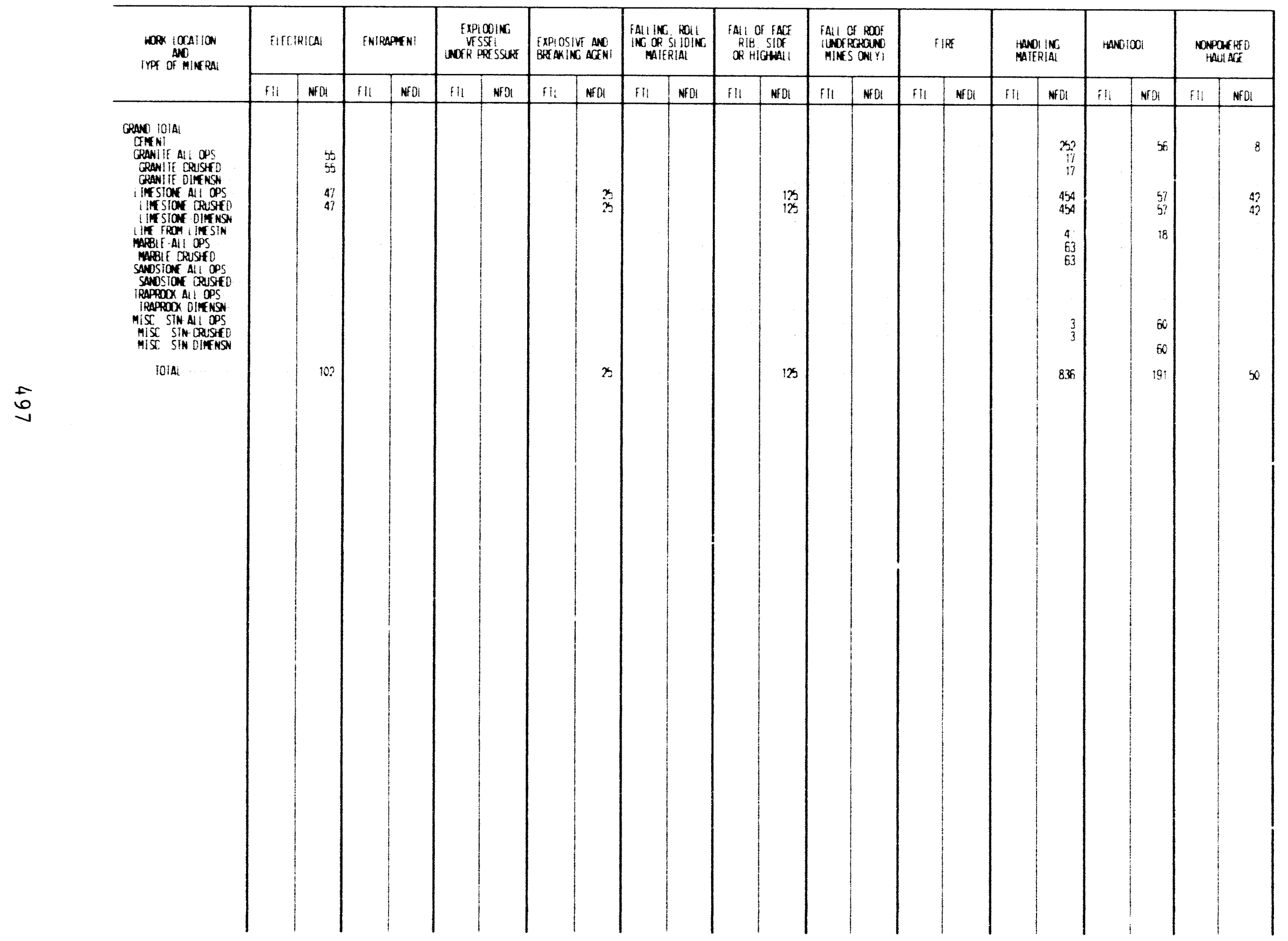




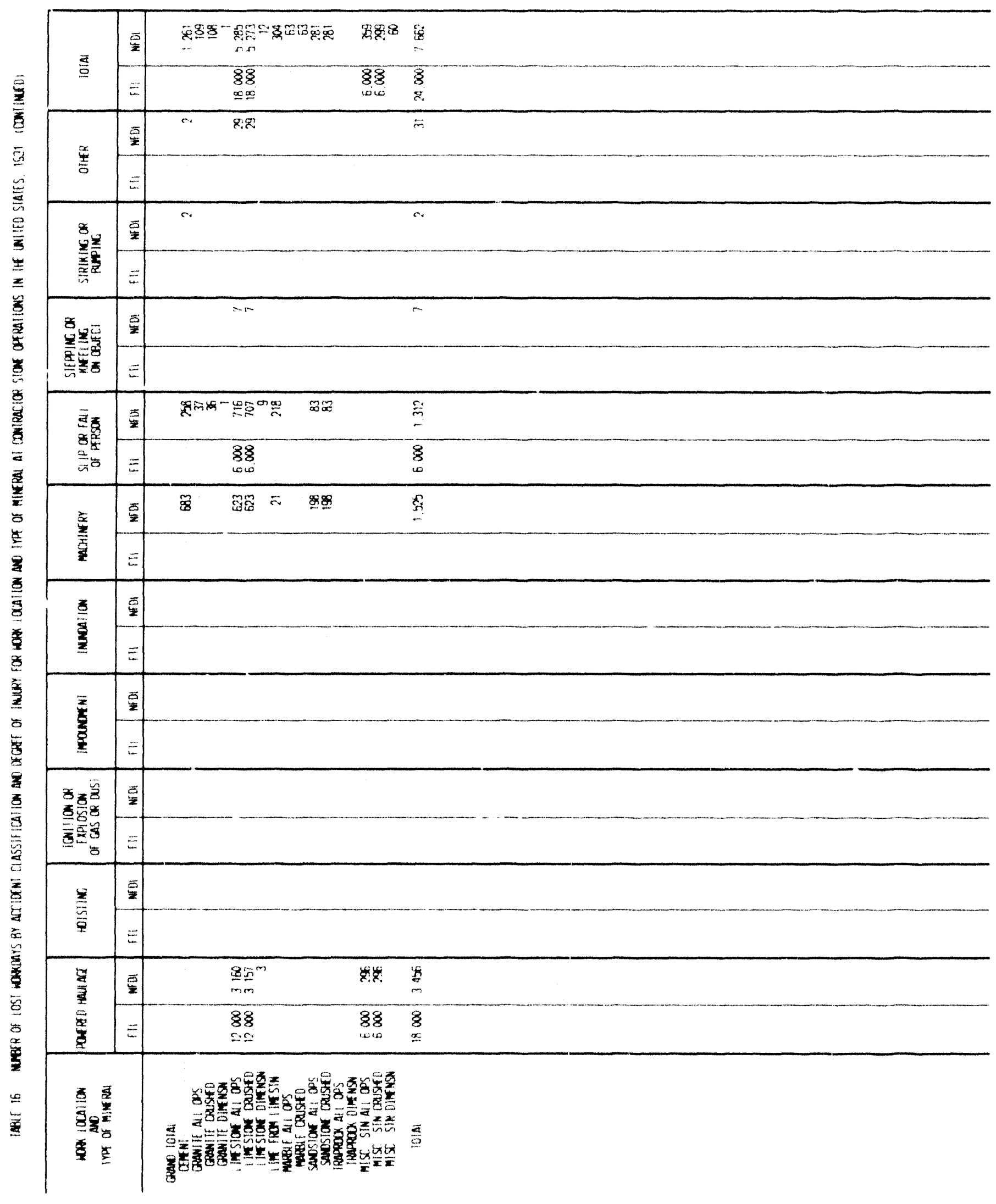




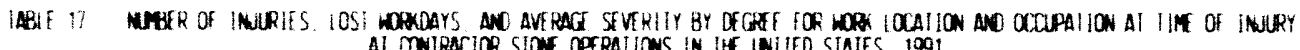

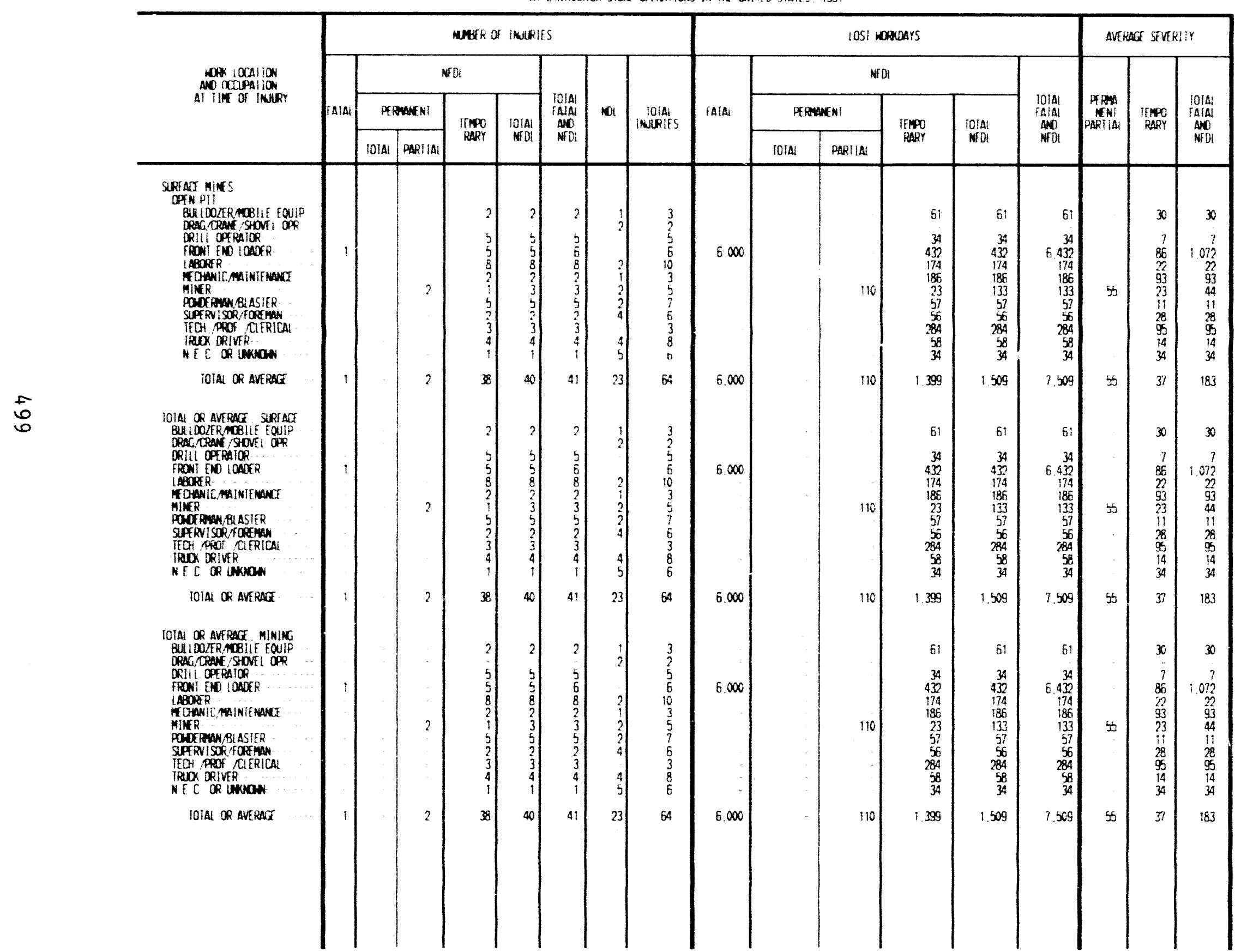




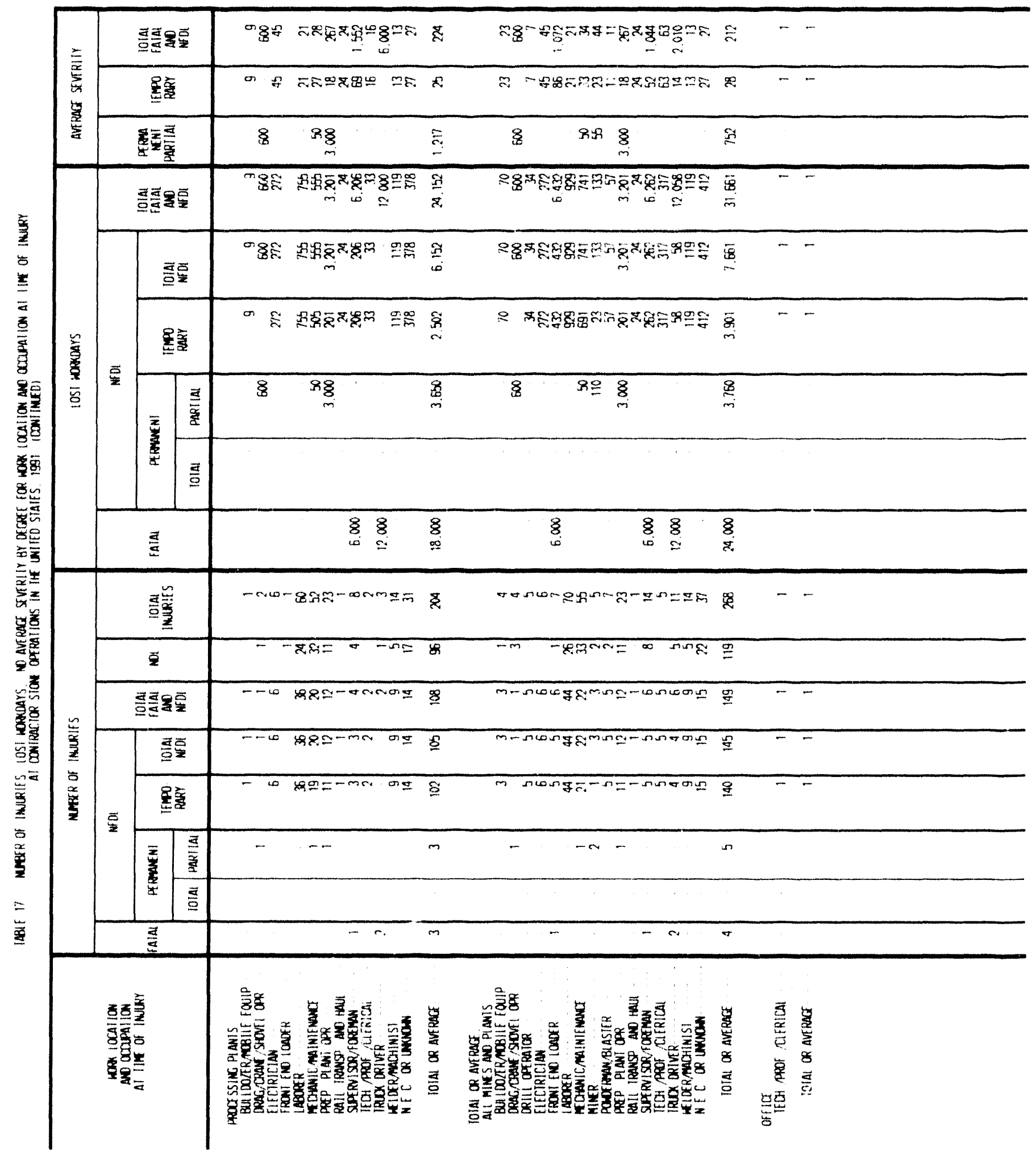




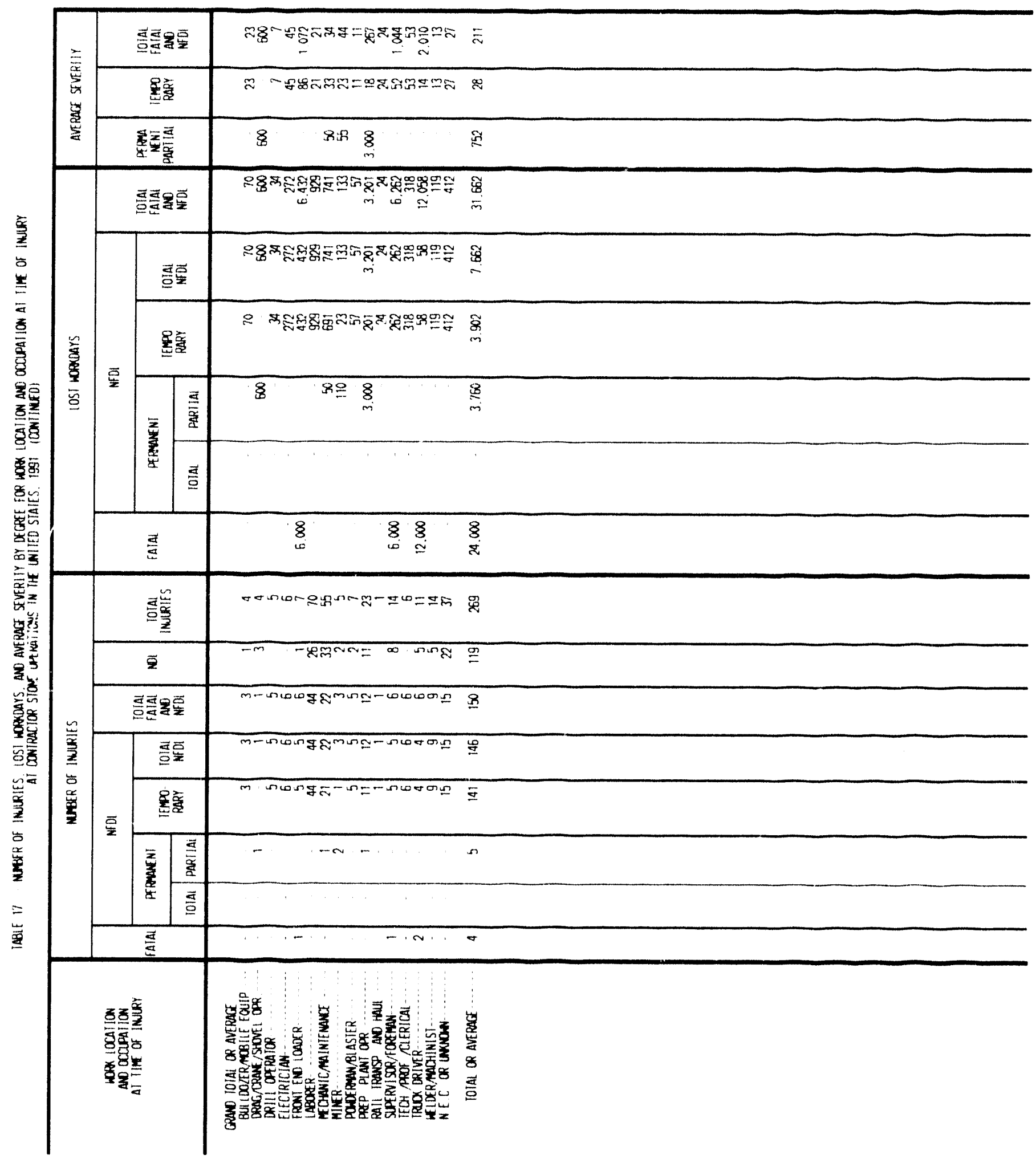




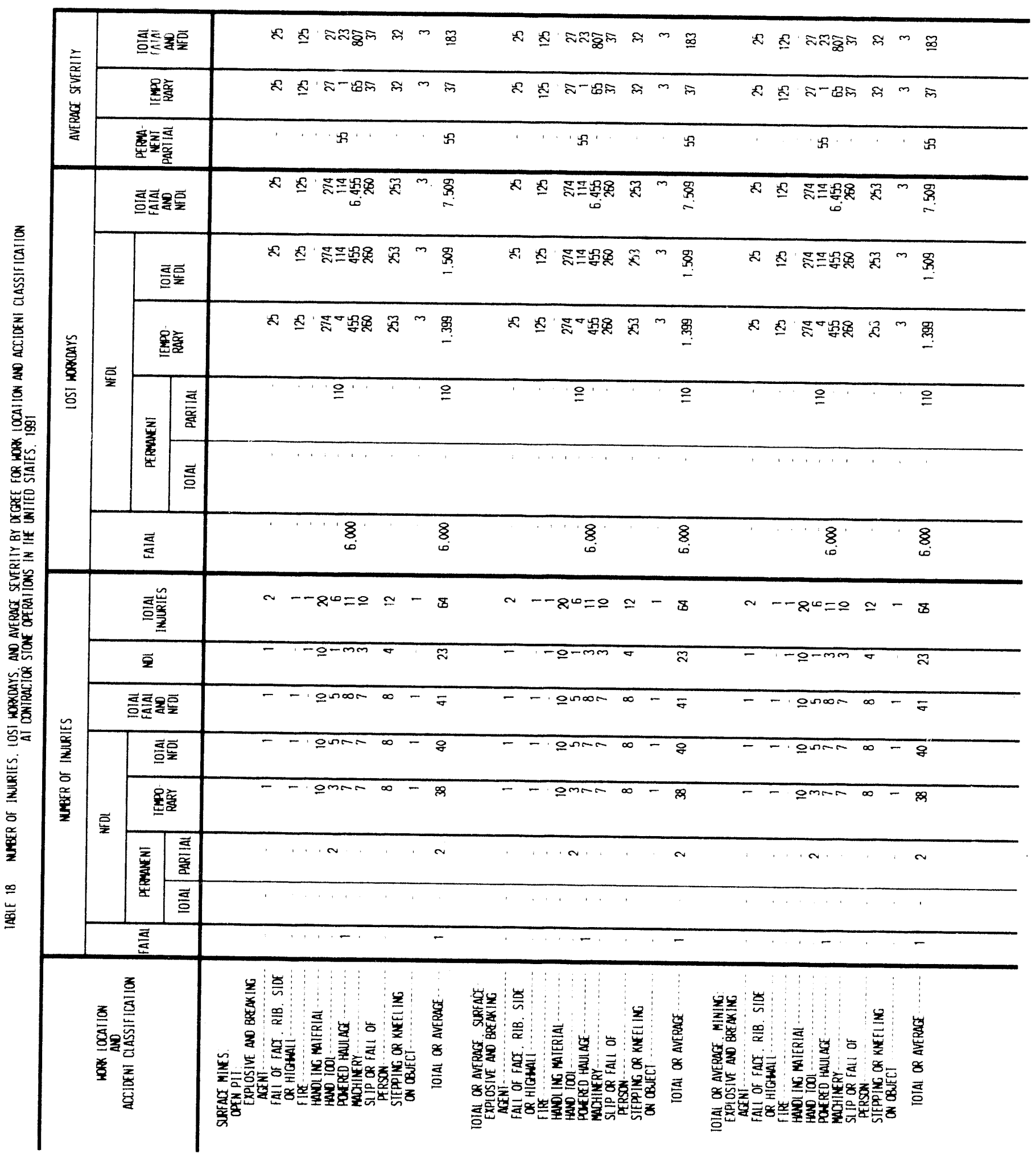




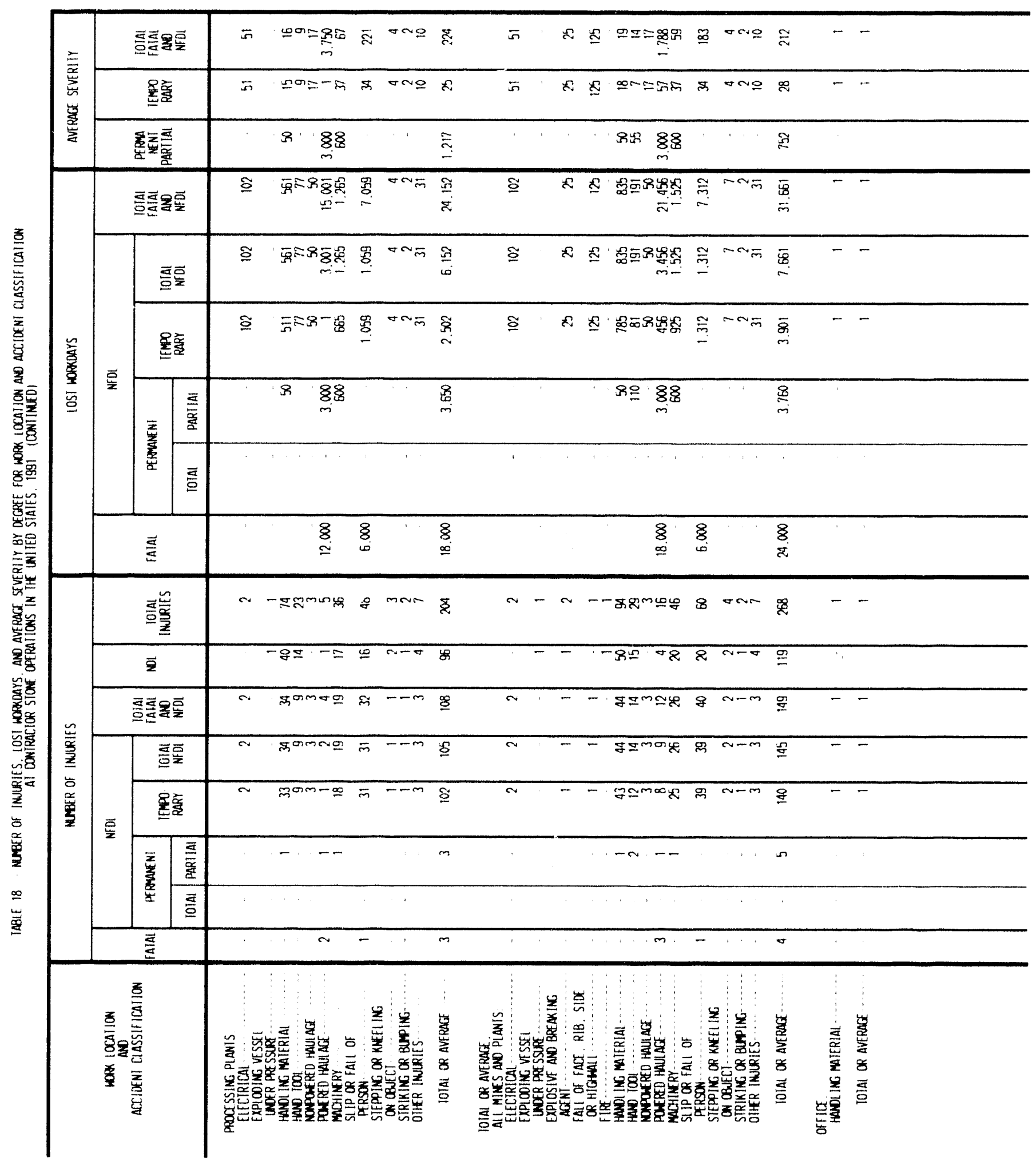




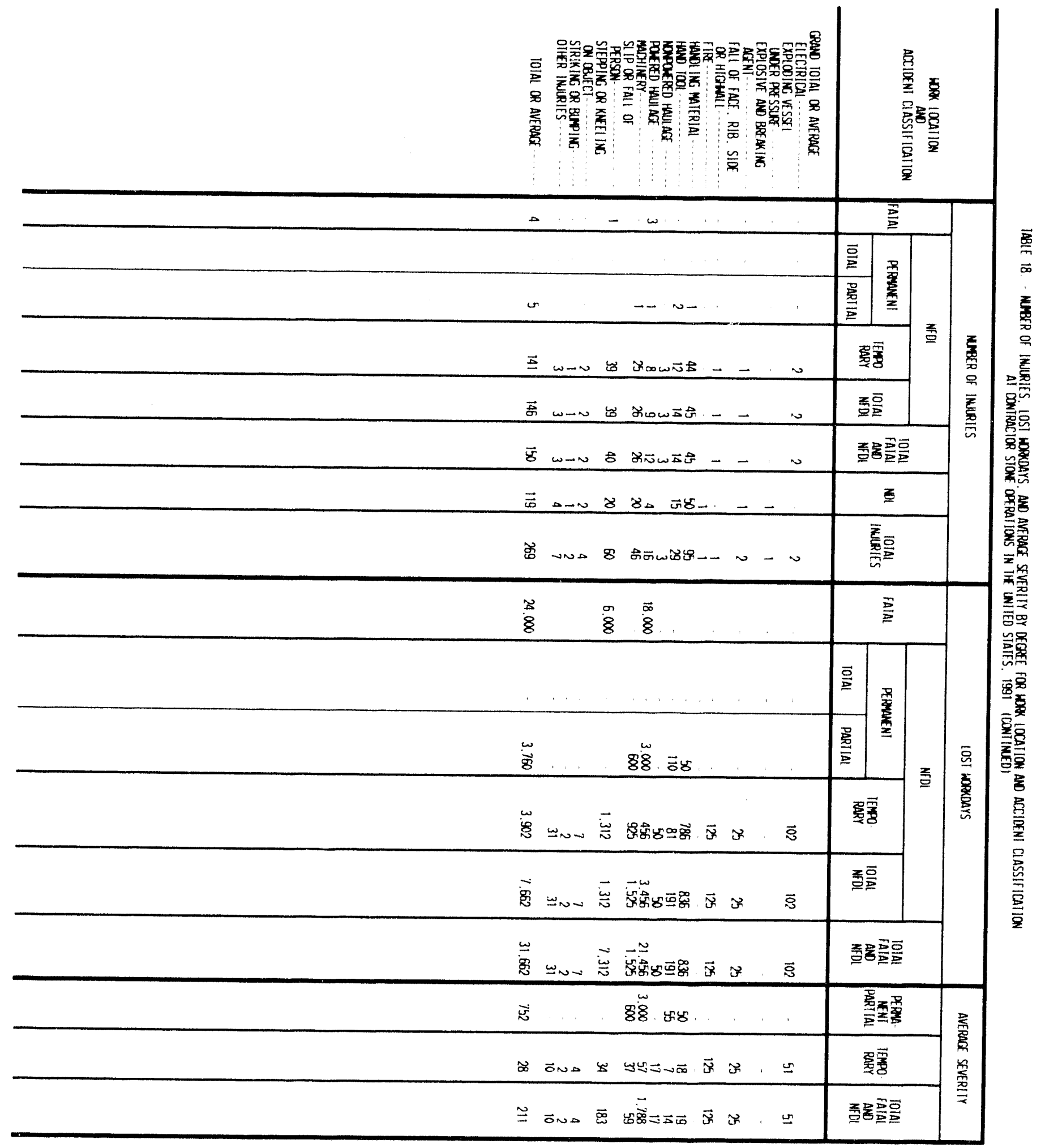




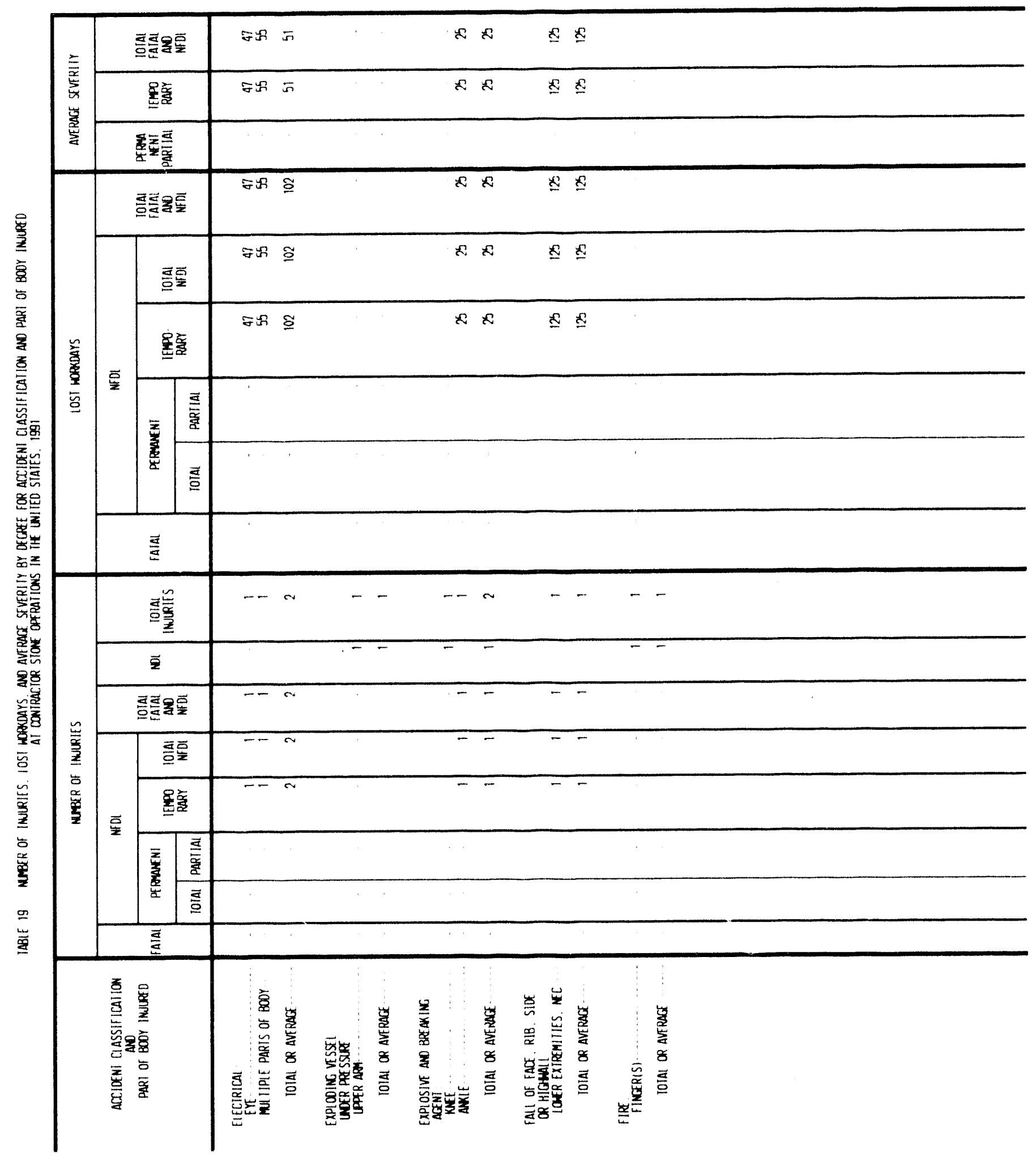



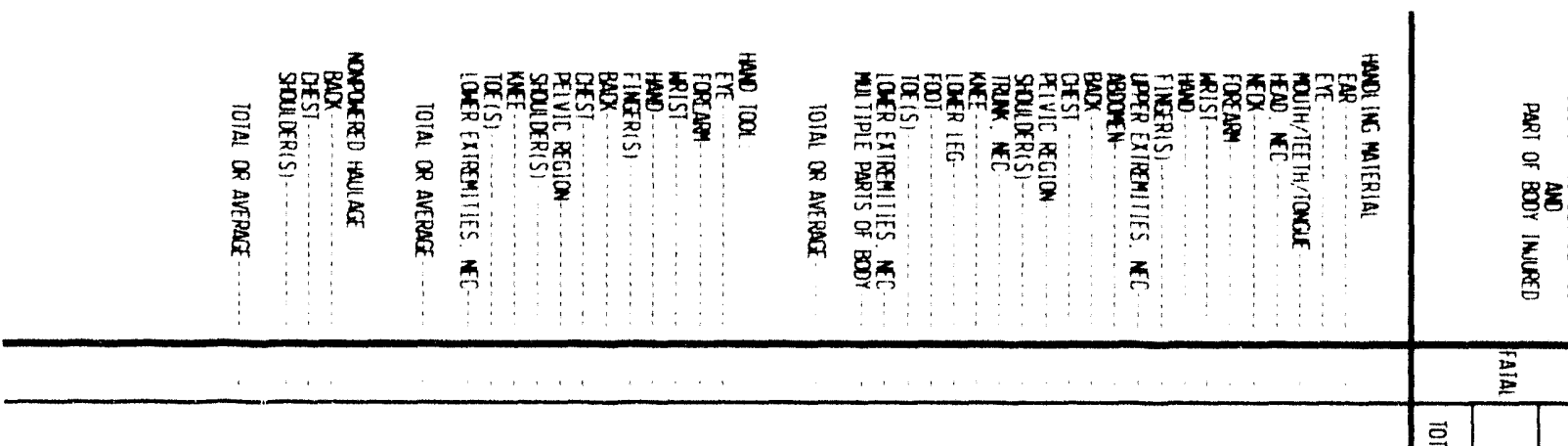

毫

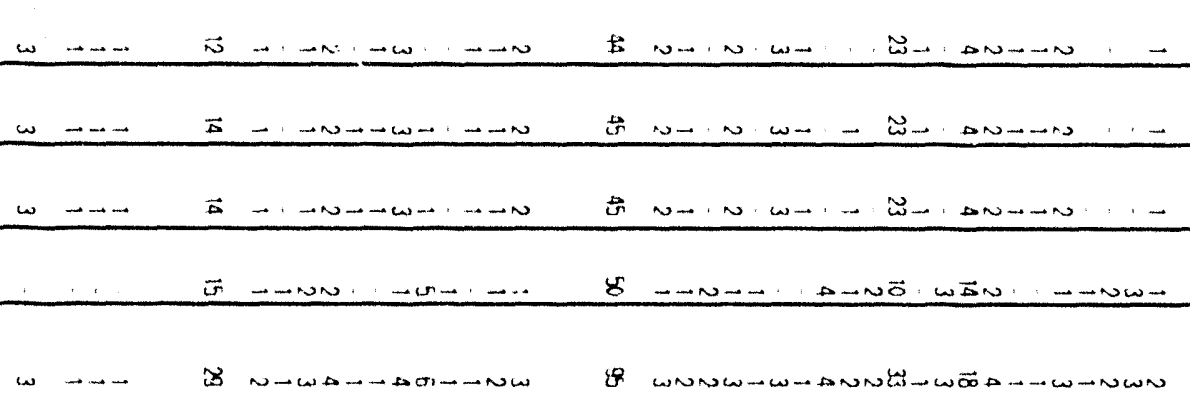

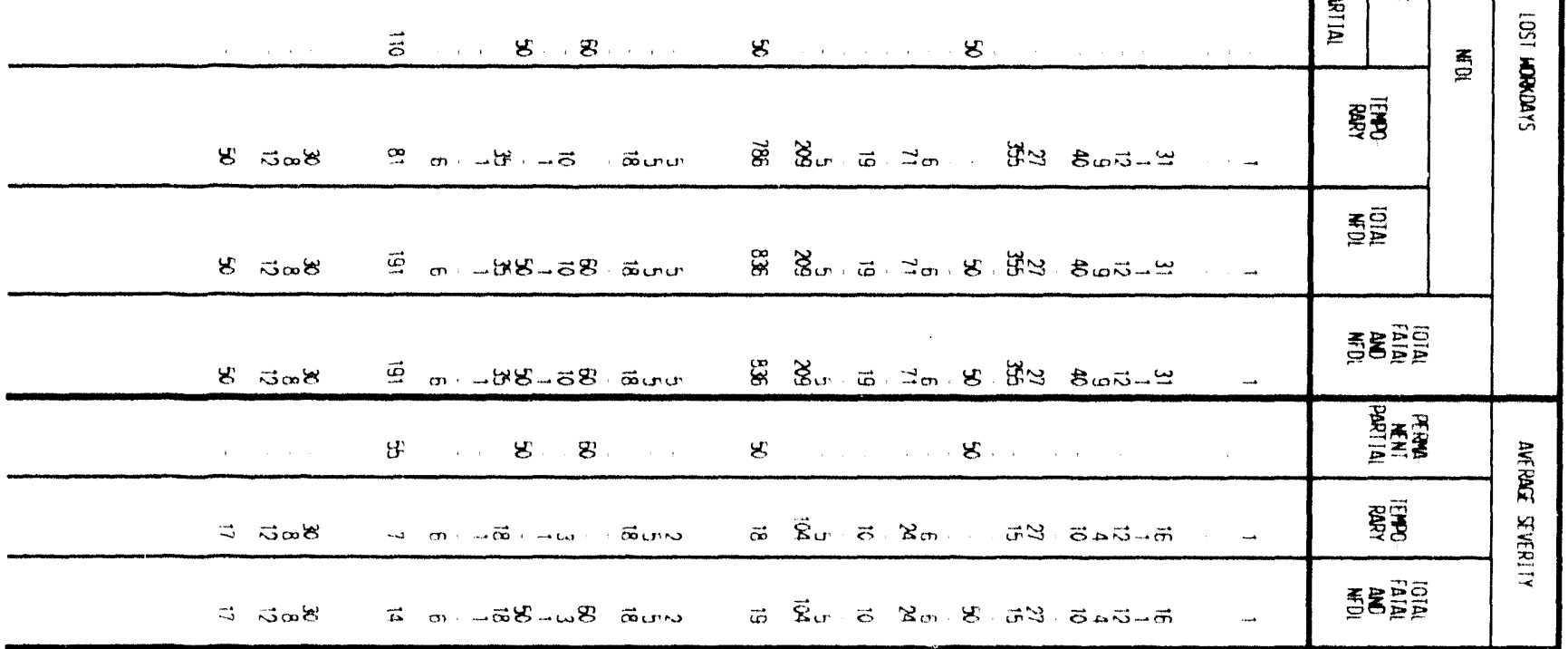




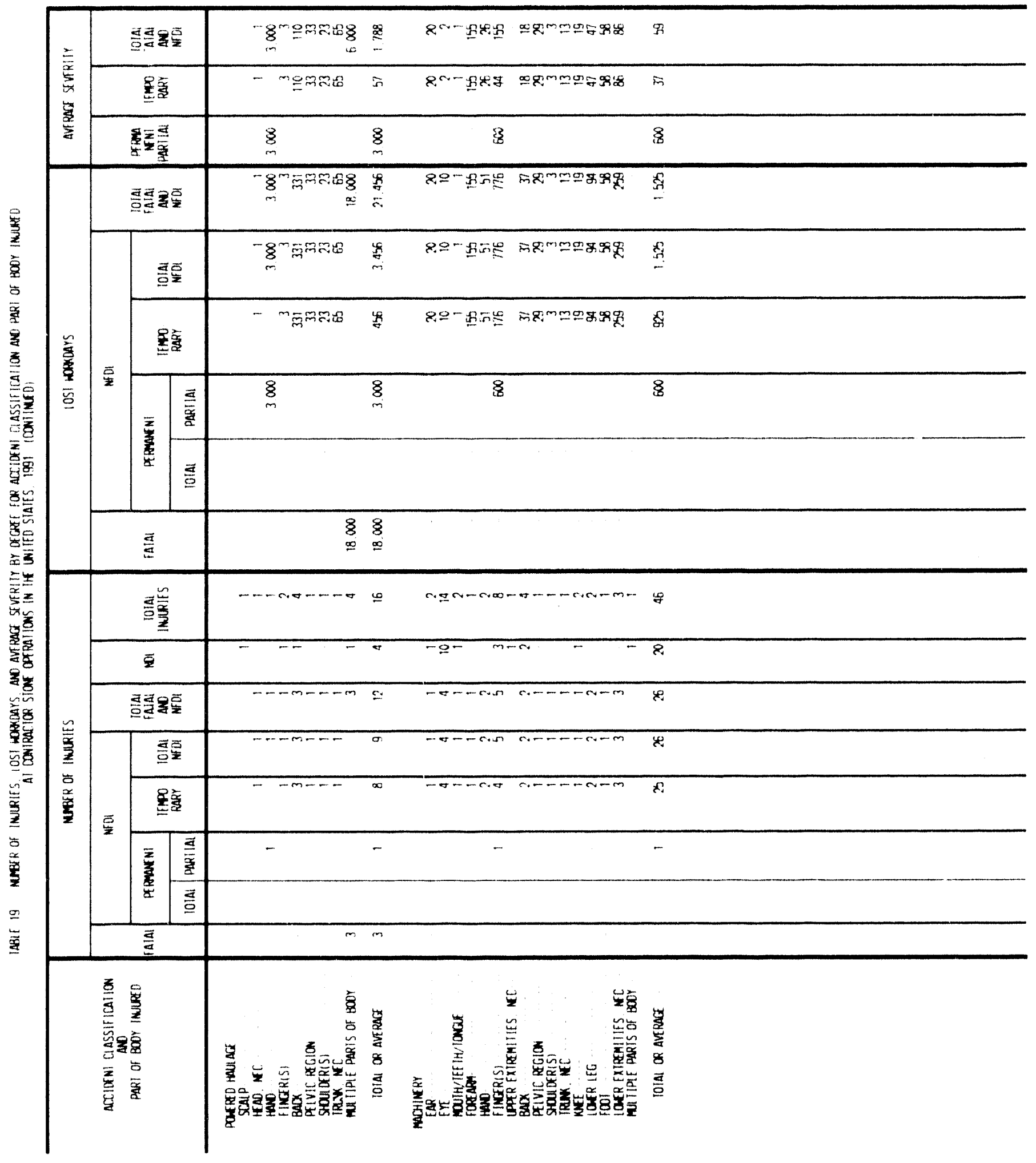




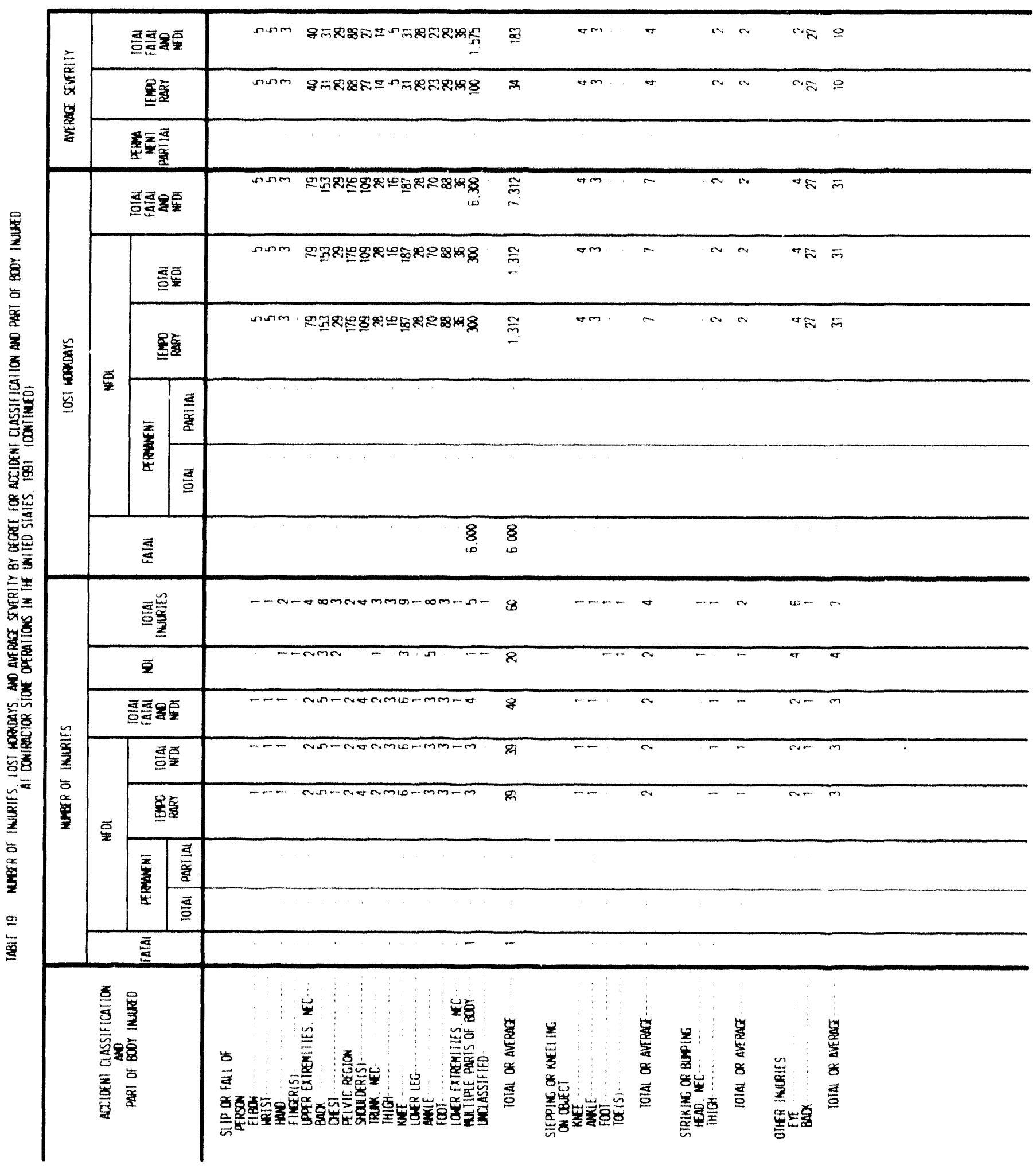




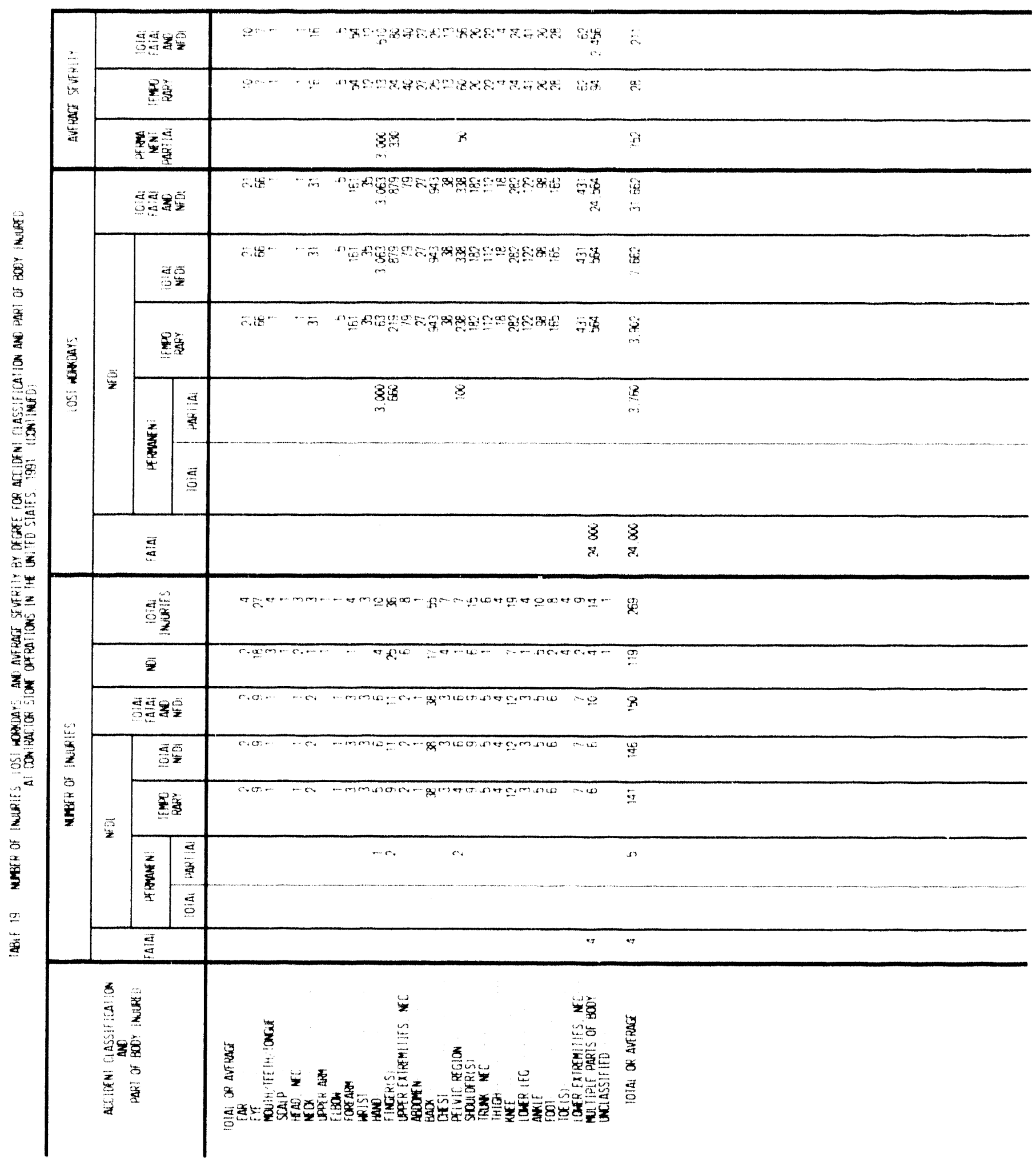




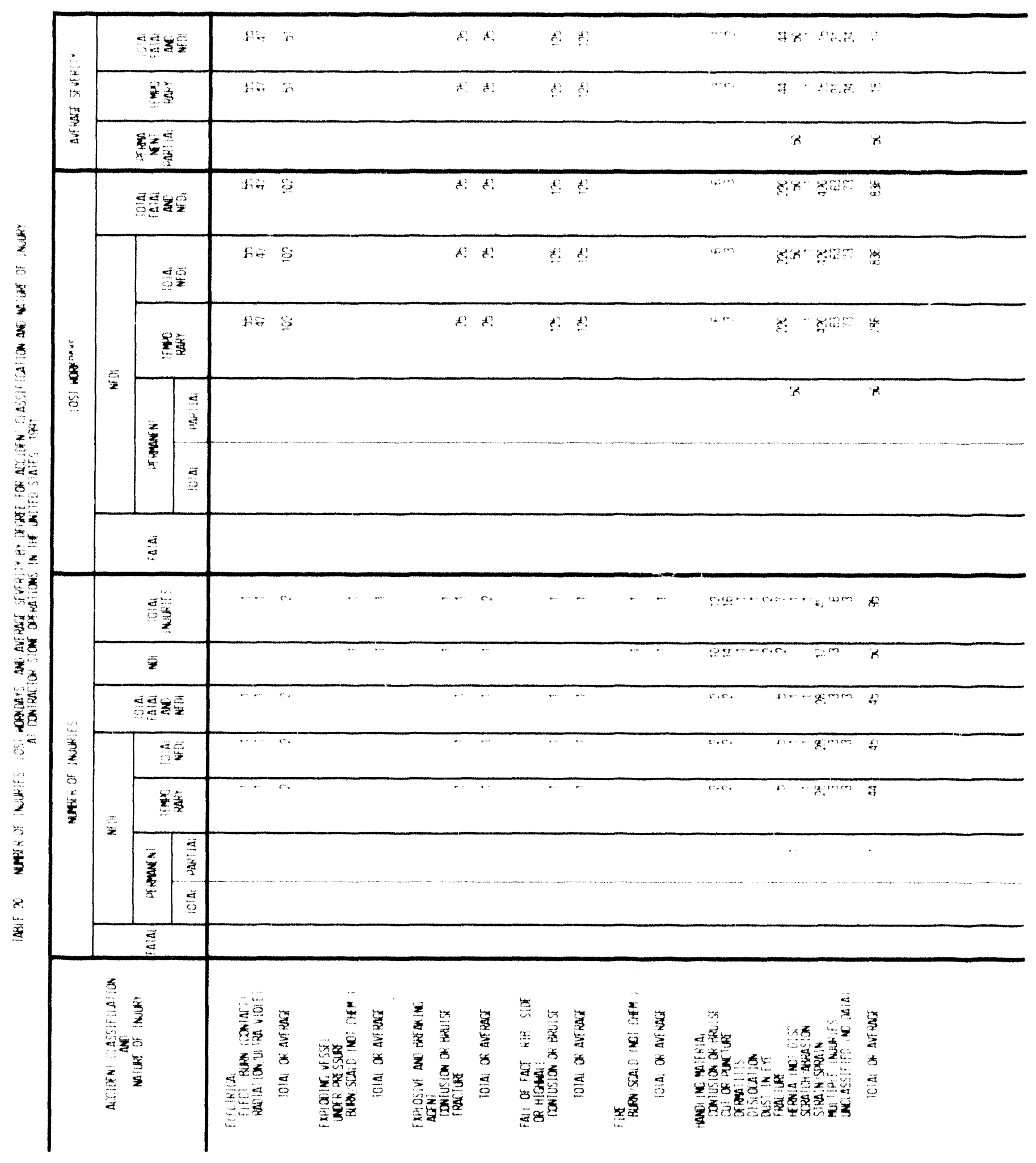




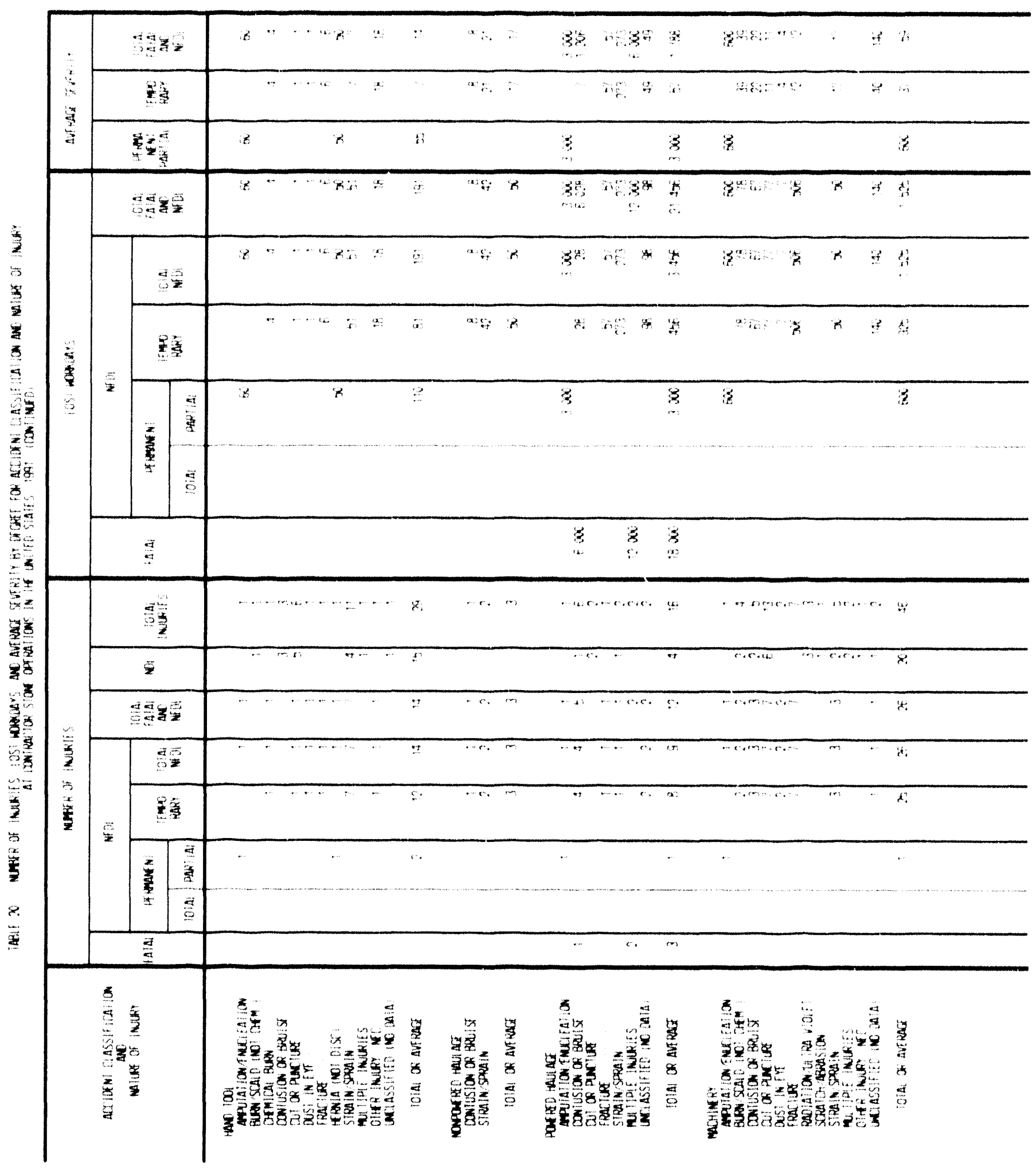




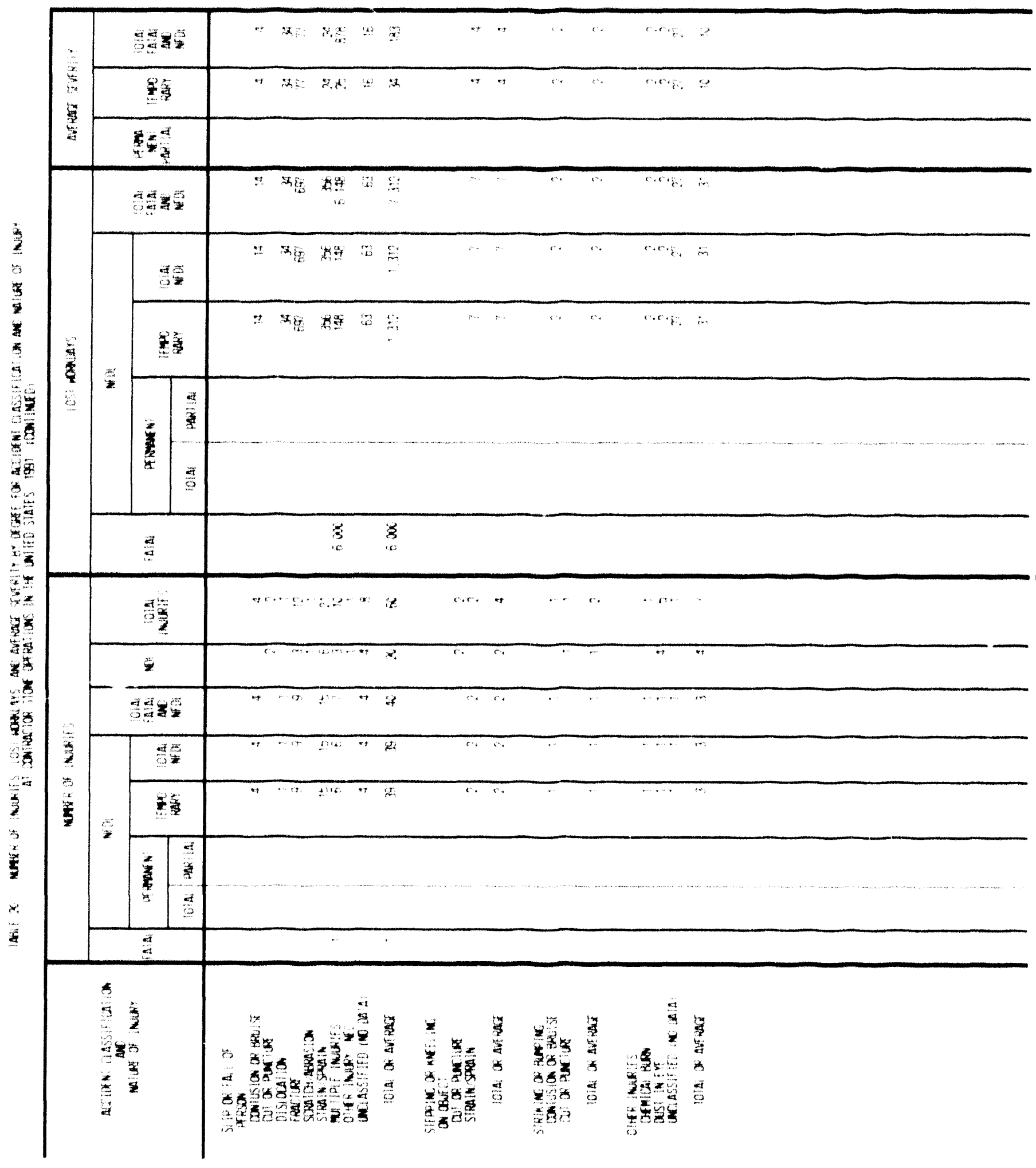




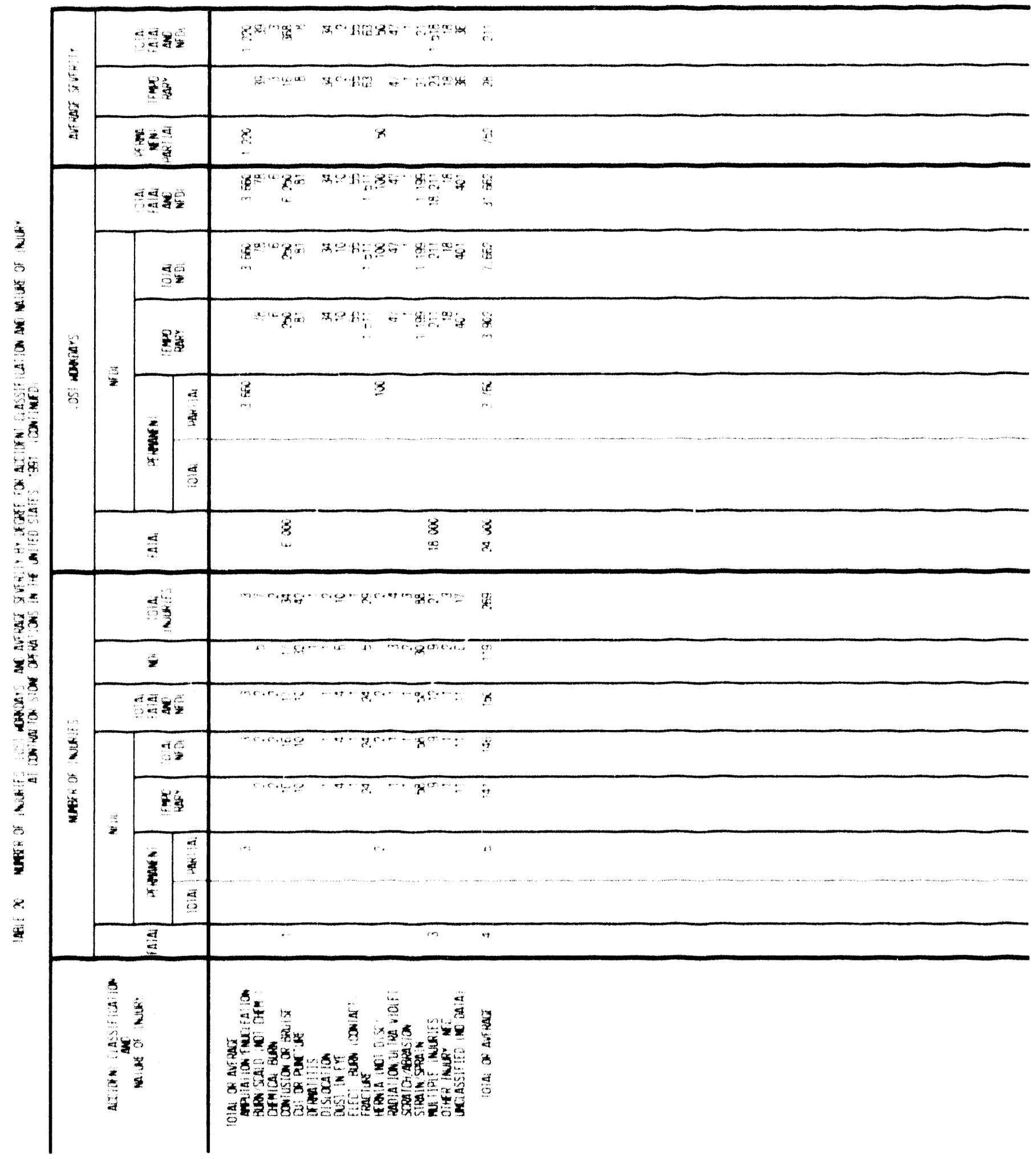




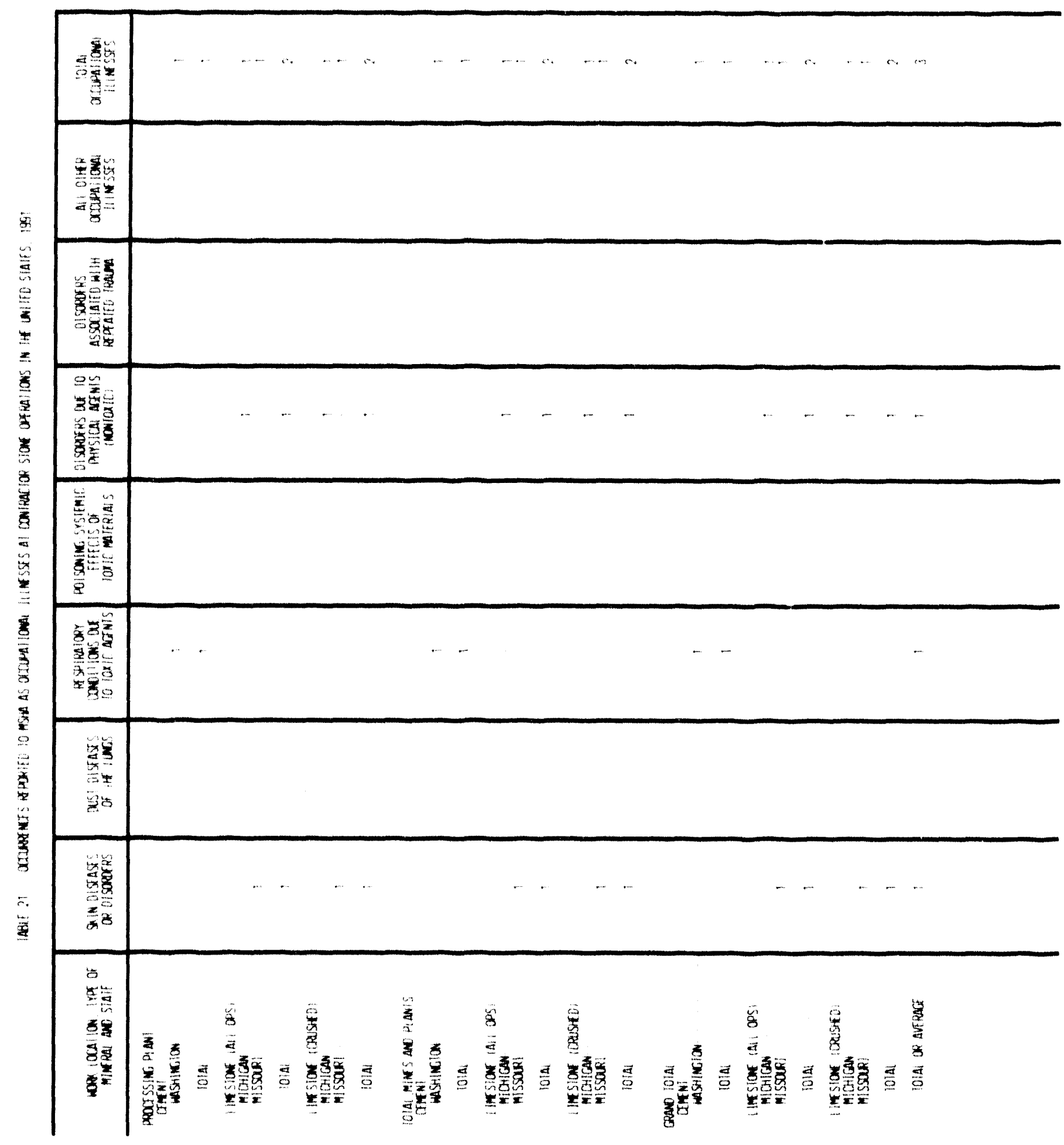




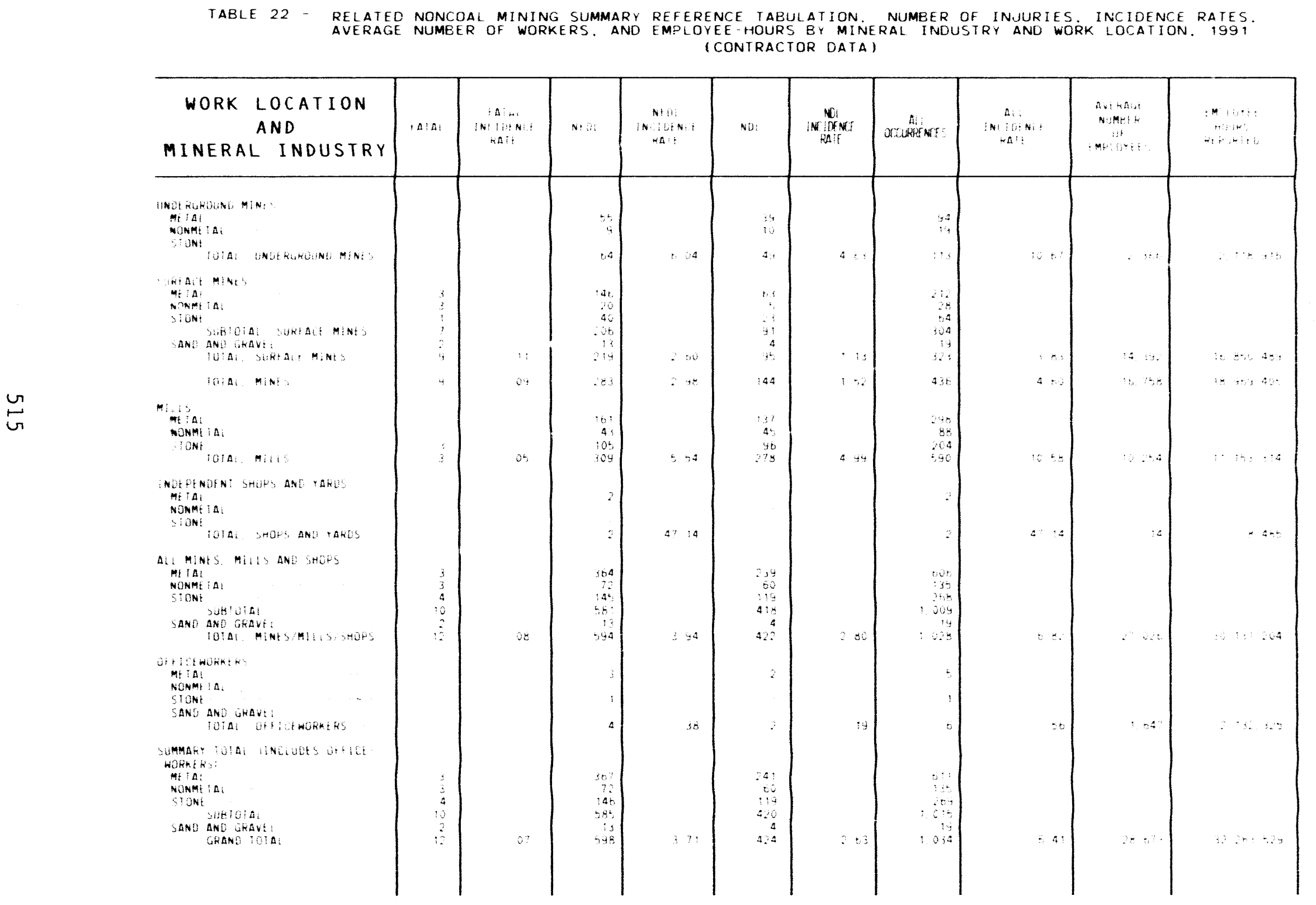


TABLE 23 - RELATED COAL MINING SUMMARY REFERENCE TABULATION, NUMBER OF INJURIES, INCIDENCE RATES, AVERAGE OF COAL MINED AND WORK LOCATION. (CONTRACTOR DATA)

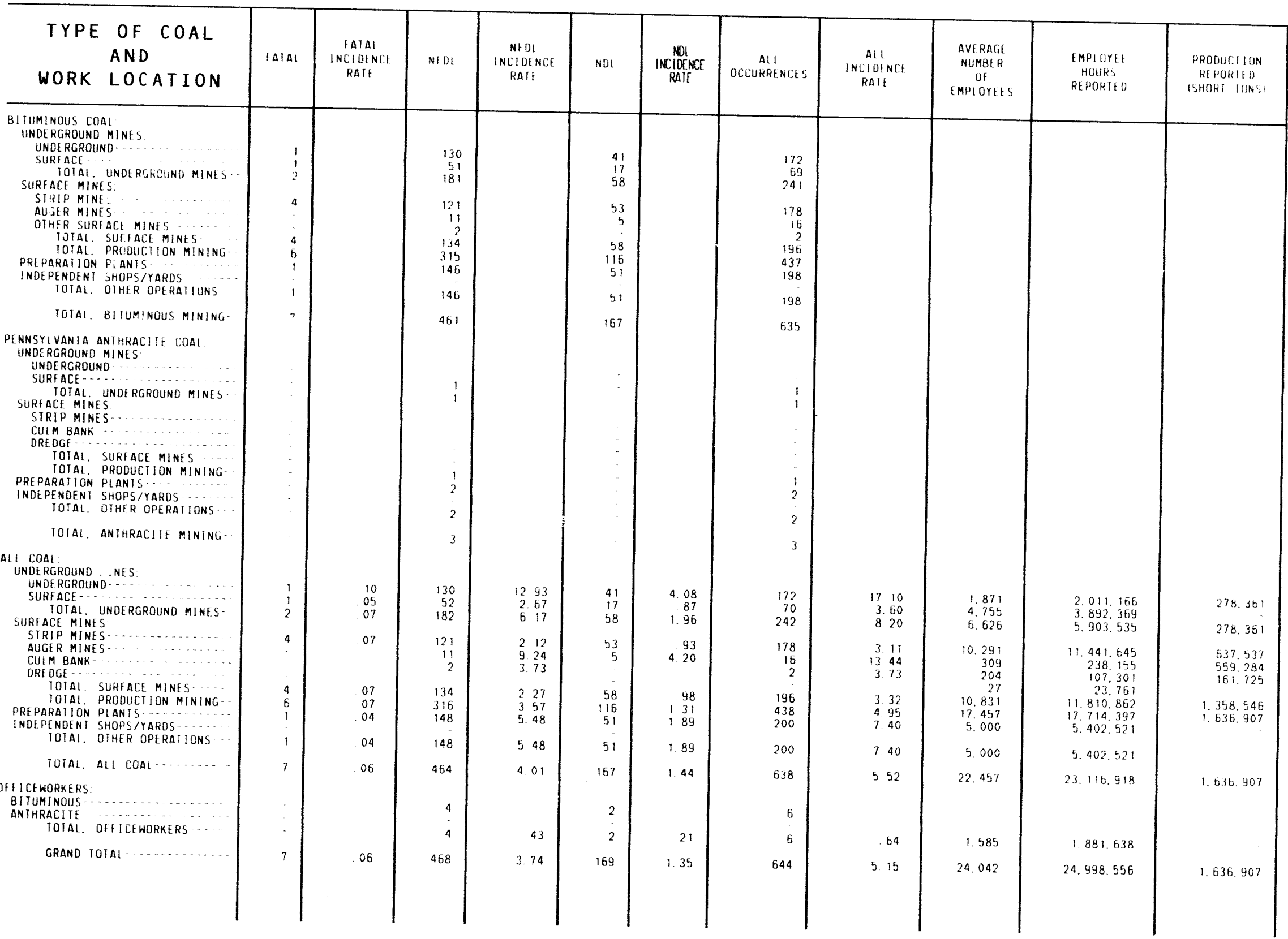



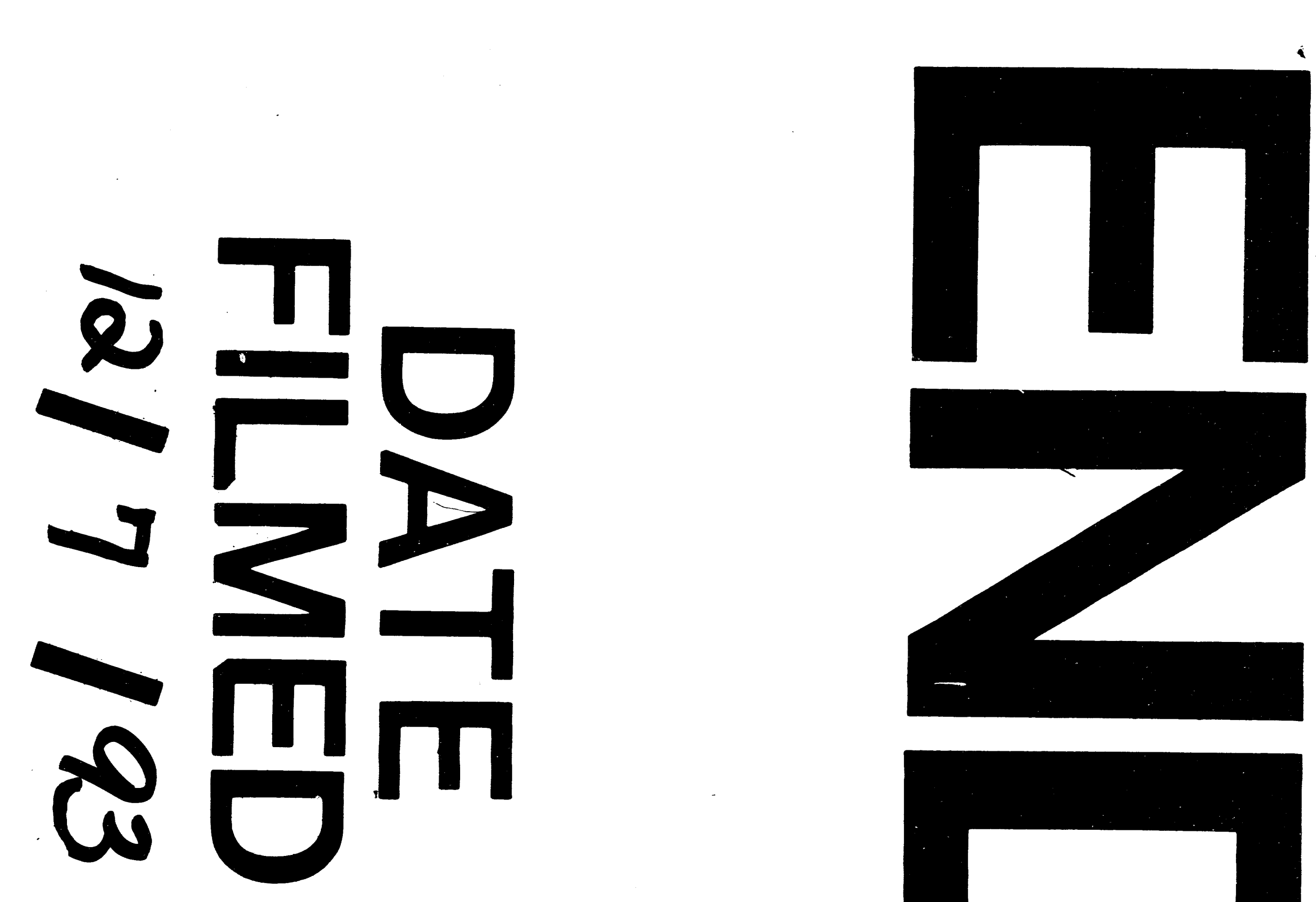

7
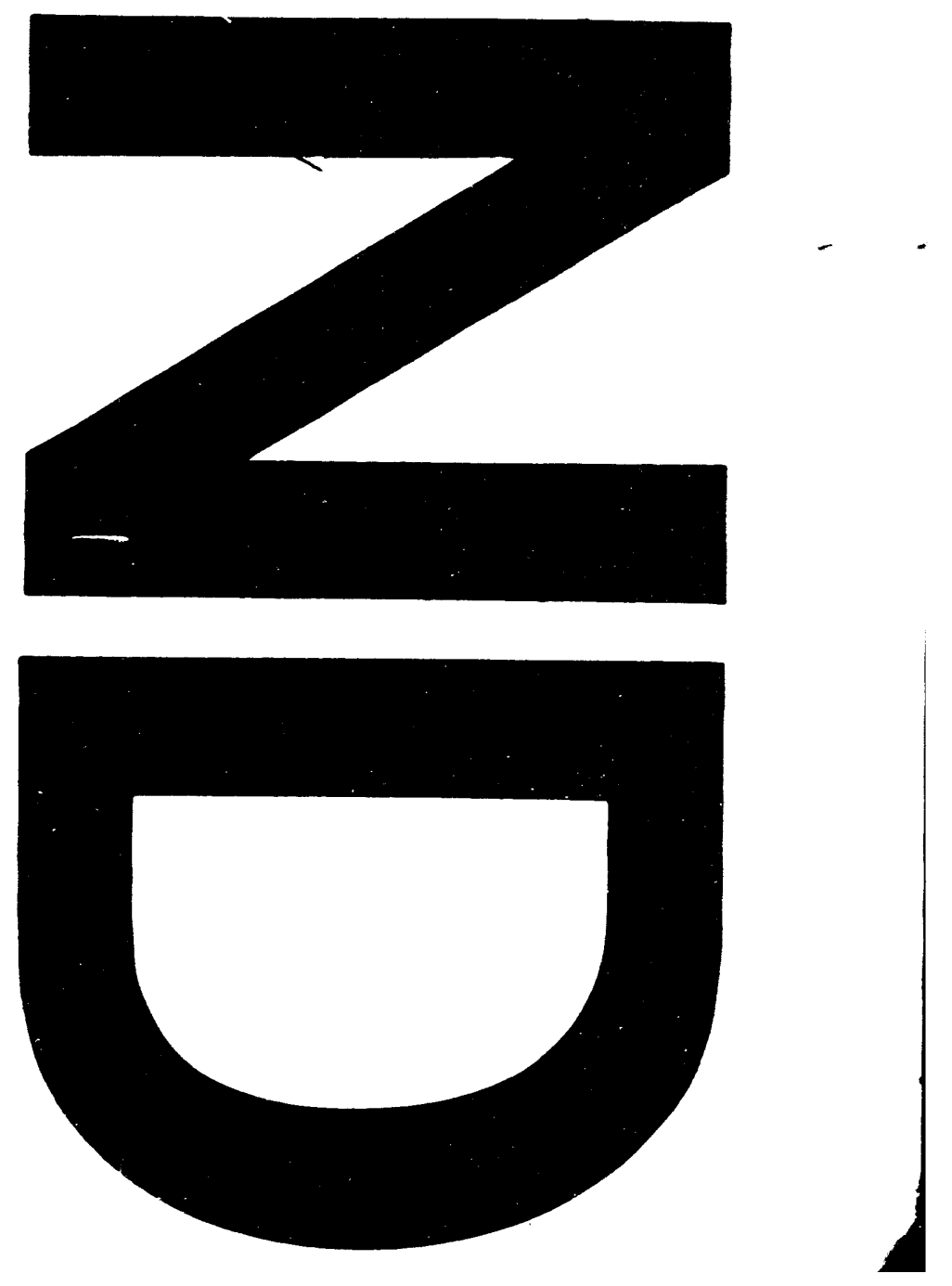
$\mid$ 